\title{
MEETING ABSTRACTS
}

\section{0th International Symposium on Intensive Care and Emergency Medicine}

\author{
Brussels, Belgium, 9-12 March 2010
}

Published: 1 March 2010

P1

Comparison of carbamylated versus recombinant erythropoietin during spinal cord ischemia/reperfusion injury

F Simon, A Scheuerle, A Soell, M Groeger, O McCook, P Radermacher,

M Georgieff, E Calzia, H Schelzig

UIm University, Ulm, Germany

Critical Care 2010, 14(Suppl 1):P1 (doi: 10.1186/cc8233)

Introduction We previously showed that erythropoietin (EPO) attenuates the morphological signs of spinal cord ischemia/reperfusion (I/R) injury in swine [1] without, however, improving neurological function. The clinical use of EPO has been cautioned most recently due to serious safety concerns arising from an increased mortality in acute stroke patients treated with EPO and simultaneously receiving systemic thrombolysis [2]. Carbamylated EPO (CEPO) is an EPO derivative without erythropoietic activity and devoid of the EPO side effects, but with apparently well maintained cytoprotective qualities [3]. We therefore tested the hypothesis whether CEPO may be equally efficient as EPO in reducing morphological as well as functional aortic occlusion-induced spinal cord I/R injury. Methods In a randomized and blinded trial pigs received either vehicle (control, $n=9$ ), EPO or cEPO, respectively ( $n=9$ each; $5,000 \mathrm{IU} / \mathrm{kg}$ over 30 minutes before and during the first 4 hours of reperfusion). Animals underwent 30 minutes of thoracic aortic balloon occlusion with catheters placed immediately downstream of the A. subclavia and upstream of the aortic trifurcation. Spinal cord function was assessed by motor evoked potentials (MEP as percentage of the amplitude before aortic occlusion) and lower limb reflexes (assessed as the subjective strength of response) for a period of 10 hours after reperfusion. Tissue damage was evaluated using Nissl staining.

Results Both EPO-treated and CEPO-treated animals presented with attenuated spinal cord injury in the Nissl staining (median (quartile) percentage of damaged neurons in the thoracic segments: control 27 $(25,44)$, CEPO $8(4,10)$, and EPO $5(5,7), P<0.001$ vs control group; in the lumbar segments: control 26 (19,32), CEPO $7(5,13)$, EPO $8(5,10), P<0.001$ vs control group). However, while only CEPO treatment was associated with recovery of the MEP amplitude to pre-occlusion values when compared with the control group $(P<0.05)$, lower limb reflex response was comparably restored stronger in both treatment groups $(P<0.05$ vs control).

Conclusions In a clinically relevant porcine model mimicking aortic crossclamping during vascular surgery repair of thoracic aortic aneurysm, cEPO protected spinal cord function and integrity as effective as EPO when applied at equipotent doses.

Acknowledgements Supported by the Deutsche Forschungsgemeinschaft (SCHE 899/2-2).

\section{References}

1. Crit Care Med 2008, 36:2143-2150.

2. Stroke 2009. [Epub ahead of print]

3. J Int Med 2008, 264:405-432.
P2

Sodium 4-phenylbutylate protects against myocardial ischemia-reperfusion injury by reducing unfolded protein response-mediated apoptosis in mice

M Okajima', M Takamura'2, S Usui', T Taniguchi', S Kaneko²

'Kanazawa University Hospital, Kanazawa, Japan; 'Kanazawa University

Graduate School of Medical Science, Kanazawa, Japan

Critical Care 2010, 14(Suppl 1):P2 (doi: 10.1186/cc8234)

Introduction Unfolded protein response (UPR)-mediated apoptosis plays a pivotal role in ischemia-reperfusion injury. Sodium 4-phenylbutyrate (PBA) has been reported to act as a chemical chaperone inhibiting UPR-mediated apoptosis triggered by ischemia in various organs other than the heart. Therefore we investigated whether PBA reduces UPR-mediated apoptosis and protects against myocardial ischemia-reperfusion injury in mice.

Methods C57BL/6 mice were subjected to 30 minutes LAD ischemia followed by reperfusion. PBA (100 mg/kg) or PBS (control) was administrated intraperitoneally just before ischemia. Apoptosis, infarct size and tissue protein levels of Grp78 and caspase-12 (UPR-mediated apoptosisassociated protein) were evaluated by TUNEL, TTC stain and western blot analyses, respectively, at 48 hours after ischemia ( $n=5$ for each group). Echocardiography was performed at 3 weeks after ischemia and the survival ratio was observed ( $n=9$ for each group).

Results Compared with PBS, PBA reduced apoptotic cells ( $30.8 \pm 0.2 \%$ vs $20.5 \pm 0.5 \%, P<0.05)$ and infarct size $(32.0 \pm 3.8 \%$ vs $13.0 \pm 2.1 \%, P<0.01)$ after ischemia-reperfusion. Grp78 and caspase-12 were increased in mice with PBS, but PBA attenuated the increase in Grp78 $(P<0.05)$ and caspase-12 $(P<0.05)$. PBA inhibited the deterioration of cardiac parameters including LVEDD $(3.35 \pm 0.08 \mathrm{~mm}$ vs $2.74 \pm 0.11 \mathrm{~mm}, P<0.01)$, LVESD $(2.30 \pm 0.08 \mathrm{~mm}$ vs $1.54 \pm 0.12 \mathrm{~mm}, P<0.01)$, and $\% \mathrm{FS}(31.3 \pm 2.2 \%$ vs $39.4 \pm$ $2.2 \%, P<0.05)$. All mice with PBA survived, but $33 \%$ animals with PBS died. Conclusions PBA maintained cardiac function and improved survival ratio after myocardial ischemia-reperfusion by reducing UPR-mediated apoptosis in mice.

References

1. Qi X, et al:: Mol Pharmacol 2004, 66:899-908.

2. Vilatoba M, et al.: Surgery 2005, 138:342-351.

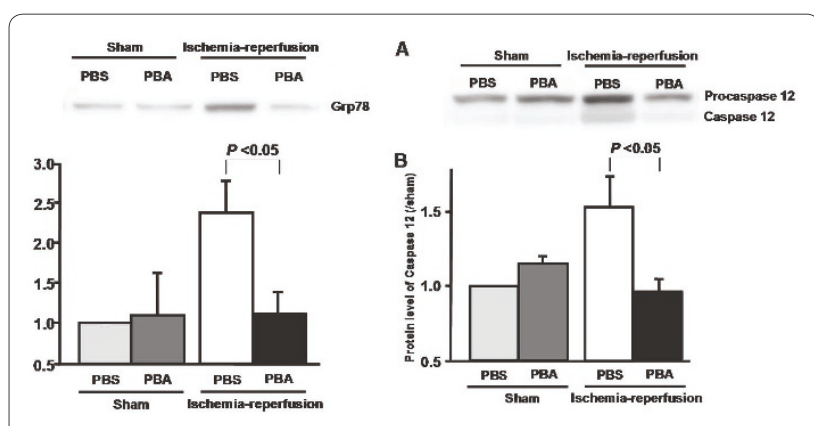

Figure 1 (abstract P2). Phenylbutyrate reduced the unfolded protein response. 
P3

Time-dependent effects of intravenous $\mathrm{H}_{2} \mathrm{~S}$ during long-term, resuscitated porcine hemorrhagic shock

H Bracht', F Simon'1, B Hauser', M Groeger', A Soell', O McCook', M Georgieff', P Radermacher1, C Szabo², E Calzia

'Ulm University, Ulm, Germany; '2University of Texas Medical Branch, Galveston, TX, USA

Critical Care 2010, 14(Suppl 1):P3 (doi: 10.1186/cc8235)

Introduction In awake, spontaneously breathing mice, inhaling hydrogen sulfide $\left(\mathrm{H}_{2} \mathrm{~S}\right)$ induced a hibernation-like metabolic state characterised by reduced energy expenditure and hypothermia [1], which protected against otherwise lethal hypoxia [2] and hemorrhage [3]. In contrast, other authors reported that inhibition of endogenous $\mathrm{H}_{2} \mathrm{~S}$ synthesis attenuated posthemorrhage organ dysfunction $[4,5]$. All these data originate, however, from unresuscitated models using a pre-treatment design. Therefore we investigated the time-dependent effect of intravenous $\mathrm{H}_{2} \mathrm{~S}$ in a clinically relevant, long-term model of porcine hemorrhage and resuscitation.

Methods After surgical instrumentation, pigs were subjected to 4 hours of hemorrhagic shock induced by removal of $40 \%$ of the calculated blood volume and thereafter by additional removal or retransfusion of blood boli as needed to maintain $\mathrm{MAP}=30 \mathrm{mmHg}$. Animals randomly received vehicle (control, $n=14$ ) or the intravenous $\mathrm{H}_{2} \mathrm{~S}$ donor $\mathrm{Na}_{2} \mathrm{~S}$ started 2 hours before hemorrhage (pre-treatment, $n=11$ ), at the beginning of blood removal (early post-treatment, $n=10$ ) or at the beginning of resuscitation (late post-treatment, $n=10$ ). In all groups the $\mathrm{Na}_{2} \mathrm{~S}$ infusion was continued over the first 10 hours of reperfusion. Resuscitation comprised retransfusion of shed blood, colloid volume expansion, and noradrenaline titrated to keep MAP at pre-shock levels. Systemic, renal and liver perfusion, $\mathrm{O}_{2}$ exchange, and organ function were assessed before and at the end of hemorrhage as well as at 10 and 22 hours of resuscitation.

Results Survival $(71 \%$ in the control vs 100,91 , and $90 \%$ in the pretreatment, early post-treatment and late post-treatment groups, respectively) was significantly improved in all treatment groups. The noradrenaline infusion rate required to maintain hemodynamic targets was significantly reduced in the early post-treatment group only, which coincided with a progressive drop in core temperature and attenuated kidney dysfunction (blood creatinine levels, creatinine clearance) in these animals.

Conclusions $\mathrm{Na}_{2} \mathrm{~S}$ application improved survival regardless of the drug timing. The less beneficial effect of pre-treatment on organ function may be due to the higher total amount of drug infused, possibly suggesting some toxicity at these doses.

Acknowledgements Supported by the German Ministry of Defence, and Ikaria Inc., Seattle, WA, USA.

\section{References}

1. Science $2005,308: 518$

2. Shock 2007, 27:370-372.

3. J Trauma 2008, 65:183-187.

4. Br J Pharmacol $2004,143: 881-889$

5. Inflamm Res 2008, 57:512-518.

P4

An ovine intensive care model of septic shock

M Chapman', M Maiden', J Fraser', C Nash', F Crichton', P Sideris², TKuchel $^{2}$

'Royal Adelaide Hospital, Adelaide, Australia; 2IMVS, Adelaide, Australia Critical Care 2010, 14(Suppl 1):P4 (doi: 10.1186/cc8236)

Introduction Translation of previous animal studies into human ICU clinical trials has frequently produced negative results. Most of these animal studies have had high baseline mortality and have not employed standardised management of sepsis as usually provided in an ICU. The aim of this study was to develop a large animal model of septic shock receiving standardised intensive care management, thus replicating the management of septic shock in humans.

Methods Eleven Marino ewes (weight 60 to $70 \mathrm{~kg}$, hemiazygous vein ligated) were anaesthetised and had radiological guided catheters inserted into the iliac, renal, and hepatic veins, coronary sinus, and the pulmonary and carotid arteries. Tracheostomy tubes were inserted and the animals mechanically ventilated while supported in a sling. Six sheep were administered intravenous E. coli (ATCC 25922) $1.0 \times 10^{8}$ orgs $/ \mathrm{kg}$ over 1 hour (septic sheep), five received placebo (nonseptic sheep). For 24 hours, animals were monitored and received sedation (midazolam + ketamine), ventilation, fluids and inotropes according to a protocol. Primary end-point was noradrenaline (NA) dose to maintain mean arterial pressure (MAP) of $75 \mathrm{mmHg}$. Secondary end-points included haemodynamic variables, respiratory, hepatic, and renal function, haematology, acid-base status and global, hind-limb, renal, hepatic and coronary oxygen extraction ratio (OER). Results Sheep were successfully instrumented, monitored and supported for 24 hours. Septic sheep required NA (mean dose $0.28 \mu \mathrm{g} / \mathrm{kg} / \mathrm{min}$ vs 0.00 , $P<0.001)$, developed a higher cardiac index $\left(6.6 \mathrm{l} / \mathrm{m}^{2}\right.$ vs $\left.4.3, P<0.05\right)$ and lower SVRI (769 dynes $/ \mathrm{m}^{2}$ vs 1,804, $P<0.05$ ). At 24 hours, septic sheep had renal impairment (creatinine $286 \mathrm{mmol} / \mathrm{l}$ vs $76, P<0.05$; urea $12 \mathrm{mmol} / \mathrm{l}$ vs $7, P<0.05$ ), metabolic acidosis ( $\mathrm{pH} 7.21$ vs $7.39, P<0.05$; lactate $10.9 \mathrm{mmol} / \mathrm{l}$ vs $1.2, P<0.01 ; \mathrm{PCO}_{2} 32$ vs $31, P=0.63$ ), coagulopathy (INR 5.9 vs 1.9, $P<0.05$; fibrinogen $0.9 \mathrm{~g} / \mathrm{l}$ vs $2.7, P<0.05)$ but preserved respiratory and hepatic function. Global OER was lower in septic sheep (0.16 vs 0.29 , $P<0.05)$ as was coronary OER $(0.36$ vs $0.68, P<0.05)$. OER did not change with sepsis in the kidney $(0.09$ vs $0.11, P=0.52)$, liver $(0.24$ vs $0.31, P=0.48)$ and hind-limb (0.31 vs $0.42, P=0.23)$.

Conclusions We have developed a large animal model of septic shock that receives intensive care support and standardised management. This model replicates much of the pathophysiology and management that occurs in human septic shock. It allows a large range of physiological parameters to be assessed when investigating new therapies for sepsis.

P5

Effects of temperature and $\mathrm{H}_{2} \mathrm{~S}$ inhalation on glucose metabolism in murine resuscitated septic shock

K Baumgart', F Wagner', V Hysa', J Vogt', U Wachter', S Weber',

M Georgieff', P Radermacher', C Szabo², E Calzia'

'Ulm University, Ulm, Germany; 'University of Texas Medical Branch,

Galveston, TX, USA

Critical Care 2010, 14(Suppl 1):P5 (doi: 10.1186/cc8237)

Introduction In awake, spontaneously breathing mice, inhaling hydrogen sulfide $\left(\mathrm{H}_{2} \mathrm{~S}\right)$ induced a hibernation-like metabolic state characterised by reduced energy expenditure and hypothermia [1], which protected against otherwise lethal hypoxia [2] and hemorrhage [3] as a result of impaired cellular energy metabolism [4]. Therefore, we investigated the metabolic effects of inhaled $\mathrm{H}_{2} \mathrm{~S}$ in our model of resuscitated murine septic shock.

Methods Sixteen hours after induction of sepsis by cecal ligation and puncture (CLP) or sham operation, anesthetized and mechanically ventilated mice received $100 \mathrm{ppm} \mathrm{H}_{2} \mathrm{~S}$ or vehicle over 5 hours at body temperatures of 38 and $27^{\circ} \mathrm{C}$, respectively. During the observation period, hyperdynamic hemodynamics were maintained by colloid resuscitation and noradrenaline infusion [5]. Endogenous glucose production was calculated from blood ${ }^{13} \mathrm{C} 6$-glucose isotope enrichment derived from the rate of appearance of stable, non-radioactive labeled $1,2,3,4,5,6-{ }^{-13} \mathrm{C} 6$ glucose during continuous isotope infusion [6]. Whole-body glucose oxidation rate was derived from the total $\mathrm{CO}_{2}$-production rate, the mixed expiratory ${ }^{13} \mathrm{CO}_{2} /{ }^{12} \mathrm{CO}_{2}$ isotope ratio and the ${ }^{13} \mathrm{C} 6$-glucose infusion rate after the steady state was achieved.

Results While endogenous glucose production was not affected by hypothermia, it was significantly higher in the septic animals when compared with the corresponding sham operated groups, most likely due to the ongoing noradrenaline infusion. In contrast, despite the catecholamine infusion and higher glucose release, whole body glucose oxidation was significantly reduced in normothermic septic animals. During hypothermia, $\mathrm{H}_{2} \mathrm{~S}$ shifted substrate towards preferential glucose utilisation, but this effect disappeared in the septic mice.

Conclusions $\mathrm{H}_{2} \mathrm{~S}$ inhalation alone does not influence glucose metabolism once temperature is maintained at normothermic levels in anesthetised and mechanically ventilated mice. The $\mathrm{H}_{2} \mathrm{~S}$-related shift of energy metabolism towards preferential carbohydrate oxidation present during hypothermia is blunted during sepsis, possibly as a result of the ongoing catecholamine treatment.

Acknowledgements Supported by the Deutsche Sepsis Gesellschaft, the DFG KFO 200, and Ikaria Inc., Seattle, WA, USA. 


\author{
References \\ 1. Science $2005,308: 518$. \\ 2. Shock 2007, 27:370-372 \\ 3. J Trauma $2008,65: 183-187$ \\ 4. JAm Soc Nephrol 2009, 20:1901-1905. \\ 5. Intensive Care Med 2009, 35:344-349. \\ 6. Crit Care Med 2010, 38:in press.
}

P6

Mitochondrial respiration and cytochrome $\mathrm{c}$ inhibition by sulfide in peritoneal macrophages in vitro: effects of temperature and $\mathrm{pH}$ M Groeger ${ }^{1}$, F Wagner ${ }^{1}$, K Baumgart ${ }^{1}$, M Huber-Lang ${ }^{1}$, M Knoeferl'1, M Georgieff', P Radermacher ${ }^{1}$, C Szabo², E Calzia

'Ulm University, UIm, Germany; ${ }^{2}$ University of Texas Medical Branch, Galveston, TX, USA

Critical Care 2010, 14(Suppl 1):P6 (doi: 10.1186/cc8238)

Introduction Hydrogen sulfide $\left(\mathrm{H}_{2} \mathrm{~S}\right)$ is a potent inhibitor of cytochrome c oxidase (COX) and, thus, of mitochondrial respiration [1]. Since $\mathrm{H}_{2} \mathrm{~S}$ was reported to induce a suspended animation-like status characterized by reduced energy expenditure and hypothermia [2], we sought to determine the effect of hypothermia on mitochondrial respiratory capacity and $\mathrm{H}_{2} \mathrm{~S}$ related $\mathrm{COX}$ inhibition. We further studied the influence of variations in $\mathrm{pH}$ on both variables.

Methods All measurements were conducted in digitonin-permeabilised cultured peritoneal macrophages using high-resolution respirometry [3] (Oxygraph-2k, Oroboros, Austria). Maximum mitochondrial respiration (1 to $2 \mathrm{Mio}$ cells $/ \mathrm{ml}$ respiration medium) was achieved in the uncoupled state by adding pyruvate, malate, glutamate and succinate as respiratory substrates. Then, in one of the two chambers of the oxygraph, mitochondrial respiration was inhibited stepwise by incremental concentrations of the $\mathrm{H}_{2} \mathrm{~S}$ donor Na2S (1 to $64 \mu \mathrm{M}$ ). In the parallel chamber, the identical inhibitor titration sequence was preceded by the inhibition of the respiratory chain by rotenone and antimycin A followed by the selective stimulation of the COX after addition of ascorbate and TMPD. COX excess capacity (\% of OXPHOS) was calculated based on the ratio of inhibition of mitochondrial respiration with full operating respiratory chain versus the COX-stimulated condition. This experimental sequence was repeated at $37^{\circ} \mathrm{C}$ and $25^{\circ} \mathrm{C}$ with a medium $\mathrm{pH}$ of 7.1 and then at $37^{\circ} \mathrm{C}$ with a pH of 6.8 and 7.7 .

Results COX excess capacity (median (quartiles)) was significantly higher at $25^{\circ} \mathrm{C}$ than at $37^{\circ} \mathrm{C}(134(113 ; 140)$ vs $61(47 ; 79))$, most likely due to the almost halved mitochondrial respiratory capacity at hypothermia (50 (37; 63) vs $95\left(81\right.$; 103) $\mathrm{pmolO}_{2} / \mathrm{s} \times$ Mio cells). Changing the medium $\mathrm{pH}$ from 6.8 to 7.7 significantly increased the COX excess capacity $(91(79 ; 103)$ vs 71 $(64 ; 82) \mathrm{pmolO}_{2} / \mathrm{s} \times$ Mio cells), which again was related to the significantly lower mitochondrial respiratory capacity with more acidic conditions (80 (70; 89) vs $94(85 ; 98)$ ).

Conclusions Our results suggest that COX excess capacity is temperature as well as $\mathrm{pH}$ dependent in peritoneal macrophages. This effect may protect cells from $\mathrm{H}_{2} \mathrm{~S}$ toxicity at low temperatures and high $\mathrm{pH}$ values.

Acknowledgements Supported by the Deutsche Forschungsgemeinschaft (KFO 200).

References

1. Science $2005,308: 518$.

2. Toxicol Sci 2002, 65:18-25

3. Biochim Biophys Acta 2006, S14:201-202.

P7

Alterations of caspase 9 mRNA gene expression in survivors and nonsurvivors of severe sepsis

M White' ${ }^{1}$ D Doherty², D Kelleher ${ }^{2}$, R McManus², T Ryan

'St James Hospital, Dublin, Ireland; ${ }^{2}$ Trinity College, Dublin, Ireland

Critical Care 2010, 14(Suppl 1):P7 (doi: 10.1186/cc8239)

Introduction Sepsis-induced lymphocyte apoptosis plays a fundamental role in the pathophysiology of sepsis. Recent animal models of sepsis have identified anomalies in the extrinsic apoptotic pathway, a key pathological occurrence in sepsis [1]. Specifically apoptosis markers such as caspases
$1,3,8,9$ and FADD have been shown to be significant in animal models of infection [2]. We investigated mRNA transcription of these markers in a human model of severe sepsis. We hypothesized that ICU mortality from severe sepsis is associated with distinctive gene expression of extrinsic apoptosis markers.

Methods A prospective observational study of patients with severe sepsis was performed. Mononuclear cells were isolated from 48 patients with severe sepsis. Total RNA was extracted from samples for day 1 of admission and again on day 7. FADD, caspase 1, 3, 8, and 9 mRNA was quantified with quantitative real-time polymerase chain reaction (GRT-PCR). Standard demographic and outcome data were recorded. Between-group comparisons were performed by Wilcoxon rank sum test. All values are stated as median and interquartile range.

Results Sixteen of the 48 patients died in the ICU. Caspase 9 mRNA copy numbers were significantly increased on day 7 in the survivor group $(5.4 \mathrm{x}$ $10^{6} ; 7.4 \times 10^{6}$ to $\left.8.9 \times 10^{6}\right)$ compared with death in the ICU group $(1.9 \times$ $10^{6} ; 3.0 \times 10^{6}$ to $\left.1.2 \times 10^{6}\right) P=0.001$. FADD, caspase 1,3 and 8 mRNA copy numbers were not significantly different between patients who died and those discharged from the ICU on either day 1 or day 7 of admission.

Conclusions Caspase 9 may be an important regulator of apoptotic mechanisms in humans with late sepsis. Pro-apoptotic mechanisms may have a role in the resolution of severe sepsis.

Acknowledgements This study is funded by the Association of Anaesthetists of Great Britain and Ireland and the Intensive Care Society Ireland. References

1. Oberholzer C, et al:: Apoptosis in sepsis: a new target for therapeutic exploration. FASEB J 2001, 15:879-892.

2. Hotchkiss R, et al: Accelerated lymphocyte death in sepsis occurs by both the death receptor and mitochrondrial pathways. J Immunol 2005 174:5110-5118.

The TLR4 antagonist CRX-526 reduces LPS-induced leukocyte activation and improves capillary perfusion of the rat intestine M Soltow ${ }^{1}$, K Zimmermann'1, D Pavlovic ${ }^{1}$, J Zhou ${ }^{2}$, S Whynot ${ }^{2}$, O Hung ${ }^{2}$, M Murphy², B Johnston², C Lehmann²

'Ernst Moritz Arndt University, Greifswald, Germany; '2Dalhousie University, Halifax, Canada

Critical Care 2010, 14(Suppl 1):P8 (doi: 10.1186/cc8240)

Introduction Toll-like receptor 4 (TLR4) represents an important mediator of endotoxin-related signal transduction. The aim of our study was to evaluate whether TLR4 inhibition after onset of experimental endotoxemia is able to improve the intestinal microcirculation, which is crucial in the pathogenesis of septic multiple organ failure.

Methods We studied four groups of animals (Lewis rats, $n=10$ per group): healthy controls (CON group), endotoxemic animals (15 mg/kg lipopolysaccharide, LPS group), endotoxemic animals treated with TLR4 antagonist (1 mg/kg CRX-526, LPS + CRX group), and CRX-526 treated controls (CRX group). Intravital microscopy of the intestinal microcirculation was performed following 2 hours of observation in all animals. Blood samples were taken for cytokine measurements at the end of the experiments.

Results Following 2 hours of endotoxemia we observed a significant increase of leukocyte adhesion in the intestinal submocosal venules (for example, V1 venules: CON $20.4 \pm 6.5 \mathrm{n} / \mathrm{mm}^{2}$, LPS $237.5 \pm 36.2 \mathrm{n}$ / $\left.\mathrm{mm}^{2}, P<0.05\right)$. Capillary perfusion of the muscular and mucosal layers of the intestinal wall was significantly reduced (for example, longitudinal muscular layer: CON $112.5 \pm 5.9 \mathrm{~cm} / \mathrm{cm}^{2}$, LPS $\left.71.3 \pm 11.0 \mathrm{~cm} / \mathrm{cm}^{2}\right)$. TLR4 inhibition reduced leukocyte activation (V1 venules: $104.3 \pm 7.8 \mathrm{n} / \mathrm{mm}^{2}$ ) and improved capillary perfusion (longitudinal muscular layer: $111.0 \pm$ $12.3 \mathrm{~cm} / \mathrm{cm}^{2}$ ) significantly. Cytokine release was not affected.

Conclusions Administration of the TLR4 antagonist CRX-526 improved intestinal microcirculation in a post-treatment model of experimental endotoxemia. The TLR4 pathway may be a target in clinical Gram-negative sepsis. References

1. Cristofaro et al:: Drugs 2006, 66:15.

2. Fort et al.: J Immunol 2005, 74:6416

3. Moue et al.: Biochim Biophys Acta 2008, 1780:134.

4. Zanotti et al:: Am J Physiol Lung Cell Mol Physiol 2009, 297:L52. 
P9

Effects of lipopolysaccharide on isolated muscle mitochondrial respiration are dose and time dependent

S Brandt', S Djafarzadeh², J Takala², SM Jakob²

'University of Bern, Switzerland; '2Department of Intensive Care Medicine,

University of Bern, Switzerland

Critical Care 2010, 14(Suppl 1):P9 (doi: 10.1186/cc8241)

Introduction There is evidence that mitochondrial dysfunction plays a role in sepsis-related tissue damage. Several studies described the uptake and endocytosis of lipopolysaccharide (LPS) by various cells. LPS has been localized in different parts of the cytoplasm, including at close proximity to or within mitochondria [1]. Whether effects of LPS on mitochondrial respiration are time and/or dose dependent is unknown.

Methods Quadriceps muscle biopsy was taken from seven anaesthetized pigs. Mitochondria were isolated using differential centrifugation and immediately incubated with $0.1,1,10,50$ and $100 \mu \mathrm{g}$ LPS per mg mitochondria protein on ice for 2 and 4 hours. Respiration rates were determined polarographically using glutamate and succinate as substrates to test the function of complex I and II. Respiration Control Ratio (State 3/ State 4) was derived for each substrate. Repeated-measures ANOVA was used to analyze time and dose effect of LPS on respiration rates.

Results The results are shown in Figure 1.

Conclusions In vitro, LPS has time-dependent and dose-dependent effects on muscle mitochondrial respiration. This has consequences for design and interpretation of experimental studies.

\section{References}

1. Diaz-Laviada I, et al.: Histochem J 1991, 23:221-228.

P10

Clinical severity and local inflammatory responses in animal models of sepsis

S Saeed, D Gilroy, M Singer

University College London, UK

Critical Care 2010, 14(Suppl 1):P10 (doi: 10.1186/cc8242)

Introduction Severe sepsis carries high morbidity and mortality. Preclinical research predominantly utilises animal models although their reproducibility may vary, thus impairing understanding of disease. We sought to determine the reproducibility of two murine models by assessing clinical severity and local immune cell response 24 hours after septic insult.
Methods Intraperitoneal faecal slurry (FS) or zymosan was given to induce acute peritonitis in 11 and 12 male C57/BI mice ( 8 to 12 weeks, 18 to $32 \mathrm{~g}$ ). A control group received saline only $(n=5)$. In surviving animals at 24 hours, clinical severity was scored as severe, moderate or mild according to appearance and alertness. Peritoneal lavage was performed to obtain immune cells. Analysis by antibody labelling (F4/80, GR-1, CD3 and CD19) for fluorescence-assisted cell sorting identified numbers of macrophages, neutrophils, T and B cells. Logistic regression (odds ratio, OR) was used to determine the relationship of cell numbers with severity (reported if $P<0.05$ ). Results Clinical severity varied markedly despite similar dosing (see Table 1). At 24 hours, total intraperitoneal immune cells increased in both models and with clinical severity (OR 0.83). Neutrophils predominated after septic insult and also rose with severity (OR 0.75). Compared with control, macrophage populations did not change in either model while $B$ and $T$ lymphocytes fell. A cell population that expressed both F4/80 and GR-1 - that is, markers for macrophages and neutrophils, respectively - occurred only in the FS model.

Table 1 (abstract P10). Number of animals according to clinical severity (with inclusive mortality)

\begin{tabular}{lccc}
\hline & Severe & Moderate & Mild \\
\hline Faecal slurry & $6(2)$ & 0 & 5 \\
Zymosan & $4(2)$ & 5 & 3 \\
\hline
\end{tabular}

Conclusions Individual variability occurs in both faecal and zymosan peritonitis models as shown by heterogeneous clinical responses and local immune cell numbers to the same dose in similar animals. The cellular immune response in both models is consistent with current understanding of infection-induced inflammation. Neutrophils, but not macrophages, rose in proportion to worsening clinical severity. The significance of F $4 / 80^{+} / \mathrm{GR}-1^{+}$ cells in the FS model requires further evaluation.

P11

Adenosine increases during human experimental endotoxemia, but does not influence the immune response and subsequent organ injury B Ramakers, N Riksen, B Franke, P Pickkers, H Van der Hoeven

Radboud University Nijmegen Medical Centre, Nijmegen, the Netherlands

Critical Care 2010, 14(Suppl 1):P11 (doi: 10.1186/cc8243)

Introduction Although the innate immune response protects the host from invading pathogens, an excessive response may also lead to
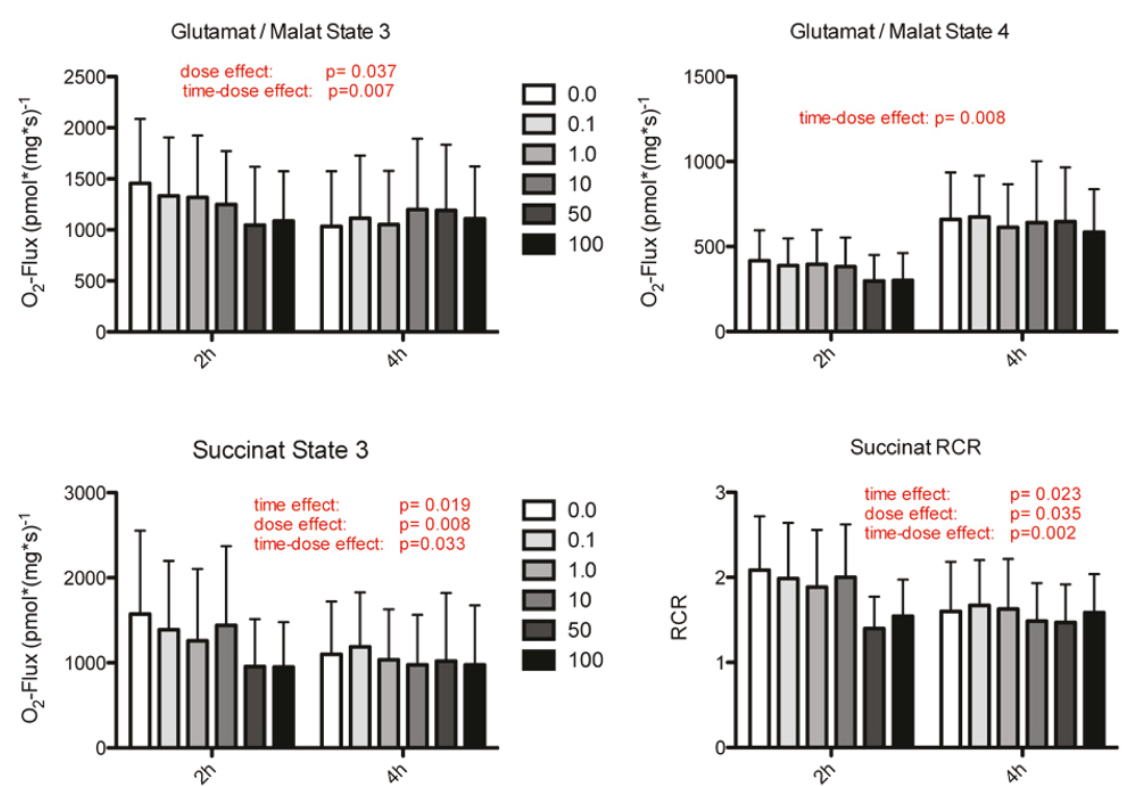

Figure 1 (abstract P9). Mean \pm SD. Boxes: $\mu \mathrm{g} / \mathrm{mg}$ LPS. 
collateral damage to normal tissues. Adenosine has been proposed as an immunomodulator capable of inhibiting inflammation and preventing tissue injury. The C34T nonsense mutation in the AMP deaminase 1 (AMPD1) gene is thought to increase the endogenous adenosine concentration and has been associated with improved prognosis and survival in ischemic heart disease. Caffeine on the other hand, acting as a nonselective adenosine receptor antagonist, could diminish adenosine-mediated effects. The present study evaluated the endotoxemia-induced adenosine response, subclinical renal damage and endothelium dysfunction in healthy male subjects. Furthermore, we investigated whether the LPSinduced inflammatory response is attenuated by AMPD1 and enhanced by caffeine, as well as its effects on markers of endothelium activation (plasma ICAM, VCAM) and renal damage (urinary excretion of GSTA1-1 and GSTP1-1).

Methods Thirty healthy male subjects received 2 ng $/ \mathrm{kg}$ E. coli LPS. Three groups were evaluated in a double-blind randomized controlled setting; a LPS-placebo group ( $n=10)$, LPS-placebo in AMPD1 subjects $(n=10)$, and a LPS-caffeine group ( $4 \mathrm{mg} / \mathrm{kg}, n=10)$.

Results During endotoxemia, the adenosine concentration increased from $10.0 \mathrm{ng} / \mathrm{ml}$ (9.0 to 15.3 ) at baseline to $15.5 \mathrm{ng} / \mathrm{ml}$ (13.0 to 22.3) 2 hours after LPS infusion ( $P=0.003$; Friedman). The response was similar between LPS groups. The increase in proinflammatory and anti-inflammatory cytokines (TNFa, IL-6, IL-10 and IL 1RA) was similar in the three groups. Experimental endotoxemia resulted in endothelial dysfunction, measured by an increase in adhesion molecules and subclinical renal injury as measured by GSTA11 and GSTP1-1. Inflammation induced subclinical end-organ damage was not influenced by either the AMPD1 SNP or treatment with caffeine.

Conclusions Human experimental endotoxemia induces an increase in circulating cytokine levels and subclinical endothelial and renal damage. Acute systemic inflammation is also associated with an increase in endogenous adenosine concentrations. Modulation of the adenosine metabolism through the presence of the AMPD1 and administration of caffeine does not affect the innate immune response and its subsequent subclinical organ dysfunction.

P12

Bacterial load plays a crucial role for survival in experimental peritonitis and modulates immunoparalysis of monocytes

S Atmatzidis', K Louis², A Pistiki', I Koutelidakis' ${ }^{1}$, T Adamis²,

E Giamarellos-Bourboulis ${ }^{2}$

'Thessaloniki, Medical School, Thessaloniki, Greece; ${ }^{2}$ ATTIKON University

Hospital, Athens, Greece

Critical Care 2010, 14(Suppl 1):P12 (doi: 10.1186/cc8244)

Introduction Peritonitis is the prototype of polymicrobial sepsis with kinetics of bacterial growth differing from those of other infections leading to sepsis. The effect of the extent of leaking of gut content in the response of the host was studied.

Methods A total of 21 rabbits were studied divided into two groups; A: high-load peritonitis; and B: normal load peritonitis. After a midline abdominal incision, the ileocecal calve was ligated. Three holes were performed in the cecum wall of group A followed by masturbation to drain cecal content in the peritoneal cavity. Two holes without masturbation were performed in group B. After closure of the abdominal cavity, blood was sampled at 2, 4, 24 and 48 hours. Peripheral blood mononuclear cells (PBMCs) were isolated and stimulated in microplate wells with $10 \mathrm{ng} / \mathrm{ml}$ LPS. Concentrations of TNFa were estimated in supernatants by a bioassay in L929 fibrosarcoma cell line. In parallel, monocytes were separated from lymphocytes by plastic adherence. Apoptosis was estimated after staining with ANNEXIN-V and PI and flow cytometric analysis. Tissue bacterial growth was estimated after death.

Results Mortality after 14 days was $84.6 \%$ in group A and $62.5 \%$ in group B (log-rank: $3.83, P=0.050$ ). Mean respective rates of apoptosis of lymhocytes of groups A and B were 32.2 and $44.5 \%$ at 2 hours; 33.5 and $51.9 \%$ at 4 hours $(P=0.028) ; 35.6$ and $39.1 \%$ at 24 hours; and 28.5 and $43.7 \%$ at 48 hours $(P=0.029)$. Mean respective rates of apoptosis of monocytes of groups A and B were 48.2 and $64.1 \%$ at 2 hours $(P=0.036)$; 57.9 and $66.3 \%$ at 4 hours; 47.3 and $69.9 \%$ at 24 hours $(P=0.041)$; and 60.5 and $73.5 \%$ at 48 hours. Respective TNFa released ex vivo from PBMCs isolated at 24 hours from groups A and B after LPS stimulation was 2,579.1 and $31.3 \mathrm{pg} / \mathrm{ml}(P=0.048)$. Mean respective $\log _{10}$ of enterobacteriaceae of groups $A$ and $B$ were 6.52 and $1.39 \mathrm{cfu} / \mathrm{ml}$ in liver $(P=0.012) ; 7.79$ and 1.43 $\mathrm{cfu} / \mathrm{ml}$ in spleen $(P=0.012)$; and 7.98 and $1.79 \mathrm{cfu} / \mathrm{ml}$ in the right kidney $(P=0.012)$.

Conclusions Experimental peritonitis with enormous bacterial leaking from the gut is accompanied by reduced survival, increased tissue bacterial growth and reduced apoptosis of lymphocytes and monocytes. Peritonitis with low bacterial leaking is characterized by immunoparalysis of PBMCs. These results may project in the efficacy of immunotherapy in abdominal sepsis.

\section{P13}

Decreased whole blood TNFa production capacity after acute alcohol exposure and LPS stimulation ex vivo

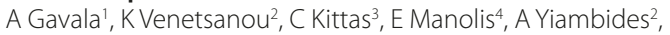

P Myrianthefs $^{2}, G$ Baltopoulos ${ }^{2}$

${ }^{1} K A T$, Kifisia, Greece; ${ }^{2}$ CU, KAT Hospital, School of Nursing, Athens University, Athens, Greece; ${ }^{3}$ Medical School, Athens University, Athens, Greece; ${ }^{4}$ School of Nursing, Athens University, Athens, Greece

Critical Care 2010, 14(Suppl 1):P13 (doi: 10.1186/cc8245)

Introduction Acute alcohol exposure is related to increased susceptibility to infections [1]. The purpose of the study was to investigate the effect of acute exposure to different alcohol concentrations in whole blood TNFa production capacity after LPS stimulation ex vivo in healthy men.

Methods Whole blood was taken from healthy volunteers and was placed in tubes containing EDTA and immediately transferred to the lab. Heparinized blood samples diluted 1:10 in RPMI 1640 culture medium $(100 \mu \mathrm{l}$ whole blood added in $900 \mu \mathrm{l}$ RPMI 1640). Samples were preincubated with 0, 5, 12.5, 25, 50, 100 and $200 \mathrm{mM}$ alcohol (EtOH) for 10 minutes at room temperature. After incubation, 500 pg LPS was added in each sample for 4 hours at $37^{\circ} \mathrm{C}$. At the end of the process, samples were centrifuged $(1,800 \mathrm{rpm}, 5$ minutes, r.t.). Culture supernatants were collected and stored at $-70^{\circ} \mathrm{C}$ until measurements. TNFa levels were determined in culture supernatant with ELISA [2].

Results We studied 17 healthy males volunteers aged $36.9 \pm 1.6$ (X \pm SEM). TNFa levels are shown in Figure 1. There was no TNFa production detected in samples without alcohol in the absence of LPS stimulation (control). TNFa production was significantly decreased at a dose of alcohol of $50 \mathrm{mM}$ after LPS stimulation $(P<0.05)$ but more apparently at doses of 100 and $200 \mathrm{mM}$ alcohol $(P<0.001)$ compared with LPS-induced samples. Conclusions Alcohol is related to inhibition of TNFa production of whole blood stimulated with LPS ex vivo [3]. This effect occurred shortly after alcohol exposure. Our observation indicates a suppression of proinflammatory response during acute alcoholic intoxication which may be related to increased susceptibility to infections.

\section{References}

1. Brown LA, et al:: Alcohol Clin Exp Res 2006, 30:1624-1631.

2. Myrianthefs P, et al: Cytokine 2003, 24:286-292

3. Nair M, et al:: Alcohol Clin Exp Res 1994, 18:602-607.

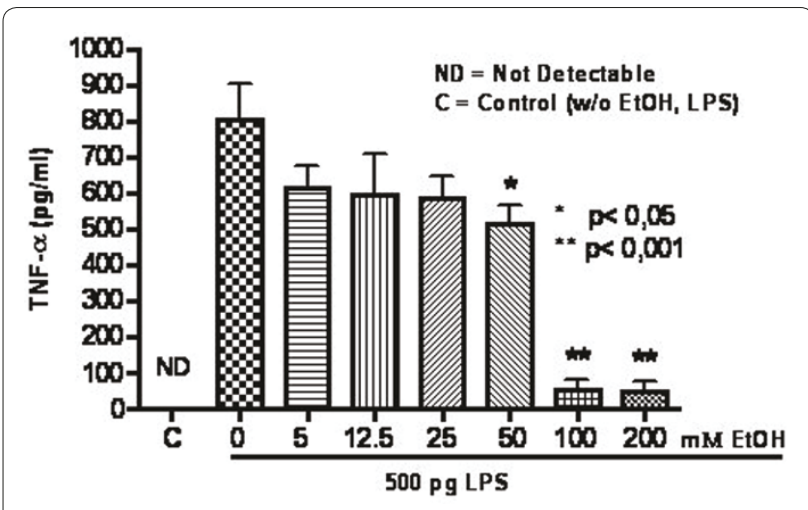

Figure 1 (abstract P13). 
P14

Leukocyte immunophenotyping: methodological aspects

J Jämsä, V Huotari, ER Savolainen, H Syrjälä, T Ala-Kokko

Oulu University Hospital, University of Oulu, Finland

Critical Care 2010, 14(Suppl 1):P14 (doi: 10.1186/cc8246)

Introduction Flow cytometric analysis of leukocyte surface receptors has been performed and shown beneficial, for example to characterize infectious and septic patients $[1,2]$. For many surface antigens the results may vary depending on the sampling temperature, the anticoagulant used and the storage of the sample before analysis [3]. In order to obtain reliable data on leukocyte immunophenotyping for patient diagnostic purposes, we wanted to carry out a thorough evaluation on these methodological issues with a wide range of leukocyte surface antigens.

Methods Four blood samples, two using acid citrate dextrose (ACD) and two using heparin as an anticoagulant, were taken from five ICU patients with severe sepsis and from five healthy volunteers. The patients and the healthy volunteers were combined into one study population $(n=10)$. The samples were taken, processed and stored either at $+4^{\circ} \mathrm{C}$ or at room temperature. The surface antigen staining and flow cytometry were performed immediately after sample collection or after 6 or 24 hours at the above-mentioned temperatures. Antibodies of interest were for monocytes and neutrophils CD11 b and CD64, for monocytes CD14, CD40, CD80, human leukocyte antigen (HLA)-DR, and for lymphocytes (CD4+ and CD8 ${ }^{+} \mathrm{T}$ cells, B cells, and NK cells) CD69. The flow cytometry analysis was done in three different time points, after 1, 6 or 24 hours of sampling. Interassay standardization and fluorescence quantifications were performed using microspheres.

Results The fluorescence intensities were higher at room temperature compared with $+4^{\circ} \mathrm{C}$ and they increased after storage (Figure 1). The effect was observed especially for CD11b-antigen in monocytes and neutrophils and for HLA-DR in monocytes.

Conclusions According to our results, flow cytometry leukocyte analysis using $C D$ antigens should be performed using $+4^{\circ} \mathrm{C}$ temperature throughout the measurement including sample collection, preparation and storage, and the analysis should be performed within 6 hours.

\section{References}

1. Payden et al:: Minerva Anest 2009, 75:484-493

2. Nuutila et al:: Hum Immunol 2009, 70:237-243

3. Li et al:: EurJ Haematol 2000, 65:57-65.

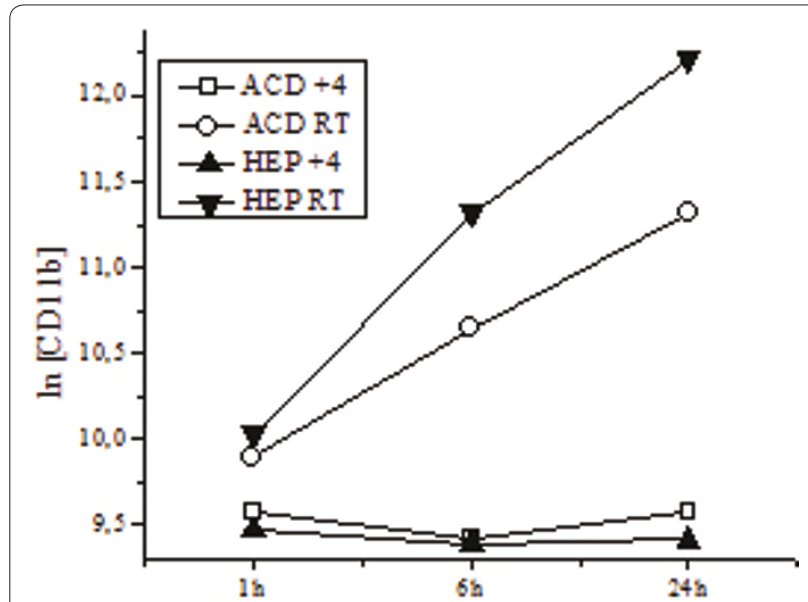

Figure 1 (abstract P14). CD11b fluorescence intensity for neutrophils $(n=10)$.
P15

Phenotypical analysis of peripheral human T lymphocytes in early sepsis

M Nalos, B Santner-Nanan, L Weisbrodt, AS Mclean, R Nanan Sydney Medical School - Nepean, The University of Sydney, Penrith, Australia Critical Care 2010, 14(Suppl 1):P15 (doi: 10.1186/cc8247)

Introduction T lymphocytes are crucial immune cells. We analysed T-cell subsets phenotypes and tested, on a single cell level, their ability to produce key cytokines in early human sepsis.

Methods Whole blood was collected from septic patients on ICU admission. Peripheral blood mononuclear cells (PBMC) were isolated and T-cell subsets analysed. To study cytokine production, PMBC were cultured in the presence of PMA/ionomycin $(50 / 750 \mathrm{ng} / \mathrm{ml})$ in supplemented RPMI 1640 for 5 hours. Intracellular cytokines IL-4, IL-10, IL-17, IFNy were stained in $\mathrm{CD}^{+} / \mathrm{CD}^{+}{ }^{+} \mathrm{CD}^{+} / \mathrm{CD}^{+}$cells using flow cytometry for both. The number of cytokine producing cells was compared with age/sex-matched healthy human volunteers. Data are expressed as mean \pm SEM

Results There were 12 patients (66 years old, six males, APACHE II-24, eight survivors) and nine volunteers. We found a relative increase in the frequency of Treg cells while the proportion of $\mathrm{CD}^{+}$cells remained unchanged in septic patients. The PMA/ionomycin lead to maximal T-cell stimulation, testing the ability of individual cell subsets to produce cytokines. Septic patients displayed reduction of IFNy $(10.5 \pm 0.8 \%$ vs $14.7 \pm 1.9 \%, P<0.01)$ and a tendency to higher number of IL-10 (1.7 \pm $0.3 \%$ vs $0.5 \pm 0.1 \%, P=0.10$ ) producing $C^{2} 4^{+}$cells, while the proportion of IFNy-positive CD8 ${ }^{+}$cells increased $(42.8 \pm 5.8 \%$ vs $28.1 \pm 4.9 \%, P=0.03$ ). However, the overall CD8 ${ }^{+}$T-cell population was reduced $(14.29 \pm 1.6 \%$ vs $25 \pm 1.2 \%$ ) following ex vivo activation in patients. The number of IL-4 and IL-17 staining cells was unchanged (Figure 1).

Conclusions Our results confirm a relative increase of Treg [1] and a skew towards Th2 lineage in the CD4 ${ }^{+}$cells. The highly activated CD8 ${ }^{+}$cells appear to be more susceptible to activation-induced cell death.

\section{References}

1. Venet F et al:: Intensive Care Med 2009, 35:678-686.

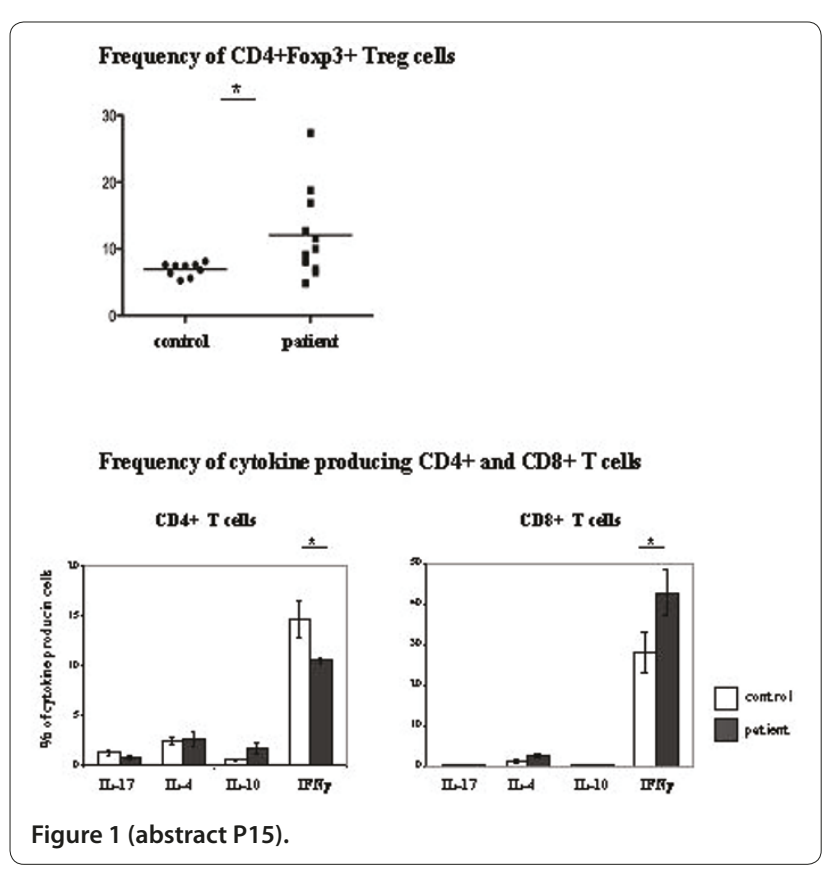




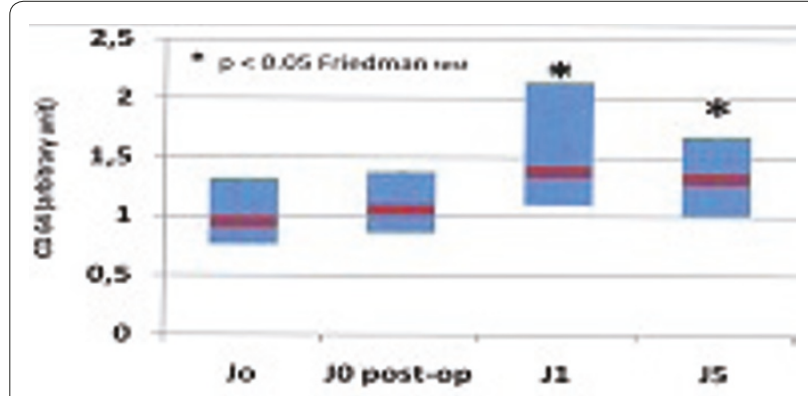

Figure 1 (abstract P16). CD64 kinetics (units).

P16

CD64, a marker of leucocyte activation kinetics after uncomplicated cardiac surgery

S Djebara, P Cauchie, A Alewaeters, A Daper, E Fosse, K Zouaoui Boujelta, P Biston

CHU Charleroi, Charleroi, Belgium

Critical Care 2010, 14(Suppl 1):P16 (doi: 10.1186/cc8248)

Introduction The aim of this study was to assess the CD64 kinetics after normal cardiac surgery, an essential step to define the potential use of CD64 as an early and specific infectious marker in this postoperative setting. CD64 is a high-affinity receptor for the Fc portion of IgG. It is weakly expressed on the polynuclear neutrophils (PMNs) at rest $[1,2]$ but increases specifically after bacterial stimulation. Thus, CD64 analysis is proposed as an early infectious marker.

Methods Prospective study realised in the medico-surgical ICU of CHU Charleroi (Belgium). Twenty-two patients (mean age $64 \pm 13$ years) scheduled for cardiac surgery were included in the analysis. The CD64 expression on neutrophils was quantified by the haematologic cell dyn sapphire method (Abott, USA). C-reactive protein, leukocyte count, white blood cells, platelets and temperature were also monitored. Values are expressed as median (25th to 75th) percentile.

Results CD64 index (Figure 1) slightly rose from the day 0, median value 0.86 units (1.06 to 1.38 units) to day 5 after the surgery, 1.01 (1.32 to 1.68 units). However, this increase was moderate, and the median values did not exceed the usual threshold of 1.5 units. Conversely, CRP showed a large increase from normal values on day 0 to $8,67 \mathrm{mg} / \mathrm{dl}$ (6.64 to $13.56 \mathrm{mg} / \mathrm{dl})$ on day 5 . No significant correlation $(P>0.05)$ was found between CD64 and the others parameters of inflammation (CRP, leukocyte count, PMNs, platelets and temperature).

Conclusions Our results show that the neutrophil CD64 expression after cardiac surgery with ECC is only moderately increased. The role of this new biomarker in the early diagnosis of infection (after major surgery) should be assessed in prospective studies.

\section{References}

1. Loan-Facsinay A, de Kimpe SJ, Hellwig SMM, et al.: FcyRI(CD64) contributes substantially to severities of arthritis, hypersensitivity responses, and protection from bacterial infection. Immunity 2002, 16:391-402.

2. Harpaz Y, Chothia C: Many of the immunoglobulin superfamily domains in cell adhesion molecules and surface receptors belong to a new structural set which is close to that containing variable domains. J Mol Biol 1994, 238:528-539.

P17

Interferon-gamma reverses sepsis-induced immunoparalysis of monocytes in vitro

M Mouktaroudi, A Savva, A Pistiki, M Raftogiannis, A Antonopoulou,

E Giamarellos-Bourboulis

ATTIKON University Hospital, Athens, Greece

Critical Care 2010, 14(Suppl 1):P17 (doi: 10.1186/cc8249)

Introduction It is currently being understood that most of the agents modulating host response in sepsis have failed because they act to refrain an over-exaggerated immune response whereas immunoparalysis takes place on the time of their administration. The effect of IFNy on immunoparalysis of monocytes in sepsis was assessed.
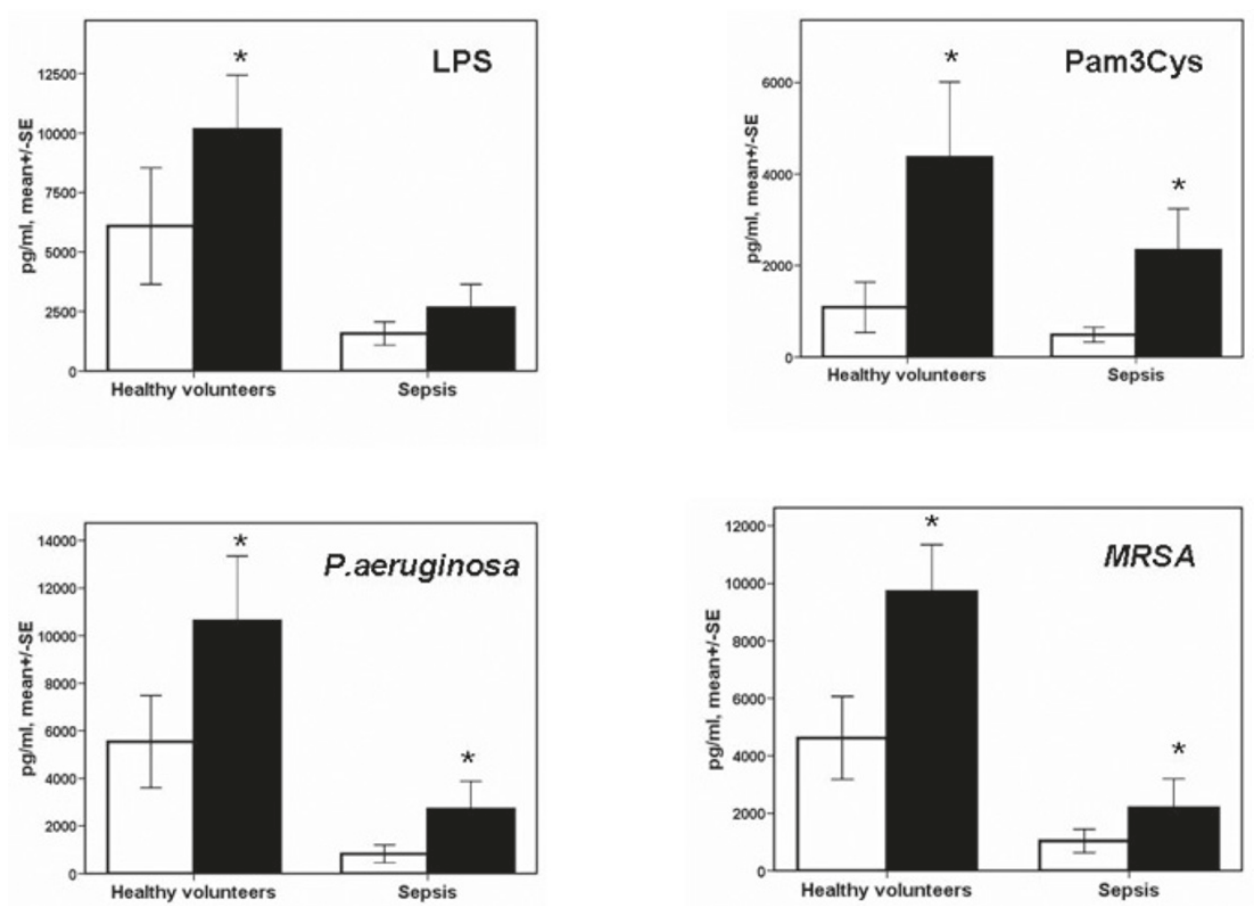

Figure 1 (abstract P17). Effect of IFNy on the release of TNFa. 
Methods Blood was isolated within the first 24 hours from the advent of signs of sepsis from 10 healthy donors and from 33 patients; 14 with sepsis and 19 with severe sepsis/shock. Peripheral blood mononuclear cells (PBMCs) were isolated and stimulated with $10 \mathrm{ng} / \mathrm{ml} \mathrm{LPS;} 5 \mu \mathrm{g} / \mathrm{ml}$ Pam3Cys; and heat-killed isolates of Candida albicans, multidrug-resistant Pseudomonas aeruginosa and methicillin-resistant Staphylococcus aureus (MRSA). Stimulations were done in the absence and presence of $10 \mathrm{ng} /$ $\mathrm{ml}$ IFNy. Concentrations of cytokines were estimated in supernatants by an enzyme immunoassay.

Results Effects of IFNy on the release of TNFa are shown in Figure 1. Open bars represent stimulation in the absence of IFNy and solid bars stimulation in the presence of IFNY. Asterisks signify differences compared with the absence of IFNy. IL-6 and IL-1 $\beta$ had similar kinetics to TNFa.

Conclusions IFNy reverses in vitro sepsis-induced immunoparalysis of monocytes. The type of affected cytokine depends on the agonist and probably reflects the mechanism of action of IFNY.

P18

Early hydrocortisone treatment counteracts circulatory derangement but not cytokine response in porcine endotoxemia

ES Söderberg', ML Lipcsey', JS Sjölin², AL Larsson³, ME Eriksson

'Section of Anaesthesiology and Intensive Care, Uppsala, Sweden; ' Section of Infectious Diseases, Uppsala, Sweden; '3ection of Clinical Chemistry, Uppsala, Sweden

Critical Care 2010, 14(Suppl 1):P18 (doi: 10.1186/cc8250)

Introduction We evaluated whether treatment with hydrocortisone administered just after establishment of endotoxin-mediated circulatory dysfunction would have anti-inflammatory and shock reversing effects. Establishment of endotoxin-mediated circulatory dysfunction was defined as the moment when the mean pulmonary arterial pressure (MPAP) reached the double baseline value.

Methods All pigs were anesthetized and given endotoxin infusion during the 6 hours of the experiment. Eight pigs were randomized to the hydrocortisone group, receiving hydrocortisone at $5 \mathrm{mg} / \mathrm{kg}$ intravenously, or to the control group, receiving a corresponding volume of saline, as soon as the MPAP doubled the baseline value. $P<0.05$ was considered significant.

Results No difference in baseline data was noted between the groups. No differences in TNFa (Figure 1), IL-6 and core temperature were seen between the groups. Pigs in the hydrocortisone group had significantly higher mean arterial pressure and systemic vascular resistance index during the 6-hour experimental period than pigs in the control group, while heart rate was significantly lower in the hydrocortisone group at 1 to 6 hours as compared with controls.

Conclusions Early treatment with hydrocortisone, administered after the onset of endotoxemia, counteracted circulatory deterioration, but did not affect the plasma levels of proinflammatory cytokines in this model. Thus, TNFa and IL-6 might not be involved in the development of circulatory dysfunction during the early phase of experimental endotoxemia.

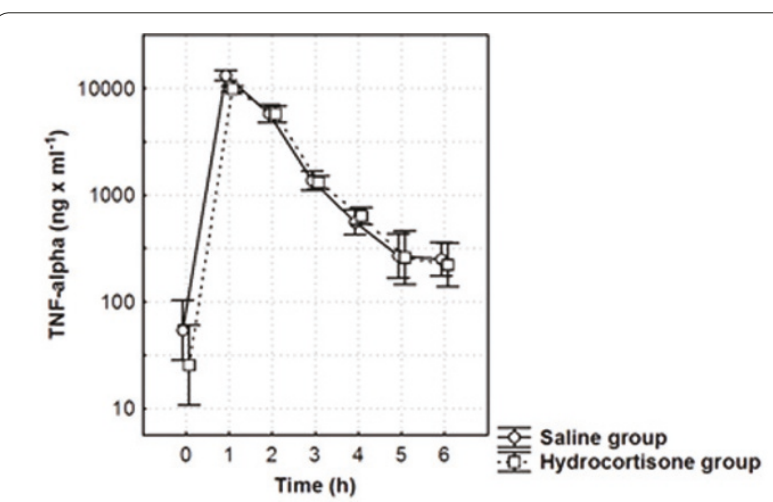

Figure 1 (abstract P18). TNFa over time (mean \pm SEM).
P19 CD40L is selectively expressed on platelets from thrombocytopenic septic patients

AM Montalbano', F Turani², EC Ciotti', MF Fede' ${ }^{1}$ MP Piperno', PD David', DF Fiume', GC Caiazza', SN Natoli', FL Leonardis', AB Bergamini' 'University Tor Vergata, Rome, Italy; '2European Aurelia Hospital, Rome, Italy Critical Care 2010, 14(Suppl 1):P19 (doi: 10.1186/cc8251)

Introduction It has been recently hypothesized that septic microangiopathy is caused or at least promoted by the interaction between endothelial surface receptor CD40 and its ligand CD40L, expressed by activated platelets. This interaction produces procoagulative changes in endothelial cells, endothelial apoptosis, subendothelial matrix exposition and microthrombi formation. Since virtually all septic patients show a certain degree of coagulation abnormalities, we hypothesized that low platelet count is associated with a different degree of CD40L expression and that this could correlate with the severity of disease.

Methods To determine the influence of sepsis on levels of platelet-derived CD40L expression, we performed a prospective observational study in a polyvalent university hospital ICU. Eighteen consecutively septic patients were enrolled in the study, independently of the platelet count and the severity of disease (SOFA score). Flow cytometry of fresh blood from septic patients $(n=18)$ and age-matched controls $(n=8)$ was performed for membrane-bound CD40L and CD62P on circulating platelets.

Results Flow cytometry demonstrated low levels of CD62P in controls while the levels in patients were high. $\mathrm{CD} 4 \mathrm{~L}^{+}$platelets were selectively found from patients with thrombocytopenia (platelet count $\leq 60,000$ / $\mathrm{mm}^{3}$ ). Furthermore a direct correlation between CD40L expression and the SOFA score was found in patients with sepsis and thrombocytopenia compared to patients with sepsis without thrombocytopenia.

Conclusions These results suggest that CD40L expression on platelets is somehow related to the degree of thrombocytopenia and possibly can be a marker of the severity of sepsis. Although the role of endothelialderived CD40/platelet-derived CD40L interaction is not fully understood during sepsis, the expression of CD40L on platelets could be related to the severity of organ disease due to the possible bursting of endothelial damage through this pathway. Further investigation is needed to determine whether platelets CD40L contributes to endothelial and subsequent organ damage, its role in thrombocytopenia and its correlation with the outcome of sepsis. The microvascular injury seems to be a central event in sepsis, so understanding the mechanisms underlying its development is crucial for the individuation of new and specific therapeutic strategies.

\section{P20}

Neuronal NOS-inhibition in the setting of septic cardiomyopathy A Van de Sandt', R Windler', A Gödecke', J Ohlig ${ }^{3}$, S Becher', TRassaf', J Schrader², M Kelm', M Merx

'Department of Cardiology, Pulmonology and Vascular Medicine, University Hospital Düsseldorf, Germany; ${ }^{2}$ Department of Cardiovascular Physiology, Heinrich-Heine-University, Düsseldorf, Germany; ${ }^{3}$ RWTH, Aachen, Germany Critical Care 2010, 14(Suppl 1):P20 (doi: 10.1186/cc8252)

Introduction Nitric oxide (NO) plays a central role in the pathogenesis of sepsis. Recently, we demonstrated that endothelial NOS (eNOS) contributes to endogenous NO-production and modulates inflammation, associated with preserved cardiac function resulting in prolonged survival of eNOS ${ }^{-1-}$ compared with wild-type (WT) mice. The role of neuronal NOS (nNOS) in septic cardiomyopathy remains unclear. This study's aim is to elucidate the influence of nNOS in the presence/absence of eNOS on cardiac function and survival in a clinically relevant model of sepsis.

Methods Inhibition of nNOS was achieved via continuous application of the selective nNOS-inhibitor Vinyl-L-NIO (VL-NIO) $(0.02 \mathrm{mg} / \mathrm{kg}$ BW/hour) using an osmotic mini pump. B6/c57 WT and eNOS ${ }^{-1-}$ mice were rendered septic by cecum ligation and puncture (CLP). After 12 hours heart function was analyzed using a pressure-volume catheter placed in the left ventricle. For catecholamine responsiveness, norepinephrine was applied $(0.4 \mu \mathrm{g} / \mathrm{kg}$ $\mathrm{BW} /$ minute, intraperitoneally). NOx-levels in plasma were measured using high-pressure performing liquid chromatography.

Results Inhibition of nNOS via VL-NIO application resulted in significantly reduced nitrate plasma levels and prolonged survival (WTCLP + VL-NIO $=38$ 
hours vs WTCLP $=29$ hours). However, cardiac function and norepinephrine responsiveness were not improved compared with untreated septic WT. In contrast to WT, application of $\mathrm{VL}-\mathrm{NIO}$ in $\mathrm{eNOS}^{-/-}$had no influence on plasma nitrate levels, while cardiac function and survival were significantly impaired compared with untreated septic eNOS ${ }^{-1}$. Impaired cardiac function was accompanied by decreased survival time (eNOS ${ }^{-1-} \mathrm{CLP}+$ $\mathrm{VL}-\mathrm{NIO}=29.5$ hours vs eNOS ${ }^{-1-} \mathrm{CLP}=69.5$ hours).

Conclusions Pharmacologic inhibition of nNOS result in significant reduction of plasma nitrate levels and prolonged survival compared with untreated septic WT despite unimproved septic cardiomyopathy. In contrast, the significant survival benefit of septic eNOS ${ }^{-/-}$compared with septic WT was abrogated by pharmacologic nNOS inhibition. Furthermore, the latter developed severe septic cardiomyopathy. Whether eNOS acts as modulator of nNOS in this setting remains to be clarified by further studies.

\section{P21}

Urinary albumin excretion is elevated in sepsis, but does not correlate with circulating VEGF-A levels

S Basu', M Bhattacharyya', T Chatterjee², S Todi', A Majumdar ${ }^{1}$

'AMRI Hospitals, Kolkata, India; 'Jadavpur University, Kolkata, India

Critical Care 2010, 14(Suppl 1):P21 (doi: 10.1186/cc8253)

Introduction In critically ill patients admitted with SIRS, endothelial dysfunction leads to increased capillary permeability. In the glomerulus, it manifests as increased albumin excretion. Microalbuminuria is a common finding in various acute conditions like sepsis, trauma and surgery. Studies have shown increased levels of vascular endothelial growth factor-A (VEGF-A), a potent vascular permeability inducing agent, in LPS-induced endotoxemia, severe sepsis and septic shock. The pathogenic mechanism of the glomerular leakage of albumin in acute inflammatory conditions remains to be clarified. We wished to investigate the causative role of VEGF-A, in this regard which might have therapeutic implications.

Methods Prospective study in the 43-bed ICU of a tertiary care hospital. Of the consecutive patients admitted to the ICUs between September 2008 and January 2009, 597 patients were included, after excluding patients with ICU stay <24 hours, pregnancy, menstruation, anuria, hematuria and proteinuria due to renal and post- renal diseases. Of these, 30 consecutive patients with sepsis, severe sepsis and septic shock (sepsis group) and 30 randomly chosen patients without sepsis were recruited for the VEGF-A study. Spot urine samples for the albumin-creatinine ratio (ACR, mg/g) and serum forVEGF-A (ELISA) were collected on ICU admission. Correlation was analyzed using Spearman's correlation coefficient.

Results Sixty critically ill patients, with a median age (IQR) of 60 years (48 to 72), 39:21 male:female ratio, median APACHE II score (IQR) of 16 (7 to 23), and 3 median days of ICU stay, had a median ACR (IQR) of $125 \mathrm{mg} / \mathrm{g}$ (51.6 to 239.1) and a median VEGF-A (IQR) of $111 \mathrm{pg} / \mathrm{ml}$ (54.3 to 286.9) on ICU admission. The median ACR on admission in the sepsis group $(n=30)$ of $161.8 \mathrm{mg} / \mathrm{g}$ was significantly higher than the median ACR $(78.3 \mathrm{mg} / \mathrm{g})$ of the group without sepsis $(n=30)(P=0.011)$. On statistical analysis, no significant correlation was obtained between levels of urinary ACR and serum VEGF-A ( $P=0.327)$ in the entire group. Analysis for correlation between ACR and VEGF-A in the sepsis patients $(P=0.396)$ and in the group without sepsis $(P=0.518)$ yielded similar results.

Conclusions Despite a strong physiologic rationale, our pilot study did not show an association between microalbuminuria and VEGF-A in critically ill patients. Larger studies are needed to come to a definitive conclusion.

\section{P22}

High mobility group box protein-1 preconditions isolated rat hearts and protects human pulmonary and renal cells against hypoxic injury CM Walshe, D O'Toole, B Higgins, JG Laffey, LG Kevin

National University of Ireland, Galway, Ireland

Critical Care 2010, 14(Suppl 1):P22 (doi: 10.1186/cc8254)

Introduction High mobility group box protein-1 (HMGB) is released by activated monocytes/macrophages during sepsis. Levels correlated with mortality in patients and anti-HMGB antibodies prevented endotoxininduced death in animal models [1]. In marked contrast to these findings are reports of cytoprotective effects because HMGB protected liver from ischemia/reperfusion (IR) [2] and HMGB-transgenic mice were resistant to myocardial infarction [3]. Despite these reports, little is known of the role or mechanism of HMGB. We aimed to further evaluate HMGB in rat hearts and in pulmonary and renal cell cultures.

Methods Isolated hearts received HMGB/vehicle before 30 minutes ischemia and 120 minutes reperfusion. In separate studies, rats were rendered septic by caecal ligation and puncture (CLP) prior to IR. Left ventricular (LV) function was measured with a latex LV balloon. Infarct size was measured by TTC staining. For human cell culture studies, A549 alveolar cells or HK-2 renal tubular cells were treated with HMGB/vehicle before incubation in normoxia or hypoxia. Fluorimetric caspase-3 activity was used as measure of apoptosis. Cytochrome C was measured by ELISA. For HK-2 cells, the MTT assay was used to test viability.

Results HMGB, prior to cardiac IR, preserved developed (d) LVP at 120 minutes reperfusion compared with controls $(44 \mathrm{mmHg}$ vs $27 \mathrm{mmHg}$, $P<0.05)$ and infarct size, expressed as \%V weight \pm SEM, was reduced (31 \pm 5.4 vs $44 \pm 4.2, P<0.05), n=6 /$ group. Hearts from CLP rats had reduced baseline dLVP $(59 \mathrm{mmHg} \pm 5.1$ vs $97 \pm 5.3, P<0.05)$ but infarct sizes after IR were significantly reduced $(27 \pm 8.8, P<0.05$ vs $44 \pm 6.2, P<0.05)$. In A549 cells, HMGB inhibited apoptosis (49\% reduced caspase-3 activity (8.6 vs 17.1 a.u., $P<0.01)$ ). This was associated with reduced cytochrome $C$ release (250 vs $389 \mathrm{pg} / \mathrm{ml}, P<0.05)$. Using MTT assay, HMGB protected against hypoxia-induced cell death vs controls (viability $75 \%$ vs $61 \%, P<0.05$ ).

Conclusions $H M G B$, or sepsis, similarly precondition the heart against IR injury. HMGB has anti-apoptotic effects that protect against hypoxic renal and alveolar cell injury. We conclude that HMGB has potent anti-ischemic effects in multiple organs and may function as an innate cytoprotective mediator in sepsis.

\section{References}

1. Wang H, et al: Science 1999, 285:248-251.

2. Tsung A, et al.: J Exp Med 2005, 201:1 135-1143.

3. Kitahara T, et al.: Cardiovasc Res 2008, 80:40-46.

\section{P23}

Early persisting elevation of plasma Pentraxin 3 is associated with mortality and with coagulation impairment in severe sepsis and septic shock

A Coppadoro', T Mauri', G Bellani', N Patroniti', M Cugno², A Grassi',

G lapichino², L Gattinoni², A Mantovani², A Pesenti'

'Universita' degli Studi di Milano-Bicocca, Monza, Italy; ${ }^{2}$ Universita' degli Studi di Milano, Italy

Critical Care 2010, 14(Suppl 1):P23 (doi: 10.1186/cc8255)

Introduction Pentraxin 3 (PTX3) is an inflammatory mediator produced by a variety of tissue cells, like neutrophils, macrophages, myeloid dendritic, endothelial and epithelial cells [1]. During sepsis a massive inflammatory activation occurs, which often leads to an important coagulation system dysfunction. PTX3 upregulates coagulation promoters in vitro [2]. PTX3 may represent an early marker of severity and outcome and may be related to coagulation impairment in septic patients.

Methods We studied 90 patients affected by severe sepsis or septic shock previously enrolled in a prospective trial regarding the impact of glycemic control on inflammation and coagulation. At enrollment we recorded sepsis signs, plasminogen activator inhibitor 1 (PAl-1, an inhibitor of fibrinolysis) activity and concentration, prothrombin fragments $1+2$ $(F 1+2)$ concentration (a marker of coagulation activation), disease severity and organ dysfunctions. We measured plasma PTX3 levels at enrollment and everyday until day 7. Mortality was recorded at day 90 .

Results Although not different on day 1, PTX3 remained significantly higher in nonsurvivors than in survivors over the first 5 days $(P=0.002$ by general linear model). On day 1, septic shock patients had higher PTX3 levels than patients with severe sepsis ( $274.41 \pm 321.92$ vs $114.95 \pm 129.93$ $\mathrm{ng} / \mathrm{ml}, P=0.029, t$ test). Day 1 PTX3 was significantly correlated with SAPS II score $\left(R^{2}=0.08, P=0.006\right.$, linear regression), platelets count $\left(R^{2}=0.14\right.$, $P<0.001)$ and SOFA score $\left(R^{2}=0.16, P<0.001\right)$. Day 1 PTX3 was correlated with PAI-1 concentration $\left(R^{2}=0.211, P<0.001\right)$ and activity $\left(R^{2}=0.231\right.$, $P<0.001)$ and with $\mathrm{F} 1+2$ concentration $\left(R^{2}=0.09, P=0.045\right)$.

Conclusions Persisting high levels of circulating PTX3 over the first days from sepsis onset may be associated with mortality. PTX3 correlates with severity of sepsis and with sepsis-associated coagulation dysfunction. 


\author{
References \\ 1. Mauri T, et al.: Crit Care Med 2008, 36:2302-2308. \\ 2. Napoleone E, et al.: J Leukoc Biol 2004, 76:203-209.
}

P24

Intravenous immunoglobulins prevent breakdown of the

blood-brain barrier in experimental sepsis

F Esen', E Senturk1, P Ergin Ozcan', B Ahishali', N Arıcan', N Orhan', O Ekizoğlu², D Ustek', M Kucuk', M Kaya' ${ }^{1}$

'Istanbul University Istanbul Medical Faculty, Istanbul, Turkey; ${ }^{2}$ Sadi Konuk

Training and Research Hospital, Istanbul, Turkey

Critical Care 2010, 14(Suppl 1):P24 (doi: 10.1186/cc8256)

Introduction The effect of intravenous immunoglobulin preparations immunoglobulin $\mathrm{G}(\mathrm{lg} \mathrm{G})$, immunoglobulin $\mathrm{G}$ enriched with $\lg A$ and $\lg M$ (IgGAM) - on blood-brain barrier (BBB) integrity and survival rate was comparatively investigated in septic rats.

Methods Sepsis was induced by cecal ligation and perforation (CLP) in Sprague-Dawley rats. The animals received either lgG $(250 \mathrm{mg} / \mathrm{kg}$, intravenously) or lgGAM (250 mg/kg, intravenously) 5 minutes before CLP surgery. All rats were observed for behavioral changes for 24 hours after CLP operation. To show the alterations in BBB integrity, Evans blue (EB; $69 \mathrm{kDa})$ dye extravasation was determined for quantitative measurement of BBB permeability, and horseradish peroxidase (HRP; $40 \mathrm{kDa}$ ) extravasation was evaluated to provide ultrastructural evidence for the transport pathways involved in BBB permeability. Immunohistochemistry was performed to show the alterations in immunoreactivity for tight junction protein occludin.

Results A high mortality rate (34\%) was noted in septic animals and the mortality rate was decreased to $15 \%$ and $3 \%$ by $\lg$ G and IgGAM, respectively. Both IgG and IgGAM alleviated the sickness behavior in septic rats and the animals were observed to be healthy and active. Increased extravasation of EB dye into brain tissue of septic animals was markedly decreased by both IgG and IgGAM. Occludin immunoreactivity remained essentially unchanged in all groups including CLP. In addition, strong staining for HRP was seen around vessels located in the cerebral cortex and the hippocampus in septic animals. Increased luminal and abluminal vesicles containing electron-dense HRP reaction product were noted in the cytoplasm of endothelial cells in the cerebral cortex and hippocampus of septic rats, emphasizing an increased BBB permeability predominantly by transendothelial route. In these animals, tight junctions were ultrastructurally intact suggesting that paracellular pathway of BBB is not responsible for the BBB breakdown in sepsis. Following IgG or IgGAM treatment, no ultrastructural evidence of leaky capillaries in brain was observed in septic animals indicating the blockade of the transcellular pathway.

Conclusions The present study indicates that lgG and IgGAM improve the integrity of the BBB and inhibit CLP-induced sickness behavior in rats.

\section{P25}

\section{Early use of immunoglobulin in septic shock}

IC Cavazzuti, LR Rinaldi, LD Donno, SB Braccini, SB Busani, MG Girardis Policlinico di Modena, Italy

Critical Care 2010, 14(Suppl 1):P25 (doi: 10.1186/cc8257)

Introduction In addition to the interventions suggested by the guidelines of the Surviving Sepsis Campaign, in the recent years other therapeutic options have been proposed and tested for patients with severe sepsis and septic shock. Polyclonal intravenous immunoglobulins seem to be an interesting and useful option because of its activity in neutralizing endotoxin and in modulating the host immune response. In this retrospective study we assessed the effects of the early use of polyclonal intravenous immunoglobulins enriched with IgA-M (IgGAM) in addition to standard therapies on clinical outcome of patients with septic shock.

Methods In January 2008, IgGAM was introduced into our ICU protocol for the management of septic shock patients (within 24 hours after shock appearance and at the dosage of $5 \mathrm{ml} / \mathrm{kg}$ for 3 days). From January 2008 to September 2009, we studied 52 consecutive patients with septic shock admitted to our ICU. In each patient we recorded the SAPS II and SOFA scores, the compliance to the 6-hour and the 24-hour sepsis bundles, the compliance to IgGAM use, the length of stay in the ICU and the 30-day mortality rate.

Results Due to low compliance of medical staff to protocol application, IgGAM has been used only in 27 patients (IgGAM group). At ICU admission, the severity scores were similar in patients with and without (Control group) IgGAM therapy (SAPS II: control 54 (22 to 99), IgGAM: 59 (39 to 101); $P>0.05$ and SOFA: control 9 (2 to 17); IgGAM 9 (4 to 15); $P>0.05$ ). The compliance to 6-hour and 24-hour bundles was also similar in the two groups, apart from compliance to fluid therapy that was larger in the IgGAM group ( $85 \%$ vs 56\% in Control group). The length of stay in the ICU was $15 \pm 9$ days in the lgGAM group and $18 \pm 31$ days in the Control group $(P>0.05)$; the 30 -day mortality was lower $(P<0.05)$ in the IgGAM group (25\%) than in the Control group (52\%).

Conclusions These preliminary data indicate that the early use of IgGAM, associated with interventions proposed by the SSC guidelines, reduces mortality of patients with septic shock.

References

1. Ballow M: Mechanism of action of intravenous immune serum globulin in autoimmune and inflammatory diseases. J Allergy Clin Immunol 1997, 100:151-157.

2. Werdan K: Mirror, mirror on the wall, which is the fairest meta-analysis of all? Crit Care Med 2007, 35:2852-2854.

\section{P26}

Low levels of immunoglobulin $\mathrm{G}$ in patients with sepsis or septic shock: a signum mali ominis?

S Dietz, C Lautenschlaeger, U Mueller-Werdan, KWerdan

University Clinics of the Martin-Luther-University Halle-Wittenberg, Halle (Saale), Germany

Critical Care 2010, 14(Suppl 1):P26 (doi: 10.1186/cc8258)

Introduction The role of intravenous immunoglobulin therapy in patients with sepsis and septic shock is still controversially discussed. In a retrospective analysis of the data from the SBITS-trial [1] we investigated whether the initial level of serum lgG on admission to the hospital in patients with sepsis and septic shock (before the first administration of the first dose of intravenous immunoglobulins) could be seen as a prognostic parameter for the primary outcome, lethality on day 28 , or the secondary endpoints, lethality on day 7 or on the ICU.

Methods A total of 543 were included in the trial: the patients were divided into four groups (quartiles) based on the 25th, 50th and 75th percentiles of their initial level of lgG on admission to the hospital. The first group contained 140 patients with a range in the IgG serum level up to $6.1 \mathrm{~g} / \mathrm{dl}$. The second and third groups contained 134 patients and 136 patients, respectively, the immunoglobulin $\mathrm{G}$ levels were greater than $6.1 \mathrm{~g} / \mathrm{dl}$ to $8.4 \mathrm{~g} / \mathrm{dl}$ and greater than $8.4 \mathrm{~g} / \mathrm{dl}$ to $11.9 \mathrm{~g} / \mathrm{dl}$. The fourth group containing 133 patients had IgG levels higher than $11.9 \mathrm{~g} / \mathrm{dl}$. In a logistic regression model we adjusted for potential confounders (that is, sex, age, APACHE II score, presence of shock on admission).

Results On basis of this model we could show that lethality between the first and second groups did not differ significantly with the lethality in the third (reference) group with physiologic levels of IgG on day 0. Surprisingly the lethality of the fourth group, with lgG levels higher than $11.9 \mathrm{~g} / \mathrm{dl}$, was significantly higher compared with the reference quartile (OR 1.69, Cl 1.01 to $2.81, P=0.047$ )

Conclusions Our post-hoc analysis did show no prognostic relevance of a low level of serum lgG on admission to hospital for the 543 patients with sepsis and septic shock. Thus the initial serum level of IgG seems to be of no aid in making the decision to initiate therapy with intravenous immunoglobulins for these patients. Whether a high level of serum lgG on admission is an independent risk factor for an increased lethality in critically ill patients, as shown in our analysis, needs to be investigated in further studies.

\section{Reference}

1. Werdan K, et al.: Score-based immunoglobulin $\mathrm{G}$ therapy of patients with sepsis: the SBITS study. Crit Care Med 2007, 35:2693-2701. 
P27

Background factors in patients receiving immunoglobulin administration and changes in sepsis markers

T Ikeda', K Ikeda', H Taniuchi', S Suda', Y Takahashi²

'Tokyo Medical University, Hachioji Medical Center, Tokyo, Japan; '2Sannoudai Hospital, Ibaraki, Japan

Critical Care 2010, 14(Suppl 1):P27 (doi: 10.1186/cc8259)

Introduction The potentially envisaged actions of intravenous immunoglobulin (IVlg) on severe infectious disease include virus or toxin neutralizing action, opsonic effect, complement bacteriolytic activity, and enhancement of sensitivity to antibiotics. In the case of severe infectious disease, antibiotics are often supplemented with administration of IVIg. The aim of this study is that the changes in sepsis markers followed by IVlg administration are investigated with severe sepsis or severe septic shock patients.

Methods The subjects were 52 patients admitted to an ICU with a diagnosis of severe sepsis or septic shock and from whom informed consent had been obtained for the present study. IVIg was administered intravenously for 3 days $(5.0 \mathrm{~g} /$ day) and measurements were undertaken before administration (Day 1), on the day after completion of administration (Day 4), and on Day 7. The items measured were IL-6, C-reactive protein (CRP) and, as indices of vascular endothelial cell activation, plasminogen activator inhibitor-1 (PAl-1) and adhesion molecules (endothelial leukocyte adhesion molecule-1 (ELAM-1)); the effect of IVlg administration on these markers was then studied. The IVIg studied was polyethylene glycol-treated human immunoglobulin injection fluid (2.5 g, $50 \mathrm{ml}$, one vial).

Results The APACHE II score was $25.3 \pm 7.8$, the SOFA score $9.5 \pm 3.5$, and the survival rate after 28 days $76.9 \%$. The values before IVIg administration were: procalcitonin $28.8 \pm 44.5$ (median value 13.6) $\mathrm{ng} / \mathrm{ml}$, CRP $16.3 \pm 8.3$ (median value 16.0) $\mathrm{mg} / \mathrm{dl}$, IL-6 8,873 $\pm 19,362$ (median value 744) $\mathrm{pg} / \mathrm{ml}$, PAl-1 $374 \pm 428$ (median value 178) ng/ml, and ELAM-1 $198 \pm 359$ (median value 61$) \mathrm{ng} / \mathrm{ml}$. All values were thus elevated. After the completion of IVlg administration, the level of mediators other than ELAM-1 (procalcitonin, CRP, IL-6, PAI-1) decreased significantly.

Conclusions The results of the present study found significant decreases of IL-6 and PAI-1 resulting from immunoglobulin administration, but did not indicate a protective effect on vascular endothelial cell function.

P28

C1-esterase inhibitor (C1INH) implication in systemic inflammation in sepsis

A Igonin, N Lazareva, VY Uvarov

BioGenius Research Centre, Moscow, Russia Federation

Critical Care 2010, 14(Suppl 1):P28 (doi: 10.1186/cc8260)

Introduction $\mathrm{C} 1 \mathrm{INH}$ is the most potent endogenous regulator of compliment as well as intrinsic coagulation pathways and the kallekrein-kinin system.

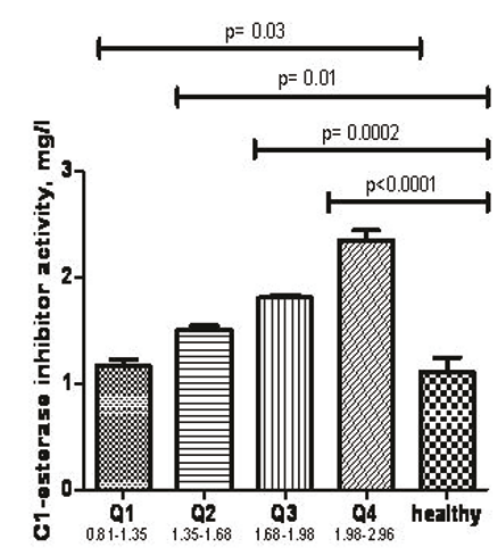

groups

Figure 1 (abstract P28). C1/NH activity in patients with sepsis analyzed in quartiles (Q1, Q2, Q3, Q4) in comparison with healthy individuals.
Methods C1INH systemic activity was studied in patients who were enrolled within 48 hours after onset of sepsis (ACCP, 1992). The analysis of $\mathrm{C} 1 \mathrm{INH}$ activity in quartiles (Q) was conducted in terms of RCT of human purified C1INH (Bicizar, Russia).

Results Sepsis patients $(n=40)$ responded with an increase of $\mathrm{C} 1 \mathrm{INH}$ activity in comparison with healthy individuals (Figure 1). Thirty percent of Q1 patients had ARDS and septic shock whereas in Q4 everyone showed only signs of sepsis. The CRP level was higher in Q1 patients (243.4 \pm $39.9 \mathrm{mg} / \mathrm{l})$ than in Q4 $(144.0 \pm 20.07 \mathrm{mg} / \mathrm{l} ; P=0.04)$, whereas the $\mathrm{C} 4$ subunit was lower in Q1 $(0.19 \pm 0.04 \mathrm{~g} / \mathrm{l})$ than in Q4 $(0.32 \pm 0.04 \mathrm{~g} / \mathrm{l} ; P=0.05)$.

Conclusions Inability to upregulate $\mathrm{C} 1 \mathrm{INH}$ activity in sepsis was associated with enhanced systemic inflammation, higher number of ARDS and septic shock cases.

\section{P29}

Prognostic value of B-type natriuretic peptide in critically ill patients with new onset of fever: preliminary study

V Soulountsi', V Voutsas', P Kontou', A Giakamozis', K Hatzimanolis², T Lazaridis' ${ }^{1}$ V Hatsiou'², M Mpitzani ${ }^{1}$

'ICU, G. Papanicolaou, Thessaloniki, Greece; '2Microbiology Laboratory,

G. Papanicolaou Hospital, Thessaloniki, Greece

Critical Care 2010, 14(Suppl 1):P29 (doi: 10.1186/cc8261)

Introduction The purpose of the study is the evaluation of B-type natriuretic peptide (BNP) as a predictor of septic complications and ICU mortality in patients with a new onset of fever during the first 3 days of hospitalization in the ICU.

Methods Thirty-one ICU patients (21 males and 10 females) with new onset of fever and leukocytosis within the first 3 days of ICU admission were prospectively included in the study. Exclusion criteria were heart or renal failure, chronic obstructive pulmonary disease and head trauma. Serial plasma samples were taken on days 1, 2 and 4 after the onset of fever for BNP level measurement. BNP values were correlated with severity scores (Acute Physiology and Chronic Health Evaluation (APACHE II) and Sequential Organ Failure Assessment (SOFA)), the progression to septic shock and the final outcome.

Results According to the clinical and laboratory findings within the first 3 days of hospitalization, the patients included in the study were divided into three groups: Group A = systemic inflammatory response syndrome (SIRS) (seven patients), Group B = sepsis (14 patients) and Group C = septic shock (10 patients). The BNP value on days 1 and 2 was significantly associated with the SOFA Max value $(P<0.001)$. The BNP value on day 4 was significantly associated with ICU mortality $(P=0.006)$. The optimal cutoff BNP value for differentiating between nonsurvivors and survivors was estimated to be $203.55 \mathrm{pg} / \mathrm{ml}$ (sensitivity $=100 \%$, specificity $=61.1 \%$ ). In Group B patients, BNP value on day 2 was significantly higher in patients who finally progressed to septic shock $(P=0.001)$. The optimal cutoff BNP value for identifying these patients was estimated to be $212.45 \mathrm{pg} / \mathrm{ml}$ (sensitivity $=85.7 \%$, specificity $=64.3 \%$ ).

Conclusions In ICU patients with new onset of fever during the first 3 days of ICU hospitalization, the BNP value on day 4 seems to be a good predictor of ICU mortality. In patients with sepsis, a cut-off BNP value of $212.45 \mathrm{pg} / \mathrm{ml}$ on day 2 could be a predictor of progression to septic shock. Due to the small number of patients included in our study, further studies are needed to confirm these findings.

\section{P30}

Microalbuminuria evaluated as a biomarker in patients with septic shock

C Grion, L Cardoso, C Carrilho, J Altafin, S Barros, L Carvalho, J Festti,

F Mansano, T Okamoto, K Uehara, J Dias, GC Silva

Hospital Universitário de Londrina, Brazil

Critical Care 2010, 14(Suppl 1):P30 (doi: 10.1186/cc8262)

Introduction The level of microalbuminuria is thought to reflect the severity of inflammation during acute phase response and may have prognostic value with regard to survival. This study aimed to describe urinary albumin excretion in patients with septic shock and changes in microalbuminuria levels in the first 3 days of treatment. 
Methods Prospective observational study of 49 consecutive patients with septic shock admitted to a general ICU. During the first 24 hours after severe sepsis was diagnosed, we collected data to calculate APACHE II and SOFA scores and urinary sample to measure urinary albumin. Another sample of urine was collected on the third day of septic shock. Microalbuminuria was measured by immunoturbidimetry with a limit of detection of $3.5 \mathrm{mg} / \mathrm{l}$. The study was approved by the local Ethic Committee.

Results The mean age was $65.8 \pm 15$ years and male sex was more frequent (63.3\%). Pneumonia (59.2\%) was the most common source of sepsis. Mean APACHE II and SOFA scores on the first day of sepsis were $24.4 \pm 7.7$ and $8.7 \pm 3.7$, respectively. Observed mortality was $71.4 \%$. Median urinary albumin was $7.0 \mathrm{mg} / \mathrm{l}$ (IQT: 4.7 to $60.3 \mathrm{mg} / \mathrm{l})$ for survivors and $15.5 \mathrm{mg} / \mathrm{l}$ (IQT: 7.9 to $77.1 \mathrm{mg} / \mathrm{l})$ for nonsurvivors on the first day of septic shock. Urinary albumin decreased from $7.0 \mathrm{mg} / \mathrm{I}$ (IQT: 4.7 to $60.3 \mathrm{mg} / \mathrm{l}$ ) to $3.9 \mathrm{mg} / \mathrm{l}$ (IQT: 3.6 to $44.1 \mathrm{mg} / \mathrm{l})$ in survivors during the first 3 days of septic shock, and in nonsurvivors remained at the same level from $15.5 \mathrm{mg} / \mathrm{l}$ (IQT: 7.9 to $77.1 \mathrm{mg} / \mathrm{l})$ to $14.5 \mathrm{mg} / \mathrm{l}(\mathrm{QQT}: 3.9$ to $45.3 \mathrm{mg} / \mathrm{l})$ in the period of observation. Conclusions Patients with septic shock showed high frequency of microalbuminuria. Levels of urinary albumin showed a tendency to decrease in survivors, suggesting prognostic significance.

\section{References}

1. Thorevska N, et al:: Crit Care Med 2003, 31:1075-1081.

2. Abid O, et al:: Chest 2001, 120:1984-1988.

P31

Evaluation of automated hematologic VCS parameters in severe sepsis and septic shock: a case-control study

A Cortegiani', A Di Benedetto', L Marino', G Virga', R Chiaramonte' C Sarno ${ }^{2}$, SM Raineri', A Giarratano

'University of Palermo, Italy; ${ }^{2}$ Fondazione Istituto S. Raffaele, Cefalù (PA), Italy

Critical Care 2010, 14(Suppl 1):P31 (doi: 10.1186/cc8263)

Introduction A cheap and quick hematologic diagnostic parameter for detection of sepsis would have both economic and therapeutic benefits. The Coulter LH series hematology analyzer uses the VCS technology (Volume, Conductivity, Laser Scatter) providing information about cell volume, size, internal structure, and surface morphology. Many authors analyzed the clinical usefulness of VCS parameters in reactive neutrophils for detection of sepsis. An increase in mean cell volume and a decrease in mean light scatter in septic patients have been described. Our aim is to verify the correlation between VCS parameters and sepsis.

Methods We enrolled 92 patients admitted in our ICU and divided them into two groups: Controls (48) and Sepsis (44). We collected blood samples immediately after making the diagnosis. All samples were analyzed by Coulter LH 500 hematology analyzer. We realized ROC curves for each V, C, S parameter.

Results The AUC was 0.816 (95\% Cl, 0.722 to 0.889 ) for mean- $V$ value; 0.546 ( $95 \%$ Cl, 0.439 to 0.650) for mean-C value; 0.604 ( $95 \%$ Cl, 0.497 to 0.704 ) for mean $\mathrm{S}$ value (Figure 1). The correlation with sepsis was a linear positive one for mean-V (strong) and mean-C (weak) instead of a linear negative one for mean-S. The best cut-off value for mean- $V$ was > 163 (Sens: 84.09\%;

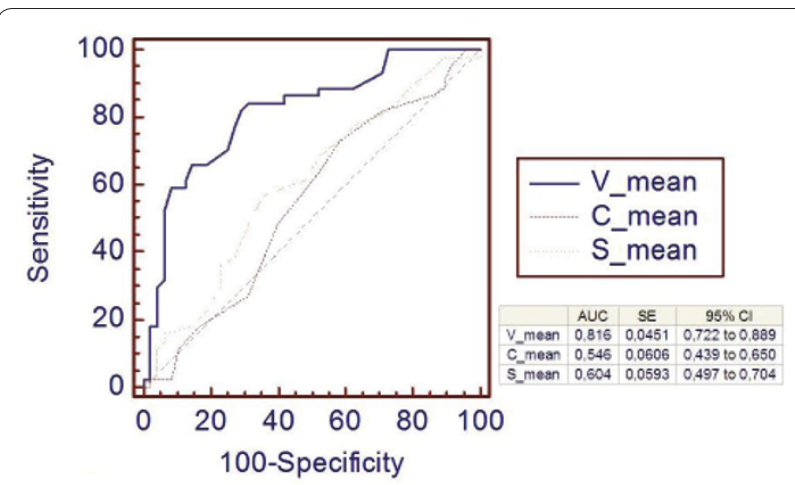

Figure 1 (abstract P31).
Spec: 68.75\%); >137 for mean-C (Sens: 72.73\%; Spec: 41.67\%); <134 for mean-S (Sens: 56.82\%; Spec: 64.58\%).

Conclusions Our study shows the high diagnostic performance of mean-V and the weak one of mean- $C$ and mean- $S$ in septic patients. The clinical usefulness of mean- $V$ is strengthened by the quickness and cheapness of its analysis. It could be tested daily or more as a valid laboratorial help for critical patient's clinical assessment.

\section{P32}

Use of CD64 for the diagnosis of sepsis: a case-control study

A Cortegiani', L Marino', A Di Benedetto', G Virga', G Evangelico', C Sarno ${ }^{2}$, SM Raineri' ${ }^{1}$

${ }^{1}$ University of Palermo, Italy; ${ }^{2}$ Fondazione Istituto S. Raffaele, Cefalù (PA), Italy Critical Care 2010, 14(Suppl 1):P32 (doi: 10.1186/cc8264)

Introduction CD64 is the high-affinity receptor of IgG. It is upregulated by inflammatory cytokines on neutrophils. The upregulation of CD64 is linked with PMN activation in SIRS or sepsis. Our aim is to verify these correlations. Methods We enrolled 48 ICU critical patients and three groups were created: SIRS- (17), SIRS ${ }^{+}$(13), Sepsis (17). We evaluated CD64 X-mean values among the three groups as shown in Figure 1. We used a $t$ test to check the correlation between uprising CD64 and different groups. We verified the correlation CD64/SOFA (Figure 2), WBC, NE, and patients' age using $r$.

Results We found a CD64 higher level in sepsis compared with SIRS $^{-}(t=$ 8.095; $P<0.001)$ and with $\mathrm{SIRS}^{+}(t=4.938 ; P<0.001)$. There were a high positive linear correlation between CD64 and SOFA score $(r=0.806$;
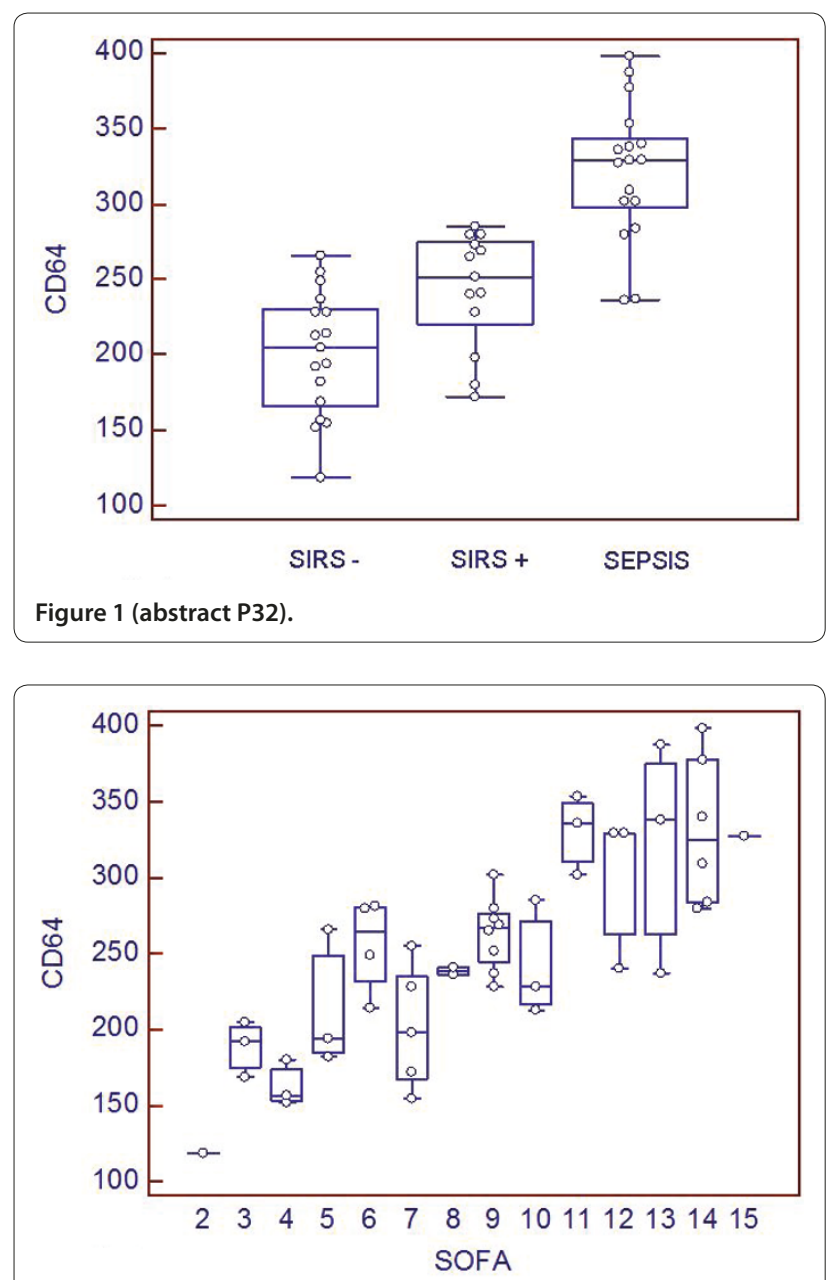

Figure 2 (abstract P32). 
$P<0.0001)$, a weak linear positive one with WBC count, a weak linear positive correlation with NE count; no correlation with age.

Conclusions Our study shows that CD64 can identify SIRS/sepsis. We found a correlation between CD64/SOFA; no influence of WBC, NE and age was detected.

P33

Endotoxin assay with endotoxin scattering photometry is one of the diagnostic markers of sepsis

Y Kase', ${ }^{1}$ Obata $^{2}$

' Jikei University School of Medicine, Tokyo, Japan; '2Microbial Chemistry

Research Foundation, Tokyo, Japan

Critical Care 2010, 14(Suppl 1):P33 (doi: 10.1186/cc8265)

Introduction Endotoxin scattering photometry (ESP) is one of a newly developed clinically applicable method for endotoxin assay. Although ESP is an analogous assay of turbidimetric method, it enables one to detect very small amounts of endotoxin within 1 hour. This is because ESP can directly detect clotting enzyme product, coagulin, which is the first appearance of Limulus amebocyte lysate (LAL) cascade and the precursor of gel clots.

Methods To study the correlates and prognostic significance of the endotoxin assay with ESP, we enrolled a total of 80 people consisting of three groups which were normal healthy volunteers $(n=35)$, patients admitted to the emergency department (ED) $(n=21)$ and patients admitted to the ICU $(n=24)$. To examine whether endotoxin is influencing in the severity of disease, we used sepsis/SIRS stratification which was defined by the American College of Chest Physicians/Society of Critical Care Medicine (ACCP/SCCM)

Results Of all 80 people, 33.7\% ( $n=27)$ was sepsis. The levels of endotoxin were significantly higher in the patients with sepsis (median, $18.4 \mathrm{pg} / \mathrm{ml}$ interquartile range, 3 to $54.1 \mathrm{pg} / \mathrm{ml}$ ) than in the people without sepsis $(0.227 \mathrm{pg} / \mathrm{ml} ; 0.031$ to $0.493 \mathrm{pg} / \mathrm{ml})$. The area under the receiver operating characteristic curve was 0.951 in the patients with sepsis. Cut-off concentrations for optimum prediction of sepsis with ESP were endotoxin $>7.06 \mathrm{pg} / \mathrm{ml}$ (diagnostic efficiency: 92.6\%).

Conclusions Endotoxin assay with ESP is one of the diagnostic markers of sepsis because the levels of endotoxin in patient with sepsis are clearly high. Endotoxin assay with ESP concentration higher than $7.06 \mathrm{pg} / \mathrm{ml}$ indicates sepsis.

\section{References}

1. Obata T, Nomura M, Kase Y, Sasaki H, Shirasawa Y: Early detection of the Limulus amebocyte lysate reaction evoked by endotoxins. Anal Biochem 2008, 373:281-286

\section{P34}

\section{Immature platelet fraction as predictive index of sepsis}

C Scaramucci, R De Blasi, S Tribuzi, A Carlini

II Faculty of Medicine - University of Rome 'La Sapienza'S. Andrea, Rome, Italy Critical Care 2010, 14(Suppl 1):P34 (doi: 10.1186/cc8266)

Introduction The incidence of sepsis is reported around 37\% in European ICUs [1]. The mortality rate depends on the severity of organ failure, up to $65 \%$ if four or more organs are involved. Multiple organ failure (MOF) is due to microcirculatory dysfunction with microthrombosis resulting from coagulation disorders including platelets' activation. An early diagnosis should identify the microcirculatory dysfunction before MOF became clinically evident. The diagnosis of sepsis is commonly based on clinical criteria, pathogen identification and use of markers like procalcitonin $(P C T)$ and C-reactive protein (PCR) associated with infection. The aim of our study is to evaluate whether the routine measurement of immature platelet fraction (IPF), considered a precocious marker of platelet production, is associated with sepsis and its severity and/or whether it could be used as a predicting marker of sepsis.

Methods We enrolled 66 consecutive patients admitted to the ICU, dividing them into two groups: septic $(n=44)$ and no septic $(n=22)$. The severity of sepsis was evaluated. The exclusion criterion was a platelet count $<150,000 / \mathrm{mm}^{3}$. Blood count, coagulation, PCR, PCT, and IPF were collected every day.

Results The IPF values between septic $(4.6 \pm 3.1)$ and no septic patients $(3.3 \pm 1.5)$ did not differ $(P=0.16)$. No correlation was found between IPF
Table 1 (abstract P34)

\begin{tabular}{lccc}
\hline & No sepsis & Sepsis & Mann-Whitney \\
\hline IPF (\%) & $5.4 \pm 2.9$ & $3.3 \pm 1.5$ & $P=0.04$ \\
PCT (ng/ml) & $0.68 \pm 1.1$ & $0.57 \pm 1.3$ & $P=0.4$ \\
PCR (mg/dl) & $9.57 \pm 10.2$ & $11.7 \pm 10$ & $P=0.46$ \\
\hline
\end{tabular}

values and the severity of septic condition (no sepsis $11.7 \pm 10.1$; sepsis $14.3 \pm 10.5$; severe sepsis $10.5 \pm 9.1$; septic shock $19.5 \pm 12.4 ; P=0.3$ ). When we considered only subjects who did not have sepsis at the ICU admission we found that patients who developed sepsis during the recovery had IPF values higher than patients who did not develop sepsis (Table 1).

Conclusions From our results IPF cannot be considered a marker of sepsis. Conversely it could be used as predictive index of sepsis because it can identify patients who will develop sepsis.

\section{References}

1. Vincent et al.: Sepsis in European intensive care units: results of the SOAP study. Intensive Care Med 2006, 34:344-353.

\section{P35}

Serial estimation of cytokine markers and their correlation with mortality in elderly patients with sepsis

R Bhanot, J Kaur, M Rao, T Ramachandran, K George, D Veliath

Pondicherry Institute of Medical Sciences, Pondicherry, India

Critical Care 2010, 14(Suppl 1):P35 (doi: 10.1186/cc8267)

Introduction Sepsis is a serious health problem in the elderly with a high degree of mortality. The study of pathophysiology of sepsis has lead to the development of cytokine markers which help in establishing diagnosis, quantifying the severity and assessing the response to therapy. TNFa and IL-6, a pleotropic cytokine, are among the most well-studied proinflammatory cytokines in sepsis. However, very limited data are available in elderly subjects regarding the markers for sepsis. The present study was undertaken to establish the cytokine profile in sepsis in the elderly and develop markers for prediction. The influence of gender on cytokine production and mortality in elderly patients with sepsis was considered in particular.

Methods In this prospective study, 50 elderly patients, age above 65 years, satisfying the systemic inflammatory response syndrome (SIRS) criteria were included. Serial estimations of TNFa and IL-6 were done on days 1, 3 and 7 of ICU admission.

Results The results were drawn on the basis of gender and survival. A correlation of APACHE score, TNFa values and IL-6 values on day 1, day 3 and day 7 between survivors and nonsurvivors was also documented, which showed reducing levels of IL-6 levels from days 1 to 7 in the survivor group $(P<0.01)$. The TNFa level was significantly low on day 1 in the nonsurvivor female group $(P<0.00001)$

Conclusions Cytokine profiling for TNFaand IL-6 level in elderly patients with sepsis helps in prognosticating the outcome. Female gender was an independent predictor of increased morality in critically ill patients with documented infection.

\section{References}

1. Martin GS, et al:: Crit Care Med 2006, 34:15-21

2. Ulloa L, et al:: Trends Mol Med 2005, 11:56-63.

3. Oberholzer A, et al:. Shock 2005, 23:488-493.

\section{P36}

Influence of TIMP-1/MMP-9 ratio on the severity and mortality in sepsis

L Lorente', M Martín², J Solé-Violán 3 , J Blanquer ${ }^{4}$, L Labarta 5 , C Díaz',

J Borreguero', J Páramo’

${ }^{1}$ Hospital Universitario de Canarias, Santa Cruz de Tenerife, Spain; ${ }^{2}$ Hospital Universitario Nuestra Señora de Candelaria, Santa Cruz de Tenerife, Spain; ${ }^{3}$ Hospital Universitario Dr Negrín, Las Palmas de Gran Canaria, Spain;

${ }^{4}$ Hospital Clínico Universitario de Valencia, Spain; ${ }^{5}$ Hospital San Jorge de Huesca, Spain: ${ }^{6}$ Hospital Insular, Las Palmas de Gran Canaria, Spain 'CIMA-University of Navarra, Pamplona, Spain

Critical Care 2010, 14(Suppl 1):P36 (doi: 10.1186/cc8268)

Introduction The role of matrix metalloproteinases (MMPs) and tissue inhibitors of matrix metalloproteinases (TIMPs) in sepsis remains unclear. 
Previously reported have been higher circulating levels of TIMP-1 in septic patients than in controls [1], higher PAI-1 levels in nonsurviving than in surviving septic patients [2], and a positive correlation between TIMP-1 and PAI-1 in apparently healthy adult individuals [3]. The objective of this study was to determine the association between TIMP-1/MMP-9 ratio and PAI-1, and its influence on the severity and mortality of patients with sepsis.

Methods This was a multicenter, observational and prospective study carried out in six Spanish ICUs. We included 192 (125 surviving and 67 nonsurviving) patients with severe sepsis. Serum levels of MMP-9, TIMP-1 and plasma levels of PAl-1 were measured at the time of diagnosis. Results Nonsurviving septic patients presented higher levels of TIMP-1 $(P<0.001)$, higher TIMP-1/MMP-9 ratio $(P<0.001)$, higher levels of PAI-1 $(P<0.001)$, higher SOFA score $(P<0.001)$, higher lactic acid levels $(P<0.001)$ and lower platelet count $(P<0.001)$ than surviving ones. TIMP-1/MMP-9 ratio positively correlated with PAl-1 $(P<0.001)$, lactic acid levels $(P<0.001)$, SOFA score $(P<0.001)$, INR ratio $(P<0.001)$, aPTT $(P<0.001)$, and negatively with platelet count $(P<0.001)$. The mortality prediction by TIMP-1/MMP-9 ratio (AUC $=0.66 ; 95 \% \mathrm{Cl}=0.58$ to $0.74 ; P<0.001$ ) was similar to that provided by other markers of severity such as lactic acid (AUC $=0.67 ; 95 \% \mathrm{Cl}=0.58$ to $0.75 ; P<0.001$ ) and SOFA score (AUC $=0.71 ; 95 \% \mathrm{Cl}=0.64$ to $0.79 ; P<0.001$ ). Conclusions The TIMP-1/MMP-9 ratio may be of great pathophysiological significance in terms of severity and mortality in sepsis, and may represent a biomarker to predict the clinical outcome of septic patients. The novel finding of our study is the association between TIMP-1/MMP-9 ratio and PAI-1 levels in sepsis, which can contribute in the organ dysfunction and mortality of sepsis.

Acknowledgements Study supported, in part, by the grants FUNCISPI-42/07 and GTEI-SEMICYUC-2009.

\section{References}

1. Hoffmann U, et al: Scand J Infect 2006, 38:867-872.

2. Hermans PW, et al: Lancet 1999, 354:556-560.

3. Aznaouridis K, et al.: Atherosclerosis 2007, 195:212-215

\section{P37}

Cytokine response in severe sepsis: predicting and modelling the course of illness

J Malaska', M Kratochvil' ${ }^{2}$, M Kyr $^{2}$, P Jabandziev², F Otevrel ${ }^{2}$, K Muriova² $^{2}$, M Fedora ${ }^{2}$, V Sramek ${ }^{3}$, J Michalek², P Sevcik

'University Hospital Brno, Czech Republic;' University Hospital Brno and Faculty of Medicine Masaryk University, Brno, Czech Republic; 'St Anne's

University Hospital Brno and Faculty of Medicine Masaryk University, Brno, Czech Republic

Critical Care 2010, 14(Suppl 1):P37 (doi: 10.1186/cc8269)

Introduction Severe sepsis remains one of the most threatening conditions in intensive care. During the progression of sepsis from early hit to multiorgan failure proinflammatory and anti-inflammatory cytokines are released. Cytokines can be used as biomarkers to determine the specific patterns of sepsis progression and association with mortality [1]. These biomarkers were successfully used as predictors in animal studies [2]. Data from humans, especially comparison between children and adults, are limited. Hence, in this study we widely describe systemic cytokine response in this type of patient population.

Methods Prospective study of 37 subjects (20 children, 17 adults) hospitalized with severe sepsis in intensive care. We measured CRP, procalcitonin, TNF, IL-1 $\beta$, IL-4, IL-6, IL-8, IL-10, IL-12, TREM-1. ANOVA models were specified using Proc Mixed. Study was fully approved by an ethics committee.

Results We identified a correlation of CRP levels with mortality or presence of shock (Figure 1). We found a distinct feature of CRP in adults with pronounced dynamic dichotomy in these subjects. Levels of IL-6 were significantly different in adult patients in the context of shock states. High IL-6 levels in the beginning of sepsis were associated with shock during progression of illness. Highest risk of death was in adult patients associated with TREM-1 sustained high after 48 hours after sepsis onset (Figure 2). Otherwise, there was no correlation with death, shock states and SOFA score for PCT, TNF, IL-1 $\beta, I L-4, I L-8, I L-10$, and IL-12.

Conclusions Response of circulating factors in patients with severe sepsis is heterogeneous in the adult and children population and has some distinct features according to the dynamics of CRP, IL-6 and TREM-1.

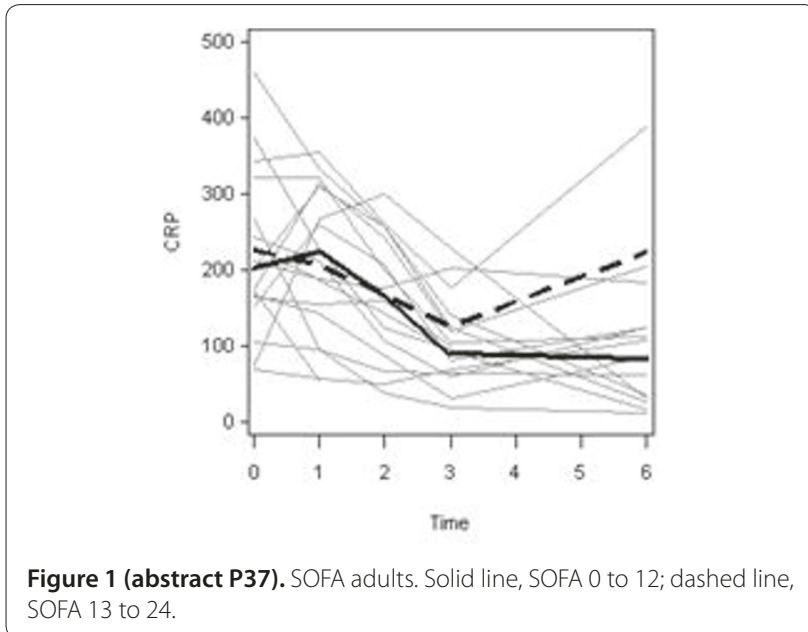

SOFA 13 to 24

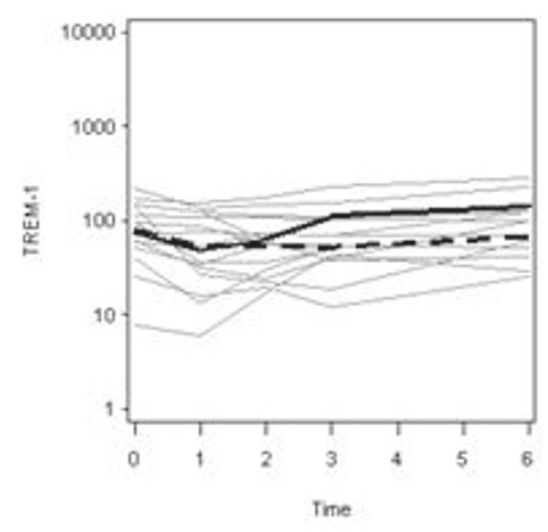

Figure 2 (abstract P37). Adults. Solid line, exitus; dashed line, survived.

Acknowledgements Supported in part by the Internal Grant Agency of the Ministry of Health NR 9297-3 and NR 9894-4.

References

1. Kellum JA, et al:: Arch Int Med 2007, 167:1655-1663.

2. Osuchowski et al:: Crit Care Med 2009, 37:1567-1573.

\section{P38}

Time course of IL- 6 and LBP, candidate biomarkers of sepsis in surgical critical care

E Kipnis, L Ogé, B Soudan, B Leroy, B Vallet, G Lebuffe Centre Hospitalier Régional Universitaire de Lille, France

Critical Care 2010, 14(Suppl 1):P38 (doi: 10.1186/cc8270)

Introduction The outcome of sepsis in critically ill patients relies on rapid identification and therapy. However, identification of sepsis in these patients is challenging, and focus has shifted towards biological surrogates as diagnostic/therapeutic biomarkers. IL-6 and lipopolysaccharide binding protein (LBP) may have such a potential.

Methods Retrospective analysis of daily IL-6 and LBP serum levels over the course of a 3-month laboratory feasibility study of large-scale chemiluminescent immunometric assays on unused residuals of routine patient blood samples from the surgical critical care unit. We determined the time course of IL- 6 and LBP over septic events defined as 4 days before and after the initiation of antimicrobial therapy as day $0(n=40)$. Variations over time of IL-6 and LBP in both absolute values and amplitude were measured between day -4 to day 0 and day 0 to day +4 . Candidate biomarker variations were assessed for coherence with clinical evolution of sepsis to determine biomarker-like behavior. 
Results Only amplitudes of variation were coherent with clinical evolution of sepsis rather than variations in absolute values or values relative to published thresholds. Coherence between patient evolution and biomarker time course was 93\% for IL-6 and 85\% for LBP. IL-6 levels always increased to over at least $50 \%$ of baseline (51 to $2,523 \%$ ) before initiation of antibiotics. IL- 6 decreased by at least $45 \%$ (45 to $98 \%$ ) of the maximal value when sepsis resolved successfully with antimicrobial therapy in $92 \%$ of the cases. Stagnating IL-6 levels always paralleled absence of resolution of sepsis. No pre-antimicrobial initiation increase of LBP was observed. LBP always decreased by at least 16\% (16 to 85\%) of the maximal value when sepsis resolved successfully with antimicrobial therapy. LBP increase after initiation of antimicrobials or LBP stagnation paralleled absence of clinical resolution. Concordant patterns of both IL- 6 and LBP were always coherent with clinical evolution but only concerned $35 \%$ of the cases.

Conclusions IL- 6 and LBP behave as biomarkers over the course of sepsis in surgical critical care patients. Only dynamic monitoring may yield any information relevant to diagnosis or treatment follow-up. It remains to be shown how well these biomarkers perform through other study designs.

\section{P39}

Prognostic value of melatonin in patients with sepsis: a comparison of survivors and nonsurvivors

K Alan, D Memis, G Altun, N Sut

Trakya University, Edirne, Turkey

Critical Care 2010, 14(Suppl 1):P39 (doi: 10.1186/cc8271)

Introduction Our objective was to investigate the plasma levels of melatonin and the prognostic value of these markers in patients with sepsis. This is a prospective study of 131 patients over 22 years old with sepsis in the ICU.

Methods The patients were divided into two groups containing 72 patients as survivors (Group I) and 59 patients as nonsurvivors (Group II). The blood collection was performed in the night (02.00 to 04.00 hours) and the Acute Physiologic and Chronic Health Evaluation II (APACHE II) score calculated on the day of hospitalisation, second day and the last day (discharge or death). The samples were centrifuged methodically and plasma samples were preserved at $-200^{\circ} \mathrm{C}$. After reaching the target quantity, all plasma samples were studied with RIA method at room temperature.

Results Statistically significant high APACHE II values were determined in Group II compared with Group I on the all days $(P<0.05)$. Statistically significant low nocturnal plasma melatonin values were determined in Group II compared with Group I on the last day $(P<0.05)$. In Group II, duration of mechanical ventilation and stay of ICU are longer, and at the last days of biochemistry parameters failed (elevation of urea, creatinine, serum glutamic oxaloacetic transaminase and serum glutamic pyruvic transaminase) $(P<0.05)$

Conclusions We conclude that from plasma melatonin values it is impossible to predict the prognosis but during the follow-up these values can demonstrate the status of prognosis in septic patients. There are several factors affecting these melatonin values; thereby we propose that further research should be performed.

P40

Serum C-reactive protein as a prognostic variable in elective surgery ICU patients: especially valuable following esophagectomy M Van Genderen, H De Geus, D Gommers, IVan Bomme Erasmus MC, Rotterdam, the Netherlands

Critical Care 2010, 14(Suppl 1):P40 (doi: 10.1186/cc8272)

Introduction Serum C-reactive protein (CRP) is synthesized in response to inflammatory status. Elevated CRP levels are associated with an increased risk of multiorgan failure in ICU patients. Preoperative elevation of serum CRP is a prognostic indicator in gastric and colorectal surgery. The aim of this study was to determine serum CRP as prognostic variable in patients undergoing esophagectomy with gastric tube reconstruction in contrast to other ICU admitted patients.

Methods Data were collected retrospectively for a total of 208 patients admitted to the ICU following elective surgery from October 2007 to December 2008. Patients included underwent esophagectomy with

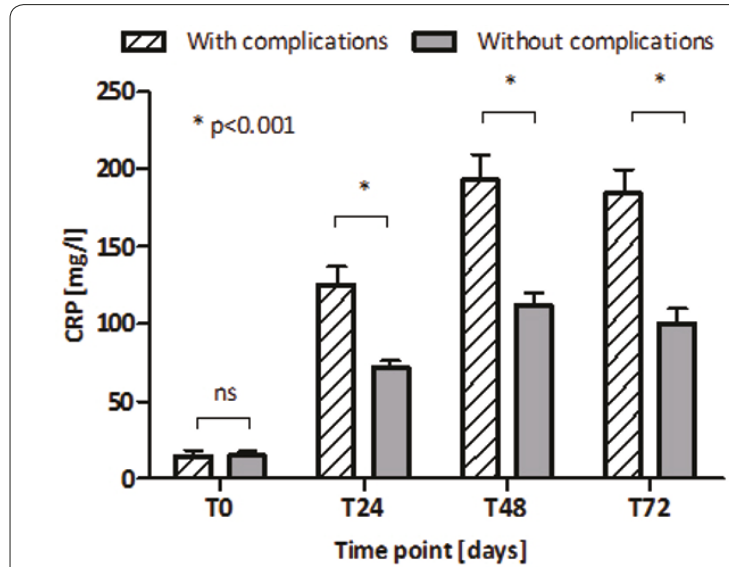

Figure 1 (abstract P40). CRP and complications in all groups.

gastric tube reconstruction, liver transplantation, hemihepatectomy, neuro- and abdominal aneurysm surgery. Postoperative serum markers were measured and the relation between the course of postoperative serum CRP, development of complications and prognosis of the patients was investigated.

Results Postoperative serum CRP was significantly higher at T24 in patients undergoing esophagectomy with gastric tube reconstruction, compared with all other patients. Higher serum CRP levels correlated with the occurrence of complications in the heterogeneous ICU population but especially in the esophagectomy patients. Within this group, serum CRP levels at T24 and T48 were significantly higher in patients with a postoperative pneumonia, which in itself was associated with increased 1 -year mortality.

Conclusions Postoperative serum CRP levels can easily be monitored in the ICU in order to identify patients at risk for the development of postoperative complications. Especially in the esophagectomy patients, the occurrence of postoperative complications is associated with reduced survival.

P41

Role of procalcitonin as a diagnostic and prognostic marker of infectious diseases in the emergency department: preliminary data L Magrini, V Mura, F Travaglino, O Piras, E Ferri, S Di Somma

A. O. S.Andrea, Rome, Italy

Critical Care 2010, 14(Suppl 1):P41 (doi: 10.1186/cc8273)

Introduction An observational study was conducted in a teaching hospital to evaluate the diagnostic and prognostic value of procalcitonin together with C-reactive protein (CRP) in assessing the presence of infection/sepsis in patients arriving in the emergency department (ED). The objective was to improve diagnostic and therapeutic time to intervention by the emergency physician.

Methods Three hundred and five patients were studied (145 male, 160 female, mean age 67.2 years). Vital parameters were recorded during patient's stay in the ED. Hemocultures and biological fluids cultures were performed before antibiotic therapy. CRP and procalcitonin were performed at arrival in the ED, and after 5 days of antibiotic therapy.

Results Median PCT and CRP values at arrival in the ED were $0.3 \mathrm{ng} / \mathrm{ml}$ and $12.9 \mathrm{mg} / \mathrm{dl}$, after 5 days they were $0.09 \mathrm{ng} / \mathrm{ml}$ and $4.2 \mathrm{mg} / \mathrm{dl}$ with a significant statistical difference in both markers $(P<0.001)$. PCT was higher in sepsis vs infections (respiratory, urinary, gut or skin infections) (3.1 vs $0.2 \mathrm{ng} / \mathrm{ml}, P<0.001$ ). PCT pre-therapy was significantly higher in septic nonsurvivors vs septic survivors ( $1.0 \mathrm{vs} 0.2 \mathrm{ng} / \mathrm{ml}, P<0.001)$, and there was a significant $\mathrm{PCT}$ increase after 5 days in nonsurvivors vs survivors (3.6 vs $0.07 \mathrm{ng} / \mathrm{ml})(P<0.001)$. CRP results did not show a statistical significance, except for CRP measured after 5 days $(3 \mathrm{mg} / \mathrm{dl})$ vs CRP pre-therapy in survivors $(12 \mathrm{mg} / \mathrm{dl}, P<0.001$ ). PCT (cut-off $0.5 \mathrm{ng} / \mathrm{ml}$ ) ROC curve showed an AUC $0.72(P<0.001)$, with a sensitivity $75 \%$ as a diagnostic marker for 

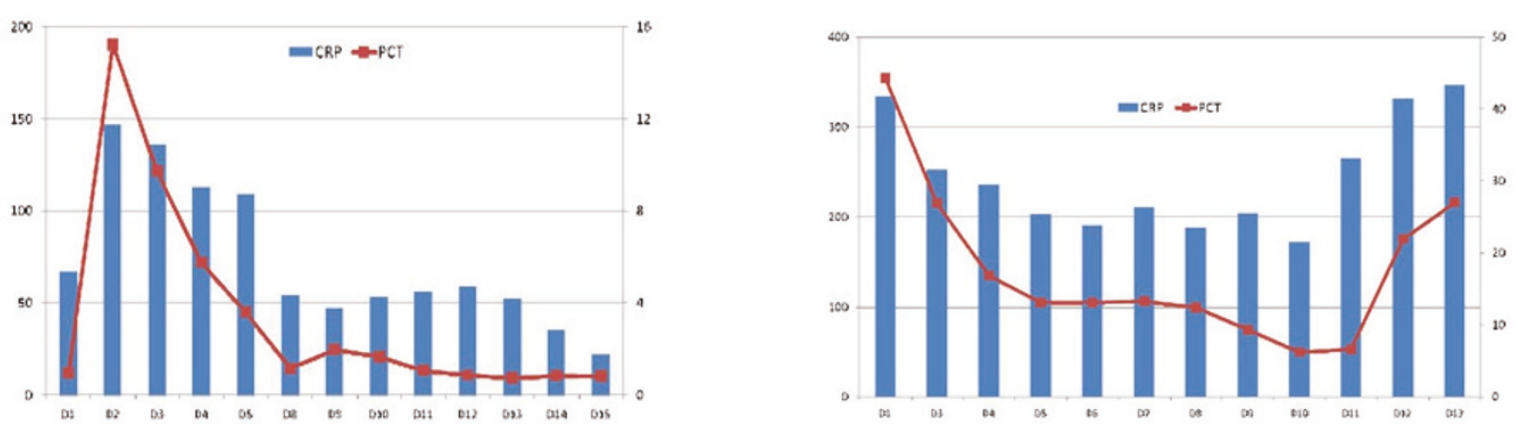

Figure 1 (abstract P42). Example 1 (left): sepsis secondary to hydronephrosis. This graph clearly shows the rise in procalcitonin (PCT) during early onset of sepsis and its fall during antibiotic treatment. Antibiotics were discontinued when the PCT level fell to $80 \%$ of its peak value on day 8 . Example 2 (right): laparoscopic cholecystectomy complicated by bile peritonitis. Good response to antibiotics initially, but PCT started to rise on day 12 which prompted us to change antibiotics. Serial PCT is useful to assess the response to treatment.

infection. In septic patients, PCT ROC curve had an AUC $0.70(P<0.001)$ if considered as a prognostic factor of death (cut-off $1 \mathrm{ng} / \mathrm{ml})$. Although pre-therapy PCT values were not particularly sensitive, nor specific for predicting deaths, PCT in therapy (cut-off $0.5 \mathrm{ng} / \mathrm{ml}$ ) had an AUC 0.79 (sensitivity $75 \%$, specificity $76 \%$ in predicting events) $(P<0.0001)$. This was higher than CRP (AUC 0.73, $P<0.0004$ ).

Conclusions $P C T$, with CRP, is useful to make an initial rapid infection diagnosis for patients presenting to the ED, and also to discriminate it from sepsis. Our results are in agreement with other authors [1,2]. A PCT cut-off value of $0.5 \mathrm{ng} / \mathrm{ml}$ shows high sensitivity as a diagnostic infection marker, but also as a prognostic marker for outcomes. PCT with CRP are useful tools to assess a fast empiric therapy, and to ameliorate the prognosis of infectious/septic in ED patients.

\section{References}

1. Hausfater P, et al:: Clin Infect Dis 2002, 34:895-901.

2. Balci C, et al:: Crit Care 2003, 7:85-90.

\section{P42}

Evaluation of procalcitonin at James Paget Intensive Care Unit A Brodbeck, N Elumogo, G Rajendran James Paget University Hospital NHS Foundation Trust, Great Yarmouth, UK Critical Care 2010, 14(Suppl 1):P42 (doi: 10.1186/cc8274)

Introduction Procalcitonin (PCT) offers the possibility of differentiating bacterial infection from viral or non-infectious reactions. PCT can be used to support decision-making on initiation or discontinuation or change of antibiotic therapy. Our aim was to determine the usefulness of PCT in our ICU. Methods PCT was measured once daily for all patients admitted to the ICU. Results were reviewed daily by intensivists and microbiologists and an appropriate decision about antibiotic therapy was made. We considered to stop antibiotics if the PCT was $<0.5 \mathrm{ng} / \mathrm{ml}$ or had decreased to $80 \%$ of the peak value. CRP and clinical progression were also taken into consideration. Results One hundred and fourteen PCT values were obtained from 27 patients over $18 \mathrm{ICU}$ days. PCT concentrations were lower than $0.5 \mathrm{ng} / \mathrm{ml}$ in 49 samples whilst CRP values were higher with a median of $107.5 \mathrm{mg} / \mathrm{l}$. Antibiotics were discontinued or not initiated following low PCT values in 38 of these samples despite high CRP. For two patients who were already on antibiotics, a rise in PCT was noticed which prompted us to change the antibiotics. No new microbiologically proven systemic infection was identified in any of the patients with low PCT values. See Figure 1.

Conclusions Frequently, discrepancy between PCT and other inflammatory markers were noticed, suggesting an inflammatory response or nonbacterial infection and not necessarily an indication for antibiotics. Regular assessment of PCT, when interpreted with clinical context, was helpful not only to decrease the duration of antibiotics but also to change the antibiotic regimen.

\section{References}

1. Shehabi Y, et al:: Crit Care 2008, 12:211.

2. Hochreiter M, et al.: Crit Care 2009, 13:R83.

\section{P43}

Procalcitonin facing the postoperative culture results after liver transplantation

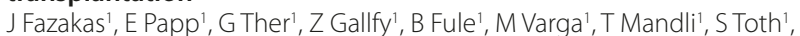

J Fazakas², L Kobori', M Arkosy ${ }^{3}$

'Semmelweis University, Budapest, Hungary; ${ }^{2 B a j c s y}$ Zsilinszky Hospital,

Budapest, Hungary; ${ }^{3}$ Soprponi Erzsébet Oktató Kórház, Sopron, Hungary

Critical Care 2010, 14(Suppl 1):P43 (doi: 10.1186/cc8275)

Introduction The early inflammatory response after liver transplantation (LTX) could be diagnosed by the simultaneous interpretation of procalcitonin (PCT) levels and culture results. The aim of this prospective, non-interventional study was to evaluate the prognostic significance of the PCT levels in the term of etiology (infection, colonization and noninfectious inflammatory states).

Methods The PCT measurements and cultures were performed before surgery and on the first five postoperative days in 114 liver transplanted patients. The relationship between the absolute PCT levels $(n=762)$ and culture results $(n=474)$ was studied. The patients were divided into two groups according to the infection or colonization with Gram-positive (GP) or Gram-negative (GN) bacteria. Statistical analysis was done with the SPSS program.

Results In 21 patients negative cultures were associated with PCT elevation (PCT: $3.77 \pm 3.22 \mathrm{ng} / \mathrm{ml}$ ), positive cultures were found in 107 patients, in 58 patients this was colonization (PCT $<0.5 \mathrm{ng} / \mathrm{ml}$ ). There significant differences between the PCT elevation according to the etiology culture (GN, $n=36$, PCT: $17.55 \pm 11.3 \mathrm{ng} / \mathrm{ml} ; \mathrm{GP}, n=222$, PCT: $16.54 \pm 6.58 \mathrm{ng} / \mathrm{ml} ; P<0.02)$.

Conclusions In the early phase after liver transplantation, the GP culture occurred more frequently; the GN culture could be associated with higher PCT elevation than those found in case of GP culture.

\section{P44}

Can follow-up procalcitonin measurements shorten the periods of antibiotic administrations for septic patients?

TWada, A Hagiwara, R Sasaki, T Sato, K Kobayashi, A Inaka, T Sakamoto,

Y Hagiwara, T Inagaki, K Katsuno, R Takegawa, A Kimura

International Medical Center of Japan, Sinjyuku Tokyo, Japan

Critical Care 2010, 14(Suppl 1):P44 (doi: 10.1186/cc8276)

Introduction To examine whether we can safely shorten the periods of antibiotic administration for septic patients with procalcitonin (PCT) measurements compared with no PCT measurements.

Methods The participants were septic patients including (1) hospitalized to our ICU from February 2009 to November 2009, (2) administered antibiotics for 4 days and over, and (3) stopped the antibiotics during their ICU stays. We treated the patients from February to June without PCT measurements (Group A). On the other hand, we treated the patients from July to November referring to serum PCT level (Group B). In group B, when 
the serum PCT level decreases under $0.5 \mathrm{ng} / \mathrm{ml}$, we stopped the antibiotic administrations if their systemic conditions were getting better.

Results Twelve patients were in Group A, and 15 patients were in Group B. The ages were $65 \pm 18$ in Group A, and $64 \pm 23$ in Group B. The APACHE II scores were $20.4 \pm 6.3$ in Group A, and $18.5 \pm 7.5$ in Group B. There were no significant differences. The periods of antibiotic administrations were $14.1 \pm 7.7$ days in Group A, and $9.7 \pm 6.0$ days in Group B. The periods in Group B tended to be significantly shorter than in Group A $(P=0.049)$. The C-reactive protein (CRP) levels were $3.5 \pm 4.2 \mathrm{mg} / \mathrm{dl}$ in Group A, and $6.5 \pm 4.1$ in Group B when the antibiotic administrations were stopped. The CRP levels in Group B were higher than in Group A ( $P=0.071)$. Two patients in Group B were repeated antibiotic administrations, while they were discharged without any complications.

Conclusions The follow-up PCT measurements safely shorten the periods of antibiotic administrations for septic patients. We believe that PCT has good cost-benefit effects for critically ill patients.

P45

Efficiency diagnostic and advantages of procalcitonin and C-reactive protein in the early diagnosis of sepsis

M De la Torre-Prados, A García-de la Torre, A García-Alcántara,

C Ortíz-García, A Enguix-Armada

Hospital Virgen de la Victoria, Málaga, Spain

Critical Care 2010, 14(Suppl 1):P45 (doi: 10.1186/cc8277)

Introduction The goal of our study is to assess the diagnostic profitability of procalcitonin (PCT) in septic shock and another biomarker as C-reactive protein (CRP)

Methods Case-control study. During 2009 we proceeded to select the sample: 54 patients (ICU) and 61 controls. PCT and CRP were set in the first 24 hours besides the diagnosis and other analytical (glucose, lactate, leukocytes, platelets, white blood cells, LIC cells and ALY cells) and clinical parameters. There were realized diagnostic output curves (ROC), area under the curve (AUC), confidence interval (95\%), cut-off and the comparison between AUC

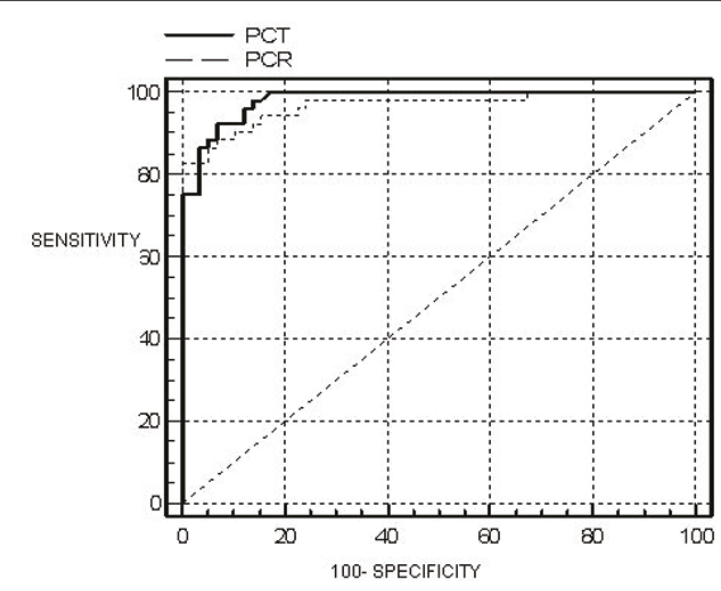

Figure 1 (abstract P45). Comparison between AUC not significantly different for PCT and CRP $(P=0.445)$.

Table 1 (abstract P45). Output curve results: PCT/CRP, sensitivity, specificity

\begin{tabular}{lcc}
\hline & PCT & CRP \\
\hline Mean & $24 \mathrm{ng} / \mathrm{ml}$ & $227 \mathrm{mg} /$ day \\
AUC & 0.983 & 0.965 \\
$95 \% \mathrm{Cl}$ & 0.93 to 0.99 & 0.91 to 0.99 \\
Sensitivity & $92.6 \%$ & $91.1 \%$ \\
Specificity & $93.1 \%$ & $100 \%$ \\
\hline
\end{tabular}

Results Fifty-four septic patients were assessed, 66\% were males; mean age, 63 years. Eighty-eight percent was diagnosed as septic shock and $11 \%$ severe sepsis. Seventy-six percent were medical patients. Positive blood cultures in $42.5 \%$. Sepsis origin: respiratory $46 \%$, neurological $5 \%$, digestive $37 \%$ and urinary $3 \%$. Average SOFA score was 10.4 .

Conclusions PCT and CRP have the same efficiency in early sepsis diagnosis. The PCT and CRP efficiency diagnostic together is significant but small. We suggest using both with the doubt of sepsis.

P46

\section{Biomarkers of sepsis in burn patients}

H Oueslati', K Bousselmi', I Rahmani', A Mokline', J Haddad', Y Yahyia',

R Ghanem², A Messadi'

'Burn and Trauma Center, Tunis, Tunisia; ${ }^{2}$ Microbiology Department, Tunis, Tunisia

Critical Care 2010, 14(Suppl 1):P46 (doi: 10.1186/cc8278)

Introduction Sepsis is still the major cause of death in the late posttraumatic period in patients with major burns. Diagnosis of sepsis remains difficult in these patients where signs of sepsis may be present in the absence of real infection. This study attempted to assess whether the plasma procalcitonin (PCT) level was related to sepsis in burned patients. Methods PCT was measured over the entire course of stay in patients with predictive signs of sepsis according to Burn French Society Criteria (SFETB) for the presence of infection. The patients were assigned to two groups depending on the clinical course and outcome: $A$ = no septic patients, $B=$ septic patients. Optimum sensitivity, predictive values, and area under the receiver operating characteristic $(\mathrm{ROC})$ curve were evaluated.

Results Over a 6-month period starting from 1 July 2008 to 31 December 2008, 157 patients were admitted. Sixty-two were investigated, 40 in group $\mathrm{A}$ and 22 in group B. Procalcitonin was significantly higher in the septic group $(7.26 \pm 7 \mathrm{ng} / \mathrm{ml})$ compared with the no septic group $(0.25 \pm 0.32 \mathrm{ng} /$ $\mathrm{ml}$ ). Area under the curve was 0.94 on the day of sepsis diagnosis. A PCT cut-off value of $0.75 \mathrm{ng} / \mathrm{ml}$ was associated with the optimal combination of sensitivity (85\%), specificity (87\%), positive predictive value (91\%), and negative predictive value (73\%). In surviving septic patients the PCT value was significantly lower than in deceased septic patients $(3.5 \pm 0.87 \mathrm{ng} /$ $\mathrm{ml}$ vs $10.18 \pm 9.6 \mathrm{ng} / \mathrm{ml})$. The PCT cut-off value for optimum prediction of outcome in septic patients was $3.66 \mathrm{ng} / \mathrm{ml}$ with sensitivity (91\%), specificity $(75 \%)$, positive predictive value $(78 \%)$, and negative predictive value (90\%).

Conclusions Procalcitonin appears to be a powerful marker of sepsis in burn patients. It is sensitive, specific, reliable and easy to measure. A high PCT concentration (>3.66 ng/ml) would indicate poor outcome in septic patients.

References

1. Gramm HJ, et al:: A biological marker of the inflammatory response with prognostic properties. Clin Intens Care 1995, 6(Suppl):71.

2. Zeni F, et al:: Procalcitonin serum concentrations and severity of sepsis. Clin Intens Care 1994, 5(Suppl):2.

\section{P47}

Age-related differences of outcomes in patients hospitalized with community-acquired pneumonia are not explained by differences in immune response

S Kale, S Yende, L Kong, A Perkins, JA Kellum, AN Vallejo, AB Newman, DC Angus

University of Pittsburgh School of Medicine, Pittsburgh, PA, USA

Critical Care 2010, 14(Suppl 1):P47 (doi: 10.1186/cc8279)

Introduction We hypothesized that the higher risk of severe sepsis and mortality observed after infection in prior epidemiologic studies are due to age-related differences in immune response.

Methods We analyzed 2,183 subjects from a multicenter observational cohort of patients hospitalized with community-acquired pneumonia (CAP). We divided subjects into five age groups $(<50,50$ to 64,65 to 74 , 74 to 84,285 ). To compare immune response, we measured circulating inflammatory (IL-6 and IL-10), coagulation-fibrinolysis (D-dimer, factor IX (F-IX), thrombin-antithrombin complex (TAT), antithrombin (AT) and plasminogen-activator inhibitor (PAI)), and cell surface markers (TLR2, 

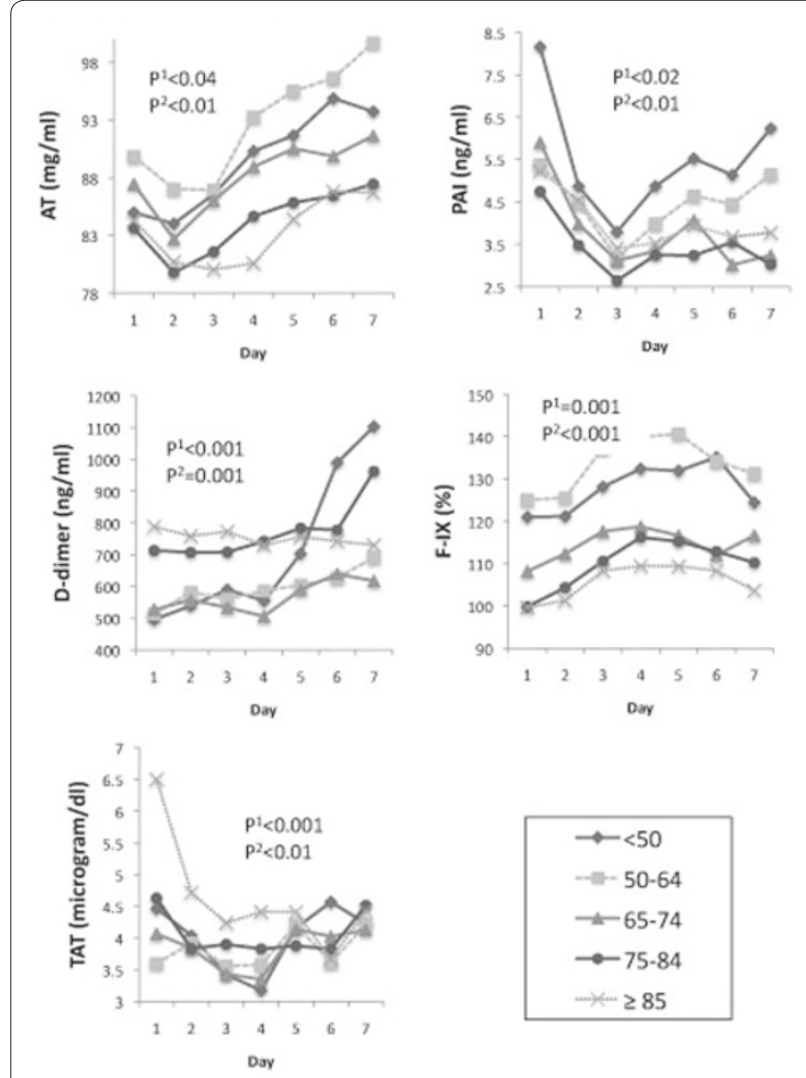

Figure 1 (abstract P47). Modest differences in circulating concentration of coagulation-fibrinolysis markers on ED presentation (day 1) and over the first 7 days. $P^{1}$, day $1 P$ value from ANOVA model adjusted for sex, race, co-morbidity, and smoking status; Tobit models used for censored data. $P^{2}$, day 1 to $7 P$ value from mixed effects model adjusted for sex, race, comorbidity, and smoking status; Tobmit models used for censored data.

TLR4, HLADR) on emergency department (ED) presentation and during the first week of hospitalization.

Results Older subjects had higher rates of severe sepsis (14.8\%, 23.0\%, $26.3 \%, 34.0 \%, 42.3 \%)$ and 90 -day mortality $(1.8 \%, 3.8 \%, 9.2 \%, 18.2 \%, 19.8 \%$, per age group $(P<0.001))$. However, there were no age-related differences in inflammatory or cell surface markers and only modest differences in coagulation-fibrinolysis abnormalities on ED presentation and over the first week $(P<0.05)$ (Figure 1).

Conclusions Despite large differences in outcomes, patients hospitalized with CAP displayed no age-related differences in circulating inflammatory or cell surface markers and modest differences in circulating coagulationfibrinolysis markers.

P48

Cost-effectiveness of a rapid and accurate test for diagnosing infection in severe sepsis and septic shock patients

TWalke', D Chalfin', J Lee', E Rivers ${ }^{3}$

'Altarum Institute, Ann Arbor, MI, USA; '2 Abbott Diagnostics, Abbott Park, IL,

USA; ${ }^{3}$ Henry Ford Hospital, Detroit, MI, USA

Critical Care 2010, 14(Suppl 1):P48 (doi: 10.1186/cc8280)

Introduction Prompt and accurate pathogen identification is crucial for treatment of severe sepsis and septic shock (SS/SS). Blood cultures (BC) require 24 to 48 hours and often yield inaccurate results. Effective pathogen identification allows for timely, targeted antimicrobial therapy.

Methods A SS/SS Markov model [1] was developed to assess the costeffectiveness (CE) of a rapid and accurate diagnostic test compared with the current standard. In the model, patients can transition between no SIRS,

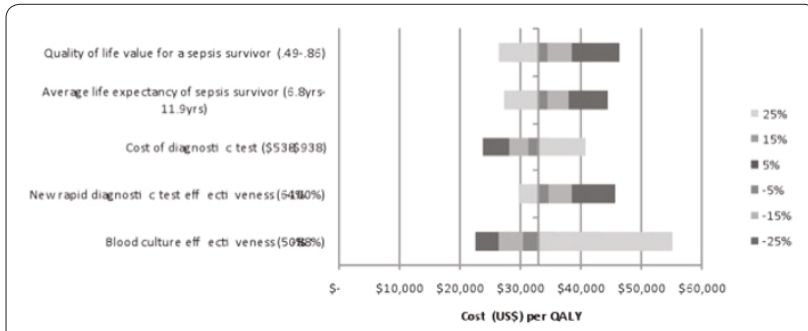

Figure 1 (abstract P48). Sensitivity analysis on CE (Base Case CE = $\$ 32,939 / \mathrm{QALY})$.

SIRS, sepsis, severe sepsis and septic shock or exit the model via discharge or death. Key literature assumptions are as follows: $91 \%$ effectiveness of empiric therapy, 70\% BC effectiveness, 90\% new test effectiveness, \$750 cost/test, 9.5 years average life expectancy of sepsis survivor, 0.69 qualityadjusted life-year (QALY) for survivors, and turnaround times of 24 hours for the new test and 48 hours for standard BC.

Results The model shows that the new test would be cost-effective when compared with current BC, at a cost of $\$ 32,939$ per QALY (day 28). These results are compelling across a range of assumptions and almost all estimates fall below the US CE threshold of $\$ 50,000$ to $\$ 100,000 /$ QALY (Figure 1). Varying most assumed values by up to $25 \%$ had a moderate impact on CE. CE is sensitive to empiric test effectiveness $>95 \%$. More rapid diagnostic information lowered the cost/QALY.

Conclusions Preliminary results show that a rapid and accurate test would be considered cost-effective across many assumptions and would reduce the mortality associated with delayed diagnosis.

\section{References}

1. Rangel-Frausto et al:: Clin Infect Dis 1998, 27:185.

\section{P49}

A multicentre study of bacteraemia using a new commercial universal 16S rDNA PCR test

S Sakka', N Wellinghausen², A Kochem'1, C Disque ${ }^{3}$, H Mühl'3 , S Gebert², J Winter ${ }^{2}$, J Matten ${ }^{4}$

${ }^{1}$ Medical Center Cologne-Merheim, Cologne, Germany; ${ }^{2}$ Universitätsklinikum Ulm, Germany; ${ }^{3}$ Hochschule Bremerhaven, Germany: ${ }^{4}$ MVZ Leverkusen, Cologne, Germany

Critical Care 2010, 14(Suppl 1):P49 (doi: 10.1186/cc8281)

Introduction Bloodstream infection is a life-threatening condition with a high mortality rate, especially in intensive care and neutropenic patients. Standard diagnostics is based on blood culturing (BC). However, limitations of $B C$ include relatively low sensitivities and a long time-toresult for the identification of the pathogen, generally over 2 days and more. On the grounds of data from a multicentre study using a universal 16S rRNA gene PCR assay, SepsiTest ${ }^{\mathrm{TM}}$, molecular diagnosis is discussed as a rapid and sensitive tool for the detection and identification of pathogens supportive of BC. A new commercial PCR test, SepsiTest $t^{T M}$, for direct detection of bacteria in whole blood was compared with $B C$ in terms of sensitivity, specificity, predictive values and time to positivity (TTP) of bacterial infections of the bloodstream of critically ill patients.

Methods The test, SepsiTest ${ }^{\text {TM }}$ (Molzym, Bremen), comprises the extraction and $16 \mathrm{~S}$ rRNA gene PCR detection of bacterial DNA in whole blood samples. Bacteria in positive samples were identified by sequence analysis of the amplicon. In a prospective multicentre study, 342 blood samples from 187 patients with systemic inflammatory response syndrome (SIRS), sepsis, or neutropenic fever were included.

Results Compared with $\mathrm{BC}$, the diagnostic sensitivity and specificity of PCR/sequencing was $87.0 \%$ and $85.8 \%$, respectively. The positivity rate of PCR/sequencing (25.7\%) was higher than BC (15.8\%). Of 31 PCR/ sequencing-positive, BC-negative patients, most of whom received antibiotics, the PCR results of 25 were judged as true or possible to bacteraemia. Using a routine testing workflow, time to positivity of the PCR test was on average decreased by 40 hours for anaerobe/fastidious infections and by 54 hours for yeast infections. 
Conclusions The PCR approach enables the detection and identification of bacteraemia in blood samples within a few hours. Despite the indispensability of $\mathrm{BC}$ diagnostics, the rapid detection of bacteria by SepsiTest ${ }^{T M}$ appears to be a valuable tool, allowing earlier pathogenadapted antimicrobial therapy in critically ill patients.

P50

\section{Time to antibiotics in sepsis}

R Appelboam, RTilley, J Blackburn

Royal Devon and Exeter Hospital, Exeter, UK

Critical Care 2010, 14(Suppl 1):P50 (doi: 10.1186/cc8282)

Introduction Mortality from sepsis increases with delay in administration of antibiotics. The Surviving Sepsis Guidelines suggest that antibiotics are administered within 1 hour of diagnosis of severe sepsis and septic shock. This retrospective study investigated the time to appropriate antibiotics in patients with sepsis and identifies reasons for delay where possible.

Methods All adult patients in our institution who had positive blood cultures in 2008 were identified. Included were patients with sepsis, severe sepsis, or septic shock. Excluded were the isolation of low-pathogenicity organisms from blood culture (for example, coagulase-negative Staphylococcus). Case notes were reviewed to identify the time between diagnosis of sepsis and administration of antibiotics. Time zero was taken as the time recorded in the notes by the doctor that the patient was seen. Times of prescription and administration of antibiotics were taken from the prescription chart.

Results There were 1,477 sets of positive blood cultures in 1,098 patients. Following exclusions there were 503 episodes of positive blood cultures in 494 patients. Three hundred and seventy-five fulfilled the criteria and were analysed: 52 did not fulfil entry criteria, in 16 cases sepsis was not suspected, and 60 sets of notes were incomplete or unavailable. Average age was 69 years. Mean time between diagnosis and antibiotic administration was 8.1 hours, median 4 hours. A delay of 3 hours or more between diagnosis and administration of antibiotics occurred in 218 cases (58\%). The 28-day mortality for all patients overall was $21 \% ; 19 \%$ in the no-delay group compared with $24 \%$ in the delayed group. The 1-year mortality was $35 \%$ for both groups. Causes of delay in administration included prescription error, awaiting senior review, and patient movement between departments.

Conclusions These retrospective data demonstrate that there is a delay in administering antibiotics patients with sepsis. These data are likely to be an underestimation as an accurate time zero is difficult to ascertain.

\section{References}

1. Kumar A, Roberts D, Wood KE, et al.: Crit Care Med 2006, 34:1589-1596

2. Dellinger RP, Levy MM, Carlet JM, et al: Crit Care Med 2008, 36:296-327.

3. Bone RC, Sibbald WJ, Sprung CL: Chest 1992, 101:1481-1483.

\section{P51}

Utility of an antibiotic guideline in hospital-associated infections

M Shankar-Hari, D Wyncoll

Guy's and St Thomas' NHS Foundation Trust, London, UK

Critical Care 2010, 14(Suppl 1):P51 (doi: 10.1186/cc8283)

Introduction Prompt initiation of appropriate antibiotic therapy improves outcome in critically ill patients [1]. In a tertiary-level ICU, we evaluated the appropriateness of, and adherence to an antibiotic guideline (based on local bacterial epidemiology) in CDC-defined hospital-associated infections.

Methods We conducted a 6-month prospective observational study (April 2008 to October 2008) in consecutive ICU admissions of patients who satisfied investigator-adjudicated classification of ICU-acquired infections according to CDC criteria [2]. We assessed patient characteristics including severity of illness at admission, ICU length of stay (LOS), appropriateness of initial antibiotic choice as judged by in vitro sensitivity results and appropriateness of the current guideline. Results are presented as mean (SD) or median as appropriate.

Results During the study period there were 101 antibiotic starts in 65 patients with sepsis secondary to ICU-acquired infections. Medical patients formed $44 \%$ of the study cohort; whilst $23 \%$ of patients were general surgical and the remaining 33\% were post cardiothoracic surgery. The age and admission APACHE II score of the study cohort was 61.8 (16.3) years and 18.4 (5.6). The median LOS and ICU mortality of the cohort was 24 days and $27.6 \%$. The most common CDC reportable diagnosis was clinical or microbiological confirmed pneumonia (PNU1/PNU2/LRI) $(n=57)$, followed by intra-abdominal infection (SSI-GIT) $(n=10)$ and urinary tract infection (SUTI) $(n=8)$. The culture positivity rate was $71.2 \%$. The appropriateness of the ICU antibiotic guideline is summarised in Table 1. Monotherapy was used in $52.5 \%$ of episodes. The median length of antibiotic treatment with positive cultures was 7 days, and 5 days for culture negative episodes. In sepsis episodes with negative culture, antibiotics were stopped within 3 days in $17 \%$ of the episodes.

Table 1 (abstract P51). Appropriateness of guideline-based antibiotic therapy

\begin{tabular}{lccc}
\hline Parameter & $\begin{array}{c}\text { Septic shock } \\
(\boldsymbol{n}=\mathbf{3 5})\end{array}$ & $\begin{array}{c}\text { Severe sepsis } \\
(\boldsymbol{n}=\mathbf{3 0})\end{array}$ & $\begin{array}{c}\text { Sepsis } \\
(\boldsymbol{n}=\mathbf{3 6})\end{array}$ \\
\hline Positive cultures & $23(66 \%)$ & $25(83 \%)$ & $24(67 \%)$ \\
Appropriateness & $18 / 23(78 \%)$ & $21 / 25(84 \%)$ & $14 / 24(58 \%)$ \\
\hline
\end{tabular}

Conclusions The study cohort had a high culture positivity rate $(71 \%)$ in ICU-acquired sepsis. Our antibiotic guidelines gave an optimal empiric initial therapy in over $74 \%$ of episodes, with more than $50 \%$ of antibiotics started being monotherapy.

References

1. Kumar et al: Crit Care Med 2006, 34:1589-1596.

2. Horan et al: Am J Infect Control 2008, 36:309-332.

\section{P52}

Assessment of antibiotic prescriptions in two French intensive care units

MP Parisot' ${ }^{1}$ CT Tassin², AL Lepape 2 , RG Gauzit

${ }^{1}$ Hotel Dieu, Paris, France; ${ }^{2}$ CHU Lyon Sud, Lyon, France

Critical Care 2010, 14(Suppl 1):P52 (doi: 10.1186/cc8284)

Introduction In the setting of antimicrobial policy, the French authorities recommend assessing the quality of antibiotic therapy [1]. The aim of this study is to assess the adequacy of antibiotic therapy in the ICU, to good practice guidelines and unit protocols. The three steps of prescription are analysed: choice of treatment, modalities of prescription and treatment follow-up.

Methods This preliminary observational trial was conducted in two ICUs (October 2005 to August 2006 and March to August 2009). All patients with curative antibiotherapy were prospectively included. For each patient, we collected individual data of treatment and we evaluated them according to the recommendations of good use published in the literature $[1,2]$.

Results One hundred and thirteen patients were included. Infections were community-acquired infection in 30\% of the cases. Respiratory tract infections were the most frequently encountered, followed by intraabdominal infections. The three most frequently isolated pathogens were P. aeruginosa, E. coli and S. aureus.

Initial antibiotic therapy, $n=113$ : lack of sample 14\%, inadequacy with unit protocol $13 \%$, too broad spectrum $7 \%$, not justified association $8 \%$, wrong posology $20 \%$, incorrect route of administration $1 \%$, wrong interval $6 \%$, lack of plasmatic dosage $(n=46) 7 \%$.

Treatment follow-up, $n=108$ : wrong delay of reappraisal 14\%, no adaptation to microbiological results $10 \%$, lack of de-escalation $5 \%$, no reassessment of posology $3 \%$, wrong posology (if change of treatment) $2 \%$, wrong interval (if change of treatment) $3 \%$, unjustified duration $10 \%$. Conclusions The rate of prescriptions that did not conform to at least one criterion in this study was over $70 \%$. This high rate is partly explained by the high number of assessment criterions $(n=15)$. The most frequent criterion of inadequacy was a wrong initial dosage, followed by the initiation of an antibiotherapy without a microbiologic sample, a wrong delay of reassessment, and a nonconformity of treatment to unit protocols. That last criterion must be assessed in a more qualitative way, as some transgressions could be justified.

\section{References}

1. Stratégie d'antibiothérapie et prévention des résistances bactériennes en établissement de santé [www.has-santefr]

2. Van der Meer JW, et al.: Clin Microbiol Infect 2001, 7(Suppl 6):12. 
P53

Occurrence of alert pathogens in the clinical materials and consumption of antibiotics in the ICU, in the years 2007 and 2008

KWawrzyniak, A Mikucka, A Deptuła, E Gospodarek, K Kusza

Nicolaus Copernicus University in Toruń, Collegium Medicum, Bydgoszcz,

Poland

Critical Care 2010, 14(Suppl 1):P53 (doi: 10.1186/cc8285)

Introduction A continuous pressure of antibiotics and chemotherapeutics in ICU patients promotes isolation of multidrug-resistant (MDR) strains. Treatment of infections caused by microorganisms resistant to available drugs often results in a therapeutic failure. The purpose of this work was a retrospective comparison of the rates of isolation of particular MDR pathogen groups in 2007 vs 2008 in ICU patients hospitalised in a university hospital 11-bed reference department, and of antibiotic consumption in the analysed years.

Methods Data concerning microbiological testing were analysed based on the WHONET software, version 5.4. The analysis rejected repeated identical culture results that did not reflect a subsequent case of infection with a given MDR pathogen, but only persistence of the same strain in a given patient.

Results MDR pathogen stains most frequently were isolated from the bronchoalveolar lavage (BAL) material. Carbapenem-resistant Gramnegative rods represented the highest rate $62 \%$ in 2007 and $64 \%$ in 2008); these included Pseudomonas aeruginosa strains (57\% and 35\%, respectively). Escherichia coli (11\% in 2007), Klebsiella pneumoniae (12\% in 2008) and Enterobacter cloacae (9\% in 2008) predominated among ESBL-producing intestinal rods. A minor increase of the rate of the ESBL(+) strains was found in 2008. Single MRSA strains were isolated in the analysis period, no VRE were cultured. The highest antimicrobial drug consumption (DDD/100 person-days) in 2007 pertained to meropenem (20.6), piperacillin/tazobactam (20.0), cephazolin and vancomycin (11.6 and 11.1). The respective values for the year 2008 were as follows: piperacillin/tazobactam (20.8), voriconazole (19.7), meropenem (14.6).

Conclusions A 47\% increase in the MDR pathogen isolation rate was found in the year 2008. MDR nonfermenting rods represent the largest therapeutic and epidemiological challenge among the isolated MDR pathogens. In the subsequent analysed year, first emergence of carbapenem-resistant Acinetobacter baumanii strains was noted.

\section{References}

1. Rossolini GM, Mantengoli E: Antimicrobial resistance in Europe and its potential impact on empirical therapy. Clin Microbiol Infect 2008, 14:2-8.

\section{P54}

Treatment cost of secondary peritonitis in Germany: a comparative study of medical cost incurred for tigecycline therapy and standard regimens

J Kresimon', U Theidel2 ${ }^{2}$, R Runge ${ }^{2}$, R Rychlik', W Krueger ${ }^{3}$

'Institute of Empirical Health Economic, Burscheid, Germany; ${ }^{2}$ Wyeth Pharma

$\mathrm{GmbH}$, Muenster, Germany; ${ }^{3}$ Clinics of Constance, Germany

Critical Care 2010, 14(Suppl 1):P54 (doi: 10.1186/cc8286)

Introduction Secondary peritonitis is the most frequent form of peritonitis, characterized by a high disease burden and a high mortality rate. Choice of adequate antibiotics is an independent factor for survival. The aim of this study was to compare treatment of secondary peritonitis with tigecycline (TG) with standard regimens (SR) from an economical standpoint.

Methods After ethics committee approval, the study was performed as prospective, non-interventional cohort trial in 23 medical centers in Germany. Patients could be included if suffering from severe secondary peritonitis treated in an ICU. Patients with pregnancy, aged below 18 years and milder forms of diseases (APACHE II score $<15$ ) were not eligible. In order to compare treatment with $\mathrm{TG}$ with $\mathrm{SR}$, the following data were documented: demographic data, disease severity scores, causing microorganisms, laboratory parameters, and length of stay (LOS). Patients were analysed according to initial antibiotic choice except for perioperative prophylaxis. In order to balance for differences in co-morbidities and severity of disease, a matched-pairs analysis was performed based on logistic regression analysis.

Results A total of 178 patients were enrolled (49 TG/129 SR). After logistic regression analysis and matching for gender, age \pm 3 years, APACHE
II score \pm 1 and (non)existence of liver cirrhosis, arterial sclerosis and coronary heart disease, 15 matched pairs were built. Compared with the SR group, in the TG group was a tendency for higher creatinine, urea and glucose levels, higher number of co-morbidities (3.3 vs 3.0, NS) and higher number of pathogens isolated on initial surgery ( $2.2 \mathrm{vs} 1.6, \mathrm{NS})$. There was a higher number of discharges 9/15 in the TG group (vs 7/15 SR, NS) and $6 / 15$ patients died (vs 4/15 SR, NS). Considering these factors, there was a trend for shorter LOS in patients treated with TG (11 days vs 18 days and 8 days vs 16 days for survivors and nonsurvivors, respectively, NS) and total costs of ICU stay were significantly lower in the TG group ( $€ 8,832$ vs $€ 15,482, P=0.023)$

Conclusions In our non-interventional study, tigecycline tended to be used in patients with more severe co-morbidities. In spite of this, there was a trend for shorter LOS and treatment costs were significantly lower, which make tigecycline an attractive treatment, also from a pharmacoeconomical standpoint.

\section{P55}

Colistin monotherapy versus therapy combination

B Charra, A Benslama, H Ezzouine, S Motaouakkil

CHU Ibn Rochd, Casablanca, Morocco

Critical Care 2010, 14(Suppl 1):P55 (doi: 10.1186/cc8287)

Introduction The purpose of our study was to compare the effectiveness of colistin monotherapy and colistin combination in the treatment of nosocomial infections with multi-resistant germs.

Methods Retrospective study including 63 patients realized during 3 years from January 2006 to December 2008 in the medical ICU of University Hospital Ibn Rochd, Casablanca, Morocco. The study includes only the patients who suffer from nosocomial infection with multi-resistant germs, all of the sites are concerned. The patients have been divided into groups: group 1 including 30 patients treated by colistin only, and group 2 including 33 patients treated by the association colistin-rifampicin. The colistin has been administered intravenously and/or in nebulization and/ or in an intrathecal way according to the considered infectious site. The main criterion of judgment was the rate of mortality in the resuscitation unit, the second criteria of judgment were the ventilator weaning, the introduction of the vasoactive drugs and the supervening of side effects. Results Sixty-three patients judged as appropriate, have been included. The mean age of the patients was about $43.62 \pm 17.34$ years and APACHE 2 score at the admission was about $15 \pm 5.69$. The total mortality caused by infection was about $41.27 \%$. The basic characteristics of the two groups were similar. The mortality in group 1 was about $36.66 \%$, and about $69.69 \%$ in group $2(P=0.001)$, the rate of introduction of vasoactive drugs was about $23.33 \%$ in group 1 versus $48.48 \%$ in group $2(P=0.03)$. In group $1,6,66 \%$ of the patients developed renal failure, against $12.12 \%$ of the patients in group $2(P=0,46)$. With the rifampicin, $27.27 \%$ of the patients of group 2 presented cytolysis.

Conclusions This study suggests that colistin represents a good therapeutic alternative for the treatment of nosocomial infection with multi-resistant germs. However, our study is not without limits; it is a retrospective study, absence of randomization and the control group of patients.

\section{P56}

\section{Efficacy and safety of once daily dosing of colistin to critically ill}

\section{patients}

A Skiada', J Pavleas², TTopalis², K Georgiou², D Siggouna²

G Thomopoulos², GL Daikos', G Floros²

'University of Athens, Greece; 'Laiko General Hospital, Athens, Greece

Critical Care 2010, 14(Suppl 1):P56 (doi: 10.1186/cc8288)

Introduction Although colistin has been used extensively in critically ill patients infected with multidrug-resistant (MDR) organisms, our knowledge about the pharmacokinetic and pharmacodynamic parameters correlating with efficacy is very limited and little consensus exists on the optimum dosing regimen of this agent. In our center, during the past 2 years several patients treated with colistin were given the total daily dose once every 24 hours. The aim of this study was to analyze the efficacy and safety of this dosing regimen in critically ill patients. 
Methods The medical files of all patients who were admitted to the ICU in 2008 and who received colistin for an infection due to MDR bacteria were retrospectively analyzed. Criteria for inclusion were: dosing regimen of colistin 9 million units (MU) every 24 hours (colistin methanesulphonate - CMS, Norma, 1 vial = 1 MU, 1 mg = 13,300 U), administration of CMS for at least 7 days and normal renal function at initiation of treatment with CMS (serum creatinine $\leq 1 \mathrm{mg} / \mathrm{dl}$ or creatinine clearance $>80 \mathrm{ml} / \mathrm{min}$ ). Mortality was evaluated at 28 days.

Results Thirteen patients were included in the study (69\% male, median age 71 years). The underlying diseases were surgical in seven patients and medical in six patients. The median APACHE and SOFA scores on the first day of CMS administration were 15 and 10, respectively. Eleven patients had nosocomial pneumonia, two had peritonitis and one osteomyelitis. Causative bacteria were isolated in 10 patients (six Acinetobacter baumanii, three Pseudomonas aeruginosa and one Klebsiella pneumonia) and were all MDR. Of the 10 isolated bacteria, six were susceptible only to colistin, while the other four were also susceptible either to aztreonam or sulbactam. In cases of susceptibility of the isolate only to colistin, it was given in combination with a carbapenem, while in the other cases either aztreonam or sulbactam was added. The median length of CMS administration was 11 days (mean 19; range 7 to 79 days). Median serum creatinine on initiation and completion of treatment with CMS was 0.7 and $0.9 \mathrm{mg} / \mathrm{dl}$, respectively $(P=0.357)$. No cases of neurotoxicity were found. Mortality at 28 days was $15 \%$.

Conclusions The total daily dose of $9 \mathrm{MU}$ of CMS, given as one dose every 24 hours in critically ill patients, seems to be efficient and safe, without resulting in increased nephrotoxicity or other adverse events. A prospective study should be done to confirm the findings we have presented herein.

\section{P57}

A comparison of the effect on renal function of flucloxacillin and vancomycin antibiotic prophylaxis in cardiac surgery patients TO Brougham, K Giraud, A Vuylsteke

Critical Care Unit, Cambridge, UK

Critical Care 2010, 14(Suppl 1):P57 (doi: 10.1186/cc8289)

Introduction This study compared the effect on renal function of flucloxacillin and vancomycin antibiotic prophylaxis for elective firsttime coronary artery bypass grafting (CABG) surgery, using both direct biochemical markers and indirect clinical outcome measures. Recent evidence has suggested that vancomycin may be nephrotoxic in patients undergoing cardiac surgery.

Methods A retrospective observational study of patients undergoing elective first-time CABG was performed, covering a 13-month period. All patients received prophylactic antibiotics: flucloxacillin $1 \mathrm{~g}$ pre-operatively and three $1 \mathrm{~g}$ doses post-operatively. Patients who were MRSA-positive, MRSA unknown or penicillin allergic received an alternative regimen: vancomycin $1 \mathrm{~g}$ pre-operatively and $1 \mathrm{~g}$ post-operatively. Exclusion criteria: preoperative creatinine $>133 \mathrm{mmol} / \mathrm{l}$, any antibiotics other than prophylaxis and haemodynamic support except $<5 \mu \mathrm{g} / \mathrm{kg} /$ hour dopamine.

Results Of 1,413 patients in the study period, 415 met the study criteria: 360 patients received flucloxacillin and 55 patients received vancomycin. There were no significant differences between the two groups in sex, age, BMI, euroSCORE, diabetes status, ejection fraction, pre-operative creatinine, eGFR, sodium, or potassium. Comparing change in renal function pre-operatively to post-operatively, there were no significant group differences in change in: creatinine ( $\mathrm{mmol} / \mathrm{l} ;$ VAN median 0 (IQR 11); FLU -2 (19); $P=0.22)$, eGFR (ml/min; VAN 0 (14); FLU 2.4 (19.3); $P=$ 0.22 ), sodium (mmol/l; VAN 1 (4); FLU 1 (4); $P=0.28$ ). Change in potassium differed significantly (mmol//; VAN 0.7 (0.9); FLU $0.5(0.7) ; P<0.05)$. In clinical outcome measures, the groups were similar. Most patients in both groups stayed in ITU for 1 day and there was no significant difference in the number of patients staying for longer than 1 day (VAN 7/55 (13\%); FLU $29 / 360(8 \%) ; P=0.30)$. There was no difference in hospital length of stay (days; VAN 7 (4); FLU 6 (3); $P=0.19$ ).

Conclusions In elective first-time CABG patients, there is no significant difference in change in renal function between those given vancomycin antibiotic prophylaxis and those given flucloxacillin prophylaxis, as assessed by creatinine, eGFR and sodium levels, and indirect clinical outcome measures. Potassium increased more in the vancomycin group but the clinical significance of this is unclear. Our data suggest that prophylactic vancomycin does not impair renal function relative to flucloxacillin.

P58

Microdialysis study of meropenem cerebral distribution in patients with acute brain injury

C Dahyot-Fizelier', I Timofeev², S Marchand', W Couet', P Hutchinson², B Debaene', D Menon², O Mimoz', A Gupta ${ }^{2}$

'University Hospital, Poitiers, France; ${ }^{2}$ Addenbrooke's Hospital, Cambridge, UK Critical Care 2010, 14(Suppl 1):P58 (doi: 10.1186/cc8290)

Introduction Antibiotic dosing recommendations are usually based on plasma or cerebral spinal fluid pharmacokinetic (PK) studies. However, as infections mainly occur in extracellular tissue fluid (ECF), corresponding unbound ECF antibiotic concentrations are responsible for the antimicrobial effect. Because of the blood-brain barrier, the cerebral antibiotic distribution is thought to be reduced compared with tissues without any physiological barrier. This study aims to determine meropenem (MPM) unbound concentrations in the brain and compare them with MPM concentrations in plasma to explore cerebral distribution of MPM in patients with acute brain injury.

Methods After local ethic approval and written informed consent, two brain-injured patients, sedated, mechanically ventilated, receiving MPM for an infection and monitored by cerebral microdialysis (CMA 71, membrane length $10 \mathrm{~mm}$, membrane diameter $0.6 \mathrm{~mm}$, molecular cut-off $100 \mathrm{kDa}$; CMA, Stockholm, Sweden) were enrolled. The PK study succeeded to $1 \mathrm{~g}$ meropenem over 30 minutes and brain dialysates and blood samples were collected over 420 minutes. Probe recoveries were evaluated individually by retrodialysis. MPM was assayed by HPLC coupled with tandem mass spectrometry (LC-MS/MS).

Results For each of the two patients, the MPM brain AUC is much lower than plasma AUC and accordingly brain to serum AUC ratios are respectively 0.73 for Patient 1 (P1) and 0.14 for Patient 2 (P2). MPM concentration versus time curves in brain are delayed (time-to-peak in $\mathrm{P} 1=100$ minutes, in $\mathrm{P} 2=80$ minutes) and present a smooth peak compared with the corresponding curves in plasma (Figure 1). Mean probe recoveries are respectively $19 \pm 7 \%$ for $\mathrm{P} 1$ and $29 \pm 7 \%$ for $\mathrm{P} 2$.

Conclusions The MPM brain AUC is much lower than plasma AUC for the two patients enrolled, consistent with the PK theory in the presence of tissue with efflux transporters. More patients are needed to better understand MPM brain distribution characteristics.

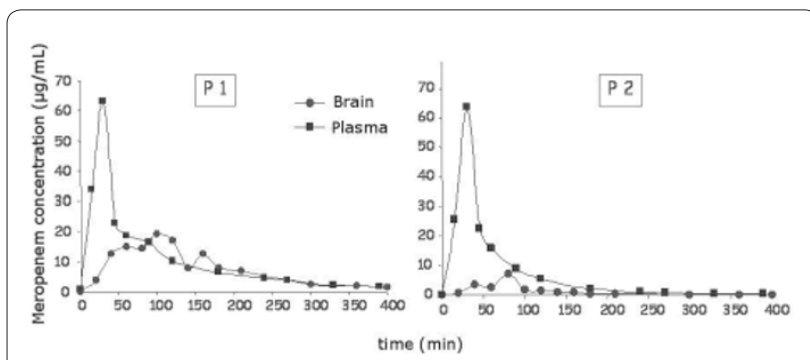

Figure 1 (abstract P58). Individual MPM plasma $(\bigcirc)$ and brain ( concentrations versus time in $\mathrm{P} 1$ and $\mathrm{P} 2$.

\section{P59}

Effects of two regimens of antibiotic prophylaxis on colonizing flora of the respiratory tract in patients with cardiovascular surgery

EM Berg, R Wesselink, M Tersmette

Sint Antonius Hospital, Nieuwegein, the Netherlands

Critical Care 2010, 14(Suppl 1):P59 (doi: 10.1186/cc8291)

Introduction Antibiotic prophylaxis in cardiovascular (CV) surgery has greatly reduced the rate of postoperative infections. First-generation or second-generation cefalosporines are used for this purpose. Our hospital changed its antibiotic prophylaxis protocol for elective CV surgery in June 2007 from cefuroxime to cefazolin in accordance with national guidelines 
[1]. The aim of this study was to explore the effect of changing this antibiotic prophylaxis protocol on microbial flora in sputum.

Methods Retrospective study. All patients admitted $>10$ days on the ICU after elective CV surgery between 1 June 2006 and 1 June 2007 (Group 1) were compared with the same group of patients between 1 July 2007 and 1 August 2008 (Group 2). Patients received single-dose prophylaxis (bypass surgery), or 2 days of prophylaxis (cardiac prosthetic surgery). Group 1 received cefuroxime and Group 2 received cefazolin. Patients did not receive selective digestive tract decontamination. Isolated pathogens from elective sputum cultures of all patients were registered and compared between groups after dividing pathogens into six classes based on the profile of intrinsic antibiotic susceptibility. Comparative data between groups were analyzed with Pearson's X test.

Results One hundred and fifty-eight patients had positive sputum cultures. Comparing total amounts of positive cultures, there was no significant difference between colonization with pathogenic micro-organisms between Group 1 ( $n=77$ patients) and Group 2 ( $n=76$ patients). In Group 1 more colonization with pathogens from class 4 ( $\beta$-lactamase-producing Enterobacteriaceae and Pseudomonas aeruginosa) was observed compared with Group 2 (45\% vs 34\%, $P=0.079$ ). This nonsignificant difference between groups was mainly attributable to a difference in colonization with Pseudomonas. In the other classes of pathogens, no differences were observed. No difference in postoperative wound infections was noted between groups.

Conclusions Prophylactic use of cefazolin instead of cefuroxime after $\mathrm{CV}$ surgery resulted in a trend towards reduction of colonization of the respiratory tract with intrinsic $\beta$-lactam-resistant microbial flora in patients with prolonged ICU stay, without adverse effects on the incidence of postoperative wound infection.

Reference

1. van Kasteren MEE, et al:: Ned Tijdschr Geneeskd 2000, 144:2049-2055.

P60

Prevalence of infections among patients admitted to ICUs in Nordic countries and the Netherlands in comparison with Mediterranean countries: a report from the EPIC II study

H Hanberger', H Gill', H Njimi'2, S Walther', JL Vincent ${ }^{2}$

'Antibiotic Research Unit, Linkoping, Sweden; 'Erasme Hospital, Brussels, Belgium

Critical Care 2010, 14(Suppl 1):P60 (doi: 10.1186/cc8292)

Introduction The prevalence of infection is high among patients admitted to the ICU and one of the main causes of mortality. The aim of this study was to determine the burden of infectious diseases in patients admitted to ICUs in Nordic countries and the Netherlands in comparison with Mediterranean countries.

Methods The EPIC I| 1-day point prevalence study of infections and demographics of critically ill patients was performed on 8 May 2007. A comparison of severity score, source of infection, co-morbidity and patient outcome was done for patients admitted to ICUs in low antibiotic resistance countries (LARC) (Denmark, Finland, the Netherlands, Norway, Sweden) and high antibiotic resistance countries (HARC) (Greece, Israel, Italy, Malta, Portugal, Spain, Turkey).

Results The number of patients included was 2,270 in HARC and 581 in LARC. On the day of the study, $45.3 \%$ of patients in LARC were considered as infected compared with $51.6 \%$ in HARC $(P=0.007)$. The mean SAPS II scores were (95\% CI) 32.7 (31.6 to 33.8) in LARC and 35.6 (35 to 36.2) in HARC $(P<0.001)$. Of all patients in LARC/HARC, $27.0 \% / 33.9 \%(P=$ $0.002)$ had a respiratory infection, $10.8 \% / 9.8 \%(P=0.45)$ an abdominal infection, $7.2 \% / 10.4 \%(P=0.023)$ a blood infection, $2.8 \% / 3.2 \%(P=0.60)$ a skin infection, $2.1 \% / 3.4 \%(P=0.09)$ a catheter infection, 3.8\%/6.6\% ( $P=$ $0.012)$ a genitourinary infection and $0.7 \% / 1.7 \%(P=0.08)$ a CNS infection. Chronic renal failure was seen in $2.6 \%$ of patients in LARC and $7.3 \%$ in HARC $(P<0.001)$ and the corresponding prevalence of chronic obstructive disease was $13.4 \%$ and $20.2 \%$, respectively $(P<0.001)$. Admissions were more often seen from $\mathrm{OR} / \mathrm{monitoring}$ and less often from emergency rooms among patients admitted to ICUs in LARC compared with HARC. Median ICU length of stay (LOS) in LARC (IQR) was 5 days (1 to 19) and in HARC was 12 days (3 to 31) $(P<0.001)$. The corresponding hospital LOS was 16 days ( 7 to 38 ) and 24 days ( 10 to 54$)$, respectively $(P<0.001)$. The ICU mortality rates in LARC/HARC were $14.9 \% / 19.9 \%(P=0.008)$ for all patients and for infected patients $21.2 \% / 25.4 \%(P=0.17)$, whereas the corresponding hospital mortality rates were $20.8 \% / 26.7 \%(P=0.005)$ for all patients and for infected patients $29.6 \% / 34.3 \%(P=0.16)$. The prevalence of MRSA was $0.8 \%$ among patients in LARC $(5.8 \%$ in HARC) $(P<0.001)$ and the prevalence of $P$. aeruginosa was $9.9 \%$ and $14.0 \%(P=0.07)$, respectively. Conclusions Infections and serious co-morbidities were more prevalent among patients admitted to ICUs in HARC than in LARC. This was associated with a longer stay and greater mortality in the ICU.

\section{References}

1. Vincent IL, et al:. JAMA 1995, 274:639-644.

\section{P61}

Risk factors for the development of carbapenem-resistant Klebsiella pneumoniae infections in critically ill patients

KZ Vardakas, DK Matthaiou, E Antypa, A Grammatikos, E Chasou,

E Antoniadou

G. Gennimatas, Thessaloniki, Greece

Critical Care 2010, 14(Suppl 1):P61 (doi: 10.1186/cc8293)

Introduction We sought to study the characteristics and outcomes of ICU patients with carbapenem-resistant (CRKp) and carbapenem-sensitive (CSKp) K. pneumoniae infections.

Methods A retrospective cohort of patients requiring ICU treatment. The study was conducted in an eight-bed ICU between January 2006 and September 2009

Results During the study period, 104 patients were diagnosed with K. pneumoniae infection. The mean age of patients was $66.3 \pm 14.3$ years. Fifty-one (49\%) were males. The mean APACHE II score was $17.9 \pm 6.9$. The median duration of hospital stay until the infection was 28 days. Fortyeight patients (46.2\%) had bacteremia, 27 (30\%) urinary tract infections, 15 (14.4\%) pneumonia, seven (6.7\%) peritonitis and seven (6.7\%) skin and soft tissue infections. Fifty-eight (56.9\%) and 39 (39\%) patients had previous and concurrent infections, respectively. Seventy-six patients (73.1\%) died. The univariate analysis showed that prior hospitalization $(P=0.049)$, dialysis $(P=0.034)$, and history of urologic neoplasia $(P=0.041)$ were associated with the development of carbapenem-resistant infections. No independent risk factors were found in the multivariate analysis. APACHE II score $(P=0.003)$, need for dialysis $(P=0.034)$, shock prior and after the infection $(P=0.006$ and $P<0.001$, respectively), respiratory distress prior and after the infection $(P=0.021$ and $P=0.032$, respectively), multiorgan failure prior and after the infection ( $P=0.02$ and $P=0.003$, respectively), treatment failure $(P<0.001)$, and acidosis after the development of infection $(P=0.003)$ were associated with death in the univariate analysis. Shock after the infection $(P=0.016)$ and treatment failure $(P=0.001)$ were independent predictors of mortality in the multivariate analysis. No difference in mortality was found between patients with CRKp and CSKp isolates.

Conclusions Infection due to K. pneumoniae in the ICU is associated with high mortality. Infection treatment and hemodynamic support of the patient may be important determinants of the clinical course in critically ill patients with such infections.

\section{Reference}

1. Falagas ME, et al:: Risk factors of carbapenem-resistant Klebsiella pneumoniae infections: a matched case control study. J Antimicrob Chemother 2007, 6:1124-1130.

P62

Outcome of unimicrobial versus polymicrobial sepsis

N Abed', A Hussein', S Salaheldine', A Hassan², M Mahfouz', H Khaled Nagi', H Khaled Nagi ${ }^{1}$

'Cairo University, Cairo, Egypt; ${ }^{2}$ National Heart Institute, Cairo, Egypt

Critical Care 2010, 14(Suppl 1):P62 (doi: 10.1186/cc8294)

Introduction Polymicrobial sepsis is associated with immunosuppression caused by the predominance of anti-inflammatory mediators and profound loss of lymphocytes through apoptosis, and so deaths directly related to sepsis are twofold higher in polymycrobial sepsis. The aim of study is to compare unimicrobial versus polymicrobial sepsis as regards microbiology, complications, length of stay, mortality, factor affecting mortality. 
Methods One hundred and one patients with sepsis were studied and divided into two groups. The first group: unimicrobial, where one organism was isolated from cultures; and the second group: polymicrobial, where more than one organism was isolated from the cultures.

Results The first group (unimicrobial) was 48 cases (47.5\%) and the second group (polymicrobial) was 53 cases $(52.47 \%)$. Both groups has similar positive sputum cultures (32/48 (66\%) versus $38 / 53(71 \%), P$ value 0.6$)$, but positive blood culture was significantly higher in the second group (27/53 (51\%) vs $7 / 48(14 \%), P$ value 0.0001$)$. There was also a significantly higher incidence of UTI and wound infection in the second group (49.7\% vs $6.3 \%$ for UTI, $P$ value 0.0001 and $20.7 \%$ vs $2.1 \%, P$ value 0.004 for wound infection, respectively). The commonest detected organisms were Staphylococci and Klebsiella (48, 22 out of 101 patients); 16/48 (33\%) in unimicrobial vs $32 / 53(60 \%)$ in polymicrobial ( $P$ value 0.007$)$ for staph, and $7 / 48(14.5 \%)$ in unimicrobial vs $15 / 53(28 \%)$ in polymicrobial for Klebsiella ( $P$ value 0.09 ). Incidence of Acinetobacter, Candida and E. coli in the second group were significantly higher $10 / 53$ in poly vs $2 / 48$ in unimicrobial ( $P$ value 0.032 ), and $10 / 53$ vs $0 / 48$ ( $P$ value 0.002 ) and $6 / 53$ vs $0 / 48$ ( $P$ value 0.016 ), respectively. Need for mechanical ventilation in the second group was significantly higher than the first group (46/53 (86\%) vs $31 / 48$ (64\%), $P$ value 0.009). The mean hospital stay was longer in the second group ( $26.9 \pm 15.4$ vs $17.4 \pm$ $9.3, P$ value 0.001$)$. Mortality was significantly higher in the second group $(P$ value 0.012$)$. Factors increasing mortality in both groups were DCL ( $P$ value 0.007 in unimicrobial and 0.036 in polymicrobial); vasopressors ( $P$ value 0.001 and 0.002 , respectively); mechanical ventilation ( $P$ value 0.00 and 0.00 , respectively), and severe sepsis and septic shock with significant $P$ value (0.019) and (0.008), respectively, for the first group and (0.003) and (0.002), respectively, for the second group.

Conclusions Polymicrobial sepsis shows higher risk for complication, length of stay and mortality than unimicrobial.

Reference

1. Aliaga et al.: Eur J Clin Microbiol Infect Dis 2000, 19:871-874.

P63

De-escalation practice pattern in an Indian intensive care unit

A Bhakta, M Bhattacharyya, STodi

AMRI Hospitals, Kolkata, India

Critical Care 2010, 14(Suppl 1):P63 (doi: 10.1186/cc8295)

Introduction Antibiotic de-escalation is thought to be beneficial by reducing the selection pressure for resistance. This study was carried out to identify variables that influence de-escalation practices.

Methods Prospective observational study during a 1-year period (July 2008 to June 2009) in a 50-bed ICU in a tertiary care hospital. Any patient admitted to the ICU during this period in whom at least one specimen was sent for microbiological culture at or before starting antibiotics was included in the study and subsequently followed up for antibiotic change according to the culture sensitivity report. Antibiotics covering Gramnegative organisms were ranked as per Figure 1.

\begin{tabular}{|l|c|}
\hline \multicolumn{1}{|c|}{ Antibiotic } & Rank \\
\hline Colistin/ Polymyxin B & 9 \\
\hline Group 2 Carbapenem & 8 \\
\hline Piperacillin-tazobactam & 7 \\
\hline Cefoperazone-sulbactam & 6 \\
\hline Group 1 Carbapenem & 5 \\
\hline $3^{\text {rd }}$ and 4 ${ }^{\text {th }}$ gen Cephalosporins & 4 \\
\hline Quinolones & 3 \\
\hline Aminoglycosides & 2 \\
\hline Others & 1 \\
\hline
\end{tabular}

Figure 1 (abstract P63). Antimicrobial therapy ranking.
Results Seven hundred and seventy-eight patients were included, of whom cultures were positive in 551 (70.8\%) and negative in 227 (29.2\%) cases. In 350 (44.9\%) patients, neither escalation nor de-escalation of therapy was done. Overall escalation of therapy occurred in 192 (24.7\%) patients and de-escalation in 236 (30.3\%). The mortality rate was lowest among patients in whom therapy was de-escalated (8.9\%) compared with categories of no change (14.2\%) or escalation (23.4\%). De-escalation occurred more frequently among patients in whom no pathogen was isolated (45.8\%) compared with culture-positive cases (23.9\%) and in $18.3 \%$ of patients with growth of drug-resistant pathogens, compared with $38.9 \%$ of patients with susceptible pathogens. De-escalation occurred most frequently where an antibiotic having only Gram-positive coverage was included in the initial empiric therapy (60.3\%), and in cases where third-generation cephalosporin was the initial empiric therapy (54.4\%).

Conclusions This study highlights no change of therapy as the most prevalent practice pattern of antibiotic use. Mortality remained low in patients in whom therapy was de-escalated. Variables favoring deescalation practices were non-isolation of pathogen, growth of susceptible organisms, and stopping of Gram-positive coverage.

P64

Incidence, identification of multidrug-resistant pathogens and impact on the outcome after cardiac surgery

A Tasouli, K Papadopoulos, G Stravopodis, C Panagiotou, J Kriaras,

S Geroulanos, G Saroglou

Onassis Cardiac Surgery Center, Athens, Greece

Critical Care 2010, 14(Suppl 1):P64 (doi: 10.1186/cc8296)

Introduction Multidrug-resistant (MDR) pathogens constitute an emerging threat with increasing incidence and uncertain outcome. On the other hand, patients undergoing open heart surgery represent a vulnerable population.

Methods Evaluation of the incidence and identification of MDR pathogens after cardiac surgery in 2,803 patients for a 2-year period. Examination of the clinical features of patients with MDR infection. Elucidation of the impact on the outcome.

Results In 18 patients (0.64\%) consisting of 12 males and six females, at least one MDR pathogen was isolated. Gram-positive pathogen was certified in five patients (27.8\%) and Gram-negative in 13 patients (72.2\%). Specifically, four patients were infected with vancomycin-resistant enterococcus (VRE), six with Klebsiella pneumoniae, three with Acinetobacter spp., one with VRE and Acinetobacter spp., four with Acinetobacter and K. pneumoniae. Low output syndrome $\left(\mathrm{Cl}<2.01 / \mathrm{min} / \mathrm{m}^{2}\right)$ was common in all these patients and essentially contributed to the deterioration of clinical situation with dependence on inotropic support, prolonged mechanical ventilation (>10 days), acute renal failure and need for haemodilution (66.6\%). Therefore, the ICU and hospital stay is prolonged ( $>20$ days and $>30$ days, accordingly) and pathogenesis of MDR infection is provoked after 20 days of ICU stay. Consequently, nine patients with MDR infection (50\%) died. All were critically ill patients with multiple organ dysfunction syndrome under broad-spectrum antimicrobials with hospital-acquired bloodstream MDR bacteremia.

Conclusions Infection with MDR pathogens, while rare, constitutes a notable prognostic marker of increased mortality after cardiac surgery. It is worth noting that the higher mortality rate is mainly attributable to the severe co-morbidity in haemodynamically compromised patients. Management must concentrate on the implementation of effective preventative strategies.

\section{P65}

Surveillance of ICU-acquired infections in Belgium: 2008 reference data I Morales, B Catry, K Mertens

Scientific Institut of Public Health, Brussels, Belgium

Critical Care 2010, 14(Suppl 1):P65 (doi: 10.1186/cc8297)

Introduction This paper aims to provide reference data for pneumonia (PN) and bloodstream infections (BSI) acquired in Belgian ICUs taking patients' characteristics into account. This information could enlighten policy decisions. This 2008 set of indicators is the first yielded by the ICU Belgian surveillance (NSIH-ICU) since 2001. 
Methods The NSIH-ICU is a voluntary, patient-based reporting system set up in 1992 to follow ICU-acquired infections and guide prevention. It establishes a national risk-adjusted benchmark for infection rates, antibiotics use and invasive device-use ratios through uniform case definitions, data-collection methods, data entry and analysis. Only patients staying more than 2 days in the ICU are included. These aggregated database means are derived from the NSIH-ICU 2008 report.

Results In 2008, 4,355 patients and 35,802 patient-days were reported by 19 ICUs. The age mean was 67.2 years; mean length of stay 8.1 days; mean SAPS II score 43.8; the proportion of patients with antibiotics at admission was $47.1 \%$; medical admissions represented $63.4 \%, 4.2 \%$ were immunocompromised patients; trauma was reported in $6.7 \%$ of cases. Overall ICU mortality was $8.5 \%$. The proportion of intubated patients was $44.4 \%$, with central venous catheters (CVC) $68.3 \%$ and with urinary catheters 58.3\%. Invasive-device use rates were: 358.5 intubation-days, 746.5 CVC-days and 644.2 urinary catheter-days per 1,000 patient-days. The ICU-acquired PN/100 patients was 9.8, 16.5/1,000 patient-days and the intubation-associated PN was 11.1/1,000 intubation days. ICU-acquired BSI was 3.6/100 patients, 5.3/1,000 patient-days and the catheter-related BSI was 4.3/1,000 catheter-days.

Conclusions The 2008 report provided Belgian hospitals with comparative ICU-acquired infection data adjusted for patients' intrinsic and extrinsic infection risks. The participation changed across time and could cause a selection bias. Outlier verification is ongoing and efforts to increase the participation are being made. Indicator rates could be related to protocol modification, demographic trends and changes in patient mix linked to restructuration of medical services or in clinical practice patterns. Some are vulnerable to continuous medical and nursing in-service training, for example at surveying the antibiotics use and improving invasive devices use. Further studies could assess to what extent modifications in clinical practice and/or external interventions might cause changes in the patient mix.

\section{P66}

Risk of acquiring drug-resistant (MDR) Gram-negative isolates with previous exposure to antibiotic

A Bhakta, M Bhattacharyya, S Todi

AMRI Hospitals, Kolkata, India

Critical Care 2010, 14(Suppl 1):P66 (doi: 10.1186/cc8298)

Introduction Prior antimicrobial therapy is one of the most important factors leading to the acquisition of MDR organisms. Formulating antibiotic policy and choice of empirical antibiotic selection will be helped by knowing the association of MDR Gram-negative organisms with a previous exposure of particular antibiotic.

Methods Prospective observational study during January 2008 to June 2009 in a 50-bed ICU in a tertiary care hospital. Specimens sent after 2 days of the start of the antibiotic and no more than 90 days from the stop date have been included in the study. Analyses were based on those specimens that resulted in detection of MDR Gram-negative organism. Observed relative risk (RR) of an antibiotic class was computed with respect to an MDR infection. RR was computed as the ratio of the risk of the event (acquiring the infection) occurring in the exposed group vs in the nonexposed group. A logistic regression model was used where multiple antibiotics were applied.

Results A total of 1,072 specimens from 500 patients met the criteria as specified above. Of these, 423 (39.4\%) specimens resulted in detection of MDR bacteria, $186(17.4 \%)$ resulted in detection of non-MDR bacteria and no bacteria were detected in the remaining 463 (43.2\%) specimens. Of the total 423 cases of MDR acquisitions, ESBL Enterobacteriaceae (151 or $35 \%$ ), MDR Acineto ( 89 or $21 \%$ ) and MDR Pseudo (58 or $14 \%$ ). Risk of isolating ESBL Enterobacteriaceae was highly significant with the prior exposure to third-generation cephalosporin $(\mathrm{RR}=5.8$ and $P<0.001)$. Risk of isolating MDR Acinetobacter spp. was highly significant with the exposure to piperacillin-tazobactam ( $R R=2.7$ and $P<0.001$ ). Risk of isolating MDR Pseudomonas spp. was significant with the exposure to Group 2 carbapenem ( $R R=2.2$ and $P>0.001)$. Group 1 carbapenem, aminoglycosides have not been found to have significant association with any individual MDR organisms.

Conclusions Previous exposure to antibiotics leads to increased acquisition of MDR organisms. There is a significant association of isolating

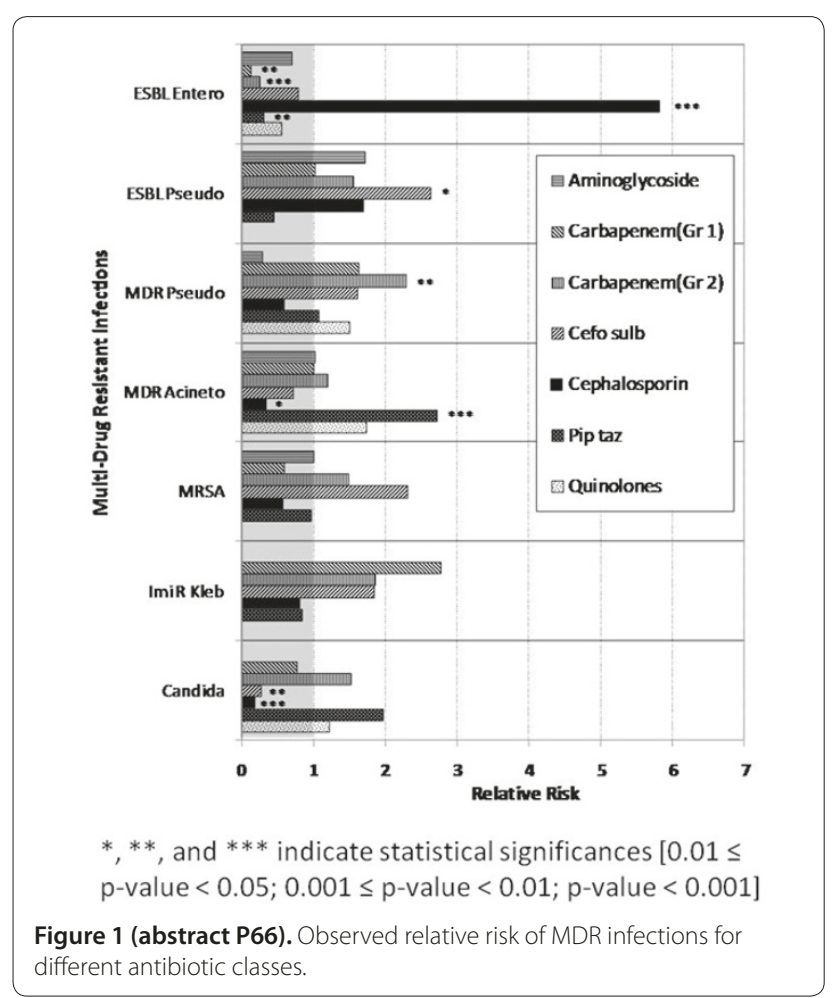

different MDR organisms with previous exposure to a particular class of antibiotic.

\section{P67}

Ozone fumigation successfully controlled and eradicated multidrug-resistant Acinetobacter baumanii from an intensive care unit

R Stümpfle, A Castello-Cortes, F Coogan, PB Nielsen

Northwick Park Hospital, London, UK

Critical Care 2010, 14(Suppl 1):P67 (doi: 10.1186/cc8299)

Introduction The emergence of multidrug-resistant Acinetobacter baumanii (MDRAB) poses a serious threat to patients on the ICU. The production of metallo- $\beta$-lactamase leaves colistin as the only therapeutic option. Outbreaks due to MDRAB can persist for months. Traditional decontamination methods fail to deal with this level of colonisation and contamination effectively. We tackled a recent outbreak of MDRAB effectively using gaseous ozone. To our knowledge it is the first time ozone has been used to control an outbreak of MDRAB.

Methods An external company (Hydrozone Environmental Ltd) was hired to perform the fumigation. The ICU was divided into three decontamination areas using heavy-duty polythene sheets. Patients were in turn relocated from contaminated to clean areas before each area was sealed and fumigated. Humidity levels within were raised to 70 to $80 \%$ using a humidifier. An Ozone Ultra Pro $16 \mathrm{~g} /$ hour ozone generator with ozone destruct capability, operated remotely, delivered ozone to a target concentration of $>2.0 \mathrm{ppm}$ for 15 minutes. A fan was used to achieve even dispersal. For safety reasons perimeter ozone concentrations were monitored with a UV photometer and kept below $0.05 \mathrm{ppm}$. The efficacy of the fumigation was measured by environmental microbiological sampling before and after fumigation.

Results All fumigated areas received ozone concentrations of 4.62 to 5.66 ppm for 21 to 32 minutes. Ozone was not detected outside the treatment areas. Prior to fumigation, 72 (38\%) of 188 environmental samples were MDRAB-positive. Following fumigation, nine (5\%) of 158 samples were positive. Most of these samples were from nontouch areas, for example ceiling, above door frame with significant dust collection and without daily cleaning. Considering that dust may impede ozone penetration, 
basic cleaning was improved and duration of fumigation was increased to 30 minutes. Subsequent samples all proved negative and no further cross-infections occurred.

Conclusions This is the first study using ozone fumigation as a disinfectant to control an outbreak in an ICU. Ozone fumigation is an effective tool for eradicating MDRAB from heavily contaminated clinical sites. Used in conjunction with good infection control measures, ozone can be used to control outbreaks such as MRSA, Clostridium difficile, Norovirus and swine flu.

\section{P68}

Viral infections in the ICU: should we search for them?

M Sousa, M Monteiro, V Alves, C Granja

Hospital Pedro Hispano, Matosinhos, Portugal

Critical Care 2010, 14(Suppl 1):P68 (doi: 10.1186/cc8300)

Introduction Respiratory viral infections are usually self-limited in adults but 4 to $30 \%$ can be clinically severe and lead to intensive care needs. The aim of this study was to determine the impact of viral respiratory infections in an intensive care setting and the role of systematic viral testing in patients admitted to an ICU.

Methods A retrospective analysis of all 114 viral tests of respiratory samples of 98 patients admitted to the ICU for four consecutive years was performed. Molecular biology test and immunofluorescence assay for adenovirus, influenza A and B, parainfluenza 1 to 3, metapneumovirus and syncytial respiratory virus (SRV) were performed in tracheal aspirate (TA) (89\%) and bronchoalveolar lavage (BAL) (11\%). SAPS II was used as the severity index. Patients were stratified according to the primary diagnostic. Results Viral tests were performed in 98 patients, $60 \%$ were male, mean age 58 years old and $45 \%$ had previous respiratory disease. SAPS II was 48. Ninety-two percent need mechanical ventilation (MV) for 8.5 days. Primary diagnostics were community-acquired pneumonia (CAP) (50\%) or tracheobronchitis (32\%), chronic pulmonary disease exacerbations (6\%), aspiration pneumonia (3\%), nosocomial infection (5\%), septic shock (3\%) and meningitis (1\%). Length of stay (LOS) was 11 days and ICU mortality was $17.5 \%$. Virus identification was positive in $13(12.2 \%)$ respiratory samples of 12 patients, 12 in TA and one in BAL. Twelve were identified in the winter. Demographic variables, LOS, co-morbidities and severity index of patients with viral infection were similar to the main group. Influenza A (in three CAP, one tracheobronchitis, one nosocomial infection and one septic shock), metapneumovirus (two tracheobronchitis and one CAP), influenza $\mathrm{B}$ (one CAP), parainfluenza 3 (one CAP) and SRV (one tracheobronchitis) were identified. In these patients, C-reactive protein was higher and leucocytes were lower. Bacterial co-infection was identified in 33\% of the patients, all of them with acquired community pneumonia. Antibiotic step down was done in $62 \%$ of the patients with isolated viral infection. In these patients LOS and days of mechanical ventilation were 9.6 and 6.2, respectively, and invasive ventilation-associated pneumonia was reduced. Conclusions Although viral tests should not be required for all ICU patients, respiratory samples for viral tests should be performed in patients with tracheobronchitis/pneumonia requiring intensive care, especially in the winter. Positive identification of viral agents could be useful in antibiotic policy.

\section{P69}

\section{Oseltamivir dosing with haemofiltration}

M Tomlin', B Skinner', J Fennell', EP Pelosi', SK Khoo ${ }^{2}$, NL Lindegardh³ 'Southampton University Hospitals NHS Trust, Southampton, UK; '2University

of Liverpool, UK; ${ }^{3}$ Mahidol University, Bangkok, Thailand

Critical Care 2010, 14(Suppl 1):P69 (doi: 10.1186/cc8301)

Introduction In the current pandemic it is likely that some patients will be admitted to hospital and require respiratory support including mechanical ventilation. These patients are likely to have a profound systemic inflammatory response syndrome (SIRS); consequently they may have multiorgan failure (MOF) requiring renal replacement therapy (RRT) with haemofiltration. Two questions then arise - what dose of oseltamivir (Tamiflu) should we give these patients to shorten the duration of the H1N1 infection? How should we modify the dose in response to altered renal drug clearance and in those requiring RRT?
Methods A young adult female patient with $\mathrm{H} 1 \mathrm{~N} 1$ infection and MOF was given oseltamivir $75 \mathrm{mg}$ BD nasogastrically. Failure to respond changed the risk: benefit ratio and justified doubling the dose despite uncertainties over an overall reduced clearance. Enteral nutrition absorption was uncertain, and thus we sampled her blood to ensure adequate oseltamivir absorption and that activation of the pro-drug was not inhibited. We undertook serial sampling for blood concentration assay to determine the pharmacokinetic parameters in this difficult scenario. Blood samples were collected in plain serum tubes (without sodium fluoride). The samples were spun and refrigerated within half an hour, then batched and shipped to Bangkok for drug concentration measurement.

Results We report both the parent oseltamivir phosphate (OP) and that of the active metabolite oseltamivir carboxylate (OC). OP levels were low at 10 to $77 \mathrm{ng} / \mathrm{ml}$. but OC concentrations were high at 2,600 to 5,000 ng/ml. Conclusions The population normal parameter for half-life OP is 1 hour and for $\mathrm{OC}$ it is 3 to 5 hours. A single dose of $150 \mathrm{mg} \mathrm{OP}$ is expected to achieve an OP level of 50 to $150 \mathrm{ng} / \mathrm{ml}$ and an OC level of 1,000 to $1,500 \mathrm{ng} / \mathrm{ml}$. Our slightly low OP levels are likely to be due to ex vivo hydrolysis in the collection tube due to a lack of esterase inhibitor. The high OC levels are most probably due to reduced renal elimination despite being on haemofiltration. Our concerns were focused on the possibility of viral mutation (subsequently shown to be negative) or poor enteral absorption/activation. What we found was that $150 \mathrm{mg} \mathrm{BD}$ produces more than adequate $\mathrm{OC}$ levels to treat $\mathrm{H} 1 \mathrm{~N} 1$ infection.

\section{P70}

\section{Efficacy and utility of a protocol for pre-emptive antimycotic}

therapy

SG Milanov, G Georgiev, VTodorova, L Kozarov, M Milanov

Pirogov Emergency Institute, Sofia, Bulgaria

Critical Care 2010, 14(Suppl 1):P70 (doi: 10.1186/cc8302)

Introduction Invasive candidiasis (IC) is associated with increasing morbidity and mortality in critically ill patients. This, in conjunction with difficulties in diagnosis, underscores the need for novel treatment strategies based on the identification of significant risk factors for IC. The aim of the study was to evaluate the efficacy and safety of a protocol for pre-emptive antimycotic treatment.

Methods A randomized prospective controlled trial was carried out in a general ICU for 2 years. After the implication of the inclusion and exclusion criteria, patients were submitted to block randomization and stratified on the basis of their initial SAPS II expanded score. We have developed a protocol for pre-emptive antimycotic treatment. Having reviewed the current literature, we combined the most significant risk factors for IC with tree major clinical criteria for persistent nonbacterial sepsis and assumed this algorithm as an indication for starting pre-emptive therapy. According to the protocol, antimycotic therapy was started on the day of inclusion in the treatment group and only with proven IC in the control group. Initial data were gathered on demographic characteristics of the patients, proven risk factors for IC-related mortality (malnutrition, non-albicans colonization, creatinine clearance) and severity of inflammatory response and organ dysfunction. Dynamics of SIRS and SOFA, subsequent Candida isolates, ventilator-free days, length of ICU stay, outcome and eventual adverse reactions were followed.

Results A total of 110 patients (equal in both groups) were enrolled. No statistically significant differences in the basal characteristics of the patients, length of ICU stay and the number of ventilator-free days were found. The delta SOFA score was significantly lower in the treatment group $(P=0.019)$. The in-hospital mortality was $38.2 \%$ in the treatment group vs $61.8 \%$ in the control group $(P=0.013)$. The associated with pre-emptive therapy relative risk was $0.62(95 \% \mathrm{Cl}=0.4$ to 0.94$)$. Significant differences between the Kaplan-Meyer estimates of survival were found (log-rank test $P=0.007)$. A total of $15(13.6 \%)$ adverse reactions were observed among treated patients in both groups which was not associated with higher mortality risk

Conclusions The implementation of the developed protocol reduced the degree of organ dysfunction severity and was associated with significant survival benefit. 
P71

Amphotericin B used as a continuous infusion is safer

J Streefkerk, S Gijsberts, J Ros, J Rommes, P Spronk

Gelre Ziekenhuizen, DZ Apeldoorn, the Netherlands

Critical Care 2010, 14(Suppl 1):P71 (doi: 10.1186/cc8303)

Introduction Many patients in an ICU are intestinal carriers of yeasts with an inherent risk of infections if bowel perforation occurs. Resistance to azoles is increasing, while treatment with conventional amphotericin B (AM-B) is associated with potential toxicity. AM-B given in a continuous 24hour infusion may be less toxic compared with the conventional 4-hour to 6-hour infusion rate of AM-B [1,2]. AM-B given in a continuous 24-hour infusion is only evaluated in a tertiary care setting, with a predominance of immunocompromised patients. We evaluated the feasibility and safety of continuous AM-B treatment in critically patients with suspected or proven yeast or fungal infections.

Methods This is an observational retrospective analysis for the side effects in consecutive patients treated with AM-B from January 2003 to December 2008 in our ICU department. During the investigation period patients received amphotericin B: $40 \mathrm{mg} / 24$ hours or a lower dose if the MDRD clearance was less than $60 \mathrm{ml} / \mathrm{min}$. During the treatment the dose was adjusted to the desired therapeutic range of 200 to $1,000 \mu \mathrm{g} / \mathrm{l}$ according to measured plasma levels.

Results The mean treatment duration was $12.3 \pm 6.3$ days with a dose of $32.1 \pm 12.2 \mathrm{mg} / 24$ hours. Of the 10 patients who died, seven died after the termination of the AM-B therapy, without signs of an active yeast or fungal infection. Within the first week three patients died, necropsy was carried out in one case, demonstrating a PCP infection, and in two patients a role of antibiotic failure cannot be ruled out. All other patients with proven yeast or fungal infection demonstrated a clinical recovery of their infection. The AM-B concentration was measured in 113 blood samples, nine samples had a level $<200 \mu \mathrm{g} / \mathrm{l}$ and 10 a level $>1000 \mu \mathrm{g} / \mathrm{l}$. Renal impairment, defined as more than 1.5 times the creatinine at the start of the treatment with $A M-B$, occurred in $9 \%$ and was not evaluable in $6 \%$ due to unresolving renal replacement therapy dependency at the start of AM-B. None of the evaluated patients developed a creatinine more than 2.0 times the baseline value. Temporary elevation of liver enzymes was seen in $23 \%$, without the need for dose modification. Hypokalemia $\left(\mathrm{K}^{+}\right.$ $<3.0 \mathrm{mmol} / \mathrm{l}$ ) was observed in one patient.

Conclusions Compared with the conventional infusion rate of $A M-B$, we conclude that continuous 24-hour infusion seems a feasible and safer treatment alternative in patients with invasive yeast or fungal infections.

\section{References}

1. Walsch TJ, et al:: N Engl J Med 1999, 340:764-771.

2. Wingard JR, et al: Clin Infect Dis 1999, 29:1402-1407.

\section{P72}

\section{Anidulafungin compared with fluconazole therapy in critically ill} patients

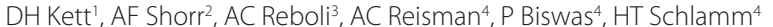
'University of Miami/Jackson Memorial Hospital, Miami, FL, USA; ${ }^{2}$ Washington Hospital Center, Washington, DC, USA; ${ }^{3}$ University of Medicine and Dentistry of New Jersey - Robert Wood Johnson Medical School, Camden, NJ, USA; ${ }^{4}$ ffizer Inc., New York, USA

Critical Care 2010, 14(Suppl 1):P72 (doi: 10.1186/cc8304)

Introduction The 2009 IDSA Treatment Guidelines for Candidiasis favor an echinocandin for initial treatment of candidemia in patients with severe illness. In a prospective, randomized study anidulafungin resulted in improved global response (GR) and a trend toward improved survival compared with fluconazole [1]

Methods Retrospective analysis in patients classified as severely ill at study entry: treatment initiated in an ICU (Group 1), APACHE II score $\geq 15$ (Group 2) or presence of severe sepsis (Group 3). Within groups, anidulafungin was compared with fluconazole for GR rate at the end of intravenous therapy and 14-day and 28-day mortality.

Results In Group 1 ( $n=89)$, GR was $63.3 \%$ vs $45.0 \%$ (95\% Cl: -2.2 to 38.8); Group $2(n=113)$, GR was $68.3 \%$ vs $46.0 \%$ ( $95 \%$ Cl: 4.3 to 40.2$)$; and Group 3 ( $n=118$ ), the GR was $67.7 \%$ vs $51.8 \%$ (95\% Cl: -1.6 to 33.5$)$, in patients with MOD $(n=45)$, the GR was $76.2 \%$ vs $29.2 \%$ (95\% Cl: 21.3 to 72.8 ) anidulafungin versus fluconazole, respectively. Across groups,

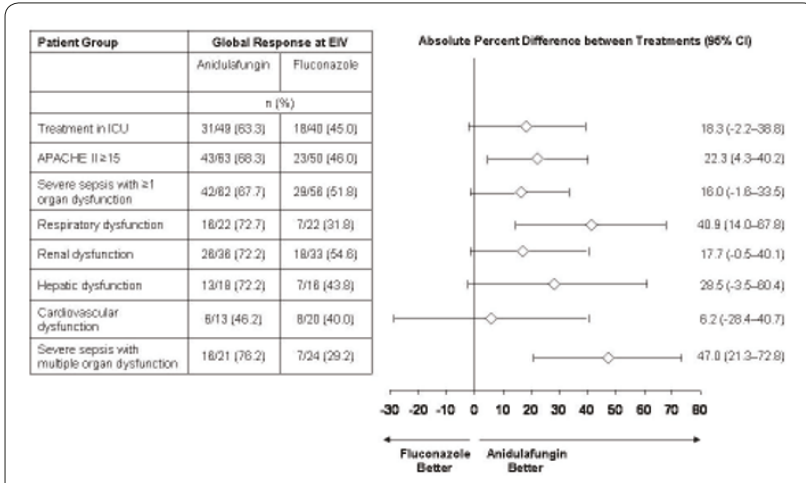

Figure 1 (abstract P72). Global response to treatment.

an association with anidulafungin use and lower day 14 mortality was suggested (12.2\% to $14.3 \%$ for patients receiving anidulafungin vs $19.6 \%$ to $28.0 \%$ for those receiving fluconazole) $(P=N S)$. See Figure 1 .

Conclusions In patients with severe illness, anidulafungin was associated with greater GR than fluconazole, significantly so for those with APACHE II score $\geq 15$ or with MOD, supporting the IDSA Guidelines.

Reference

1. Reboli AC, et al.: NEngl J Med 2007, 356:2472-2482.

\section{P73}

Increase in systolic blood pressure and improvement in laboratory parameters following polymyxin B-immobilized fiber treatment in septic shock

Y Sakamoto', K Mashiko', T Obata², H Matsumoto', Y Hara', N Kutsukata', $\mathrm{HYokota}^{3}$

'Chiba Hokusoh Hospital, Nippon Medical School, Chiba, Japan; 'Microbial Chemistry Reaearch Foundation, Tokyo, Japan; ${ }^{3}$ Nippon Medical School, Tokyo, Japan

Critical Care 2010, 14(Suppl 1):P73 (doi: 10.1186/cc8305)

Introduction Direct hemoperfusion using a polymyxin B-immobilized fiber column (DHP-PMX; Toray Industries Inc., Tokyo, Japan) was first developed in 1994 and has since been used for the treatment of septic shock.

Methods A total of 47 patients with septic shock who received DHPPMX for 2 to 6 hours were retrospectively reviewed to examine any improvement in sepsis-related factors after DHP-PMX and to analyze the relationship between any such improvement and increase in SBP.

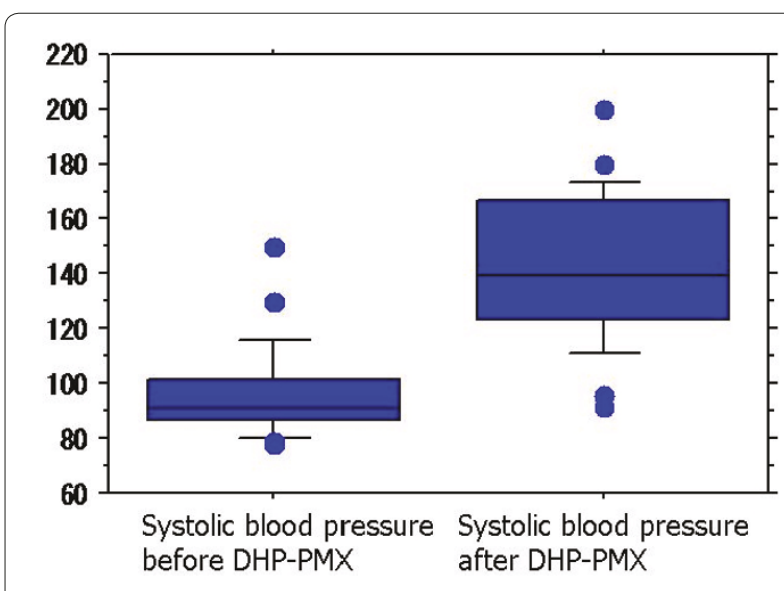

Figure 1 (abstract P73). Changes in systolic blood pressure in patients treated with DHP-PMX in whom HMBG-1 level increased. $P<0.0001$. 
In addition, we analyzed the effectiveness of long-term DHP-PMX (5 to 6 hours) with respect to improvement in sepsis-related factors.

Results $\mathrm{N}$-arachidonoylethanolamine (AEA), 2-arachidonoyl glycerol (2-AG), and plasminogen activator inhibitor-1 (PAI-1) were significantly improved after DHP-PMX treatment. SBP increased significantly in the group showing improved high mobility group box protein 1 (HMGB-1) level $(P<0.0122)$. AEA, 2-AG and HMGB-1 were improved in all four patients who were treated with long-term DHP-PMX.

Conclusions We observed a relationship between hemodynamic improvement and a decrease in serum HMGB-1 level in septic shock patients treated with DHP-PMX. We suggest that long-term DHP-PMX improves sepsis-related factors.

\section{References}

1. Sakamoto Y, et al:: ASAIO J 2007, 53:646-650.

P74

Selection of acute blood purification therapy according to lipid mediator adsorption and blood purification in patients with septic shock

Y Sakamoto', K Mashiko', T Obata ${ }^{2}$, H Yokota $^{3}$

'Chiba Hokusoh Hospital, Nippon Medical School, Chiba, Japan; ${ }^{2}$ Microbial

Chemistry Research Foundation, Tokyo, Japan; ${ }^{3}$ Nippon Medical School,

Tokyo, Japan

Critical Care 2010, 14(Suppl 1):P74 (doi: 10.1186/cc8306)

Introduction Direct hemoperfusion using a polymyxin B-immobilized fiber column (DHP-PMX) has been used for the treatment of septic shock. As an alternative method for acute blood purification therapy, continuous venovenous hemodiafiltration (CVVHDF) has been reported to be an effective clinical treatment for critically ill patients; however, the optimal column for performing CVVHDF remains controversial. On the other hand, recently, one of the lipid mediators, endocannabinoids (N-arachidonoylethanolamine (AEA) and 2-arachidonoyl glycerol (2-AG)) have been reported with a blood pressure decreased effect.

Methods We investigated 14 polymethylmethacrylate (PMMA) membrane hemofilters and three polyacrylonitrile (PAN) membrane hemofilters after use in patients with septic shock. Therefore, in clinical study, we used CVVHDF after DHP-PMX to treat 32 patients with septic shock. To determine the optimal acute blood purification therapy, we subsequently divided the patients into two groups: group A underwent CVVHDF using a PMMA membrane hemofilter after undergoing DHP-PMX $(n=25)$; group B underwent CVHDF using a PAN membrane hemofilter after undergoing DHP-PMX $(n=7)$. In addition, the levels of endocannabinoids (AEA, 2-AG) were measured. The severity scores and the improvement of endocannabinoids were compared between the two groups.

Results Endocannabinoids (AEA, 2-AG) were adsorbed more in the PMMA column (AEA; $506.3 \pm 680.2 \mathrm{ng} /$ column, 2-AG; $23.0 \pm 38.5 \mu \mathrm{g} /$ column) than in the PAN column (AEA; $1.5 \pm 0.7 \mathrm{ng} /$ column, 2-AG; $0.1 \pm 0.1 \mu \mathrm{g} /$ column). The average Acute Physiology and Chronic Health Evaluation (APACHE) ॥ score and the average sepsis-related organ failure assessment (SOFA) score did not differ significantly between the two groups. Group A showed a better outcome compared with Group B $(P=0.05)$. In addition, only group A showed a significant improvement in the blood AEA level on day $1(P=0.0185)$.

Conclusions Our study suggests that the PMMA column might be the better column for performing CVVHDF after DHP-PMX treatment, as suggested by the adsorption and blood purification of endocannabinoids. Reference

1. Sakamoto Y, et al:: ASAIO J 2008, 54:129-132.

\section{P75}

Prognostic assessment in community-acquired pneumonia by pneumonia severity scores and biomarkers

F Dusemund', W Albrich', P Schuetz², B Müller

'Kantonsspital Aarau, Switzerland; ${ }^{2}$ University Hospital Basel, Switzerland

Critical Care 2010, 14(Suppl 1):P75 (doi: 10.1186/cc8307)

Introduction Classical biomarkers like C-reactive protein (CRP) or the leucocyte count are only inaccurate tools for predicting the severity of community-acquired pneumonia (CAP). Procalcitonin (PCT) was found to predict 28-day mortality in CAP (area under the curve (AUC) 0.8) similar to the combination of PCT and CRB65 score (AUC 0.83) [1]. Novel prognostic biomarkers, such as Pro-ET1 and MR-ProADM, were shown to correlate with CAP severity [2]. We aimed to compare the diagnostic accuracy to predict mortality and ICU admission of clinical severity scores, biomarkers and their combination.

Methods Nine hundred and twenty-five CAP patients enrolled in the ProHOSP trial [3] were analyzed by assessing clinical severity scores (SMARTCOP, PSI, CURB65) and biomarker levels (PCT, MR-ProADM, Pro-ET1). Receiver operating characteristic curves for 30-day mortality and ICU admission were used to calculate and compare the different predictive values.

Results The AUC for the prediction of 30-day mortality was 0.84 with SMART-COP, 0.82 with PSI, 0.72 with CURB65, 0.59 with PCT, 0.75 with MR-ProADM and 0.75 with ProET1. ICU admission was predicted best by SMART-COP (AUC 0.83), compared with the other severity scores and biomarkers (PSI: 0.68, CURB65: 0.65, PCT: 0.7, Pro-ET1: 0.73, ProADM: 0.72). The combination of SMART-COP and MR-ProADM was superior to SMARTCOP alone (AUC 0.84, $P=0.04$ ).

Conclusions The combination of MR-PrOADM with SMART-COP significantly improved the prediction of ICU admission. Prognostic biomarkers should complement the clinical assessment of patients with LRTI to improve allocation of healthcare resources to high-risk patients.

\section{References}

1. Krüger S, et al.: Eur Respir J 2008, 31:349-355.

2. Schuetz P, et al:: BMC Inf Dis 2008, 8:22-30

3. Schuetz P, et al.:. JAMA 2009, 302:1059-1066.

\section{P76}

\section{Current progress in pneumonia research}

T Smelaya', V Moroz', A Goloubev', L Salnikova², A Rubanovich²

'V.A. Negovsky Research Institute of General Reanimatology RAMS, Moscow, Russia Federation; ${ }^{2}$ N.I. Vavilov Research Institute of General Genetics,

Moscow, Russia Federation

Critical Care 2010, 14(Suppl 1):P76 (doi: 10.1186/cc8308)

Introduction Diagnosis and treatment of acute pneumonia $(\mathrm{Pn})$ is a problem of high significance in modern medicine. The incidence of complicated and lethal acute Pn has increased [1]. Candidate genes are of great importance in the course of acute diseases and their complications. Cytokines and xenobiotic detoxication genes are the most investigated. The aim of the investigation was to study genetic predisposition to acute Pn.

Methods Results of the associative DNA polymorphism studies in 243 patients with acute community-acquired Pn are presented; 178 healthy individuals formed a control group. Genetic variability of the candidate loci was studied: renin-angiotensin ACE system gene, chemokine receptor CCR5 gene and four genes controlling xenobiotic detoxification (CYP1A1, GSTM1, GSTT1, GSTP1). Multiplex polymerase chain reaction was utilized for genotyping of insertion-deletion polymorphism on loci ACE (287 nucleotide pairs) and CCR5 (deletion of 32 nucleotide pairs). The odds ratio index was used to describe the degree of association of the genotypes with the diseases. Statistical analysis was done by means of Fisher's exact test and the online program SNPStats (http://bioinfo.iconcologia.net).

Results An increased predisposition to Pn development was registered in homozygotes in deletion at the ACE locus $(\mathrm{OR}=1.8 ; P=0.013)$, positive genotypes of the GSTM1 locus ( $O R=1.7 ; P=0.010)$ and homozygotes in allele 606T of the CYP1A1 gene $(\mathrm{OR}=1.6 ; P=0.020)$.

Conclusions A combination of positive genotypes of the GSTM1 locus and homozygotes in allele 606T of the CYP1A1 gene (OR=1.9, $P=0.006$; incidence in controls $>20 \%$ ) presented with a most effective prognostic power.

\section{Reference}

1. Sinopalnikov Al, et al: Military Med J 1996, 2:30-33.

\section{P77}

Bacterial colonization and infections of the lower respiratory tract in an interdisciplinary ICU

M Sartzi, M Agrafiotis, P Peppa, F Nanu, M Charitidi, I Basiliadis, G Kallitsi, M Michalia, P Clouva-Molyvdas

General Hospital Thriassio Elefsinas, Magoula, Greece

Critical Care 2010, 14(Suppl 1):P77 (doi: 10.1186/cc8309)

Introduction Tracheal intubation and mechanical ventilation are often associated with infection of the lower respiratory tract (LRT). The morbidity 
associated with the progression of airway colonization (AC) to ventilatorassociated tracheobronchitis (VAT) and from VAT to ventilator-associated pneumonia (VAP) has been analyzed but not been fully elucidated. We endeavored to study the relationship between $A C$ and development of infections (VAT and VAP) of the LRT in ICU patients.

Methods Retrospective study of 400 consecutive ICU patients ventilated $>48$ hours in the past 4 years. Patients age, gender, APACHE II, prior illness, cause of admittance, length of stay (LOS), time of mechanical ventilation (MV), outcome, time of appearance of AC and infection of the LRT were registered. MODS and CPIS were measured at onset of infection (VATVAP) and 3 days after. MODS was also calculated on the day AC appeared. Bronchial secretions were cultured at admission, at least once a week and whenever there was a change in the amount and quality of bronchial secretions or clinical infection was suspected. The Mann-Whitney test was used for statistical analysis and statistical significance was set at $P<0.05$.

Results From the 400 studied patients 68 (17\%) were colonized and 153 (48.25\%) developed infections: 54 (13.5\%) VAT and 99 (24.7\%) VAP. Colonization appeared after $3.1 \pm 0.8$ days of ICU admission exclusively with Gram-negative microorganisms. Twenty-five (36.7\%) of the colonized patients developed infections of the LRT (with the same pathogen): 8 (11.7\%) VAT after $4.6 \pm 2.7$ days and 17 (25\%) were diagnosed with VAP after $9.8 \pm 1.8$ days. From the eight VAT patients, four developed VAP (50\%) after $5.4 \pm 0.4$ days. None of the colonized patients died. The colonized patients who developed infections were elderly $(P<0.05)$, more severely ill $(P<0.04)$ and had at the time of infection a diagnosis higher temperature $(P<0.01)$, more severe leukocytosis $(P<0.02)$ but not statistically significant organ dysfunction $(P>0.3)$. The appearance of VAP caused more severe organ dysfunction $(P<0.002)$, longer MV duration $(P<0.01)$ and longer $\operatorname{LOS}(P<0.001)$ but did not influence mortality. On the day of AC detection MODS was $2.4 \pm 0.5$, on VAT detection MODS was $4.8 \pm 0.9$ and on VAP appearance MODS was $9.6 \pm 0.7$. CPIS at VAT detection was $3.9 \pm 0.9$ and at VAP detection $6.1 \pm 0.5$ (day I) and $7.2 \pm 0.8$ (on day III).

Conclusions $\mathrm{AC}$ resulted in LRT infections in only one-third of our patients and the majority of LRT infections are not preceded by AC.

\section{P78}

Rehydration does not affect pulmonary immune responses to influenza or susceptibility to secondary bacterial pneumonia RD Sanders', A Godlee', JC Goulding', M Maze'2, T Hussell 'Imperial College London, UK; ${ }^{2}$ UCSF, San Francisco, CA, USA Critical Care 2010, 14(Suppl 1):P78 (doi: 10.1186/cc8310)

Introduction In our murine model of influenza, significant weight loss occurs up to day 7 post-infection [1]. We sought to determine whether weight loss from influenza could be altered by rehydration and whether this affects pulmonary immune responses.

Methods Adult BALB/c mice were infected with X31 (H3N2) influenza (1:80) via the intranasal route and randomized to intraperitoneal rehydration with $20 \mathrm{ml} / \mathrm{kg}$ compound sodium lactate (CSL), normal saline (NS) or no rehydration (NR) starting on day 3 following infection and continued for 4 days ( $n=5$ /group). On day 7, mice were challenged with $1 \times 10^{6}$ Streptococcus pneumoniae (serotype 2). Two further cohorts of mice were challenged with different doses of influenza and rehydrated from day 3 to 7 to investigate pulmonary immune responses in the absence of bacteria. Mice were infected with 1:80 ( $n=10 /$ group) influenza and rehydrated once daily or 1:60 ( $n=5 /$ group) influenza and rehydrated twice (1:60) daily with $20 \mathrm{ml} / \mathrm{kg}$ CSL or not. Daily weight, survival following secondary bacterial pneumonia, number of colony-forming units (48 hours after bacterial challenge) from peripheral blood, lung, and nasal wash and cellularity in lung compartments were measured.

Results Rehydration did not affect weight loss following 1:80 influenza infection (naïve mice $(+0.3 \pm 0.4 \mathrm{~g})$, influenza plus NR $(-1.58 \pm 0.4 \mathrm{~g})$, influenza plus CSL $(-1.1 \pm 0.7 \mathrm{~g})$ and influenza with NS $(-1.3 \pm 0.4 \mathrm{~g}))$. A repeat experiment with CSL once daily or twice daily did not alter weight loss compared with NR $(P>0.05)$. Survival or CFU counts following bacterial pneumonia did not differ between the groups $(P>0.05)$. The total number or activational status of bronchoalveolar, lung macrophages/monocytes and lymphocytes was not affected by rehydration following influenza infection or 48 hours following bacterial pneumonia ( $P>0.05 ; P<0.05$ vs naïve mice). Conclusions Rehydration does not affect immunity or pathophysiology in a murine influenza infection model. Assuming these results can be extrapolated to the clinical setting, our findings support the use of conservative fluid resuscitation strategies in patients with influenza. Reference

1. Snelgrove et al: Nat Immunol 2008, 9:1074-1083.

\section{P79}

Bacteremic nosocomial pneumonia cases from the ATTAIN studies

E Rubinstein', SL Barriere'2, FC Genter'2, GR Corey ${ }^{3}$, C Luna ${ }^{4}$, A Lentnek ${ }^{5}$, ME Stryjewski ${ }^{6}$

'University of Manitoba, Winnipeg, Canada; ${ }^{2}$ Theravance, Inc., South San Francisco, CA, USA; ${ }^{3}$ DCRI, Durham, NC, USA; ${ }^{4}$ University of Buenos Aires, Argentina; ${ }^{5}$ Wellstar Infectious Disease, Marietta, GA, USA; ${ }^{6}$ CEMIC, Buenos Aires, Argentina

Critical Care 2010, 14(Suppl 1):P79 (doi: 10.1186/cc8311)

Introduction Bacteremic pneumonia is associated with worse outcome including higher mortality. The ATTAIN program compared telavancin (TLV), a lipoglycopeptide antibiotic, with vancomycin (VAN) for treatment of nosocomial pneumonia (NP) due to Gram-positive pathogens including MRSA. This subgroup analysis examined the baseline characteristics and clinical outcomes in bacteremic HAP cases.

Methods ATTAIN 1 and 2 were methodologically identical, randomized, double-blind, phase 3 studies. Adult patients with NP due to presumed or confirmed Gram-positive pathogens were randomized (1:1) to TLV $10 \mathrm{mg} /$ $\mathrm{kg}$ intravenously every 24 hours or VAN $1 \mathrm{~g}$ intravenously every 12 hours (adjusted per site-specific guidelines) for 7 to 21 days. The modified alltreated (MAT) population consisted of patients who received $\geq 1$ dose of study medication and who had a respiratory pathogen recovered from baseline cultures. Bacteremic NP was defined by the identification of a pneumonia-causing pathogen in the blood or of the same pathogen in lung and blood with identical susceptibility profiles. Clinical outcomes were assessed at test-of-cure (TOC) 7 to 14 days after end of study treatment.

Results All MAT patients with bacteremic NP $(n=73)$ were included in this analysis. At baseline, more TLV patients than VAN patients were in the ICU (TLV 74\%, VAN 62\%) and had ventilator-associated pneumonia (TLV $59 \%$, VAN 44\%); APACHE II scores were similar between groups (mean \pm SD, TLV $16 \pm 6$, VAN $17 \pm 6$ ). S. aureus was the most common pathogen (TLV 76\%, VAN 69\%) and included MRSA (TLV 41\%, VAN 49\%). Cure rates for TLV and VAN were $44 \%$ and $36 \%$, respectively (difference TLV - VAN (95\% $\mathrm{Cl})=7.3 \%(-15.9 \%, 30.5 \%))$. On-study mortality was similar, $41 \%$ in each treatment group. Incidences of adverse events (AE) were similar between groups, except for nausea (TLV 21\%, VAN 3\%) and vomiting (TLV 15\%, VAN, $0 \%$ ). Proportions of patients who discontinued the study medication due to AEs were similar (TLV 12\%, VAN 13\%).

Conclusions TLV and VAN had similar cure rates in a subgroup of ATTAIN patients with bacteremic NP. The safety profiles of TLV and VAN were mostly comparable in these patients.

\section{P80}

Late ventilator-associated pneumonia: analysis of baseline characteristics and clinical outcomes in the ATTAIN studies E Rubinstein', SL Barriere ${ }^{2}$, FC Genter ${ }^{2}$, GR Corey ${ }^{3}$, PC Lee ${ }^{4}$, T Lalani ${ }^{3}$ 'University of Manitoba, Winnipeg, Canada; ${ }^{2}$ Theravance, Inc., South San Francisco, CA, USA; ${ }^{3} D C R I$, Durham, NC, USA; ${ }^{4}$ Baystate Medical Centre, Springfield, MA, USA

Critical Care 2010, 14(Suppl 1):P80 (doi: 10.1186/cc8312)

Introduction Pneumonia is a leading cause of death associated with hospital-acquired infections. Late ventilator-associated pneumonia (VAP), defined as disease onset after $\geq 4$ days of mechanical ventilation, is associated with worse outcomes than other forms of hospital-acquired pneumonia (HAP).

Methods ATTAIN 1 and 2 were methodologically identical, randomized, phase 3 studies of telavancin (TLV) $10 \mathrm{mg} / \mathrm{kg}$ intravenously every 24 hours vs vancomycin (VAN) $1 \mathrm{~g}$ intravenously every 12 hours for treatment of HAP, including VAP. VAN doses could be adjusted per investigative site guidelines. Clinical outcome was assessed at the test-of-cure (TOC) visit 7 to 14 days after the end of study treatment. Baseline characteristics, outcomes, and safety in the late VAP sub-group were analyzed in the 


\begin{tabular}{|c|c|c|c|}
\hline & \multicolumn{2}{|c|}{ Patients with late VAP } & \multirow[b]{2}{*}{$\begin{array}{c}\text { Difference } \\
{[95 \% \mathrm{Cl}]}\end{array}$} \\
\hline & $\begin{array}{c}\text { TLV } \\
n / N(\%)\end{array}$ & $\begin{array}{c}\text { VAN } \\
n / \mathbb{N}(\%)\end{array}$ & \\
\hline Age $(y)$, mean \pm SD & $56 \pm 19.1$ & $60 \pm 17.5$ & \\
\hline APACHE II, mean $\pm S D$ & $17 \pm 5.5$ & $17 \pm 5.2$ & \\
\hline $\mathrm{CLCr} \leq 50 \mathrm{~mL} / \mathrm{min}$ & $26 / 112(23.2)$ & $22 / 85(25.9)$ & \\
\hline \multicolumn{4}{|l|}{ Cure rates at TOC: } \\
\hline $\begin{array}{l}\text { MAT } \\
\text { ME }^{2}\end{array}$ & $\begin{array}{c}63 / 112(56.3) \\
36 / 40(90.0)\end{array}$ & $\begin{array}{l}40 / 85(47.1) \\
14 / 26(53.8)\end{array}$ & $\begin{array}{r}9.2(-5.0,23.4) \\
35.4(11.3 .53 .5)^{\circ}\end{array}$ \\
\hline
\end{tabular}

Figure 1 (abstract P80).

modified all-treated population (MAT; patients with baseline respiratory pathogen(s) who received $\geq 1$ dose of study medication) and the microbiologically evaluable population (ME; protocol adherent MAT patients with baseline Gram-positive pathogen(s)). Patients with mixed Gram-positive/Gram-negative infections were excluded in this analysis.

Results A total of 197 late VAP cases were analyzed. Baseline characteristics, including the APACHE I| scores, were balanced between the treatment groups (Figure 1). At least one adverse event (AE) was reported by $95 \%$ (106/112) and $93 \%(79 / 85)$ of MAT patients in the TLV and VAN groups, respectively, and $21 \%(23 / 112)$ of the TLV group and $22 \%$ (19/85) of the VAN group died during the study.

Conclusions In this exploratory sub-group analysis, numerically higher cure rates were observed for TLV than for VAN in patients with late VAP. Incidences of reported AEs and mortality rates were similar between the TLV and VAN groups.

\section{P81}

\section{On the diagnosis of acute respiratory distress syndrome in} nosocomial pneumonias

A Kuzovlev, WV Moroz, AM Goloubev

V.A. Negovsky Research Institute of General Reanimatology, Moscow, Russia Federation

Critical Care 2010, 14(Suppl 1):P81 (doi: 10.1186/cc8313)

Introduction Clinical practice deals highly frequently with patients presenting a concomitant occurrence of acute respiratory distress syndrome (ARDS) and nosocomial pneumonia (NPn). Timely diagnosis of ARDS and NPn in such circumstances is problematic, but it provides a possibility of differential treatment. The aim of the investigation was to elucidate the value of the oxygenation index (OI), extravascular lung water index (EVLWI), pulmonary vascular permeability index (PVPI) and central hemodynamics indexes in the diagnosis of ARDS in NPn.

Methods Thirty-eight cancer and severely traumatized patients were enrolled in the prospective clinical investigation. The patients were split into three groups according to the ARDS and NPn diagnostic criteria: group 1 (ARDS + NPn), group 2 (NPn), group 3 (no ARDS, no NPn). ARDS was diagnosed by means of the Lung Injury Score (LIS), the AmericanEuropean Consensus Conference on ARDS criteria (1992), and the criteria of the V.A. Negovsky Research Institute of General Reanimatology (2006). All patients were investigated with a complex protocol, key elements of which were EVLWI, PVPI and central hemodynamics indexes measured by the transpulmonary thermodilution (Pulsion PiCCO plus, Pulsion Medical Systems, Germany). The data were analyzed by Statistica 7.0 ( $M \pm S D$, Newman-Keuls test, correlations). $P<0.05$ was considered statistically significant.

Results Patients of group 1 on the day of enrollment presented with a significantly lower OI (160.9 $\pm 51.7 \mathrm{mmHg}$ vs $239.5 \pm 96.7 \mathrm{mmHg})$ and static pulmonary compliance $(46.3 \pm 13.7 \mathrm{ml} /$ water $\mathrm{cm}$ vs $72.4 \pm 23.1 \mathrm{ml} /$ water $\mathrm{cm})$ and significantly higher EVLWI $(12.7 \pm 4.7 \mathrm{ml} / \mathrm{kg}$ vs $7.6 \pm 1.6 \mathrm{ml} / \mathrm{mg})$ and LIS ( $2.22 \pm 0.67$ scores vs $1.68 \pm 0.58$ scores) in comparison with group 2 patients. The patients of group 2 presented with an EVLWI within the physiological limits over the whole investigation period. PVPI calculated by three existing methods was available within the physiological limits even in the patients with a profound pulmonary edema. There were no significant differences between the groups in central hemodynamics parameters.

Conclusions The OI, EVLWI, static pulmonary compliance and LIS made it possible to timely diagnose ARDS in NPn. Patients with NPn without ARDS presented with EVLWI within the physiological limits. A complex analysis of these indexes must be performed to diagnose ARDS in NPn. Physiological limits of the PVPI require an additional investigation due to an insufficient diagnostic value. Volumetric indexes of central hemodynamics should be measured to confirm the noncardiogenic nature of pulmonary edema.

P82

Genetic variability at the surfactant proteins $A$ and $D$ in susceptibility and severity of pneumonia

J Solé-Violán', M Garcia-Laorden', F Rodriguez de Castro'

O Rajas², J Blanquer³, L Borderías', P Saavedra 5, J Aspa², M Briones³, J Marcos-Ramos ${ }^{6}$, E Herrera-Ramos ${ }^{1}$, J Ferrer', I Sologuren', J Rello7, C Rodriguez-Gallego'

${ }^{1}$ Hospital Dr Negrín, Las Palmas de Gran Canaria, Spain; ${ }^{2}$ Hospital de la Princesa, Madrid, Spain; ${ }^{3}$ Hospital Clínico, Valencia, Spain: ${ }^{4}$ Hospital San Jorge, Huesca, Spain; 'University of Las Palmas de Gran Canaria, Las Palmas, Spain; ${ }^{6}$ Hospital General de Lanzarote, Spain; ${ }^{7} J o a n$ XXIII University Hospital, Tarragona, Spain

Critical Care 2010, 14(Suppl 1):P82 (doi: 10.1186/cc8314)

Introduction Genetic variability of the pulmonary surfactant proteins $A$ and D may affect clearance of microorganisms and the extent of the inflammatory response. The genes of these collectins (SFTPA1, SFTPA2 and SFTPD) are located in a cluster at 10q21-24, near to the gene coding for mannose-binding lectin (MBL2), another collectin involved in innate immunity. The aim of this study was to evaluate the association of variability at SFTPA1, SFTPA2 and SFTPD with susceptibility to and severity of community-acquired pneumonia (CAP). Another objective was to evaluate the existence of linkage disequilibrium among SFTPA1, SFTPA2, SFTPD and MBL2.

Methods Nonsynonymous polymorphisms of SFTPA1, SFTPA2, SFTPD and MBL2 were analysed in 682 CAP patients and 769 controls. Haplotypes were inferred and linkage disequilibrium (LD) was characterized. The effect of genetic variability on SP-A and SP-D serum levels was studied. Results Haplotypes SFTPA1 6A2 $(P=0.0009)$, SFTPA2 1 A0 $(P=0.0017)$, and SFTPA1-SFTPA2 6A2-1A0 $(P=0.0005)$ were under-represented in patients, whereas haplotypes SFTPA2 1A10 $(P=0.00007)$ and SFTPA1-SFTPA2 6A3-1A ( $P=0.00065)$ were over-represented. We observed the existence of LD among the studied genes. Chromosomes carrying the SFTP-D aa1 1-C allele with $6 A 2-1 A 0$ and the XA variant of MBL2 were found to be even more under-represented in patients $(P=0.00008) .1 \mathrm{~A} 10$ and $6 \mathrm{~A}-1 \mathrm{~A}$ were associated with higher mortality, and also with multiorgan dysfunction syndrome (MODS) and acute respiratory distress syndrome (ARDS), respectively. SFTPD aa11-C allele was associated with development of MODS and ARDS.

Conclusions We report for the first time an association between genetic variants of SFTPA1, SFTPA2 and SFTPD with the susceptibility, severity and outcome of pneumonia.

\section{P83}

Successful strategy to reduce ventilator-associated pneumonia

AL Manoel, I Boszczowski, AH Andrade, L Bierrenbach, ETaira, AC Baruzzi

Hospital Municipal Dr. Moysés Deutsch, São Paulo, Brazil

Critical Care 2010, 14(Suppl 1):P83 (doi: 10.1186/cc8315)

Introduction VAP rates in Brazil are higher than those related in Europe and USA. The study objective was to examine the effect of the Institute for Healthcare Improvement's ventilator bundle plus oral decontamination with chlorhexidine (ODC) in the incidence of VAP in an ICU.

Methods The study was conducted in a 20-bed, medical-surgical ICU. Criteria for nosocomial pneumonia are those from the CDC. Strategy was to implement the IHI's ventilator bundle plus ODC. The goals were the ICU team adhesion of $80 \%$ achieved in the ninth month after bundle implementation and 98\% after 1 year of follow-up. These measures included five strategies to prevent ventilator-associated pneumonia: 30 to $45^{\circ}$ elevation of the head of the bed, adequate sedation level (Ramsay 2 or 3), DVT/PE prevention, peptic ulcer prophylaxis and oral decontamination with chlorhexidine $0.12 \%$.

From February 2009 onwards, the ICU nursing staff and ICT performed a daily checklist in order to observe the five issues accomplishment. If any item was found to be inadequate it was promptly corrected. 


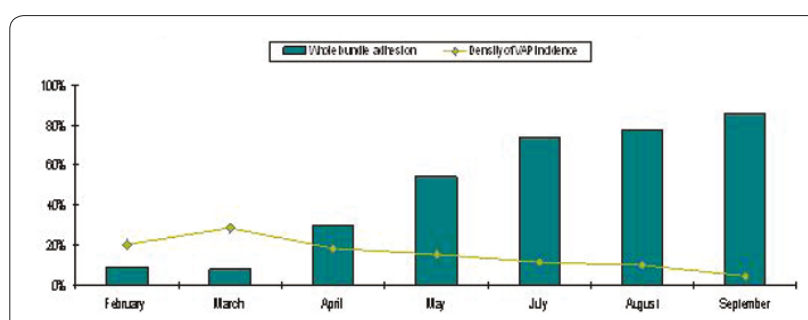

Figure 1 (abstract P83). Proportion of bundle adhesion and density of incidence of ventilator-associated pneumonia.

Results In February and September, adhesion to the whole bundle was 9\% and $86 \%$, respectively $(P<0.001)$ (Figure 1$)$. VAP density was proportionally lower to bundle adhesion in the same period, 20 per 1,000 ventilation/day and 4.5 , respectively.

Conclusions Initial VAP rates were extremely high even for Brazilian benchmarks. Although we could not implement expensive technologies like continuous aspiration of subglottic secretions, the ICU team and ICT efforts were crucial for satisfactory results, as well the administrative board support, which turned this issue into an institutional priority. Our goals are to reduce even more, implementing the 'ventilator bundle - getting to zero' program, and maintaining a continued effort to sustain these results.

\section{P84}

Ventilator-associated pneumonia rate and ventilator bundle compliance in a district general hospital

EWinkley, E Sykes

Northumbria Healthcare NHS Trust, Ashington, UK

Critical Care 2010, 14(Suppl 1):P84 (doi: 10.1186/cc8316)

Introduction An observational study to establish the incidence of ventilator-associated pneumonia (VAP), and ventilator care bundle (VCB) compliance. Neither has previously been quantified at our institution. VAP is a nosocomial pneumonia presenting in patients mechanically ventilated for $\geq 48$ hours [1]. Use of microbiological data in conjunction with the Clinical Pulmonary Infection Score (CPIS) improves VAP diagnostic specificity [1]. VCBs reduce VAP rates, in some cases to zero. The Department of Health VCB is one such collection of evidence-based interventions [2].

Methods A 3-month (April 2009 to June 2009) prospective observational study, in an eight-bed critical care unit in a district general hospital. All mechanically ventilated patients, age $\geq 18$, intubated $>48$ hours were included. Pregnant or immunosuppressed patients were excluded. Patients treated with antibiotics for suspected or confirmed VAP were identified. CPIS was calculated on day 0 and day 3 of treatment. VCB compliance was recorded weekly in all patients.

Results A total of 190 ventilator-days were identified with no cases of VAP. The VAP rate per 1,000 ventilator-days is 0 . Sixty-nine percent of cases achieved $100 \%$ VCB compliance. Four of the six VCB elements were 100\% compliant (Table 1). All incidents of noncompliance had valid clinical reasons.

Conclusions The VAP rate at Wansbeck General Hospital is zero. Compliance with a recognised VCB is high. The previous VAP rate was unknown. The impact of the VCB and the short study duration are unclear. Continuous data collection has been implemented to establish whether such results are representative and sustainable. Use of the CPIS to limit inappropriate antibiotic in suspected VAP is planned.

\section{References}

1. Calandra T, et al.: Crit Care Med 2005, 33:1538-1548.

2. [http://www.clean-safe-care.nhs.uk/index.php?pid = 4]
P85

Oral chlorhexidine to prevent nosocomial pneumonia in critically ill patients: a systematic review and meta-analysis

RJ Pugh, P Rathbone

Glan Clwyd Hospital, Rhyl, UK

Critical Care 2010, 14(Suppl 1):P85 (doi: 10.1186/cc8317)

Introduction This systematic review aims to evaluate evidence from randomised controlled trials (RCTs) for oral chlorhexidine in preventing nosocomial pneumonia in intubated mechanically ventilated critically ill adults. Use of oral chlorhexidine appeals since it should reduce bacterial aspiration from the orophayrnx. A number of RCTs have recently been published on this topic.

Methods Search of Medline, Embase, Cochrane library, grey literature registers, conference proceedings and reference lists for RCTs comparing chlorhexidine with placebo or standard care for prevention of pneumonia in the critically ill. Outcomes: episode of nosocomial respiratory tract infection (RTI), mortality, duration of mechanical ventilation (MV) and length of ITU stay (ITU LOS). Review Manager 4.2 (Nordic Cochrane Centre) was used for data synthesis. Effect estimates (odds ratio for dichotomous and weighted mean difference for continuous data) were calculated using a random effects model.

Results Fourteen studies were identified, three involving patients undergoing cardiac surgery (1,841 patients) and 11 involving patients in noncardiothoracic ITUs (1,497 patients; see Table 1). Five studies (including two cardiac studies) found a significant reduction in episodes of nosocomial RTI in the chlorhexidine-treated group versus placebo or standard care. Pooled data indicated a significant reduction in nosocomial RTI in the treatment group among all patients, and among cardiac and noncardiac sub-groups (odds ratio 0.57 ( $95 \% \mathrm{Cl} 0.42$ to 0.77 ), 0.52 (0.37 to 0.75 ) and 0.6 (0.4 to 0.89), respectively). However, no significant differences in mortality, duration of mechanical ventilation or ITU stay were demonstrated. Significant heterogeneity ( $R^{2}$ statistic $>40 \%$ ) was detected for all outcomes except mortality.

Table 1 (abstract P85). Effect estimates

\begin{tabular}{lccc}
\hline & Number of studies & Participants & Effect \\
\hline RTI & 14 & 3,338 & OR 0.57 \\
Mortality & 11 & 3,233 & OR 1.2 \\
MV days & 6 noncardiac & 1,027 & WMD +1.34 \\
ITU LOS days & 4 noncardiac & 702 & WMD -0.51 \\
\hline
\end{tabular}

Conclusions Use of oral chlorhexidine is associated with reduction in nosocomial respiratory tract infection in intubated mechanically ventilated critically ill adults.

P86

Impact of routine multiple site decontamination in intubated patients on ICU-acquired infections

C Camus, S Lavoué, A Gacouin, S Marque, A Gros, Y Le Tulzo

Hôpital Pontchaillou, Rennes, France

Critical Care 2010, 14(Suppl 1):P86 (doi: 10.1186/cc8318)

Introduction We have shown that a the combination of selective digestive decontamination with topical antibiotics (SDD) and a decontamination regimen using nasal mupirocin with chlorhexidine bodywashing (M/C) markedly reduced acquired infections (Al) in intubated patients as compared with SDD alone, $\mathrm{M} / \mathrm{C}$ alone or none [1]. We report the surveillance of $\mathrm{Al}$ in our ICU before and after the implementation of multiple site decontamination (MSD) as a routine prevention procedure.

Table 1 (abstract P84). Ventilator care bundle compliance

\begin{tabular}{lcccccc}
\hline Element & $\begin{array}{c}30 \text { to } 40^{\circ} \\
\text { head elevation }\end{array}$ & $\begin{array}{c}\text { Sedation } \\
\text { hold }\end{array}$ & $\begin{array}{c}\text { DVT } \\
\text { prophylaxis }\end{array}$ & $\begin{array}{c}\text { Gl } \\
\text { prophylaxis }\end{array}$ & $\begin{array}{c}\text { All } \\
\text { Humidification }\end{array}$ & $\begin{array}{c}\text { Tubing } \\
\text { elements }\end{array}$ \\
\hline Compliance (\%) & 100 & 88 & 81 & 100 & 100 & 100 \\
\hline
\end{tabular}


Methods MSD was implemented in June 2007. We compared the incidence rates of Al (expressed per 1,000 patient-days (\%) or per 1,000 device-days for device-related Al) between the last 1-year period before (period A, 7,723 patient-days) and the first 1-year period after (period B, 7,646 patient-days) MSD use was started.

Results In period B (MSD) versus period A (no MSD), there was a reduction in the rates of total $\mathrm{Al}(9.4 \%$ vs $23.6 \%)$, bloodstream infections $(1.0 \%$ v v $4.0 \%$ ), ventilator-acquired pneumonia (5.6\%o vs $19.1 \%$ ) (all $P<0.01$ ), and to a less extent catheter-related urinary tract infection ( $1.0 \%$ vs $2.2 \%$, $P=0.11)$. Multivariate analysis with the Cox regression model showed that period $\mathrm{A}$ (OR 2.34 (1.64 to 3.20)) and the presence of a central venous catheter (OR 2.07 (1.22 to 3.57)) were the two independent risk factors for Al. In period $\mathrm{B}$, there was a statistically significant reduction in the rates of $\mathrm{Al}$ involving S. aureus $(1.0 \%$ vs $3.0 \%)$, coagulase-negative staphylococci $(0.4 \%$ vs $1.3 \%$ ), Candida (0.4\%o vs 1.3\%) and aerobic Gram-negative bacilli (5.0\%o vs $15.8 \%$ ), especially Enterobacteriaceae (3.4\%o vs $9.4 \% 0)$, Pseudomonas aeruginosa (1.4\% vs 3.9\%), S. maltophilia (0 vs 1.7\%) and Acinetobacter sp. (0 vs $0.8 \%$ ) (all $P \leq 0.05)$. MRSA Al rates were also lower ( $0.3 \%$ vs $0.9 \%$, NS) and no Al due to VRE occurred during both period. The rates of Al involving antimicrobial-resistant Enterobacteriaceae were lower in period B for 12 antimicrobials tested, especially for ticarcillin (2.1\%o vs $6.5 \%)$, ticarcillin/ clavulanate ( $0.8 \%$ vs $4.0 \%$ ) , cefotaxime $(0.3 \%$ vs $2.2 \%$ ) and colistin $(0.5 \%$ o vs $1.8 \%$ ) (all $P \leq 0.02$ ). Similar findings were observed for antimicrobialresistant $P$. aeruginosa although differences were not significant. Overall, the rate of $\mathrm{Al}$ involving organisms defined as multidrug-resistant (MDROs) markedly decreased in period B (2.5\% vs 9.1\%, $P<0.001)$.

Conclusions Routine use of MDS was associated with a strong reduction in ICU AI. The reduction was consistently observed for all principal classes of pathogens, including MDROs. No new MDRO emerged.

\section{Reference}

1. Camus C: Crit Care Med 2005, 33:307-314

P87

Ventilator-associated pneumonia caused by Pseudomonas aeruginosa and respiratory colonization by Candida spp.

F Antonicelli, R Festa, F Idone, F Di Muzio, R Maviglia, M Antonelli

Università Cattolica del Sacro Cuore, Roma, Italy

Critical Care 2010, 14(Suppl 1):P87 (doi: 10.1186/cc8319)

Introduction This study aims to test the association of an increased mortality in critically ill patients with ventilator-associated pneumonia (VAP) caused by Pseudomonas aeruginosa in patients with a previous respiratory tract colonization by Candida spp. compared with that of patients with a VAP by $P$. aeruginosa without Candida spp. isolation.

Methods A retrospective study on all 5,236 critically ills patients admitted to the ICU of a university hospital from 2001 to 2008, of which 1,097 received mechanical ventilation and in whom Pseudomonas and/or Candida were isolated in quantitative cultures from the Mini-BAL [1].

Results $P$. aeruginosa and Candida spp. were present at the same time in 295 patients ( $P-C$ group), P. aeruginosa only in 92 patients ( $P$ group) and Candida spp. only in the remaining 710 patients (C group). SAPS II was $44.27 \pm 14.8$ (mean \pm SD), $39.07 \pm 14.61$ and $39.07 \pm 14.61$, respectively, for P-C, P and C groups. The SAPS II score of the C group was significantly higher than the $P$ group $(P<0.05)$, the SAPS II score of the $C$ group was higher than the P-C group $(P>0.05)$, and the SAPS II score of the $P$ group was lower than the P-C group $(P>0.05)$. The population with Candida spp. isolation alone was older (70.25 years vs 63.17 (group P-C) and 60.22 (group P), $P<0.05$ ). The group with Pseudomonas associated with Candida spp. had a mortality rate higher than the $P$ group $(57.96 \%$ vs $6.05 \%$, $P<0.05)$. The length of stay in the ICU of patients with Candida spp. and $P$. aeruginosa was not different from the group who had $P$. aeruginosa alone ( $26.22 \pm 31.80$ vs $29.15 \pm 25.66, P>0.05$ ). Duration of hospitalization in the group with only $P$. aeruginosa was longer than the other two groups (79.38 days vs 51.85 of the group $P-C$ and 28.40 of group $C, P<0.05$ ).

Conclusions Candida colonization seems to increase the risk for Pseudomonas infection [2]. Patients who developed VAP due to P. aeruginosa with previous colonization of the respiratory tract by Candida spp. were older, with a higher SAPS II score and had a high mortality.

\section{References}

1. Koenig SM, Truwit JD: Clin Microbiol Rev 2006, 19:637-657.

2. Azoulay E, et al:: Chest 2006, 129:110-117.
P88

Time course of RT-PCR positivity in H1N1-induced ARDS

E Mannelli', M Murtigni', M Andreani', S Cianferoni', D Colosimo', G Zagli², M Bonizzoli², A Azzi ${ }^{3}$, A Peris ${ }^{2}$

'Postgraduate School of Anesthesia and Intensive Care, University of Florence, Italy; ${ }^{2}$ Careggi Teaching Hospital, Florence, Italy; ${ }^{3}$ University of Florence, Italy Critical Care 2010, 14(Suppl 1):P88 (doi: 10.1186/cc8320)

Introduction The aim of this study was to analyze the correlation between antiviral therapy efficacy and the negative profile of the RT-PCR made on pharyngeal swab, subglottic aspiration, and bronchoalveolar lavage in patient affected by ARDS caused by H1N1 infection.

Methods A prospective analysis was performed on 11 patients admitted to the ICU of a tertiary referral center (Careggi Teaching Hospital, Florence, Italy). All patients underwent daily RT-PCR monitoring on pharyngeal swab, subglottic aspiration, and bronchoalveolar lavage. All patients were treated with oral administration of oseltamivir (75 mg twice daily) and inhaled zanamivir (10 mg twice daily) since ICU admission. Six patients were treated with extracorporeal membrane oxygenation (ECMO) due to their critical respiratory conditions. Two of them resulted co-infected by legionella pneumophila.

Results As shown in Figure 1, RT-PCR from pharyngeal swab at ICU admission failed to demonstrate the viral infection in four patients, whereas RT-PCR from bronchoalveolar lavage had a sensibility of $100 \%$. Similarly, the time course showed that RT-PCR from pharyngeal swab resulted negative in an average time of 3 days after therapy start, while RT-PCRs from bronchoalveolar lavage continued to permit infection monitoring and therapy regimen conduction. None of RT-PCRs on subglottic aspiration samples resulted positive. All patients recovered and were discharged alive from ICU in spontaneous breathing.

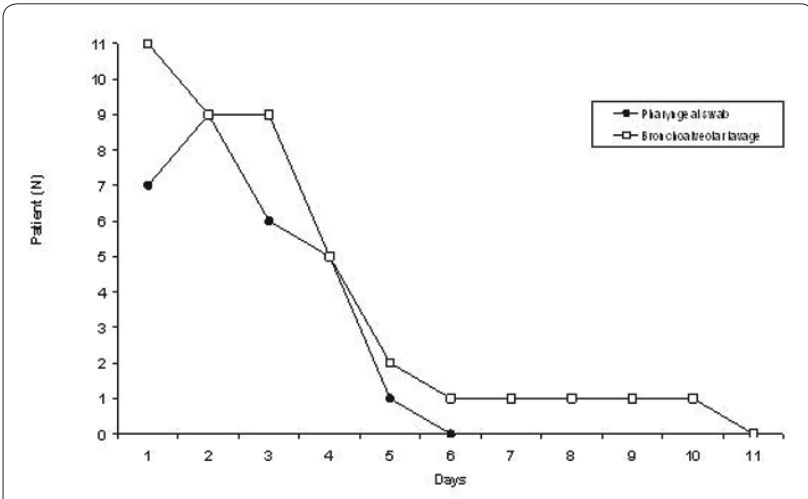

Figure 1 (abstract P88). Time course of RT-PCR positivity for $\mathrm{H} 1 \mathrm{~N} 1$ infection.

Conclusions In our experience, the most reliable method to diagnose and monitor H1N1 infection was RT-PCR from bronchoalveolar lavage, since pharyngeal swab do not offer enough sensibility, either for antiviral therapy initiation or for antiviral therapy management. Samples from subglottic aspiration can be avoided due to a low sensibility.

\section{P89}

Novel swine influenza A: most frequent ultrasonographic lung patterns

LTutino ${ }^{1}$, F Barbani', G Cianchi², S Batacchi², L Perretta ${ }^{2}$, R Cammelli², M Bonizzoli2, R Spina' ${ }^{2}$ G Zagli2, A Peris ${ }^{2}$

'Postgraduate School of Anesthesia and Intensive Care, Faculty of Medicine,

Florence, Italy; ${ }^{2}$ Anaesthesia and Intensive Care Unit of Emergency

Department, Careggi Teaching Hospital, Florence, Italy

Critical Care 2010, 14(Suppl 1):P89 (doi: 10.1186/cc8321)

Introduction Bedside lung ultrasound is able to identify with high sensibility and sensitivity most pulmonary pathological patterns and is widely adopted in critically ill patients' daily management. It is a feasible 
and reliable method for the identification of the lung pathological patterns caused by H1N1 influenza infection.

Methods The study took place in the ICU of a regional referral center, with ECMO availability, for respiratory failure (Careggi Teaching Hospital, Florence, Italy). Eight patients admitted for H1N1-induced ARDS (September to October 2009) underwent daily LU examination. The examination was standardized with a procedure ad hoc in order to achieve complete and comparable reports for every patient. Patients were examined supine, taking lateral and anterior views and, if possible, on one side. Intercostal spaces were used as acoustic windows. Every single examination and its finding have been reported in our ICU database.

Results Pleural thickness was present in 100\% of cases, mostly related to little and multiple pleural consolidations. Anyway, pleural gliding was present most of the time (87.5\%), even if with a visible decrease of its movement. This was replaced with the lung pulse only in the presence of important lung consolidation. Alveolar interstitial syndrome (AIS) was always present on the whole lung, ranging from moderate to severe (100\%), with high positivity at the base, posteriorly (100\%). White lung appeared in every patient, most at the lung base and in the middle fields, posteriorly. As far as consolidation is concerned, its presence was confirmed in 100\% of the patients, associated with satellite multiple subpleural consolidations in $37.5 \%$ of patients. Basal lung was always involved (100\%), followed by middle (50\%) and apical fields(25\%). Bronchogram was present in $100 \%$ of patients in bigger consolidations. Their aspect was aerial and only in one patient, with severe consolidations, turned into a fluid one. Anechoic pleural effusions were found in $37.5 \%$ of patients. No cases of pneumothorax were detected.

Conclusions In this group of patients, H1N1 infection shows different lung patterns altogether, where the most frequent seemed to be severe basal posterior AIS, multiple subpleural lung consolidations, and multiple parenchymal consolidation with bronchogram. The presence of spared areas did not seem to belong to H1N1 LU patterns.

\section{P90}

Outcome and prognostic factors in patients with HIV-negative pneumocystis pneumonia requiring mechanical ventilation

EChoi

Asan Medical Center, Seoul, Republic of Korea

Critical Care 2010, 14(Suppl 1):P90 (doi: 10.1186/cc8322)

Introduction Pneumocystis pneumonia (PCP) in HIV-negative patients frequently presents as fulminant respiratory failure and is associated with a high mortality rate when the patient requires mechanical ventilation. The aims of this study were to evaluate the outcome and prognostic factors in the patients with HIV-negative PCP requiring mechanical ventilation (MV). Methods We retrospectively reviewed the medical records and collected the HIV-negative patients who were microbiologically confirmed as PCP and required MV in ICU over a 10-year period in a tertiary care teaching hospital.

Results A total of 51 patients were identified. Mean age was $55.4 \pm 15.0$ years. Mean APACHE II score at ICU admission was $25.7 \pm 5.8$. The 28-day mortality and in-hospital mortality were $45.8 \%$ and $66.7 \%$, respectively. Between survivors and nonsurvivors, there were no significant differences in baseline characteristics, APACHE II score, $\mathrm{PaO}_{2} / \mathrm{FiO}_{2}$ ratio, and absolute neutrophil counts on ICU admission day. Also the mortality was not different in relation to the presence of barotrauma, application of noninvasive ventilation, timing of susceptible antibiotic administration, changing or not to salvage regimens, presence of cytomegalovirus coinfection and even the microbiologic persistency in follow-up specimens. Based on the types and intensity of previous immunosuppressive therapy, we classified patients into three subgroups: patients receiving low-dose steroid maintenance \pm other immunosuppressive agents (LS), which represent previously stable organ transplants; another group consisting of patients receiving recent intensive chemotherapy (CTx); and the other group refers to patients receiving high dose (defined as $>2$ weeks at least $1 \mathrm{mg} / \mathrm{kg}$ dose) steroid therapy \pm other immunosuppressive agents (HS). Significant differences of outcome were observed among the three different groups (28-day mortality: $\mathrm{LS}=22.2 \%, \mathrm{CTx}=29.4 \%, \mathrm{HS}=71.4 \%$, $P=0.01 ; 60$-day mortality: $\mathrm{LS}=33.3 \%, \mathrm{CT} x=64.7 \%, \mathrm{HS}=81.0 \%, P=0.04)$. Conclusions Our data showed that the mortality of fulminant HIVnegative PCP requiring MV was significantly different according to the types and intensity of previous immunosuppressive treatment despite similar clinical features on ICU admission.

\section{P91}

Acute respiratory failure from pandemic influenza $A(H 1 N 1)$ in an intensive care unit in southern Brazil

S Beduschi Filho, D Siqueira, SC Carvalho Flores, I Yoshiko Masukawa, L Kretzer, AC Burigo Grumann, I Silva Maia, M Pimentel Pincelli

Hospital Nereu Ramos, Florianópolis, Brazil

Critical Care 2010, 14(Suppl 1):P91 (doi: 10.1186/cc8323)

Introduction In the winter of 2009 the influenza A (H1N1) pandemic reached Brazil, affecting severely its southern states. A total 68,806 cases of suspected pandemic influenza A infection had been reported in Brazil and $91 \%$ of the specimens tested were positive. Incidence of pandemic influenza A in southern Brazil was 137/100,000. In the southern Brazilian state of Santa Catarina, Nereu Ramos Hospital is the referral center for the treatment of infectious diseases, and it provided eight intensive care beds for the management of acute respiratory failure from suspected cases of influenza A during the 2009 pandemic.

Methods We collected retrospective data on the epidemiological features, clinical course and ventilatory characteristics of patients with laboratoryconfirmed influenza A (H1N1) infection who were admitted to our ICU during the winter of 2009

Results Thirty-four adult patients were admitted to our ICU with acute respiratory failure and suspected pandemic influenza A infection. Of these, 14 cases tested positive for influenza A ( $\mathrm{H} 1 \mathrm{~N} 1)$. The majority of the patients were male $(61.5 \%)$ with median age of 27.5 years and interquartile range (IR) of 26.5 to 48.5. The APACHE II median was 14.5 (IR 10.0 to 18.25) with median predicted mortality of 20.0 (IR 10.73 to 27.7 ). Risk factors were: obesity (23.1\%), obstructive respiratory diseases (15.4\%); pregnancy, immunosuppressive and neuromuscular disorders were present in one patient each. All 14 patients presented severe respiratory failure, with a median lowest $\mathrm{PaO}_{2} / \mathrm{IOF}$ of 113.3 (IR 83.53 to 159.55 ) and $92.3 \%$ of them requiring mechanical ventilation. The patients in mechanical ventilation were all ventilated in pressured-controlled mode, demanded high PEEP levels (mean of $18.5 \pm 5.16 \mathrm{cmH}_{2} \mathrm{O}$ ), presented high peak inspiratory pressure (mean of $37.83 \pm 7.35 \mathrm{CmH}_{2} \mathrm{O}$ ) and required elevated IOF $(81.15 \pm 20.83 \%)$. A total of $57.1 \%$ of the patients were submitted to lung recruitment, while the prone position was used in $42.8 \%$ of them. The median ICU stay was 13.0 days (IR 4.75 to 23.50) and the median hospital stay was 16.5 days (IR 9.75 to 25.0). The ventilatory-associated pneumonia (VAP) rate was unusually high (69.2\%) as well as the mortality rate during the intensive care stay (38.5\%).

Conclusions Patients admitted to our ICU with confirmed pandemic influenza A infection presented severe acute respiratory failure and an unusually high incidence of VAP and mortality rates.

Reference

1. Perez-Padilla R, et al:: Pneumonia and respiratory failure from swine-origin influenza A (H1N1) in Mexico. N Engl J Med 2009, 361:680-689.

\section{P92}

Extracorporeal membrane oxygenation for influenza A (H1N1): experience in a regional referral center

A Pasquini', S Di Valvasone², S Biondi², S Batacchi', G Cianchi', M Ciapetti', M Bonizzoli', R Spina', L Turrisi', E Mascitelli³, M Bonacchi', G Zagli', GF Gensini', A Peris'

'Careggi Teaching Hospital, Florence, Italy:'2Postgraduate School of Anesthesia and Intensive Care, Florence, Italy; ${ }^{3}$ University of Florence, Italy Critical Care 2010, 14(Suppl 1):P92 (doi: 10.1186/cc8324)

Introduction The novel influenza A H1N1 virus can cause, in a restricted subgroup of infected patients, an acute respiratory failure not responding to conventional treatment. In selected cases, extracorporeal membrane oxygenation (ECMO) has been applied with a $21 \%$ mortality rate. Here we report the experience of the ICU of a regional referral center for ECMO (Careggi Teaching Hospital, Florence, Italy).

Methods An Emergency Medical Service has been established for the novel pandemic influenza to guarantee the possibility of ECMO initiation in the peripheral hospitals by our ECMO Team and the subsequent transport 
on extracorporeal circulation to our referral center. The ECMO Team is composed of an intensivist, a cardiac surgeon, a cardiologist, a perfusionist and a nurse. All of the different figures were properly trained and formed on ECMO treatment. According to our internal protocol, eligible patients for ECMO treatment are aged 15 to 70 years old with acute respiratory failure with one of the following conditions: a ratio $\mathrm{PaO}_{2} / \mathrm{FiO}_{2}<60$ or $\mathrm{pH}<7.20$ under protective ventilation conditions. ECMO insertion was achieved percutaneously and a high-flow approach (5 to $6 \mathrm{l} /$ minute) was initially established according to patient need. ECMO device is a Rotaflow Maquet Centrifugal Pump with a Quadrox-D oxygenator (Maquet, Rastatt, Germany) and biocoated circuits. H1N1 infection was monitored by RTPCR examination on pharyngeal swab and bronchial aspirate. Antiviral therapy was conducted by oral administration of oseltamivir $(75 \mathrm{mg}$ twice daily), and inhaled zanamivir (10 mg twice daily).

Results From October 2009 to November 2009, six patients with influenza A (H1N1) have been treated with ECMO support. Three patients were cannulated in the district hospital and transported safety to our ICU by our ECMO Team. Median SAPS II at admission, median age and sex were as listed as follows: 46,35 years old and five males/one female. All patients had an acute respiratory failure accompanied by a multiorgan dysfunction. ECMO was established and maintained for 200 hours. All patients were successfully weaned from ECMO support, extubated and discharged from our ICU. No major procedure-related complications were observed.

Conclusions Well-timed ECMO use in cases of influenza A H1N1 acute respiratory failure could improve overall the survival rate.

\section{P93}

Budd-Chiari syndrome complicated by abdominal compartment syndrome: evidence of central hypovolaemia?

D Joshi, S Saha, W Bernal, J Wendon, G Auzinger

King's College Hospital, London, UK

Critical Care 2010, 14(Suppl 1):P93 (doi: 10.1186/cc8325)

Introduction Budd-Chiari syndrome (BCS) is characterised by hepatic venous outflow obstruction (HVOO) leading to post-sinusoidal portal hypertension, congestion of the liver with caudate lobe hypertrophy. In addition to intra-abdominal hypertension (IAH), caused by severe ascites, HVOO may aggravate the cardiovascular disturbances seen in patients with decompensated disease. The aim was to study the haemodynamic response to abdominal decompression in BCS compared with patients with decompensated cirrhosis.

Methods Ten patients with BCS admitted to the Liver ICU, King's College Hospital were studied. Transpulmonary thermodilution cardiac output monitoring and calculation of volumetric indices of preload was performed with the PiCCO system. Haemodynamic variables and IAP were analysed pre and post intervention. The control group comprised of cirrhotic patients with IAH requiring abdominal paracentesis (PC).

Results Ten patients with BCS were studied, median age 39 years (range (R) 20 to 52); eight had liver transplantation and two had a surgical shunt procedure. Eight patients (PC), median age 59 (33 to 65), underwent abdominal paracentesis for tense ascites. IAP was raised in both groups pre intervention (23, R 17 to 40, BCS vs 26, R 20 to 40, PC). ITBVI remained low in the BCS group (632, R 453 to 924) pre intervention despite aggressive volume resuscitation (median positive FB10L, R 0.5 to 39). Post intervention, a reduction in IAP was seen in both groups (BC $P<0.001$, $P C P<0.0001)$. ITBVI increased (633, R 453 to 924 vs 736, R 512 to 1,110, $P=0.001$ ) in BCS patients. No change in ITBVI was noted (pre 870, R 598 to 1,619 vs post $1,036, R 763$ to 1,762 ) in the $C P$ group despite albumin replacement. An increase in $\mathrm{Cl}$ and SVI was noted in both groups: BCS (Cl $P=0.003, \mathrm{SVI} P=0.007), \mathrm{CP}(\mathrm{Cl} P=0.005, \mathrm{SVI} P=0.02)$. There was an inverse relationship between IAP, CI $(P=0.003), \mathrm{SVI}(P=0.004)$ and ITBVI $(P=0.01)$ in BCS patients. In the CP group, IAP did not correlate with ITBVI.

Conclusions Compared with cirrhotic patients with ascites, patients with $\mathrm{BCS}$ and IAH have evidence of central hypovolaemia. We postulate that in addition to raised IAP, hepatic venous obstruction and caudate lobe hypertrophy limit venous return in patients with BCS. Reduction in IAP restores preload with improvement in cardiac output.

\section{References}

1. Cheatham et al.: Intensive Care Med 2007, 33:951-962.

2. Malbrain et al:: Intensive Care Med 2006, 32:1722-1732.

\section{P94}

Adenosine signalling has a protective role in intestinal ischemia and reperfusion injury

K Zimmermann'1, T Krieg'2, M Soltow', D Pavlovic', J Zhou ${ }^{3}$, S Whynot ${ }^{3}$,

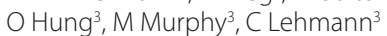

'Ernst Moritz Arndt University Greifswald, Germany; ${ }^{2}$ Department of

Cardiology, Cambridge, UK: ${ }^{3}$ Dalhousie University, Halifax, Canada

Critical Care 2010, 14(Suppl 1):P94 (doi: 10.1186/cc8326)

Introduction Gut ischemia and reperfusion (IR), for example in small bowel transplantation or following resuscitation, may result in severe impairment of the intestinal microcirculation. Potential sequelae are mucosal damage, loss of barrier function, bacterial translocation, systemic inflammation, multiple organ failure and death. We hypothesized that extracellular adenosine signalling has a protective role in intestinal IR injury. Using intravital microscopy we investigated the effects of the adenosine receptor agonist NECA (5'-N-ethyl carboxamide adenosine) on leukocyte-endothelial interactions and capillary perfusion in the intestinal microcirculation following intestinal IR.

Methods Six groups of animals ( $n=7$ per group) were studied: control (CON), NECA, IR (30 minutes of intestinal ischemia, 2 hours of reperfusion), $I R+N E C A, I R+N E C A+M R S 1754$ (adenosine $A_{2 B}$ receptor antagonist), IR $+\mathrm{NECA}+\mathrm{DPCPX}$ (adenosine $A_{1}$ receptor antagonist). All substances were administered immediately after declamping of the superior mesenteric artery. Intravital microscopy was performed after 2 hours of reperfusion. Leukocyte adhesion (rolling/firm adherence) and functional capillary density (FCD) were measured offline by blinded investigators.

Results Following IR we observed a significant increase of leukocyte adherence in the intestinal submucosal venules (for example, $\mathrm{V} 1$ venules: CON $20.1 \pm 5.8 \mathrm{n} / \mathrm{mm}^{2}$, IR $237.8 \pm 24.2 \mathrm{n} / \mathrm{mm}^{2}, P<0.05$ ). Capillary perfusion of the muscular layers of the intestinal wall was reduced (for example, longitudinal muscular layer: CON $112.3 \pm 5.3 \mathrm{~cm} / \mathrm{cm}^{2}, I R \quad 92.4 \pm 8.4 \mathrm{~cm} /$ $\mathrm{cm}^{2}$ ). NECA administration reduced significantly leukocyte adherence (V1 venules: $67.8 \pm 6.8 \mathrm{n} / \mathrm{mm}^{2}, P<0.05$ ) and improved capillary perfusion (longitudinal muscular layer: $113.9 \pm 8.3 \mathrm{~cm} / \mathrm{cm}^{2}$ ). Administration of the adenosine $A_{2 B}$ receptor antagonist completely reversed the NECA effects (for example, leukocyte adherence V1 venules: $228.8 \pm 33.2 \mathrm{n} / \mathrm{mm}^{2}$ ), whereas $A_{1}$ receptor inhibition only partially abolished the action of NECA (for example, leukocyte adherence $V 1$ venules $124.5 \pm 14.5 \mathrm{n} / \mathrm{mm}^{2}$ ).

Conclusions The data support the hypothesis, that adenosine signalling is involved in intestinal IR injury. The adenosine $A_{2 B}$ receptors are more important than adenosine $A_{1}$ receptors since inhibition of $A_{2 B}$ receptor by MRS1754 completely reversed the effect of the adenosine receptor agonist NECA.

\section{References}

1. Förster et al:: Basic Res Cardiol 2006, 101:319-326.

2. Solenkova et al:: Am J Physiol 2005, 290:H441-449.

3. Hart et al:. I Immunol 2009, 182:3965-3968.

\section{P95}

Hemodynamic states during the course of norepinephrine weaning E Kipnis, H Tytgat, N Bruneau, B Leroy, G Lebuffe, B Tavernier, B Vallet

Centre Hospitalier Régional Universitaire de Lille, France

Critical Care 2010, 14(Suppl 1):P95 (doi: 10.1186/cc8327)

Introduction Norepinephrine is a standard of care in the hemodynamic management of septic shock concomitantly to fluid administration. However, no guidelines exist concerning norepinephrine weaning. Furthermore, norepinephrine may rapidly restore macrocirculatory hemodynamics through stressed volume without treating the underlying volume deficit, especially in the absence of optimal volume expansion. Norepinephrine weaning may reveal latent/persisting need for volume expansion in apparently stable patients.

Methods Observational study of norepinephrine weaning in seven resuscitated SICU septic shock patients. Weaning was performed in $0.1 \mathrm{mg} /$ hour decrements every 5 to 15 minutes until hemodynamic instability, defined as a mean arterial pressure (MAP) $\leq 70 \mathrm{mmHg}$, interrupted the process, at which point pulse pressure variation (PPV) was measured and transthoracic echocardiography (TTE) performed as per standard procedures. Upon instability with PPV $\geq 13 \%, 250 \mathrm{ml}$ fluid challenge was performed and the MAP increase allowed pursuit of weaning, whereas 
instability with PPV $<13 \%$ halted weaning. Nonparametric correlations were sought between norepinephrine decrements and variations over weaning (and fluid challenge) in PPV, arterial compliance, and indexed systolic ejection volume (iSEV). Global resuscitation endpoints (serum lactate, $\mathrm{SCVO}_{2}$ ) were compared before and after the entire weaning process.

Results Two behaviors were observed. First, norepinephrine weaning unmasked latent preload dependency in steps characterized by marked PPV increase (16.5\%, 8 to 36), correlated to norepinephrine decrements $\left(\rho=0.971 ; r^{2}=0.745 ; P=0.005\right)$ resulting in PPV $\geq 13 \%$ and fluid challenge increasing the MAP and allowing pursuit of weaning. Second, weaning revealed norepinephrine dependency in steps halted by instability and characterized by slight PPV increases (3.9\%, 0.3 to 8.4), resulting in PPV $<10 \%$, and a decrease in iSEV that was correlated to norepinephrine decrements $\left(\rho=0.943 ; r^{2}=0.830 ; P=0.001\right)$. Globally, weaning decreased serum lactate to under $2 \mathrm{mmol} / \mathrm{l}$ with $\mathrm{ScvO}_{2}$ maintained above $70 \%$.

Conclusions Hemodynamic stability through norepinephrine use in septic shock might be at the cost of occulting residual fluid requirements which can be revealed during norepinephrine weaning.

\section{P96}

Threshold levels of extreme body surface area that may cause a misrepresentation of cardiac performance

WT McGee', BH Nathanson²

'Baystate Medical Center, Springfield, MA, USA; ${ }^{2}$ OptiStatim, LLC, Longmeadow, MA, USA

Critical Care 2010, 14(Suppl 1):P96 (doi: 10.1186/cc8328)

Introduction Stroke volume (SV) and cardiac output (CO) are standardized into the stroke index (SI) and cardiac index (CI) by dividing by the patient's body surface area (BSA). Commonly used algorithms in hemodynamic management suggest diverse therapeutic interventions based on low, high, or normal SI or Cl. Morbid obesity is increasingly common. When morbidly obese patients have their SV or CO indexed, high absolute values become low $\mathrm{SI}$ and $\mathrm{Cl}$, and may be misleadingly low. This would then cause therapeutic interventions opposite to their needs.

Methods BSA was derived using the Mosteller formula on the metric equivalent of simulated patients ranging from $5 \mathrm{ft}$ to $7 \mathrm{ft}$ and $100 \mathrm{lbs}$ to $700 \mathrm{lbs}$. A priori, we defined normal $\mathrm{CO}=4$ to 8 , normal $\mathrm{Cl}=2.5$ to 5.0 , normal SV $=60$ to $100 \mathrm{ml} /$ beat, and normal SI $=33$ to 47 . Algebraic analysis was used to determine BSA levels that would classify an SV or CO as abnormal.

Results Critical BSA thresholds (T) are presented in Table 1. For example, at $S V=100$, a BSA higher than 3.03 (to the second decimal place) would classify the patient as having a low SI.

\section{Table 1 (abstract P96)}

\begin{tabular}{lccc}
\hline SV & T & CO & T \\
\hline 90 & 2.72 & 7 & 2.80 \\
100 & 3.03 & 8 & 3.20 \\
110 & 3.33 & 9 & 3.60 \\
120 & 3.64 & 10 & 4.00 \\
130 & 3.94 & 11 & 4.40 \\
\hline
\end{tabular}

Conclusions Patients with extreme BSAs are increasingly encountered in the ICU, especially larger BSAs related to obesity. We provide threshold values where extreme BSAs will classify high SV or CO values as low indexed values. The ranges considered normal for SI and CI may be inappropriate for patients with extreme BSAs, particularly in the obese. We caution against relying solely on the $\mathrm{SI}$ and $\mathrm{Cl}$ to assess hemodynamic performance. Instead, the SV and CO along with other physiological parameters should also be considered before making therapeutic decisions.
P97

Cardiac output measurement is feasible in the presence of left-to-right shunt with ultrasound dilution method: a validation study in lambs

S Vrancken, W De Boode, J Hopman, S Singh, K Liem

Radboud University Nijmegen Medical Centre, Nijmegen, the Netherlands

Critical Care 2010, 14(Suppl 1):P97 (doi: 10.1186/cc8329)

Introduction It remains a great challenge to measure systemic blood flow in critically ill newborns, especially during the transitional period with intracardiac and extracardiac shunts. Due to technical restraints, size limitations, the necessity for a relatively large amount of blood withdrawn and possible indicator toxicity, many methods of cardiac output monitoring are not feasible, hence cardiac output is generally estimated from indirect parameters of systemic blood flow. In a former study we assessed the agreement for cardiac output using the ultrasound dilution method (UDCO) and ultrasound transit time-based measurement of main pulmonary blood flow in a juvenile piglet model without shunts [1]. In the present study we analyzed the influence of a left-to-right shunt on the agreement between UDCO and ultrasonic transit time pulmonary blood flow in a juvenile lamb model.

Methods In this prospective, experimental animal study, which was approved by the Ethical Committee on Animal Research of the Radboud University Nijmegen, we placed a Gore-Tex ${ }^{\circledR}$ shunt between the left pulmonary artery and the descending aorta in eight randombred newborn lambs (3.5 to $8.3 \mathrm{~kg}$ ). This aortopulmonary shunt was intermittently opened and closed while cardiac output was manipulated by creating hemorrhagic hypotension. Ultrasound dilution cardiac output (Q-UDCO) - using repeated injection of $1.0 \mathrm{ml} / \mathrm{kg}$ isotonic saline at body temperature - was compared with pulmonary blood flow (Q-MPA) invasively measured by a perivascular flow probe around the main pulmonary artery.

Results A total of 220 measurements were performed. Bias, defined as Q-UDCO minus Q-MPA, was calculated for each measurement. With an open shunt there was a significant left-to-right shunt (mean Qp-/Os- ratio 1.8; range 1.6 to 2.6). Mean bias (SD) was 6.80 (18.0) $\mathrm{ml} / \mathrm{kg} / \mathrm{minute}$ with a closed shunt and $11.1(19.8) \mathrm{ml} / \mathrm{kg} / \mathrm{minute}$ with an open shunt. Limits of agreement ( $\pm 1.96 \mathrm{SD}$ ) were $\pm 35.2 \mathrm{ml} / \mathrm{kg} /$ minute and $\pm 38.8 \mathrm{ml} / \mathrm{kg} /$ minute, respectively. Percentage error was $22.0 \%$ and $24.2 \%$ for measurements with a closed and open shunt, respectively.

Conclusions Cardiac output measurement with the UDCO method is reliable and easily applicable in ventilated juvenile lambs, even in the presence of a significant left-to-right shunt.

\section{Reference}

1. de Boode WP, et al:: Pediatr Crit Care Med 2009, 9. [Epub ahead of print]

P98

The PAPIKAS trial: a comparative clinical trial of pulmonary catheter versus the PiCCO device during therapy of patients with acute heart failure and cardiogenic shock

T Schwab, B Schmid, S Richter, C Bode, HJ Busch

University Hospital, Freiburg, Germany

Critical Care 2010, 14(Suppl 1):P98 (doi: 10.1186/cc8330)

Introduction Haemodynamic measurement plays an important role in the treatment of patients with acute heart failure and cardiogenic shock (CS). The pulmonary catheter (PAC) is a common device for enhanced haemodynamic measurement. The aim of this study was to evaluate the comparability of the PiCCO device with the PAC during the treatment of patients with CS; for example, the use of intra-aortic counterpulsion (intraaortic balloon pumping (IABP)), and therapeutic hypothermia after cardiac arrest.

Methods Seventy-seven measurements were taken in 11 critically ill patients during the therapy of cardiogenic shock. The cardiac index $(\mathrm{Cl})$, stroke volume (SVI), and systemic vascular resistance (SVRI) were measured by pulmonary catheter and PiCCO device, as well as the pulmonary aortic occlusion pressure (PAWP) with a pulmonary catheter and the global end-diastolic volume (GEDI) with the PiCCO device, and registered in a commercially available computer system.

Results A good correlation between the two techniques in all data, as well as during the use of IABP or during hypothermia was shown. The 
mean bias between Clpa and Clart was 0.042. The calculated mean error was $24.89 \%, 26.17 \%$ and $19.08 \%$, respectively. There was also a good convergence between the measurements of vascular resistance in overall data and during IABP. Significant correlations were found for SVIpa, SVIpi, SVRIpa, SVRIpi to CIpa. For GEDI there was a significant correlation with Clpa for data collected during cooling. No significant correlation between PAWP und Clpa was found.

Conclusions The data show interchangeability for the two methods using intermitting measurements during therapy of cardiogenic shock even during intra-aortic counterpulsation and therapeutic hypothermia. The cardiac output seems to be related to the GEDI as a preload parameter; no relationship between PAOP and $\mathrm{Cl}$ can be shown. The usability of the less invasive PiCCO device for the enhanced hemodynamic measurement in patients with acute heart failure and cardiogenic shock is ensured.

\section{P99}

Bioreactance versus PICCOTD/PC in critically ill septic shock patients G Monti', G Pizzilli', M Cecconi², A Rhodes², S Vesconi', P Brioschi', M Pulici', G Casella

'Niguarda Hospital, Milan, Italy; '2St George's Hospital, London, UK

Critical Care 2010, 14(Suppl 1):P99 (doi: 10.1186/cc8331)

Introduction We designed this study to compare the performance in cardiac output (CO) monitoring capabilities of two devices in refractory septic shock patients (RSS Pts): non-invasive transthoracic bioreactance (NICOM) and pulse contour analysis (PICCO PC) coupled to transpulmonary thermodilution (PICCOTD).

Methods We included RSS Pts in vasopressor/inotrope need monitored with both devices. Triplicate measurements of CO by PICCOTD were used to measure $\mathrm{CO}$ at baseline and to calibrate PulseCO. The $\mathrm{CO}$ values recorded simultaneously minute-by-minute by the two systems were compared at baseline (nonperturbated system), in response to a passive leg-raising maneuver ( $P L R=$ leg elevation to $45^{\circ}$ for 2 minutes starting from a supine position) and PEEP test (10 and $15 \mathrm{cmH}_{2} \mathrm{O}$ for 10 minutes each) (perturbated system). We used PICCOTD/PC as the reference technology evaluating the accuracy and estimating the precision of both devices.

Results Continuous CO recording with both devices was performed on 12 consecutive RSS Pts (Nep + Epi $=0.66 \pm 0.15 \mu \mathrm{g} / \mathrm{kg} /$ minute, all ventilated with TV $<8 \mathrm{ml} / \mathrm{kg}$ ). At baseline (nonperturbated system), correlation analysis of NICOM vs PiCCOTD CO showed $r^{2}$ of $0.78(P<0.001)$. BlandAltman analysis evidenced a mean bias of $0.08 \mathrm{l} / \mathrm{min}$ (LOA -1.31 to 1.49 ). The mean CO was $6.01 \pm 1.48 \mathrm{l} / \mathrm{min}$. In a perturbated system the bias of NICOM vs PICCO PC was respectively-0.05 I/min (LOA -1.52 to 1.42 ) and $0.3 \mathrm{l} / \mathrm{min}$ (LOA -2 to 2.6 ) during PLR and PEEP test. The percentage error was $<30 \%$ in $92 \%$ of patients at baseline (nonperturbated system), in $92 \%$ of patients during PLR and in 74\% during PEEP test. In a nonperturbated system the CO precision (calculated as 2 SD/mean over 10 consecutive measurements) was $6.5 \pm 6 \%$ and $6.7 \pm 9 \%$ for NICOM and PiCCOTD, respectively (NS). Precision for NICOM and PICCOPC was respectively 6.8 $\pm 13 \%$ and $4.7 \pm 10 \%$ during PLR and $7 \pm 15 \%$ and $7.6 \pm 15 \%$ during PEEP test.

Conclusions Although limited to a small number of patients, NICOM and PICCO PC, calibrated by TD, seem to have comparable accuracy and precision in CO monitoring in RSS Pts even in a perturbated system.

\section{References}

1. Squara P: Intensive Care Med 2007, 33:1191-1194.

2. Squara P: Crit Care 2009, 13:R125.

\section{P100}

NICOM vs LiDCO ${ }^{T M}$ plus during changes in cardiac output in critically ill patients

M Cecconi', G Monti², S Vesconi², M Hamilton'1, M Grounds ${ }^{1}$, A Rhodes

'St George's Hospital, London, UK; '2Niguarda Hospital, Milan, Italy

Critical Care 2010, 14(Suppl 1):P100 (doi: 10.1186/cc8332)

Introduction NICOM (Cheetah) is a new non-invasive cardiac output (CO) monitor based on bioreactance. We tested the agreement of NICOM vs LiDCO ${ }^{\text {TM }}$ plus in terms of absolute CO values and of the ability to track changes after therapeutic intervention in critically ill patients.

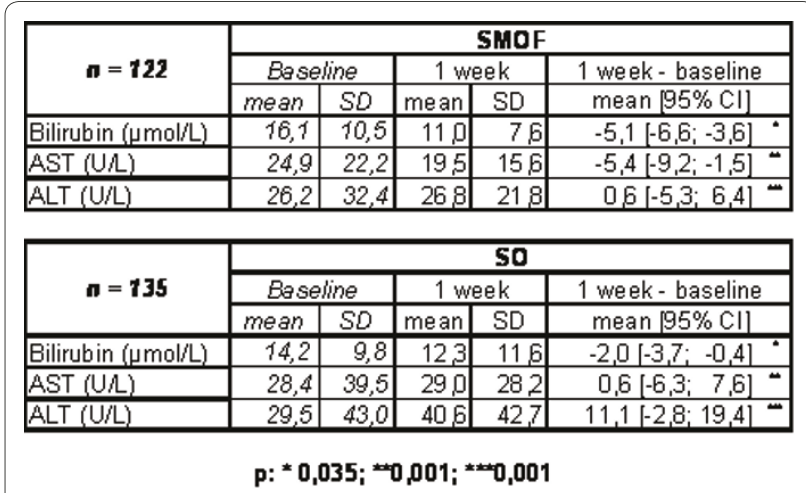

Figure 1 (abstract P100). ROC analysis for NICOM CO in R and NR.

Methods Patients requiring haemodynamic monitoring were monitored with LiDCO ${ }^{\mathrm{TM}}$ plus and NICOM. Three measurements of CO by LiDCO were used to measure $\mathrm{CO}$ at baseline and to calibrate PulseCO. After fluid challenges ( $F C=250 \mathrm{ml}$ colloid/5 minutes) or inotropic therapy alterations, the change in stroke volume (SV) for PulseCO and NICOM CO was recorded. Patients able to increase $S V \geq 10 \%$ as measured by PulseCO were considered responders (R). Bland-Altman analysis was performed for NICOM vs LiDCO CO at baseline. The coefficient of variation (CV) and percentage error (PE) were calculated. $\triangle S V$ of NICOM vs PulseCO was analysed with correlation analysis. In patients receiving $F C$, a $R O C$ analysis was performed to detect the sensitivity and specificity of NICOM to track $\triangle$ SV as measured by PulseCO CO.

Results Baseline haemodynamics in 30 patients enrolled: BA analysis for NICOM vs LiDCO CO showed a mean bias of $-0.18 \mathrm{l} /$ minute (LOA -2.5 to 2.16). Mean CO was $5.8 \mathrm{l} /$ minute, PE was $41 \%$. Mean LiDCO CO CV was $6.8 \%$. A total of 81 pairs of data were collected after FC or inotrope dose change. Correlation analysis showed $r^{2}$ of $0.55(P<0.0001)$ for changes post FC and $r^{2}$ of $0.54(P<0.006)$ post inotrope dose changes. ROC analysis for NICOM CO in R and NR after a FC showed an area under the curve (AUC) of $0.8(P<0.0001$; Figure 1). An increase in NICOM SV $>8 \%$ showed a sensitivity of $76 \%$ and a specificity of $75 \%$ to predict PulseCO changes $>10 \%$.

Conclusions NICOM demonstrated a moderate agreement with LiDCO but showed excellent agreement with PulseCO in tracking CO changes following therapeutic interventions.

\section{P101}

Effect of cardiac arrhythmias on PulseCO calibration and performance

M Jonas', E Mills², CWolff', T O'Brien²

'Southampton University Hospital, Southampton, UK; ${ }^{2}$ LiDCO Ltd, London, UK Critical Care 2010, 14(Suppl 1):P101 (doi: 10.1186/cc8333)

Introduction Arrhythmias are common among high-risk surgical and ICU patients. The PulseCO pressure waveform algorithm is used for both LiDCO ${ }^{\mathrm{TM}}$ plus and LiDCOrapid hemodynamic monitors, which are frequently used to estimate cardiac output (CO) in critically ill patients. Cardiac arrythmias could increase the variation of both the lithium dilution (LiDCO) and/or the PulseCO measurement. At set-up the algorithm is calibrated by comparing the PulseCO CO estimate, averaged over 30 seconds, with a known CO (normally LiDCO) to generate a calibration factor (CF) [1]. This study was designed to explore the effect of arrythmias on the accuracy of CF generation in the PulseCO monitor.

Methods $\mathrm{LiDCO}{ }^{\mathrm{TM}}$ plus hemodynamic data files were obtained retrospectively from a university hospital medical/surgical ICU. Files were separated into those records with and without arrhythmia - defined as heart rate variation $>5 \%$ during at least one additional CF determination after monitor set-up. Previous studies have established the coefficient of variation (CV) of a single LiDCO determination at 8\% [2] and the PulseCO measurement at 2.4\% [3]. A combined $\mathrm{CV}$, reflecting the effect of calibration, is estimated at $8.5 \%$, resulting in an expected precision for the CF of 17\%. Data were analysed for variation in CF against HRV using linear regression and the Student's $t$ test. 
Results Twenty-eight records were collected and analysed. Twenty-one records contained 32 post set-up calibration events. Of these 17 occurred with HRV $\leq 5 \%$ (median $=1 \%$, range: 0 to $5 \%$ ) and 15 occurred while HRV $>5 \%$ (median: $19 \%$, range: 7 to $26 \%$ ). The average variation in CF during HRV was $5.4 \pm 4.0 \%$ and for high HRV was $8.9 \pm 8.1 \%$ of the initial value. The $t$ test indicated no difference in the mean variation of CF $(P=0.162)$ or median. There was no correlation between HRV and CF variation $\left(r^{2}=\right.$ 0.002). Ninety-one per cent (29/32) of the observed CF variation were less than $17 \%$ of the initial CF value.

Conclusions CF determinations are not significantly affected by HRV. PulseCO estimates CO acceptably in the presence of arrhythmias. Interpretation of the data is enhanced by using at least 30 second averaging. References

1. Rhodes A, et al:. Functional Hemodynamic Monitoring Update in Intensive Care and Emergency Medicine, volume 42, 2005:183-192.

2. Cecconi M, et al: Intensive Care Med 2009, 35:498-504.

3. Kemps H, et al.: J Appl Physiol 2008, 105:1822-1829.

\section{P102}

PulseCO consistency: variation in calibration factor over $\mathbf{2 4}$ and $\mathbf{4 8}$ hours E Mills', M Jonas ${ }^{2}$, C Wolff', T O'Brien'

'LiDCO Ltd, London, UK; '2Southampton University Hospital, Southampton, UK Critical Care 2010, 14(Suppl 1):P102 (doi: 10.1186/cc8334)

Introduction The PulseCO pressure waveform algorithm is used for both LiDCO ${ }^{\text {TM}}$ plus and LiDCOrapid hemodynamic monitors to estimate cardiac output (CO). The accuracy of this CO estimate is achieved by comparing (calibrating) the PulseCO with a known CO, for example from the lithium dilution or LiDCO which scales the software algorithm and reduces the bias to the actual CO [1]. This calibration/scaling factor (CF) is patient specific and should not significantly change over a period of a few days as it reflects the patient's aortic capacitance.

Methods LiDCO ${ }^{\text {TM}}$ plus hemodynamic data files were obtained retrospectively from a university hospital medical/surgical ICU. Files were screened to obtain records with at least 24 hours of continuous hemodynamic data and at least one calibration at 24 hours following the initial set-up calibration. Data were analysed for change in CF from initial calibration over 24 and up to 48 hours. Relative change in CF was analyzed for correlation with changes in SVR and CO.

Results Twenty-one records contained at least 24 hours of data with a calibration at 24 hours. Eleven contained at least 48 hours of data and a further calibration at 48 hours. The 32 calibrations were reviewed for consistency of PulseCO and LiDCO results. The average variation in CF over 24 hours $(7.4 \pm$ $6.9 \%($ mean \pm SD) $)$ and 48 hours $(6.5 \pm 6.2 \%)$ were similar $(P=0.362)$. Average changes in SVR were $24.7 \pm 27.4 \%$ (range: 0 to $130 \%$ ); average CO changed by $21.4 \pm 17 \%$ (range: 0 to $57 \%$ ) over 24 hours. There was no correlation between the variation in CF and changes in either CO or SVR.

Conclusions It is known that the estimated coefficient of variation of a single measurement of LiDCO is 8\% [2] and PulseCO is 2.4\% [3]. An orthogonally combined $C V$, reflecting the effects on $C F$, is estimated at $8.5 \%$, or precision of $17 \%$. Ninety-one per cent (29/32) of the observed CF changes were less than $17 \%$ of the initial CF. The PulseCO CF remains constant despite changes in SVR of up to $130 \%$ and CO of up to $57 \%$ over periods of 24 to 48 hours.

\section{References}

1. Rhodes A, et al:: Functional Hemodynamic Monitoring Update in Intensive Care and Emergency Medicine, volume 42, 2005:183-192.

2. Cecconi M, et al:: Intensive Care Med 2009, 35:498-504.

3. Kemps H, et al.: J Appl Physiol 2008, 105:1822-1829.

\section{P103}

Performance of cardiac output measurement derived from arterial pressure waveform analysis in patients undergoing triple-H-therapy of cerebral vasospasms after subarachnoidal hemorrhage

SM Metzelder, R Kopp, M Fries, M Reinges, S Reich, R Rossaint, G Marx, S Rex RWTH Aachen, Germany

Critical Care 2010, 14(Suppl 1):P103 (doi: 10.1186/cc8335)

Introduction The validity of an arterial waveform-based device for measuring cardiac output (CO) without the need for invasive calibration
(FloTrac/Vigileo) in patients needing large doses of vasoactive medication has not yet been thoroughly studied. We performed the present study to assess the validity of both the second-generation and the thirdgeneration software compared with transpulmonary thermodilution CO measurement using the PiCCO technology in patients undergoing triple-H-therapy (hypertonia, hypervolemia, hemodilution) of cerebral vasospasms after subarachnoidal hemorrhage.

Methods Twenty-three patients (18 females and five males) were included in this study. All of them were suffering from a subarachnoidal hemorrhage (Hunt\&Hess grade I to V) due to rupture of a cerebral aneurysm. Triple-H-therapy was initiated for the treatment of cerebral vasospasm. Simultaneous $\mathrm{CO}$ measurements by bolus thermodilution and the FloTrac/Vigileo device were obtained at baseline as well as 2 hours, 6 hours, 12 hours, 24 hours, 48 hours and 72 hours after inclusion. A percentage error of $30 \%$ or less was established as the criterion for method interchangeability.

Results Patients received vasoactive support with $0.53 \pm 0.46 \mu \mathrm{g} / \mathrm{kg} /$ minute norepinephrine, resulting in a mean arterial pressure of $104 \pm$ $13.6 \mathrm{mmHg}$ and a systemic vascular resistance index of 1,741.17 \pm 432.50 dyn.s/ $/ \mathrm{cm}^{5} / \mathrm{m}^{2}$. One hundred and fifty-one CO-data pairs were analyzed. Transpulmonary thermodilution CO ranged from 5.18 to $14.28 \mathrm{l} /$ minute (mean $8.61 \pm 1.93 \mathrm{l} /$ minute) and FloTrac/Vigileo CO ranged from 4.1 to $13.7 \mathrm{l} /$ minute (mean $7.62 \pm 1.79 \mathrm{l} / \mathrm{minute}$ ). Bias and precision (1.96SD of the bias) were $0.99 \mathrm{l} /$ minute and $2.46 \mathrm{l} /$ minute, resulting in an overall percentage error of $28.55 \%$. Subgroup analysis revealed a percentage error of $29.53 \%$ for 67 data pairs measured using the second-generation FloTrac software and $26.44 \%$ for 84 data pairs analyzed by the third-generation software.

Conclusions In patients undergoing triple-H-therapy and needing extensive vasoactive support, CO values obtained by arterial waveform analysis showed good agreement with intermittent transpulmonary thermodilution CO measurements, which was improved by the introduction of a new software generation.

References

1. Mayer J, Boldt J, Wolf MW, Lang J, Suttner S: Cardiac output derived from arterial pressure waveform analysis in patients undergoing cardiac surgery: validity of a second generation device. Anesth Analg 2008, 106:867-872, table.

P104

Influence of systemic vascular resistance on cardiac output measured by new non-invasive cardiac output monitor

KYamashita, TYamatabe, H Abe, M Yokoyama

Kochi Medical School, Kochi, Japan

Critical Care 2010, 14(Suppl 1):P104 (doi: 10.1186/cc8336)

Introduction Early manipulation of hemodynamic variables has been reported to be able to improve the outcome of patients. Cardiac output (CO) was an important parameter to understand the status of hemodynamics. Recently, the pulse-contour method is widely used to estimate $\mathrm{CO}$, because it is non-invasive and easy to use. However, the change in the systemic vascular resistance (SVR) affects CO measured by the pulse-contour method. In this study, we evaluated the new noninvasive cardiac output monitor [1] based on pulse pressure analysis combined with pulse-wave transition time (estimated continuous CO; es(CO) compared with the conventional thermodilution method under a clinical setting.

Methods Twenty-five surgical patients who underwent cardiac or vascular surgery (ASA physical status 2) were enrolled in this study. After anesthesia induction, radial arterial catheter and pulmonary artery catheter were inserted. Intermittent cardiac output (ICO) was measured by thermodilution method in triplicate and averaged $\left(<5^{\circ} \mathrm{C}\right.$ saline $10 \mathrm{ml}$ in each measurement) using Vigilance (Edwards Life Science, Irvine, CA, USA). Echocardiogram, pulse oximetry and arterial blood pressure were also monitored and connected to personal computer to calculate esCCO. Bland and Altman plots were used to evaluate the percentage difference in CO in relation to SVR.

Results One hundred matched sets of data were obtained. The limit of agreement (bias \pm 2 SD of bias) was $-2.9 \pm 31.9 \%$. Only five measurements were exceeded - 30\% against ICO. Each SVR was 1,159, 1,376, 1,418, 2,567, 3,150 dyn $\cdot s / \mathrm{m}^{5}$ 
Conclusions The difference in most CO was within $\pm 30 \%$ against the reference values, although CO was underestimated by esCCO under the relative high SVR. COs estimated by esCCO were acceptable under the clinical setting. Therefore, we concluded that esCCO was a useful algorithm to estimate $\mathrm{CO}$.

\section{References}

1. J Clin Monit Comput 2004, 19:313-330.

\section{P105}

Comparison of cardiac output values; pulse contour methods against thermodilution technique

R De Wilde, B Geerts, P Van den Berg, J Jansen

Leiden University Medical Center, Leiden, the Netherlands

Critical Care 2010, 14(Suppl 1):P105 (doi: 10.1186/cc8337)

Introduction We evaluated the performance of the FloTrac/Vigileo system (FCO) and simultaneously obtained cardiac output (CO) values with the PiCCOplus (PCO), LiDCO ${ }^{T M}$ plus (LCO), Vigilance continuous pulmonary artery thermodilution (CCO) against the intermittent pulmonary artery thermodilution technique (ICO).

Methods Data were collected during standard postoperative care in 28 cardiac surgery patients. The cardiac output data were collected at 1 hour (T1), 2 hours (T2), 4 hours (T3), 8 hours (T4), 12 hours (T5), 24 hours (T6), 36 hours (T7), and 48 hours (T8) after ICU admission. The number of observations per patient varied between four and eight. Data were analyzed with Bland-Altman statistics.

Results Reference cardiac output (ICO) ranged from 2.90 to $8.70 \mathrm{l} /$ minute, mean value of $5.12(S D=1.02) \mathrm{l} /$ minute. Agreement between methods against the reference method are also expressed in a percentage (LOA\%); that is, at low CO a small error and at high CO a higher error is observed. The distribution of errors is different among the methods (Figure 1). This is confirmed by Levine's statistics, which showed significant ( $F$ value $=20.5$, $P<0.001)$ unequal homogeneity of the variances of the four methods. For CCO bias and limits of agreement are 0.31 and -0.99 to 1.61 . Bias and limits of agreement of $\mathrm{FCO}, \mathrm{LCO}$ and $\mathrm{PCO}$ are $0.59,-1.37$ to $2.55 ;-0.05,-1.99$ to 1.89 and $-0.16,-2.60$ to $2.28 \mathrm{l} /$ minute, respectively.
Conclusions The performance of pulse contour methods has significantly increased in the past few years, which makes comparisons with older publications invalid. The auto-calibrated FloTrac/Vigileo system can replace the initially PAC-calibrated LiDCO and PiCCO system.

P106

Continuous cardiac output measurement: effect of time

R De Wilde, B Geerts, P Van den Berg, J Jansen

Leiden University Medical Center Leiden, the Netherlands

Critical Care 2010, 14(Suppl 1):P106 (doi: 10.1186/cc8338)

Introduction Calibration turns pulse contour methods of cardiac output measurement from continuous to intermittent. We evaluated the necessity to recalibrate pulse contour cardiac output methods; LiDCO ${ }^{T M}$ plus (LCO), FloTrac/Vigileo (FCO), PiCCO (PCO) and continuous CO measurement with pulmonary artery catheter (Vigilance) (CCO).

Methods In 28 cardiac surgery patients, data were collected at 1 hour (T1), 2 hours (T2), 4 hours (T3), 8 hours (T4), 12 hours (T5), 24 hours (T6), 36 hours (T7), and 48 hours (T8) after ICU admission. Devices were only calibrated at start of the investigation period. To compute effect against time, at each time point, the CO values from LCO, FCO, PCO and CCO were subtracted, and compared with COtd (ICO). Effect of time was quantified by calculating the slope values using linear regression. Slope values of the regression line were tested against a horizontal line (no effect).

Results No change with time was found for CCO (slope $=0.02 \mathrm{l} /$ minute/ hour, $95 \% \mathrm{Cl}-0.12$ to $0.17, P=0.763$ ) nor for $\mathrm{LCO}$ (slope $=0.011 \mathrm{l} /$ minute/ hour, $95 \% \mathrm{Cl}-0.11$ to $0.03, P=0.322$ ). Time effect for $\mathrm{PCO}$ was (slope $=$ $-0.017 \mathrm{l} /$ minute/hour, $95 \% \mathrm{Cl}-0.032$ to $-0.001, P=0.036$ ) and for FCO (slope $=0.029 \mathrm{l} / \mathrm{minute} /$ hour, $95 \% \mathrm{Cl} 0.003$ to $0.055, P=0.027$ ). For the LCO system, the data range indicated by the $95 \% \mathrm{Cl}$ crosses the threshold value of $10 \%$ at 2,12 and 24 hours, implying more than $2.5 \%$ of the data points are outside the chosen 10\% limits at these time points (Figure 1). This occurs with PCO from 1 hour to 24 hours, with FCO at 4, 8, 12 and 24 hours, and with $\mathrm{CCO}$ at 4,12 and 24 hours.

Conclusions PiCCO and FloTracNVigileo showed an effect with time. For $\mathrm{PiCCO}$, our findings are in accordance with the publications of Boyle and Hanzaoui.
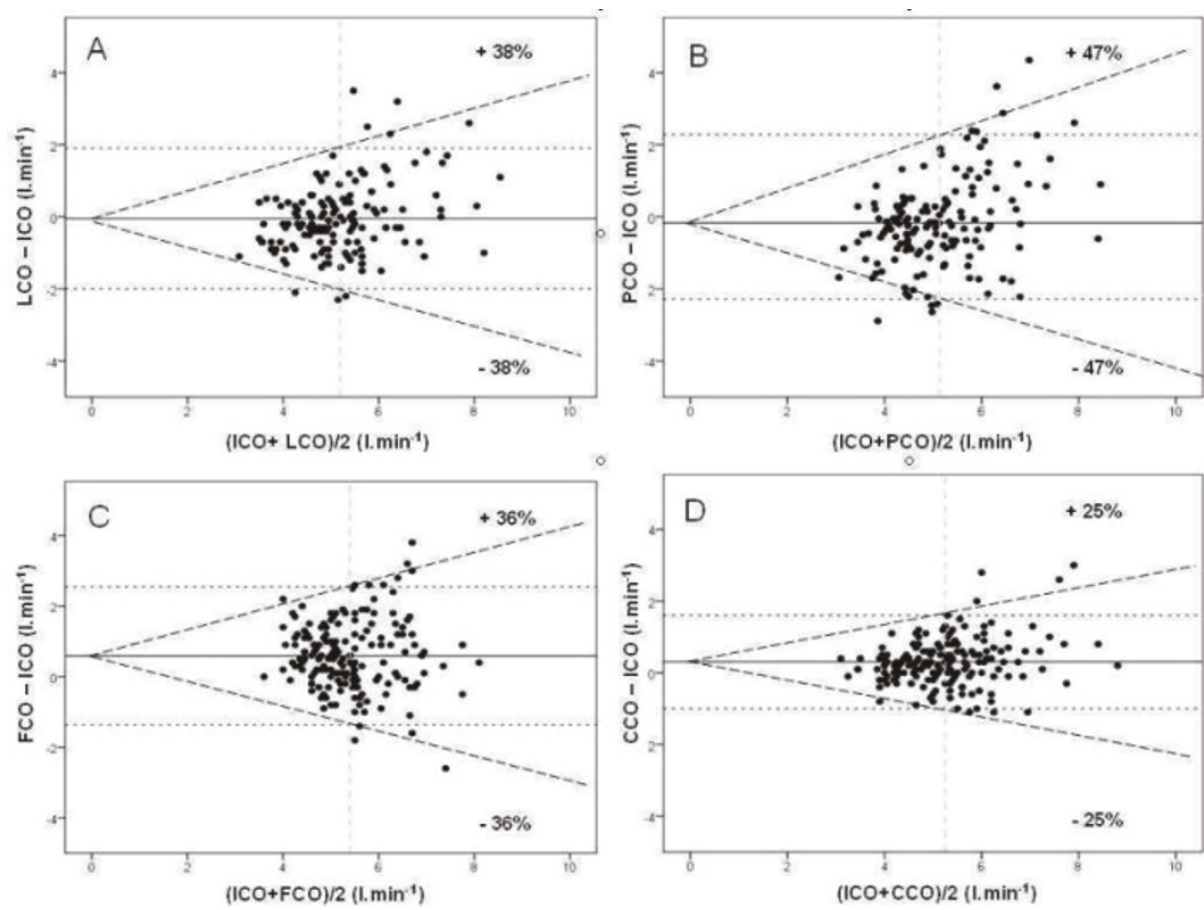

Figure 1 (abstract P105) 

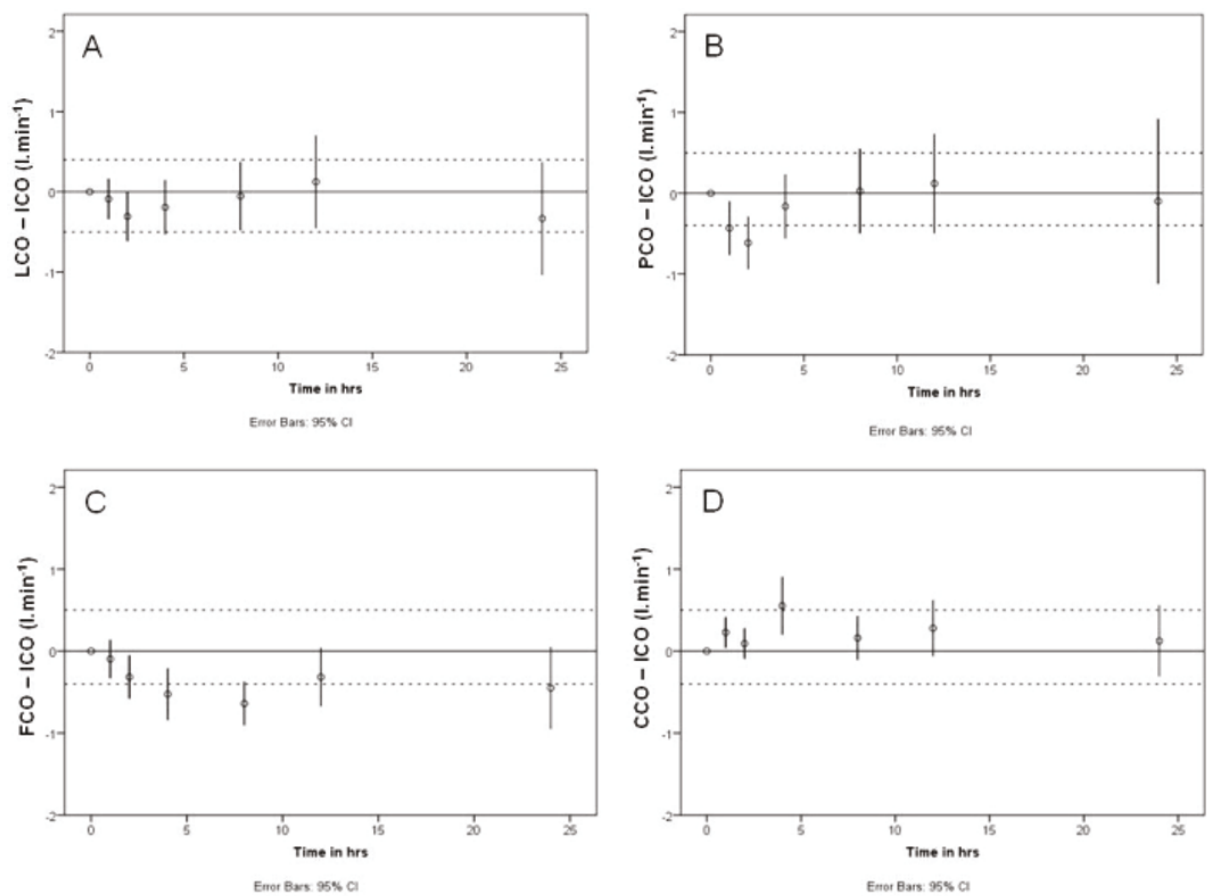

Figure 1 (abstract P106). Stability range of $\pm 10 \%$ indicated by dashed lines.

\section{P107}

A comparison in cardiac output data: a random effects model for repeated measures

R De Wilde, B Geerts, PVan den Berg, J Jansen

Leiden University Medical Center, Leiden, the Netherlands

Critical Care 2010, 14(Suppl 1):P107 (doi: 10.1186/cc8339)

Introduction A random effects model can be used to estimate the withinsubject variation after accounting for other observed and unobserved variations, in which each subject has a different intercept and slope over the observation period. On the basis of the within-subject variance estimated by the random effects model, Bland-Altman plots can be created.

Methods In 28 cardiac surgery patients, cardiac output data LiDCO ${ }^{\mathrm{TM}}$ plus, PICCO, FloTracNigileo pulse contour and CCO (PAC-Vigilance) was collected at 1 hour (T1), 2 hours (T2), 4 hours (T3), 8 hours (T4), 12 hours (T5), 24 hours (T6), 36 hours (T7), and 48 hours (T8) after ICU admission and compared against intermitted thermodilution COtd (ICO). Within patient variation was calculated using Linear Mixed Models (SPSS). Percentage error is calculated as: $\mathrm{PE}=\left[(2 . \mathrm{SD}\right.$ of $\mathrm{CO}$ difference $\left.) /\left(\mathrm{CO}_{\text {mean }}\right)\right] \times 100 \%$.

\begin{tabular}{|c|c|c|c|c|c|c|}
\hline & \multicolumn{2}{|c|}{ Cardiac Output } & \multicolumn{3}{|c|}{ difference } & \multirow{3}{*}{$\begin{array}{c}\text { LOA } \\
\text { lower upper } \\
\text { L.min } \text { min }^{-1}\end{array}$} \\
\hline & \multirow{2}{*}{$\begin{array}{l}\text { Mean } \\
\text { L'min }\end{array}$} & \multirow{2}{*}{$\frac{\text { SD }}{L \cdot \min ^{-1}}$} & \multirow{2}{*}{$\begin{array}{c}\text { Bias } \\
\mathrm{L} \cdot \mathrm{min}^{-1}\end{array}$} & \multicolumn{2}{|c|}{ precision } & \\
\hline & & & & $L \cdot \min ^{-1}$ & $\%(\mathrm{PE})$ & \\
\hline \multicolumn{7}{|c|}{ Pooled data $n=179 / 151\left(^{*}\right)$} \\
\hline LiDCO* & 5.15 & 1.26 & -0.05 & 0.94 & 18.82 & -2.00 to 1.90 \\
\hline PiCCO* & 5.03 & 1.37 & -0.16 & 1.22 & 23.93 & -2.61 to 2.29 \\
\hline Vigileo & 5.70 & 0.96 & 0.59 & 0.98 & 18.09 & -1.37 to 2.54 \\
\hline $\mathrm{CCO}$ & 5.41 & 1.11 & 0.31 & 0.65 & 12.37 & -0.99 to 1.61 \\
\hline \multicolumn{7}{|c|}{ Random effect model $n=28$} \\
\hline LiDCO & 5.15 & 0.84 & 0.06 & 0.48 & 9.3 & -0.90 to 1.02 \\
\hline $\mathrm{PiCCO}$ & 5.10 & 0.37 & -0.04 & 0.55 & 10.8 & -1.14 to 1.06 \\
\hline Vigileo & 5.42 & 0.68 & 0.59 & 0.78 & 14.7 & -1.00 to 2.19 \\
\hline $\mathrm{CCO}$ & 5.27 & 0.79 & 0.31 & 0.36 & 6.9 & -0.42 to 1.03 \\
\hline
\end{tabular}

Figure 1 (abstract P107). Bland-Altman statistics from CO data: random effects model (LMM).
Results The results of the random effects model on continuous cardiac output data are presented in Figure 1.

Conclusions The variation of the differences of the original measurement will be underestimated by this practice because the measurement error is, to some extent, removed. The bias between these two methods will not be affected by averaging the repeated measurements.

\section{Reference}

1. Myles PS, Cui J: Br J Anaesthesia 2007, 99:309-311.

\section{P108}

In vivo validation of a new transpulmonary thermodilution method to assess global end-diastolic volume and extravascular lung water K Bendjelid', R Giraud', N Siegenthaler', F Michard²

'Geneva University Hospitals, Geneva, Switzerland;' 2 Edwards Lifesciences, Nyon, Switzerland

Critical Care 2010, 14(Suppl 1):P108 (doi: 10.1186/cc8340)

Introduction The downslope time (DSt) is part of the equation used to derive global end-diastolic volume (GEDV) and extravascular lung water (EVLW) from a transpulmonary thermodilution curve. DSt may be affected by recirculation phenomena of the cold indicator, as those observed in case of valvular regurgitation. Our goal was to validate a new transpulmonary thermodilution method that does not depend on DSt.

Methods Eleven anesthetized and mechanically ventilated pigs (90 to $110 \mathrm{~kg}$ ) were instrumented with a central venous catheter and a right (PulsioCath; Pulsion, Munich, Germany) and a left (VolumeView; Edwards Lifesciences, Irvine, CA, USA) thermodilution femoral arterial catheter. The right femoral catheter was connected to a $\mathrm{PiCCO}_{2}$ monitor (Pulsion) and used to measure COp (cardiac output), GEDVp and EVLWp using the method based on the equation: GEDV = COp x (MTt - DSt). The left femoral catheter was connected to the EV1000 monitor (Edwards) and used to measure COe, GEDVe and EVLWe using the new method based on the equation: GEDVe = $f(S 2 / S 1) \times C O e \times M T t$, where S1 and S2 are respectively the maximum upslopes and down-slopes of the dilution curve. One hundred and thirty-seven measurements were done during inotropic stimulation (dobutamine), during hypovolemia (bleeding), during hypervolemia (fluid overload), and after inducing acute lung injury (oleic acid). 
Results COp and COe ranged from 3.1 to 15.4 and from 3.4 to $15.1 \mathrm{l} /$ minute, respectively. COp and COe were closely correlated $\left(r^{2}=0.99\right)$, mean bias $( \pm$ SD) was $0.18 \pm 0.29$ and percentage error was $7 \%$. GEDVp and GEDVe ranged from 701 to 1,629 and from 774 to $1,645 \mathrm{ml}$. GEDVp and GEDVe were closely correlated $\left(r^{2}=0.79\right)$, mean bias was $-11 \pm 78$ and percentage error was 14\%. EVLWp and EVLWe ranged from 507 to 2379 and from 495 to 2,222 ml. EVLWp and EVLWe were closely correlated $\left(r^{2}=\right.$ $0.97)$, mean bias was $-5 \pm 72$ and percentage error was $15 \%$.

Conclusions In animals, and over a very wide range of values, the new transpulmonary thermodilution method is as reliable as the PiCCO method to assess cardiac output, cardiac preload and lung water during inotropic stimulation, bleeding, volume loading and lung injury.

P109

Cardiac index derived from arterial pressure waveform: uncalibrated analysis vs once-only calibrated analysis

H Einwaechter, S Hiemer, M Treiber, B Saugel, V Phillip, RM Schmid,

W Huber

Klinikum rechts der Isar der TU München, Germany

Critical Care 2010, 14(Suppl 1):P109 (doi: 10.1186/cc8341)

Introduction As the need for recalibration and the best time point to recalibrate has been a matter of debate since the introduction of the PiCCO monitor (Pulsion, Germany), we set out to analyze the performance of its pulse contour (PC) analysis without recalibration over a 24-hour period. Methods We studied the cardiac index (CI) over a 24-hour period in eight nonoperative patients admitted to our ICU. Seven CI measurements (median number; every 4 hours) by thermodilution (TD) were performed in each patient (in triplicate), PC-derived data were recorded continuously. We used a special PiCCOplus monitor with a disabled auto-recalibrate feature; that is, TD did not lead to an automatic calibration of the PC analysis. Calibration was only performed manually once at the start of the analysis for each patient. Later TD measurements were recorded but had no effect on the CIPC. An additional comparison was performed using the FloTrac/Vigileo System (Edwards, USA), which does not need manual recalibration and instead recalibrates itself every 60 seconds based on the arterial waveform. The FloTrac/Vigileo monitor used was a secondgeneration device (software version 1.14). Both the pressure transducers of the PiCCO and the FloTrac were connected in series to the same femoral artery catheter

Results A total of 59 TD measurements of $\mathrm{Cl}$ (CITD) were compared with FloTrac Cl measurements (CIFloTrac) and PC-derived $\mathrm{Cl}$ measurements (CIPC) from the PiCCO device. CITD ranged from 2.1 to $7.6 \mathrm{l} /$ minute $/ \mathrm{m}^{2}$ (mean $4.3 \pm 1.39 \mathrm{l} /$ minute $/ \mathrm{m}^{2}$ ). PC (PiCCO) compared with TD: bias and precision (1.96SD of the bias) were $0.18 \mathrm{l} / \mathrm{minute} / \mathrm{m}^{2}$ and $\pm 1.35 \mathrm{I} /$ minute/ $\mathrm{m}^{2}$, the percentage error was $30.9 \%$. FloTrac compared with TD: bias and precision were $-0.75 \mathrm{l} / \mathrm{minute} / \mathrm{m}^{2}$ and $\pm 1.79 \mathrm{l} /$ minute $/ \mathrm{m}^{2}$, the percentage error was $46.2 \%$. The mean absolute value of the difference |CITD - CIPC| and |CITD - CIFloTrac| was $0.48 \pm 0.49 \mathrm{l} / \mathrm{minute} / \mathrm{m}^{2}$ for $\mathrm{PiCCO}$ pulse contour values and $0.97 \pm 0.76 \mathrm{l} /$ minute $/ \mathrm{m}^{2}$ for FloTrac/Nigileo values, respectively. This higher difference of CIFloTrac from CITD was significant $(P=0.0002)$ and remained significant even when only data from the last time point (24 hours) was analyzed $(P=0.03)$.

Conclusions $\mathrm{PC}$-derived $\mathrm{Cl}$ values obtained over a 24-hour period from the PiCCO device after only one manual calibration provide significantly better estimates of $\mathrm{Cl}$ than measurements by the FloTrac/Vigileo device, even after a calibration-free interval of 24 hours.

\section{P110}

Cardiac output monitoring during abdominal aortic cross clamping: a comparison between Vigileo/FloTrac system and transoesophageal Doppler

D Simion, M Lecca, M Dan, A Martini, N Menestrina, L Gottin

University of Verona, Verona, Italy

Critical Care 2010, 14(Suppl 1):P110 (doi: 10.1186/cc8342)

Introduction Cardiac output (CO) monitoring is one of the key points in the hemodynamic evaluation of critically ill patients, and can be useful in various settings of high-risk surgery. There is a lack of evidence that the extensive use of invasive devices in the hemodynamic monitoring has a good impact in terms of outcome [1], and less invasive systems have been proposed. Our aim was to compare the CO estimated by Vigileo/FloTrac with the blood flow in thoracic aorta as measured by transoesophageal Doppler in patients undergoing open abdominal aortic aneurysm repair, during the aortic cross-clamping (AoX) phase. We have measured the Augmentation Index (Al), a parameter related to vascular stiffness, using the applanation tonometry method, in order to have a better understanding of the effect of AoX on blood pressure waves.

Methods We enrolled 10 consecutive patients (10 men; age $66 \pm 6$ years) undergoing elective open AAA repair (ASA II to III) under general anesthesia. Radial arterial access was used for semi-invasive determination of blood pressures and CO (APCO) with the Vigileo. An esophageal Doppler was positioned after clinical stabilization. Applanation tonometry was measured just before and after the aortic clamping.

Results We found a significant $(P<0.05)$ increase in $C O$ reported by Vigileo/FloTrac system in the post-clamping phase, when compared with the pre-clamping and basal phases, while the blood flow in thoracic aorta resulted decreased, according with the theory of redistribution of fluids in the splanchnic venous vasculature [2]. There was an important contribution of the wave reflection to the aortic pulse pressure wave after the AoX, as expressed by a significant increase in the Al.

Conclusions The Vigileo/FloTrac system appears to overestimate CO after AoX when compared with the measure of blood flow in thoracic aorta, and this result could be influenced by the pulse pressure wave reflection occurring after clamping. In high-risk surgical settings, other situations of rapid change of systemic resistance vessels could be similarly misread, thus suggesting the necessity of a more tailored Vigileo algorithm.

\section{References}

1. Shah MR et al:: Impact of the pulmonary artery catheter in critically ill patients: meta-analysis of randomized clinical trials. JAMA 2005, 294:1664-1670.

2. Gelman S: The pathophysiology of aortic cross-clamping and unclamping. Anesthesiology 1995, 82:1026-1060.

\section{P111}

Stroke volume index assessment using two minimal invasive devices during hemodynamic postoperative optimization M Costa, T Cecconet, P Chiarandini, L Pompei, STomasino, S Buttera, G Della Rocca

University of Udine, Italy

Critical Care 2010, 14(Suppl 1):P111 (doi: 10.1186/cc8343)

Introduction Postoperative hemodynamic optimization has been proved to reduce morbidity in high-risk patients [1]. Nowadays stroke volume index (SVI) monitoring is available with different less invasive techniques that have shown different levels of agreement and precision with the pulmonary artery catheter [2]. The aim of this study was to evaluate agreement and precision between SVI obtained with a calibrated (LiDCO ${ }^{\text {TMp }}$ plus; LiDCO Ltd, Cambridge, UK) and an uncalibrated pulse contour analysis device (FloTrac/Vigileo; Edwards Lifesciences, Irvine, CA, USA), in patients undergoing postoperative hemodynamic optimization. Methods Patients undergoing a hemodynamic optimization protocolized care according to a previous published trial [1] to reach an oxygen delivery

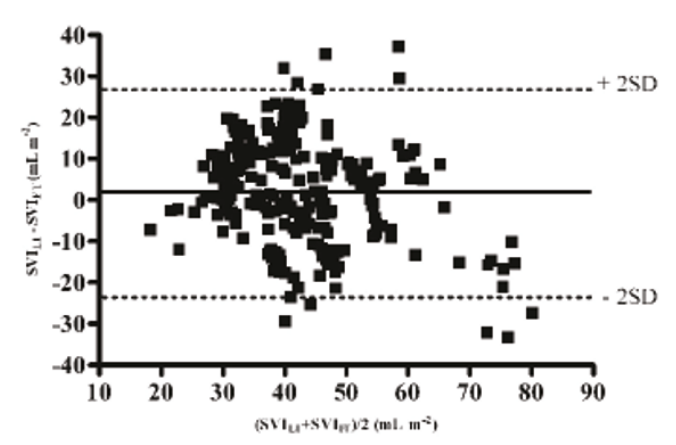

Figure 1 (abstract P111). Bland and Altman analysis. 
index $>600 \mathrm{ml} /$ minute $/ \mathrm{m}^{2}$ after abdominal surgery were enrolled. After calibration of the LiDCO ${ }^{\mathrm{TM}}$ plus, SVI data obtained from LiDCO ${ }^{\mathrm{TM}}$ plus, and FloTrac/Vigileo (version 1.07) were recorded every 15 minutes for 6 hours. Agreement and precision between SVI obtained with LiDCO (SVI $)$ and SVI obtained with Vigileo (SVI ) were evaluated with Bland and Altman analysis.

Results Thirteen patients (nine males, four females), mean age 68.5 $( \pm 28.3)$ years were enrolled into the study. Two hundred and seventy SVI data pairs were analyzed. Bias was $1.79 \mathrm{ml} / \mathrm{m}^{2}$, with a precision (1.96SD of the bias) of $25.1 \mathrm{ml} / \mathrm{m}^{2}$ (Figure 1).

Conclusions SVI obtained from the uncalibrated low-invasive, pulse contour analysis technique seems as accurate as the calibrated technique in a clinical hemodynamic protocolized setting to increase oxygen delivery in postoperative high-risk patients.

\section{References}

1. Pearse R, et al: Crit Care 2005, 9:R687-R693.

2. Hofer CK, et al:: Curr Opin Crit Care 2007, 13:308-331.

\section{P112}

Non-invasive cardiac output monitoring in children:

clinical validation

Y Vandormael, T Vu, M Gewillig, D Vlasselaers

University Hospitals Leuven, Belgium

Critical Care 2010, 14(Suppl 1):P112 (doi: 10.1186/cc8344)

Introduction Continuous noninvasive cardiac output (CO) provides valuable information for patient management. Non-invasive cardiac output monitoring (NICOM) measures CO based on chest bioreactance and is validated in adults [1]. Validated data in children are lacking. Our objective was to evaluate NICOM in children with pulmonary artery catheter thermodilution (PAC) as reference.

Methods Paired CO values using NICOM and TD were recorded during cardiac catheterization in children with congenital heart disease. Children with intracardiac or extracardiac shunts were excluded. PAC was inserted through the femoral vein and CO was measured after bolus injection of $5 \mathrm{ml}$ iced saline. NICOM was connected in accordance with the manufacturers' directrix.

Results Nineteen pairs of CO measurements were collected in nine patients. Mean age was 4.6 years (range: 0 to 12 years) and mean weight $16.8 \mathrm{~kg}$ (range: 4.8 to $34 \mathrm{~kg}$ ). Cardiac diagnosis was dilated cardiomyopathy or interventional procedures. Mean CO values were $2.18 \mathrm{l} /$ minute (PAC) and $1.88 \mathrm{l} /$ minute (NICOM). Correlation between two methods was significant $(r=0.826 ; P=0.0005)$. Bland-Altman analysis shows a mean difference between the reference method and NICOM of $+0.33 \mathrm{l} /$ minute. Ninety-five percent of measurements were inside the limits of agreement $( \pm 1.96 \mathrm{SD})$ but these limits were broad ( -1.24 to $1.96 \mathrm{l} / \mathrm{min}$ ite) (Figure 1). Conclusions $\mathrm{CO}$ measurements with NICOM and PAC show a significant correlation. Bland-Altman demonstrates an acceptable agreement;

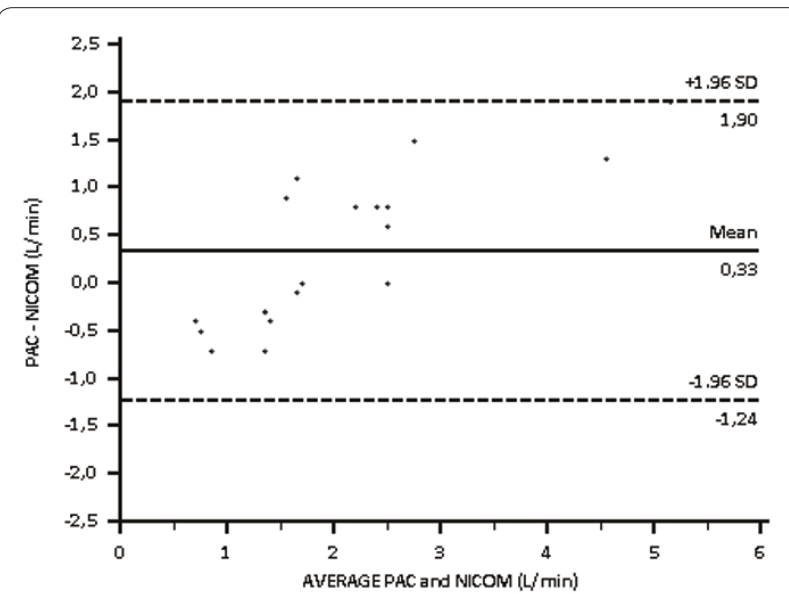

Figure 1 (abstract P112). Bland-Altman analysis. however, the limits of agreement are broad. Depending on the CO range, NICOM reveals a trend to systematically overestimate or underestimate CO. Additional studies in larger and more heterogeneous pediatric patient populations are warranted for further validation.

References

1. Squara P, et al:: Intensive Care Med 2007, 33:1191-1194.

\section{P113}

Response of NICOM stroke volume to passive leg raising to predict fluid responsiveness in critically ill patients with spontaneous breathing activity

B Lamia', A Cuvelier', PL Declercq'1, LC Molano', MR Pinsky², JF Muir ${ }^{' R o u e n}$ University Hopsital, Rouen School of Medecine, Rouen, France; 2 University of Pittsburgh Medical Center, Pittsburgh, PA, USA

Critical Care 2010, 14(Suppl 1):P113 (doi: 10.1186/cc8345)

Introduction Volume responsiveness cannot be predicted by the respiratory variation in arterial pressure in hemodynamically unstable patients with spontaneous breathing activity. The NICOM device (Reliant ${ }^{\oplus}$; Cheetah) (bioreactance technique) is a totally non-invasive hemodynamic monitoring technique for the measurement of stroke volume. Our objective was to test whether volume responsiveness could be predicted by the response of stroke volume measured by the NCOM device to passive leg raising $(P L R)$ in patients with spontaneous breathing activity. Methods Prospective study in the respiratory critical care of a university hospital. Eleven patients with spontaneously breathing activity considered for volume expansion. An increase in stroke volume index (SVi) of 15\% or more after volume expansion defined a responder patient. We measured the response of the bioreactance stroke volume to PLR and to saline infusion (500 $\mathrm{ml}$ over 15 minutes).

Results The proportional changes in NiCOM-SVi induced by PLR were correlated with the proportional changes in NICOM-SVi induced by volume expansion $(r=0.67, P=0.02$ ). The proportional changes in NICOM-cardiac index (CI) induced by PLR were also correlated with the proportional changes in NICOM-Cl induced by volume expansion $(r=0.63$, $P=0.03$ ). A PLR-induced increase in stroke volume of $9 \%$ or more predicted an increase in stroke volume of $15 \%$ or more after volume expansion with a sensitivity of $100 \%$ and a specificity of $80 \%$.

Conclusions The response of NICOM-stroke volume to PLR was a good predictor of volume responsiveness. In our hemodynamically unstable patients with spontaneous breathing activity, fluid responsiveness can be assessed totally non-invasively with a bioreactance device.

P114

Global end-diastolic volume and its correlation to cardiac index inside and outside normal values

W Huber, S Mair, B Saugel, V Phillip, H Einwaechter, R Schmid

Klinikum rechts der Isar der Technischen Universität München, Germany Critical Care 2010, 14(Suppl 1):P114 (doi: 10.1186/cc8346)

Introduction Transpulmonary thermodilution (TPTD)-derived volumetric parameters such as global end-diastolic volume (GEDI) and ELWI have been established as hemodynamic cornerstones for assessment of preload (GEDI) and pulmonary hydration. Normal values of GEDI have been created more than a decade ago based on studies in pre-selected patients. Therefore, it was the aim of our prospective study to investigate the correlation of GEDI to cardiac index (CI) in clinical routine.

Methods Over a 6-month period all 1,574 routine TPTD measurements in 78 consecutive patients (APACHE II: $23.5 \pm 8.6$ ) of an internal ICU with a PiCCO catheter were prospectively documented and analysed: correlation (Spearman) and multiple regression analysis; SPSS 17.0.

Results Including all 1,574 measurements, Cl was univariately correlated to GEDI $(r=0.251 ; P<0.001), \mathrm{dP} \quad(r=0.221 ; P<0.001)$ and heart rate $(r=$ $0.102 ; P<0.001)$, but not to $C V P(r=0.001 ; P=0.962)$. The correlation of GEDI, $\mathrm{dP}_{\text {max }}$ and heart rate to $\mathrm{Cl}$ was confirmed in multivariate analysis $(P<0.001$ for all three variables). Changes in $\mathrm{Cl}$ (Delta-Cl) were univariately correlated to changes in GEDI $(r=0.414), \mathrm{dP} \quad(r=0.240)$ and ELWI $(r=0.152 ; P<0.001$ for all comparisons). In a multivariate analysis of all measurements, Delta$\mathrm{Cl}$ was independently associated with changes in GEDI $(P<0.001), \mathrm{dP}_{\text {max }}$ $(P<0.001)$ and $\operatorname{CVP}(P=0.017)$. Subgroup analysis of all measurements 
with GEDI below the lower normal level $680 \mathrm{ml} / \mathrm{kg} / \mathrm{m}^{2}$ demonstrated an independent association of $\mathrm{Cl}$ to GEDI $(P<0.001), \mathrm{dP}_{\text {max }}(P<0.001)$ and ELWI $(P=0.041)$ but not to CVP. Similarly, Delta-Cl was independently associated with changes in GEDI and $\mathrm{dP}_{\text {max }}(P<0.001)$. Similar results were found for the measurements with GEDI within the normal range $(680$ to $800 \mathrm{ml} / \mathrm{kg} /$ $\left.\mathrm{m}^{2}\right)$ : significant and independent correlation of $\mathrm{Cl}$ to GEDI $(P<0.017)$ and $\mathrm{dP}_{\text {max }}(P<0.001)$. Changes in $\mathrm{Cl}$ were independently correlated to changes in GEDI $(P<0.001), \mathrm{dP}_{\text {max }}(P<0.001)$ and $\mathrm{CVP}(P=0.035)$. Interestingly, even in measurements with GEDI $>800 \mathrm{ml} / \mathrm{kg} / \mathrm{m}^{2}, \mathrm{Cl}$ was independently correlated to GEDI $(P=0.009)$ and $\mathrm{dP} \quad(P<0.001)$. Changes in $\mathrm{Cl}$ in this group were independently associated with changes in $\mathrm{dP}_{\max }$ and GEDI $(P<0.001)$.In the subgroup of measurements with GEDI $>1,000 \mathrm{ml} / \mathrm{kg} / \mathrm{m}^{2}$ there was no correlation of any parameter to $\mathrm{Cl}$, however changes in $\mathrm{Cl}$ were independently correlated to changes in GEDI $(P<0.001)$ and $\mathrm{dP}_{\max }$ $(P=0.003)$.

Conclusions $\mathrm{GEDI}$ and $\mathrm{dP}_{\max }$ and their changes have an independent and positive correlation to $\mathrm{Cl}$ and its changes even in patients with increased GEDI.

\section{P115}

Relationship of stroke volume variation, pulse pressure variation and global end-diastolic volume in patients undergoing brain surgery

A Rieß1', S Wolf2, C Lumenta², L Schürer², P Friederich'

'Klinikum Bogenhausen, Department of Anesthesiology, München, Germany;

2 Department of Neurosurgery, München, Germany

Critical Care 2010, 14(Suppl 1):P115 (doi: 10.1186/cc8347)

Introduction Monitoring intravascular volume in patients with intracranial pathology is often mandatory for maintaining hemodynamic stability $[1,2]$. Cyclic changes in cardiac stroke volume $[1,3]$ and pulse pressure induced by positive pressure ventilation as well as target values of global end-diastolic volume index (GEDVI) $\left(\mathrm{ml} / \mathrm{m}^{2}\right)$ [2] allow volume therapy quidance. The relationship between stroke volume variation (SVV) (\%) and pulse pressure variation (PPV) (\%), as well as between SVV or PPV and values of GEDVI has not been established in patients with intracranial pathology.

Methods In this prospective investigation the correlation between dynamic and static hemodynamic parameters of 38 patients undergoing brain surgery was studied. Measurements were performed using the PiCCO technology. For statistical analysis, nonparametric correlation analysis and hypothesis testing were applied.

Results SWV correlated significantly with PPV $\left(r_{2}=0.87, P<0.001\right)$. Neither $\operatorname{SWV}\left(r_{2}=0.14, P=0.13\right)$ nor PPV $\left(r_{2}=0.07, P=0.81\right)$ correlated with GEDVI. Threshold values for SW $(9.5 \%, 11.6 \%)$ as well as for PPV (12.5\%) allowed discrimination between groups with significantly different values of stroke volume index, while failing to discriminate between groups with significantly different values of GEDVI. Dichotomizing the patients into groups of GEDVI $\leq 680 \mathrm{ml} / \mathrm{m}^{2}$ and $>680 \mathrm{ml} / \mathrm{m}^{2}$ resulted in groups with significantly different values of stroke volume index as well while failing to discriminate between groups with significantly different values of SWV and PPV.

Conclusions Static (GEDVI) and dynamic (SVV, PPV) parameters of cardiac preload may reflect different properties of the cardiovascular system. The combination of SWV, PPV, and GEDVI may offer more precise information on the cardiovascular system than either parameter alone.

\section{References}

1. Berkenstadt $H$, et al: Anesth Analg 2001, 92:984-989

2. Mutoh T, et al: Stroke 2009, 40:2368-2374

3. Marik PE, et al: Crit Care Med 2009, 37:2642-2647.

\section{P116}

The value of pulse pressure variation to predict volume response in patients ventilated with low VT

GF Friedman, CD Costa, SR Vieira, L Fialkow

Hospital de Clínicas de Porto Alegre, Brazil

Critical Care 2010, 14(Suppl 1):P116 (doi: 10.1186/cc8348)

Introduction The prediction value of pulse pressure variation $(\triangle P P)$ in patients ventilated with low VT is not well studied. A $\triangle \mathrm{PP}$ of 12 to $13 \%$ is validated as a predictor of volume response in several studies, but in patients ventilated with VT $>8 \mathrm{ml} / \mathrm{kg}$. One study has shown that the $\triangle P P$ of 12 to $13 \%$ does not predict volume response in patients ventilated with a low VT. We hypothesized that a lower cut-off value for $\triangle \mathrm{PP}$ can predict volume response in patients with low VT.

Methods Thirty-seven adult patients mechanically ventilated with a tidal volume $<8 \mathrm{ml} / \mathrm{kg}$ (PBW), without cardiac arrhythmias, with a pulmonary artery catheter and a peripheral arterial catheter were included. An increase in cardiac index (thermodilution)) $>15 \%$ output after a fluid challenge (Crystalloid 1,000 ml or Colloid $500 \mathrm{ml}$ ) was considered a positive response.

Results Seventeen patients were responders. The ROC curve showed that the best cut-off value for $\triangle P P$ was $10 \%$ (ROC area $=0.74,95 \%$ Cl: 0.51 to 0.9 : sensitivity $53 \%$, specificity $95 \%$, positive likelihood ratio 9.4 and negative 0.34).Twelve patients consisted of a heterogeneous group of patients (liver transplant, acute pancreatitis, aortic surgery). Among 25 septic shock patients, a $\triangle \mathrm{PP}>10 \%$ showed a ROC area of 0.84 (sensitivity $78 \%$, specificity $93 \%$ ). In any case, the greater $\triangle P P$, the greater the fluid response. $\triangle \mathrm{PP}>10 \%$ was a better predictor than CVP or PAOP.

Conclusions $\triangle P P$ has a limited value in patients ventilated with low VT. However, a $\triangle P P>10 \%$ may help identify septic shock patients that will respond to a fluid challenge.

\section{References}

1. Vincent $\mathrm{J}$, et al:: Pulse pressure variations to predict fluid responsiveness: Influence of tidal volume. Intensive Care Med 2005, 31:517-523.

\section{P117}

Pre-ejection period is a reliable parameter to estimate cardiac preload in a hemorrhagic shock

R Giraud, N Siegenthaler, JA Romand, K Bendjelid

Hôpitaux Universitaires de Genève, Switzerland

Critical Care 2010, 14(Suppl 1):P117 (doi: 10.1186/cc8349)

Introduction We have already demonstrated that in mechanically ventilated patients, the respiratory change in the pre-ejection period $(\triangle P E P)$ is a reliable dynamic index for the prediction of increase in cardiac output after volume infusion [1]. However, in an animal study, Kubitz and colleagues showed that the pre-ejection period is not sensitive to the changes in intravascular volume status [2].

Methods This study investigated the influence of changes in intravascular volume status on $\triangle P E P$. In 17 pigs, ECG, arterial pressure and cardiac output derived from a Swan-Ganz catheter were recorded. Measurements were performed during normovolaemic conditions, after haemorrhage $(25 \mathrm{ml} /$ $\mathrm{kg}$ ) and following re-transfusion $(25 \mathrm{ml} / \mathrm{kg})$ with constant tidal volume $(10 \mathrm{ml} / \mathrm{kg})$ and respiration rate $(15 /$ minute)

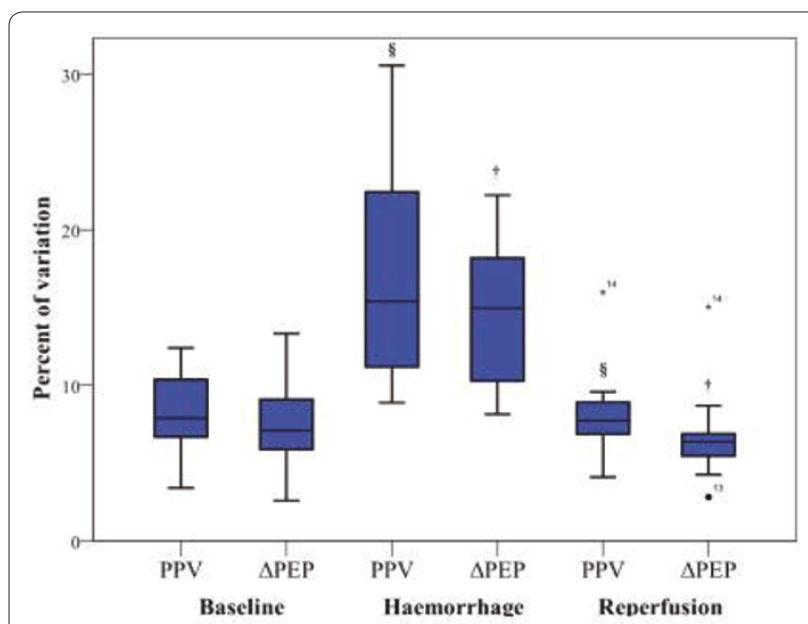

Figure 1 (abstract P117). Box plot representing pulse pressure variations (PPV) (\%) and pre-ejection period variations ( $\triangle \mathrm{PEP})(\%)$ at the three times of the protocol. ${ }^{\circledR} P<0.05,{ }^{+} P<0.05$. 
Results At baseline, respiratory changes in pulse pressure (PPV) and $\triangle \mathrm{PEP}$ were both $<12 \%$. PPV significantly correlated with $\triangle P E P(r=0.96, P<0.001)$. Volume loss induced by haemorrhage increased PPV and $\triangle P E P$. Moreover, during this state, PPV correlated with $\triangle P E P$ with a significant correlation coefficient $(r=0.88, P<0.001)$. Retransfusion significantly decreased PPV and $\triangle P E P$ and PPV significantly correlated with $\triangle P E P(r=0.94, P<0.001)$ (Figure 1).

Conclusions Available correlations between PPV and $\triangle$ PEP at each time of the study were observed, meaning that $\triangle P E P$ is a reliable parameter to estimate the changes in intravascular volume status.

\section{References}

1. Bendjelid K, Suter PM, Romand JA: The respiratory change in preejection period: a new method to predict fluid responsiveness. J Appl Physiol 2004, 96:337-342.

2. Kubitz JC, Kemming GI, Schultheib G, Starke J, Podtschaske A, Goetz AE, Reuter DA: The influence of cardiac preload and positive end-expiratory pressure on the pre-ejection period. Physiol Meas 2005, 26:1033-1038

\section{P118}

Predictive value of pulse pressure variation for fluid responsiveness after maneuver to change tidal volume in patients with protective ventilatory strategy

F Machado, F Freitas, M Assuncao, B Mazza, M Jackiu

Federal University of São Paulo, Brazil

Critical Care 2010, 14(Suppl 1):P1 18 (doi: 10.1186/cc8350)

Introduction After the early phase of sepsis, excessive fluid administration may worsen pulmonary edema and prolong mechanical ventilation [1]. Accurately predicting fluid responsiveness obviates unnecessary fluid loading, and helps to detect patients who may benefit from a volume expansion. Pulse pressure variation (DPP) is a reliable predictor of fluid responsiveness in mechanically ventilated patients only when tidal volume is at least $8 \mathrm{ml} / \mathrm{kg}$ [2]. The aim of this study was to evaluate the predictive value of DPP for fluid responsiveness after a maneuver to change tidal volume to $8 \mathrm{ml} / \mathrm{kg}$ in patients ventilated with $6 \mathrm{ml} / \mathrm{kg}$.

Methods Prospective clinical study in 40 patients ventilated with $6 \mathrm{ml} / \mathrm{kg}$ after resuscitation phase of severe sepsis and septic shock. Fluid challenge was indicated by the attending physician $(7 \mathrm{ml} / \mathrm{kg}$ of $6 \%$ hydroxyethyl starch 130/0.4). Complete hemodynamic measurements including DPP (DPP $6 \mathrm{ml} / \mathrm{kg}$ ) were obtained at baseline. The tidal volume was then changed to $8 \mathrm{ml} / \mathrm{kg}$ and the DPP (DPP $8 \mathrm{ml} / \mathrm{kg}$ ) was measured after 5 minutes. The ventilatory settings were returned to $6 \mathrm{ml} / \mathrm{kg}$ before fluid challenge. Patients whose cardiac output (CO) increased by $\geq 15 \%$ were considered to be fluid responders. Receiver operating characteristic (ROC) curve analysis was used to evaluate the predictive value of DPP.

Results In 19 patients (responders), CO increased by $>15 \%$ after fluid infusion. Fluid responsiveness was better predicted with DPP $6 \mathrm{ml} / \mathrm{kg}$ (ROC curve area $0.92 \pm 0.05$ ) than with pulmonary artery occluded pressure $(0.56 \pm 0.09)$ and right atrial pressures $(0.74 \pm 0.08)$. Increasing tidal volume to $8 \mathrm{ml} / \mathrm{kg}$ did not improved prediction as the ROC curve area with DPP $8 \mathrm{ml} / \mathrm{kg}$ was $0.94 \pm 0.03$. The best cut-off values defined by the ROC curve analysis was $6.5 \%$ and $10.5 \%$ for DPP $6 \mathrm{ml} / \mathrm{kg}$ and DPP $8 \mathrm{ml} / \mathrm{kg}$, respectively. Conclusions The maneuver to change tidal volume to $8 \mathrm{ml} / \mathrm{kg}$ in patients ventilated with protective ventilatory strategy to better predict fluid responsiveness is not useful. Fluid responsiveness can be correctly predicted in patients ventilated with tidal volume of $6 \mathrm{ml} / \mathrm{kg}$.

\section{References}

1. Wiedemann HP, et al: N Engl J Med 2006, 354:2564-2575

2. De Backer D, et al:: Intensive Care Med 2005, 31:517-523.

\section{P119}

Does stroke volume increase after a fluid challenge? A study on the management of patients undergoing major head and neck free flap surgery: preliminary data

L Wijayasiri, D Garewal, M Khpal, A Rhodes, A Dewhurst, M Cecconi

St George's Hospital, London, UK

Critical Care 2010, 14(Suppl 1):P119 (doi: 10.1186/cc8351)

Introduction Major head and neck surgery involving reconstructive free flaps for oropharyngeal cancers are complex and prolonged operations during which appropriate fluid management can become difficult. Potential adverse effects of fluid mismanagement in this group of patients include flap hypoperfusion related to hypovolaemia or flap oedema and deterioration in alveolar-arterial gradients related to excessive fluid administration. This study looked at ongoing standard practice, to determine whether the use of a cardiac output monitor could improve fluid management in this subset of patients.

Methods Single-blinded, prospective observational study conducted on consecutive adult patients undergoing major head and neck reconstructive free flap surgery. All patients were anaesthetised by the same individual, using a standardised technique. Volume-controlled positive pressure ventilation was initiated in all patients. Patients received maintenance crystalloid fluids at a rate of $5 \mathrm{ml} / \mathrm{kg} / \mathrm{hour}$. Additional fluid challenges ( $250 \mathrm{ml}$ crystalloid boluses given over 5 minutes) were administered at the discretion of the anaesthetist. A priori criterion of fluid responsiveness was defined as an increase in stroke volume (SV) $>10 \%$ as measured with the LiDCOrapid after a fluid challenge. The anaesthetist was blinded to the LiDCOrapid data and observations were made by an independent investigator. Data were collected on: heart rate (HR), mean arterial pressure (mAP) and central venous pressure (CVP).

Results Forty-seven fluid boluses were assessed. The median age of the patients was 72 years (range 68 to 81 years) and their median weight was $71.2 \mathrm{~kg}$ (range 63 to $88 \mathrm{~kg}$ ). The ventilatory set-up used a median tidal volume of $7 \mathrm{ml} / \mathrm{kg}$ (range 450 to $600 \mathrm{ml}$ ). Fifteen out of 47 (32\%) of the fluid challenges were positive when assessed against the change in stroke volume. When comparing the fluid responders with the nonresponders, there were no differences in HR ( $62 \pm 10 \mathrm{bpm}$ vs $62 \pm 10 \mathrm{bpm}), \mathrm{mAP}$ ( $63 \pm$ $6 \mathrm{mmHg}$ vs $63 \pm 7 \mathrm{mmHg})$ or CVP $(8 \pm 3 \mathrm{mmHg}$ vs $10 \pm 3 \mathrm{mmHg})$.

Conclusions This preliminary report suggests that only $32 \%$ of fluid challenges given in theatre were effective in increasing the SV $>10 \%$ and potentially two-thirds of the fluid challenges may have been detrimental. This can be seen only if SV is monitored continuously. If further data confirm this, LiDCOrapid may be useful to guide fluid administration in order to optimise the SV, thereby minimizing the risk of fluid overload.

\section{P120}

Optimizing stroke volume and oxygen delivery in elective abdominal aortic surgery

J Bisgaard', E Rønholm', T Gilsaa', P Toft ${ }^{2}$

'Kolding Hospital, Kolding, Denmark; ${ }^{2}$ Odense University Hospital, Odense, Denmark

Critical Care 2010, 14(Suppl 1):P120 (doi: 10.1186/cc8352)

Introduction Patients undergoing elective abdominal aortic surgery (EAAS) are at risk of developing complications due to preoperative co-morbidity, surgical trauma, blood loss and inflammatory injury [1]. Individualized goal-directed therapy (IGDT) has been proposed to improve outcome in patients undergoing high-risk surgery [2]. The aim of this study was to investigate whether IGDT, targeting stroke volume (SV) and oxygen delivery ( $\mathrm{DO}_{2}$ ), can be performed safely in EAAS.

Methods Sixty-three EAAS patients were randomized to IGDT or conventional therapy. The LiDCO ${ }^{\mathrm{TM}}$ plus system was used for SV and DO monitoring. SV was optimized by $250 \mathrm{ml}$ fluid challenges intraoperatively and the first 6 hours postoperatively. DO, was optimized 6 hours postoperatively targeting a DO,l level of $600 \mathrm{ml} / \mathrm{min}^{2} / \mathrm{m}^{2}$, by infusion of dobutamine, if necessary. Hemodynamic data were collected at baseline (t0) preoperatively (t1), before aortic cross-clamping ( $\mathrm{t} 2)$, at the end of surgery ( $\mathrm{t} 3$ ), and the first 6 hours postoperatively ( $p 1$ to $p 6$ ). All patients were monitored with fivelead ECG during dobutamine infusion and dosage was reduced at signs of ischemia or heart rate $>20 \%$ above baseline. Dobutamine dosage was limited to a maximum of $10 \mu \mathrm{g} / \mathrm{kg} /$ minute.

Results The mean SVI level was $19.4 \%$ higher at $\mathrm{p} 4$ to $\mathrm{p} 6$ in the IGDT group compared with the control group $(P=0.02)$, and $12.1 \%$ higher in the entire intervention period (t1 to p6) $(P=0.07)$. The mean $\mathrm{DO}_{2}$ I was $18.0 \%$ higher at p4 to $\mathrm{p} 6(P=0.01)$ and $12.9 \%$ higher in the entire intervention period ( $\mathrm{t}$ 1 to p6) in the IGDT group $(P=0.03)$. Mean arterial pressure and heart rate did not differ significantly ( $P=0.12$ and $P=0.21)$. There was no difference in the frequency of postoperative cardiac complications between the groups. Conclusions The results of this study demonstrate that IGDT targeting SV and $\mathrm{DO}_{2}$ can be performed safely in patients undergoing EAAS. Whether this intervention is beneficial is being evaluated in the current study. 


\section{References}

1. Dardik et al:: Results of elective abdominal aortic aneurism repair in the 1990s: A population-based analysis of 2335 cases. J Vasc Surg 1999, 30:985-995.

2. Bundgaard-Nielsen et al:. Monitoring of perioperative fluid administration by individualized goal-directed therapy. Acta Anaestesio/ Scand 2007, 51:331-340.

P121

Oxygen saturation determined from the mouth is an early indicator of central hypovolemia in humans

A Lima, M Alamyar, C Ince, J Bakker

Erasmus MC University Medical Centre, Rotterdam, the Netherlands

Critical Care 2010, 14(Suppl 1):P121 (doi: 10.1186/cc8353)

Introduction Studies have suggested that tissue oxygenation $\left(\mathrm{StO}_{2}\right)$ measured on the thenar muscle is not sensitive to track acute changes in hemodynamics due to reductions in central blood volume. We aimed to investigate the feasibility of $\mathrm{StO}_{2}$ measurements in the mouth as a quantitative indicator comparable with $\mathrm{StO}_{2}$ measurements obtained from the thenar eminence during changes in central blood volume (CBV). Methods We performed a head-up tilt (HUT) test in 10 healthy volunteers as an experimental model to reduce $\mathrm{CBV}$. $\mathrm{StO}_{2}$ was continuously measured using two devices (InSpectra model 650): a multiple depth optical probe was placed over the thenar eminence $(15$ and $25 \mathrm{~mm}$ ) and a 1-mm probe was placed in the mouth. Subjects were placed on an electrically driven tilt table with a footboard. After 5 minute baseline measurements in the supine position, the table was tilted up to $70^{\circ}$ and returned to the supine position after 10 minutes. $\mathrm{StO}_{2}$ readings were analyzed at the lowest stroke volume value.

Results All subjects (mean age: $23 \pm 6$; six males) tolerated well the supine and head-up positions. Cardiac output significantly decreased in the HUT position; simultaneous decrease in $\mathrm{StO}_{2}$, was observed in the mouth, but not in the thenar. The general results of the HUT test are shown in Tables 1 and 2 .

Table 1 (abstract P121). Hemodynamics in the baseline and in the head-up tilt (HUT)

\begin{tabular}{lcc}
\hline & Baseline & HUT \\
\hline HR & 69 & $85^{*}$ \\
MAP & 91 & 93 \\
CO & 5.6 & $4.9^{*}$ \\
\hline
\end{tabular}

* $P<0.05$ vs baseline.

Table 2 (abstract P121). $\mathrm{StO}_{2}$ in the baseline and in the head-up tilt (HUT)

\begin{tabular}{lccc}
\hline Baseline & HUT & Baseline 2 & StO $_{2}$ 15 thenar \\
\hline $76 \pm 6$ & $75 \pm 6$ & $78 \pm 7$ & $\mathrm{StO}_{2} 25$ thenar \\
$76 \pm 5$ & $75 \pm 6$ & $78 \pm 8$ & $\mathrm{StO}_{2}$ buccal \\
$88 \pm 3$ & $78 \pm 3^{*}$ & $87 \pm 3$ & \\
\hline
\end{tabular}

* $P<0.05$ vs baseline 1 and 2 .

Conclusions Near-infrared spectroscopy can be used in the mouth to track changes in central blood volume. Our results showed that buccal $\mathrm{StO}_{2}$ is an earlier indicator of acute hemodynamic responses to the HUTinduced central hypovolemia than thenar $\mathrm{StO}_{2}$.

\section{P122}

\section{Plethysmographic indices as predictors of fluid responsiveness: a} meta-analysis

CM Marano, FC Cavallaro, CS Sandroni, AD Dell'Anna, MA Antonelli

Catholic University School of Medicine, Rome, Italy

Critical Care 2010, 14(Suppl 1):P122 (doi: 10.1186/cc8354)

Introduction The aim of the study was to assess the ability of respiratory variations in pulse oximetry plethysmographic waveform amplitude $(\triangle P O P)$ to predict the increase of cardiac output (CO) after fluid infusion (fluid responsiveness).

Methods Two authors independently performed a search iterated unti November 2009 in MEDLINE, Embase and Cochrane Database using keywords: 'fluid OR preload OR volume responsiveness', 'cardiovascular monitoring,',fluid challenge,',functional hemodynamic monitoring,' dynamic indices OR indexes,' 'delta POP', 'pleth index', 'plethysmographic waveform'. Predictive value of $\triangle P O P$ was estimated by: difference in mean value of $\triangle P O P$ between responders and nonresponders; correlation coefficient between pre-infusion $\triangle \mathrm{POP}$ and $\mathrm{CO}$ increase after fluids; and sensitivity, specificity and area under the ROC curve (AUC) for $\triangle P O P$ to predict a responder state. A meta-analysis was performed using Comprehensive Meta-Analysis v. 2.2. Pooled values of diagnostic odds ratio (DOR), sensitivity, specificity and summary ROC (SROC) curve were calculated using MetaDiSC v. 1.4. Heterogeneity was evaluated with $Q$ and $l^{2}$ tests.

Results From the initial dataset of 839 records we identified six articles [1-6] on 135 patients to whom 195 fluid boluses were administered. All patients were in sinus rhythm and adapted to a ventilator. An increase of cardiac index or stroke volume index $>10$ to $15 \%$ defined responders. Mean responder rate was 58.9\%. Pooled difference in mean was 10\% (95\% CI 5.8 to 14.2), correlation coefficient 0.59 (0.41 to 0.73 ), AUC 0.85 (0.76 to 0.94 ), sensitivity $81.1 \%$ (72.5 to 87.9 ), specificity $78.6 \%$ (68.3 to 86.8 ), DOR 21.7 (6.3 to 74.4), area under SROC 0.88 (0.80 to 0.96). The best threshold value for identification of responders was 9.5 to $15 \%$. Heterogeneity was significant for all evaluations.

Conclusions $\triangle P O P$ is a non-invasive dynamic parameter able to predict fluid responsiveness with only a moderate level of accuracy. The main limitation of this analysis is heterogeneity between included studies.

\section{References}

1. Cannesson M, et al: Anesthesiology 2007, 106:1105-1111.

2. Cannesson M, et al:: Br J Anaesth 2008, 101:200-206.

3. Feissel M, et al: Intensive Care Med 2007, 33:993-999.

4. Wyffels PA, et al:: Anesth Analg 2007, 105:448-452.

5. Natalini $G$, et al:: Anesth Analg 2006, 103:1478-1484.

6. Solus-Biguenet $H$, et al:: Br J Anaesth 2006, 97:808-816.

P123

Maintenance of cardiac index within normal range is associated with mortality reduction in patients undergoing major urological surgery

PS Szturz, JM Maca, JT Tichy, PS Sukenik, VC Chylek, PS Sklienka, JJ Jahoda, RK Kula

Faculty Hospital Ostrava, Czech Republic

Critical Care 2010, 14(Suppl 1):P123 (doi: 10.1186/cc8355)

Introduction Hemodynamic optimisation based on flow variables allows an early detection and correction of possible occult organ hypoperfusion in patients undergoing major surgery. Shoemaker described a markedly decreased cardiac index $(\mathrm{Cl})$ in nonsurvivors, which remained significantly below the values compared with survivors during the surgery. The aim of this study was to evaluate the length of ICU stay, overall in-hospital stay and the postoperative outcome in a group of patients undergoing major urological surgery, while the $\mathrm{Cl}$ is maintained within the normal range during intraoperative period.

Methods Patients were randomised into groups the day before surgery conventional management group (decision about fluid therapy and vasoactive support was based on internal guidelines to preserve normal macrohemodynamic variables), and protocol group. Each patient in the protocol group received an oesophageal Doppler probe (TED) (Hemosonic 100; Arrow International, USA) after the start of general anaesthesia and then hemodynamic optimisation (fluid management and vasoactive drugs), according to TED variables, was performed to keep CI between 2.6 and $3.8 \mathrm{l} / \mathrm{minute} / \mathrm{m}^{2}$.

Results We enrolled 230 patients. The control group: $n=115$ and the protocol group: $n=115$. High-risk criteria surgery was fulfilled in $43 \%$ patients in protocol group and $45 \%$ in control group. There were no significant differences in baseline variables between both groups (age, gender, length of surgical procedure, estimated blood loss and also in intraoperative values of MAP and (VP). In the protocol group was observed a high frequency of $\mathrm{Cl}<2.6 \mathrm{l} / \mathrm{minute} / \mathrm{m}^{2}$ after induction of anesthesia $75 \%$ with fast recovery of $\mathrm{Cl}$. The volume of fluids (Ringer's solution and HES 6\% 130/0.4) administered during surgery was lower in the control group (medians: $2,800 \mathrm{ml}$ vs $3,800 \mathrm{ml}, P<0.05$ ). Amount of used blood units (RBC 71 vs 133, $P=0.001$; FFP 71 vs $142, P<0.001$ ) was higher in control group. Significant differences have also been found in the use of vasoactive 

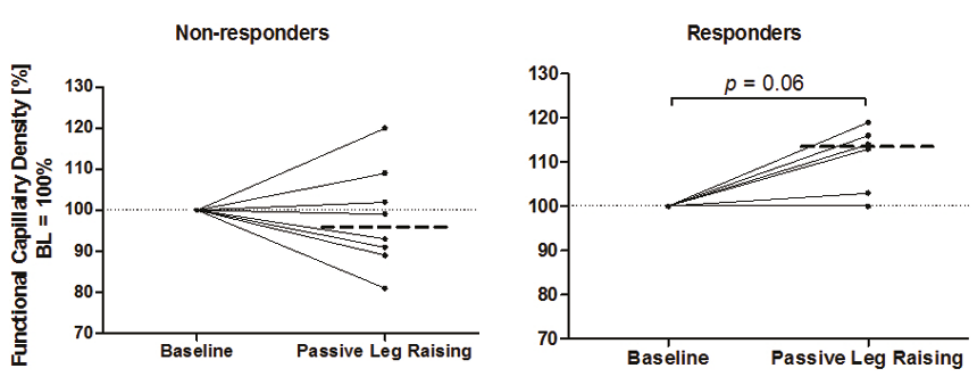

Figure 1 (abstract P124). Functional capillary density. Dotted line, median.

agents. Total number of postoperative complications $(P<0.001)$, the ICU stay and overall in-hospital stay (medians: 2 vs 3 days, $P=0.041$, and 9 vs 11 days, $P=0.014)$, in-hospital mortality ( $2.6 \%$ vs $10.4 \%, P=0.029)$ were in favour of the protocol group of patients.

Conclusions Hemodynamic optimisation guided by TED can improve postoperative outcome in patients after major urological surgery. Maintaining normal values of $\mathrm{Cl}$ is an applicable target of intraoperative therapeutic intervention.

P124

Effect of a preload challenge on peripheral perfusion in critically ill patients

E Klijn, S Niehof, J Bakker, C Ince, J Van Bommel

Erasmus Medical Center, Rotterdam, the Netherlands

Critical Care 2010, 14(Suppl 1):P124 (doi: 10.1186/cc8356)

Introduction Fluid therapy in ICU patients serves to maintain tissue perfusion and is directed at increasing cardiac stroke volume (SV) through an increase in preload: fluid responsiveness. Although there is increasing evidence that the regional blood flow cannot be predicted from global hemodynamic measurements, the relation between SV and parameters of peripheral perfusion is not clear. The aim of our study was to evaluate the effect of an increase in preload on commonly used parameters of peripheral perfusion.

Methods Hemodynamically unstable patients with clinically suspected fluid responsiveness underwent a passive leg raising (PLR) test, which consisted of 5 minutes of rest in a semirecumbent position of $30^{\circ}$, followed by 5 minutes PLR (lower limbs elevated at $30^{\circ}$ and trunk in supine position). SV was measured continuously by pulse contour analysis using PiCCO (Pulsion). Peripheral perfusion was measured continuously with sidestream dark field imaging (sublingual area) and laser Doppler flowmetry (LDF) (finger).

Results Sixteen patients (age: 63 years (55 to 72), APACHE II: 25 (20 to 28), SOFA: 10 (7 to 13)) were included in our study. Of these 16 patients, six (38\%) increased their SV by $>10 \%$ in response to a PLR. Flow indices (LDF and sublinguale microcirculatory flow) did not change. However, there was a trend in increase of the functional capillary density in the responders (see Figure 1).

Conclusions These data suggest that increasing SV in hemodynamically unstable patients might improve peripheral perfusion, however only in the sublingual area and not in all patients. There was no relation between systemic circulation and peripheral perfusion: it remains to be investigated whether optimizing SV actually results in improved tissue perfusion.

\section{P125}

Distensibility index of inferior vena cava diameter in ventilated septic and trauma patients with shock

N Parenti', D Sangiorgi ${ }^{2}$, A Pigna $^{3}$, C Coniglio ${ }^{4}$, F Cancellieri $^{4}$, G Gordini ${ }^{4}$, R Melotti ${ }^{3}, \mathrm{G}$ Di Nino ${ }^{3}$

${ }^{1}$ Hospital Santa Maria della Scaletta Imola, Bologna, Italy; ${ }^{2}$ Università, Bologna, Italy; ${ }^{3}$ Policlinico Sant'Orsola, Bologna, Italy; ${ }^{4}$ Ospedale Maggiore, Bologna, Italy

Critical Care 2010, 14(Suppl 1):P125 (doi: 10.1186/cc8357)

Introduction We evaluated the distensibility index of the inferior vena cava (dIVC\%) in ventilated septic and trauma patients with shock before and after fluid therapy. There are no data on this index in patients in shock post trauma.

Methods This is a prospective study conducted in two ICUs between September 2008 and May 2009. Inclusion criteria were: shock (systolic arterial pressure below $90 \mathrm{mmHg}$ and/or perfusion of vasopressor amines) related to severe sepsis or to trauma. The inferior vena cava diameter at end-expiration (IVCD ${ }_{\max }$ ) and at end-inspiration (IVCD ${ }_{\min }$ ) was measured by echocardiography using a subcostal approach. The distensibility index of the IVC was the ratio of IVCD max $_{\text {min }}-I V C D_{\text {min }} /$ IVCD min (dIVC\%). Cardiac index (Cl) was calculated by analysis of the arterial pressure wave (FloTracNigileo; Edwards). Measurements were performed at baseline and after a volume expansion using $7 \mathrm{ml} / \mathrm{kg}$ colloid and $20 \mathrm{ml} / \mathrm{kg}$ crystalloid for septic and trauma patients, respectively. Patients were separated into responders (increase in $\mathrm{Cl} \geq 15 \%$ ) and nonresponders (NR) after fluid therapy. The Wilcoxon and Mann-Whitney tests were used to compare paired values. Statistical significance was tested at an a level of 0.05 .

Results Eleven patients in shock (five septic, six trauma; six responder, five NR) were included. The median age was 62 years (range 28 to 78 years) and mean SAPS II score was $52 \pm 30$ SD. There were no significant differences between responders (R) and NR regarding age, gender, and risk scores. Among all patients, at baseline, median $\mathrm{Cl}$ and dIVC\% were $2.6 \mathrm{l} /$ minute/ $\mathrm{m}^{2}$ and $29 \%$, respectively. Volume expansion significantly increased the median Cl from 2.6 (2 to 3.3$)$ to $3(2.1$ to 4$) \mathrm{l} / \mathrm{minute} / \mathrm{m}^{2}(P=0.005)$ and decreased dIVC\% from $29.4 \%$ to $12.6 \%(P=0.003)$. The median dIVC\% in $\mathrm{R}$ was higher than NR: $31.3 \%$ vs $17 \%(P<0.05)$. Fluid therapy decreased more dIVC\% in R than in NR: R $31 \%$ to $12 \%(P=0.03)$, NR $17 \%$ to $12 \%(P=0.04)$. The dIVC\% showed similar trend in both groups of septic shock (SS) and trauma shock (TS) patients before and after fluid therapy: dIVC\% 27\% in SS and 24\% in TS before fluid therapy; $15 \%$ in SS and 11\% in TS after therapy. Conclusions Our data suggest that dIVC\% is a sensitive index of fluid responsiveness in septic and trauma patients in shock. Limitations: few patients.

\section{References}

1. Barbier C, et al:: Respiratory changes in inferior vena cava diameter are helpful in predicting fluid responsiveness in ventilated septic patients. Intensive Care Med 2004, 30:1740-1746.

P126

Aortic $\mathrm{dP} / \mathrm{dt}_{\text {max }}$ accurately reflects left ventricular contractility when effective preload independence is achieved

P Morimont', B Lambermont ${ }^{1}$, T Desaive', N Janssen'1, G Chase ${ }^{2}$, V D'Orio'

'University of Liege, Belgium; 'University of Canterbury, New Zealand

Critical Care 2010, 14(Suppl 1):P126 (doi: 10.1186/cc8358)

Introduction Myocardial depression occurs in 40\% of patients presenting with sepsis. In critically ill patients, the peak first derivative of aortic pressure (Ao_dP/dt ${ }_{\text {max }}$ ) derived from a fluid-filled catheter has been commonly used by clinicians for decades to assess directional change in left ventricular (LV) contractility. However, this parameter remains questionable because of its preload sensitivity. The aim of this study was to test whether Ao_dP/dt represents an accurate method for assessing LV contractility when preload independence, based on dynamic indices, is achieved.

Methods LV pressure-volume data obtained with a conductance catheter and invasive aortic pressure obtained with a fluid-filled catheter were continuously recorded in six anaesthetized and mechanically 


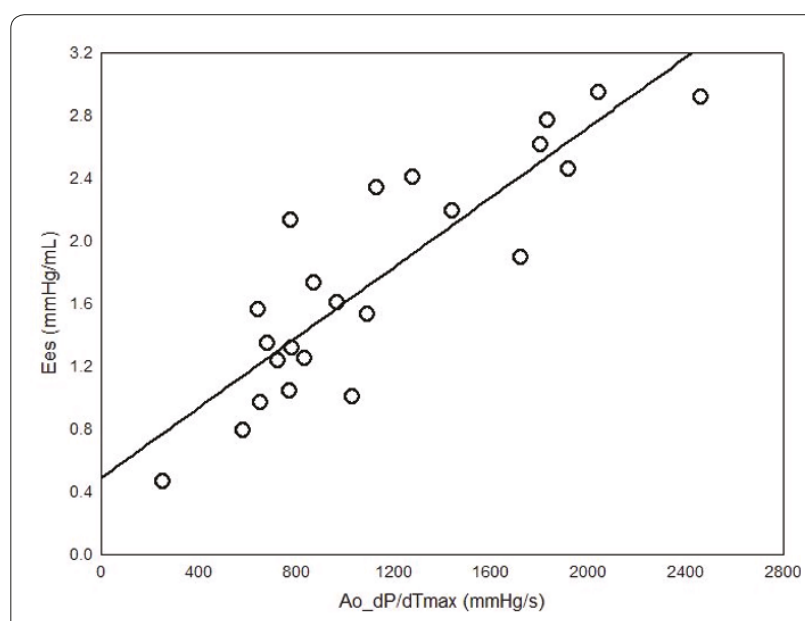

Figure 1 (abstract P126). Correlation between $\mathrm{E}_{\mathrm{es}}$ and $\mathrm{Ao}_{-} \mathrm{dP} / \mathrm{dt}$ when $\mathrm{PPV}<11 \%$. Incidence of ICR-BSI in WCNN intensive therapy unit.

ventilated pigs. After a stabilization period, endotoxin was infused to induce septic shock. Fluid administration was continuously controlled by preload responsiveness by holding pulse pressure variation (PPV) $<13 \%$. Catecholamines were transiently administrated during shock. Ao_dP/dt was compared with end-systolic elastance $\left(E_{e s}\right)$, the gold standard method for assessing LV contractility.

Results Endotoxin-induced septic shock and catecholamine infusion lead to significant variations in LV contractility. The best correlation $\left(r^{2}=0.76\right)$ and agreement between Ao_dP/dT ${ }_{\text {max }}$ and $E_{e s}$ were obtained when PPV $<11 \%$ (Figure 1).

Conclusions Ao_dP/dT is a minimally invasive and accurate method for assessing LV contractility when effective preload independence, defined as PPV $<11 \%$, is achieved.

\section{References}

1. Sharma AC: Shock 2007, 28:265-269.

2. Pinsky MR, et al.: Curr Opin Crit Care 2005, 11:235-239.

P127

Cardiac cycle efficiency: a new index for cardiac work estimation tested during aortic valve plasty

S Romagnoli, M Romano, C Lazzeri, G Santoro, F Meucci, D Quattrone,

D Dini, A De Gaudio

Careggi Hospital, Florence, Italy

Critical Care 2010, 14(Suppl 1):P127 (doi: 10.1186/cc8359)

Introduction The pressure recording analytical method (PRAM) is the only pulse contour method that does not need any calibration since it estimates in vivo, beat to beat, the impedance of the cardiovascular system [1-3]. Cardiac cycle efficiency (CCE) is a novel parameter that is directly related to the cardiovascular impedance. Since the aortic valve contributes to impedance, we hypothesized during an aortic valvuloplasty, performed for severe aortic stenosis, the cardiovascular impedance may decrease by the reduction of the transvalvular gradient.

Methods In a cath lab, five consecutive patients undergoing aortic valve plasty for severe aortic stenosis were monitored by means of PRAM during the procedure. Systolic (SAP), diastolic, and mean (MAP) arterial pressures, stroke volume (SV), heart rate (HR), cardiac output (SV x HR), CCE, and dP/ $\mathrm{dt}$ were continuously collected and afterwards analyzed. The stroke work $(\mathrm{SW}=\mathrm{SV} \times \mathrm{MAP})$ and minute work $(\mathrm{MW}=\mathrm{SBP} \times \mathrm{CO})$ were also measured.

Results After the ballooning maneuver (valvuloplasty), the maximal gradient measured with intraventricular and intraaortic catheters significantly decreased (146 (37) vs $43(12) \mathrm{mmHg} ; \quad P<0.0001)$. The CCE significantly improved from $-0.72(0.4)$ to $0.02(0.19) \mathrm{U} ; P<0.0001$. Peripheral $\mathrm{dP} / \mathrm{dt}$ increased from $0.8(0.27)$ to $1.16(0.23) \mathrm{mmHg} / \mathrm{ms} ; P=$ 0.001 , while $\mathrm{SW}$ and $\mathrm{MW}$ did not show substantial modifications (4.06 (1.03) vs 4.02 (1.4) I x mmHg; NS, and 549.8 (111.5) vs 544.6 (153.4) I x $\mathrm{mmHg}$; NS, respectively).
Conclusions CCE demonstrated to be a very sensitive estimation of cardiovascular impedance since it shows a significant improvement after the valvuloplasty in spite of unchanged values of both SW and MW. In other words, the energy expenditure of the whole cardiovascular system significantly reduced after the aortic valve stenosis is corrected. Moreover, our data strongly suggest that peripheral $\mathrm{dP} / \mathrm{dt}_{\max }$ is deeply affected by aortic valve stenosis.

References

1. Romagnoli S, Romano SM: Estimation of hemodynamic parameters by arterial waveform: available technologies. Anesth Analg in press.

2. Maus TM, Lee DE: Arterial pressure-based cardiac output assessment. $J$ Cardiothorac Vasc Anesth 2008, 22:468-473.

3. Romano SM, Pistolesi M: Assessment of cardiac output from systemic arterial pressure in humans. Crit Care Med 2002, 30:1834-1841.

P128

Nonocclusive mesenteric ischemia following cardiothoracic surgery N Kiyonaga, T Yasuda, T Yonemitsu, M Nagaoka, T Oryoji, M Nakahara, N Okayama, T Kikuchi, T Imabayashi, Y Kakihana, Y Kanmura Kagoshima University Hospital, Kagoshima City, Japan Critical Care 2010, 14(Suppl 1):P128 (doi: 10.1186/cc8360)

Introduction Nonocclusive mesenteric ischemia (NOMI) following cardiac surgery carries a high mortality. This disease is difficult to diagnose. Early diagnosis is thought to be of paramount importance as the only chance of improving the survival rate in these patients. Therefore, we were retrospectively studied all cases of NOMI to evaluate what were the sensitive markers and what were the risk factors contributing to the occurrence of $\mathrm{NOMI}$.

Methods We retrospectively reviewed 279 patients undergoing cardiothoracic surgery from August 2007 to July 2008. Six of these patients (2.2\%) developed NOMI postoperatively and the data for them were retrospectively evaluated in detail.

Results In all cases of NOMI, they were diagnosed at laparotomy. The mean age was 69.7 years ( 50 to 83 years old), and the male:female ratio was 1:1. One patient received off-pump coronary artery bypass surgery, and five underwent thoracic aortic surgery. Hemodialysis was initiated in one of six patients before operation, while the continuous hemodiafiltration was initiated in all patients postoperatively. After operation, high-dose catecholamines were necessary in five of six patients for long periods because of severe hypotension. In four of six patients, abdominal pain was the presenting symptom. The rest of two patients had a nonspecific presentation because they were ventilated and sedated. All patients presented abdominal distension and their abdominal $\mathrm{X}$-rays showed paralytic ileus features. The serum values of AST, LDH, CK, and lactate were slightly elevated in most patients. Five of six patients died from septic shock and multiple organ failures, and the mortality rate of patients with NOMI was $83 \%$. Potential risk factors contributing to the occurrence of NOMl and sensitive markers might be the following: continuous hemodiafiltration (6/6); hypotension (5/6); high-dose catecholamines (5/6); dehydration (6/6); abdominal pain (4/6); paralytic ileus patterns of abdominal X-rays (6/6).

Conclusions The increase of NOMl incidence following cardiothoracic surgery might be related to continuous hemodiafiltration, hypotension, dehydration, and uses of high-dose catecholamines. Identification of patients at NOMI risk and prevention of hypovolemic hypotension and use of vasodilator may help to reduce the incidence of NOMI.

\section{References}

1. Klotz S, Vestring T, Rotker J, et al:: Dagnosis and treatment of nonocclusive mesenteric ischemia after open heart surgery. Ann Thorac Surg 2001, 72:1583-1586

P129

Etiology, comorbidity and prognosis of hospitalized patients with congestive heart failure

Y Zhao, J Li, Q Xue, L Gao, Q Xu, XWu

Institute of Geriatric Cardiology, Beijing, China

Critical Care 2010, 14(Suppl 1):P129 (doi: 10.1186/cc8361)

Introduction Congestive heart failure (CHF) is a growing public health problem in China, mainly because of aging of the population and the 
increased prevalence of CHF in the elderly. The aim of present study was to investigate the major causes, comorbidities and in-hospital mortality of patients with $\mathrm{CHF}$.

Methods A retrospective study was performed in 6,960 patients (4,352 males, 2,608 females) with a validated primary discharge diagnosis of CHF hospitalized from 1 January 1993 through 31 December 2007, at Chinese PLA General Hospital in Beijing. The patients were divided into five groups based on the number of etiologies and comorbidities from one to five or more than five. A comparative analysis was performed to explore the major causes, comorbidities and in-hospital mortality of patients among the groups.

Results The mean $( \pm$ SD) age of patients was $53 \pm 17$ years in the onecomorbidity group, $60 \pm 16$ years in the two-comorbidity group, $65 \pm$ 14 years in the three-comorbidity group, $70 \pm 13$ years in the fourcomorbidity group and $72 \pm 11$ years in the five-comorbidity group. The major causes of hospitalized patients with CHF were coronary artery disease (44.9\%), vavular heart disease (27.5\%), cor pulmonale (9.6\%) and cardiomyopathy (7.4\%). The comorbidities of CHF were hypertension (38.6\%), atrial fibrillation (23.1\%), diabetes mellitus (18.3\%), pneumonia $(11.6 \%)$ and renal failure (7.1\%). The single comorbidity was predominant in younger patients while multiple comorbidity was predominant in the elderly $(P<0.001)$. The most common single etiology was vavular heart disease, the most common triple etiology was coronary artery disease complicated with hypertension and diabetes mellitus. Cox regression analysis showed higher hospital mortality rates associated with increased numbers of etiology and comorbidity (hazard ratio (HR) from $0.98,95 \% \mathrm{Cl}$ 0.71 to 1.36 to HR $1.59,95 \% \mathrm{Cl} 1.23$ to 2.05 , to HR $1.90,95 \% \mathrm{Cl} 1.43$ to 2.51 , to HR $2.47,95 \% \mathrm{Cl} 1.81$ to $3.35, P<0.001)$.

Conclusions This study demonstrates that the older a hospitalized patient with CHF is, the more comorbidities they have. The major causes and comorbidities of hospitalized patients with CHF are coronary artery disease, vavular heart disease, cor pulmonale, cardiomyopathy, hypertension, atrial fibrillation, diabetes mellitus, pneumonia and renal failure. The single etiology or comorbidity is predominant in younger patients, while multiple etiology or comorbidity is predominant in the elderly. A higher in-hospital mortality rate of CHF is associated with an increased number of etiologies and comorbidities.

P130

Factors associated with intensive care morbidity following surgical repair of tetralogy of fallot

A Aralihond, A Krishnaiah, B Mimic, K Brown

Great Ormond Street Hospital, London, UK

Critical Care 2010, 14(Suppl 1):P130 (doi: 10.1186/cc8362)

Introduction The outcome of surgical repair of tetralogy of fallot (TOF) is a useful benchmark for the assessment of congenital cardiac surgical programs. The aim of this study was to describe postoperative morbidity following TOF repair and assess the factors linked to a longer duration of postoperative ventilation.

Methods Retrospective study of all patients admitted to cardiac intensive care between January 2003 and December 2008 following classic TOF repair. More complex repairs were excluded. Factors were investigated for a relationship with intubation hours using linear regression analysis. Intubation hours were log transformed for the analysis since the data were skewed.

Results A total of 174 children were included, 97 (56\%) male, of whom 31 (18\%) had previous palliation with a BT shunt and 23 (13\%) had extra medical problems. The median age at repair was 8.9 (range 1.6 to 112.8 ) months and the median weight was $8.2(3.5$ to 28.5$) \mathrm{kg}$. The median cardiopulmonary bypass (CPB) time was 101 (42 to 292) minutes and 94 (54\%) required transannular patch. The median postoperative intubation time was 23 ( 0 to 566) hours and 11 patients $(6 \%)$ did not require postoperative ventilation. The median ICU stay was 66 (13 to 791) hours and there was one early death before hospital discharge. One hundred and seventy (95\%) patients received an inodilator. Postoperative complications included renal failure and fluid overload requiring peritoneal dialysis (PD) in 31 (17\%); junctional ectopic tachycardia (JET) in 29 (16\%); other arrhythmias in $10(6 \%)$, delayed chest closure in six (3\%) of which two had emergency mediastinal exploration due to bleeding and ECMO in one patient. A multiple regression model for the outcome measure intubation hours indicated that younger age at repair $(P=0.03)$, associated medical problems $(P=0.04)$, longer CPB time $(P=0.057)$, JET, PD, noradrenalin use and adrenaline use $(P<0.01$ for all) were independently linked with longer postoperative ventilation times.

Conclusions Younger children, those with extra medical problems and children that required higher levels of inotropes (probably reflecting low cardiac output syndrome), had longer intubation times. The commonest and most important complications that influenced duration of ventilation were JET and PD. In our series, mortality and length of stay were comparable with other published data.

\section{P131}

Sildenafil attenuates pulmonary vascular remodeling and upregulates Kv1.5 mRNA expression in pulmonary hypertension secondary to left-to-right shunt in rats

L Hu, LHTan

Children's Hospital, Zhejiang University School of Medicine, Hangzhou, China Critical Care 2010, 14(Suppl 1):P131 (doi: 10.1186/cc8363)

Introduction Sildenafil is a phosphodiesterase-type-5 inhibitor and selectively decreases pulmonary artery pressure. So far, the mechanism underlying sildenafil's effects on pulmonary vascular remodeling and potassium channel activity in pulmonary artery smooth muscle cells (PASMCS) has not been clearly addressed in pulmonary hypertension secondary to increased pulmonary blood flow.

Methods A total of 27 male SD rats were randomly divided into a sham group $(n=9)$, a shunt group $(n=9)$ and a shunt + sildenafil group $(n=9)$. A left-to-right shunt was established by performing an abdominal aorta to inferior vena cava fistula both in the shunt group and the shunt + sildenafil group. Rats in the shunt + sildenafil group received oral sildenafil $10 \mathrm{mg} / \mathrm{kg} /$ day, whereas the rats in the sham group and the shunt group were fed with normal saline of the same volume. Eleven weeks later, mean pulmonary artery pressure (mPAP) was measured. Meanwhile, the ratio of right ventricular mass to left ventricular plus septal mass $(\mathrm{RV} /(\mathrm{LV}+\mathrm{S}))$ was detected as a marker of the degree of right ventricular hypertrophy. The relative medial thickness (RMT) of middle and small pulmonary muscularized arteries was calculated as a sign of pathological changes of pulmonary vasculature. The voltage-gated potassium channel Kv1.5 mRNA expression of pulmonary vasculature was detected using real-time PCR.

Results Eleven weeks later, the rats in the shunt group developed pulmonary hypertension as evidenced by significantly increased $\mathrm{mPAP}, \mathrm{RV} /(\mathrm{LV}+\mathrm{S})$, as well as higher RMT of middle and small pulmonary muscularized arteries (all $P=0.01$ ). In addition, the rats in shunt group had decreased Kv1.5 mRNA expression in pulmonary vasculature $(P=0.01)$. The rats in the shunt + sildenafil group had a significantly decreased MPAP, and RV/(LV+S) ratio, and a lower RMT as well (all $P=0.01$ ), whereas the levels of Kv1.5 mRNA expression were significantly upregulated $(P=0.01)$. Furthermore, there were no statistically significant difference in MPAP, in $\mathrm{RV} /(\mathrm{LV}+\mathrm{S})$ ratio, RMT of middle and small pulmonary muscularized arteries and Kv1.5 mRNA expression between the shunt + sildenafil group and the sham group (all $P=N S$ ).

Conclusions Oral sildenafil attenuated pulmonary vascular remodeling and upregulated Kv1.5 mRNA expression in the rats with pulmonary hypertension secondary to left-to-right shunt.

P132

Validation of echocardiographic indices of right ventricular afterload: an experimental, open pericardium pig model

A Knook, D Reis Miranda, J Van Bommel, A Bogers, D Duncker, D Gommers, J Bakker

Erasmus Medical Centre, Rotterdam, the Netherlands

Critical Care 2010, 14(Suppl 1):P132 (doi: 10.1186/cc8364)

Introduction Pulmonary vascular resistance (PVR) has been used to assess right ventricular afterload; however, this is considered meaningless due to several physiologic shortcomings. Echocardiography has often been used to assess right ventricular (RV) afterload, but this has never been validated as an index of RV afterload. The purpose of this study is to evaluate echocardiographic indices of RV afterload in an experimental, open-pericardium pig model with induced, variable main pulmonary artery stenosis. 
Methods Eleven anesthesized pigs were instrumented for the measurement of arterial blood pressure, central venous pressure, RV and pulmonary pressure. An ultrasonic flowprobe (MA14PAX; Transonic) was positioned on the main pulmonary artery to obtain pulmonary flow. Distal to the flowprobe, a balloon-occluder was positioned facilitating gradual constriction of the pulmonary artery. To obtain a stepwise pressure difference increment over the banding of $10 \mathrm{mmHg}$ at each measurement, we gradually inflated the balloonoccluder. After 10 minutes, all invasive hemodynamic data were registered and an epicardial echocardiography was performed to obtain tricuspid flow velocities, isovolumetric and isovolumetric relaxation time. To calculate the TEI index during one heartbeat, echocardiographic measurements were synchronized with flowprobe measurements to obtain ejection time. The E/E' ratio was obtained with tissue Doppler echocardiography of the lateral tricuspid annulus. All echocardiographic measurements were performed in triple and averaged. The ejection period and mean pulmonary acceleration and cardiac output were calculated from the pulmonary flow curve derived from the ultrasonic flowprobe. Resistance over the pulmonary banding was calculated by pressure gradient divided by cardiac output.

Results Of the 11 pigs, two pigs died prematurely, one inferior caval vein rupture, and one due to asystole while inserting the pulmonary artery catheter. After pulmonary banding, central venous pressure did not increase significantly. Mean acceleration correlated with the resistance over the banding $(r=0.59, P<0.0001)$. The TEl index and E/E' neither correlated with the pressure gradient over the banding nor flow.

Conclusions Mean acceleration correlated with RV afterload in this model.

P133

Activation of apoptotic pathways in experimental acute afterload-induced right ventricular failure

C Dewachter', L Dewachter', B Rondelet', P Fesler', S Brimioulle', F Kerbaul $^{3}$, R Naeije ${ }^{1}$

'Faculty of Medicine, ULB, Brussels, Belgium; '2Lapeyronie Hospital, Montpellier, France; ${ }^{3}$ La Timone University Hospital, Marseille, France

Critical Care 2010, 14(Suppl 1):P133 (doi: 10.1186/cc8365)

Introduction The pathobiology of persistent right ventricular (RV) failure observed after an acute increase in pulmonary artery pressure $\left(\mathrm{P}_{\mathrm{pa}}\right)$ remains incompletely understood. We hypothesized that these severe complications might be related to an activation of apoptotic pathways.

Methods Fourteen anesthetized dogs were randomised to a transient 90 minutes pulmonary artery constriction or to a SHAM operation, followed 30 minutes later by hemodynamic measurements including effective arterial elastance $(E)$ to estimate RV afterload and end-systolic elastance $\left(E_{e s}\right)$ to estimate RV contractility, and sampling of cardiac tissue to assess apoptosis by real-time quantitative polymerase chain reaction, enzyme-linked immunosorbent assay and immunohistochemistry.

Results Transient increase in $P_{p a}$ persistently increased $E_{a}$ from $0.75 \pm 0.08$ to $1.37 \pm 0.18 \mathrm{mmHg} / \mathrm{ml}$, and decreased $\mathrm{E}$ from $1.06 \pm 0.09$ to $0.49 \pm 0.09$ $\mathrm{mmHg} / \mathrm{ml}, E_{\mathrm{es}} / \mathrm{E}_{\mathrm{a}}$ from $1.44 \pm 0.06$ to $0.34 \pm 0.03$ and cardiac output from $3.78 \pm 0.16$ to $1.46 \pm 0.10 \mathrm{l} /$ minute, indicating RV failure. As compared with the SHAM-operated group, and with left ventricular tissue in animals with persistent RV failure, there were decreased gene expressions of RV and septal BCl-2, with no changes in the gene expressions of Bax and Bak, and an increase in the $\mathrm{Bax} / \mathrm{BCl}-2$ ratio. $\mathrm{RV}$ and septal $\mathrm{BCl}-\mathrm{XL}$, and $\mathrm{RV} \mathrm{BCl-w}$ gene expressions were decreased as compared with the SHAM-operated group. There were activations of RV caspases 8 and 9, and of RV and septal caspase 3. Diffuse RV and septal apoptosis was confirmed by TUNEL staining. There were also increased RV and septal protein expressions of TNFa.

Conclusions Acute afterload-induced persistent RV failure appears to be related to an early activation of apoptotic pathways, and to a myocardial increase of TNFa.

\section{P134}

High incidence of severely prolonged QT interval after cardiac surgery M Biry, U Schurr, S Ritter, K Baenziger, A Zollinger, M Genoni

Triemli City Hospital, Zurich, Switzerland

Critical Care 2010, 14(Suppl 1):P134 (doi: 10.1186/cc8366)

Introduction A prolonged QT interval may be associated with torsades de pointes and lead to sudden cardiac death. The aim of this study was to determine both the frequency of QT prolongation and administration of QT prolonging drugs perioperatively in cardiac surgery.

Methods This prospective observational study included 82 patients (mean age 67 years) undergoing elective cardiac surgery (coronary bypass grafting 37, valve surgery 29, combination 16 ). The QT interval was manually measured in lead II and V2 of a 12-lead electrocardiogram (ECG) and corrected for heart rate (corrected QT interval, QTc, calculated from Bazett's formula) on the day before surgery, immediately after surgery, daily until day 5 and before hospital discharge. A QTc interval >440 ms in men and $>460$ ms in women in either ECG lead II or V2 was defined as moderately prolonged. A QTc interval $>500 \mathrm{~ms}$ was considered to be moderately prolonged in patients showing complete left or right bundle block, and severely prolonged in patients without complete bundle block. All administered drugs that may prolong the QT interval were identified and classified according to the QTdrugs.org Advisory Board: drugs with a definite risk of torsades de pointes (class 1, for example amiodarone, sotalol, haloperidol) or with a possible risk (class 2, for example granisetron, risperidone).

Results A total of 489 ECG were analyzed (mean six ECG per patient). The QTC interval was moderately prolonged in 40/82 patients before surgery (48.8\%). Five out of 40 patients (12.5\%) had received drugs from class 1 preoperatively and $1 / 40(2.5 \%)$ from class 2 . The QTc interval was severely prolonged in $3 / 82$ patients before surgery (3.7\%), of which none had received QT prolonging drugs preoperatively. In 38/82 patients (46.3\%) moderate QTc prolongation was newly discovered in at least one postoperative ECG. Seventeen put of 38 patients (44.7\%) received drugs from class 1, and 3/38 (7.9\%) from class 2 perioperatively. In 16/82 patients (19.5\%) severe QTc prolongation newly appeared in at least one postoperative ECG. Seven out of 16 patients (43.8\%) received drugs from class 1 and $1 / 16$ (6.3\%) from class 2 perioperatively.

Conclusions Severe QT interval prolongation $>500$ ms occurs in onequarter of cardiac surgical patients in the perioperative period while moderate prolongation occurs in most of them. In nearly one-half of these cases, QT prolonging drugs like amiodarone and haloperidol may be involved. Before and during administration of such drugs in cardiac surgical patients perioperatively, assessment of the QT interval from routine ECG is recommended.

\section{P135}

\section{Endothelial protein C receptor expression after cardiopulmonary} bypass in adult cardiac surgical patients

R Hajek', J Ruzickova², L Slavik', V Krcova', M Simek', I Fluger

'University Hospital Olomouc, Czech Republic; ${ }^{2}$ Hospital Prerov, Czech

Republic

Critical Care 2010, 14(Suppl 1):P135 (doi: 10.1186/cc8367)

Introduction Endothelial protein $C$ receptor (EPCR) is a transmembrane glycoprotein primarily localised on vessel endothelium. EPCR binds protein $C(P C)$ on the endothelial surface and presents it to the thrombin/ thrombomodulin complex. Thrombin activates PC to APC, which exerts anticoagulant and anti-inflammatory effect. Proteolysis of membranebound EPCR releases soluble EPCR which can be detected in plasmatic circulation. This reaction indirectly correlates with thrombin generation and can be interpreted as marker of endothelial activation during cardiopulmonary bypass (CPB).

Methods In a prospective study, the group of 35 adult patients (mean age 68 years) scheduled for cardiac surgery with standard cardiopulmonary bypass (duration $80 \pm 30$ minutes, heparin dose $3 \mathrm{mg} / \mathrm{kg}$, mild hypothermia about $34^{\circ} \mathrm{C}$ ) was evaluated. The markers of endothelial activation and coagulation parameters (EPCR, PAl-1, antithrombin, tPA, PTT, aPTT, fibrinogen, platelets and thromboelastography (TEG)) immediately before and after surgery were recorded. We hypothesized that the EPCR level (detected with ELISA monoclonal antibodies labeled with myeloperoxidase, $450 \mathrm{~nm}$ reader - detection limit $10 \mathrm{ng} / \mathrm{ml}$ soluble EPCR) increases after CPB and correlates with other markers of endothelial activation.

Results The cut-off value of EPCR was set down $200 \mathrm{ng} / \mathrm{ml}$. In 17\% of patients the value above the cut-off ( $366 \mathrm{ng} / \mathrm{ml}$ before, $209 \mathrm{ng} / \mathrm{ml}$ after CPB) was detected. The EPCR value decreases after CPB $(150.7 \pm 124$ vs $99.4 \pm$ 70.6) whereas tPA increases (1.5 vs $9.3 \mathrm{ng} / \mathrm{ml}$ ) and PAl-1 was unchangeable. A trend to hypocoagulation after CPB was noticed ( $\triangle$ fibrinogen $-1.04 \mathrm{~g} / \mathrm{l}$, 
$\triangle \mathrm{aPTT}+7.7 \mathrm{sec}, \Delta$ platelets $-67 \mathrm{G} / \mathrm{l})$ but no correlation between EPCR and TEG parameters (coagulation index $\Delta \mathrm{Cl}-1.46, \Delta \mathrm{R}+0.22$ minutes, $\Delta \mathrm{a}^{\prime}-3.9^{\circ}$, $\triangle M A-9.3 \mathrm{~mm}$ ) was recorded. No correlation between EPCR and CPB duration was recorded as well.

Conclusions CPB is not associated with increase of EPCR - a novel endothelial marker - measured immediately after operation. We need further studies to explain mechanisms of endothelial protection in this clinical setting.

\section{References}

1. Esmon CT: The endothelial protein C receptor. Curr Opin Hematol 2006, 13:382-385.

2. Thiyagarajan $M$, Cheng T, Zlokovic V: Endothelial cell protein C receptor: role beyond endothelium? Circ Res 2007, 100:155-157.

3. Lopez-Sagasetza J, Montes R, Puy C, et al: Binding of factor Vlla to the endothelial cell protein C receptor reduces its coagulant activity. J Thromb Haemost 2007, 5:1813-1816.

\section{P136}

Urapidyl for hypertension control in severe pre-eclampsia: a comparative study with nicardipine

R Vizitiu', M Krauss-Grignard², V Garcia', L Valentin², E Samain², P Diemunsch 'Hôpitaux Universitaires de Strasbourg, France; ${ }^{2}$ Hôpital Saint Jacques, Besançon, France

Critical Care 2010, 14(Suppl 1):P136 (doi: 10.1186/cc8368)

Introduction During severe pre-eclampsia (PE), control of blood pressure (BP) is crucial in order to prevent systemic complications. Currently approved treatments such as nicardipine $(\mathrm{N})$ and dihydralazine have drawbacks. Since hypertension in PE is associated with increased sympathetic activity, urapidil $(U)$, a peripheral a antagonist, has potential for $\mathrm{BP}$ control in $\mathrm{PE}$, but no controlled comparison of $\mathrm{U}$ with $\mathrm{N}$ is available. This preliminary randomized controlled trial aims to compare efficacy and safety of $U$ and $N$ to reduce $B P$ in severe PE.

Methods After IRB approval and signed informed consent, 18 women with severe PE without previous antihypertensive treatment were randomized to $\mathrm{U}$ or $\mathrm{N}$ groups. The therapeutic goal was to achieve a mean BP (MBP) between 105 and $125 \mathrm{mmHg}$. The $\mathrm{U}$ patients first received $\mathrm{U} 6.25 \mathrm{mg}$ boluses every 5 minutes until the diastolic BP dropped below $105 \mathrm{mmHg}$, followed by a $4 \mathrm{mg} /$ hour infusion adjusted as needed. In the $\mathrm{N}$ group, patients first received a $\mathrm{N} 1 \mathrm{\gamma} / \mathrm{kg} /$ minute infusion until a 15\% reduction in mean $\mathrm{BP}$, followed by a $\mathrm{N} 0.75 \mathrm{\gamma} / \mathrm{kg} /$ minute infusion adjusted as needed. Non-invasive BP was assessed every 5 minutes during treatment titration and then every 15 minutes. The time needed to reach the therapeutic goal was registered. The main endpoint was the achievement of the BP goal in 2 hours or less. Tolerance was assessed by the number of episodes of hypotension $(\mathrm{HO}$ ) (defined as MBP below $100 \mathrm{mmHg}$ ) and side effects. Severe $\mathrm{HO}$, defined as MBP below $80 \mathrm{mmHg}$ or two episodes of $\mathrm{HO}$, was considered as treatment failure and led to exclusion. Further assessment was limited to safety, amount of ocytocics used and neonatal evaluation by ICU paediatricians until discharge from ICU. Results were compared using analysis of variance. Side effects were compared with the chi-square test.

Results One $U$ patient was excluded from the efficacy assessment due to a protocol violation. The main endpoint was reached in all 17 patients, after 50 minutes in both groups. During the first 2 hours, the needed treatment adjustment median value was 1 ( 0 to 10 ) in the $U$ group and 1 ( 0 to 13 ) in the $\mathrm{N}$ group. Side effects attributable to the study treatment were observed in six of the nine cases in the $\mathrm{N}$ group and in one of the nine cases in the $U$ group $(P<0.02)$. There were no severe side effects or neonatal side effects. Conclusions No difference in efficacy could be shown in this preliminary series. Both treatments were easy to titrate. Fewer side effects were recorded in the $\mathrm{U}$ group. Further studies are needed in order to compare $\mathrm{U}$ and $\mathrm{N}$.

P137

Comparison of the effects of furosemide, captopril and lorazepam on noncomplicated hypertensive patients in the emergency department A Uzun, L Yamanel, O Cinar, H Hasman, B Comert

Gulhane Military Medical Academy, Ankara, Turkey

Critical Care 2010, 14(Suppl 1):P137 (doi: 10.1186/cc8369)

Introduction Patients frequently refer to the emergency department with hypertension and related disorders. If hypertensive crisis is not diagnosed in these patients, urgent treatment is not necessary. However, taking patient satisfaction into consideration, the emergency physicians usually discharge these patients after lowering the blood pressure with various medications [1]. This prospective, randomized, placebo-controlled study is designed in order to compare the effects of captopril, furosemide and lorazepam on lowering blood pressure and increasing patient satisfaction. Methods One hundred patients with uncomplicated hypertension were included in the study. All were randomized into four groups: (1) captopril group, (2) furosemide group, (3) lorazepam group, (4) placebo group. The blood pressure was measured at baseline, 30th, 60th and 90th minute. The patient satisfaction was assessed with a visual analog scale (VAS) at baseline and 90th minute.

Results Captopril $(23.64 \mathrm{mmHg})$, lorazepam $(24.90 \mathrm{mmHg})$ and furosemide $(24.10 \mathrm{mmHg}$ ) were found similarly effective in lowering blood pressure when we compare baseline and 90th minute, but all three drugs were superior to placebo $(15.94 \mathrm{mmHg})(P<0.05)$ (Table 1). When patient satisfaction was assessed with the VAS, captopril (30.12 mm), furosemide $(28.04 \mathrm{~mm})$ and lorazepam $(32.88 \mathrm{~mm}$ ) were statistically similar and all three drugs were superior to placebo $(22.76 \mathrm{~mm})$.

Table 1 (abstract P137). Mean arterial pressure

\begin{tabular}{lccc}
\hline Group & MAP $(\mathbf{m m H g})$ & SD $(\mathbf{m m H g})$ & Level of significance \\
\hline Furosemide & 24.1 & 10.7 & 19.9 to 28.5 \\
Captopril & 23.6 & 11.7 & 18.8 to 28.4 \\
Lorazepam & 24.9 & 10.1 & 20.7 to 29.1 \\
Placebo & 15.9 & 10.1 & 11.7 to 20.1 \\
Total & 22.1 & 11.1 & 19.9 to 24.3 \\
\hline
\end{tabular}

Conclusions In conclusion, all three drugs can be used in subjects referred to the emergency department with uncomplicated hypertension. They are similarly effective in both lowering the blood pressure and increasing patient satisfaction.

\section{References}

1. Chiang WK: Asymptomatic hypertension in the ED. Am J Emergency Med 1998, 16:701.

P138

Incidence, risk factors and outcome of hypertensive crises in critically ill patients: a retrospective survey

C Chelazzi, G Villa, V Selmi, AR De Gaudio

University of Florence, Italy

Critical Care 2010, 14(Suppl 1):P138 (doi: 10.1186/cc8370)

Introduction Hypertensive crises $(\mathrm{HC})$ are common among patients admitted to emergency rooms [1]. However, data are lacking about prevalence in the critically ill admitted to the ICU. The aim of the study was to assess the rate of $\mathrm{HC}$ in a cohort of patients admitted to a medicalsurgical ICU, to look for risk factors for $\mathrm{HC}$ and to assess outcome of patients with $\mathrm{HC}$.

Methods Data have been collected from clinical charts of patients consecutively admitted to a mixed ICU (1 January 2008 to 31 January 2009). HC diagnosis was made based on JNC definitions [2]. Patients were divided into two groups, the HC group and the control group. Differences between groups were evaluated regarding age, gender distribution, admission diagnosis, SAPS II score, ICU length of stay, ICU mortality, and inhospital mortality $(P<0.05)$. Clinical and behavioural conditions associated with $\mathrm{HC}$ were considered in the two groups and differences tested for statistical significance $(P<0.05)$.

Results Of a total of 409 patients, 63 had one or more HC (15.4\%). Conditions significantly associated with $\mathrm{HC}$ were age, cigarette smoking, cancer surgery, vasculopathy, medical indication to ICU admission, coronary artery disease, and chronic atrial fibrillation. HC patients showed higher ICU length of stay and ICU mortality.

Conclusions $\mathrm{HC}$ were common among critically ill patients admitted to the ICU, irrespective of their admission diagnosis. Conditions strongly 
associated with $\mathrm{HC}$ included cigarette smoking, cancer and age. $\mathrm{HC}$ were associated with worse outcomes.

\section{References}

1. Zampaglione B, Pascale C, Marchisio M, Cavallo-Perin P: Hypertensive urgencies and emergencies. Prevalence and clinical presentation. Hypertension 1996, 27:144-147.

2. Joint National Committee on Prevention, Detection, Evaluation, and Treatment of High Blood Pressure; National Heart, Lung, and Blood Institute; National High Blood Pressure Education Program Coordinating Committee: Seventh report of the Joint National Committee on Prevention, Detection, Evaluation and Treatment of High Blood Pressure. Hypertension 2003, 42:1206-1252.

\section{P139}

Comparative study to evaluate blood pressure levels measured by an invasive method versus a non-invasive method in patients in an intensive care unit

S Kiffer Macedo, T Bissoli, L Carvalho Dias, M Rodrigues Lacerda, R Paiva Barbosa de Castro, D Leite Cordeiro, R Janetti Carrara, PV Falcão Duarte

Hospital Sao José do Avai, Itaperuna, Brazil

Critical Care 2010, 14(Suppl 1):P139 (doi: 10.1186/cc8371)

Introduction The blood pressure is an important variable in medical practice. A mistaken measurement can change the management of some diseases, modifying the patient's prognosis. Therefore a correct definition and control of blood pressure is an essential item in an ICU environment. Methods A prospective and comparative study of the blood pressure assessed by arterial catheterization (pressure referenced to atmospheric pressure at mid-chest level and adequate curve in the monitor) and by non-invasive blood pressure device (using the cuff of the monitor with automatic oscillometric measurement) in the upper and lower limbs.

Results Included were 52 patients with average age of 54.7 years. In the right arm (RA), a difference between IBP and NIBP larger than or equal to $20 \mathrm{mmHg}$ in systolic blood pressure (SBP) (plus or minus) occurred in $26.5 \%$ of the patients; $14.3 \%$ in diastolic blood pressure (DBP) and $8.7 \%$ in mean blood pressure (MBP). In the left arm (LA), 30.6\% for SBP; $4.1 \%$ for DBP and $4.2 \%$ for MBP. In the left leg (LL), 40\% for SBP, $17.8 \%$ for DBP and $17.8 \%$ for MBP. In the right leg (RL), $47.8 \%$ for SBP; $8.7 \%$ for DBP and $17.4 \%$ for MBP.

Conclusions There were differences between BP measured by invasive and non-invasive methods mainly in SBP. Lower limbs presented a larger difference, which means that non-invasive methods in this place are not reliable. The DBP in both methods presented less difference.

\section{References}

1. Crit Care 2006, 10:R64

P140

Chronic atrial fibrillation in intensive care unit treated with electrical cardioversion: is it appropriate?

M Murtigni', E Mannelli', D Colosimo', S Cianferoni', S Degl'innocenti', J Parodo ${ }^{2}$, M Bonizzoli², G Zagli2, A Peris ${ }^{2}$

'Postgraduate School of Anesthesia and Intensive Care, University of Florence, Italy: ${ }^{2}$ Careggi Teaching Hospital, Florence, Italy

Critical Care 2010, 14(Suppl 1):P140 (doi: 10.1186/cc8372)

Introduction Chronic atrial fibrillation is known to be an important comorbidity factor in critically ill patients admitted to the ICU. The aim of this study was to evaluate whether electrical cardioversion in the ICU can reduce mortality of patients with chronic atrial fibrillation.

Methods From January 2005 to November 2009, 50 patients with history of chronic atrial fibrillation admitted to the ICU were retrospectively studied. Patients were divided into two groups: the first group included patients who underwent electrical cardioversion (CVE group, $n=10$ ) and were discharged in sinus rhythm; the second included patients who did not undergo electrical cardioversion and were discharged with chronic atrial fibrillation (CAF group, $n=40$ ). In both groups, mortality, antiarrhythmic drug therapy and oral anticoagulation regimen were monitored. The first group's follow-up evaluated maintenance of sinus rhythm. The number of

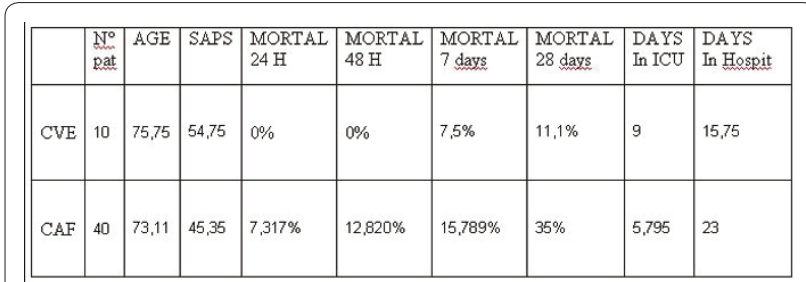

Figure 1 (abstract P140).

DC shocks required for the maintenance of sinus rhythm in patients of the CVE group was also collected. The main outcome parameter considered was 28-day mortality.

Results Patients of both groups resulted in similar demographic and clinical parameters. In patients included in the CVE group, the maintenance of sinus rhythm was achieved with one DC shock in $66.6 \%$, two DC shocks in $22.2 \%$ and three DC shocks in $11.1 \%$ of cases. The mortality rate at 24 hours was $0 \%$ while at 28 days was $11.1 \%$, whereas patients in the CAF group had a 28-day mortality rate of $35 \%$. See Figure 1.

Conclusions Our pilot study indicates that the improvement of ventricular performance with the contribution of atrial systole might improve critically ill patients' outcome. Considering the small number of cases, a prospective study, based on these preliminary results, is ongoing.

\section{P141}

\section{Applanation tonometry application in ICU patients with acute} cardiogenic edema

N Menestrina', A Martini', D Simion', M Dan², L Gottin

'University Hospital, Verona, Italy; ${ }^{2}$ Ospedale Civile, Verona, Italy

Critical Care 2010, 14(Suppl 1):P141 (doi: 10.1186/cc8373)

Introduction The incidence of acute cardiogenic pulmonary edema (ACPE) is increasing and it is now one of the leading causes of morbidity and mortality in our society. As the global population of European countries is getting older, the impact of arterial stiffness is becoming more and more important in the pathophysiology of ACPE, besides coronary vascular disease and valvular disease. The aim of the study was to evaluate whether acute modifications in elastic artery distensibility can be involved in the pathogenesis of ACPE.

Methods Six consecutive patients (four men and two women; age $76 \pm$ 7.1 years) were admitted to our ICU with the clinical diagnosis of ACPE. All patients were studied with transthoracic echocardiography (TTE) and by evaluation of pulse wave analysis (PWA) and pulse wave velocity (PWV) with the applanation tonometry method, performed at admission and after clinical stabilization.

Results In all patients, left ventricular systolic function was not significantly reduced when evaluated with transthoracic echocardiography, while a diastolic dysfunction was always demonstrated. We have recorded a significant $(P<0.05)$ decrease in blood pressure values after clinical stabilization. Estimation of the central aortic pressure waveform by mathematical transformation of radial tonometry pressure was similarly reduced. Other tonometric parameters, such as the Augmentation Index, which represents the contribution of the wave reflection to the global pulse pressure wave, and PWV, which is inversely correlated to the arterial compliance, were also significantly decreased, when compared with the values at the admission time. The subendocardial viability ratio (SEVR), an indirect index of myocardial perfusion relative to cardiac workload, increased after treatment with vasodilatators.

Conclusions Our data confirm the determining role of the increased arterial stiffness in the pathogenesis of ACPE, and how a therapeutic strategy able to ameliorate this target can be associated with a clinical improvement for the patient. The applanation tonometry could be an interesting method to evaluate these patients in the ICU setting.

\section{Reference}

1. Nichols WN, O'Rourke MF: McDonalds Blood Flow in Arteries: Theoretical, Experimental and Clinical Principles. 4th edition. London: Hodder Arnold; 1998. 
P142

A better prognosis for septic patients with left ventricular dysfunction?

P Carrilho-Ferreira, A Pais-de-Lacerda, H Côrte-Real, C França

Hospital de Santa Maria, Lisbon, Portugal

Critical Care 2010, 14(Suppl 1):P142 (doi: 10.1186/cc8374)

Introduction Several studies using angiographic or echocardiographic methods have shown that in septic shock (SSh) or severe sepsis (SeS), cardiac abnormalities, which are often documented [1], seem to have a relevant prognostic significance [2,3].

Methods A retrospective study was conduced during 6 months (April to September 2009) in an ICU of a university tertiary hospital, identifying all patients with SSh or SeS and reviewing their echocardiographic data. All the patients with known or clinical/echocardiographic evidence of significant prior cardiac disease were excluded from this analysis.

Results Forty-nine patients were identified with SSh or SeS from a total of 211 ICU patients (23.2\%). Among these, 26 had echocardiograms done during their ICU stay (53.1\%). We analyzed 19 echocardiograms from 15 patients. The remaining 11 patients were excluded, having ischemic heart disease (seven patients) and significant valvular heart disease (four patients). Within the SSh/SeS group of patients, those with echocardiograms were younger (mean age 56.3 vs 64.4) and more often women $(53.3 \%$ vs $36.7 \%)$. The ICU mortality was nevertheless similar in the two groups (46.7\% vs $46.9 \%)$. The primary sites of infection were the lungs and abdomen (26.7\% each) or unknown (20\%). The agents most frequently isolated were Gram-negative bacilli (26.7\%). In 53.3\% of cases there was no identified agent. Echocardiographic evaluation revealed significant changes in $26.7 \%$ of the patients, with the remaining being considered normal. Of those with significant changes, $75 \%(n=3)$ showed impairment of left ventricular (LV) systolic function with global hypokinesia simultaneously with diastolic dysfunction. Either abnormality was not found in any other patients. The mortality rate during ICU stay was lower in the group of patients with systolic and diastolic LV dysfunction compared with those with no apparent modification of LV function (0 vs $58.3 \%$ ). The right ventricle was dilated and showed high systolic pressures present in only one patient.

Conclusions This study reinforces the concept that changes in systolic and diastolic functions are common in septic shock and severe septic patients, and that the development of these changes (as an adaptation mechanism) seems to correlate inversely with the acute mortality rate during ICU stay $[2,4]$.

\section{References}

1. Zanotti-Cavazzoni SL, et al:: Curr Opin Crit Care 2009, 15:392-397.

2. Parker MM, et al:: Ann Intern Med 1984, 100:483-490.

3. Poelaert J, et al: Intensive Care Med 1997, 23:553-560.

4. Jardin F, et al: Chest 1999, 116:1354-1359.

\section{P143}

Microvascular effects of pulsatile versus nonpulsatile perfusion during cardiopulmonary bypass

PW Elbers', J Wijbenga ${ }^{2}$, F Solinger ${ }^{2}$, A Yilmaz ${ }^{2}$, M Van Iterson²,

EVan Dongen ${ }^{2}, \mathrm{C} \mathrm{Ince}^{3}$

'OLVG, Amsterdam, the Netherlands; ${ }^{2} S t$ Antonius Hospital, Nieuwegein, the Netherlands; ${ }^{3}$ Academic Medical Center, Amsterdam, the Netherlands

Critical Care 2010, 14(Suppl 1):P143 (doi: 10.1186/cc8375)

Introduction While advantages of pulsatile perfusion (PP) during cardiopulmonary bypass (CPB) in terms of clinical outcome remain the subject of debate, possible benefits are generally thought to occur via improvements in microvascular flow [1]. However, this is currently not supported by human clinical data. Therefore we used real-time human microvascular imaging to test our hypothesis that pulsatile perfusion would enhance microvascular perfusion.

Methods We used sidestream dark field imaging to record video clips of the human microcirculation in 16 patients undergoing routine CPB for cardiac surgery. Following administration of cardioplegia, CPB was continued in either pulsatile (PP, $n=8$ ) or nonpulsatile (NP) mode. After 10 minutes, microvascular recordings were made. The perfusion mode was then switched from PP to NP or vice versa. Ten minutes later, a second series of microvascular video recordings were obtained. Global hemodynamic and laboratory data were recorded and the energy equivalent pressure (EEP) and pulse pressure (both mean \pm SD) were calculated to quantify PP generated surplus energy. Microvascular analysis was performed both for smaller and larger microvessels with a diameter cut-off of $20 \mu \mathrm{m}$. Assessments included perfused vessel density (PVD) (mean \pm SD, 95\% confidence intervals of the difference between NP and PP (95\% CID)) and the Microvascular Flow Index (MFI) (mean \pm SD and interquartile range). Results Pulsatile perfusion resulted in higher pulse pressure ( $27 \pm 6$ vs $7 \pm$ $2 \mathrm{mmHg}, P<0.0001)$ and CPB circuit EEP $(184 \pm 33$ vs $150 \pm 27 \mathrm{mmHg}, P$ $<0.0001$ ) as compared with NP while MAP was similar between these perfusion modes ( $52 \pm 11$ vs $56 \pm 13 \mathrm{mmHg}, P=0.09$ ). Both for small and larger microvessels, we found no differences in indices of microvascular perfusion between PP and NP. Small microvessel PVD was similar between $\mathrm{PP}$ and NP $(6.65 \pm 1.39$ vs $6.83 \pm 1.23 / \mathrm{mm} ; 95 \% \mathrm{CID}-0.50$ to $0.87 / \mathrm{mm}, P=$ 0.58). The same was true for larger microvessel PVD $(2.16 \pm 0.64$ vs $1.96 \pm$ $0.48 ; 95 \%$ CID -0.50 to $0.11, P=0.20)$. Similarly, the MFI did not differ between groups either for smaller (3.00 (2.83 to 3.00) vs 3.00 (2.75 to 3.0), $P=0.41$ ) and larger microvessels (3.00 (2.75 to 3.00$) 3.00$ (3.00 to 3.00 ), $P=0.50$ ).

Conclusions PP during CPB does not alter human microvascular perfusion using standard equipment in routine cardiac surgery despite yielding higher CPB circuit energy equivalent pressure and patient pulse pressure. Reference

1. Murphy GS, et al:: Anesth Analg 2009, 108:1394.

\section{P144}

Microcirculation and intravascular coagulopathy in patients with severe sepsis and septic shock

R Wimmer, S Franz, S Siebelist, M Uschner, C Seidelmann, H Ebelt,

S Hettwer, J Wilhelm, H Loppnow, KWerdan

Universitätsklinikum Halle (Saale), Halle, Germany

Critical Care 2010, 14(Suppl 1):P144 (doi: 10.1186/cc8376)

Introduction Sidestream darkfield imaging (SDF) technology is a new method to visualize directly microcirculation. In this study we examined the perfusion of the oral mucosa in patients with severe sepsis and septic shock in order to evaluate the link between the severity of illness, microcirculation and intravascular coagulopathy.

Methods Microcirculation was analysed in 46 ICU patients with clinical suspicion for sepsis and procalcitonin $>2 \mathrm{ng} / \mathrm{ml}$. Microcirculation was recorded daily over a 7-day period (d0 to d6). Each day, at least five single film clips were taken by SDF and analysed with special software on a PC. We used the functional vessel density and distribution of the vessel diameters as well-established parameters to qualify microcirculation. Laboratory parameters, hemodynamic data and vital signs were also registered. The degree of disseminated intravascular coagulopathy was evaluated by calculating the overt DIC score (according to ISTH), and severity of illness was determined using SAPS $I I$ and the SOFA score.

Results During the stay in the ICU we observed a continuous descent of the DIC score (1.82 \pm 1.91 on d0 vs $1.3 \pm 1.94$ on $\mathrm{d} 6)$ as well as a decline of SAPS II $(63.1 \pm 14.1$ on d0 vs $57.1 \pm 18.4$ on d6) and SOFA score (12.2 \pm 3.9 on d0 vs $9.2 \pm 5.8$ on d6). The percentage of smallest vessels below $25 \mu \mathrm{m}$ diameter decreased initially from $79.5 \pm 21.9 \%$ (d1) to rise up then persistently $86.2 \pm 10.3 \%$ (d6). Nonsurvivors $(n=11$ ) had, compared with survivors $(n=35)$, a significantly higher initial DIC score on day $0(1.3 \pm 1.6$ vs $2.05 \pm 4.1 ; P<0.05)$

Conclusions Our data show a clear relation between the time courses of $\mathrm{DIC}$ and microcirculation in patients with severe sepsis and septic shock. This finding is in line with previous reports stating the profound impact of DIC on microcirculation and finally on patient outcome.

P145

Prevalence and prognostic significance of cardiac abnormalities in the ICU: an echocardiographic study

A Dell'Anna, C Sandroni, F Cavallaro, C Marano, M Antonelli

UCSC School of Medicine, Rome, Italy

Critical Care 2010, 14(Suppl 1):P145 (doi: 10.1186/cc8377)

Introduction The aim was to describe the prevalence and type of cardiac abnormalities in ICU patients detected using echocardiography and to assess whether those abnormalities are correlated with ICU survival. 
Methods Prospective cohort study. Consecutive patients hospitalized in the UCSC medical-surgical ICU for $>24$ hours were evaluated using transthoracic echocardiography (TTE). Patients admitted for postoperative monitoring or in an agonal state were excluded. Transesophageal echocardiography (TEE) was used when TTE was technically impossible. Measured parameters included: volume, thickness, global and regional kinesis of the left ventricle $(\mathrm{LV})$, systolic function of the right ventricle (RV), and valvular function.

Results From 1 March to 30 June 2009 a total of 100 patients (median age 68.5 years (IQR 50 to 77.5$)$ ) were included in the study. Medium SAPS II was $46 \pm 15$. ICU mortality rate was $29 \%$. Median LOS in ICU was 14 days. TTE was technically feasible in 97 patients; the other three patients underwent TEE. Significant cardiac abnormalities were found in 66 patients, 41 of whom had no history of cardiac disease. The most frequent abnormalities were: tricuspidal regurgitation (45.3\%), regional asynergies of LV (32\%), left heart failure (30.2\%) and mitral regurgitation (28\%). The median left ventricular ejection fraction (EF) in our population was 50\% (IQR 15 to 72\%). Left ventricular end-diastolic volume was $100 \mathrm{ml}$ (IQR 78 to $130 \mathrm{ml}$ ). Mean tricuspid annular systolic excursion (TAPSE) was lower in patients who died (18.9 vs $20.8 \mathrm{~mm} ; P=0.09$ ). TAPSE was significantly correlated to $\mathrm{EF}$ ( $r$ coefficient $=0.48 ; P<0.0001)$. Pulmonary artery systolic pressure was significantly higher in patients who died than in those who survived to the ICU $(49.8 \pm 13.0 \mathrm{mmHg}$ vs $35.0 \pm 12.8 \mathrm{mmHg} ; P=0.045)$. On logistic regression, prediction of ICU mortality was better (AUC $=0.901$ vs 0.787) when cardiac abnormalities detected by echocardiography were added to a model based on age and SAPS II.

Conclusions In our study, a complete echocardiographic assessment was possible using TTE in $97 \%$ of patients. Two-thirds of our population had echocardiographic abnormalities, $62.1 \%$ of whom were previously unknown. The presence of $L V$ asynergies and reduced RV systolic function were associated with a worse prognosis. Detection of cardiac abnormalities increased prediction of ICU mortality based on logistic regression.

\section{P146}

BNP and NT-proBNP in patients with acute myocardial infarction complicated by cardiogenic shock: results from the IABP Shock trial H Lemm'1, R Prondzinsky', A Geppert'², M Russ', K Huber' ${ }^{1}$, KWerdan', M Buerke' 'Martin-Luther-University Hospital, Halle, Germany; 'Wilhelminenspital, Vienna, Austria

Critical Care 2010, 14(Suppl 1):P146 (doi: 10.1186/cc8378)

Introduction B-type natriuretic peptide (BNP) and N-terminal proBNP (NT-proBNP) are routine diagnostic and monitoring markers in patients with heart failure. LV function and prognosis of these patients have been shown to be reflected by BNP and NT-proBNP levels. However, in patients with cardiogenic shock after myocardial infarction, the relationship and relevance of these markers has not been elucidated.

Methods The IABP Shock trial was as a monocentric, randomised and prospective clinical trial to determine the role of therapeutic intraaortic balloon pump (IABP) counterpulsation after primary percutaneous transluminal coronary angioplasty $(\mathrm{PCl})$ in acute myocardial infarction (AMI) complicated by cardiogenic shock. Cardiac catheterization was performed in 40 patients within 12 hours of onset of hemodynamic instability. Creatinine, hemodynamic parameters and survival were determined. Further, BNP and NT-proBNP levels were measured on admission as well as 24,48 and 72 hours afterwards.

Results BNP levels detected differences in treatment regarding LVunloading under IABP $(632 \pm 194 \mathrm{pg} / \mathrm{ml}$ vs $1,370 \pm 475 \mathrm{pg} / \mathrm{ml}, P<0.05)$. However, there was no significant difference between the group treated with IABP and no IABP with regard to NT-proBNP levels. Interestingly, NTproBNP levels clearly differentiated between survivors and nonsurvivors $(4,590 \pm 1,230 \mathrm{pg} / \mathrm{ml}$ vs $14,370 \pm 4,886 \mathrm{pg} / \mathrm{ml}, P<0.05)$, contrary to no significant difference between survivors and nonsurvivors with regard to BNP levels (NS). Elevated levels of NT-proBNP in patients with cardiogenic shock might be more dependent on impaired renal function, which might reflect additional organ dysfunction (creatinine $>200 \mu \mathrm{mol} / \mathrm{l}$ vs creatinine $\leq 200 \mu \mathrm{mol} / \mathrm{l}: 24,965 \pm 9,567 \mathrm{pg} / \mathrm{ml}$ vs $7,246 \pm 2,650 \mathrm{pg} / \mathrm{ml}, P<0.05)$.

Conclusions In myocardial infarction complicated by cardiogenic shock, levels of BNP and NT-proBNP both provide valuable additional information. BNP seems to closely reflect the cardiac status and effects of therapy, while NT-pro-BNP seems a good indicator for prognosis in patients with cardiogenic shock by its dependency on organ dysfunction.
P147

Understanding vital-sign abnormalities in critical care patients

S Hugueny', DA Clifton'1, M Hravnak², MR Pinsky², L Tarassenko'

'University of Oxford, UK; ${ }^{2}$ University of Pittsburgh, PA, USA

Critical Care 2010, 14(Suppl 1):P147 (doi: 10.1186/cc8379)

Introduction We have previously shown how a data fusion index, based on the integration of vital signs continuously monitored in a stepdown unit (SDU), can identify the cardiorespiratory instability that often precedes adverse events [1]. We show how the correlations between vital signs, captured in a data fusion index based on a probabilistic model of normality [2], change during times of cardiorespiratory instability.

Methods An observational study was carried out in a 24-bed SDU, in which vital signs from 326 patients were continuously recorded [1]. The existing standard of care used single-channel Medical Emergency Team (MET) activation criteria to determine when individual vital signs became abnormal. Retrospective evaluation of the continuous vital sign data identified that MET criteria were exceeded on 238 nonartefactual occasions. One hundred and eleven of these were indicative of sufficient cardiorespiratory instability that they should have required an MET call (critical events). Vital-sign dynamics during these events were compared with data from stable periods, using order statistics and covariance analysis. Results We found the probability distributions of the most extreme and the median data for each parameter over 1-minute intervals. The bivariate Gaussian distributions of best fit for data from critical events (abnormal respiratory rate $(R R)$ or heart rate $(H R)$ ) show significant differences in covariance when compared with those calculated for data intervals from stable patients (see Figure 1, in which covariance is indicated by ellipse orientation). Under normal conditions (black ellipses), RR is correlated with HR. During critical events, tachycardia occurs with little or no variation in RR (red ellipses); tachypnoea occurs with little or no variation in HR (blue ellipses).

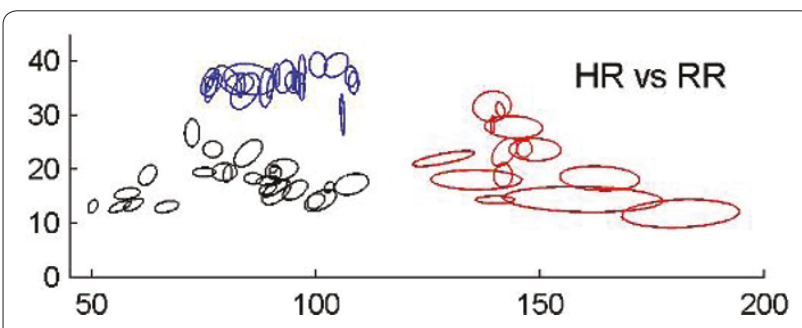

Figure 1 (abstract P147).

Conclusions Dynamics and correlations that exist in vital signs during periods of stability change significantly during periods of abnormality. A statistical approach that integrates vital signs and captures correlations between them will be sensitive to cardiorespiratory deterioration.

\section{References}

1. Hravnak M, et al:: Arch Intern Med 2008, 168:1300.

2. Tarassenko et al.: Br J Anaesth 2006, 97:64.

P148

Tissue microdialysis in critically ill septic patients: associations with sepsis severity and mortality

M Theodorakopoulou', S Orfanos ${ }^{1}$, A Diamantakis', ETsafou²,

G Karkouli', N Nikitas³, I lkonomidis', I llias'1, I Tsangaris', A Armaganidis',

I Dimopoulou ${ }^{1}$

${ }^{1}$ Attikon University Hospital, Athens, Greece; ${ }^{2}$ Athens Veterans Hospital,

Athens, Greece; ${ }^{3}$ Department of Therapeutics, Alexandra General Hospital,

Athens, Greece

Critical Care 2010, 14(Suppl 1):P148 (doi: 10.1186/cc8380)

Introduction In vivo microdialysis (MD) is a bedside sampling method that permits continuous analysis of a patient's extracellular tissue chemistry without consuming blood. MD is performed by implanting a commercially available catheter that mimics a blood capillary at the site of interest. 
Methods A total of 35 (20 men) mechanically ventilated septic patients having a median age of 60 years were studied. All patients met the ACCP/ SCCM consensus criteria for sepsis. Upon sepsis onset, a microdialysis catheter (CMA 60; CMA, Solna, Sweden) was inserted into the subcutaneous adipose tissue of the upper thigh. The dialysate samples were collected in microvials and were analyzed immediately for glucose, pyruvate, lactate, and glycerol using a mobile CMA ISCUS analyzer. The lactate/pyruvate $(L / P)$ ratio was automatically calculated. Measurements were performed six times per day during the first 6 days from sepsis onset. The daily mean values of MD measurements were calculated for each patient.

Results Sepsis severity of the study group was graded as follows: sepsis $(n=5)$, severe sepsis $(n=2)$ and septic shock $(n=28)$. APACHE and SOFA scores at study entry were $23 \pm 4$ and $8 \pm 3$, respectively. Overall, 18 patients died yielding an ICU mortality rate of $51 \%$. MD revealed that at study entry patients with septic shock had higher lactate ( $3.5 \pm 1.6 \mathrm{Vs} 1.8 \pm$ $0.9, P=0.01)$, and higher pyruvate ( $204 \pm 137$ vs $95 \pm 68, P=0.04)$ levels compared with septic patients without shock. In contrast, the two groups had similar values for glucose ( $5.9 \pm 2.7$ vs $4.3 \pm 2.9, P=0.18)$, glycerol (369 \pm 225 vs $252 \pm 171, P=0.21)$, and $L / P$ ratio $(83 \pm 289$ vs $75 \pm 150, P=0.92)$. Septic shock correlated with SOFA on day $1(r=0.55, P=0.001)$, APACHE $\|$ $(r=0.42, P=0.01)$, lactate on day $1(r=0.48, P=0.004)$, lactate on day $2(r=$ $0.50, P=0.002)$, pyruvate on day $1(r=0.040, P=0.018)$, and with pyruvate on day $2(r=0.35, P=0.04)$. Nonsurvivors had higher glycerol $(426 \pm 236$ vs $253 \pm 157, P=0.01)$ at study entry and on day 1 ( $437 \pm 260$ vs $282 \pm 169$, $P=0.04)$ compared with survivors. Nonsurvivors also had higher pyruvate levels on day $1(170 \pm 99$ vs $104 \pm 65, P=0.03)$ and on day $2(150 \pm 81$ vs $97 \pm 61, P=0.04)$. Logistic regression analysis showed that APACHE II (OR $=$ $1.312, P=0.06)$ and glycerol on day $1(\mathrm{OR}=1.005, P=0.04)$ independently predicted patient outcome.

Conclusions MD seems to be a safe and promising tool in grading sepsis severity and in predicting early mortality in critically ill patients.

\section{P149}

\section{Delayed increase in inflammation and thenar $\mathrm{VO}_{2}$ in marathon} runners

A Lima', J Weigel', E Hoorn'1, E Klijn'1, R De Jonge', N Morgenthaler², U Haagen'2, S Hofdom', J Van Ochten', C Ince', J Bakker'

'Erasmus MC University Medical Centre, Rotterdam, the Netherlands; BRAHMS, Berlin, Germany

Critical Care 2010, 14(Suppl 1):P149 (doi: 10.1186/cc8381)

Introduction The muscle-specific inflammatory response after strenuous exercise has some similarity with those observed in sepsis and it is used as a mirror of sepsis-specific inflammatory response. Near-infrared spectroscopy (NIRS) has been used to quantify sepsis-induced metabolic alterations by measuring variations of tissue oxygen saturation $\left(\mathrm{StO}_{2}\right)$ during a vascular occlusion test (VOT). We aimed to investigate whether NIRS dynamic variables could reflect the magnitude of inflammatory process that follows strenuous exercise in marathon runners.

Methods VOT-NIRS (InSpectra Model 650) measurement was performed before, at the finish line and on the day after the marathon in 13 runners (10 males/three females). VOT-derived NIRS traces were analyzed for $\mathrm{StO}_{2}$ downslope $\left(\mathrm{R}_{\text {dec }} \mathrm{StO}_{2}\right)$ and $\mathrm{StO}_{2}$-upslope $\left(\mathrm{R}_{\text {inc }} \mathrm{StO}_{2}\right)$. Blood samples were collected to measure $C$-reactive protein (CRP), procalcitonin (PCT), white blood cells (WBC), creatine kinase (CK), and lactate. Data are mean \pm SD (in Table 1 , median (IQR)). $P<0.05$ was considered statistically significant.

Results The results of NIRS dynamic and hemodynamic variables are shown in Table 1. $\mathrm{R}_{\text {dec }} \mathrm{StO}_{2}$ was significantly increased in the day after the marathon. Significant increases in lactate $(2.7 \pm 0.5$ vs $1.2 \pm 0.3)$ and WBC

Table 1 (abstract P149). NIRS and hemodynamic variables

\begin{tabular}{lccc}
\hline & Baseline & End line & Day after \\
\hline $\mathrm{StO}_{2}$ & $80(8.0)$ & $69(8.0)^{*}$ & $81(7.0)$ \\
$\mathrm{R}_{\text {dec }} \mathrm{StO}_{2}$ & $9.5(7.0)$ & $9.4(9.0)$ & $13(7.0)^{*}$ \\
$\mathrm{R}_{\text {inc }} \mathrm{StO}_{2}$ & $4.1(3.0)$ & $2.3(3.0)^{*}$ & $4.0(3.4)$ \\
$\mathrm{HR}$ & $58(24)$ & $87(60)^{*}$ & $62(26)$ \\
MAP & $93(14)$ & $88(29)$ & $87(20)$ \\
\hline
\end{tabular}

Data are median (IQR). ${ }^{*} P<0.05$. $\left(14 \times 10^{3} \pm 4\right.$ vs $\left.5 \times 10^{3} \pm 2\right)$ were observed after the completion of the marathon. Serum CRP, PCT and CK were significantly increased on the day after the marathon $(0.6 \pm 0.6$ vs $12 \pm 8 ; 0.09 \pm 0.2$ vs $0.7 \pm 0.9 ; 398 \pm 198$ vs $1,932 \pm 1,620$ ), indicating the muscle-specific inflammatory response as a result of muscle damage.

Conclusions Delayed increase in thenar $\mathrm{VO}_{2}$ is associated with musclespecific inflammatory response after strenuous exercise as a result of the muscle damage.

P150

Value of peripheral tissue oxygen tension in animal experimental models of haemorrhagic and septic shock

L Van Wagenberg, D Ramnarain, J Kesecioglu, L Leenen

University Medical Centre Utrecht, the Netherlands

Critical Care 2010, 14(Suppl 1):P150 (doi: 10.1186/cc8382)

Introduction Current clinical parameters on efficiency of resuscitation are often insufficient, and unable to reliably assess tissue hypoxia. Considerable interest has been aimed to direct monitoring of tissue oxygen tension $\left(\mathrm{ptO}_{2}\right.$ ). However, no golden standard has yet been found. Animal experimental models have shown interesting results in hypovolemic and septic shock and have lead to some interesting clinical studies. The purpose of this review is to evaluate the current value of $\mathrm{ptO}_{2}$ monitoring in animal experimental studies under various pathophysiological conditions.

Methods An electronic literature search on Pubmed and Embase databases was conducted to find relevant articles on tissue oxygenation and hemorrhage, trauma and endotoxemia. An initial amount of 7,876 articles were retrieved. After applying inclusion and exclusion criteria and critical appraisal, 48 articles were ranked on their level of evidence.

Results After screening of the 48 articles, 19 articles were found to be suitable to answer our goal. Ten articles discussed tissue oxygenation in a hemorrhagic model and nine articles in a model of endotoxemia. No relevant articles concerning $\mathrm{ptO}_{2}$ and experimental trauma were found. Eight articles compared splanchnic $\mathrm{ptO}_{2}$ measurements in relation to $\mathrm{ptO}_{2}$ measured in several peripheral sites, like subcutaneous and skeletal muscle tissue. A positive correlation between these locations was found in seven articles. A decrease in both peripheral and central tissue oxygen pressure $\left(\mathrm{ptO}_{2}\right)$ was found during hemorrhage. Importantly, peripheral $\mathrm{ptO}_{2}$ changed earlier during hemorrhage before any hemodynamic parameter did. In contrast, only one article found an earlier change in hemodynamic parameters than in peripheral $\mathrm{ptO}_{2}$. Nine articles discussed $\mathrm{ptO}_{2}$ in a model of septic shock. Only two studies compared peripheral $\mathrm{ptO}_{2}$ measurements with splanchnic $\mathrm{ptO}_{2}$ measurements. Both sites had decreased $\mathrm{ptO}_{2}$ values in endotoxemia and both were found to change before any changes in hemodynamic parameters.

Conclusions A significant relation between intestinal $\mathrm{ptO}_{2}$ and peripheral $\mathrm{ptO}_{2}$ was found in studies on experimental models of hemorrhagic and septic shock. This suggests that peripheral sites like the subcutaneous tissue or skeletal muscles tissue are reliable locations for measurements of $\mathrm{ptO}_{2}$.

\section{P151}

Incidence of a low tissue oxygen saturation in a mixed population of critically ill patients

A Veening ${ }^{1}$, C Ince $^{2}$, J Bakker ${ }^{2}$

'Hutchinson Technology Inc., Rotterdam, the Netherlands; ${ }^{2}$ Erasmus MC

University Medical Center, Rotterdam, the Netherlands

Critical Care 2010, 14(Suppl 1):P151 (doi: 10.1186/cc8383)

Introduction Tissue oxygen saturation ( $\mathrm{StO}_{\text {; }}$, normal value $87 \pm 6 \%$ [1]) measured with NIRS has been associated with disease severity in trauma [1]. The incidence of $\mathrm{StO}_{2}<75 \%$ that has been associated with organ failure [2] and its clinical consequences for ICU patients are unknown.

Methods $\mathrm{StO}_{2}$ was measured on the thenar eminence (InSpectra $\mathrm{StO}_{2}$ Monitor Model 650; Hutchinson Technology) of all patients present in the ICU during 1 week. All patients were measured only once (on Monday when already admitted, or on the day of admission). Data are presented as mean \pm SD unless otherwise specified.

Results Forty-one patients (age $53 \pm 19$ years; 63\% males) were included. Fifty-nine percent of the patients were admitted following surgery. Median 


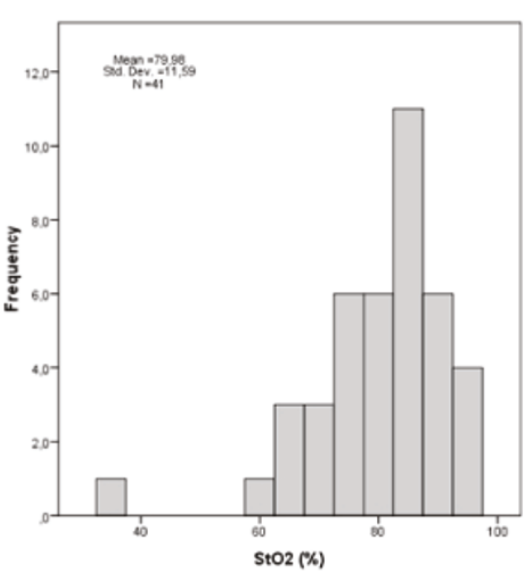

Figure 1 (abstract P151). Frequency distribution of $\mathrm{StO}_{2}$.

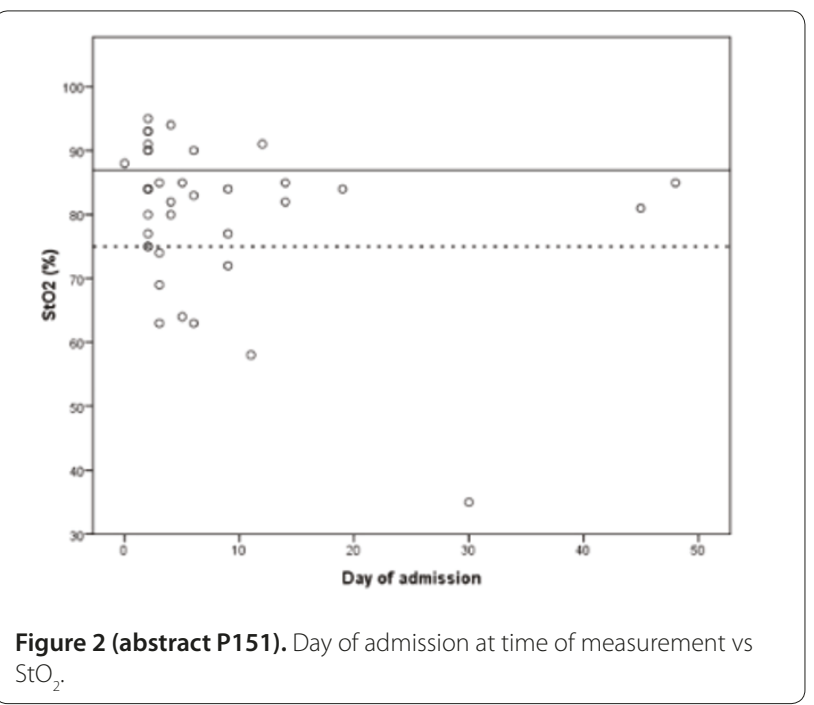

$\mathrm{StO}_{2}$ was $83 \%$ (IQR: 75 to 88 ) (Figure 1). No relationship was found between day of admission and measured $\mathrm{StO}_{2}$ (Figure 2). Thirty-one patients (76\%) had $\mathrm{StO}_{2}$ below normal (Figure 2, top line). Twelve (29\%) patients had $\mathrm{StO}_{2}$ $\leq 75 \%$ (Figure 2, dotted line). Seven patients had $\mathrm{StO}_{2}<70$; mortality of these patients was $29 \%$ vs $9 \%$ in patients with $\mathrm{StO}_{2}>70 \%$ (NS).

Conclusions The incidence of an abnormal $\mathrm{StO}_{2}$ in critically ill patients is high. Also very low $\mathrm{StO}_{2}$ values are frequently found and may be associated with increased mortality.

\section{References}

1. J Trauma 2005, 58:806-813.

2. JTrauma 2007, 62:44-54.

P152

Use of near-infrared spectroscopy during a vascular occlusion test to assess the microcirculatory response during fluid responsiveness E Futier', B Vallet², E Robin², M Vignaud', J Constantin', J Bazin'

'University Hospital of Clermont-Ferrand, France; ${ }^{2}$ University Hospital of Lille, France

Critical Care 2010, 14(Suppl 1):P152 (doi: 10.1186/cc8384)

Introduction Adequate volume expansion for hypovolemia should improve cardiac preload and stroke volume leading to improved microvascular blood flow, tissue perfusion and oxygenation. The dynamic

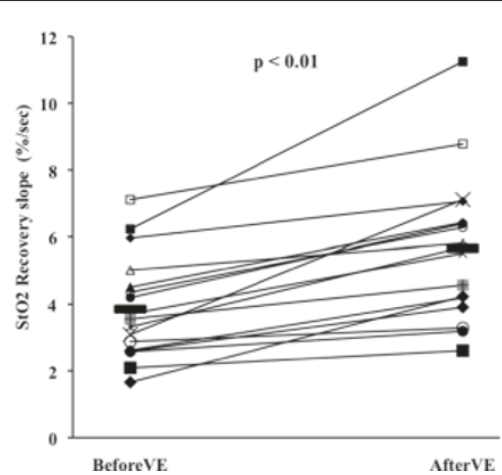

Figure 1 (abstract P152).

recovery slope of tissue oxygen saturation $\left(\mathrm{StO}_{2}\right)$ during a standardized vascular occlusion test (VOT) is proposed to reflect microvascular reactivity. We aimed at evaluating the dynamic $\mathrm{StO}_{2}$ variations during fluid challenge in high-risk surgical patients.

Methods Thirteen adult Caucasian patients, ASA II to III, undergoing major abdominal surgery, were included. In all, cardiac output (CO), stroke volume (SV), pulse pressure variation (PPV), and standard hemodynamic parameters were continuously recorded. Fluid responsiveness was defined as an increase in SV >15\%, after a $500 \mathrm{ml}$ colloid (HEA 130/0.4) infusion over 10 minutes. $\mathrm{StO}_{2}$ measurements and VOT (sphygmomanometer inflated until $>50 \mathrm{mmHg}$ above systolic pressure and kept inflated until $\mathrm{StO}_{2}$ decreased to $40 \%$ ) were performed after anaesthesia induction (baseline), and before (PPV $>13 \%$, therefore defining hypovolemia) and after fluid challenge.

Results At baseline, no patient was preload dependent (mean PPV $7.9 \pm 2 \%$ ), and none required vasopressors during the procedure. Baseline mean $\mathrm{StO}_{2}$ was $85 \pm 6 \%$, while CO and SV were $4.9 \pm 0.9 \mathrm{l} /$ minute and $68 \pm 21 \mathrm{ml}$, respectively. During hypovolemia, compared with baseline, there was no difference in both $\mathrm{StO}_{2}(85 \pm 6$ to $84 \pm 9 \%, P=0.76)$ and $\mathrm{StO}_{2}$ desaturation rate $(-9.6 \pm 1.6$ to $-11 \pm 2.7 \% /$ minute, $P=0.10)$, while the $\mathrm{StO}_{2}$ recovery slope was lowered $(5.1 \pm 1.6$ to $3.8 \pm 1.5 \% /$ second, $P=0.04)$. Patients were all responders to fluid challenge. After volume expansion, SV ( $84 \pm 20$ vs $62 \pm 12 \mathrm{ml}, P<0.01)$ and $C O(6.5 \pm 2$ vs $4.8 \pm 1.2 \mathrm{l} /$ minute, $P<0.01)$ were higher, with no significant changes in both $\mathrm{StO}_{2}$ and $\mathrm{StO}_{2}$ desaturation rate. Fluid challenge induced a $46 \%$ increase in $\mathrm{StO}_{2}$ recovery slope $(P<0.01$; Figure 1$)$, which was comparable with baseline.

Conclusions Whilst redistribution of blood flow may occur during circulatory failure (vasoconstriction of lesser vital organs), impairment of microcirculation and tissue hypoperfusion indicators are of particular importance. Both hypovolemia and intravascular volume expansion are associated with significant modifications in the dynamic recovery slope of $\mathrm{StO}_{2}$. Future studies are needed to better clarify its potential clinical applications.

P153

Influence of mechanical ventilation on intestinal microcirculation and inflammation in septic rats

D Henzler', M Haeusler', O Gil', R Chankalal'2, N Ismaiel'2, C Lehmann² 'QEll Health Sciences Center, Halifax, Canada; ${ }^{2}$ Dalhousie University, Halifax, Canada

Critical Care 2010, 14(Suppl 1):P153 (doi: 10.1186/cc8385)

Introduction Although the adverse effects of mechanical ventilation on circulation and intestinal organ perfusion are well known, the influence of assisted breathing versus controlled ventilation has not been investigated. We hypothesized that pressure support ventilation causes less decrease in gut perfusion and less neutrophil adhesion than controlled ventilation. Methods Male SD rats $(n=40)$ were anesthetized and tracheotomized and 10 animals each ventilated in pressure support (PSV) or pressure controlled (PCV) mode. PCV animals were paralyzed. After 5 hours of ventilation, intravital microscopy was performed. Endotoxin $(15 \mathrm{mg} / \mathrm{kg}$ lipopolysaccharide) was given to 20 rats, 20 animals served as controls. 
Results There were no differences in baseline hemodynamics or gas exchange. Animals were stable over the whole observation period with minimal changes in blood pressure and oxygenation. In PCV, the percentage of functional capillaries in the longitudinal mucosal layer was lower $(57 \pm 39 \%)$ than in PSV $(87 \pm 33 \%)$. In sepsis, there was a further decrease in PCV and a major decrease in PSV. Leukocyte adhesion in the intestinal submocosal venules (for example, $\mathrm{V} 1$ venules) was higher in PCV $(228 \pm 119)$ than in PSV $(93 \pm 65)$ in controls, but not in septic animals.

Conclusions In septic rats with preserved macrohemodynamic stability, intestinal perfusion was impaired after LPS infusion. Pressure-controlled ventilation had a significant influence on microcirculation and neutrophil adhesion in controls. In septic animals, pressure support ventilation did not attenuate these adverse effects as compared with PCV.

\section{P154}

$\mathrm{ScvO}_{2}$ and $\mathrm{Pcv}-\mathrm{aCO}_{2}$ as complementary tools for goal-directed therapy during high-risk surgery

E Futier', B Vallet², E Robin'², J Constantin', J Bazin'

${ }^{1}$ University Hospital of Clermont-Ferrand, France; ${ }^{2}$ University Hospital of Lille, France

Critical Care 2010, 14(Suppl 1):P154 (doi: 10.1186/cc8386)

Introduction Central venous oxygen saturation $\left(\mathrm{S}_{\mathrm{CVO}}\right)$ has been shown to be a useful therapeutic target in septic shock or high-risk surgery. The central venous-to-arterial carbon dioxide difference $\left(\mathrm{PCv}_{\mathrm{aCC}}\right)$ has been proposed as a complementary tool for goal-directed therapy (GDT) in septic shock. We tested the hypothesis that both $\mathrm{ScvO}_{2}$ and $\mathrm{Pcv}-\mathrm{aCO}_{2}$ could be used as complementary tools for GDT during high-risk surgery. Methods Seventy adult patients, ASA I to III, undergoing major abdominal surgery, were randomly assigned to $6 \mathrm{ml} / \mathrm{kg} / \mathrm{hour}$ (R-GDT group, $n=36$ ) or $12 \mathrm{ml} / \mathrm{kg} /$ hour (C-GDT group, $n=34$ ) of crystalloids. Additional boluses of HES (130/0.4) were given to maintain respiratory variation in peak aortic flow velocity ( $\triangle \mathrm{PV}$ ) below $13 \%$. In both groups, $\mathrm{ScVO}_{2^{\prime}}$, ardiac output (CO), oxygen delivery $\left(\mathrm{DO}_{2} \mathrm{i}\right), \mathrm{Pcv}-\mathrm{aCO}$ and postoperative complications were blindly recorded.

Results At baseline, there were no differences in hemodynamic variables, $\mathrm{ScvO}_{2}(79 \pm 7$ vs $80 \pm 6, P=0.37)$ and $P \mathrm{Cv}-\mathrm{aCO}_{2}(6 \pm 3$ vs $6 \pm 2, P=0.95)$. The total volume of fluid perfused was larger in the C-GDT group than in the R-GDT group $(P<0.01)$. The two groups showed no differences in intraoperative blood loss, blood transfusion, mean $\mathrm{CO}$ and mean $\mathrm{DO}_{2} \mathrm{i}$ values. Overall, postoperative complications were increased in the R-GDT group $(P<0.01)$, especially postoperative sepsis occurred more often $(P=$ 0.0064).

Minimal $\mathrm{ScVO}_{2}\left(\mathrm{minScvO}_{2}\right)$ was higher in the C-GDT group (72 \pm 6 vs $69 \pm$ $6 \%, P=0.04$ ). In patients with complications, $\mathrm{minScvO}_{2}$ was significantly reduced $(72 \pm 6$ vs $67 \pm 6 \%, P=0.0017)$. $\mathrm{minScvO}_{2}<70 \%$ was independently associated with sepsis (OR $4.2(95 \% \mathrm{Cl} 1.1$ to 14.4$), P=0.025)$. Intraoperative mean PCV-aCO was higher in the R-GDT group ( $7 \pm 3$ vs $5 \pm 2 \mathrm{mmHg}$, $P<0.01)$. In patients who develop sepsis, $\mathrm{PCV}-\mathrm{aCO}_{2}$ was higher than in patients who did not $(8 \pm 2$ vs $5 \pm 2 \mathrm{mmHg}, P<0.01)$. In patients with $\mathrm{ScvO}_{2}>70 \%$ and who develop sepsis, $\mathrm{PcV}-\mathrm{aCO}_{2}$ was also significantly higher $(P<0.01)$. The area under the ROC curve was 0.758 ( $95 \% \mathrm{Cl} 0.71$ to 0.81) for discrimination of patients with $\mathrm{ScvO}_{2}>70 \%$ who did and did not develop sepsis, with $5 \mathrm{mmHg}$ as the best threshold value.

Conclusions $\mathrm{SCvO}_{2}$ reflects important changes in oxygen delivery in relation to oxygen needs during the perioperative period and might help guiding GDT better than $\triangle \mathrm{PV}$ alone. $\mathrm{P} \mathrm{CV}-\mathrm{aCO}$ appears a useful tool to identify persistent hypoperfusion when GDT is associated with $\mathrm{ScvO}_{2}$ $>70 \%$.

\section{P155}

Prognostic value of central venous-arterial $\mathrm{pCO}_{2}$ difference in severe sepsis and septic shock patients

F Machado, A Guarnieri, FG Freitas, M Assuncao, B Mazza, M Jackiu Federal University of São Paulo, Brazil

Critical Care 2010, 14(Suppl 1):P155 (doi: 10.1186/cc8387)

Introduction Global indices of tissue perfusion include lactate and central venous oxygen saturation $\left(\mathrm{SvCO}_{2}\right)$. The mixed venous-arterial $\mathrm{pCO}_{2}$ difference $\left(\Delta \mathrm{CO}_{2} \mathrm{~m}\right)$ cannot serve as a marker of tissue hypoxia. It is a marker of venous flow adequacy to remove the total $\mathrm{CO}_{2}$ produced by peripheral tissues [1]. Substitution of a central for a mixed venousarterial $\mathrm{pCO}_{2}$ difference is acceptable [2]. The objective was to assess the relationship between $\triangle \mathrm{CO}_{2}$ and SOFA score variation in patients with severe sepsis and septic shock. Lactate and $\mathrm{ScO}_{2}$ were also evaluated.

Methods Prospective observational study in patients with severe sepsis and septic shock admitted to an adult tertiary ICU. The management of sepsis was carried out as proposed by Surviving Sepsis Campaign guidelines. The earliest simultaneous measurement of lactate, $\mathrm{SvcO}_{2^{\prime}}$ and $\triangle \mathrm{CO}_{2}$ were obtained when clinically indicated (TO) and after 8 to 12 hours (T8-12). The SOFA score was determined at T0 and after 24 hours (T24) and $\triangle$ SOFA (T24-T0) was calculated. The patients were classified according to their parameters at TO $\left(\Delta \mathrm{CO}_{2} \geq 6\right.$ vs $<6$, lactate $\geq 28 \mathrm{mg} / \mathrm{dl}$ vs $<28 \mathrm{mg} / \mathrm{dl}$, $\mathrm{SvCO}_{2} \geq 70 \%$ vs $<70 \%$ ) and their trends after 8 to 12 hours (adequate vs not adequate). Those groups were compared regarding their $\triangle$ SOFA score. The Student's $t$ test was used for analysis and results were considered significant if $P<0.05$.

Results Forty-three patients were included with mean of $60.2 \pm 18.6$ years, time from intensive care admission to T0 $10.3 \pm 9.3$ hours and duration of organ dysfunction before enrollment $21.4 \pm 15.6$ hours. APACHE II, SOFA TO, and SOFA T24 scores were $18.8 \pm 6.5,7.9 \pm 3.8$, and $8.0 \pm 4.3$, respectively. $\triangle \mathrm{CO}_{2}$ at TO $(P=0.60)$ and its trend $(P=0.36)$ are not related to $\triangle \mathrm{SOFA}$. The analysis of $\mathrm{SvCO}_{2}$ also showed no significant finding. Patients with high $(\geq 28 \mathrm{mg} / \mathrm{dl})$ blood lactate concentration at T0 $(P=0.03)$ and not adequate in blood lactate concentration at T8-12 $(P=0.04)$ had an increase in SOFA score.

Conclusions Patients with hyperlactatemia in the beginning of admission, which persists with increased levels of lactate, evolve to a worsening of SOFA score in 24 hours. $\triangle \mathrm{CO}_{2}$ and $\mathrm{SvCO}_{2}$ and its trends are unrelated to the SOFA score in 24 hours.

\section{References}

1. Lamia B, et al:: Minerva Anestesio/ 2006, 72:597-604

2. Cuschieri J, et al: Intensive Care Med 2005, 31:818-822.

\section{P156}

Veno-arterial carbon dioxide gradient at the early stage of septic shock

J Mallat, P Salaun, G Gasan, LTronchon, D Thevenin

Hospital of Lens, France

Critical Care 2010, 14(Suppl 1):P156 (doi: 10.1186/cc8388)

Introduction The measurement of cardiac output and its adequacy to tissue needs are essential for hemodynamic evaluation. In a recent review it was stated that the venous-to-arterial carbon dioxide difference $\left(\mathrm{dPCO}_{2}\right)$ could be considered a marker of adequacy of venous blood flow to remove the total $\mathrm{CO}_{2}$ produced by the peripheral tissues. Several studies have already shown that $\mathrm{dPCO}_{2}$ and the cardiac index $(\mathrm{Cl})$ are inversely correlated in critically ill patients. However, the $\mathrm{APCO}$ can be influenced not only by $\mathrm{Cl}$, but also by other factors governing $\mathrm{CO}_{2}$ production and $\mathrm{CO}_{2}$ elimination. The aim of this work was to study the behaviour of $\mathrm{dPCO}_{2}$ measured from central venous blood, and its evolution during the early stage of septic shock.

Methods Forty-six patients with septic shock were prospectively included. $\mathrm{dPCO}$, was calculated by the difference between the arterial $\mathrm{PCO}_{2}$ and the $\mathrm{PCO}_{2}$ from central venous blood. A value of $\mathrm{dPCO}_{2}>6 \mathrm{mmHg}$ was considered high. The $\mathrm{Cl}$ was measured by transpulmonary thermodilution. $\mathrm{ScvO}_{2}$ and serum lactate were obtained. Patients were separated into a normal $\mathrm{dPCO}_{2}$ group and a high $\mathrm{dPCO}_{2}$ group. The results were compared by Student $t$ test or Mann-Whitney test. $P<0.05$ was chosen as significance. Results At inclusion 24 patients (52\%) had a high $\mathrm{dPCO}_{2}$. These patients had a lower $\mathrm{Cl}\left(3.5 \pm 1.1 \mathrm{vs} 4.2 \pm 0.99 \mathrm{l} / \mathrm{min} / \mathrm{m}^{2}, P=0.04\right)$ and lower $\mathrm{ScvO}_{2}$ $(57 \pm 17$ vs $71 \pm 8 \%, P<0.001)$ than patients with normal $\mathrm{dPCO}_{2}$. No difference was found in $\mathrm{PaCO}_{2}$ or $\mathrm{PaO}_{2} / \mathrm{FiO}_{2}$ levels between both groups, suggesting a similar $\mathrm{CO}_{2}$ elimination. Thirteen patients had a decrease in $\mathrm{dPCO}_{2}$ from above to below $6 \mathrm{mmHg}$ (from $8 \pm 2$ to $5 \pm 1 \mathrm{mmHg}, P=$ 0.001 ) associated with an increase in $\mathrm{Cl}$ (from $3.7 \pm 1.3$ to $4.2 \pm 1.3 \mathrm{l} / \mathrm{min} /$ $\left.\mathrm{m}^{2}, P=0.003\right)$. Conversely, 11 patients had an increase in $\mathrm{dPCO}_{2}$ from below to above $6 \mathrm{mmHg}$ (from $3.8 \pm 2.4$ to $7.7 \pm 2.6 \mathrm{mmHg}, P=0.002$ ). In these patients, $\mathrm{Cl}$ decreased (from $4.3 \pm 1.3$ to $3.5 \pm 1 \mathrm{l} / \mathrm{min} / \mathrm{m}^{2}, P=$ $0.013)$ equally. A negative correlation was established between $\mathrm{APCO}_{2}$ and $\mathrm{Cl}\left(r^{2}=0.40, P<0.001\right)$ and between changes in $\mathrm{PPCO}_{2}$ and change in $\mathrm{Cl}$ 
$\left(r^{2}=0.16, P=0.008\right)$ during the study. The lactate level was similar in both groups. The $\mathrm{ScvO}_{2}$ was correlated with $\mathrm{Cl}\left(r^{2}=0.44, P<0.001\right)$.

Conclusions $\mathrm{APCO}$, patients with septic shock seem to be related principally to $\mathrm{Cl}$. $\mathrm{dPCO}_{2}$ might be a marker of tissue perfusion adequacy to patient's metabolism and could be a resuscitation target for management of septic shock patients.

\section{P157}

Venous-arterial $\mathrm{PCO}_{2}$ difference as an early predictor of organ dysfunction in the ICU

E Biagioni, E Boni, M Girardis

Azienda Ospedaliera Universitaria Policlinico di Modena, Italy

Critical Care 2010, 14(Suppl 1):P157 (doi: 10.1186/cc8389)

Introduction The early identification and scrupulous monitoring of tissue dysoxia can improve the management of critical patient. In this light, the final product of aerobe and anaerobe metabolism (that is, carbon dioxide) can provide useful information on adequacy of tissue perfusion and metabolism $[1,2]$. The aim of our study was to evaluate whether the venous-arterial $\mathrm{PCO}_{2}$ gradient provides useful information on tissue dysfunction in patients admitted to the ICU.

Methods We retrospectively studied 135 patients admitted to our ICU in 2006 with a length of stay $>24$ hours and a gas analysis from arterial and central venous blood at admission. Venous-arterial $\mathrm{PCO}_{2}$ gradient $\left(\triangle \mathrm{pCO}_{2}\right)$, organ dysfunction in the first 24 hours and ICU mortality were collected. Organ dysfunction was defined as a SOFA score $\geq 2$ for each organ. The patients were subdivided and compared on the basis of $\triangle \mathrm{pCO}$, value: $\triangle \mathrm{pCO}_{2} \geq 6 \mathrm{mmHg}$ (High group) and $\triangle \mathrm{pCO}_{2}<6 \mathrm{mmHg}$ (Normal group). Results Thirty-nine patients (29\%) showed a $\triangle \mathrm{pCO}_{2} \geq 6 \mathrm{mmHg}(\triangle \mathrm{pCO}$ $9.6 \pm 3.1)$. In the Normal group the mean $\triangle \mathrm{pCO}_{2}$ value was $1.2 \pm 4.1$. The High group showed a larger rate (44\%) of respiratory failure than the Normal group (25\%) Similarly, cardiovascular dysfunction was observed in $49 \%$ of the patients of the High group and only in 19\% of the Normal group $(P<0.05)$. Renal failure was also slightly larger in the High group $(31 \%)$ than in the Normal group (22\%) $(P>0.05)$. As expected, patients of the High group showed an ICU mortality (33\%) three times larger $(P<0.05)$ than patients of the Normal group (12\%).

Conclusions The above data support the hypothesis that $\triangle \mathrm{pCO}_{2}$ can provide useful information on the tissue perfusion and metabolism in ICU patients and can be used as a reliable biomarker for early prediction of organ dysfunction

\section{References}

1. Mekontso $\mathrm{A}$, Castelain $\mathrm{V}$, Anguel $\mathrm{N}$, et al:: Combination of venoarterial $\mathrm{PCO}_{2}$ difference with arteriovenous $\mathrm{O}_{2}$ content difference to detect anaerobic metabolism in patients. Intensive Care Med 2002, 28:272-277.

2. Lamia $\mathrm{B}$, Monnet $\mathrm{X}$, Teboul $\mathrm{L}$ : Meaning of arterio-venous $\mathrm{PCO}_{2}$ difference in circulatory shock. Minerva Anestesiol 2006, 72:597-604.

\section{P158}

\section{Lactate and $\mathrm{ScvO}_{2}$ combinations do not predict mortality in ICU} patients

A Ali, S Gearay, D Bennett, M Terblanche

St Thomas's Hospital, London, UK

Critical Care 2010, 14(Suppl 1):P158 (doi: 10.1186/cc8390)

Introduction Lactate (LCT) is used to identify critically ill patients at risk of death. Low central venous oxygen saturation $\left(\mathrm{SvO}_{2}\right)$ is also associated with increased mortality. It is physiologically plausible that patients with low $\mathrm{SVO}_{2}$ and high LCT do worse versus those with only one of $\mathrm{SvO}_{2}$ or LCT abnormal, or with both normal. However, the prognostic value of combining $\mathrm{LCT}$ and $\mathrm{SVO}_{2}$ is unknown. We studied the association between ICU mortality and combinations of $\mathrm{SVO}_{2}$ and LCT abnormality.

Methods We used a retrospective single-centre cohort methodology using data obtained from the comprehensive electronic clinical information system $\left(\mathrm{CareVue}^{\mathrm{TM}}\right)$. All patients who underwent LCT and $\mathrm{SVO}_{2}$ measurement (from internal jugular or subclavian vein) during the first 24 hours after ICU admission (2004 to 2009) were included. Baseline (demographic, physiological), daily follow-up (physiological, SOFA) and 30-day mortality data were recorded. Worst admission values were used to combine $\mathrm{SVO}_{2} / \mathrm{LCT}$ into four groups (SL groups: 0 to 3 ) dichotomised by mean $\left(\mathrm{SvO}_{2}\right.$ ) and median (LCT): $0-\mathrm{N} / \mathrm{N} ; 1-\mathrm{L} / \mathrm{N} ; 2-\mathrm{N} / \mathrm{H} ; 3-\mathrm{L} / \mathrm{H}$. Descriptive analysis used standard statistical techniques. Variables individually associated $(P<0.20)$ with $\mathrm{SVO}_{2}$ and/or LCT, and mortality were included in a multivariate logistic regression model (with mortality as the dependent variable) using forward stepwise inclusion. Variables with adjusted $P<0.05$ remained in the final model.

Results A total of 1,544 patients were included. Mean (SD) $\mathrm{SvO}_{2}$ was 63.6 (11.3) and median (IQR) LCT 3.85 (4.3). Overall mortality was 20.2\%. Univariate analysis showed a statistically significant association between $S L$ group and mortality $(P<0.001)$. This association did not remain significant in multivariate analysis: only LCT (not $\mathrm{SVO}_{2}$ ) was associated with mortality when adjusted for other variables.

Conclusions In this population LCT but not $\mathrm{SvO}_{2}$ predicts 30-day ICU mortality. These data does not support the hypothesis that patients with low $\mathrm{SVO}_{2}$ and high LCT do worse compared with when only one of $\mathrm{SVO}_{2}$ or $L C T$ is abnormal, or if both are normal.

\section{P159}

Early changes in lactate and central venous saturation combinations do not predict subsequent changes in organ performance

A Ali, S Gearay, D Bennett, M Terblanche

St Thomas' Hospital, London, UK

Critical Care 2010, 14(Suppl 1):P159 (doi: 10.1186/cc8391)

Introduction High lactate (LCT) identifies critically ill patients, predicts risk of mortality and guides resuscitation. Low central venous oxygen saturation $\left(\mathrm{SvO}_{2}\right)$ is associated with mortality and is used as a resuscitation target. A change in LCT, but not in $\mathrm{SVO}_{2}$, has been shown to relate to outcome. It is physiologically plausible that those with low $\mathrm{SvO}_{2}$ and high LCT do worse versus those with only one of $\mathrm{SvO}_{2}$ or LCT abnormal, or with both normal. Early changes in these combinations may also determine subsequent changes in organ performance. We hypothesised that changes in combinations in $\mathrm{SVO}_{2}$ and LCT from day 1 to day 2 predicted changes in total SOFA between day 2 and day 4.

Methods We used a retrospective cohort methodology using data obtained from the electronic clinical information system. All included patients underwent LCT and $\mathrm{SvO}_{2}$ measurement (from internal jugular or subclavian vein) in the fist 24 hours after ICU admission. Baseline (demographic, physiological), daily follow-up (physiological, SOFA) and 30-day mortality data were recorded. Worst admission values were used to combine $\mathrm{SVO}_{2} / \mathrm{LCT}$ into four groups (SL groups: 0 to 3) dichotomised by mean $\left(\mathrm{SvO}_{2}\right.$ ) and median (LCT): $0-\mathrm{N} / \mathrm{N} ; 1-\mathrm{L} / \mathrm{N} ; 2-\mathrm{N} / \mathrm{H} ; 3-\mathrm{L} / \mathrm{H}$. Variables individually associated $(P<0.20)$ with a change in total SOFA between day 2 and day 4 were included in a multivariate linear regression model (with change in total SOFA as the dependent variable) using forward stepwise inclusion. Variables with adjusted $P<0.05$ remained in the final model.

Results A total of 1,544 patients were included. Complete data on all change variables were available for 675 patients. Mean (SD) $\mathrm{SvO}_{2}$ was 63.6 (11.3) and median (IQR) LCT 3.85 (4.3). Mean (SD) APACHE II and age were $22.4(6.2)$ and 64.9 (15.9), respectively, and $61.9 \%$ were male. The mean fall (SD) in total SOFA from day 2 to day 4 was 1.05 (2.78). APACHE $\|$ (adj. $P<0.001$ ), day 1 SL group (adj. $P=0.019)$ and SOFA (adj. $P<0.001$ ), and $C$-reactive protein $>209 \mathrm{mg} / \mathrm{l}$ (adj. $P=0.006$ ) were independently associated with $\triangle$-SOFA. Changes in SL group were not associated with improvements in organ function (adj. $P=0.12$ ).

Conclusions APACHE II, SL group and total SOFA (both on day 1) and C-reactive protein $>209 \mathrm{mg} / \mathrm{l}$ were associated with worsening organ function between day 2 and day 4. Improvement in combinations of $\mathrm{SvO}_{2}$ and LCT were not associated with changes in organ performance.

\section{P160}

Regional lactate and oxygen saturation gradients: preliminary experimental data

A Pereira, P Rehder, C Dias, L Poli de Fuqueiredo, E Silva Universidade de São Paulo, Brazil

Critical Care 2010, 14(Suppl 1):P160 (doi: 10.1186/cc8392)

Introduction Regional differences among blood flow and oxygen uptake are widely cited but not well explained. The lactate shuttle 


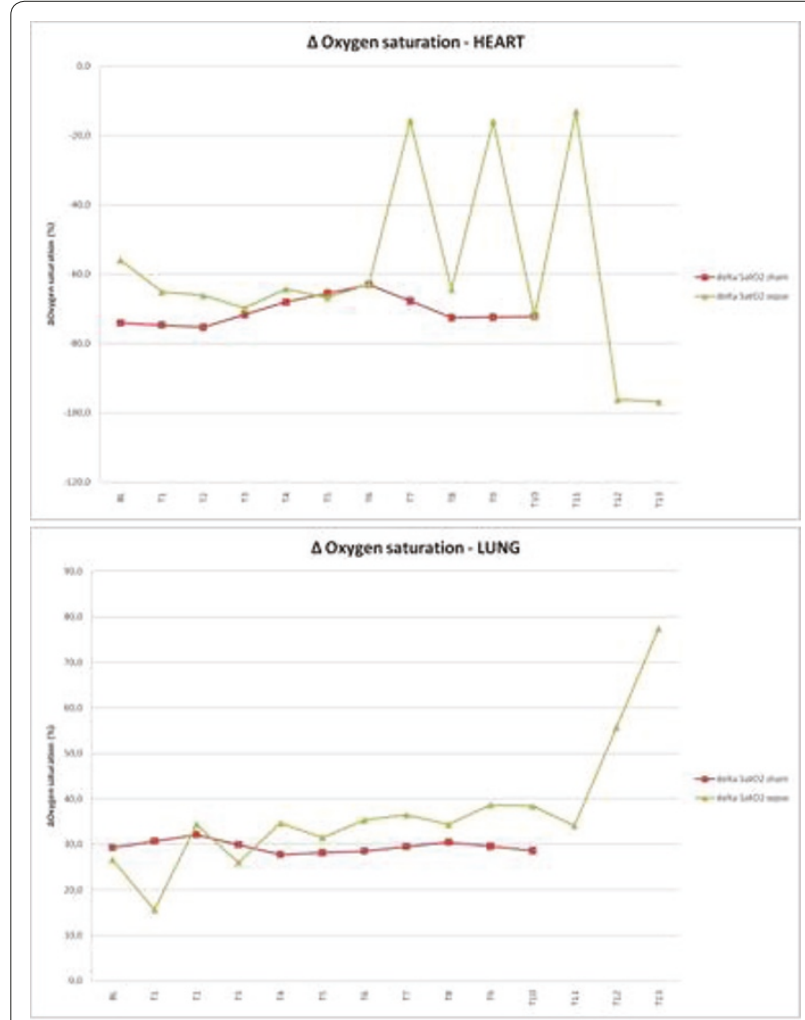

Figure 1 (abstract P160). Oxygen saturation gradients - heart and lung.

concept, alternative sets of lactate production, nowadays, challenges our comprehension about oxygen delivery and consumption. Recent studies have shown the prognostic value of oxygen and lactate gradients measured from central venous to pulmonary artery circulation. Our data represent one sample of our 4-year experimental research, trying to build a conceptual framework related to oxygen and lactate dynamic in different hypoxic states.

Methods Sixteen large white pigs $(35 \mathrm{~kg}$ ) and seven shams were studied. After anesthesia and full monitorization (EKG, MAP, etCO $2_{2^{\prime}}$ continuous gas analysis, Doppler portal flow, small bowel tonometry, liver $\mathrm{tpO}_{2}$ and urine output), abdominal sepsis was induced by fecal peritonitis.

Results See Figures 1 and 2.

Conclusions Our preliminary data show high rates of oxygen extraction rates in coronary circulation and suggest apparent lactate consumption by heart and lactate production by the lung, during experimental sepsis.

\section{P161}

\section{Examination of the utility of serum lactate and base deficit in} hemorrhagic shock

K Jonishi', Y Sakamoto', Y Ueno², H Matsumoto', Y Hara', N Kutsukata', K Mashiko' ${ }^{1}$ H Yokota $^{3}$

'Chiba Hokusoh Hospital, Nippon Medical School, Chiba, Japan; 'Tsukuba Medical Center, Ibaraki, Japan; ${ }^{3}$ Nippon Medical School, Tokyo, Japan Critical Care 2010, 14(Suppl 1):P161 (doi: 10.1186/cc8393)

Introduction Serum lactate and base deficit are markers of tissue ischemia and are used to assess the adequacy of resuscitation. Recent reports have shown that they are also useful markers associated with the severity and prognosis of sepsis and trauma; however, there are few reports regarding their use in hemorrhagic shock. We examined the utility of serum lactate and base deficit about the patients with upper gastrointestinal hemorrhagic shock. We thought that upper gastrointestinal hemorrhage cases reflected hemorrhagic clinical conditions of hemorrhagic shock rather than trauma. Methods We retrospectively reviewed the cases of 152 consecutive patients with upper gastrointestinal hemorrhage and analyzed the relationships

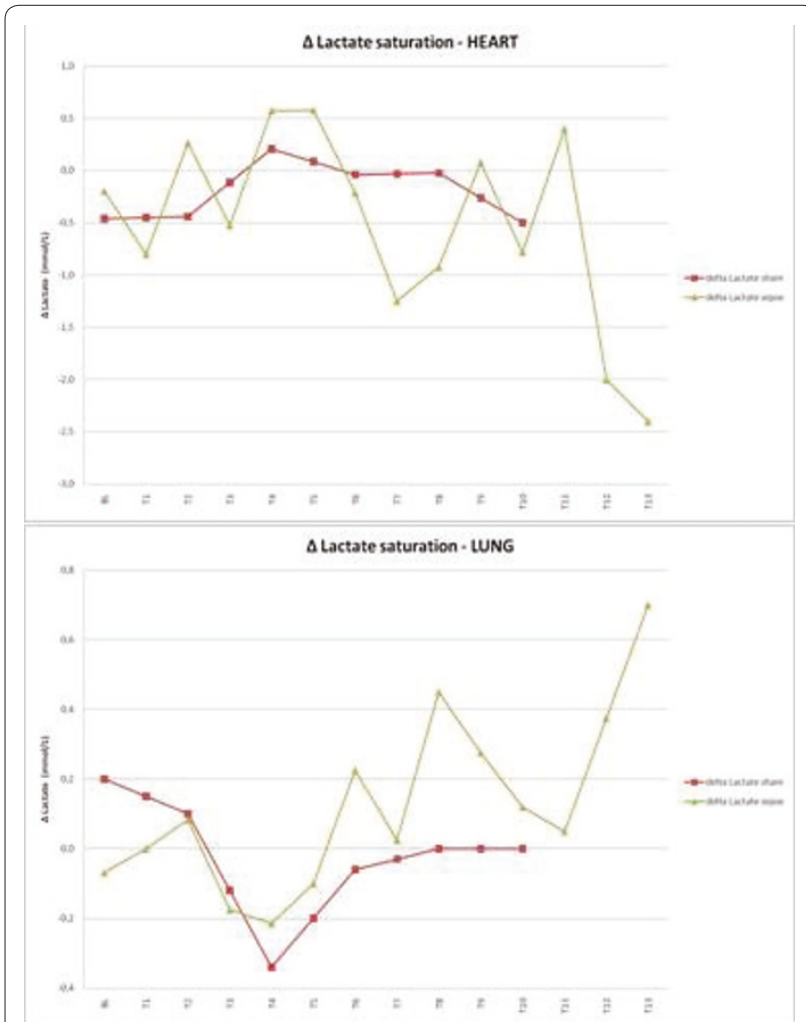

Figure 2 (abstract P160). Lactate gradients - heart and lung.

between levels of serum lactate, base deficit and hemorrhagic shock on admission. We divided the patients into two groups according to whether their shock index (systolic blood pressure/pulse) was less than 1 (shock group, $n=57 ; 37.5 \%$ ) or greater than 1 (nonshock group, $n=95 ; 62.5 \%$ ).

Results Among the 152 patients included in the analysis, 97 had gastric ulcers, 18 had esophageal varices, 17 had duodenal ulcers, seven had acute gastric mucosal lesions, six had gastric carcinoma, five had MalloryWeiss syndrome, and two had gastric varices. The study cohort comprised $27 \%$ women $(n=41)$ and $73 \%$ men $(n=111)$. The median age was $62.2 \pm$ 15.1 years. On admission, the average serum lactate level was $3.4 \pm 4.2$ $\mathrm{mmol} / \mathrm{l}$, and the base deficit was $-2.4 \pm 5.1 \mathrm{mmol} / \mathrm{l}$. The serum lactate level and base deficit were significantly higher in the shock group than in the nonshock group ( $5.8 \pm 5.5$ vs $2.0 \pm 2.4 \mathrm{mmol} / \mathrm{l}, P<0.001$, and $-4.4 \pm 5.8$ vs $-1.0 \pm 4.1 \mathrm{mmol} / \mathrm{l}, P=0.002$, respectively).

Conclusions We observed a relationship between serum lactate level, base deficit value and hemorrhagic shock in patients with upper gastrointestinal hemorrhage.

\section{References}

1. Mikkelsen ME, et al::Crit Care Med 2009, 37:1670-1677.

2. Smith I, et al: Intensive Care Med 2001, 27:74-83.

\section{P162}

Metformin increases platelet lactate production by inhibiting mitochondrial function

A Protti', A Lecchi', F Fortunato', F Rosini', A Artoni', N Greppi', A Gjoka', G Mistraletti², G Comi', L Gattinoni'

'Fondazione IRCCS Ospedale Maggiore Policlinico, Mangiagalli e Regina Elena, Università degli Studi, Milan, Italy; ${ }^{2}$ Ospedale San Paolo, Università degli Studi, Milan, Italy

Critical Care 2010, 14(Suppl 1):P162 (doi: 10.1186/cc8394)

Introduction Metformin improves glucose control by inhibiting hepatocyte mitochondrial complex I [1]. The pathogenesis of metforminassociated lactic acidosis is poorly understood and whether drug accumulation per se can cause hyperlactatemia remains unclear. The aim 
Table 1 (abstract P162)

\begin{tabular}{|c|c|c|c|c|c|}
\hline & No metformin & Metformin $0.01 \mathrm{mM}$ & Metformin $1 \mathrm{mM}$ & Metformin $100 \mathrm{mM}$ & $P$ value \\
\hline Lactate (mM) & $5 \pm 1$ & $5 \pm 1$ & $10 \pm 2$ & $21 \pm 4$ & $<0.001$ \\
\hline $\mathrm{FL} 2 / \mathrm{FL} 1$ & $5.6 \pm 1.5$ & $4.6 \pm 1.1$ & $4.6 \pm 0.7$ & $1.8 \pm 0.7$ & $<0.001$ \\
\hline $\mathrm{CS}$ (nM/minute/mg) & $102 \pm 27$ & $94 \pm 15$ & $99 \pm 20$ & $105 \pm 10$ & 0.74 \\
\hline
\end{tabular}

PRP was incubated with metformin at the reported final concentrations $(n=4)$.

of the present study is to clarify whether metformin can increase cellular lactate production by inhibiting mitochondrial function in tissues other than the liver.

Methods Platelet-rich-plasma (PRP) from healthy volunteers was incubated for 72 hours with different doses of metformin. The plasma lactate concentration was then measured. The proportion of normally polarized vs abnormally depolarized platelet mitochondria (FL2/FL1) was assessed by flow cytometry after staining with JC-1. Platelet respiratory chain complex I activity and citrate synthase (CS) activity, a marker of mitochondrial content, were measured by spectrophotometry. Data are presented as means \pm SD. Analysis was performed with one-way ANOVA.

Results Main results are reported in Table 1.

Conclusions Metformin can cause hyperlactatemia by impairing platelet mitochondrial function.

Reference

1. El-Mir MY, Nogueira V, Fontaine E, Avéret N, Rigoulet M, Leverve X: J Biol Chem $2000,275: 223-228$

P163

Artifactual lactic acidosis from point of care analysis: mind the gap

D Brindley, P Brindley

University of Alberta Hospital, Edmonton, Canada

Critical Care 2010, 14(Suppl 1):P163 (doi: 10.1186/cc8395)

Introduction A patient presented with severe acidosis, point-of-care (POC) lactate of $42 \mathrm{mmol} / \mathrm{l}$. This led to suspicion of mesenteric ischemia and potential need for laparotomy. However, plasma lactates were $<4 \mathrm{mmol} / \mathrm{l}$ and ethylene glycol (EG) ingestion was subsequently diagnosed. We therefore wished to determine why discrepant lactates occur and whether this lactate gap (that is, the difference between lactate measured using two common methods) could be clinically useful.

Methods We phlebotomized blood, added various concentrations of EG metabolites, and tested with the five most common lactate analyzers.

Results Glycolate caused massive artifactual lactate elevation with the Radiometer 700 POC analyzer even at low concentrations. Another major EG metabolite, glyoxylate, but not oxalate (or formate), caused similar elevation. The iSTAT and Bayer POC analyzers and Beckman and Vitros laboratory analyzers caused minimal lactate elevations. The lactate gap was determined by comparing the Radiometer with any of the other analyzers. Conclusions We demonstrated how inappropriate laparotomy or delayed-therapy might occur if clinicians are unaware of this phenomenon (now dubbed the lactate gap) or if hospitals only have access to a single analyzer. We also showed how the lactate gap can be exploited: to expedite treatment, diagnose late EG ingestion, and terminate dialysis. By comparing lactate results from the iSTAT or Bayer versus the Radiometer EG ingestion can be diagnosed at POC, thereby further expediting diagnosis and treatment ahead of plasma EG.

P164

Serum lactate on admission to intensive care and outcome: an observational cohort study

A Mackay, H Simpson, L Strachan, T Quasim, M Booth

Glasgow Royal Infirmary, Glasgow, UK

Critical Care 2010, 14(Suppl 1):P164 (doi: 10.1186/cc8396)

Introduction Lactate is an important substrate for intermediary metabolism and allows movement of carbon and reducing power between cells [1]. Its use as a measure of end organ perfusion and as a marker of tissue hypoxia in the critically ill is well established. It is suggested that a rise in serum lactate is a sensitive indicator of poor prognosis in this group [2]. We wished to identify whether or not this was the case in our patient population.

Methods A retrospective case note review of 504 consecutive admissions to Glasgow Royal Infirmary ICU was undertaken over an 18-month period. Serum lactate on initial blood gas analysis following admission to intensive care was retrieved from the clinical information system. Demographic, APACHE II score and outcome data were retrieved from the Ward Watcher system in the ICU.

Results Complete data were available for 454 patients. Demographics of the patient group were as follows: $65 \%$ were male with a mean age of $53.8 \pm 1.5$ years. Average length of stay was $6.1 \pm 0.46$ days with a median APACHE II score of 18 (IQR 12 to 24). Crude ICU and hospital mortalities were $28 \%$ and $34.7 \%$, respectively. Mean lactate was $2.46 \pm 0.24 \mathrm{mmol} / \mathrm{l}$. We constructed an ROC curve for admission lactate vs ICU mortality with an area under the curve (AUC) $=0.66, P<0.001$. ROC curve for APACHE $\|$ vs ICU mortality gives AUC $=0.80, P<0.001$. Using univariate analysis and chi-square testing we found that, using different thresholds for lactate level, we were able to establish a relative risk of death for those exceeding this level as follows (RR, $P$ value): $>2 \mathrm{mmol} / /$ (RR 1.29, $P<0.001),>4 \mathrm{mmol} / \mathrm{l}$ (RR 1.63, $P<0.001),>6 \mathrm{mmol} / \mathrm{l}$ (RR 2.08, $P<0.001),>8 \mathrm{mmol} / \mathrm{l}$ (RR 3.43, $P<0.001),>10 \mathrm{mmol} / \mathrm{l}(\mathrm{RR} 3.43, P<0.001)$.

Conclusions While using ROC analysis suggests serum lactate is of moderate predictive value for ICU mortality, applying serial increasing lactate cutoff points and comparing those above and below these points suggests a strong relationship between increasing lactate level and increased risk of ICU mortality.

\section{References}

1. Leverve XM: Lactate in the intensive care unit. Crit Care 2006, 9:622-623.

2. Bakker J, et al:: Blood lactate levels are superior to oxygen delivery variables in predicting outcome in human septic shock. Chest 1991, 99:956-962.

P165

Physicochemical acid-base parameters and mortality in 1,070 ICU patients: a retrospective cohort study

N Jones, S Gearay, D Bennett, M Terblanche

St Thomas' Hospital, London, UK

Critical Care 2010, 14(Suppl 1):P165 (doi: 10.1186/cc8397)

Introduction Acid-base disorders are common in ICU patients and are associated with increased mortality and morbidity. A physicochemical (PHYSCO) approach to acid-base abnormalities based on principles described by Stewart and Figge may explain various derangements better than traditional approaches. Recent data suggest an increased inorganic ion difference is associated with lower mortality in septic patients. We determined the effects of baseline and changes in PHYSCO parameters suggested by Stewart and Figge on 30-day mortality in a large cohort of unselected ICU patients.

Methods We performed a retrospective cohort study using data obtained from the electronic clinical information system. All patients who underwent lactate and $\mathrm{SvO}_{2}$ measurement (from internal jugular or subclavian vein) during the first 24 hours after ICU admission were included. Baseline, daily follow-up and 30-day mortality data were recorded. The worst recorded were used to calculate, for each day and for the change from day 1 to day 3, the following PHYSCO parameters: apparent strong ion difference (SIDa), inorganic SIDa (iSIDa; excludes lactate), effective SID (SIDe: includes pCO, albumin, $\mathrm{pH}$ and phosphate) and strong ion gap (SIG; SIDa minus SIDe). Descriptive analysis used standard statistical techniques and univariate logistic regression (dependent variable 30-day mortality) assumed statistical significance at $P<0.05$. 
Results A total of 1,544 patients were included. Complete data to calculate day 1 and day 3 parameters were available in 1,070 and 656 patients, respectively. Overall 30-day mortality was $21.3 \%$. Mean (SD) age and APACHE II score were 63.0 (16.4) and 21.6 (6.9), respectively. For survivors versus nonsurvivors there were significant differences in baseline $\mathrm{SIDa}(P=$ $0.03)$, iSIDa, SIDe, lactate and base excess (all $P<0.001)$, but not in SIG $(P=$ $0.1)$. An increased SIDa $(P=0.015)$ and SIDe $(P<0.001)$, and decreased iSIDa and lactate (all $P<0.001$ ) but not SIG $(P=0.09$ ) were predictive of mortality. For day 1 to day 3 , only $\triangle$-SIG was weakly predictive of mortality (OR 1.03 per 1 unit increase, $P=0.04,95 \% \mathrm{Cl}=1.00$ to $1.05, n=656$ ) but there was no difference between survival groups $(P=0.051)$.

Conclusions This study confirms recent data suggesting baseline differences (but not changes over 3 days) in the relationships between strong and weak ions and $\mathrm{pCO}$ (reflected in SIDa, lactate, and impacts on mortality). The different effects observed for SIG and BE imply a significant unmeasured acid load influencing mortality. Bias and confounding may affect these findings and they should therefore be confirmed prospectively.

\section{P166}

Accuracy of predicting arterial blood gases following mechanical ventilator adjustment

H Al-Otaibi', N Bedforth², R Mahajan', J Hardman

'The University of Nottingham, UK: ${ }^{2}$ Nottingham University Hospitals NHS

Trust, Nottingham, UK

Critical Care 2010, 14(Suppl 1):P166 (doi: 10.1186/cc8398)

Introduction Clinicians adjust mechanical ventilator (MV) in critical care units to optimise arterial blood gases (ABG). The present study evaluates their accuracy in predicting ABG values following MV adjustment.

Methods Thirty-one sets of data were collected from 16 patients requiring mechanical ventilation on the ICU. Each set consisted of two subsets before and after MV adjustment. Each of subset consisted of the following variables: $\mathrm{FIO}_{2}$, respiratory rate (breaths/minute), tidal volume $(\mathrm{ml} / \mathrm{kg})$, body temperature $\left({ }^{\circ} \mathrm{C}\right)$, haemoglobin concentration $(\mathrm{g} / \mathrm{dl})$ ), and $A B G$ values $\left(\mathrm{pH}, \mathrm{PaCO}_{2}, \mathrm{PaO}_{2}\right.$, base excess). All data, with the exception of ABG values after MV adjustment, were presented to clinicians. Thirty-three clinicians (11 respiratory therapists (RTs), 11 nurses, and 11 medical staff) were asked to predict the new ABG values as they would in clinical settings. All data are presented as mean \pm SD unless stated otherwise.

Results All datasets include changes in $\mathrm{FIO}_{2^{\prime}}$ while only 13 datasets include changes in minute ventilation. Increases in $\mathrm{FIO}_{2}$ were $0.19 \pm 0.04$ and decreases were $-0.12 \pm 0.04$. Size of change of minute ventilation was $1.38 \pm 0.95 \mathrm{l} /$ minute. The absolute magnitudes of change for measured $\mathrm{pH}, \mathrm{PaCO}_{2}$, and $\mathrm{PaO}_{2}$ were $0.024 \pm 0.027,0.40 \pm 0.37 \mathrm{kPa}$ and $5.91 \pm 3.35$ $\mathrm{kPa}$, respectively. The magnitudes of change for predicted $\mathrm{pH}, \mathrm{PaCO}_{2}$, and $\mathrm{PaO}_{2}$, respectively, were $0.025 \pm 0.033,0.34 \pm 0.23 \mathrm{kPa}$, and $3.45 \pm 2.03 \mathrm{kPa}$ for medical staff; $0.024 \pm 0.021,0.40 \pm 0.41 \mathrm{kPa}$, and $3.91 \pm 1.75 \mathrm{kPa}$ for RTs and $0.022 \pm 0.015,0.32 \pm 0.22 \mathrm{kPa}$, and $3.21 \pm 2.1 \mathrm{kPa}$ for nurses. The $95 \%$ limits of agreement between predicted and measured size of changes for $\mathrm{pH}, \mathrm{PaCO}$, and $\mathrm{PaO}_{2}$, respectively, were 0.043 to $-0.041,0.724$ to -0.596 $\mathrm{kPa}$, and 3.22 to $-8.14 \mathrm{kPa}$ for medical staff; 0.036 to $-0.036,0.66$ to -0.66 $\mathrm{kPa}$, and 2.64 to $-6.64 \mathrm{KPa}$ for RTs; and 0.036 to $-0.076,0.74$ to $-0.9 \mathrm{kPa}$, and 3.91 to $-9.31 \mathrm{kPa}$ for nurses. Correlation between duration of clinicians clinical experience and errors in predicting size of change for $\mathrm{pH}_{1} \mathrm{PaCO}_{2}$ and $\mathrm{PaO}_{2}(-0.0225,-0.054$, and 0.173 , respectively) were weak and not statistically significant.

Conclusions Clinicians show considerable variation in predicting $A B G$ values. Medical staff and nurses underestimate the magnitude of $\mathrm{PaCO}_{2}$ changes whereas RTs show some accuracy. All clinicians have a large bias in predicting the size of $\mathrm{PaO}_{2}$ changes.

\section{P167}

Use of capnography and the availability of airway equipment on UK intensive care units

A Georgiou, S Gouldson, A Amphlett

Frenchay Hospital, Bristol, UK

Critical Care 2010, 14(Suppl 1):P167 (doi: 10.1186/cc8399)

Introduction Provisional data from the 4th National Audit Project of the Royal College of Anaesthetists has identified that at least $20 \%$ of major airway events take place on the ICU and that these are more frequently fatal than events elsewhere (TM Cook, personal communication). Over $23 \%$ of these incidents relate to the instigation or maintenance of an artificial airway [1]. Both the use of capnography and the immediate availability of airway rescue devices to avert or manage such incidents have been recommended. This study seeks to determine the adherence to these recommendations, the availability of such equipment on UK ICUs and to identify areas for safety improvement.

Methods Every adult general, hepatobiliary and satellite ICU (grouped together as GICU), neurointensive care unit (NICU) and cardiac intensive care unit (CICU) in the UK was telephoned, thereby covering every area in the UK where ventilated adult patients are routinely cared for outside the operating theatre. Data were obtained from the nurse in charge over a 3-month period in 2009

Results In total, 315 of 328 contacted units responded (96\% response). Capnography during intubation: $32 \%$ units use always, $43 \%$ sometimes and 25\% never. Capnography for continuous monitoring of intubated patients: 25\% units use always, 35\% sometimes and 40\% never. Of note, $45 \%$ of NICUs never use capnography for continuous monitoring (where doing so may prove most beneficial). Basic airway equipment (oropharyngeal airways, laryngoscopes and bougies), alternative means of patient ventilation and equipment checking procedures are seen on 97 to $100 \%$ of ICUs. A standard laryngeal mask (LMA) is available on $97 \%$ of ICUs. More advanced airway rescue devices are seen less commonly: $71 \%$ have a McCoy laryngoscope and 55\% have a bougie through which ventilation is possible. Forty-three per cent have an intubating LMA and $21 \%$ have supraglottic airway with a gastric port immediately available. Forty-nine per cent of units have a bronchoscope on the ICU. Seventy per cent of units have equipment for emergency trans-tracheal access. CICUs are least likely to use capnography or have airway rescue equipment immediately available.

Conclusions Basic standards of airway safety are not being met on UK ICUs and are far from the standards being routinely employed in the operating theatre. The potential airway difficulties presented by the ICU patient, together with the lack of routine use of capnography, exposes these patients to significant risks that may be easily addressed.

\section{Reference}

1. Thomas et al.: Anaesthesia 2009, 64:358-365

\section{P168}

Combined oximetry and transcutaneous capnography is a reliable non-invasive monitoring in patients after cardiac surgery

W Baulig, RR Roediger, O Theusinger, D Rusch, D Spahn

University Hospital Zurich, Switzerland

Critical Care 2010, 14(Suppl 1):P168 (doi: 10.1186/cc8400)

Introduction The aim of this study was to validate the revised V-Sign ${ }^{\mathrm{TM}} 2$ ear lobe sensor for combined assessment of pulse rate (PR), pulse oximetry $\left(\mathrm{SpO}_{2}\right)$ and transcutaneous carbon dioxide tension $\left(\mathrm{PtCCO}_{2}\right)$ in adults after cardiac surgery.

Methods In 20 patients coevally readings of blood gases were compared with values of the $\mathrm{V}$-Sign ${ }^{\mathrm{TM}} 2$ ear lobe sensor. Measurements were performed during periods of hypercapnia, normocapnia and hypocapnia and then in intervals of 30 minutes up to 5 hours. Agreement was assessed by Bland-Altman analysis and single regression analysis.

Results Two hundred and ninety-six data pairs were analysed. Detection failures for $\mathrm{PtcCO}$, were 1.3\%, for $\mathrm{SpO}_{2}$ 10\% and for PR 5\%. PtcCO, and $\mathrm{PaCO}_{2}$ ranged between 3.58 and $7.70 \mathrm{kPa}$. Mean bias and limits of agreement ( $\mathrm{LOA}$ ) between $\mathrm{PtcCO}$, and $\mathrm{PaCO}$, was excellent (Table 1). The best agreement between $\mathrm{PtcCO}_{2}$ and $\mathrm{PaCO}_{2}$ was found during periods of

Table 1 (abstract P168). Agreement between V-Sign ${ }^{\mathrm{TM}} 2$ sensor and co-oximetry and ECG

\begin{tabular}{lcccc}
\hline Parameter & $\boldsymbol{n}$ (data pairs) & $\boldsymbol{r}^{2}$ & Mean bias & $\begin{array}{c}\text { Limits of } \\
\text { agreement }\end{array}$ \\
\hline $\mathrm{PtcCO}_{2}-\mathrm{PaCO}_{2}(\mathrm{kPa})$ & 296 & 0.92 & +0.14 & $-0.45 /+0.73$ \\
$\mathrm{~V}-\mathrm{Sign}^{\mathrm{TM}} \mathrm{SpO}_{2}-\mathrm{SaO}_{2}(\%)$ & 270 & 0.59 & -1.68 & $-6.81 /+3.45$ \\
$\mathrm{~V}-\mathrm{Sign}^{\mathrm{TM}} \mathrm{PR}-\mathrm{ECG}(\mathrm{bpm})$ & 285 & 0.98 & +1.22 & $-3.31 /+5.75$ \\
\hline
\end{tabular}


hyperventilation and hypoventilation with a mean bias (LOA) of $+0.18 \mathrm{kPa}$ $(-0.31 /+0.67 \mathrm{kPa})$ and $0.07 \mathrm{kPa}(-0.45 /+0.59 \mathrm{kPa})$, respectively. At about 5 hours no relevant drift was observed between $\mathrm{PtcCO}$ and $\mathrm{PaCO}$. V-Sign ${ }^{\mathrm{TM}}$ $2 \mathrm{SpO}_{2}$ moderately agreed and slightly underestimated $\mathrm{SaO}_{2}$ determined by direct oximetry, whereas pulse rate values detected by the $\mathrm{V}$-Sign ${ }^{\mathrm{TM}} 2$ ear lobe sensor agreed well with those of the ECG (Table 1).

Conclusions Transcutaneous capnography using the $V$-Sign ${ }^{\mathrm{TM}} 2$ ear lobe sensor reliably detects the ventilation state of patients after cardiac surgery. This non-invasive approach has the potential to reduce the number of arterial blood gas samples.

\section{P169}

Computerized lung sound monitoring to assess effectiveness of physiotherapy and secretion removal: a feasibility study

G Ntoumenopoulos', Y Glickman²

'Guy's and St Thomas' NHS Foundation Trust, London, UK; ${ }^{2}$ Deep Breeze Ltd, Or-Akiva, Israel

Critical Care 2010, 14(Suppl 1):P169 (doi: 10.1186/cc8401)

Introduction Intubation and mechanical ventilation impair secretion clearance and can lead to lung collapse, consolidation and ventilatorassociated pneumonia [1]. There is, however, no valid diagnosis of secretion retention in the intubated and ventilated patient. Vibration response imaging (VRI) is a commercially developed acoustic lung imaging system that displays breath sound distribution [2]. VRI should be able to identify relationships between specific breath sound distributions and secretion retention. This preliminary study investigated the changes in VRI measurements before and after chest physiotherapy in adult intubated and mechanically ventilated patients.

Methods Intubated and ventilated adult patients who were receiving chest physiotherapy were investigated. Lung sound amplitude at peak inspiration was measured immediately before and after chest physiotherapy using two arrays of sensors attached to the patient's back in a supine position. Chest physiotherapy included combinations of closed airway suctioning, saline lavage, postural drainage, manual techniques and/or lung hyperinflation, dependent upon clinical indications. Means are compared with the Wilcoxon matched-pairs signed-ranks test.

Results A total of 16 patients were included in the study $(12$ males four females, age $65 \pm$ 14). Patients were predominantly ventilated with continuous positive airway pressure and pressure support. Following physiotherapy, total lung sound amplitude at peak inspiration decreased twofold from $37 \pm 58.10^{6}$ to $18 \pm 23.10^{6}$ arbitrary units (AU), with significant reduction in the left lung $(P=0.03)$. Furthermore, the difference in sound amplitude between right and left lungs significantly decreased posttreatment compared with pretreatment $(P=0.03)$.

Conclusions Computerized lung sound monitoring may be useful to assess secretion retention and the effectiveness of secretion removal in mechanically ventilated patients. Further investigation is, however, necessary in order to distinguish between secretion-related effects and changes due to other factors such as airflow rate and pattern.

\section{References}

1. Konrad et al:: Chest 1994, 105:237-241

2. Jean et al: Anesth Analg 2008, 107:1243-1247.
P170

Analysis of sound waves recorded with the VRI pulmonary acoustic monitoring system in critically ill mechanically ventilated patients

S Lev', YA Glickman², M Shapiro', PS Singer'

'RMC, Petach Tiqva, Israel; '2Deep Breeze, Or Akiva, Israel

Critical Care 2010, 14(Suppl 1):P170 (doi: 10.1186/cc8402)

Introduction Posterior auscultation of mechanically ventilated patient is challenging. Environmental ICU noises make the detection of subtle auscultatory features difficult. Digitalized pulmonary acoustic monitoring has recently been introduced in the ICU. We explored the possibility to listen to sound waves recorded by the VRI system.

Methods Lung sounds were recorded for 20 seconds using two arrays of 17 piezoelectric contact sensors attached to the patient's back in a supine position. Sound files were archived in a WAV format for offline analysis. Three physicians and one trained respiratory therapist, blinded to the patient's clinical status, provided their findings while listening to sound waves recorded by 12 sensors distributed from apex to base, six from each lung. Breath sounds were categorized as normal versus abnormal and adventitious lung sounds were characterized as wheezes, rhonchi or crackles. Findings were compared with anterior stethoscope auscultation performed at the time of the recording by one of the physicians.

Results Eighteen critically ill mechanically ventilated patients (age $65 \pm$ 17) were enrolled in this study. Chest radiography (CXR) findings included consolidation in 13 patients. There was an agreement among at least three clinicians in the normal/abnormal classification. Furthermore, in 10 out of these 15 patients (67\%), this assessment based on sound wave analysis was in agreement with anterior stethoscope auscultation. Finally, the physician who performed both sound wave analysis and stethoscope auscultation reported crackles/rhonchi in five out of 13 patients with consolidation (38\%) during sound wave analysis but only in one patient during auscultation (8\%).

Conclusions The level of interobserver variability for sound wave characterization was comparable with levels usually reported during stethoscope auscultation. However, in patients with consolidation, a higher number of crackles/rhonchi was reported during posterior sound wave analysis than during anterior stethoscope auscultation. This discrepancy may be either due to the anterior/posterior orientation or to the higher sensitivity of the offline sound wave analysis.

P171

Lung tissue properties obtained by optical alveolar elastometry

D Schwenninger', H Runck', S Schumann', K Möller²

'University Medical Center of Freiburg, Germany; ${ }^{2}$ Furtwangen University, VS-Schwenningen, Germany

Critical Care 2010, 14(Suppl 1):P171 (doi: 10.1186/cc8403)

Introduction Knowledge of micromechanical properties of lung parenchyma is essential for understanding macroscopic lung mechanics. The first results of in vivo and in situ measurements using an endomicroscopic device [1] are reported. The degree of local lung deformation in dependence of locally applied pressure is shown.

Methods An endoscopic system including two concentric trocars was constructed to apply a defined local pressure within the field of view $\left(P_{\text {fov }}\right)$.
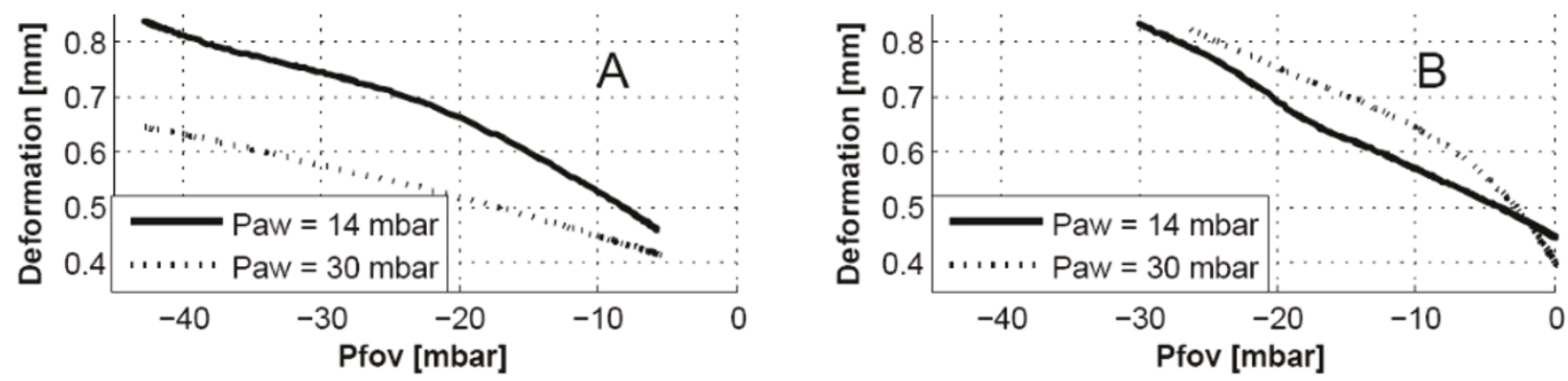

Figure 1 (abstract P171) 
The endoscopic system was placed between the ribs of mechanically ventilated rats with the tip placed on the pleura. $P_{\text {fov }}$ was applied to a circular area on to the lungs' surface and led to a local deformation. By adjusting the flow rate of the flushing fluid from the outer trocar to the inner trocar, $P_{\text {fov }}$ was varied. The resulting local deformation of lung parenchyma was optically measured by estimating the diameter of ceramic particles, fixed on the pleura for that purpose. Deformation was measured at different levels of airway pressure $\left(\mathrm{P}_{\text {aw }}\right)$ with $\mathrm{P}_{\text {fov }}$ ramped from 0 to -40 mbar.

Results The healthy lung (Figure 1A) parenchyma showed less deformation caused by fluidic pressure compared with the lavaged lung (Figure 1B). In contrast to the lavaged lung, the deformation of the healthy lung was less when higher $P$, was present.

Conclusions Micromechanical properties of lung parenchyma can be analyzed in vivo at an alveolar level. The healthy lung parenchyma appears to be stiffer (less deformation) at higher $\mathrm{P}_{\text {aw }}$. The stronger deformation and less dependence on airway pressure in the lavaged lung support the hypothesis that small lung compliance in lavaged lungs might not be reasoned by stiff lung parenchyma, but rather by regional collapse.

\section{References}

1. Schwenninger $\mathrm{D}$, et al.: IEEE Trans Biomed Eng in press.

P172

Combined monitoring of functional residual capacity and compliance may avoid hyperinflation and cardiac depression in ARDS

F Turani, L Cococcia, R Barchetta, F Mounayerfi, E Di Marzio, M Falco, A Marinelli

European Aurelia Hospital, Rome, Italy

Critical Care 2010, 14(Suppl 1):P172 (doi: 10.1186/cc8404)

Introduction Combined monitoring of functional residual capacity (FRC) and compliance of the respiratory system $\left(C_{r}\right)$ at the bedside may allow alveolar recruitment and detect hyperinflation [1]. The aims of this study were to evaluate the FRC by a modified nitrogen multiple washout technique (NMBW) combined with the study of $C_{\mathrm{r}^{\prime}}$ to set best PEEP on data of FRC and Crs, and to evaluate by echocardiography study the cardiac response to different levels of PEEP.

Methods Thirty patients with ALI/ARDS were ventilated with an Engstrom carestation ventilator (GE Healthcare, Helsinki, Finland) in accordance with the ARDS net guidelines. FRC measurement was carried out with the COVX module integrated within the ventilator (GE Healthcare) by the NMBW technique. Every patient had a basal FRC measurement and then three measurements at PEEP 15/10/5 $\mathrm{cm} \mathrm{H}_{2} \mathrm{O}$ during a de-recruiting maneuver. At every step we studied the changes of $\mathrm{FRC}, \mathrm{C}_{\mathrm{r}^{\prime}} \mathrm{PaO}_{2} / \mathrm{FIO}_{2}$ ratio and performed a transthoracic echocardiography (Agilent 5500; Hewlett and Packard) to evaluate the integral of velocity time of left ventricular outflow tract (LVOT VTI). All data are reported as mean \pm SD. ANOVA was used to compare changes during the time.
Results Table 1 presents the main results of the study. Best PEEP was set at $10 \mathrm{cmH}_{2} \mathrm{O}$, at which level the decrease of FRC and improvement of $C_{r s}$ indicates the start of de-recruitment and end of overdistention.

Table 1 (abstract P172)

\begin{tabular}{lccc}
\hline & PEEP 15 & PEEP 10 & PEEP 5 \\
\hline FRC ml & $2,975 \pm 644$ & $2,412 \pm 585^{*}$ & $2,059 \pm 556^{* *}$ \\
$\mathrm{C}_{\mathrm{rs}} \mathrm{ml} / \mathrm{cm}$ & $33 \pm 8$ & $39 \pm 9^{*}$ & $45 \pm 10^{* *}$ \\
$\mathrm{PaO}_{2} / \mathrm{FlO} \mathrm{F}_{2}$ & $310 \pm 62$ & $291 \pm 78$ & $185 \pm 89^{\dagger}$ \\
LVOTVTl cm & $16.3 \pm 2$ & $20 \pm 3^{*}$ & $22 \pm 6^{* *}$ \\
\hline
\end{tabular}

${ }^{*} P<0.05 .{ }^{* *} P<0.01$ vs PEEP $15 .{ }^{\dagger} P<0.001$ vs PEEP 15 and 10.

Conclusions Increased levels of PEEP improve FRC. Combined monitoring of FRC and $C_{r s}$ may detect hyperinflation. Hyperinflation impairs LV systolic function.

References

1. Lambermont B, et al:: Crit Care 2008, 12:R91.

P173

Electrical impedance tomography at two thoracic levels provides detailed information about ventilation distribution in the cranial to caudal direction

I Bikker, C Preis, J Bakker, D Gommers

Erasmus Medical Center, Rotterdam, the Netherlands

Critical Care 2010, 14(Suppl 1):P173 (doi: 10.1186/cc8405)

Introduction Electrical impedance tomography (EIT) is a promising new tool for bedside monitoring of regional lung ventilation. Several studies have focused on the ventilation distribution and relationship with regional lung volume on a lower, caudal lung level. However, no information is available at a higher, cranial lung level.

Methods EIT (EIT Evaluation Kit 2; Dräger, Lübeck, Germany) was measured at cranial and caudal lung levels in 10 patients after cardiothoracic surgery. Patients were fully sedated and mechanically ventilated and a PEEP trial was performed at four PEEP levels $\left(15,10,5\right.$ and $\left.0 \mathrm{CmH}_{2} \mathrm{O}\right)$.

Results The center of gravity index decreased after lowering the PEEP level at both the caudal and cranial lung levels (Figure 1 right). Whereas the tidal volume impedance variation divided by tidal volume increased at the cranial lung level and decreased at the caudal lung level during the step-wise reductions in PEEP (Figure 1 left).

Conclusions During decremental PEEP steps, the ventilation distribution not only shifts from a dorsal to ventral direction, but also from the caudal to cranial direction.
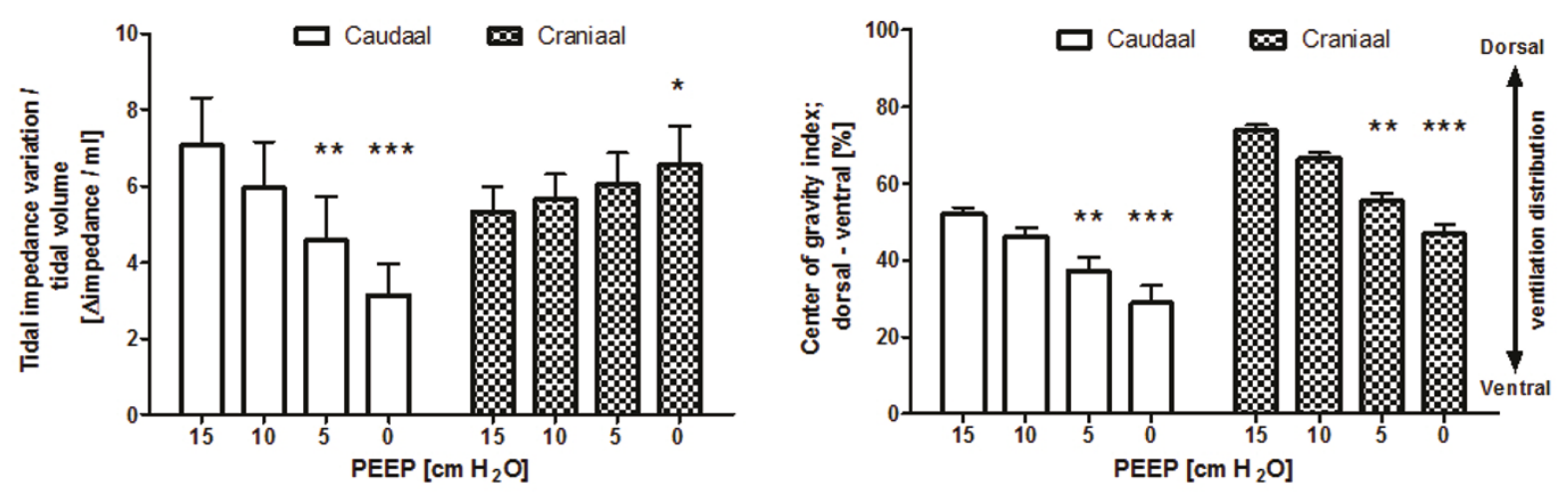

Figure 1 (abstract P173). 
P174

Changes in regional ventilation determined by volume and pressurecontrolled ventilation as assessed by electrical impedance tomography in an experimental model of acid-induced acute lung injury CA Holms, DA Otsuki, J Noel-Morgan, M Kahvegian, DT Fantoni, JO Auler Jr Faculdade de Medicina da Universidade de São Paulo, Brazil Critical Care 2010, 14(Suppl 1):P174 (doi: 10.1186/cc8406)

Introduction Electrical impedance tomography (EIT) is a promising bedside tool for monitoring regional lung ventilation during mechanical ventilation [1]. The purpose of this study was to assess regional lung ventilation by EIT in a pig model of acid-induced acute lung injury (ALI) during pressurecontrolled (PCV) and volume-controlled ventilation (VCV).

Methods Ten anesthetized and tracheotomized female pigs (25 to 30 $\mathrm{kg}$ ) were randomized into two groups: mechanical ventilation by PCV (pressure adjusted to achieve and maintain $8 \mathrm{ml} / \mathrm{kg}$ tidal volume, $n=$ 5) or VCV $(8 \mathrm{ml} / \mathrm{kg}, n=5)$ in $\mathrm{FiO}_{2} 50 \%$. ALI was induced in both groups by intratracheal instillation of $4 \mathrm{ml} / \mathrm{kg}$ hydrochloric acid $(\mathrm{HCl}) 0.1 \mathrm{~N}$. Impedance changes (Draeger, Germany) were recorded by a 16-electrode belt placed at the level of the sixth intercostal space, and four horizontal, equally-sized regions of interest (ROIs) were defined for offline data analysis. Measurements were performed before (T-BL) and after lung injury (T-ALI). Lung tidal volume $(\mathrm{Vt})$, pulmonary static compliance $\left(\mathrm{C}_{\text {stal }}\right)$ pulmonary mean artery pressure (mPAP), peak pressure $(\mathrm{P})$, respiratory rate (RR) and $\mathrm{PaO}_{2} / \mathrm{FiO}_{2}$ ratio were also recorded. Statistical analysis was based on two-way ANOVA followed by the Tukey test for analysis of data within and between groups, and a $t$ test was used analysis of impedance changes induced by ALI.

Results After ALI, both PCV and VCV groups showed significant decreases in the $\mathrm{PaO} / \mathrm{FiO}$ ratio $(479.6 \pm 46.5$ to $244.5 \pm 64.7$ and $428.8 \pm 22.9$ to $212.4 \pm 26.7)$ and $C_{\text {stat }}(29.2 \pm 5.1$ to $13.2 \pm 2.3$ and $26.6 \pm 3.4$ to $12.3 \pm 3.2)$ and significant increases in $P_{p}(14.2 \pm 1.9$ to $25.6 \pm 2.6$ and $13.8 \pm 0.8$ to $24.6 \pm 4.16)$ and mPAP (19.6 \pm 0.5 to $35.2 \pm 5.8$ and $19.4 \pm 1.5$ to $26.8 \pm$ 4.5). Between groups, only mPAP was statistically different at T-ALI. Global impedance decreased slightly after ALI in both groups, with no statistical significance. At T-ALI, there were statistically significant differences in tidal distributions of ROIs $2(38.9+2.6 \%$ to $42.4+3.9 \%)$ and $3(39.7+3.1$ to $35.1 \pm 4.3 \%$ ) of animals in the VCV group, when compared with T-BL. No significant difference was found in the PCV group.

Conclusions EIT assessment of ventilation revealed a ventral shift in the tidal distribution in the VCV group after ALI, while PCV preserved tidal distribution observed at baseline.

Acknowledgements Grants from FAPESP (08/55376-7 and 08/56792-4) and LIM08/FMUSP.

Reference

1. Frerichs I, et al:: Physio/ Meas 2007, 28:S261-S267.

P175

Changes in lung tidal distribution assessed by electrical impedance tomography in a model of hemorrhagic shock with endotoxemia and resuscitation

J Noel-Morgan, DT Fantoni, DA Otsuki, CA Holms, JO Auler Jr

Faculdade de Medicina da Universidade de São Paulo, Brazil

Critical Care 2010, 14(Suppl 1):P175 (doi: 10.1186/cc8407)

Introduction Electrical impedance tomography (EIT) is a non-invasive bedside imaging tool with the potential to assess regional lung ventilation reliably [1]. The purpose of this study was to monitor changes in lung impedance in a pig model of severe shock followed by fluid resuscitation. Methods Twelve anesthetized, mechanically ventilated $(8 \mathrm{ml} / \mathrm{kg}$, PEEP $5 \mathrm{cmH}_{2} \mathrm{O}$ ), supine pigs were submitted to acute hemorrhagic shock with infusion of endotoxin. Animals were allocated to positive control (PC, $n=$ 6) or treatment group with lactated Ringer's to achieve and maintain pulse pressure variation 13\% and mean arterial pressure $65 \mathrm{mmHg}(\mathrm{PPV}, n=6)$. Ventilatory and hemodynamic parameters were recorded at baseline, 1 hour after hemorrhagic shock (Tshock), and hourly for 3 hours (T1 to T3) A 16-electrode belt was placed at the level of the sixth intercostal space for EIT measurements (Dräger, Germany). Offline analysis was based on four horizontal regions of interest (ROIs) over the ventrodorsal lung area. Statistical analysis was based on two-way ANOVA followed by the Tukey test $(P<0.05)$.
Results At Tshock there was hemodynamic compromise, statistical decrease in lung compliance $\left(C_{\text {stat }}\right)$ and significant increases in pulmonary vascular resistance index, mean pulmonary artery pressure and peak pressure $\left(P_{\text {peak }}\right)$, with no statistical difference between groups. Endpoints and hemodynamic stability were achieved in the PPV group in $117 \pm$ 28 minutes. $C_{\text {stat }}$ continued to deteriorate and $P_{\text {peak }}$ continued to rise in both groups from T1 to T3. When compared with PC, the PPV group had significant impedance increases in ROls 1 and 2 at T2 and T3 and, at T3 the increase in ROI 1 was also statistically greater than Tshock within the group. Statistical decrease in the percentage tidal distribution in ROI 3 and increase in $\mathrm{ROI} 2$ of the PPV group, in relation to the PC group, were also noted.

Conclusions Despite re-establishment of hemodynamic adequacy in PPV group and although ventilatory parameters were similar in both groups over time, resuscitation as performed in the study induced significant changes in tidal impedance toward nondependent lung regions, implying greater lung impairment in treated animals.

Acknowledgements Grants from FAPESP 08/50063-0, 08/50062-4 and LIM08/FMUSP.

Reference

1. Victorino et al.: Am J Respir Crit Care Med 2004, 169:791-800.

\section{P176}

Estimating the intratidal compliance profile with the new gliding-SLICE method

S Schumann'1, B Burcza', C Haberthuer², M Lichtwarck-Aschoff3, J Guttmann

'University Medical Center, Freiburg, Germany; ${ }^{2}$ Kantonsspital, Luzern,

Switzerland; ${ }^{3}$ University of Uppsala, Sweden

Critical Care 2010, 14(Suppl 1):P176 (doi: 10.1186/cc8408)

Introduction At the bedside, but even in most research work, the analysis of respiratory system mechanics is limited to quasi-static conditions excluding any insight into what happens during the breath. The new gliding-SLICE method helps looking into the breath. It is a further development of the SLICE method for calculating compliance and resistance of subsequent intratidal volume ranges (slices) of the pressurevolume (PV) loop by multiple linear regression analysis in a continuous way. This allows for detecting intratidal compliance and resistance nonlinearity during ongoing ventilation. Our objective was to determine whether the nonlinear intratidal compliance profile hints at what level to set PEEP and tidal volume (VT) to make lung ventilation protective.

Methods $\ln 12$ piglets, atelectasis was induced by application of negative pressure. The PV relationship and the ECG signal were recorded during mechanical ventilation at different levels of end-expiratory pressure (PEEP: $0,5,10$ and $15 \mathrm{cmH}_{2} \mathrm{O}$ ) and a VT of $12 \mathrm{ml} / \mathrm{kg} \mathrm{BW}$. Using the gliding-SLICE method [1], intratidal compliance profiles were calculated and compared with the conventional quasi-static compliance.

Results In contrast to quasi-static compliance, the gliding-SLICE method revealed pronounced intratidal nonlinearity of the compliance profile under ongoing ventilation (Figure 1). At low levels of PEEP, intratidal compliance increased in the low volume range, remained at a high level while further volume was delivered, and finally decreased with volume $>6 \mathrm{ml} / \mathrm{kg}$ BW. With higher levels of PEEP, intratidal compliance decreased from the onset of inspiration.

Conclusions The gliding-SLICE method gives detailed insights into the intratidal course of compliance during uninterrupted ventilation. From
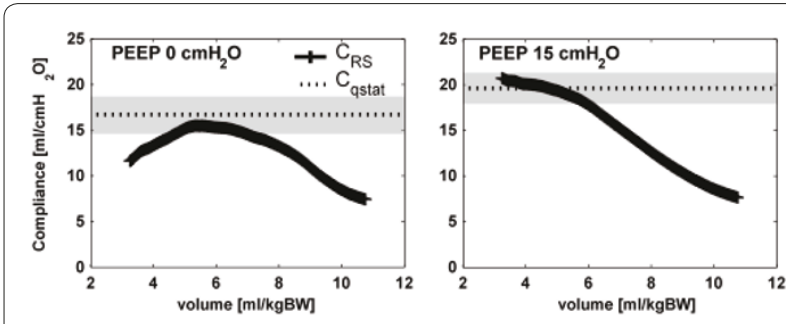

Figure 1 (abstract P176). 
the profile of the intratidal compliance, the occurrence of intratidal recruitment and/or overdistension can be identified.

\section{Reference}

1. Schumann S, Burcza B, Haberthür C, Lichtwarck-Aschoff M, Guttmann J: Physiol Meas 2009, 30:1341-1356.

P177

Atelectasis and overdistension are detected by heartbeat-induced disturbances in the pressure-volume loop

S Schumann', L Vimlati'2, K Moeller', A Wahl', R Kawati², J Guttmann', M Lichtwarck-Aschoff

'University Medical Center, Freiburg, Germany; ${ }^{2}$ University of Uppsala,

Sweden; ${ }^{3}$ HS Furtwangen University, Furtwangen, Germany

Critical Care 2010, 14(Suppl 1):P177 (doi: 10.1186/cc8409)

Introduction Heartbeats transfer mechanical energy to the lungs causing flow and pressure disturbances that appear as cardiogenic oscillations (COS) at the airway opening. Here we adopt a new approach for analyzing respiratory system mechanics. We consider the beating heart as a natural intrathoracic mechanical oscillator transferring mechanical energy to the lungs that travels across the lung parenchyma. COS therefore convey information on the mechanical conditions of the lung parenchyma that they cross.

Methods $\ln 25$ piglets with either healthy or atelectatic lungs, the pressurevolume relationship and the ECG signal were recorded during mechanical ventilation at different levels of end-expiratory pressure (PEEP: 0, 5, 10 and $15 \mathrm{cmH}_{2} \mathrm{O}$ ). The heartbeat-related disturbance of the PV loop was quantified as the maximal compliance following an R-wave in the ECG signal, as determined by the gliding-SLICE method. Atelectasis was assessed by CT. Results The intratidal pattern of heartbeat-induced $C_{\cos }$ changed with PEEP and atelectasis in a characteristic way. With PEEP and tidal volume levels assumed to be lung protective $C_{\cos }$ was high with little intratidal changes, compared with atelectasis and overdistension that were signaled by low $C$. that either increased (atelectasis) or decreased (overdistension) intratidally. The systolic pressure variations did not parallel the $C_{\text {cos }}$ pattern, hinting at a negligible impact of hemodynamics on the inspiratory $C_{\text {cos }}$ pattern.

Conclusions Heartbeats induce fluctuations in the PV loop and, as a consequence, peaks in compliance, which show characteristic patterns depending on the presence of atelectasis or overdistension. The gliding-SLICE method has the potential to detect those intratidal nonlinearities without requiring additional technical equipment making use of the ECG signal and the pressure and flow signals already required for controlling the ventilator.

\section{P178}

Transpulmonary pressure and ventilation distribution measured with EIT during a PEEP trial in porcine acute lung injury

I Bikker, B Vd Berg, P Specht, J Bakker, D Gommers

Erasmus Medical Center, Rotterdam, the Netherlands

Critical Care 2010, 14(Suppl 1):P178 (doi: 10.1186/cc8410)

Introduction Setting the optimal level of positive end-expiratory pressure (PEEP) in the ICU is still a matter of debate. Talmor and colleagues used the transpulmonary pressure calculated from the oesophageal balloon to set PEEP in a recent randomized controlled study. This strategy aims at preventing alveolar collapse by counterbalancing the gravitational force of the lung by an equal or higher PEEP. We evaluated the relation between ventilation distribution measured by EIT and transpulmonary pressure during a PEEP trial in porcine ALI.

Methods Eight pigs $(30 \mathrm{~kg}$ ) were studied during a PEEP trial before and after the induction of acute lung injury (ALI) with oleic acid. Global lung parameters, regional compliance, and oesophageal pressure were recorded at the end of each PEEP step. Regional compliance was calculated by dividing the tidal impedance variation (EIT Evaluation Kit 2; Dräger, Lübeck, Germany) by the applied driving pressure.

Results Transpulmonary pressures were negative at $0 \mathrm{cmH}_{2} \mathrm{O}$ PEEP and became positive during the stepwise increase of PEEP at $5 \mathrm{~cm}_{2} \mathrm{O}$ before, and $10 \mathrm{CmH}_{2} \mathrm{O}$ PEEP after the induction of ALI (Figure 1). Optimum regional compliance was different between the ventral (nondependent) and dorsal (dependent) regions of interest (ROI). In the healthy lung, optimum PEEP was 10 in the dorsal ROI and 5 in the ventral ROI, whereas after ALI this was 15 in the dorsal ROI and 5 in the ventral ROI.

Conclusions If EIT is measured at a caudal lung level, optimal EIT PEEP in the dependent lung exceeds the PEEP required for positive transpulmonary pressures as used in the Talmor study, whereas in the nondependent lung optimal EIT PEEP is equal before ALI and lower after ALI. We speculate that this is probably influenced by the location of the EIT slice in the cranial to caudal direction.

\section{P179}

A prospective pilot study of the effect of neutrophil elastase on the pulmonary vascular permeability in patients with pneumonia TTagami', RTosa', H Hirama', K Yonezawa', M Omura', G Akiyama', T Masuno ${ }^{2}$, Y Yamamoto², S Kushimoto ${ }^{2}$, H Yokota $^{2}$

'Aizu Chuo Hospital, Aizuwakamatsu, Fukushima, Japan; 2Nippon Medical

School, Tokyo, Japan

Critical Care 2010, 14(Suppl 1):P179 (doi: 10.1186/cc8411)

Introduction Some cases of pneumonia may lead to hypoxemia with acute respiratory failure, acute lung injury (ALI) or acute respiratory distress syndrome (ARDS), which may require intensive management. Neutrophil elastase is thought to be one of the causes of ALI/ARDS, because it raises the pulmonary capillary permeability, lyses of pulmonary connective tissue proteins, and produces leukocyte chemotactic factors. However, a causal relationship between the plasma neutrophil elastase level and changes in the pulmonary capillary permeability has not been established in patients with pneumonia, one of the most serious diseases underlying ALI/ARDS. Therefore, the objective of this study was to determine whether an increase in plasma neutrophil elastase is related to elevation of the pulmonary capillary permeability in patients with pneumonia.

Methods Patients with pneumonia who were hospitalized from November 2008 to April 2009 and had $\mathrm{PaO}_{2} \leq 60$ in room air with no need for mechanical ventilation were prospectively enrolled in the study. Plasma neutrophil elastase levels were collected via blood samples at baseline; 1, 3 , and 7 days after the start of the study. Of those enrolled, patients with
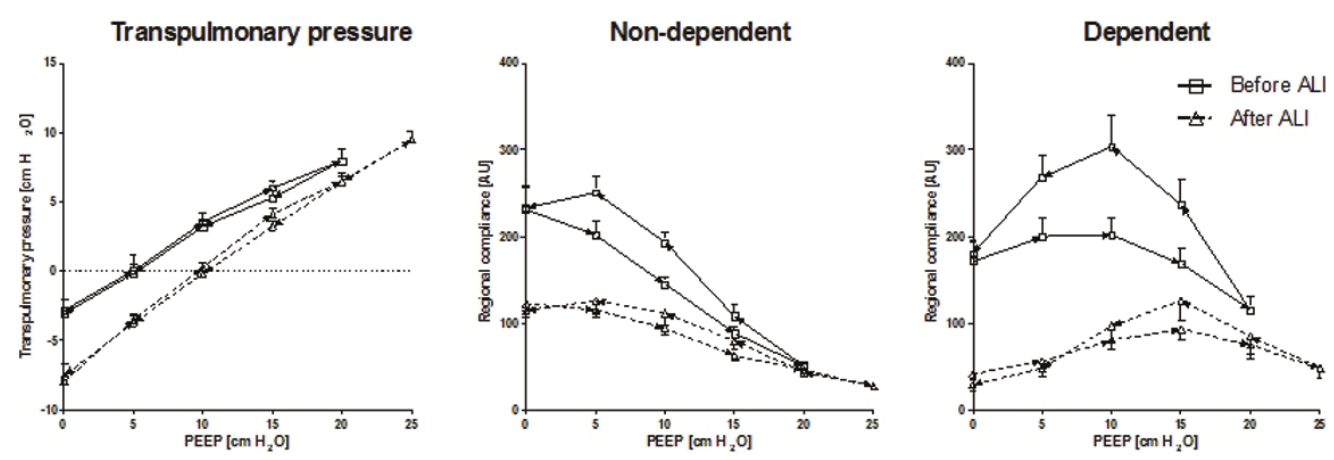

Figure 1 (abstract P178) 
$\mathrm{PaO}_{2} / \mathrm{FiO}_{2} \leq 150$ also had their extravascular lung water index (EVLWi) and pulmonary vascular permeability index (PVPI) monitored using the PiCCO system (Pulsion, Munich, Germany) when required by their attending physician. Statistical analysis was performed using the Spearman correlation coefficient $(R)$ with $P \leq 0.05$ assumed to be significant.

Results Fourteen patients were enrolled in the study. In six of these patients, the EVLWi and PVPI were measured simultaneously. At baseline, the elastase level and the PVPI showed a strong and significant correlation $\left(R_{\mathrm{s}}=1.000, n=6, P<0.05\right)$. All of the plot data of the six patients showed strong correlations of the elastase level with the EVLWi $\left(R_{s}=0.750, n=25\right.$, $P<0.01)$ and the PVPI $\left(R_{\mathrm{s}}=0.811, n=25, P<0.01\right)$.

Conclusions The plasma neutrophil elastase level and the PVPI measured by $\mathrm{PiCCO}$ were strongly correlated in patients with pneumonia. This suggests that a rise in the blood level of elastase may elevate the PVPI, resulting in an increased EVLWi. (UMIN Clinical Trials Registry: ID UMIN000002803.)

\section{P180}

Evaluating the fibroproliferative response to ventilator-induced lung injury

G Curley', M Contreras', B Higgins', D O'Toole', C O'Kane², JG Laffey'

'National University of Ireland, Galway, Ireland; '2Queens University Belfast, UK

Critical Care 2010, 14(Suppl 1):P180 (doi: 10.1186/cc8412)

Introduction Acute lung injury (ALI), and its more severe subset acute respiratory distress syndrome (ARDS), are a major cause of mortality in the ICU [1]. Mechanical ventilation, a supportive therapy necessary to sustain life in many cases, may contribute to and worsen ALI, termed ventilatorinduced lung injury (VILI). Fibroproliferation is an early response to lung injury [2]. Indeed, dysregulated repair resulting in pulmonary fibrosis may be at the heart of ventilator dependence in ARDS. Characterising the role of excessive lung stretch in contributing to aberrant repair mechanisms would assist in developing strategies to hasten recovery from ARDS.

Methods Male Sprague-Dawley rats were anaesthetized, orotracheally intubated and subjected to injurious ventilation until a defined worsening of compliance was noted. The rats were then recovered and extubated. The level of ongoing injury/repair was characterised at time periods of 6, 24 and 48 hours and at 4, 7 and 14 days. Systemic oxygenation, lung compliance, wet/dry ratio, BAL total protein, cytokines and cell count and histological analysis was carried out at each time point.

Results The results demonstrated a time-course-dependent improvement in compliance and oxygenation, together with clearance of neutrophilic infiltration at 96 hours. TNFa, and IL-1 $\beta, I L-6$ and IL-10 were significantly elevated in BAL fluid early post injury. Although total lung collagen remained similar at all time points, evidence of an early fibroproliferative response was present in the form of transforming growth factor- $\beta$ activation and pro-collagen I and III peptide mRNA levels. Matrix metalloproteinase 3 and 9 zymography demonstrated increased levels of these matrikines. Histologic assessment of injury revealed increased alveolar tissue fraction up to and including 96 hours post injury. Myofibroblasts were present in a-smooth muscle actin stained sections in significantly increased numbers post injury.

Conclusions This rat model of repair of VILI demonstrates some of the mechanisms by which excessive lung stretch can contribute to fibroproliferation in ARDS and will serve to improve our knowledge of aberrant lung tissue remodelling as well as provide a useful paradigm for testing strategies to hasten recovery in ALI.

\section{References}

1. Rubenfeld GD, Herridge MS: Chest 2007, 131:554-562.

2. Marshall RP, et al:: Am J Respir Crit Care Med 2000; 162:1783-1788.

\section{P181}

Effects of severe hemorrhage on pulmonary mechanics in ventilated pigs with ARDS

N Siegenthaler', R Giraud', D Morel', CWiklund², K Bendjelid' ${ }^{1}$ Hôpital Cantonal Universitaire, Genève, Switzerland; ${ }^{2}$ Karolinska University Hospital, Stockholm, Sweden

Critical Care 2010, 14(Suppl 1):P181 (doi: 10.1186/cc8413)

Introduction The effects of hemorrhagic shock on respiratory system mechanics have rarely been investigated and published data are

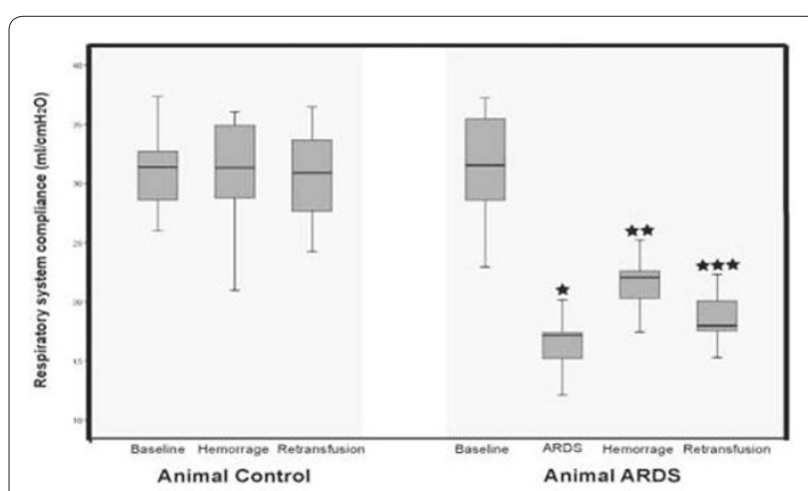

Figure 1 (abstract P181). Respiratory system compliance. Tidal volume, $10 \mathrm{ml} / \mathrm{kg}$.

controversial. Pulmonary compliance depends in part on intrapulmonary blood and interstitial fluid volume. When compliance is severely reduced, small modification of these components may have important effects. The present analysis explored the effect of hemorrhagic shock on respiratory system mechanics and oxygenation parameters in a model of pigs with ARDS.

Methods We evaluated the dynamic respiratory system compliance $\left(C_{\mathrm{rs}}=\right.$ VT / (inspiratory airways pressure - PEEP)) of 14 domestic pigs. Animals were mechanically ventilated: tidal volume $(\mathrm{VT})$ set at $10 \mathrm{ml} / \mathrm{kg}$; respiratory rate at $15 \mathrm{bpm}$; PEEP at $0 \mathrm{cmH}_{2} \mathrm{O}$. Animals were separated into a control group $(n=9)$ and an ARDS group $(n=5)$. ARDS was induced by lung lavage with $\mathrm{NaCl} 0.9 \%$. During hemorrhage $40 \%$ of the total blood volume was removed. The blood was then infused during the re-transfusion phase.

Results In the control group, $\mathrm{C}_{\mathrm{rs}}\left(\mathrm{ml} / \mathrm{cmH}_{2} \mathrm{O}\right)$ did not change during hemorrhage or re-transfusion (Figure 1). In the ARDS group, $C$ decreased with lung lavage (31.2 \pm 5.7 (baseline) to $16.4 \pm 3.0$; ${ }^{*} P<0.01$ ). After hemorrhage $C$ increased $(21.5 \pm 2.9 ; * *<0.001$ compared with lavage) and then decreased again after re-transfusion $\left(18.7 \pm 2.7\right.$; $\left.{ }^{* * *} P<0.05\right)$. In the same group $\mathrm{PaO}_{2} / \mathrm{FiO}_{2}(\mathrm{mmHg})$ decreased after ARDS (469 \pm 50 (baseline) to $105 \pm 38 ; P<0.001$ ), increased during hemorrhage $(218 \pm$ $105 ; P<0.05)$ and did not change after re-transfusion $(207 \pm 125 ; P=0.82)$. The shunt fraction (\%) decreased during hemorrhage in the ARDS group (26.2 \pm 14.9 (lavage) to $6.4 \pm 6.6 ; P<0.05$ ) but did not change significantly after re-transfusion $(13.9 \pm 17.0 ; P=0.3)$.

Conclusions Acute reduction of blood volume is associated with an increase of respiratory system compliance and oxygenation parameters. Reduction of intrapulmonary blood and interstitial fluid volume or thoracic cage compliance could be responsible for this effect.

P182

Higher versus lower positive end-expiratory pressure in acute lung injury and acute respiratory distress syndrome: systematic review and individual patient data meta-analysis

MB Briel', MO Meade ${ }^{2}$, A Mercat ${ }^{3}$, RG Brower', D Talmor ${ }^{5}$, AS Slutsky ${ }^{6}$, DJ Cook ${ }^{2}$, L Brochard 7 , JC Richard ${ }^{8}$, F Lamontagne ${ }^{9}$, T Stewart ${ }^{6}$, GH Guyatt ${ }^{2}$ 'University Hospital Basel, Switzerland; '2McMaster University, Hamilton, Canada; ${ }^{3}$ University Hospital Angers, France; ${ }^{4}$ Johns Hopkins University, Baltimore, MD, USA; ${ }^{5}$ Harvard Medical School, Boston, MA, USA; ${ }^{6}$ University of Toronto, Canada: 7 Paris 12 University, Creteil, France; ${ }^{8}$ University Hospital Charles Nicolle, Rouen, France; 'University of Sherbrooke, Canada Critical Care 2010, 14(Suppl 1):P182 (doi: 10.1186/cc8414)

Introduction Trials comparing higher versus lower levels of positive end-expiratory pressure (PEEP) in adults with acute lung injury or acute respiratory distress syndrome (ARDS) were underpowered to detect small but important effects on mortality, overall or in any subgroups.

Methods We searched MEDLINE, Embase, and the Cochrane Central Register for trials randomly assigning adults with acute lung injury or ARDS to higher versus lower levels of PEEP (minimal difference of $3 \mathrm{cmH}_{2} \mathrm{O}$ over first 3 days), while using low tidal volume ventilation, and comparing mortality. Data from 2,299 individual patients in three trials 
were analyzed using uniform outcome definitions. We tested prespecified effect modifiers using multivariable hierarchical regression, adjusting for important prognostic factors and clustering effects.

Results Overall, there were 374 hospital deaths (32.9\%) in the higher PEEP group and 409 (35.2\%) in the lower PEEP group (adjusted relative risk, 0.94; $95 \%$ confidence interval $(\mathrm{Cl}), 0.86$ to $1.04 ; P=0.25)$. Treatment effects varied with the presence or absence of ARDS, defined by a ratio of partial pressure of oxygen to fractional inspired oxygen concentration equal to or less than $200 \mathrm{mmHg}$ (interaction $P=0.02$ ). The relative risks of hospital mortality for patients with and without ARDS were $0.90(95 \% \mathrm{Cl}, 0.81$ to $1.00, P=0.049)$ and $1.37(95 \% \mathrm{Cl}, 0.98$ to $1.92, P=0.065)$, respectively. Patients with ARDS were more likely to achieve unassisted breathing earlier (hazard ratio, 1.16 ( $95 \% \mathrm{Cl}, 1.03$ to $1.30, P=0.01$ ); whereas the hazard ratio for time to unassisted breathing was $0.79(95 \% \mathrm{Cl}, 0.62$ to $0.99, P=0.04)$ in patients without ARDS at baseline. Rates of pneumothorax and the use of neuromuscular blockers, vasopressors and corticosteroids were similar.

Conclusions Higher levels of PEEP are likely to improve survival for patients with ARDS, but not for patients with less severe acute lung injury.

\section{P183}

Effect of different recruitment maneuvers on bacterial translocation

P Ergin Ozcan, I Edipoğlu, IO Akıncı, E Senturk, S Baylan, AA Cagatay,

F Esen, L Telci, N Cakar

Istanbul Faculty of Medicine, Istanbul University, Istanbul, Turkey

Critical Care 2010, 14(Suppl 1):P183 (doi: 10.1186/cc8415)

Introduction Experimental and clinical studies have shown beneficial effects of recruitment maneuvers (RMs) (sustained inflation (SI) or SIGH) on ventilatory and gas exchange parameters. In this study we investigated the effect of different RMs on bacterial translocation from lung to blood.

Methods Thirty-two rats were anesthetized, after tracheotomy was performed ventilation was started with $10 \mathrm{cmH}_{2} \mathrm{O} \mathrm{P} \mathrm{aw}^{\prime} \mathrm{O} \mathrm{cmH}_{2} \mathrm{O}$ PEEP, 60 breaths/minute, I/E: 1/2 on pressure-controlled ventilation (PCV) mode. After cannulation of the carotid artery was performed, a baseline blood gas sample was taken. Subsequently $0.5 \mathrm{ml}$ of $10^{5} \mathrm{cfu} / \mathrm{ml}$ Pseudomonas aeruginosa was inoculated through the tracheotomy tube and PEEP was increased to $3 \mathrm{CmH}_{2} \mathrm{O}$ and ventilated for 30 minutes before randomization. Then rats were randomized into four groups: G1; SI was performed as $40 \mathrm{CmH}_{2} \mathrm{OPEEP}$ and $\mathrm{OP}$ for 20 seconds, four times in an hour (15-minute intervals), G2; SI was performed as $20 \mathrm{cmH}_{2} \mathrm{O}$ PEEP and $\mathrm{O} \mathrm{P}_{\text {aw }}$ for 40 seconds, four times in an hour (15-minute intervals), G3; SIGH was performed four times in 1 hour (15-minute intervals) as $40 \mathrm{cmH}_{2} \mathrm{O} \mathrm{P}, 3 \mathrm{mH}_{2} \mathrm{O}$ PEEP for 60 seconds, G4; control group that was ventilated with $\mathrm{P}_{\text {aw }} 10 \mathrm{cmH}_{2} \mathrm{O}$, PEEP $3 \mathrm{cmH}_{2} \mathrm{O}$ during the study period. Multiplication of pressure and pressure performing time for each study group were equal. Blood cultures were taken at baseline, 15 minutes after randomization, which is after each RM for the first hour, and last blood culture was taken after 60 minutes from the fourth RM. Then rats were sacrificed with intra-arterial sodium thiopental, and the lungs were extirpated; the left lung was taken for measurement of the wet weight/dry weight ratio (WW/DW).

Results There were no differences in baseline $\mathrm{pH}, \mathrm{PaO}_{2}, \mathrm{PaCO}_{2}, \mathrm{MAP}, \mathrm{HR}$ among groups. But $\mathrm{PaO}_{2}$ were decreased in groups $\mathrm{G} 1, \mathrm{G} 2$, and $\mathrm{G} 3$, but only in $\mathrm{G} 3$ was statistically significant to compared baseline values. The WW/DW ratio was found higher in G3 when compared with G1, and G2, but this difference was not significant. The amount of positive blood culture was higher in $\mathrm{G} 3$ at early study periods.

Conclusions SIGH as a recruitment maneuver causes a high probability of bacterial translocation from the lung to the bloodstream.

\section{P184}

Nonlinear recruitment model with viscoelastic component fit respiratory mechanics in ARDS

C Schranz', J Guttmann², K Möller

'Department of Biomedical Engineering, Furtwangen University,

Villingen-Schwenningen, Germany; ${ }^{2}$ Section of Experimental Anesthesiology, University Hospital Freiburg, Germany

Critical Care 2010, 14(Suppl 1):P184 (doi: 10.1186/cc8416)

Introduction Alveolar recruitment/de-recruitment (R/D) seems to play an important role in the development of VILI [1]. Many clinicians base their determination of PEEP settings during mechanical ventilation of ARDS/ ALI patients on an estimate of alveolar recruitability [2]. This project aims to establish an online tool that provides estimates of R/D in patients at the bedside.

Methods In volume-controlled ventilated piglets as ARDS models, the airway pressure $\mathrm{P}_{\text {aw }}$ (SI-Special Instruments, Nördlingen, Germany) and the flow rate $\mathrm{Q}(\mathrm{F}+\mathrm{G} \mathrm{GmbH}$, Hechingen Germany) were continuously recorded at $200 \mathrm{~Hz}$. The pressure curve shows high nonlinearity being a suspect of recruitment effects during inspiration and a relaxation process during the end inspiratory pause. Based on the obtained data, the parameters of the linear viscoelastic model [3] are calculated by a LSE fitting process. As the parameter $C$ represents a static constant compliance of the lung. this model is not capable of reproducing the nonlinear effects during inspiration. To improve on this, the constant compliance $C_{\text {, }}$ is replaced by a nonlinear pressure-dependent compliance describing recruitment and dilatation as proposed by Hickling [1].

Results Since the nonlinear model has far more variable parameters to be optimized in the fitting process than the linear model, an approach via fitting the linear model first is helpful. Therefore, the estimated parameters of the linear model fit can be used as starting values for fitting the nonlinear model where the focus can be put on the recruitment phenomena. With the new nonlinear model, using the estimated values of $R_{1}, R_{2}, C_{2}$ from the linear model (Figure 1 left), it is now possible to reproduce the nonlinear characteristics (Figure 1 right).

Conclusions Using this new model it is possible to fit nonlinear behavior due to alveolar recruitment separately from viscoelastic effects with minimized error.
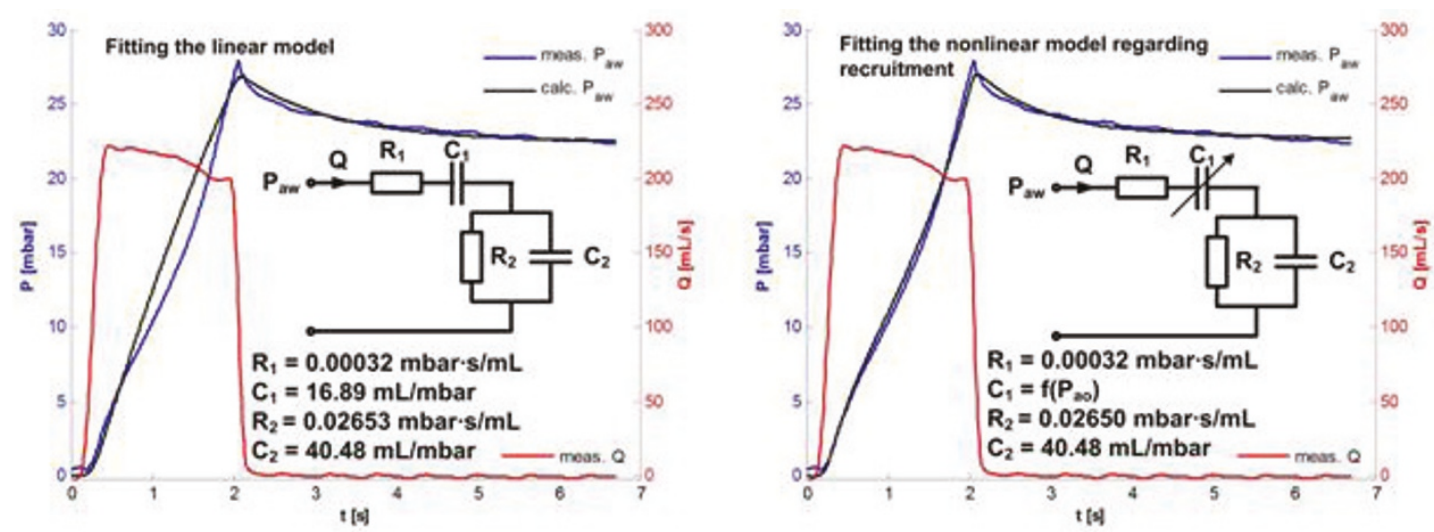

Figure 1( abstract P184). 


\section{References}

1. Hickling KG: Am J Respir Crit Care Med 2001, 163:69-78

2. Gattinoni L, et al:: N Engl J Med 2006, 354:1775-1786.

3. Bates JH, et al.: J Appl Physiol 2002, 93:705-713.

\section{P185}

Lung recruitment and PEEP response in ARDS-related $\mathrm{H} 1 \mathrm{~N} 1$ virus patients

D Chiumello' ${ }^{1}$ C Mietto², V Berto², A Marino², E Gallazzi², D Tubiolo'

'Fondazione IRCCS, 'Ospedale Maggiore Policlinico Mangiagalli Regina Elena' di Milano, Milan, Italy; ${ }^{2}$ Università degli Studi di Milano, Milan, Italy

Critical Care 2010, 14(Suppl 1):P185 (doi: 10.1186/cc8417)

Introduction Starting in April 2009 the swine-origin influenza (H1 N1) virus is considered a worldwide pandemic and is associated with a large number of patients admitted to intensive care. In a proportion of these patients, acute respiratory distress syndrome (ARDS) can develop. We described the case of two patients that presented ARDS related to H1N1 virus confirmed by a nasal swab tested with real-time reverse transcription-polymerase chain reaction. Using a lung $\mathrm{CT}$ scan we measured the potential of lung recruitment and the response to positive end-expiratory pressure (PEEP) in terms of gas exchange and respiratory mechanics.

Methods Patients underwent a lung CT scan at an airway pressure of 5 and $45 \mathrm{CmH}_{2} \mathrm{O}$; lung profiles were outlined and quantitative analysis was performed with custom-made software. The potential of lung recruitment was computed as the amount of nonaerated lung tissue at $5 \mathrm{cmH}_{2} \mathrm{O}$ in which aeration can be restored at an airway pressure of $45 \mathrm{cmH}_{2} \mathrm{O}$. The PEEP test was performed at two PEEP levels ( 5 and $15 \mathrm{cmH}_{2} \mathrm{O}$ ), maintaining constant the minute ventilation and the inspiratory oxygen fraction; gas exchange and respiratory mechanics were recorded 20 minutes after each PEEP change.

Results Two sedated and paralyzed subjects with acute lung injury (ALI)/ ARDS (age $46 \pm 5$ years, BMl $38.6 \pm 5.5 \mathrm{~kg} / \mathrm{m}^{2}, \mathrm{PaO}_{2} / \mathrm{FiO}_{2} 187 \pm 30 \mathrm{mmHg}$, PEEP $17 \pm 1 \mathrm{cmH}_{2} \mathrm{O}$; both patients were obese and one of them suffered from diabetes) were enrolled. One patient required extracorporeal membrane oxygenation support for 5 days. Lung CT scans were performed $5 \pm 3$ days after $\mathrm{ICU}$ admission. The total lung weight at $5 \mathrm{cmH}_{2} \mathrm{O}$ was $1,598 \pm 142 \mathrm{~g}$. The percentage of potentially recruitable lung was $24 \pm$ $8 \%$ of the total lung weight, corresponding to an absolute weight of 371 $\pm 109 \mathrm{~g}$ recruitable lung tissue. $\mathrm{A} 15 \mathrm{cmH}_{2} \mathrm{O}$ compared with $5 \mathrm{cmH}_{2} \mathrm{O}$ of PEEP improved the compliance of the respiratory system from $42.2 \pm 7.0$ to $46.6 \pm 2.1 \mathrm{ml} / \mathrm{cmH}_{2} \mathrm{O}$ and the gas exchange from $\mathrm{PaO}_{2} 62.7 \pm 0.1$ to $123.2 \pm$ $61.8 \mathrm{mmHg}$ and $\mathrm{PaCO}_{2} 47.1 \pm 0.9$ to $45.9 \pm 3.2 \mathrm{mmHg}$, respectively.

Conclusions The ARDS-related H1N1 virus presented, in the early phase, a high potentially recruitable lung in which elevated PEEP levels can be beneficial.

\section{P186}

Maximal recruitment strategy minimizes tidal recruitment in severe ARDS: a CT scan study

G Matos'1, J Borges², V Okamotoㅇ, C Carvalho², M Amato², C Barbas'

${ }^{1}$ Hospital Albert Einstein, São Paulo, Brazil;' ${ }^{2}$ Universidade de São Paulo, Brazil

Critical Care 2010, 14(Suppl 1):P186 (doi: 10.1186/cc8418)

Introduction The maximal recruitment strategy (MRS) is considered an efficient maneuver to reverse nonaerated lung in ARDS patients. There is no information regarding tidal recruitment during MRS.

Methods Early and severe ARDS patients were submitted to MRS guided by thoracic CT: A - recruitment phase (PEEP 10 to $45 \mathrm{cmH}_{2} \mathrm{O}$ and constant driving pressure $15 \mathrm{cmH} 2 \mathrm{O}$ ); $\mathrm{B}$ - PEEP titration phase (PEEP 25 to $10 \mathrm{cmH}_{2} \mathrm{O}$ ). Images were obtained at end expiration and inspiration. Global and regional quantitative $C T$ analyses from each step of PEEP were compared [1].

Results Analyses of 12 patients showed that MRS reduced significantly the global amount of nonaerated tissue $(54 \pm 8 \%$ to $7 \pm 6 \%, P<0.01)$, tidal recruitment ( $4 \pm 4 \%$ to $1 \pm 1 \%, P=0.029)$ (Figure 1$)$. Most dependent regional tidal recruitment significantly increased from PEEP 10 to $20 \mathrm{cmH}_{2} \mathrm{O}$ $(2 \pm 3 \%$ to $11 \pm 7 \%, P<0.01)$, but significantly decreased after MRS $\left(11^{2} \pm\right.$ $7 \%$ to $2 \pm 2 \%, P<0.01)$. High PEEP $\left(25 \mathrm{cmH}_{2} \mathrm{O}\right)$ was necessary to sustain recruitment.

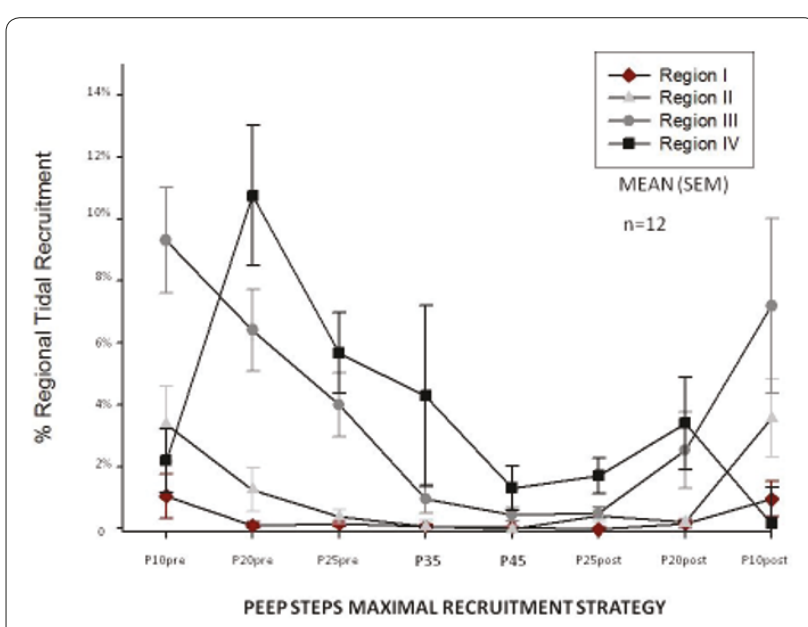

Figure 1 (abstract P186). Percentage of regional (I to IV) tidal recruitment during all steps of the MRS protocol.

Conclusions MRS decreased nonaerated areas and tidal recruitment. Increasing PEEP without full recruitment may cause lung injury exacerbation in the severe ARDS population.

References

1. Borges JB, et al:: Reversibility of lung collapse and hypoxemia in early acute respiratory distress syndrome. Am J Respir Crit Care Med 2006, 174:268-278.

P187

Mechanical ventilation with different alveolar pressures improves alveolar recruitment: a model study

ZZhao', J Guttmann², K Möller'

${ }^{7}$ Furtwangen University, Villingen-Schwenningen, Germany; ${ }^{2}$ University

Medical Center Freiburg, Germany

Critical Care 2010, 14(Suppl 1):P187 (doi: 10.1186/cc8419)

Introduction In patients with acute respiratory distress syndrome (ARDS) tidal ventilation is always inhomogeneous, with atelectasis in dependent lung and overdistention in nondependent lung. The aim of our study was to develop a flow pattern that simultaneously produces locally different alveolar pressures, in order to recruit collapsed alveoli and at the same time to prevent hyperinflation of already open alveoli.

Methods We modified Horsfield's model of the canine lung [1] to predict alveolar pressure differences between the collapsed and opened alveoli. The morphometric model has an asymmetrical branching airway system with 47 orders, whereby order 47 corresponds to the trachea. Each branch is terminated with a viscoelastic tissue unit and there are in total 150,077 acini for the whole model. Using this model, atelectasis of alveoli was simulated by reducing the diameter of small airways (order 1,2 and 3 ) up to $90 \%$ of their original sizes. The tissue damping factor $G$ was $10^{4}$ times higher in the collapsed alveoli than that in normal alveoli. The shunt gas compression compliance $C_{g}$ for the alveolus was $10^{6}$ times smaller. Different percentages of atelectatic area from 0 to $90 \%$ were simulated. Flow distribution and airway input impedance were calculated at different frequencies from 0.1 to $5 \mathrm{~Hz}$. Alveolar pressure differences were obtained by analyzing the products of flow distribution and alveolar input impedance.

Results The alveolar pressure $P_{\text {alv }}$ reduced as the frequency increased (for both collapsed and open alveoli). Compared with the situation at 0.2 $\mathrm{Hz}, \mathrm{P}_{\text {alv }}$ reduced to $9.1 \pm 1.0 \%$ at $4 \mathrm{~Hz}$. On the other hand, $\mathrm{P}_{\text {alv }}$ increased as the percentage of atelectatic area increased (for all frequencies). $P_{\text {alv }}$ increased to $267 \pm 6 \%$ when $70 \%$ of alveoli collapsed compared with $10 \%$ of collapse. The pressure differences between collapsed and open alveoli increased as the frequency increased. At $0.2 \mathrm{~Hz}$ the differences were $<3 \%$, while at $4 \mathrm{~Hz}$ the differences were $>20 \%$ (relative to the lower value). 
Conclusions Increasing the high-frequency contents of the inspiratory flow pattern may help to develop a pressure difference between collapsed and open alveoli. The low-frequency contents of the flow pattern, however, may be still needed to guarantee the minimum ventilation.

Reference

1. Horsfield K, et al.: J Appl Physiol 1982, 52:21-26.

P188

Decreasing tidal volume from 6 to $4 \mathrm{ml} / \mathrm{kg}$ : feasibility and effects on repeated opening and closing

J Retamal, J Libuy, M Jimenez, M Delgado, C Besa, G Bugedo, A Bruhn

Pontificia Universidad Católica de Chile, Santiago, Chile

Critical Care 2010, 14(Suppl 1):P188 (doi: 10.1186/cc8420)

Introduction Low tidal volumes $(\mathrm{Vt})$ are thought to protect the lung by avoiding overdistension. We recently have shown that Vt may also influence repeated opening and closing (O/C) [1]. The goal of this study was to determine whether decreasing Vt from 6 to $4 \mathrm{ml} / \mathrm{kg}$ was effective to reduce $\mathrm{O} / \mathrm{C}$, and whether it was possible to maintain alveolar ventilation at such low Vt.

Methods Cross-over study at two Vt levels: 6 versus $4 \mathrm{ml} / \mathrm{kg} \mathrm{IBW}$. We included ALI/ARDS patients, ventilated $<48$ hours, and who would have a chest computed tomography (CT) scan. For the $4 \mathrm{ml} / \mathrm{kg}$ arm: we replaced the heat and moisture exchange filter by a heated humidifier, and the respiratory rate was increased to keep minute ventilation constant. The protocol had two parts: one bedside and other in the CT room. Both Vts were applied in a random order. For the bedside protocol each Vt arm was applied for 30 minutes. Data on lung mechanics and gas exchange were taken at baseline and 30 minutes. For the $\mathrm{CT}$ scan protocol each $\mathrm{Vt}$ arm was applied for 5 minutes and then a dynamic CT ( 4 images/second for 8 seconds) was taken at each Vt at a fixed transverse region at the lower third of the lungs. Afterwards, CT images were analyzed by software (MALUNA) and repeated $\mathrm{O} / \mathrm{C}$ was determined as nonaerated tissue variation between inspiration and expiration, expressed as a percentage of lung tissue weight.

Results We analyzed nine patients (six male), who had a median age of 39 (21 to 72 ) years, APACHE II score 14 (5 to 23) and SOFA score 9 (6 to 15). All patients had a pulmonary origin of their ARDS and were on their first day of ventilation. At baseline patients had a $\mathrm{PaO}_{2} / \mathrm{FiO}_{2}$ ratio of 141 (71 to 280), compliance of 32 (17 to 43$) \mathrm{ml} / \mathrm{cmH}_{2} \mathrm{O}$, and PEEP of 12 (10 to 16) $\mathrm{cmH}_{2} \mathrm{O}$. In the Vt arms 4 and $6 \mathrm{ml} / \mathrm{kg}$, Vts were 260 (210 to 300) and 350 (310 to 400) $\mathrm{ml}$, respectively $(P<0.01)$, respiratory rates were $37(31$ to 42$)$ and $25(21$ to 28) breaths per minute $(P<0.01)$, and $\mathrm{PaO}$ levels were 84 (54 to 148) and 83 (61 to 162) $\mathrm{mmHg}(P=0.3)$. PEEP and $\mathrm{FiO}_{2}$ were kept constant. $\mathrm{PaCO}_{2}$ did not significantly increase with $\mathrm{Vt} 4$ but repeated $\mathrm{O} / \mathrm{C}$ (delta nonaerated tissue) consistently decreased (Figure 1).

Conclusions Decreasing Vt from 6 to $4 \mathrm{ml} / \mathrm{kg}$ reduces repeated $\mathrm{O} / \mathrm{C}$ Hypercapnia can be effectively prevented by decreasing the instrumental dead space and increasing the respiratory rate.

Acknowledgements Grant was Fondecyt 11060350

Reference

1. Bruhn A, et al:: Intensive Care Med 2009, 35(Suppl 1):S227.
P189

Barriers to providing lung-protective ventilation to patients with ALI/ARDS

RH Passos, RH Oliveira, MQ Trabuco, RR Rodrigues, SP Souza, PB Batista Hospital São Rafael, Salvador, Brazil

Critical Care 2010, 14(Suppl 1):P189 (doi: 10.1186/cc8421)

Introduction Acute lung injury (ALI) and acute respiratory distress syndrome (ARDS) are associated with significant morbidity and mortality. Mechanical ventilation is the cornerstone of supportive therapy. However, the optimal strategy of ventilation and adjunctive therapies are still evolving. There is evidence to support the use of volume-limited and pressure-limited lung-protective ventilation but practice variability in the clinical management is still a concern mainly in sicker patients. The purpose of this study was to examine the ventilatory prescriptions of clinicians caring for patients with ALI/ARDS.

Methods We prospectively examined demographic characteristics, APACHE II score and choice of ventilatory settings at the day of diagnosis of ALI/ARDS (AECC criteria) of 84 mechanically ventilated patients from May 2007 through October 2009 in a mixed medical-surgical critical care unit.

Results ALI/ARDS occurred in $7.1 \%$ of admissions and $16.1 \%$ of all mechanically ventilated patients. Sepsis was the main cause. All patients were ventilated with pressure control mode. Median tidal volume/ predicted body weight was 6.1 (3.5 to 9.9) $\mathrm{ml} / \mathrm{kg}$, plateau pressure was 26 (15 to 55) $\mathrm{CmH}_{2} \mathrm{O}$ and mean PEEP was 12 (5 to 25) $\mathrm{CmH}_{2} \mathrm{O}$. Univariate analysis showed sepsis-related ALI/ARDS $(P=0.026)$ and APACHE II score $>18(P<0.001)$ were barriers to initiate lung-protective ventilation. In multivariate analysis, APACHE score $>18$ was an independent predictor. Ventilatory settings were significantly different in this subset of patients ( $n=26$ ). The tidal volume/predicted body weight (6.2 (3.5 to 9.9) vs 5.1 ( 4.3 to 8.5$) \mathrm{ml} / \mathrm{kg}$ ), PEEP (15 (10 to 25$)$ vs 7 (6 to 12$) \mathrm{cmH}_{2} \mathrm{O}$ ) and plateau pressure (38 (32 to 55) vs 22 ( 15 to 30 ) $\mathrm{CmH}_{2} \mathrm{O}$ ) were higher in the group with APACHE II score $>18(P<0.001)$. $\mathrm{PaO}_{2} / \mathrm{FiO}_{2}^{2} \mathrm{pH}$ and $\mathrm{paCO}_{2}$ did not differ between groups. As expected, mortality was higher in this group, $60 \%$ vs 22\% $(P<0.001)$

Conclusions Protective mechanical ventilation is not completely feasible in the subset of ALI/ARDS patients with higher risk of mortality (APACHE $\|>18$ ).

Reference

1. Acute Respiratory Distress Syndrome Network: Ventilation with lower tidal volumes as compared with traditional tidal volumes for acute lung injury and the acute respiratory distress syndrome. N Engl J Med 2000, 342:1301.

\section{P190}

Influence of aetiology of acute respiratory distress syndrome and early differences in oxygenation on outcome

M Shankar-Hari', Cl Olvera², S Webb², S Phelps², S Batson², A Puddicome², R Marshall2, D Howell', G Bellingan²

'Guy's and St Thomas' NHS Foundation Trust, London, UK; '2University College Hospital, London, UK

Critical Care 2010, 14(Suppl 1):P190 (doi: 10.1186/cc8422)

Introduction Studies evaluating the relationship between the severity of hypoxemia in patients with acute respiratory distress syndrome (ARDS)
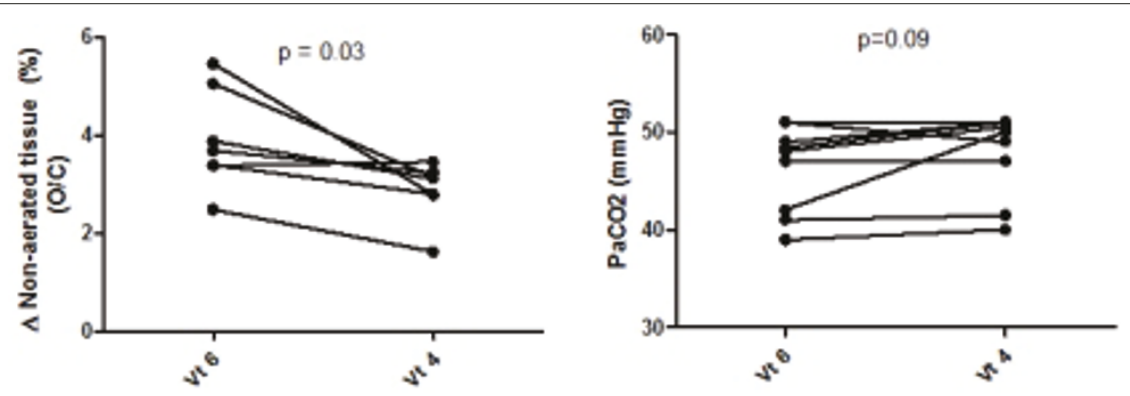

Figure 1 (abstract P188). 
and hospital outcome have not found a consistent outcome association. We hypothesized that severity of hypoxemia on admission after optimal ventilation may predict hospital outcome if the ARDS patients are categorised based on the primary etiology: pulmonary ARDS (ARDS) and extra pulmonary ARDS (ARDS ). Our aim was therefore to ascertain the relationship between hospital outcome and the severity of hypoxemia in patients with early ARDS (days 1 to 3 following admission) after categorising based on etiology.

Methods We used a prospective cohort study design and enrolled 151 consecutive patients with a primary diagnosis of ARDS on the day of admission, admitted over a 2-year period to our adult general ICU. Patients enrolled in other clinical interventional trials were excluded from the study. Protocol-based management of mechanical ventilation was used to achieve optimal ventilation. Two authors independently designated patients as ARDS or ARDS exp and a third reviewed any conflicts (only four cases). Patients were then subcategorised by severity of hypoxaemia based on $\mathrm{PaO}_{2} / \mathrm{FiO}_{2}$ ratio ( $\mathrm{P} / \mathrm{F}$ ratio) intervals. All other clinical interventions were at the discretion of treating clinician.

Results The hospital mortality in all patients included in the study $(n=$ 151) with ARDS was $44.1 \%$. The patients classified as ARDS had a higher hospital mortality (50.6\%) compared with ARDS (36.4\%), but the difference was not statistically significant $(P=0.12)$. Nonsurvivors with ARDS had a significantly lower P/F ratio on day 1 of ARDS diagnosis compared with survivors (12.05 \pm 4.46 vs $15.39 \pm 4.97 \mathrm{kPa}$; $P=0.002)$

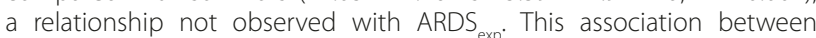
mortality and hypoxaemia in early ARDS persisted at even more serve levels of hypoxaemia $(<15 \mathrm{kPa},<12.5 \mathrm{kPa}$, and $<10 \mathrm{kPa} ; P=0.01, P=0.003$ and $P=0.002$, respectively), while in ARDS the effect of hypoxaemia on mortality was not observed.

Conclusions Our findings indicate that the hypoxaemia burden as assessed by $\mathrm{P} / \mathrm{F}$ ratio intervals despite optimal ventilatory support on the day of ICU admission predicts increased risk of death in ARDS; but not in ARDS . Interventional trials need to account for the influence of etiology and hypoxaemia burden on outcome prior to concluding this as a negative intervention.

\section{P191}

\section{Acute lung injury in TBI patients with SIRS: role of vascular} endothelial damage

TSaito', H Kushi², T Miki', J Sato', A Yoshino', KTanjo'

'Nihon University, School of Medicine, Tokyo, Japan; 'Nihon University, College of Humanities and Sciences, Tokyo, Japan

Critical Care 2010, 14(Suppl 1):P191 (doi: 10.1186/cc8423)

Introduction The pathological organ dysfunction that occurs in patients with SIRS or sepsis is believed to be related to vascular endothelial damage. We investigated the role of vascular endothelial damage in the occurrence of acute lung injury in severe TBI patients with SIRS.

Methods The subjects were 20 severe TBI patients with SIRS. The P/F ratio was calculated from arterial blood gas analysis data obtained for seven consecutive days from the time of admission. Peripheral blood samples were collected four times to measure the serum levels of IL-6 and IL-8, and the levels of ICAM-1 and granulocyte elastase (GE) as markers of vascular endothelial damage.

Results The P/F ratio decreased with time and was below 300 from day 4 onwards. However, the survivors maintained a P/F ratio of 300 or more. In the patients with a fatal outcome, the ratio continued to decline and the subjects developed acute lung injury on day 3. From day 5 onward they showed a significant decrease of the ratio, with values of 200 or less and symptoms of adult respiratory distress syndrome. The levels of IL-6, IL-8, ICAM-1, and GE increased after admission, but then decreased again in the survivors. In the patients who died, these levels continued to rise and there was a significant increase of IL-6, IL-8, and ICAM-1 after 1 week. The correlation between the blood level of IL- 6 and the level of IL-8, ICAM-1, or GE was strong, while that between the IL-8 level and the ICAM-1 or GE levels was also strong and that between the ICAM-1 and GE level was weaker. In contrast, the correlations between the $\mathrm{P} / \mathrm{F}$ ratio and the blood levels of IL-6, IL-8, ICAM-1, or GE were moderate and negative. Therefore, inverse correlations were noted between the P/F ratio and all these parameters.
Conclusions By determining the changes of humoral mediators, we demonstrated that vascular endothelial damage was involved in the occurrence of the pathological state of acute lung injury, which occurs in severe TBI patients with SIRS.

\section{P192}

Mechanical ventilation aggravates transfusion-related acute lung injury induced by MHC class I antibodies

AP Vlaar', EK Wolthuis', JJ Hofstra', JJ Roelofs', L Boon², MJ Schultz' R Lutter', NP Juffermans'

'Academic Medical Center, Amsterdam, the Netherlands; ${ }^{2}$ Bioceros, Utrecht, the Netherlands

Critical Care 2010, 14(Suppl 1):P192 (doi: 10.1186/cc8424)

Introduction Transfusion-related acute lung injury (TRALI) occurs more often in critically ill patients than in a general hospital population, possibly due to the presence of underlying inflammatory conditions that may prime pulmonary neutrophils. Mechanical ventilation (MV) may be a risk factor for developing TRALI. We determined the influence of MV on the development of TRALI, combining a murine MV model causing ventilatorinduced lung injury with a model of antibody-induced TRALI $[1,2]$.

Methods BALB/C mice $(n=84)$ were ventilated for 5 hours with low $(7.5 \mathrm{ml} / \mathrm{kg})$ or high $(15 \mathrm{ml} / \mathrm{kg})$ tidal volume, a positive end-expiratory pressure of $2 \mathrm{cmH}_{2} \mathrm{O}$ and a fraction of inspired oxygen of $50 \%$. After 3 hours of MV, TRALI was induced by infusion of MHC-I antibodies $(4.5 \mathrm{mg} /$ $\mathrm{kg}$ ), controls received vehicle. Nonventilated animals receiving vehicle, isotype or $\mathrm{MHC}-\mathrm{I}$ antibodies served as additional controls.

Results All animals receiving MHC-I antibodies developed TRALI within 2 hours. In mice in which TRALI was induced, MV with low tidal volumes aggravated pulmonary injury as evidenced by an increase in neutrophil influx, pulmonary and systemic levels of cytokines and lung histopathological changes compared with unventilated controls. The use of high tidal volume ventilation resulted in a further increase in protein leakage and pulmonary edema.

Conclusions MV synergistically augmented lung injury during TRALI, which was even further enhanced by the use of injurious ventilator settings. Results suggest that MV may be a risk factor for the onset of TRALI and may aggravate the course of disease.

References

1. Wolthuis EK, Vlaar AP, Choi G, et al:: Mechanical ventilation using noninjurious ventilation settings causes lung injury in the absence of preexisting lung injury in healthy mice. Crit Care 2009, 13:R1.

2. Looney MR, Su X, Van Ziffle JA, et al: Neutrophils and their Fcy receptors are essential in a mouse model of transfusion-related acute lung injury. J Clin Invest 2006, 116:1615-1623.

P193

Adaptive support ventilation may be inappropriate for patients with ALI/ARDS after recruitment: an observational study F Paulus, DA Dongelmans, DP Veelo, JM Binnekade, MJ Schultz Academic Medical Center, Amsterdam, the Netherlands Critical Care 2010, 14(Suppl 1):P193 (doi: 10.1186/cc8425)

Introduction With adaptive support ventilation (ASV), a microprocessorcontrolled mode of mechanical ventilation, the ventilator adapts tidal volume (VT) size based on the Otis least work of breathing formula. In recent studies in patients with ALL/ARDS, ASV applied VT of 7.3 (6.7 to 8.8) $\mathrm{ml} / \mathrm{kg}$ ideal body weight (IBW). It is unclear whether an open-lung approach was used in these studies. Lung recruitment improves lung compliance, and as a consequence may allow the ventilator to apply too large a VT with ASV.

Methods Ten consecutive patients with ALI/ARDS, ventilated in accordance with our local protocol dictating frequent recruitment maneuvers, were observed while the ventilator was switched from pressure control ventilation (PC) to ASV. Thereafter, all patients were subjected to an additional standard recruitment procedure. The primary endpoint was VT before and after switch of the ventilator, and after standard recruitment.

Results Four patients suffered from ALI, six patients from ARDS. Seven patients had an extrapulmonary cause for ALI/ARDS. VT increased from $6.5 \pm 0.8 \mathrm{ml} / \mathrm{kg} \mathrm{IBW}$ to $9.0 \pm 1.6 \mathrm{ml} / \mathrm{kg} \mathrm{IBW}(P<0.01)$ after switch from PC 
to ASV. Additional recruitment after switch of the ventilator did not affect VT size $(9.3 \pm 1.4 \mathrm{ml} / \mathrm{kg}$ IBW, $P>0.05)$. In seven patients ASV applied VT $>8 \mathrm{ml} / \mathrm{kg} \mathrm{IBW}$, in one patient VT even increased to $>12 \mathrm{ml} / \mathrm{kg} \mathrm{IBW}$.

Conclusions Patients with ALI/ARDS may be ventilated with too large a VT when subjected to ASV. Our results contrast findings of previous studies on ASV in patients with ALI/ARDS, probably because we frequently use recruitment maneuvers.

\section{P194}

Receptor for advanced glycation end products is associated with systemic and organ-specific severity of acute lung injury and acute respiratory distress syndrome

A Pradella', T Mauri', S Masson², G Bellani', A Coppadoro', F Magni', M Bombino ${ }^{1}$, N Patroniti', R Latini ${ }^{2}$, A Pesenti ${ }^{1}$

'San Gerardo Hospital and University of Milan-Bicocca, Monza, Italy; Istituto di Ricerche Farmacologiche Mario Negri, Milan, Italy

Critical Care 2010, 14(Suppl 1):P194 (doi: 10.1186/cc8426)

Introduction Receptor for advanced glycation end products (RAGE) is an acute phase inflammatory mediator primarily expressed by alveolar type I cells in the lung. The association between circulating RAGE and severity of acute lung injury and acute respiratory distress syndrome (ALI/ARDS) has been recently suggested by both experimental and human studies. The purpose of our study is to study the significance of plasma RAGE levels over time and of bronchoalveolar lavage fluid (BALf) RAGE in ALI/ARDS patients.

Methods We enrolled 21 patients admitted to a single general ICU of a university-affiliated hospital affected by ALI/ARDS (1994 Consensus Conference criteria). We measured plasma RAGE levels twice on the first 2 days from intubation, then every 3 days for the first month and then once a week, until ICU discharge or death $(n=188)$. We also measured RAGE levels in BALf obtained by means of a standardized technique when clinically indicated $(n=22)$. At each sampling time we recorded data on ventilator settings, gas exchange, organ function and blood cell counts. Results Day 1 plasma RAGE levels (normal values $<170 \mathrm{pg} / \mathrm{ml}$ ) were high (median 1,588 pg/ml, IQR 780 to 2,398 pg/ml) and then lowered over time. When all samples were considered, plasma RAGE levels were significantly higher in patients with blood platelet count $<100 \times 10^{3} / \mu$ l, with plasma creatinine level $\geq 2 \mathrm{mg} / \mathrm{dl}$ and with SOFA score $\geq 5(P=0.029, P=0.001$ and $P=0.045$, respectively). Plasma RAGE increased also with the number of organ failures $(P=0.004)$. Moreover, circulating RAGE was significantly higher in patients with $\mathrm{PaO}_{2} / \mathrm{FiO}_{2}<150$ and with PEEP $\geq 10 \mathrm{cmH}_{2} \mathrm{O}(P=$ 0.045 and $P<0.001$, respectively). RAGE was present in BALf (median 174 $\mathrm{pg} / \mathrm{ml}$, IQR 46 to 1,476 pg/ml). Interestingly, plasma and BALf RAGE levels were not correlated $(P=0.2)$. BALf RAGE was significantly higher in patients with $\mathrm{PaO}_{2} / \mathrm{FiO}_{2}<150 \mathrm{mmHg}$ and with administered tidal volume:ideal body weight ratio $>6 \mathrm{ml} / \mathrm{kg}(P=0.021$ and $P=0.009$, respectively). Patients with culture-positive BALf had higher BALf RAGE levels in comparison with culture-negative BALf $(P=0.014)$.
Conclusions In ALI/ARDS patients, circulating RAGE is high on day 1, decreases over time and may be related to systemic and lung injury severity. BALf RAGE obtained from these patients may be associated with lung dysfunction and infection.

P195

Mild hypothermia was protective in a physiological model of ventilator-induced lung injury by reducing inflammation but not by reducing the respiratory rate

H Aslami, M Schultz, N Juffermans

Laboratory of Experimental Intensive Care and Anesthesiology, Amsterdam,

the Netherlands

Critical Care 2010, 14(Suppl 1):P195 (doi: 10.1186/cc8427)

Introduction In animal models of ventilator-induced lung injury (VILI), mild hypothermia was found to be protective by reducing pulmonary inflammation and possibly by reducing mechanical strain by applying lower respiratory rates. However, models are hampered by severe alkalosis or an ex vivo design. In a physiological model of VILI, we investigated whether hypothermia protects from VILI by reducing respiratory rates, or by reducing inflammation.

Methods In rats, VILI was induced using a peak inspiratory pressure (PIP) of $23 \mathrm{cmH}_{2} \mathrm{O}$ and zero PEEP. Controls were ventilated with a PIP of $12 \mathrm{cmH}_{2} \mathrm{O}$ and PEEP of $5 \mathrm{cmH}_{2} \mathrm{O}$. Hypothermia $\left(32^{\circ} \mathrm{C}\right)$ was induced by external cooling, controls were maintained at $37^{\circ} \mathrm{C}$. Normo-pH (7.3 to 7.4) or strict normocapnia ( 4.5 to $5.0 \mathrm{kPa}$ ) was achieved by adjusting the respiratory rate according to blood gases drawn every 30 minutes. After 4 hours of

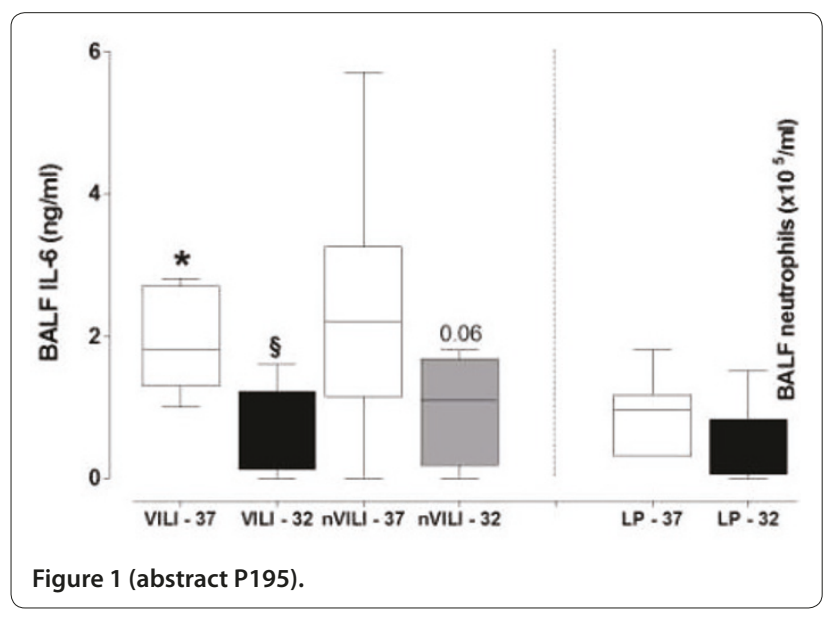

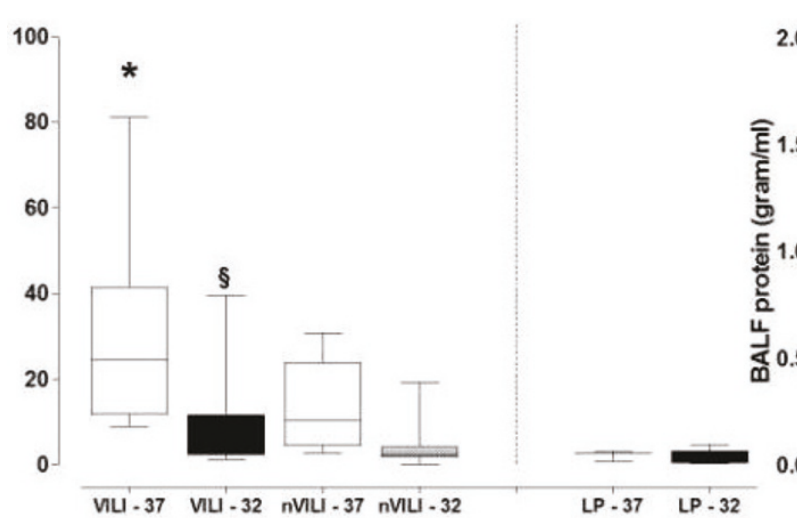

Figure 2 (abstract P195).
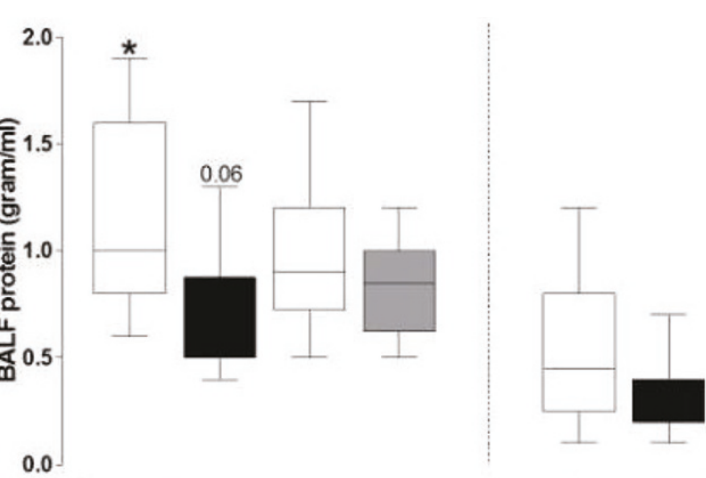

VIL, 37 VILI, 32 nVLI, 37 nVIL, 32

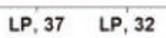


ventilation, bronchoalveolar lavage (BAL) was done. Statistics include Kruskal-Wallis and Mann-Whitney $U$ tests.

Results A physiological model of VILI was established. In the normo-pH group, hypothermia decreased pulmonary IL-6 and neutrophil influx and tended to decrease pulmonary protein leak (Figure 1). In the normocapnia group, hypothermia allowed for lower respiratory rates compared with normo-pH (11 \pm 1 vs $17 \pm 2$ breaths/minute) (Figure 2). However, this did not further reduce parameters of lung injury.

Conclusions Hypothermia was protective in a physiological model of VILI, by reduction of inflammation, but not by reducing the repetitive strain of respiratory cycles.

\section{P196}

\section{Effect of induced hypothermia on respiratory parameters in}

\section{mechanically ventilated patients}

H Aslami, J Binnekade, S Huissoon, N Juffermans

Laboratory of Experimental Intensive Care and Anesthesiology, Amsterdam, the Netherlands

Critical Care 2010, 14(Suppl 1):P196 (doi: 10.1186/cc8428)

Introduction Therapeutic hypothermia is applied to reduce hypoxiainduced organ injury. In the past decades, the use of hypothermia has increased in critically ill patients who are mechanically ventilated (MV). Data on the effect of hypothermia on gas exchange and lung mechanics in these patients are limited. In this retrospective study, we describe the effect of induced hypothermia and rewarming on respiratory parameters in patients after a cardiac arrest.

Methods Patients with a Glasgow Coma Scale $<8$ after resuscitation for a cardiac arrest in whom hypothermia was applied (32 to $34^{\circ} \mathrm{C}$ ), were enrolled. Patients with $\mathrm{PaO} / \mathrm{FiO}_{2}$ ratio $<200$ or $\mathrm{MV}$ with positive endexpiratory pressure (PEEP) $>15 \mathrm{cmH}_{2} \mathrm{O}$ were excluded. Ventilator settings and arterial blood gasses were retrieved from the electronic patient database during hypothermia and at every ${ }^{\circ} \mathrm{C}$ grade increase during rewarming. Statistics include $z$ paired $t$ test

Results From a cohort of 98 patients, 35 patients were excluded, leaving 62 patients for analysis. During hypothermia, arterial $\mathrm{pCO}_{2}$ decreased, while end tidal (et) $\mathrm{CO}_{2}$ was low at unchanged minute volume ventilation (Figure 1). Hypothermia increased the P/F ratio from $255 \pm 55$ to $283 \pm$ 12 at unchanged PEEP $(P<0.05)$, while fluid balances were positive in all patients $(2.5 \pm 1.6 \mathrm{I})$. After rewarming, arterial $\mathrm{pCO}_{2}$ was unchanged while etCO increased. The P/F ratio after rewarming was unchanged compared with the start of hypothermia, while lower PEEP levels were applied (7.0 \pm $0.4 \mathrm{cmH}_{2} \mathrm{O}$ vs $6.1 \pm 0.3 \mathrm{cmH}_{2} \mathrm{O}, P<0.05$ ).

Conclusions Induced hypothermia improved ventilation and oxygenation in critically ill patients. Hypothermia may be considered in patients with acute lung injury, in whom low minute ventilation results in severe hypercapnia.

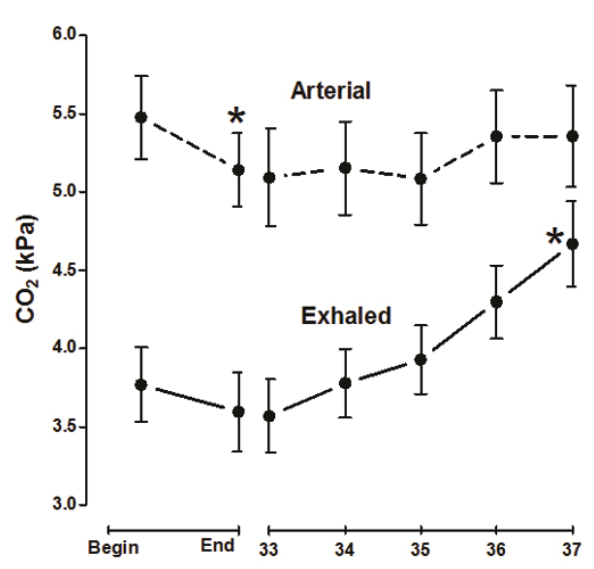

Figure 1 (abstract P196).
P197

Extracorporeal life support service in a regional referral center: the Florence experience

S Biondi', A Pasquini², S Batacchi², G Cianchi², M Ciapetti², S Di Valvasone', S Bacci ${ }^{2}$, L Varegliano ${ }^{2}$, M Solaro², M Bonizzoli², R Spina ${ }^{2}$, M Bonacchi ${ }^{3}$, G Zagli², C Nozzoli' ${ }^{2}$ A Peris ${ }^{2}$, GF Gensini ${ }^{3}$

'Postgraduate School of Anesthesia and Intensive Care, University of Florence, Italy; ${ }^{2}$ Careggi Teaching Hospital, Florence, Italy; ${ }^{3}$ University of Florence, Italy Critical Care 2010, 14(Suppl 1):P197 (doi: 10.1186/cc8429)

Introduction Extracorporeal life support (ECLS) represents a therapeutic choice in cases of acute reversible lung and/or heart failure. An emergency ICU can safely manage both veno-arterial ( $\mathrm{V}-\mathrm{A}$ ) and veno-venous ( $\mathrm{V}-\mathrm{V}$ ) ECLS.

Methods In our experience, the ECLS treatment implementation started on April 2008. The ECLS team is composed of intensivists, cardiac surgeons, cardiologists, perfusionists and nurses. All of these figures were trained in ECLS management. An Emergency Medical Service has been equipped to guarantee rapid and safe ECLS positioning in peripheral hospitals and transportation to our referral center by the ECLS team. According to our internal protocol, eligible patients for $\mathrm{V}$ - $\mathrm{V}$ ECLS treatment are aged 15 to 70 years old and affected by ARDS with a $\mathrm{PaO} / 2 / \mathrm{FiO}_{2}$ ratio $<60$ or $\mathrm{pH}$ $<7.20$, under a protective ventilation setting. The use of ECLS for cardiac support is reserved for those cases of cardiac shock refractory to standard treatments and cardiac arrest not responding to advanced cardiac life support (ACLS). The ECLS device used is a Rotaflow Maquet Centrifugal Pump with a Quadrox-D oxygenator (Maquet, Rastatt, Germany) and biocoated circuits.

Results From April 2008 to November 2009, 21 patients were treated with ECLS. In 13 patients V-V ECLS was established due to severe ARDS not responding to conventional treatment. Median SAPS II at admission was 49 and median duration was 235 hours. Three out of 13 patients were cannulated by the ECLS team in peripheral hospitals and safety transported. The intra-ICU survival rate was $62 \%$. ECLS for cardiac support was performed in eight patients: four cases of intrahospital cardiac arrest, four cases of cardiogenic shock. Median stay of V-A ECLS was 110 hours. In cases of $\mathrm{V}$-A ECLS due to cardiac arrest, no positive outcome was observed. The median stay of ACLS maneuvers before ECLS start was 62.5 minutes In cases of cardiogenic shock undergoing ECLS, two out of four patients were discharged from our ICU.

Conclusions A complete competence acquisition for ECLS management makes this system a safe and feasible technique. The possibility to guarantee a safe treatment must involve different specialists and properly trained nurses. We found the importance of a well-timed start of ECLS.

P198

Interhospital ground transportation of severe acute respiratory distress syndrome patients on extracorporeal membrane oxygenation: Monza's experience

S Isgro', M Milan'1, A Zanella', M Bombino'1, G Foti', M Giani', S Abd El Aziz El Sayed Deabi ${ }^{1}$ N Patroniti², A Pesenti²

${ }^{1}$ AO San Gerardo Monza, Italy; ${ }^{2}$ Milano-Bicocca University, Milan, Italy Critical Care 2010, 14(Suppl 1):P198 (doi: 10.1186/cc8430)

Introduction Severe acute respiratory distress syndrome (ARDS) patient transportation is an extremely high-risk procedure. We report our experience in transferring these patients to our centre while on extracorporeal membrane oxygenation (ECMO).

Methods After telephone referral and bed availability confirmation, patients matching entry criteria for ECMO are evaluated for transportation to our centre. A skilled crew consisting of two expert plus one training physician, one expert plus one training ICU nurse and one ECMO specialist reaches the referral hospital for re-evaluation. If eligible, cannulation, ECLS circuit set up and ECLS start are accomplished. Ground transport is performed with a specially equipped ambulance, endowing enlarged oxygen, fuel and energy supplies. The ambulance is loaded with all of the items required for cannulation and ECMO circuit set up, additional oxygen and a nitric oxide tank. Entry criteria are: potentially reversible respiratory failure, Murray Score $\geq 3$ or respiratory acidosis with $\mathrm{pH}<7.2$, no intracranial bleeding and absolute contraindication to heparinization. 
Results Between 2004 and 2009 our crew evaluated for transfer on ECMO 15 ARDS patients (10 males), age $38 \pm 15$ years, BMI $28 \pm 7$, APACHE ॥ score $26 \pm 9$, SOFA score $9 \pm 4$, Oxygenation Index $39 \pm 17$. The average distance was $133 \pm 124 \mathrm{~km}$. Two patients improved after NO trial and were transferred without ECMO. All of the other patients underwent venovenous ECMO: 11 with cannulation of femoral veins, one femoral-jugular veins and one with a DL cannula in the jugular vein. ECMO settings were (mean \pm SD) BF $2.9 \pm 0.8$, GF $3.6 \pm 1.6, \mathrm{GFFiO} 2$ 1. Data have been recorded 30 minutes before and 1 hour after ECLS began: Vv-ECMO granted a better clearance of $\mathrm{pCO}_{2}(75 \pm 20.5$ vs $49.7 \pm 7.9 \mathrm{mmHg}, P<0.01)$, thus improving the $\mathrm{pH}(7.279 \pm 0.10$ vs $7.41 \pm 0.06, P<0.01)$ and mean pulmonary arterial pressure $(41 \pm 11$ vs $31 \pm 5 \mathrm{mmHg}, P<0.05)$ and allowing a reduction in respiratory rate ( $28 \pm 11$ vs $9 \pm 4, P<0.01)$, minute ventilation $(10.2 \pm 4.6$ vs $3.3 \pm 1.7 \mathrm{l} / \mathrm{min}, P<0.01)$ and mean airway pressure $(26 \pm 6$ vs $22 \pm 5$ $\left.\mathrm{CmH}_{2} \mathrm{O}, P<0.01\right)$. Arterial $\mathrm{pO}_{2}$, mean blood pressure and heart rate did not show significant variations. After ECMO began, vasoconstrictor therapy (being administered to five patients) was quickly tapered. Neither clinical nor technical major complications were reported.

Conclusions ECMO employment at referral centers enabled longdistance, high-risk ground transportation.

\section{P199}

\section{Effects of hypertonic saline on a pig model of acute lung injury} induced by hydrochloric acid instillation

CA Holms', DA Otsuki', M Kahvegian', J Noel-Morgan', C Massoco², DT Fantoni ${ }^{1}$, P Gutierrez ${ }^{3}$, JO Auler Jr ${ }^{1}$

'Faculdade de Medicina da Universidade de São Paulo, Brazil; ' Genoa, São

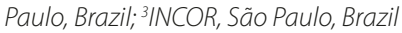

Critical Care 2010, 14(Suppl 1):P199 (doi: 10.1186/cc8431)

Introduction Controversy exists over the possible beneficial effects of hypertonic saline (HS) in pulmonary inflammatory response, particularly in neutrophil immunomodulation [1,2]. This study was designed to investigate possible benefits of HS in the treatment of pigs submitted to experimental acute lung injury.

Methods Twelve anesthetized, tracheotomized pigs (25 to $30 \mathrm{~kg}$ ) were mechanically ventilated by pressure, adjusted to $8 \mathrm{ml} / \mathrm{kg}$ tidal volume, with $\mathrm{FiO}_{2} 50 \%$, and submitted to intratracheal instillation of $4 \mathrm{ml} / \mathrm{kg}$ hydrochloric acid $(\mathrm{HCl}) 0.1 \mathrm{~N}$. They were then randomized to $\mathrm{ALI}$ group $(n=7)$ or $\mathrm{ALI}+\mathrm{HS}$ group $(n=5)$, where animals of the latter group received $4 \mathrm{ml} / \mathrm{kg}$ intravenous hypertonic saline, 15 minutes after injury. Hemodynamic parameters, pulmonary compliance $\left(C_{\text {stat }}\right)$, peak pressure $(P$,$) , plateau pressure \left(P_{p}\right)$ and tidal volume $\left(V_{t}\right)$ were analyzed at baseline (TBL), 15 minutes after $\mathrm{HCl}$ instillation (TALI) and hourly thereafter for 4 hours (T0 to T3). Bronchoalveolar lavage was performed at the end of the observation period for flow cytometry analysis of neutrophil burst activity. Postmortem histopathology of the right diaphragmatic lung was also performed in all animals.

Results After TALI, animals of both groups presented significant increases in $P_{\text {peak }}$ and $P_{\text {plat }^{\prime}}$ and a decrease in $C_{\text {stat }}$ at all time points, when compared with TBL. $V_{t}$ was preserved in both groups over time. There were significant differences between groups $\mathrm{ALI}$ and $\mathrm{ALI}+\mathrm{HS}$, respectively, in: central venous pressure (T0, T1 and T2), pulmonary artery occlusion pressure (T1 and T2) and pulmonary vascular resistance index (TALI). In the ALI group, significant differences related to TBL were found in mean arterial pressure (T1), mean pulmonary artery pressure (TALI, TO, T1, T2 and T3) and pulmonary vascular resistance index (TALI, T0, T3). In the ALI + HS group, there were significant differences related to TBL in mean arterial pressure ( T0, T1, T2), mean pulmonary artery pressure (TALI, T0, T1, T2, T3), cardiac index $(T 0, T 1, T 2)$ and pulmonary vascular resistance index $(T 1, T 2, T 3)$. No differences were found between groups regarding histopathology and flow cytometry analyses.

Conclusions HS produced no significant benefit in the studied parameters regarding the lungs, in the proposed model of ALI.

Acknowledgements Grants from FAPESP 08/55376-7 and 08/56792-4. References

1. Roch A et al:: Intensive Care Med 2007, 33:1645-1654.

2. Deree J et al.: J Trauma 2007, 62:104-111.
P200

Conventional mechanical ventilation can injury intact lungs in severe trauma patients

O Ignatenko, D Protsenko, A Yaroshetskiy, B Gelfand

State Medical Unversity of Russia, Moscow, Russia Federation

Critical Care 2010, 14(Suppl 1):P200 (doi: 10.1186/cc8432)

Introduction Conventional mechanical ventilation (MV) may cause additional lung injury in ALI/ARDS due to overdistention of aerated lung regions (high Vt) and cyclic lung reopening (low PEEP level). Hyperproduction of inflammatory mediators is one of the side effects in these cases. This factor could delay or prevent resolution of respiratory failure $[1,2]$. However, it is not clear whether conventional mechanical ventilation damages intact lungs. The aim of this study was to evaluate the effects of conventional and protective mechanical ventilation on intact lungs in patients with severe trauma.

Methods A prospective, randomized controlled trial in trauma patients with mechanical ventilation for extrapulmonary indications. The protocol was approved by the local ethics committee. Seventy-eight patients were randomized to conventional (Vt 10 to $12 \mathrm{ml} / \mathrm{kg}$ IBW, PEEP $5 \mathrm{cmH}_{2} \mathrm{O}-n=$ 39) or protective ( $V t 5$ to $6 \mathrm{ml} / \mathrm{kg} \mathrm{IBW}$, PEEP $10 \mathrm{cmH} O-n=39$ ) mechanical ventilation. TNFa, IL-1 $\beta$ and $\mathrm{IL}-6$ levels in plasma and BAL fluids were measured on 1, 2, 3, 5 and 7 days of MV. Frequency of ALI (AECC criteria) and VAP were evaluated. The endpoints of this study were the length of MV, LOS in ICU and outcome on 28 days.

Results In first 3 days ALI was revealed in 26 patients $(66.6 \%)$ in the conventional and 10 patients $(26.5 \%)$ in the protective MV groups ( $P=$ 0.001; OR 4.375, 95\% CI 2.227 to 8.189). ARDS occurred in four patients $(10,2 \%)$ of the conventional MV group (LIS > 2$)$ and no one in the protective MV group $(P<0.0001)$. Levels of TNFa, $I L-1 \beta$ and $I L-6$ in BAL fluids were significantly higher in the conventional MV group from 1 to 7 days with maximal increase on day $3(542 \pm 44 / 91 \pm 11 ; 315 \pm 35 / 86 \pm 10 ; 1,092 \pm$ $160 / 111 \pm 18, P<0.0001)$. No differences were found in levels of TNFa, IL-1 $\beta$ and IL-6 in plasma samples. VAP occurred in 31 patients $(83.7 \%)$ of the conventional and nine patients (23\%) of the protective MV groups ( $P=$ $0.0001 ; \mathrm{OR} 17.2,95 \% \mathrm{Cl} 5.5$ to 54.3$)$. The length of MV was $17.4 \pm 6$ vs 12.8 $\pm 3(P=0.0001 ;$ OR $4.2,95 \%$ Cl 1.5 to 11.5$)$, LOS in the ICU was $21.9 \pm 5.6$ vs $15.75 \pm 2.9(P=0.0002 ;$ OR $2.0,95 \% \mathrm{Cl} 0.18$ to 23.6$)$. The 28 -day mortality was not significantly different in the groups.

Conclusions Conventional MV for more than 72 hours in patients with severe trauma and intact lungs can cause lung injury, and increase duration of MV and LOS in the ICU.

References

1. Dreyfuss D, Saumon G: Ventilator-induced lung injury: lessons from experimental studies. Am J Respir Crit Care Med 1998, 157:294-323.

2. Parker JC, Hernandez LA, Peevy KJ: Mechanisms of ventilator-induced lung injury. Crit Care Med 1993, 21:131-143.

\section{P201}

Protective effect of methylprednisolone on ventilator-induced diaphragm dysfunction is dose dependent

K Maes', A Agten'1, A Smuder², SK Powers², M Decramer'1, G Gayan-Ramirez ${ }^{1}$ ${ }^{1}$ KU Leuven, Belgium; ${ }^{2}$ University of Florida, Gainesville, FL, USA

Critical Care 2010, 14(Suppl 1):P201 (doi: 10.1186/cc8433)

Introduction Administration of $80 \mathrm{mg} / \mathrm{kg}$ methylprednisolone has been shown to prevent controlled mechanical ventilation (CMV) diaphragm dysfunction in rats, partly by inhibiting the calpain system [1]. The current experiments determined whether lower doses of corticosteroids will also provide protection against ventilator-induced diaphragm dysfunction.

Methods Rats were assigned to a control group or to 24 hours of CMV receiving a single injection of saline or $5 \mathrm{mg} / \mathrm{kg}$ (low MP) or $30 \mathrm{mg} / \mathrm{kg}$ (high MP) of methylprednisolone.

Results Diaphragm force production was decreased after CMV but significantly more in the low MP group while similar to controls in the high MP group. Atrophy of the type lla fibers was only present in the low MP group. Atrophy of the type IIx/b fibers was more severe in the low MP group than in the CMV group while no atrophy was observed in the high MP group. Diaphragm calpain activity was increased after CMV (+93\%, $P<0.05$ vs C) and in the low MP group ( $+83 \%, P<0.05$ vs C), while it was similar to controls in the high MP group. Expression of calpastatin was 
decreased in the CMV and the low MP group $(-18 \%, P<0.05$ vs $C)$ but its level was preserved to control levels in the high MP group. Analysis of the caspase-3 mediated cleavage of a-spectrin revealed that CMV induced a significant rise in caspase-3 activity when compared with C (+194\%, $P<0.001)$. Caspase- 3 activity was similarly increased in the MP-5 and the MP-30 groups ( $+96 \%$ and $+78 \%$ respectively, $P<0.05$ vs $C$ ) but this increase was significantly less compared with that of CMV. Significant negative correlations were found between calpain activity and diaphragm force $(-0.50<r<-0.41, P<0.05)$ as well as with CSA of the type IIX/b fibers $(r=$ $-0.57, P<0.02$ ). Significant positive correlations were observed between calpastatin and diaphragm force $(0.43<r<0.54, P<0.05)$ and calpastatin and CSA of the type IIx/b fibers $(r=0.57, P<0.02)$.

Conclusions The ability of corticosteroids to protect against CMV-induced diaphragmatic contractile dysfunction and atrophy are dose dependent with only high doses of corticosteroids providing protection.

Acknowledgements Supported by Astra Zeneca Pharmaceuticals and FWO Vlaanderen.

Reference

1. Maes et al:: Am J Respir Crit Care Med 2008, 178:1219-1226.

P202

Alveolar recruitment with non-invasive mechanical ventilation (C-PAP) in patients with nonobstructive respiratory failure

VTomicic', R Moreno', V Hidalgo', P Vargas', J Keymer', A Fuentealba', G Hormazabal', R Perez', J Molina', J Borges

'Clinica Alemana de Santiago, Chile; ' University of São Paulo, Brazil

Critical Care 2010, 14(Suppl 1):P202 (doi: 10.1186/cc8434)

Introduction Non-invasive mechanical ventilation (NIMV) is able to reduce reintubation especially in patients with exacerbation of COPD. Results have not been reached in critically ill patients with nonobstructive respiratory failure (NORF). However, the NIMV in most of these studies has been applied without making an effort to open the lung and adjusting the C-PAP after opening up the lung using a clinical approach. Our aim was to evaluate the effects of applying recruitment manoeuvres (RM) with C-PAP and titrate it according to clinical decremental C-PAP trial in patients with NORF.

Methods NORF patients for whom NIMV was indicated between January 2008 and July 2009 were included and submitted to the NIMV-RM protocol when a trained team was available. Bi-PAP and a full face mask were used. The inclusion criteria were at least two of the following: respiratory rate (RR) $>30$, accessory muscle activity, saturation $\leq 90 \%$ with $\mathrm{FiO}_{2} \geq 50 \%$ and consolidation areas on thorax X-ray. Gradual increasing of C-PAP $\left(2 \mathrm{cmH}_{2} \mathrm{O}\right)$ was used from 10 to $20 \mathrm{cmH}_{2} \mathrm{O}$. Each level of C-PAP was sustained for 5 minutes according to tolerance. The C-PAP after RM was adjusted when the maximal tidal volume $(\mathrm{VT})$ was reached, pulse oximetry $(\mathrm{PO})$ did not show any substantial change and when the patient was comfortable. Demographic data, APACHE $\|$ score and lung injury score (LIS) were measured. Cardiac rate (CR), RR, arterial pressure (AP), $P O$, minute ventilation (VE) and percentage of mask leak (PML) were recorded through the RM. Arterial blood gases were measured pre-RM, 1, 12 and 24 hours after RM. Variables are expressed as median (range). ANOVA by repeated measures or Kruskal-Wallis was used, $P<0.05$ was considered significant.

Results Fourteen patients were included. Age, APACHE II and LIS were: 56 (17 to 80); 14 (4 to 21 ) and 2 (1.3 to 2.7). The $\mathrm{PaO} / \mathrm{FiO}_{2}$ ratio increased from $169.1 \pm 69.7$ (basal) to $261 \pm 106$ after 1 hour of RM $(P=0.02)$.Improvement was preserved at 12 and 24 hours, $280 \pm 69$ and $295 \pm 73$, respectively. The C-PAP level 1 hour after RM was $14.9 \pm 2.4$, and $14.1 \pm 1.9$ at 24 hours post RM. The hemodynamic stability, RR, AP, PML and VE did not change during and after the RM.

Conclusions RM with gradual increments of C-PAP is safe. RM in patients with NORF could be an alternative to rescue patients with poor outcome with NIMV alone.

\section{P203}

\section{$\mathrm{N}$-acetylcysteine attenuates ventilator-induced diaphragm}

\section{dysfunction in rats}

A Agten'1, K Maes' ${ }^{1}$, A Smuder², SK Powers², M Decramer',

G Gayan-Ramirez ${ }^{1}$

'KU Leuven, Belgium; '2University of Florida, Gainesville, FL, USA

Critical Care 2010, 14(Suppl 1):P203 (doi: 10.1186/cc8435)
Introduction Controlled mechanical ventilation (CMV) results in diaphragmatic dysfunction. Oxidative stress is an important contributor to ventilator-induced diaphragm dysfunction, since 18 hours of CMV lead to increased protein oxidation and increased lipid peroxidation. We hypothesized that administration of an antioxidant, $\mathrm{N}$-acetylcysteine (NAC), would restore the redox balance in the diaphragm and prevent the deleterious effects of CMV.

Methods Anesthetized rats were submitted for 24 hours to either spontaneous breathing while receiving $150 \mathrm{mg} / \mathrm{kg}$ NAC (SBNAC) or saline (SBSAL) or to CMV while receiving $150 \mathrm{mg} / \mathrm{kg}$ NAC (MVNAC) or saline (MVSAL).

Results After 24 hours, diaphragm forces were significantly lower in MVSAL compared with all groups. Administration of NAC completely abolished this decrease such that forces produced in the MVNAC group were comparable with those of both SB groups. Protein oxidation was significantly increased in MVSAL $(+53 \%, P<0.01)$ and was restored in MVNAC. Diaphragm caspase-3 activity was significantly increased in MVSAL compared with SBSAL $(+279 \%, P<0.001)$. Caspase-3 activity was also increased in the MVNAC group $(+158.5 \%, P<0.01)$ but to a significantly lesser extent compared with that of MVSAL. Calpain activity was significantly increased after CMV $(+137 \%, P<0.001$ vs SBSAL), while it was similar to SB groups in the MVNAC group. Significant negative correlation was found between calpain activity and diaphragm tetanic force $(r=-0.48, P=0.02)$

Conclusions These data show that the administration of NAC was able to preserve the diaphragm from the deleterious effects of CMV. NAC inhibits the increase in oxidative stress and proteolysis and reduces the decrease in force generating capacity of the diaphragm.

\section{P204}

In vitro muscle contraction force measurements on isolated and entire rat diaphragms

C Armbruster, C Dassow, K Gamerdinger, J Guttmann, M Schneider, S Schumann

University Medical Center Freiburg, Germany

Critical Care 2010, 14(Suppl 1):P204 (doi: 10.1186/cc8436)

Introduction Inactivity of the diaphragmatic muscles during mechanical ventilation leads to atrophy and contractile dysfunction. Up to now, in vitro force measurements were performed only on single diaphragmatic muscle strips. Our intention was to find out how mechanical and electrical stimulation influences the condition of the diaphragm as a whole organ. To determine the status of the diaphragm, muscle contraction forces were measured on entire rat diaphragms.

Methods We used an earlier described bioreactor [1] as the cultivation and measurement device for the whole rat diaphragm. The bioreactor consists of a pressure chamber and a supply chamber that are separated by a very flexible and soft membrane [2]. On this membrane the sample diaphragm is placed. By application of certain gas volumes (0 to $1.5 \mathrm{ml}$ ) in the pressure chamber, diaphragms are deflected to various levels of pretension. Diaphragms were electrically stimulated at each deflection level 10 times $(750 \mathrm{~ms}$ duty cycle, $100 \mathrm{~ms}$ stimulation time, $5 \mathrm{~ms}$ pulse width, $200 \mathrm{~Hz}$ frequency). Pressure changes caused by muscle contraction were recorded inside the pressure chamber and muscle contraction forces were calculated. After initial force measurements, diaphragms were exposed for 6 hours to one of four different treatments: nonstimulated storage (control), cyclic mechanical deflection, electrical stimulation every 20 minutes, combination of cyclic deflection and electrical stimulation. After 6 hours another force measurement was performed. Supernatants were collected after 6 hours and investigated for IL-6 activity.

Results Depending on the level of deflection of the diaphragms, muscle contraction force increased from $0.1 \mathrm{~N}$ (volume $0.6 \mathrm{ml}$ ) to $0.7 \mathrm{~N}$ (volume $1.5 \mathrm{ml}$ ). A larger pretension of the diaphragm resulted in larger muscle contraction force. After treatment, muscle contraction force decreased in all groups. Muscle contraction force was smallest in the passive control group $(0.05 \mathrm{~N})$, larger and similar in the electrically stimulated $(0.1 \mathrm{~N})$ and combination $(0.09 \mathrm{~N})$ groups and largest in the mechanically deflected group (0.15 N). IL-6 activity increased after 6 hours of treatment.

Conclusions We conclude that it is possible to perform force measurements on whole rat diaphragms in our in vitro model. Additionally, the diaphragms can kept alive for $>6$ hours to apply different stimulation 
treatments. The diaphragmatic muscle force generation depends on the pretension level of the diaphragm as well as on the treatment history. References

1. Schumann et al: J Biomed Mater Res B Appl Biomater 2008, 86B:483-492.

2. Armbruster et al:: J Biomed Mater Res B Appl Biomater 2009, 91:700-705.

P205

Useful of implementation of neurally adjusted ventilatory assist in critically ill patients

MJ Sucre, A De Nicola

San Leonardo Hospital, Castellamamre di Stabia, Italy

Critical Care 2010, 14(Suppl 1):P205 (doi: 10.1186/cc8437)

Introduction Neurally adjusted ventilatory assist (NAVA) is a new mode of assisted mechanical ventilation that uses the signal obtained from electromyography activity of the diaphragm (EAdi) to control the mechanical ventilator that delivers assist in proportion to the patient's respiratory drive. This study evaluated the monitoring Edi on conventional mode response, during the switch to pressure support assist and during the change to NAVA: a mode of predicting weaning from ventilation.

Methods Sixteen adult patients ventilated with SERVO-I (Maquet) on pressure control or Bivent were randomized; we placed the specialized naso/orogastric tube (Edi catheter) and watched the Edi signal. PSV was set to obtain a Vt/kg of 6 to $8 \mathrm{ml} / \mathrm{kg}$ with an active inspiration. Were studied the peak airway pressure $\left(\mathrm{P}_{\mathrm{aw}}\right)$ and breathing pattern. Were registered the Edi minimum and Edi maximum for determining PEEP and peak pressure, respectively, $\mathrm{Vt}$, respiratory rate, $\mathrm{FiO}_{2}$ and blood gases serially.

Results We observed synchrony between Edi signal and ventilator breaths in pressure control or Bivent, and when the patients have started spontaneous breaths ( $T$ signal) we move to pressure support. Low Edi minimum (0 to 1 ) was associated with overdistending the diaphragm and required decreasing the PEEP, contrary to high Edi minimum that was associated with higher tonic activity and dictated to raise the PEEP.

During NAVA, the pressure delivered was proportional to the Edi. The NAVA level was continuously readjusted in proportion to the predicted inspiratory effort from the Edi signal. At the highest assist level, we found lower $\mathrm{Vt} / \mathrm{kg}(6.1 \pm 3 \mathrm{ml} / \mathrm{kg}$ vs $8.1 \pm 1.8, P<0.001)$, and higher breathing frequency $(19 \pm 6.9$ vs $11 \pm 7, P<0.001)$ and peak EAdi $(11.8 \pm 8.5$ vs $8.1 \pm$ $7.7, P<0.002)$ in NAVA than in PSV.

Conclusions The asynchrony during mechanical ventilation increases the cost of care, length of days on mechanical ventilation, and other morbidities associated with increased ICU stay. The Edi monitoring helps to understand other modes of ventilation and has been a great tool to decide the timing of a switch to NAVA and predict an early weaning. Systematically increasing the NAVA level reduces the respiratory drive, unloads respiratory muscles, and offers a method to determine an assist level that results in sustained unloading, low Vt, and stable respiratory function when implemented for 3 hours. NAVA gives us the opportunity to augment these patients' own drive to breathe enough to recover more quickly.

\section{References}

1. Brander L, et al: Chest 2009, 135:695-703.

2. Sinderby C, Beck J: Clin Chest Med 2008, 29:329-342.

P206

Twenty-four-hour neurally adjusted ventilatory assist and volume cycled ventilation have similar effects on respiratory neuromuscular function in a porcine model of fecal peritonitis

R Schroeder', KA Ackermann', D Tuchscherer', S Djafarzadeh'1,

WJ Z゙'Graggen', C Sinderby², SM Jakob', J Takala', L Brander'

'University Hospital of Bern, Switzerland; '2St Michael's Hospital, University of Toronto, Canada

Critical Care 2010, 14(Suppl 1):P206 (doi: 10.1186/cc8438)

Introduction Sepsis and muscle inactivity may lead to neuromuscular dysfunction. Neurally adjusted ventilatory assist (NAVA) converts diaphragm electrical activity (EAdi) into airway pressure (Paw) and preserves respiratory muscle activity [1]. We hypothesised that respiratory neuromuscular function would be better preserved with NAVA compared with volume cycled ventilation (VCV) in early sepsis.
Methods Twenty-eight pigs (40.0 $(37.6 ; 41.8)$ kg; median (quartiles)) were randomized ( $n=7$ per group) to fecal peritonitis or nonseptic controls ventilated for 24 hours with either NAVA or VCV (nonparalyzed, Vt 6 to $8 \mathrm{ml} / \mathrm{kg}$, rate adjusted to suppress EAdi). Fluids and norepinephrine (NE) were used based on protocols to keep mean arterial pressure (MAP) $>50 \mathrm{mmHg}$. Before sepsis and after 24 hours, transcutaneous supramaximal stimulations of the cervical phrenic nerve (tPNS) and expiratory occlusion manoeuvres (eOM) were performed. Latency and amplitude of diaphragm compound muscle activity potentials (CMAP) were measured during tPNS; maximal deflections of Paw $(\triangle P a w)$, esophageal $(\triangle P e s)$, and transdiaphragm ( $\triangle \mathrm{Pdi}$ ) pressures were measured during repetitive tPNS (40 Hz, 100 stimuli) and eOM.

Results Hemodynamic parameters were not different among groups before sepsis and remained stable in controls. MAP did not change in septic NAVA but decreased in septic VCV animals from $91(74 ; 95) \mathrm{mmHg}$ before sepsis to $58(57 ; 68) \mathrm{mmHg}$ at 24 hours ( $P<0.001$, MANOVA t-g interaction). The heart rate increased $(P<0.001)$ and stroke volume decreased $(P=$ 0.034 ) in both septic groups. Fluid balance was not different among septic groups. Three septic NAVA and six septic VCV animals received NE. CMAP latency and amplitude, as well as $\triangle \mathrm{Paw}, \triangle \mathrm{Pes}$, and $\triangle \mathrm{P}$ di were not different among all groups during repetitive tPNS and eOM before sepsis and after 24 hours $(P=N S)$.

Conclusions Respiratory neuromuscular function is not affected by the mode of ventilation during the first 24 hours of abdominal sepsis. Early effects of sepsis on neuromuscular function are not reflected in respiratory muscle strength. NAVA may reduce the need for hemodynamic support in early sepsis.

Acknowledgements Supported by SNF 3200B0-113478; Stiftung Anästhesiologie und Intensivmedizin, Bern.

Reference

1. Brander et al:: Chest 2009, 135:695-703.

P207

Effects of conventional and closed-loop neurally adjusted ventilatory assist in intubated spinal cord injured patients J Spahija', M De Marchie², KTruflandier', A Moga ${ }^{3}$, P Bellemare', S Delisle', M Albert ${ }^{1}$

'Hôpital du Sacré-Coeur de Montréal, Canada; 'Jewish General Hospital, Montreal, Canada; ${ }^{3}$ McGill University, Montreal, Canada Critical Care 2010, 14(Suppl 1):P207 (doi: 10.1186/cc8439)

Introduction This study aimed to evaluate the effect of high versus low tidal volumes (VT) during volume control (VC) ventilation and neurally adjusted ventilatory assist (NAVA) in spinal cord injured (SCI) patients. We hypothesized that VC with higher VT would significantly unload and deactivate the diaphragm, whereas NAVA would not.

Methods Seven intubated C3 to C7 SCl patients (age: $28 \pm 12$ years) were studied. Using VC (Servo300), VT was increased progressively (not exceeding $40 \mathrm{CmH}_{2} \mathrm{O}$ or 2 I VT) (high volume) and then lowered until 6 $\mathrm{ml} / \mathrm{kg}$ or intolerance (low volume). NAVA was then progressively adjusted targeting similar peak pressures. Finally subjects received 15 minutes each of: high VC, low VC, high NAVA, and low NAVA in randomized order.

Results Despite comparable increases in peak airway pressure (Pmo), VT increased with VC but was unaltered with NAVA. Inspiratory and total breath durations were lower and respiratory rate higher with NAVA compared with VC during both assist levels. VC resulted in significant diaphragm unloading and deactivation whereas both were maintained during high and low NAVA. Complete diaphragm deactivation in more than $80 \%$ of breaths was observed during VC in five of the patients.

Table 1 (abstract P207)

\begin{tabular}{lcccccc}
\hline & $\begin{array}{c}\text { Low } \\
\text { volume }\end{array}$ & $\begin{array}{c}\text { High } \\
\text { volume }\end{array}$ & $\begin{array}{c}\text { Low } \\
\text { NAVA }\end{array}$ & $\begin{array}{c}\text { High } \\
\text { NAVA }\end{array}$ & $\begin{array}{c}P \text { v V } \\
\text { vs NAVA level }\end{array}$ \\
\hline VT (I) & $0.6 \pm 0.2$ & $1.3 \pm 0.2$ & $0.6 \pm 0.2$ & $0.6 \pm 0.2$ & $<0.001$ & $<0.001$ \\
Peak Pmo $\left(\mathrm{cmH}_{2} \mathrm{O}\right)$ & $19.2 \pm 3.2$ & $34.9 \pm 2.6$ & $18.4 \pm 5.9$ & $31.5 \pm 5.9$ & NS & $<0.001$ \\
Mean EAdi $(\%)$ & $3.3 \pm 4.3$ & $0.8 \pm 1.7$ & $6.8 \pm 4.2$ & $4.6 \pm 3.0$ & 0.004 & 0.03 \\
Mean Pdi $\left(\mathrm{CmH}_{2} \mathrm{O}\right)$ & $2.5 \pm 3.1$ & $-1.3 \pm 2.1$ & $4.3 \pm 3.4$ & $2.6 \pm 2.6$ & 0.02 & 0.009 \\
\hline
\end{tabular}

$\mathrm{VT}$, tidal volume; EAdi, diaphragm activation; Pdi, transdiaphragmatic pressure. 
Conclusions VC ventilation results in significant diaphragm unloading and deactivation especially at high assist levels, whereas diaphragm activation (EAdi) is maintained with NAVA.

\section{P208}

A level of neurally adjusted ventilatory assist corresponds to low levels of continuous positive airway pressure and pressure support ventilation in patients preparing to be extubated from mechanical ventilation

Y Klai-on, A Navasakulpong, A Wattanathum

Phramongkutklao Hospital, Bangkok, Thailand

Critical Care 2010, 14(Suppl 1):P208 (doi: 10.1186/cc8440)

Introduction Neurally adjusted ventilator assist (NAVA) is a new mode of mechanical ventilation which delivers ventilator assist in proportion to electrical activity of the diaphragm (EAdi), as assessed by trans-esophageal electromyography, and an adjustable supporting level called the NAVA level. NAVA can be used as a supporting and weaning mode of ventilation [1-3]. However, a level of NAVA corresponding to continuous positive airway pressure (CPAP) and pressure support ventilation (PSV) in patients ready for extubation has not been established.

Methods We carried out a prospective study in the medical ICU at Phramongkutklao Hospital involving patients who were considered by their physicians to be ready for extubation. Patients initially received PSV of $5 \mathrm{cmH}_{2} \mathrm{O}$, PEEP of $5 \mathrm{cmH}_{2} \mathrm{O}$, and $\mathrm{FiO}$ of $40 \%$ for 15 minutes (PS). Subsequently, each patient received a trial of NAVA mode with PEEP of $5 \mathrm{cmH}_{2} \mathrm{O}$, and $\mathrm{FiO}_{2}$ of $40 \%$ for 15 minutes (NAVA), and, finally, a 30-minute spontaneously breathing trial without ventilatory support (SBT). During these trials, the minute volume (MV), respiratory rate, EAdi, and airway pressure were measured. We determined the corresponding NAVA level with the level of PSV of $5 \mathrm{cmH}_{2} \mathrm{O}$ and PEEP of $5 \mathrm{cmH}_{2} \mathrm{O}$ using the NAVA level matching MV $( \pm 10 \%)$ received from the PSV.

Results The NAVA level corresponding to the PSV of $5 \mathrm{cmH}_{2} \mathrm{O}$ and PEEP of $5 \mathrm{cmH}_{2} \mathrm{O}$ was $0.52 \mathrm{cmH}_{2} \mathrm{O} / \mu \mathrm{V}(0.2$ to 1.2). The airway pressure during NAVA was $5.97 \mathrm{cmH}_{2} \mathrm{O}$ (3.2 to 8) above PEEP level. The peak EAdi was not significantly different between the PS, NAVA, and SBT $(11.58,12.06$ vs $12.65 \mu \mathrm{V} ; P=0.6)$. Also, there was no significant difference in MV during the PS, NAVA, and SBT.

Conclusions NAVA mode can be used as a weaning mode by decreasing the NAVA level. The reduced level of NAVA to $0.5 \mathrm{cmH}_{2} \mathrm{O} / \mu \mathrm{V}$ did not load respiratory muscle more than a low level of PSV and SBT. Thus, a NAVA level of $0.5 \mathrm{cmH}_{2} \mathrm{O} / \mathrm{\mu V}$ might be the level that is safe to discontinue ventilator support in patients without problems for resuming spontaneous breathing.

\section{References}

1. Navalesi P, et al:: Curr Opin Crit Care 2003, 9:51-58.

2. Sinderby C, et al: Chest 2007, 131:711-717.

3. Beck J, et al: Pediatr Res 2007, 61:289-294.

\section{P209}

Towards partially automated ventilation: adapting decision-making according to medical preferences

S Lozano-Zahonero', D Gottlieb², J Guttmann², K Möller'

'Furtwangen University, VS-Schwenningen, Germany; ${ }^{2}$ University Hospital

Freiburg, Germany

Critical Care 2010, 14(Suppl 1):P209 (doi: 10.1186/cc8441)

Introduction A novel methodology is proposed to adapt decisionmaking strategies into our fuzzy-based expert system, AUTOPILOT-BT [1]. The special features of this approach are: knowledge from clinical experts can be extracted in a setup simulating daily ICU routine; an automated process serves to obtain the required information and to create new fuzzy sets; and the fuzzy controller from AUTOPILOT-BT employs the newly derived fuzzy rules. Thus, the knowledge base can easily be modified and the resulting mechanical ventilation therapies may be adapted to individual preferences of the clinician.

Methods The methodology consists of: (i) Acquisition of decision-making strategies from single or groups of anesthesiologists. This can either be done with a questionnaire or with a PC-based program simulating the doctors every day situation in diagnosis. (ii) Definition of fuzzy membership

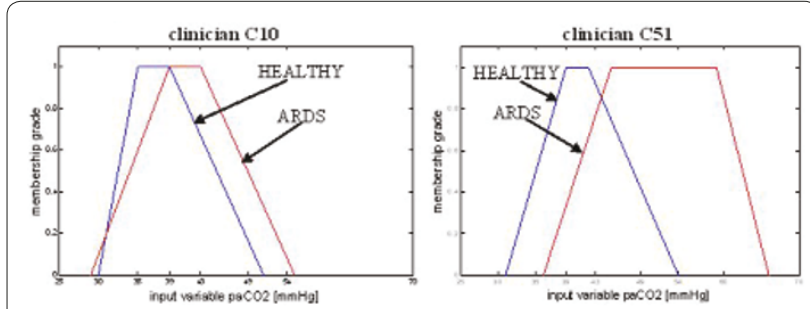

Figure 1 (abstract P209). Normal ventilation for healthy vs ARDS patients fuzzy sets for $\mathrm{paCO}_{2}$ given from two clinicians: (left) $\mathrm{C10}$, (right) $\mathrm{C} 51$.

functions based on the acquired knowledge (fuzzification of the input). (iii) Construction of fuzzy inference rules and defuzzification or calculation of change in ventilator settings (controller action). This approach allows implementing clinician-dependent decision-making that reflects individual preferences. Thus guidelines from EBM can be used but as well ICU specifics can be realized, for example different levels of acceptable hypercapnia in patients with acute respiratory distress syndrome (ARDS).

Results Exemplarily Figure 1 shows, for healthy and ARDS lungs, the difference between two fuzzy sets for our $\mathrm{paCO}_{2}$ controller given from two clinicians (C10 and C51) with different expertise in mechanical ventilation. With the newly designed fuzzy sets, our AUTOPILOT-BT reacts according to the clinicians' preferences, but still minimizes the time in which the patient is not ventilated within the specified limits.

Conclusions The system automatically implements the know-how of medical experts in ventilation management if the clinicians are willing to interact with the query system. The resulting strategy is mainly influenced by the expertise, experience and demands of the clinician. Thus the AUTOPILOT-BT, has the potential to select established guidelines or to adapt the system to modified ventilation therapies. Further clinical trials will test the actual clinical efficiency of different controllers.

References

1. Lozano S, et al:: Tech Health Care 2008, 16:1-11.

P210

Postoperative pulmonary complications in adult elective surgery patients in the US: severity, outcomes and resources use

WL Linde-Zwirble', JD Bloom², RS Mecca ${ }^{3}$, DM Hansel/ ${ }^{4}$

'ZD Associates LLC, Perksaie, PA, USA; ${ }^{2}$ Covidien, Boulder, CO, USA; ${ }^{3}$ University of California at Irvine, Orange, CA, USA; ${ }^{4}$ Massachusetts General Hospital, Boston, MA, USA

Critical Care 2010, 14(Suppl 1):P210 (doi: 10.1186/cc8442)

Introduction Postoperative pulmonary complications (PPCs) are associated with adverse outcomes and added resource use. However, PPCs vary in severity, and so may have very different outcomes.

Methods We selected adult elective surgical cases from the Premier database for 2008. The PPC conditions identified were: pneumonia; respiratory failure; bronchospasm; tracheobronchitis; pleural effusion; pulmonary collapse; ARDS; and pneumothorax. We stratified PPC cases into levels: those with bronchospasm and no other PPC (BS), those with respiratory failure (RF) and all remaining (NRF). We examined incidence, length of stay, hospital mortality, and total cost. US national projections were made using Premier supplied projection weights. We calculated incremental resource use and outcomes by comparing cases with and without PPCs for each surgical condition and summarizing across all conditions.

Results There were 738,039 cases in our cohort. At least one PPC was identified in 85,435 . BS occurred on $6.4 \%$ of cases $(45,005)$, NRF in $3.8 \%$ $(27,796)$ and RF in $1.7 \%$ of cases $(12,634)$. BS was not associated with an increase in the risk of death (one added death per 5611 cases), but had significant increases in resource use: one added ICU admission per 14 cases; one added ICU day per five cases; and one added hospital day and \$1,563 added cost per case. NRF was associated with a modest increase in risk of death (one added death per 157 NRF cases) and substantial increases in resource use: one added ICU admission for every six cases; 0.66 ICU days per case; and three hospital days and $\$ 5,771$ added cost per NRF case. RF was associated with greatly increased risk of death (one added death per 
10 cases) and larger increases in resource use: one added ICU admission per two cases; 5 ICU days, 8 hospital days and \$24,000 in added cost per RF case. Projecting to the US population there were 583,300 PPC cases, associated with 9,500 added deaths, 92,200 added ICU admissions, 584,200 added ICU days, 867,400 added floor days and $\$ 3.42$ billion US in added costs. PPC add $\$ 717$ to the average cost of elective surgery in the US.

Conclusions PPCs are very common, occurring in one in eight elective postoperative patients. While BS was not associated with added deaths, all three PPC strata were associated with substantial increase in resource use. Better strategies for the prevention and management of PPCs could lead to greatly improved outcomes and substantial savings.

\section{P211}

Increased duration of mechanical ventilation is associated with decreased diaphragmatic force

G Hermans, A Agten, D Testelmans, M Decramer, G Gayan-Ramirez

UZ Leuven, Belgium

Critical Care 2010, 14(Suppl 1):P211 (doi: 10.1186/cc8443)

Introduction Respiratory muscle weakness is an important risk factor for prolonged mechanical ventilation, and may be part of critical illness related polyneuropathy and myopathy. Animal data also strongly point to atrophy and weakness of the diaphragm due to mechanical ventilation itself, called ventilator-induced diaphragmatic dysfunction. Recently, measuring transdiaphragmatic pressure following magnetic stimulation (TwPdi BAMPS) was introduced in the ICU to evaluate diaphragm function $[1,2]$. We aimed to evaluate reproducibility of twitch TwPdi BAMPS in critically ill, mechanically ventilated patients. We also aimed to describe the relationship between TwPdi and duration of mechanical ventilation.

Methods Prospective observational study in a medical ICU of a university hospital. TwPdi BAMPS was measured in critically ill and mechanically ventilated patients. Briefly, the phrenic nerves were stimulated bilaterally from the anterior approach, at the posterior border of the sternocleidomastoid muscle, at the level of the cricoids using two figureof-eight $45 \mathrm{~mm}$ magnetic coils (Magstim, Dyfed, Wales) and a bistim (Magstim, Dyfed, Wales). A custom-built occlusion valve was used to create isometric conditions during stimulation. Oesophageal and abdominal pressure changes were measured using balloon catheters (UK Medical, Sheffield, UK) inserted through the nose after local anaesthesia.

Results Nineteen measurements were made in a total of 10 patients at various intervals after starting mechanical ventilation. In seven patients, measurements were made on at least two occasions with a minimal interval of 24 hours. The between-occasion coefficient of variation of TwPdi was 9.7\%, which is comparable with data from healthy volunteers. Increasing duration of mechanical ventilation was associated with a logarithmic decline in TwPdi $(R=0.69, P=0.038)$. This association was also found when cumulative time on pressure control ventilation $(R=0.71, P=$ $0.03)$ and pressure support ventilation $(P=0.05, R=0.66)$ were considered separately, as well as for cumulative dose of propofol $(R=0.66, P=0.05)$ and piritramide $(R=0.79, P=0.01)$

Conclusions Increased duration of mechanical ventilation is associated with a logarithmic decline in diaphragmatic force. These findings are compatible with the concept of ventilator-induced diaphragmatic dysfunction. The observed decline may also be due to the cumulative dose of sedatives/analgesics or other co-factors, such as sepsis.

\section{References}

1. Watson AC, et al: Crit Care Med 2001, 29:1325-1331

2. Laghi F, et al:: Am J Respir Crit Care Med 2003, 167:120-127.

\section{P212}

Haemodynamics and volhaemic stage in patients with respiratory disorders to thermal injury

K Shatovkin, I Shlyk

Djanelidze Research Institute of Emergency Medicine, Saint-Petersburg, Russia Federation

Critical Care 2010, 14(Suppl 1):P212 (doi: 10.1186/cc8444)

Introduction The aim of the investigation was to study the parameters of haemodynamics, gas exchange and volhaemic status in patients with severe thermal injury.
Methods The trial has covered 30 injured patients aged from 21 to 60 years with 25 to $78 \%$ skin burns and $\mathrm{PO}_{2} / \mathrm{FiO}_{2}<300$. Patients were randomized into two groups. The first group was $n=13, \mathrm{PO} / \mathrm{FiO}_{2}<300$, 24 to $72 \mathrm{~h}$ after burn. The second group was $n=17, \mathrm{PO}_{2} / \mathrm{FiO}_{2}<300,4$ to 11 days after burn. Injured persons were subjected to evaluation of cardiac index (CI), intrathoracic blood volume index (ITBI), extravascular lung water index (ELWIO and pulmonary vascular permeability index (PVPI) by single transpulmonary thermodilution (PiCCOplus; Pulsion Medical Systems, Germany), calculation of oxygen delivery $\left(\mathrm{DO}_{2}\right)$, and colloidal osmotic pressure of plasma. Grade of pulmonary damage was evaluated by Murrey, severity of dispragia by SOFA. Correlation analysis was performed using Pearson and Spearman criteria $(r ; P)$. Differences were significant at $P<0.05$.

Results Hypoxaemia $\left(\mathrm{PO}_{2} / \mathrm{FiO}_{2}=259 \pm 19\right)$ in the injured of the first group was developing at the background of reduction of $\mathrm{Cl}=3.2 \pm 0.5 ; \mathrm{ITB}=751$ $\pm 114 ; \mathrm{DO}_{2}=596 \pm 124(r=-0.92 ; P=0.02)$. ELWI level was normal $(7.3 \pm$ 0.8). In the second group, reduction of $\mathrm{PO}_{2} / \mathrm{FiO}_{2}$ has been developing at the background of burn sepsis (average SOFA point $=7.0 \pm 1.9$, Murrey $=1.6 \pm 0.4)$ and was caused by increasing of ELWI up to $9.0 \pm 1.4(r=$ $-0.66 ; P=0.002)$ and correlated with PVPI $(r=0.57 ; P=0.01)$. There was no statistically significant correlation ELWI with ITBI and colloidal osmotic pressure of plasma $(r=0.13, r=-0.42)$.

Conclusions Gas exchange disorders in patients of the first group were caused by lack of perfusion and misbalance between oxygen delivery and demand. Reduction of oxygenation index in patients from the second group was accompanied by ELWI at the background of alteration of intravascular penetration and sepsis.

P213

Invasive mechanical ventilation in cancer patients: prior non-invasive ventilation is a poor prognostic factor

A Meert, T Berghmans, N Nayer, M Paesmans, E Markiewicz, M Hardy,

J Sculier

Institut Jules Bordet, Bruxelles, Belgium

Critical Care 2010, 14(Suppl 1):P213 (doi: 10.1186/cc8445)

Introduction Prior non-invasive ventilation (NIV) is associated with an increased mortality in patients with haematological malignancies and acute respiratory failure treated by invasive mechanical ventilation (IMV).

Methods We have assessed whether NIV failure is an independent prognostic factor for hospital discharge in a general cancer population treated by IMV. One hundred and six patients with solid tumours and 58 patients with haematological malignancies were eligible for this retrospective study; 41 were treated by NIV before IMV.

Results The main indications for mechanical ventilation were sepsis/ shock (35\%), acute respiratory failure (33\%), cardiopulmonary resuscitation (16\%) and neurologic disease (10\%). Respectively, 35\%, 28\% and 24\% of the patients were extubated, discharged from the ICU and from the hospital. For patients treated with NIV prior to IMV, the rates were $22 \%$, $17 \%$ and $10 \%$, respectively. In multivariate analysis, three variables were independently associated with a decreased probability of being discharged from the hospital: NIV use before IMV (OR $=0.30,95 \% \mathrm{Cl}: 0.09$ to $0.95 ; P=0.04)$; leucopenia $(\mathrm{OR}=0.21,95 \% \mathrm{Cl}: 0.06$ to $0.77 ; P=0.02)$ and serum bilirubin $>1.1 \mathrm{mg} / \mathrm{dl}(\mathrm{OR}=0.38,95 \% \mathrm{Cl}: 0.16$ to $0.94 ; P=0.04)$.

Conclusions NIV failure before IMV is an independent poor prognostic factor in cancer patients treated by IMV.

P214

Laryngeal angioedema: do we need a new treatment algorithm in the ER?

K Stelter ${ }^{1}$, A Leunig 1 , M Jacob ${ }^{1}$, M Bas $^{2}$

'Ludwig Maximilians University, Munich, Germany; ${ }^{2}$ Technical University, Munich, Germany

Critical Care 2010, 14(Suppl 1):P214 (doi: 10.1186/cc8446)

Introduction Laryngeal angioedema (AE) could be life-threatening and is frequently due to allergic reactions. Other causes, that is bradykininmediated conditions, have to be taken into account. The aim of our survey is to provide information on the incidence, diagnosis and potentially new treatment options for laryngeal AE. 


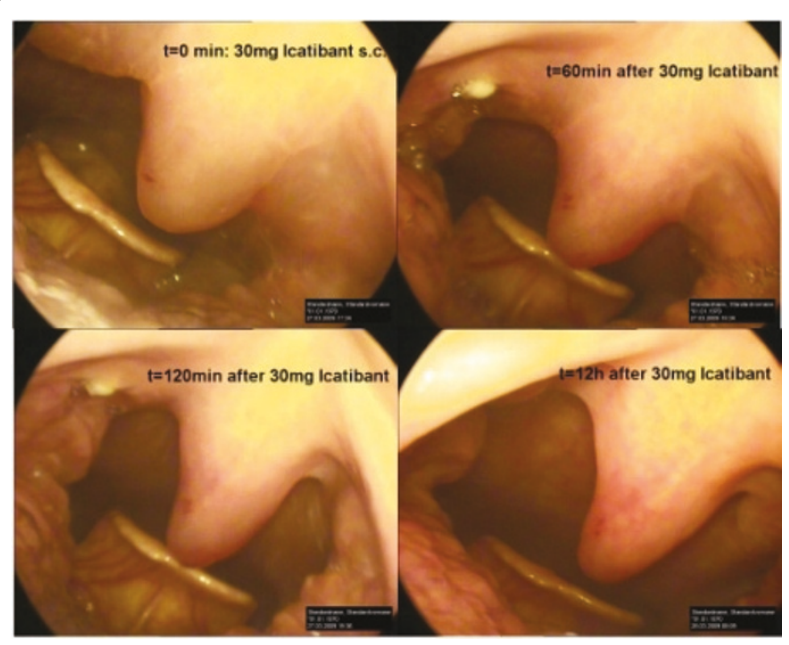

Figure 1 (abstract P214). Effect of $30 \mathrm{mg}$ icatibant s.c. on a laryngeal edema due to ACEI.

Methods The data for 102 patients who were treated for swelling of the larynx at the Department of Otorhinolaryngology of Ludwig-MaximiliansUniversity of Munich from 2004 to 2008 were evaluated in a retrospective survey.

Results Causes of laryngeal AE were in 26 patients an allergic reaction, in 33 cases were due to radiotherapy after cancer, 32 patients took an angiotensin-converting enzyme inhibitor (ACEl), one patient had a hereditary angioedema (HAE) and in 10 patients are unknown. All patients independent from the underlying cause were treated with high-dose intravenous steroids, 69 patients with antihistamines, 33 with epinephrine (inhalation or i.m.), two patients with ACEl-induced AE were successfully treated with icatibant. Ten patients needed an intubation, 11 a tracheotomy, one patient an emergency coniotomy to preserve the airway. All 11 tracheotomy patients suffered from cancer. Nine out of 10 intubated patients took an ACEI and were extubated on the ICU after 2 to 7 days.

Conclusions According to our experience $80 \%$ of the patients with laryngeal swelling and upper airway obstruction react well to the standard therapy with steroids, epinephrine and antihistamines, indicating that the AE is histamine induced. In contrast these drugs have almost no effect on bradykinin-induced angioedema; that is, AE induced by ACEI. There is preliminary evidence that the bradykinin $B_{2}$ receptor antagonist icatibant that is approved for treatment of HAE type I and II may also be effective in ACEl-induced edema.

\section{P215}

Comparison of two insertion techniques of the ProSeal laryngeal mask airway: standard versus $90^{\circ}$ rotation in pediatric patients M Yun', J Hwang', A Oh', M Han', $\mathrm{H} \mathrm{Kim}^{2}, \mathrm{H} \mathrm{Na}^{1}$, S Lee ${ }^{2}$

'Seoul National University Bundang Hospital, Seongnam, Republic of Korea; ${ }^{2}$ Seoul National University Hospital, Seoul, Republic of Korea

\section{Critical Care 2010, 14(Suppl 1):P215 (doi: 10.1186/cc8447)}

Introduction This study compared two insertion techniques of the ProSeal laryngeal mask airway in pediatric patients.

Methods A total of 92 pediatric patients (American Society of Anesthesiologists physical status I or II; age 3 to 12 years) undergoing ophthalmologic surgery were randomly allocated to the standard or rotational technique groups. In the standard technique group $(n=46)$, ProSeal laryngeal mask airway insertion was performed by a single experienced user using digital manipulation. In the rotational technique group $(n=46)$, the ProSeal laryngeal mask airway was rotated counterclockwise through $90^{\circ}$ in the mouth and advanced until the resistance of the hypopharynx was felt, and then straightened out in the hypopharynx $(n=80)$. The ease of insertion was assessed by the success rate at the first attempt. Heart rate and mean blood pressure were recorded 1 minute before and 1 minute after insertion. Postoperative complications were noted.

Results The success rate of insertion at the first attempt was higher for the rotational technique $(95.7 \%$ vs $76.1 \%, P<0.001)$. The overall success rate that is, successful insertion within three attempts - was 100\% for the both techniques. Systolic, diastolic and mean blood pressure and heart rate increased significantly with the standard technique $(P<0.001)$. Systolic blood pressure and heart rate increased significantly with the rotational technique $(P<0.01)$. The incidence of blood staining $(8.7 \%$ vs $23.9 \%, P=$ 0.048) was lower with the rotational technique.

Conclusions The rotational technique is more successful than the standard technique and is associated with less pharyngeal mucosal trauma, as evidenced by a lower incidence of mucosal bleeding.

\section{Reference}

1. Hwang JW, Park HP, Lim YJ, Do SH, Lee SC, Jeon YT: Comparison of two insertion techniques of ProSeal laryngeal mask airway: standard versus 90-degree rotation. Anesthesiology 2009, 110:905-907.

P216

Endotracheal intubation in the critically ill: complication rate and use of intubation aids

A Verstraete, K Colpaert, J Nollet, J Decruyenaere

Ghent University Hospital, Ghent, Belgium

Critical Care 2010, 14(Suppl 1):P216 (doi: 10.1186/cc8448)

Introduction Intubation in the ICU is associated with a high complication rate. No data exist about how frequently intubation aids are used in the ICU. In our ICU we have the availability of different intubation aids, and perform an annual training course on difficult intubation strategy. We wanted to evaluate the incidence of difficult intubation, together with the complication rate and the use of intubation aids in our ICU.

Methods We performed a prospective cohort study in a 56-bed academic tertiary care hospital of all patients requiring intubation during their ICU stay during a 5-month observation period in 2008. Standardized data forms were used to collect detailed information on the intubating physicians, supervisors, techniques, medications and complications.

Results We enrolled 120 patients requiring immediate intubation in $37.5 \%$, semi-urgent (<30 minutes) intubation in $50.8 \%$, and semi-elective intubation in $11.7 \%$. All intubations were successful. Difficult intubation was anticipated in $17.5 \%$ of all cases, although only $28 \%$ of these effectively proved to be difficult. Immediate intubation was possible in $74.2 \%$ of all cases, and difficult intubation (at least three attempts) occurred in $6.6 \%$. Furthermore, $4.2 \%$ of intubations required 10 minutes or more. Nonexperts experienced more complications, and performed successful intubation in only $37.7 \%$. One-third of all intubations were supervised by an intensive care physician, although this was not associated with a decrease of complication rate (65\% vs $57.5 \%$ ). Techniques most commonly used for difficult intubation management were change in intubator (48.4\%), change in patient position (29\%), BURP maneuver (19.3\%) and use of a Gum elastic bougie (19.3\%). Overall risk of complications occurring was $76.6 \%$. Hypoxia was most common (40\%), followed by hypotension (31.6\%), heart rhythm disorders (4.1\%) and oesophageal intubation (3.3\%). Neuromuscular blockade was used in all but one patient. Hypnomidate was used in $31.6 \%$ of intubations, preferentially in patients with shock. The rate of complications or mortality did not significantly differ according to the hypnotic drug used.

Conclusions Intubation in the ICU is frequently associated with complications, most commonly hypoxia and hypotension. The use of the Gum elastic bougie is required in $19.3 \%$ of cases, and results in achieving a $100 \%$ successful intubation rate.

\section{P217}

Intensity and adequacy of laryngoscope light in seven intensive care units

J Hodd, S Gupta, N Rahman, P Young

Cleveland Clinic, Cleveland, OH, USA

Critical Care 2010, 14(Suppl 1):P217 (doi: 10.1186/cc8449)

Introduction The objective of the study was to determine the adequacy of illumination of laryngoscopes in seven critical care units (CCU) (two 


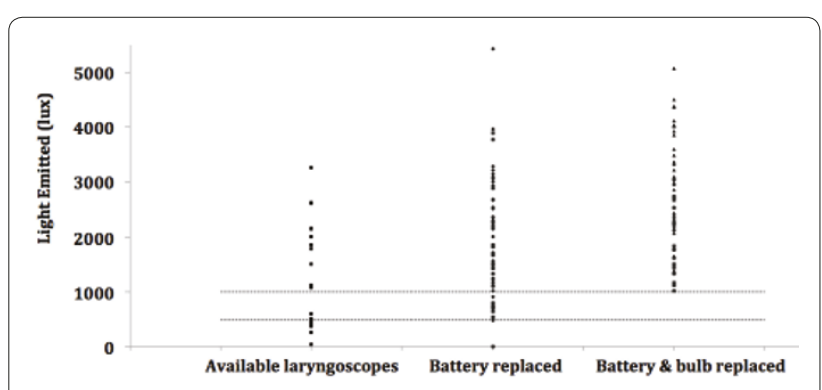

Figure 1 (abstract P217). Laryngoscope illumination.

medical, three surgical, two neurosurgical). Laryngoscopes in the Cleveland Clinic are routinely checked twice daily by healthcare workers. Confirmation of the presence of bulb illumination in ambient room light, however, may not be a sufficient test. In the CCU environment successful tracheal intubation may be time sensitive, and failure of adequate illumination of the larynx impedes visualization. Adequacy of lighting is subjective; however, the Health and Safety Executive suggests 500 Lux as a minimum for work requiring the perception of fine detail [1].

Methods In a single-day spot audit, all clinically available laryngoscopes were evaluated for brightness. Battery voltage was measured with a voltmeter (Innova 3320; Fountain Valley, CA, USA), then the batteries were replaced for new prior to re-measuring of illumination. The bulb was then replaced for new and the illumination measured again. Illumination measurements were taken from tip of the blade at $90^{\circ}$ using a luxometer (Model CA813; AEMC instruments, Foxborough, MA, USA) in a lightproof box.

Results Forty-six laryngoscopes were tested. All had traditional vacuum incandescent bulbs. Twelve (26\%) fell below 1,000 Lux and six (13\%) fell below the 500 Lux minimum. The failures were corrected by battery replacement in 25\% and by bulb replacement in the remaining $75 \%$ (see Figure 1). Conclusions Simply checking laryngoscopes for the presence of illumination on a regular basis is insufficient to ensure best or even adequate function. Poor function is as frequently related to bulb dysfunction as battery fatigue. Institutions should consider quality control and maintenance programs or consider more advanced laryngoscopic lighting (for example, LED or halogen bulbs).

References

1. Goodwin et al:: Anaesthesia 2006, 61:792-799.

\section{P218}

Airtraq: new device in patients at increased risk for difficult tracheal intubation

STomasino, ETricarico, C Gonano, E Zompicchiatti, L D'Orlando

ASS n3 Alto Friuli, Tolmezzo, Udine, Italy

Critical Care 2010, 14(Suppl 1):P218 (doi: 10.1186/cc8450)

Introduction A novel single-use indirect laryngoscope, the Airtraq (A), has been demonstrated to be of promise in the normal and simulated difficult airway [1]. We compared the ease of awake intubation using the A against the fiberoptic bronchoscope (F) in patients at increased risk for difficult tracheal intubation [2].

Methods In this randomised, controlled, clinical trial we enrolled patients at increased risk for difficult tracheal intubation, undergoing surgical operations requiring tracheal intubation. With ethics committee approval and written, informed consent, patients were randomly assigned to Fibroscope-guided tracheal intubation ( $F$ group) or Airtraq-guided intubation (A group). All patients, maintained in spontaneous breathing all through the procedure, received awake intubation performed by one of three anesthetists expert in difficult airway management. All patients received a topical airway anesthesia with $2 \%$ lidocaine and total intravenous anesthesia (TIVA) performed with propofol c.i. with an effecter site concentration of $1.5 \mu \mathrm{g} / \mathrm{ml}$.

Results We enrolled 30 patients, 15 in the F group and 15 in the A group. All patients were successfully intubated in both groups. In group A we assessed a short time and a small amount of attempts of intubation with a statistically significant difference between the two groups $(P<0.05)$. No difference was noted between the two groups in hemodynamic setting, saturation, Ramsey score and airway-trauma-related side effects.

Conclusions Our experience demonstrated that the Airtraq could be used during awake sedations and may be a promising alternative device for difficult airway management as a valid alternative to the traditional fiberoptic bronchoscope.

\section{References}

1. Maharaj CH, Costello JF, Harte BH, Laffey JG: Evaluation of the Airtraq and Macintosh laryngoscopes in patients at increased risk for difficult tracheal intubation. Anaesthesia 2008, 63:182-188.

2. SIAARTI Study Group: Recommendations for airway control and difficult airway management. Minerva Anestesio/ 2005, 71:217.

\section{P219}

\section{Evaluation of a new tracheostomy technique for morbidly obese} patients on an intensive care unit

A Mallick', A Bodenham', C Waldmann², M Verch ${ }^{3}$, F Brettner ${ }^{4}$, J Diebolt ${ }^{5}$, M Meier $^{6}$

'Leeds General Infirmary, Leeds, UK; 2 The Royal Berkshire Hospital, Reading, UK: ${ }^{3}$ Herzchirurgie Heidelberg, Germany; ${ }^{4}$ H Barmherzige Bruder, Munich, Germany; ${ }^{5}$ Hôpital Haute Pierre, Strasbourg, France; ${ }^{6}$ University of Alberta Hospital, Edmonton, Canada

Critical Care 2010, 14(Suppl 1):P219 (doi: 10.1186/cc8451)

Introduction Tracheostomy in morbidly obese patients is generally performed by surgical technique. Although PDT has a low complication rate in nonobese patients, it is not technically possible to use standard PDT kits in morbidly obese patients with thick necks. The skin to trachea distance renders traditional-length tracheostomy tubes or dilators too short. Recently a tracheostomy kit (Uniperc, Smith Medicals, UK), designed for obese patients has become available. This kit consists of an S-shaped dilator and a long adjustable reinforced tracheostomy tube. The objective of this study was to evaluate the safety and complications of this new technique in morbidly obese patients.

Methods The study was approved and patients' relatives were informed of the use of the new device. A total of 32 (male:female 21:11) morbidly obese (BMI mean $36 \pm 4.3$ ) patients (aged 27 to 57 years) were enrolled. Patients were anaesthetised and ventilated with 100\% oxygen. PDT was carried out under endoscopic control with stomal dilation using the single stage S-shaped dilator and a reinforced adjustable flange tracheostomy tube size 7 to $9 \mathrm{~mm} \mathrm{ID}$ as required. Measurements were taken including the distance from skin to the trachea using the graduated introducer needle and the initial small dilator, neck circumference, and length of the tracheostomy tube at the skin. Complications including accidental extubation, paratracheal placement, pneumothorax, major or minor bleeding, hypoxia, transient hypotension, subcutaneous emphysema or death were recorded. Patients were followed up with the inner cannula changed every day.

Results Thirty-two patients underwent PDT using this new kit. Patients were ventilated for a mean of 10 days (range 3 to 37) prior to tracheostomy. The mean neck circumference was $46.3 \mathrm{~mm}$ (37 to 55). The depth of the trachea from skin was a mean of $39 \mathrm{~mm}$ (15 to 80). The operators had no problem in stomal dilation using the single-stage dilator. The length of the tracheostomy tube adjusted at the skin ranged from 8 to $20 \mathrm{~cm}$ with a mean of $12 \mathrm{~cm}$. No major complications were observed except two minor bleeding episodes and transient desaturation in one patient. The tracheostomy tube was decannulated after a mean of 25 days (6 to 82).

Conclusions Bedside PDT can be performed in morbidly obese ICU patients using this new single-stage dilator and the adjustable flange reinforced tracheostomy tube with a low rate of complications. Further studies with longer-term follow-up are required.

P220

Our percutaneous tracheostomy experiences using the rotation-

dilatation screw method

C Kaymak, N Ozcan, H Basar, A Ozcan

Ankara Training and Research Hospital, Ankara, Turkey

Critical Care 2010, 14(Suppl 1):P220 (doi: 10.1186/cc8452)

Introduction A tracheostomy procedure is indicated for urgent airway control, to avoid complications of lengthened endotracheal intubation 
and to make weaning easier. Today, percutaneous tracheostomy techniques are preferred to classical surgical tracheostomy and the use is spreading. Ciaglia's multiple dilatation technique, the Blue Rhino single dilatation technique, the Griggs dilatation forceps technique and the rotation-dilatation screw technique are the most preferred techniques for percutaneous tracheostomy $[1,2]$. The aim of this study is to retrospectively assess the techniques used for tracheostomy, tracheostomy time after intensive care admittance, length of intensive care stay and tracheostomy complications in our clinic.

Methods After approval of the hospital ethics committee, files of the patients on whom the tracheostomy was performed between 2007 and 2009 were evaluated retrospectively. Demographic data of the patients, tracheostomy time after intensive care admittance, length of total intensive care stay and related complications were registered.

Results Files of 35 patients were evaluated on whom tracheostomy was performed between 2007 and 2009. The male-to-female ratio was 17/18, mean age of the patients was $57.4 \pm 20.1$ years ( 16 to 81 years, min to max), mean intensive care stay was $33.4 \pm 26.4$ days ( 5 to 103 days, min to max). Mean tracheostomy time after intensive care admittance was $13 \pm 8$ days ( 2 to 45 days, min to max). The rotation-dilatation screw technique was used for 34 patients and the Griggs dilatation forceps technique was preferred for one of the patients. No complication was observed for any of the patients.

Conclusions Studies comparing the ease of performance, early and late complications and long-term results of different percutaneous tracheostomy techniques do not exist. Different advantage/disadvantage ratios are reported in literature related to early and late tracheostomy performance time after admittance. In our clinic practice, the decision for the tracheostomy time is different for each patient. The mean tracheostomy time shows our clinic prefers late tracheostomy. In the aspect of complications, we did not observe complications reported in the literature.

\section{References}

1. Ciaglia P, et al: Chest 1985, 87:715-719.

2. Sengupta N, et al.: Anesth Analg 2004, 99:188-192.

P221

Single-step vs balloon dilatation tracheostomy: a pilot study on

20 tracheostomies

L Perretta', S Biondi², I Guerri², N Maccarone², L Tutino', A Nella', M Linden',

R Cammelli', G Cianchi', M Bonizzoli', R Spina', G Zagli', A Peris'

'Careggi Teaching Hospital, Florence, Italy; 'Postgraduate School of

Anesthesia and Intensive Care, University of Florence, Italy

Critical Care 2010, 14(Suppl 1):P221 (doi: 10.1186/cc8453)

Introduction The Ciaglia Blue Rhino percutaneous dilatational tracheostomy (PDT) technique, introduced in 1998 as a modification of the original procedure described in 1985, has been indicated as procedure of choice (level 2C) because of its technical simplicity and short procedure time. Recently, a modified balloon dilatation technique (Ciaglia Blue Dolphin) has been introduced with the intention to increase feasibility and safety. Here we report our initial experience in Ciaglia Blue Dolphin tracheostomy in comparison with a prospective control group of Ciaglia Blue Rhino procedures, which are actually the goal-standard in our ICU.

Methods Twenty consecutively admitted patients in the Emergency Department ICU (Careggi Teaching Hospital, Florence, Italy) requiring percutaneous dilatational tracheostomy (PDT) were treated with Ciaglia Blue Dolphin (first 10, group D) and Ciaglia Blue Rhino (second 10, group R). Demographic, clinical and procedural data were collected. PDTs were all performed with bronchoscopic video guidance. The Mann-Whitney test was used to compare continuous variables.

Results Patients of the two groups were similar for mean age (51.2 and 53.4 years old, respectively), body mass index (25.1 vs 24.8 , respectively) and severity of illness (SAPS II: 44.3 vs 45.4, respectively). Group D showed a significant higher procedural duration (measured from tracheal puncture to tracheostomy tube positioning) than group $\mathrm{R}$ (4.3 minutes vs 2.6 minutes; $P<0.01$ ).

Conclusions Despite recent reports in literature, we did not found substantial advantage in the use of Ciaglia Blue Dolphin with respect to the wider accepted Ciaglia Blue Rhino. Moreover, the higher cost of the new kit should be taken into consideration. In our opinion, complications such as tracheal posterior wall injury, pneumothorax, and pneumomediastinum can be avoided using the bronchoscopic video guidance.
P222

Recruitment maneuver for prevention of alevolar derecruitment during percutaneous dilation tracheostomy

F Franchi, Al Di Chiaro, M Fiorillo, S Scolletta, P Mongelli, G Coratti, L Cubattoli

University of Siena, Italy

Critical Care 2010, 14(Suppl 1):P222 (doi: 10.1186/cc8454)

Introduction Alveolar derecruitment is an early complication of percutaneous dilational tracheostomy (PDT). The aim of this prospective study was to evaluate the efficacy of performing a recruitment maneuver (RM) before tracheostomy, in order to prevent the alveolar derecruitment in critically ill patients.

Methods We enrolled 16 patients admitted to the ICU between December 2008 and July 2009, with $\mathrm{PaO}_{2} / \mathrm{FiO}_{2}$ ratio $\leq 3$ who underwent PDT. We used the Ciaglia/Blue Rhino technique with endoscopic guidance throughout the procedure. Patients were ventilated before and during PDT on volumecontrolled mechanical ventilation (tidal volume of $6 \mathrm{ml} / \mathrm{kg}$ body weight) and $\mathrm{FiO}_{2}$ set at 1. Patients were randomized into two groups: MRa group (seven patients who underwent RM 10 minutes before PDT) and no-MRa group (nine patients without application of RM before PDT). RM was performed by imposition of continuous positive airway pressure (CPAP) of $40 \mathrm{CmH}_{2} \mathrm{O}$ for 40 seconds. We collected gas exchange, respiratory and hemodynamic data at four times: 1 hour before RM (T1), 5 minutes after RM (T2), 5 and 30 minutes after PDT (T3 and T4, respectively).

Results The average duration of the technique was similar in both groups: $20 \pm 3.5$ minutes (MRa) vs $17.5 \pm 3.2$ minutes (no-MRa). MR induced a statistically significant increase $(P<0.05)$ in $\mathrm{PaO} / / \mathrm{FiO}$, ratio of $\mathrm{MRa}$ patients at T2, T3, T4 (Table 1). We did not find significant differences of respiratory and hemodynamic data between groups; MR did not induce cardiovascular instability: the mean cardiac output was $5.2 \pm 2.2$ vs $4.9 \pm$ $1.5 \mathrm{l} / \mathrm{min}$ (MRa vs no-MRa, respectively).

Table 1 (abstract $\mathrm{P} 222$ ). $\mathrm{PaO}_{2} / \mathrm{FiO}_{2}$ in $\mathrm{MRa}$ and in no-MRa groups

\begin{tabular}{lcccc}
\hline $\mathrm{PaO}_{2} / \mathrm{FiO}_{2}$ & $\mathrm{~T} 1$ & $\mathrm{~T} 2$ & $\mathrm{~T}$ & $\mathrm{~T}$ \\
\hline MRa & $182.8 \pm 121.5$ & $464.1 \pm 119.7^{*}$ & $360 \pm 138.8^{*}$ & $360.4 \pm 79.7^{*}$ \\
no-MRa & $247.1 \pm 121.9$ & $228.1 \pm 116.3$ & $126.5 \pm 76.9$ & $163.5 \pm 84.5$ \\
\hline
\end{tabular}

${ }^{*} P<0.05$.

Conclusions RM with CPAP performed before PDT could be performed safely to prevent alveolar derecruitment due to PDT.

P223

An audit of timing of tracheostomy formation in neurosurgical patients: how long should we wait?

P Bearfield, B Agarwal, S Ward, D Martin, S Shaw

Royal Free Hospital, London, UK

Critical Care 2010, 14(Suppl 1):P223 (doi: 10.1186/cc8455)

Introduction In neurosurgical patients requiring ventilation on the ICU, a tracheostomy is frequently formed to facilitate airway protection and weaning from the mechanical ventilator. However, the issue of when to form a tracheostomy remains contentious. In order to better inform our decision-making processes we audited practice within our own institution. Methods A retrospective study was conducted in which the ICU charts of all neurosurgical patients admitted to a tertiary referral ICU during the calendar year 2007 were reviewed. Patients who did not require mechanical ventilatory support or who died within 7 days of admission were excluded. Demographic data, diagnosis, duration of mechanical ventilation, ICU day of stay on which tracheostomy was formed, and ICU length of stay were recorded and the data analysed accordingly.

Results A total of 106 patients were included, 65 male and 41 female. The mean age was 49 years. Sixty-three patients were able to be separated from the mechanical ventilator within 7 days of commencement of ventilation via a cuffed oral endotracheal tube. Of the remaining 43 patients, 34 (79\%) went on to undergo tracheostomy formation as determined by the attending intensivist. In this group the median time of tracheostomy formation was 13 days. The median time from tracheostomy formation to separation from the mechanical ventilator was 3 days. Of those patients 
who could not be extubated within 7 days of ventilatory support, nine (14\%) were successfully separated from the mechanical ventilator without the need for a tracheostomy. There were no significant differences in age or diagnosis between the two groups.

Conclusions Our data suggest that the failure of a neurosurgical patient to separate from the mechanical ventilator within 7 days is predictive of the eventual requirement for tracheostomy formation. In light of this we intend to expand our sample size over a 5-year period and subject the data to multivariate regression.

\section{References}

1. [www.tracman.org.uk]

2. Griffiths J, Barber VS, Morgan L, Young JD: Systematic review and metaanalysis of studies of the timing of tracheostomy in adult patients undergoing artificial ventilation. Br Med J 2005, 330:1243-1246.

P224

Percutaneous dilational tracheotomy in adult burn patients

P Zeyneloglu, A Pirat, C Aydogan, G Arslan, M Haberal

Baskent University Faculty of Medicine, Ankara, Turkey

Critical Care 2010, 14(Suppl 1):P224 (doi: 10.1186/cc8456)

Introduction Few studies have focused on the safety and potential benefits of tracheotomy in burn patients. The purpose of this study is to determine the safety and efficacy of percutaneous dilational tracheotomy (PDT) in patients with burn injury.

Methods A retrospective chart review was completed for patients admitted to Baskent University Burn Institute, from January 2003 through December 2008, who underwent tracheotomy during their hospitalization. Parameters recorded included demographics, extent of burn, timing of tracheotomy and presence of inhalation injury. Other variables measured were the arterial blood gas findings and lung compliance before and after PDT and PDT-related complications and mortality.

Results Out of 325 patients, 17 patients ( $67 \%$ male) with a mean age of $33.1 \pm 15.9$ years and a mean total body surface area involvement of $51 \pm$ $16 \%$ with a $40 \%$ of inhalation injury underwent PDT. Tracheotomies were performed within 48 hours after endotracheal intubations. All PDTs were performed at bedside by experienced staff anesthesiologists with direct bronchoscopic guidance. The site of intended tracheotomy was affected in 52\% of patients. There were no procedural failures and no PDT-related complications and deaths. The calculated lung compliance and $\mathrm{PaO}_{2}: \mathrm{FiO}_{2}$ ratio improved after PDT $\left(36.2 \pm 12.9\right.$ vs $47.3 \pm 16.5 \mathrm{ml} / \mathrm{cmH}_{2} \mathrm{O}$ and $243 \pm$ 81 vs $350 \pm 104$, respectively).

Conclusions The results suggest that PDT is an efficacious and safe technique for airway management in patients with burn injuries. Besides improved ventilatory mechanics, PDT may also benefit the patient with severe burns by earlier mobilization and ease of suctioning, oral intake and ability to communicate by mouthing words.

\section{P225}

\section{Safety of percutaneous tracheostomy in trauma patients}

IM Maldonado, SB Becker

Maimonides Medical Center, Brooklyn, NY, USA

Critical Care 2010, 14(Suppl 1):P225 (doi: 10.1186/cc8457)

Introduction Percutaneous tracheostomy is a common procedure in many trauma ICUs. A concern about the safety of performing percutaneous tracheostomy in patients with no cervical spine clearance or cervical spine injury has limited its adoption for some surgeons. Most experts recommend the use of fiberoptic bronchoscopy during the insertion of percutaneous tracheostomy.

Methods: From January 2001 to December 2007 we retrospectively evaluated the medical records of all patients with blunt trauma who required tracheostomy in a level II trauma center. Data were gathered from a trauma registry database and medical records. Patients were divided in two groups, open tracheostomy (OT) and percutaneous tracheostomy (PT). Patient age, Injury Severity Score (ISS), type of tracheostomy insertion method, cervical spine clearance status prior to tracheostomy, presence of cervical spine injury, use of bronchoscopy assistance in percutaneous tracheostomy, and immediate complications post tracheostomy were recorded for each patient.
Results The total number of tracheotomies during the study period was 220, of which 125 (56\%) were PT and 95(44\%) were OT. Both groups were similar in age, sex and ISS distribution. Of the OT group, 60 (63\%) were done in patients with no cervical spine clearance or cervical spine injury. There were no immediate complications reported in the OT group. The PT group had 63 cases (50.4\%) done with no preoperative cervical spine clearance or positive for cervical spine injury. The PT group underwent the procedure without bronchoscopy assistance in $95 \%$ of the cases. Two cases (1.5\%) in the PT group were reported with postoperative bleeding from the tracheostomy site that did not required intervention. Both cases were PT done without bronchoscopy assistance and did not have preoperative cervical spine clearance. No other immediate complications were reported.

Conclusions The results of this study suggest that PT is safe in trauma patients without preoperative cervical spine clearance or with cervical injuries as compared with the OT group. Most of the PT cases were done without bronchoscopy assistance (95\%). This finding suggests the need for further study to clarify the role of bronchoscopy assistance in PT.

P226

Safety of balloon dilatational tracheostomy with the Ciaglia Blue Dolphin device

A De Nicola, MJ Sucre

San Leonardo Hospital, Castellamamre di Stabia, Italy

Critical Care 2010, 14(Suppl 1):P226 (doi: 10.1186/cc8458)

Introduction Percutaneous dilatational tracheostomy (PDT) has gained widespread acceptance in many ICU and trauma centers as a viable alternative approach. Numerous investigative reports show that all traditional techniques for PDT have similar complications. The most dangerous complication is posterior tracheal wall damage during insertion of the dilational device employing downward pressure. The Ciaglia Blue Dolphin (CBD) is an innovative device for PDT using radial balloon dilation. This technique has the potential advantage of less tracheal damage over other methods by avoiding downward pressure to enter the trachea $[1,2]$. Methods Case series of consecutive ICU patients with acute respiratory failure who required tracheostomy for failure to wean and continued mechanical ventilatory support. We report our clinical experience of 22 percutaneous tracheostomies performed using the CBD system (Cook, Bloomington, IN, USA). Informed consent was obtained from each patient, or family member. All procedures were performed under direct visualization using a fibreoptic bronchoscope on anaesthetised patients. The technique relies on the employ of a balloon-tipped catheter loaded over the guidewire. The balloon was inflated with saline up to 11 atmospheric pressure for 30 seconds to create a tracheostoma. A preloaded tracheostomy tube was then inserted through the stoma following balloon deflation.

Results Bedside PDT with the CBD system was successfully performed in the ICU for all 22 patients. Tracheostomies were performed within a mean time of $4.1 \pm 1.4$ minutes of the tracheal needle entry and with negligible blood loss in all patients. The procedure was not associated with a decrease in $\mathrm{SpO}_{2}$ at the time of balloon inflation. Procedural complications were limited to one patient requiring the repetition of balloon inflation for tracheostomy tube placement. There were no other immediate medical or technical problems.

Conclusions The CBD has been shown to be a safe, quick and successful alternative to the traditional PDT (PercuTwist, guidewire dilating forceps, and so forth) that have a tendency to go too far and perforate the tracheal rings: the intensivist performing PDT should be aware when making the decision as to which approach to use. In our hospital, the CBD method has become the procedure of choice.

\section{References}

1. Byhahn C, et al:: Crit Care 2008, 12(Suppl 2):P333.

2. Gromann TW, et al:: Anesth Analg 2009, 108:1862-1866. 
P227

Influence of the cuff pressure on the swallowing reflex in tracheostomized ICU patients

R Amathieu, D Luis, W Kamoun, V Slavov, G Dhonneur

CHU APHP Jean Verdier, Bondy, France

Critical Care 2010, 14(Suppl 1):P227 (doi: 10.1186/cc8459)

Introduction Because recovery of an efficient swallowing reflex is a determining factor for patient's spontaneous rehabilitation of airway protective reflexes, we have studied the influence of cuff pressure of the tracheostomy tube on the swallowing reflex elicited in tracheostomized ICU patients.

Methods Twelve conscious eupneic adult patients were studied. Simultaneous electromyography of submental muscles [1] and acceleromyography of laryngeal elevation [2] were measured during reflex swallows elicited by pharyngeal injection of distilled water. After cuff deflation, instrumental characteristics of the swallowing reflex (latency time, electromyography and acceleromyography peak and amplitude) were measured at 10,15,20,25,30,40,50, and $60 \mathrm{cmH}_{2} \mathrm{O}$ of air-filled induced randomly imposed cuff pressure.

Results Cuff pressure was inversely correlated to both electromyography and acceleromyography characteristics of the swallowing reflex. Latency time of the swallowing reflex and cuff pressure were linearly correlated. Instrumental characteristics of the swallowing reflex deteriorated for a cuff pressure threshold $>22 \mathrm{cmH}_{2} \mathrm{O}$ (extremes 15 to 40 ).

Conclusions We have demonstrated that instrumental characteristics of the swallowing reflex were influenced by cuff pressure in the tracheostomy tube. Increasing cuff pressure affected both the setting and the muscular activity of submental muscles during reflex swallows. Our data suggest that peripheral mechanical constraints and centrally controlled inhibition may be involved in cuff pressure rise induced deterioration of the swallowing reflex. Our report has several implications for intensive care daily clinical practice. In case of dysphagia, swallowing function should be evaluated and re-educated with a cuff pressure tidily controlled at $20 \mathrm{cmH}_{2} \mathrm{O}$.

\section{References}

1. Nishino T: J Appl Physio/ 1991, 70:888-993.

2. Reddy NP: J Rehabil Res Dev 2000, 37:361-372.

\section{P228}

\section{Microaspirations during mechanical ventilation: polyurethane} versus polyvinyl cuffed endotracheal tubes

P Bulpa, S Bouhon, F Schryvers, J Jamart, P Evrard, I Michaux, A Dive,

TVander Borght, B Krug

Mont-Godinne University Hospital, Yvoir, Belgium

Critical Care 2010, 14(Suppl 1):P228 (doi: 10.1186/cc8460)

Introduction Mechanically ventilated (MV) patients are prone to develop ventilator-associated pneumonia. One of the major risk factors is microaspirations of supraglottic secretions past the endotracheal tube cuff (usually in polyvinyl (PV)). A novel polyurethane (PUE) cuff was designed to minimize these leakages. We therefore compared the sealing capacities of the two tubes in MV patients.

Methods Twenty-nine consecutive MV patients (mean age \pm SD: $68 \pm$ 13, 21 males), were randomly allocated to receive either a PV (HI-LO Evac, Mallinckrodt) or a PUE (SEALGUARD Evac, Mallinckrodt) cuffed endotracheal tube (size: 9 for men; 8 or 8.5 for women, as a rule). We excluded patients with emergency intubation, unstable haemodynamics, severe respiratory failure or patients with history of tracheal/laryngeal disease. In each patient, cuff pressure was maintained at $30 \mathrm{cmH} \mathrm{O}_{\text {, }}$ and ventilator parameters were set to plateau pressure $\leq 30 \mathrm{cmH}_{2} \mathrm{O}_{\text {; }}$ patients were fasting and placed in a strict semirecumbent position $\left(45^{\circ}\right)$. Radioactivity of tracheal aspirates was assessed sequentially (hourly samplings from T0 to T6 hours, then T8 hours and T12 hours) after injection of $74 \mathrm{MBq}^{99 \mathrm{mTC}-D T P A}$ diluted in $5 \mathrm{ml}$ of $0.9 \% \mathrm{NaCl}$ just above the cuff via the aspiration channel of the tube. Additionally, kinetics of respiratory tract contamination was followed by simultaneous pulmonary images using a scintillation camera. Data were blindly analysed by nuclear physicians. The study was approved by the hospital ethics committee and informed consent was obtained from relatives.

Results Sixteen PUE and 13 PV cuffed tubes were compared. The study was performed $3.2 \pm 2.8$ days after intubation and $8.3 \pm 9.6$ days after ICU admission (mean $\pm \mathrm{SD}$ ). Ventilator parameters were the following: volume control or pressure support but one on $\mathrm{T}$ tube, $\mathrm{FiO}_{2}$ was $0.43 \pm 0.14$, PEEP $6 \pm 2 \mathrm{cmH}_{2} \mathrm{O}$. Leakages were observed in $11 / 29$ patients (38\%), with similar rate of aspiration in PUE (5/16) and PV (6/13) groups ( $P=N S)$. Leakages were more frequently observed in female $(7 / 8)$ than in male patients $(4 / 21)(P<0.001)$. There was a trend to decreased frequency of aspiration in patients with larger tubes (size 9 vs 8.5: $P=0.062$ ).

Conclusions Both PUE and PV cuffed endotracheal tubes are poorly effective in preventing microaspirations in MV patients. Tube size and/or gender may be more important than cuff composition in the prevention of aspiration during MV.

\section{P229}

Do endotracheal tubes prevent microaspiration?

P Lichtenthal ${ }^{1}$, U Borg $^{2}$, D Maul ${ }^{3}$

'University of Arizona, Tucson, AZ, USA; ' ${ }^{2}$ Covidien, Boulder, CO, USA;

${ }^{3}$ Pre-Clinical Research Services, Fort Collins, CO, USA

Critical Care 2010, 14(Suppl 1):P229 (doi: 10.1186/cc8461)

Introduction For decades it was assumed that cuffed endotracheal tubes prevented aspiration. However, currently used cuffed tubes do not prevent the development of post-intubation pulmonary complications caused by microaspiration [1,2]. A new taper-shaped cuffed tube (TaperGuard (TG)) has recently been introduced. The aim of this study was to compare this tube with a traditional tube $(\mathrm{Hi}-\mathrm{Lo}(\mathrm{HL}))$ in an aspiration pig model.

Methods Fourteen pigs between 65 and $75 \mathrm{~kg}$ were studied. The pigs were randomly intubated with either TaperGuard (tapered cuff) or HiLo (barrel cuff) tubes. The tube size was either 7.0 or 8.5 depending on weight. Cuff pressures were maintained between 24 and $27 \mathrm{~cm}$ water. After intubation, $0.3 \mathrm{ml} / \mathrm{kg}$ acidic blue dye (ph 2.5) was placed on top of the cuff. The animals were ventilated for 3 hours. The animals were then sacrificed and the tracheobronchial tree and lungs examined. Aspiration was characterized as follows: dye leak, ulceration/erosion, hemorrhagic pneumonia, bronchitis/bronchiolitis.

Results Of the 14 pigs, one had to be excluded due to accidental cuff deflation. Tube sizes were evenly distributed amongst the groups. Cuff pressures were equal: TG 23.7, HL $25.2-P<0.2$. As seen in Table 1, the incidence of microaspiration was significantly less for TG in the Blue Dye and bronchitis groups.

Table 1 (abstract P229). Incidence of microaspiration

\begin{tabular}{lccc}
\hline & Hi-Lo & TaperGuard & P value \\
\hline Dye leak & $7 / 7$ & $1 / 6$ & 0.005 \\
Ulceration & $5 / 7$ & $1 / 6$ & 0.07 \\
Hemorrhagic pneumonia & $5 / 7$ & $1 / 6$ & 0.07 \\
Bronchitis & $5 / 7$ & $0 / 6$ & 0.016 \\
\hline
\end{tabular}

Conclusions The TG provided significant microaspiration protection compared with the conventional tube in the dye and bronchitis categories. Although not statistically significant, the difference in the other two categories may be of clinical significance. Further clinical studies are necessary to confirm this point.

References

1. Oikkonen M, Aromaa U: Leakage of fluid around low-pressure tracheal tube cuffs. Anaesthesia 1997, 52:567-569.

2. Rawlinson E, Minchom A: Pulmonary aspiration. Anaesth Intensive Care Med 2007, 8:365-367.

P230

A cuff-leak pressure test is a simple method for assessing severe laryngeal edema in postoperative patients

H Hayami, S Nagai, S Ohama, A Sakurai, Y Sugawara, H Yamada,

O Yamaguchi, Y Koide

Yokohama City University Medical Center, Yokohama, Japan

Critical Care 2010, 14(Suppl 1):P230 (doi: 10.1186/cc8462)

Introduction The cuff-leak test has been proposed as a clinical method for predicting stridor or respiratory distress caused by laryngeal edema 
following extubation. However, the true incidence of laryngeal edema in postoperative patients is not clear. We assessed the relationship between upper airway obstruction and the values of cuff-leak pressure in postoperative patients.

Methods One hundred and fifty-eight postoperative patients (123 elective, 35 emergency) were included. After ventilator weaning was accomplished, we measured the airway pressure at which a sound of cuff leakage was audible by a cuff pressure monitor. In 28 cases, the cuff leak pressure was measured during both awake and sedated states.

Results The cuff leak pressure value was $12.8 \pm 10.1$ (median 10) $\mathrm{mmHg}$ for elective cases and $12.6 \pm 10.0$ (median 10) for emergency cases (NS). Six patients (3.8\%) were not extubated because of high leak pressure and the value was $40.5 \pm 16.0$ (24 to 60) $\mathrm{mmHg}$. One hundred and fortythree patients were extubated and nine of those (6.3\%) were diagnosed as laryngeal edema by laryngoscopy. Seven of those (5.0\%) needed reintubation, one was for the reason of massive sputa, three were for granuloma formation, and three (2.1\%) were diagnosed as severe laryngeal edema. Patients who developed severe laryngeal edema had a higher leak pressure $(27.2 \pm 22.7 \mathrm{mmHg})$ than those who did not, and all of such patients had pressure above $20 \mathrm{mmHg}$. The sensitivity and the specificity of the test using a threshold value of $20 \mathrm{mmHg}$ for severe laryngeal edema were $97.2 \%$ and $40.0 \%$, respectively. The occurrence of severe laryngeal edema was not associated with age, gender, perioperative weight gain, duration of translaryngeal intubation, inner diameter of the endotracheal tube, or serum albumin concentration. However, the cuff leak pressure value $>20 \mathrm{mmHg}$ was associated with gender (female, $P=0.02$ ) and inner diameter of the endotracheal tube $(P=0.0017)$ by multivariate regression. In 28 patients, the cuff leak pressure was $17.8 \pm 10.6$ during the awake state and $9.9 \pm 4.6 \mathrm{mmHg}$ under sedative. Because tonus of larynx is considered to be related to cuff leak pressure, it is useful to measure the cuff leak pressure in a sedated state if the patient had a high value in the awake state.

Conclusions Cuff leak pressure values $<20 \mathrm{mmHg}$ at any time are useful to rule out severe laryngeal edema. It may be useful to measure the pressure in a sedated state if the value in the awake state is high.

\section{P231}

Code critical airway teams improves patient safety

G Sample, P Mccabe, TVandruff

Washington Hospital Center, Washington, DC, USA

Critical Care 2010, 14(Suppl 1):P231 (doi: 10.1186/cc8463)

Introduction It takes an experienced multiprofessional team to handle emergent airway situations. We created a Code Critical Airway team to manage airway emergencies. Critical (difficult) airway emergencies are increasing due to advances in medical treatments, obesity and sleep apnea. Difficult airway events are low volume and high risk, requiring expert skill and communication. In the United States a leading level I adverse patient safety event is 'death or serious disability associated with airway management' [1].

Methods Utilizing a complex framework with the goal of successful airway management outcomes, the project was divided into six components: patient safety, includes the development of an airway management plan focused on improved team efficiency and culture change from Panic Button to Bridge to Safety; patient assessment, includes the development of educational programs focusing on early recognition of potential critical airway patients; teamwork, includes the development of improved communication, paging and handoff processes; performance improvement, includes after-action reviews of each event to assess and address system and process issues; equipment, includes the development of standardized airway equipment at the bedside; and team simulation training, includes consistency in communication, handoff, teamwork and equipment use

Results The average time to establish an airway across all techniques has been drastically reduced (55 minutes to 22 minutes), of special note is the dramatic improvement in the time to reintubation (18 minutes to 5 minutes). The number of surgical airways required reduced from five pre-project to zero in phase two. Thirty-one percent of the airway patients were obese or had a short thick neck. We have seen an increase in our respiratory distress calls versus respiratory arrest calls, reinforcing the benefit of proactive over reactive calling. Code Critical Airway education programs improved the number of appropriate Code Critical Airways being called, as well as improved overall Code Critical Airway Team response time. As a by-product of this process, reliability of available appropriate airway equipment is now $100 \%$.

Conclusions This project made significant process improvements in the areas of patient safety, team communication, equipment availability and response efficiency. Hospitals should develop a specialized airway team to decrease adverse airway events.

\section{References}

1. Office of Health Care Quality: Maryland Hospital Patient Safety Program Annual Report Fiscal Year 2006. Annapolis, MD: Office of Health Care Quality; 2007.

2. Rochlen $\mathrm{L}$, et al:: Proceedings from the Annual Meeting of the American Society Anesthesiologists. Designated Airway Emergency Team May Improve Survival Rates at Hospital Discharge; 17-21 October 2009; New Orleans, LA.

P232

A prospective evaluation of hemodynamic indexes to predict weaning from mechanical ventilation in patients after cardiac surgery F Galas, L Hajjar, T Lara, E Nozawa, M Feltrim, M Sundin, R Kalil, J Otavio Heart Institute, São Paulo, Brazil

Critical Care 2010, 14(Suppl 1):P232 (doi: 10.1186/cc8464)

Introduction Weaning from mechanical ventilation represents an important issue after cardiac surgery because a delayed extubation can burden the patient's recovery health, increasing the risk of infections and the length of hospital stay. Recognized parameters such as minute ventilation, negative inspiratory force, maximal inspiratory pressure ratio, and Tobin index do not predict very well the success of extubation in these patients. We tested whether hemodynamic parameters and indexes predict better successful extubation after cardiac surgery.

Methods We prospectively evaluated 100 patients undergoing cardiac surgery with pump admitted to the InCor ICU and monitored with Swan-Ganz. Exclusion criteria were congenital diseases, transplantation procedures, chronic renal failure, and ejection fraction lower than $20 \%$.

Results Patients were considered for extubation if they tolerated a T-piece for 30 minutes. From 100 patients, 88 were successfully extubated and 12 patients failed. There was no difference between groups regarding baseline characteristics, including ejection fraction and co-morbidities. Univariate analysis showed in the successful group a lower duration of pump (55 minutes $\times 90$ minutes, $P=0.038)$, a lower length of stay in ICU $(12 \times 32$ days, $P<0.0001)$, a lower BNP level $(144 \times 422, P=0.002)$, and a higher $\mathrm{PO}_{2} / \mathrm{FiO}_{2}$ $(312 \times 266, P=0.043)$ and a higher VeRT (time recovery minute ventilation) 4 $\times 2, P<0.01$. Multivariate analysis showed high levels of $\mathrm{BNP}$ and of $\mathrm{PO}_{2} / \mathrm{FiO}_{2}$ at admission are strong predictors of successful weaning.

Conclusions After cardiac surgery, lower levels of BNP and higher $\mathrm{PO}_{2} /$ $\mathrm{FiO}_{2}$ are predictors of successful weaning. This suggests that adequate hemodynamic optimization must be achieved in patients to improve outcomes as early weaning of mechanical ventilation.

\section{References}

1. Epstein SK, et al.: Effect of failed extubation on the outcome of mechanical ventilation. Chest 1997, 112:186-192.

2. Epstein SK: Decision to extubate. Intensive Care Med 2002, 28:535-546.

\section{P233}

\section{Rapid shallow breathing index predicts readiness to wean?}

R Janssen-Dean, KVrints, I Blum, B Speelberg

St Anna Hospital Geldrop, the Netherlands

Critical Care 2010, 14(Suppl 1):P233 (doi: 10.1186/cc8465)

Introduction The rapid shallow breathing index (RSBI) has been shown to be a parameter that predicts successful weaning. A RSBI of less than 105 is a predictor for successful weaning. We investigated whether the RSBI could be a useful parameter in our patient population.

Methods From June 2008 until March 2009 all patients who had been mechanically ventilated on our six-bed ICU were studied. Only the patients who were invasively mechanically ventilated, for longer than 6 hours, who had no missing information and did not die whilst on the ventilator were included in this study. RSBls were measured by these patients at two different time points of weaning, firstly at the point of reduction in pressure support and then at the point of extubation. The RSBI was measured using 
the ventilator (Maquet servo-i) by reducing the pressure support level to zero and adjusting the PEEP to $5 \mathrm{cmH}_{2} \mathrm{O}$. A significance level $<0.05$ was considered significant. The nonparametric Kruskall-Wallis test was used to analyse the collected data.

Results One hundred and nineteen patients were ventilated over this time period: 74 patients did not meet the inclusion criteria; 45 were included. At the time point of further reduction in pressure support, the RSBI showed significant differences $(P=0.038)$ between the group who was ready for weaning and the group who was not ready for weaning. In the group with successful reduction of PS $(n=28)$ the RSBI was $83.54 \pm 32.12$, and in the group without successful reduction of PS $(n=2)$ the RSBI was $158.50 \pm$ 38.89. At the point of extubation the RSBI was significant in predicting successful extubation $(P<0.001)$. In the group who was successfully extubated $(n=24)$ the RSBI was $75.25 \pm 21.62$, and in the group who was unsuccessfully extubated $(n=5)$ the RSBI was $155.20 \pm 25.23$.

Conclusions At the moment of extubation, the RSBI showed significant low values in the successful group. This was also for the time point further reduction in pressure support level.

\section{P234}

Increasing pressure support during vibrocompression is useful during respiratory therapy?

W Naue, A Guntzel, A Silva, R Condessa, R Oliveira, S Vieira

Hospital de Clínicas de Porto Alegre, Brazil

Critical Care 2010, 14(Suppl 1):P234 (doi: 10.1186/cc8466)

Introduction ICU inpatients are susceptible to events of transient hypoxemia. The physical therapist makes use of bronchial hygiene techniques to solve or alleviate this problem. The goal of this paper was to compare two physiotherapy techniques: vibrocompression versus vibrocompression plus increase of $10 \mathrm{cmH}_{2} \mathrm{O}$ in inspiratory pressure (IP) during pressure support ventilation (PSV).

Methods After being placed in the supine position in bed, with head angle elevation at $30^{\circ}$, patients were randomized to: group 1 (G1): vibrocompression for 10 minutes in the chest, or group 2 (G2): vibrocompression plus increase of $10 \mathrm{CmH}_{2} \mathrm{O}$ in IP in PSV for 10 minutes in the chest. Clinical variables and APACHE II score were registered. Parameters analyzed at the beginning (1) and at the end (2) of the protocol were: variation of peak pressure $(\triangle \mathrm{PP}=$ $\mathrm{Pp} 2-\mathrm{Pp} 1)$, variation of tidal volume $(\Delta \mathrm{VT}=\mathrm{VT} 2-\mathrm{VT} 1)$, variation of dynamic compliance $(\Delta C$ dyn $=C$ dyn2 $-C$ dyn 1$)$. The amount of variation of mucus secretion aspirated $(\triangle \mathrm{Sa}=\mathrm{Sa} 2-\mathrm{Sa} 1)$ at the end was also measured. The results are showed in mean \pm SD.

Results Both groups (G1: $n=30$ and G2: $n=39$ ) were similar in clinical characteristics and APACHE II score. The variation of variables in G1 and G2, were respectively: $\triangle \mathrm{Pp}\left(\mathrm{cmH}_{2} \mathrm{O}\right)=0.58 \pm 1.41$ and $-0.10 \pm 0.98, P=0.02 ; \Delta \mathrm{VT}$ $(\mathrm{ml})=28.90 \pm 119.40$ and $64.26 \pm 93.82, P=0.96 ; \Delta \mathrm{Cdyn}\left(\mathrm{cmH}_{2} \mathrm{O}\right)=3.03 \pm$ 9.56 and $4.26 \pm 8.24, P=0.89 ; \Delta \mathrm{Sa}$ (g) $0.29 \pm 1.73$ and $0.93 \pm 2.28, P=0.36$.

Conclusions We found no differences between groups, except in peak pressure (Pp2 >Pp1) that was greater in G1. This result could be probably due to decreased airway resistance.

\section{References}

1. Stiller K: Chest 2000, 118:6.

2. Passam F, et al: Respiration 2003, 70:4.

\section{P235}

Predicting success in weaning from mechanical ventilation in patients with and without cardiac disease

L Cassel, C Hahn, S Vieira

Hospital de Clínicas de Porto Alegre, Brazil

Critical Care 2010, 14(Suppl 1):P235 (doi: 10.1186/cc8467)

Introduction Failure in weaning from mechanical ventilation (MV) is frequent (25 to 30\%) and associated with high mortality. Indexes predicting success can be helpful clinically. However their predictive capacity can be low, principally in patients with cardiac disease. The goal of this study is to evaluate weaning predictor indexes in patients with cardiac disease during weaning from MV.

Methods We included patients with and without cardiac disease under MV for at least 48 hours, submitted to spontaneous breathing trial (SBT) for 30 minutes, extubated according to clinical decision and followed for
48 hours. They were evaluated concerning age, sex, clinical characteristics, length of hospital and ICU stay and of MV. At the first and 30th minutes from SBT there were analyzed: arterial blood gases, hemodynamic and respiratory parameters as respiratory rate (f), tidal volume (VT), rapid shallow breathing index (f/NT), maximal inspiratory and expiratory pressures. Comparisons were done between this group of patients and success $x$ failure, defining failure as a return to MV in the first 48 hours.

Results Four hundred and thirteen patients were studied, 81 with cardiac disease and 332 without. Overall mortality rate was 14\%. Return to MV occurred in $19.7 \%$. The most important differences comparing patients with cardiac disease with the control group were: lower mortality rate $(21 \% \times 13.5 \%, P<0.006)$, shorter length of ICU stay $(9 \pm 3 \times 16 \pm 13$ days). Comparing patients who failed in the weaning process with and without heart disease $(20 \% \times 20 \%, P<0.55)$. Comparing $\mathrm{f} / \mathrm{NT}$ in 30th minute of patients who have had success with those who failed the weaning process $(60 \pm 38 \times 74 \pm 47, P<0.012)$, a lower increase in $\mathrm{f} / \mathrm{VT}(\Delta \mathrm{f} / \mathrm{NT})(4 \pm 27 \times 11 \pm$ $33, P<0.075)$ during the test.

Conclusions $\mathrm{In}$ this group of patients a great number failed in the weaning process, showing, as expected, a higher mortality rate. Parameters most related to failure in the literature were higher age, longer length of ICU stay, mortality and fNT. In this study, just the last parameter was sensitive principally in the 30th minute, and higher increase in $f / N T(\Delta f / N T)$ during the test, demonstrating that patients with cardiac disease not fail more than others during the weaning process, as well as the efficiency of the test to predict success in weaning.

Members of Weaning Study Group R Wickert, LG Borges, ME Alves, ACT Silva, R Condessa, MB Blom, R Zancanaro, F Callefe, KB Pinto, K Hartmann, P Pinheiro, ES Oliveira, C Trevisan.

References

1. Frutos-Vivar F, et al:: Chest 2006, 130:1664-1671.

2. Tanios MA, et al:: Crit Care Med 2006, 34:2530-2535.

\section{P236}

Implementation of a standardised weaning protocol in patients with prolonged mechanical ventilation in a post-acute care ICU K Schneider', F Oehmichen', M Pohl', M Ragaller ${ }^{2}$

'Bavaria Klinik Kreischa, Germany; ${ }^{2}$ University Hospital Dresden, Germany

Critical Care 2010, 14(Suppl 1):P236 (doi: 10.1186/cc8468)

Introduction Due to the improvement of nearly all fields of acute care in the past decades, the burden of long-term dependency from mechanical ventilation (MV) increases incessantly. The aim of this study was to implement and test a standardised weaning protocol in patients suffering from long-term mechanical ventilation.

Methods After approval by the local ethics committee and informed consent, 644 patients were enrolled in a prospective cohort study in 1 year. The mean time on the ventilator before inclusion was 39.4 ( 6 to 357) days. The reasons for long-term MV were: cerebral 33.1\%; cardiovascular $31.5 \%$; pulmonary $28.7 \%$ and $5.6 \%$ neuromuscular diseases. The weaning protocol started with $6 \times 5$ minutes spontaneous breathing (intermission of mechanical ventilation) on day 1 and was increased stepwise up to 24 hours spontaneous breathing on day 22. If there was no improvement in weaning steps over 5 days or there were more than three steps backwards the patient was switched to the individual weaning approach. The weaning protocol was carried out by the previously trained ICU staff as well as by specialized physiotherapists.

Results A total of $77.3 \%(n=498)$ of the patients could be weaned off the ventilator by the first effort. Out of these, $85.9 \%$ of the patients were weaned by the protocol whereas $14.1 \%$ needed an individual approach. The mean time of weaning was 17 (5 to 67) days using the protocol and 29 (1 to 88) days due to the individual approach. In 12.6\% both weaning procedures failed and these patients were discharged from the hospital into a home-care ventilation program. In total, $10.1 \%$ of the patients died on mechanical ventilation during their ICU stay. Patients who had needed a second weaning effort $(n=111)$ were weaned in $36.4 \%$ by the protocol, transferred to home-care ventilation (28.8\%) or died (35.1\%).

Conclusions Using the standardised weaning protocol, more than three-quarters of the prolonged mechanically ventilated patients could be weaned off the ventilator in a mean of 17 days. Only $14.1 \%$ needed an individual strategy that takes 29 days until complete release from the ventilator. Therefore we suggest this kind of weaning protocol as a useful 
tool in patients with prolonged mechanical ventilation in a post-acute care facility.

\section{References}

1. Scheinhorn DJ, et al:: Chest 2001, 119:236-242.

\section{P237}

Comparison of pressure control and pressure support modes for non-invasive mechanical ventilation in acute hypercapnic respiratory failure

M Aydogdu, G Gursel, F Yıldırım, STasyurek, F Aykan, GTeksut, S Nazik, A Demir Gazi University Medical Faculty, Ankara, Turkey

Critical Care 2010, 14(Suppl 1):P237 (doi: 10.1186/cc8469)

Introduction Non-invasive positive pressure ventilation (NIPPV) has generally been accepted as beneficial to use in acute hypercapnic respiratory failure (AHCRF), but it is not still standardized how to perform it, and with which ventilation modes. In this study it was aimed to compare the efficiency of pressure control (PCV) and pressure support ventilation (PSV) modes on patients with AHcRF.

Methods A prospective randomized controlled study, performed in a pulmonary ICU of a university hospital. Patients who were admitted with the diagnosis of AHCRF were included in the study consecutively and they were randomized to have either NIPPV with PSV or PCV for the first 2 hours. Then NIPPV was stopped for 4 hours to achieve a basal $\mathrm{PaCO}_{2}$ level, and after this waiting period NIPPV was continued with the other mode. The mode that leads to a greater decrease in $\mathrm{PaCO}_{2}$ value was accepted as the successful mode.

Results A total of 40 patients with mean age of $69 \pm 12$ years were included in the study. In only two (5\%) patients were endotracheal intubation and mechanical ventilation needed. Among them, only one (2.5\%) patient died. After NIPPV there was a significant decrease in $\mathrm{PaCO}_{2}$ level with both PSV and PCV modes. But when $\triangle \mathrm{PaCO}_{2}\left(\mathrm{PaCO}_{2}\right.$ after NIPPV - $\mathrm{PaCO}_{2}$, baseline) of both groups was compared, no statistically significant difference was identified. Among the 20 patients that NIPPV was started with PSV, in nine (45\%) of them PSV was assessed as successful and continued; in 11 of them (55\%) NIPPV was switched to PCV $(P>0.05)$. Among the 20 patients in which NIPPV was started with PCV, in one of them NIPPV was unsuccessful ending up with intubation, in 16 of them PCV $(84 \%)$ was successful $(P=0.051)$. The presence of apnea and apnea plus hypopnea with the initial mode was found as the factor affecting the assessment of PCV as the successful mode $(P<0.05)$.

Conclusions Both PSV and PCV can be used efficiently in the treatment of AHcRF of various causes. But in the presence of apneas and hypopneas (either central or obstructive) PCV can be preferred instead of PSV.

\section{P238}

\section{Non-invasive CPAP with face mask: comparison between different}

\section{devices}

A Galimberti, G Mistraletti, F Rapido, M Gomarasca, B Salihovic, L D'Amato, T Crespi, C Reali Forster, P Spanu, G lapichino

Università degli Studi di Milano, Milan, Italy

Critical Care 2010, 14(Suppl 1):P238 (doi: 10.1186/cc8470)

Introduction This study compared the reliability and the efficacy of three different non-invasive CPAP systems, Ventumask ${ }^{\otimes}$ low flow (LF), Ventumask ${ }^{\circledR}$ high flow (HF) and Boussignac ${ }^{\circledR}$ CPAP valve (B), both in a lung model and in healthy subjects.

Methods Lung model: pneumatic simulator first set at respiratory rate 16/ minute with $400 \mathrm{ml}$ tidal volume (L1), then RR 40/minute with $800 \mathrm{ml}$ tidal volume (L2). Human study: 10 healthy subjects were asked to make an expiratory pause (T1), five breaths at tidal volume (T2), and a short sequence of tachypnea (T3). The differences between set and effective PEEP and $\mathrm{FiO}_{2}$ were used as indexes of reliability. The minimum airway pressure below the PEEP level and the airway pressure swing around PEEP were used as indexes of efficacy. In the lung simulator protocol, the pressure-time product was measured and then correlated to the other efficacy indexes.

Results Lung model: HF showed a tendency towards a more stable PEEP $(P=0.067)$. In B a significant fall in the $\mathrm{FiO}_{2}$ value from L1 $(96.9(95.5 ; 97.6) \%)$ to $L 2(54.2(50.4 ; 56.9) \%)$ was observed $(P<0.001)$. The airway pressure swing around PEEP was greater in LF and B compared with HF in L1 $(P<0.001)$, while during $\mathrm{L} 2$ it was lower in $\mathrm{B}(P=0.007)$. The pressure-time product was better described by the airway pressure swing around PEEP $\left(r^{2}=0.95\right)$ rather than the minimum airway pressure below the PEEP level $\left(r^{2}=0.85\right)$. Human study: a lower difference between set and effective $\mathrm{FiO}_{2}$ was registered with $\mathrm{HF}$ $(P<0.001)$. Ventumask ${ }^{\circledast}$ systems showed an overall higher minimum airway pressure below the PEEP level compared with $\mathrm{B}(P<0.001)$. During T3, HF $\left(+10.1(+7.4 ;+13.2) \mathrm{CmH}_{2} \mathrm{O}\right)$ showed a smaller airway pressure swing around PEEP relative to LF $\left(+11.9(+10.1 ;+16.6) \mathrm{CmH}_{2} \mathrm{O}, P<0.001\right)$ but not to $\mathrm{B}(+12.2$ $\left.(+6.6 ;+15.1) \mathrm{cmH}_{2} \mathrm{O}, P=0.40\right)$. During the whole respiratory sequence, an increase in end-expiratory pressure was observed. This increase was greater in $B$ valve than in HF and LF $(P<0.001)$.

Conclusions According to observations, HF seemed to be the most reliable device. In conditions of high flow demand, $\mathrm{B}$ reached $\mathrm{FiO}_{2}$ values lower than expected. The dynamic hyper-pressurization, higher with $\mathrm{B}$, is probably due to the relationship between expiratory flow and the resistance offered by the expiratory valve. B, even if handy and lightweight, is less reliable in terms of flow supply and PEEP stability.

\section{P239}

Effects of non-invasive mechanical ventilation on hemodynamic and gas exchanges parameters after cardiac surgery in patients with $300>\mathrm{PaO}_{2} / \mathrm{FiO}_{2}>150$

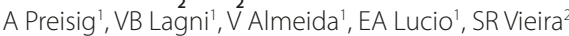

'Irmandade da Santa Casa de Porto Alegre, Brazil;' Hospital de Clínicas de Porto Alegre, Brazil

Critical Care 2010, 14(Suppl 1):P239 (doi: 10.1186/cc8471)

Introduction Non-invasive mechanical ventilation (NIV) has been used in hypoxic postoperative cardiac patients but more studies are necessary to clarify its respiratory and hemodynamic effects. Therefore, our objective was to study its effects in the oxygenation index $\left(\mathrm{PaO}_{2} / \mathrm{FiO}_{2}\right)$ and in hemodynamic variables in this group of patients.

Methods This is a randomized trial in which all postoperative cardiac patients having a pulmonary artery catheter and showing a $\mathrm{PaO}_{2} / \mathrm{FiO}_{2}$ between 150 and 300 (with $\mathrm{FIO}_{2}$ 0.31), 1 hour after extubation, were included. The intervention group used NIV with a bilevel positive airway pressure (an inspiratory pressure to generate a tidal volume of $6 \mathrm{ml} / \mathrm{kg}$ and an expiratory pressure of $7 \mathrm{CmH}_{2} \mathrm{O}$ ) with $\mathrm{FIO}_{2} 0.4$ during 3 hours. The control group used oxygen by Venturi mask in order to keep good oxygenation. In both groups, measurements were done in a basal situation $\left(\mathrm{FiO}_{2}, 0.31\right)$, in the first hour after beginning the treatment $\left(\mathrm{FiO}_{2}, 0.4\right)$ and 1 hour after the end of the intervention ( $\mathrm{FiO}_{2}$ 0.31). Variables studied included: $\mathrm{pH}, \mathrm{PaO}_{2}$, $\mathrm{PaCO}_{2}$ and $\mathrm{PaO} / \mathrm{FiO}_{2}$ ratio, heart rate $(\mathrm{HR})$, mean arterial pressure (MAP), pulmonary capillary wedge pressure (PCWP), central venous pressure (CVP), mean pulmonary arterial pressure (MPAP) and cardiac output (CO).

Results Forty-two patients were included in the study period. The mean age was $65.7 \pm 10$ years. The basal variables were similar in the two groups. There was an increase in the $\mathrm{PaO}_{2} / \mathrm{FiO}_{2}$ ratio in the NIV group in the first hour $(P<0.05,95 \% \mathrm{Cl} 74.8$ to 6.6$)$ and 1 hour after stopping the treatment ( $P<0.05,95 \% \mathrm{Cl} 90.7$ to 10.8 ) compared with the control group. There were no significant changes in hemodynamic parameters, $\mathrm{pH}$ and $\mathrm{PaCO}_{2}$ during NIV compared with the control group.

Conclusions There were no changes in hemodynamic variables during the NIV period. However, these results suggest that NIV improved oxygenation even 1 hour after stopping the treatment.

\section{References}

1. Zarbock A, et al:: Chest 2009, 135:1252-1259.

2. De Santo LS, et al.: J Thorac Cardiovasc Surg 2009, 137:342-346.

3. Kindgen-Milles D, et al:: Chest 2005, 128:821-828

4. Matte P, et al:: Acta Anaesthesio/ Scand 2000, 44:75-81.

\section{P240}

Non-invasive positive pressure ventilation in burns

J Haddad, A Mokline, I Rahmani, H Oueslati, I El Jami, K Brini, K Bousselmi, A Messadi

Burn and Trauma Center, Tunis, Tunisia

Critical Care 2010, 14(Suppl 1):P240 (doi: 10.1186/cc8472)

Introduction Acute respiratory failure is a common complication of severely burn-injured patients. Non-invasive positive pressure ventilation 
(NIPPV) has been used with success in hypercapnic and hypoxaemic acute respiratory failure. However, the outcome of NIPPV with burn patients is less well documented. The purpose of this study is to report our experience with NIPPV in a series of burn-injured patients.

Methods The records of all burn patients from July 2008 to September 2009, in whom NIPPV was used in the intensive burn care unit, were reviewed. The criteria for selecting patients for NIPPV included a combination of the following factors: patients with acute respiratory failure, haemodynamically stable, conscious and cooperative with their treatment. There had to be no need for endotracheal intubation.

Results Thirty-one patients were treated with NIPPV. Nineteen were female. Mean age was 44.22 years, mean total body surface area (TBSA) was $38.37 \%$. NIPPV was used to treat hypoxia in 21 patients, hypercapnia in four patients and both of them in six patients. The mean $\mathrm{PaO}_{2} / \mathrm{FiO}_{2}$ ratio before NIPPV was 188.53. NIPPV was used to treat ARDS in 12 patients, pneumonia in eight patients, atelectasis in six patients and cardiogenic oedema in five patients. The mean $\mathrm{PaO} / / \mathrm{FiO}_{2}$ ratio after NIPPV was 256.43 . Intubation was successfully avoided in 12 out of the 31 (38.7\%) patients. All of these patients progressed to self-ventilation status following NIPPV. Conclusions The use of NIPPV with burn-injured patients is, as yet, unclear because little work has been documented. In our experience, the use of NIPPV can lead to avoid the need for endotracheal intubation and mechanical ventilation.

Reference

1. Smailes ST: Burns 2002, 28:795-801.

P241

Non-invasive mechanical ventilation in the weaning process: continuous hemodynamic monitoring

F Franchi, C Palomba, E Mastrocinque, L Cubattoli, P Mongelli, E Casadei University of Siena, Italy

Critical Care 2010, 14(Suppl 1):P241 (doi: 10.1186/cc8473)

Introduction The purpose of this prospective study is to describe the hemodynamic and cardiac variation during a trial of non-invasive ventilation (NIV) in the process of weaning from ventilatory support. A continuous hemodynamic monitoring was performed with a pulse contour method, the MostCare (Vytech Health, Laboratoires Pharmaceutiques Vygon, Ecouen, France). This device could be used to identify the early warnings of cardiovascular dysfunction, which may contribute to unsuccessful weaning.

Methods Fourteen patients, admitted to our ICU between January and July 2009, were included in the study: six intubated on respiratory failure due to cardiogenic pulmonary edema (CPE; $52 \pm 18$ years, four male $(\mathrm{M})$, two female (F)), eight intubated on chronic obstructive pulmonary disease (COPD; $71 \pm 16$ years, four $M$, four F). The NIV trial was performed with a face mask: pressure support (PS) $=5$ to $10 \mathrm{cmH}_{2} \mathrm{O}$, positive endexpiratory pressure (PEEP) $=5$ to $7 \mathrm{cmH}_{2} \mathrm{O}$. Cardiovascular variables and gas exchange data were measured at three defined points in time: 1 hour before the extubation (T1), continuously during the NIV trial (a mean value was calculated and expressed as T2), and at the end of the NIV trial with the patient in spontaneous breathing (T3).

Results The variation of cardiac index (Cl) was: T1 $2.53 \pm 0.43 \mathrm{ml} / \mathrm{minute} / \mathrm{m}^{2}$ CPE vs $3.2 \pm 0.64 \mathrm{ml} / \mathrm{minute} / \mathrm{m}^{2}$ COPD; T2 $2.93 \pm 0.93 \mathrm{ml} / \mathrm{minute} / \mathrm{m}^{2}$ CPE vs $2.9 \pm 1.33 \mathrm{ml} / \mathrm{minute} / \mathrm{m}^{2} \mathrm{COPD} ; \mathrm{T} 31.96 \pm 0.61 \mathrm{ml} / \mathrm{minute} / \mathrm{m}^{2}$ CPE vs $3.3 \pm$ $1.37 \mathrm{ml} / \mathrm{minute} / \mathrm{m}^{2}$ COPD. The variation of $\mathrm{Cl}$ depended on the variation of stroke volume (SV), while the heart rate (HR) did not change during the trial. We calculated the oxygen delivery $\left(\mathrm{DO}_{2}\right)$ by the correlation of $\mathrm{Cl}$ and gas exchange: $T 1767 \pm 218 \mathrm{ml} /$ minute CPE vs $839 \pm 134.3 \mathrm{ml} /$ minute COPD, T2 $917 \pm 417 \mathrm{ml} /$ minute CPE vs $701 \pm 349 \mathrm{ml} /$ minute COPD, T3 $760 \pm 404$ $\mathrm{ml} /$ minute CPE vs $753 \pm 340 \mathrm{ml} /$ minute COPD. The variation of $\mathrm{Cl}$ and $\mathrm{DO}_{2}$ observed within and among the two groups was never significant.

Conclusions Both groups of patients were successful in achieving spontaneous ventilation. In our opinion, continuous hemodynamic monitoring may provide helpful beat-to-beat information and it might be used, combined with the gas exchange and oxygen saturation monitoring, during the weaning process as a predictor of cardiovascular instability or respiratory failure. Moreover, continuous hemodynamic monitoring enables one to be aware of the variation of systemic oxygen delivery, and these data could be used to value critically ill patients during the weaning process.
P242

Influence of severe obesity on non-invasive ventilation strategies and responses in patients with acute hypercapnic respiratory failure in ICU

G Gursel', M Aydogdu', G Gulbas², S Ozkaya3, STasyurek', F Yıldırım' 'Gazi University Medical Faculty, Ankara, Turkey; Inonu University Medical Faculty Pulmonary Diseases Department, Malatya, Turkey; ${ }^{3}$ Rize University Medical Faculty Pulmonary Diseases Department, Rize, Turkey Critical Care 2010, 14(Suppl 1):P242 (doi: 10.1186/cc8474)

Introduction Obesity rates are increasing in the general population and it is also prevalent in ICUs. Patients are sometimes admitted to ICUs for hypercapnic respiratory failure or cor pulmonale but in general they are admitted for pneumonia, excessive daytime sleepiness, heart failure, COPD or asthma attacks or pulmonary embolism; and hypercapnic respiratory failure is noticed during this period. On the other hand, optimal non-invasive mechanical ventilation strategy is not known during their ICU treatment. The aim of this study is to assess the differences between NIV strategies and outcomes between obese and nonobese patients with acute hypercapnic respiratory failure.

Methods In this retrospective cohort study, 73 patients were studied and all of them were ventilated with a face mask. Patients divided into two groups as obese $\left(B M I>35 \mathrm{~kg} / \mathrm{m}^{2}\right)$ and nonobese $\left(B M I<35 \mathrm{~kg} / \mathrm{m}^{2}\right.$ ), and whether necessary pressure, volume, mode, ventilator and time to reduce $\mathrm{PaCO}_{2}$ below $50 \mathrm{mmHg}$ were significantly different in obese and nonobese patients was investigated.

Results Mean age of the patients was $66 \pm 14$ years and mean admission APACHE II score was $18 \pm 4 ; 41$ (56\%) of them were female. ICU admission reasons for the obese patients were significantly more frequently pulmonary edema and less frequently pulmonary infections $(P=0.003$ and 0.043 , respectively) than the nonobese patients. While there were no significant differences across the groups between the ventilators, modes, and inspiratory pressure levels, obese patients required higher end-expiratory pressure levels and more time to reduce the $\mathrm{PaCO}_{2}$ level below $50 \mathrm{mmHg}$ than the nonobese group. Length of NIV and ICU stay, intubation and the mortality rate were similar across the groups.

Conclusions These results suggest that improvement of hypercapnia in obese patients may require higher PEEP levels and longer times than the non-obese ones during acute hypercapnic respiratory failure attack.

P243

A simple predictive scoring system for prolonged mechanical ventilation in severe sepsis and septic shock

N Saito, Y Sakamoto, K Mashiko

Chiba Hokusou Hospital, Nippon Medical School, Chiba, Japan

Critical Care 2010, 14(Suppl 1):P243 (doi: 10.1186/cc8475)

Introduction Prolonged mechanical ventilation (MV) is associated with high morbidity and mortality in septic patients. However, limited data are available on the prediction of prolonged MV. We conducted an observational cohort study aimed at developing a prolonged MV predictive scoring system for severe sepsis and septic shock.

Methods We retrospectively analyzed 120 consecutive patients with severe sepsis or septic shock who were ventilated for more than 72 hours between January 2005 and October 2009. Clinical features and physiologic parameters were examined for more than 15 days for use as predictors of MV. Patients were divided into two groups: group 1, those requiring MV for $<15$ days; group 2, those requiring MV > 15 days.

Results The mean $( \pm$ SD) age and SOFA scores were $65.3 \pm 16.7$ years and $10.0 \pm 3.9$, respectively, and $35 \%$ required prolonged MV. Univariate analysis indicated that the length of ICU and hospital stays, hospital mortality, the rate of transfusion, incidence of ARDS, ventilator-associated pneumonia (VAP), other nosocomial infection (NI) and drug-resistant bacteria, the ratio of steroid therapy and muscle relaxant use, and the mean $\mathrm{PaO}_{2} / \mathrm{FiO}_{2}$ ratio during the first 3 days after admission were significantly different between the two groups. The independent predictors for prolonged MV were ARDS (OR $5.24(P=0.001 ; 95 \% \mathrm{Cl}: 1.9$ to 14.1$))$, VAP (OR $7.75(P<0.001 ; 95 \% \mathrm{Cl}$ : 2.7 to 22.0$)$ ), and transfusion (OR $2.84(P=0.036 ; 95 \% \mathrm{Cl}: 1.0$ to 7.5$))$ Using these results, we were able to develop a prolonged MV predictive scoring system. This simplified clinical risk assessment tool was developed from 
the results of univariate and multivariate analysis, with scoring based on a cut-off point related to the adjusted odds ratio. Prolonged MV score: VAP $=7$ points; ARDS $=5$ points; transfusion $=2$ points; incidence of drugresistant bacteria, steroid therapy, muscle relaxant use, other $\mathrm{NI}$, the mean $\mathrm{PaO} / \mathrm{FiO}_{2}$ ratio during the first 3 days after admission $<220=$ each 1 point. The area under the receiver operating characteristic curve for prolonged MV in this scoring system was 0.866 ( $P<0.001 ; 95 \%$ Cl: 0.802 to 0.931$)$.

Conclusions The simple predictive scoring system for prolonged MV in severe sepsis/septic shock developed here will therefore help in planning the long-term care of such patients.

P244

Simplified approach to ICU severity scoring with MPM and EUROSCORE

JH Reeves, JA Barrett, G Hawdon, F Hawker

Cabrini Hospital, Malvern, Australia

Critical Care 2010, 14(Suppl 1):P244 (doi: 10.1186/cc8476)

Introduction Severity scoring is a powerful tool for quality control in the ICU. We introduced a new severity scoring system to our ICU. The aim of this report is to describe the database and present the results of the first 12 months.

Methods We analysed needs for database and severity scoring. We chose on-admission scoring with EUROSCORE for cardiac surgical patients, and Mortality Prediction Model II time zero for other patients. Data were collected via highly simplified forms onto a spreadsheet. Demographic entry was by ward clerk, on-admission severity scoring was by resident medical staff, and risk of death calculation and data cleaning was by senior attending medical staff. Individual patient risk of death was presented as a logit. Combined risk of death for the whole cohort was calculated from the arithmetic mean of individual logits. We estimated the time to completion of each step in data acquisition to calculate a total time spent per patient. Results There were 1,355 admissions, mean (SD) age 69.2 (15.9) years, $57.1 \%$ male; median (range) length of stay $23.1(1.7$ to $1,882.5)$ hours. Fiftyfour per cent of patients were ventilated for a median (range) 7.5 (0.8 to 1,877 ) hours. Predicted mortality for cardiothoracic and noncardiothoracic patients combined was $8.64 \%$ and the observed mortality was $2.8 \%$. EUROSCORE-predicted mortality for the 535 cardiothoracic patients was $5.72 \%$ and the observed mortality was $0.75 \%$ (four patients). MPMpredicted mortality for the 820 noncardiothoracic patients was $11.23 \%$ and the observed mortality was 4\% (34 patients). Estimated time to complete the severity scoring form was 30 seconds, to enter a new patient on the database was 90 seconds and to calculate risk of death and check data integrity was 90 seconds. Total was 3.5 minutes per patient.

Conclusions Data collection/analysis is essential for quality management in the ICU. Proprietary systems are expensive. Traditional scoring systems (APACHE II) are poorly calibrated to some case mixes. To overcome these problems, we devised a simple, inexpensive and highly valuable ICU database. The key features were well calibrated, on-admission severity scoring, highly simplified forms, a basic spreadsheet and collaborative staff involvement. Senior medical staff performed the final data checking. The project provided abundant high-quality data with a total input of 3.5 minutes per patient. We are trialling the database in a large ICU in China and we would welcome input from other ICUs that would like to copy our methods.

\section{P245}

Validation of a computerised system to calculate the sequential organ failure assessment score

C Bourdeaux', M Thomas' ${ }^{2}$ T Gould ${ }^{2}$, Z Evans ${ }^{2}$, D Bryant ${ }^{2}$

'Frenchay Hospital, Bristol, UK' '2Bristol Royal Infirmary, Bristol, UK

Critical Care 2010, 14(Suppl 1):P245 (doi: 10.1186/cc8477)

Introduction The sequential organ failure assessment (SOFA) score was introduced with the aim of quantifying the severity of illness, based on organ dysfunction, serially over time. A previous study has suggested that the reliability and accuracy of SOFA scoring by intensive care physicians is good but there may be room for improvement [1]. Manual calculation of the score can be time consuming. We developed a computer program that derives a SOFA score from the electronic patient record, the Innovian system (Draeger, Germany). To date, no automated method for SOFA score calculation has been validated. We validated the automated SOFA score collection method in order to assess its accuracy and reliability. We also measured the time for manual collection of SOFA scores in order to assess the resource saving potential of the computer system.

Methods Fifty patient records were selected from the database as a stratified random sample in order to represent the patient population of our teaching hospital adult ICU. Two ICU physicians calculated a total SOFA score and an individual organ score on each of the 50 patient records. A gold standard SOFA score was then generated after discussion between the two physicians with the aid of a third adjudicating ICU physician. SOFA scores generated by the computer were compared with the gold standard to assess accuracy. Reasons for inaccurate scoring were recorded.

Results SOFA scores varied from 1 to 15 in this sample, the mean SOFA score for the Gold standard was 8.1 and the standard deviation was 2.9. The agreement between the different ratings was very good. The computer score had a better agreement with the gold standard score (Pearson correlation coefficient 0.92), compared with the individual physician scores (Pearson correlation coefficient 0.890 and 0.895). The computer calculated the SOFA score correctly in 41 cases and the physicians calculated the SOFA score correctly in 32 cases. The average time to calculate a SOFA score was 4.91 minutes and was not significantly different between the physicians.

Conclusions The results show that this computer system is highly accurate at calculating SOFA scores from the electronic patient record and is more accurate than physicians. The time saved is considerable.

Reference

1. Arts DGT, de Keizer NF, Vroom MB, de Jonge E: Reliability and accuracy of Sequential Organ Failure Assessment (SOFA) scoring. Crit Care Med 2005, 33:1988-1993.

P246

Sequential Organ Failure Assessment scores and mortality

C Borni, L Meynaud-Kraemer, G Laplatte, HL Lessire

CH Pasteur, Colmar, France

Critical Care 2010, 14(Suppl 1):P246 (doi: 10.1186/cc8478)

Introduction In 2005, in a multicenter study in 79 ICUs in Spain, Cabré and colleagues [1] found patients older than 60 years and with SOFA score higher than 9 for at least 5 days unlikely to survive, suggesting that it could provide a basis for deciding whether to withhold or withdraw life support. We tried to control their hypothesis in our patient population.

Methods We reviewed the data of the patients older than 60 years and with a LOS higher than 5 days admitted to our ICU in 2007. We calculated the daily SOFA score of the patients with an initial SOFA score higher than 9. IGSII, LOS and mortality were compared between patients with a SOFA score higher than 9 for at least 5 days (SOFA(+) group) and the other selected patients (SOFA(-) group).

Results In 2007, 430 patients were admitted to our ICU. One hundred and forty were older than 60 years and remained more than 5 days in the ICU. Eleven of these patients had a SOFA score higher than 9 during 5 days or more. In the SOFA(+) group, LOS (22.6 \pm 13.1 vs $10.8 \pm 6.9$ days) and mortality $(55 \%$ vs $33 \%)$ are significantly higher $(P<0.05)$. The mortality of $55 \%$ is in good agreement with the predicted IGSII mortality but far less than the $100 \%$ predicted by Cabré and colleagues.

Conclusions A SOFA score higher than 9 for at least 5 days in patients older than 60 years seems useless to define futility in our patient population. It is applicable to only less than 3\% of the 430 patients admitted in 2007. The mortality rate of the SOFA(+) group increased but remained far from values permitting to withdraw or withhold intensive care. A weakness of the use of sequential SOFA score to predict outcome is that some therapeutic options can influence the value of the SOFA score.

\section{Reference}

1. Cabré L, et al:. Intensive Care Med 2005, 31:927-933 
P247

Bacteremia, APACHE II and need for renal replacement therapy are independent predictors of mortality in critically ill transplanted patients with cirrhosis

C Karvellas', F Pink'2, M McPhail², G Auzinger'2, W Bernal'2, I Eltringham², J Wendon ${ }^{2}$

'University of Alberta, Edmonton, Canada; '2King's College Hospital, London, UK Critical Care 2010, 14(Suppl 1):P247 (doi: 10.1186/cc8479)

Introduction Our objective was to determine what physiological and biochemical factors predict development of bacteremia in transplanted cirrhotic patients (TP) and, upon diagnosis of bacteremia, what is the natural history of bacteremic patients (BTP) versus controls (TP).

Methods Data collected prospectively and entered into a dedicated physiology database for all transplanted cirrhotic patients admitted to a specialist liver intensive therapy unit (LITU) were examined from January 2003 to July 2005 for incidence of bacteremia and survival.

Results In total, 362 patients were transplanted for decompensated cirrhosis (64\% elective, 36\% in-hospital). Thirty-eight (10\%) suffered bacteremia (BTP) while 324 (90\%) patients did not (TP). Forty-nine per cent of isolates were Gram-positive organisms, 45\% Gram-negatives and $6 \%$ fungemia. Median time to first bacteremia was 11.5 days. On admission (univariate), BTP patients had significantly higher APACHE ॥ scores ( 23 vs $11, P<0.001)$, worse acidosis ( $\mathrm{pH} 7.27$ vs $7.35, P<0.001)$ and greater degrees of encephalopathy (GCS 12 vs $15, P<0.001$ ). During their LITU course BTP patients had significantly greater requirements for renal replacement therapy (RRT) (68\% vs $17 \%, P<0.001)$, mechanical ventilation (median 15 days vs 2 days, $P<0.001)$ and a longer median LITU stay (21 vs 3 days, $P<0.001)$. Survival to hospital discharge was worse in the BTP group (55\% vs $95 \%, P<0.001)$. Multivariate analysis (logistical regression) was performed modelling with APACHE II. APACHE II (odds ratio 1.06), bacteremia (4.01) and requirement for RRT pre or post transplant (12.5) were independent predictors of mortality. The APACHE II model performed well (AUROC 0.817).

Conclusions In transplanted cirrhotic patients, bacteremia was associated with increased severity of illness on admission, greater requirements for organ support and independently adversely impacted on survival. Higher APACHE II score and the requirement of RRT during LITU stay were also independently predictive of mortality.

\section{P248}

External validation of six mortality prediction systems for intensive care unit patients

M Verhofstad', TTimmers², LP Leenen², KG Moons ${ }^{3}$

'St Elizabeth Hospital, Tilburg, the Netherlands; ${ }^{2}$ Universitair Medical Centre Utrecht, the Netherlands; ${ }^{3}$ Julius Centre for Health Sciences and Primary Care, Utrecht, the Netherlands

Critical Care 2010, 14(Suppl 1):P248 (doi: 10.1186/cc8480)

Introduction This study investigates the prognostic quality of six prediction models (APACHE II score, SAPS II, SAPS II (Expanded), SAPS 3, MPM and MPM IIO) for assessment in an adult surgical ICU in the Netherlands.

Methods Of all single admissions to the surgical ICU of the St Elisabeth Hospital between 1995 and 2000, data to calculate the results of six prediction models were prospectively documented. To evaluate discrimination and calibration, receiver operating characteristic (ROC) curves, area under the characteristic (AUC) curve and the HosmerLemeshow goodness-of-fit test were performed.

Results The data of 1,821 patients were applied to all six models. Accurate overall mortality prediction was found for the APACHE II, SAPS II, SAPS 3 and MPM models. Discrimination was best for the SAPS 3 model and worst for the APACHE II and MPM models with AUC of $0.81,0.77$ and 0.77 , respectively (Figure 1). Calibration was poor for the six prediction systems, varying between 23 (SAPS 3) and 233 (SAPS II (Expanded)).

Conclusions The SAPS 3 prediction mortality model is the best validated model for a Dutch surgical ICU population of six models tested. The other general prognostic models for ICU patients underestimate the risk of dying.

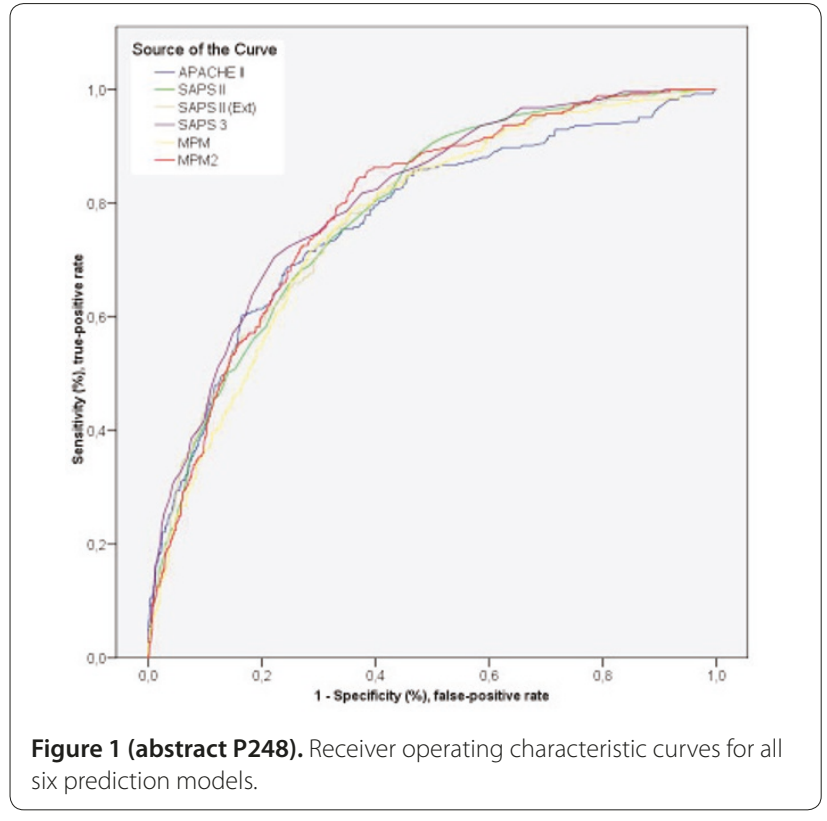

\section{P249}

Prognostic accuracy of severity score for prolonged ICU length of stay $>72$ hours in general surgical ICU: a prospective study S Kongsayreepong, N Lomarat, S Thamtanavit Siriraj Hospital, Mahidol University, Bangkok, Thailand Critical Care 2010, 14(Suppl 1):P249 (doi: 10.1186/cc8481)

Introduction Prolonged ICU stay is associated with high morbidity, mortality and costs $[1,2]$. Prediction of this prolonged stay will provide information for physician and family and help with resource allocation. Even though, available severity scoring system ie. APACHE II, APACHE III, MPM, SAPS II, MODS scores are widely accepted for evaluating outcomes in the ICU population. But these models might be inaccurate when apply to subpopulation and might not predict prolonged length of stay. The objective of this study was to refine ASA physical status and five severity scores (APACHE II, APACHE III, SOFA, SAPS, MOFS) in order to predict prolonged length of stay in the adults patients admitted to the general surgical ICU.

Methods This prospective observational study was done in 948 adult surgical patients admitted to the general surgical ICU at Siriraj Hospital during 1 January 2005 to 31 December 2005. A prolonged length of stay was defined as staying in the ICU $>72$ hours. Preoperative ASA physical status and five severity scores as APACHE II, APACHE III, SOFA, SAPS, MOFS on day 1 were registered and calculated. We analyzed the relationship of each severity score and outcome as prolonged ICU length of stay.

Results Prolonged length of stay represented $20.1 \%$ of ICU admissions. Among severity scores, ASA physical status IV $(P<0.001)$, emergency ASA $(P<0.001)$, APACHE III $(P=0.025)$ were significantly associated with prolonged ICU length of stay ( $>72$ hours) while MODS was most likely associated with this prolonged length of stay $(P=0.059)$. From multivariate analysis, only ASA physical status IV (OR 6.503 (2.314 to 18.275), $P<0.001$ ) and emergency ASA (OR 4.52 (2.86 to 7.12$), P<0.001)$ were significantly associated with prolonged ICU length of stay ( $>72$ hours).

Conclusions The study was conducted in 948 adult surgical patients admitted to the general surgical ICU at a 3rd referral university hospital. Among all of the severity scores, ASA physical status IV and emergency ASA were significantly correlated with prolonged ICU length of stay $>72$ hours.

\section{References}

1. Halpern NA, Betts L, Greenstein R: Federal and nationwide intensive care units in health care costs: 1986-1992. Crit Care Med 1994, 22:2001-2007.

2. Arabi Y, Venkatesh S, Haddad S, Al Shimemeri A, Al Malik S: A prospective study of prolonged stay in the intensive care unit: predictors and impact on resource utilization., prospective, mixed ICU, $>14$ days. Int $J$ Qual Health Care 2002, 14:403-410. 
P250

Perioperative scores to predict mortality in surgical oncologic patients: a review of 1,362 cases

C Simoes ${ }^{1}$, L Hajjar'2, M Carmona', F Galas'1, J Auler Jr'

'Instituto do Cancer do Estado de São Paulo, Brazil; 'Heart Institute, São Paulo, Brazil

Critical Care 2010, 14(Suppl 1):P250 (doi: 10.1186/cc8482)

Introduction Surgical outcomes depend on many factors, including baseline characteristics and differences in surgical and anesthetic practices. Few data are available in general surgical oncological patients. We aimed to evaluate the predictive value of Porthsmouth POSSUM and American Society of Anesthesiologists (ASA) scores in oncologic patients submitted to surgical procedures.

Methods Data were collected from a database filled with demographic characteristics, neoplasm definitions, co-morbidities and data from intraoperative room and hospital mortality. The predicted mortality risk was calculated using Porthsmouth POSSUM and ASA physical status grading.

Results From 1,362 surgical patients, the observed general hospital mortality was $2.34 \%$. ASA grading underestimated the mortality for low-complexity patients, overestimated in patients with severe systemic disease (ASA III), and was a strong predictor of mortality in patients with life-threatening conditions (ASA IV) (Table 1). On the other hand, the Porthsmouth POSSUM scoring system overestimated mortality in all groups (Table 2).

Table 1 (abstract P250). Observed and predicted mortality of oncologic patients evaluated through ASA physical status grading

\begin{tabular}{lccc}
\hline ASA & Number & Observed & Predicted \\
\hline ASA I & 209 & $4(1.91 \%)$ & $1(0.05 \%)$ \\
ASA II & 867 & $10(1.15 \%)$ & $1(0.4 \%)$ \\
ASA III & 247 & $7(2.83 \%)$ & $11(4.5 \%)$ \\
ASA IV & 39 & $11(28.2 \%)$ & $10(25 \%)$ \\
Total & 1,362 & $32(2.34 \%)$ & \\
\hline
\end{tabular}

Table 2 (abstract P250). Observed and predicted mortality of oncologic patients evaluated through Portsmouth POSSUM scoring

\begin{tabular}{lccc}
\hline Number & Observed & Predicted & 0 to $\mathbf{5}$ \\
\hline 0 & 0 & $0(3.1 \%)$ & 5 to 30 \\
1,055 & $15(1.42 \%)$ & $142(13.5 \%)$ & 30 to 100 \\
307 & $17(5.53 \%)$ & $149(48.7 \%)$ & 0 to 100 \\
1,322 & $32(2.34 \%)$ & & \\
\hline
\end{tabular}

Conclusions In surgical oncologic patients, Porthsmouth POSSUM and ASA are not good predictors of mortality. These data suggest that a more sensible and specific score is needed in this population.

Reference

1. Wakabayashi H, et al.: Int J Surg 2007, 5:323-327.

P251

Scoring patients with a suspected infection in the emergency department (ED): comparison of the ED-specific MEDS score with APACHE II and SOFA score

J Wilhelm, S Hettwer, D Hammer, M Schürmann, M Amoury, H Ebelt, K Werdan

University Hospital Halle (Saale), Germany

Critical Care 2010, 14(Suppl 1):P251 (doi: 10.1186/cc8483)

Introduction Several scores exist for characterization of critically ill patients, developed in part for septic patients especially for the ICU patient (for example, Acute Physiology and Chronic Health Evaluation, APACHE II; Sequential Organ Failure Assessment, SOFA), but also for the septic patient in the emergency department (ED) (Mortality in Emergency Department Sepsis, MEDS). The practicability of these scores in the ED is discussed controversially. The aim of our study was to directly compare these scores in the setting of an ED on a cohort of patients with suspected infection. Methods Patients with suspected severe infection from which blood cultures were taken were included in the study. Procalcitonin (PCT) levels were determined, and APACHE II, MEDS and SOFA scores were calculated at admission. Endpoints were 28-day-mortality and necessity for therapy on an ICU. Receiver operating characteristic (ROC) curve analysis was performed to compare the ability of the three scores to predict the endpoints.

Results We analyzed 211 patients. Eighty-two patients (43.6\%) had a PCT value $\geq 2 \mathrm{ng} / \mathrm{ml}$ and were considered septic. Mean values of APACHE II, MEDS and SOFA score were $16.1 \pm 8.5,7.4 \pm 4.7$, and $3.4 \pm 3.1$ respectively. Tables 1 and 2 show the area under the curve (AUC) values of the ROC curves for mortality (Table 1) and ICU therapy (Table 2).

Table 1 (abstract P251). ROC analysis for mortality

\begin{tabular}{llcc}
\hline Score & AUC & Standard error & Significance \\
\hline APACHE II & 0.804 & 0.045 & $<0.001$ \\
MEDS & 0.785 & 0.048 & $<0.001$ \\
SOFA & 0.708 & 0.051 & 0.006 \\
\hline
\end{tabular}

Table 2 (abstract P251). ROC analysis for ICU therapy

\begin{tabular}{llcc}
\hline Score & AUC & Standard error & Significance \\
\hline APACHE II & 0.757 & 0.038 & $<0.001$ \\
MEDS & 0.648 & 0.042 & 0.001 \\
SOFA & 0.731 & 0.039 & $<0.001$ \\
\hline
\end{tabular}

Conclusions All three scores are useful prognostic factors for mortality and for ICU therapy in the ED, with usually lower patients' severity of infection than in the ICU. The ICU-validated APACHE II and SOFA scores were of similar prognostic value as the ED-specific MEDS score.

P252

Validation of the new proposed Emergency Trauma Score (EMTRAS)

M Mangini', S Di Valvasone ${ }^{2}$, C Greco ${ }^{2}$, A Ognibene', G Cappuccini', R Spina', RTartaglia ${ }^{3}$, G Zagli', A Peris ${ }^{1}$

'Careggi Teaching Hospital, Florence, Italy: 2Postgraduate School of Anesthesia and Intensive Care, University of Florence, Italy; ${ }^{3}$ Centre for Clinical Risk Management and Patient Safety of Tuscany Region, Florence, Italy Critical Care 2010, 14(Suppl 1):P252 (doi: 10.1186/cc8484)

Introduction Recently, Raum and colleagues [1] generated and validated a new trauma score (Emergency Trauma Score, EMTRAS) based on age, prehospital GCS, prothrombin time and base excess. Each parameter is subdivided into four classes, scored from 0 to 3 points. Scores of each class are summed to obtain the EMTRAS, ranging from 0 to 12. Here we present preliminary results of a study with the aim to validate this new scoring system and to compare it with other commonly used illness scores.

Methods One hundred and fifty trauma patients admitted to the regional referral trauma center (Careggi Teaching Hospital, Florence, Italy) were studied. Predictive value of the EMTRAS score was compared with the Injury Severity Score (ISS), Revised Trauma Score (RTS), Trauma Injury

Table 1 (abstract P252). Area under the curve (AUC) for EMTRAS and other scoring systems

\begin{tabular}{lll}
\hline Variable & AUC & $95 \% \mathrm{Cl}$ \\
\hline EMTRAS & 0.809 & 0.74 to 0.87 \\
ISS & 0.758 & 0.68 to 0.83 \\
RTS & 0.818 & 0.74 to 0.88 \\
TRISS & 0.909 & 0.84 to 0.96 \\
SAPS II & 0.845 & 0.78 to 0.90 \\
\hline
\end{tabular}


Severity Score (TRISS) and Simplified Acute Physiology Score (SAPS) II. Logistic regression analysis was performed with MedCalc version 10 (MedCalc Software, Mariakerke, Belgium).

Results The areas under the curves confirmed a good reliability of the EMTRAS score if compared with other validated trauma scores and with nontrauma-specific scores such as SAPS II (Table 1). In particular, patients with EMTRAS scores of 5, 6 and 7 had a more major risk of death (odds ratio) of 2.3, 4 and 16, respectively, than patients with EMTRAS score below 5 .

Conclusions Our preliminary results confirm that EMTRAS has a good correlation with mortality risk. All four parameters of the score are simply available in a small amount of time, allowing physicians to quickly estimate trauma patients' severity, before other examinations like CT scan are performed.

\section{Reference}

1. Raum MR, et al:: Crit Care Med 2009, 37:1972-1977.

\section{P253}

A new severity score for community-acquired pneumonia:

PARB score

N Uchiyama, R Suda, S Yamao, Y Tomishima, T Jinta, N Nishimura,

N Chohnabayashi

St Luke's International Hospital, Tokyo, Japan

Critical Care 2010, 14(Suppl 1):P253 (doi: 10.1186/cc8485)

Introduction Community-acquired pneumonia (CAP) is one of the most common diseases encountered in the emergency department (ED). For the ED physician, it is quite difficult to estimate the severity of CAP patients in the ED because most CAP patients present at an earlier stage of disease. Although several societies such as ATS, IDSA and BTS have established severity scores for CAP, those scores are too complicated to calculate easily, especially in a busy department such as the ED. Therefore, a new simple and useful severity score for CAP is necessary for ED physicians. The purpose of our study is to develop a new severity score for CAP using 30-day mortality or patients needing more than 2 weeks oxygen therapy as the main outcomes.

Methods We derived a prediction rule for outcomes by analyzing data on 243 adult inpatients with CAP between March 2006 and November 2008 in our hospital. Patients' charts were abstracted to collect data relating to demographics, history, coexisting illnesses, laboratory results, and radiographic findings. Significant predictors of outcomes were identified through logistic regression analyses.

Results The following were independently associated with outcomes: presence of pleural effusions (P) (OR 5.0 (95\% Cl: 1.70 to 14.85)); albumin (A), <3.0 g/dl (OR 8.7 (1.43 to 53.29)); respiratory rate (R), >30/minute (OR 8.0 (1.62 to 39.64)); BUN (B), >25 mg/dl (OR 3.4 (1.07 to 11.07)). Patients were assigned according to whether they had these factors or not from class 0 to class IV (class 0 means the CAP patient had no these factors, class IV means the CAP patient had all these factors). Patients in risk class 0 to IV had a predicted probability of death or $>2$ weeks oxygen therapy of $0.03 \%$, $0.08 \%, 52.2 \%, 75.0 \%$ and $100 \%$, respectively. The sensitivity and specificity of this model were $36.0 \%$ and $99.0 \%$, respectively. Accuracy of this model was $91.9 \%$. The area under the ROC curve was 0.8705 .

Conclusions The PARB score can accurately predict patients with CAP who are at risk for death or who will need more than 2 weeks oxygen therapy. This score may help ED physicians provide adequate treatment for patients with CAP.

\section{References}

1. Fine MJ, et al:: N Engl J Med 1997, 336:243-250

2. Lim WS, et al:: Thorax 2003, 58:377-382
P254

Modified Early Warning Score and identification of patients with severe sepsis

L Zavatti, E Barbieri, E Amateis, M Girardis

Azienda Ospedaliera-Universitaria Policlinico di Modena, Italy

Critical Care 2010, 14(Suppl 1):P254 (doi: 10.1186/cc8486)

Introduction The early identification of patients with severe sepsis is a key point to provide the correct treatment and is actually based on clinical evaluation. In hospital wards, the Modified Early Warning Score has been proposed as a system to identify early signs of deterioration and for outreach team activation. However, MEWS sensibility in early recognition of septic patients is still unknown. The aim of this study was to evaluate whether MEWS $\geq 4$ is an adequate threshold for early identification of patients with severe sepsis.

Methods In 51 patients with severe sepsis (no septic shock) evaluated by our sepsis team, between November 2007 and December 2008 in the emergency department and no ICU wards, we collected the SOFA score and MEWS at the time of first evaluation, the ICU admission rate and the 30-day mortality rate. We subdivided and compared patients into two groups on the basis of a MEWS value $\geq 4$.

Results In the 22 (43\%) patients with a MEWS value $\geq 4$, the SOFA score $(5.9 \pm 2.9)$ was similar to that measured in the $29(57 \%)$ patients with MEWS value $<4(5.24 \pm 2.6)$ whereas the ICU admission rate was larger (54\%) than in patients with MEWS value <4 (24\%) $(P=0.03)$. In patients with MEWS value $\geq 4$ the 30 -day mortality rate was slightly lower (15\%) than in patients with MEWS value $<4(24 \%)(P>0.05)$.

Conclusions The above data indicate that a MEWS value $\geq 4$ has a very low sensitivity (43\%) for identification of patients with severe sepsis and therefore it should be used with caution for this aim and also as a criterion for ICU admission of septic patients (7/19 of patients admitted to the ICU with a MEWS value $<4$; sensitivity $63 \%$, specificity $68 \%$ ).

\section{Reference}

1. Subbe CP, Kruger M, Rutherford P, Gemmel L: Validation of a Modified Early Warning Score in medical admission. QJM 2001, 94:521-526.

\section{P255}

Impact of a systematic MEWS introduction on preoperative and postoperative evaluation in urgent/emergency surgery

N Maccarone1, I Guerri', M Franchi', C Fricelli', L Perretta², G Zagli², R Spina ${ }^{2}$, M Linden ${ }^{2}$, M Bonizzoli², A Peris ${ }^{2}$

'Postgraduate School of Anesthesia and Intensive Care, University of Florence, Italy; ${ }^{2}$ Careggi Teaching Hospital, Florence, Italy

Critical Care 2010, 14(Suppl 1):P255 (doi: 10.1186/cc8487)

Introduction The Modified Early Warning Score (MEWS) has been used in medical wards as a decision-making instrument. Here we show a pilot study on MEWS introduction as a routine method for care improvement in urgency/emergency surgical patients.

Methods Patients enrolled underwent urgent/emergency surgery due to trauma, appendicitis, cholecystitis, gut perforation or ischemia. Data collection with MEWS involved patients admitted for between April and October 2009. It was measured during preoperative evaluation and within 24 hours after surgery. To evaluate the role of MEWS utilization we use as outcome criteria: hospital length of stay (LOS), mortality, ICU or subintensive care unit (SCU) need. The MEWS group was compared with a control historical group (January 2008 to March 2009).

Results Groups were similar in surgical diagnosis and demographic characteristics. Patients of the control group showed a higher mortality rate compared with the MEWS group (6.7 vs 3.7). The number of SCU admissions after surgery passed from $9.6 \%$ to $15.4 \%$ after MEWS

Table 1 (abstract P255)

\begin{tabular}{|c|c|c|c|c|c|c|c|}
\hline & Number & $\begin{array}{l}\text { Age, years } \\
\text { (mean) }\end{array}$ & $\begin{array}{c}\text { ASA } \\
\text { (mean) }\end{array}$ & $\begin{array}{l}\text { Hospital LOS, } \\
\text { days (mean) }\end{array}$ & $\begin{array}{c}\text { Mortality, } \\
\%(n)\end{array}$ & $\begin{array}{c}\text { ICU admission, } \\
\%(n)\end{array}$ & $\begin{array}{c}\text { SCU admission, } \\
\%(n)\end{array}$ \\
\hline Control group & 714 & 54.8 & 1.9 & 9.6 & $6.7 \%(48)$ & $9.6 \%(69)$ & $12.9 \%(92)$ \\
\hline
\end{tabular}


introduction, whereas need for an ICU bed decreased from 12.9\% to 10.6\% (Table 1). Linear regression between high MEWS score and hospital LOS resulted significant $(P<0.001)$.

Conclusions These preliminary results indicate that MEWS, used in preoperative evaluation and follow-up in urgent/emergency surgical patients, can have an important impact on patient care and mortality.

\section{References}

1. Cei M, et al:: Int J Clin Pract 2009, 63:591-595.

2. Gardner-Thorpe J, et al:: Ann R Coll Surg Engl 2006, 88:571-575.

3. Subbe CP, et al:: QJM 2001, 94:521-526.

\section{P256}

\section{Clinical predictors of physiological deterioration}

M Shehata, A Hussein, A Farouk, H Khaled Nagi

Cairo University, Cairo, Egypt

Critical Care 2010, 14(Suppl 1):P256 (doi: 10.1186/cc8488)

Introduction To study the ability and the feasibility of the Modified Early Warning Score (MEWS) as a screening tool to predict the high-risk critically ill patients who may develop cardiorespiratory arrest, and to compare between the MEWS and the Simplified Acute Physiology Score II (SAPS II) regarding the sensitivity, specificity and more easy to be applied.

Methods Randomized, prospective study in the critical care medicine department in the Cairo University Hospitals including 100 critically ill patients newly admitted to the ICU. The MEWS is calculated daily during the period of the stay in the ICU with determination of the score Max, and SAPS II is calculated during the first 24 hours.

Results The MEWS score max grade of 8 or more was associated with the highest rate of arrest at which the sensitivity was $78.9 \%$, specificity was $93.5 \%$, efficacy was $88.00 \%$, with area under the curve (AUC) $=0.928$. The SAPS II - Expanded grade of 50 or more was associated with the highest rate of arrest at which the sensitivity was $71.1 \%$, specificity was $100 \%$, efficacy was $89.00 \%$, with AUC $=0.872$. Comparing the MEWS and SAPS II reveals that the MEWS has more sensitivity but less specificity than SAPS II and efficacy is almost the same. Regarding the simplicity and applicability: MEWS is easier, faster and simpler.

Conclusions The MEWS score is a useful screening tool to predict the high-risk patients who have high probability to develop cardiorespiratory arrest among the critically ill patients in the ICU. It is simple and practical, has more sensitivity than SAPS II and therefore should be recommended in clinical practice.

\section{Reference}

1. Le Gall et al:: Crit Care 2005, 9:R645-R652.

\section{P257}

Modified Early Warning Scores: inaccurate summation or inaccurate assignment of score?

M Edwards', H McKay², CVan Leuvan'², I Mitchell ${ }^{2}$

'Australian National University, Canberra, Australia;' 2 The Canberra Hospital,

Garran, Australia

Critical Care 2010, 14(Suppl 1):P257 (doi: 10.1186/cc8489)

Introduction Modified Early Warning Scores (MEWS) have been utilised to improve the recognition and medical response to patient deterioration. Their usefulness is, in part, dependent upon their accuracy and to date only small datasets have been analysed to determine the accuracy of MEWS [1]. The aim of our study was to determine the accuracy of MEWS and the reasons for any inaccuracies found in a large dataset.

Methods A prospective observational study of 9,672 vital sign sets and MEWS recordings in 315 adult medical and surgical patients admitted to four wards in both a tertiary and a metropolitan hospital over a 4-month period. Individual vital sign MEWS and total MEWS was assessed for accuracy against a computer-generated individual vital sign MEWS and total MEWS using the vital sign dataset and a modified MEWS algorithm. Results Of the 9,672 total MEWS recorded, 3,504 (3,504/9,672, 36\%) had discrepancies between the nurse-recorded and computer-generated total MEWS. Of these, 3,029 $(3,029 / 3,504,86 \%)$ underestimated the computergenerated total MEWS. In the majority of the total MEWS inaccuracies $(2,443 / 3,504,69.7 \%)$, the summation of the total MEWS from the individual MEWS was correct but the individual vital sign MEWS assignment was inaccurate. In 711 (711/3,504, 20.3\%) cases the individual vital sign MEWS assignment was correct but the summation for a total MEWS was incorrect, and in 350 (350/3,504, 10\%) cases both the individual vital sign MEWS assignment and summation for a total MEWS was incorrect.

Conclusions The underscoring between nurse-recorded and computergenerated MEWS indicates that the paper-based MEWS system is less likely to trigger a timely medical review and appropriate treatment of a deteriorating patient. Further education and regular auditing on the assignment of the individual vital sign MEWS may improve the accuracy of the score given that the summation of the score was not the predominant issue. Equally, an electronic system to calculate and summate the individual vital sign MEWS may reduce the total MEWS error.

\section{Reference}

1. Prytherch DR, et al:: Resuscitation 2006, 70:173-178.

\section{P258}

Analysis of Modified Early Warning System scores and intraoperative factors on the incidence of sepsis and septic shock after elective major surgery

PA Hampshire, A Guha, A Strong, D Parsons, P Rowan

Royal Liverpool and Broadgreen University Hospital, Liverpool, UK

Critical Care 2010, 14(Suppl 1):P258 (doi: 10.1186/cc8490)

Introduction Severe sepsis is an important cause of morbidity and mortality following major surgery. Factors that are associated with an increased risk of sepsis following surgery include emergency surgery, patient co-morbidities, allogeneic blood transfusion and degree of surgical insult [1]. Physiological track-and-trigger systems are widely used to identify deteriorating patients. The Modified Early Warning System (MEWS) is one such system, but has not been studied in regard to predicting the development of sepsis after surgery. Although high MEWS scores are associated with increased hospital mortality, the sensitivity of MEWS and other physiological track and trigger scores for predicting death or admission to intensive care is low [2].

Methods We carried out a prospective cohort study on 101 patients undergoing elective major surgery in a large university teaching hospital. The patients were followed up for 10 days, and the incidence of sepsis and septic shock was documented. MEWS scores were recorded daily for each patient. Admissions to critical care were documented, along with critical care length of stay.

Results Twenty-seven (27\%) patients developed sepsis and nine (9\%) developed septic shock. Factors associated with the development of sepsis were intraoperative blood transfusion $(P=0.013)$, duration of operation $(P=0.004)$ and a postoperative MEWS score greater than 3 $(P=0.0003)$. Using multivariate logistic regression analysis, a MEWS score greater than 3 after surgery was the only factor that remained significantly associated with sepsis (odds ratio 4.89, $P=0.003$ ). Although a high MEWS score was associated with sepsis after surgery, only five (19\%) patients who developed sepsis had an abnormal MEWS score prior to (mean 4.6 days) sepsis being diagnosed.

Conclusions The routine use of MEWS scores in postoperative elective surgical patients may help to identify those patients at risk of developing sepsis.

\section{References}

1. Veltkamp SC, Kemmeren JM, Graaf YVD, Edlinger M, Werken CVD: Prediction of serious complications in patients admitted to a surgical ward. Br J Surg 2002, 89:94-102.

2. Gao H, McDonnell A, Harrison DA, et al.: Systematic review and evaluation of physiological track and trigger warning systems for identifying at-risk patients on the ward. Intensive Care Med 2007, 33:667-679.

P259

Temporal variability of medical emergency team calls

J Tourtier, E Fontaine, J Vandenbossche, S De Rudnicki, E Falzone, M Borne, Y Auroy

Hopital Val-de-Grâce, Paris, France

Critical Care 2010, 14(Suppl 1):P259 (doi: 10.1186/cc8491)

Introduction We evaluated temporal variability of telephone calls to the medical emergency team (MET), as well as patient outcome. 


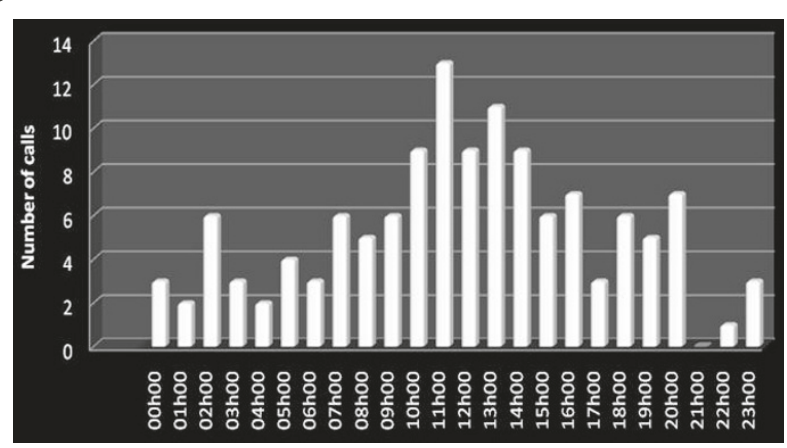

Figure 1 (abstract P259). Occurrence of calls throughout the day.
Methods All calls over a 48-month period between December 2005 and November 2009 were included. The time of telephone calls was noted, and occurrence throughout the day was studied. We compared the number of calls between 8:00 and 20:00 and between 20:00 and 8:00, using a Wilcoxon test. Patient's age, gender, reason for call, and outcome were entered into the ICU's database.

Results In the 4-year period, there were 136 calls, amongst which 17 were erroneous (12.5\%). Figure 1 shows the occurrence of calls throughout the day. Between 8:00 and 20:00 and between 20:00 and 8:00, there were respectively 93 and 43 calls $(P=0.02)$. The immediate causes of intervention were cardiac arrest in 35\%, respiratory depression in $8 \%$, and decreased level of consciousness in $43 \%$ of cases (other: 14\%). Eighteen percent of patients died during MET intervention. Amongst survivors, 31\% were transferred in the ICU. Involved patients'age was $67 \pm 17$ (male: 53\%). Conclusions Reporting data might have less accuracy during nights. Nevertheless, despite difficulty of capturing such data, our series suggests that there is a temporal variability of medical emergency team calls. The fact that the majority of calls occurred between 8:00 and 20:00 may probably be a reflection of the level of supervision and the general amount of patient activity within the hospital during the day.

\section{P260}

Medical emergency team use and outcomes in an Australian private hospital

J Barrett, J Reeves, T Bucknall, M Levinson

Cabrini Hospital, Melbourne, Victoria, Australia

Critical Care 2010, 14(Suppl 1):P260 (doi: 10.1186/cc8492)

Introduction Medical emergency teams (METs) are widespread in Australian public hospitals and are becoming more common in private hospitals. The epidemiology of MET calls and patient outcomes post MET have not been well studied in the private hospital setting.

Methods Prospective observational study of all inpatient MET calls in a 510-bed private teaching hospital for a 9-month period ending in August 2009. Information was collected on a standard data form after each MET call. Admission, discharge and mortality data were obtained from the hospital administrative database.

Results There were 302 MET calls and 17,419 admissions with a MET call rate of $1.7 \%$ of admissions. Mean (SD) age was 72.9 (17.0) and $42.9 \%$ of patients were male. The rate of Code Blue calls was $0.26 \%$ and of unexpected (non-NFR) deaths $0.09 \%$ of admissions. The MET call rate was higher for medical than surgical patients ( $2.4 \%$ vs $1.1 \%, P<0.005)$. Inpatient mortality was $24.8 \%$ and patients receiving a MET call had an increased mean length of stay compared with the hospital average (16.1 vs 5.4 days, $P<0.005)$. Activation of MET calls was delayed by more than 15 minutes in $7.6 \%$ of calls. Of the patients who died, only $16 / 69(23 \%)$ were transferred to the ICU post MET call. Median (range) time from MET call to death was 1.8 (0 to 76.6) days.

Conclusions The MET rate in our hospital overall is consistent with a maturing MET system. The higher MET rate in medical vs surgical patients is unexpected compared with the published experience in Australian public hospitals. This may reflect a difference in the patient population or a difference in the pre-MET ward management in our hospital. Whilst inpatient mortality was high, patients who died were mostly managed without transfer to the ICU and the non-NFR death rate was low. This is consistent with a significant overlap between MET and end-of-life care. Alternatively the high mortality could reflect that MET as an intervention occurs too late in these patients to favourably affect outcomes. A more detailed understanding of patients dying after MET calls is required to determine the best ongoing strategy to manage this high-risk group of patients.

\section{P261}

Continuously improving patient safety by a rapid response system V Van Bruggen, P Barendrecht, A Geense, E Van Dijk, M Achilleos, I Saris, M Meijer, A Deijkers, G Verwoerd, M Taks, E Oskam, R So Albert Schweitzer Hospital, Dordrecht, the Netherlands Critical Care 2010, 14(Suppl 1):P261 (doi: 10.1186/cc8493)

Introduction Resulting from the Dutch VMS Safety Program 'Prevent injury, work safely', we recently started to implement a rapid response system (RRS) in our hospital. The RRS consists of: a warning signs pocket card, a Rapid Response Team (RRT) and an evaluation system. The purpose of the RRS is to recognize and treat the patients with clinical warning signs early on the ward to reduce preventable hospital-wide avoidable injury. We present the first results of the implementation of the RRS.

Methods Data regarding the RRT calls and the evaluations were collected from 1 May 2008 until 30 April 2009 (total: 114 calls). We were interested in: the cause of the RRT call, the results of the call and the advice following the evaluation of the calls.

Results The main reasons for the calls were respiratory failure (47\%), hemodynamic problems (31\%), neurological problems (7\%) or a combination of problems in $15 \%$. In $45 \%$ of the calls the patient was admitted to the ICU, medium care unit (MCU) or cardiac care unit (CCU). In 55\% of the calls the patient stayed on the ward. During the call $14 \%$ of the patients who stayed on the ward obtained a do-not-resuscitate or a do-not-transfer-to-the-ICU order. These decisions were always taken in close cooperation with the doctor taking care of the patient on the ward. Evaluations were performed in $76 \%$ of the calls. In $45 \%$ of the calls the warning signs were recognized in time. Problems identified were late identification of clinical deterioration of the patient (30\%) and failure to treat to a patient with clinical warning signs due to failures in planning (6\%) or communication (26\%).

Conclusions $\ln 45 \%$ of the RRT calls, transfer of the patient to the ICU/MCU/ CCU is required. Hospital-wide improvement initiatives should focus on: timely recognition of clinical deterioration of the patient; failures in planning and communication; and timely agreed restrictive measurements on the ward.

\section{P262}

Role of critical care outreach to provide the intention to treat of hematological disorders: a pilot study

I Guerri', C Greco'1, L Perretta², G Cianfaldoni², G Longo², S Batacchi²,

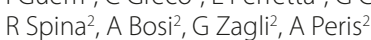

'Postgraduate School of Anesthesia and Intensive Care, University of Florence, Italy; ${ }^{2}$ Careggi Teaching Hospital, Florence, Italy

Critical Care 2010, 14(Suppl 1):P262 (doi: 10.1186/cc8494)

Introduction Hematological malignancy patients admitted to the ICU due to complications are at high risk of death. In the second half of 2008 we started Critical Care Outreach (CCO) activity. The purpose of this team was to start intensive care early as possible for avoiding mechanical ventilation and multiorgan failure for improving intensive treatment outcome.

Methods One hundred and twenty-six hematological patients from 2003 to November 2009 were included in the study. We divided patients into three groups: (1) pre-CCO group: 56 patients from 2003 to 31 December 2007; (2) transition group (2008): 22 patients admitted to the ICU and three treated in the hematology ward; (3) post-CCO group (January to November 2009): 18 patients admitted to the ICU and 27 patients treated in the haematology ward. In the post-CCO group 10 of the 27 patients were admitted to the ICU and 17 of these were treated in hematology ward, and nine of these received NIV (Figure 1). We defined six criteria for activation of the CCO team: radiological signs of pneumonia, organ 


\begin{tabular}{|c|c|c|c|c|c|c|c|c|}
\hline & 2003 & \multicolumn{2}{|c|}{2004} & 2005 & 20 & 2007 & 2008 & 2009 \\
\hline $\begin{array}{l}\text { Patients } \\
\text { Number }\end{array}$ & 10 & \multicolumn{2}{|c|}{11} & 12 & 1. & 11 & 22 & 18 \\
\hline $\begin{array}{c}\text { ICU } \\
\text { Mortality }\end{array}$ & $60 \%$ & \multicolumn{2}{|c|}{$54,5 \%$} & $75 \%$ & 58. & $63,3 \%$ & $54.5 \%$ & $33.3 \%$ \\
\hline $\begin{array}{l}\text { Intention } \\
\text { To Treat }\end{array}$ & $25 \%$ & \multicolumn{2}{|c|}{$40 \%$} & $33 \%$ & 0 & $100 \%$ & $90 \%)$ & $41 \%$ \\
\hline $\begin{array}{l}\text { Mechanical } \\
\text { Ventilation }\end{array}$ & $60 \%$ & \multicolumn{2}{|c|}{$72.7 \%$} & $83,3 \%$ & 91 & $82 \%$ & $55 \%$ & $28 \%$ \\
\hline $\begin{array}{l}\text { Patients } \\
\text { Number }\end{array}$ & \multicolumn{2}{|c|}{$\begin{array}{l}\text { Consultancy } \\
\text { Number }\end{array}$} & \multicolumn{3}{|c|}{$\begin{array}{c}\text { Consultancy Median } \\
\text { Time }\end{array}$} & $\begin{array}{c}\text { ICU } \\
\text { Admitted } \\
\text { Patients }\end{array}$ & $\begin{array}{c}\text { Patients } \\
\text { Treated in } \\
\text { Hematology } \\
\text { Ward }\end{array}$ & $\begin{array}{c}\text { NIV } \\
\text { Treated } \\
\text { Patients }\end{array}$ \\
\hline 27 & \multicolumn{2}{|l|}{91} & \multicolumn{3}{|c|}{$52 \mathrm{~min}$} & $37 \%$ & $63 \%$ & $53 \%$ \\
\hline
\end{tabular}

Figure 1 (abstract P262).

failure, positive microbiological test, need for increase in oxygen therapy, SIRS progression, and Modified Early Warning Score (MEWS) $>3$ [1]. Primary outcome is the ability of the $\mathrm{CCO}$ to provide the intention to treat hematological malignancies.

Results After CCO introduction, intention to treat was guaranteed at $90 \%$ in 2008 and at $41 \%$ in 2009. Comparing the number of criteria of activation, during 2008 and 2009 intensivists were activated earlier, with a lower number of criteria. The ICU mortality rate passed from $60 \%$ in 2003 to $54.5 \%$ in 2008 and $33.3 \%$ in 2009. Also the need for mechanical ventilation decreased after CCO introduction.

Conclusions The introduction of CCO and activation criteria improves the intention to treat and reduces ICU admissions and mortality.

Reference

1. Subbe CP, Kruger M, Rutherford P, Gemmel L: QJM 2001, 94:521-526.

\section{P263}

Score to door time: a benchmarking tool for rapid response systems C Subbe', L Durham², J Welch³, K Oglesby'

'Wrexham Maelor Hospital, Wrexham, UK; ${ }^{2}$ NorthTyneside General Hospital,

North Shields, UK; ${ }^{3}$ University College Hospital, London, UK

Critical Care 2010, 14(Suppl 1):P263 (doi: 10.1186/cc8495)

Introduction Delay in recognition and treatment of deteriorating patients is a significant contributor to mortality of emergency admissions to intensive care (ICU) [1]. Rapid response systems (RRS) seek to improve this process. Service evaluation of RRS might be hampered by the lack of comparative quality assurance tools. The aim of this international collaborative audit was to test a benchmarking tool for the process of emergency admissions to the ICU in analogy to the door-to-needle time in cardiology.

Methods Data were collected in August and September 2009. Collaborators were asked to audit data from a minimum of five emergency admissions to the ICU (range 3 to 35 admissions). The Score 2 Door time was defined as the time between first trigger of the local track-and-trigger system and time of admission to intensive care. Collaborators were asked to give possible causes for delays in transfer to the ICU. Observations were risk-stratified with an age-adjusted Modified Early Warning Score (MEWS-A) [2]. Data were anonymised and hospitals were coded in a random fashion.

Results Up to 22 September, data for 125 patients from 17 hospitals had been submitted. Of these, four were non-UK hospitals (US x 1, Denmark $x$ 1, Australia $\times 2$ ) using medical emergency team type of systems and UK hospitals used a summary score. Median age was 67 years. Mean APACHE II score on admission to the ICU was 18 (SD 8). The median Score 2 Door time was 3:15 (range 0:09 to 19:45). Median time was shorter in the four non-UK hospitals (1:29 vs 4:05). Sicker patients (as evidenced by a higher MEWS-A trigger) did not have shorter Score 2 Door times. The main quoted causes for delays in ICU admission were transient improvement of condition ( $n=$ 4), immediate treatment on the ward $(n=9)$, a procedure ( $n=12$; that is, surgery, computer tomography), a shortage of intensive care beds at the time of referral $(n=11)$ or waiting for a senior review $(n=14)$.

Conclusions Organisational and other delays in ICU admission might reduce the impact of RRS on care of deteriorating patients on general wards. The Score 2 Door time is a pragmatic tool to quantify and benchmark these delays in order to identify obstacles to patient flow and improve care.

\section{References}

1. McQuillan P, et al.: Br Med J 1998, 316:1853-1858.

2. Subbe CP, etal: OJM 2001, 94:521-526.

\section{P264}

A comparison of injected lidocaine $2 \%$ and no analgesia for peripheral intravenous cannula insertion in the emergency department

G Papakitsos', T Papakitsou², A Kapsali'

${ }^{1}$ General Hospital Arta, Greece; ${ }^{2}$ General Hospital Messologi, Greece

Critical Care 2010, 14(Suppl 1):P264 (doi: 10.1186/cc8496)

Introduction Many patients who present to the emergency department require placement of a peripheral intravenous line, which frequently causes pain and anxiety. We compare pain and anxiety associated with peripheral intravenous cannula insertion after pretreatment with no local anaesthesia or subcutaneously injected, buffered $2 \%$ lidocaine.

Methods In a randomized study, peripheral intravenous cannulas were inserted in 185 patients arriving in the emergency department. In random order, insertion sites were pretreated with nothing or injected, buffered lidocaine. After each intravenous insertion, subjects recorded pain, anxiety, and preference (as patient and provider) for each technique on a 10-point numeric rating scale. Higher scores indicated greater pain, anxiety and preference.

Results Median pain scores were 7 (5 to 8) without local anaesthesia and 1 ( 1 to 2 ) with injected, buffered lidocaine. Median anxiety scores were 4 (3 to 7) without local anaesthesia and 2 ( 1 to 3 ) with injected, buffered lidocaine. Most intravenous placement attempts were successful, regardless of technique. Eighty-seven per cent of subjects indicated they would always request buffered lidocaine for peripheral intravenous insertion. In the emergency department or other busy practice setting, injected, buffered lidocaine has the advantage of being immediately effective.

Conclusions Pain and anxiety associated with peripheral intravenous insertion is significantly reduced by using topical injected, buffered lidocaine. Injected, buffered lidocaine reduces intravenous insertion pain more than pretreatment with no local anaesthesia, without affecting success.

\section{Reference}

1. McNaughton C, et al:: A randomized, crossover comparison of injected buffered lidocaine, lidocaine cream, and no analgesia for peripheral intravenous cannula insertion. Ann Emerg Med 2009,54:214-220.

\section{P265}

High-sensitive troponin T measurement for patients with acute chest pain: improvement of diagnostics?

S Popp, S Herdtle, H Pohlmann, M Poravas, D Umarov, R Bach, T Bertsch, MChrist

Klinikum Nueremberg, Germany

Critical Care 2010, 14(Suppl 1):P265 (doi: 10.1186/cc8497)

Introduction Highly precise laboratory methods have been developed to determine cardiac troponin levels (cTn) according to the universal definition of acute myocardial infarction (MI). We examined the impact of lowering the level of detection of cTn measurement in consecutive patients presenting to the emergency department (ED) of a large German community hospital.

Methods $\mathrm{CTnT}$ levels were measured using a standard assay (Roche $\mathrm{cTnT}$ fourth generation; cutoff $\geq 0.04 \mathrm{ng} / \mathrm{ml}$ ) and a high-sensitive assay (Roche cTnThs; cutoff $\geq 0.014 \mathrm{ng} / \mathrm{ml}$ ). Patients were categorized to have an acute $\mathrm{Ml}$ according to the universal definition using the old or the new cutoff point of cTnT levels (cTnT $\geq 0.04 \mathrm{ng} / \mathrm{ml}$ or cTnThs $\geq 0.014 \mathrm{ng} / \mathrm{ml}$ ).

Results We analyzed 137 consecutive patients with chest pain admitted to the ED (age: $65.7 \pm 15.5$ years; $63.5 \%$ male; in-hospital mortality: $0.7 \%$ ). Totals of $36 \%, 22 \%$ and $42 \%$ of patients presented within 2 hours, 2 to 6 hours and $>6$ hours of chest pain. Using the old cutoff point, 30 patients have been classified as acute Ml (type $1 \mathrm{Ml}$ : 67\%; type $2 \mathrm{Ml}$ : 33\%). Using the new cutoff point (TnThs $\geq 0.014 \mu \mathrm{g} / \mathrm{l})$, diagnosis of acute Ml significantly 
increased by $116 \%$ ( $n=65$; type $1 \mathrm{Ml}$ : 54\%; type $2 \mathrm{Ml}$ : 46\%; $P<0.05$ ). Type $1 \mathrm{Ml}$ increased by $75 \%$, which was reflected by a $57 \%$ decrease of patients formerly classified as instable angina. Of note, increases of $\mathrm{MI}$ diagnosis were mainly reflected by a 200\% increase of type $2 \mathrm{MI}$ (for example, acute heart failure, tachycardia or hypertensive crisis). Numbers of patients with noncardiac chest pain were not significantly changed with the use of the new cutoff point. AUC of admission CTnT levels were 0.76 and 0.96 for the cTnT fourth generation and CTnThs $(P<0.05)$. At admission, sensitivity and specificity of cTnThs were $93 \%$ and $94 \%$ for the detection of acute MI, while they were $32 \%$ and $96 \%$ using CTnT fourth generation.

Conclusions Using lower cutoff points for the definition of Ml as suggested by current recommendations, the rate of chest pain patients with acute MI doubled. Because the increased rate of patients categorized as acute $\mathrm{Ml}$ is mainly reflected $\mathrm{Ml}$ type 2 , the ED triage decision at lower cutoff levels of CTnT levels should include patient history, physical examination, 12-lead ECG and cardiac imaging.

\section{P266}

\section{Abstract withdrawn}

\section{P267}

Correlation of lung comet score with decreased gas exchange in patients with acute pulmonary edema: a pilot study

G Guiotto', F Dello Vicario', R Marino', F Paladino', S Scott², F Schiraldi'

'San Paolo Hospital, Napoli, Italy; ${ }^{2}$ Gold Coast Hospital, Southport, Australia

Critical Care 2010, 14(Suppl 1):P267 (doi: 10.1186/cc8499)

Introduction The aim of this study was to determine the correlation between $B+$ line score and blood gas analysis (BGA) parameters in patients with acute cardiogenic pulmonary edema (ACPE). The presence of $B+$ line artifacts on lung US is correlated with increased extravascular lung water. In patients with acute decompensated heart failure the resolution of $\mathrm{B}+$ lines can be used to monitor resolution of pulmonary congestion. A simple nine-point comet score has been proposed to quantify B+ lines and monitor ACPE treatment [1].

Methods Twenty-one patients with ACPE were submitted to lung US and BGA on presentation at 2 and 24 hours after admission. Lung US was done in nine thoracic areas and scored one point if $\mathrm{B}+$ lines were present. A score of 10 was given for the presence of multiple coalesced comets in all fields (white lung). BGA values were used to calculate $\mathrm{PO}_{2} / \mathrm{FiO}_{2}(\mathrm{P} / \mathrm{F})$ and A-a gradient.

Results A total of 64 scans were performed with simultaneous BGA. Ninety-eight percent of patients presenting with white lung were severely hypoxic $\left(\mathrm{PO}_{2}<50 \mathrm{mmHg} ; \mathrm{P} / \mathrm{F}<200\right)$. Figure 1 illustrates a strong linear correlation between reduction in comet score and improvement in $\mathrm{P} / \mathrm{F}$

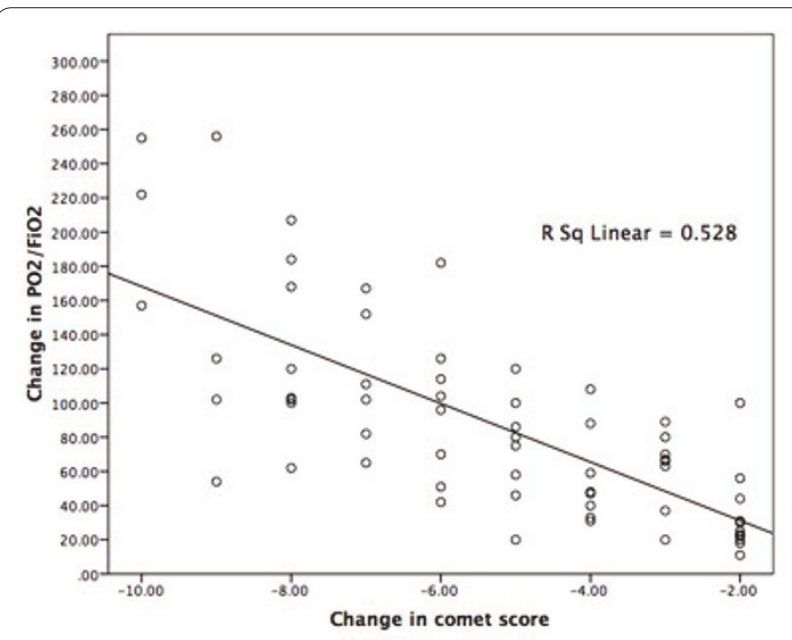

Figure 1 (abstract P267). Correlation between reduction in comet score and improvement in P/F. $(r=-0.73 ; P<0.001)$. A decrease in comet score of 2 points corresponded to a minimum increase of 20 points in $P / F(P<0.05)$. The correlation between comet score and A-a gradient was less striking significant $(r=$ $-0.51 ; P=0.05)$, perhaps because patients with underlying lung disease were not excluded from this pilot study.

Conclusions There is a strong negative correlation between lung comet score and gas exchange in patients with ACPE. In these patients, serial thoracic US may reduce the need for repeated invasive BGA monitoring and further help tailor therapy.

\section{Reference}

1. Volpicelli G, Caramello V, Cardinale L, et al:: Bedside ultrasound of the lung for the monitoring of acute decompensated heart failure. Am J Emerg Med 2008, 26:585-591.

P268

Magnitude of first medical contact in the long-term prognosis of patients treated with STEMI

D Becker, G Barczi, G Szabo, A Horvath, B Merkely

Semmelweis University, Budapest, Hungary

Critical Care 2010, 14(Suppl 1):P268 (doi: 10.1186/cc8500)

Introduction Numerous STEMI patients are treated successfully in the Central PCI on call system. Up until this time we do not have data about the first medical contact's - National Emergency Service or other medical staff - impact on long-term prognosis. It is also unknown how the method of transport to the $\mathrm{PCl}$ center (direct or secondary) influences the prognosis. Our aim was to evaluate the magnitude of first medical contact and the type of transport in the short-term and long-term prognosis.

Methods We evaluated the 30-day and 1-year mortality data of 1,890 consecutive patients who were treated with primary PCI due to STEMI. We selected the patients according to the first medical contact: National Ambulance Service or other medical staff, and according to the type of transport: direct or secondary transport. At the follow-up procedure we used the university's and the National Health Insurance Found's database. Results The 1,890 patients' follow-up rate was $100 \%$. First, $34.7 \%$ of the patients $(656 / 1,890)$ called for Emergency Service directly. This rate was even lower in working hours $(30.6 \%$ - 205/671). It was also observed that as the time delay was increasing from the onset of symptoms, the number of people who called for Emergency Service was lower. Between 10 and 12 hours it was just 12.2\% (19/155). Thirty-day mortality (Ambulance Service versus other staff): $7.3 \%$ (48/656) vs 9.5\% (117/1232) - $P=0.1$. One-year mortality: $12.7 \%(83 / 656)$ vs $16.1 \%(199 / 1232)-P=$ 0.04 . Second, $44.4 \%$ of the patients $(838 / 1,890)$ were transported directly to the $\mathrm{PCl}$ center (direct transport). Thirty-day mortality (direct versus secondary transport): 60/838 (7.2\%) vs 105/1,052 (9.9\%); 1-year mortality: $106 / 838(12.6 \%)$ vs $176 / 1,052$ (16.7\%). The difference is significant at 30 days and at 1 year also. The 30 -day mortality rate is higher with $24 \%$, the 1 -year mortality rate is higher with $15 \%$ among those patients who were admitted after a secondary transport.

Conclusions The long-term prognosis is significantly better among those STEMI patients who are transported directly to the $\mathrm{PCl}$ center and where the first medical contact is the National Ambulance Service.

P269

Ultrasound-guided hematoma block and fracture reduction: a new way to go forward

M Majeed, A Mukherjee, R Paw

Russells Hall Hospital, Dudley, UK

Critical Care 2010, 14(Suppl 1):P269 (doi: 10.1186/cc8501)

Introduction The conventional treatment for most distal radial fractures is closed reduction and cast immobilization. Conventionally the hematoma block is used to reduce the fractures but it is done blindly and radiographic techniques have been essential and effective in monitoring these reductions [1]. Radiation-free ultrasonography, however, can provide both real-time and dynamic multiple-plane images with a small and simpleto-use transducer that can be operated with only one hand. We therefore wanted to see whether the real-time and dynamic multiple-plane 
observation capabilities of ultrasonography would allow an ED physician to perform under direct vision a hematoma block and closed reduction without multiple attempts of either as are frequently required when only conventional techniques are used.

Methods Sonographically guided hematoma block and closed reduction was performed in three patients in the emergency department for an acute distal radial fracture. The efficacy of this method was evaluated and compared with that of conventional techniques.

Results The ultrasound images helped giving the hematoma block under direct vision and delineated the fractures as accurately as did the conventional radiographs. All parameters measured on the ultrasound images showed substantial restoration of anatomic alignment after reduction. Under direct vision, the hematoma block helped reduce the number of attempts and was less painful and achieved more patient satisfaction.

Conclusions Sonography is an accurate, simple, time-saving, less painful and radiation-free tool. Reducing badly displaced or angulated forearm fractures in the emergency department can be difficult. Multiple attempts at reduction may be required, with repeated trips to the radiology department, before an adequate reduction is achieved [2]. Therefore ultrasound-guided hematoma block and reduction of difficult forearm fractures reduces the number of needle attempts and allows the physician to assess the adequacy of the reduction at the patient's bedside.

\section{References}

1. Sonography for monitoring closed reduction of displaced extra-articular distal radial fractures. J Bone Joint Surg Am 2002, 84:194-203.

2. Ultrasound guided reduction of pediatric forearm fractures in the ED. Am J Emerg Med 2000, 18:72-77.

P270

Pulmonary edema in the prehospital setting: the importance of early treatment

S Lourenco, J Figueira Coelho, A Martins Baptista

Curry Cabral Hospital, Lisbon, Portugal

Critical Care 2010, 14(Suppl 1):P270 (doi: 10.1186/cc8502)

Introduction Patients with acute cardiogenic pulmonary edema require rapid assessment and therapy to prevent progression to respiratory failure and cardiovascular collapse. Lisbon city has emergency medical teams that respond to situations where the life of the patient is at risk and whose goal is to begin treatment if indicated and assure transport to the hospital in the best conditions possible. We studied the intervention of one of these teams on patients with acute pulmonary edema.

Methods Medical records of the activity of our prehospital emergency vehicle from April 2005 to October 2009 were analyzed. All records of patients having a clinical diagnosis of acute pulmonary edema at the scene were considered. Incomplete records were excluded.

Results In the 55 months considered our emergency vehicle attended 9,856 calls, from which 493 (5\%) were for acute pulmonary edema. Patients ages range from 20 to 99 years, with a mean age of 75.8 years, and $47.8 \%$ were males. A total of $58.6 \%$ of calls were made on the night period (from $8 \mathrm{pm}$ to $8 \mathrm{am}$ ), most between $8 \mathrm{pm}$ and $0 \mathrm{pm}$. Risk factors included arterial hypertension (62.3\%), ischemic heart disease $(43.2 \%)$, diabetes mellitus (31.8\%), heart failure $(23.3 \%)$, atrial fibrillation $(13.2 \%)$ and renal failure (9.9\%). On arrival of the prehospital medical team at the scene, 24 patients (4.9\%) required immediate intubation and five evolved to cardiac arrest, four of whom were successfully resuscitated and transported to hospital. Conclusions Our prehospital medical teams attended a large number of patients with pulmonary edema during this period. We recorded a small number of patients requiring mechanical intubation and a high rate of survival on those suffering from cardiac arrest in this context. We believe this is largely because of the decreased response time that prehospital emergency offers in this situation, allowing the institution of early treatment.

\section{References}

1. Dickstein $\mathrm{K}$, et al: ESC quidelines for the diagnosis and treatment of acute and chronic heart failure 2008: the Task Force for the diagnosis and treatment of acute and chronic heart failure 2008 of the European Society of Cardiology. Eur J Heart Fail 2008, 10:933-989.

2. Tresch DD, et al:: Out-of-hospital pulmonary edema: diagnosis and treatment. Ann Emerg Med 1983, 12:533-537.
P271

Efficacy of bedside ultrasonography in early diagnosis of patients admitted to the emergency department with dyspnea

E Dogan, S Girisgin, D Dündar, B Cander

Selcuk University Meram Faculty of Medicine, Konya, Turkey

Critical Care 2010, 14(Suppl 1):P271 (doi: 10.1186/cc8503)

Introduction We designed our study to find a useful USG pattern for diagnosis and assessment of dyspnea. We also compared utilities of chest radiograms in a supine position, thorax CT and bedside USG techniques.

Methods In Selcuk University Meram Medical Faculty, during the year 2009, 60 patients who had acute dyspnea were included in our study (30 patients with chest trauma and 30 patients with nontraumatic causes). We designed our study as prospective. We tried to determine pathologies that have positive and negative Sliding Lung Sign (SLS) in USG examinations. We also investigated its reliability in differential diagnosis of acute dyspnea. Results First of all we performed chest radiograms on all patients in a supine position. After that we performed CT scans. All of the results were interpreted by a detached radiologist. Then we performed bedside thoracic USG to find SLS by physicians in the ER. We conceded CT results as the golden standard and compared the results with chest radiograms and USG findings. The SPSS 13.0 program and $X^{2}$ test were used for statistical analysis $(P<0.05)$. According to our findings, SLS in USG examinations has a sensitivity of $84 \%$ and a specificity of $97 \%$ with a $97 \%$ positive predictive value and $83 \%$ negative predictive value.

Conclusions Throughout the years, USG examinations were defined as inappropriate in lung examinations but in our study we reached a conclusion that they are a necessity. Bedside sonographic examination allows impetuous diagnosis. But every respiratory failure has its own sonographic pattern so mistakes are inevitable. Nevertheless we believe that if profound studies like ours keep coming and if more and more clinicians begin to use USG examinations, the SLS diagnostic value will rise and become a routine element in the ER.

References

1. Razzaq QM: Use of the 'sliding lung sign' in emergency bedside ultrasound. Eur J Emerg Med 2008, 15:238-241.

2. Blaivas M, Lyon M, Duggal S: A prospective comparison of supine chest radiography and bedside ultrasound for the diagnosis of traumatic pneumothorax. Acad Emerg Med 2005, 12:844-849.

3. Wüstner A, Gehmacher O, Hämmerle S, Schenkenbach C, Häfele H, Mathis G: Ultraschalldiagnostik beim stumpfen Thoraxtrauma. Ultraschall Med 2005, 26:285-290.

P272

Auscultation in flight: comparison of amplified and traditional stethoscopes

J Tourtier, S Coste, E Fontaine, N Libert, M Viaggi, E Forsans, M Borne Hopital Val-de-Grâce, Paris, France

Critical Care 2010, 14(Suppl 1):P272 (doi: 10.1186/cc8504)

Introduction Many air medical transport programs use pulse oximeters, end-tidal carbon dioxide monitors and other devices as indirect measures of respiratory and cardiac status. Thus, these methods do not replace cardiac auscultation during flight, which may be needed to identify sudden critical change. And the ability to compare left-sided and right-sided breath sounds may be essential to confirm the appropriate placement of endotracheal tubes as well as to diagnose pneumothorax. The ability for auscultation during air medical transport is compromised by high ambient noise levels. The aim of this study was to assess the capabilities of a traditional and an amplified stethoscope (which is expected to reduce background and ambient noise) to assess heart and breath sounds during medical transport in a FALCON 50 plane.

Methods A prospective, double-blind, randomized study was performed. We tested one model of traditional stethoscope (Littman ${ }^{\circledR}$ cardiology III) and one model of amplified stethoscope (Littman 3100). The sound level was amplified in six of the eight increments of the amplified stethoscope. Practitioners on board were all experienced in air medical transport. They had normal audiologic testing. We studied 18 heart and lung auscultations, during real medical evacuations on board the Falcon 50 (medically configured; Dassault aviation). For each, the quality of 
auscultation was described using a numeric rating scale (ranging from 0 to 10, 0 corresponding to'I hear nothing', 10 to'I hear perfectly'). Comparisons were accomplished using a $t$ test for paired values.

Results Age of patients was $42 \pm 11$ years, $78 \%$ were males. The body mass index was $29.5 \pm 4.7$. For cardiac auscultation, the value of the rating scale was $5.7 \pm 1.4$ and $6.5 \pm 1.8$, respectively, for the traditional and amplified stethoscope $(P=0.027)$. For lung sounds, quality of auscultation was estimated at $3.6 \pm 2.3$ for the traditional stethoscope, at $3.9 \pm 2.9$ for the amplified stethoscope $(P=0.193)$.

Conclusions We concluded that flight practitioners in the Falcon 50 are more able to hear cardiac sounds with an amplified than with a traditional stethoscope, whereas there is no significant difference concerning breath sound auscultation. These findings suggested to the investigators that Falcon 50 noise and human breath sounds might share a common or substantially overlapping frequency spectrum, with amplification of one necessarily amplifying the other. To assess this hypothesis, further studies are needed to evaluate the sound frequency spectrum in the medically configured Falcon 50.

\section{P273}

Performance of ventilators at simulated altitude: study of fraction of inspired oxygen

J Tourtier, S Ramsang, E Forsans, S De Rudnicki, A Chrissment, J Schaal, M Borne

Hopital Val-de-Grâce, Paris, France

Critical Care 2010, 14(Suppl 1):P273 (doi: 10.1186/cc8505)

Introduction We studied the performance of two respirators employing an advanced turbine delivery system: LTV 1000, Tbird VSO2. We assessed the ability of the ventilators to deliver to a normal lung model a set fraction of inspired oxygen $\left(\mathrm{FiO}_{2}\right)$ at different simulated cabin altitudes.

Methods We used a decompression chamber to mimic the hypobaric environment at a range of simulated cabin altitudes of 1,500, 2,000 and 3,000 meters $(4,000,5,333,8,000$ feet). A model of normal lung was used. Concerning the Tbird VSO2, cabin altitude was input. Ventilators were tested with $\mathrm{FiO}_{2}$ set at $50 \%$ and $\mathrm{Vt}$ set at $700 \mathrm{ml}$. We noted the effective $\mathrm{FiO}_{2}$ assessed by the ventilators (paramagnetic analysis). We measured the $\mathrm{FiO}_{2}$ really delivered with a dedicated instrument of the French physiological laboratory of aviation and space medicine of the Air Force. Comparisons of preset and assessed $\mathrm{FiO}_{2}$ to actual measured $\mathrm{FiO}_{2}$ were accomplished using a $t$ test for each altitude.

Results Figure 1 shows the data with $\mathrm{FiO}_{2}$ set at $50 \%$.

Conclusions On one hand, both ventilators showed a moderate variation between $\mathrm{FiO}_{2}$ set and delivered. On the other hand, variations between $\mathrm{FiO}_{2}$ delivered and assessed are high, suggesting inefficacity of ventilators' paramagnetic analysis in hypobaric conditions.

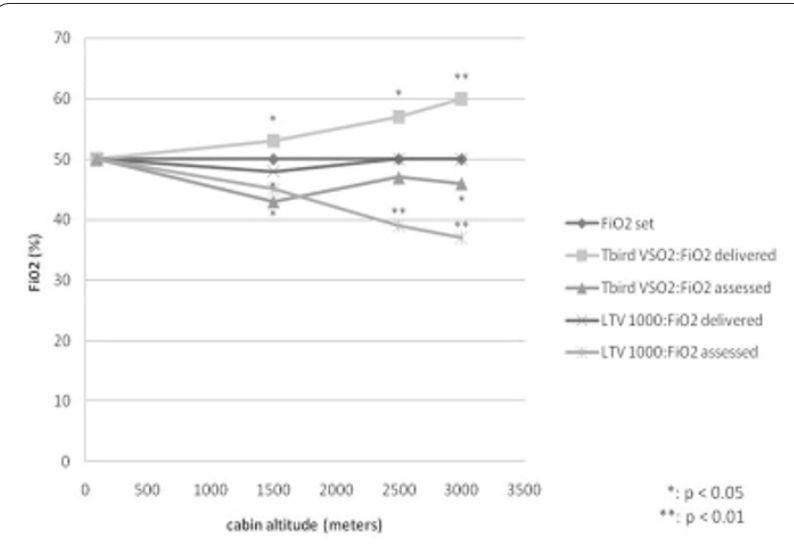

Figure (abstract P273).
P274

Aeromedical evacuation of the French Army: survey of the past 10 years

S Coste, J Tourtier, C Daudé, M Viaggi, E Morgand, M Borne

Hopital Val-de-Grâce, Paris, France

Critical Care 2010, 14(Suppl 1):P274 (doi: 10.1186/cc8506)

Introduction The Military Medical Service of the French Forces insures the support of 29,000 French servicemen spread around the world each year and performs as such their medical evacuation by air towards France to guarantee their adequate treatment as soon as possible. This knowledge is sometimes also used for a national civilian abroad. We propose to describe the epidemiology of medical evacuation (MEDEVAC) made in the past 10 years.

Methods We analysed data for every patient evacuated since 1 January 2000 and noted the destination, the number of patients per flight, the age, the gender, the status (military or civilian), the pathology, the presence of a specialist in the medical team (intensivist, cardiologist, psychiatrist) and the recipient hospital. The presence of an intubated ventilated patient or transfusion in flight was studied.

Results A total of 420 strategic MEDEVACs were carried out in 10 years (per year: $42 \pm 6.9$ ) in favor of 529 patients, among whom $90 \%$ were French servicemen. Each flight carried between one and 15 patients. Europe represents $42 \%$ of the original location of evacuation, Africa 39\% and the Middle East 17\%. An anesthetist-resuscitator reinforced the medical team in $51.7 \%$ of the evacuations. The patients with respiratory assistance are present in $19.8 \%$ of the flights and transfusion occurred in $3.1 \%$ of the flights. Traumatic pathology is predominant (49.5\% of patients), divided into three major causes nearly equally: road traffic accident, ballistic and others. Burns represent a significant proportion (6.8\%) of the causes of evacuation. Other causes are represented by $30.2 \%$ for medical conditions ( $n=160$ among which 59 cardiac affections), 10.2\% for nontraumatic surgical affections and $2 \%$ for psychiatry. Finally $25.5 \%$ of the patients were accommodated in an ICU, $6 \%$ in a specific center for treatment of burns, $35.3 \%$ in a service of surgery and $24.6 \%$ in medicine.

Conclusions If the number of MEDEVACs is relatively stable over the past decade, destinations and causes are changing. Africa remains an usual location of evacuation, the part of Europe tends to decrease for the benefit of the Middle East, which became in 2009 the predominant destination (21/47) stretching out the duration of flights. The causes of evacuations testify to the intensity of the commitment of French forces through traumatic affections, and in particular of ballistic origin, which are in 2009 the main traumatic affections (15/31). The corollary is an increase of the appeal to the resuscitators to deal with trauma patients.

\section{P275}

\section{Comparison of two disaster drills' management performed by} trained and not-trained students: key times evaluation

FL Barra, L Carenzo, PL Ingrassia, M Tengattini, F Prato, D Colombo, F Della Corte

University of Eastern Piedmont 'Amedeo Avogadro', Novara, Italy

Critical Care 2010, 14(Suppl 1):P275 (doi: 10.1186/cc8507)

Introduction The aim of this report is to compare two disaster exercises' management of students with different backgrounds. To our knowledge nobody has ever compared two exercises, probably because of the difficulty in their evaluation. We implemented a tool for an objective evaluation [1] and we used it for this purpose.

Methods Both drills represented a ceiling collapse over a crowded room with a similar amount of casualties and similar severity index. The START triage system was used.

The trained students $(T)$ were attending the European Master in Disaster Medicine (EMDM), while the not-trained students (NT) were at the beginning of an introductory course in disaster medicine. During the exercises we recorded key victim-provider interaction times [2] using victim-based data collection. Each victim had their own data card to record triage and time information.

Results In this preliminary report we present data regarding the scene length of stay (LOS) and triage to collecting area/advanced medical post time (T-AMP). The LOS was 67.5 (50.0 to 111.0$)$ minutes ( 25 to 75 IQR) for T as compared with 145.0 (110.0 to 150.0) minutes (25 to 75 IQR) for NT 
( $P$ <0.001). Stratification according to assigned triage code showed no difference for high-priority codes (reds and yellows) as opposed to the green code (55.0 (47.0 to 75.0) minutes for T vs 145.0 (141.0 to 155.0) minutes for NT with $P$ <0.01). T-AMP was 10.0 (3.0 to 34.5) minutes for T as compared with 63.5 (19.5 to 104.3) minutes for NT $(P<0.001)$. Stratification according to triage code showed no difference for red codes between $T$ and NT but showed a difference for yellow codes (36.5 (15.0 to 82.0) vs 71.0 (30.0 to 99.0) minutes) and green codes (7.0 (3.0 to 12.0) vs 85.0 (17.3 to 115.0) minutes) with $P<0.01$

Conclusions Both teams evacuated red codes before the yellow ones in similar time. T-AMP was shorter considering global, yellow and green codes for T as opposed to NT. Global and green LOS was also shorter in the T group as opposed to NT. Training seems to influence global exercise management, less affecting red codes but with an impact on yellow and green evacuation strategies.

\section{References}

1. Ingrassia PL, et al.: Evaluation of medical management during a mass casualty incident exercise: an objective assessment tool to enhance direct observation. J Emerg Med 2009. [Epub ahead of print]

2. Green $\mathrm{GB}$, et al:: Generic evaluation methods for disaster drills in developing countries. Ann Emerg Med 2003, 41:689-699.

P276

Acute severe asthma: performance of ventilator at simulated altitude J Tourtier, E Falzone, J Schaal, L Franck, G Edjo Nkilly, A Chrisment, M Borne Military Hospital Val-de-Grâce, Paris, France

Critical Care 2010, 14(Suppl 1):P276 (doi: 10.1186/cc8508)

Introduction Exacerbation of asthma can appear during air transport. Severe patients, not responding to conventional therapy can require ventilator support. We evaluated the performance of two transport ventilators, built with turbine technology - the T-bird VSO2 and the LTV 1000 - for use during aeromedical evacuation of acute severe asthma. We have assessed the ability of both ventilators to deliver to an acute severe asthma model a tidal volume (Vt) set at different simulated altitudes, by changing the ambient air pressure.

Methods We used a decompression chamber to mimic the hypobaric environment at a range of simulated cabin altitudes of 1,500, 2,500 and 3,000 meters $(4,000,6,670,8,000$ feet). Ventilators were tested with realistic parameters. Vt was set at $700 \mathrm{ml}$ and $400 \mathrm{ml}$ in an acute severe asthma lung model. The protocol included three measurements for each simulated altitude. Comparisons of preset to actual measured values were accomplished using a $t$ test for each altitude.

Results Figure 1 summarizes the data. With altitude, the T-bird VSO2 showed a decrease in volume delivered. Comparisons of actual delivered $\mathrm{Vt}$ and set $\mathrm{Vt}$ demonstrated a significant difference starting at 1,500 $\mathrm{m}$ for a Vt set of $700 \mathrm{ml}$, at 2,500 m for Vt set of $400 \mathrm{ml}$, with a negative variation of more than $10 \%$ compared with tidal volume set at respectively 3,000 and 2,500 m. With decreasing barometric pressure, the LTV 1000 showed mostly an increase in volume delivered. Comparisons of actual delivered $\mathrm{Vt}$ and set $\mathrm{Vt}$ demonstrated a significant difference at 1,500 $\mathrm{m}$ for a $\mathrm{Vt}$ set of $700 \mathrm{ml}$, at 2,500 $\mathrm{m}$ for a $V$ test of $400 \mathrm{ml}$. The delivered tidal volume remained within $10 \%$ of the set $\mathrm{Vt}$.

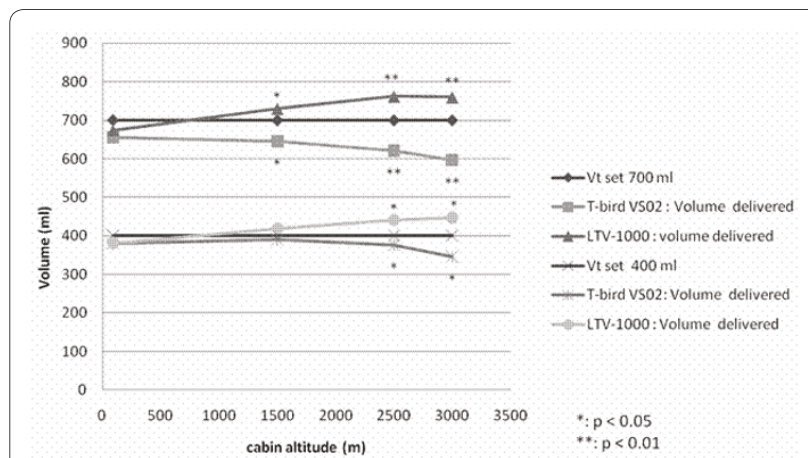

Figure 1 (abstract P276). Acute severe asthma, Vt delivered in altitude.
Conclusions The T-bird VSO2 progressively delivered lower volumes as barometric pressure decreased; whereas the LTV 1000 showed a moderate increase in volume delivered for the acute severe asthma lung model, with increasing altitude, but maintained the delivered volume within 10\% of the set Vt up to $3,000 \mathrm{~m}$.

P277

Validation of a new French-language triage algorithm: the ELISA

scale

J Jobé1, A Ghuysen'1, P Gérard², G Hartstein', V D'Orio'

'University Hospital, Liège, Belgium; ${ }^{2}$ University of Liège, Belgium

Critical Care 2010, 14(Suppl 1):P277 (doi: 10.1186/cc8509)

Introduction For some time, most emergency departments have been experiencing severe overcrowding which could lead to reductions in the quality of care. Consequently, nurse triage tools have been developed with the aim of getting 'the right patient to the right resources, at the right place and at the right time.' However, differences in emergency medical services in different countries have limited the generalisation of preexisting triage systems; for this reason, specific algorithms corresponding to local characteristics are needed. Accordingly, we developed a specific French-language triage system called ELISA (Echelle Liegeoise d'Index de Sévérité à l'Admission - Liege admission severity scale). We aimed to test its validity in the present study.

Methods The ELISA is a five-category (U1 to U5) nursing triage algorithm, using flow charts based on vital signs and anamnestic data. Each category is associated with a pre-established time delay for first medical contact. We tested inter-rater and intra-rater agreement respectively. As concerns inter-rater agreement, nine triage nurses sorted the same 100 patients. The assigned classifications compared the reference assignment and were analyzed with Cohen's kappa coefficient. Intra-rater agreement was tested by asking 30 triage nurses to sort the same 10 patients at two different times ( $\mathrm{T} 1$ was early morning and $\mathrm{T} 2$ was late night). We compared the classification at T1 and 2 with the null hypothesis being no difference and determined whether differences were nurse dependent.

Results Statistical analysis of intra-rater agreement revealed that the mean difference between classification at T1 and T2 did not significantly depend on the nurse $(P=0.3487)$. This result allowed pooling of the data and testing to determine whether the mean was different from zero. We used the Sign test and found that classification at T1 was not different from classification at T2 $(P=0.581)$. As concerns inter-rater agreement, we used Cohen's kappa coefficient, which revealed an almost perfect agreement between classification by nurses and the reference.

Conclusions The need for a specific triage tool in our emergency department led us to develop a new French-language triage scale. The present study demonstrates that this scale is a valid triage tool with very good inter-rater and intra-rater agreement. Such results now allow a future study to evaluate its efficiency.

P278

Examining the suitability of medical admissions to the emergency short stay ward of a large UK hospital

E Hartley, E Hood, N Bashir, R Zahir

New Cross Hospital, Wolverhampton, UK

Critical Care 2010, 14(Suppl 1):P278 (doi: 10.1186/cc8510)

Introduction In 1996, a UK audit suggested suboptimal involvement of consultant care in acute medicine [1]. Acute medicine has since evolved as a specialty to improve medical inpatient care in the first 48 hours [2]. Many hospitals now incorporate an acute short stay unit through which up to $70 \%$ of medical admissions can be directed [3]. These units reduce the length of hospital inpatient stay [4].

Methods We conducted a review of case notes for 100 consecutive medical admissions to the Emergency Short Stay ward (ESS). Data collected included diagnoses on admission and discharge, length of stay on ESS and discharge destination. On reviewing each initial medical clerking, an assessment was made regarding the suitability of the admission to ESS, standardized against local criteria.

Results Data on 100 patients (age 17 to 89) were collected. Eighty-nine per cent were admitted from the medical admissions unit, most commonly 
for respiratory and cardiac problems. Average length of stay on ESS was 2 days. Twenty-five per cent of patients remained on the ward for over 72 hours and $6 \%$ for over 5 days. Whilst $68 \%$ of patients were discharged home from ESS, $32 \%$ of patients were later transferred to a different ward. When compared with the available admissions criteria, 27\% of all admissions to ESS were found to be inappropriate. Most (18/27) of these patients were later transferred to a different inpatient ward. Twenty-six per cent of inappropriate admissions remained on ESS for $>48$ hours. The total number of medical admissions to the hospital on each day of the study did not affect the number of inappropriate transfers to ESS.

Conclusions The 24-bed Emergency Short Stay Ward at our UK hospital is for assessment and treatment of selected acute conditions requiring an inpatient stay $<48$ hours. ESS was not always being used appropriately according to the admission criteria defined by the ward managers. Hospital admissions of $>72$ hours are associated with significant increase in workload [5]. It is important that a short stay ward does not increase the length of admission by becoming a transit lounge for patients before transfer to a specialty lead ward.

\section{References}

1. Houghton A, et al.: J R Coll Phys Lond 1996, 30:551-559.

2. Dowdle JR: Emerg Med J 2004, 21:652-653.

3. Cooke MW, et al.: Emerg Med J 2003, 20:138-142.

4. Downing H, et al:: Clin Med 2008, 8: 8-20.

5. Rainer TH, et al.: J Accid Emerg Med 1996, 13:325-329.

\section{P279}

A study on reliability of a new triage emergency method among nursing students

N Parenti', D Sangiorgi ${ }^{2}$, V Serventi ${ }^{3}$, L Sarli ${ }^{3}$

'Hospital Santa Maria della Scaletta Imola, Bologna, Italy; 'Università

Bologna, Italy; ${ }^{3}$ Università Parma, Italy

Critical Care 2010, 14(Suppl 1):P279 (doi: 10.1186/cc8511)

Introduction We analysed triage reliability among nursing students at an Italian university before and after a course on a new Triage Emergency Method, TEM v2, which showed good reliability in a previous study [1]. Few studies compared triage reliability in nursing students. To our knowledge there are no Italian studies on this topic.

Methods This is an observational study conducted in the University of Parma using a triage scenarios database used in previous studies $[1,2]$. Fifty students attending the third year of a nursing course were selected to assign a triage level to 105 paper scenarios, without triage protocols (before course) and with TEMv2 (1 month after a 2-hour course on triage and TEMv2). To prevent communication between participants, they assigned triage codes in different rooms, and in the presence of two investigators. The triage scenarios were given randomly to the participants. We measured: the inter-reliability using the weighted kappa statistics; the complete disagreement (when nurses of the same group assigned to the same scenario triage codes that differed in more than two priority levels); the complete agreement (when all five nurses assigned the same triage code) before and after the course.

Results Of the 105 patients included in triage scenarios, 66 (63\%) were women, the mean age was 43.7 years ( $S D \pm 26.3$ ), and 22 were under the age of 18 years. The most frequent presentation at triage was minor trauma (30\%). There were 30 hospital admissions: 27 in non-intensive wards and three in ICUs. The mean age of students was $24 \pm 3$ SD. Few participants attended triage training before $(18 \%)$ and they declared scarce triage knowledge. Inter-rater reliability was $k=0.42$ ( $95 \% \mathrm{Cl}: 0.37$ to 0.46$)$ and $k=0.61(95 \% \mathrm{Cl}: 0.56$ to 0.67$)$ before the course (without triage protocol) and after the course (with TEMv2), respectively. Complete disagreement occurred in $98 \%$ of scenarios evaluated before and in $64 \%$ after the course. Complete agreement was always zero.

Conclusions Our data suggest that TEM v2 improves triage reliability among nursing students. It seems to be easy to understand and to use.

\section{References}

1. Parenti $\mathrm{N}$, et al:: Reliability and validity of two four-level emergency triage systems. Eur J Emerg Med 2009, 16:115-120.

2. Parenti $\mathrm{N}$, et al:: Affidabilità e validità di un Metodo di Triage di Pronto Soccorso a 4 codici di priorità. Emerg Care J 2008, 5:30-33.
P280

Aggression and violence against staff of ambulance services

A Auer

Vienna Ambulance Service, Vienna, Austria

Critical Care 2010, 14(Suppl 1):P280 (doi: 10.1186/cc8512)

Introduction Aggression and violence in the workplace have increasingly become topical for various professions in the past years. Studies, however, are mostly published for the areas of psychiatry and education. The first part of the study investigates the question of how emergency services in general deal with aggressive assaults against their staff. In the second part, the monitoring system for aggression incidents of the Vienna Ambulance Service will be presented and evaluated.

Methods By the means of an email questionnaire, 35 emergency medical services and EMS training facilities were interrogated about their procedures in case of aggression incidents. The monitoring system of the Vienna Ambulance Service, which is based on the SOAS-R questionnaire, was statistically evaluated.

Results Emergency medical services survey: 21 of the 35 included organizations returned the questionnaire with usable answers. For $80 \%$ of the responding organizations, aggression against their staff is an issue; $50 \%$ installed a staff reporting system and $58 \%$ offer de-escalation courses to their staff. Vienna Ambulance Service: during the analysis period of 2 years, 203 aggressive and/or violent assaults were reported within the Vienna Ambulance Service. In relation to the total number of 268,528 treated patients, the rate of incident is $0.08 \%$. Males committed, with $71.7 \%$, most of these aggressions. With regard to diagnoses, the diagnostic group of alcohol-related incidents was most frequent with 59 cases. Most events took place on weekends and in the evening hours. Verbal aggression was with $86.2 \%$ predominant; however, more than $20 \%$ of the incidents lead to physical injuries of the medical personnel.

Conclusions Aggression against emergency medical service staff is brought up for discussion in many organizations. Despite several approaches in different states of development, standardized reporting systems are the exception. The aggression incident reporting formula of the Vienna Ambulance Service was proved to be practical for recording aggression events as well as for evaluation of de-escalation education. The study showed that aggression is a serious problem in emergency medical services and appropriate precautions need to be institutionalized.

\section{P281}

Analysis of the disease spectrum of treated patients after Wenchuan earthquake

Z He, YWangy, L Huang

First Hospital Affiliated to General Hospital of PLA, Beijing, China

Critical Care 2010, 14(Suppl 1):P281 (doi: 10.1186/cc8513)

Introduction To investigate the data of patients treated by this field hospital, and to analyze the rule of spectrum of diseases in Wenchuan earthquake.

Methods Sexes, ages and diagnoses of the patients were collected and the percentage was respectively calculated. The amount tendencies of the time distribution of patients were also analyzed.

Results Sex differences of patients were not obvious in the total 2,331 cases. The amount in the group 15 to 60 years old, 1,535 (65.9\%) cases, was significantly higher than those of other groups, but the relative ratios of the group 0 to 14 years old, $382(16.4 \%)$ cases, and the group $>60$ years old, $414(17.7 \%)$ cases, were higher than that of 15 to 60 years old. The top four diseases consist of respiratory system 601 (25.8\%), trauma (fracture, wound or injury) 586 (24.9\%), dermatology system 295 (12.7\%) and others 243 (10.4\%). The amount of internal medicine patients exceeded that of surgery. Conclusions The reasonable allocations of medical personnel and drugs after earthquake may help to improve the remedy results and to prevent waste of medical resource during future disasters.

\section{References}

1. Hogan DE, Burstein JL: Disaster Medicine. Lippincott Williams \& Wilkins; 2003:8.

2. Zhongjie He: The platinum ten mins - new ideal and concept. Chinese J Crit Care Med 2004, 24:745-746.

3. Watts J: China's health challenges after the earthquake. Lancet 2008, 371:1825-1826.

4. Conly J, Johnston B: Natural disasters, corpses and the risk of infectious diseases. Can J Infect Dis Med Microbiol 2005, 16:269-270. 
P282

Large-scale implementation of a critical care surge capacity management program

BW Lawless', J Trpkovski²

'St Michael's Hospital, Toronto, Canada; ${ }^{2}$ Critical Care Secretariat, Toronto, Canada

Critical Care 2010, 14(Suppl 1):P282 (doi: 10.1186/cc8514)

Introduction In 2003 the outbreak of severe acute respiratory syndrome (SARS) in some jurisdictions around the world highlighted a number of areas in healthcare planning that could be improved for dealing with such disasters. In Canada, one of the noted system challenges was the significant impact that an increase in the number of critically ill patients had on access to care and to other hospital services. Many jurisdictions have undertaken large-scale pandemic planning; however, there is a paucity of tools available to help hospitals deal with the day to day challenges of surges in patient volumes. As part of a larger comprehensive Critical Care Strategy designed to improve access to care, improve the quality of care and improve health system integration, and in partnership with hospitals and healthcare workers, the Ontario Ministry of Health and Long-Term Care designed and implemented a critical care Surge Capacity Management Program.

Methods A review of the literature was completed to determine existing models for surge capacity planning. Surges were then classified as minor, moderate or major, depending upon the increase in demand above available services and the length of time a surge situation persisted. A framework was built to incorporate surge planning on five key elements: management, physical space, human resources, equipment and technology, and processes. A demonstration project to test the model was carried out in 18 hospitals. Hospitals collected data on patient flow processes across the organization in conjunction with daily data capture in the Critical Care Information System. Based on initial success, the program was approved for a province-wide implementation.

Results Critical Care Surge Resource Teams that included front-line care providers, and a senior team sponsor and a physician champion were formed to implement surge capacity plans using the standardized framework and tools. Hospitals developed plans to handle minor surges that involved increasing to $115 \%$ above their normal operating capacity. This program has involved 201 critical care units in 150 hospitals across the province.

Conclusions This program represents one of the largest implementations of a standardized Surge Capacity Program for managing critical care resources in a time of crisis. The program has seen practical use during the recent experience with $\mathrm{H} 1 \mathrm{~N} 1$ with improved access to care for patients.

\section{P283}

Standard X-rays for the victims of severe trauma: time for a change E Cingolani, C Siddi, G Ranaldi, L Nocilli, S Rogante, A Stasolla, G Nardi Azienda Ospedaliera San Camillo Forlanini, Rome, Italy Critical Care 2010, 14(Suppl 1):P283 (doi: 10.1186/cc8515)

Introduction International guidelines for trauma care still recommend the traditional advanced trauma life support investigations for the primary survey: plain radiographs of the chest, cervical spine and pelvis with FAST ultrasounds. However, an increasing amount of data suggest that plain $X$-rays have excessively low sensitivity. Cervical $X$-ray may miss up to $35 \%$ of the spine injuries, chest $X$-ray may miss as many as many as $50 \%$ of traumatic pneumothorax and a significant percentage of pelvic fractures may not be detected on the pelvis $X$-ray. CT scan and extended emergency ultrasound (EUs) have a much higher sensitivity if compared with plain X-rays. Moreover, EUs is even less time consuming. Therefore since 2004 we have adopted EUs and/or total body CT scan as the firstline diagnostic tools for major trauma (MT) victims. In a benchmarking analysis of Italian trauma centers (TCS), our hospital ranked first for patient mortality and long-term disability [1]. These results were associated with the shortest diagnostic time. The aim of this study is to assess whether these results were associated with a real change in the diagnostic process. Methods In order to evaluate the diagnostic approach to MT we retrospectively analyzed all MT cases (ISS >15) admitted to our TC over a 2-year time span. All investigations performed in the emergency department (ED) are entered in an electronic shift just before being performed. Data for all investigations requested within 2 hours from admission were analyzed.

Results From 1 November 2007 to 31 October 2009, 743 MT patients were admitted to the ED. EUs was performed in 515 patients (69\%), total body CT scan in 679 (91\%). Patients who were not submitted to CT either died soon after admission or were rushed to the operating room on the basis of EUs results. Thirty-eight patients had a chest X-ray taken in the ED (5.1\%), $11(1.5 \%)$ a pelvic $X$-ray and only three a cervical spine $X$-ray.

Conclusions Although many international guidelines for trauma care still recommend traditional plain radiographic investigations, our TC as well as many other European institutions with a high volume of trauma patients have adopted a different strategy based on the extended use of EUs and CT scan. This may improve the accuracy of the diagnosis in the stabilized patients and reduce time to the operating room in the highly instable ones. We suggest that a change in the recommended guidelines should be considered.

\section{Reference}

1. Di Bartolomeo S, et al:: Injury 2007, 38:305-311.

\section{P284}

Focused assessment with sonography for trauma in the prehospital setting

R Egashira, N Saito, H Matumoto, K Mashiko

Chiba Hokuso Hospital, Nippon Medical School, Chiba, Japan

Critical Care 2010, 14(Suppl 1):P284 (doi: 10.1186/cc8516)

Introduction Previous studies have focused on the accuracy of the focused assessment with sonography for trauma (FAST) examination performed in the emergency department (ED), but have not evaluated FAST performed in the field setting (field FAST). We aimed to evaluate the accuracy of field FAST by comparison with FAST performed in the ED.

Methods A total of 1,380 trauma patients were admitted to our hospital by air ambulance between April 2005 and October 2009. We compared the sensitivity and specificity of field FAST with those of FAST in the hospital setting. The presence of pericardial and intraperitoneal fluid was confirmed by the medical records.

Results Of these patients, 317 blunt trauma patients (mean age, $43.9 \pm$ 23.8 (SD) years) were examined by field FAST and enrolled in the study; all participants were also examined by FAST in the ED. The mean Injury Severity Score was $16.1 \pm 15.4$ (SD). Forty-seven patients (14.8\%) were ultimately diagnosed with pericardial and intraperitoneal fluid. Field FAST detected 12 of these cases. The sensitivity of field FAST was significantly lower than that of FAST in the ED $(P<0.01)$, whereas the specificities of FAST in the two settings were not significantly different. Emergency surgery was performed in nine of the 12 patients who were positive with field FAST.

Table 1 (abstract P284)

\begin{tabular}{lccc}
\hline & Field FAST & ED FAST & $P$ value \\
\hline Sensitivity & $28.9 \%$ & $66.7 \%$ & $<0.01$ \\
Specificity & $97.0 \%$ & $96.6 \%$ & 0.76 \\
\hline
\end{tabular}

Conclusions Because the sensitivity of field FAST is low, repeated examination in ED is needed to improve diagnostic sensitivity. However, field FAST may be an effective tool for early detection of critical blunt trauma and as a rapid indicator for surgery.

P285

Clinicopathogenetic aspects of systemic inflammatory response development in polytrauma

I Ustyantseva, O Khokholva, V Agadzhanyan, O Petukhova, Y Zhevlakova

Federal State Medical Prophylactic Institution 'Scientific Clinical Center of

Miners' Health Protection', Leninsk-Kuznetsky, Russia Federation

Critical Care 2010, 14(Suppl 1):P285 (doi: 10.1186/cc8517)

Introduction The aim of the study was identification of common regularities and pathogenetic significance of changes in the ratio between primary and secondary inflammatory mediators in development of systemic inflammatory response syndrome (SIRS) in polytrauma. 
Methods Three hundred and eighty-seven patients with polytrauma hospitalized to the ICU were examined. There was infection confirmed by microbiology in 175 (pneumonia, bronchitis, osteomyellitis, acute urethritis, and so forth); 212 patients had no infection (acute respiratory distress syndrome, disseminated intravascular coagulation, fat embolism, and so forth). In $87 \%$ two or more signs of SIRS were noted according to the criteria of the Consensus Conference ACCP/SCCM (2003). C-reactive protein (CRP) in venous blood serum was measured on 1,3,5, 7, 10, 15 and 17 days after admission using the biochemical analyzer HITACl-912 with reagents Tina-Quant CRP (Roche Diagnostic GMBH), lipopolysaccharidebinding protein (LPSBP) and cytokines TNFa, IL-2R, IL-6, IL-8, IL-10 using the immunochemiluminescent analyzer IMMULITE with the reagents DPC (USA). Constant variables were compared using Student's $t$ test and the Mann-Whitney $U$ test. The differences were significant at $P<0.05$.

Results On admission, increased serum LPSBP and TNFa were noted in all patients with infection compared with the patients without it (1.8 and 3 times respectively, $P<0.01$ ). Moreover, the infectious patients had the apparent increase of IL-2R, IL-6, IL-8 and CRP: 5, 13, 18 and 5 times, respectively $(P<0.05)$. At that, generalized manifestation of inflammatory reaction in the infectious patients was characterized by at mean fivefold increase in IL-8 on day 3 of the follow-up. It was not accompanied by adequate anti-inflammatory response that was indicated by an absence of IL-10 dynamics. A direct correlation was found between an increase in inflammatory mediator concentration (IL-6, IL-8, IL-10) and respiratory rate $(r=0.73 ; 0.46 ; 0.59)$, CRP and heart rate $(r=0.48, P<0.05)$, and an inverse correlation between inflammatory mediators (TNFa, IL-2R, LPSPB) and arterial pressure level $(r=-0.42 ;-0.63 ;-0.48)$ and respiratory function values (vital lung capacity and forced expiratory volume, $r=-0.42$ ).

Conclusions Hyperproduction of proinflammatory cytokines is closely connected with ventilation disturbances and clinical variants of the posttraumatic period course in patients with polytrauma.

P286

Diagnostic and prognostic significance of serum apoptosis markers in the patients with severe trauma injury

EG Grigoryev', AR Radivilko', AK Kiseleva², YC Churliaev², GV Vavin'

'Kemerovo State Medical Academy, Kemerovo, Russia Federation;'2Branch of Scientific Institute of General Reanimatology RAMS, Novokuznetck, Russia

Federation

Critical Care 2010, 14(Suppl 1):P286 (doi: 10.1186/cc8518)

Introduction The aim of the investigation was to analyze the diagnostic and prognostic significance of serum proapoptosis and antiapoptosis markers in the patients with severe trauma injury.

Methods Thirty-three patients with severe trauma injury were enrolled into our investigation (we excluded patients with severe brain injury, the middle age was $39 \pm 8$, initial TRISS scale $7.8 \pm 1.8$, APACHE $\| 14 \pm 5$ ). All of the patients demonstrated signs of systemic inflammatory response, in 12 patients the infectious pulmonary complications were revealed and in 21 patients the infectious complications were not developed. Venous blood serum EDTA samples were investigated every day during a week in the $-25^{\circ} \mathrm{C}$ freeze. We investigated antiapoptotic markers (soluble Fas-L, soluble APO-1/Fas, Cu/Zn superoxide dismutase), proapoptotic markers (protein p53, protein $\mathrm{BCl}-2$ ), and high-sensitivity CRP (ELISA, Bender Medsystem).

Results We observed an increase in proapoptotic markers in patients with infectious complications from the first day in the ICU, antiapoptotic markers (Cu/Zn superoxide dismutase) were lower with regard to reference points. The patients demonstrated a maximum of Fas- $L$, protein p53, and protein $\mathrm{BCl}-2$ (near $50 \%$ relative to first day) on the second day. In patients without infection complications we identified a decreased level of proapoptotic markers and an increased level of antiapoptotic markers (SOD, soluble Fas-L, soluble APO-1/Fas). High-sensitivity CRP correlated with the severity of infectious complications.

Conclusions Serum proapoptotic and antiapoptotic markers have demonstrated diagnostic and prognostic significance in the patients with severe trauma injury.

\section{Reference}

1. De Freitas I, Fernández-Somoza M, Essenfeld-Sekler E, et al: Serum levels of the apoptosis-associated molecules, tumor necrosis factor-alfa/tumor necrosis factor type-I receptor and Fas/FasL, in sepsis. Chest 2004, 125:2238-2246.
P287

Predictors of mechanical ventilation after burn injury

A Pirat, P Zeyneloglu, A Kundakci, C Aydogan, G Arslan, M Haberal

Baskent University Faculty of Medicine, Ankara, Turkey

Critical Care 2010, 14(Suppl 1):P287 (doi: 10.1186/cc8519)

Introduction It is important to predict the need for mechanical ventilation (MV) in burn patients in order to improve their management and also to optimize resource ultilization. However, there are scant data on the predictors of MV in burn patients. The purpose of this study was to determine the risk factors for MV in a cohort of our burn patients.

Methods A retrospective chart review was completed for patients admitted to Baskent University Burn Institute, from January 2003 through December 2008. Patients were divided into two groups based on whether they required MV during their first 3 days of hospitalization or not. After comparing the two groups for the recorded variables, a binary logistic regression model was developed using statistical and clinical significance to identify the risk factors for MV.

Results Out of 160 patients, 33 patients (21\%) required MV during their first 3 days of hospitalization. Patients who required MV were similar to those who did not in terms of demographic features. Patients who required MV were significantly different from those who did not regarding the mean burn percentage $(56 \% \pm 23 \%$ vs $19 \% \pm 15 \%, P<0.001)$, mechanism of burn (flame, $73 \%$ vs $37 \%, P=0.001)$, inhalational injury ( $27 \%$ vs $3 \%, P<0.001)$, and burn affecting head and neck (72\% vs 37\%, $P<0.001)$. Binary logistic regression revealed that inhalational injury $(\mathrm{OR}, 8.4 ; 95 \% \mathrm{Cl}, 1.1$ to 66.1 ; $P=0.042)$ and burn percentage $(\mathrm{OR}, 1.1 ; 95 \% \mathrm{Cl}, 1.1$ to $1.2 ; P<0.001)$ were independent risk factors for MV.

Conclusions Our results demonstrate that inhalational injury and burn percentage are predictors of MV in our series of burn patients. Particularly, patients with inhalational injury were eight times more likely to require MV during their first 3 days of hospitalization in our series.

P288

Impact of regional imaging guidelines for head injury and unconscious polytrauma patients

R Gibbs ${ }^{1}$, R Walker ${ }^{2}$, EThomas

'Musgrove Park Hospital, Taunton, UK; ${ }^{2}$ Torbay Hospital, Torbay, UK; ${ }^{3}$ Derriford Hospital, Plymouth, UK

Critical Care 2010, 14(Suppl 1):P288 (doi: 10.1186/cc8520)

Introduction Inadequate or delayed imaging of the unconscious patient with traumatic brain injury or of the unconscious polytrauma patient may lead to catastrophic consequences. The need for comprehensive imaging needs to be balanced against the patient's condition, the resources available and excessive radiation exposure. We have introduced regional evidence-based guidelines in the Southwest region of the UK. The aim of these guidelines was to standardise practice, minimise delayed or missed diagnosis of serious injuries, facilitate treatment of associated injuries (such as head injuries) and to obviate the need for repeated imaging.

Methods The notes of all unconscious polytrauma patients transferred from the emergency department or other hospitals and all patients transferred to our institution for ongoing neurosurgical care were retrospectively reviewed over a 1-year period. Adherence to the imaging protocol was assessed and the need for any further radiological investigations within 48 hours was also documented.

Results A total of 46 patients were identified who fulfilled the criteria for the introduced guidelines. Of these patients, 21\% were transferred from other hospitals while the remainder was admitted from the onsite emergency department. Eighty-three per cent of all eligible patients adhered to the protocol. Two patients (4\%) required further radiological investigation in the 48 hours following admission. Both had isolated head injury and required further imaging to exclude cervical injuries following inadequate imaging which did not follow protocol. One further patient (2\%) required repeated imaging to exclude mesenteric injury.

Conclusions Regional adoption of imaging guidelines aimed at obtaining early comprehensive imaging of both head-injured and polytrauma patients results in high compliance and a low rate of re-imaging.

References

1. Trauma: Who Cares? NCEPOD; 2009.

2. Effect of whole-body CT during trauma resuscitation on survival: a retrospective, multicentre study. Lancet 2009, 373:1455-1461. 
P289

Blunt vascular injuries of the carotid and vertebral arteries: should we screen the asymptomatic trauma patients at high risk?

Preliminary results of a prospective cohort study

G Nardi, C Alessandrini, C Siddi, S Rogante, L Nocilli, G Ranaldi,

E Cingolani, V Miele

Azienda Ospedaliera S Camillo Forlanini, Rome, Italy

Critical Care 2010, 14(Suppl 1):P289 (doi: 10.1186/cc8521)

Introduction Blunt cerebrovascular injuries $(\mathrm{BCVI})$ are more common than previously reported and, if not promptly recognized and treated, may have devastating sequelae. When these injuries are diagnosed before the onset of stroke, and patients receive early antiplatelet or anticoagulation therapy, a substantial reduction in BCVI-related neurologic events has been demonstrated [1]

Methods The study setting was a level 1 trauma center with a catchment population of more than 2 million people, admitting 350 major traumas yearly. To assess the incidence of asymptomatic BCVI in the severely injured patients, we planned a prospective cohort study. According to the study design, all of the severely injured patients presenting with at least one of the following criteria [2] were submitted to a screening 16-channel CT angiography within 24 hours from admission: diffuse axonal injuries; fracture of the cervical vertebrae or of the skull base; Lefort II or III or other severe facial fractures.

Results During the first 5 months of the study, 24 major trauma patients (ISS $>15$ ) with at least one of the above listed risk factors were submitted to a screening $C T$ angiography for BCVI. All of the patients were sedated and artificially ventilated. None of them was symptomatic for stroke. Three patients (12.5\%) in this high-risk group had an asymptomatic BCVI: pseudoaneurysm (one), traumatic stenosis (one) or dissection (one) of the carotid artery. They were immediately treated with antiplatelet therapy (clopidogrel + aspirin). They experienced no episodes of cerebral infarction and no cerebral haemorrhage.

Conclusions There are limited data in the literature on traumatic BCVI. The available data as well as the preliminary results of our prospective study show that $\mathrm{BCV}$ are more common than previously recognized. Aggressive screening and earlier therapy may significantly reduce complications and improve patient outcomes.

\section{References}

1. Edward N, et al: J Am Coll Surg 2007, 204:1007-1015.

2. Utter GH, et al.: J Am Coll Surg 2006, 203:838-848.

\section{P290}

Effect of $8.4 \%$ sodium bicarbonate on raised intracranial pressure after traumatic brain injury

C Bourdeaux, J Brown

Frenchay Hospital, Bristol, UK

Critical Care 2010, 14(Suppl 1):P290 (doi: 10.1186/cc8522)

Introduction The aim of this study was to determine the efficacy of 8.4\% sodium bicarbonate for intracranial pressure (ICP) reduction in adult patients with severe traumatic brain injury (TBI) and intracranial hypertension.

Methods The study examined 10 episodes of ICP $>20 \mathrm{mmHg}$ in seven patients with severe TBI. Eighty-five milliliters of $8.4 \%$ sodium bicarbonate was infused over 30 minutes when osmotherapy was indicated after standard care. ICP, mean arterial pressure (MAP), and cerebral perfusion pressure (CPP) were recorded at baseline and then continuously for 6 hours. Serum $\mathrm{pH}, \mathrm{pCO}_{2^{\prime}}\left(\mathrm{Na}^{+}\right)$, and $\left(\mathrm{Cl}^{-}\right)$were measured at baseline, 30 minutes, 60 minutes and then hourly for 6 hours. Serum osmolality was measured at baseline and at 6 hours in three patients. All other care was identical to the institutional protocol for the management of raised ICP.

Results At the completion of the infusion, the mean ICP was reduced to $36.2 \%$ of baseline, from $28.5 \mathrm{mmHg}( \pm 2.62)$ to $10.33 \mathrm{mmHg}( \pm 1.89)$. Mean ICP remained below $20 \mathrm{mmHg}$ for 6 hours (Figure 1). CPP was increased after the infusion due to the effect on ICP. MAP did not change. Mean $\mathrm{pH}$ was elevated at $t=30$ minutes (from $7.45 \pm 0.02$ to $7.50 \pm 0.02$ ) and remained elevated for the duration of the study period. Serum $\left(\mathrm{Na}^{+}\right)$ increased (from $145.4+1.9 \mathrm{mOsm} / \mathrm{l}$ to $147.1+1.9 \mathrm{mOsm} / \mathrm{l}$ ) at 30 minutes. $\mathrm{pCO}_{2}$ did not change. Osmolality was elevated.

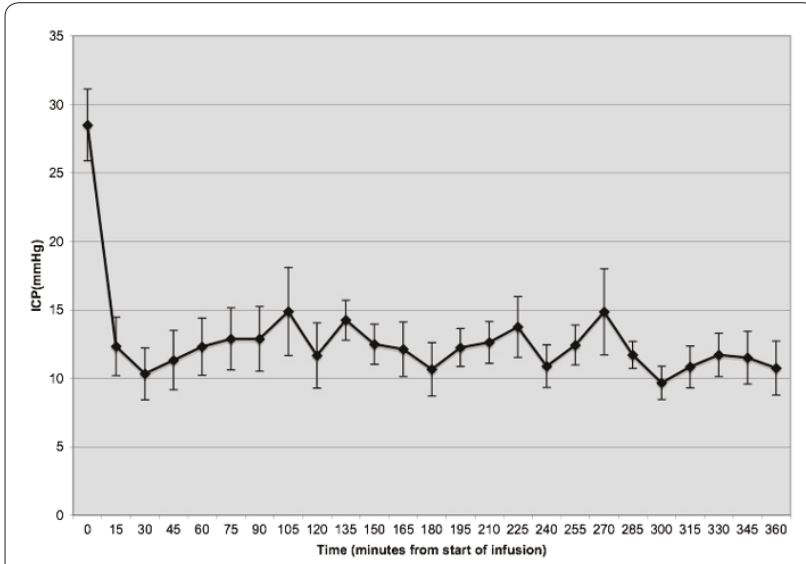

Figure 1 (abstract P290).

Conclusions Eighty-five milliliters of $8.4 \%$ sodium bicarbonate infused over 30 minutes is effective at reducing sustained ICP $>20 \mathrm{mmHg}$ to within accepted treatment targets (ICP $<20 \mathrm{mmHg}$ ) for at least 6 hours.

\section{P291}

Hypernatremia in pediatric patients with severe traumatic brain injury D Azovskiy', A Lekmanov², S Pilyutik', E Gegueva²

'Speranskiy Children's Hospital, Moscow, Russia Federation; ${ }^{2}$ Moscow Research Institute of Pediatric Surgery, Moscow, Russia Federation

Critical Care 2010, 14(Suppl 1):P291 (doi: 10.1186/cc8523)

Introduction Water-electrolyte imbalance and endocrine disorders make the problem of maintaining patients with severe traumatic brain injury (TBI) more difficult. A plasma sodium level $\geq 160 \mathrm{mmol} / \mathrm{l}$ is associated with $75 \%$ mortality. The purpose of this investigation was to find the relationship between hypernatraemia and the rate of unfavorable outcomes in children with TBI.

Methods A total of 77 children $<18$ years of age with TBI (admission GCS score $<8$ ) were divided retrospectively into three groups: Group A included children without hypernatraemia $(n=51)$, Group B children with hypernatraemia $(n=14)$ and Group C $(n=12)$ children with hypernatraemia and polyuria. Group C was considered the group of patients with central diabetes insipidus (CDI). Hypernatraemia was defined as a twice elevation of the plasma sodium level over $149 \mathrm{mmol} / \mathrm{l}$ within 24 hours, while polyuria was defined as an increase in the hourly diuresis of more than $3 \mathrm{ml} / \mathrm{kg} /$ hour in no less than 6 hours.

Results The mean sodium level at admission was $140.1 \pm 4.1 \mathrm{mmol} / \mathrm{l}$. Hypernatraemia was detected in 26 patients (33.8\%). The mean duration of the period of hypernatraemia in Group B was 4 days ( 3 to 6 days), while the mean sodium level during the period of hypernatraemia was $158.3 \pm 3.3$ $\mathrm{mmol} / \mathrm{l}(\mathrm{max} 176.8 \mathrm{mmol} / \mathrm{l})$. The duration of the period of hypernatraemia in Group C was 4.5 days with $\max 181.1 \mathrm{mmol} / \mathrm{l}$ and average $161 \pm 4.7 \mathrm{mmol} / \mathrm{l}$. Polyuria was diagnosed in $15.5 \%$ of the cases. The highest diuresis in this group was $4.1 \mathrm{mmol} / \mathrm{kg} /$ hour, mean $3.7 \pm 0.5 \mathrm{ml} / \mathrm{kg} / \mathrm{hour}$. Such changes were considered a manifestation of CDI. All 12 patients in Group C received desmopressin (DDAVP) for more than 48 hours (mean $56.8 \pm 4$. 5 hours). The doses were 0.025 to $0.2 \mathrm{mg} /$ day. In four out of 14 children in Group B (29\%), an increase hourly diuresis up to $3 \mathrm{ml} / \mathrm{kg} /$ hour was considered the onset of CDI; thus, they were also prescribed DDAVP. Unfavorable outcomes (GOS score 1 to 3) during a 30-day assessment were observed only in Groups B and C. In a comparison of unsuccessful outcomes between Groups B and C, there was an increase in the unfavorable outcome rate in patients of Group C (with hypernatraemia and polyuria) - 10 children (84\%) and Group B four children (28\%). The risk factor in the comparison between patients of Groups $B$ and $C$ was $0.3, P<0.05$

Conclusions Our results demonstrate that hypernatraemia increases the rate of unfavorable outcomes in children with TBI. Thirty-day outcomes were worse with CDI patients. Presumably, the used of DDAVP prevents dehydration and CDI advance. 
P292

Use of transcranial Doppler in patients with severe traumatic brain injury

D Ziegler, G Cravens, G Angles, R Gandhi, P Cho

John Peter Smith Hospital, Fort Worth, TX, USA

Critical Care 2010, 14(Suppl 1):P292 (doi: 10.1186/cc8524)

Introduction Secondary ischemic insult after severe traumatic brain injury (TBI) is correlated with poor outcome. Transcranial Doppler sonography (TCD) permits a non-invasive measurement of cerebral blood flow. The purpose of this study is to determine the usefulness of TCD in patients with severe TBI.

Methods TCD was performed on 73 patients with severe TBI, defined as a Glasgow Coma Scale of 8 or less on admission. All patients were on mechanical ventilation. TCD was performed on hospital days 1, 2, 3 and 7. Hypoperfusion was defined by having two out of three of the following: mean velocity of the middle cerebral artery less than $35 \mathrm{~cm} /$ second, diastolic velocity of the middle cerebral artery less than $20 \mathrm{~cm} /$ second and a pulsatility index greater than 1.4. Vasospasm was defined by the following: mean velocity of the middle cerebral artery greater than $120 \mathrm{~cm} / \mathrm{second}$ and/or Landegaard index greater than 3 .

Results Thirty-four patients (64\%) had normal measurements. Thirteen were discharged home, 16 were discharged to a long-term care facility and five died. Two of these patients were comatose and their families requested withdrawal of care. The other three died from brain death. Eighteen patients (25\%) had hypoperfusion and all 18 progressed to brain death. Twenty-one patients (29\%) had vasospasm. Four of these patients were discharged home, 11 to a long-term care facility and six died. The vasospasm was detected on hospital day 1 in three patients, hospital day 2 in seven patients, hospital day 3 in four patients and hospital day 7 in seven patients. Nimodipine was administered in six patients and all six were discharged to a long-term care facility. However, in one patient, nimodipine caused hemodynamic instability and was discontinued. In 15 patients, nimodipine was not given. Six of these patients expired from brain death. Twelve of 21 patients (57\%) with subarachnoid hemorrhage on computed tomography had vasospasm.

Conclusions Most patients with normal measurements can be expected to survive. Patients with hypoperfusion have a poor prognosis. In patients with vasospasm, the use of nimodipine should be considered; however, further studies are needed to determine safety and efficacy. TCD may be useful in determining early prognosis. Further studies are also needed to determine whether TCD can improve outcome in patients with severe TBI.

\section{P293}

\section{Abstract withdrawn}

P294

Effect of hyperbaric oxygen on cerebral oxygen saturation in patients with sub-acute traumatic brain injury

L Liu, B Zhou, B Liu

Second Affiliated Hospital of Soochow University, Suzhou, China

Critical Care 2010, 14(Suppl 1):P294 (doi: 10.1186/cc8526)

Introduction How hyperbaric oxygen ( $\mathrm{HBO}$ ) affects injured brain tissue is not yet confirmed. We suppose that HBO could make the oxygen stay longer in the injured tissue, because of poor circulation or perfusion of blood in the injured area. This mechanism may help to explain the longer effect of $\mathrm{HBO}$, after $\mathrm{HBO}$ treatment. That is the purpose of our study.

Methods Patients suffering from sub-acute traumatic brain injury, who would be treated with $\mathrm{HBO}$, were enrolled in the study. They were divided into two groups: frontal lobe lesion and nonfrontal lobe lesion groups $(n=20)$ depending on their iconography check (X-CT or MR). The regional cerebral oxygen saturation $\left(\mathrm{rSO}_{2}\right)$ was measured by a Somanetics INVOS 5100 monitor before and after 90 minutes treatment with HBO (15 minutes compression, 40 minutes breathing oxygen of $\mathrm{FiO}_{2} 99 \%$ by mask, 10 minutes rest, 15 minutes decompression). Meanwhile, the arterial blood was sampled to measure blood gas analysis and cytokine.

Results The parameter of $\mathrm{rSO}_{2}$ showed no significant difference following HBO treatment in two groups $(t=0.352, P>0.05)$; however, there were inconceivable results in blood gas analysis. Partial pressure of oxygen $\left(\mathrm{PaO}_{2}\right)$ was significantly decreased after $\mathrm{HBO}$ treatment $(P<0.05)$, although these changes did not take effect on the clinical manifestation. Otherwise, the measurement of cytokines (TNF, IL-1) before and after HBO has no difference $(P>0.05)$ in all groups.

Conclusions $\mathrm{HBO}$ has no effect on brain oxygen saturation, after HBO treatment. But $\mathrm{PaO}_{2}$ was significantly decreased following $\mathrm{HBO}$. The mechanism needs to be studied further.

\section{Reference}

1. Allen BW, et al:: Two faces of nitric oxide: implications for cellular mechanisms of oxygen toxicity. J Appl Physiol 2009, 106:662-667.

\section{P295}

Brain biomarkers and brain tissue oxygenation: changes and correlations following severe head injury

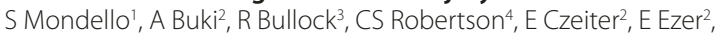
FC Tortella ${ }^{5}$, KK Wang $^{1}$, RL Hayes ${ }^{1}$

'Banyan Biomarkers and University of Florida, Alachua, FL, USA:²University of Pecs, Hungary; ${ }^{3}$ University of Miami, FL, USA; ${ }^{4}$ Baylor College of Medicine, Houston, TX,

USA; ${ }^{5}$ Walter Reed Army Institute of Research, Silver Spring, MD, USA

Critical Care 2010, 14(Suppl 1):P295 (doi: 10.1186/cc8527)

Introduction This study evaluated relationships between CSF levels of brain biomarkers, glial fibrillary acidic protein (GFAP), ubiquitin C-terminal hydrolase (UCH-L1) and $\mathrm{a}_{\|}$-spectrin breakdown (SBDP145), partial pressure of brain tissue oxygen ( $\mathrm{ptiO}_{2}$ ) and brain temperature (Licox system) during the first 24 hours and for up to 10 days following severe TBI.

Methods We studied 27 severe TBI patients having CSF drainage and invasive monitoring of partial brain tissue oxygen tension $\left(\mathrm{PbtO}_{2}\right)$ and brain temperature using the Licox (Integra Neurosciences, Plainsboro, NJ, USA) probe. CSF SBDP145, UCH-L1 and GFAP levels were measured by quantitative ELISA assay on admission and every 6 hours thereafter for a maximum of 10 days. Using a double lumen bolt, $\mathrm{ptiO}_{2}$ and temperature were measured with the Licox. This study focused on the recordings of the first 24 hours following injury (27 patients), as well as preliminary data from four patients for 10 days.

Results The total duration of monitoring was 1,512 hours. During the first 24 hours, biomarker levels decreased while levels of $\mathrm{PbrO}_{2}$ increased. All three biomarkers correlated with $\mathrm{PbrO}_{2}(P<0.0001, P=0.016$ and $P=0.023$, respectively). After the first 24 hours, there were statistically significant changes in levels of brain biomarkers (SBDP145, UCH-L1 and GFAP) as well as in levels of $\mathrm{ptiO}_{2}$ (respectively, $P=0.025, P<0.0001, P=0.033$, $P<0.0001$ ). However, the correlation between biomarkers and brain tissue oxygenation was sustained, and for UCH-L1 improved $(P<0.0001)$. No significant correlations between biomarker levels and brain temperature were found. There were no complications from the monitoring.

Conclusions Our findings show that CFS levels of SBDP145, UCH-L1 and GFAP are related to brain tissue oxygenation in acute and possibly the subacute ( $\leq 10$ days post injury) phases of severe TBI. Future studies will more directly address relationships between changes in tissue oxygenation and biochemical markers of injury following severe TBI. CSF levels of biomarkers and brain tissue oxygenation could yield insights into pathophysiological events following severe TBI and aid in clinical assessments of severe TBI patients.

Table 1 (abstract P294)

\begin{tabular}{|c|c|c|c|c|c|c|}
\hline \multirow[b]{2}{*}{ Group } & \multicolumn{2}{|c|}{$\mathrm{pH}$} & \multicolumn{2}{|c|}{$\mathrm{PaO}_{2}(\mathrm{mmHg})$} & \multicolumn{2}{|c|}{$\mathrm{PaCO}_{2}(\mathrm{mmHg})$} \\
\hline & Pre & Post & Pre & Post & Pre & Post \\
\hline Frontal lobe lesion & $7.42 \pm 0.02$ & $7.45 \pm 0.04$ & $135.4 \pm 29.77$ & $102.12 \pm 9.08$ & $43.69 \pm 4.58$ & $40.81 \pm 7.53$ \\
\hline Nonfrontal lobe lesion & $7.41 \pm 0.03$ & $7.42 \pm 0.04$ & $128.2 \pm 34.22$ & $107.2 \pm 24.00$ & $43.93 \pm 4.88$ & $43.00 \pm 4.80$ \\
\hline
\end{tabular}


P296

Cortex and deep grey matter have different sensitivities to hypoxia-hypotension and traumatic brain injury: a combined $\mathrm{PtiO}_{2}$ and microdialysis study in rats

A Odri, B Vigué, J Duranteau, T Geeraerts

$\mathrm{CHU}$ de Bicetre, Le Kremlin Bicetre, France

Critical Care 2010, 14(Suppl 1):P296 (doi: 10.1186/cc8528)

Introduction After traumatic brain injury $(\mathrm{TBI})$, structural lesions are heterogeneous, but the spatial heterogeneity of consequences of insults as hypoxia-hypotension (HH) and/or TBI has never been studied. The objective of this study was to compare the effect of standardized insults $(\mathrm{HH}, \mathrm{TBI}$ and both) on brain energy metabolism in two different regions: frontal cortex and thalamus.

Methods Twenty-eight Sprague-Dawley rats were randomized into four groups: Sham, TBI (impact acceleration alone, $450 \mathrm{~g}$ weight drop from $1.8 \mathrm{~m}$ ), $\mathrm{HH}$ (blood depletion to mean arterial pressure $40 \mathrm{mmHg}, \mathrm{FiO}_{2} 10 \%$, 15 minutes) and TBI-HH (TBI followed by HH, 45 minute delay). Cerebral perfusion pressure (CPP) was continuously and invasively measured. Brain microdialysis and $\mathrm{PtiO}_{2}$ probes were both inserted stereotaxically in the right thalamus and frontal cortex.

Results Except during the $\mathrm{HH}$ phase, CPP was always greater than $60 \mathrm{mmHg}$. During the hour following the $\mathrm{HH}$ period, a significant increase in cerebral lactate/pyruvate ratio (Figure 1), glycerol and glutamate was observed. This increase was higher in the cortex than in the thalamus in all groups subjected to $\mathrm{HH}(P<0.001)$. In the TBI-HH groups, the increase in glycerol in the cortex was significantly higher compared with the $\mathrm{HH}$ group $(P<0.001)$, as well as thalamic and cortical glutamate. During 15 minutes following the $\mathrm{HH}$ phase (after reinjection and reoxygenation), an increase in $\mathrm{PtiO}$ was observed in the cortex and thalamus, but with different profiles (lower increase in the cortex) (Figure 1).

Conclusions Different profiles of cerebral response to $\mathrm{HH}$ and TBI were observed with higher sensitivity in the cortex than in the thalamus. The post-ischemic hyperemia seems to be altered in the traumatized cortex but conserved into the thalamus and nontraumatized brain.

\section{P297}

Diagnosis of carotid and vertebral artery injury in major trauma with head injury

A Circelli', M Ciapetti², R Spina ${ }^{2}$, A Pasquini², L Migliaccio², M Pierini ${ }^{3}$, G Zagli², A Peris²

'Postgraduate School of Anesthesia and Intensive Care, University of Florence, Italy; ${ }^{2}$ Careggi Teaching Hospital, Florence, Italy; ${ }^{3}$ Mechanical and Technology Department, University of Florence, Italy

Critical Care 2010, 14(Suppl 1):P297 (doi: 10.1186/cc8529)

Introduction The purpose of this study is to describe a single institutional experience in diagnosis and management of patients with blunt cerebrovascular injury (BCVI) after severe traumatic brain injury (TBI).
Methods We considered blunt major trauma patients consecutively admitted from January 2008 to October 2009 to our ICU of a regional referral hospital (Careggi Teaching Hospital, Florence, Italy). Patients were screened on the basis of Memphis criteria to identify risk factors for BCVI: complex cervical spine fractures, neurological conditions not sustained by imaging, Horner's syndrome, LeFort II/III fractures, basilar skull fractures with petrous/ neck soft tissue injury. We modified this protocol to investigate all petrous fracture and to consider also cervical spine fractures (subluxation, transverse foramen, upper cervical spine involvement). In all patients judged at risk for BCVI, a 64-slice angio-CT scan (CTA) was performed.

Results During the study period, 266 patients were admitted to the ICU for BMT with an ISS >15; 162 patients (60.9\%) presented TBI or cervical spine fracture. According to our proposed modified Memphis criteria, 46 patients showed risk factors for BCVI (17.3\% of the total of BMT; $28.4 \%$ of $\mathrm{TBI}$ ), and six of them had carotid injury (2.2\% of all BMT; $13.0 \%$ of patients with risk factors). No vertebral injury was detected. Anticoagulant therapy within 72 hours was initiated in all patients. No complications occurred and all patients were discharged from the ICU. Clinical examination during the 6-month follow-up showed no neurological deficiency related to previous vascular injury.

Conclusions A higher percentage of BCVI was observed using the proposed modified Memphis criteria if compared with other studies $(2.2 \%$ vs $1 \%)$. The mortality rate was lower than previously reported for patients with BCVI (0\% vs 13\%) and it could be explained by the early diagnosis and anticoagulant therapy initiation. An extended screening protocol application allows prompt recognition of $\mathrm{BCVI}$. In regard of the higher percentage of BCVI found in our population, an advanced study on protective devices (for example, helmets) is actually in progress with the aim to reduce biomechanical effects of head injury on extracranial vessels.

\section{P298}

Decompressive craniectomy in severe intracranial hypertension after brain injury: early or late?

S diValvasone ${ }^{1}$, S Biondi ${ }^{2}$, A Nella ${ }^{2}$, L Migliaccio ${ }^{2}$, M Ciapetti ${ }^{2}$, G Cianchi ${ }^{2}$ M Bonizzoli2, G Zagli2, F Mariotti2 ${ }^{2}$, F Posteraro ${ }^{3}$, A Peris $^{2}$

'Postgraduate School of Anesthesia and Intensive Care, University of Florence, Italy; ${ }^{2}$ Careggi Teaching Hospital, Florence, Italy; ${ }^{3}$ Auxilium Vitae, Volterra, Italy Critical Care 2010, 14(Suppl 1):P298 (doi: 10.1186/cc8530)

Introduction Decompressive craniectomy is indicated for treatment of severe intracranial hypertension. However, this procedure is invasive and potentially associated with complications. We present a preliminary result of a study comparing early and late decompressive craniectomy in severe traumatic brain injury.

Methods Patients studied were all admitted to the ICU of a tertiary referral center (Careggi Teaching Hospital, Florence, Italy) during 4 years (2005 to 2009). In total, data of 62 brain-injured patients, who underwent decompressive craniectomy, were retrospectively examined and included in two groups based on decompressive craniectomy execution:
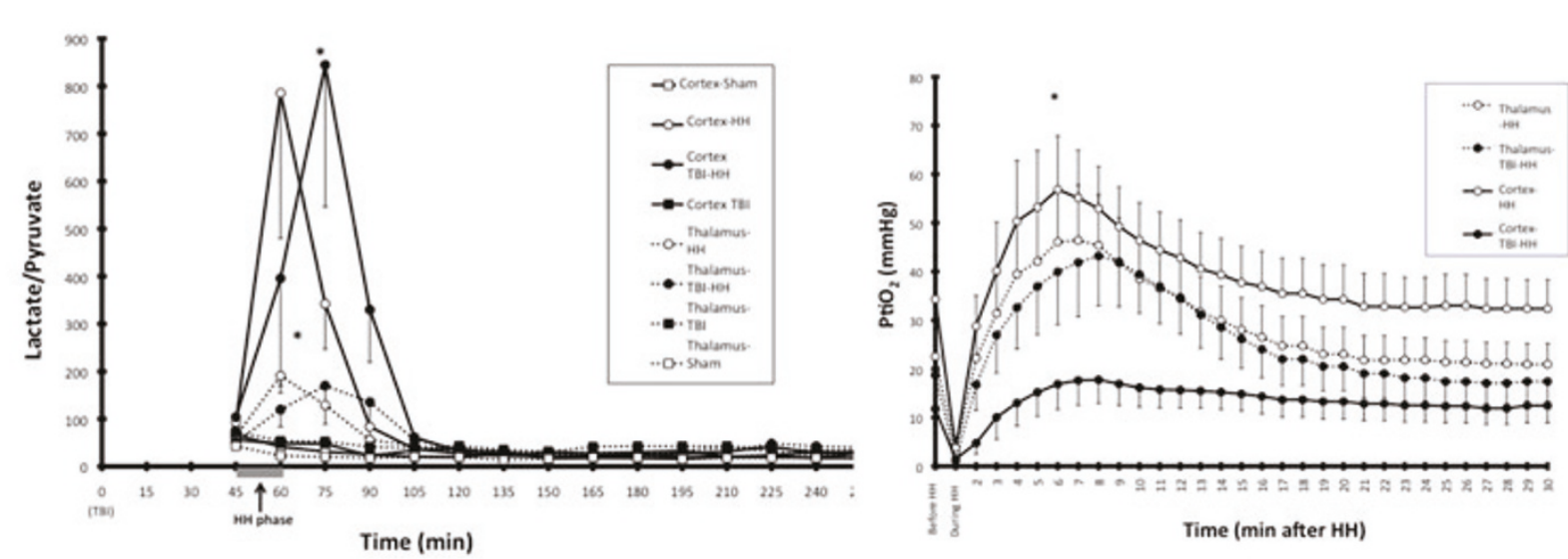

Figure 1 (abstract P296). Brain lactate/pyruvate ratios and $\mathrm{PtiO}_{2}$ in the thalamus and cortex. 


\begin{tabular}{|c|c|c|}
\hline & Group $A(N=41)$ & Group B $(N=21)$ \\
\hline Age & $44 \pm 18$ & $36 \pm 19$ \\
\hline Male sex, N (\%) & $31(75 \%)$ & $16(76 \%)$ \\
\hline ISS & $33 \pm 11$ & $33 \pm 9$ \\
\hline IRISS & $63.4 \pm 28.4$ & $62.6 \pm 20.3$ \\
\hline RTS & $5.7 \pm 1.5$ & $6.3 \pm 1.3$ \\
\hline SAPSII & $46.1 \pm 12.5$ & $42.4 \pm 9.2$ \\
\hline ICULOS & $16.6 \pm 8.9$ & $18=10.1$ \\
\hline HospitalLOS & $21.9 \pm 21.1$ & $27.6 \pm 19.9$ \\
\hline ICU mortality, $\mathrm{N}(\%)$ & $12(29.2 \%)$ & $6(28.5 \%)$ \\
\hline Hospitalmortality, $N(\%)$ & $16(39 \%)$ & (33.5\%) \\
\hline
\end{tabular}

Figure 1 (abstract P298). Demographic and clinical characteristics of both groups.

early decompressive craniectomy group (decompressive craniectomy performed within 24 hours after brain injury, group $A ; n=41$ ) and late decompressive craniectomy (later than 24 hours, group $B ; n=21$ ). For all patients, demographic, scores, clinical data, length of stay and final outcome were collected from the institutional database. Traumatic lesions were compared at admission using the Marshall score and 24 hours after decompressive craniectomy execution with CT scan. The Glasgow Outcome Scale (GOS) at 6 months was also collected.

Results Demographic and clinical characteristics of groups are shown in Figure 1 (data expressed as mean \pm SD). Patients who underwent decompressive craniectomy within 24 hours after injury (group A) had a significant worst Marshall score if compared with group $B(3.1 \pm 0.7$ vs $2.4 \pm 0.8$, respectively; $P<0.05)$, but also showed a significant enlargement of contusions compared with group B $(52.7 \%$ vs $16.6 \%$; $P<0.01)$. ICU/ hospital length of stay and mortality were not significantly different between groups. The GOS evaluated at 6 months showed a good recovery of surviving patients in both groups (3.7 \pm 1.0 in group $A$ and $3.2 \pm 0.9$ in group B).

Conclusions Our data, limited by the retrospective nature of the study, do not encourage an early decompressive treatment of severe intracranial hypertension. Decompressive craniectomy should be considered in case of lack of response to a medical, even intensive, approach.

\section{References}

1. Neurosurg Focus 2009, 26:E7.

2. Curr Opin Crit Care 2007, 13:163-168

P299

\section{Abstract withdrawn}

\section{P300}

Optic nerve ultrasound for detecting intracranial hypertension

V Orzalesi, L Bucciardini, A Amadori, S Marchiani, P Innocenti

AOU Careggi, Florence, Italy

Critical Care 2010, 14(Suppl 1):P300 (doi: 10.1186/cc8532)

Introduction The purpose of this study is to verify the reliability of optic nerve ultrasound $(\mathrm{ONU})$ for detecting intracranial hypertension $(\mathrm{IH})$. $\mathrm{IH}$ is a frequent and potentially fatal complication of severe head injury. Actually, intraventricular catheters represent the gold standard for ICP measurements. Unfortunately they present serious complications and relative contraindications like thrombocytopenia or coagulopathy. Computed tomography and transcranial Doppler (TCD) represent noninvasive methods to evaluate ICP but they present drawbacks. ONU has been recently suggested as a non-invasive tool to diagnose $\mathrm{IH}$. Its reliability has been showed in patients with severe head injury or with intracranial hemorrhage $[1,2]$.

Methods The study was conducted on 10 patients admitted to the neurointensive care unit for moderate/severe head injury in the period January to June 2009 (Group 1). Ten healthy subjects were enrolled as the control group (Group 2). Estimated ICP (elCP) was calculated with TCD using the equation proposed by Czosnyka and colleagues [3]. It was measured twice a day for 3 consecutive days. In the same sonographic session, the optic nerve sheath diameter (ONSD) was measured in the sagittal and transverse plane $3 \mathrm{~mm}$ behind the papilla, in both eyes.

Results No significant differences have been found between groups regarding age and sex. The ONSD distribution is shown in Figure 1. ONSD, eICP and ICP values were significantly higher in Group 1 than in Group 2. Linear regression analysis identified a significant relationship

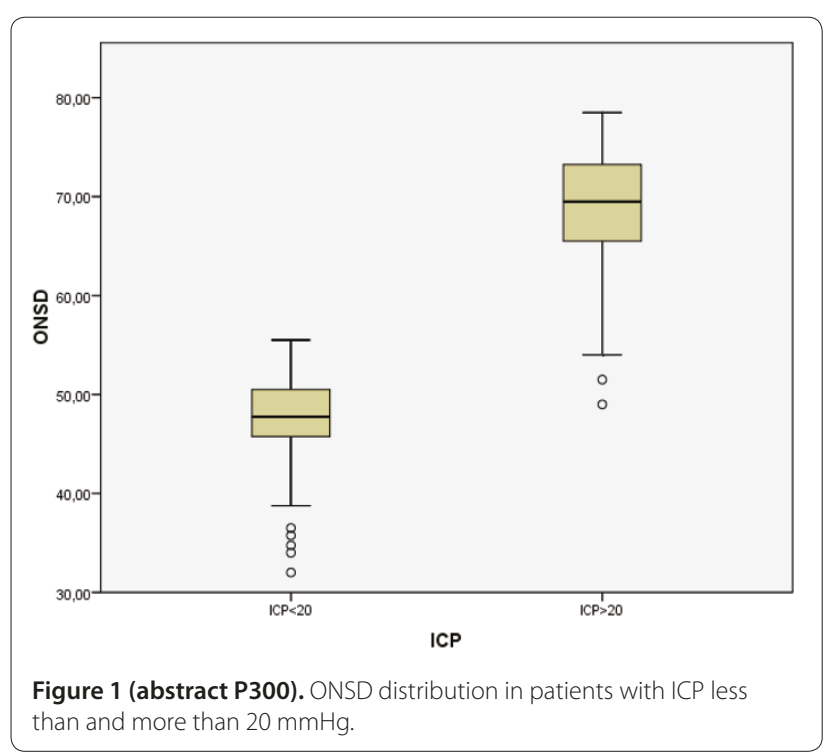


between ONSD and ICP ( $r=0.588)$. By calculating the receiver operating characteristic curve, an ONSD value of $5.35 \mathrm{~mm}$ resulted as the optimal cut-off point with a sensitivity of $95.1 \%$ and a specificity of $96.2 \%$.

Conclusions ONSD measurements correlated with invasive and noninvasive (TCD) measurements of ICP. It is a useful, non-invasive bedside tool to diagnose $\mathrm{IH}$. It is safe, easy to perform and can rapidly give reproducible information on patients'ICP.

\section{References}

1. Moretti R, et al:: Reliability of optic nerve ultrasound for the evaluation of patients with spontaneous intracranial hemorrhage. Neurocrit Care 2009. [Epub ahead of print]

2. Geeraerts, et al:: Non-invasive assessment of intracranial pressure using ocular sonography in neurocritical care patients. Intensive Care Med 2008, 34:2062-2067.

3. Czosnyka M, et al.: Cerebral perfusion pressure in head-injured patients: a noninvasive assessment using transcranial Doppler ultrasonography. J Neurosurg 1998, 88:802-808.

\section{P301}

Measurement of neuronal specific enolase as a predictor of outcome in patients suffering from severe traumatic brain injury JW Gatson, AH Idris, PE Pepe, JP Minei, J Wigginton

University of Texas Southwestern Medical Center at Dallas and the Parkland Health and Hospital System, Dallas, TX, USA

Critical Care 2010, 14(Suppl 1):P301 (doi: 10.1186/cc8533)

Introduction Traumatic brain injury (TBI) is a multiphasic disease. Following the initial impact, secondary injury, including oxidative stress and inflammation, is thought to contribute significantly to death and disability. This ongoing damage in the penumbra of the brain leads to the demise of neuronal populations, and ultimately decreased brain function. Identification of various neural markers such as neuronal specific enolase (NSE) at various time points following TBI may help us better understand the magnitude of secondary injury following $\mathrm{TBI}$, and may help predict outcome in these patients.

Methods Early serial cerebrospinal fluid (CSF) samples were collected from patients with severe TBI (GCS 3 to 8) who required placement of a therapeutic ventriculostomy. Following ventriculostomy placement, CSF samples were obtained every 4 hours for the first 24 hours post-injury, then every 8 hours for post-injury days 2 to 5 . The levels of NSE were then measured using the ELISA method.

Results Following sample analysis in 11 patients, we found that in TBI patients that had a good neurological outcome, the levels of NSE in the CSF rose early (between 25 and $60 \mathrm{ng} / \mathrm{ml}$ ) within 12 to 16 hours following the initial injury. The NSE levels then rapidly decreased to normal levels $(\sim 5 \mathrm{ng} / \mathrm{ml})$ at approximately 20 hours following injury, with these levels persisting until day 5 (day of final sample collection). Patients with a poor outcome (lack of ability to return to pre-injury activities or death) showed significantly higher levels of NSE persisting out to 5 days post injury, with late levels ranging from 35 to $50 \mathrm{ng} / \mathrm{ml}$.

Conclusions In TBI patients with a good outcome, there was an increase in NSE levels in the CSF noted at early time points ( 16 hours), which abated at approximately 20 hours after injury. In TBI patients with a poor clinical outcome, CSF levels of NSE were significantly elevated at later time points over the first 5 days post injury.

\section{P302}

Hyperphosphorylated neurofilament NF-H, S100 $\beta$ and NSE as indicators of severity of brain injury in children

J Žurek, L Marek, P Košut, M Fedora

Faculty of Medicine, Masaryk University, University Children's Hospital, Brno, Czech Republic

Critical Care 2010, 14(Suppl 1):P302 (doi: 10.1186/cc8534)

Introduction The aim of the study was to determine whether serum levels of biomarkers - hyperphosphorylated neurofilament NF-H, S100 $\beta$ protein and NSE - correlate with severity of brain injury and outcome in children with traumatic brain injury (TBI).

Methods Thirty-eight patients with TBI were enrolled in the prospective study. TBI was verified by computerized tomography according to the Marshall classification. Venous blood samples were taken after admission and every 24 hours for a maximum of 6 consecutive days. Serum NF-H concentrations were quantified by ELISA, S100ß and NSE by electrogenerated chemiluminescence. The outcome was evaluated 6 months after TBI using the Glasgow Outcome Scale (GOS) in all patients. Results Serum concentrations of NF-H (median 86 vs 12; $P=0.01$ ), S100 $\beta$ (median 3.47 vs $0.54 ; P=0.001$ ), and NSE (median 193.2 vs $37.03 ; P=0.001$ ) were higher in nonsurvivors than survivors. Serum NF-H (median 86 vs 12; $P=0.004$ ), S100 $\beta$ (median 3.47 vs $0.54 ; P=0.001$ ) and NSE (median 193.2 vs 37.03; $P=0.001$ ) was significantly higher in patients with unfavourable outcome (GOS $\leq 3$ vs GOS $>3$ ). Spearman's rank correlation coefficient on first day of hospitalization S100B $\times$ NSE $=0.58(P<0.05), \mathrm{GCS} \times \mathrm{GOS}=0.50$ $(P<0.05), \mathrm{GOS} \times$ Marshall classification $=0.46(P<0.05)$.

Conclusions Plasma levels of hyperphosphorylated neurofilament, $\mathrm{S} 100 \beta$ and NSE correlate with severity of TBI and may be useful as predictors of outcome in children with TBI.

\section{P303}

A simple hospital discharge score predicts Glasgow Outcome Scale at 12 months in patients with traumatic brain injury

GW Fuller, P Yeoman

Queens Medical Centre, Nottingham, UK

Critical Care 2010, 14(Suppl 1):P303 (doi: 10.1186/cc8535)

Introduction This study examines whether a hospital discharge score predicts future Glasgow Outcome Scale (GOS) following traumatic brain injury (TBI). Loss to follow-up is a major problem in TBI studies and an early prediction score could be used for interpolation of missing outcome data or as a surrogate outcome measure.

Methods Adults admitted to the Queens Medical Centre between 1993 and 2002 with TBI and GCS $<12$ were included prospectively in the Nottingham Head Injury Register. At hospital discharge the modified Barthel Index was recorded by nursing staff and a Discharge Disability Score (DDS) calculated: 1. Independent, 2. Dependent <24 hour care, 3. Fully dependent, 4. Dead [1]. Glasgow Outcome Scale (GOS) was calculated at 12 months by the patient's general practitioner [2]. Unfavourable outcome was defined as DDS 3, 4 or GOS 1, 2, 3. Correlation between DDS and GOS was measured and the ability of DDS to predict GOS at 12 months in survivors was assessed using logistic regression. Sensitivity and specificity of dichotomised DDS for prediction of unfavourable outcome at 12 months was also calculated.

Results Data were available on 1,227 patients. A highly correlated linear relationship was evident between DDS and GOS (spearman correlation coefficient $0.77, P<0.0001$ ). Unfavourable outcome measured by DDS showed sensitivity of $73 \%$ (95\% Cl 69 to $76 \%$ ) and specificity of 56\% (53 to $59 \%$ ) for prediction of unfavourable outcome at 12 months defined by GOS. DDS showed a strong association with GOS at 12 months for survivors at discharge, with fully dependent patients having 15 times higher odds of an unfavourable outcome. Adjusting for known TBI prognostic factors attenuated the strength of the association, however DDS remained a statistically significant strong predictor of outcome.

Conclusions DDS is strongly associated with 12-month GOS and could potentially be used to replace missing data or provide a surrogate outcome measure in TBI trials. A valid prediction score may also aid the clinician's ability to discuss patient prognosis at hospital discharge.

\section{References}

1. Shah S, et al: J Clin Epidemiol 1989, 42:703-709.

2. Jennet B, et al: Lancet 1975, 1:480-484.

\section{P304}

Outcome of aggressive treatment for blunt and penetrating traumatic cardiac arrest

Y Moriwaki, M Iwashita, Y Tahara, S Arata, N Harunari, T Kosuge, H Toyoda, N Suzuki

Yokohama City University Medical Center, Yokohama, Japan

Critical Care 2010, 14(Suppl 1):P304 (doi: 10.1186/cc8536)

Introduction The outcome of patients with cardiac arrest due to blunt trauma (BT-CPA) and penetrating trauma (PT-CPA) is very poor. The aim of this study is to clarify the outcome of patients with CPA on arrival due 
to BT-CPA and PT-CPA treated with our strategy including emergency department thoracotomy (EDT).

Methods This study is a population-based case series observational study. We have taken three approaches to these patients: our private aggressive treatment strategy (resuscitation for 30 minutes, aggressive infusion using sheath introducer into the subclavian vein, and EDT); in-hospital system supporting this aggressive resuscitation (logistic issue such as the close location between ED and the room for catheter intervention and CT, and direct entrance to the OR by exclusive lift, and common instruments interchangeable between ED and OR including the bed); and prehospital EMS in our city (CPA patients are transferred in about 7 minutes to the nearest of selected 11 hospitals which can receive and treat CPA patients). Results For the past 10 years, of 478 BT-CPA and 30 PT-CPA patients, 76\% and $70 \%$ were witnessed, and $21 \%$ and $37 \%$ were CPA after scene. A total of $85 \%$ and $77 \%$ underwent EDT. Although $34 \%$ and $60 \%$ achieved ROSC, only $18 \%$ and $40 \%$ went to the ICU, TAE room, and OR (admitted), and only $2.7 \%$ and $17 \%$ were discharged (survivors). Restricted in eight witnessed patients showing VF as the initial cardiac rhythm in BT-CPA, 38\% were admitted and 13\% survived. No PT-CPA patients showed VF. Restricted in 134 witnessed patients showing PEA in BT-CPA and 10 in PTCPA, $28 \%$ and $70 \%$ were admitted and $1 \%$ and $30 \%$ survived. Two hundred and twenty witnessed patients showing no life sign (asystole) in BT-CPA and 12 in PT-CPA, $8 \%$ and $25 \%$ were admitted, and $3 \%$ and $17 \%$ survived. The initial rhythm of survivors was asystole in 70\% in BT-CPA and 40\% in PT-CPA. Although the time interval from arrival at the hospital to ROSC of survivors tended to be shorter than for nonsurvivors in BT-CPA, there was no difference in this interval in PT-CPA. The longest interval was 43 and 30 minutes in BT-CPA and PT-CPA.

Conclusions The expected outcome of BT-CPA and PT-CPA patients is hopeless. However, we cannot and should not give up on resuscitating them merely because they are CPA and they do not show any life sign at the scene. We consider the indication of aggressive resuscitation in every individual case by his/her individual condition.

\section{P305}

Using flow-rate limiting bag-valve-mask device without training

J Nurmi', M Castrén²

${ }^{1}$ Helsinki University Central Hospital, Helsinki, Finland; '2Karolinska Institutet, Stockholm, Sweden

Critical Care 2010, 14(Suppl 1):P305 (doi: 10.1186/cc8537)

Introduction The Smart Bag (SB, O-Two Medical Technologies Inc. Canada) is a bag-valve-mask resuscitator (BVM) equipped with a valve limiting flow-rate and peak airway pressure to decrease hyperventilation and gastric insufflation. We studied this device used by hospital staff without training to evaluate the possibility to change the standard BVM to the SB in hospital wards where positive pressure ventilation is rarely needed and training is sporadic.

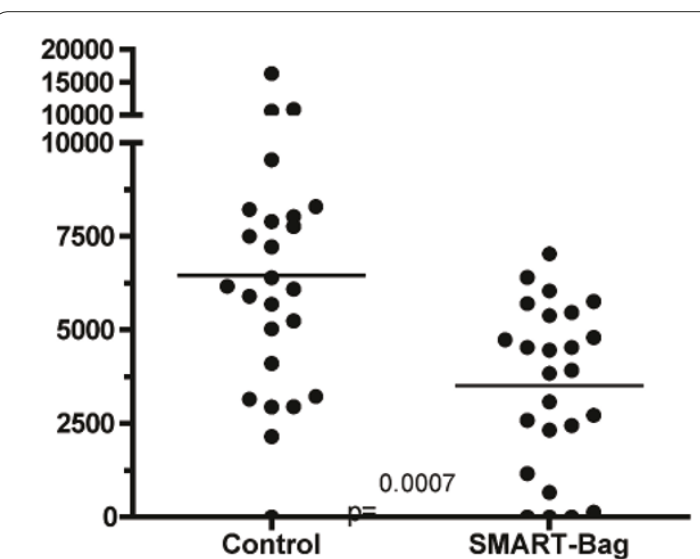

Figure 1 (abstract P305). Minute ventilation ( $\mathrm{ml}$ ) in the Smart Bag group and the control group.
Methods The participants (12 physicians, 38 nurses) were randomised to use the standard BVM or SB to ventilate a resuscitation training manikin over 1 minute. The mechanism of the SB was shortly described in the SB group but no hands-on training was provided. Participants were asked to ventilate the patients as they would do if patients have a pulse but do not breathe. Tidal volumes were registered to a computer connected to the manikin.

Results The medians of minute ventilation were 6.1 (interquartile range 2.6 to 8.1 ) and 3.9 (IQR 1.7 to 5.4) litres per minute in the SB and control groups, respectively (Figure 1). Hyperventilation $>10 \mathrm{l} /$ minute occurred only in the control group $(P=0.23)$

Conclusions Using the SB without previous hands-on training is possible for the majority of nursing and medical staff and decreases hyperventilation in comparison with the standard BVM.

\section{P306}

Out-of-hospital cardiac arrest and resource utilisation in the ICU

A Manning, M Purcell-Jones, A Saayman, M Wise

University Hopsital of Wales, Cardiff, UK

Critical Care 2010, 14(Suppl 1):P306 (doi: 10.1186/cc8538)

Introduction Survival rates for out-of-hospital cardiac arrests (OHCA) remain poor and typically of the order of $10 \%$ or less [1]. In the community, bystander CPR and public automated external defibrillators have been promoted, whilst in hospital therapeutic hypothermia and coronary reperfusion are employed to improve outcome. Few studies have assessed how this group of patients impacts on critical care resources [2].

Methods Patients with a spontaneous circulation following OHCA admitted to the critical care unit of a university teaching hospital between 1999 and 2009 were analysed retrospectively.

Results A total of 1,656 patients presented to the emergency department following OHCA, of which 217 were admitted to the ICU. The ICU and hospital survivals were $41 \%$ and $32.7 \%$, respectively. In the period 1999 to 2003, ICU and hospital survival rates were $37.5 \%$ and $22.9 \%$, respectively. In the period 2004 to 2009, ICU and hospital survival rates were $43.8 \%$ and $40.5 \%$, respectively. At admission to the ICU, the mean APACHE II score was 22.82, mean $\mathrm{pH} 6.87$ and $\mathrm{FIO}_{2} 52.6 \%$. In those patients who survived hospital discharge, the mean APACHE II score was 19.71, mean pH 6.97 and $\mathrm{FIO}_{2} 47.1 \%$, whilst in nonsurvivors they were $24.33,6.83$ and $55.4 \%$, respectively. Overall ICU and hospital lengths of stay (LOS) were 5.41 and 13.72 days and the average TISS score was 221. In those who survived to hospital discharge, ICU and hospital LOS were 10.1 and 32.4 days and the average TISS score was 380. Amongst patients who died in hospital, the mean ICU and hospital LOS were 3.1 and 4.7 days, the average TISS was 144

Conclusions Overall one-third of patients admitted to the ICU in the period 1999 to 2009 with OHCA were discharged from hospital. This increased to $40.5 \%$ in the period from 2004 with almost all patients discharged from the ICU surviving to hospital discharge. Nonsurvivors amongst the cohort are unsurprisingly more seriously ill with higher APACHE II scores and deranged physiology. The cost of treating survivors of OHCA in ICU is considerably higher for survivors compared with nonsurvivors. However, as patients who are discharged from ICU usually leave hospital (2004 to 2009), this would appear to be an appropriate utilisation of resources.

References

1. Sayre et al:: Prehosp Emerg Care 2009, 13:469-477

2. Graf J, et al:: Crit Care 2008, 12:R92.

P307

Comparison of external cardiac compression on the floor and in a dental chair

G Papakitsos, A Kapsali, T Papakitsou

General Hospital Arta, Greece

Critical Care 2010, 14(Suppl 1):P307 (doi: 10.1186/cc8539)

Introduction Dental treatment is often stressful for patients and causes accidental systemic symptoms. In such situations, cardiopulmonary resuscitation (CPR) may be required. If external cardiac compression (ECC) in the dental chair is not effective, the patient should be moved onto the floor, which needs at least two or three persons and 10 to 15 seconds. 
In the current study, therefore, we investigated the efficacy of ECC in the dental chair in comparison with ECC on the floor.

Methods Two dentists and two nurses with experience of ECC participated in this study; $30 \pm 5$ years old, $160 \pm 5 \mathrm{~cm}$ in height, $65 \pm 7 \mathrm{~kg}$ in weight. Before the study, they were educated about CPR and performed ECC for 5 minutes on the resuscitation manikin in two different situations; on the floor and in the dental chair. On separate days, they repeated these ECC procedures on the floor and in the dental chair again. The depth of compression and the percentage of adequate compression were evaluated. In addition, each participant commented on the preferable situation in the questionnaire after each set of ECC.

Results Four dental personnel performed ECC five times on the floor and five times in the dental chair and commented on the preferable setting five times. The efficacy of ECC was evaluated by the average depth and the percentage of ECC with adequate depth; $39.8 \pm 8.2 \mathrm{~mm}$ and $46.8 \pm 48.8 \%$ on the floor and $34.4 \pm 6.9 \mathrm{~mm}$ and $41.7 \pm 42.7 \%$ in the dental chair. The percentage of ECC with adequate depth was higher for the floor setting than that of the dental chair setting, although it did not reach statistical significance $(P=0.079)$. In the 20 questionnaires, three of them preferred the dental chair setting, two of them were no difference between both settings and 15 of them preferred the floor setting.

Conclusions ECC on the floor can be performed effectively and easy and we can start CPR immediately after moving the patient onto the floor.

Reference

1. Yokoyama T, et al.: Efficacy of external cardiac compression in a dental chair. Eur J Anaesthesiol 2008, 25(Suppl 44):2-5.

\section{P308}

\section{Effect of telephone CPR on the rate of bystander CPR for} out-of-hospital cardiac arrest in a typical urban city in Japan

Y Moriwaki, Y Tahara, H Toyoda, T Kosuge, M Iwashita, S Arata, M Toh, STakagi, N Harunari, N Suzuki

Yokohama City University Medical Center, Yokohama, Japan

Critical Care 2010, 14(Suppl 1):P308 (doi: 10.1186/cc8540)

Introduction Today, the emergency medical service (EMS) system has been developing in each country. However, it is not known whether people are willing to perform CPR and whether they are prepared to perform CPR under telephone CPR advice. In this study, we examined how Japanese citizens are interested in the importance of immediate CPR and defibrillation, and how they understand this importance.

Methods Patients with out-of-hospital cardiac arrest due to nontraumatic etiology treated in our center for the past 2 years were enrolled. Cardiac arrest after the scene was excluded. Patients' records from our emergency department were reviewed. In Japan, the ambulance service, dispatch service and emergency life-saving technician (ELST) belong to the fire department. ELSTs perform not only advanced CPR for CPA patients on the job but also education of call takers in the central operation center under medical control about the importance of the recognition of CPA and advice for immediate bystander CPR.

Results A total of 747 patients were enrolled. Telephone CPR advice was performed for bystanders of 336 (45\%) patients and 304 bystanders actually performed CPR (90\%). Five percent (40) of all 747 cases underwent voluntarily bystander CPR before telephone advice, 40\% underwent bystander CPR according to telephone advice, and 4\% (32) did not undergo CPR against telephone CPR advice. In 344 patients with bystander CPR, 33\% reached ROSC, 11\% survived more than 24 hours, and 3\% were discharged, and in 391 patients without bystander CPR, 30\%, 11\%, and 6\%, respectively. Restricted in 302 witnessed patients, of 125 with bystander CPR, 52\% reached ROSC, 16\% survived more than 24 hours, and 3\% were discharged, and in 177 without bystander CPR, 44\% reached ROSC, 14\% survived more than 24 hours, and 1\% were discharged, respectively.

Conclusions Most people are willing to perform CPR. Bystanders are prepared to perform CPR with telephone CPR advice to help them. However, bystander CPR is not always adequate, resulting in no distinct effect of CPR on survival rate. We should educate citizens beforehand and guide bystanders with more proper and quick advice by telephone.

P309

Time factors rather than type and/or nature of CPR modify the prognosis of out-of-hospital cardiac arrests witnessed by bystanders

H Inaba, T Yachida, M Enami, Y Takei, Y Goto, K Ohta

Kanazawa University Graduate School of Medicine, Kanazawa, Japan

Critical Care 2010, 14(Suppl 1):P309 (doi: 10.1186/cc8541)

Introduction The purpose of the study was to analyze the effects of the type and nature of CPR on the prognosis of out-of-hospital cardiac arrests (OHCAs).

Methods We analyzed 1,612 OHCAs, witnessed by citizens and handled by the dispatch system in Ishikawa, Japan, from 1 April 2003 to 31 March 2008. Bystander CPR was classified into four groups according to type (CC only or CC + MMV) and nature (under one's own initiative or telephoneassisted instruction)

Results The presence of bystander CPR significantly augmented the 1 -month survival rate. However, there were no significant differences among the four groups of CPR. The multivariate logistic regression analysis identified three time factors including intervals of collapse-to-call, call-tofirst CPR, call-to-arrival to patients as independent factors associated with 1-month survival. See Figures 1 and 2.

Conclusions Significance of correctable time factors rather than type of CPR should be considered in the future guideline revision.

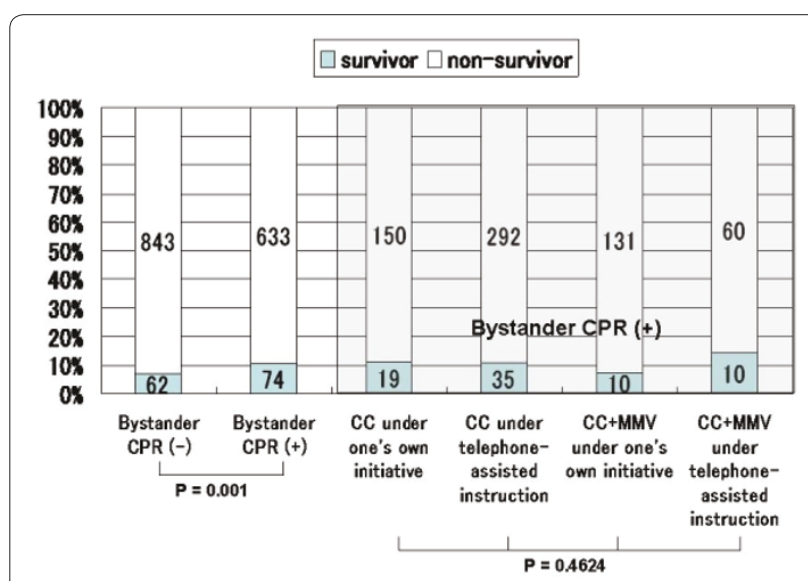

Figure 1 (abstract P309). Effect of bystander CPR on the 1-month survival rate.

\begin{tabular}{|c|c|c|c|c|c|}
\hline \multirow[b]{2}{*}{ Factors related to survival } & \multicolumn{2}{|c|}{ 19-survival } & \multicolumn{2}{|c|}{$\begin{array}{l}\text { Mono-variate } \\
\text { enalysis } \\
\end{array}$} & \multirow{2}{*}{$\begin{array}{l}\text { Odds ratio } \\
\text { (95\% C.1.) } \\
\text { Multiple } \\
\text { logistc regression } \\
\text { Anaysis }\end{array}$} \\
\hline & $\begin{array}{c}\text { Yes } \\
(N=136)\end{array}$ & $\begin{array}{c}\text { No } \\
(N=1476)\end{array}$ & p & $\begin{array}{l}\text { Odds } \\
\text { ratio for } \\
\text { survival }\end{array}$ & \\
\hline Region - Central (urban) \% & $70.6 \%$ & $52.6 \%$ & $<0.001$ & 2.160 & $1.47(0.45-0.95)$ \\
\hline$A g \theta(y)^{*}$ & $66(55-77)$ & $77(66-85)$ & $<0.001^{* *}$ & & $0.10(0.03-0.33)$ \\
\hline Sex - female \% & $31.6 \%$ & $36.8 \%$ & 0.264 & & $1.35(0.87-2.07)$ \\
\hline Place - Home \% & $60.3 \%$ & $64.3 \%$ & 0.347 & & $1.41(0.90-2.22)$ \\
\hline Initial rhythm - shockable \% & $51.2 \%$ & $10.3 \%$ & $<0.001$ & 9.123 & $8.31(5.09-13.78)$ \\
\hline Cause of arrest - cardac \% & $64.7 \%$ & $49.1 \%$ & $<0.001$ & 1.904 & $0.78(0.48-1.25)$ \\
\hline Bystander CPR \% & $100 \%$ & $100 \%$ & 0.0287 & & $C \mathrm{C}:$ \\
\hline No & $45.6 \%$ & $57.1 \%$ & & & $0.81(0.42-1.56)$ \\
\hline$\triangle C$ & $14.7 \%$ & $12.9 \%$ & & & CO+MMV: \\
\hline $\mathrm{CC}+\mathrm{MMV}$ & $39.7 \%$ & $30.0 \%$ & & & $1.08(0.47-2.37)$ \\
\hline CPR under telephone-assisted instruction \% & $46.3 \%$ & $41.1 \%$ & 0.233 & & $1.298(0.83-2.03)$ \\
\hline Interval of collapse to emergency call (min)" & $1(1-3)$ & $2(0-4)$ & 0.042 & & $0.95(0.92-0.99)^{*}$ \\
\hline Interval of call to arrival at patient's site (min)" & $6(5-8)$ & $7(6-10)$ & $<0.001^{\prime \prime}$ & & $0.86\left(0.79-0.94^{*}\right.$ \\
\hline Interval of call to first CPR (min) $)^{*}$ & $2(0-6)$ & $5(1-8)$ & $<0.001^{* *}$ & & $0.96(0.90-0.99)^{\prime \prime}$ \\
\hline
\end{tabular}


P310

Use of the laryngeal tube by paramedics during prehospital resuscitation: an observational study

G Papakitsos', T Papakitsou², A Kapsali'

'General Hospital Arta, Greece; 'eneral Hospital Messologi, Greece

Critical Care 2010, 14(Suppl 1):P310 (doi: 10.1186/cc8542)

Introduction In the current guidelines of the ERC, tracheal intubation has become less important for persons not trained and different supraglottic airway devices are recommended. The present investigation deals with the application of the laryngeal tube (LT) during prehospital resuscitation by paramedics.

Methods The study was observational during a period of 2 years (2007 to 2009). We registered all cardiac arrest situations in which the LT had been applied according to the ERC guidelines 2005. All participants had completed an obligatory course in emergency medicine, but had not been specifically trained in endotracheal intubation; they were therefore designated as unfamiliar in using the endotracheal tube to secure the airway. Primary outcome measures were placement time and successful placement. Study endpoint was the total 'no flow time', which is defined as the time without chest compression in the first period of cardiac arrest. Results During the defined period, 53 resuscitation attempts recorded on standardised data sheets were included. The LT was used in $87 \%$ of all cardiac arrest situations. Overall, the LT was successfully and significantly faster inserted (about 9.4 seconds) in more than $96 \%$ of all cases, on the first attempt. In $99 \%$ of all cases, no problems concerning ventilation of the patient were described. During the cardiac arrest simulation, establishing and performing first ventilation took an average of 12.5 seconds with the LT. Use of the LT during cardiac arrest significantly reduced the 'no flow time' about 87.6 seconds.

Conclusions As an alternative airway device recommended by the ERC, the LT may enable airway control rapidly and effectively. Additionally, by using the LT, a reduced 'no-flow-time' and a better outcome may be possible. LT may be a good alternative airway device for providing and maintaining a patent airway during resuscitation.

Reference

1. Wiese $\mathrm{CH}$, et al:: The use of the laryngeal tube disposable (LT-D) by paramedics during out-of-hospital resuscitation-an observational study concerning ERC guidelines 2005. Resuscitation 2009, 80:194-198.

P311

The i-gel supraglottic airway as an alternative in the preclinical emergency medical aid? A comparative study on a manikin

JTiesmeier, M Emmerich

Krankenhaus Bad Oeynhausen, Germany

Critical Care 2010, 14(Suppl 1):P311 (doi: 10.1186/cc8543)

Introduction Endotracheal intubation (ET) is the recognised gold standard for securing an airway in the realm of preclinical emergency medical aid. Supraglottic airway devices present a valid alternative in the case of an elevated incidence of difficult airway securing and intubation preconditions. Due to positive experiences with the $\mathrm{i}$-gel supraglottic airway, we - through a joint paramedic (PM) and doctor (DR) team - have compared its use under standardised conditions with the classic laryngeal mask (CLMA) and the laryngeal tube (LT) using an airway simulator.

Methods Seventy-one probands (40 PM and 31 DR) took part in this study. The airway simulator used was the Ambu ${ }^{\oplus}$ Airway Man. Timekeeping started with the laying aside of the anaesthesia bag after successful ventilation of the airway simulator and ended after having ensured sufficient ventilation. Participants used three different devices (i-gel size 4, CLMA size 3 and LT size 4) each five times. Additionally, an interval time was measured after successful ventilation with the i-gel. Without interruption, participants continued the experiment with two interval ventilations and conducted a secondary ET through the i-gel. Timekeeping ended in this case after the first ventilation.

Results Prior to the study $95 \%$ of PM had previous experience with the CLMA, $77.5 \%$ with the LT and $0 \%$ with the i-gel. Respective percentages for DR were $61.2 \%$ for the CLMA, 38.7\% for the LT and 32.2\% for the i-gel $(P<0.001$ respectively). Across the entire group of 71 study participants, users required an average of 16.6 seconds for the successful insertion of the CLMA, 15 seconds for the LT and 8.48 seconds for the i-gel. When comparing the PM with the DR, the PM required 16.5 seconds for the $C L M A$ (vs 16.9 seconds, $P=0.693$ ), 14.9 seconds for the LM (vs 16.8 seconds, $P=$ 0.08 ) and 7.8 seconds for the $i$-gel (vs 9.4 seconds, $P=0.001$ ). The total time needed for secondary ET was on average 25.8 seconds (PM 24.2 seconds, DR 27.9 seconds, $P<0.001)$. Error-free insertion at all five incidents was achieved by $73.3 \%$ of DR and by $46.1 \%$ of PM $(P<0.001)$.

Conclusions Sufficient ventilation was achieved $50 \%$ faster through the i-gel when compared with the CLMA and the LT. The correct usage of the i-gel can be learnt quickly. The i-gel allows the options of secondary ET.

\section{Reference}

1. Emmerich M: Use of the i-gel laryngeal mask for management of a difficult airway. Anaesthetist 2008, 57:779-781.

\section{P312}

Retention of skills during emergency airway management training: a 12-month follow-up

H Fischer', A Fast' ${ }^{1}$, E Kajcsa', M Fleck'1, R Greif ${ }^{2}$

'General Hospital Vienna, Austria; 'University Hospital Bern, Switzerland

Critical Care 2010, 14(Suppl 1):P312 (doi: 10.1186/cc8544)

Introduction Besides the gold standard endotracheal tube, supraglottic airway devices are alternatives for emergency airway management [1]. The goal of the study was to identify airway devices that provide successful ventilation, even 12 months after training in manikins.

Methods In 2008, 288 medical students were trained to use laryngeal mask airways (LMAs) Unique, ProSeal, Supreme, I-Gel and bag-mask ventilation (BM) on manikins (Ambu). Students successful on the first attempt ( $n=$ 190) for all devices in random order were included and tested 12 months later without further training. The insertion time to first successful ventilation, tidal volume (TV) and gastric inflation were assessed.

Results The time (in seconds) needed for the I-Gel and the Unique was significant longer in 2009 (BM $20088.2 \pm 4.7$ vs $20098.4 \pm 5.4, P=0.882$; I-Gel $200810.2 \pm 2.7$ vs $200911.7 \pm 4.9 ; P=0.008$; Supreme $200815 \pm 3.7$ vs $200915.5 \pm 4.6, P=0.147 ;$ Unique $200816.5 \pm 3.6$ vs $200918.2 \pm 5.5 ; P=$ $<0.001$; ProSeal $200817.7 \pm 4.9$ vs $200917.7 \pm 5.2, P=0.856)$. A higher rate of gastric inflation for BM and the I-Gel was seen in comparison of 2008 with 2009 (BM 10.5\% vs 18.6\%, $P=0.012$; I-Gel $0.5 \%$ vs 4.2\%, $P=0.039$; Supreme $0.5 \%$ vs $2.2 \%, P=0.375$; Unique $3.7 \%$ vs $5.8 \%, P=0.607$; ProSeal $4.7 \%$ vs $1.2 \%, P=0,18$ ). About $60 \%$ of the TVs were insufficient with little improvement for the Supreme and Proseal (Figure 1).

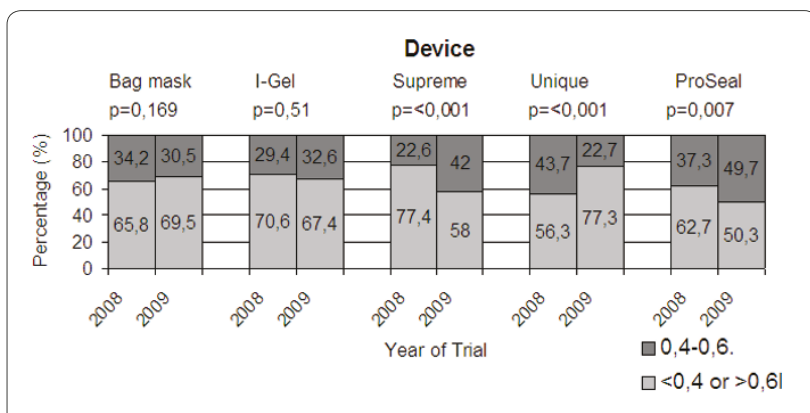

Figure 1 (abstract P312). Tidal volume (correct range 0.4 to $0.6 \mathrm{l}$ ).

Conclusions One year after training, time for successful ventilation for all devices was lower than 25 seconds. This is acceptable compared with the gold standard endotracheal tube, but due to the rising gastric inflation rate and high amount of insufficient tidal volume, shorter intervals of training maybe necessary.

\section{References}

1. Nolan JP, et al:: Resuscitation 2005, 67(Suppl 1):S54-S56. 
P313

Factors correlated with the advanced life support simulation performance of senior medical students

KH Park, YJ Kang

Jeju National University Hospital, Jeju-si, Republic of Korea

Critical Care 2010, 14(Suppl 1):P313 (doi: 10.1186/cc8545)

Introduction Even though medical education using simulation for medical students is increasing and suggests positive outcomes, little is known about factors associated with the performance of advanced life support simulation. We investigated the correlation between various factors and simulation performance using senior medical students.

Methods Fourth-year medical students were enrolled and divided into six groups. Each group underwent a 2-week emergency medicine training. This study was conducted consecutively with a 2-week break between each group from March to July, 2009. Every student had an advanced cardiac life support (ACLS) simulation performance test as a moderator, scored by two ACLS instructors certified by the American Heart Association. The following data were examined: basic life support (BLS) practice, personality characteristics survey, case presentation, one posttraining written test, and two plan tests for the Korean Medical Licensing Examination (KMLE). The statistical significance was defined as $P<0.05$. Results Forty-six students participated. No correlation was found between ACLS performance scores and personality type or medical knowledge as measured by the post-training written test and the two plan tests for the KMLE. However, scores of the BLS examination and the case presentation were moderately correlated with the ACLS performance scores: $r=0.390$, 0.402 , respectively $(P<0.01)$.

Conclusions The ACLS simulation performance was correlated with the BLS skills and the case presentation. This supports the idea that the ability to manage resuscitation is associated with BLS skills and the capability to analyze and coordinate patient management. This result provides guidance for education of medical students.

P314

Aging augments unwllingness to the initial three links in chains of survival

H Inaba, M Enami, T Yachida, Y Takei, O Oyama, K Ohta

Kanazawa University Graduate School of Medicine, Kanazawa, Japan

Critical Care 2010, 14(Suppl 1):P314 (doi: 10.1186/cc8546)

Introduction The purpose of the study was to clarify the influence of aging on attitudes toward the initial three links in chains of survival.

Methods We gave questionnaires to attendants of compulsory programs for basic life support (BLS) or driving technique at the beginning in authorized driving schools. The questionnaires included their backgrounds. We studied their willingness in four hypothetical scenarios related to the initial three links: early emergency call, cardiopulmonary resuscitation (CPR) under one's own initiative, telephone-assisted chest compression and use of AED. The respondents were divided into young (17 to 29 years, $n=6,122$ ), middle-aged (29 to 59 years, $n=827$ ), older person ( $>59$ years, $n=15,743)$ groups.

Results There were significant differences in gender, occupation, residential area and experience of BLS training, and knowledge of AED use between the three groups. The proportions of respondents who are willing to perform the desirable BLS actions were lowest in the older person group (Table 1). Multiple logistic regression analysis confirmed that aging is one of the independent factors relating to negative attitude in all the scenarios. Gender, occupation, resident area, experience of BLS training, and knowledge for AED use were other independent factors relating to negative attitude to some of the scenarios.

Conclusions The aged population is more negative to the chain of survival. More are willing to follow the telephone-assisted direction for chest compression. The BLS training should be modified for them to gain confidence and to be aware of the significance and benefit of early call.

\section{P315}

Prediction of the success of cardiac resuscitation: a pattern classification approach based on ECG spectral and temporal features

M Baronio', F Baronio², M Campi², G Amicucci', A Caré2 , A Sorgato³, G Perone ${ }^{4}$, A Candiani ${ }^{4}$

'Ospedale S. Orsola Brescia, Italy; ${ }^{2}$ Department of Information Engineering, University of Brescia, Italy; ${ }^{3}$ Division of Cardiology, Ospedale S. Orsola, Brescia, Italy; ${ }^{4}$ Spedali Civili, University of Brescia, Italy

Critical Care 2010, 14(Suppl 1):P315 (doi: 10.1186/cc8547)

Introduction The ECG tracings recorded during a ventricular fibrillation (VF) using an automated external defibrillator (AED) contain useful information predictive of shock outcome. The focus is on the VF waveforms' morphology. The amplitude and the spectral properties of VF may predict the likelihood of successful defibrillation [1-4]. In almost all previous studies, the amplitude or the spectral properties of the ECG tracings have been singularly used. However, these approaches have led to methods lacking sufficient predictive power.

Methods Five hundred patients with out-of-hospital cardiac arrest on arrival in an emergency room were examined. The rhythm was identified as VF and confirmed by two trained investigators. ECG data were stored in modules in digitized form over a period of 20 minutes and were analyzed retrospectively. ECG traces containing CPR artefacts were removed by digital filtering. Times of collapse, dispatch, scene arrival, CPR, and initial defibrillation were determined from dispatch records, recordings of arrest events, interviews with bystanders, and hospital records. The preshock VF waveform morphology was studied and different parameters of VF ECG signals were extracted. We then introduced a pattern classification machine that combines the amplitude and spectral features simultaneously.

Results The use of the pattern classification machine which combines amplitude and spectral features of VF ECG signals shows an improved predictive power as compared with other methods.

Conclusions This technique could help to determine which patients should receive shock first and which should receive a period of CPR prior to shock, thereby increasing the probability of survival. The potential impact of this research is high in the direction of generating a new methodology able to increase the probability of survival after a cardiac crisis.

\section{References}

1. Strohmenger H: Chest 1997, 111:584-589.

2. Sherman LD: Resuscitation 2006, 69:479-486.

3. Hamprecht F: Resuscitation 2001, 50:297-299.

4. Eftestol T: Resuscitation 2005, 67:55-61.

Table 1 (abstract P314). Difference among the three age groups in the proportion of respondents who are willing to perform the desirable BLS actions

\begin{tabular}{|c|c|c|c|c|}
\hline \multirow[b]{2}{*}{ Scenario } & \multicolumn{3}{|c|}{ Age group } & \multirow[b]{2}{*}{ Chi-square test $P$ value } \\
\hline & 17 to 29 years & 29 to 59 years & $>59$ years & \\
\hline CPR under their own initiative & $78.9 \%(4,810 / 6,094)$ & $71.9 \%(589 / 819)$ & $57.1 \%(8,186 / 14,346)$ & $<0.001$ \\
\hline Telephone-assisted chest compression & $94.6 \%(5,766 / 6,898)$ & $92.5 \%(757 / 818)$ & $84.6 \%(12,112 / 14,316)$ & $<0.001$ \\
\hline Early 119 call & $91.2 \%(5,556 / 6,092)$ & $91.2 \%(747 / 819)$ & $79.4 \%(12,130 / 15,270)$ & $<0.001$ \\
\hline Use of AED & $56.8 \%(3,386 / 5,963)$ & $48.4 \%(388 / 801)$ & $21.4 \%(2,832 / 13,285)$ & $<0.001$ \\
\hline
\end{tabular}


P316

Dispatcher-assisted telephone cardiopulmonary resuscitation using a French compression-only protocol: performance of volunteers with or without prior life support training

A Ghuysen', S Stipulante' ${ }^{2}$, D Colas' ${ }^{1}, V D^{\prime}$ Orio'

${ }^{1}$ CHU Liège - ULG, Liège, Belgium; ${ }^{2}$ CS 100 Liège, Belgium

Critical Care 2010, 14(Suppl 1):P316 (doi: 10.1186/cc8548)

Introduction Although early bystander-initiated cardiopulmonary resuscitation (CPR) is a key factor in survival improvement from out-ofhospital cardiac arrest, only one-third of victims actually receive CPR before arrival of emergency medical services. Dispatcher-assisted telephone CPR might improve the frequency and performance of bystanders'resuscitation efforts. Due to the recent interest in hands-only CPR protocol [1] and the lack of any French validated algorithm, we designed the present study in order to validate a new French protocol called ALERT (Algorithme Liègeois d'Encadrement à la Réanimation par Téléphone).

Methods We tested the efficacy of the ALERT protocol on CPR performances in a manikin model of cardiac arrest. Participants $(n=110)$ were divided into four groups. Previously untrained subjects, distributed into two sub-groups depending on whether they were beneficiating from telephone assistance (PUG group, $n=30$ ) or not (PUNG group, $n=30$ ), were compared with previously trained nurses students distributed in two other sub-groups: with (PTG group, $n=25$ ) or without (PTNG group, $n=25$ ) telephone assistance.

Results Global evaluation of CPR performances in untrained volunteers benefiting from telephone assistance revealed a significant improvement as compared with unguided untrained volunteers. Indeed, they approached CPR performance levels of previously trained nurse students with no dispatcher's guidance. Previously trained nurses with telephone assistance gained the best CPR scores, notably because of an improvement in the quality of airway management.

Conclusions Our results indicate that use of the ALERT algorithm by dispatchers may improve the CPR performance in both previously untrained volunteers and in volunteers with prior basic life support training.

Reference

1. Hallstrom AH, et al:: N Engl J Med 2000, 342:1546-1553.

\section{P317}

CPR team members' attitudes towards family presence during cardiopulmonary resuscitation

N Kianmehr, M Mofidi, H Rahmani, A Forouzan

Iran University of Medical Sciences, Tehran, Iran

Critical Care 2010, 14(Suppl 1):P317 (doi: 10.1186/cc8549)

Introduction The concerns about allowing family members to be present during resuscitation are a matter of debate in many countries. The purpose of this study was to determine the opinions of Iranian health-care providers about family-witnessed resuscitations.

Methods The study population consisted of CPR team members in four teaching hospitals. We developed a questionnaire, assessing their opinions, using 20 questions and also considering their comments.

Results Of the total 200 participants, 77\% oppose family-witnessed resuscitation. There was no significant difference among opponents about FWR based on sex, age, number of CPR exposures and the experience of respondents $(P>0.05)$. Emergency physicians were more likely advocates for FWR than other specialties $(P=0.004)$. All of the anesthesiologists opposed FWR. Most reasons for opposition were fear of psychological trauma to family members, interference with patient care, increased staff stress and violation with patient confidentiality.

Conclusions CPR team members in Iran do not believe in the presence of relatives during resuscitation. Informing healthcare providers on the benefit of witnessed resuscitation and public education of CPR would be a fundamental element for implementing a formal program.

\section{References}

1. Bae H, Lee S, Jang HY: The ethical attitude of emergency physicians toward resuscitation in Korea. J Emerg Med 2008, 34:485-490.

2. Maclean SL, Guzzetta CE, White C et al:. Family presence during cardiopulmonary resuscitation and invasive procedures: practices of critical care and emergency nurses. J Emerg Nurs 2003, 29:208-221.

3. Tucker TL: Family presence during resuscitation. Crit Care Nurs Clin North Am 2002, 14:177-185.
P318

Prehospital therapeutic hypothermia induced with cold infusions improves haemodynamic stability in nonshockable cardiac arrest patients

A Truhlar1, R Skulec ${ }^{2}$, J Seblova², L Zabka³, V Cerny

'University Hospital, Hradec Kralove, Czech Republic; ${ }^{2}$ Central Bohemian

Region EMS, Kladno, Czech Republic; ${ }^{3}$ Hradec Kralove Region EMS, Hradec Kralove, Czech Republic

Critical Care 2010, 14(Suppl 1):P318 (doi: 10.1186/cc8550)

Introduction Recent studies report an increase of asystole and pulseless electrical activity (PEA) as the first monitored cardiac arrest rhythms after arrival of the Emergency Medical Services [1]. The asystolic patients presumably undergo ischaemia for a longer time and may benefit from treatment reducing hypoxic brain injury. Therapeutic hypothermia (TH) has expanded into prehospital care to be initiated as soon as possible. Rapid cold crystalloid infusion is the most frequent method; however, severe haemodynamic instability is its contraindication. The aim of the study was to assess the adverse effects of prehospital volume expansion in patients with initial nonshockable rhythms when used in a setting with only restricted cardiovascular monitoring.

Methods Thenonshockable patients enrolled in a prospectivenonrandomized PRE-COOL trial (PRE-hospital COOLing in cardiac arrest patients) were rapidly administered cold normal saline (5 to $30 \mathrm{ml} / \mathrm{kg}$ ) intravenously and analysed for body temperature, haemodynamic variables (blood pressure, heart rate, shock index), incidence of circulatory complications (recurrence of cardiac arrest, pulmonary oedema), and outcome. Hypothermic patients (group A, $n$ = 19) were compared with matched historic controls (group B, $n=22$ ).

Results In treatment group A, 1,021 $\pm 526 \mathrm{ml}(13.2 \pm 6.3 \mathrm{ml} / \mathrm{kg})$ of $4^{\circ} \mathrm{C}$ cold normal saline was administered before hospital admission. Body temperature decreased by $1.32 \pm 0.71^{\circ} \mathrm{C}$ until arrival at hospital (calculated rate $1.08 \pm 0.44^{\circ} \mathrm{C} / 30$ minutes). The incidence of hypotension requiring vasopressors (noradrenaline or dopamine) significantly decreased in patients given cold intravenous fluids compared with control group B: $31.6 \%$ vs $63.6 \%(P=0.04)$. No patient developed pulmonary oedema within 24 hours. Recurrence of cardiac arrest was comparable in both groups (3/19 vs 3/22), shock index at hospital arrival was 0.92 vs $0.97(P=$ 0.78 ), and favorable outcome (cerebral performance category 1 or 2 ) $26.3 \%$ vs $13.6 \%(P=0.31)$.

Conclusions The early prehospital administration of cold infusions is safe and contributes to haemodynamic optimization in patients resuscitated from nonshockable rhythms. Incidence of hypotension requiring vasopressors was significantly reduced in the treated group. No pulmonary oedema was observed.

Acknowledgements Supported by grant IGA MH CZ NS10383-2/2009 and research project MZO 00179906.

Reference

1. Hallstrom A, et al: Resuscitation 2009, 80:975-976.

\section{P319}

Trans-nasal cooling during CPR: a single-center experience

FS Taccone, JL Vincent

Erasme Hospital, Brussels, Belgium

Critical Care 2010, 14(Suppl 1):P319 (doi: 10.1186/cc8551)

Introduction Trans-nasal cooling started during cardiopulmonary resuscitation (CPR) has shown to improve the return to spontaneous circulation (ROSC) and survival rate in an experimental prolonged cardiac arrest model. A multicenter randomized trial (PRINCE) has also suggested an improved neurological outcome in patients receiving trans-nasal cooling during CPR in the prehospital setting when compared with those treated by conventional hypothermia on hospital arrival, provided a delay between collapse and CPR of less than 10 minutes.

Methods Patients with witnessed cardiac arrest and a downtime less than 20 minutes were randomized to prehospital intra-arrest cooling versus standard ACLS care. Trans-nasal cooling (RhinoChill, BeneChill Inc., CA, USA) was initiated using a mixture of volatile coolant fluid with oxygen delivered into the nasopharynx for rapid evaporative heat transfer. Cooling was continued during CPR and, for patients who achieved ROSC, until initiation of systemic cooling at hospital. Resuscitation was continued for at least 30 minutes. All patients were then cooled at the hospital. 
Results Twenty-four patients were included, but one in the treatment group was excluded from the per-protocol analysis because of DNR orders. Patients randomized to treatment group $(n=9)$ or standard care $(n=14)$ had similar demographics, initial rhythm, time from collapse to CPR and ALS arrival. Median time from collapse to cooling initiation was 19 minutes. In total, 6/9 (66\%) treated and 6/14 (42\%) control patients achieved ROSC. Three patients (33\%) in the treatment group survived to hospital discharge with CPC 1 to 2, while only one (7\%) of the control group patients had good neurological outcome. No serious adverse events occurred in treated patients.

Conclusions Trans-nasal cooling seems to be safe and feasible in a prehospital setting. These single-center data confirm that trans-nasal cooling may improve the ROSC rate as well as good neurological outcome if started in patients with a short delay between collapse and CPR.

\section{P320}

Intra-arrest cooling using a novel intra-nasal cooling method for immediate induction of therapeutic hypothermia in Germany H Busch', H Fritz ${ }^{2}$, F Eichwede ${ }^{3}$, B Inderbitzen ${ }^{4}$, D Barbut ${ }^{4}$, T Schwab IIntensive Care Unit Heilmeyer, Freiburg, Germany; ${ }^{2}$ Krankenhaus MarthaMaria Halle-Dölau, Halle/Saale, Germany; ${ }^{3}$ Medizinisches Zentrum Aachen, Würselen, Germany; ${ }^{4 B e n e c h i l l, ~ S a n ~ D i e g o, ~ C A, ~ U S A ~}$

Critical Care 2010, 14(Suppl 1):P320 (doi: 10.1186/cc8552)

Introduction Recent investigations have demonstrated improved neurological outcome after therapeutic hypothermia in patients after successful resuscitation. The time course and duration to achieve target temperature may be an important factor to influence patient's outcome. To determine the safety and efficacy of intranasal cooling during ongoing resuscitation, for immediate induction of therapeutic hypothermia, the Pre-Resuscitation Intra-Nasal Cooling Effectiveness (PRINCE) study involved 200 patients in Europe, using a non-invasive nasal catheter that sprays evaporating coolant liquid into the nasal cavity. Here we demonstrate data from all German participating sites.

Methods All patients who were deemed eligible for advance cardiac life support (ACLS) were included as long as the arrest was witnessed and cardiopulmonary resuscitation (CPR) was initiated within 20 minutes of collapse. Patients were randomized to treatment or control groups. The trial was designed to determine the safety and effectiveness of early cooling initiated at the site of arrest. Survival and time to target temperature were documented.

Results Data are presented as the mean \pm SD or median (interquartile range $(25,75 \%))$. Mean age was $67.8 \pm 14$ years in the intervention group and $65.4 \pm 13.9$ years in the control group. On average, cooling therapy was started in $33 \pm 12$ minutes in the RhinoChill ${ }^{\mathrm{TM}}$ group and $170 \pm 97$ minutes in the control group. Temperatures at hospital admission were significant lower in the RhinoChill ${ }^{\mathrm{TM}}$ group. Time to target tympanic temperature, reflecting brain temperature, were significant faster in the RhinoChill ${ }^{\mathrm{TM}}$ group $(211 \pm 124$ minutes vs $424 \pm 217$ minutes; $P<0.05)$. Adverse events occurred in 12 patients. None was related to the cooling therapy. In the intervention group five patients (20\%) survived and three patients (12\%) had a CPC of 1 to 2 . In the control group only four patients (12.5\%) survived and one patient (3.1\%) had a CPC of 1 to 2.

Conclusions Using the intranasal cooling method, cooling was much faster and earlier in treated patients. Neurologically intact survival and discharge rates were higher in treated patients. Transnasal cooling for the induction of therapeutic hypothermia during prehospital resuscitation is feasible and highly effective in lowering brain temperature rapidly. The method offers the possibility for immediate introduction and realization of mild hypothermia in the field.

P321

Therapeutic hypothermia after cardiac arrest: implementation in UK intensive care units

A Binks', R Murphy', A Padkin², J Nolan²

'Bristol Royal Infirmary, Bristol, UK; '2Royal United Hospital, Bath, UK

Critical Care 2010, 14(Suppl 1):P321 (doi: 10.1186/cc8553)

Introduction In 2003 the International Liaison Committee on Resuscitation (ILCOR) published guidelines recommending all patients admitted

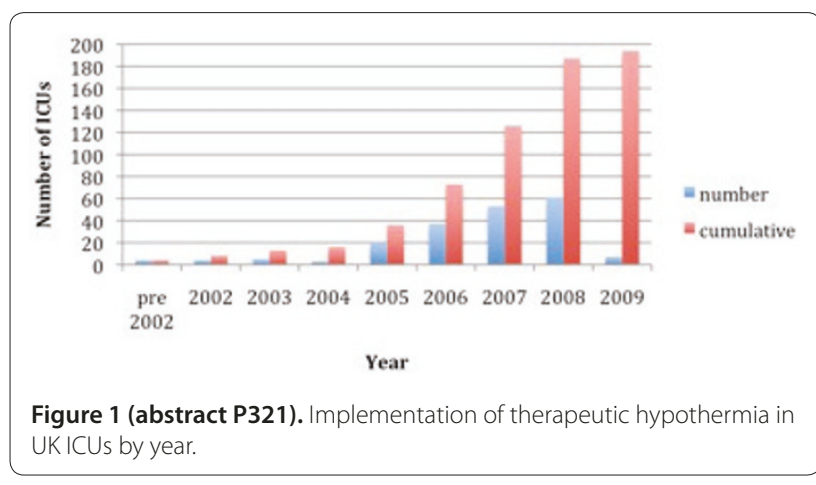

comatose to hospital following an out-of-hospital cardiac arrest (OHCA) with an initial rhythm of ventricular fibrillation should be treated with therapeutic hypothermia for a period of 12 to 24 hours [1]. We aimed to determine how many units are using hypothermia as part of their postcardiac arrest management and when each of the ICUs implemented the therapy. Other objectives were to determine what cooling techniques are being used, the target temperature and the duration of cooling used.

Methods The 248 UK ICUs listed in the Directory of Critical Care Services were contacted by telephone by one of the authors. The duty ICU consultant or nurse in charge was asked a standardised set of questions. Results We obtained responses from 244 of 248 (98.3\%) of ICUs. Currently, 209 (85.6\%) ICUs are using hypothermia as part of post-cardiac arrest management. Since 2003, there has been an increase annually in the number of units using hypothermia, with the majority of units starting in 2007 or 2008 (Figure 1). Of the units who use hypothermia as part of their post-cardiac arrest management, $99 \%$ are using it to treat patients after OHCA from shockable rhythms, $60.6 \%$ are using it to treat patients after OHCA from nonshockable rhythms, and $63.5 \%$ are using it after in-hospital cardiac arrest. Cooling is initiated with intravenous cold fluid in $71 \%$. The commonest technique to maintain hypothermia is to use ice; used by $55 \%$ of units. Most ICUs (89.9\%) aim for a temperature of 32 to $34^{\circ} \mathrm{C}$ and the duration of hypothermia is 24 hours in 141 ICUs (67.5\%).

Conclusions Generalised implementation of this clinical guideline in the UK has taken about 5 years but now the majority of ICUs are using therapeutic hypothermia as part of post-cardiac arrest management.

\section{Reference}

1. Nolan J: Circulation 2003, 108:118-121.

\section{P322}

Hospital-wide therapeutic hypothermia protocol significantly reduces the time to induce hypothermia but does not improve outcomes

P Hayden, AA James

Medway NHS Foundation Trust, Gillingham, UK

Critical Care 2010, 14(Suppl 1):P322 (doi: 10.1186/cc8554)

Introduction Therapeutic hypothermia (TH) is a reliable, evidence-based modality to improve neurological outcome following out-of-hospital cardiac arrest. Often TH is induced in the ICU and as a result the time from cardiac arrest (CA) to induction of $\mathrm{TH}\left(\mathrm{TH}_{\mathrm{st}}\right)$ and achieving target temperature $\left(\mathrm{CA}_{\mathrm{ta}}\right)$ is delayed. Preliminary data have shown that delaying TH may worsen outcome [1]. We sought to investigate whether a hospitalwide approach to $\mathrm{TH}$ after CA would reduce delays and result in improved outcomes.

Methods We conducted a retrospective analysis of all TH interventions from 2007 to 2008. Following this we implemented a hospital-wide approach to TH and re-evaluated in 2008 to 2009. The hospital-wide approach included an educational programme, TH guidelines in the resuscitation room in the ED, and a cooling pack stored in the ICU which could be taken immediately to the post-CA patient.

Results CA-ROSC and CA-Hosp delays were similar between groups. The hospital-wide approach significantly reduced the time to initiate $\mathrm{TH}$ and time to achieve target temperature (see Table 1). The small sample size may have prohibited demonstration of outcome differences. 
Table 1 (abstract P322). Differences between groups pre and post the hospital-wide approach

\begin{tabular}{lccc}
\hline & 2007 to 2008 & 2008 to 2009 & Pvalue \\
\hline$n$ & 16 & 26 & \\
CA-ROSC & 28.5 minutes & 24.1 minutes & \\
CA-Hosp & 59.1 minutes & 50.1 minutes & \\
CA-TH & 306.2 minutes & 133.2 minutes & $<0.0001$ \\
CA-TH & 527.3 minutes & 252.7 minutes & 0.005 \\
HospSurv & 7 & 13 & 0.69 \\
\hline
\end{tabular}

Conclusions A hospital-wide approach to $\mathrm{TH}$ following cardiac arrest significantly reduces delays to achieve the target temperature, although this did not improve outcomes at our institution. Initiation of prehospital TH may be an ideal solution to further reduce this delay, and ongoing trials may demonstrate improved outcomes.

\section{Reference}

1. Nozari A, et al.: Critical time window for intra-arrest cooling in a dog model. Circulation 2006, 113:2690-2696.

\section{P323}

Induced hypothermia after cardiac arrest for 12 hours: single-centre experience

R Sykora ${ }^{1}, \mathrm{R}$ Janda ${ }^{2}$

'Charles University, Medical School and Teaching Hospital, Plzen, Czech

Republic; ${ }^{2}$ Hospital of Karlovy Vary, Czech Republic

Critical Care 2010, 14(Suppl 1):P323 (doi: 10.1186/cc8555)

Introduction Induced hypothermia $(\mathrm{IH})$ for 12 to 24 hours is a standard part of post-resuscitation care. We present a cohort of patients with outof-hospital or in-hospital cardiac arrest (OHCA, IHCA) from our ICU who underwent 12 to 14 hours of $\mathrm{H}$. Moreover we aimed at neurological outcome in a subgroup of patients with initial ventricular fibrillation (VF) and ST-elevation acute myocardial infarction (STEMI) undergoing coronary intervention ( $\mathrm{PCl})$.

Methods We retrospectively evaluated patients with nontraumatic cardiac arrest admitted to our ICU from 2006 to 2009. A standardised therapeutic protocol including $\mathrm{IH}$ was implemented. $\mathrm{IH}\left(32\right.$ to $34^{\circ} \mathrm{C}$ ) was started within 30 minutes after restoration of spontaneous circulation (ROSC), before $\mathrm{PCl}$, if performed. $\mathrm{H}$ was maintained for 12 to 14 hours, than passive rewarming followed. Endovascular cooling device was not used. Cerebral performance category (CPC) at hospital discharge was evaluated as a main outcome.

Results Total number of patients was $88(\mathrm{OHCA} n=63$; IHCA $n=25)$, mean age 57 years, 65\% men. ICU mortality was 32\% and hospital mortality $53 \%$. Initial ECG rhythm was VF in 59\%, asystole in 32\% and pulseless electrical activity in 9\%. Coronary angiography was performed in 59\% and intervention in $41 \%$ of all cases. The required target temperature was achieved in $76 \%$. Complications were ventilator-associated pneumonia in 6\%, tracheobronchitis in 15\% and gastrointestinal bleeding in 2\%, no severe bradyarhythmias or iontogram dysbalance was recorded. In a group of 23 patients with VF/STEMI, PCl after initiation of $1 \mathrm{H}$ was performed. The required target temperature was achieved in $91 \%$. The ICU survival rate was $87 \%$ and survival to discharge from hospital was 78\%. Good neurological outcome (CPC 1 or 2) in this group at hospital discharge was in $83 \%(n=15)$.

Conclusions Induced hypothermia has to be started as soon as possible after ROSC. Cooling of patients after cardiac arrest should continue during all therapeutic interventions. Twelve hours of induced hypothermia after cardiac arrest could offer comparable neurological outcomes to 24 hours, especially in the subgroup of patients with VF/STEMI.

Acknowledgements Supported by a research project MSM 0021620819.
P324

Survival following adult cardiac arrest in intensive care units: a 5-year retrospective analysis

S Bagshaw, P Brindley, D Kutsogiannis

University of Alberta Hospital, Edmonton, Canada

Critical Care 2010, 14(Suppl 1):P324 (doi: 10.1186/cc8556)

Introduction This study aims to provide survival data for adult patients following attempted cardiopulmonary resuscitation (CPR) in critical care units. We hope objective data can facilitate dialogue and decision-making. Methods We retrospectively reviewed CPR records from 1 January 12000 to 30 April 2005 from all critical care units of the tertiary care hospitals of Edmonton, Canada. Full inpatient medical records were reviewed for further details.

Results Of 529 arrests, 59.4\% had return of spontaneous circulation, $29.5 \%$ survived to critical care unit discharge, $26.3 \%$ survived to hospital discharge, $20.5 \%$ survived to 3 months, and $19.9 \%$ to 6 months. Pulseless electrical activity (PEA) represented 29.7\%, ventricular tachycardia (VT) 20.8\%, asystole (ASY) 15.7\%, bradycardia 15.5\%, ventricular fibrillation (VF) $13.4 \%$, supraventricular tachycardia $2.8 \%$, and respiratory arrest $2.1 \%$. A total $36.7 \%$ of arrests occurred between 08:01 and 16:00 hours, 33.0\% occurred between 00:01 and 08:00 hours and 30.3\% between 16:01 and 24:00 hours. There was no significant association between arrest time and survival. There was a significant association between longer CPR duration and increased mortality $(P<0.001)$. GSICU cardiac arrest patients had statistically higher critical care unit mortality than CCU or CVICU patients (75.5\% vs $70.0 \%$ vs $45.7 \% ; P=0.002$ ). ASY/PEA were significantly more common in GSICUs, whereas VF and VT were more common in CVICU $(P=$ 0.001). Mortality was significantly higher following PEA/ASY regardless of location. Multivariate analysis showed no significant association between survival and age, gender or arrest time. In contrast, survival was significantly lower with increased APACHE II score (O.R 1.07, $P<0.001)$, prolonged CPR (O.R.1.03, $P<0.01$ ), and ASY/PEA (O.R. 5.44, $P<0.001$ ). No patient with an APACHE $\| \geq 30$ survived to hospital discharge following PEA.ASY, and no patient, regardless of arrest type, survived to hospital discharge with an APACHE II $>45$.

Conclusions Initial survival following in-hospital cardiac arrest is higher than from noncritical care units, and therefore should be considered differently. Survival was higher still from cardiac ICUs compared with other critical care units. Admission APACHE II, type of arrest, and resuscitation duration provide useful prognosticators, especially in combination. In contrast, age, gender, and arrest time do not. Despite encouraging early survival, less than one-quarter survived to hospital discharge, and less than one-in-six were alive at 1 year.

\section{P325}

Effects of bed height and time on the quality of chest compressions during cardiopulmonary resuscitation

A Lewinsohn, PB Sherren, DS Wijayatilake

Queens Hospital, London, UK

Critical Care 2010, 14(Suppl 1):P325 (doi: 10.1186/cc8557)

Introduction The 2005 International Liaison Committee on Resuscitation gave a detailed update on best practice for CPR with a discussion around appropriate patient and rescuer position, based largely on expert opinion [1]. The objectives of this study were: to demonstrate a difference in chest compression effectiveness with bed height, and provide a suggestion for an optimal and achievable bed height for effective chest compressions; and to demonstrate fatigue during chest compressions, and provide a suggestion for an upper time limit for effective chest compressions.

Methods Exclusion criterion for this trial; no previous basic life support training in the past 4 years, or refused consent to participate. A modified Laerdal manikin was connected to a Dragor ventilator (to measure intrathoracic pressures generated). The manikin was placed on a hospital trolley, and CPR was performed by candidates at three different bed heights: mid thigh; anterior superior iliac spine; and xiphisternal area. Chest compressions were continuous and asynchronous with ventilation, and allowed to continue for 30 seconds before recordings were taken.

Results One hundred and one subjects took part. The differences in intrathoracic pressures generated at different bed heights were compared using ANOVA variance testing for multiple groups, and were statistically 


\begin{tabular}{|l|c|c|}
\hline \multicolumn{3}{|c|}{} \\
\hline Bed Height & Mean Pmax at 30 seconds & Standard Deviation \\
\hline 1) Xiphisternal area & $15.36^{\circ}$ & 2.52 \\
\hline 2) ASIS & $17.69^{*}$ & 2.91 \\
\hline 3) Mid Thigh & $18.89^{*}$ & 2.80 \\
\hline
\end{tabular}

Figure 1 (abstract P325). Effects of bed height position on intrathoracic pressure generated after 30 seconds of CPR. $n=101$. *Statistically significant $(P<0.05)$.

\begin{tabular}{|c|c|c|}
\hline \multirow{3}{*}{$\begin{array}{c}\text { Pmax Mean } \\
\text { Standard Deviation }\end{array}$} & 30 seconds CPR & 2 minutes CPR \\
\hline & $17.52^{\circ}$ & $14.5^{\circ}$ \\
\hline & 3.64 & 2.89 \\
\hline
\end{tabular}

Figure 2 (abstract P325). Mean intrathoracic pressures generated during CPR at 30 seconds as compared with 2 minutes. *Statistically significant $(P<0.05)$.

significant with $P<0.01$ (Figure 1). We also found that the effectiveness of CPR decreased $17 \%$ over a 2 -minute period (Figure 2).

Conclusions The most effective bed height position, allowing rescuers to achieve the highest intrathoracic pressures during CPR, was with the patient chest in line with the rescuer's mid-thigh. The rescuer performing CPR should change every 2 minutes.

\section{Reference}

1. Resuscitation 2005, 67:187-201.

\section{P326}

New results about early therapeutic hypothermia in sudden cardiac death and following return of spontaneous circulation

FR Righetti, AL Luzzani, EP Polati

'Ospedale Policlinico Universitario, Verona, Italy

Critical Care 2010, 14(Suppl 1):P326 (doi: 10.1186/cc8558)

Introduction Therapeutic hypothermia is defined as controlled cooling of body temperature for therapeutic purposes. At present, the use of mildmoderate hypothermia $\left(32\right.$ to $34^{\circ} \mathrm{C}$ ) in patients with neurological damage is increasing in the ICU. In this regard, recent studies suggest that it is able to improve the neurological outcome in patients with anoxic cerebral damage following sudden cardiac death (SCA) [1]. The aim of this study is to assess the role of therapeutic hypothermia on neurological outcome in patients who experienced SCA with ensuing return of spontaneous circulation (ROSC).

Methods Ninety adult patients, aged between 18 and 85, referred to our ICU after SCA due to cardiac disease with following ROSC were randomly allocated to the following treatment groups: patients in group 1 were treated immediately after admission with therapeutic hypothermia plus standard treatment, patients in group 2 received only standard treatment. All patients at entry presented with GCS 3. Neurological outcome was assessed on discharge and after 6 months, by means of GOS scale $(0=$ dead, 1 = vegetative, 2 = severely disabled, $3=$ moderately disabled, $4=$ good recovery). We consider scores 0 to 1 as unfavourable outcome, scores from 2 to 4 as favourable outcome. To compare the two groups we used the Mann-Whitney $U$ test of for continuous variables, the chi-square test for qualitative variables.

Results Patients in group 1 (45 patients) and in group 2 (45 patients) resulted statistically comparable for sex $(P=0.14)$ and age $(P=0.68)$ and presentation ECG rhythm $(P=0.42)$ and APACHE II score $(P=0.34)$. At discharge from the ICU, $66.6 \%$ of patients in group 1 presented favourable neurological outcome (GOS 2 to 4 ) versus 35\% of patients in group 2. At 6 months, $70 \%$ of patients in group 1 presented favourable neurological outcome (GOS 2 to 4 ) versus $38.3 \%$ of patients in group 2. The mortality was $16.6 \%$ in group 1 versus $36.6 \%$ in group $2(P=0.003)$. The patients who improved their GOS values were $64 \%$ in group 1 and $18 \%$ in group $2(P<0.005)$.

Conclusions Our study demonstrated that early treatment with therapeutic hypothermia in the patient who had SCA improves neurological outcome. It is independent of the rhythm of presentation. Besides, we reached statistical significance.

\section{Reference}

1. Polderman $\mathrm{KH}$, et al:: Therapeutic hypothermia and controlled normotermia in the intensive care unit: practical considerations, side effects, and cooling methods. Crit Care Med 2009, 37:1101-1120.

P327

Infection rates among cooled patients post cardiac arrest

A Binks, MJ Thomas

Bristol Royal Infirmary, Bristol, UK

Critical Care 2010, 14(Suppl 1):P327 (doi: 10.1186/cc8559)

Introduction We conducted a retrospective study looking at infection rates in patients treated with therapeutic hypothermia (TH) following cardiac arrest who were admitted to the ICU of the Bristol Royal infirmary since May 2007. TH is recommended in all patients presenting with persistent coma following cardiac arrest. One complication of TH is the risk of infection. Hypothermia suppresses the immune system by inhibiting the release of proinflammatory cytokines and by suppressing the chemotactic migration of leukocytes and phagocytosis [1]. In patients with traumatic brain injury, $\mathrm{TH}$ for more than 48 hours is associated with a higher risk of infection, but not if the period of hypothermia is less than 24 hours [2]. In patients following cardiac arrest, infection is common, reported in up to 73\% of patients [3].

Methods Data were collected retrospectively, and information about ICU length of stay, whether they had a protected catheter (PC) specimen taken, whether they had any positive cultures and whether they were treated with antibiotics was extracted. Data for ICU length of stay was also obtained. Infections were defined as $>10^{5}$ colony-forming units (cfu) grown from either PC specimens or from peripheral blood cultures.

Results We identified 82 patients treated with TH post cardiac arrest. Nineteen (29\%) had proven infection either on PC or on peripheral blood cultures. PC specimens were taken in 21 (25\%) patients. Of the 21 patients who had PC specimens taken, 16 (76\%) had proven infection. We also found that an increase in ICU length of stay was associated with increased infection rates, $44 \%$ in patients with a length of stay of greater than 3 days and $55 \%$ in patients with a length of stay of greater than 4 days.

Conclusions We have shown, in our ICU, that of patients who were treated with $\mathrm{TH}$ following cardiac arrest, 29\% had a proven infection. On PC sampling, there was a much higher rate of infection with $76 \%$ of patients with positive cultures. This suggests that the risk of infection in patients treated with therapeutic hypothermia post cardiac arrest is higher than that for patients who are cooled post traumatic brain injury.

\section{References}

1. Polderman KH: Intensive Care Med 2004, 30:556-575

2. Polderman KH: Crit Care Med 2009, 37:S186-S202.

3. Tsai MS, et al.: Intensive Care Med 2005, 31:621-626.

\section{P328}

Strategies to implement a therapeutic hypothermia program

S Blosser, N Cavarocchi, S Ettinger, IC Gilchrist, R Reichwein, S Bressler, J Burkholder, E Sarsfield, K Zagari

Penn State Hershey Medical Center MCH075, Hershey, PA, USA

Critical Care 2010, 14(Suppl 1):P328 (doi: 10.1186/cc8560)

Introduction In this presentation we will review the process used for developing a therapeutic hypothermia program and our outcomes during the first year. Despite the fact that therapeutic hypothermia has been shown to improve survival and neurologic outcome after cardiac arrest [1], it is underused [2] and has been only slowly introduced in many institutions. One of the reasons that it is not more widely used may be the difficulty of implementation of a program.

Methods A multidisciplinary team was assembled in 2007 to explore the opportunity for therapeutic hypothermia in patients after cardiac arrest. Pertinent literature was reviewed and a guideline and electronic orders were drafted. The team investigated all available options for cooling and chose a method that was non-invasive and did not require a large financial investment by the institution. Nurses were sent to an international program to learn about hypothermia and become advocates 
and educators. Education was done for caregivers in the prehospital setting, emergency department, cardiac catheterization laboratory, and adult ICUs. Advertising to community and other hospitals was done by newspaper, radio, and television. The team continued to meet regularly to assess and modify the program based on experience.

Results Prior to the start of this program, therapeutic hypothermia was essentially not utilized at the institution. Twenty-one patients were cooled after cardiac arrest and resuscitation at our institution in the first year (2008), with few missed opportunities. Fifteen (71\%) survived, and 12 (57\%) had good neurologic outcome. (Post-cardiac arrest survival rates without hypothermia in the literature range from less than 5\% to 35\% [1].) Challenges included altering historic practice patterns, integrating sectors of the healthcare delivery system that had historically drifted apart, and interacting with extra-institutional regulations governing prehospital care. Conclusions The therapeutic hypothermia program at our institution is successful in improving patient outcome after cardiac arrest. The elements that contributed to a successful program included: teamwork; multidisciplinary nature of the team; promoting awareness of the program; buy-in by nurses who championed the program in the $\mathrm{ICU}_{\text {; }}$ positive patient outcomes; and low financial investment for the institution. References

1. Bernard SA, et al:: N Engl J Med 2002, 346:557-563.

2. Brigham BL, et al:: Resuscitation 2009. [Epub ahead of print]

\section{P329}

A comparison of core and tympanic temperature measurement in the critically ill

B Sanderson, L Lim, K Lei, J Smith, L Camporota, R Beale

Guys and St Thomas'NHS Foundation Trust, London, UK

Critical Care 2010, 14(Suppl 1):P329 (doi: 10.1186/cc8561)

Introduction Measurement of body temperature using the tympanic method (TTM) represents a standard non-invasive method which best approximates core temperature (CTM). However, published evidence increasingly casts doubt on the agreement between TTM and CTM. The aim of this study was to assess the agreement of these two methods of temperature measurement in critically ill patients.

Methods We recruited 20 consecutive critically ill patients, who required indwelling thermistor-tipped arterial catheters for haemodynamic monitoring. For each patient we simultaneously collected the core temperature, obtained from the arterial catheter, and tympanic temperatures in the left and right ears, in the supine position. Tympanic temperature was recorded using the ear setting (TTMe) and the core setting tympanic temperature (TTMC) in accordance with the manufacturer's instructions (Genius ${ }^{\text {TM }} 2$ Kendall, Tyco Healthcare, MA, USA). Local ethics committee approval was waived and the study was conducted as a service improvement audit. Bland-Altman analysis was used to measure agreement between the two set of measurements.

Results From the 20 patients we made 102 paired measurements Comparison of the TTM in the right and left ears resulted in high variability in the recorded temperature with a percentage error of $34.4 \%$. In view of this observed large variability, the mean TTM between the two ears was used for subsequent comparison with core temperature. Comparison of core measurements against tympanic measurements using ear settings (TTMe) showed a small positive mean bias (limits of agreement (LOA); percentage error) of $0.3^{\circ} \mathrm{C}\left(-0.4\right.$ to $\left.1.1^{\circ} \mathrm{C}, 2.4 \%\right)$. However, when the CTM and TTM were compared using the core temperature setting of the thermometer, the bias $(\mathrm{LOA})$ increased to $-0.97^{\circ} \mathrm{C}\left(-1.6\right.$ to $\left.-0.4^{\circ} \mathrm{C}\right)$, creating a larger bias than the unadjusted TTM.

Conclusions Tympanic temperature measurements showed a large random variability compared with core temperatures and suggest bilateral averaged tympanic measurements may be necessary. In our series, using the core setting increased error and increased the bias between the two methods. Our findings raise the possibility that the diagnosis of fever and the need for further investigation may be affected by the choice of temperature measurement site and device setting used.
P330

Can we predict neurological prognoses with computed tomography just after resuscitation?

T Mochizuki', N Otani', O Takahashi², S Ishimatsu'

'St Luke's International Hospital, Tokyo, Japan;'2St Luke's Life Science Institute,

Tokyo, Japan

Critical Care 2010, 14(Suppl 1):P330 (doi: 10.1186/cc8562)

Introduction Noncontrast computed tomography (CT) is often used to evaluate a primary catastrophic brain injury after cardiac arrest. On day 3 after resuscitation, cerebral damage presents as swelling on CT; however, there is no strong evidence associated with how prognosis is predicted on CT performed just after resuscitation. We assessed whether the ratio (in Hounsfield units $(\mathrm{HU})$ ) of grey matter (GM) to white matter (WM) on CT performed just after resuscitation can be used as a predictor of patient outcome.

Methods We utilized the electronic medical record to examine admission to the ICU after resuscitation from out-of-hospital cardiac arrest. Brain CTs performed just after resuscitation were re-evaluated. Patients were excluded if they had head trauma, cerebral vessel disease and were 16 years of age or younger. Clinical outcome was assessed at the time of discharge using the Pittsburgh cerebral performance category (CPC). On noncontrast CTs, HU were measured as GM in the regions of bilateral caudate head, putamen, globus pallidaus and cortical layer of the precentral sulcus, and as WM in the regions of the periventricular WM. We defined good neurological prognosis as CPC 1 to 2 and an unfavorable prognosis as CPC 3 to 5. Each ratio of average GM to WM was calculated, and they were compared with a good outcome and an unfavorable outcome.

Results In 1,057 episodes of out-of-hospital cardiac arrest, 296 were resuscitated and admitted to our ICU; 64 were excluded, and the final study sample consisted of 232 patients. CPC 1 to 5 at hospital discharge was $16 \%, 6 \%, 6 \%, 12 \%$ and $60 \%$. The mean time from arrival to perform brain CT was 66 (25 to 330) minutes. The HU of caudate head, putamen and globus pallidaus were much higher in good outcome than in unfavorable outcome ( $P<0.001, t$ test). The HU of cortical layer of the precentral sulcus and periventricular WM were not significantly different. The caudate head/WM ratio and globus pallidaus/WM ratio were much higher in good outcome $(P<0.01, t$ test). Using ROC analysis, we determined that a caudate head/WM ratio $>1.21$ or a globus pallidaus/WM ratio $>1.17$ was $90 \%$ predictive of a good outcome in this study.

Conclusions The caudate head/WM ratio and globus pallidaus/WM ratio by brain $C T$ just after resuscitation are useful to predict the postresuscitation neurological prognosis.

\section{Reference}

1. Wijdicks EFM, et al:: Neurology 2006, 67:203-210.

\section{P331}

Unintended consequences of technology improvement

W McGee, PM Mailloux

Baystate Medical Center, Springfield, MA, USA

Critical Care 2010, 14(Suppl 1):P331 (doi: 10.1186/cc8563)

Introduction Technological improvements in ventilator design may confound the diagnosis of brain death. We describe a series of patients clinically perceived to be breathing spontaneously even though they satisfied the criteria for brain death. The misdiagnosis of cerebral function in these patients delayed the diagnosis of brain death and subsequent organ donation. Resource utilization, patient/family suffering, and staff morale are all negatively impacted when this occurs.

Methods The ventilator triggering mode was recorded in all patients. Patients were identified by the authors with a high likelihood of brain death and receiving pressure support ventilation. Patients without cranial nerve function underwent formal apnea testing.

Results Seven patients were identified with cessation of cranial nerve function, but thought to have spontaneous breathing activity. All patients were on pressure support ventilation with flow triggering. Formal apnea testing was performed (Table 1).

Conclusions Improvement in triggering ventilation may confound the diagnosis of brain death. Considering the unmet need for transplantation and the negative impact of not recognizing death on families, staff, resource utilization and both patients and potential recipients of organs mandates a simple solution to eliminate these problems. We propose that 
Table 1 (abstract P331)

\begin{tabular}{lccc}
\hline Patient & Injury & Apnea & Outcome \\
\hline 42 years old, male & Gunshot & Yes & Donor \\
27 years old, male & TBI & Yes & DCD \\
73 years old, male & TBI & Yes & Donor \\
52 years old, female & ICH & Yes & No \\
66 years old, male & Anoxia & Yes & No \\
56 years old, female & Anoxia & Yes & Donor \\
43 years old, male & Gunshot & Yes & Donor \\
\hline
\end{tabular}

$\mathrm{DCD}$, donor after cardiac death; $\mathrm{TBI}$, trauma brain injury; ICH, intracranial hemorrhage.

in all patients being evaluated for brain death once cranial function has ceased an apnea test should be carried out promptly with the patient disconnected from the ventilator.

\section{P332}

\section{Bispectral Index Scale alterations in brain death patients}

V Grosomanidis', B Fyntanidou', K Kotzampassi², EThomaidou', V Ourailoglou', G Thoma', C Skourtis ${ }^{1}$

'Department of Anaesthesiology and ${ }^{2}$ Department of Surgery, University of

Thessaloniki Medical School, Thessaloniki, Greece

Critical Care 2010, 14(Suppl 1):P332 (doi: 10.1186/cc8564)

Introduction Brain death (BD) is defined as the total and irreversible loss of brain function, including the brain stem. According to current Greek guidelines, low BD diagnosis is determined only by clinical criteria, thus electroencephalogram (EEG), cerebral angiography or transcranial Doppler are not considered mandatory for BD confirmation. The Bispectral Index Scale (BIS) is a multifactorial parameter derived from the EEG, which allows monitoring of the hypnotic component of anaesthesia. Furthermore, it has been reported that there is a good correlation between BIS and neurological status in unsedated coma patients. In addition, it has been recently suggested that BIS is associated with the Glasgow Coma Score (GCS) and could serve as a good predictor tool for the outcome after head trauma. Moreover it has been reported that BIS can be used in the early detection of $\mathrm{BD}$. The aim of the present study was to record the BIS alterations in BD patients.

Methods Thirty brain injury patients (24 males and six females, with a mean age of $48.4 \pm 12.3$ years) who were considered as being BD according to the standard clinical criteria were included in the study. Eleven of them became organ donors. Continuous BIS monitoring (BIS; Aspect Medical Systems Inc., Natick, MA, USA) was performed for 24 to 32 hours (mean 26.7 hours).

Results All patients were haemodynamically stable, normothermic and without any electrolytical disturbance. Ten patients showed BIS 0 continuously. In the remaining 20 patients (66.6\%) BIS fluctuations over 30 continuing for more than 30 minutes were recorded. These values then dropped to the BIS 0 level after different time periods. These alterations were compatible with EEG activity and not associated with external stimulus such as surgical or other manipulations.

Conclusions BIS monitoring is a continuous, simple method, easily interpreted. Although it has been suggested to be a good predictor tool of early detection of $\mathrm{BD}$, there is a possibility of increased BIS values in BD patients. The anaesthesiologist should be aware of these situations and in no case should the BIS findings decrease the validity of standard clinical criteria of BD determination.

\section{P333}

Prognosis in patients presenting with nontraumatic coma

S Forsberg, J Höjer, U Ludwigs

Karolinska Institute, Stockholm, Sweden

Critical Care 2010, 14(Suppl 1):P333 (doi: 10.1186/cc8565)

Introduction Studies of patients presenting with coma are limited and little is known about the prognosis of these cases [1-3]. The aim of this study was to investigate the acute and long-term prognosis after an episode of nontraumatic coma.

Methods Adults admitted consecutively to an emergency department in Stockholm, Sweden between February 2003 and May 2005 with a Glasgow Coma Scale (GCS) score of 10 or below were enrolled prospectively. All available data were used to explore the cause of the impaired consciousness on admission. Patients surviving hospitalization were followed up for 2 years regarding survival.

Results The final study population of 865 patients had the following eight different coma etiologies: poisoning $(n=329)$, stroke $(n=213)$, epilepsy ( $n=113)$, circulatory failure $(n=60)$, infection $(n=56)$, metabolic disorder $(n=44)$, respiratory insufficiency $(n=33)$, and intracranial malignancy $(n=$ 17). The hospital mortality rate among the 865 patients was $26.5 \%$, varying from $0.9 \%$ for epilepsy to $71.7 \%$ for circulatory failure. The accumulated total 2-year mortality rate was $43.0 \%$, varying from $13.7 \%$ for poisoning to $88.2 \%$ for malignancy. The level of consciousness on admission also influenced the prognosis: a GCS score of 3 to 6 .

Conclusions The prognosis in patients presenting with nontraumatic coma is serious and depends largely both on the level of consciousness on admission and on the etiology of the coma. Adding the suspected coma etiology to the routine coma grading of these emergencies may more accurately predict their prognosis.

\section{References}

1. Martikainen K, Seppä K, Viita P, Rajala S, Laippala P, Keränen T: Transient loss of consciousness as reason for admission to primary health care emergency room. Scand J Prim Health Care 2003, 21:61-64.

2. Kanich W, Brady WJ, Huff JS, Perron AD, Holstege C, Lindbeck G, Carter CT: Altered mental status: evaluation and etiology in the ED. Am J Emerg Med 2002, 20:613-617.

3. Levy DE, Bates D, Caronna JJ, et al.: Prognosis in nontraumatic coma. Ann Intern Med 1981, 94:293-301.

P334

Dynamic changes in cerebral oxygenation by two methods during cardiac surgery and postoperative cognitive decline

Y Kakihana, N Kiyonaga, TYasuda, T Imabayashi, T Ohryoji, M Nakahara,

N Okayama, Y Kanmura, T Kikuchi, T Yonemitsu

Division of Intensive Care Medicine, Kagoshima University Hospital,

Kagoshima, Japan

Critical Care 2010, 14(Suppl 1):P334 (doi: 10.1186/cc8566)

Introduction Recently, near-infrared time-resolved spectroscopy (TRS), which is quite effective in quantitative monitoring tissue oxygenation, because it offers the actual measurement of photon migration in the tissues, and the photon mean path length is easily obtained from the center of gravity of the temporal profile, has been developed. In this study, we investigated whether the changes in the cerebral oxygen saturation ( $\mathrm{T}-\mathrm{SO}_{2}$ ) obtained with the TRS and the jugular venous oxygen saturation $\left(\mathrm{SjVO}_{2}\right)$ predicted cognitive decline after cardiac surgery.

Methods With institutional approval and informed consent, we studied 10 patients (68.7 \pm 6.1 years) undergoing cardiac surgery under cardiopulmonary bypass (CPB). T-SO, was continuously monitored using a TRS-10 (Hamamatsu Photonics KK, Hamamatsu, Japan). For measurement of $\mathrm{SjVO}_{2}$, a $5.5 \mathrm{Fr}$ oximetry catheter was inserted by retrograde cannulation of the right internal jugular vein. The values of $\mathrm{T}_{-} \mathrm{SO}_{2}$ and $\mathrm{SjvO}_{2}$, were compared with each point: before CPB, 5 minutes after the onset of CPB, before aorta clamp, after aorta clamp, rewarming, aorta declamp, end of rewarming, and end of CPB. The cognitive decline was evaluated by Mini Mental State Examination before and 7 days after the operation. The statistical analysis was performed by repeated-measures ANOVA followed by Fisher's PLSD. $P<0.05$ was considered statistically significant.

Results Four of 10 cases showed postoperative cognitive decline. The mean values of $\mathrm{T}_{-} \mathrm{SO}_{2}$ and $\mathrm{SjvO}_{2}$ during operation in patients without postoperative cognitive decline $(n=6)$ were $63.9 \pm 4.6 \%$ and $60.5 \pm 9.7 \%$, respectively, and there were no statistical significances between these values. However, the mean values of $\mathrm{T}_{-} \mathrm{SO}_{2}$ and $\mathrm{SjvO}_{2}$ during operation in patients with postoperative cognitive decline $(n=4)$ were $62.8 \pm 5.6 \%$ $\left(\mathrm{T}-\mathrm{SO}_{2}\right)$ and $70.4 \pm 14.9 \%\left(\mathrm{SjVO}_{2}\right)$, and showed significant differences between two values $(P=0.0024)$, and at the rewarming period, the values of $\mathrm{SjvO} 2$ was significantly higher than those of $\mathrm{TSO}_{2}(87.9 \pm 6.3 \%$ vs $65.0 \pm$ $5.3 \%, P=0.0014)$. 
Conclusions Our results demonstrate a lack of agreement between $\mathrm{SjvO}_{2}$ and $\mathrm{T}-\mathrm{SO}_{2}$ for monitoring cerebral oxygenation at the occurrence of postoperative cognitive decline. Therefore, we conclude that the two methods are not interchangeable, and the TRS instrument may be useful for obtaining accurate values for cerebral oxygen saturation under CPB.

P335

Acetazolamide-induced cerebrovascular reactivity is impaired in sepsis-associated encephalopathy

S Szatmari, TVégh, C Molnár, C Antek, B Fülesdi

University of Debrecen, Hungary

Critical Care 2010, 14(Suppl 1):P335 (doi: 10.1186/cc8567)

Introduction The pathophysiology of sepsis-associated encephalopathy (SAE) is not entirely clear. One of the possible underlying mechanisms is the alteration of the cerebral microvascular function induced by the systemic inflammation. The aim of the present work was to test whether cerebral vasomotor reactivity is impaired in patients with SAE.

Methods Patients fulfilling the criteria of clinical sepsis and showing disturbance of consciousness of any severity were included. Nonseptic persons without previous diseases affecting cerebral vasoreactivity served as controls. Transcranial Doppler blood flow velocities were measured at rest and at 5, 10, 15 and 20 minutes after intravenous administration of $15 \mathrm{mg} / \mathrm{kg}$ BW acetazolamide. The time course of the acetazolamide effect on cerebral blood flow velocity (cerebrovascular reactivity, CVR) and the maximal vasodilatory effect of acetazolemide (cerebrovascular reserve capacity, (RC) were compared among the groups.

Results Fourteen patients with SAE and 20 controls were included. Absolute blood flow velocities after administration of the vasodilator drug were higher among control subjects than in SAE. Assessment of the time course of the vasomotor reaction showed that patients with SAE reacted slower to the vasodilatory stimulus than control persons. When assessing the maximal vasodilatory ability of the cerebral arterioles to acetazolamide during vasomotor testing, we found that patients with SAE reacted to a lesser extent to the drug than did control subjects (CRC SAE: $46.2 \pm 15.9 \%$, CRC controls: $29.4 \pm 15.8 \%, P<0.01$ ).

Conclusions We conclude that cerebrovascular reactivity is impaired in patients with SAE.

\section{References}

1. Wilson JX, Young GB: Sepsis-associated encephalopathy: evolving concepts. Can J Neurol Sci 2003, 30:98-105.

2. Matta BF, Stow PJ: Sepsis-induced vasoparalysis does not involve the cerebral vasculature: indirect evidence from autoregulation and carbon dioxide reactivity studies. Br J Anaesth 1996, 76:790-794.

3. Settakis $\mathrm{G}$, Molnár C, Kerényi L, et al:: Acetazolamide as a vasodilatory stimulus in cerebrovascular diseases and in conditions affecting the cerebral vasculature. Eur J Neurol 2003, 10:609-620.

P336

Epidemiological analysis of patients with cerebral aneurysms submitted to embolization at São José do Avaí Hospital

S Kiffer Macedo, CM Primo de Siqueira, S Boechat Primo de

Siquiera, L Amaral de Oliveira, L Carvalho Dias, N Raissa da Matta,

E Abreu Reis Vargas de Almeida, L Bonani Brito, A D'Ávila Carvalho, N Ferreira Bastos

São José do Avaí Hospital, Itaperuna, Brazil

Critical Care 2010, 14(Suppl 1):P336 (doi: 10.1186/cc8568)

Introduction Cerebral aneurysms affect between 1 and $5 \%$ of the adult population and are responsible for significant rates of morbidity and mortality. The treatment of intracranial aneurysms evolved substantially since the introduction of endovascular neurosurgery by Guglielmi detachable coils (GDC) in the 1990s. The ablation overtook a clipping as the initial method in many centers, including Brazil, because of the safety and feasibility of this method.

Methods This cohort retrospective study analyses clinical and epidemiological variables. It was conducted from the database of patients submitted to ablation in the neurosurgery department of São José do Avaí Hospital in the period of December 2006 to November 2009.
Results We studied 1,504 patients submitted to ablation. There 1,120 were females $(74.46 \%)$ and 384 males. The average age was 52 years. Hunt-Hess scale prevalence: $1-67.88 \%, 2-18.62 \%, 3-8.19,4-4.92 \%, 5-2.32 \%$, and Fisher: $1-62.58 \%, 2-7.91 \%, 3-17.08 \%, 4-7.58 \%$. The main risk factors involved in the cerebral vascular accident were: systemic arterial hypertension $40.4 \%(n=608)$ and smoking 30.8\% $(n=463)$. The arteries more involved were: posterior communicant $=381(25.33 \%)$, median cerebral $=296(19.61 \%)$ and anterior communicant $=254(16.87 \%)$. A total $72.67 \%$ of patients presented only one aneurysm $(n=1,093)$ and $27.33 \%$ two or more aneurysms $(n=411)$. We observed an occurrence of 1,217 (80.91\%) ruptured aneurysms and 287 incidental. The sizes of the aneurysms were: $46.30 \%$ standard aneurysms (between 5 and $25 \mathrm{~mm}$ ), $47.95 \%$ smaller than $5 \mathrm{~mm}$ and $5.75 \%$ giant. The aneurysms were narrow neck in $37.78 \%$ of the cases $(n=1,082)$, and large in $14.34 \%(n=412)$. Among the 218 events that occurred, there were coils into the vascular lumen in 96 cases (6.38\%), bleeding in 58 (3.85\%) and others. The materials used were: 334 balloons and 136 stents. Angiographic vasospasm occurred in 178 patients.

Conclusions There was a predominance of females for the occurrence of cerebral vascular aneurysmatic accident. The average age was 52 years. Systemic hypertension and smoking showed strong association with the rupture of intracranial aneurysms. The arteries of the previous segment were those that had higher incidence of aneurysms. More than onehalf of the patients did not have complications during the procedure. Embolization of cerebral aneurysms was revealed to be a low lethality method.

\section{P337}

\section{Frequency/prevalence analysis of risk factors on aneurysmal} subarachnoid hemorrhage

S Kiffer Macedo, CM Primo de Siqueira, S Boechat Primo de Siqueira, R Mendoza Nuss, L Carvalho Dias, A Cedro Souza, G Vieira Rangel Pereira Hospital São José do Avaí, Itaperuna, Brazil

Critical Care 2010, 14(Suppl 1):P337 (doi: 10.1186/cc8569)

Introduction Subarachnoid hemorrhage $(\mathrm{SAH})$ is a catastrophic clinical event in which $2 / 3$ of spontaneous $\mathrm{SAH}$ are characterized by rupture and bleeding of cerebral aneurysm.

Methods After institutional approval and informed consent, this prospective observational study took place from April 2008 to November

\begin{tabular}{|c|c|c|}
\hline FACTORS & Frequency-o (n) & (ac9590) \\
\hline Average Age & 55,3 & 0.00064 \\
\hline \multicolumn{3}{|l|}{ Skin Color } \\
\hline White & $46,2 \%(54)$ & $36,9-55,6$ \\
\hline Blsck: & $24,8 \%(29)$ & $17,3-33,6$ \\
\hline Brown & $29,1 \%(34)$ & $21,0 \cdot 38,2$ \\
\hline \multicolumn{3}{|l|}{ Gender } \\
\hline Femala & $73,4 \%(94)$ & $64,9 \cdot 80,9$ \\
\hline \multirow{2}{*}{\multicolumn{3}{|c|}{ Dyslipidemia }} \\
\hline & & \\
\hline Yas & $10,9 \%(14)$ & $6,1-17,7$ \\
\hline No & $89,1 \%(114)$ & $82,3-93,6$ \\
\hline \multicolumn{3}{|l|}{ Arterial Hypertension } \\
\hline Yes & $72,7 \%(93)$ & $64,1-80,5$ \\
\hline No & $27,3 \%(35)$ & $19,8-35,9$ \\
\hline \multicolumn{3}{|l|}{ Dinbetes Mellitas } \\
\hline Yes & $14,8 \%(19)$ & $9,2-22,2$ \\
\hline No & $85.2 \%(109)$ & $77,8-90,8$ \\
\hline \multicolumn{3}{|l|}{ Alcoholise } \\
\hline Yos & $12,6 \%(16)$ & $7,4-19,7$ \\
\hline No & $87,4 \%(111)$ & $87,4-92,6$ \\
\hline \multicolumn{3}{|l|}{ Smoking Habit } \\
\hline Yas & $44,5 \%(57)$ & $35,7-53,6$ \\
\hline No & $55,5 \%(71)$ & $46,4 \cdot 64,3$ \\
\hline \multicolumn{3}{|l|}{ Sedentary } \\
\hline Yes & $25,8 \%(33)$ & $18,5-34,3$ \\
\hline No & $74,2 \%(95)$ & $65,6-81,5$ \\
\hline \multicolumn{3}{|l|}{ Obesity } \\
\hline Yes & $11,7 \%(15)$ & $6,7-18,6$ \\
\hline & $88,3 \%(113)$ & $81,4-93,3$ \\
\hline \multicolumn{3}{|l|}{$\begin{array}{l}\text { Oral Contracep tive } \\
\text { Mlethod * }\end{array}$} \\
\hline $\begin{array}{l}\text { Metsod } \\
\text { Yet }\end{array}$ & $5,3 \%(5)$ & $1,7 \cdot 12,0$ \\
\hline No & $94,7 \%(89)$ & $88,0-98,3$ \\
\hline
\end{tabular}

Figure 1 (abstract P337). 
2009, involved all adult patients with spontaneous SAH admitted to the $I C U$, evaluating the frequency/prevalence of some factors such as: gender, age, skin color, arterial hypertension (AH), smoking habit, diabetes mellitus (DM), alcoholism, dyslipidemia, sedentary and use of oral contraceptive method.

Results We observed a total of 128 patients, average age of 55.3 years, and obtained as the main results of frequency with respective confidence intervals (Cl): gender, female $73.4 \%(n=94)(\mathrm{Cl} 64.9$ to 80.9$)$, male $26.6 \%$ $(n=34)$ (Cl 19.1 to 35.1$)$; skin color, white $46.2 \%(n=54)$ (Cl 36.9 to 55.6$)$, black 24.8\% $(n=29)(\mathrm{Cl} 17.3$ to 33.6), brown $29.1 \%(n=34)(\mathrm{Cl} 21.0$ to 38.2$)$; $\mathrm{AH}$, yes $72.7 \%(n=93)(\mathrm{Cl} 64.1$ to 80.5$)$, no $27.3 \%(n=35)(\mathrm{Cl} 19.8$ to 35.9$)$; smoking habit, yes $44.5 \%(n=57)(\mathrm{Cl} 35.7$ to 53.6$)$, no $55.5 \%(n=71)$ (C 46.4 to 64.3$)$; diabetes mellitus, yes $14.8 \%(n=19)(\mathrm{Cl} 9.2$ to 22.2$)$, no $85.2 \%$ ( $n=109)$ (Cl 77.8 to 90.8); alcoholism, yes $12.6 \%(n=16)(\mathrm{Cl} 7.4$ to 19.7$)$ no $87.4 \%(n=112)$ (Cl 87.4 to 92.6); dyslipidemia, yes 10.9\% $(n=14)(\mathrm{Cl} 6.1$ to 17.7$)$, no $89.1 \%(n=114)$ (Cl 82.3 to 93.9$)$; sedentary, yes $25.8 \%(n=33)$ (Cl 18.5 to 34.3), no 74.2\% ( $n=95)(\mathrm{Cl} 65.6$ to 81.5$)$; obesity, yes $11.7 \%(n=$ 15) (Cl 6.7 to 18.6), no 88.3\% ( $n=113$ ) (Cl 81.4 to 93.3); oral contraceptive method (analysis obtained only for female gender population), yes $5.3 \%$ $(n=5)(\mathrm{Cl} 1.7$ to 12.0), no 94.7\% ( $n=89)(\mathrm{Cl} 88.0$ to 98.3). See Figure 1.

Conclusions We can show that among all RF, female gender, $\mathrm{AH}$ and smoking habit had greatest prevalence index; the factor skin color had a wide distribution from its variants; other RF as DM, alcoholism, dyslipidemia, sedentary, and use of oral contraceptives had not obtained significant prevalence on patients with aneurysmal SAH.

Reference

1. Krishna V, Kim DH: J Neurosurg 2007, 107:522-529.

P338

Monitoring regional brain oxygen saturation in patients with clipped aneurysms of the anterior circle of Willis

R Gendek', K Kusza²

${ }^{1} 10$ th Military Clinical Hospital, Bydgoszcz, Poland; ${ }^{2}$ University of Nicolaus Copernicus, Collegium Medicum of Ludwik Rydygier, Bydgoszcz, Poland Critical Care 2010, 14(Suppl 1):P338 (doi: 10.1186/cc8570)

Introduction The objective of this work was to assess the usefulness of monitoring regional brain oxygen saturation $\left(\mathrm{rSO}_{2}\right)$ in the identification of intraoperative episodes of cerebral ischemia during the surgical clipping of brain aneurysms. Moreover it aimed to verify whether this kind of monitoring affects the incidence of postoperative neurological deficits.

Methods We monitored 50 patients undergoing cerebral aneurysm clipping with the use of Somanetics INVOS Cerebral Oximeter 4100. The alarm threshold of $\mathrm{rSO}_{2}$ was defined as a minimum of $20 \%$ drop from baseline, triggering intervention aimed at increasing $\mathrm{rSO}_{2}$ values.

Results Eleven cases of postoperative deterioration of neurological status were noted, of which six were reversible. Additional intraoperative events, such as numerous surgical clip displacements, temporary clipping, aneurysm ruptures, and trapping occurred in 16 cases. On the operated side, a rapid, lasting increase of $\mathrm{rSO}_{2}$ values was noted in nearly one-half of the cases; implying an artifact caused by the neurosurgical procedure. On the nonoperated side, seven cases of $\mathrm{rSO}_{2}$ values dipping below the alarm threshold were noted. In all cases intervention led to an increase and normalization of $\mathrm{rSO}_{2}$ values in a relatively short time. Since the values registered in the frontal lobe on the nonoperated side were considered representative of the entire brain, the balance of cerebral oxygen supply and consumption was seen as intraoperatively preserved. The comparison between two groups of patients, with and without neurological deficits developed in the postoperative period, revealed no disparities in cerebral oximetry values on the nonoperated side, nor in the range of other monitored physiological parameters. In 10 out of 16 cases involving additional intraoperative events, deterioration of neurological status was noted (62.5\%), while in the remaining cases only one such occurrence was registered (2.95\%).

Conclusions Monitoring regional brain oxygen saturation on the operated side is a method hampered by a large percentage of false results, and thus is a monitoring tool of little prognostic value. Assuming that the frontal lobe on the nonoperated side is representative of the entire brain with regard to $\mathrm{rSO}_{2}$ measures, the maintenance of oxygen supply and demand balance does not safeguard the brain against ischemic lesions in the supply area of the operated artery.
P339

Early prognosis value of the first 24 hours mean plasma S100 $\beta$ protein level after subarachnoid aneurysmal hemorrhage

D Frasca, A Proust, C Millet, B Debaene, O Mimoz, C Dahyot-Fizelier University Hospital, Poitiers, France

Critical Care 2010, 14(Suppl 1):P339 (doi: 10.1186/cc8571)

Introduction Plasma S100 $\beta$ protein (PS100 $\beta$ ) was described as a biological marker of white substance damage and brain ischemia. Studies of new neuroprotective approaches in patients with traumatic brain injury and subarachnoid aneurysmal hemorrhage would benefit from the development of such markers. The aim of this study was to evaluate the first 24 hours mean plasma PS100 $\beta$ for predicting outcome at ICU discharge after subarachnoid aneurysmal hemorrhage (SAH).

Methods From August 2006 to August 2008, 100 patients (46 men and 54 women; mean age $52 \pm 11$ years) were admitted for SAH and treated by surgical clipping or coiling within 2 days after admission. SAPS II, World Federation of Neurological Surgeons (WFNS), Fisher and Glasgow Outcome Scale (GOS) were scored at ICU discharge. Plasma S100ß concentrations were assayed at admission (hours 0,6 and 24) and daily up to day 12 (routine procedure). Statistical analysis relied on the mean first 24 hours PS100 $\beta$ and the main outcome criterion was the GOS score at discharge dichotomized as poor (GOS 1 to 3) or good (GOS 4 to 5).

Results Poor outcome was associated with high mean first 24 hours PS100 $\beta$, high initial WFNS and Fisher scores in univariate analysis $(P<0.0001)$. The best cut-off for the mean first 24 hours $5100 \beta$ value was $0.15 \mu \mathrm{g} / \mathrm{l}$ (specificity $0.60,95 \%$ confidence interval $(\mathrm{Cl}) 0.52$ to 0.67 ; sensitivity $0.85,95 \% \mathrm{Cl} 0.79$ to 0.89 ; area under the curve $0.83,95 \% \mathrm{Cl} 0.69$ to 0.92 ; Figure 1).

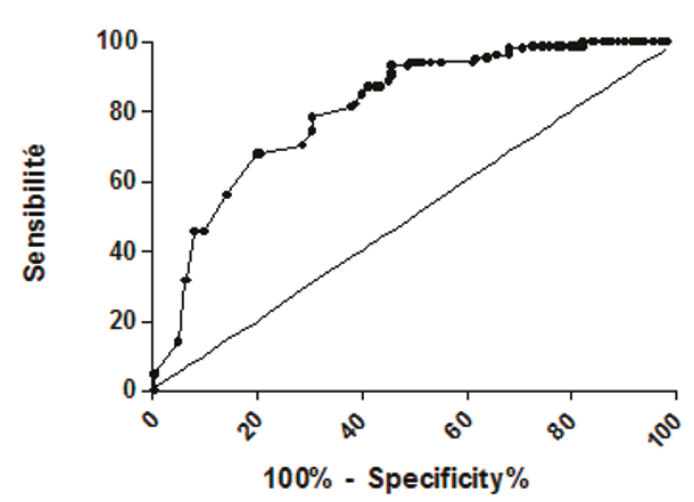

Figure 1 (abstract P339). ROC curve of the first 24 hours mean PS100 $\beta$ value and outcome at ICU discharge.

Conclusions The mean PS100 $\beta$ value assessed during the first 24 hours is a prognostic tool complementary to initial clinical evaluation in $\mathrm{SAH}$ patients.

\section{P340}

Altered aortic stiffness associated with stress cardiomyopathy in patients with aneurysmal subarachnoid haemorrhage

I Papanikolaou', M Fragou², P Zotos², D Karakitsos², A Patrianakos ${ }^{3}$,

E Zakinthinos ${ }^{1}$, A Karabinis ${ }^{2}$

University Hospital of Larissa, Greece; ${ }^{2}$ General Hospital of Athens, Greece;

${ }^{3}$ University Hospital of Heraklion, Crete, Heraklion, Greece

Critical Care 2010, 14(Suppl 1):P340 (doi: 10.1186/cc8572)

Introduction We investigated whether possible alterations in aortic stiffness were associated with stress cardiomyopathy (SC) in patients with subarachnoid haemorrhage (SAH).

Methods Thirty-seven patients with SAH (aged 42.65 \pm 13.07, 23 males) were included in the study. The severity of SAH was estimated by HuntHess and Fisher scales, respectively. The left ventricular ejection fraction (LVEF) was measured by the Simpson's biplane method of disks; aortic 
stiffness was assessed by sonographic measurements of the carotid to femoral pulse wave velocity (PWV). The above sonographic measurements along with BNP measurements were obtained upon admission and repeated measurements were performed $20 \pm 2$ days following the acute phase of SAH. All patients were mechanically ventilated under the same conditions during all measurements.

Results Patients with severe SAH showed depressed LV function and increased aortic stiffness. Both Hunt-Hess and Fisher scales were associated with $\operatorname{LVEF}(r=-0.460, P=0.004$ and $r=-0.512 ; P=0.001$, respectively) and PWV values $(r=0.359, P=0.029$ and $r=0.363 ; P=0.027$, respectively). On admission, 14 patients (38\%) manifested SC with increased BNP values as compared with those without cardiac alterations (841.43 \pm 561.04 vs $145.04 \pm 106.02 \mathrm{pg} / \mathrm{ml}, P<0.001)$. These subjects exhibited increased PWV as compared with patients with normal LV function $(12.79 \pm 2.02$ vs 9.63 $\pm 2.89 \mathrm{~m} / \mathrm{second}, P=0.001)$. PWV correlated to BNP values $(r=0.489, P=$ 0.002). Follow-up measurements revealed a significant increase in LVEF and decrease in PWV as compared with baseline measurements (62.64 \pm 9.18 vs $57.94 \pm 12.55 \%, P=0.005$ and $9.37 \pm 2.07$ vs $10.71 \pm 2.96 \mathrm{~m} / \mathrm{second}$, $P=0.001$, respectively). In this series, brain death was documented clinically in seven cases. An ongoing study including a larger number of patients with $\mathrm{SAH}$ analyzes the prognostic value of echocardiographic and BNP measurements.

Conclusions Alterations of the heart-vessel coupling may be due to catecholamine-induced mechanisms. Stress cardiomyopathy associated with altered aortic stiffness during the acute phase of SAH may be transient phenomena.

\section{P341}

Effect of packed red blood cell transfusion on cerebral oxygenation and metabolism after subarachnoid hemorrhage

P Kurtz, R Helbok, J Claassen, J Schmidt, L Fernadez, R Stuart, ES Connoly, K Lee, SA Mayer, N Badjatia

Columbia University Medical Center, New York, USA

Critical Care 2010, 14(Suppl 1):P341 (doi: 10.1186/cc8573)

Introduction Anemia is frequently encountered in critically ill patients and adversely affects cerebral oxygen delivery and metabolic function. However, there is limited evidence to support the use of packed red blood cell (PRBC) transfusion to optimize brain homeostasis after subarachnoid hemorrhage. The objective of this study was to investigate the effect of PRBC transfusion on cerebral oxygenation and metabolism in patients with subarachnoid hemorrhage.

Methods Prospective observational study in a neurological ICU of a university hospital. Nineteen PRBC transfusions were studied in 15 consecutive patients with subarachnoid hemorrhage that underwent multimodality monitoring (intracranial pressure, brain tissue oxygen and cerebral microdialysis). Data were collected at baseline and during 12 hours after transfusion. The relationship between $\mathrm{Hb}$ change and lactate/pyruvate ratio (LPR) and brain tissue oxygen $\left(\mathrm{PbtO}_{2}\right)$ was tested in univariate and multivariable analyses.

Results PRBC transfusion was administered on median post-bleed day 8. The average $\mathrm{Hb}$ concentration at baseline was $8.1 \mathrm{~g} / \mathrm{dl}$ and increased by $2.2 \mathrm{~g} / \mathrm{dl}$ after transfusion. After transfusion initiation, $\mathrm{PbtO}_{2}$ increased between hours 2 and 4 and this rise was maintained until hour 10. LPR did not change during the 12 hours of monitoring. Multivariable analysis demonstrated that, after adjusting for peripheral oxygen saturation, cerebral perfusion pressure and LPR, the change in $\mathrm{Hb}$ concentration was independently and positively associated with change in $\mathrm{PbtO}_{2}$ (adjusted b estimate $=1.39$ (95\% confidence interval 0.09 to 2.69$) ; P=0.036$ ). No relationship between change in LPR and change in $\mathrm{Hb}$ was found.

Conclusions Transfusion of PRBC results in $\mathrm{PbtO}_{2}$ improvement without a clear effect on cerebral metabolism after subarachnoid hemorrhage.

P342

Comparison of surgical or endovascular treated aneurismal SAH

patients with a special emphasis on cerebral vasospasm

I Akinci, C Kamar, E Guresti, A Sencer, S Sencer, T Kiris

Istanbul Medical Faculty, Istanbul, Turkey

Critical Care 2010, 14(Suppl 1):P342 (doi: 10.1186/cc8574)

Introduction The aim of this study was to evaluate the incidence of delayed cerebral ischemia (DCI) and outcome in SAH patients who were admitted for surgical or endovascular procedures to treat cerebral aneurysm.

Methods One hundred consecutive patients were evaluated according to their demographic data, severity of illness on admission, incidence of $\mathrm{DCl}$, outcome and choice of treatment. The $t$ test, Mann-Whitney $\mathrm{U}$, and chi-square tests were used for statistical evaluation.

Results Patients' demographic data were as shown in Table 1. Clinical vasospasm diagnosis was made by the existence of three signs which are headache, neurological deficit, and worsening of Glasgow Coma Score (GCS). Due to this evaluation 28 of the patients demonstrated DCI. While 14 of them were in the clipping group (23.3\%), the other 14 were in the coiling group (35\%). But $\mathrm{HHH}$ therapy and CSF drainage with lumber puncture was started more liberally, in 57 and 35 patients respectively, due to at least two of the following findings or clinician's decision: headache, agitation, elevated leukocyte level (without infection), new motor deficit and worsening in GCS. The severity of cases on admission in the clipping and coiling groups were similar for both. Although mortality and morbidity rates were higher in the coiling group there was no statistical difference in both groups (Table 2).

\section{Table 1 (abstract P342). Demographic data}

\begin{tabular}{lc}
\hline Age & $51 \pm 11.2$ \\
WFNS & $1.3 \pm 0.6$ \\
Fisher & $2.0 \pm 1.0$ \\
H\&H & $1.8 \pm 0.9$ \\
Rankin & $1.3 \pm 1.5$ \\
GOS & $4.4 \pm 1.1$ \\
Hospital stay & $16.1 \pm 12.0$ \\
HHH therapy & $58 \%$ \\
Clipping & $60 \%$ \\
Coillin & $38 \%$ \\
Clip + Coil & $2 \%$ \\
Mortality & $5 \%$
\end{tabular}

GOS, Glasgow outcome score; H\&H, Hunt and Hess.
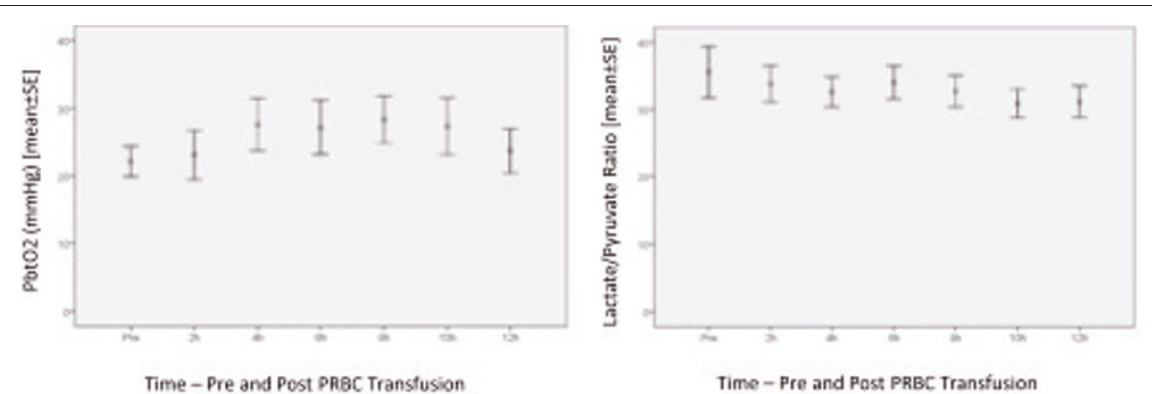

Figure 1 (abstract P341). 
Table 2 (abstract P342)

\begin{tabular}{lccc}
\hline & Clipping & Coiling & $P$ \\
\hline Age & $50.3 \pm 11.6$ & $52.6 \pm 10.2$ & NS \\
WFNS & $1.2 \pm 0.6$ & $1.3 \pm 0.7$ & NS \\
Fisher & $2.0 \pm 1.1$ & $2.0 \pm 0.8$ & NS \\
H\&H & $1.8 \pm 0.4$ & $1.7 \pm 1.0$ & NS \\
Rankin & $1.1 \pm 1.2$ & $1.7 \pm 1.8$ & NS \\
GOS & $4.6 \pm 0.9$ & $4.3 \pm 1.2$ & NS \\
Hospital stay & $17.1 \pm 12.2$ & $15.1 \pm 11.7$ & NS \\
DCI & $23.3 \%$ & $35 \%$ & 0.25 \\
Mortality & $3.4 \%$ & $7.5 \%$ & 0.38 \\
\hline
\end{tabular}

GOS, Glasgow outcome score; H\&H, Hunt and Hess.

Conclusions This study demonstrated that although mortality, morbidity rates and $\mathrm{DCl}$ incidence were lower in the surgical group, there was no statistical significance.

\section{P343}

Prediction of cerebral vasospasm in patients with aneurysmal subarachnoid hemorrhage using jugular bulb oximetry monitoring: preliminary results

R Souissi', A Boubakker', H Souissi², A Abdelrazek', M Badri', S Bziouech', H Jemel ${ }^{1}$

'National Institute of Neurology, La Rabta, Tunisia; 'La Rabta Hospital, La Rabta, Tunisia

Critical Care 2010, 14(Suppl 1):P343 (doi: 10.1186/cc8575)

Introduction Cerebral vasospasm (CV) is the leading cause of death and disability in patients with subarachnoid hemorrhage (SAH). We suppose that clinical vasospasm disturbances could be detected by the increase in cerebral oxygen extractions $\left(\mathrm{AVDO}_{2}\right)$, so in this study we tried to predict clinical CV appearances using jugular bulb oximetry monitoring.

Methods Open prospective clinical study, from July 2009 to November 2009, in which 23 consecutive patients who underwent surgery for ruptured aneurysms were included; a jugular bulb catheter was placed in all patients. To calculate AVDO, arterial and jugular bulb blood samples were taken every 12 hours. Demographics, neurological examination, WFNS grade, Fisher modified classification, aneurysm characteristics, vasospasm, and hemodynamic changes were collected. All patients presenting clinical vasospasm were treated by hypertensive, hemodilution and hypervolemic therapy ( $3 \mathrm{H}$ therapy). Adequate statistical tests were used.

Results From 23 patients, eight had neurologic deficits (35\%), in all these patients $\mathrm{AVDO}_{2}$ significantly increased approximately 12 hours before the onset of clinical deficits $(P<0.01)$ and it was improved after administering $3 \mathrm{H}$ therapy. Two patients (8\%) had clinical vasospasm but did not demonstrate a significant rise in $\mathrm{AVDO}_{2}(P<0.68)$, all other patients $(57 \%)$ did not present clinical vasospasm nor significant rising in $\mathrm{AVDO}_{2}$.

Conclusions Increases in $\mathrm{AVDO}_{2}$ using jugular bulb oximetry monitoring could predict clinical vasospasm in aneurysmal SAH hours before its onset. A larger number of patients would confirm these results.

P344

Magnesium use on prophylaxis of vasospasm morbidity and mortality rate in subarachnoid hemorrhage

S Kiffer Macedo, CM Primo de Siqueira, S Boechat Primo de Siqueira, R Mendoza Nuss, L Carvalho Dias, A Cedro Souza, G Vieira Rangel Pereira Hospital São José do Avaí, Itaperuna, Brazil

Critical Care 2010, 14(Suppl 1):P344 (doi: 10.1186/cc8576)

Introduction Cerebral aneurysms are an important cause of morbidity and mortality of which the most severe consequence is their rupture. Subarachnoid hemorrhage (SAH) has arterial vasospasm as one of the most serious complications.

Methods After institutional approval and informed consent, a prospective, randomized, single-blind study was carried out between February 2008 and November 2009. The study evaluated Mg use on patients from Beds 1

\begin{tabular}{|c|c|c|}
\hline FEATURES & Average Age (interval) & Average of Mg level \\
\hline Group I $\mathbf{n}=\mathbf{5 5}$ & $50,6(19-79)$ & 2,27 \\
\hline Group II $\mathbf{n}=\mathbf{5 2}$ & $51,7(26-77)$ & 1,91 \\
\hline
\end{tabular}

Figure 1 (abstract P344).

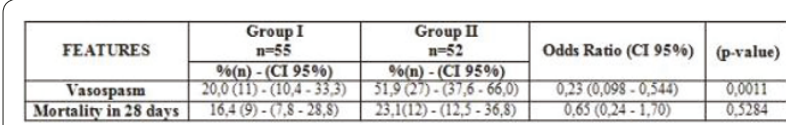

Figure 2 (abstract P344).

to 4 and the control group from Beds 5 to 8. The serum measure of Mg was made by colorimetric method in order to reach a measurement between 2.5 and $3.5 \mathrm{mg} / \mathrm{dl}$, using a solution of $\mathrm{Mg} \mathrm{2 \%} \mathrm{(SG} \mathrm{5 \% ,} 400 \mathrm{ml}+\mathrm{MgSO}_{4}$ $10 \%, 100 \mathrm{ml} / 24$ hours), during the first 14 days of the event (aneurysm rupture). Admission criteria: patients diagnosed with SAH confirmed by CT or cerebral angiography and $\Delta t<96$ hours. Exclusion criteria: patients with $\mathrm{SAH}$ and $\Delta \mathrm{T}>96$ hours; patients who presented a vasospasm episode in less than 24 hours of $\mathrm{Mg}$ solution infusion.

Results In a previous study evaluation a total of 107 patients were analysed with $n=55$ in Group 1 and $n=52$ in Group 2 (Figures 1 and 2). The main results were: Group 1 - vasospasm frequency $20.0 \%$, confidence interval $(\mathrm{Cl})=10.4 \%$ to $33.3 \%$; and mortality $16.4 \%$ in 28 days, $\mathrm{Cl}=7.8 \%$ to 28.8 ; Group 2 - vasospasm frequency $51.9 \%, \mathrm{Cl}=37.6 \%$ to $66.0 \%$; and mortality $23.1 \%$ in 28 days, $\mathrm{Cl}=12.5 \%$ to $36.8 \%$. The analysis for the vasospasm showed odds ratio $(\mathrm{OR})=0.23, \mathrm{Cl}=0.098 \%$ to $0.544 \%$ and $P=0.0011$, and for mortality: $\mathrm{OR}=0.65, \mathrm{Cl}=0.24 \%$ to $1.70 \%$ and $P=0.5284$.

Conclusions According to the outcome, we can conclude that Group 1 obtained a greater protection on the vasospasm incidence in comparison with Group 2 but showed no difference in mortality. The $P$ value was significant for vasospasm but was still not significant for mortality.

\section{References}

1. Veyna RS, et al.: J Neurosurg 2002, 96:510-514

2. Knobel E, et al.: Condutas no Paciente Grave. São Paulo: Atheneu; 2006:871-883.

\section{P345}

Effects of sinvastatin in prevention of vasospasm in nontraumatic subarachnoid hemorrhage: preliminary data

S Kiffer Macedo, CM Primo de Siqueira, S Boechat Primo de Siqueira,

Y Brum Bello, A Campeão, L Carvalho Dias

Hospital São José do Avaí, Itaperuna, Brazil

Critical Care 2010, 14(Suppl 1):P345 (doi: 10.1186/cc8577)

Introduction Vasospasm is the main cause of death and cognitive deficits in patients with subarachnoid hemorrhage after rupture of the aneurysm $(\mathrm{aSAH})$. Some trials have shown that statins in the acute phase of aSAH reduces the incidence, morbidity and mortality of cerebral vasospasm.

Methods We realized a prospective, randomized, nonblind study, with the use of $80 \mathrm{mg}$ SVT (at night) in the first 72 hours of the beginning of bleeding, and a control group that did not use SVT, for 21 days, between January and December 2008. Informed consent was obtained for all patients. CT scans was performed as control and another CT scan in patients with altered neurological signals. In the presence of changes suggestive of vasospasm or correlation in clinical and CT scans, the patients were taken for cerebral arteriography examination followed by an angioplasty procedure if necessary. Liver and renal function, LDL cholesterol evaluated weekly, and CK total evaluated every 3 days. Exclusion criteria: liver and renal disease, pregnant elevation of serum transaminases (three times the value of normal), creatinine $\geq 2.5$, rhabdomyolysis or CK total $\geq 1,000 \mathrm{U} / \mathrm{l}$.

Results We excluded two patients with bleeding for more than 72 hours. There was no significant change in the levels of CK total, renal or liver function. We included 20 patients, 11 in the SVT group and nine in the control group. Mortality was eight patients (38\%), six patients in the control group and two from the SVT group. Vasospasm was confirmed by 
cerebral arteriography examination in four patients in the control group and one patient in the SVT group. All patients who died had Fisher scale IV. Conclusions SVT at a dose of $80 \mathrm{mg}$ was effective in reducing the mortality (18.1\% against 66\%) compared with the group that did not use SVT, and also decreased the incidence of cerebral vasospasm despite higher APACHE II score in the group that used SVT (14.3 vs 10.7). There was less morbidity in the SVT group with an average of scale of Glasgow 3.25 vs 2.1 .

\section{Reference}

1. Lynch JR, Wang H, et al: Simvastatin reduces vasospasm after aneurysmal subarachnoid hemorrhage: results of a pilot randomized clinical trial. Stroke 2005, 36:2024-2026.

P346

Addition of new criteria to Sequential Organ Failure Assessment for patients with subarachnoid hemorrhage

S Kiffer Macedo, N Ferreira Bastos, J Flores Paz, T De Carvalho Pessamilio,

R Barbosa Castro, M Rodrigues Lacerda

Hospital São José do Avaí, Itaperuna, Brazil

Critical Care 2010, 14(Suppl 1):P346 (doi: 10.1186/cc8578)

Introduction Sequential Organ Failure Assessment (SOFA) was originally created for sepsis, but their quality is now used in other medical conditions. Therefore, we add some criteria to the index to assess patients with subarachnoid hemorrhage.

Methods Informed consent for each patient/family; APACHE II (criteria for admission) and SOFA weekly, serum glucose, lactate, calcium, sodium and magnesium; and measurement of axillary temperature and diuresis hourly as the additional prognostic index SOFA. The study enrolled 103 patients diagnosed with SAH, confirmed by computed tomography. These patients were divided into two groups according to their development in the ICU: Group I - patients who had good evolution (out of ICU), and Group II patients who progressed to death in the ICU.

Results Among 103 patients, 74 (71.84\%) were female and 29 (28.16\%) were male. The APACHE $\|$ for admission varied from 2 to 34 , with an average of 15.5. The spent time maximum in the ICU was 49 days (two patients). Group I had 64 patients (62.14\%) and the 39 remaining patients $(37.86 \%)$ were classified as Group II. See Figures 1 and 2.

Conclusions We can conclude that the group with patients with SAH is predominantly female (74:29). The APACHE II in Group I was 10.9, while Group II was 17.9. Regarding the criteria used to assess patients with SAH was observed that the only criterion which showed statistical significance in the prediction of death was the serum sodium $(P=0.002)$. The other

\begin{tabular}{|c|c|c|c|c|}
\hline \multirow[t]{2}{*}{ Characteristcs } & \multicolumn{2}{|c|}{ Group I } & \multicolumn{2}{|c|}{ Group II } \\
\hline & $\frac{\%(n)}{62.14(64)}$ & $\begin{array}{l}\text { IC 95\% } \\
496-705\end{array}$ & $\%(\mathbf{n})$ & IC $95 \%$ \\
\hline Age (yea & & & & \\
\hline $\begin{array}{l}\text { Average } \\
\text { Jnterval }\end{array}$ & 52,3 & - & 52 & $\cdots$ \\
\hline Female & $68,75(44)$ & $59,0-83,9$ & $76,92(30)$ & $60,8-89,9$ \\
\hline SAH & $9,4(6)$ & $1,1-15,1$ & $0(0)$ & $0-9,7$ \\
\hline $\begin{array}{l}\text { Fischer III } \\
\text { Fischer IV }\end{array}$ & $\begin{array}{l}46,9(30) \\
40,7(26)\end{array}$ & $\begin{array}{l}38,8-66,3 \\
28,7-55,9\end{array}$ & $\begin{array}{l}43,6(17) \\
56,4(2)\end{array}$ & $\begin{array}{l}2,5,5-59,2 \\
40,8-74,5\end{array}$ \\
\hline $\begin{array}{l}\text { APACHE II (Admission) } \\
\text { Average }\end{array}$ & 10,8 & & 17 & \\
\hline$\geq 20$ & $9,4(6)$ & $4,1-22,2$ & $35,9(13)$ & $20,8-5$ \\
\hline
\end{tabular}

Figure 1 (abstract P346).

\begin{tabular}{|c|c|c|c|c|c|}
\hline \multirow[t]{2}{*}{ Criteria } & \multicolumn{2}{|l|}{$\begin{array}{l}\text { Group I } \\
\mathrm{n}=55\end{array}$} & \multicolumn{2}{|c|}{$\underset{n=36}{\substack{\text { Group II } \\
\mathrm{n}=36}}$} & \multirow[b]{2}{*}{$(p)$} \\
\hline & $\begin{array}{l}\text { Frequency \% } \\
\text { (IC95\%) }\end{array}$ & OR & $\begin{array}{l}\text { Frequence\% } \\
\text { (IC95\%) }\end{array}$ & OR & \\
\hline Arterial lactate $\geq 2,0$ & $46,7(37,1-64,6)$ & 1,0 & $51,3(32,9-67,1)$ & 0,96 & 0,5 \\
\hline $\begin{array}{c}\text { Serum calcium } \\
(<1,12 \text { or }>1,32)\end{array}$ & $51,6(35,4-62,9)$ & 1,0 & $56,4(38,1-72,1)$ & 1,3 & 0,3 \\
\hline $\begin{array}{c}\text { Serum glucose } \\
(<70 \text { or }>150(\mathrm{mg} \mathrm{dl}))\end{array}$ & $32,8(13,2-37,0)$ & 1,0 & $38,5(20,8-53,8)$ & 1,8 & 0,1 \\
\hline $\begin{array}{c}\text { Serum magnesium } \\
(<1,7 \text { or }>2,4)\end{array}$ & $14,6(4,1-22,2)$ & 1 & $2,6(0-9,7)$ & 0 & 0,04 \\
\hline $\begin{array}{l}\text { Serum sodium } \\
(<135 \text { or }>145)\end{array}$ & $20,8(13,2-37,0)$ & 1,0 & $56,4(38,1-72,1)$ & 4,0 & 0,002 \\
\hline $\begin{array}{c}\text { Hourly diuresis } \\
(<0,5 \text { or }>1,5 \mathrm{ml} / \mathrm{kg} \mathrm{h})\end{array}$ & $71,9(65,0-88,2)$ & 1,0 & $66,7(54,8-85,8)$ & 0,7 & 0,3 \\
\hline Axillary temperature & $17,2(7,8-28,8)$ & 1,0 & $32,3(16,3-48,1)$ & 2,2 & 0,06 \\
\hline
\end{tabular}

Figure 2 (abstract P346). criteria evaluated did not have statistical significance in predicting the prognosis of patients.

\section{Reference}

1. Knobel E: Condutas no Paciente Grave. Volume 1. 3rd edition. São Paulo: Editora Atheneu; 2006:871-883.

P347

Cardiac output augmentation with fluid resuscitation improves brain tissue oxygenation after severe brain injury

R Helbok, P Kurtz, J Claassen, J Schmidt, L Fernadez, R Stuart, ES Connoly, N Badjatia, SA Mayer, K Lee

Columbia University Medical Center, New York, USA

Critical Care 2010, 14(Suppl 1):P347 (doi: 10.1186/cc8579)

Introduction The objective of this study was to investigate the relationship between cardiac output response to a fluid challenge and changes in brain tissue oxygen pressure $\left(\mathrm{PbtO}_{2}\right)$ in patients with severe brain injury. Methods Prospective observational study conducted in a neurological ICU in a university hospital. Seventy-eight fluid challenges were administered to 17 consecutive comatose patients that underwent multimodality monitoring of cardiac output, intracranial pressure (ICP), and $\mathrm{PbtO}_{2}$. The relationship between cardiac output and $\mathrm{PbtO}_{2}$ was analyzed with logistic regression utilizing GEE with an exchangeable correlation structure.

Results Of the 78 fluid boluses analyzed, 34 (44\%) resulted in a $\geq 10 \%$ increase in cardiac output. Median absolute $(+5.4 \mathrm{vs}+0.7 \mathrm{mmHg})$ and percentage (20\% vs $3 \%$ ) changes in $\mathrm{PbtO}_{2}$ were greater in cardiac output responders than in nonresponders within 30 minutes after the end of the fluid bolus infusion. In a multivariable model, a cardiac output response was independently associated with $\mathrm{PbtO}_{2}$ response (adjusted odds ratio $15.4,95 \% \mathrm{Cl} 1.9$ to $122.0, P=0.01$ ) after adjusting for mean arterial pressure,

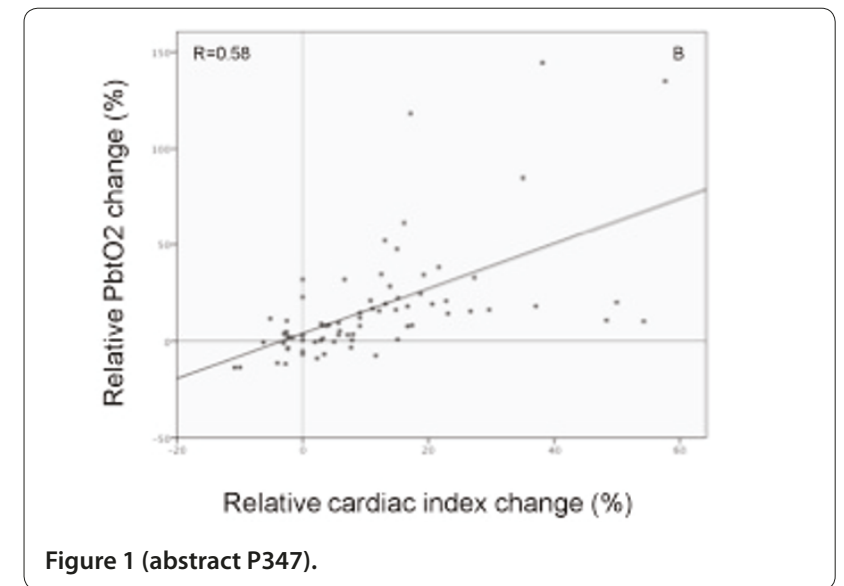

Figure 1 (abstract P347).

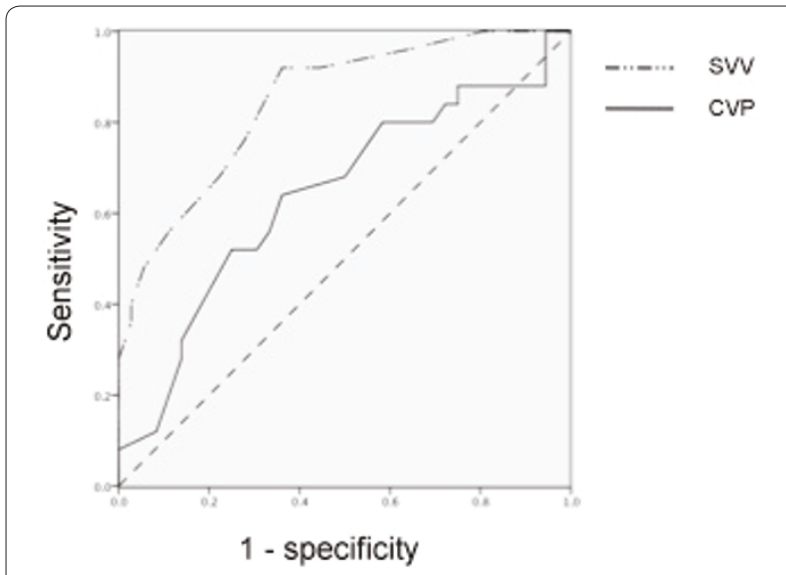

Figure 2 (abstract P347). 
intracranial pressure and end-tidal $\mathrm{CO}_{2}$. Stroke volume variation showed a good ability to predict cardiac output response with an area under the ROC curve of 0.85 with a best cutoff value of $8 \%$. See Figures 1 and 2 .

Conclusions Bolus fluid resuscitation resulting in augmentation of cardiac output can improve cerebral oxygenation after severe brain injury.

P348

Multicentre experience of using $\mathrm{ICM}^{+}$for investigations of cerebrovascular dynamics with near-infrared spectroscopy

P Smielewski', M Czosnyka', C Zweifel', K Brady², C Hogue², L Steiner³, P Hutchinson', D Mennon', J Pickard'

'University of Cambridge, UK; ${ }^{2} J$ ohns Hopkins Hospital, Baltimore, MD, USA;

${ }^{3}$ University Hospital, Basel, Switzerland

Critical Care 2010, 14(Suppl 1):P348 (doi: 10.1186/cc8580)

Introduction $\mathrm{ICM}^{+}$software encapsulates our 20 years' experience in brain monitoring. It collects data from a variety of bedside monitors and produces time trends of parameters defined using configurable mathematical formulae. To date it is being used in nearly 40 clinical research centres worldwide. We present its application for continuous monitoring of cerebral autoregulation using near-infrared spectroscopy (NIRS).

Methods Data from multiple bedside monitors are processed by $\mathrm{ICM}^{+}$ in real time using a large selection of signal processing methods. These include various time and frequency domain analysis functions as well as fully customisable digital filters. The final results are displayed in a variety of ways including simple time trends, as well as time window based histograms, cross histograms, correlations, and so forth. All this allows complex information from bedside monitors to be summarized in a concise fashion and presented to medical and nursing staff in a simple way that alerts them to the development of various pathological processes.

Results One hundred and fifty patients monitored continuously with NIRS, arterial blood pressure (ABP) and intracranial pressure (ICP), where available, were included in this study. There were 40 severely headinjured adult patients, $27 \mathrm{SAH}$ patients (NCCU, Cambridge); 60 patients undergoing cardiopulmonary bypass (John Hopkins Hospital, Baltimore) and 23 patients with sepsis (University Hospital, Basel). In addition, MCA flow velocity (FV) was monitored intermittently using transcranial Doppler. FV-derived and ICP-derived pressure reactivity indices (PRx, Mx), as well as NIRS-derived reactivity indices (Cox, Tox, Thx) were calculated and showed significant correlation with each other in all cohorts. Errorbar charts showing reactivity index PRx versus CPP (optimal CPP chart) as well as similar curves for NIRS indices versus CPP and ABP were also demonstrated.

Conclusions $\mathrm{ICM}^{+}$software is proving to be a very useful tool for enhancing the battery of available means for monitoring cerebral vasoreactivity and potentially facilitating autoregulation guided therapy. Complexity of data analysis is also hidden inside loadable profiles, thus allowing investigators to take full advantage of validated protocols including advanced processing formulas.

\section{P349}

Cerebral oxygen saturation and cerebral blood flow are relatively stable during single-lung ventilation, if normocapnia is maintained TVégh, S Szatmári, M Juhász, I László, I Takács, B Fülesdi

University of Debrecen, Hungary

Critical Care 2010, 14(Suppl 1):P349 (doi: 10.1186/cc8581)

Introduction Previously a report has suggested that administration of a lung-protective strategy for one-lung ventilation (OLV) results in oxygen desaturation of the brain parenchyma. The aim of the present work was to test whether the maintenance of normocapnia during a protective OLV strategy results in alteration of cerebral blood flow and cerebral oxygen saturation.

Methods Data were obtained from 15 patients undergoing thoracic surgery and necessitating OLV of more than 1 hour. Cerebral oxygen saturation (Satcereb) was continuously monitored by the INVOS $5100 \mathrm{C}$ Cerebral Oxymeter System along with measurement of cerebral blood flow velocity (MCAV) by transcranial Doppler sonography. Arterial blood samples were taken for blood gas analysis in the awake state, in the supine position after induction during DLV, in the lateral decubitus position during DLV and every 15 minutes during OLV.

Results Satcereb increased significantly when DLV was started with $\mathrm{FiO}_{2}$ 1.0 and remained stable during the course of the study. When ventilation was changed from DLV to OLV, no significant change was observed. A significant decrease of cerebral oxygen saturation was found compared with the value observed during DLV in the lateral decubitus at the time point of 60 minutes after the start of OLV. No significant changes in the MCAV were observed throughout the course of the thoracic surgical procedure.

Conclusions OLV does not result in clinically relevant decreases in cerebral blood flow and cerebral oxygen saturation during application of lung-protective ventilation if normocapnia is maintained.

\section{P350}

Factors affecting the transient hyperaemic response test of the cerebral autoregulation

MS Sitina, V Cerny

University Hospital, Hradec Kralove, Czech Republic

Critical Care 2010, 14(Suppl 1):P350 (doi: 10.1186/cc8582)

Introduction The transient compression of the internal carotid artery (ICA) results in dilatation of cerebral arterioles by effect of autoregulation. In the THR test, the increase in middle cerebral artery (MCA) blood flow immediately after release of transient compression of the ICA is measured, which is proportional to cerebral autoregulation. The aim of the study was to determine the significance of several factors of the test - blood flow inertia (acceleration), dimensions (length and radius) of ICA, patency of the Circle of Willis (WC), and asynchrony between release of ICA compression and arterial pressure pulsations (phase shift).

Methods We constructed a mathematical model of the elements included in the THR test and simulated, using various parameters, blood flow in cerebral vessels and the increase in flow velocity in the MCA after release of ICA compression. We simulated two extreme situations - a perfectly patent (all afferent and efferent arteries meet in one point) and a completely isolated Circle of Willis (ICA bifurcates into only the anterior and middle cerebral arteries). The series of ordinary differential equations describing the THR test was solved by MATLAB R2008a. The solution assumed an absence of autoregulation (effect of studied factors is independent of autoregulation) and the rigidity of all vessels.

Results The effects of acceleration (inertia), dimensions of ICA and patency of WC are negligible. The effect of phase shift (Figure 1) can significantly decrease the immediate post-release MCA flow, which can entirely nullify the increase in post-release MCA flow caused by autoregulation.

Conclusions The effect of phase shift can significantly alter the results of the THR test. For practical purposes we recommend calculation of THR test parameters from the second peak of post-release MCA flow, or using the highest post-release MCA flow from several THR tests.

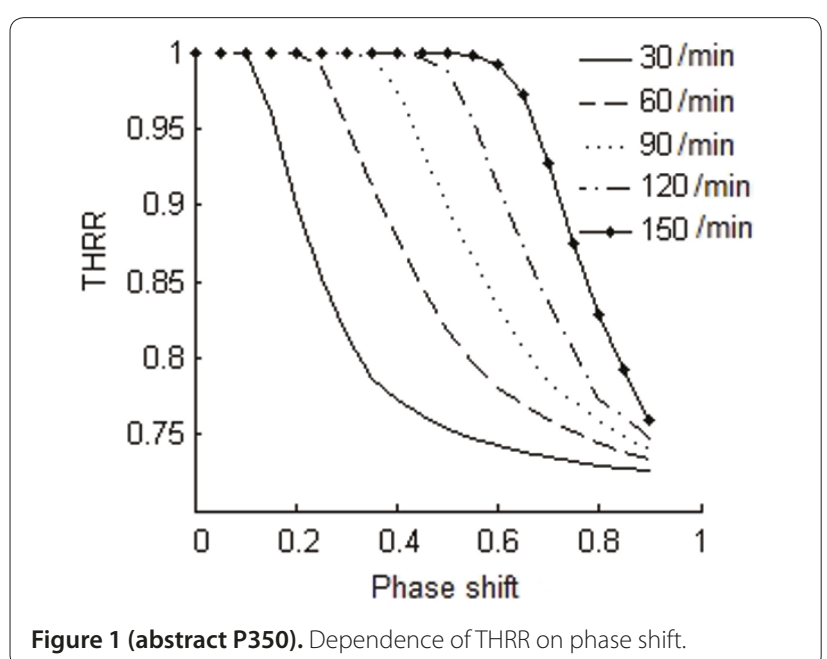


P351

Infection associated with the external ventricular derivation catheter: preliminary data

S Kiffer Macedo, CM Primo de Siqueira, S Boechat Primo de Siqueira, B Almeida Simão Da Mata, S Ferrari Salles, L Reggiani de Almeida, F Oliva Battisti

Hospital São José do Avaí, Itaperuna, Brazil

Critical Care 2010, 14(Suppl 1):P351 (doi: 10.1186/cc8583)

Introduction The purpose of this study was to evaluate the total drainage time, the risk of infection associated with external ventricular derivation (EVD) and the most prevalent infective agent during the use of EVD drainage in patients on the ICU.

Methods During 15 months (8 August 2008 to 28 October 2009), all patients on the neurovascular ICU who had an EVD inserted were studied prospectively. Each 3 days we analysed cerebral spinal fluid (CSF) samples for cell count, biochemistry and sputum. CSF cultures were evaluated three times a week combined with an antibiogram. Not included were the cultures that persisted with the same result (agent), considering only a new agent until negativity of the culture and clinical resolution. We excluded patients admitted with EVD from another hospital.

Results Were included 46 patients, in which were obtained a total number of 66 EVDs. Thirty-five EVDs $(53,03 \%)$ had associated ICP monitoring. A total $65.21 \%$ of patients were female. The mortality of patients was $36.95 \%$ (17 deaths). Among infected catheters, infection was confirmed in approximately 7.1 days after the EVD was installed. However, the catheters discharged with negative culture or without growth of a new agent remained approximately 6.15 days on the brain ventricle of patients. Registered were 20 positive bacteriological cultures of CSF (30.30\%) among the total EVDs. The most prevalent organisms were Acinetobacter baumanii on nine cultures (45\%), Pseudomonas aeruginosa on six cultures (30\%), Serratia rubidaea on one culture (5\%), Streptococcus oralis on one culture (5\%), Klebsiella ornithinolytica on one culture (5\%), Estafilococcus coagulase negative on one culture(5\%), Stenotrophonas maltophylia on one culture (5\%).

Conclusions Some previous studies show a substitution from positive Gram agents to negative Gram agents on infections associated with EVD. The study has shown a prevalence of $P$. aeruginosa and $A$. baumanii on infection associated with the EVD catheter, which are also more prevalent in the ICU environment. This study reinforces that, in case of suspected CNS infection associated with catheter EVD (meningitis, ventriculitis, or abscess), we can start empirical antimicrobial coverage for Gram-negative infections, including intraventricular administration (intrathecal) as Polymixin B.

\section{P352}

fMRI default mode connectivity in vegetative state and locked-in syndrome patients

A Soddu', A Vanhaudenhuyse', M Boly', MA Bruno', L Tshibanda², C Phillips', M Stanziano 3 , S Ovadia Caro', Y Nir ${ }^{5}$, P Maquet', M Papa 3 , R Malach ${ }^{4}$, Q Noirhomme', S Laureys

'Coma Science Group, Liege, Belgium; ' Neurology Department, CHU Sart Tilman Hospital, University of Liège, Belgium: ${ }^{3}$ Pubblica Clinica e Preventiva, Second University of Naples, Italy; ${ }^{4}$ Department of Neurobiology, Weizmann Institute of Science, Rehovot, Israel; ${ }^{5}$ Department of Psychiatry, University of Wisconsin, Madison, WI, USA

Critical Care 2010, 14(Suppl 1):P352 (doi: 10.1186/cc8584)

Introduction The aim of this study is to assess fMRI resting-state cerebral connectivity in vegetative state and locked-in syndrome patients by means of a user-independent method. Resting baseline (or default mode) activity is thought to be related to awareness of the internal world (that is, mind wandering, and so forth) and encompasses posterior cingulate/precuneal, anterior cingulate/mesiofrontal and posterior lateral parietal cortices.

Methods We here present a novel clinical application for a userindependent default mode network analysis. Resting state data were acquired for seven vegetative state patients (age range 16 to 87 years), one locked-in syndrome patient (aged 24 years) and seven healthy subjects (25 to 65 years). Patients' diagnosis was based on Coma Recovery Scale assessment prior and following scanning. Data were pre-processed and analyzed using independent component analysis as implemented in Brain Voyager. Connectivity studies employed 13 target regions of interest defined on an average default mode map calculated from an independent dataset of 20 controls (21 to 60 years). Resting state connectivity was assessed by calculating the number of functional connections within the default mode map for each subject. Next, Student $t$ tests compared patients with controls at the group level $(P<0.05)$.

Results Compared with controls, vegetative patients showed a lower total number of edges (that is, connections; $24 \pm 4$, range 19 to 31 and $13 \pm 3$, range 8 to $15, P<0.001)$ and less functional connections with the precuneus ( $6 \pm 1$ and $2 \pm 2 ; P<0.001)$. The default mode network shows a reduced connectivity in vegetative patients as compared with controls mainly between the precuneus and medial prefrontal cortex ventral. Finally, for the locked-in syndrome patient the connectivity graph counted 21 total number of effective connections and five effective connections with the precuneus, which was not significantly different from healthy controls.

Conclusions The connectivity study showed no overlap between the number of effective connections or edges in vegetative state patients and in controls. Connectivity graph analysis identified anterior-posterior midline disconnections in the vegetative state, in line with previous studies emphasizing the critical role of the precuneus/PCC and mesiofrontal cortices in the emergence of conscious awareness.

P353

Incidence of cerebral salt wasting syndrome: comparison in subarachnoid haemorrhagic compared with head trauma

FD Di Sacco', GB Bernardeschi', AD Demi', CC Conversano², EL Lensi², FF Forfori', FG Giunta'

'Pisa University, Pisa, Italy; '2Siena University, Pisa, Italy

Critical Care 2010, 14(Suppl 1):P353 (doi: 10.1186/cc8585)

Introduction Cerebral salt wasting syndrome (CSWS) is characterized by severe hyponatriemia, caused by the reduction of sodium reabsorption at the level of proximal tubule, with associated polyuria and hypovolemia. For decades, this syndrome was confused with the syndrome of inappropriate secretion of antidiuretic hormone (SIADH), but in CSWS the antidiuretic hormone is physiologically secreted. The only way to make a difference in the diagnosis is the assessment of the extracellular volume, not easily measurable in critical care patients. We designed this study to assess the frequency, the onset and the duration of the symptoms of CSWS in patients hospitalized in post-neurosurgery critical care.

Methods In the 2-year period 2008 to 2009 in the post-neurosurgery ICU, 131 patients were studied retrospectively; 63 patients had subarachnoid haemorrhage (SAH) caused by the rupture of a cerebral aneurysm and 68 patients had severe head trauma (HI). CSWS diagnosis was done evaluating sodiemia, serum osmolality, sodiuria 24 hours and central venous pressure. The time from the onset of hyponatriemia during ICU stay and duration of symptoms was also evaluated.

Results Patients developed CSWS in $12.7 \%$, of whom $7.9 \%$ within 1 week from admission and $4.8 \%$ after. Among the patients with $\mathrm{HI}, 5.8 \%$ developed CSWS, of whom 2.9\% within the first week while the other $2.9 \%$ after. CSWS symptoms lasted for more than 1 week in $11.1 \%$ of patients with $\mathrm{SAH}$, and less only in $1.6 \%$, while in $\mathrm{HI}$ the symptoms lasted less than 1 week in $2.9 \%$ and more than 1 week in $2.9 \%$ of patients.

Conclusions Differential diagnosis of ICU hyponatriemia is important in determining patient outcome. CSWS treatment required an appropriate fluid and salt replacement to normalize hydroelectrolytic balance. CSWS has a different distribution in patients with subarachnoid haemorrhage compared with traumatic head injury. Furthermore, the syndrome starts earlier and lasts longer in SAH compared with $\mathrm{HI}$.

\section{P354}

Sodium dysbalances in neurointensive care: results of a 5-year prospective study

$\checkmark$ Spatenkova', A Kazda², P Suchomel'

${ }^{1}$ Regional Hospital, Liberec, Czech Republic; ${ }^{2} 1$ st Faculty of Medicine, Charles University, Prague, Czech Republic

Critical Care 2010, 14(Suppl 1):P354 (doi: 10.1186/cc8586)

Introduction Sodium dysbalances represent important complications in neurointensive care. The aim of this study was to analyse sodium 
dysbalances and their relationship to outcome in the neurologicneurosurgical care unit (NNICU) over a period of 5 years.

Methods We prospectively evaluated patients with brain diseases, who developed serum sodium below $135 \mathrm{mmol} / \mathrm{l}$ (hyponatremia) or above $150 \mathrm{mmol} / \mathrm{l}$ (hypernatremia). We compared the incidence of cerebral complications, Glasgow Outcome Scale upon discharge from the NNICU and mortality in the NNICU between these two groups.

Results In the 5-year observation period, serum sodium dysbalances occurred in 378 (24\%) patients. The majority of them had hyponatremia (245 patients, 65\%); hypernatremia was less frequent, in 133 (35\%) patients. Hypernatremic patients stayed in the NNICU longer $(P=0.035)$, onset of hypernatremia arose in patients with significantly lower Glasgow Coma Scale $(P=0.001)$. These patients had more cerebral complications $(P<0.001)$, worse Glasgow Outcome Scale upon discharge from the NNICU $(P<0.001)$, higher mortality in the NNICU $(P=0.003)$ and higher incidence of pulmonary edema $(P=0.021)$. They received more antiedematic therapy $(P<0.001)$ and diuretics $(P<0.001)$. On the other hand, hyponatremia was more frequent upon entry to the NNICU $(P<0.001)$ and arose later after brain damage $(P<0.001)$ in comparison with hypernatremia.

Conclusions In neurointensive care, hypernatremia was a prognostically more serious and less frequent sodium dysbalance than hyponatremia.

\section{P355}

Impact of hypernatremia on patients with severe traumatic brain injury M Shehata, D Ragab, M Khaled, M Hegazy, A Hussein, H Khaled Cairo University, Cairo, Egypt

Critical Care 2010, 14(Suppl 1):P355 (doi: 10.1186/cc8587)

Introduction Hypernatremia is frequently encountered in the neurocritical ICU and its effect can badly affect mortality. The objective of this study was to verify whether the occurrence of hypernatremia during the ICU stay increases the risk of death in patients with severe traumatic brain injury (TBI).

Methods Randomized prospective study of 100 patients with TBI in neurocritical care units at Cairo University Hospital. Hypernatremia is defined as serum sodium above $145 \mathrm{mmol} / \mathrm{l}$. The major outcome was death in the ICU. Logistic regression models were used to assess independent factors that could affect patients' mortality including hypernatremia, age, desmopressin and Glasgow Coma Score.

Results We included in the study $100 \mathrm{TBI}$ patients (mean age $35.8 \pm 21.3$ years); males $86 \%$. Extradural hematoma (EDH) was documented in 27 patients (27\%), subarachnoid (SAH) in 20 patients $(20 \%)$, intracerebral $(\mathrm{ICH})$ in 19 patients (19\%), cerebral contusion in 17 patients $(17 \%)$, brain oedema in 15 patients (15\%), and subdural hematoma in two patients (2\%). Glasgow Coma Scores (range 3 to 10); 60 patients were mechanically ventilated; $10 \%$ were diabetic and $22 \%$ were hypertensive. Hypernatremia was documented in 40 patients (40\%) of the total TBI patients. The total inhospital mortality was 36/100 (36\%), 10 of them had normal sodium levels all through their in-hospital course and 26 patients were hypernatremic. After adjustment for the baseline risk, the incidence of hypernatremia over the course of the ICU stay was significantly related to increased mortality (hazard ratio $3.2(P=0.0001)$ ). However, there was positive correlation between serum sodium levels and duration of the ICU stay (Spearman correlation coefficient 0.5 and $P=0.002$ )

Conclusions Hypernatremia in patients with severe TBI is associated with an increased risk of death, and a longer ICU stay. This association is independent of other outcome predictors including age and Glasgow Coma Score. Strategies to prevent hypernatremia in neurocritical ICUs should be encouraged.

Reference

1. Adrogué HJ, Madias NE: N Engl J Med 2000, 342:1493-1499.
P356

Prevalence and clinical setting of SIADH in neurosurgery: comparison between subarachnoid haemorrhage and head trauma FD Di Sacco ${ }^{1}$, AD Demi ${ }^{1}$, GB Bernardeschi', CC Conversano², EL Lensi², FF Forfori', FG Giunta ${ }^{1}$

${ }^{1}$ Pisa University, Pisa, Italy; ${ }^{2}$ Siena University, Pisa, Italy

Critical Care 2010, 14(Suppl 1):P356 (doi: 10.1186/cc8588)

Introduction The syndrome of inappropriate secretion of antidiuretic hormone (SIADH) is a very important disorder in neurosurgery, and it is one of the most common causes of hyponatriemia in acute brain injury. SIADH should be suspected in any patient with hyponatremia, hypoosmolarity, urine osmolality above 100 mosmol/kg H $\mathrm{H}_{2} \mathrm{O}$, concentration of sodium in the urine usually above $40 \mathrm{mEg} / \mathrm{l}$, and clinical euvolemia. This study was designed to assess the frequency of this syndrome in the postneurosurgery ICU, the time of onset and the duration of symptoms.

Methods In a 2-year period (2008 to 2009), 131 patients, hospitalized in a post-neurosurgery ICU for TBI or ESA were retrospectively studied. According to the previous definition, 63 patients had subarachnoid haemorrhage $(\mathrm{SAH})$ from rupture of aneurysm and 68 patients had severe head trauma (HI). Diagnosis of SIADH was confirmed by $\mathrm{pH}$, sodiemia, plasmatic and urinary osmolality, sodiuria of 24 hours and central venous pressure. The time to onset of hyponatremia during hospitalization in ICU was evaluated, and its duration despite adequate treatment (fluid restriction and eventually hypertonic saline).

Results Patients developed SIADH in 5.34\%. Three patients had a subarachnoid hemorrhage and four patients were hospitalized for head trauma. The onset of this syndrome was within the first week in two patients with SAH and in only one with traumatic head injury. With regard to the duration of symptoms, SIADH lasted more than a week in the most of patients, with no differences between the two groups.

Conclusions In patients with hyponatriemia, an adequate diagnosis of SIADH is very important in order to improve the outcome, especially in the ICU. In this study we found no differences in the SIADH setting between patients with subarachnoid haemorrhage and in patients with traumatic head injury. But, owing to the small number of patients with this syndrome enrolled, this trend has to be confirmed in further studies.

\section{P357}

Risk factors for venous thromboembolism in Japanese patients with severe blunt trauma

Y Masuda, N Saito, KMashiko

Chiba Hokusou Hospital, Nippon Medical School, Chiba, Japan

Critical Care 2010, 14(Suppl 1):P357 (doi: 10.1186/cc8589)

Introduction Venous thromboembolism (VTE) is a common complication after major trauma, and has a high rate of morbidity and mortality. However, racial disparities in incidence of as well as risk factors for VTE exist between Asian and western populations. The risks for VTE after major trauma in Japanese patients remain unknown. Our objective was to identify risk factors for VTE after severe blunt trauma in Japanese patients. Methods We conducted a prospective cohort study to investigate risk factors for VTE in patients who were admitted to our hospital because of blunt trauma between April 2008 and July 2009. We performed VTE screening by duplex ultrasonographic scan for all blunt trauma patients who had bed rest $\geq 3$ days and recorded Injury Severity Score (ISS) $\geq 9$ within the first 7 days after admission. We initiated pharmacologic VTE prophylaxis, first with low-dose unfractionated heparin and then with lowdose warfarin. The chi-square test was used for categorical variables and the $t$ test for continuous variables.

Results A total of 101 patients (69 males, 32 females) with blunt trauma (median age $48.48 \pm 20.5$ (SD) years and mean ISS of $20.0 \pm 10.2$ ) were enrolled in this study. Pharmacologic VTE prophylaxis was initiated in 61 of these patients (60.4\%). Ten patients (10.0\%) experienced VTE. Univariate analysis of potential factors for VTE identified significant associations between thrombosis and the following factors: transfusion, major surgery, the presence of a central venous line in the femoral vein, and direct traction of lower limb ( $P<0.05$ for all these factors).

Conclusions The incidence of VTE in Japanese patients with severe blunt trauma was $10.0 \%$. These risk factors have all been given in the results above. 
P358

Safety of heparin prophylaxis for venous thromboembolism in patients with neurotrauma

G Puri, P Joseph

Postgraduate Institute of Medical Education and Research, Chandigarh, India

Critical Care 2010, 14(Suppl 1):P358 (doi: 10.1186/cc8590)

Introduction Patients with neurotrauma are at high risk for VTE. The safety of early anticoagulation for neurotrauma has not been established. Our study was based on the hypothesis that unfractionated heparin can be safely administered to most patients with neurotrauma forVTE prophylaxis. Methods Prospective, single-cohort, observational study. All adult patients with neurotrauma admitted to a tertiary care teaching hospital in India from March to December 2007 received unfractionated heparin beginning approximately 24 hours after hospital admission until discharge. Brain CT scans were performed at admission, 24 hours after admission, and at variable intervals thereafter based on clinical course. Patients were excluded for coagulopathy, expected brain death or discharge within 48 hours. Complications of heparin prophylaxis were defined as expansion of existing intracranial haemorrhage or development of a new haemorrhagic lesion on follow-up CT after beginning heparin use.

Results Twenty-one of 57 (36.8\%) adult neurotrauma patients were eligible for heparin therapy. Their distribution of CT findings is presented in Table 1. Serial CT scan had progression of lesion in four of the 26 patients not on heparin therapy and in two of the 21 patients on heparin $(P>0.05)$. No difference in complications between patients with $\mathrm{ICH}$ on heparin $(2 / 14)$ and not on heparin $(1 / 13)$ were identified $(P>0.05)$. The mortality in the heparin group was $3 / 21(14.3 \%)$. Both the patients who developed worsening of CT findings after initiation of heparin therapy survived hospitalization. None of the patients suffered from DVT.

Table 1 (abstract 358). Distribution of CT findings and heparin therapy

\begin{tabular}{lcc}
\hline CT & No heparin & Heparin \\
\hline Normal & 8 & 0 \\
DBE & 8 & 3 \\
Contusion & 13 & 3 \\
Hge & 27 & 14 \\
\# Skull & 1 & 1 \\
\hline
\end{tabular}

Conclusions Heparin can be safely used for VTE prophylaxis in patients with head injury when started 24 hours after hospital admission.

\section{P359}

Prevalence and incidence of proximal deep vein thrombosis in critically ill patients

A Panitchote, W Chaiyasoot, C Permpikul

Mahidol University, Bangkok, Thailand

Critical Care 2010, 14(Suppl 1):P359 (doi: 10.1186/cc8591)

Introduction The epidemiology of DVT in critically ill medical patients has not been studied in Thailand. The aim of this study was to assess the prevalence and incidence rate of proximal DVT in these patients and to determine the factors associated with the development of this condition. Methods A single-center, prospective observational study was performed in critically ill medical patients who developed sepsis or new organ failure requiring organ support. DVT was detected by ultrasonography with color Doppler imaging performed within 48 hours after the patients met the inclusion criteria, and serial studies at days 7, 14 and 28 (or earlier if clinically indicated) were carried out.

Results Of the 170 patients, 87 were male (51.2\%). The mean age was 59.95 years (range 18 to 94), and the mean APACHE II score was 21.34 (range 4 to 42 ). Acute respiratory failure and sepsis were the leading diagnoses. Prevalence and incidence of proximal DVT were $14.1 \%$ (95\% CI 9.6 to 20.15) and $8.82 \%(95 \% \mathrm{Cl} 5.4$ to 14.0$)$, respectively. Univariate analysis of factors associated with DVT showed female sex $(P=0.017)$, age $\geq 50$ years $(P=$ $0.017)$, renal replacement therapy ( $P=0.005)$ and femoral venous catheter $(P=0.002)$ while multivariate analysis showed female sex $(P=0.007 ; \mathrm{OR}=$ $4.43,95 \% \mathrm{Cl} 1.5$ to 13.08$)$, age $\geq 50$ years $(P=0.027 ; \mathrm{OR}=6.12,95 \% \mathrm{Cl} 1.23$ to 30.49), femoral venous catheter $(P<0.001 ; \mathrm{OR}=11.77,95 \% \mathrm{Cl} 3.11$ to
44.53) and the absence of platelet transfusion ( $P=0.015$; OR $=0.056,95 \%$ $\mathrm{Cl} 0.005$ to 0.58 ). Clinical probability at the first 48 hours but not at days 7 , 14 and 28 was strongly correlated with the development of proximal DVT. The duration of mechanical ventilation, the hospital length of stay and the ICU mortality were not affected by the presence of DVT.

Conclusions Proximal DVT of lower extremities in critically ill medical Thai patients are not uncommon. Thromboprophylaxis study is needed in order to investigate the risk and benefit.

References

1. Geerts WH, et al.: Prevention of venous thromboembolism: the Eighth ACCP Conference on Antithrombotic and Thrombolytic therapy. Chest 2008, 133(Suppl):381S-453S.

2. Cook DJ, et al.: Deep venous thrombosis in medical-surgical ICU patients: prevalence, incidence and risk factors. Crit Care Med 2005, 33:1565-1571.

\section{P360}

Trauma patients with prophylactic IVC filter and chemical prophylaxis: a descriptive study

MM Perreault, J Yan, A Karamaoun, E Villeneuve, T Razek

The Montreal General Hospital, Montreal, Canada

Critical Care 2010, 14(Suppl 1):P360 (doi: 10.1186/cc8592)

Introduction Trauma patients at high risk of thrombosis and bleeding often have a retrievable inferior vena cava filter (IVCF) inserted prophylactically shortly after admission for the prevention of pulmonary embolism. Despite their increasing use in trauma, controversy exists regarding patient selection, benefits and safety. In addition, chemical prophylaxis should be initiated as soon as determined to be safe to do so. Limited data exist describing the use of chemical prophylaxis and the timing of it in relation to IVCF placement in this patient population. We aim to describe such use and timing in relation to IVCF placement.

Methods All trauma patients with a retrievable prophylactic IVCF inserted over 3.5 years with an age $\geq 18$ and an ISS $\geq 15$ were enrolled into the descriptive study. Patients were identified using the local trauma registry and data collected from the registry and patient chart.

Results One hundred and three patients with a prophylactic IVCF are described. Mean age was $42.8 \pm 17.9$ years, $68 \%$ were male and they had a mean ISS of $38.6 \pm 12$. A total of $36.9 \%$ had $\geq 3$ injuries. A total of $60.2 \%$ had an injury with maximum AIS score $\geq 5$ and the type of injury associated with maximal AIS was head (53.4\%), pelvic (19.4\%), thoracic (15.5\%) and spinal cord injury (11.7\%). Shock was present on admission in $8.7 \%$ patients, 98.1\% required ICU admission, $88.4 \%$ required intubation and $27.2 \%$ patients required $\geq 4$ PRBC over the first 24 hours. The retrievable IVCF was inserted prophylactically after a mean of $1.7 \pm 1.9$ days (range 0 to 9 days) of hospital admission. Chemical prophylaxis was initiated $5.7 \pm 5.1$ days after IVCF in 77.7\% patients, and dalteparin or enoxaparin were the initial agents of choice in $93.8 \%$ while heparin was chosen in $6.2 \%$ of cases. The time from hospital admission to the start of chemical prophylaxis ranged from 6.3 to 8.5 days.

Conclusions Chemical prophylaxis was initiated in the majority of trauma patients with an IVCF, albeit $22 \%$ still did not receive any. When administered, a low-molecular-weight heparin was selected. Although rapidly inserted after admission, there was considerable delay between insertion of IVCF and initiation of chemical prophylaxis. The reasons for this delay require further investigation.

\section{Reference}

1. Kaufman JA, et al:. Guidelines for the use of retrievable and convertible vena cava filters. Surg Obes Relat Dis 2006, 2:200-212.

\section{P361}

Clinical signs and risk factors of acute deep vein thrombosis of lower extremities in patients after abdominal surgery for cancer: efficiency and safety of different types of anticoagulant therapy O Tarabrin', V Suslov², S Kalinchuk³, S Tarasenko', V Grubnik',

S Shcherbakov', D Gavrichenko ${ }^{3}$

'Odessa Medical University, Odessa, Ukraine; ${ }^{2} T h e$ Institute of Urology, Kiev,

Ukraine; ${ }^{3}$ Regional Clinical Hospital, Odessa, Ukraine

Critical Care 2010, 14(Suppl 1):P361 (doi: 10.1186/cc8593)

Introduction The background of our research was to conduct a retrospective analysis of clinical signs and risk factors for acute deep vein 
thrombosis (ADVT) of lower extremities, and to compare the effectiveness and safety of therapy of LMWH bemiparin and UFH in patients after abdominal surgery for cancer.

Methods For the period from 2008 to 2009 the diagnosis of ADVT was set for 362 patients, among them 179 (49.4\%) men and 183 (50.6\%) women. Among patients with ADTV with primary localization in ileofemoral and popliteal segments treated in the department of vascular surgery, senior persons more than 60 years old prevailed (63.9\%). All patients with ADVT received therapy with anticoagulants. Bemiparin sodium was prescribed in 257 (70.9\%) cases, UFH in 71 (19.6\%) and in 34 (9.5\%) others LMWH. We analysed efficiency and complications after antithrombotic therapy in a hospital period. A complete coagulation screen, activated clotting time, thromboelastography and low-frequency haemoviscoelastography (HVG) were performed to reveal coagulation disturbances.

Results In $83.7 \%$ of cases the most frequent symptoms of disease are edema of an extremity (in $78.1 \%$ ) and pain syndrome $74.7 \%$, which at a monosymptomal variant (39.4\% patients) are marked in $66.3 \%$ and $44.4 \%$ accordingly. The most frequent risk factors (RF) were prolonged (more than 8 days) immobilization and malignant tumors. Therapy of UFH and LMWH was effective enough (on average, a good result obtained in more than in $77 \%$ cases). Bemiparin treatment was related to considerably less hemorrhagic complications than treatment of UFH $(P<0.05)$, which concerned all of types of such complications.

Conclusions Treatment of with bemiparin as compared with UFH accompanied reduced frequency of all hemorrhagic complications by 2.1 times, serious 2.7 times and moderate 3.1 times. HVG is an effective pointof-care monitor for routine clinical practice and clinical research.

\section{P362}

Multidrug sedation as a risk factor for deep vein thrombosis in the intensive care unit

F Barbani', L Tutino', S Batacchi' ${ }^{2}$, A Cecchi², M Bonizzoli², M Boddi ${ }^{3}$

G Zagli², A Peris²

'Postaraduate School of Anesthesia and Intensive Care, University of Florence, Italy; ${ }^{2}$ Careggi Teaching Hospital, Florence, Italy; ${ }^{3}$ Department of Critical Care Medicine and Surgery, University of Florence, Italy

Critical Care 2010, 14(Suppl 1):P362 (doi: 10.1186/cc8594)

Introduction Critically ill patients are at high risk for deep vein thrombosis (DVT). Among ICU DVT risk factors, the role of muscle relaxant (MR) has been clearly shown [1] but the possible influence of sedative medications (SM) is still under debate. This study was aimed at investigating whether the use of different SM may affect DVT incidence in ICU patients.

Methods We retrospectively enrolled all patients admitted to a mixed medical-surgical trauma ICU from January 2007 to August 2008 who showed a $>72$ hours length of stay and were $>18$ years old; exclusion criteria were DVT at admission and/or within the previous 6 months, thrombophilic profile, high haemorrhagic risk, admission from another ICU. For studying ICU lower limb DVT incidence, compressive ultrasonography (CUS) was performed by three trained ultrasonographers twice a week until discharge. SM and MR administration was registered daily: the drugs considered are presented in Figure 1. MR-treated patients were excluded from statistical analysis; the difference in SM administration in the No DVT group vs the DVT-positive group was assayed by chi-squared test.

Results Over the period of study, 380 patients were enrolled. Lower limb DVT was diagnosed in 23 of 380 patients, with a DVT incidence of $6.05 \%$.

\begin{tabular}{|c|c|c|c|}
\hline & $\begin{array}{c}\text { No DVT } \\
n=252 \\
\text { Frequency (\%) }\end{array}$ & $\begin{array}{c}\text { DVT } \\
\text { n=7 } \\
\text { Frequency }(\%)\end{array}$ & $\mathbf{p}$ \\
\hline Propofol & $170(67.46)$ & $5(71.43)$ & $\mathrm{ns}$ \\
\hline Benzodinzepine & $70(27.78)$ & $2(28.57)$ & $\mathrm{ns}$ \\
\hline Morp hine & $140(55.55)$ & $5(71.43)$ & $\mathrm{ns}$ \\
\hline Fentanyl / Remifentanil & $110(43.65)$ & $4(57.14)$ & $\mathrm{ns}$ \\
\hline Sedated Patients & $195(77.38)$ & $6(85.71)$ & $\mathrm{ns}$ \\
\hline At least $2 . S M$ & $166(65.87)$ & $6(85.71)$ & $\mathrm{ns}$ \\
\hline At least 3 SM & $80(31.74)$ & $3(42.86)$ & $\mathrm{ns}$ \\
\hline At least 4 SM & $20(7.94)$ & $1(14.29)$ & $\mathrm{ns}$ \\
\hline
\end{tabular}

MRs were administered in 121 patients and they were associated with DVT incidence $(P<0.001)$. Statistical analysis results performed on 259 patients are shown in Figure 1: none of the considered SM resulted to be associated with DVT incidence, nor sedation as a whole or the number of SM administered.

Conclusions Our study confirms prior findings [1] on the MR role as a DVT risk factor. Nevertheless, SM administration in patients not treated with MR is not associated with increased DVT risk.

References

1. JThromb Haemost 2009. [Epub ahead of print. DOI: 10.1111/j.1538-7836.2009.03664.x]

\section{P363}

\section{New method diagnostics for coagulation disorders after surgery}

O Tarabrin', V Suslov', V Grubnik', S Kalinchuk³, P Pustovoyt ${ }^{3}$,

S Shcherbakov', D Gavrichenko ${ }^{3}$

'Odessa Medical University, Odessa, Ukraine; ${ }^{2 T}$ The Institute of Urology, Odessa, Ukraine; ${ }^{3}$ Regional Clinical Hospital, Odessa, Ukraine

Critical Care 2010, 14(Suppl 1):P363 (doi: 10.1186/cc8595)

Introduction Despite the evidence of perioperative hypercoagulability in cancer patients, there are no consistent data evaluating the extent, duration, and specific contribution of platelets and procoagulatory proteins by in vitro testing. This study compared efficacy of haemoviscoelastography versus thromboelastography for monitoring of coagulation imbalance.

Methods Two hundred and forty-one patients undergoing surgery for abdominal cancer were examined for the efficacy of a variety of coagulation tests. A complete coagulation screening, thromboelastography (TEG) and haemoviscoelastography (HVG) were performed before and at the end of surgery.

Results We calculated the elastic shear modulus of standard MA (Gt) and HVG MA (Gh), which reflect the total clot strength and procoagulatory protein component, respectively. The difference was an estimate of the platelet component (Gp). There was a 16\% perioperative increase of standard MA, corresponding to a $51 \%$ increase of Gt $(P<0.05)$ and a $79 \%$ to $87 \%$ contribution of the calculated Gp to Gt. We conclude that serial standard thromboelastography and the HVG viscoelastic test may reveal the independent contribution of platelets and procoagulatory proteins to clot strength. Using multiple linear regression, all coagulation, TEG and HVG variabilities were used to model postoperative hypercoagulation. Results showed that some components of the TEG failed to identify hypercoagulation $(r<0.2, P>0.75)$. All components of the HVG test reflect postoperative coagulopathies.

Conclusions Hypercoagulability is not reflected completely by standard coagulation monitoring and TEG, and seems to be predominantly caused by increased platelet reactivity. HVG provides a fast and easy-toperform bedside test to quantify in vitro coagulation, and may be useful in determining the coagulation status of cancer patients perioperatively.

\section{P364}

Thrombocytopenia in surgical ICU patients

O Yhiaoui, M Metref

Military Hospital, Algiers, Algeria

Critical Care 2010, 14(Suppl 1):P364 (doi: 10.1186/cc8596)

Introduction The aim was to study the incidence, the etiology and to assess the severity of thrombocytopenia in surgical ICU (SICU) patients admitted to the central army hospital of Algiers (Algeria).

Methods We conducted a retrospective study; all admitted patients during the year 2008 were enrolled in the study (351 patients). All charts were reviewed and different hemograms recorded from admission until discharge. One hundred and fifty-three thrombocytopenia cases defined as a platelet count (plt) $<150 \times 10^{9}$ were reported (43.6\%).

Results Among 351 patients (227 survivors, 124 died), 153 developed thrombocytopenia, 96 males and 57 females. The onset of thrombocytopenia was for the majority of patients $(98 \%)$ at 24 to 72 hours after ICU admission. Only $26 \%$ were severe (plt $<50 \times 10^{9}$ ) and $3 \%<20 \times 10^{9}$ ). The etiopathology is multifactorial and difficult to obtain precisely: $77 \%$ 
of cases were hemodynamically unstable multitrauma patients (with or without ARDS and fat embolism), and necessitated volume and blood product administration through an intravascular device; sepsis was present in $47 \%$ of cases; disseminated intravascular coagulopathy in 33\%; and five thrombocytopenia cases were related to drug administration with late onset after the 5th day. Reported mortality was 57\% $\left(P<10^{-6}\right)$ compared with the overall mortality $(35.3 \%)$, related to the severity of underlying diseases and the associated co-morbidities.

Conclusions Thrombocytopenia is frequent among chirurgical patients, especially multitrauma patients with cardiovascular instability where aggressive volume and blood product administration is needed.

\section{P365}

Platelet count as a prognostic marker in intensive care

A Mackay, J Erskine, K Connor, M McCusker, S Noble

Victoria Infirmary, Glasgow, UK

Critical Care 2010, 14(Suppl 1):P365 (doi: 10.1186/cc8597)

Introduction Thrombocytopenia is a common occurrence in the critically ill population [1]. We aimed to determine the incidence, severity and prognosis of thrombocytopenia in our ICU.

Methods A retrospective laboratory result review of 330 consecutive admissions to the Victoria Infirmary ICU was undertaken. Demographics, APACHE II score and outcome data were retrieved from the Ward Watcher system in the ICU. We compared survivors' and nonsurvivors' platelet levels on admission to the ICU and the trough level in the ICU and looked at correlation with the length of stay and duration of mechanical ventilation. Data were analysed using Student's $t$ test, Pearson correlation coefficient and chi-squared test where appropriate.

Results Complete data were available for 274 patients. Population demographics were as follows $-61.3 \%$ male, mean age $56.3 \pm 2.1$ years, median APACHE II 20 (IQR 15 to 27), crude ICU mortality 25.6\% and mean length of stay $5.2 \pm 0.7$ days. Incidence of thrombocytopenia (platelet count $\left.<150 \times 10^{\%} / 1\right)$ was $29.8 \%$ on admission to the ICU, increasing to 46.9\% when considering the entire ICU stay. Comparing survivors and nonsurvivors, nonsurvivors had a lower trough platelet count $\left(140 \times 10^{\%} /\right.$ vs $\left.181 \times 10^{9} / 1, P=0.005\right)$. Patients with platelet counts less than $50 \times 10^{9} / 1$ have the highest mortality ( $45.7 \%$ vs $27.6 \%, P=0.006)$. Platelet data were used to construct an ROC curve, demonstrating an area under the curve of $0.66, P<0.001$. Platelet count correlated negatively with APACHE II ( $r=$ $-0.20, P<0.001)$ but did not correlate significantly with length of stay or duration of mechanical ventilation.

Conclusions Thrombocytopenia occurs in $47 \%$ of our ICU patients. It correlates significantly with severity of illness as measured by APACHE II and decreasing platelet count correlates with increasing mortality, with the highest mortality in those with a trough ICU platelet count of less than $50 \times 10^{9} / \mathrm{l}$. The ROC characteristics also demonstrate that the platelet count is a useful predictor of mortality.

\section{Reference}

1. Strauss R, et al:: Newly developed thrombocytopenia in medical intensive care patients. Crit Care 2000, 4(Suppl 1):P23.

\section{P366}

Evaluation of the particle immunofiltration anti-platelet factor 4 rapid assay in MICU patients with thrombocytopenia

DM Andrews, DH Kett, Y Fajardo Aquino, GF Cubillos, AS Castelbalnco, DL Seckinger

University of Miami/Jackson Memorial Hospital, Miami, FL, USA

Critical Care 2010, 14(Suppl 1):P366 (doi: 10.1186/cc8598)

Introduction Heparin-induced thrombocytopenia (HIT) is a lifethreatening and limb-threatening, immune-mediated, prothrombotic disease resulting from an interaction between heparin and platelet factor 4 (PF4). Due to the many causes of thrombocytopenia in critically ill patients, the diagnosis of HIT is difficult, requiring both a high clinical suspicion and confirmatory testing. The ELISA test is the most commonly performed method to detect anti-PF4 antibodies; however, the ELISA results generally take one or more days to report. The particle immunofiltration assay (PIFA) test has the advantage of being done rapidly; with results generally available within 1 hour.
Methods Starting in July 2009, patients in our MICU were screened daily for thrombocytopenia; defined as either a platelet count that decreased by at least 30\% from baseline or an absolute platelet count less than 150,000 . Patients with thrombocytopenia underwent both PIFA and GTI ELISA testing for anti-PF4 antibodies. PIFA is a qualitative test reporting results as positive or negative. The GTI ELISA test reports an optical density $(\mathrm{OD})$, with an OD greater than 0.40 considered positive. Patients were followed through hospitalization.

Results One hundred and thirty-eight patients were admitted to the MICU, with 47 developing thrombocytopenia. The PIFA results were negative in 22 patients, positive in 21 patients and inconclusive in four patients. For all patients with a negative PIFA result, the GTI ELISA test was negative. All four inconclusive PIFA tests had negative GTI ELISA tests. In patients with PIFA-positive results, two GTI ELISA tests were positive and 19 tests were negative. A serotonin release assay (SRA) was done in 13 patients with discordant PIFA/GTI ELISA tests, of which one SRA result was positive. None of the 47 patients with thrombocytopenia developed thrombotic complications.

Conclusions Our analysis suggests that a negative PIFA test can quickly exclude the presence of anti-PF4 antibodies and therefore HIT as the cause of the thrombocytopenia. The PIFA test can be used as a rapid screening procedure for patients with possible HIT, and, when negative, decrease the exposure of thrombocytopenic MICU patients to alternative anticoagulation. The clinical significance of a positive PIFA test requires further study and must be evaluated in conjunction with clinical judgment and, as appropriate, additional testing for anti-PF4 antibodies.

\section{P367}

Heparin-induced thrombocytopenia incidence in the ICU: preliminary results

F Barbani', R Marcucci'2, S Degl'Innocenti ${ }^{3}$, A Rogolino'2, S Fedi' ${ }^{2}$, A Gori², M Bonizzoli3 , G Zagli3 ${ }^{3}$, A Peris ${ }^{3}$, R Abbate ${ }^{2}$

'Postgraduate School of Anesthesia and Intensive Care, University of Florence, Italy: ${ }^{2}$ Department of Critical Care Medicine and Surgery, University of

Florence, Italy; ${ }^{3}$ Careggi Teaching Hospital, Florence, Italy

Critical Care 2010, 14(Suppl 1):P367 (doi: 10.1186/cc8599)

Introduction Heparin-induced thrombocytopenia (HIT) is a lifethreatening and limb-threatening immune-mediated prothrombotic complication caused by heparinic drugs. The aim of this study was to evaluate the incidence of HIT in a mixed ICU population.

Methods Patients admitted to the ICU of a regional referral center (Careggi Teaching Hospital, Florence, Italy) who underwent unfractionated heparin (UFH) or low-molecular-weight heparin (LMWH) administration were prospectively observed from October 2008. Exclusion criteria: haematological malignancy, platelet count $<50,000 / \mathrm{mm}^{3}$ before anticoagulant treatment. After anticoagulant administration start, patients showing a fall of platelet count $>50 \%$ or a nadir $<150,000 / \mathrm{mm}^{3}$ were clinically evaluated with the 4T score (4Ts) [1]: cases with a 4Ts of at least 2 were investigated with the laboratory antigen assay, the ELISA. Positive tests underwent to a functional confirmation test, the heparin-induced platelet activation (HIPA) test [2].

Results Preliminary results refer to the period from the start to August 2009. Patients admitted to the ICU were 369; 327 enrolled for the study. Clinical evaluation with 4Ts was performed for 31 patients, 23 of 31 (74\%) had a value of at least 2; eight of 23 were on UFH and the remaining 15 on LMWH. Potential etiologies of thrombocytopenia have been considered: 21 patients were septic, three were on extracorporeal membrane oxygenation, two were admitted to the ICU due to cardiogenic shock. The ELISA test resulted positive in three of 23 (13\%) suspected HIT cases; none of the three cases resulted positive in the HIPA test, so HIT was excluded. The medium 4Ts of the 23 suspected HIT cases was $4.1 \pm 1.7$ whereas $4 \mathrm{TS}$ did not differ between patients with positive and negative ELISA test.

Conclusions HIT incidence in the ICU is extremely rare, with no cases shown in this study. HIT was suspected in 23 of 327 patients $(7.03 \%)$, but the HIT incidence was $0.0 \%$. ICU patients show numerous potential etiologies of thrombocytopenia and in this population sepsis was the most common.

References

1. Warkentin TE, et al: Curr Hematol Rep 2003, 2:148-157.

2. Greinacher A, et al.: Thromb Haemost 1991, 66:734-736. 
P368

Treatment of the coagulation deficit during extracorporeal circulation in a porcine model by a prothrombin complex concentrate

F Kaspereit, S Hoffman, I Pragst, G Dickneite

CSL Behring, Marburg, Germany

Critical Care 2010, 14(Suppl 1):P368 (doi: 10.1186/cc8600)

Introduction Many procedures in cardiac surgery require extracorporeal circulation (EC). During EC, blood is pumped from a venous line to an oxygenator, cooled and infused back via an arterial line. This procedure is known to compromise the coagulation system, especially under prolonged hypothermia. The aim of the present study was to investigate the effects of EC and hypothermia on the coagulation system in a porcine model. The efficacy of a substitution therapy with a prothrombin complex concentrate (PCC) regarding normalization of coagulation and decrease in hemorrhage was evaluated.

Methods A total of 17 male anesthetized pigs were included in the study. EC was performed in 12 animals by a hollowfiber oxygenator with a priming solution containing saline, HES and heparin. Five animals without EC served as a control. The coagulation system was characterized by thromboelastography (TEG), thrombin generation, coagulation factor levels, platelet numbers and function (aggregation). A GoreTex patch was inserted into the carotid artery, bleeding occurred from the stitch channels. The effects of intravenous substitution therapy by PCC (Beriplex $\mathrm{P} / \mathrm{N}, 30 \mathrm{U} / \mathrm{kg}, n=6)$ compared with placebo $(n=6)$ on bleeding from the stitch channels was investigated.

Results EC and hypothermia compromised the coagulation system. Coagulation factor levels were decreased, TEG and thrombin generation became pathologic. Platelet count and aggregation were decreased. Blood loss from the stitch channels of the GoreTex patch was doubled in the EC group when compared with animals without EC. After substitution therapy with PCC, blood loss decreased significantly from $83.0 \pm 48.4 \mathrm{ml}$ in the $E C+$ placebo to $27.2 \pm 35 \mathrm{ml}$ in the $E C+P C C$ group $(P<0.025$, Wilcoxon test; Figure 1). PCC normalized the impaired coagulation.

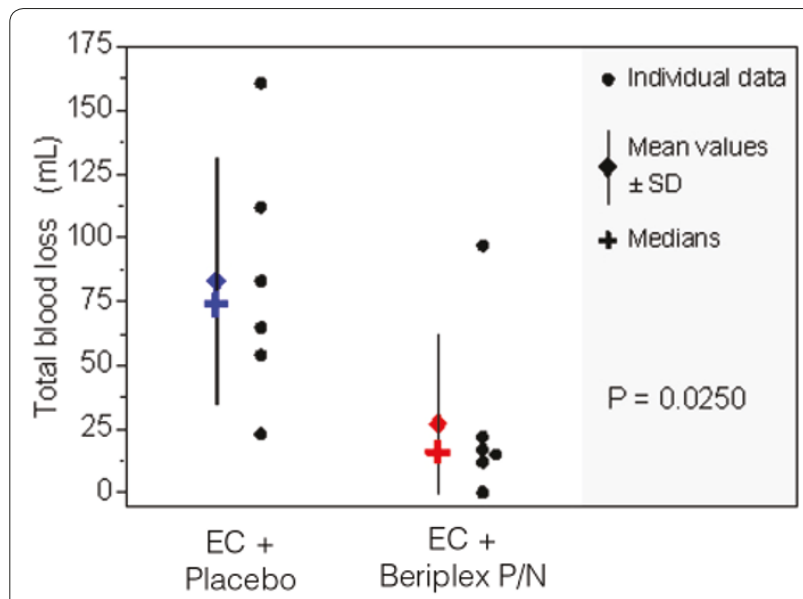

Figure 1 (abstract P368). Blood loss from a GoreTex Patch after EC.

Conclusions Hypothermia and EC led to a compromised coagulation system, resulting in increased hemorrhage. The deficit in coagulation could be overcome by substitution therapy with PCC. Elevated blood loss from stitch channels was decreased significantly after treatment with PCC. It was concluded that PCC should be beneficial in patients undergoing cardiovascular surgery with an EC-induced coagulopathy.
P369

Fibrinogen in dilutional coagulopathy: a dose study in pigs

J Martini', S Maisch', W Martini², W Streif', D Fries'

'Medical University Innsbruck, Austria; ${ }^{2}$ US Army Institute for Surgical

Research, San Antonio, TX, USA

Critical Care 2010, 14(Suppl 1):P369 (doi: 10.1186/cc8601)

Introduction Dilutional coagulopathy following massive bleeding is the result of clotting factor dilution and impaired fibrin polymerization after infusion of colloidal plasma expanders. Although fibrinogen has been clinically used to treat dilutional coagulopathy $[1,2]$, the effects of fibrinogen dosages on normalizing coagulation function is unclear. This study investigated the effect of six different fibrinogen dosages (range: 37.5 to $600 \mathrm{mg} / \mathrm{kg}$ ) on ROTEM ${ }^{\oplus}$ parameters and overall blood loss in a pig model for dilutional coagulopathy.

Methods Forty-two pigs underwent a $60 \%$ hemodilution with Voluven ${ }^{\oplus}$ (HES130/0.4). A standardized bone injury was performed after the completion of hemodilution. Animals were then randomized to receive $37.5,75,150,300,450$ or $600 \mathrm{mg} / \mathrm{kg}$ fibrinogen (FGTW, LFB, France) or $500 \mathrm{ml}$ saline. Four hours after fibrinogen administration a standardized liver injury was performed. Animals were then observed for 2 hours or until death. Blood loss was measured and tissue samples were collected at the end of the study. Hemodynamic and coagulation parameters were measured at baseline (BL), after hemodilution, 15 minutes, 1, 2 and 4 hours after drug infusion and 2 hours after liver injury or right before the animal's death. Statistical significance was set at $P<0.05$.

Results Fibrinogen dosages of $150 \mathrm{mg} / \mathrm{kg}$ and higher completely reversed dilutional coagulopathy: the maximum clot firmness (MCF) which was decreased after hemodilution ( $36 \pm 3$ vs $65 \pm 4 \mathrm{~mm}$ at $B L, P<0.05$ ), returned to $B L$ levels after fibrinogen administration $(69 \pm 5 \mathrm{~mm})$. Blood loss from bone and liver injury significantly decreased with increased fibrinogen dosages: $42 \pm 19$ (sham), $34 \pm 14$ (75 mg/kg), $29 \pm 13$ (150 mg/kg) and $28 \pm 10 \mathrm{ml} / \mathrm{kg}$ BW $(600 \mathrm{mg} / \mathrm{kg})$. No thrombotic events occurred.

Conclusions In a swine model of $60 \%$ hemodilution with bone and liver injury, fibrinogen administration (150 mg/kg and above) normalized MCF and decreased blood loss. Infusions of 12 times the dosage recommended in humans did not induce hypercoagulability.

\section{References}

1. Fenger-Eriksen C, et al: J Thromb Haemost 2009, 75:795-802.

2. Mittermayr M, et al.: Anesth Analg 2007, 105:905-917.

\section{P370}

Tranexamic acid reduces blood loss in postpartum haemorrhage A Ducloy-Bouthors' ${ }^{1}$, F Broisin'2 ${ }^{2}$ H Keita ${ }^{3}$, S Fontaine ${ }^{4}$, S Depret ${ }^{1}$, F Legoeff ${ }^{5}$, L Mandelbrot ${ }^{3}$, R Rudigoz $^{2}, B$ Jude

'Centre Hospitalier Regional Universitaire, Lille, France; ${ }^{2}$ Hôpital Croix Rousse HCL, Lyon, France; ${ }^{3}$ Hôpital Louis Mourrier APHP, Colombes, France; ${ }^{4}$ Maternité Monaco CH, Valenciennes, France; ${ }^{M}$ Maternité Paul Gellée, Roubaix, France Critical Care 2010, 14(Suppl 1):P370 (doi: 10.1186/cc8602)

Introduction Postpartum haemorrhage (PPH) is a leading cause of maternal death. Present guidelines for PPH management focus on uterotonic treatments, while the potential efficacy of antifibrinolytic drugs has been poorly explored in this indication.

Methods After approval by the ethics committee, we randomly assigned women with PPH $>800 \mathrm{ml}$ after vaginal delivery to receive intravenous tranexamic acid (TXA) $(4 \mathrm{~g}$ within 1 hour, followed by $1 \mathrm{~g} /$ hour during 6 hours) (TXA group) or no antifibrinolytic treatment (Control group). Monitoring and management were performed according to the guidelines. Additional procoagulant treatments (coagulant concentrates, fresh frozen plasma, platelets) were used only when PPH became intractable ( $>2,500 \mathrm{ml}$ or $>500 \mathrm{ml} / 30$ minutes). Embolization or surgery was performed when considered clinically necessary. Blood loss, maternal morbidity and safety were collected at four time points: inclusion (T1), $\mathrm{T} 1+30$ minutes (T2), $\mathrm{T} 1+2$ hours (T3), and $\mathrm{T} 1+6$ hours (T4). The study plan, based on the hypothesis that TXA would induce a $20 \%$ blood loss reduction with an a risk of $5 \%$ and a $\beta$ risk of $10 \%$, required the inclusion of 72 patients in each group. Statistical analysis used the SAS system.

Results One hundred and forty-four patients were included, 72 in the TXA group and 72 in the control group. Management data were similar between the two groups. In the TXA group, as compared with the control 
group: blood loss was 46\% lower between T2 and T3 $(P=0.026)$, and $49 \%$ lower between T2 and T4 ( $P=0.0012)$; bleeding duration was shorter ( $31 \pm$ 28 vs $65 \pm 95$ minutes, $P=0.004)$; and maternal morbidity was reduced (hemoglobin drop $>4 \mathrm{~g} / \mathrm{dl}: n=15$ vs $34, P<0.001$; blood transfusion requirement: $n=14$ vs 20, $P=$ NS; overall number of transfused packed red blood cells: $n=42$ vs 62, $P<0.001$; number of severe PPH (Charbit criteria 1): $n=23$ vs $36, P=0.028)$. Invasive procedures were needed in four women in the TXA group and in seven women in the control group $(P=N S)$. Transient visual and digestive side effects were observed more frequently in the TXA group than in the control group (17 vs $4 P=0.028$ ).

Conclusions These results show, for the first time, that TXA, when administered early in the management of PPH, reduces blood loss and maternal morbidity, with only minor and transient side effects. As TXA is a readily available and cheap hemostatic agent, we strongly suggest that it should be further investigated as an adjuvant treatment in PPH.

\section{Reference}

1. J Thromb Haemost 2007, 5:266-273.

\section{P37'}

Regional variation of the off-licence use of rFVIla for patients with uncontrolled haemorrhage in England

MH Spivey, RL Eve, MR Duffy

Derriford Hospital, Plymouth, UK

Critical Care 2010, 14(Suppl 1):P371 (doi: 10.1186/cc8603)

Introduction In 1999 the first publication appeared of the use of recombinant factor VIIa (rFVIIa) for uncontrolled haemorrhage [1]. Since then there has been widespread international use of rFVIla in this setting. Consensus guidelines have been published [2], but there is much variation in its administration [3].

Methods Review of the blood bank databases of all six acute hospitals in the southwest of England between January 2000 and February 2009 was undertaken to reveal all prescriptions of rFVIla. Case notes were analysed and the administration of all blood products for 24 hours either side of rFVIla administration was extracted from the blood bank databases.

Results Eighty-two patients were identified who had received off-licence rFVIla. Of these, full data were available on 67 patients. There was unequal use between hospitals; Plymouth had $33 \%$ of use compared with Taunton, with only $4 \%$. A total $65.7 \%$ of patients were male and the mean age was 56.6 years. The mean APACHE II score was 18.4 ( \pm 7.4 ) and $40.3 \%$ patients died in $\leq 96$ hours. The mean dosage administered was $80.9 \mu \mathrm{g} / \mathrm{kg}( \pm 21.3)$. The number of annual prescriptions shows a bimodal pattern with peaks in 2005 and 2008. The distribution of usage was predominantly with general and vascular surgical patients (39\%), but trauma (14.9\%), obstetrics (13.4\%) and cardiac surgery (13.4\%) were the next most frequent specialties. rFVIIIa was given after a mean of 17.6 ( \pm 11.2) units of packed red cells, $8.8( \pm 5.9)$ units of fresh frozen plasma and $2.3( \pm 1.9)$ pooled platelets. Following rFVIla transfusion of all blood products was markedly less, and only three patients received a repeat dose of $\mathrm{rFV}$ lla. The prothrombin time decreased from a mean of 18.9 seconds to 13.9 seconds following rFVIla. Thirty-six per cent of patients had $<50$ platelets at administration and $40 \%$ were acidotic with $\mathrm{pH}<7.2$. Sixty-eight per cent patients had a haematocrit $<0.25$ and $30 \%$ of patients were hypothermic.

Conclusions Off-label prescribing of rFVlla varies widely across the southwest region. Its use as an adjunctive therapy for uncontrolled haemorrhage has often been unsuccessful. Our analysis of prescribing rFVIla in this setting reflects the practice of the larger Australian series in terms of indications and dosage; however, the mortality at 28 days of $32 \%$ is lower than in our series [3]. There is still heterogeneity in prescribing rFVIla and its use does not conform to European guidelines [2]

\section{References}

1. Kenet G, et al:: Lancet 1999, 354:1879.

2. Vincent JL, et al:: Crit Care 2006, 10:R120.

3. Willis CD, et al.: Int Med J 2009. [Epub ahead of print. PMID: 19712199]
P372

Early use of factor VIla in cardiac surgery is associated with lower rates of blood transfusions without impact on outcome

F Galas, L Hajjar, F Zampieri, A Roquim, R Kalil, V Guimaraes, J Auler Jr Heart Institute, São Paulo, Brazil

Critical Care 2010, 14(Suppl 1):P372 (doi: 10.1186/cc8604)

Introduction Bleeding after cardiac surgery is a common state, associated with adverse effects. Factor VIla is a recombinant factor able to restore blood coagulation without significant adverse effects. We aimed to evaluate whether the early use of factor VIla reduces bleeding and the rates of transfusion.

Methods We studied 241 patients submitted to cardiac surgery who presented bleeding after heparin reversal and a first replacement of clotting factors. From these, 81 patients were submitted to valve procedures, 51 coronary artery bypass surgery (CABG), 60 valve + CABG, 35 aortic ascending surgery and 14 cardiac transplant. From these, 131 received factor VIla early (in the intraoperative room) and 110 received the drug in the ICU.

Results Both groups of patients presented a statistically significant reduction of blood transfusion after receiving factor Vlla $(P<0.001)$. The group of patients undergoing early use presented a lower rate of reoperations due to bleeding $(P<0.01)$, and received less units of red blood cells $(P<0.01)$, fresh frozen plasma $(P<0.01)$, cryoprecipitate and platelets $(P<0.01)$. Also, we detected a low incidence of infection in the group who received the factor in intraoperative room.

Conclusions Early use of factor VIla in patients who bleed after cardiac surgery is associated with lower rates of reoperations, lower rates of transfusion and lower incidence of infection. This suggests the drug should not be used as a last therapeutic tool and may be indicated earlier in the management of bleeding.

\section{Reference}

1. Napolitano LM, et al:: Red blood cell transfusion in adult trauma and critical care. Crit Care Med 2009, 37:3124-3157.

P373

Low-frequency hemoviscoelastography in monitoring coagulation disorders after abdominal surgery for cancer

O Tarabrin', V Grubnik1, P Pustovoyt², O Osipenko², S Shcherbakov', D Gavrichenko²

'Odessa Medical University, Odessa, Ukraine; ${ }^{2}$ Regional Clinical Hospital, Odessa, Ukraine

Critical Care 2010, 14(Suppl 1):P373 (doi: 10.1186/cc8605)

Introduction Venous thromboembolism is of the most common complications in cancer patients and may be due to the hypercoagulable state of malignancy and to its surgical treatment.

Methods Patients undergoing planned curative open surgery for abdominal cancer received MEDNORD (Ukraine Co. analyzer) analysis $(H V G)$, a viscoelastic test that measures clot formation and includes information on the cellular as well as the plasmatic coagulation system. We examined the efficacy of a variety of coagulation tests. A complete coagulation screen, activated clotting time, thromboelastography (TEG) and haemoviscoelastography (HVG) were performed before surgery, at the end of surgery, and on postoperative days 1,2,3, and 7; they were analyzed for the reaction time and the maximal amplitude (MA).

Results We calculated the elastic shear modulus of standard MA (Gt) and HVG MA $(\mathrm{GH})$, which reflect the total clot strength and procoagulatory protein component, respectively. The difference was an estimate of the platelet component (Gp). There was a 14\% perioperative increase of standard MA, corresponding to a $48 \%$ increase of Gt $(P<0.05)$ and an 80 to $86 \%$ contribution of the calculated Gp to Gt. We conclude that serial standard thromboelastography and the HVG viscoelastic test may reveal the independent contribution of platelets and procoagulatory proteins to clot strength. Using multiple linear regressions, all coagulation, TEG and HVG variables were used to model postoperative hypercoagulation. However, three components of the routine coagulation assay, including bleeding time, prothrombin time and platelet count, could be modeled to show prolonged postoperative hypercoagulability $(P<0.01)$. We conclude that all components of the HVG test reflect postoperative coagulopathies; these results suggest that it may be useful in determining the coagulation status of cancer patients perioperatively. 
Conclusions Postoperative hypercoagulability, occurring for at least 1 week after major cancer abdominal surgery, may be demonstrated by the HVG viscoelastotest. This hypercoagulability is not reflected completely by standard coagulation monitoring and the TEG HVG viscoelastotest provides a fast and easy-to-perform bedside test to quantify in vitro hemocoagulation.

\section{P374}

Reliability of the HemoCue ${ }^{\circledR}$ hemoglobinometer in critically ill patients

D Frasca, C Dahyot-Fizelier, A Medard, L Laksiri, F Petitpas, O Mimoz University Hospital, Poitiers, France

Critical Care 2010, 14(Suppl 1):P374 (doi: 10.1186/cc8606)

Introduction In the ICU, the hemoglobin concentration is currently estimated by portable hemoglobinometer and in emergency transfusion a decision can be based on that result. The aim of the study was to compare the HemoCue ${ }^{\circledast}$ with laboratory determination of the blood hemoglobin concentration in critically ill patients with a high risk of bleeding.

Methods A prospective observational study was conducted in three adult surgical ICUs of a university hospital. After ethic committee approval, 198 consecutive patients requiring 1,166 determinations of hemoglobin concentration were included. Hemoglobin was measured from an arterial blood sample at the laboratory $\left(\mathrm{Hb}_{\mathrm{m}}\right)$ and estimated at the bedside $\left(\mathrm{Hb}_{\mathrm{ea}}\right)$ using the same blood sampling and a portable hemoglobinometer (HemoCue $\left.{ }^{\oplus} \mathrm{Hb} 201+\right)$. Simultaneously, a capillary measurement $\left(\mathrm{Hb}_{\text {e.cap }}\right.$ ) was performed at the bedside using the same device.

Results The mean difference (bias, Figure 1) between $\mathrm{H}_{\text {becap }}$ and $\mathrm{Hb}$ was $-0.2 \mathrm{~g} / \mathrm{dl}(95 \% \mathrm{Cl},-0.3$ to -0.1$)$ and limits of agreement $-1.3 \mathrm{~g} / \mathrm{dl}^{\mathrm{m}}(95 \%$ $\mathrm{Cl},-1.4$ to -1.2$)$ to $1.7 \mathrm{~g} / \mathrm{dl}(95 \% \mathrm{Cl}, 1.6$ to 1.9$)$. Discrepancies between $\mathrm{Hb}_{\text {ecao }}$ and $\mathrm{Hb}_{\mathrm{m}}$ were greater than $1 \mathrm{~g} / \mathrm{dl}$ in $30.8 \%$ of cases. The accuracy of HemoCue ${ }^{\oplus}$ was not affected by the unit of hospitalization, the patients severity assessed by SAPS II score on admission, the site of lancet puncture (finger or ear), the administration of norepinephrine, the presence of hypothermia (body temperature $<36.3^{\circ} \mathrm{C}$ ) or by a hemoglobin level below $10 \mathrm{~g} / \mathrm{dl}$ or $8 \mathrm{~g} / \mathrm{dl}$. The bias between $\mathrm{H}_{\text {bea }}$ and $\mathrm{Hb}$ was $-0.1 \mathrm{~g} / \mathrm{dl}(95 \% \mathrm{Cl}$, -0.2 to 0.2$)$ and limits of agreement $-1.1 \mathrm{~g} / \mathrm{dl}(95 \% \mathrm{Cl},-1.2$ to -1.0$)$ and 1.0 $\mathrm{g} / \mathrm{dl}(95 \% \mathrm{Cl}, 0.9$ to 1.1$)$.

Conclusions Capillary HemoCue ${ }^{\oplus}$ is not accurate enough to base therapeutic decisions such as blood transfusion on. The performance of the method is improved with the use of arterial blood.

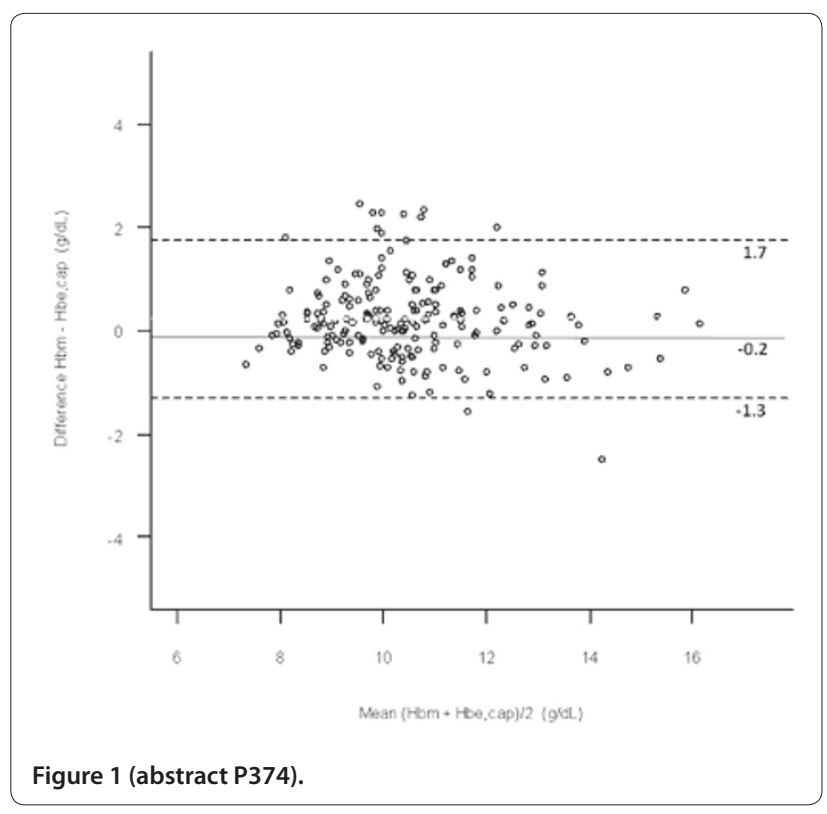

P375

Effect of transfusion, leukocyte-depleted blood product on onset of new septic shock and mortality in septic shock

H Hayami, S Nagai, A Sakurai, K Okamura, J Yamada, H Kawakami,

K Fujimoto, O Yamaguchi, Y Koide

Yokohama City University Medical Center, Yokohama, Japan

Critical Care 2010, 14(Suppl 1):P375 (doi: 10.1186/cc8607)

Introduction A number of studies have observed decreased survival associated with transfusion. Leukocyte-mediated immunosuppression may contribute to postoperative infectious complications. There is evidence for a benefit with the use of leukocyte reduced transfusion, but it is unclear among septic shock patients. In Japan, leukocyte-depleted blood products have been used since 2007. We assessed the effect of transfusion and the efficacy of the use of leukocyte-depleted blood products for new onset of septic shock and mortality among patients with septic shock.

Methods A total of 101 septic shock patients at a single university general ICU were enrolled in the study. A target hemoglobin ( $\mathrm{Hb})$ concentration of $10 \mathrm{~g} / \mathrm{dl}(\mathrm{Ht}$ 30\%) was considered for allogeneic red cell transfusion (to maintain central venous saturation $>70 \%$ ) during the early phase of severe sepsis, and $\mathrm{Hb}$ was kept in the range of 7 to $9 \mathrm{~g} / \mathrm{dl}$ in the stable phase except for the patients with particular disease (acute coronary syndrome, and so forth). Fresh frozen plasma was used to keep PT-INR $<1.67$, and platelet transfusion was done due to the patient's condition (for example, postoperative, and so forth).

Results Eighty-six patients (85\%) received transfusion (group T: age $68 \pm$ 12, APACHE $\|$ 25.9 \pm 9.5 ) and 15 received no blood product (group NT: age $63 \pm 14$, APACHE II $27.4 \pm 3.2$. . Frequent sites of infection were the lung $(46 \%)$, peritoneum $(17 \%)$, mediastinum (12\%), and gastrointestinal tract (7\%) (NS between groups). Overall mortality for group T vs group NT was $27 / 86(31 \%)$ vs $3 / 15(20 \%)(P=0.54)$. Onset of new septic shock for group T vs group NT was $27 / 86(31 \%)$ vs $4 / 15(27 \%)(P=0.71)$. Survivors received $26.6 \pm 31.2$ Japanese units of total blood product and $44.6 \pm 35.6$ units for patients who died, respectively (1 American unit is almost equivalent to 2 Japanese units) $(P=0.013)$. For group T, 56 patients received leukocytereduced blood product (LRB) and 29 received ordinary product (Cont.). Overall mortality for LRB vs Cont. was 40/56 (71\%) vs 18/29 (62\%) $(P=0.38)$. Conclusions The total amount of blood products received was highly associated with increased mortality, but there was no obvious adverse effect of transfusion to the onset of new septic shock. No association between overall mortality and the use of leukocyte depleted blood was identified in patients with septic shock.

\section{P376}

Transfusion Requirements After Cardiac Surgery (TRACS) study: preliminary data of a randomized controlled clinical trial L Hajjar', F Galas', M Sundin', R Nakamura', C Silva', T Mauad², P Pomerantzeff', P Bueno', R Kalil Filho', JL Vincent', J Auler Jr 'Heart Institute, São Paulo, Brazil;'25ão Paulo University, São Paulo, Brazil; 3Erasme Hospital, Brussels, Belgium Critical Care 2010, 14(Suppl 1):P376 (doi: 10.1186/cc8608)

Introduction There are no prospective studies comparing outcomes between restrictive or liberal strategies in cardiac surgery. This study is a double-blind randomized study to determine whether a restrictive strategy of red cell transfusion and a liberal strategy produced equivalent results in patients submitted to cardiac surgery.

Methods Until November 2009 we enrolled 380 patients undergoing elective cardiac surgery and randomly assigned 185 patients to a restrictive strategy of transfusion, in which red cells were transfused if the hematocrit concentration dropped below 24\%, and 195 patients to a liberal strategy, in which transfusions were given when the hematocrit fell below $30 \%$. Both transfusion strategies were followed into the operative room and during the ICU stay. We compared the rates of complications and death from all causes during the hospital stay and clinical outcomes of patients after 90 days. Adult patients, after written informed consent, were allocated if they would be submitted to elective primary surgery or were redone adult cardiac surgical patients for coronary artery bypass grafting, valve procedure or combined procedures.

Results Overall, hospital mortality was similar in the two groups ( $4.7 \%$ vs $5.3 \%, P=0.11)$. The rates of complications were similar in the two groups 
(28.3\% in the restrictive-strategy group and $26.1 \%$ in the liberal-strategy group, $P=0.33)$. The ICU stay was significantly lower in the restrictivestrategy group ( 2.2 days vs 4.1 days, $P=0.05$ ). There was no difference in 90-day mortality between groups.

Conclusions A restrictive strategy of red cell transfusion is at least as effective as and possibly superior to a liberal transfusion strategy in patients submitted to cardiac surgery.

\section{References}

1. Napolitano LM, et al: Clinical practice guideline: red blood cell transfusion in adult trauma and critical care. Crit Care Med 2009, 37:3124-3157.

2. Hébert PC, et al: Controversies in RBC transfusion in the critically ill. Chest 2007, 131:1583-1590.

P377

Analysis of red cell transfusion practices in patients without active haemorrhage over a 12-month period in a UK intensive care unit

RL Eve, MH Spivey, S Hart, MR Duffy

Derriford Hospital, Plymouth, UK

Critical Care 2010, 14(Suppl 1):P377 (doi: 10.1186/cc8609)

Introduction The deleterious effects of red blood cell (RBC) transfusion are well known [1] and restrictive transfusion practices are safe in patients without active haemorrhage [2]. Our objective was to determine transfusion practices in critically ill patients without evidence of ongoing bleeding to establish our conformity with published guidelines [3].

Methods All adult ICU patients receiving RBC transfusions between 1 September 2008 and 31 August 2009 were included in the analysis. Data were collected on demographics, APACHE II score, ICU and hospital length of stay (LOS), ICU and hospital mortality, presence of ischaemic heart disease (IHD), and pre/post-transfusion haemoglobin concentrations in $\mathrm{g} / \mathrm{dl}([\mathrm{Hb}])$. Subgroup analyses were performed for patients with IHD, age $<55$ years or APACHE $\| \leq 20$. We analysed patients with IHD because benefit from liberal transfusion has not been confirmed [1] whereas the latter two subgroups have shown a mortality benefit with restrictive transfusion [2]. Results A total of 1,723 patients were admitted to the ICU during the study period. Two hundred and five patients (11.9\%) received RBCs, of whom 47 had active bleeding and were excluded from further analysis. The remaining 158 patients (9.2\%) received a total of 477 units RBCs (median 2.0 units/patient, IQR 1.0 to 3.8). Median pre-transfusion [Hb] was 7.7 (IQR 7.3 to 8.2 ) with a post-transfusion [Hb] of 9.2(IQR 8.5 to 9.8). Median ICU and hospital LOS in days was 10.9 (IQR 4.9 to 18.8) and 26.9 (IQR 14.0 to 45.0). ICU and hospital mortalities were $34.2 \%$ and $44.9 \%$, respectively. Patients with acute IHD had a pre-transfusion [Hb] of 8.3(IQR 7.8 to 8.8) with a posttransfusion [Hb] of 9.4 (IQR 9.2 to 10.2) whilst patients with chronic IHD had values of 7.9 (IQR 7.4 to 8.4) and 9.4 (IQR 8.5 to 9.8), respectively. In patients aged $<55$ years, pre/post-transfusion values were 7.5 (IQR 7.1 to 8.0 ) and 9.2 (IQR 8.5 to 10.0 ), respectively, and patients with APACHE $\| \leq 20$ had values of 7.7 (IQR 7.3 to 8.2) and 9.2 (IQR 8.5 to 9.8), respectively.

Conclusions $[\mathrm{Hb}]$ transfusion triggers were $>7$ in all subgroups and post-transfusion $[\mathrm{Hb}]$ was $>9$. Transfusion strategies were too liberal. It is important to restrict transfusions to limit morbidity and mortality and to make efficient use of RBCs [4]. This study demonstrates the importance of regular audit and will be used to inform local guidelines.

\section{References}

1. Marik PE, etal: Crit Care Med 2008, 36:2667-2674.

2. Hebert PC, et al:: N Engl J Med 1999, 340:409-417.

3. Thomas D, et al:: Red Cell Transfusion 2 [http://www.aagbi.org/publications/ guidelines/docs/red_cell_08.pdf]

4. Joseph BG, et al: Transfusion 2009, 49:2060-2069.

\section{P378}

Effects of red blood cell transfusion in central venous saturation and lactate levels in patients with septic shock

BF Mazza, M Assunção, F Freitas, M Jackiu, FR Machado

Universidade Federal de São Paulo, Brazil

Critical Care 2010, 14(Suppl 1):P378 (doi: 10.1186/cc8610)

Introduction Transfusion of red blood cells has been used with the objective of improving oxygen delivery to the tissues in the critically ill patient. However, the appropriate hemoglobin $(\mathrm{Hb})$ level is still controversial in septic shock patients [1,2]. The objective of this study was to evaluate the effect of red blood cell transfusion on the central venous saturation $\left(\mathrm{SvCO}_{2}\right)$ and lactate levels in patients with septic shock with two different levels of $\mathrm{Hb}$

Methods Patients with less than 48 hours of septic shock and Hb levels under $9.0 \mathrm{~g} / \mathrm{dl}$ were randomized to maintain $\mathrm{Hb}$ over $9.0 \mathrm{~g} / \mathrm{dl}$ (Group 1) or over $7.0 \mathrm{~g} / \mathrm{dl}$ (Group 2). Before and up to 1 hour after each transfusion, $\mathrm{Hb}$, lactate and $\mathrm{SvCO}_{2}$ were determined. Results were expressed as median ( $25 \%$ to $75 \%$ percentile) or percentage and analyzed through chi-square or Mann-Whitney test. $P<0.05$ was considered significant.

Results Forty-six patients were included with a total of 74 transfusions, being 39 in Group 1 and 35 in Group 2, without any difference in demographic or hemodynamic data between the groups. Before and after $\mathrm{Hb}$ levels were 8.5 (8.2 to 8.7) $\mathrm{g} / \mathrm{dl}$ and 9.4 (9.1 to 9.5$) \mathrm{g} / \mathrm{dl}$ in Group $1(P=0.000)$ and 6.8 (6.6 to 6.9) $\mathrm{g} / \mathrm{dl}$ and $7.6(7.4$ to 7.8$) \mathrm{g} / \mathrm{dl}$ in Group 2 $(P=0.000)$. A reduction in median lactate after transfusion was found in Group 2 patients (22.0 (18.1 to 29.1) mg/dl and 20.0 (16.3 to 25.2$) \mathrm{mg} / \mathrm{dl}$, $P=0.005$ ) but not in Group 1 (17.2 (16.3 to 23.9$) \mathrm{mg} / \mathrm{dl}$ and 18.1 (15.4 to $21.8) \mathrm{mg} / \mathrm{dl}, P=0.23)$. The same occurred regarding $\mathrm{SvcO}$, with improved values only in Group 2 (68.0 (64.0 to 72.0$) \%$ and 72.0 (69.0 to 75.0$) \%$ in Group 2, $P<0.000$ ) and not in Group 1 (72.0 (69.0 to 74.0$) \%$ and $72.0(71.0$ to 73.0$) \%, P=0.98)$. In patients with altered lactate levels a reduction was found regardless of the basal levels of hemoglobin $(P=0.002$ and $P=0.001$ for Groups 1 and 2, respectively). The same was demonstrated in patients with $\mathrm{SvCO}_{2}<70 \%(P=0.007$ and $P=0.000$ for Groups 1 and 2 , respectively). On the other hand, patients with normal levels of both perfusion parameters did not present a worsening of those variables, either in Group 1 or Group 2.

Conclusions Red blood cell transfusion improved tissue perfusion in patients with signs of hypoperfusion regardless of basal levels of $\mathrm{Hb}$. Transfusion did not seem to worsen tissue perfusion in patients without signs of hypoperfusion, even in the group with $\mathrm{Hb}$ levels above $9.0 \mathrm{~g} / \mathrm{dl}$.

\section{References}

1. Hebert PC: NEngl J Med 1999, 340:409-417.

2. Rivers E: N Engl J Med 2001, 345:1368-1377.

\section{P379}

\section{Massive transfusion in paediatric trauma: a single-centre} experience

R Hearn, M Tecle, S Rajan, N Edmonds

The Royal London Hospital, London, UK

Critical Care 2010, 14(Suppl 1):P379 (doi: 10.1186/cc8611)

Introduction Uncontrolled haemorrhage is a leading cause of early mortality in trauma. Administration of blood products, packed red cells and fresh frozen plasma (PRBC:FFP) in the ratio of 1:1 is associated with improved mortality in major haemorrhage in adult trauma. However, there is little evidence available for the management of major haemorrhage in the context of paediatric trauma.

Methods We retrospectively analysed the local paediatric trauma database of all children following trauma call activation on arrival at the emergency department of The Royal London Hospital, a major urban trauma centre in London. The study period was over 15 months, May 2008 to August 2009. During this period, there has been no major haemorrhage policy within the centre for paediatric trauma. We collected data on demographic profile, injury severity scores, blood products transfused in the first 24 hours and the outcomes. We defined massive transfusion as the requirement of packed red cells $>40 \mathrm{ml} / \mathrm{kg}$ in the first 4 hours or $>80 \mathrm{ml} / \mathrm{kg}$ in the first 24 hours.

Results Two hundred and twenty-seven children presented to the emergency department during this period following major trauma call activation. The median age at presentation was 10.2 years. Thirteen (5.7\%) children had major haemorrhage. The median ISS was 35 (IQR 10 to 60). All but one were males. Three had penetrating trauma, one of whom made it to theatre, but all died. Four had emergency damage control surgery. Abnormal results were seen in three patients, each having one abnormal result (INR $=1.9$ and $\mathrm{APTT}=86$, low Hb $=7.6$, thrombocytopaenia $=63$ ). Eight of the 13 patients received additional blood products such as fresh frozen plasma, platelets and cryoprecipitate. However, no patient received the ratio of blood products of PRBC:FFP of 1:1 as practised in adult trauma. Two patients had no admission bloods done. Worsening coagulation 
parameters were seen in two patients when measured post transfusion and the remaining 11 patients did not have routine monitoring of blood parameters post transfusion. Eight (62\%) patients died, of which seven died in the emergency department.

Conclusions Major haemorrhage is associated with a very high mortality in severely injured children. We recommend rigorous monitoring of laboratory parameters to guide appropriate administration of blood products. There is a need for instituting a major haemorrhage policy in paediatric trauma and consideration of point-of-care testing of blood parameters.

\section{P380}

Cytokine levels evaluation during acute isovolemic anemia M Kahvegian', DT Fantoni², DA Otsuki', CA Holms', CO Massoco³, JO Auler Jr

'Faculdade de Medicina da Universidade de São Paulo, Brazil;' ${ }^{2}$ School of Veterinary Medicine, Universidade de São Paulo, Brazil; 3 Sales Gomes Consulting, São Paulo, Brazil

Critical Care 2010, 14(Suppl 1):P380 (doi: 10.1186/cc8612)

Introduction To date there is poor evidence that documents the benefit of red blood cell transfusion on clinical outcome. Acceptance of a lower transfusion trigger in the perioperative period requires detailed information regarding the effects of volume replacement in different organs and systems. Since fluids can have a widely divergent impact on the immune response, this study was undertaken to determine the cytokine levels in a model of acute isovolemic anemia (AIA) with hydroxyethyl starch (HES) and gelatin (GEL).

Methods Twenty-one pigs were anesthetized, instrumented and randomized into three groups: Control, HES and GEL. Animals in the HES and GEL groups were submitted to acute isovolemic anemia to a target hematocrit of 15\% with volume replacement performed with HES 130/0.4 and GEL at a 1:1 ratio. The withdrawn blood was returned to the animals 120 minutes after the end of AIA. TNF, IL-1, IL- 6 and IL-10 measurements were performed with blood samples collected at the femoral vein at the following time points: Baseline, after instrumentation (INST), immediately after AIA (AIA), 60 minutes after AIA (60AIA), 120 minutes after AIA (120AIA) 60 minutes after blood infusion (60BI) and 120 minutes after blood infusion (120BI). The cytokines were measured in serum by a commercially available ELISA with specific monoclonal antibodies. Two-way analyses of variance (ANOVA) and a Tukey test were used to assess its significance $(P<0.05)$.

Results TNF varied significantly among the groups in different time points (AIA: Control, $91 \pm 16$ and GEL, $221 \pm 61 ; P<0.05 ; 60 \mathrm{AIA}$ : Control, $76 \pm$ 15; HES, $172 \pm 42 ; P<0.05$ and GEL, $323 \pm 77 ; P<0.001$; 120AIA: Control, $98 \pm 6$ and GEL, $304 \pm 66 ; P<0.01 ; 120 \mathrm{Bl}:$ Control, $71 \pm 7$ and GEL, $211 \pm$ 71; $P<0.05)$. In relation to IL-1, only in the GEL group (224 \pm 56 ) was verified a statistical significant at time point 60AIA when compared with Control $(84 \pm 17 ; P<0.05)$. When IL-6 levels were studied, GEL presented a statistically significant at 60AIA (GEL, $331 \pm 65$ ) when compared with Control (146 $\pm 19 ; P<0.05)$. Serum IL-10 levels in GEL-treated animals were statistically significantly elevated at 60AIA (Control, $17 \pm 3$ and GEL, $46 \pm$ 12; $P<0.01$ ) and 120BI (Control, $21 \pm 5$ and GEL, $59 \pm 11 ; P<0.05$ ).

Conclusions Fluid replacement influences the cytokine measurements during acute isovolemic anemia expressed by serum TNF, IL-1, IL-6 and IL-10 increases, especially in GEL group.

\section{P381}

Haemodynamic alterations in community-acquired sepsis at early stage

S Hettwer, JWilhelm, M Schürmann, H Ebelt, KWerdan

Martin-Luther-University, Halle (Saale), Germany

Critical Care 2010, 14(Suppl 1):P381 (doi: 10.1186/cc8613)

Introduction Community-acquired sepsis at an early stage is common, but haemodynamic alterations remain unclear. The aim of the study was to characterize cardiovascular alterations in patients of our ProFS (monocentric observational) study, which was to characterize patients with sepsis in the emergency department.

Methods Systemic vascular resistance (SVR) and cardiac output (CO) were measured non-invasively using a TaskForce monitor (CNSystems, Graz, Austria) after admission, 24 hours and 72 hours. Indexed values were calculated (SVRI (dyn:second $\left./ \mathrm{cm}^{5} / \mathrm{m}^{2}\right), \mathrm{Cl}\left(1 /\right.$ minute $\left./ \mathrm{m}^{2}\right)$ ). Procalcitonin $(\mathrm{PCT}, \mathrm{ng} / \mathrm{ml}$ ) was measured in serum.

Results A sample of 64 patients of 208 included patients received haemodynamic examination. Mean age was $61.8 \pm 18.0$ years, $62.7 \%$ were male. Patients were divided by $\mathrm{PCT}<2$ and $\geq 2$. Age, gender and previous medical history were comparable in both groups. The heart rate was $99.8 \pm 21.6$ vs $104.6 \pm 23.0 /$ minute $(P=N S)$ and the mean artery pressure was $89.5 \pm 15.6$ vs $81.6 \pm 21.5 \mathrm{mmHg}(P<0.01)$. Mean SVRI in patients with $\mathrm{PCT}<2$ was $2,934 \pm 1,045$ vs $2,376 \pm 842, P<0.05$ at the time of admission. No difference was found after 24 hours $(2,959 \pm 1,002$ vs $2,924 \pm 1,324, P=$ NS) and 72 hours $(3,123 \pm 931$ vs 3,556 $\pm 1,524, P=N S)$. On the contrary, for patients with $P C T \geq 2$ the increase after 72 hours was significant $(P<0.05)$. Differences after admission could not be observed for $\mathrm{Cl}$ between patients with $\mathrm{PCT}<2$ vs $\geq 2$. Mean values after admission were $2.7 \pm 1.0$ vs $2.8 \pm 0.8$, after 24 hours $2.5 \pm 0.8$ vs $2.5 \pm 0.5$, and after 72 hours $2.3 \pm 0.7$ vs $2.3 \pm 0.6$ (all: $P=\mathrm{NS}$ ). See Figure 1

Conclusions Patients with community-acquired sepsis in the emergency department had an elevated SVRI. At the time of admission patients with high PCT had a significantly lower SVRI than patients with low PCT. Cardiac index at the time of admission was at a lower limit of normal range in all patients. These findings are in strong contrast to the classic pattern of sepsis on the ICU, where SVRI is keenly reduced and Cl elevated. They implicate that patients with sepsis in the emergency department may benefit more from application of fluid and positive inotrope substances than from vasopressor.
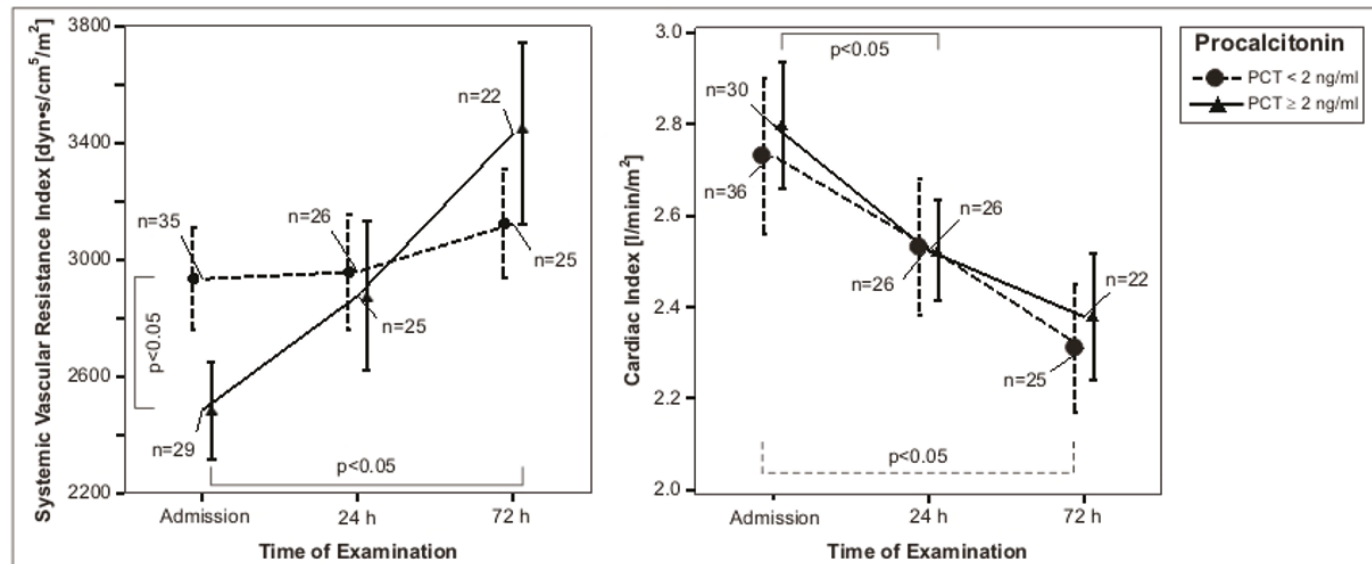

Figure 1 (abstract P381). Systemic vascular resistance index and cardiac index in sepsis in the emergency department. 
P382

Epidemiology of severe sepsis in India: an update

STodi', S Chatterjee', S Sahu'2, M Bhattacharyya

'AMRI Hospitals, Kolkata, India;' 'Kalinga Hospital, Bhubaneswar, India

Critical Care 2010, 14(Suppl 1):P382 (doi: 10.1186/cc8614)

Introduction A multicentre, prospective, observational study conducted in four intensive therapy units (ITUs) in India from June 2006 to June 2009 to determine the incidence and outcome of severe sepsis among adult patients.

Methods All patients admitted to the ITU were screened daily for SIRS, organ dysfunction and severe sepsis as defined by the ACCP and SCCM. Patient with severe sepsis were further studied.

Results A total of 5,478 ITU admissions were studied. SIRS with organ dysfunction was found in 1,385 (25\%) patients, of which 731 (52.77\%) were due to sepsis. The incidence of severe sepsis was $16.45 \%$ of all admissions. Mean age of the study population was 58.17 years (SD 18.66), of which $57.71 \%$ were male. The median APACHE II score was 13 (IQR 13 to 14) with predominant (90.93\%) medical admission. ITU mortality of all admissions was $12.08 \%$ and that of severe sepsis was $59.26 \%$. Hospital mortality and 28 -day mortality of severe sepsis were $65.2 \%$ and $64.6 \%$, respectively. The standardized mortality ratio of severe sepsis patients was 1.45. Median duration of stay in the ITU for the severe sepsis cohort who survived was 13 days (IQR 11 to 17). The number of episodes where infection was the primary reason for admission to the ITU was $86.32 \%$. Culture positivity was found in $61.6 \%$. The lung was the predominant source of sepsis (57.45\%). Gram-negative organisms were responsible for $72.45 \%$ of cases and Grampositive for $13.13 \%$. The rest were parasitic, viral and fungal infection.

Conclusions Severe sepsis was common in Indian ITUs. ITU mortality was higher compared with western literature. Gram-positive infections were less common although incidences of parasitic and viral infection were higher than in the West.

\section{P383}

Systemic inflammatory response syndrome is reduced by inline

filtration in intensive care patients

T Jack, M Boehne, BE Brent, A Wessel, M Sasse

Medical School Hannover, Germany

Critical Care 2010, 14(Suppl 1):P383 (doi: 10.1186/cc8615)

Introduction Sepsis, systemic inflammatory response syndrome (SIRS) or organ failure often complicate the clinical course on an ICU. Particulate contamination of the infusion solution may contribute to the clinical deterioration of these patients. Particles have been shown to induce thrombogenesis, deterioration of microcirculation and modulation of immunoresponse. The use of inline filtration with microfilters almost completely prevents particulate infusion. We assessed the effect of inline filtration on the reduction of major complications in critically ill children (Clinical Trials.gov ID NCT 00209768).

Methods In a randomised, prospective trial 807 paediatric patients admitted to the interdisciplinary PICU of a tertiary university hospital were assigned to either a control or an interventional group, the latter receiving inline filtration (infusion filter Pall ELD96LLCE/ NOE96E, Braun Intrapur Lipid/Intrapur Neonat Lipid) throughout the whole infusion therapy. Prior to this study, the infusion regimen was optimised to prevent precipitation and incompatibilities of solutions and drugs. Primary objectives included a reduction in the incidence of sepsis, thrombosis, SIRS, organ failure (liver, lung, kidney, circulation) and mortality.

Results eight hundred and seven children (343 female, 464 male) with a heterogeneous background of underlying diagnoses and a Gaussian distribution to either the control group (406 patients) or the inline filtration group (401 patients) were included. According to the study criteria, a significant reduction in the incidence of SIRS for the interventional group (95\% Cl, 145 versus 200 patients, $P<0.001$ ) was evident. No differences were demonstrated for the occurrence of sepsis, thrombosis, organ failure (liver, lung, kidney, circulation) or mortality between the control and interventional groups.

Conclusions The occurrence of SIRS often complicates the treatment in intensive care medicine. Inline filtration is most effective, reducing the incidence of SIRS, and offers a novel therapeutic option.
P384

Review of central venous catheter-related infections in neurointensive care patients in a tertiary referral centre CTan, P Nair, A Sule, M Rothburn The Walton Centre for Neurology and Neurosurgery, Liverpool, UK Critical Care 2010, 14(Suppl 1):P384 (doi: 10.1186/cc8616)

Introduction Intravenous catheter-related bloodstream infections (ICR-BSI) are a major contributing factor to in-hospital mortality and morbidity, extending the inpatient stay by 10 days and expenditure per patient by $£ 2,000$ to $£ 30,000$ [1]. A prospective survey was conducted in our unit on all patients with central venous catheters to ascertain the incidence of ICR-BSI, identify the organisms and determine the occurrence of infection from the various sites - femoral, internal jugular and subclavian lines.

Methods The survey was carried out over a period of 13 weeks. Data collected from patients' case notes included site of central line insertion, length of line in situ, reason for line removal and positive blood culture reports.

Results During the study period, 104 patients were treated on the unit. Fifty-two central venous lines were inserted in 36 patients (63.5\% femoral $(n=33), 32.7 \%$ internal jugular $(n=17)$ and $3.9 \%$ subclavian lines $(n=2))$. The lines were reviewed daily and removed if indicated clinically (pyrexia or raised white cell count) or if not required. A total $51.5 \%$ of femoral lines $(n=17)$ were removed due to clinical indications, as were $29 \%(n=5)$ of internal jugular and $50 \%(n=1)$ of subclavian lines. The average duration of a line remaining in situ was 4.5 days for femoral, 6 days for internal jugular and 5 days for subclavian lines. Blood cultures were taken at the time of line removal. These yielded positive results in eight femoral, seven internal jugular and one subclavian line. Our survey indicated that the incidence of ICR-BSI in our unit is $30.8 \%$ (of this $62.5 \%$ coagulase-negative staphylococci (CNS), 12.5\% E. coli and Pseudomonas each, and 6.25\% MSSA and MRSA each).

Conclusions The distribution of microorganisms causing bacteraemia is broadly similar in our unit to that in other teaching hospitals in the UK [2], in that CNS was the commonest organism isolated. However, E. coli and Pseudomonas were the next common organisms, unlike other units where Staphylococcus aureus was the second most prevalent organism. The incidence of bacteraemia from femoral lines (53.7/1,000 catheter-days) was lower than that from internal jugular lines $(68.6 / 1,000$ catheter-days) possibly due to a higher index of suspicion in the case of femoral lines and earlier removal (Figure 1). Our study highlights the fact that femoral lines, which are often the safest option for unstable patients with head injury, can be effectively managed with strict adherence to guidelines to reduce ICR-BSI.

References

1. Maki DG, Kluger DM, Crnich CJ: The risk of bloodstream infection in adults with different intravascular devices: a systematic review of 200 published prospective studies. Mayo Clin Proc 2006, 81:1159-1171.

2. Coello $R$, Charlett A, Ward V, et al.: Device-related sources of bacteraemia in English hospitals - opportunities for the prevention of hospital-acquired bacteraemia. J Hosp Infect 2003, 53:46-57.

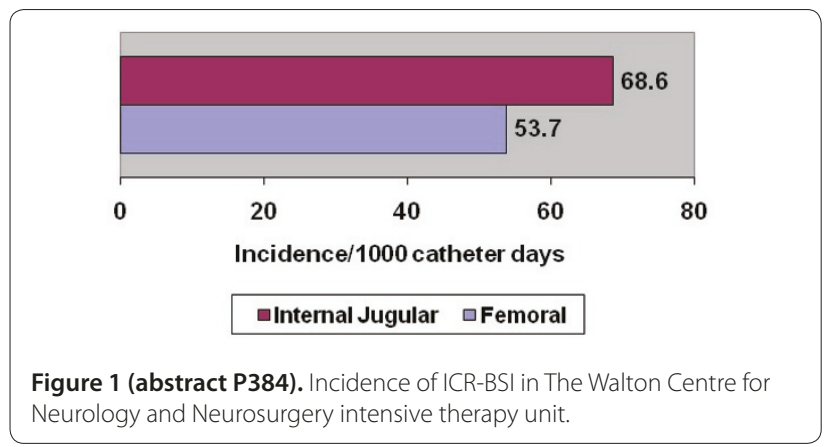


P385

Clinical impact of diagnosis methods for catheter-related bloodstream infection in ICU patients

RO Deliberato, AR Marra, OF Dos Santos, SB Wey

Hospital Israelita Albert Einstein, São Paulo, Brazil

Critical Care 2010, 14(Suppl 1):P385 (doi: 10.1186/cc8617)

Introduction The central venous catheter (CVC) is indispensable in the ICU. Catheter-related bloodstream infection (CR-BSI) is the leading cause of healthcare-associated infections and one of the most important complications from the CVC. Nowadays there are two well-known ways to diagnose CR-BSI: the standard method (withdrawal of the catheter) and the conservative method (without withdrawal of the catheter). Our objective was to compare the in-hospital mortality between the two methods in patients with CR-BSI (short-term catheter) in the ICU.

Methods This study was conducted in a 38-bed mixed ICU in a tertiary hospital. We reviewed all episodes of CR-BSI that occurred in our ICU from January 2000 to December 2008. The standard method was defined in a patient with a CVC with at least one positive blood culture obtained from a peripheral vein and a positive semiquantitative (>15 CFU) catheter segment culture whereby the same organism (species and antibiogram) was isolated from the catheter segment and peripheral blood. The conservative method was defined in a patient with a CVC with at least one positive blood culture obtained from a peripheral vein and one of the following: differential time period of CVC culture versus peripheral culture positivity of more than 2 hours, or simultaneous quantitative blood culture with a ratio $\geq 5: 1$ (CVC versus peripheral).

Results During the study period, 247 episodes of bloodstream infection were identified; of these, 192 were catheter-associated bloodstream infection and 55 were CR-BSI (39 standard method and 17 conservative method). Considering the CR-BSI patients, the mean age \pm standard variation (SD) was $64 \pm 19.29$ (years), $75 \%$ were under mechanical ventilation, 55\% were under vasopressors, $40 \%$ were under total parental nutrition, $40 \%$ were under hemodialysis, $72 \%$ were double lumen and the mean time \pm SD in place was $16.32 \pm 8.56$ (days). The in-hospital mortality of the standard method versus the conservative method did not show any difference ( $57 \%$ vs $75 \%, P=0.208)$.

Conclusions This study showed that there is no difference in the inhospital mortality between the standard method versus the conservative method in patients in the ICU with CR-BSI.

\section{References}

1. O'Grady NP, et al: Centers for Disease Control and Prevention. MMWR Recomm Rep 2002, 51:1-29.

2. Bouza et al: Clin Infect Dis 2007, 44:820-826.

\section{P386}

Catheter-related bacteremia in a jugular site with tracheostomy and in a femoral site

L Lorente, S Palmero, C Naranjo, J Jiménez, J Iribarren, C García, R Galván, J Castedo, J Martínez, M Mora

Hospital Universitario de Canarias, Santa Cruz de Tenerife, Spain

Critical Care 2010, 14(Suppl 1):P386 (doi: 10.1186/cc8618)

Introduction In previous guidelines of the Centers for Disease Control and Prevention (CDC) [1] and in the recently published Guidelines of the Society for Healthcare Epidemiology of America/Infectious Diseases Society of America (SHEA/IDSA) [2] it is recommended to avoid the femoral access to reduce the risk of catheter-related bacteremia (CRB). However, in these guidelines there are no recommendations for the catheter site regarding the presence of tracheostomy, and we have not found data about the incidence of CRB comparing a central jugular site with tracheostomy and a femoral site. The objective of this study was to determine whether a jugular site with tracheostomy may have a higher risk of CRB than a femoral site.

Methods A prospective, observational, 4-year study (from 1 May 2000 to 30 April 2004) was carried out in the medical-surgical ICU of the University Hospital of the Canary Islands (Tenerife, Spain). We included all patients undergoing a central jugular catheterization with tracheostomy or femoral venous catheterization.

Results In the period of study, were used 208 catheters in femoral access and 52 catheters in central internal jugular access with tracheostomy. There were no significant differences between patients with central jugular with tracheostomy and femoral access in age, sex, APACHE II score, diagnosis group, use of mechanical ventilation, use of antimicrobials, use of total parenteral nutrition, use of pulmonary artery catheter, and duration of the catheter. We diagnosed 16 CRB in 208 femoral catheters during 1,679 days of catheterization and $10 \mathrm{CRB}$ in 52 central internal jugular catheters with tracheostomy during 462 days of catheterization. The incidence of CRB was higher in the central internal jugular with tracheostomy than in the femoral site (21.64 vs 9.52 per 1,000 catheter-days; risk ratio $=2.27 ; 95 \%$ confidence interval $=1.04$ to $4.97: P=0.04$.

Conclusions The femoral site could be considered a more safe venous access than the central internal jugular in patients with tracheostomy to minimize the risk of CRB.

\section{References}

1. MMWR 2002, 1:1-29.

2. Infect Control Hosp Epidemiol 2008, 29(Suppl 1):S22-S30.

P387

A comparative study between conventional and antiseptic impregnated central venous catheters in neurocritical patients S Kiffer Macedo, G Castro Lima, L Bonani de Almeida Brito, A Davila Carvalho, JL Fernandes Molina Filho

Hospital São José do Avaí, Itaperuna, Brazil

Critical Care 2010, 14(Suppl 1):P387 (doi: 10.1186/cc8619)

Introduction The central venous catheter (CVC) is very useful in the ICU. A comparative study between antiseptic impregnated and standard catheters is therefore of great value.

Methods Alternating the type of CVC used in each patient, we recorded for each patient: sex, age, APACHE II score, GCS, site of the puncture, reason for withdrawal of the catheter and the type of catheter used. The tip of the catheter was cultured. The groups were divided: group I (41 patients, 54 punctures) used the standard CVC, and group II (38 patients, 54 punctures) used the impregnated CVC.

Results Sixty-two patients were included (48.38\% male). We studied 108 periods, of which 54 were standard CVCs and 54 were impregnated CVCs. The average length of stay was higher for impregnated CVCs (14.11 days) compared with standard CVCs (10.7 days). Excluding death in both groups, the length of stay of the catheter in group I was 10.86 days, compared with 15.43 days in group II. Adding all periods of catheterization, group I have an amount of 578 days, and 762 days for group II. The total duration for group II was $31.84 \%$ higher than for group I. Regarding the reason for withdrawal of the CVC, predominant were suspected infection $77.8 \%$ of the time for standard CVCs, and $49.1 \%$ of the time for impregnated CVCs. The culture of the catheter's tip was positive in 10 (18.5\%) standard CVs, against eight (15.1\%) CVCs in the impregnated group. Most patients had GCS $<9$.The average APACHE II score was 17.97 in group I, compared with 19.63 in group II. The predominant site of puncture was the subclavian vein (56.48\%). See Figure 1.

Conclusions According to our study we observed that the length of stay with use of the impregnated CVC was higher (15.43 days). The rate of colonization was higher in the standard CVC. Patients who require a CVC for long periods have benefited with the use of impregnated CVCs,

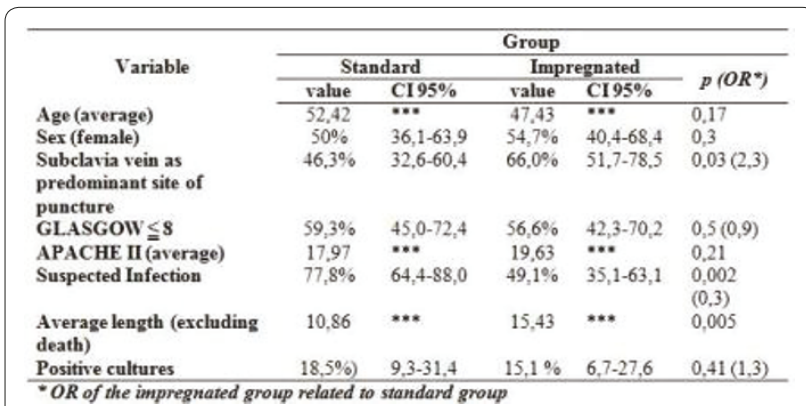

Figure 1 (abstract P387). 
because they present long-term use, lower rates of colonization, and avoiding complications related to the procedure of successive punctures and related to the permanence of the catheters. The benefit reached by the use of antiseptic impregnated catheters compensated for the initial expensive cost of $40 \%$.

P388

Comparison between peripherally inserted central venous catheters and short-term central venous catheter in patients discharged from the ICU

S Batacchi', G Zagli', S diValvasone², M Ciapetti', G Cianchi', VTucci',

G Martini', M Mostardini', S Proietti', A Peris'

${ }^{\prime}$ Careggi Teaching Hospital, Florence, Italy; ${ }^{2}$ Postgraduate School of

Anesthesia and Intensive Care, University of Florence, Italy

Critical Care 2010, 14(Suppl 1):P388 (doi: 10.1186/cc8620)

Introduction The use of peripherally inserted central catheters (PICCs) in the hospital setting has been indicated as an alternative to the shortterm central venous catheter (ST-CVC) in providing prolonged intravenous access. The aim of our study was to assess the rate of complications (deep venous thrombosis (DVT), infections) in patients discharged from the ICU with a PICC or a ST-CVC.

Methods A total of 239 patients discharged from our ICU (Careggi Teaching Hospital, Florence, Italy) have been followed (March 2007 to December 2008). PICCs were used in 114 patients (PICC group), whereas ST-CVCs were placed in 125 patients (ST-CVC group). Data, expressed as the mean, were analysed with the Mann-Whitney test and Fisher's exact test $(P<0.05)$

Results The PICC group and ST-CVC group resulted similar in age (54.3 vs 58.8), BMI (25.6 vs 26.5), SAPS II (46.2 vs 46.5), and ICU length of stay (17.6 vs 16.4). Admission diagnoses in the PICC and ST-CVC groups were also similar: major trauma (38.6\% vs $44.8 \%)$, sepsis (37.7\% vs $32 \%$ ), postsurgical complications (23.7\% vs $23.2 \%$ ). Total PICC catheter-days were 4,024, whereas total ST-CVC catheter-days were 2,764. Despite similar demographic and clinical characteristics, the PICC group showed a significantly higher rate of DVT than ST-CVC group (37.7\% vs $14.4 \%$; $P<0.01)$. This difference resulted significant also with catheter-day correction (number of events*1,000/catheter-days). The highest incidence of DTV events occurred after 15 days from placement in both groups.

Conclusions In our experience, the significant rate of DVT found using PICCs induces one to consider a presumably higher thrombogenicity of this kind of catheter. Based on our result, the use of ST-CVC in patients discharged from the ICU, who are at higher risk of DVT development in regard of the recent critically ill status, might be more appropriate.

\section{P389}

Vacuum-assisted closure of laparostomy, an efficient strategy for complicated intra-abdominal infection

NG Germano, IG Gonçalves, B Moya, R Ribeiro

Hospital S Bernardo, Setúbal, Portugal

Critical Care 2010, 14(Suppl 1):P389 (doi: 10.1186/cc8621)

Introduction The chirurgical strategy for infection control in complicated intra-abdominal infections is responsible alone for $40 \%$ reduction in mortality. The second look strategy in tertiary peritonitis reduces the mortality and morbidity of the patients. Vacuum-assisted closure therapy can potentially decrease the concentration of proinflammatory cytokines, bacterial count, management of third-space fluid and improved input and output monitoring.

Methods The authors present a revision of 60 patients with tertiary peritonitis admitted to the ICU after chirurgical infection control. All patients have septic shock at admission. Twelve percent of them were admitted with an open abdomen and vacuum-assisted closure therapy was used. The parameters evaluated were hospital mortality, ICU stay (days) and time of mechanical ventilation (days).

Results The patients submitted to vacuum-assisted closure therapy were similar to patients in whom the abdominal wall was closed, regarding the risk factors for peritoneal infection (corticotherapy, oncologic disease, renal insufficiency, hepatic insufficiency, desnutrition, hypoalbuminemia, a high APACHE II score). The patients submitted to vacuum-assisted closure therapy had a higher ICU stay and time of mechanical ventilation but lower hospital mortality.

Conclusions Vacuum-assisted closure therapy is superior to primary abdominal wall closure in patients with tertiary peritonitis.

References

1. Becker HP, et al.: Scand J Surg 2007, 96:263-271.

2. Andrabi SIH, et al.: J Ayub Med Coll Abottabad 2007, 19

\section{P390}

Comparison between left ventricular tissue Doppler strain imaging and the sequential organ failure assessment score (SOFA score) in patients with severe sepsis or septic shock

M Masaki, J Pulido, T Yuasa, S Gillespie, B Afessa, D Brown, S Mankad, J Oh Mayo Clinic, Rochester, MN, USA

Critical Care 2010, 14(Suppl 1):P390 (doi: 10.1186/cc8622)

Introduction The Sequential Organ Failure Assessment (SOFA) score is a widely used method to describe organ dysfunction/failure in critically ill patients. Although the majority of the studies use the SOFA score in outcome research, little is known about its correlation to left ventricular (LV) function in patients with severe sepsis and septic shock. The purpose of this study was to evaluate the correlation of SOFA score and LV function by tissue Doppler imaging in this patient population.

Methods Sixty patients (ages $66 \pm 15$, men $n=28$ ) admitted to the ICU with severe sepsis or septic shock were prospectively enrolled. Transthoracic echocardiography was performed within 24 hours of admission to the ICU. We measured the LV end diastolic volume index (LVEDVI), LV end systolic volume index (LVESVI), LV ejection fraction (LVEF), mitral deceleration time of E velocity (Dct) and myocardial tissue Doppler strain imaging profiles at the basal, mid and apical portions of the LV septal wall in the LV apical fourchamber view. On the ICU admission day, the SOFA scores were calculated. Significantly correlated parameters were subjected to linear regression analysis. $P<0.05$ was considered significant.

Results The mean heart rate was $90+17 \mathrm{bpm}$, mean LV volumes, mean LVEF and mean Dct were within normal limits (LVEDVl; $44.6 \pm 15.4 \mathrm{ml} / \mathrm{m}^{2}$, LVESVl; $18.7 \pm 11.5 \mathrm{ml} / \mathrm{m}^{2}$, LVEF; $58.9 \pm 15.7 \%$, Dct; $\left.175.8 \pm 50.2 \mathrm{~ms}\right)$. The mean peak systolic strain measurements were $-16.0 \pm 5.0 \%$ (basal; $-18.1 \pm$ $7.0 \%$, mid; $-15.7 \pm 5.6 \%$, apical; $-14.1 \pm 6.6 \%$ ). The mean SOFA score was $10.8 \pm 3.9$. Linear regression analysis showed correlation between the SOFA score and LVEF $(r=-0.40, P=0.02)$, Dct $(r=-0.34, P=0.007)$, mean septal strain $(r=0.40, P=0.002)$, mid-septal strain $(r=0.43, P=0.0007)$ and apical septal strain $(r=0.36, P=0.006)$.

Conclusions These results suggest that the higher SOFA score had lower LVEF, lower LV systolic strain and higher LV filling pressure in the patients with severe sepsis or septic shock. An assessment of the left ventricular longitudinal contraction by tissue Doppler strain imaging may serve as a useful tool to evaluate multiple organ failure in this patient population.

\section{P391}

Systolic and diastolic function and survival in severe sepsis and septic shock: clinical, echocardiographic, biochemical correlation G Landesberg, M Georgieva, Y Meroz, S Goodman, P Levin Hebrew University - Hadassah Medical Center, Jerusalem, Israel Critical Care 2010, 14(Suppl 1):P391 (doi: 10.1186/cc8623)

Introduction The exact mechanisms causing cardiac dysfunction in sepsis are still in major part obscure or controversial.

Methods All patients who had the criteria for severe sepsis or septic shock underwent daily transthoracic echocardiography examinations from which detailed data on left ventricular end-diastolic, end-systolic volumes areas, and diastolic measurements were obtained. In addition, numerous clinical, hemodynamic, respiratory and laboratory data were prospectively collected, including data on survival up to 2 years after admission. Blood samples were collected, centrifuged and serum separated for additional biomarker tests, mainly troponin and NT-proBNP.

Results A total of 262 patients had $712(2.5 \pm 1.4)$ transthoracic echocardiography examinations. APACHE was $20.6 \pm 7.3,41 \%$ had positive blood cultures, $>40 \%$ died within 4 months. On multivariate Cox survival analysis, the LV ejection fraction, end-diastolic volume and Em wave on tissue Doppler imaging independently predicted improved survival and 


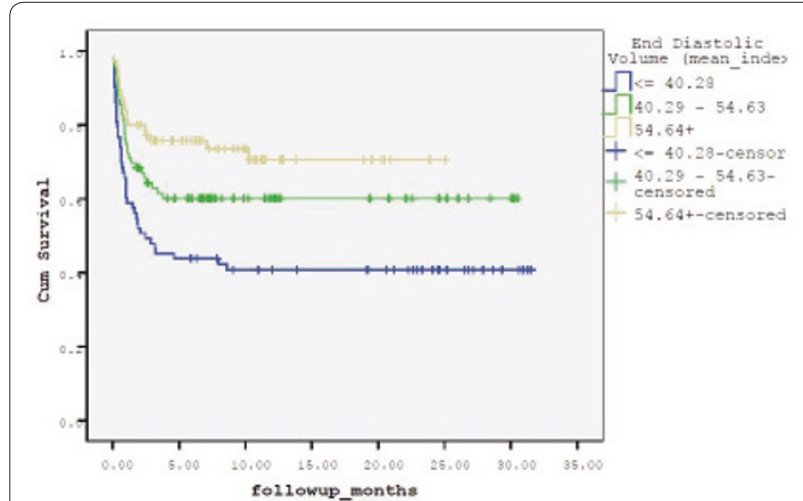

Figure 1 (abstract P391). Survival functions.

the heart rate independently predicted worse survival $(\operatorname{Exp}(B)=0.009$, $0.969,0.9,1.004$ and $P<0.0001, P<0.0001, P=0.001, P=0.002$, respectively). Only $45 \%$ of patients with end-diastolic volume index $<40 \mathrm{ml}$ survived more than 4 months, compared with $75 \%$ survival in patients with enddiastolic volume index $>54 \mathrm{ml}$.

Conclusions Decreased ejection fraction, decreased end-diastolic volume, diastolic dysfunction and tachycardia are independent predictors of early mortality in severe sepsis and septic shock.

\section{P392}

\section{Frequency and clinical spectrum of myocardial dysfunction in} severe sepsis and septic shock

JN Pulido, T Yuasa, M Masaki, S Gillespie, V Herasevich, S Mankad, B Afessa, D Brown, J Oh

Mayo Clinic, Rochester, MN, USA

Critical Care 2010, 14(Suppl 1):P392 (doi: 10.1186/cc8624)

Introduction Myocardial dysfunction is reportedly a common complication of sepsis that requires specific management. Although frequently reported in the literature, the clinical spectrum and frequency of this organ failure have not been fully appreciated. We sought to determine the frequency of myocardial dysfunction in severe sepsis and septic shock and to describe the clinical spectrum of this entity with transthoracic echocardiography.

Methods Prospective single-center study capturing all patients admitted to the ICU with severe sepsis or septic shock from May 2007 to January 2009. All patients enrolled underwent comprehensive transthoracic echocardiography on admission. The exclusion criteria included $<18$ years of age, pregnancy, documented ischemic, valvular or congenital heart disease. All patients with LV systolic dysfunction defined as LVEF <50\% received a repeat echocardiographic study at 5 days or upon dismissal from ICU.

Results One hundred and six patients were enrolled, mean age was 65 , and $50 \%$ were female. The mean SOFA score was 11 . Central venous oxygen saturation was less than $70 \%$ in $37 \%$ of patients. Twenty-nine patients (27\%) had global LV systolic dysfunction $(14=$ mild, nine $=$ moderate, six = severe), 33 patients $(31 \%)$ had RV dysfunction (18 = mild nine $=$ moderate, six = severe), 14 patients had biventricular involvement (13\%) and 39 patients (37\%) had diastolic filling abnormalities. Of the 29 patients with LV systolic dysfunction on initial examination, 28 received a follow-up echocardiogram and $96 \%$ of these patients $(n=27)$ improved in all parameters. Thirty-day mortality was 39\%, 6-month mortality $52 \%$. Myocardial dysfunction did not predict mortality.

Conclusions These results confirm that myocardial dysfunction is frequent and broad in patients with severe sepsis and septic shock. Right ventricular involvement and diastolic abnormalities should be considered as part of the clinical spectrum of this entity. There was poor correlation with myocardial dysfunction and mortality and it was reversible regardless of presentation. We should not focus only on left ventricular ejection fraction to diagnose myocardial dysfunction in sepsis, since only a small portion of these patients had isolated LV systolic dysfunction.

\section{References}

1. Dellinger RP, et al.: Crit Care Med 2004, 32:858-73

2. Parker MM, et al:: Ann Intern Med 1984, 100:483-490.

3. Court O, et al.: Crit Care 2002, 6:500-508.

\section{P393}

Effects of nitroglycerin on intestinal microcirculatory blood flow in patients with abdominal sepsis

F Roossien', EC Boerma', N Vellinga' ${ }^{1}$ N Bruins ${ }^{1}, \mathrm{C}^{1}$ Ince $^{2}$

'Medical Center Leeuwarden, the Netherlands; ${ }^{2}$ Academic Medical Center, University of Amsterdam, the Netherlands

Critical Care 2010, 14(Suppl 1):P393 (doi: 10.1186/cc8625)

Introduction In sepsis, microcirculatory blood flow is impaired. Intravenous nitroglycerin (NTG) seems to have no effect on sublingual microcirculation [1], but might have a different effect on intestinal perfusion. The aim of this prospective observational sub-study was to evaluate the effect of NTG on intestinal microcirculatory flow index (MFI) compared with sublingual MFI in the early phase of sepsis.

Methods Eight patients (>18 years) with abdominal sepsis were included. Sublingual and intestinal side-stream dark field (SDF) images were obtained and MFIs were calculated. Patients received NTG intravenously for 24 hours ( $2 \mathrm{mg} /$ hour), after fulfillment of protocol-driven resuscitation endpoints. Nonparametric tests were used to compare MFI baseline with 24 hours and for comparison within groups. Data are expressed as median (IQR).

Results At baseline, sublingual and intestinal MFI did not differ significantly (2.2 (1.2 to 1.8 ) vs 2,7 (1.1 to 2.8$), P=0.89$ ). After 24 hours, sublingual and intestinal MFI were nonsignificantly higher in comparison with baseline (2.33 (0.48 to 3 ), $P=0.94$ vs 2.75 (2.13 to 2.98$), P=0.67$ ). Furthermore, groups did not differ significantly $(P=0.46)$. However, one patient (number 13) showed a striking decrease in sublingual MFI after 24 hours, whereas intestinal MFI did not change over time. In another patient (number 25), sublingual MFI improved with a concomitant reduction in intestinal MFI over time (Figure 1).

Conclusions In this small population of septic patients we could not detect significant differences between the sublingual and intestinal microcirculation in response to NTG after a strict resuscitation protocol. However, in individuals there were marked differences in absolute values of sublingual and intestinal microcirculatory blood flow at baseline and after 24 hours, as well as in differences over time. These observations on dynamic microcirculatory changes in response to a specific

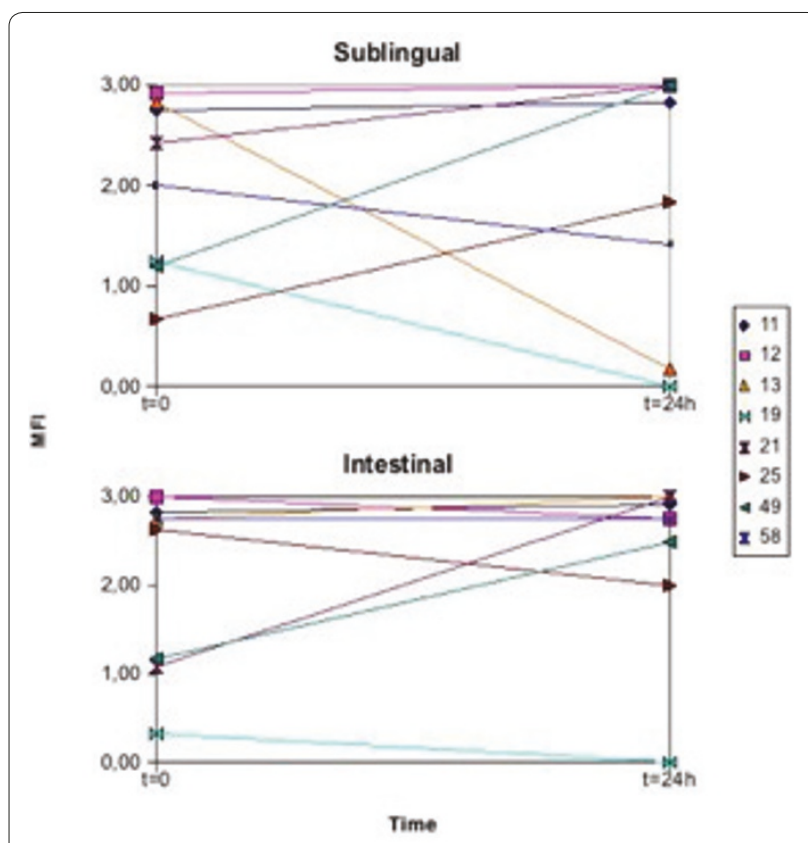

Figure 1 (abstract P393). MFI with time per patient. 
therapeutic intervention add to previous reports about heterogeneity of microcirculatory blood flow in sepsis.

\section{Reference}

1. Boerma et al:: Crit Care Med 2009. [Epub ahead of print]

\section{P394}

Diastolic dysfunction in septic shock is an independent predictor of elevated B-type natriuretic peptide and hospital mortality

D Sturgess, T Marwick, C Joyce, C Jenkins, M Jones, P Masci, D Stewart,

B Venkatesh

University of Queensland, Brisbane, Australia

Critical Care 2010, 14(Suppl 1):P394 (doi: 10.1186/cc8626)

Introduction Diastolic dysfunction as demonstrated by tissue Doppler imaging (TDI), particularly E/E' (peak early diastolic transmitral/peak early diastolic mitral annular velocity) is common in critical illness. In septic shock, the prognostic value of TDI is undefined. This study sought to evaluate and compare the prognostic significance of TDI and cardiac biomarkers (B-type natriuretic peptide (BNP); N-terminal proBNP (NTproBNP); troponin $\mathrm{T}(\mathrm{TnT})$ ) in septic shock. The contribution of fluid management and diastolic dysfunction to elevation of BNP was also evaluated.

Methods Twenty-one consecutive adult patients from a multidisciplinary ICU underwent transthoracic echocardiography and blood collection within 72 hours of developing septic shock.

Results The mean \pm SD APACHE III score was $80.1 \pm 23$.8. Hospital mortality was $29 \%$. E/E' was significantly higher in hospital nonsurvivors (15.32 \pm 2.74 , survivors $9.05 \pm 2.75 ; P=0.0002$ ). Areas under ROC curves were $E / E^{\prime} 0.94$ TnT 0.86, BNP 0.78 and NTproBNP 0.67. An E/E' threshold of 14.5 offered $100 \%$ sensitivity and $83 \%$ specificity. Adjustment for APACHE III, cardiac disease, fluid balance and grade of diastolic function, demonstrated $E / E$ ' as an independent predictor of hospital mortality $(P=0.019)$. Multiple linear regression incorporating APACHE III, gender, cardiac disease, fluid balance, noradrenaline dose, $C$-reactive protein, ejection fraction and diastolic dysfunction yielded APACHE III $(P=0.033)$, fluid balance $(P=0.001)$ and diastolic dysfunction $(P=0.009)$ as independent predictors of InBNP. Conclusions $\mathrm{E} / \mathrm{E}^{\prime}$ is an independent predictor of hospital survival in septic shock. It offers better discrimination between survivors and nonsurvivors than cardiac biomarkers. Fluid balance and diastolic dysfunction were independent predictors of BNP concentration in septic shock.

P395

Cardiac-specific troponin I and cardiovascular dysfunction in post-traumatic sepsis

G Georgiev, SG Milanov, VTodorova, L Kozarov, M Milanov

Pirogov Emergency Institute, Sofia, Bulgaria

Critical Care 2010, 14(Suppl 1):P395 (doi: 10.1186/cc8627)

Introduction Elevated serum levels of cardiac-specific troponin I (cTnl) are claimed to be associated with the degree of sepsis-related cardiac dysfunction and with the disease outcome in critically ill. We tested this association in a specific group of ICU patients with post-traumatic sepsis. Methods A prospective observational study was conducted in an ICU of a level I-equivalent trauma center between September 2008 and August 2009. No exclusion criteria were adopted. Serum cTnl concentrations were collected at peak sepsis-related cardiovascular dysfunction (defined by the highest pressure adjusted heart rate (PAR) in MODS score and by the dose of the inotrope). Data were gathered on demographics, severity of inflammatory response (SIRS criteria) and organ dysfunction (MODS score), presence of superimposed acute coronary events, other potential reasons for cTnl elevation and the disease outcome. Intergroup comparisons were made with Student's $t$ test/nonparametric alternatives. The prognostic utility of cTnl was examined by the construction of ROC curves and its levels were categorized on the basis of the defined cut-off value. Probable associations between these categories and the organ dysfunction severity, as well as the outcome, were examined with Fisher's exact test.

Results A total of 92 polytrauma patients were enrolled. The confidence intervals of PAR, MODS and SIRS were $21.26 \pm 2.72,6.99 \pm 2.94$ and $3.75 \pm$ 1.10 , respectively. Blunt cardiac injury had been diagnosed in 20 patients (21.7\%) on admission. Three patients (3.3\%) developed acute coronary events during their ICU stay and 12 patients (13.2\%) had other potential reasons for cTnl elevation. Significantly higher $\mathrm{cTnl}$ concentrations were found in the critically ill with myocardial contusion and with acute coronary events. However, the presence of blunt cardiac injury had no measurable outcome effect. Troponin I levels were significantly higher in nonsurvivors $(P=0.00)$ and very good prognostic performance of cTnl was found (AUROC $=0.82 ;$ cut-off value $=0.05 \mathrm{ng} / \mathrm{ml}$ ). The subsequent comparison between its categorized values and those of PAR and MODS showed strong positive association. Elevated cTnl concentrations $(>0.05 \mathrm{ng} / \mathrm{ml})$ significantly increased the risk of death ( $\mathrm{RR}=5.58 ; 95 \% \mathrm{Cl}=1.67$ to $20.79 ; P=0.002)$.

Conclusions In a group of patients with post-traumatic sepsis elevated serum cTnl levels proved to be a good marker of severe organ (including cardiovascular) dysfunction. They also showed reasonable prognostic value regarding the disease outcome.

P396

Both systolic and diastolic functions are impaired in critically ill patients with community-acquired sepsis

S Hettwer, M Schürmann, J Wilhelm, H Ebelt, K Werdan

Martin-Luther-University, Halle (Saale), Germany

Critical Care 2010, 14(Suppl 1):P396 (doi: 10.1186/cc8628)

Introduction Alterations in haemodynamic parameters in septic cardiomyopathy are usual. While patients with APACHE $I I$ score $<15$ have a low risk of death, patients with score $\geq 15$ have one of $>25 \%$. In a subgroup of our monocentric observational ProFS study (depiction of patients with community-acquired sepsis in the emergency department), systolic and diastolic function were evaluated concerning patients risk of death.

Methods Echocardiographic examination was performed at admission, after 24 and 72 hours. The left ventricular systolic ejection fraction (EF, \%)
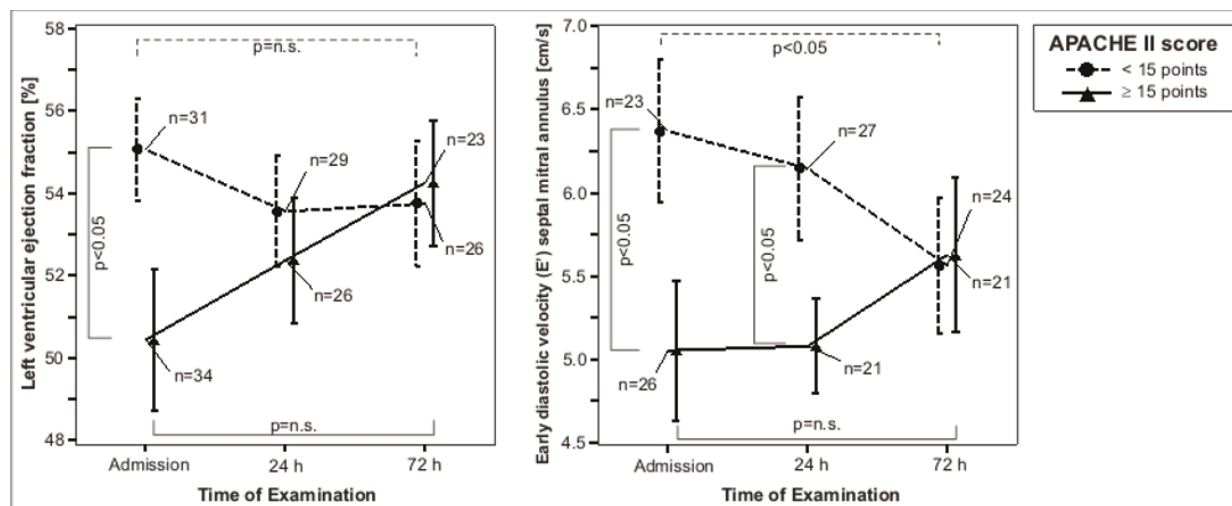

Figure 1 (abstract P396). Systolic and diastolic function indices in sepsis. n.s., not significant. 
was measured using Simpson's rule, and early peak diastolic relaxation velocity of septal mitral annulus ( $E^{\prime}, \mathrm{cm} /$ second) was measured using tissue Doppler imaging. APACHE II score was calculated at admission. By APACHE II score, two groups were formed: Iow APACHE II score (LAS) was defined as 0 to 14 patients and high APACHE II score (HAS) $\geq 15$ patients.

Results In 65 of 208 patients an echocardiographic examination was performed. Age ( $61.0 \pm 18.4$ vs $63.5 \pm 16.9$ years, $P=N S)$, male sex ( $64.5 \%$ vs $58.8 \%, P=\mathrm{NS})$ and mean artery pressure $(86.2 \pm 15.7 \mathrm{vs} 85.7 \pm 21.2 \mathrm{mmHg}$, $P=$ NS) were comparable, whereas patients with HAS had a higher heart rate $(96.5 \pm 24.5$ vs $108.2 \pm 17.1 /$ minute, $P<0.05)$. At admission, patients with HAS had a significantly lower EF than patients with LAS ( $55.1 \pm 7.0$ vs $50.5 \pm 10.1, P<0.05$ ). Difference could not be observed after 24 hours (53.6 \pm 7.2 vs $52.4 \pm 7 . \%, P=\mathrm{NS}$ ) and 72 hours ( $53.8 \pm 7.3$ vs $54.3 \pm 7.3, P=\mathrm{NS})$; see Figure 1. Patients with $\mathrm{HAS}$ showed a lower initial $\mathrm{E}^{\prime}$ than patients with LAS $(6.2 \pm 2.1$ vs $5.1 \pm 2.1, P<0.05)$ and after 24 hours $(6.1 \pm 2.2$ vs $5.1 \pm 1.3$, $P<0.05)$. Three days after admission, E'values in patients with $L A S$ and HAS were comparable $(5.6 \pm 2.0$ vs $5.7 \pm 2.1, P=\mathrm{NS})$.

Conclusions In patients with community-acquired sepsis and APACHE ॥ score $\geq 15$ points, a significantly depression of both systolic and diastolic function could be observed. After 24 hours systolic function and after 72 hours diastolic function of patients with an APACHE II score $<15$ and $\geq 15$ points were similar. This could be possibly due to alterations in afterload.

P397

Selective $\mathrm{V}_{1 \mathrm{a}}$ agonism reduces vascular leakage and cardiopulmonary dysfunction in methicillin-resistant Staphylococcus aureus sepsis S Rehberg, P Enkhbaatar, Y Yamamoto, AK Hasselbach, LD Traber, DL Traber

The University of Texas Medical Branch, Galveston, TX, USA

Critical Care 2010, 14(Suppl 1):P397 (doi: 10.1186/cc8629)

Introduction This randomized, controlled experimental trial was performed to compare the effects of the selective $V_{12}$-receptor agonist (Phe2Orn8) vasotocin (POV) and the mixed $\mathrm{V}_{12} \mathrm{~N}_{2}$-receptor agonist (Arg8) vasopressin (AVP) on vascular leakage and cardiopulmonary dysfunction associated with methicillin-resistant Staphylococcus aureus (MRSA) pneumonia-induced septic shock using an established ovine model.

Methods After instrumentation for chronic hemodynamic monitoring and tracheostomy, sheep were subjected to 48 breaths of cotton smoke $\left(<40^{\circ} \mathrm{C}\right)$ and MRSA was placed into both lungs under deep anesthesia. Following a $10 \mathrm{mmHg}$ drop in mean arterial pressure (MAP) from baseline, sheep were randomly assigned to receive intravenous infusions of either POV, AVP or the vehicle $(0.9 \% \mathrm{NaCl} ; n=6$ each). POV and AVP were titrated to keep MAP above baseline $-10 \mathrm{mmHg}$. All sheep were awake, mechanically ventilated and fluid resuscitated to maintain hematocrit at baseline $\pm 3 \%$. Data are expressed as mean \pm SEM at 24 hours. Fluids are represented as the average over time to account for individual survival times.

Results Both treatment strategies significantly reduced fluid input (AVP: $7.7 \pm 0.6 \mathrm{ml} / \mathrm{kg} / \mathrm{hour}$; POV: $5.8 \pm 0.8 \mathrm{ml} / \mathrm{kg} /$ hour), positive net fluid balance (AVP: $4.9 \pm 0.5 \mathrm{ml} / \mathrm{kg} / \mathrm{hour}$; POV: $0.4 \pm 0.6 \mathrm{ml} / \mathrm{kg} / \mathrm{hour}$ ), thoracic (AVP: $0.8 \pm 0.2 \mathrm{ml} / \mathrm{kg} /$ hour; POV: $0.3 \pm 0.1 \mathrm{ml} / \mathrm{kg} /$ hour) and abdominal fluid (AVP: $0.10 \pm 0.04 \mathrm{ml} / \mathrm{kg} / \mathrm{hour} ;$ POV: $0.01 \pm 0.01 \mathrm{ml} / \mathrm{kg} / \mathrm{hour}$ ) as well as extravascular lung water (AVP: $22.6 \pm 1.4 \mathrm{ml} / \mathrm{kg}$; POV: $15.8 \pm 1.9 \mathrm{ml} /$ $\mathrm{kg}$ ) and increased plasma oncotic pressures (AVP: $12.5 \pm 0.9 \mathrm{mmHg}$; POV: $15.9 \pm 1.1 \mathrm{mmHg})$ vs control animals $(9.6 \pm 0.4 \mathrm{ml} / \mathrm{kg} / \mathrm{hour} ; 7.7 \pm 0.4 \mathrm{ml} / \mathrm{kg} /$ hour; $2.2 \pm 0.4 \mathrm{ml} / \mathrm{kg} /$ hour; $0.29 \pm 0.13 \mathrm{ml} / \mathrm{kg} /$ hour; $22.9 \pm 2.8 \mathrm{ml} / \mathrm{kg} ; 8.9 \pm$ $0.1 \mathrm{mmHg}$, respectively). Notably, POV was superior to AVP in all these variables $(P<0.05$ each). Myocardial contractility was higher in the POV group vs AVP and control animals as suggested by higher left ventricular stroke work indexes $\left(66 \pm 3\right.$ vs $48 \pm 7$ and $43 \pm 3 \mathrm{~g} / \mathrm{m} / \mathrm{m}^{2}$, respectively: $P<0.05$ each) at lower or similar global end-diastolic volumes. Pulmonary dysfunction (decrease in $\mathrm{PaO}_{2} / \mathrm{FiO}_{2}$ ratio) was attenuated in POV (364 \pm $47 \mathrm{mmHg})$ as compared with AVP $(195 \pm 66 \mathrm{mmHg} ; P<0.05)$ and control animals $(213 \pm 76 \mathrm{mmHg} ; P<0.05)$. Whereas one AVP and three control animals had to be sacrificed prematurely, all POV-treated animals survived the 24-hour study period.

Conclusions In MRSA pneumonia-induced ovine septic shock, the selective $V_{1 a}$-receptor agonist POV is superior to the mixed $V_{1 a} N_{2}$ receptor agonist AVP in reducing vascular leakage and cardiopulmonary dysfunction when administered as a first-line vasopressor.
P398

Sepsis resuscitation bundle compliance in septic shock: improves survival and yet better late than never

$\checkmark$ Coba, HFH Sepsis Collaborative Group

Henry Ford Hospital, Detroit, MI, USA

Critical Care 2010, 14(Suppl 1):P398 (doi: 10.1186/cc8630)

Introduction Improvements in survival for acute myocardial infarction, trauma, and stroke have been realized through continuous quality improvement (CQI) initiatives that include early identification and implementation of time-sensitive therapies at the earliest stage of disease presentation. In addition, challenges in the management of severe sepsis and septic shock remain in regards to implementation of an early sepsis resuscitation bundle (RB) in order to maximize outcome benefit. We examined the impact of a hospital-wide CQI initiative on septic RB compliance and outcome and investigated the mortality benefits beyond the 6-hour recommendation period.

Methods Prospective cohort study of patients who met the definition of severe sepsis or septic shock over an 18-month period. Current sepsis RB being MAP $\geq 65, \mathrm{CVP} \geq 8$ and $\mathrm{SvO}_{2} \geq 70 \%$ goals met within 6 hours and 18 hours upon presentation.

Results The 498 severe sepsis and septic shock patients were enrolled and examined at what time period would the RB still be effective. Using a time cut-off, the Compliers at 18 hours and NonCompliers at 18 hours were then compared. There were 202 patients who had the RB completed in less than or equal to 18 hours (Compliers at 18 hours). There were 296 patients who never completed the RB within 18 hours (NonCompliers at 18 hours). The Compliers at 18 hours had a significant 10.2\% lower hospital mortality at $37.1 \%$ (22\% relative reduction) compared with the NonCompliers at 18 hours hospital mortality of $47.3 \%(P<0.03)$. Adjusting for differences in baseline illness severity, the Compliers at 18 hours had a greater reduction in predicted mortality of $26.8 \%$ vs $9.4 \%, P<0.01$. Compliance started at $30.4 \%$ and finished at $63 \%$.

Conclusions A CQI initiative for severe sepsis and septic shock with particular emphasis on the RB significantly improved bundle compliance and decreased hospital mortality. It has been previously shown that compliance to the RB within 6 hours improves outcome; however, when the time of bundle completion is extended to 18 hours, the mortality benefits are still significant. References

1. Dellinger RP, et al:: Surviving Sepsis Campaign: international guidelines for management of severe sepsis and septic shock: 2008. Crit Care Med 2008, 36:296-327.

2. Rivers $E$, et al: Early goal-directed therapy in the treatment of severe sepsis and septic shock. N Engl J Med 2001, 345:1368-1377.

\section{P399}

Effect of myocardial preservation of early goal-directed therapy on severe sepsis/septic shock patients in the ICU

GL Cai, J Yan, S Qiao

Zhejiang Hospital, Hangzhou, China

Critical Care 2010, 14(Suppl 1):P399 (doi: 10.1186/cc8631)

Introduction To investigate the effect of myocardial preservation of early goal-directed therapy (EGDT) on severe sepsis/septic shock patients in the ICU.

Methods This is a prospective and randomized controlled study, in which the total 158 severe sepsis/septic shock patients from the ICU were randomly assigned into two groups (EGDT group, $n=81$ and control group, $n=77$ ). Then the concentration of serum cardiac troponin I (cTnl), high sensitivity C-reactive protein (hs-CRP) and APACHE II score of patients were obtained on the $0,3 \mathrm{rd}, 7 \mathrm{th}$, and 14 th day after fluid resuscitation therapy.

Results The levels of cTnl were the same between the EGDT and control groups on day 0 (exceeded normal cTnl patients in two groups 0.41 vs 0.42 , normal cTnl patients in two groups 0.05 vs $0.04, P>0.05$ ), there was a dramatic decrease in exceeded normal cTnl patients of the EGDT group, in which cTnl returned to normal after EGDT (14th day: exceeded normal CTnl patients in two groups 0.08 vs $0.16, P<0.05)$; however, only a little difference in normal cTnl patients of the two groups $(P>0.05)$. The level of hs-CRP changed like $C T n l(P<0.05)$, and there was positive correlation between $C T h l$ and hs-CRP on each time $(P<0.05)$; APACHE \| scores obviously decrease in the EGDT group $(P<0.05)$. Meanwhile, the EGDT 
group have an obviously higher 28-day survival rate and longer survival time than that of the control group ( $74.1 \%$ vs $55.8 \%$; 23.5 vs 19.6, $P<0.05$ ).

Conclusions EGDT has an effect of myocardial preservation and improves the survival rate for severe sepsis/septic shock patients in the ICU.

\section{References}

1. Richard S, Hotchkiss RS, Karl IE, et al: The pathophysiology and treatment of sepsis. N Engl J Med 2003, 348:138-150.

2. Rivers E, Nguyen B, Havstad S, et al.: Early goal-directed therapy in the treatment of severe sepsis and septic shock. NEngl J Med 2001, 345:1368-1377.

P400

Impact of the sepsis bundle strategy on the outcomes of the patients suffering from severe sepsis and septic shock

ZWang

Beijing Shijitan Hospital, Beijing, China

Critical Care 2010, 14(Suppl 1):P400 (doi: 10.1186/cc8632)

Introduction Patients suffering from severe sepsis and septic shock have a high mortality. Studies have demonstrated that the septic bundle strategy decreased the mortality of severe sepsis and septic shock. The aim of this study was to compare the impact of the sepsis bundle strategy on the outcomes of the patients suffering from severe sepsis and septic shock in our emergency department.

Methods A prospective study was performed. The studied population included severe sepsis and septic shock patients entered into the Surviving Sepsis Campaign (SSC) database, who were admitted to the emergency department of an urban tertiary-care medical center during June 2008 to June 2009. They were divided into two groups based on the admission time (Group 1: June 2008 to December 2008, baseline survey of real practice in sepsis therapy before creating a protocol. Group 2: January 2009 to June 2009, creating a protocol implementing sepsis resuscitation bundle in sepsis therapy). Acute Physiology and Chronic Health Evaluation (APACHE II) scores, treatments and in-hospital mortality were compared.

Results The timing giving broad-spectrum antibiotics of ED admission was 3.67 hours in Group 1 and 2.52 hours in Group $2(P<0.01)$. Blood cultures before antibiotics administration was $39.7 \%$ in Group 1 and $56.3 \%$ in Group $2(P<0.01)$. Fluid bolus $(20 \mathrm{ml} / \mathrm{kg})$ delivered followed by vasopressors if needed was 27\% in Group 1 and $76 \%$ in Group $2(P<0.01)$. In-hospital mortality was $44.8 \%$ in Group 1 and $35.6 \%$ in group, in-hospital mortality decreased by $9.2 \%(P<0.01)$.

Conclusions Creating a protocol implementing sepsis bundles improved compliance for the bundle strategy on the treatment of severe sepsis and septic shock and decreased in-hospital mortality.

\section{P401}

Impact of Surviving Sepsis Campaign implementation in Brazil

F Machado, A Pereira, C Silva, P Schippers, E Ferreira, N Akamine, M Assuncao, BF Mazza, R Costa Filho, H Fernandes, C Grion, M Oliveira, MO Maia, E Silva

Latin America Sepsis Institute, São Paulo, Brazil

Critical Care 2010, 14(Suppl 1):P401 (doi: 10.1186/cc8633)

Introduction Sepsis mortality rates in Brazil are high $[1,2]$. Many studies have already shown that implementation of education programs based on Surviving Sepsis Campaign 6-hour (6h SSC) and 24-hour bundles successfully reduces sepsis mortality. The objective of this study was to evaluate the impact of the SSC-based program on patient outcome in Brazil. Methods Severe sepsis and septic shock patients were included in voluntary participating hospitals that have sent data for at least 2 years. The intervention was based on strategies aimed at improving compliance with the bundles. Regular reports containing compliance and mortality data were sent to the hospital in order to base local strategies of improving care. Results are presented by the semester of hospital inclusion in the campaign through 2 years, regardless of when those months occurred in the time frame of the study. Results were considered significant if $P<0.05$. Results From February 2005 and August 2009, 19 hospitals included 2,135 patients in a nonconcomitant fashion. Demographic data are restricted as in the SSC database. Septic shock patients comprised $52 \%$ of the patients with a mean number of organ dysfunctions of $2.34+1.33$, and $38.6 \%$ were admitted from the ER and $24.9 \%$ developed sepsis during their ICU stay.
Compliance to $6 \mathrm{~h}$ SSC increased from the first to the fourth semester as follows: lactate: $65.0 \%$ to $71.5 \%, P=0.01$, blood culture: $42.5 \%$ to $50.3 \%, P=$ 0.08 , antibiotics: $38.9 \%$ to $45.3 \%, P=0.02$, achieving mean arterial pressure $>65$ mmHg: $43.7 \%$ to $75.4 \%, P<0.000$, CVP achievement: $14.6 \%$ to $36.6 \%$, $P<0.000, \mathrm{SVO}_{2}$ achievement: 10.4 to $22.7, P=0.000$ and completion of the whole bundle: 8.3 to $9.0, P=0.34$. A 7\% absolute reduction (relative reduction of $11.6 \%$ ) in hospital mortality was found in the third semester of the campaign (OR 1.33 (1.04 to 1.69), $P=0.01$ ). However, in the fourth semester mortality has increased again to $60.6 \%$.

Conclusions Implementation of the SSC in Brazil has been associated with increased bundle adherence. However, the impact on the mortality rate seems not to be sustained, probably due to the difficulty associated with keeping an education program. It is important to develop strategies aiming to increase motivation in Brazilian hospitals.

References

1. Silva E, et al: Crit Care 2004, 8:R251-R260.

2. Sogayar AM, et al:: Pharmacoeconomics 2008, 26:425-434.

\section{P402}

Effect of synthetic vasopressin in septic shock using eosphageal Doppler H Elatroush, N Abed, F Ragab, M Afify

Cairo University, Cairo, Egypt

Critical Care 2010, 14(Suppl 1):P402 (doi: 10.1186/cc8634)

Introduction Noradrenaline and dopamine were the standard catecholamines used in the treatment of septic shock. Loss of response was the common problem that led to patient loss after large continuous doses of noradrenaline, which was termed catecholamine refractory septic shock. Recently vasopressin and its analog, namely terlipressin, were used in the treatment of such a catastrophic condition.

Methods In a prospective controlled study we included 40 patients with catecholamine-resistant septic shock (that is, noradrenaline dose exceeded $0.6 \mathrm{\mu g} / \mathrm{kg} / \mathrm{minute}$ ) divided into two groups: 20 patients were treated conventionally according to the Surviving Sepsis Campaign 2008, who served as a control group; and the other 20 patients were treated conventionally and when the noradrenaline dose exceeded $0.6 \mu \mathrm{g} / \mathrm{kg} /$ minute, terlipressin in a dose of $1 \mathrm{mg}$ intravenous bolus every 12 hours for a study time of 48 hours was started.

Results Terlipressin therapy was associated with increased systemic vascular resistance from $546 \pm 260$ dyne.sec/ $\mathrm{cm}^{-5}$ to $986 \pm 390$ dyne.sec/ $\mathrm{cm}^{-5}$ after 48 hours, which represents normalized arterilor tone that is expected to allow better organ bed perfusion. There was a reduction of both stroke volume and cardiac output (from $63 \pm 16 \mathrm{ml} /$ beat to $51 \mathrm{ml} /$ beat and from $78 \mathrm{l} /$ minute to $5.3 \mathrm{l} /$ minute, respectively) yet this was not associated with abnormal organ perfusion marked by improved urine output from $49 \mathrm{ml} /$ hour to $133 \mathrm{ml} /$ hour and improved global perfusion as marked by decrease lactic acidosis from $9.3 \pm 3 \mathrm{mEq} / \mathrm{l}$ to $5.7 \pm 3 \mathrm{mEq} / \mathrm{l}$, $P<0.002$. There was significant reduction of oxygen delivery ( $\mathrm{BO}_{2}$ from $848 \mathrm{ml} /$ minute to $610 \pm 47 \mathrm{ml} /$ minute after 48 hours, $P>0.02$ ). There was no effect on length of ICU stay in both groups ( $16 \pm 6$ days in the terlipressin group and $12 \pm 6$ days in control group, $P<0.06$ ). Terlipressin support showed nonsignificantly less mortality $(60 \%$ vs $70 \%$ in control group). Regarding organ function, terlipressin could improve the SOFA score from $11 \pm 3.2$ to $8 \pm 5$ with $P<0.02$.

Conclusions Terlipressin is a rather safe, inexpensive, easy to administer alternative in the treatment of septic shock. Further studies are needed to decide the ideal timing for initiation of this therapy early vs late adjuvant or as an initial treatment.

\section{Reference}

1. Vincent JL: Crit Care Clin 2006, 22:187-197.

\section{P403}

Effects of sepsis resuscitation bundle in cirrhotic patients with septic shock

L Rinaldi, E Ferrari, D Trevisan, L Donno, M Girardis

Policlinico di Modena, Italy

Critical Care 2010, 14(Suppl 1):P403 (doi: 10.1186/cc8635)

Introduction The effectiveness of the interventions recommended by the Surviving Sepsis Campaign (SSC) guidelines [1] has never been tested 
in patients with specific co-morbidities such as chronic liver disease. In this study we evaluated whether the adherence to the resuscitation sepsis bundle improved the outcome of cirrhotic patients with septic shock admitted to the ICU.

Methods The prospective observational cohort study included 38 patients with documented cirrhosis and septic shock admitted to a multidisciplinary ICU at a university hospital from January 2005 to June 2009. In each patient the compliance to four resuscitation interventions recommended by the SSC guidelines (that is, 6-hour bundle) and the 30-day mortality were measured.

Results The 6 -hour bundle was completed in $50 \%$ of the patients. In these patients the MELD and SOFA scores ( $39 \pm 11$ and $18 \pm 2$ ) were higher $(P<0.05)$ than those observed in patients without compliance to the 6 -hour bundle ( $31 \pm 12$ and vs $15 \pm 3$ ). The 30 -day mortality was $94.7 \%$ and 68.4\% $(P<0.05)$ in patients with and without 6 -hour bundle compliance, respectively. A Cox regression analysis, after adjustment for MELD and SOFA scores, indicated that none of the single sepsis interventions as well as the 6-hour bundle was independently associated with 30-day survival. Conclusions The adherence to the resuscitation interventions recommended by the SSC evidence-based guidelines did not improve the survival rate of cirrhotic patients with septic shock admitted to our ICU. Reference

1. Dellinger RP, et al: Surviving Sepsis Campaign: international guidelines for management of severe sepsis and septic shock: 2008. Crit Care Med 2008, 36:296-327.

P404

Outcomes and incidence of bleeding events associated with drotrecogin alfa: a single-centre experience of 440 patients

A Boyle, C McKenzie, S Yassin, A McLuckie, D Wyncoll

Guy's and St Thomas'NHS Trust, London, UK

Critical Care 2010, 14(Suppl 1):P404 (doi: 10.1186/cc8636)

Introduction Although drotrecogin alfa activated (DAA) was approved in 2002 for the treatment of severe sepsis, controversy has surrounded this therapy especially with respect to the increased risk of serious bleeding events (SBEs). Recently, there has been further interest in the complications of DAA following a review by Gentry and colleagues suggesting a significantly increased incidence of SBEs and death in those with baseline bleeding precautions [1]. We are one of the largest single-centre users of DAA worldwide and thus are keen to report our experience.

Methods In 2002 a scoring system, reporting tool, and supporting database were developed to track the outcome of DAA-treated severe sepsis patients in our hospital. This is maintained by the clinical pharmacists and captures data on relative contraindications, adverse events and patient outcome. Classification of data is obtained from the electronic patient record. Any DAA administration outside agreed criteria is recorded and bleeding incidents are reviewed with a consultant intensivist to confirm categorisation into serious or minor.

Results Four hundred and forty patients, including some enrolled in clinical trials, received DAA in our critical care unit over a 7-year period (2003 to 2009). The mean age was 63 years (range 17 to 92). Four hundred and twenty-eight (97.3\%) patients met agreed criteria for treatment. Twelve $(2.7 \%)$ were treated despite being in single organ failure. Mean organ dysfunction was 3.3 and over $75 \%$ of patients had at least three organ failures. The commonest sites of infection were respiratory (56\%), abdominal (29\%) and urinary tract (4\%). The 28-day, critical care and hospital mortalities were $35 \%, 39 \%$ and $46 \%$, respectively. Relative contraindications (Cls) were identified for 38 (8.6\%) patients treated with DAA. Of these, 23 (5.2\%) had an increased baseline bleeding risk, most commonly thrombocytopenia (2\%). Hospital mortality for patients with any Cl was $47 \%(n=18)$ and $48 \%(n=11)$ if existing predisposition for bleeding. In the 440 patients, 67 (15\%) bleeding events were reported, but only 19 (4\%) were considered SBEs (using the definitions from PROWESS [2]). Overall mortality for all bleeding events was $57 \%$ and $74 \%$ for patients with SBEs. Administration errors occurred in 34 (7.7\%) patients, of which three $(0.7 \%)$ were considered serious. Three hundred and eighteen (72\%) patients received DAA for at least 72 hours. Of those who did not complete the course, 81 (66\%) died.

Conclusions We have observed a low incidence of SBEs in patients treated with DAA. As expected, SBEs were associated with a poorer outcome.
We firmly believe an established scoring system and monitoring tool has facilitated effective use of DAA whilst minimising associated risks of therapy. References

1. Gentry C, et al:: Crit Care Med 2009, 37:19-25

2. Bernard G, et al: N Engl J Med 2001, 344:699-709.

P405

Activated protein C: cost matters!

T Dravid, B Pawar, B Bhurke, P Akole, P Rajhans, S Jog

Deenanath Mangeshkar Hospital, Pune, India

Critical Care 2010, 14(Suppl 1):P405 (doi: 10.1186/cc8637)

Introduction Activated protein $C(A P C)$ is an effective treatment in multiorgan failure (MOF) due to severe sepsis. Recommended duration of treatment of APC is 96 hours infusion. The cost of APC infusion for 96 hours in a $60 \mathrm{~kg}$ person is an average INR 490,000 in India $(€ 7,000)$ ( $€ 1 \sim$ INR 70 in November 2009, average value) whereas the average cost of 10 days of ICU treatment for a patient with sepsis with MOF, all inclusive, is approximately INR 140,000 in our hospital (€2,000). It is unclear whether stopping APC before 96 hours, in patients in whom MOF resolves before 96 hours, ultimately affects the final outcome in terms of survival. This is more relevant in developing countries like India where the cost of 96 hours of infusion of APC is beyond the reach of the majority of patients eligible for it. We retrospectively analysed the cost savings achieved by early stoppage of APC ensuring MOF reversal, before completion of the recommended 96 hours infusion.

Methods During a period of 3 years, patients with severe sepsis having $\geq 3$ organ failures were offered the option of APC. For those who opted for it, infusion was initiated within 24 hours of onset of MOF. APC infusion was stopped before completion of 96 hours only if septic shock, ARDS and coagulopathy was completely resolved, otherwise it was continued for 96 hours.

Results Two hundred and thirteen patients with severe sepsis having MOF were offered the option of APC infusion. Twenty patients opted for it. In seven patients APC was infused for 96 hours as per the standard recommendation. In 13 patients APC infusion could be stopped before 96 hours assuring complete reversal of MOF. They were enrolled in our study. Eleven out of 13 patients were discharged home after complete recovery. MOF did not reappear in any of these 13 patients. Two patients developed a new episode of ventilatory-associated pneumonia on day 4 and day 10, respectively, after stopping the infusion of APC and could not be salvaged. Average duration of APC infusion was $70.33 \pm 20.76$ hours. Cost of APC infusion per patient for average 70 hours was INR 347,692 $\pm 38,330$ (€4,967). Duration of ICU stay (average) was $11.61 \pm 5.18$ days. Average cost of ICU care for 11 days (excluding cost of APC) per patient was INR $144,272 \pm 86,754(€ 2,061)$. The cost saving achieved due to early stoppage of APC (per patient) was INR 132,708 $\pm 42,330(€ 1,895)$.

Conclusions Stopping APC infusion before 96 hours in patients who show rapid reversal of MOF is associated with significant cost saving without compromising the survival outcome. With this judicious early stoppage strategy, a greater number of patients can afford this effective drug.

\section{P406}

Outcome of patients receiving two or more infusions of activated protein C: a single-centre experience

A Retter', S Lippett'ㄹ, R Beale', D Wyncoll

'Guy's and St Thomas' NHS Trust, London, UK; 'Royal Sussex County Hospital, Brighton, UK

Critical Care 2010, 14(Suppl 1):P406 (doi: 10.1186/cc8638)

Introduction Patients frequently develop a second discrete episode of sepsis during an ICU stay such as ventilation-acquired pneumonia (VAP), or patients may have a second ICU admission unrelated to their initial presentation. In the PROWESS [1] study, drotrecogin alfa (DAA) could not be given twice to the same patient; yet this may be a potentially life-saving intervention when a second episode of severe sepsis occurs.

Methods We conducted a retrospective study of patients who received more than one infusion of DAA in our combined adult surgical and medical ICU. The attending ICU physician determined who received a second course of DAA. 
Results During the 6-year study period (November 2002 to October 2008) 354 patients were treated with DAA, seven patients were identified who received DAA twice and one patient received three infusions. Five patients treated with two infusions survived to be discharged home. Four patients were admitted to the ICU on two separate occasions, for these patients one infusion of DAA was given on each admission, three survived, and the median interval between admissions was 17 months (range 7 to 23). Four patients received a second infusion of DAA on the same ICU admission, the median interval between the first and second infusion was 3.5 days (range 3 to 12), and two survived. The median age was 67 years (range 41 to 77) with a median of four organ failures per patient (range 2 to 5). Those patients who received DAA twice on the same admission developed VAP or a line-related infection. Patients admitted for a second time had a different discrete episode of septic shock unrelated to their first admission. There was one major bleeding episode requiring cessation of DAA and a blood transfusion. This patient was found to have a duodenal ulcer. Six infusions of DAA were not completed, three patients died whilst receiving their second infusion, three courses were terminated due to rapid improvement in the patient's condition. No patient experienced significant bleeding during their second infusion.

Conclusions This is the first case series to report the outcome of patients who have received multiple courses of DAA. The incidence of repeat episodes of sepsis during a single admission or the precise rate of recurrent sepsis during ICU admissions is not easily identified in the current literature. Our findings are limited by the heterogeneous nature of the patient group and the lack of strict criteria defining who received a second course of DAA. A second course of DAA treatment may be justified on a case-by-case basis.

Reference

1. Bernard GR, et al:: N Engl J Med 2001, 344:699-670.

P407

Drotrecogin alfa (activated): a single-centre experience of 110 patients S Fletcher, N Pathmanathan

Bradford Teaching Hospitals, Bradford, UK

Critical Care 2010, 14(Suppl 1):P407 (doi: 10.1186/cc8639)

Introduction We present our experience with drotrecogin alfa (activated protein C (APC)) over 4 years (2005 to 2008) in 110 patients and compare the findings with a large UK audit [1].

Methods Data were collected on diagnosis, acuity, complications and outcomes. Survival was assessed using standardised mortality ratios.

Results One hundred and ten patients received APC. Two patients had two courses. Fifty-six per cent of patients received APC in the first 24 hours of ICU stay; $88 \%$ within 48 hours. Sixty-eight per cent of patients completed the 96 -hour course; $20 \%$ died during the infusion; $5 \%$ had APC stopped because of resolution of severe sepsis. Crude ICU mortality was 47\%; 9\% died after ICU discharge. Survival to hospital discharge was 44\%. The SMRs were $1.21(95 \% \mathrm{Cl} 1.02$ to 1.41$)$ using APACHE II and 1.05 (0.88 to 1.22) using the ICNARC method. Three patients had cerebral haemorrhage. Three had cerebral thrombotic events. There were no other cases of significant haemorrhage. APC usage fell over the study period 2005 to 2008 with 37, 35,26 and 14 patients per year, respectively.

\section{Table 1 (abstract P407). Patient characteristics}

\begin{tabular}{lc}
\hline Age & $57 \pm 16$ \\
Male sex & $53 \%$ \\
APACHE & $21 \pm 6$ \\
ICNARC & $29 \pm 7.3$ \\
Organ failures & 5 (4 to 5 IQR) \\
Postop & $38 \%$ \\
\hline
\end{tabular}

Conclusions Our results are similar to those of a UK-wide audit of APC usage [1]. The mortality of our patients was higher (56\% vs $45 \%)$, but our patients were probably sicker (median five organ failures vs three). Based on APACHE, actual outcome appears worse than predicted for patients receiving APC. Using the ICNARC method score, however, outcomes were similar to predicted. Rates of serious complications appeared to be similar to those experienced elsewhere. The fall in APC usage over time reflects uncertainty over its risk-benefit profile.

Reference

1. Rowan KM, et al:: Crit Care 2008, 12:R58

P408

Efficacy of recombinant human soluble thrombomodulin in disseminated intravascular coagulation

TYasuda, N Kiyonaga, T Ohryorji, M Nakahara, N Okayama, T Kikuchi, T Imabayashi, Y Kakihana, Y Kanmura

Kagoshima University Hospital, Kagoshima City, Japan

Critical Care 2010, 14(Suppl 1):P408 (doi: 10.1186/cc8640)

Introduction Soluble thrombomodulin is a promising therapeutic natural anticoagulant that is comparable with antithrombin, tissue factor pathway inhibitor and activated protein C. Recently, a recombinant human soluble thrombomodulin, composed of the active, extracellular domain of thrombomodulin, has become commercially available for patients with disseminated intravascular coagulation (DIC). However, its effect on adhesion molecule or plasminogen activator inhibitor 1 (PAI-1) in patients with DIC is not clear. To elucidate the possible effect of a soluble thrombomodulin on endothelial cell and neutrophil interaction in DIC, we investigated the efficacy of a recombinant human soluble thrombomodulin against not only soluble fibrin (SF) for the sensitive marker of DIC, but also soluble E-selectin (SES) and PAI-1 in patients with DIC.

Methods We studied 33 patients with DIC associated with sepsis. DIC was diagnosed according to the diagnostic criteria established by the Japanese Association for Acute Medicine. Seventeen of 33 patients (RTM group) were assigned a recombinant human soluble thrombomodulin for 6 consecutive days. SF, sES, and PAI-1 were measured at day 0 (before treatment), and day 7 . DIC resolution rate, clinical course of bleeding symptoms, and survival rates at 90 days were evaluated. Data were subjected to Mann-Whitney U test, Kaplan-Meier method and log-rank test $(P<0.05$ being considered statistically significant).

Results The baseline characteristics at study entry in both groups (17 treated and 16 control) were almost similar. There were no significant differences of the serum values of platelet, FDP, D-dimer and PAI-1 between two groups. However, SES and SF values in the RTM group were significantly decreased from $43.9 \pm 31.2$ (at day 0 ) to $29.46 \pm 15.08 \mu \mathrm{g} /$ $\mathrm{ml}$ (at day 7) $(P=0.044)$ and from $44.6 \pm 35.5$ (at day 0 ) to $18.5 \pm 16.6 \mu \mathrm{g} /$ $\mathrm{ml}$ (at day 7) $(P=0.039)$, respectively. Also both the DIC score and SOFA score improved significantly. There was no incidence of bleeding-related adverse events up to 7 days after the start of infusion in the RTM group. Survival rates at 90 days were 58.8\% (RTM group) vs 25.0\% (Control group). Conclusions In patients administered with recombinant human soluble thrombomodulin, sES and SF decreased and the DIC score and SOFA score improved significantly. There was no significant difference between the thrombomodulin group and Control group in survival rate, but there was a tendency that the RTM group had higher survival rate in comparison with the Control group.

\section{Reference}

1. Saito H, et al:. J Thromb Haemost 2007, 5:31-41.

\section{P409}

Clinical experience with lipopolysaccharide adsorber in cancer patients with severe sepsis and septic shock

E Gromova, M Kisselevskiy, N Anisimova, L Kuznetsova

Cancer Research Center, Moscow, Russia Federation

Critical Care 2010, 14(Suppl 1):P409 (doi: 10.1186/cc8641)

Introduction Sepsis is one of the most often met complications for cancer patients. It is caused by immunosuppression after radiotherapies and chemotherapies as extensive operations. For sepsis treatment, many experts now recommend even more often using extracorporeal detoxification methods. Since sepsis is more often caused by Gramnegative microorganisms in clinical hospitals, lipopolysaccharide (LPS) adsorption was offered for treatment of the patients.

Methods For extracorporeal detoxification of cancer patients with severe Gram-negative sepsis and septic shock (APACHE II score $28 \pm 7$ on starting 
hemoperfusion) we used LPS adsorber (Alteco ${ }^{\circledR}$ LPS Adsorber; Alteco Medical AB, Lund, Sweden). The patient's blood was taken before and after adsorption, then the serum was separated and stored at $-70^{\circ} \mathrm{C}$. We determined the LPS concentration by LAL test, lipopolysaccharide-binding protein (LBP), sIL-1 RII and SCD14 by ELISA kits (Hycult Biotechnology, the Netherlands), and proinflammatory and anti-inflammatory cytokines IL6, TNF $\beta$, IL-8, IL-2, INFY, IL-12, TNFa, IL-4, IL-5, IL-1 $\beta$ and IL-10 by ELISA kit (Bender MedSystems).

Results In all cases we saw reliable reduction of the LPS level in serum (1.8 to 2 times). After the investigation of some cytokine levels we obtained similar results: IL-6, TNF $\beta$ and IL-8 levels had been reduced 30 to $40 \%$, $87 \%$ and 62 to $76 \%$ accordingly. At the same time, IL-2, INFY, IL-12, TNFa, IL-4 and IL-5 levels in serum had not changed practically. However, in approximately $1 / 3$ cases an IL-1 $\beta$ and IL-10 concentration increase in serum was observed ( 33 and $40 \%$ accordingly). Also our research has shown that, in the most of the cases after application of this sorbent, LBP decreased insignificantly (10 to $32 \%$ ), but the sIL-1 RIl level slightly increased (10 to $18 \%$ ) in the majority of patients and considerably decreased (two times) in $1 / 3$ investigated patients. The CD14 concentration in patient serum reliably did not change before and after adsorption. The somatic status of patients was stabilized after LPS adsorption.

Conclusions After LPS adsorption with Alteco ${ }^{\oplus}$ the LPS level in patient serum significantly reduced. Simultaneously, concentrations of some cytokines (IL-6, TNF $\beta$, IL-8) were reliably decreased. But the LPS adsorber probably does not eliminate soluble receptors like SCD14 and RIL-1 from blood. So the LPS adsorption may represent a significant improvement in the treatment of cancer patients with severe Gram-negative sepsis and septic shock.

\section{P410}

\section{Apheresis of activated leukocytes with an immobilized polymyxin \\ B filter}

N Takeyama', T Kumagai', M Harada', Y Kajita', Y Miki', H Kanou², Y Inoue', T Nakagawa' ${ }^{1}, \mathrm{H} \mathrm{Noguchi}^{2}$

'Aichi Medical University, Aichi, Japan; 'Fujita Health University, Toyoake,

Japan

Critical Care 2010, 14(Suppl 1):P410 (doi: 10.1186/cc8642)

Introduction It was assumed that pathologic activation of neutrophils and monocytes is associated with sepsis, acute lung injury/ARDS, and multiple organ failure, and that removal of these cells from the circulation could reduce leukocyte-dependent tissue injury. Cartridges containing polymyxin B (PMX) immobilized to fibers (Toraymyxin; Toray Industries, Tokyo, Japan) have been developed for selective adsorption of circulating endotoxin in patients with Gram-negative bacterial infection, and this treatment has proven to be highly effective. This study examined the effect of direct hemoperfusion through filters with immobilized PMX direct-hemoperfusion (DHP) on leukocyte function and plasma levels of cytokines in patients with septic shock.

Methods We evaluated the effect of PMX-DHP on circulating leukocytes in patients with septic shock by assessing the changes of neutrophil and monocyte surface antigen expression after PMX-DHP. In another experiment, heparinized blood from patients with sepsis was passed through PMX filters in a laboratory circuit and then changes of the cell count and surface antigen expression by neutrophils and monocytes were assessed. After perfusion, neutrophils were isolated and the capacity of these cells to damage cultured endothelial monolayers was also determined.

Results We found that PMX-DHP led to an increased CXCR1 and CXCR2 expression along with a decrease of CD64 and CD11b expression by circulating neutrophils from septic patients. Plasma levels of cytokines, including IL-6, IL-8, IL-10, and high-mobility group box-1, were elevated in patients with septic shock compared with healthy controls, but cytokine levels were not altered by PMX-DHP. Ex vivo perfusion of heparinized blood from patients with sepsis through PMX filters in a laboratory circuit caused a significant decrease of the neutrophil and monocyte counts. Activated neutrophils with high CD11b/CD64 expression and low CXCR1/CXCR2 expression showed preferential adhesion to PMX filters. Neutrophils isolated from the blood after ex vivo PMX perfusion caused less damage on the endothelial cell monolayer than cells from sham-treated blood.
Conclusions PMX-DHP influences neutrophils via a mechanism that does not involve cytokines. Ex vivo results indicate that PMX-DHP selectively removes activated neutrophils and reduces the ability of circulating cells to cause endothelial damage. Selective removal of activated neutrophils by PMX-DHP may contribute to improvement of the systemic inflammatory reaction in septic patients.

P411

Therapeutic blood purification using functionalized core/shell nanomagnets

IK Herrmann', M Urner2', FM Koehler', B Beck Schimmer², WJ Stark'

'ETH Zurich, Switzerland; '2University Hospital Zurich, Switzerland

Critical Care 2010, 14(Suppl 1):P411 (doi: 10.1186/cc8643)

Introduction Direct and rapid removal of pathogens or noxious metabolites from a patient is the most straightforward cure imaginable. Dialysis and plasma filtration/exchange are the current broadly applicable methods to perform a direct removal of disease-causing factors from a patient. We describe the use of stable nanomagnets to rapidly and selectively remove heavy metal ions, overdosed steroid drugs and proteins from human blood. This nanomagnet-based purification method avoids fouling of filter membranes and benefits from a high external surface area, and a correspondingly fast diffusion.

Methods Nanomagnets equipped with heavy metal complexants, digoxin antibody fragments and entire human IL-6 antibodies were added to a series of blood samples. The nanomagnets scan blood by Brownian motion and capture their target. Afterwards, a small magnet was placed at the sample tube wall accumulating the nanomagnets in the pole region of the external magnet. The purified supernatant can then easily be decanted. The concentrations of lead, digoxin and IL-6 in blood samples were determined by standard clinical methods. Blood integrity was observed by rotation thromboelastography and monitoring of serum potassium, lactate dehydrogenase, bilirubin and haptoglobin levels. To measure the biological relevance of the IL- 6 removal, the effect on caspase-3 activation was assessed in camptothecin-stimulated neutrophils.

Results A significant decrease of lead, digoxin and IL-6 levels was measured after the blood purification procedure. The extraction using nanomagnets was in clear dose-effect dependence and could be accurately titrated. Treatment with nanomagnets did not significantly affect the integrity of blood and all levels remained in the clinical norm range. Caspase- 3 assays showed a reduced anti-apoptotic effect after IL-6 removal, underlining the biological relevance of the achieved removal efficiency.

Conclusions We demonstrate the extraction of lead, digoxin and IL-6 from whole blood as an example for the rapid treatment of heavy metal poisoning, drug overdosing and severe inflammation. The presented direct blood extraction could be combined with existing therapeutic strategies and may have major implications on the treatment of severe intoxications, sepsis (specific filtering of cytokines or toxins) [1], metabolic disorders (thyreotoxicosis) and autoimmune diseases.

References

1. Ronco C, et al:: N Eng/ J Med 2003, 348:1600-1602.

\section{P412}

\section{Coupled plasma filtration and adsorption in septic shock:} a multicentric experience

F Turani', M Falco', S Natoli², F Leonardis², M Fede², G Berlot' M Pelichovskà ${ }^{3}$

'European Aurelia Hospital, Rome, Italy; 'University of Tor Vergata, Rome, Italy; ${ }^{3}$ University of Trieste, Italy

Critical Care 2010, 14(Suppl 1):P412 (doi: 10.1186/cc8644)

Introduction Recently extracorporeal removal of mediators and endotoxins improved haemodynamics, organ dysfunction and mortality in patients with abdominal sepsis [1]. Coupled plasma filtration and adsorption (CPFA), too, may improve haemodynamics, respiratory function and mortality through removal of proinflammatory mediator. The aim of this multicentric study is to evaluate the haemodynamic response and the respiratory function, and to evaluate the reduction of inflammatory markers during CPFA. 
Methods Fifty-five septic patients were enrolled in this study. Every patient had four CPFA treatments (LINDA; Bellco-Mirandola, Italy) for 8 hours with $\mathrm{Q}_{\mathrm{b}}=200 \mathrm{ml} /$ minute, $\mathrm{Q}_{\text {utratition }}=30 \mathrm{ml} / \mathrm{kg} /$ hour and $\mathrm{Q}_{\text {. }}=20 \%$ of $\mathrm{Q}_{\mathrm{b}}$. At TO (basal), T1 (after first cycle), T2 (after second cycle), T3 (after third cycle) and T4 (after fourth cycle) we evaluated haemodynamic parameters, norepinephrine dosage, $\mathrm{PaO}_{2} / \mathrm{FiO}_{2}$ ratio, plasma IL-6, and procalcitonin $(P C T)$. The ANOVA test was used to compare changes during times study. $P<0.05$ was considered statistically significant.

Results Patients enrolled in the study have been submitted to 256 CPFA treatments for 2,650 hours. Table 1 presents the main results of the study. IV quartile of IL-6 is shown in Table 1.

Table 1 (abstract P412)

\begin{tabular}{lcc}
\hline & T0 & T4 \\
\hline $\mathrm{IL}-6(\mathrm{pg} / \mathrm{ml})$ & $850 \pm 127$ & $125 \pm 28^{*}$ \\
$\mathrm{PCT}(\mathrm{pg} / \mathrm{ml})$ & $45 \pm 9$ & $6 \pm 2^{* *}$ \\
Noradrenaline $(\mathrm{\mu g} / \mathrm{kg} / \mathrm{min})$ & $0.35 \pm 0.09$ & $0.08 \pm 0.05^{* *}$ \\
$\mathrm{PaO}_{2} / \mathrm{FIO}_{2}$ & $198 \pm 28$ & $310 \pm 92^{* *}$ \\
\hline
\end{tabular}

${ }^{*} P<0.05,{ }^{* *} P<0.01$ vs T0.

Conclusions In this large multicentric study, CPFA may improve haemodynamic status and respiratory function. Plasma filtration and adsorption of proinflammatory mediators may explain this improvement. Larger randomized controlled trials are indicated to confirm these data. Reference

1. Cruz D, et al:. JAMA 2009, 23:2445-2452.

\section{P413}

Patients admitted to the ICU for extra-abdominal disease and operated on for emergency laparotomy have significant survival

C Lentschener, J Beranger, J Charpentier, Y Ozier, J Mira

Cochin Hospital, Paris, France

Critical Care 2010, 14(Suppl 1):P413 (doi: 10.1186/cc8645)

Introduction Limited data are available concerning patients admitted to the ICU for an extra-abdominal disease and operated on for emergency laparotomy (EL) [1]. We investigated whether such patients had benefit from EL and factors likely to predict survival.

Methods EL was performed in 48 ICU patients admitted for an extraabdominal disease over the year 2008. The following variables were compared between survivors (discharged alive from hospital) and nonsurvivors: (a) sex ratio, BMl; (b) pre-existing co-morbidities considered in seven additive categories: cardiac, respiratory, renal, gastrointestinal, hepatic, diabetes, malignancies, and corticosteroid therapy; (c) preoperatively: IGS II score, mechanical ventilation (MV), vasopressor use, extra-renal epuration requirement, abdominal signs (obstruction, tenderness, contracture, compartment syndrome), records of computed tomography $(\mathrm{CT})$ and ultrasonography examinations; (d) intraoperative findings defined as perforation, infection, ischemia or necrosis of a visceral organ, pancreatitis, bowel obstruction, biliary disease, no finding. The $t$, Mann-Whitney $U$, and chi-square tests $(P<0.05)$, and multiple regression analysis $(P<0.1)$ were used.

Results Twenty-six patients (58\%) survived. EL was decided because of abdominal signs in 35 patients, lack of improvement of one or several organ failures in 44 patients, specific signs on CT scan, and/or ultrasound examination (active bleeding, subphrenic collection, bowel obstruction, pneumoperitoneum) in 22 patients, or nonspecific signs in 29 patients. EL did not identify any surgical cause in 10 patients. Groups did not differ in intraoperative findings $(P=0.2)$, preoperative creatinine level $(P=0.3)$, and preoperative anuria requiring extra-renal epuration $(P=0.2)$. Groups differed in preoperative $\mathrm{MV}(P=0.04)$, vasopressor requirement $(P=0.02)$, lactate levels $(P=0.03)$, IGS II $(P=0.0003)$, and pre-existing co-morbidities $(P=0.04)$. No such correlation was found in multivariate analysis (all $P>0.15$ ).

Conclusions Neither clinical nor laboratory variables, or operative findings predicted death following EL. The survival rate was high enough to warrant surgical EL for suspected intra-abdominal focus.

Reference

1. Sutherland FR, et al.: J Trauma 1989, 29:1982-1986.
P414

Can ICU admission be predicted?

L Orwelius, M Fredriksson, C Bäckman, J Persson, F Sjöberg

Faculty of Health Sciences, Linköping, Sweden

Critical Care 2010, 14(Suppl 1):P414 (doi: 10.1186/cc8646)

Introduction After intensive care (IC), patients report poor health-related quality of life (HRQoL). Many factors affect the patients and influence the HRQoL after discharge. One of these factors is the patient's health status before the critical care period. In a previous study we found that the IC patients have a high frequency of pre-existing diseases. However, it is unknown to what extent these pre-existing diseases affect the consumption of hospital resources (measured as days as inpatients) in the time period before admission to the ICU and during the years following it. The consumption prior to the ICU event may also be claimed to herald an increased risk for a later ICU admittance? The aim of this study was to examine the hospital care consumption of former ICU patients 3 years prior to and 3 years after the intensive care period. This was examined in relation to the pre-existing health status.

Methods Two mixed ICUs from hospitals in two cities in Sweden. A questionnaire including previous illnesses and HRQoL scale SF-36 were sent 6, 12, 24 and 36 months after discharge from the ICU and hospital. All adult patients with an ICU stay $>24$ hours were included. The institutional care consumption was assessed from the regional hospital databases

Results Six hundred and thirteen patients were included in the study. Of these $73 \%$ had pre-existing diseases. There were no significant differences between the previously healthy patients apart from younger age (mean age 52 (SD 20.7) vs 60 (SD 17.3)) and lower APACHE II scores (13.4 (SD 7.2) vs 16.4 (SD 7.7)) compared with patients with pre-existing disease. The ICU patients with pre-existing disease consumed significantly more hospital resources 3,2 and 1 year prior to admission to the ICU, and up to 3 years after discharge both regarding quantity of care (number of visits) $(P=0.001)$, length of stay $(P=0.001)$, and cost $(P=0.002)$. There were no significant differences in quantity of care, length of stay, or costs during the ICU period. Most importantly there was a significant increasing trend for the 3 years prior to the ICU event.

Conclusions Patients with co-morbidities have a significant consumption of hospital resources both prior to and after the ICU period. Interestingly, this consumption increased prior to the ICU event and seemed to herald the future deterioration of the patients. Further investigations should be made to examine to what extent ICU admissions can be predicted based on these data.

\section{Reference}

1. Orwelius L, et al:: Crit Care Med 2005, 33:1557-1564.

\section{P415}

\section{Unsuitable for ICU: what happens next?}

A McKeown, L Strachan, P Keeley, MG Booth, A Calder, A Panicker Glasgow Royal Infirmary, Glasgow, UK

Critical Care 2010, 14(Suppl 1):P415 (doi: 10.1186/cc8647)

Introduction This observational prospective study examined the outcomes and symptoms in those patients who are unsuitable for ICU admission.

Methods All patients referred to ICU for admission but deemed unsuitable were recorded by the team. These patients were reviewed by palliative care researchers. Demographic information (age, sex, diagnosis) and data on patient's symptoms of progress and outcome were collected at four time points $(24,48,72$ hours and 1 week).

Results Fifty patients were identified between January and April 2009. There was an age range of 24 to 86 (mean $=62)$. The commonest cause for referral was respiratory failure (40\%). Overwhelming sepsis requiring inotropic and ICU support was also common (28\%). Patients were most frequently deemed unsuitable for admission as they were too well at the time of referral (48\%). The second most common reason for unsuitability was co-morbidities (32\%) with nine (18\%) identified as suffering probable fatal insults. One patient was referred for assessment for ICU support post emergency surgery which was not required. A number of patients remained in hospital at 1 week post referral, with ongoing illness (32\%) or rehabilitation (8\%). Twenty-six per cent (13/50) of patients were discharged home and 34\% (17/50) died. Of those that died, 59\% were within 24 hours of ICU referral. All other deaths occurred within 1 week of referral. Nine 
of these 17 had been identified as having nonsurvivable insults at ICU referral, seven as having excess co-morbidities making ICU inappropriate, and just one identified as being too well.

Conclusions This study suggests that our ICU team is both sensitive and specific at identifying those patients who are either too well for admission or those patients for whom ICU is unlikely to be of benefit as their insult is presumed fatal. We suggest those patients whom the ICU identifies as unlikely to survive should be assessed and referred to the palliative care team, where appropriate. This should facilitate appropriate communication of this by clinical staff and allow the relevant social, psychological and spiritual preparations for death that are the hallmarks of good care of the dying.

\section{References}

1. [http://www.sicsag.scot.nhs.uk]

2. Joynt G, et al:: Intensive Care Med 2001, 27:1459-1465.

3. Lyons RA, et al: Lancet 2000, 355:595-598.

4. Thiery G, et al: J Clin Oncol 2005, 23:4406-4412.

5. Crippen D: Crit Care Med 2009, 37:1498-1499.

6. Wood G, et al:: Crit Care Med 2009, 37:1 147-1148.

\section{P416}

\section{Admission pattern and outcome in a UK weaning centre}

S Goodhand, K Stewart, L McGee, N Grey, A Williams, C Davidson, N Hart Guy's and St Thomas' Foundation Trust, London, UK

Critical Care 2010, 14(Suppl 1):P416 (doi: 10.1186/cc8648)

Introduction Although patients with weaning failure may be transferred to weaning centres, there are limited data on admission practice and clinical outcome.

Methods We collected data prospectively from March 2005 to October 2009. Results There were 286 referrals of which $20 \%$ were not accepted for transfer. Patients that were not accepted were categorised as weanable locally, unweanable, or died prior to transfer. A total 172/229 accepted patients were admitted (mean age was $60 \pm 17$ years; 118 male). Forty-six per cent of these achieved ventilator independence with $21 \%$ requiring ongoing non-invasive ventilation (NIV). The median time to wean was 17 days with $16 \%$ of patients remaining tracheostomy ventilator dependent. The unit mortality was $17 \%$. Neuromuscular disease (NMD) was the main referral group (36\%) with a low mortality rate (6\%). COPD and postsurgical patients both comprised $21 \%$ of the referrals and had respective mortalities of $37 \%$ and $25 \%$. There were no in-hospital deaths in the obesity-related respiratory failure (ORRF) group, but this was the smallest referral group (3\%). Of 57 patients declined transfer, 43 (83\%) were assessed as unweanable and nine (17\%) were assessed as weanable locally. Of 56 patients accepted but not transferred, 28 (50\%) were weaned awaiting transfer and $23(41 \%)$ died prior to transfer. Mean transfer delay was $20.3 \pm 19.2$ days and a major contributor to this delay was prolonged discharge delay following weaning (12.9 \pm 19.3 days) and extended discharge delay of tracheostomy-ventilated patients ( $77.4 \pm 51.8$ days).

Conclusions In conclusion, compared with our previous data [1], there was an absolute reduction in mortality of $10 \%$ with similar admission diagnoses, proportions achieving ventilator independence and requiring NIV, suggesting that this was not necessarily selection bias. NMD was the main referral group with a low mortality rate. Although COPD patients had the highest mortality, almost 50\% were weaned from invasive ventilation. There were no in-hospital deaths in the ORRF group, but these patients had the longest weaning time. In addition, poor outcome was observed in patients accepted but not transferred due to delay. This study highlights the need for more speciality weaning facilities in the UK.

\section{Reference}

1. Thorax 2005, 60:187-192

\section{P417}

\section{Referral to intensive care: who and when?}

A Mackay, J Erskine, P Doherty, E McMillan

Victoria Infirmary, Glasgow, UK

Critical Care 2010, 14(Suppl 1):P417 (doi: 10.1186/cc8649)

Introduction Intensive care beds in the UK are a limited resource and deciding whether or not to admit a patient to intensive care is often a difficult decision. It is increasingly felt that, as intensive care consultants are involved in the decision-making process for every admission, the consultant from the referring team should also be involved in the referral process. The Scottish Intensive Care Society Trainee Group found that this occurred in less than $50 \%$ of occasions during the Referral and Review Audit of 2007 [1]. We wished to establish what the referral process was like in our hospital.

Methods We undertook a prospective audit of all referrals to a five-bed teaching hospital ICU between April and July 2009. Data were collected regarding time of referral, seniority of referrer, involvement of referring consultant, seniority of ICU trainee, involvement of ICU consultant and whether or not the referral resulted in intensive care admission.

Results Ninety-eight referrals were received during the audit period. Of 31 medical referrals, 21 were out of hours, 16 had no consultant involvement and ultimately 27 patients were admitted. Of nine surgical patients, three were out of hours, three had no consultant involvement and seven were eventually admitted. Thirty patients were referred by the emergency department, of whom 18 were out of hours, 23 had no consultant involvement and 26 resulted in admission. Twenty-eight patients were referred by anaesthetics, all of whom had been seen by a consultant and were admitted.

Conclusions General medicine is the most significant contributor to outof-hours work of the intensive care medical staff. Over $50 \%$ of medical referrals have no referring consultant involvement, although ultimately many of these patients are admitted and are seen by an intensive care consultant within a few hours of admission. Most surgical patients are admitted postoperatively and thus are referred by a consultant anaesthetist, resulting in a very high conversion rate for referrals to admissions. Emergency medicine is increasingly a consultant delivered service with increased presence in the department into the extended working day, a fact that is sadly not reflected in our results. It is regrettable that despite repeated recommendations that intensive care admission should be on the basis of consultant to consultant referral, $43 \%$ of patients are referred to intensive care without any referring consultant involvement.

\section{Reference}

1. Scottish Intensive Care Society Annual Report 2008 [http://www scottishintensivecare.org.uk/sics/Annual\%20Report/2008\%20annual\%20 report.pdf]

\section{P418}

Acute drug overdose in an ICU: 15 years experience

L Cachafeiro, JJ Manzanares, S Yus, C Soriano, M Oliveros, M Jimenez

Hospital Universitario La Paz, Madrid, Spain

Critical Care 2010, 14(Suppl 1):P418 (doi: 10.1186/cc8650)

Introduction The aim of our study is to identify the epidemiology of patients admitted to an ICU following severe acute drug overdose.

Methods The study is a retrospective observational study of a 20-bed medical/surgical ICU during a 15-year period. We reviewed the clinical records of patients admitted from 1994 to 2008 with acute drug overdose. Data on demographics, mean length of stay, days on mechanical ventilation (MV), Glasgow Coma Scale (GCS) on admission, and mortality were recorded. The type of drug was also stated.

Results A total of 12,881 patients were admitted to our ICU from 1994 to 2008, of them 621 patients (4.8\%) had a diagnosis of acute intoxication on admission. From these 621 patients, 159 (25.6\%) were drug overdoses, which represented $1.2 \%$ from the total admissions. The median age was 33 years, the range 15 to 87 , with a standard deviation of 12.5. A total of $50.9 \%$ were male. The median GCS on admission was 6 and the APACHE II score was 16, with a range 3 to 31 and a standard deviation of 6.7. MV was required by $71.1 \%$ with a median duration of 2.38 days, with a range 0 to 45 and a standard deviation of 6.9. The median length of stay was 3.2 days, the range 0 to 41 and a standard deviation of 6.1. The types of drugs found were ethanol, cocaine, opioids (methadone, heroin, opium), LSD, ketamine, GHB, amphetamines, cannabis, and PCP. Ethanol was the most commonly encountered in $57 \%$, followed by cocaine in $37 \%$, and opioids in $27 \%$ of patients. Global mortality was $2 \%$, and $50 \%$ of these were due to ethanol.

Conclusions In our study, severe acute drug overdose accounts for a low percentage of patients admitted to the ICU. The profile is that of a middleaged male patient with acute ethanol, cocaine, or opioids overdose, in 
that order of frequency, and with rates similar to those of national and European statistics, of short length of stay (3.2 days) and a mortality inferior to that expected for the APACHE II score.

\section{References}

1. Palazon Sanchez C, et al:: Rev Esp Salud Publica 2000, 74:55-63.

2. Caballero Valles PJ, et al: An Med Interna 2008, 25:262-268.

\section{P419}

Prognostic factors for very short-term mortality in severe sepsis E Silva', R Salomao ${ }^{2}$, O Rigato ${ }^{3}$, E Ferreira², T Mohovic', S Callas ${ }^{3}$, N Tereran², M Assuncao', N Delbiagi ${ }^{3}$, F Colombari', C Silva ${ }^{4}, \mathrm{RCal}^{4}, \mathrm{~F} \mathrm{Machado}^{2}$ 'Hospital Israelita Albert Einstein, São Paulo, Brazil,' ${ }^{2}$ Federal University of São Paulo, Brazil; ${ }^{3}$ Hospital Sirio-Libanes, São Paulo, Brazil; ${ }^{4}$ Latin American Sepsis Institute, São Paulo, Brazil

Critical Care 2010, 14(Suppl 1):P419 (doi: 10.1186/cc8651)

Introduction Short-term mortality studies could be crucial for evaluating new therapies as well as to better understand pathophysiological aspects. This study aims to identify prognostic factors associated with sepsisrelated death within 4 days after the first organ dysfunction development. Methods All severe sepsis and septic shock patients over 18 years old admitted to three Brazilian ICUs were prospectively included. Demographic, clinical and outcome features, including compliance to the 6-hour Surviving Sepsis Campaign bundle (SSC-6h), were collected in an electronic clinical research form. Duration of organ dysfunction was defined as the time elapsed between the installation of the dysfunction and its diagnosis by the healthcare provider. Results were expressed as a percentage or as median and interquartiles. Univariate analysis was performed by chi-squared or Student $t$ test. $P<0.05$ was considered significant.

Results Three hundred and eighty-six patients were included, being 30 (7.8\%) in Group 1 (short-term mortality group, median time from organ dysfunction to death: 2.39 (1.75 to 3.25) days) and 356 (92.2\%) in Group 2. No difference was found between the groups regarding gender $(P=$ $0.14)$, age $(P=0.80)$, being in a public hospital $(P=0.94)$ or duration of organ dysfunction $(P=0.79)$. Variables related to severity of disease were associated with early mortality: APACHE I| score (Group 1: 21.0 (15.0 to 26.0), Group 2: 18.0 (14.0 to 22.0), $P=0.01$ ); SOFA score at admission (SOFAO) (Group 1: 9.0 (8.0 to 11.0), Group 2: 7.0 (5.0 to 9.0), $P=0.003$ ); Day 1 SOFA score (10.0 (8.0 to 12.0), Group 2: 7.0 (5.0 to 10.0), $P=0.0002)$; Day 3 SOFA score (SOFA3) (Group 1: 9.0 (8.0 to 12.0), Group 2: 7.0 (4.0 to 10.0), $P=0.006)$ and the delta between SOFA0 and SOFA3 (Group 1: $-6.5(-11.0$ to +1.0$)$, Group 2: $-1.0(-3.0$ to +9.0$), P=0.003)$. Delta SOFA with Day 1 was not different between groups. Group 1 patients had a trend towards higher number of organ dysfunction $(P=0.07)$ and more septic shock episodes $(P=0.09)$. Regarding treatment adequacy, compliance with any indicator or the entire SSC-6h bundle was not related to survival. However achievement of the CPV target was associated with a higher early mortality (Group 1: 69.6\%, Group 2: 40.6\%, $P=0.013$; OR - 3.3.4 (1.33 to 8.40)).

Conclusions In this observational study, factors related to disease severity rather than to the evidence-based treatment compliance were associated with short-term death among severe septic patients.

\section{P420}

\section{Is sepsis mortality rate driven by differences in Brazilian healthcare} systems?

F Machado', R Salomao', O Rigato', E Ferreira', T Mohovic ${ }^{3}$, G Schettino², N Tereran', M Assunçao ${ }^{3}$, R Caltabiano ${ }^{2}$, F Colombari $^{3}$, C Silva $^{4}$, R Cal ${ }^{3}$, E Silva $^{3}$

'Federal University of São Paulo, Brazil; ${ }^{2}$ Hospital Sirio-Libanes, São Paulo, Brazil; ${ }^{3}$ Hospital Israelita Albert Einstein, São Paulo, Brazil; ${ }^{4}$ Latin American Sepsis Institute, São Paulo, Brazil

Critical Care 2010, 14(Suppl 1):P420 (doi: 10.1186/cc8652)

Introduction The sepsis mortality rate is higher in Brazilian public hospitals with no clear differences in risk factors. We aim to identify risk factors that could explain why the mortality rate is different in public and private hospitals.
Methods All severe sepsis and septic shock patients over 18 years old admitted to two private ICUs (Group 1) and one public ICU (Group 2) were prospectively included. Demographic, clinical and outcome features, including compliance to the 6-hour Surviving Sepsis Campaign bundle (SSC-6h), were collected in an electronic CRF. Duration of organ dysfunction was defined as the time elapsed between the installation of the dysfunction and its diagnosis by the healthcare provider. Results were expressed as a percentage or as median and interquartiles, and $P<0.05$ was considered significant.

Results Three hundred and eighty-six patients were included (Group 1 - 210, Group 2 - 176). Patients from Group 1 were older (69.5 (53.0 to 81.0 ) years vs 59.0 ( 43.5 to 73.5 ) years, $P<0.000)$, had a higher APACHE ॥ score (19.0 (15.0 to 23.0$)$ vs 17.0 (13.0 to 23.0$), P=0.01$ ), had more organ dysfunctions (3.0 (2.0 to 4.0) vs $2.0(2.0$ to 4.00$), P=0.08$ ), and more septic shock episodes $(74.3 \%$ vs $60.8 \%, P=0.006)$. No difference was found regarding gender, site of infection, and day 1 or day 3 delta SOFA score. However, the mortality rate was higher in Group 2 than in Group 1, 60.8 and 38.3 , respectively. Compliance to the SSC-6h bundle did not explain this difference as they were better in Group 2 for lactate (70.5\% vs 49.8\%), blood culture (61.4\% vs 52.4\%), achieving MAP $>65 \mathrm{mmHg}(98.8 \%$ vs $90.5 \%)$, and completion of the bundle (15.9\% vs $4.8 \%)$. Only antibiotics (63.3\% vs $54.5 \%, P=0.10$ ) and CVP had a better performance in Group 1 ( $49.4 \%$ vs $33.3 \%, P=0.01)$. SOFA score at admission was slightly higher on Group 2 (7.0 (4.0 to 9.0) vs 8.0 (5.5 to 10.0), $P=0.004$ ). However, duration of organ dysfunction was clearly longer in Group 2 (150.0 (0.0 to 650) minutes vs 360.0 ( 0.0 to 1008.0 ) minutes, $P=0.02$ ). In all patients, we have identified some variables associated with mortality, including being in the public hospital (OR 2.46 (1.63 to 3.72), $P=0.00002$ ), as well as the median duration of organ dysfunction, SOFA at admission, age, APACHE II score and number of organ dysfunctions.

Conclusions The mortality rate was higher in the public hospital despite better SSC bundle adherence. A delay in initiating those interventions may negatively impact the outcome in patients treated in public hospital.

\section{P421}

\section{Prognoses indicators of HIV patients admitted to the ICU}

B Moya, N Germano, G Imelda

Hospital de S. Bernardo, Setúbal, Portugal

Critical Care 2010, 14(Suppl 1):P421 (doi: 10.1186/cc8653)

Introduction The level of immunity in HIV patients is associated with a high mortality, particularly when CD4+ lymphocytes are inferior to 200 cells $/ \mathrm{mm}^{3}$. Besides the immunity, other prognoses indicators are necessary. Methods The author performed a revision of 1,028 admissions to the ICU from January 2007 and November 2009. Selected were the cases of HIV patients with low immunity $\left(\mathrm{LCD} 4^{+}<200 \mathrm{cell} / \mathrm{s} / \mathrm{mm}^{3}\right)$. The following parameters were collected at admission to the ICU: viral load, LDH, albumin, hemoglobin, Hct, thrombocytopenia, presence of opportunistic infection, nutritional status, need for mechanical ventilation, renal insufficiency and history of antiretroviral therapy (ART). Demographic parameters were also collected. The primary endpoint was mortality, secondary endpoints were ICU length stay and days of mechanical ventilation.

Results Fifteen patients were selected; all of them were medical admissions mainly from the emergency department. Sixty-five percent had previous history of opportunistic infection, and only two patients had ART. The main reason to suspend ART was social and drug-dependency problems. Sepsis was diagnosed in $73 \%$ of the patients. Pneumocystis carinii pneumonia was diagnosed in $80 \%$ of the patients. Fungus infection was diagnosed in $60 \%$ of patients. One patient had pulmonary tuberculosis and another had Leishmania infection. The mortality rate was $53 \%$. The factors associated with high mortality were: P. carinii pneumonia, malnutrition and absence of ART.

Conclusions The patient's nutritional status is an independent predictor of mortality in HIV patients.

References

1. Johannessen A, Naman E, et al:: Predictors of mortality in HIV-infected patients starting antiretroviral therapy in a rural hospital in Tanzania. BMC Infect Dis 2008, 8:52.

2. Swaminathan $S$, Padmapriyadarsini $C$, et al:: Nutritional status of persons with HIV infection, persons with HIV infection and tuberculosis, and HIVnegative individuals from southern India. Clin Infect Dis 2008, 46:946-949. 
P422

Outcomes of cancer patients admitted to intensive care units with severe acute kidney injury

S Lobo, M Soares, A Torelly, P Mello, U Silva, J Teles, E Silva, P Caruso,

G Friedman, P Souza, A Rea-Neto, A Vianna, M Maia, J Salluh

Brazilian Research in Intensive Care Network - BRICNet, Rio de Janeiro, Brazil

Critical Care 2010, 14(Suppl 1):P422 (doi: 10.1186/cc8654)

Introduction Critically ill cancer patients are at increased risk for acute kidney injury $(\mathrm{AKI})$, but studies on these patients are scarce and were all single centered, conducted in specialized ICUs. The aim of this study was to evaluate the characteristics and outcomes in a prospective cohort of ICU cancer patients with AKI.

Methods Prospective multicenter cohort study conducted in ICUs from 28 hospitals in Brazil over a 2-month period. Univariate and multivariate logistic regression were used to identify factors associated with hospital mortality.

Results Out of all 717 ICU admissions, 87 (12\%) had AKI and 36\% of them received dialysis. Kidney injury developed more frequently in patients with hematological malignancies than in patients with solid tumors (26\% vs $11 \%, P=0.003)$. Ischemia/shock (76\%) and sepsis (67\%) were the main contributing factors, and kidney injury was multifactorial in $79 \%$ of the patients. The ICU mortality was 61\% (53/87) and hospital mortality was 71\% (62/87). Despite the lack of statistical significance, hospital mortality was higher in patients who received RRT later on during the ICU stay (92\%) in comparison with those who received RRT on the first day in the ICU (78\%) and those who were not dialyzed (64\%) $(P=0.105)$. End-of-life decisions (to withhold or to withstand therapies) were taken in 18 (23\%) patients. General and renal-specific severity-of-illness scores were inaccurate in predicting outcomes for these patients. In a multivariate analysis, length of hospital stay prior to ICU, acute organ dysfunctions, need for mechanical ventilation and a poor performance status were associated with increased mortality. Moreover, cancer-related characteristics were not associated with outcomes

Conclusions The present multicenter study confirmed that AKI in critically ill patients with cancer is frequent, usually multifactorial and still associated with high mortality rates. On the other hand, the current study also suggests that ICU admission and RRT should be considered in selected patients. Mortality in these patients is mostly dependent on the severity of acute illness and the performance status, rather than cancerrelated characteristics.

\section{P423}

Is intensive care beneficial for patients with haematologic malignancies?

M Nyunga, C Lemaire, E Ras, P Herbecq, I Verheyde

Centre Hospitalier de Roubaix, France

Critical Care 2010, 14(Suppl 1):P423 (doi: 10.1186/cc8655)

Introduction Despite a better prognosis for patients with haematologic malignancies (PHM) over these past years due to progress in management, intensivists are reluctant to admit those patients to the ICU because of high mortality rates, above $50 \%$. The aim of this study was to retrospectively evaluate the value of intensive care therapy of PHM and predictive factors of short-term and long-term survival with the best post-ICU quality of life. Methods The case notes of 88 PHM patients requiring admission to a 25 bed medical-surgical ICU of Roubaix Hospital, France were retrospectively reviewed during a 5-year period, 2003 to 2007. We collected demographic factors, Simplified Acute Physiology Score II (SAPS II), Logistic Organ Dysfunction System score (LODS), Charlson score, microbiology, status of underlying HM, haematologic factor data, therapeutic support, blood cell transfusion, and survival follow-up (at the first 48 hours on the ICU, at discharge from the ICU, at 3, 12 and 18 months after ICU).

Results Univariate logistic regression analysis of factors previously shown to influence survival revealed that $\mathrm{HM}$ status, Charlson score, $\mathrm{PaO}_{2} / \mathrm{FiO}_{2}$ $<200$, during mechanical ventilation, blood cell transfusion, and outcome in the first 48 hours on the ICU were significantly associated with survival $(P<0.05)$. PHM with a favourable course (treatment response) in the first 48 hours on the ICU had a better survival rate (95.7\%) than an unfavourable course $(33.3 \%)(P<0.001)$. There was a correlation between mean SAPS II, LODS and outcome at the first 48 hours on the ICU $(P=0.03)$. Using multivariate analysis identified three variables independently associated with ICU survival: two negative, mechanical ventilation $(H R=4.9)$ and LODS score $(H R=1.12)$; one protective factor, red blood cell transfusion $(\mathrm{HR}=0.7)$ without additional cost. ICU, 3-month, 12-month and 18-month survivals were respectively $69.3 \%$ (61/88), 37.5\% (33/88), 27.3\% (24/88) and $23.8 \%(21 / 88)$ with a mean performance status at $1.03 \pm 1$, a mean Karnofsky index at $77.6 \pm 15$.

Conclusions The ICU management provided benefit for PHM cases referred earlier to the ICU because of the good quality of life for long-term survivors. Therefore, the unlimited life-sustaining treatment should be re-evaluated on the first 48 hours in the ICU based on SAPS II, LODS and Charlson scores.

\section{P424}

Postoperative mortality in the Netherlands: identifying high-risk surgery

PG Noordzi

OLVG, Amsterdam, the Netherlands

Critical Care 2010, 14(Suppl 1):P424 (doi: 10.1186/cc8656)

Introduction Type of surgery is considered a risk factor of adverse outcome, but mortality rates of common procedures are scarce $[1,2]$. The objective of the study was to further identify high-risk patients by procedure-specific mortality.

Methods Data between 1991 and 2006 were obtained from Dutch national healthcare statistics. Patients age $>20$, with an elective, nonday case, open surgical procedure were enrolled. Data included main diagnosis, secondary diagnoses, dates of admission and discharge, death during admission, operations, age, gender, and co-morbidities according to ICD-9-CM. Logistic regression analyses were applied to evaluate the relationship between type of surgery and postoperative mortality.

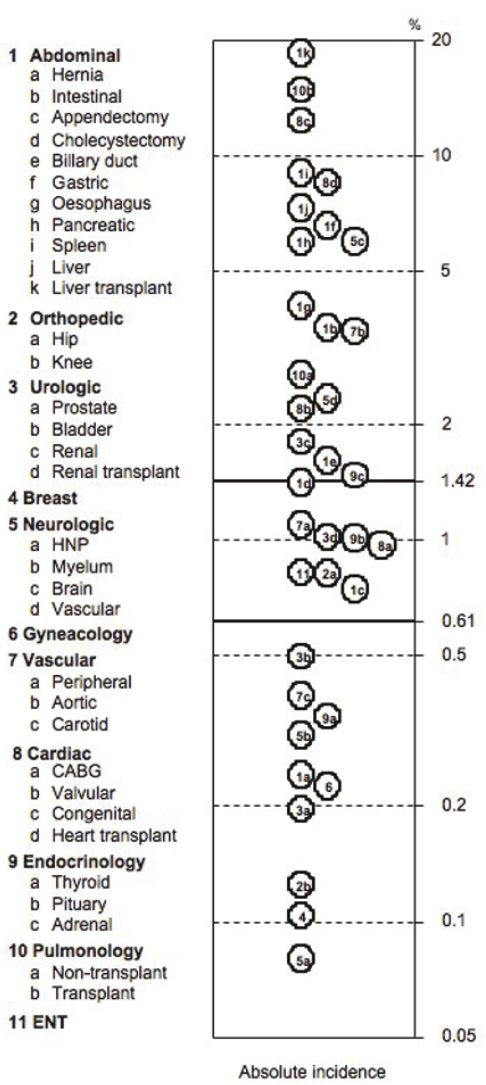

Figure 1 (abstract P424). Absolute incidence of postoperative mortality in relation to type of procedure. 
Results A total of $3,667,875$ surgical procedures, from 102 hospitals, were included. Postoperative death was observed in 67,879 patients (1.85\%). Overall, vascular surgery had highest mortality (incidence 6.0\%). Abdominal surgery was associated with $2 \%$ mortality. However, procedure-specific adjusted mortality identified spleen (adjusted OR 6.84), liver (adjusted OR 5.45), gastric (adjusted OR 4.86) and pancreatic surgery (adjusted OR 4.38) as higher risk procedures than aortic surgery (adjusted OR 2.5) (Figure 1).

Conclusions This study revealed a procedure-specific risk classification to accurately identify high-risk patients.

\section{References}

1. Lee TH, et al:: Circulation 1999, 100:1043-1049.

2. Fleisher LA: J Am Coll Cardiol 2007, 50:1707-1732.

\section{P425}

Outcomes of 1,000 patients with cancer admitted to the intensive care unit: a Brazilian prospective study

F Galas', L Hajjar², J Almeida', T Trielli', S Vieira', M Bazan'1, H Palomba', C Simoes' ${ }^{1}$, Torres $^{1}$, R Kalil-Filho', P Hoff', J Auler Jr'

'Instituto do Cancer do Estado de São Paulo, Brazil; 'Heart Institute, São Paulo, Brazil

Critical Care 2010, 14(Suppl 1):P425 (doi: 10.1186/cc8657)

Introduction ICU admission of critically ill cancer patients was controversial until recently. In the past years, advances in the management of malignancies and organ failures have improved outcomes of patients, resulting in higher rates of survival in the ICU. The aim of this study is to prospectively evaluate the characteristics, short-term and mid-term outcomes of cancer patients requiring intensive care.

Methods During 1 year, we evaluated prospectively patients with cancer admitted to the Instituto do Cancer do Estado de São Paulo. A total of 1,000 patients were included in the study; and collected data were: baseline data, risk scores, clinical status, co-morbidities, admission diagnosis, ICU interventions, ICU and hospital outcomes and 90-day outcomes.

Results From 1,000 patients, 828 (82.8\%) had solid tumors and 172 (17.2\%) had hematologic malignancies. The most frequent reasons for ICU admission were: sepsis (31\%), postoperative care (29\%) and respiratory failure (24\%). The mean APACHE II score value at the day of admission was $24.1 \pm 8.8$ (8 to 45 ). ICU mortality was $24 \%$, hospital mortality was $32 \%$ and 3 -month mortality was $41 \%$. Logistic regression analysis showed that need for mechanical ventilation (odds ratio $=6.76 ; 95 \% \mathrm{Cl}=3.56$ to 12.85), presence of metastasis (odds ratio $=3.87 ; 95 \% \mathrm{Cl}=2.06$ to 7.28 ), occurrence of acute renal failure (odds ratio $=3.92 ; 95 \% \mathrm{Cl}=1.47$ to 10.46 ) and higher SOFA scores 72 hours after admission (odds ratio $=5.76 ; 95 \%$ $\mathrm{Cl}=4.56$ to 14.85 ) were independently associated with increased hospital mortality. Three-month quality of life of patients who survived was considered unchanged in 55\% patients, worse in 25\% and better in 20\%.

Conclusions This prospective analysis of 1,000 patients with cancer needing intensive care shows high survival rates and good quality of life after ICU admission. These data encourage intensive care treatment in oncologic patients to prevent, detect and cure organ dysfunction.

\section{References}

1. Azoulay $E$, Thiery $G$, Chevret $S$, et al.: The prognosis of acute respiratory failure in critically ill cancer patients. Medicine 2004, 83:360-370.

2. Soares M, Salluh J, Torres VB, et al:: Short- and long-term outcomes of critically ill patients with cancer and prolonged ICU length of stay. Chest 2008, 134:520-526.

\section{P426}

Trend in outcome of critically ill patients with co-morbidity:

a 15-year study

B Afessa, O Gajic, MT Keegan

Mayo Clinic, Rochester, MN, USA

Critical Care 2010, 14(Suppl 1):P426 (doi: 10.1186/cc8658)

Introduction Critically ill patients with underlying co-morbidity have a high hospital mortality rate. This study describes the trend in the severityadjusted mortality of such patients over a 15-year period.

Methods We used the Acute Physiology and Chronic Health Evaluation (APACHE) III database of our institution to identify patients with solid tumors, hematology malignancy, and hepatic cirrhosis. We categorized the 15-year study period into three 5-year groups. We calculated the standardized mortality ratio (SMR) with the 95\% confidence interval (CI) for each co-morbidity.

Results During the study period, there were 73,626 ICU admissions; $92.6 \%$ white, $56.1 \%$ male, mean age 62.7 years, and $17.7 \%$ with comorbidities. The overall mortality rate of the study population was $10.2 \%$ : $7 \%$ for patients with no co-morbidity, $21.1 \%$ for solid tumors, $25.7 \%$ for hematology malignancy, and $29.2 \%$ for hepatic cirrhosis. The predicted mortality rates were $9.9 \%$ for patients with no co-morbidity, $25.6 \%$ for solid tumors, $27.6 \%$ for hematology malignancy, and $35.7 \%$ for hepatic cirrhosis. The SMR (95\%) Cl for patients without co-morbidity was 0.71 ( 0.66 to 0.75 ), 0.83 (0.79 to 0.87 ), and 0.62 (0.59 to 0.65 ) for the first, second, and third 5 -year periods, respectively. The SMR (95\%) CI for the each co-morbidity is presented in Table 1.

Table 1 (abstract P426). SMR, 95\% Cl for each co-morbidity

\begin{tabular}{lccc}
\hline Co-morbidity & First 5-year & Second 5-year & Third 5-year \\
\hline Hematology & $1.16,1.03$ to 1.30 & $0.95,0.88$ to 1.02 & $0.85,0.79$ to 0.91 \\
Cirrhosis & $1.12,0.95$ to 1.31 & $0.82,0.73$ to 0.92 & $0.70,0.62$ to 0.80 \\
Solid tumor & $0.80,0.68$ to 0.93 & $0.84,0.75$ to 0.93 & $0.82,0.74$ to 0.91 \\
\hline
\end{tabular}

Conclusions The severity-adjusted hospital mortality of critically ill patients with hematology malignancy and hepatic cirrhosis has improved over the past 15 years.

\section{P427}

Clinical profile, maternal and fetal mortality analysis of severe gestosis admitted to the ICU

E Curiel Balsera, M Prieto Palomino, G Quesada Garcia, J Muñoz Bono,

J Mora Ordoñez, MJ Ruiz de Elvira

Carlos Haya Hospital, Málaga, Spain

Critical Care 2010, 14(Suppl 1):P427 (doi: 10.1186/cc8659)

Introduction Pre-eclampsia is a severe multisystemic disease that may occur in pregnancy or in the immediate postpartum period.

Methods We included prospectively women admitted to the ICU with the diagnosis of severe gestosis, from January 1998 to October 2007. We define pre-eclampsia, eclampsia and HELLP syndrome as used by the American College of Obstetrics and Gynecology. Qualitative variables are shown as a percentage and quantitative variables as mean \pm standard deviation or median and interquartile range. We used the chi-square test, $t$ test and multivariate testing for statistical analysis of the data.

Results We report data from 262 women. Median age was 31 years (27 to 34). The admission diagnosis was pre-eclampsia $77.48 \%$, eclampsia $6.11 \%$ and HELLP syndrome $16.41 \%$. Complications registered were pulmonary oedema $9 \%$, coagulopathy $2 \%$ and renal failure $5 \%$. Delivery was by cesarean in $89.69 \%$. The median newborn weight was $1,500 \mathrm{mg}(1,000$ to 2,140$)$. We did not find significant difference on systolic pressure between preeclampsia, eclampsia or HELLP nor in uric acid levels. Gestational age was significantly lower in pre-eclampsia than in eclampsia or HELLP $(P<0.0001)$ and, additionally, the weights of the newborn were significantly lower in pre-eclampsia versus eclampsia and HELLP. Maternal mortality was 1.5\% and fetal mortality $16.3 \%$. Maternal death is associated with complications in the ICU (renal failure, heart failure or coagulopathy) $(P<0.034)$, OR 6.55 ( $95 \% \mathrm{Cl} 0.89$ to 48.12 ), but multivariate analysis shows only coagulopathy as an independent mortality risk factor $(P<0.026)$, OR $30.3(95 \% \mathrm{Cl} 1.5$ to 609.8$)$. Fetal mortality is associated in multivariate analysis with newborn weight $<1 \mathrm{~kg}(P=0.026), \mathrm{OR} 4.1$ (95\% Cl 1.1 to 14.3$)$, and higher gestational age at delivery is protective $(P=0.001)$, OR 0.68 ( $95 \% \mathrm{Cl} 0.54$ to 0.85 ).

Conclusions Women admitted to the ICU are mostly 31 years old, in the 32-week gestational age of their first pregnancy. In our environment, delivery mostly occurs in the following 5 to 7 days of admission. Maternal mortality is low, but not so fetal mortality that rises up to $16,3 \%$. Maternal mortality is associated with complications such as coagulopathy, and fetal mortality mostly with gestational age and low birth weight.

\section{Reference}

1. Sibai BM: Diagnosis and management of gestational hypertension and preeclampsia. Obstet Gynecol 2003, 102:181-192. 
P428

Obstetric critical care in developing countries: a comparison between two intensive care units

JA Rojas-Suárez', FP Paruk², JE Miranda-Quintero', AP Paternina-Caicedo ', OD De la Ossa-Mercado ${ }^{3}$

'Universidad de Cartagena, Cartagena de Indias, Colombia; ${ }^{2}$ University of Witwatersrand, Johannesburg, South Africa; ${ }^{3} G R I C I O$, Cartagena de Indias, Colombia

Critical Care 2010, 14(Suppl 1):P428 (doi: 10.1186/cc8660)

Introduction To describe both characteristics and differences of critically ill obstetric patients in two ICUs from two developing countries.

Methods A comparative study between two historic cohorts (2001 to 2002 from South Africa and 2006 to 2007 from Colombia) was designed to analyze characteristics from two ICUs in two developing countries: one in South Africa, King Edward VII Hospital (KEH) (South Africa); and another in Colombia, Clínica Maternidad Rafael Calvo (CMRC) (Colombia). Differences between both cohorts were assessed through chi-square and ANOVA. $P<0.05$ was considered statistically significant.

Results There was a high rate of obstetrical admissions in both ICUs. Three hundred and ninety-eight patients were registered during these periods of time: 214 patients were from South Africa, 184 from Colombia. The most frequent diagnosis was hypertensive disorders during pregnancy $(\mathrm{KEH}=65.4 \%, \mathrm{CMRC}=47 \% ; P<0.001)$. There were no statistical differences between the two ICUs regarding age, gestational age, ICU stay/days and mechanical ventilation days. There were statistically significant differences in APACHE II score and mortality, being highest in South Africa. All types of organ dysfunctions were lower in CMRC; with statistical significance in cardiovascular, haematologic and neurologic dysfunction. OR from mechanical ventilation was $0.02(P<0.001)$, being lower in CMRC $(25.67 \%)$. There were no statistical differences in cesarean section and haemodialysis. Conclusions Obstetric critical care is considered of special interest in these countries, where $90 \%$ of global maternal mortality can be found. Although there are common characteristics regarding admissions of these patients to ICU settings, it is also evident that differences are noted when considering demographics and interventions in obstetric critically ill patients.

\section{References}

1. Soubra SH, Guntupalli KK: Critical illness in pregnancy: an overview. Crit Care Med 2005, 33:S248-S255.

2. Martin S, Foley M: Intensive care in obstetric: an evidence-based review. Am J Obstet Gynecol 2006, 195:673-689.

3. Zeeman G: Obstetric critical care: a blueprint for improved outcomes. Crit Care Med 2006, 34:S208-S214.

\section{P429}

Co-morbidity, demographic and social factors as predictors of outcome from intensive care: an observational cohort study

A Mackay, R Docking, J Kinsella, M Booth

Glasgow Royal Infirmary, Glasgow, UK

Critical Care 2010, 14(Suppl 1):P429 (doi: 10.1186/cc8661)

Introduction Critically ill patients arrive in intensive care with various degrees of impairment in chronic health. These are recognised to have an impact upon survival and the Acute Physiology and Chronic Health Evaluation II (APACHE II) system utilises this when predicting mortality. We wished to look at the impact of many of these factors upon outcome from our intensive care.

Methods A prospective case-note review of 500 consecutive admissions to Glasgow Royal Infirmary ICU was undertaken over an 18-month period. Details of co-morbidity, social factors, regular medication and exercise tolerance were sought from the patients' case notes by hand using details of current and previous admissions, clinic letters, results of investigations and correspondence from the patient's general practitioner, using agreed criteria. Demographic, APACHE II score and outcome data were retrieved from the Ward Watcher system in the ICU. Data were analysed using a univariate analysis model and chi-square test.

Results Complete data were available for 500 patients. Population demographics were as follows $-65 \%$ male, mean age $53.8 \pm 4.1$ years, median APACHE II score 18 (IQR 12 to 24), crude ICU mortality 33.6\%. The following factors were associated with an increased mortality (odds ratio, $P$ value): age $>50$ years (OR 3.11, $P<0.001)$, cigarette smoking (OR $1.57, P=0.02)$, NYHA class $3 / 4$ (OR 2.52, $P<0.001$ ), ischaemic heart disease (OR 1.70, $P=0.01)$, peripheral vascular disease (OR 1.91, $P=0.04)$, chronic obstructive pulmonary disease (OR 2.56, $P<0.001)$, alcoholic liver disease (OR 3.86, $P<0.001$ ). The following factors were not significantly associated with increased mortality: male gender, social deprivation, unemployment, alcohol excess, intravenous drug use, polypharmacy, hypertension, diabetes, obesity or concurrent malignancy.

Conclusions These results suggest significant increased ICU mortality in the following groups: over 50 years old, smokers, patients with poor exercise tolerance, patients with $\mathrm{HD}$ or PV, patients with COPD and patients who drink alcohol to excess or those with ALD. While these results should not be used as a basis upon which to permit or refuse intensive care admission, they should be used to inform staff and patients of likely outcome from intensive care.

P430

C-Reactive protein and albumin as prognostic markers in intensive

care

A Mackay, J Erskine, O Kerr, L Marshall, A Davidson

Victoria Infirmary, Glasgow, UK

Critical Care 2010, 14(Suppl 1):P430 (doi: 10.1186/cc8662)

Introduction C-reactive protein (CRP) is an acute-phase protein, the blood levels of which increase rapidly in response to infection, trauma, ischemia, burns, and other inflammatory conditions. Serum albumin decreases in critically ill patients with similar conditions. The use of these blood tests as risk markers or as predictors of organ failure and death has been studied previously $[1,2]$ and we wished to investigate their applicability to our population.

Methods A retrospective laboratory result review of 300 consecutive admissions to the Victoria Infirmary ICU was undertaken. Demographic, APACHE II score and outcome data were retrieved from the Ward Watcher system in the ICU. We compared survivors' and nonsurvivors' levels of CRP and albumin on day of admission and looked at correlation with length of stay and duration of mechanical ventilation. Data were analysed using Student's $t$ test and the Pearson correlation coefficient where appropriate. Results Complete data were available for 287 patients. Population demographics were as follows $-61 \%$ male, mean age $56.4 \pm 2.0$ years, median APACHE II score 20 (IQR 15 to 27), crude ICU mortality $24.7 \%$. Comparing survivors and nonsurvivors, admission CRP was significantly higher in nonsurvivors (144.7 vs 113.0, $P=0.037$ ) whereas admission albumin was significantly lower in nonsurvivors ( 21.3 vs $24.7, P=0.003$ ). Admission CRP correlated positively with length of stay $(r=0.14, P=0.017)$ and APACHE $\|$ score $(r=0.13, P=0.03)$ but did not significantly correlate with duration of mechanical ventilation $(r=0.10, P=0.103)$. Admission albumin correlated negatively with length of stay $(r=-0.15, P=0.01)$, duration of mechanical ventilation $(r=-0.15, P=0.014)$ and APACHE ॥ score $(r=-0.17, P=0.004)$.

Conclusions CRP and serum albumin on admission are both predictors of intensive care mortality. CRP and serum albumin also correlate significantly with other severity markers such as length of stay and duration of mechanical ventilation and also APACHE II score.

\section{References}

1. Lobo SMA, et al: C-Reactive protein levels correlate with mortality and organ failure in critically ill patients. Chest 2003, 123:2043-2049.

2. FHY Yap et al:: Association of serum albumin concentration and mortality risk in critically ill patients. Anaesth Intensive Care 2002, 30:202-208.

\section{P431}

Statin therapy on admission to intensive care and outcome:

an observational cohort study

A Mackay, R Docking, J Kinsella, M Booth

Glasgow Royal Infirmary, Glasgow, UK

Critical Care 2010, 14(Suppl 1):P431 (doi: 10.1186/cc8663)

Introduction Statins are powerful hypolipaemic drugs that are proven to improve survival in patients with atherosclerosis. Their effects are not limited to lowering cholesterol but also include anti-inflammatory and antioxidative properties. For these reasons, it has been postulated 
that statins may reduce mortality in critically ill patients. We aimed to investigate the association between statin therapy on admission and outcome from intensive care in our ICU.

Methods A prospective case-note review of 504 consecutive admissions to Glasgow Royal Infirmary ICU was undertaken over an 18-month period. Details of statin prescription, cardiovascular co-morbidity and smoking status was sought from the patients' case notes by hand using details of current and previous admissions, clinical letters, results of investigations and correspondence from the patient's general practitioner, using agreed criteria. Demographic, Acute Physiology and Chronic Health Evaluation II (APACHE II) score and outcome data were retrieved from the Ward Watcher system in the ICU.

Results Complete data were available for 444 patients. One hundred and eleven $(25 \%)$ of these were on statin therapy on admission to intensive care. All data are expressed as mean $\pm 95 \%$ confidence interval or median (interquartile range). Differences between groups were tested using the chi-squared test, Mann-Whitney test and unpaired Student's $t$ test. Compared with patients without concurrent statin therapy, patients on statin therapy are more likely to be male $(76.6 \%$ vs $63 \%, P=0.009)$, older $(65.2 \pm 1.93$ years vs $49.7 \pm 1.86$ years, $P<0.001)$, have a higher APACHE II score (20 (15 to 25$)$ vs 16 (11 to 23$), P<0.001)$, have ischaemic heart disease ( $69.4 \%$ vs $17.1 \%, P<0.001$ ), and have hypertension ( $47.5 \%$ vs $0.22 \%$, $P<0.001)$. There was no significant difference in mortality in the ICU $(27.9 \%$ vs $27 \%, P=0.86$ ) or the hospital $(40.5 \%$ vs $31.2 \%, P=0.07)$.

Conclusions Statin therapy does not significantly alter ICU or hospital mortality in our population. There are significant differences in APACHE II scores between the groups and predicted mortality which may suggest that patients on statins fare better than predicted, based upon their co-morbidities.

\section{Reference}

1. Mekonso-Dessap A, Brun-Buisson C: Statins: the next step in adjuvant therapy for sepsis? Intensive Care Med 2006, 32:11-14.

\section{P432}

How did you sleep in the ICU?

LF Franck, DM Le Moullec, EB Batjom, NL Libert, JT Tourtier,

SR De Rudnicki, MB Borne

HIA Val de Grace, Paris, France

Critical Care 2010, 14(Suppl 1):P432 (doi: 10.1186/cc8664)

Introduction It has been proven that patients'sleep is extremely disturbed in the ICU for many reasons: illness pathophysiology and severity, pain, or the ICU environment itself. The aim of our survey was to describe the memorization of sleep disturbances during an ICU stay and the patients' reported sleep quality after critical care. We also assessed the possible underlying causes of sleep disturbances including factors related to the critical illness.

Methods A telephone interview including a random sample of 60 patients admitted to our ICU (between December 2008 and May 2009) was performed 6 to 12 months after their discharge. Eight patients were excluded (two for language barrier, two were dead, four were not joined). Conventional intensive care variables were retrospectively recorded from the ICU database and sleep disturbances were evaluated using the Basic Nordic Sleep Questionnaire. Two nonvalidated questions were analysed, one estimated the quality of sleep in the ICU and the other compared the quality of sleep before and after the ICU stay. Data were presented descriptively using logistic regression to evaluate the independent effects of sex, age, BMI at admission, SAPS II, length of stay, hemodialysis, length of mechanical ventilation, length of central venous catheterization and prescription of vasopressor agents or transfusion.

Results Among responding patients, 47\% remembered sleep disturbances in the ICU and among them $43 \%$ still declared a decreased long-term quality of sleep. In addition, $30 \%$ of patients report a worse quality of sleep after the ICU than it used to be before. No relation was found when we assessed the possible effects of intensive care variables on the sleeping patterns during critical illness.

Conclusions Sleep disturbances were described in one-half of the patients we studied during their ICU stay. As interesting (but alarming) is that one-third of the group declared having a worse quality of sleep a long time after their discharge from the ICU. The sleep disturbances were not affected by ICU factors but their high self-reported prevalence probably reveals the necessity of protocols evaluating the effects of strategies to improve sleep quality in patients' recovery of critical illness and long-term sleep.

Reference

1. Friese RS: Crit Care Med 2008, 36:697-705.

P433

Factors associated with longer stay on a surgical intensive care unit PToomtong, M Raksakietisak

Faculty of Medicine Siriraj Hospital, Bangkok, Thailand

Critical Care 2010, 14(Suppl 1):P433 (doi: 10.1186/cc8665)

Introduction The advance in medical care for surgical patients results in older patients, increasing medical problems, and complexities of operations in surgical patients. Therefore, these patients may require a longer stay in the surgical ICU (SICU). The aim of the present study was to evaluate the occurrence, clinical outcome, and predictive risk factors for the longer SICU length of stay in critically ill surgical patients in our general SICU.

Methods This study was an observation study; after the approval of the IRB, all patients admitted to the SICU from the operating theatres during the study period from 1 June to 31 December 2004 were recruited to the study. Data collection consisted of initial patient assessment and demographics, underlying problems, SICU interventions and laboratory investigations for TISS-28 and SOFA scores within the first 24 hours of ICU admission. Descriptive statistics and backward stepwise logistic regression were employed to study for independent factors associated with the longer length of SICU stay.

Results During the 6-month study period, 562 patients were admitted to the SICU with an overall mortality rate of $6.23 \%$, and the overall average length of stay in the SICU was 2.72 days. There were 99 patients staying in the SICU longer than 4 days, with a mean of 9.12 days in these patients. The independent associated factors of SICU stay longer than 4 days were perioperative cardiac complications, odds ratio (OR) 12.77 (95\% confidence interval 4.52 to 36.08$) ; P<0.01$, requirement of ventilator support, OR 6.95 (3.13 to 15.42); $P<0.01$, bleeding in the SICU, OR 4.85 (1.33 to 17.65); $P=$ 0.02 , perioperative respiratory complications, OR 2.77 (1.09 to 7.02$) ; P=$ 0.03 , emergency surgical procedures, OR 2.47 (1.35 to 4.51); $P<0.01$, new haemodynamic monitoring interventions in the SICU, OR 2.28 (1.28 to 4.08); $P<0.01$, hypotensive episodes in the SICU, OR 2.27 (1.16 to 4.45); $P=$ 0.02 , and oliguria, OR 1.82 (1.03 to 3.22); $P=0.04$.

Conclusions The SICU patients who had a longer stay are associated with emergency procedures, the development of perioperative cardiac and respiratory complications, surgical bleeding, active monitoring interventions upon SICU admission, they were more likely to be hypotensive and had oliguria in the first 24 hours of the SICU stay.

\section{Reference}

1. Higgins T: Early indicators of prolonged intensive care unit stay: impact of illness severity, physician staffing, and pre-intensive care unit length of stay. Crit Care Med 2003, 31:45-51.

\section{P434}

\section{Social support: a significant factor for health-related quality of life} after critical illness?

L Orwelius, M Fredriksson, C Bäckman, A Samuelsson, F Sjöberg

Faculty of Health Sciences, Linköping, Sweden

Critical Care 2010, 14(Suppl 1):P434 (doi: 10.1186/cc8666)

Introduction Decreased health-related quality of life (HRQoL) is well known for the previous ICU population after critical care, and many factors are of importance for this outcome. We have in a recent study shown that pre-existing disease is most important. This finding was made when comparing a control population adjusting for co-morbidities and approximately $50 \%$ of the decrease in HRQoL was attributed to COmorbidities. Therefore, there must be other factors that contribute to the HRQoL decrease. In HRQOL research it has been shown that social support also is important for the HRQoL experience. In the southeast of Sweden, two neighbouring large cities are known to have different social support patterns depending on their differences in social structure. We hypothesized that the social support is different between the patients 
coming from city A's ICU compared with city B. The aim of this study was to examine to what extent social support affected HRQoL outcome in the follow-up of these patients in general and to what extent it differed between the patients of ICUs of the different cities.

Methods Mixed ICUs from two hospitals in different cities. Questionnaires, including the social support instrument Availability of Social Integration (AVSI), the HRQoL scale SF-36, demographic data and previous illnesses, were sent 6 months after discharge to all adult patients.

Results Six hundred and thirty-three (57\%) patients returned the questionnaire, 371 from city A. The ICU patients from city B reported a significantly lower mean score in social support compared with city A, 18.3 (SD 6.3) compared with 19.6 (SD 6.5) $(P=0.013)$. The SF-36 scores in all subscales showed no significant differences between the two cities. The results demonstrate that ICU patients with pre-existing disease have eight times higher risk of a decreased physical component score, and nearly six times higher risk of a decreased mental component score than the previously healthy patients. ICU patients with lower mean social support scores had 0.16 higher risks of a decreased physical score and 0.46 higher risks of a decreased mental component score.

Conclusions This study indicates that social support has a significant, but small, effect on HRQoL in former ICU patients. Importantly, the magnitude of this effect was too small to be relevant in comparisons of HRQoL in former ICU patients from cities with different social structures within one country.

\section{Reference}

1. Orwelius L, et al:: Crit Care Med 2005, 33:1557-1564.

\section{P435}

Quality of the evaluations of clinical studies in adult critical care and emergency medicine on a specialized Internet site

T Leclerc', D Journois ${ }^{2}$

'HIA Percy, Clamart, France; ${ }^{2 H}$. Européen G Pompidou, Paris, France

Critical Care 2010, 14(Suppl 1):P435 (doi: 10.1186/cc8667)

Introduction Given the huge publication flow, tools helping intensivists to select articles worth reading and to appraise relevant information are welcome. Evaluations of selected articles on dedicated Internet sites could serve that purpose.

Methods As a first attempt to estimate the quality of such sites, we conducted a prospective observational study of www.f1000medicine.org (F1000). Consecutive adult clinical studies with a first F1000 evaluation in the critical care and emergency medicine specialty were included. We recorded predefined items describing the internal validity, clinical relevance and external coherence of the studies and corresponding information in their evaluations. The primary endpoint was conformity of the conclusions to raw study results, compared between each study and its first F1000 evaluation.

Results Fifty studies with 56 evaluations (six articles evaluated twice) were included. Conclusions conform to factual study results in 52\% of the studies, excessive in $30 \%$, out of scope in 18\%; conform in $36 \%$ of all evaluations, excessive in $25 \%$, out of scope in $39 \%$. The conformity level significantly differed between the studies and their first evaluation ( $P=0.026$, Mantel-Haenszel chi-squared test). No association between conformity level and journal impact factor was observed in included studies ( $P=0.84$, Kruskal-Wallis test), neither in their evaluations between conformity level and publication-to-evaluation time $(P=0.46)$, evaluation length $(P=0.35)$ nor strictly article-focused relative length $(P=$ 0.027 , Bonferroni correction: NS). Regarding internal validity, out of 28 prospective studies (13 interventional), five had been registered, three of which before study termination with publicly traceable protocol changes; registration was discussed in four out of 33 related evaluations. Alpha risk inflation was uncontrolled in $95 \%$ out of 41 nonpurely descriptive studies, but never discussed in their 46 evaluations. Out of 20 negative studies, 16 were underpowered (an unrecognized fact in 15 studies), but power was discussed in only five out of 25 related evaluations. Clinical relevance was discussed in only $13 \%$ of F1000 evaluations. Regarding external coherence, $52 \%$ of F1000 evaluations did not relate study results to existing literature. Conclusions Our results suggest that F1000 evaluations often provide information poorly suited to critical appraisal of clinical studies by intensivists. Basing evaluations on assessment grids could improve their overall quality.
P436

Functional status and quality of life in patients suffering severe cranioencephalic trauma at the time of discharge from the intensive care unit and 1 year after

E Curiel Balsera', M Prieto Palomino', M Delange², A Muñoz',

JF Fernandez Ortega', G Quesada García'

'Carlos Haya Hospital, Málaga, Spain; ${ }^{2}$ Hospital de la Axarquia, Velez-Málaga, Spain

Critical Care 2010, 14(Suppl 1):P436 (doi: 10.1186/cc8668)

Introduction Results obtained after management of cranioencephalic trauma (CET) are referred only in terms of morbimortality. Our objective is to analyze physical and functional status after being discharged from the ICU, and the quality of life 1 year later.

Methods Cohort study with adult patients admitted to the ICU from 2004 to 2008 with severe CET. Demographic, epidemiologic and clinical data were recorded. Validated measure scales used were GCS, GOS, ISS and the quality-of-life questionnaire PAEEC (Project for the Epidemiological Analysis of Critical Patients). Multivariate analysis identified predictor factors of mortality.

Results We included 323 patients with a median age of 32 years old, $79.6 \%$ males, who suffered severe CET secondary to traffic $(57.6 \%)$ or falling (29.8\%) accidents. Cranial computed tomography (CT) scan most frequent findings were diffuse injury III (30\%) in Marshall classification; ISS first median was 33; APACHE II, 19; length of stay, 6 days (10 days if we take away those who died very soon); days on mechanical ventilator, 8 . Total GCS at admission/discharge was 5/10 ( $P=0.001)$. GOS 4 to 5 at discharge from the ICU was $14.4 \%$ and 1 year later was $54.1 \%(P=0.001)$. Mortality in the ICU was $33.1 \%$. Predictors of mortality were the length of stay ( $P=$ $0.0001)$, GCS at admission ( $P=0.05)$, CT findings type III, IV and V $(P=0.014$, 0.001 and 0.028 , respectively), complications $(P=0.0001)$, tracheotomy $(P=0.028)$, days on ventilator $(P=0.0001)$ and APACHE $\|(P=0.025)$. One year after discharge, 159 from 216 patients answered the questionnaire (73.6\%): severe physiologic dysfunction, great dependence in activities of daily living and emotional disturbances were detected when leaving the ICU and marked as $59.3 \%, 88.6 \%$ and $70.1 \%$, while 1 year after they were $18.9 \%, 32.1 \%$ and $35.8 \%$, respectively $(P=0.0001)$

Conclusions Severe CET was usually caused by traffic accidents, affected young adults and the mortality was over 33\% in the ICU. Factors independently associated with increased mortality were coma level, type of findings in CT, complications, prolonged mechanical ventilation, length of stay, APACHE II, and the need for a tracheotomy. One year later, survivors showed a better GOS and physiologic capacity.

\section{Reference}

1. Temkin NR, Corrigan JD, Dikmen SS, Machamer J: Social functioning after traumatic brain injury. J Head Trauma Rehabil 2009, 24:460-467.

\section{P437}

Long-term ( $>6$ years) quality of life after surgical intensive care admission

TTimmers 1 , MJ Verhofstad ${ }^{2}$, LP Leenen ${ }^{1}$, KG Moons ${ }^{3}$, EF Van Beeck ${ }^{4}$ 'Universitair Medical Centre Utrecht, the Netherlands; ${ }^{2}$ St Elizabeth Hospital, Tilburg, the Netherlands; ${ }^{3}$ Julius Centre for Health Sciences and Primary Care, Utrecht, the Netherlands; ${ }^{4}$ Erasmus Medical Centre, Rotterdam, the Netherlands

Critical Care 2010, 14(Suppl 1):P437 (doi: 10.1186/cc8669)

Introduction Treatment of surgical patients in the ICU, affected by severe illnesses or injuries, should be justified by an acceptable survival and longterm quality of life (QoL). The primary aim of this study was to quantify the long-term QoL ( $>6$ years) of a large cohort of patients admitted to a surgical ICU. In addition, we aimed to explore the influence of different surgical diagnosis groups on long-term health status and to make comparisons with general population norms.

Methods QoL was measured in all surviving surgical ICU patients admitted to a Dutch teaching hospital between 1995 and 2000. Patientreported data on QoL were collected with the EuroQol-5D+ after a mean follow up of 8 (range 6 to 11) years. Patient characteristics, surgical diagnosis group, length of ICU stay and survival were prospectively registered. EQ-utility scores (EQ-us), EQ Visual Analogue Scales (EQ-VAS) 
and prevalences of domain-specific health problems were calculated. The effect of surgical diagnosis group on EQ-US/EQ-VAS was assessed by multivariable generalized linear regression analysis. Logistic regression was used to explore the influence of surgical diagnosis group on domainspecific health problems. Long-term quality of life of surgical ICU patients was compared with an age-matched and sex-matched general Dutch population using the $t$-test analysis.

Results Eight hundred and thirty-four patients survived the ICU and were available for follow up. In 598 (72\%) patients, the health-related QoL was measured. For all surgical groups combined, after 6 to 11 years nearly one-half of all patients still suffered from problems in the dimensions of mobility (52\%), usual activity (52\%), pain (57\%) and cognition (43\%). Compared with the age-matched and sex-matched general Dutch population, HRQoL was worse with a difference of 0.11 on the EQ utilities score (range 0 to 1). Oncological surgery patients had the best (EQ-us 0.83 ) and vascular patients had the worst (EQ-us 0.72) HRQoL. Trauma (odds ratio between 2.47 and 3.47) and vascular surgery (2.27 to 5.37) showed significantly increased prevalences of problems in mobility, self-care, usual activities and cognition.

Conclusions More than 6 years after a surgical ICU admission, the QoL of this patient population is largely reduced. Many patients still suffer from a variety of health problems, including decreased cognitive functioning. Treatment advances should be made to reduce the current health deficit of surgical ICU survivors compared with the general population.

\section{P438}

Children's self-reported health-related quality of life after intensive care treatment

G Colville', C Pierce ${ }^{2}$

${ }^{1}$ St George's Hospital, London, UK; ${ }^{2}$ Great Ormond Street Hospital, London, UK Critical Care 2010, 14(Suppl 1):P438 (doi: 10.1186/cc8670)

Introduction A number of studies have reported on parental/clinician reports of children's health-related quality of life (HRQoL) after intensive care treatment [1]. The aim of this study was to establish children's own views as to their outcome in this regard.

Methods A cohort of 102 children aged over 7 years, with no preexisting learning difficulties, completed the PedsQL 4.0 Pediatric Quality of Life Inventory [2] and a post-traumatic stress screener in face-to-face interviews, 3 months after discharge from the PICU. Of this group, 76 also completed questionnaires, by post or telephone, at 12 months.

Results Children's total PedsQL scores were significantly lower than those of healthy children at 3 months (PICU mean $=79.3$; healthy mean $=83.9$, $P<0.01$ ), but at 12 months they were comparable. By 12 months, the mean score for the PICU group on the physical functioning subscale had improved significantly (from 73.1 to $81.5, P<0.001$ ) but was still below normal population levels (healthy mean $=88.5, P<0.01$ ). The total PedsQL score at 12 months was not associated with PIM score or length of stay, but was significantly negatively associated with post-traumatic stress symptoms $(r=-0.40, P<0.001)$. Within-group analyses revealed that electively admitted children reported higher emotional functioning than healthy children at 3 months (PICU elective mean $=91.0$ vs healthy mean $=$ $78.5, P<0.01)$

Conclusions The self-report version of the Peds $\mathrm{QL}$ proved to be a feasible and responsive tool for assessing HRQOL in this group of PICU survivors. However, in order to assess this aspect of outcome for the majority of children admitted to the PICU, who are younger and/or have significant cognitive impairment, administration of the parent-proxy version of this questionnaire would be necessary.

\section{References}

1. Knoester H, Grootenhuis MA, Bos AP: Outcome of paediatric intensive care survivors. Eur J Paediatr 2007, 116:1119-1128

2. Varni JW, Seid M, Kurtin P: The PedsQLTM 4.0: reliability and validity of the Pediatric Quality of Life Inventory ${ }^{\mathrm{Tm}}$ version 4.0 generic core scales in healthy and patient populations. Med Care 2001, 39:800-812.
P439

Activities of daily living (Barthel Index) at discharge from the intensive care unit

CB Kancir, PK Korsgaard

Regional Hospital West Jutland, Holstebro, Denmark

Critical Care 2010, 14(Suppl 1):P439 (doi: 10.1186/cc8671)

Introduction In order to continue optimal and adequate rehabilitation care in the nursing ward, information about the functional status of patients at discharge from the ICU is needed [1]. The Barthel Index is a simple index of independence to score the ability of a patient to care for itself. It measures the patient's daily functioning, specifically the activities of daily living (ADL) and mobility [2]. The objectives of the present study were to assess the ADL of ICU patients at discharge to the ward, and to identify explanatory factors of functional status.

Methods A prospective, observational, cohort study was conducted with patients who stayed in a six-bed mixed (predominantly medical) ICU for longer than 48 hours. We used the Danish-language version of the Barthel-20 Index, which is a validated and reliable generic instrument, to measure ADL. The Barthel Index measures the capacity to perform 10 basic $A D L$ and gives a quantitative estimation of the patient's level of dependency, with scoring from 0 (totally dependent) to 20 (totally independent). All assessments were carried out by critical care nurses with an experienced physiotherapist. Patient characteristics and outcome measures were analyzed with descriptive statistics. The data are expressed as medians and interquartile ranges.

Results At discharge from the ICU, functional status (as primary outcome) was assessed in 73 patients. The overall functional status was poor (median Barthel Index 2; 0 to 9). In their ability to perform basic ADL, 63\% were severely dependent, $25 \%$ were moderately dependent, and $10 \%$ were slightly dependent on other people. The transfer from bed to wheelchair and return with major help was the ADL component that was noted in the great majority of the patients. Duration of mechanical ventilation (median 5 days; 1 to 12 days) was associated with poorer functional status at ICU discharge.

Conclusions This study provides information that is important for the planning of rehabilitation of patients at ICU discharge. After transfer to the ward, the physical resilience of critically ill patients is severely limited. This implies that prior assessment of any care needed after discharge from the ICU is very important.

References

1. van der Schaaf M, et al:: Disabil Rehabil 2008, 30:1812-1828.

2. Mahoney FI, Barthel D: Md State Med J 1965, 14:56-61.

\section{P440}

A comparison of Short Form 36 and Hospital Anxiety and Depression Scale based on patients 1 month after discharge from the intensive care unit

CB Kancir

Regional Hospital West Jutland, Holstebro, Denmark

Critical Care 2010, 14(Suppl 1):P440 (doi: 10.1186/cc8672)

Introduction Although the Hospital Anxiety and Depression Scale (HADS) and the health-related quality of life assessed by the Short Form 36 (SF-36) have been used concomitantly in several studies, extensive comparisons between HADS and the different components of SF-36 have previously been reported but not in ICU survivors. The aim of this study was to compare the scorings of anxiety and depression assessed by the HADS (HADA (Anxiety) and HADD (Depression)) with the scorings on the various domains of SF-36. Methods In a six-bed mixed (predominantly medical) ICU in a community hospital, 111 adult ICU survivors with a minimum stay of 72 hours were included. One month after ICU discharge or soon after hospital discharge they were sent the SF-36 and the HADS using regular mail. SF-36 tests eight domains. Four domains assess physical health, physical functioning $(P F)$, role limitation due to physical problems (PR), bodily pain (BP) and general health perception $(\mathrm{GH})$; while the other four assess psychological health, social functioning (SF), role limitation due to emotional problems $(\mathrm{RE})$, energy and vitality (VT) and mental health $(\mathrm{MH})$. The physical health summary score $(\mathrm{PCS})$ reflects $\mathrm{PF}, \mathrm{PR}, \mathrm{BP}$, and $\mathrm{GH}$. The mental health summary scale (MCS) reflects VT, SF, RE, and $\mathrm{MH}$. Pearson's correlation coefficients were calculated on item and scale levels to assess the associations between the HADS and the SF-36 scales. 
Results HADA and HADD were significantly associated with MCS but not with PCS. The absolute $r$ value between MCS and HADA (0.72) was significantly higher than the comparable figure between HADD and MCS (0.68). HADA explained $1 \%$ of the variance of PCS and 52\% of the variance of MCS. The comparable figures for HADD were $2 \%$ and $47 \%$, respectively. Differences of correlation coefficients were further explored by evaluation of the associations between HADS and each of the eight SF-36 subscales. Only the association between HADA and PF was not statistically significant. Relatively high $r$ values were observed between HADA (0.76) or HADD (0.65) and MH. The associations between HADA and MH exceeded significantly the analogue $r$ values as to HADD.

Conclusions This pattern of different predictions of the summary scales of SF-36 supports a clinical practice that anxiety and depression should be assessed separately. Additional use of a self-rating instrument for depression and anxiety, such as HADS, is recommended when SF-36 is used for quality of life assessment.

\section{P441}

Post-traumatic stress disorder after surgical ICU admission

C Aldecoa, S Pico, J Rico, B Vazquez, L Gomez, C Garcia-Bernardo,

J Gomez-Herreras

Universitary Hospital Rio Hortega, Valladolid, Spain

Critical Care 2010, 14(Suppl 1):P441 (doi: 10.1186/cc8673)

Introduction Post-traumatic stress disorder (PTSD) is a potentially serious psychiatric disorder that has traditionally been associated with traumatic stressors, such as participation in combat, having suffered violent assault and survival after natural disasters. Recently, investigators have reported that the experience of critical illness can also lead to PTSD, although details of the association between critical illness and PTSD remain unclear. Our objective is to determine the prevalence and severity of symptoms related to the diagnosis of PTSD in a cohort of postsurgical ICU patients and to identify potential risk factors for PTSD symptoms.

Methods A prospective cohort of patients over 18 years old, admitted to our surgical ICU for over 48 hours, between October 2002 and June 2008, were studied. Variables such as age, gender, type of admission, neoplasia, SOFA, ASA, APACHE II scores, surgical intervention, sepsis, renal failure, mechanical ventilation, cortocosteroid treatment, psychiatric history, blood product transfusions, and so forth, were recorded. The Post-Traumatic Stress Syndrome 10-Questions Inventory (PTSS-10) was used to measure the stress symptoms a year after discharge from the unit. Five hundred and one patients met the inclusive criteria, 198 died during the follow-up year after ICU outcome. Three hundred and three patients survived, 160 of them completed the questionnaire by telephone. The association of potential risk factors with PTSS-10 scores was done by logistic regression and multivariate analysis.

Results PTSD prevalence was 13\% in our unit. Statistical analysis shows the relationship between the syndrome and the following factors: female gender, age $<65$ years, mechanical ventilation, daily interruption of the sedation, previous psychiatric history and blood product transfusions. The multivariate analysis shows as independent risk factors: female gender, age $<65$ years, previous psychiatric history and blood product transfusions.

Conclusions Our results agree with other authors about prevalence and most of the risk factors. Nonetheless we have found a positive relationship between interruption of sedation and the development of symptoms of PTSD as with psychiatric history.

\section{References}

1. Cuberthson BH, et al.: Post-traumatic stress disorder after critical illness requiring general intensive care. Intensive Care Med 2004, 30:450-455.

2. Boer KR, et al.: Long-term prevalence of post-traumatic stress disorder symptoms in patients after secondary peritonitis. Crit Care 2007, 11:R30

P442

Health-related quality of life before intensive care

B Wittekamp, DTjan

Gelderse Vallei Hospital, Ede, the Netherlands

Critical Care 2010, 14(Suppl 1):P442 (doi: 10.1186/cc8674)

Introduction We hypothesise that subjects admitted to the ICU have lower self-reported health-related quality of life (HRQOL) in

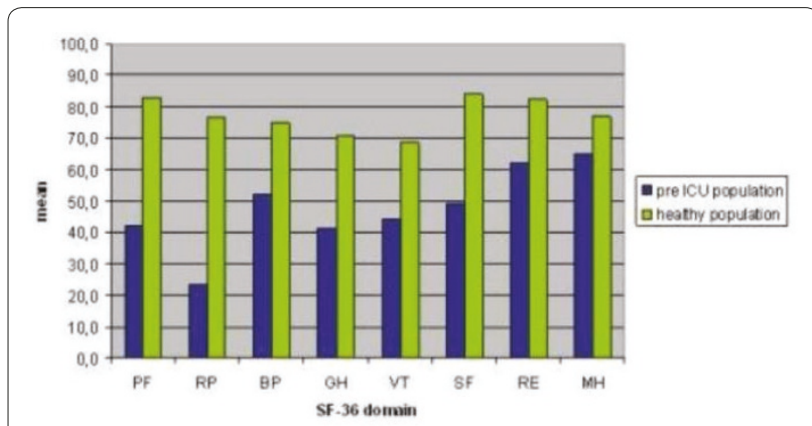

Figure 1 (abstract P442). HRQOL pre ICU compared with the healthy population.

the month before intensive care compared with the normal healthy population

Methods Potential participants were patients admitted for more than 48 hours to a 12-bed medical-surgical ICU. Patients or their proxies were asked to give informed consent and complete the SF-36. The SF-36 is a questionnaire measuring HRQOL on eight subscales [1]. The use of the SF-36 in proxies has been validated $[2,3]$. Participants were asked to complete the SF-36 based on the situation 4 weeks before ICU admission. SF-36 scores were compared with normative data ( $n=1,742)$ [1], by independent $t$ tests.

Results Fifty-one questionnaires were completed, of which 28 (51.9\%) were completed by a proxy. HRQOL before ICU admission was significantly lower on all SF-36 domains compared with the general population $(P<0.0001)$ (Figure 1). This is in line with findings in one other Dutch survey [4].

Conclusions HRQOL before admittance to the ICU is lower compared with $\mathrm{HRQOL}$ in the normal healthy population. This is likely to contribute to the diminished HRQOL after ICU discharge. To measure the influence of critical illness and ICU stay on HRQOL after an ICU stay, it is important to measure HRQOL before ICU admission.

\section{References}

1. Aaronson NK, et al: J Clin Epidemio/ 1998, 51:1055-1068.

2. Hofhuis J, et al.: Intensive Care Med 2003, 29:974-979.

3. Rogers J, et al:: Anaesthesia 1997, 52:1137-1143.

4. Hofhuis JG, et al: Chest 2008, 133:377-385.

\section{P443}

Nursing staff development: strategies for success in a unique critical care unit in a developing country

N Shwaihet, N Mohamad, E Saad

King Faisal Specialist Hospital and Research Centre, Riyadh, Saudi Arabia

Critical Care 2010, 14(Suppl 1):P443 (doi: 10.1186/cc8675)

Introduction Creation of innovative retention strategies is a major focus for nursing administration as a shortage of nurses recurs and turnover of staff becomes a problem. Retention strategies, to be effective, need to be targeted specifically to particular conditions of the nursing staff. The cardiac surgical intensive care unit (CSICU) at the King Faisal Specialist Hospital \& Research Center is a unique unit that combines adult, pediatric and neonatal cardiac surgical patients. This demands well-trained and skilled nurses to be able to look after this complex variety of patients. The big majority of nurses recruited to the CSICU have an adult educational background only. Therefore the Department of Nursing Development and Saudization together with Cardiovascular Nursing Department developed and implemented different education strategies to improve nurse's skills and competencies according to the unit's needs.

Methods The strategies implemented included: comprehensive and periodic assessment of nurses, regular inservices in the form of lectures, skill review sessions, a progressive orientation program divided into different phases, bedside teaching, provision of a wide educational references, and professional development workshops.

Results In a survey done in 2006, from a database of 64 nurses, 18 (28\%) had only adult experience. A total of 14/18 (78\%) were unable to progress to pediatric care phase (PCP). Another survey in 2007 for a database of 68 
nurses showed that 15 (22\%) had only adult experience. Eight out of 15 (53\%) could not progress to PCP. A third survey performed in 2008, for a database of 82 nurses, showed that 25 (30\%) had adult experience. Six out of 25 (24\%) were not able to progress to PCP.

Conclusions Implementing comprehensive education strategies targeting different approaches of training contributed to considerable progressive staff development and retention in the CSICU.

\section{References}

1. Aiken LH, Buchan J, Sochalski J, Nichols B, Powell M: Trends in international nurse migration. Health Affairs 2004, 23:69-77.

2. Atencio BL, Cohen J, Gorenberg B: Nurse retention: is it worth it? Nurs Econ 2003, 21:262-268, 299.

3. Barney $\mathrm{S}$ : Retaining our workforce, regaining our potential. J Healthc Manag 2002, 47:291-295.

P444

Implementing the Leapfrog standard in a developing country J Scribante', S Bhagwanjee'

'University of the Witwatersrand, Johannesburg, South Africa; ${ }^{2}$ University of Washington, Seattle, WA, USA

Critical Care 2010, 14(Suppl 1):P444 (doi: 10.1186/cc8676)

Introduction The Leapfrog standard of ICU physician staffing is regarded as the gold standard. It is suggested that hospital leaders should focus on how to implement intensivist staffing [1]. Internationally, especially in developing countries, the 'how to' poses a tremendous challenge. The Critical Care Society of Southern Africa identified the need to objectively quantify existing ICU resources in South Africa (SA) with a view to making recommendations for the future. From this audit we discuss the challenges of implementing the Leapfrog standard of ICU physician staffing in a developing country.

Methods Approval to conduct a national audit was obtained from appropriate authorities and was undertaken in 2004 and 2005 in all public and private hospitals in SA.

Results A 100\% sample was obtained. At the time there were 448 units in SA, of which $7 \%$ of ICUs in the public sector and $1 \%$ in the private sector were closed units. There were a total of 4,168 ICU and HC beds, with 30 intensivists and 4,584 nurses (25\% ICU trained) working in the ICU [2-4].

Conclusions SA ICU services face challenges and the results show the difficulty of implementing the Leapfrog standard. A strategy to deal with these challenges must be reality based and contextually appropriate. We propose short-term, medium-term and long-term recommendations that consider fiscal pressure and competing healthcare imperatives [5]. Short term: Regionalisation and integration of ICU services, development of guidelines and protocols to guide practice. Introduction of continuous professional development programs and a structured outreach program for medical and nursing staff. Active recruitment and training of personnel. Medium term: Development of telemedicine and a two-tiered ICU program. The first tier to comprise nurses and doctors with intermediate skills and experience. The second tier to comprise intensivists and fully trained ICU nurses. Both tiers to have a clear scope of practice, training and incentive schemes. A significant percentage of ICU beds are not in use due to various factors, these beds to be recommissioned. Long term: A strategy to convert open units to closed units over a period of time. New ICUs to be established as closed units. An up-to-date national ICU database to be maintained to direct future planning.

\section{References}

1. Provonost PJ, et al:: Crit Care Med 2006, 34:S18-S24.

2. Bhagwanjee S, et al: S Afr Med J 2007, 97:1311-1314.

3. Scribante J, et al:: SAfr Med J 2007, 97:1319-1322.

4. Scribante J, et al: S Afr Med J 2007, 97:1315-1318.

5. Bhagwanjee S, et al: S Afr J Crit Care 2008, 24:4-6.

\section{P445}

Impact of thiopental use on cerebral perfusion pressure in severe brain trauma: an example of caregivers' adaptation

H Mehdaoui', L Allart', B Sarrazin', R Valentino', E Balestrat', P Ravaux ${ }^{2}$

'Fort de France University Hospital, Fort de France, Martinique; 'Lille 2 University, Lille, France

Critical Care 2010, 14(Suppl 1):P445 (doi: 10.1186/cc8677)
Introduction Thiopental (TP) is used in severe brain trauma to control high intracranial pressure episodes. Deleterious side effects were described that questioned its ability to reach its objectives securely and efficiently. We analyzed the impact of TP use on cerebral hemodynamics and caregivers' behavior towards recommendations using a high-rate recording information system.

Methods A set of 813 hours of data were recorded at a rate of $0.5 \mathrm{~Hz}$. The study was observational. Deleterious episodes were detected using the Information System (IS) and validated by two experts. We detected low cerebral perfusion pressure (CPP), high intracranial (HICP) and/or low mean arterial pressure (IMAP) episodes. We used commonly admitted threshold for the ICP $(20 \mathrm{mmHg})$ and the CPP $(60 \mathrm{mmHg})$. Medical orders intended to reach the recommended objectives were analyzed.

Results Forty-eight periods stratified according to TP dose were analyzed on 16 patients. Cumulative duration with TP was 20,520 minutes and 26,294 minutes without TP. The mean dose of TP administration was $250 \pm$ $20 \mathrm{mg} /$ hour ( $3.4 \pm 1.4 \mathrm{mg} / \mathrm{kg} /$ hour). The HICP incidence was $85 \pm 16$ per 100 hours with TP vs $95 \pm 29$ without TP (NS). HICP duration was longer with TP ( $27 \pm 2$ vs $19 \pm 2$ minutes, $P<0.005)$. The IMAP incidence was the same with or without TP ( $56 \pm 19$ vs $34+8$ per 100 hours of monitoring). Duration of IMAP episodes was the same with or without TP ( $22 \pm 2$ vs $20 \pm 2$ minutes, NS). The incidence of orders intended to restore cerebral hemodynamics was equivalent in both situations (TP vs no TP, $79 \pm 24$ vs $104 \pm 29$ per 100 hours, NS). One hundred and eighty-eight medical orders were analyzed. Use of catecholamine was more frequent with TP (57\% ( $n=$ $44)$ vs $43 \%(n=33), P=0.034)$.

Conclusions The use of TP complies with the recommendations (prolonged HICP). It did not result in a significant increase in cerebral hypoperfusion episodes. We showed evidence of adaptations to its use by physicians. The doses are lower than the doses prescribed earlier in the literature and the use of catecholamine is more frequent during TP infusion. This could result in a better control of deleterious side effects of TP and a better compliance with recommendations. Our IS was efficient in physicians' orders'analysis.

\section{P446}

Knowledge of ICU nurses regarding selected care areas commonly guided by protocols

H Perrie, S Schmollgruber

University of the Witwatersrand, Johannesburg, South Africa

Critical Care 2010, 14(Suppl 1):P446 (doi: 10.1186/cc8678)

Introduction Pain management, glycaemic control and weaning from mechanical ventilation are nursing care areas that can impact on patient outcome and are commonly guided by protocols. However, in order to ensure safe, optimal management of patients, even when care is guided by protocols, nurses require a sound knowledge base. This study attempted to describe the knowledge of ICU nurses regarding pain management, glycaemic control and weaning from mechanical ventilation, to compare the difference in knowledge between ICU-trained and non-ICU-trained nurses and to describe the impact of years of ICU experience on this knowledge.

Methods A prospective, descriptive, non-interventional study method was used. Approval was obtained from the ethics committee and other relevant authorities. The questionnaire used was developed and validated by two groups of ICU nursing experts. The study population included all consenting ICU nurses working in the ICUs of the five hospitals participating in this study.

Results There were 136 participants in the study (68 ICU trained and 68 non-ICU trained). The mean score obtained was $47.56 \%$ (SD 11.61), the ICUtrained participants obtaining 50.11 (SD 11.96) and the non-ICU trained obtaining 45.01 (SD 10.75). This difference was statistically significant ( $P=$ 0.0099). The difference between the two groups for the section on pain management was not statistically significant $(P=0.408)$ with ICU-trained participants achieving a mean score of $45.07 \%$ (SD 16.01) and non-ICUtrained participants 42.86 (SD 14.91). The difference between the two groups for the other two sections was statistically significant $(P=0.025$ and $P=0.006$, respectively) with the ICU-trained group achieving $51.26 \%$ (SD 11.74) and the non-ICU trained 46.16 (SD 14.34) for the glycaemic control section and 53.99\% (SD 18.18) for the ICU-trained participants and $46.01 \%$ (SD 15.18) for non-ICU-trained participants for the section 
pertaining to weaning from mechanical ventilation. The correlation between knowledge and years of ICU experience was poor ( $r=0.137)$. Conclusions This study found a lack of knowledge regarding the care areas tested, a small, but statistically significant difference between ICUtrained and non-ICU-trained nurses and a poor correlation between knowledge and years of ICU experience.

\section{P447}

Pharmacist clinical interventions in the ICU

NG Hunfeld, PH Melief, RM Van Hest, BE Bosma

HagaTeaching Hospital, Den Haag, the Netherlands

Critical Care 2010, 14(Suppl 1):P447 (doi: 10.1186/cc8679)

Introduction We wanted to investigate the number and nature of pharmacotherapeutical interventions of a dedicated hospital pharmacist during rounds in the ICU.

Methods A pharmacist specially trained in ICU practice participated in the patient rounds twice a week (from July to December 2008). The pharmacist focused on optimalization of pharmacotherapy based on ICU guidelines and standards as well as general pharmacotherapy. Medication overviews were generated from the ICU Patient Data Management System and were analyzed for each patient during the patient rounds. Interventions were made to comply with guidelines and to avoid interactions, contraindications, overtreatment and undertreatment and to recognize side effects. All pharmacist interventions were collected in a database and the percentage of interventions leading to a change in pharmacotherapy was analyzed.

Results Fifty patient rounds were attended (160 patients, mean age: 64 , male: 59\%, mean APACHE II score: 25 , mean length of stay: 8.7 days, number of drug orders analyzed: 4,227$)$. A total of 342 interventions was made in $66 \%$ of patients (mean: 2.1 interventions/patient), resulting in a change in pharmacotherapy in $67 \%$ of the interventions. The kind of interventions were: start drug: $18 \%$, stop drug: $15 \%$, reduction of dosage: $3 \%$, increase of dosage: $4 \%$, toxicology: $12 \%$, interaction: $6 \%$, contra-indication: $9 \%$, adverse events: $3 \%$, double medication: $4 \%$, switch in accordance to guidelines: $6 \%$, software: $2 \%$, dose reduction because of renal failure: $6 \%$ and other (for example, infusion incompatibility or administration by feeding tube): $13 \%$. Classes of drugs most involved were: antiobiotics, gastrointestinal drugs and anticoagulants.

Conclusions Frequent presence of a dedicated pharmacist during patient rounds in the ICU results in interventions in pharmacotherapy in $66 \%$ of the admitted patients. The mean number of interventions per patient is 2.1. Interventions lead to improved compliance with ICU guidelines and pharmacotherapy standards and warrants monitoring of pharmacotherapy. Interventions probably lead to improved efficacy and safety of pharmacotherapy, although future controlled research will have to prove to what extent drug-induced harm is prevented.

\section{P448}

Adverse drug events in the critical care unit

S Seynaeve, D Reyntiens, D Vandenplas, W Verbrugghe, B Claes, P Jorens

University Hospital Antwerp, Edegem, Belgium

Critical Care 2010, 14(Suppl 1):P448 (doi: 10.1186/cc8680)

Introduction Mistakes and errors may occur during the care process, particularly in the ICU as characterized by its large number of drugs administered to the single patient. One of the most important influencing factors on intramural morbidity and mortality is indeed a harmful or unpredicted reaction to a drug, a so-called adverse drug event (ADE) [1]. By far the largest proportion (70\%) of these ADEs is dose related [1]. The objectives of this study are to measure the frequency and severity of ADEs in the ICU and to determine the influence of severity of illness and nursing workload on their prevalence.

Methods A cross-sectional study in the ICU of a tertiary referral hospital, based on a retrospective chart review. A tool was developed for measuring the incidence and characteristics of ADEs based on the Global Trigger Tool for Measuring Adverse Drug Events [2]. If an ADE was identified the severity was evaluated using the categories based on the system for classifying medication errors by the National Coordination Council for Medication Error Reporting and Prevention [3]. The severity of illness was calculated using the Sepsis-related Organ Failure Assessment score [4] and the nursing workload by the classical Therapeutic Intervention Scoring System-28 [5].

Results The review of 1,009 nursing days in 79 patients revealed 230 ADEs, which occurred in a total of 175 nursing days. The most commonly identified ADE was a hypoglycemia of $<50 \mathrm{mg} / \mathrm{dl}(n=75)$, followed by hypokalemia $(n=67)$. Ninety-six percent of the ADEs were classified as category $E$, whereas only $4 \%$ of ADEs were classified as category $F$. The mean severity of illness and nursing workload scores were significant higher on nursing days when an ADE occurred $(P<0.001$ and $P=0.002$, respectively).

Conclusions ADEs are common in the ICU. The lack of a golden standard for reporting and collecting ADEs makes it difficult to compare with other studies and to assess the real value of this study. However, these date strongly and clearly indicate the influence of severity of illness and nursing workload on the prevalence of ADEs.

\section{References}

1. Nebeker JR, et al:: Ann Intern Med 2004, 140:795-801.

2. Rozich JD, et al: Qual Saf Health Care 2003, 12:194-200.

3. National Coordinating Council for Medication Error Reporting and Prevention [www.nccmerp.org]

4. Vincent JL, et al:: Intensive Care Med 1996, 22:707-710.

5. Miranda DR, et al:: Crit Care Med 1996, 24:64-73.

\section{P449}

Evaluation of appropriateness of intensive care unit admissions: the GIVITI's StART approach

R Rosi', P Malacarne ${ }^{2}$, S Livigni', D Radrizzani ${ }^{4}$, M Postiglione ${ }^{5}$, S Piva 6 , S Di Gangi ${ }^{7}$

${ }^{1}$ AOUS, Siena, Italy; ${ }^{2} \mathrm{H}$ S Chiare, Pisa, Italy; ${ }^{3}$ H S Giovanni Bosco, Torino, Italy; ${ }^{4}$ H Legnano (MI), Milan, Italy; ${ }^{5}$ L Loreto Nuovo, Napoli, Italy; ${ }^{6}$ Spedali Civili, Brescia, Italy; ${ }^{7}$ stituto Mario Negri Ranica, Bolognia, Italy Critical Care 2010, 14(Suppl 1):P449 (doi: 10.1186/cc8681)

Introduction Appropriate use of ICU resources is mandatory. When a patient is admitted to a unit able to provide a higher (lower) level of care than required, a waste (overuse) of resources can be advocated. StART is an approach to identify possible mismatches between the level of care actually delivered, assumed to correspond to what is clinically required, and the level of care deliverable by the unit.

Methods ICU beds are classified by levels of care deliverable as High (ventilator, monitor, and 720 minutes nurse time) and Low (monitor and 360 minutes nurse time) [1]. The level of care actually delivered is classified as High (invasive or non-invasive ventilation, or two vasoactive drugs, or at least two of the following: one vasoactive drug, dialysis, respiratory support), Low (single vasoactive drug, or dialysis, or respiratory support) and Ordinary (none of the above) [2]. Mismatches between the level of beds available and the level of care delivered were evaluated both on admission and for each ICU-day of 4,237 patients in 28 ICUs. An ICU-day was judged as inappropriate, even without mismatch, if an Ordinary patient was present.

Results Eighteen percent of admissions resulted inappropriate ( $9 \%$ overuse, $9 \%$ waste of resources). Patients receiving $\mathrm{H}$ level of care (58\% of the total) were appropriately admitted in $88 \%$ of cases. There were 271 patients admitted for Ordinary care (6\%). They stayed in the ICU 2.5 days on average, and $95 \%$ of them had no complications. Sixty-eight percent of the ICU-days were inappropriate: $12 \%$ for overuse and $56 \%$ for waste of resources. Forty-two percent of the latter days were only due to the presence of Ordinary patients ( $43 \%$ of them were instead admitted as $\mathrm{H}$ or $L$ patients).

Conclusions Most of the ICU admissions (82\%) were appropriate; the inappropriateness was mostly due to Ordinary patients treated. The short ICU stay and the absence of complications of these patients strengthen the inappropriateness of their admission, which was probably due to the lack of intermediate care units in Italy. The situation is completely different considering the ICU stay: the inappropriateness rose up to $68 \%$, mainly due to the presence of Ordinary patients. Almost one-half of them were patients recovered from sicker condition, who were probably difficult to discharge from the ICU.

\section{References}

1. lapichino G, et al:: Min Anest 2007, 73:501-506.

2. lapichino $G$, et al:. Intensive Care Med 2001, 27:131-136. 
P450

Noise in the critical care environment

N Haskins, J Soldan

University Hospital of Wales, Cardiff, UK

Critical Care 2010, 14(Suppl 1):P450 (doi: 10.1186/cc8682)

Introduction The subject of excessive noise levels within critical care is not new and has been identified on numerous occasions in the literature. Research illustrates the detrimental effect of noise on patients both physically and psychologically. Our interest in this field stemmed from Fundamentals of Care audits which described noise as being too high and disturbing for patients.

Methods The extent of the problem was initially explored by undertaking an audit of noise using an on-the-spot soundmeter. This showed that noise levels were in excess of the World Health Organisation recommendations for a patient care environment [1]. This confirmed the need to address the subject of noise within our critical care unit. Noise levels were then investigated further using a Soundlog device. This enabled us to measure noise levels continuously over multiple 24-hour periods. The device measures the noise level every second and then records the average measurement every 5 minutes. Noise levels were audited over a 6-week period in a variety of locations in an effort to gain an accurate assessment of noise levels. In doing this, patterns and themes in levels of noise could be identified and analysed.

Results The audit showed that noise levels were consistently between 52 and $65 \mathrm{~dB}$ over a 24-hour period, which is above the WHO guidance of $35 \mathrm{~dB}$ and $40 \mathrm{~dB}$ limit.

Conclusions The current noise levels within our unit exceed the recommendations so action needs to be taken to address this. It is a busy unit with some environmental noise origins but also a high density of staff and relatives, so with this in mind we are developing a project plan to enable us to explore the current levels of noise and its sources, identify those sources that are avoidable or open to modification and enable staff to consider their role and responsibility in relation to noise reduction. By considering the issue of noise and developing strategies that result in sustainable reductions in noise levels, we are aiming to improve the patients' experience of their critical care stay. We anticipate that there will be both practical and behavioural solutions to issues that arise, some of which can be addressed by quick-fix solutions while others will require longer term work.

\section{Reference}

1. World Health Organisation 1999 Guidelines for Community Noise [whqlibdoc.who.int/hq/1999/a68672.pdf]

\section{P451}

\section{Allergy documentation and transfer within critical care}

K Hatton', N Barrett ${ }^{2}$, J Lim² ${ }^{2}$, C McKenzie ${ }^{2}$

${ }^{\prime}$ Chelsea and Westminster NHS Foundation Trust, London, UK; ${ }^{2}$ Guy's and

St Thomas' NHS Foundation Trust, London, UK

Critical Care 2010, 14(Suppl 1):P451 (doi: 10.1186/cc8683)

Introduction Allergies to medication are common and potentially lifethreatening [1]. Patients enter critical care with incomplete information about their history. It is essential for safety that accurate allergy status is documented early in the critical care stay. This clinical audit (CA) was undertaken in a 43-bed, level 3 critical care unit to explore compliance with local guidelines on allergy documentation.

Methods Critical care patient episodes were obtained retrospectively for a 1-month period. Timing of allergy documentation and drug prescribing was noted from the critical care electronic system (ICIP). Allergy status prior to the critical care admission and after discharge was noted from the ward drug chart. Additional allergy data were identified from the hospital electronic patient record (EPR). The CA was repeated 1 year after implementation of recommendations.

Results Patient episodes were collated (initial CA $n=58$, repeat CA $n=79$ ). A known drug/nondrug allergy was stated in $29.3 \%$ patient episodes during the initial CA and $39.2 \%$ patient episodes in the repeat CA. Allergy status was incomplete 24 hours after critical care admission for two patients at the initial CA with a reduction to zero during the repeat. Allergy status was incomplete prior to prescribing of a new drug in critical care (excludes fluids, drugs required for emergency intubation) for $51.7 \%$ of patient episodes in the initial CA. This figure reduced to $19.0 \%$ in the repeat CA. Concordance between EPR and ICIP allergy at the outset was 68.8\%, which increased to $76.5 \%$ in the repeat CA. Concordance with the ward drug chart preadmission and ICIP was $77.6 \%$, increased to $93.9 \%$ at re-audit.

Conclusions This CA suggests that up to one in three critical care patients have a known allergy. The potential for harm is high. More than onehalf of patients admitted to critical care did not have an allergy status documented prior to prescribing a new drug. There was significant discordance between the paper medication chart and ICIP allergy. A number of factors were introduced following initial findings, including making the allergy status mandatory on ICIP, not allowing the admission summary to be saved prior to allergy documentation and ensuring current allergy documentation on EPR.

\section{Reference}

1. Bates DW, et al.: JAMA 1995, 274:29-34.

\section{P452}

Use of an anonymous medication incident reporting system on a critical care unit

H Dillon, M Rosbergen, S Hutchinson

Norfolk and Norwich University Hospital, Norwich, UK

Critical Care 2010, 14(Suppl 1):P452 (doi: 10.1186/cc8684)

Introduction An anonymous error reporting system was trialled to establish whether error reporting rates would improve and to allow the identification of medication incidents. The National Patient Safety Agency (NPSA) feedback report for the Norfolk and Norwich Hospital shows that, compared with similar organisations, medication error reporting is low. A review of the medication incidents reported for the critical care facility in 2007 and 2008 had only 19 events. Error rates in the ICU are typically higher than in other acute settings, one study reporting 10.5 medication errors per 100 bed-days [1]. Patients in critical care typically receive highrisk medications. Error reporting is integral to identifying common errors and medication risk reduction.

Methods A medication reporting form was developed to run alongside the official hospital incident reporting system for 2 weeks. Forms were distributed throughout the critical care facility. All members of the multidisciplinary team were asked to anonymously complete a form every time a medication error, or near miss, occurred. After 2 weeks, the submitted forms were analysed by the project team.

Results In total 112 reports were submitted. The largest numbers of incidents reported were due to prescribing (67\%) errors followed by administration (15\%), documentation (7\%), electronic prescribing problems $(6 \%)$, storage (3\%) and monitoring (2\%). Within prescribing incidents there were four recurring themes: duplication of therapy, wrong/inappropriate drug choice, incorrect dose/regimen and omission. The numbers of incidents reported increased as the week progressed, with the largest number occurring on a Friday (24\%). A review of the types of medicines involved showed that insulins, proton pump inhibitors, anticoagulants, vancomycin, and epidurals were recurring themes, reflecting NPSA alerts. When graded for significance, two were found to be harmful, 56 significant and 54 insignificant.

Conclusions There is clearly considerable under-reporting of medication errors in critical care via the official incident reporting system. One of many reasons for poor official reporting of errors is the lack of tangible local response to those errors. It is therefore important that the areas highlighted by our project are effectively addressed, not only to improve safety but also to encourage further reporting and work to develop a culture of incident reporting within critical care.

\section{Reference}

1. Valentin A, et al:. Intensive Care Med 2006, 32:1591-1598.

\section{P453}

Three years experience of a quality improvement programme

R Innes, S Burt, F Miles, S Watkins

Musgrove Park Hospital, Taunton, UK

Critical Care 2010, 14(Suppl 1):P453 (doi: 10.1186/cc8685)

Introduction As part of a safer patient initiative under the auspices of the Institute of Health improvement (IHI), Boston, we took part in a 2-year project to improve the safety and quality of care in adult intensive care. 


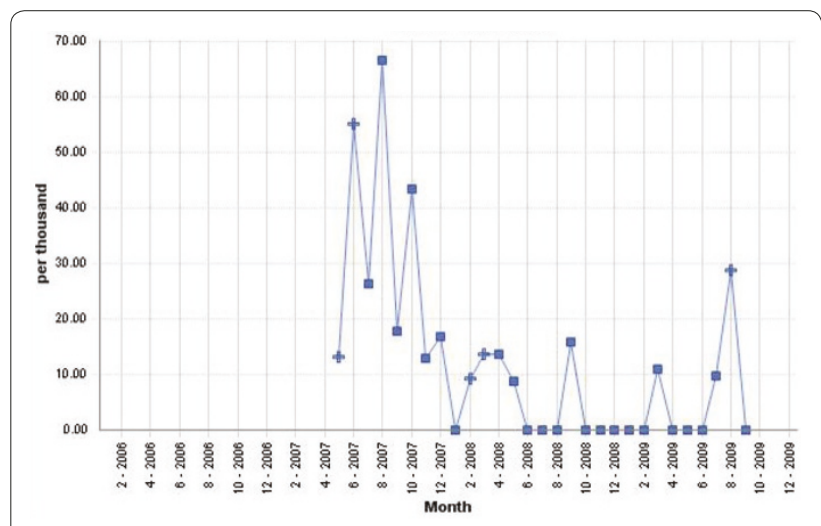

Figure 1 (abstract P453). Ventilator-associated pneumonias per 1,000 ventilator-days. C03: Musgrove Park Hospital, Taunton and Somerset NHS Trust. CCO1 - VAP rate.

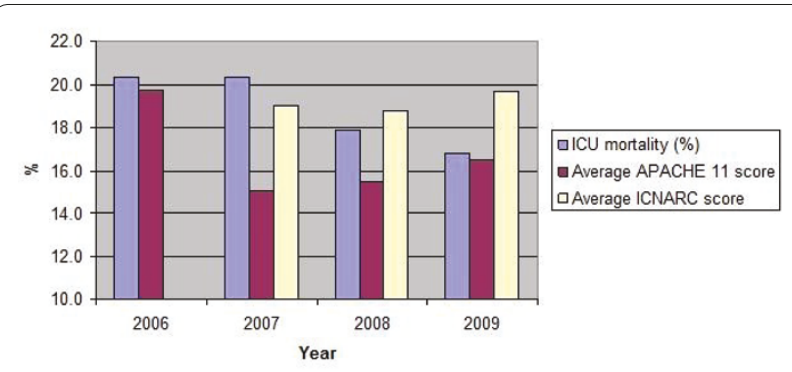

Figure 2 (abstract P453). Intensive care mortality between 2006 and 2009.

Methods Between October 2006 and October 2008 the $\|H\|$ coached and advised our institution on mechanisms to improve quality of care. The aim was to develop systems of healthcare that were reliable at least 95\% of the time. The changes to be introduced - central line bundle, ventilator bundle, hand hygiene, daily patient goals - were led by a small multidisciplinary team chaired by a hospital executive (finance director) who met every 2 weeks for 2 years for short meetings ( $1 / 2$ hour). Our aim was to reduce nosocomial infection and mortality.

Results It took $\sim 12$ months to introduce reliable process in all areas. Hand hygiene improved from $\sim 70 \%$ compliance to consistently $>95 \%$. MRSA acquisition on the ICU reduced from about $10 \%$ of patients prior to October 2006 to 0.3\%. VAP was reduced (Figure 1). The average unadjusted mortality was reduced year on year despite similar mean APACHE II and ICNARC scores (Figure 2).

Conclusions Reliable evidence-based processes for patient care can significantly reduce nosocomial infection and mortality. The use of executive nonmedical hospital personnel experienced in the mechanisms of introducing change may be beneficial.

\section{P454}

Improving team work during bedside rounds: using daily goals and best practices

M Adamson, L Kure, B Duncan, N Holmes, AA Amara

Sunnybrook Health Sciences Centre, Toronto, Canada

Critical Care 2010, 14(Suppl 1):P454 (doi: 10.1186/cc8686)

Introduction Communication between healthcare professionals is a key step for patient safety, its failure accounting for over $60 \%$ of root causes in sentinel events [1]. Bedside rounds are important for teamwork communication and can be improved by an explicit approach [2] and by process-oriented information tools to organize and direct interprofessional rounds [3].

Methods As part of a quality improvement project, we conducted an observation of the documentation of daily goals (DG) and best practices
(BP) in a step-down unit (both tools have been previously added to patient flowsheets), before and after the introduction of a structured rounds process and team education. Our hypothesis was that these important tools were used before rounds, without input from all team members. Rounds were observed on two separate periods and the observer would take notes of whether DG and BP were documented or not and whether discussion took place before documentation. Differences in proportions between the two periods were analyzed with Fisher's exact test. $P<0.05$ was considered significant.

Results We observed 100 bedside interactions on each period. Documentation remained unchanged for DG (pre 55\% vs post 53\%, $P>0.05$ ) and BP (pre $57 \%$ vs post $48 \%$ ); however, the second period had an improved documentation after team discussion (DG: pre 2\% vs post $31 \%$, $P<0.001$; BP pre $0 \%$ vs post $33 \%, P<0.001$ )

Conclusions The intervention aided in increasing documentation after discussion, implying an increased communication among the interprofessional team. About 50\% of patients still will not have documentation after bedside rounds. Patient information was not collected, therefore our study is limited in providing information on clinical outcomes. Further research should focus on how to best implement these tools, how to qualitatively assess the content of daily goals and to demonstrate effects on patient-centered outcomes.

\section{References}

1. Arora V, Johnson J, Lovinger D, Humphrey HJ, Meltzer DO: Communication failures in patient sign-out and suggestions for improvement: a critical incident analysis. Qual Saf Health Care 2005, 14:401-407.

2. Dodek PM, Raboud J: Explicit approach to rounds in an ICU improves communication and satisfaction of providers. Intensive Care Med 2003 29:1584-1588

3. Gurses AP, Xiao Y: A systematic review of the literature on multidisciplinary rounds to design information technology. J Am Med Inform Assoc 2006, 13:267-276.

P455

Stress induced by simulation-based training in anesthesiologist residents

T Geeraerts, P Roulleau, G Cheisson, K Aidan, M Leguen, J Duranteau, D Benhamou

CHU de Bicetre, Le Kremlin Bicetre, France

Critical Care 2010, 14(Suppl 1):P455 (doi: 10.1186/cc8687)

Introduction The use of a high-fidelity simulator can lead to very realistic clinical situations sometimes difficult to manage psychologically. The aim of this study was to evaluate the psychological stress induced by simulationbased training and the associated skills in anesthesiologist residents.

Methods A cohort of 27 residents was studied. The psychological stress just before and after the simulation session was quantified by autoevaluation scale (numeric scale 0 to 10 ) and by salivary amylase sampling [1]. Nontechnical skills were quantified by analysing videotapes and scoring the Anaesthetist NonTechnical Skills [2].

Results The median stress numeric scale before the simulation session was 5 (ranging 2 to 8$)$, and after was 7 (2 to 10) $(P=0.0004)$ (Figure 1$)$. The stress scale before the session was significantly lower in residents who already

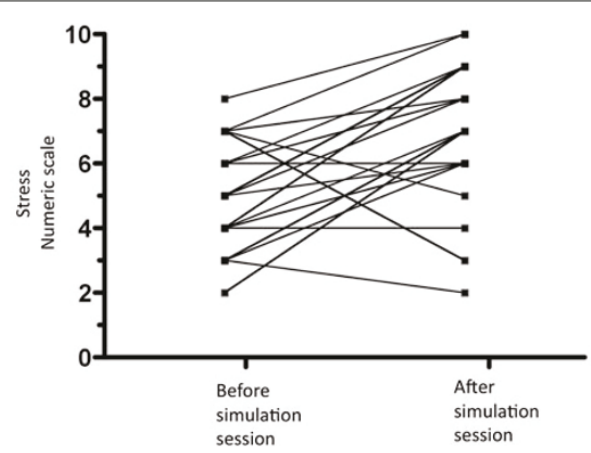

Figure 1 (abstract P455). 
underwent simulation-based training ( $P=0.04)$. In $48 \%$ of residents, stress scales after the simulation session were above $8 / 10$. Salivary amylase after the session was significantly higher than before $(P=0.008)$, corresponding to a 2.2-fold increase. They were no significant relationships between psychological stress parameters and nontechnical skills.

Conclusions Psychological stress before the simulation session, but especially after simulation, appears to be high in anesthesiologist residents, and particularly in those who performed a simulation session for the first time. This fact should be considered when organising such simulation-based teaching.

\section{References}

1. Dantzer R, et al: Psychoneuroendocrinology 2009, 34:1.

2. Yee B, et al:: Anesthesiology 2005, 103:241-248.

\section{P456}

Impact of an education program in respiratory therapy quality of

care

WA Pinto, HB Rossetti, A Araujo, HM Do Carmo, AP Acerbi, MV Santos,

JJ Sposito, M Rabelo, SS Matos, M Assuncao, F Machado

Federal University of São Paulo, Brazil

Critical Care 2010, 14(Suppl 1):P456 (doi: 10.1186/cc8688)

Introduction The respiratory therapist has a central role in the management of critically ill patients. The objective of this study was to evaluate the impact of an education program aiming to improve quality of care of respiratory therapy.

Methods A before-after study was designed to assess compliance to 15 respiratory therapy indicators, chosen due to their relevance in patient care and availability for objective measurement, in all patients admitted to the ICU regardless of mechanical ventilation or not. Compliance was assessed during 1 month before implementation of the education program. The educational process comprised of meetings, group training, written manuals and individual feedback for noncompliance situations. After 6 months of the implementation process, compliance assessment was again performed. Results were expressed as percentages and comparisons were performed through the chi-square test. Results were considered significant if $P<0.05$.

Results In both periods 80 patients were observed, with a total of 1,212 observations in the before period (BP) and 1,369 observations in the after period (AP). Compliance rates improved for almost all indicators as follows: chart registration $(C R)$ of weight/height $(B P=97.7 \% ; A P=99.4 \% ; P=$ 0.0026), $C$ R of weight-predicted tidal volume $(B P=73.3 \% ; A P=94.7 \% ; P=$ 0.000), $C R$ of orotracheal tube fixation level $(B P=69.5 \% ; A P=93.6 \% ; P=$ $0.000)$, $C R$ of humidification line change date $(B P=83 \% ; A P=89.8 \% ; P=$ $0.000)$, $C R$ of chest $X$-ray pattern $(B P=86.8 \% ; A P=96.9 \% ; P=0.000) ; C R$ of clinical decisions during round ( $\mathrm{BP}=91.3 \% ; \mathrm{AP}=98.7 \% ; P=0.000$ ), actual compliance to clinical decisions during rounds $(\mathrm{BP}=79.77 \%$; $\mathrm{AP}=98.8 \%$; $P=0.000$ ), correct utilization of mechanical ventilation humidification ( $\mathrm{BP}=$ 75.9\%; $\mathrm{AP}=96.4 \% ; P=0.000$ ), actual tidal volume between 6 and $8 \mathrm{ml} / \mathrm{kg}$ predicted body weight $(B P=82.0 \% ; A P=96.3 \% ; P=0.000)$, adequacy of ventilators alarms $(\mathrm{BP}=69.5 \% ; \mathrm{AP}=95.4 \% ; P=0.000)$, actual plato pressure $<30 \mathrm{mmH}_{2} \mathrm{O}(\mathrm{BP}=93.2 \% ; \mathrm{AP}=95.7 \% ; P=0.0241)$. Some indicators did not show a statistically significant improvement: $C R$ of secretion aspect $(\mathrm{BP}=94.4 \% ; \mathrm{AP}=94.2 \% ; P=0.861)$, $\mathrm{CR}$ of circuit change date (before $=$ $90 \%$; after $=92.2 \% P=0.0848$ ) and $C R$ of round changes (BP $=92.0 \%$. $A P=$ $92.6 \% ; P=0.551)$. Only $C R$ of cuff pressure had a lower compliance on the $\mathrm{AP}(\mathrm{BP}=87.5 \% ; \mathrm{AP}=79.4 \% ; P=0.0005)$.

Conclusions An education program based on protocol-driven actions and training is able to improve compliance in respiratory therapy quality indicators. The clinical relevance of this compliance improvement still needs to be established.

\section{P457}

Use of the FAST HUG checklist in Mexican critically ill patients

JC Cerda, V Sanchez, F Perez, E Fernandez

HSJ Tec de Monterrey, Mexico

Critical Care 2010, 14(Suppl 1):P457 (doi: 10.1186/cc8689)

Introduction There have been several efforts to improve the quality of care of ICU patients. The use of checklists has been linked to improvements in ICU care. In 2005, Vincent and colleagues published the FAST HUG checklist strategy [1]. We decided to study the level of adherence of Mexican physicians to the measures proposed in this checklist.

Methods We conducted a 3-month prospective observational study in the adult ICU of an academic hospital in northeastern Mexico. Patients were sorted into one of the two study groups: group 1 had a critical care physician on charge of the ICU management, while group 2 had a noncritical care physician on charge. We measured the adherence of the physicians to the FAST HUG checklist items. We arbitrarily defined good compliance as having fulfilled $>4$ items.

Results One hundred and forty-seven patients were admitted to the ICU during the study period, but only 129 had complete data and were evaluated. There were 86 patients in group 1, while group 2 had 43 patients. Group 1 patients had higher age, SOFA and APACHE score on admission. We analyzed the subgroup of patients who had an initial APACHE score between 11 and 25, as they are usually the ones most likely to benefit from FAST HUG measures. These results are described in Table 1. We noticed that most of the group 1 patients had severe sepsis, while most of the group 2 patients had ischemic heart disease, which clearly explains the differences in mechanical ventilation and length of ICU stay.

Table 1 (abstract P457). Variables of the subgroup APACHE II 11 to 25

\begin{tabular}{lccc}
\hline Variable & Group 1 & Group 2 & P value \\
\hline Age & $64 \pm 10$ & $69 \pm 17$ & 0.25 \\
APACHE II & $18 \pm 5$ & $15 \pm 4$ & 0.08 \\
SOFA on admission & $5 \pm 3$ & $3.3 \pm 2$ & 0.12 \\
SOFA at discharge & $2.4 \pm 4$ & $1.7 \pm 2$ & 0.82 \\
ICU length of stay & $10.5 \pm 9$ & $4.6 \pm 3$ & $0.04^{*}$ \\
Mechanical ventilation days & $6.7 \pm 9.6$ & $1.8 \pm 3$ & 0.11 \\
FAST HUG & $6.4 \pm 0.9$ & $3.8 \pm 1.4$ & $0.001^{*}$
\end{tabular}

Group 1 had a critical care physician in charge of ICU management, while group 2 had a noncritical care physician in charge. We observed the same severity of illness in this group with APACHE II between 11 and 25 points and better adherence to the FAST HUG items among intensive care physicians.

Conclusions We found a better adherence to the FAST HUG items among intensive care physicians.

\section{Reference}

1. Vicent $\mathrm{JL}$, et al:: Give your patient a fast hug (at least) once a day. Crit Care Med 2005, 33:1225-1229.

\section{P458}

Bay rooms vs single-bed rooms in intensive care unit nosocomial infections: a case-control study

E Bigazzi, L Turrisi, G Zagli, P Pecile, M Bonizzoli, A Peris

Careggi Teaching Hospital, Florence, Italy

Critical Care 2010, 14(Suppl 1):P458 (doi: 10.1186/cc8690)

Introduction Nosocomial infections remain a clinical challenge in the ICU. Based on definitions by the Centers for Disease Control and Prevention of Atlanta, nosocomial infections are defined as infection occurring within 48 to 72 hours after hospital admission. In ICU settings, a single-bed room location has been suggested as a structural organization helpful to prevent nosocomial infection diffusion. Here we show preliminary results of a study conducted in our ICU before and after a change from open bay to single-bed room locations.

Methods Microbiological data were collected from ICU patients hosted in bay rooms in the ICU (April 2006 to April 2007) and compared with data collected from patients hosted in the subsequent period (May 2007 to May 2008), after the single-bed ICU room re-allocation. For analysis, we considered the prevalence of microorganisms isolated and the difference in blood culture/tracheal aspirate positivity.

Results The cohorts examined resulted in similar demographic and clinical data. As shown in Figure 1, microorganisms isolated did not change significantly in the two periods. Figure 2 summarizes the differences in tracheal aspirate/blood culture isolation among the two periods.

Conclusions We cannot confirm that a single-bed room location can prevent nosocomial infection diffusion. A limit of this study, as in previous 


\begin{tabular}{|l|cc|}
\hline & & \\
\hline Microor ganism (\%) & $\mathbf{0 1 / 0 4 / 2 0 0 6 - 3 0 / 0 4 / 2 0 0 7}$ & $\mathbf{0 1 / 0 5 / 2 0 0 7 - 3 1 / 0 5 / 2 0 0 8}$ \\
\hline acinetobacter baumannii & 0.2 & 0.7 \\
species aspergillus & 0.0 & 1.8 \\
species candida & 26.4 & 31.8 \\
\hline species enterobacter & 5.0 & 3.7 \\
\hline species enterococcus & 5.4 & 2.3 \\
\hline escherichia coli & 5.1 & 10.2 \\
species klebsiella & 6.1 & 4.3 \\
\hline pseudomonas aeruginosa & 13.4 & 13.4 \\
\hline serratia marcescens & 3.7 & 0.5 \\
\hline staphilococcus aureus & 16.8 & 13.7 \\
staphylococcus coagul ase & 0.8 & 0.1 \\
staphylococcus epidermidis & 4.8 & 4.5 \\
\hline other & 12.3 & 13 \\
\hline
\end{tabular}

Figure 1 (abstract P458).

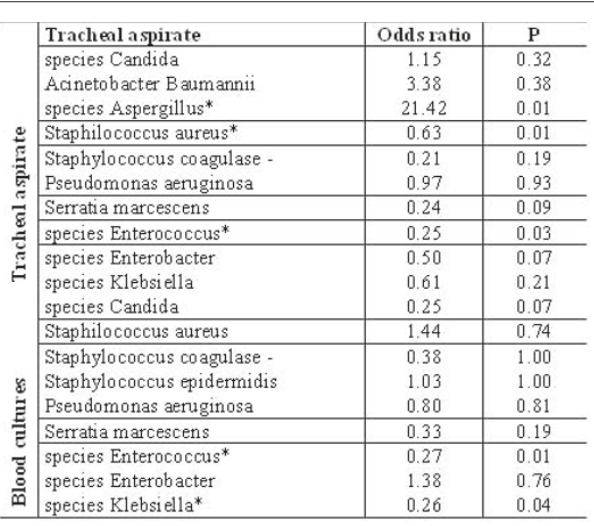

Figure 2 (abstract P458).

published works, remains the bias related to patients admitted from other wards or after a prolonged emergency room stay.

\section{P459}

Impact of quality improvement process on healthcare-associated infection in the ICU in a tertiary care hospital in India

M Bhattacharyya, A Bhakta, S Todi

AMRI Hospitals Kolkata, India

Critical Care 2010, 14(Suppl 1):P459 (doi: 10.1186/cc8691)

Introduction Healthcare-associated infection (HCAl) has a significant contribution to mortality, morbidity and cost of treatment in critically ill patients. The objective of this study was to conduct a process (quality improvement) and outcome (pre and post implementation) surveillance, HCAl being taken as the major variable.

Methods This was a prospective observational study carried out from June 2005 to September 2009. Process implementation was started from May 2006 and included compliance with hand hygiene, urinary catheter care, central line care and VAP bundle. HCAI rates were recorded prospectively every month from June 2005. We recorded the rates of central line-related bloodstream infection (CLI), catheter-associated urinary tract infection (UTI), hospital-acquired pneumonia (HAP) and ventilator-associated pneumonia (VAP) per 1,000 device-days as per CDC-NNIS definition $[1,2]$. Results Various infection rates showed numerical improvement after the implementation of the quality improvement $(\mathrm{QI})$ process (Figure 1). The differences were statistically significant for two of these four endpoints ( $P$ for HAP $=0.029$ and for UTI $=0.013$ ) and in others there was a trend towards improvement. Device utilization rates associated with these endpoints before and after the implementation remained unchanged, confirming that the drop of infection rates was not influenced by any reduction/increase of respective device utilizations (Figure 1). The average compliance rate during the study period for hand hygiene was 77.92, for urinary catheter care 98.24, for central line care 96.62 and for VAP bundle 91.54 .

Conclusions Implementation and continuous surveillance of the $\mathrm{Q}$ process improved nosocomial HCAl in our hospital.

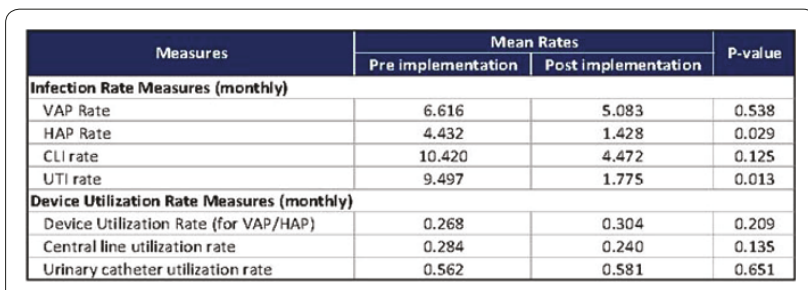

Figure 1 (abstract P459). Comparing mean infection rates before and after the quality improvement process.

\section{References}

1. Garner JS, Jarvis WR, Emori TG, Horan TC, Hughes JM: CDC definitions for nosocomial infections, 1988. Am J Infect Control 1988, 16:128-140.

2. Emori TG, Culver DH, Horan TC, et al: National nosocomial infections surveillance system (NNIS): description of surveillance methods. Am 」 Infect Control 1991, 19:19-35.

P460

Teaching airway resuscitation to novices: a randomized controlled simulator mannequin study

PBrindley, C Needham

University of Alberta Hospital, Edmonton, Canada

Critical Care 2010, 14(Suppl 1):P460 (doi: 10.1186/cc8692)

Introduction The sniffing position is widely promoted for teaching airway positioning prior to intubation attempts, but whether this analogy results in novices placing the head and neck appropriately has not been evaluated. An alternate analogy - win with the chin - or simple anatomic instructions may perform better.

Methods Randomized controlled study comparing different instructions for enabling novices to adequately position a simulator mannequin's head and neck. Study volunteers included medical students and PGY1 residents in surgery and internal medicine. Subjects independently positioned a mannequin based upon their understanding of four randomly-assigned written instructions for: the sniffing position; the win with the chin analogy; anatomic instructions; and no instructions (control). Digital photographs following each instruction were analyzed by two airway experts for adequacy of overall positioning, and for the three components of airway positioning.

Results There were 81 volunteers. The positioning was adequate most often (43.2\%) following the win with the chin analogy as compared with the other instructions (37.0\% anatomic instructions; $19.8 \%$ control; $14.8 \%$ sniffing position analogy). Positioning following the sniffing position instructions was not different from no instruction $(P=0.53)$. The win with the chin and anatomic instructions were better than no instructions $(P=$ 0.002 and $P=0.023$, respectively).

Conclusions The win with the chin analogy resulted in adequate airway positioning significantly more often than the sniffing position or control, and maintained atlanto-occipital extension when compared with providing anatomic instructions. Overall, win with the chin was a superior teaching analogy and could replace the sniffing position analogy.

\section{P461}

Impact of ultrasound guidance in central venous catheterization by inexperienced trainees: a prospective observational study

S Yamao, R Suda, Y Tomishima, N Uchiyama, T Jinta, N Nishimura, S Ohde, N Chohnabayashi

St Luke's International Hospital, Tokyo, Japan

Critical Care 2010, 14(Suppl 1):P461 (doi: 10.1186/cc8693)

Introduction Central venous catheterization is a common procedure, but can potentially result in serious complications. Previous studies have shown that inexperienced trainees have lower success rates than experienced operators. Little is known about the efficacy of ultrasound (US) guidance in central venous catheter (CVC) insertion by inexperienced trainees. 
Methods A prospective, observational study, performed in a Japanese training hospital. All patients who were inserted CVCs by inexperienced trainees (postgraduate year 1 to 6) between 1 June 2008 and 31 October 2009 were included. The following parameters were evaluated: the rate of successful insertions, the incidence of complications, the site of CVC insertion, the method of US use, and the postgraduate year of the trainees. We defined success of CVC insertion if a trainee completes catheterization without complication and personnel exchange. We compared the success rate and the complication rate with both insertion sites and US techniques; Group SA (surface anatomy) underwent CVC insertion using the landmark technique, Group indirect-ultrasound (iUS) using ultrasound image-based skin surface marking, and Group direct-US (dUS) using realtime ultrasound guidance. The chi-test was used for analysis and $P<0.05$ was considered statistically significant.

Results In total 593 CVCs were inserted, 527 via the internal jugular vein (IJV), and 66 via the subclavian vein (SV). The IJV approach includes 52 patients (9.9\%) in Group SA, 145 patients (27.5\%) in Group iUS, and 330 patients (62.6\%) in Group dUS. The SV approach, on the other hand, consisted of 26 patients (39.4\%) in Group SA, and 40 patients (60.6\%) in Group dUS. The success rate of CVC insertion of the IJV approach linearly rose in Group SA, Group iUS and Group dUS $(76.9 \%, 81.4 \%, 88.2 \%, P=$ 0.033), but in the SV approach there is no significant difference between Group SA and Group dUS (76.9\%, 75.0\%, $P=0.83$ ). Five patients who underwent real-time US via the SV developed pneumothorax while no one in Group SA had the complication $(P=0.57)$.

Conclusions The use of ultrasound quidance improves the success rate of CVC insertion via the IJV by inexperienced trainees. However, in the SV approach the efficacy of ultrasound guidance is not proven, and the incidence of pneumothorax increased in our study.

\section{P462}

Performance analysis of an ICU according to GiViTI's StART approach

R Rosi', V Peccianti', E Fatighenti' ${ }^{1}$ M Giardino², D Crespi², A Anghileri²

'AOUS, Siena, Italy; 'Istituto Mario Negri, Ranica (BG), Italy

Critical Care 2010, 14(Suppl 1):P462 (doi: 10.1186/cc8694)

Introduction ICU beds are a limited resource; their appropriate use is mandatory: only patients sick enough should be admitted to the ICU, and all admitted patients should receive the adequate level of care.

Methods From 1 January 2008 to 9 March 2009, 716 admissions to a seven-bed general ICU were analyzed according to GiViTl's StART approach, to evaluate appropriateness of the ICU admission and stay. Available resources (ICU beds) were classified as High Level (HL) or Low Level (LL), according to equipment and staffing. Patients may require High $(H)$, Low $(L)$, or Ordinary $(O)$ levels of care (HP, LP, OP, respectively) [1]. OPs should not be admitted to the ICU and can stay in the ICU only briefly, following $\mathrm{HL}$ or $\mathrm{LL}$ periods. One nurse can attend two HPs, or one HP and two LPs, or four LPS, provided that each bed can be controlled easily (not in different rooms, for example) [2]. For each day shift, matching of required and available care was analyzed: mismatch is expressed as nurse deficit: positive values indicate excess of care (too many nurses for not too sick patients), zero suggests that assistance requirements are met, with negative values available care is less than patients' needs.

Results In this ICU, all seven beds are fully equipped. Of the $716 \mathrm{ICU}$ admissions, 20 (2.8\%) were inappropriate OPs. One hundred and ninetyseven further ICU-days of the 2,711 available (7.3\%) were used for OPs (mostly due to delayed discharges); altogether 217/3,427 (6.3\%) ICUdays were inappropriately used for OPs. In the analysis of resources and requirements matching, if the seven beds were in the same room, available assistance would meet requirements in most nurse shifts, even if the staff would have worked at maximum almost always; in fact, in this ICU, four beds are in a room and there are three single-bed rooms - therefore, according to the suggested standards, a mean 1.9 nurse deficit per shift is observed. The relevance of this observation on patient outcome can be speculated only.

Conclusions The relevance of the StART approach is the definition of inappropriateness both from excess and insufficiency of care points of view: both admissions and ICU stays of OPs and/or too many too sick patients for the amount of care available are equally inappropriate. Moreover, the available care is determined both by the number of ICU nurses and by logistic constraints: seven beds in an open space are different from four beds in a room and three beds in single rooms, and in fact this ICU pays a two nurse deficit per shift to its design.

References

1. lapichino G, et al:: Minerva Anestesiol 2005, 71:273-280.

2. Iapichino G, et al: Minerva Anestesiol 2007, 73:501-506.

\section{P463}

Simulation training at the point of care to decrease the incidence of airway complications on the intensive care unit

SC Cook, JJ Gatward, FE Kelly, J Bell, FM O'Higgins, MJ Thomas

Bristol Royal Infirmary, Bristol, UK

Critical Care 2010, 14(Suppl 1):P463 (doi: 10.1186/cc8695)

Introduction A recent analysis of critical incidents reported to the National Patient Safety Agency (NPSA) by ICUs in the UK revealed a significant number of airway incidents in critical care [1]. We recently performed an online survey with responses from 305 trainees from across the UK regarding airway management and complications on the ICU. The results revealed a high number of adverse airway events including 27 deaths. A large number of junior trainees (many of whom cover the ICU alone at night) expressed low confidence levels with airway management in critical care, and the majority of all trainees felt inexperienced with tracheostomy care and emergencies. Simulation has been recommended as a suitable way of providing critical incident training [2], and simulation at the point of care has been shown to be especially powerful and effective [3].

Methods In response to our survey, we set up a multidisciplinary simulation-based airway teaching programme for all staff on our unit. A Resusci-Annie ${ }^{\text {TM }}$ manikin with an Airway Trainer ${ }^{T M}$ head (Laerdel, Norway) is set up in a bed space on our ICU. Realistic patient monitoring is achieved with the Laerdel SimMan ${ }^{T M}$ software (free from the Laerdel website). We have written a number of scenarios in response to incidents and training deficiencies highlighted by our survey. Scenarios are run with ICU staff from all disciplines acting within their normal roles. Realism is enhanced by staff practicing in their usual working environment, using the unit's own equipment.

Results So far, we have trained $43 \mathrm{ICU}$ staff. Feedback has been excellent, with scores ranging from 8.5 to 10 . Participants were especially positive about the multidisciplinary aspect of the training and on practising uncommonly encountered scenarios in their normal workplace.

Conclusions We have created a multidisciplinary airway training programme to practice critical incident drills at the point of care. This teaching method is low cost, simple and can be set up quickly and spontaneously during quiet periods on the ICU.

\section{References}

1. Thomas AN, et al: Anaesthesia 2009, 64:358-365.

2. Wheeler DW, et al.: Br J Anaesth 2009, 103:145-147.

3. Weinstock P, et al:: Pediatr Crit Care Med 2009, 10:176-181.

\section{P464}

Management of needle stick injuries from incapacitated patients

L Burrows, A Padkin

Royal United Hospital, Bath, UK

Critical Care 2010, 14(Suppl 1):P464 (doi: 10.1186/cc8696)

Introduction This survey investigated current UK intensive care practice in testing patients for blood-borne viruses following needle stick injury when the patient lacks capacity for consent. Needle stick injuries are a common occurrence in the National Health Service, with an estimated incidence of 113 to 623/10,000 healthcare workers/per year [1]. Current UK law $[2,3]$ does not permit the testing of a patient's infective status for the benefit of a healthcare worker involved without the patient's consent. Incapacitated patients cannot give consent. The General Medical Council (GMC) guidance for the management of needle stick injuries from incapacitated patients reflects this [4].

Methods A questionnaire was sent to the clinical lead of the 225 general ICUs within England, Wales and Northern Ireland. Responses were anonymous. 
Results Ninety-nine consultants replied out of 225 (44\% response rate). Sixty-two of the 99 consultants (63\%) reported incidences of needle stick injuries to their staff from incapacitated patients in the past year. In six incidents the patient was not tested because their full blood-borne virus status was already known. Thirty-six of the remaining 56 incidents (64.5\%) stated that patients were tested without consent. Sixty-one per cent of consultants (22 of 36 ) reported that the patient was informed once conscious that the test had been performed. Two consultants discussed the decision to perform the test with the family prior to proceeding. One patient, not tested at the time of injury, refused consent on regaining capacity. Sixteen patients out of the 62 incidents were blood-borne viruspositive (26\%), six being new diagnoses following the needle stick injury (37.5\%).

Conclusions Despite the recent change in legislation, the majority of incapacitated patients are being tested for blood-borne viruses when unable to give consent. This has significant beneficial effects for the healthcare worker involved; a negative test allowing prophylactic antiviral treatment to be discontinued and minimises anxiety. This survey highlights the need for further discussion involving healthcare professionals, the legal profession and legislators regarding needle stick injuries from incapacitated patients. Is there a duty of care to the healthcare worker that is not acknowledged in current legislation?

\section{References}

1. Trim JC, et al:.J Hosp Infect 2003, 53:237-242.

2. Human Tissue Act 2004 [http://www.opsi.gov.uk/acts/acts2004/ ukpga_20040030_en_1.htm]

3. Mental Capacity Act 2005 [http://www.opsi.gov.uk/acts/acts2005/ ukpga_20050009_en_1]

4. Update to Serious Communicable Disease guidance [http://www.gmc-uk. org/guidance/update_serious_communicable_diseases.asp]

P465

Stress and fatigue among anaesthesia and intensive care doctors in Lithuania

A Macas, A Kolciniene, I Vetaite, D Bilskiene, A Mikalauskas

Kaunas University of Medicine, Kaunas, Lithuania

Critical Care 2010, 14(Suppl 1):P465 (doi: 10.1186/cc8697)

Introduction Evidence shows that anaesthesia and intensive care is a stressful occupation and it may have an impact on anaesthetists' and intensive care doctors' well-being. The goal of the study was to evaluate stress and fatigue and their correlation with working conditions among anaesthesiologists and intensive care specialists employed in Kaunas city (Lithuania) hospitals.

Methods One hundred and forty-two doctors of anaesthesia and intensive care departments were interviewed using a standard questionnaire in 2008. It consisted of three parts: the first part covered the demographic and social situation, second - stress experienced during work, third - risk of fatigue.

Results The response rate was $71 \%$. Average age of respondents was 41 years, $66.2 \%$ were female. A total of $25.4 \%$ had working experience $<5$ years and $23.2 \%$ working experience of 11 to 15 years. The workload of $49.3 \%$ of respondents was 37 to 54 hours per week (mean number of on-call nights per month $-6.5 ; 15.3 \%$ of respondents had $>9$ on-call night shifts). Often and very often stress was experienced by $24.6 \%$ of doctors; symptoms of fatigue were demonstrated by $57 \%$ of respondents. Respondents older than 55 years emphasized increased blood pressure, while younger respondents - up to 34 years - reported inability to concentrate, decreased objectiveness, unstable mood, difficulty in making decisions. Respondents living without partners experienced chest pain, anger and nuisance. Doctors experience less stress if they are satisfied with their work and if their workload is adequate, while increasing pressure by hospital administration to decrease costs of care increased risk of fatigue. If a doctor is employed for more 40 hours per week he faces difficulties concentrating, while more than 60 hours per week causes interference with home life.

Conclusions Every fourth anaesthesiologist experienced stress during work, while more than one-half had symptoms of moderate fatigue. Growing workload increased doctor's stress. Pressure by hospital administration and limited freedom in decisions increased incidence of fatigue. Work satisfaction reduced risk of stress and fatigue. Stress and fatigue significantly correlated with the state of physical health.
P466

Implementation of daily goals in the ICU reduces length of ICU stay and errors of omission in patient care

JM Binnekade', A Brunsveld², S Arbous², MG Dijkgraaf', J Horn'1, J Sloot',

A Balzereit', MB Vroom'

'Academic Medical Center, Amsterdam, the Netherlands; ${ }^{2}$ LUMC, Leiden, the

Netherlands

Critical Care 2010, 14(Suppl 1):P466 (doi: 10.1186/cc8698)

Introduction In the ICU, daily goals have improved the effectiveness of communication within care teams [1]. The study objective was to investigate the effect of explicit daily goals in ICU practice on the length of intensive care stay, length of hospital stay, duration of mechanical ventilation and errors of omission in patient care.

Methods A multicenter, sequential cohort, pretest-post-test study was performed. During the pretest phase, daily goals were formulated by staffphysicians, evaluated by a research physician and kept blinded for the rest of the ICU team. Subsequently, daily goals were broadly implemented. During the post-test phase, daily goals were routine practice. Patient data were collected one full year before (as baseline reference) and during the pretest and post-test phases. During pretest and post-test, medical charts (random subsets of 10 patients a week) were reviewed for errors of omission, defined as deviations from our care protocols. Patient data were compared with data from nonparticipating academic ICUs, extracted from the National Intensive Care Evaluation database.

Results Two academic ICUs participated in the study. Patients included: baseline reference $n=3,641$; pretest $n=3,401$, and post-test 3,426. ICU mean (SD) length of stay (days) differed significantly between the pretest phase 5.7 (2) and the post-test phase 4.3 (2.3), difference 1.4 days (95\% Cl 1.3/1.5). The post-ICU length of hospital stay decreased in the post-test phase, mean difference 2.3 days $(95 \% \mathrm{Cl} 1.2 / 3.4)$. Median (IQR) duration of mechanical ventilation during pretest was 2 ( 1 to 4 ) days and during posttest was 1 ( 1 to 4 ) days, $P<0.001$. The probability of an error of omission was significant larger in the pretest phase then in the post-test phase, RR $6.5(5.7 / 7.4)$

Conclusions The results showed a significant decrease in ICU LOS, duration of mechanical ventilation and length of post-ICU stay following DGF implementation. Also the chance of errors of omission to occur was six times lower, which indicates a significant increase in protocol adherence. The drawback of the study design (historic control group and a pretest and post-test for comparison) was limited by comparing the study results with other academic centers during the same time frame.

\section{Reference}

1. Pronovost PJ, et al:. J Crit Care 2003, 18:71-75.

\section{P467}

Use of single patient rooms to decrease acquisition of antibioticresistant bacteria in the ICU

PD Levin, M Golovanevski, AE Moses, CL Sprung, S Benenson

Hadassah Hebrew University Hospital, Jerusalem, Israel

Critical Care 2010, 14(Suppl 1):P467 (doi: 10.1186/cc8699)

Introduction The role of isolation rooms in decreasing bacterial transmission between ICU patients has been debated. A recent change in ICU location allowed for further investigation.

Methods Old ICU A and Old ICU B were open-plan seven-bed and four-bed units. In March 2007, ICU A moved to single patient rooms (New ICU A eight beds). ICU B remained unchanged (New ICU B). The same physicians cover both ICUs. Patient distribution is based on available space only. Demographic and microbiological data were collected on consecutive patients staying $>48$ hours. Culture of resistant organisms (MRSA, VRE, ESBL, resistant Acinetobacter, or carbapenem-resistant Gram-negatives) in surveillance or clinical cultures was measured. New ICU A was compared with open-plan New ICU B to assess the effect of single rooms. New ICU A was compared with Old ICU A to assess change vs historical controls and New and Old ICU B were compared with show control group stability. Results Data were collected on 62, 62, 44 and 39 patients from Old ICU A, New ICU A, Old ICU B and New ICU B. Patient populations in New ICU A and $B$ were similar. ICU acquisition of resistant organisms was lower in New ICU A (3/62, 5\% vs $7 / 39,18 \%$ New ICU B patients, $P=0.043)$. This finding was confirmed using survival analysis $(P=0.011)$. Comparing New with 
Old ICU A showed lower APACHE II scores (18 \pm 9 vs $21 \pm 9, P=0.028)$, preICU antibiotic use $(20 / 62,32 \%$ vs $37 / 62,60 \%, P=0.001)$, pre-ICU hospital admission $(21 / 62,34 \%$ vs $37 / 62,60 \%, P=0.004)$ and ICU admission prevalence of resistant organisms (11/62, $18 \%$ vs $25 / 62,40 \%, P=0.006)$. On Cox regression only the admitting ICU was significant for acquisition of resistant organisms (Old vs New ICU A: HR 4.07, 95\% Cl 1.03 to 16.06, $P=0.025)$. Comparing New with Old ICU B showed no changes. Adequate hand hygiene amongst the same physicians was found on 140/242 (58\%) occasions in New ICU A vs 23/66 (35\%) in New ICU B $(P<0.001)$.

Conclusions The change in ICU design to single patient rooms significantly decreased acquisition of multiresistant organisms. Whether the ICU design or improved infection control awareness led to change could not be determined. This is the first study to measure the effect of ICU design on acquisition of a range of resistant organisms while including a contemporaneous control ICU environment.

\section{P468}

Time to first review of new admissions to critical care by the consultant intensivist

A Dhrampal

Norfolk and Norwich University Hospital, Norwich, UK

Critical Care 2010, 14(Suppl 1):P468 (doi: 10.1186/cc8700)

Introduction The National Confidential Enquiry into Patient Outcome and Death (NCEPOD) recommends that all new admissions to the ICU should be reviewed by a consultant intensivist within 12 hours [1]. This early review may reduce the ICU length of stay (LOS), but does not appear to affect mortality [2]. Patient severity of illness (APACHE II scores) may also influence patient outcome.

Methods The database of our clinical information system (MetaVision, iMDsoft) was interrogated and 116 new consecutive critical care admissions were retrospectively reviewed. We ascertained the time to first review (in hours) by a consultant intensivist from MetaVision entry logs. Patient outcomes including mortality and LOS were also captured for patients reviewed early ( $<12$ hours) and late ( $>12$ hours). The admission APACHE II score was also calculated. Continuous and ordered categorical variables are expressed as the median and analysed by the Mann-Whitney $U$ test. Outcome binary variables were analysed by the chi-squared test $P<0.05$ was considered statistically significant

Results The early reviewed group $(n=53)$ had a median first review time of 4.35 hours (IOR 1.70 to 6.90 ), median LOS of 46 hours (IQR 22 to 86 ) and mortality of $17 \%(n=9)$. The late review group $(n=63)$ had a median first review time of 16.55 hours (IQR 14.35 to 18.88), median LOS of 25 hours (IQR 20 to 46$)$ and mortality of $1.6 \%(n=1)$. The median admission APACHE II score for the early group and late group was 14 (IQR 10 to 24) and 10 (IQR 8 to 16), respectively. There was no significant difference in LOS between the two groups $(P=0.137$, two-sample Mann-Whitney $U$ test). The early group had a significantly higher mortality $(P=0.03$, chi-squared test) and admission APACHE II score ( $P=0.04$, two-sample Mann-Whitney U test).

Conclusions We were unable to demonstrate that early consultant intensivist review of new critical care admissions reduces the LOS or mortality. Despite early consultant intensivist review, this group had a significantly higher mortality. A significantly higher severity of illness in the early group may account for this higher mortality.

\section{References}

1. An Acute Problem? A Report of the National Confidential Enquiry into Patient Outcome and Death; 2005 [www.ncepod.org.uk]

2. Mullen P, et al: Timing of first review of ICU admissions by consultant intensivists in a UK district general hospital. Crit Care 2009, 13(Suppl 1):P476.

\section{P469}

Analysis of critical care referrals

M Dallison, $\mathrm{H}$ Jones, $\mathrm{P}$ Matthews

Morriston Hospital, Swansea, UK

Critical Care 2010, 14(Suppl 1):P469 (doi: 10.1186/cc8701)

Introduction Anecdotal evidence at our hospital suggested an increase in the number of patients referred for admission to the ITU by junior doctors without consultation with senior doctors from their own specialty.
Recent UK guidance has recommended consultant-to-consultant referral for critical care patients [1]. Our ITU welcomes the timely referral of at-risk patients. However, excessive numbers of poor referrals have contributed to the prolonged absence of critical care team members from the unit. At a time of increasing difficulty in maintaining adequate levels of medical cover because of regulations concerning hours of work and training requirements, there is concern that this may adversely affect the efficient and safe management of the ITU.

Methods All referrals were prospectively recorded for a 6-week period. The following data were recorded: date/time of referral, grade/specialty of the referring doctor, involvement of the referring consultant, indication for the referral, referral to assessment time, duration of the assessment and whether the referral led to admission.

Results Fifty-seven per cent of referrals were out of working hours. The sources of the referrals were as follows: emergency codes 29\% and specialty referrals $71 \%$. The admission rates to the ITU for the referrals were cardiac arrests 33\%, trauma calls $75 \%$, specialty referrals $42 \%$. Referral rates according to grade (excluding emergency codes): consultant 14\%, senior trainee $49 \%$, junior trainee $37 \%$. Admission rates according to grade of referral: consultant $54.6 \%$, senior trainee $42.1 \%$, junior trainee $34.4 \%$. The mean time to review of referral was 20.2 minutes. The mean time for assessment for all referrals was 38.8 minutes. This was 52.3 and 28.9 minutes for patients admitted to the ITU and not admitted, respectively. The mean assessment and management times for the code calls were: cardiac arrest 42 minutes and trauma calls 81.2 minutes.

Conclusions Our data suggest that consultant-to-consultant referral would reduce ITU referrals that do not require admission, and decrease the time that ITU trainees are off the unit.

Reference

1. An Acute Problem? A Report of the National Confidential Enquiry into

Patient Outcome and Death; 2005 [www.ncepod.org.uk]

\section{P470}

Communication with intensive care patients

R Likar', V Langhold², A Kovinjalo', H Trabe'

'LKH Klagenfurt, Austria; ${ }^{2}$ FH Kaernten, Feldkirchen, Austria

Critical Care 2010, 14(Suppl 1):P470 (doi: 10.1186/cc8702)

Introduction Communication with conscious patients who are on oxygen and cannot verbally communicate due to temporary loss of speaking ability has proven to be anywhere from problematic to extremely difficult for the nursing staff trying to understand the needs of the patients.

Methods In order to improve the situation of conscious patients on oxygen, it is first necessary to conduct a status quo analysis to determine which communication aids can be implemented in the care of such patients. For this reason, a questionnaire was designed and carried out with the staff of the ICU.

Results The questionnaire of the intensive care staff showed that from $31.7 \%$ (20 staff members), the use of diverse electronic aids was indicated 10 times as a means for improvement in the communication situation of conscious patients on oxygen. A further question regarding communication aids that the intensive care staff would like - but are not available in the ward - resulted in the computer being named as the most wanted communication aid. In cooperation with the engineering secondary school (HTL Mössingerstraße) a special keyboard was developed especially to suit the needs of conscious patients on oxygen and an operating system was installed on computers the general hospital made available.

Conclusions The results indicated that the communication between the patients and the nursing staff using the computer showed a $50 \%$ improvement in understanding the needs of the patient from the point of view of the nursing staff. Thirty percent of the patients had tracheotomies and $20 \%$ were aphasia patients.

\section{References}

1. Bazlen U: Pflege bei Lungenerkrankungen. In Pflege heute. Lehrbuch und Atlas für Pflegeberufe. 2nd edition. Edited by Menche N, Bazlen U, Kommerell T. München: Urban \& Fischer Verlag; 2001:703-742.

2. Besendorfer A: Das Erleben von Patienten auf Intensivstationen. In Fokus Intensivpflege. Pflegewissenschaftliche Erkenntnisse zu Critical Care Nursing. Edited by Abt-Zegelin A. Hannover: Schlütersche Verlagsgesellschaft; 2004:95-158. 
P471

Quality of transfers of critically ill patients within the hospital

C Tjen, D Rowland, A Dhrampal, S Hutchinson

Norfolk and Norwich University Hospital, Norwich, UK

Critical Care 2010, 14(Suppl 1):P471 (doi: 10.1186/cc8703)

Introduction Transferring critically ill patients between clinical areas is recognised to be potentially hazardous [1] and associated with poor outcome [2]. This study addresses the standard of intrahospital transfer of sick patients within our Trust including monitoring, equipment availability, personnel and training.

Methods We surveyed senior ward staff on how they would conduct the theoretical transfer of a deteriorating, hypoxic, shocked patient to the ITU. Monitoring and equipment deemed necessary and its perceived availability were recorded, as was transfer personnel. We then prospectively reviewed actual transfers of patients with Early Warning Scores (EWS) of 3 or greater.

Results Theoretical transfer data were collected from 19 wards. Most $(74 \%)$ requested oxygen saturation $\left(\mathrm{SpO}_{2}\right)$ monitoring, while less than one-third wanted either non-invasive blood pressure or ECG monitoring. Some wards expressed a need to borrow equipment, while others felt this would lead to delay. Three wards considered any monitoring unnecessary. Of 13 wards declining a defibrillator/cardiac monitor, two did so due to lack of familiarity. Prospective data were gathered from 32 transfers between September and November 2009. EWS ranged from 3 to 9. The actual transfer monitoring mirrored the initial survey, but for $\mathrm{SpO}_{2}$ monitoring (44\% vs $74 \%$, respectively). A doctor was included by $10 \%$ for the theoretical transfer and in 16\% of actual transfers. Less than one-half of actual transfers had a trained member of the transfer team. These patients were better monitored but the standard of transfers did not correspond to EWS. Patients with higher EWS were neither better monitored nor accompanied. Out of hours activity comprised $21 \%$ of actual transfers. The bulk of patients were from the admission units and the majority went to critical care or radiology.

Conclusions There is considerable movement of sick patients around the hospital. Many transfers are performed by untrained staff, without adequate monitoring, and many are out of hours. There is poor understanding of risks of transfer and of appropriate monitoring. The data suggest that deficits are due partly to equipment unavailability - we are conducting a further audit to determine this. We propose additional investment and training and are compiling intrahospital transfer guidelines according to EWS.

\section{References}

1. Waydhas C: Crit Care 1999, 3:R83-R89.

2. Voight L, et al:: I Intensive Care Med 2009, 24:108-115.

\section{P472}

Are bariatric patients putting a strain on intensive care beds?

W Rutherford, H Wydell, S McAuliffe, C Peters

Homerton University Hospital, London, UK

Critical Care 2010, 14(Suppl 1):P472 (doi: 10.1186/cc8704)

Introduction This study looks at the characteristics and outcomes of patients admitted to an ICU after bariatric surgery and considers the staffing and financial implications for the National Health Service (NHS). There is currently increased government incentive to treat obese patients with bariatric surgery with the aim of reducing co-morbidities in the future. Methods A retrospective analysis of bariatric surgery patients requiring an ICU admission between 1 February 2007 and 31 August 2009. The study was carried out in a nine-bed mixed medical and surgical adult ICU providing level 2 and level 3 critical care to a 430-bed hospital in East London with a regional laparoscopic bariatric surgery service. Medical records were reviewed and information including gender, body mass index (BMI), age, reason for admission, treatment required and length of stay in the unit was collected. APACHE II and SOFA scores were calculated. Results A total of 502 bariatric operations were performed during this period. Twenty-four (4.8\%) patients required ICU admission at a cost of $£ 2,500(€ 2,750)$ per day for an average of 8.7 days (range 1 to 48). The average length of stay for all elective admissions was 2 days and for emergency admissions was on average 10 days. Cumulatively, bariatric patients spent a total of 209 days on the unit costing £522,500 ( $€ 575,300)$.
The average weight was $146 \mathrm{~kg}$ (range 71 to $320 \mathrm{~kg}$ ). The average BMl was 53.3 (range 32.1 to 102.1). The age range was 24 to 69 (mean 48) with an average APACHE II score of 13 and average SOFA score of 5. Four admissions (17\%) were elective, due to severe co-morbidities, extreme weight or anticipated airway difficulties. Seven patients needed to go back to theatre one or more times for re-exploratory surgery due to postoperative complications. Three patients remained intubated for 19 days or more, two of whom required a tracheostomy. Three patients died; two from intra-abdominal haemorrhage and one from sepsis.

Conclusions This is a high-risk cohort of patients, with $4.8 \%$ requiring an ICU admission. The need for patients to return to theatre for re-exploratory surgery was associated with longer ICU stays and multiple complications. This demand on critical care services is likely to increase as bariatric surgery continues to grow as a specialty and the NHS will need to increase investment in ICUs accordingly.

\section{P473}

Five-year evolution of drug prescribing in a university adult intensive care unit

P Voirol, P Eggimann, C Carron, A Pannatier, R Chiolero, JB Wasserfallen University Hospital (CHUV), Lausanne, Switzerland

Critical Care 2010, 14(Suppl 1):P473 (doi: 10.1186/cc8705)

Introduction Drug prescription is difficult in ICUs as prescribers are many, drugs expensive and decisions complex. In our ICU, specialist clinicians (SC) are entitled to prescribe a list of specific drugs, negotiated with intensive care physicians (ICP). The objective of this investigation was to assess the 5-year evolution of quantity and costs of drug prescription in our adult ICU and identify the relative costs generated by ICP or SC.

Methods Quantities and costs of drugs delivered on a quarterly basis to the adult ICU of our hospital between 2004 and 2008 were extracted from the pharmacy database by ATC code, an international five-level classification system. Within each ATC first level, drugs with either high level of consumption, high costs or large variations in quantities and costs were singled out and split by type of prescriber, ICP or SC. Cost figures used were drug purchase prices by the hospital pharmacy.

Results Over the 5-year period, both quantities and costs of drugs increased, following a nonsteady, nonparallel pattern. Four ATC codes accounted for $80 \%$ of both quantities and costs, with ATC code B (blood and haematopoietic organs) amounting to $63 \%$ in quantities and $41 \%$ in costs, followed by ATC code J (systemic anti-infective, 20\% of the costs), ATC code N (nervous system, $11 \%$ of the costs) and ATC code C (cardiovascular system, 8\% of the costs). Prescription by SC amounted to $1 \%$ in drug quantities, but $19 \%$ in drug costs. The rate of increase in quantities and costs was seven times larger for ICP than for SC (Figure 1 overleaf). Some peak values in costs and quantities were related to a very limited number of patients.

Conclusions A 5-year increase in quantities and costs of drug prescription in an ICU is a matter of concern. Rather unexpectedly, total costs and cost increases were generated mainly by ICP. A careful follow-up is necessary to try influencing this evolution through an institutional policy co-opted by all professional categories involved in the process.

\section{P474}

A semiquantitative assessment of the effects of delayed discharge from the intensive care unit of survivors of critical illness

P Hopkins, J Shrosbree, M Mekki, C Bell, A Feehan, K Peters

Kings Health Partners, London, UK

Critical Care 2010, 14(Suppl 1):P474 (doi: 10.1186/cc8706)

Introduction One consequence of pressure on inpatient hospital bed numbers is a delay in the discharge of critically ill patients to the ward. Although this delay in discharge has been reported in a number of countries, the clinical and economic consequences of this delay have not been studied [1,2].

Methods We examined data from a mixed London ICU (32 beds) using the medtrack software tool (MASH Ltd) in addition to real-time mapping of ICU bed utilisation and patient flows. We defined delayed discharge as beginning 3 hours after the actual medical/nursing decision to discharge. We described a set of criteria a priori, to assess the effect of delayed 


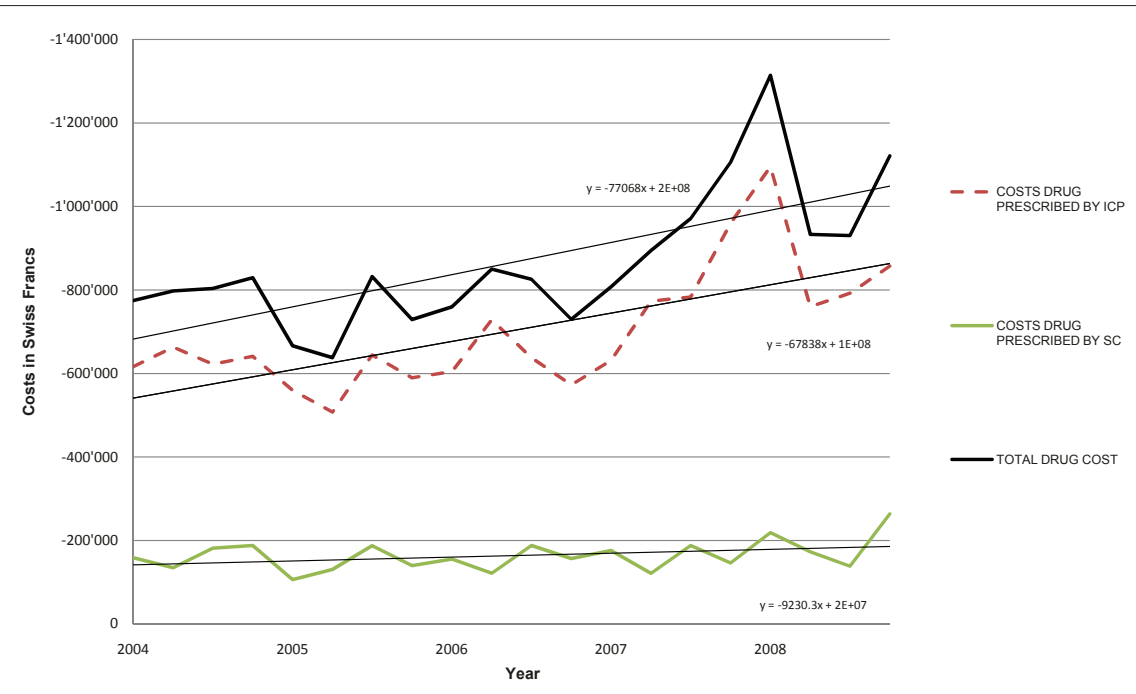

Figure 1 (abstract P473). Costs by type of prescriber.

patient discharge, searching for both beneficial and harmful effects. We also analysed the economic consequences of discharge delay using the medtrack capture of the critical care minimum dataset and available tariffs. Results In the 6-month analysis period, there were 15,320 hours of delayed discharge. This resulted in the postponement/delay of 45 operations for patients requiring level 3 intensive care and 177 operations for patients requiring level 2 intensive care. One hundred and fourteen tertiary referrals were refused because of bed blockage. Seventy-two patients were discharged between the hours of 22:00 and 06:00. There was a mean delay of 5.3 hours between identifying a patient requiring unplanned admission to critical care and their actual admission due to delayed discharge. Only 21 patients acquired a hospital-acquired organism while awaiting discharge (seven infections). Family/patient interviews suggested that the negative effects of noise and exposure to the resuscitation and/or death of ICU patients were offset by a perceived benefit of receiving critical care nursing for a prolonged period. This potential benefit was reflected by a $72 \%$ reduction in the readmission of critically ill patients to critical care. The estimated cost of delayed discharge was $£ 342,000$.

Conclusions Delayed discharge of patients to the ward following an episode of critical illness is a common and increasing problem. This delay is not benign and should be considered when prioritising bed utilisation in acute hospitals.

\section{References}

1. Williams T: Aust Health Rev 2004, 28:87-96.

2. Levin PD: J Crit Care 2003, 18:206-211.

\section{P475}

Robotics telepresence in acute care facilities during influenza

AH1N1 outbreak in Mexico

GV Vazquez de Anda', A Salinas Arnaut², R Camacho Beiza², O Macedo², N Estrada ${ }^{2}$, L Romero'

'Universidad Autonoma del Estado de Mexico, Toluca, Mexico; ${ }^{2}$ nstituto de Salud del Estado de Mexico, Toluca, Mexico

Critical Care 2010, 14(Suppl 1):P475 (doi: 10.1186/cc8707)

Introduction The main objective of this study is to show the experience of using telepresence with robots in acute care facilities (ACFs) at community hospitals (CHs) during an influenza AH1N1 outbreak in Mexico.

Methods Three CHs with 60 beds each were equipped with a telepresence system that includes: high-speed connectivity, wireless Internet access, a RP-7i robot (InTouch Health, Santa Barbara, CA, USA) and a central computer based at a high-care hospital. The $\mathrm{CH}$ s give medical care to people without social security and are far away from the capital city at not less than $63 \mathrm{~km}$ (Atlacomulco $63 \mathrm{~km}$, Valle de Bravo $85 \mathrm{~km}$ and Tejupilco $90 \mathrm{~km}$ ). The program was designated to assist physicians in ACFs, which include emergency rooms (ERs) and ICUs during the second wave of an influenza AH1N1 outbreak in Mexico. The program includes: rounds at the ACF and isolation area for patients with influenza-like syndrome (ILS) every day from 9 am to $15 \mathrm{pm}$ from Monday to Friday given by the $\mathrm{MPH}$ specialist team (intensivist), mechanical ventilation supervision (MV), transfer from CHs to high-care hospitals, length of stay (LOS) and mortality. Additionally, interventions in critically ill patients were recorded.

Results From 3 October to 30 November, 56 patients with ILS were assisted by the MPH team: three patients in Atlacomulco CH, six in Valle de Bravo $\mathrm{CH}$ and 47 in Tejupilco $\mathrm{CH}$. The mean age was 45.3 years old (from 19 years to 84 years), 32 were female (57\%). A confirmatory test for AH1N1 was documented in 10 patients (17.8\%). Patients assisted in the ER, three; in the ICU, nine; in the isolation area for influenza, 44 patients. The mean LOS for all patients was 3.6 days, 3.4 days for patients in the isolation area, 6.5 days in the ICU and 1 day in the ER. There were five patients on mechanical ventilation (8.9\%) and two patients were pregnant (3.5\%). Mortality was observed in five patients (8.9\%) and the mortality rate in the ICU was 50\%. Additionally, two patients were transferred to a high-care hospital (one died). All patients who went on ventilatory support died during the first day on MV.

Conclusions This preliminary report shows that the practice of telepresence using robots at acute care facilities during an influenza outbreak is feasible at $\mathrm{CH}$ s in Mexico.

P476

Association between out of hours discharge from the ICU and subsequent readmission

S Gopal, L Terry, C Corbett

Royal Wolverhampton Hospitals NHS Trust, Wolverhampton, UK

Critical Care 2010, 14(Suppl 1):P476 (doi: 10.1186/cc8708)

Introduction Readmission to the ICU may be considered a benchmark quality indicator on an ICU. Previous studies have assessed the impact of out of hours discharge from the ICU and subsequent outcome $[1,2]$. We hypothesized that patients who were discharged from the ICU after hours were at higher risk of readmission to the ICU than patients discharged during daylight hours.

Methods The database of a 15-bed medico-surgical ICU of a teaching hospital was retrospectively interrogated from the period 1 October 2007 to 31 July 2009. Patients who died whilst on the ICU were excluded from the study. Demographic details were collected as well as the date and time of discharge from the ICU. Readmissions to the ICU were defined as readmissions if they occurred during the same hospital admission episode. Out of hours discharges were defined as discharges occurring between the hours of 20:00 and 09:00. Logistic regression analysis was 
used to assess the relationship between timing of discharge from the ICU and risk of readmission.

Results The database contained 1,257 patients during the study period, of whom 207 died whilst on the ICU. A total of 1,050 patients were therefore included in the study. Two hundred and forty-nine patients (24\%) were discharged between 20:00 and 09:00. Ninety-five patients (9\%) were discharged and subsequently readmitted to the ICU during the same hospital admission episode. Compared with patients discharged between the hours of 09:00 and 20:00, patients discharged between the hours of 20:00 and 09:00 were 2.74 ( $95 \% \mathrm{Cl} 1.7$ to 4.3 ) times more likely to be readmitted to the ICU during the same admission episode, $P<0.001$. This relationship remained true even after adjusting for potential confounders age, gender. OR 2.75 (95\% Cl 1.7 to 4.3).

Conclusions Compared with patients discharged during the day, patients discharged during the night are at a significantly increased risk of readmission to the ICU. Timing of discharge from ICU may therefore be used as a quality indicator in benchmarking quality of care on an ICU.

\section{References}

1. Priestap FA, Martin CM: Impact of ICU discharge time on patient outcome. Crit Care Med 2006, 34:2946-2951

2. Hahane T, Keegan MT, Seferian EG, Gajic O, Afessa B: The association between nighttime transfer from the intensive care unit and patient outcome. Crit Care Med 2008, 36:2232-2237.

P477

Sequential Organ Failure Assessment in pandemic planning

R Shahpori, T Stelfox, C Doig, P Boiteau, D Zygun

Alberta Health Services, Calgary, Canada

Critical Care 2010, 14(Suppl 1):P477 (doi: 10.1186/cc8709)

Introduction The H1N1 pandemic has highlighted the importance of reliable and valid triage instruments for scarce resources during periods of high demand. Christian and colleagues have proposed a triage protocol that utilizes a Sequential Organ Failure Assessment (SOFA) score >11 to exclude patients from critical care resources quoting an associated mortality of more than $90 \%$ [1]. We sought to assess the hospital mortality associated with this SOFA threshold and the resource implications of such a triage protocol.

Methods This retrospective cohort study included consecutive ICU patients admitted to any one of our three tertiary-care adult multisystem ICUs from January 2003 to December 2008. Patients were excluded if they were admitted for routine postoperative monitoring (ICU stay < 48 hours) or postoperative cardiac surgery. SOFA was collected daily by an electronic bedside clinical information system (QS; GE Medical Systems).

Results A total of 10,204 patients (69,913 patient-days) were included. Mean age was 59. Mean admission APACHE was 19.1. Mortality was $25 \%$. Median ICU LOS was 4 days. A total $13.4 \%$ of the cohort (representing $9 \%$ of total patient-days) had an initial SOFA $>11$. Mortality in patients with an initial SOFA score $>11$ was 59\% (95\% Cl 56\%, 62\%). Figure 1 demonstrates increased mortality associated with SOFA $>11$ during the ICU stay to a maximum of $78 \%(95 \% \mathrm{Cl} 68 \%, 86 \%)$ on day 14 . The mortality associated with an initial SOFA $>11$ across diagnostic categories (ICNARC) varied from $29 \%$ for poisoning to $67 \%$ for neurological patients. Mortality associated with an initial SOFA >11 was lowest for those patients 18 to 20 years old (37\%) and highest for those $>80$ years old (75\%). Mortality exceeded $90 \%$ when the initial SOFA was $>20$. However, only $0.2 \%$ of patients had an initial SOFA $>20$.

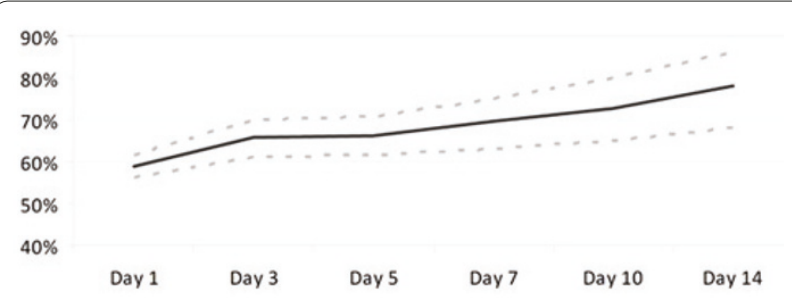

Figure 1 (abstract P477). Hospital mortality associated with SOFA >11 during the ICU stay.
Conclusions A SOFA score > 11 was not associated with a hospital mortality $>90 \%$ at any time during the ICU stay. Age and diagnostic category represent potential modifying factors in the association of SOFA $>11$ and hospital mortality. Only a small proportion of patients have the extreme initial SOFA values associated with a hospital mortality $>90 \%$, limiting the usefulness of SOFA as a triage instrument for pandemic planning.

\section{Reference}

1. Christian et al: CMAJ 2006, 175:1377-1381.

P478

Failure mode and effect analysis in interhospital transport of critically ill patients: a tool to design checklists for handovers in the mobile intensive care unit

EVan Lieshout, A Hamming, D Dongelmans, S Smorenburg

Academic Medical Center, Amsterdam, the Netherlands

Critical Care 2010, 14(Suppl 1):P478 (doi: 10.1186/cc8710)

Introduction The complex interhospital transport of critically ill patients bears multiple risks for patient safety, including handovers and reliance on mobile medical equipment. The hypothesis of the present study was that multiple handovers of both equipment and patients in critical care transport would demonstrate omissions and a failure mode and effect analysis (FMEA) would unravel its safety priorities.

Methods FMEA of the process of interhospital ground critical care transport in the Amsterdam region was performed by three clinical experts to estimate failures in handovers of patients and equipment. Deviations of the optimal process were documented by observations of 16 transports. A questionnaire was used to evaluate the multiple handovers of these transports by the escorting critical care registered nurses and intensivists and included attitude evaluation towards future structured checklists.

Results The FMEA revealed that the highest risk priority numbers (above 45) were all related to the medical equipment control before start of transport. During transport observations the most frequent deviations in the process were related to omission of the defibrillator equipment check before transport (in nine out of 16 transports, 56\%), handover of medication dose (25 of 39 syringe pumps, 64\%) and location of infusion lines (39 of 54 lines, $72 \%$ ). Nurses and intensivists demonstrated a positive attitude towards implementing handover checklists to improve the quality of the transport (29 of 34 respondents, $85 \%$ ).

Conclusions FMEA seems an applicable tool for risk assessment in critical care. The safety of interhospital critical care transport seems to be suboptimal by lack of standardized handovers. The check of medical equipment should have the highest priority in the optimization of safety in critical care transport. Handovers of information in critical care transport could possibly benefit from checklists.

\section{P479}

Selective neurogenic blockades and perioperative immune reactivity in patients undergoing lung resection

EViviano', M Renius', J Rückert'1, A Bloch'1', C Meisel', A Harbeck-Seu', W Boemke', M Hensel' ${ }^{2}$, K Wernecke ${ }^{1,3}$, C Spies $^{1}$

'Charité-University Medicine Berlin, Germany; ${ }^{2}$ Parkklinik Weissensee, Berlin Germany; ${ }^{3}$ Sostana GmbH, Berlin, Germany

Critical Care 2010, 14(Suppl 1):P479 (doi: 10.1186/cc8711)

Introduction Pain is the most relevant factor for prolonged hospital stay after thoracic surgery and is associated with stress known to alter the Th1/Th2 ratio ( $T h=T$ helper cells) in the immediate postoperative period. Thoracic epidural block (TEB), central $a_{2}$-receptor stimulation via intravenous clonidine application and stimulation of opioid receptors can decrease either pain and/or stress and might therefore influence this immune imbalance. The primary endpoint of the current study was the perioperative Th1/Th2 balance in lung surgery. The secondary endpoints aimed to the incidence of pain and pneumonia.

Methods After approval by the ethics committee and informed consent a total of 60 patients was randomized to receive double-blinded either remifentanil intravenously, or remifentanil + clonidine intravenously, or ropivacaine epidurally. Pain intensity was assessed by the numeric rating scale (NRS). The Th1/Th2 ratio was measured using a cytometric bead 
array. Pneumonia was diagnosed according to the hospital-acquired pneumonia criteria of the American Thoracic Society.

Results The Th1/Th2 ratio adjusted for baseline differed between groups over time $(P=0.012)$. At the end of surgery there was no significant difference between the remifentanil and the remifentanil + clonidine groups $(P=0.679)$ but a significantly lower ratio in the ropivacaine group compared with the remifentanil $(P=0.004)$ and the remifentanil + clonidine groups $(P=0.019)$. NRS scores immediately after surgery were lower in the ropivacaine group compared with the remifentanil group and the remifentanil + clonidine group but achieved only borderline statistical significance. None of the patients developed pneumonia.

Conclusions Intraoperative TEB decreases the Th1/Th2 ratio and provides better pain therapy immediately after surgery.

\section{P480}

Adverse effects of beta adrenergic blockers during spinal anesthesia with $0.5 \%$ bupivacaine

JVukelić, M Rakarić Poznanović

University Hospital of Traumatology Zagreb, Croatia

Critical Care 2010, 14(Suppl 1):P480 (doi: 10.1186/cc8712)

Introduction Protocol for perioperative usage of beta blockers was used in only $9 \%$ of major clinics in the USA. It is a very common practice to discontinue beta blockers during the perioperative period or to maximally reduce their doses. The main purpose of this paper is to investigate the incidence of adverse effects of beta blockers during large-scale trauma and orthopedic surgeries in spinal anesthesia.

Methods In this open prospective study we evaluated the adverse effects of beta blockers in ASA II and III research subjects who received beta blockers 1 to 12 hours preoperatively, $n=37$, mean age $67 \pm 12$, with adjacent metabolic syndrome. Results were compared with the same number of patients and ASA group, mean age $57 \pm 18$, who have not received beta blockers. Patients from both groups received midazolam premedication 30 minutes before arriving in the operating room and spinal anesthesia with $0.5 \%$ bupivacaine. Patient surveillance was conducted by continued EKG, blood pressure, pulse (all non-invasive) and $\mathrm{SpO}_{2}$ monitoring. Systolic and diastolic pressure and pulse were recorded every 10 minutes. Results were statistically tested $(M \pm S D, t$ test).

Results The results are shown in Figure 1. The group receiving beta blockers shows a higher percentage of patients with hypertension on arrival in the operating room for $11 \%$ and hypotension after the start of spinal anesthesia. Occurrence of bradycardia ( $H R<60 /$ minute) was increased with statistical significance for $24 \%(P<0.05)$, with use of atropine for $27 \%$ $(P<0.05)$. The occurrence of arrhythmias shows a statistical increase in the same group, for $27 \%(P<0.05)$, as well as nausea for $29 \%(P<0.05)$.

Conclusions Due to their adverse effects, beta blockers should be discontinued before spinal anesthesia and surgical procedures with significant circulating volume loss.

\section{References}

1. Mangano DT, et al: N Engl J Med 1996, 335:1713-1720

2. Priebe JH: Yearbook of Intensive Care and Emergency Medicine; 2008:101-110.

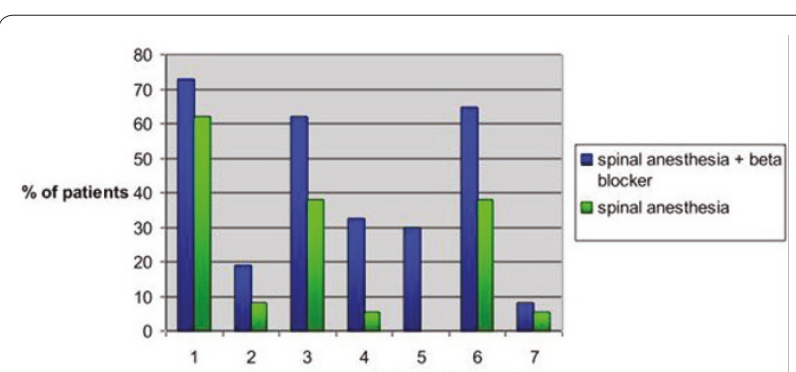

Figure 1 (abstract P480). Adverse beta blocker effects. 1. Preoperative hypertension. 2. Intraoperative hypotension. 3. Intraoperative bradycardia. 4. Arrhythmia. 5. Nausea. 6. Atropine applied. 7. Ephedrine applied.
P481

Remifentanil for postoperative analgo-sedation: effects on glycaemia and HOMA score

C Chelazzi', G Consales², L Margiacchi', AR De Gaudio

University of Florence, Italy; ${ }^{2}$ Az USL 4, Prato, Italy

Critical Care 2010, 14(Suppl 1):P481 (doi: 10.1186/cc8713)

Introduction Surgical-related neuroendocrine stress response extends over the postoperative period, inducing insulin resistance and hyperglycaemia [1]. Remifentanil continuous infusion, reducing pain and anxiety, may reduce postoperative insulin resistance and result in better glycaemic control. The aim of the study was to assess trends in glycaemia and HOMA scores in postoperative patients during remifentanil-driven, postoperative analgo-sedation.

Methods Enrolled patients were those consecutively admitted to a surgical ICU after major abdominal interventions during a 1-month period (July 2009). Group R patients underwent analgo-sedation with continuous remifentanil infusion at the recommended doses [2]. Glycaemia and HOMA scores were evaluated at admission time (TO) and after 12 and 24 hours. Control patients were those who underwent morphine-based continuous analgo-sedation (M Group). Differences in glycaemias and HOMA scores across the two groups were tested with a Student $t$ test $(P<0.05)$.

Results The total number of enrolled patients was 100. No differences were found across the groups regarding general and clinical data, particularly age, prevalence of diabetes, and VAS and Ramsay scores during analgosedation. No differences were found in glycaemia at T0 and T12, while at T24 the R group patients showed lower glycaemia than controls ( $99.4 \pm$ 24.7 vs $104.2 \pm 17.4 \mathrm{mg} / \mathrm{dl}$, respectively; $P=0.018$ ). No differences either could be found in HOMA scores at T0 and T12, while at T24 the R group patients had lower scores than control patients (1.962 \pm 0.19 vs $2.414 \pm$ 0.81 , respectively; $P=0.019$ ).

Conclusions Remifentanil-based analgo-sedation was associated with lower glycaemia and HOMA score in patients following major abdominal surgery, particularly at 24 hours from ICU admission. HOMA scores showed that morphine-treated patients were insulin resistant, although normoglycaemic. A better control of the neuroendocrine stress response in patients analgo-sedated with remifentanil might explain these results.

References

1. Langouche $L$ : The dynamic neuroendocrine response to critical illness. Endocrinol Metab 2006, 35:777-791.

2. Willhelm W: The place for short-acting opioids: special emphasis on remifentanil. Crit Care 2008, 12:S5.

P482

Adding ultralow-dose oral naltrexone to morphine: a double-blind, randomized clinical trial

S Farahmand ${ }^{1}$, O Ahmadi $^{2}$

'Tehran University of Medical Sciences, Tehran, Iran; ${ }^{2}$ Esfahan University of

Medical Sciences, Esfahan, Iran

Critical Care 2010, 14(Suppl 1):P482 (doi: 10.1186/cc8714)

Introduction Ultralow-dose opioid antagonists can enhance opioid analgesia and prevent tolerance in rodent nociceptive pain assays.

Methods A randomized, double-blind controlled trial is designed to investigate whether the addition of $5 \mathrm{ml}$ ultralow-dose naltrexone $(1 \mu \mathrm{g}$ in a liter of sterile water) to morphine $(0.05 \mathrm{mg} / \mathrm{kg})$ changes the total opioid requirement and side effects.

Results Two hundred and sixty-seven patients (18 to 45 years old) with moderate extremities trauma entered the study, Pain control measurements were evaluated every 15 minutes for the first hour, then every 30 minutes for the second and third hours and finally at the fourth hour. Efficacy was measured by the 11-point numerical pain rating scale. The following side effects were evaluated: sedation, nausea, vomiting and pruritus. We found that opioid requirements did not differ significantly between groups. The morphine + naltrexone group on average required $0.04 \mathrm{mg}$ more morphine during the 4 hours than the morphine group. Conclusions The morphine + naltrexone group had a lower incidence of nausea than the morphine + placebo group. However, the incidence of vomiting, pruritus and sedation between the two groups were similar. The combination of ultralow-dose naltrexone and morphine in moderate 
extremities trauma did not affect opioid requirements, but it decreased the incidence of nausea.

\section{References}

1. Crain SM, Shen KF: Antagonists of excitatory opioid receptor functions enhance morphine's analgesic potency and attenuate opioid tolerance/ dependence liability. Pain 2000, 84:121-131.

2. Cepeda MS, Africano JM, Manrique AM, Fragoso W, Carr DB: The combination of low dose of naloxone and morphine in PCA does not decrease opioid requirements in the postoperative period. Pain 2002, 96:73-79.

\section{P483}

Four-day sedation using sevoflurane: fluoride and renal function KWagner, A Becker, T Meier, H Kehnscherper, HM Benad Klinikum Suedstadt Rostock, Germany

Critical Care 2010, 14(Suppl 1):P483 (doi: 10.1186/cc8715)

Introduction Volatile anesthetics for sedation in the ICU are currently undergoing a revival, mainly due to the recent availability of a novel delivery system, the anesthetic conserving device (ACD). Cytochrome P450 metabolic degradation of isoflurane and sevoflurane leads to the generation of inorganic fluoride and hexafluoro isopropanol. Historically, data derived from methoxyfluorane defined a fluoride nephrotoxicity threshold of $50 \mu \mathrm{mol} / \mathrm{l}$. Therefore, we investigated the feasibility and safety, focusing on kidney function, of 4-day sevoflurane-ACD sedation.

Methods Approval of the State Ethics Committee was obtained. Visceral surgical patients either received sedation via sevoflurane-ACD $(n=20)$ or were intravenously sedated (sufentanil, propofol, $n=20$ ). Serum fluoride, renal, liver, pulmonary, cardiovascular function and depth of sedation were measured.

Results Serum fluoride concentrations increased significantly during sevoflurane-ACD sedation (Figure 1). Renal function parameters and urinary output did not indicate deterioration of kidney function. Catecholamine support and pulmonary function were not different between the groups at equivalent depths of sedation.

Conclusions Inhalational sevoflurane sedation subsequently leads to a transient, marked increase of serum fluoride concentrations. The methoxyflurane fluoride nephrotoxicity threshold was largely surpassed without apparent deleterious effect on renal volume regulation and detoxification. The sedational use of the volatile anesthetic sevoflurane up to 4 days is safe and feasible.

\section{Reference}

1. Roehm et al:: Anesth Analg 2009, 108:1848-5184.

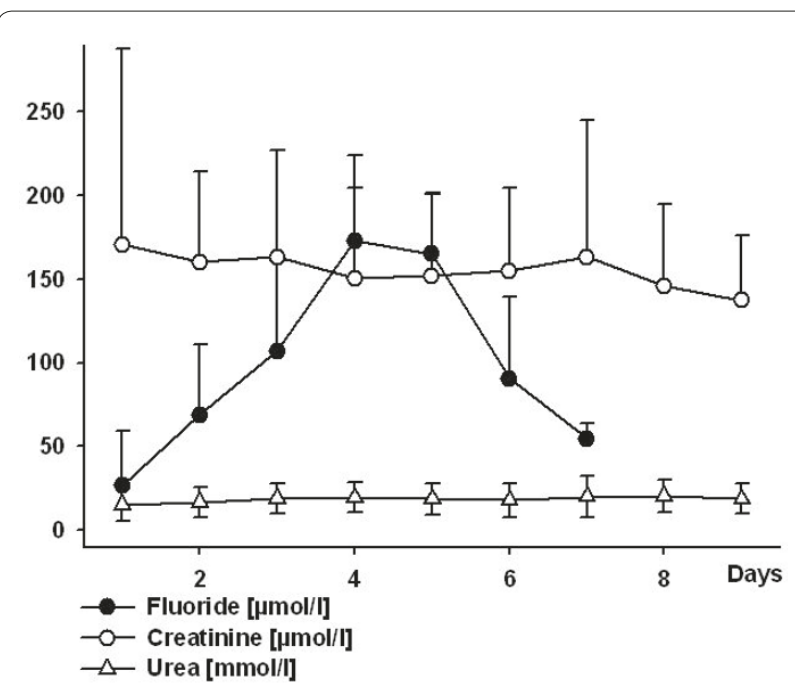

Figure 1 (abstract P483)
P484

Beneficial effect of sevoflurane on endotoxin-induced pulmonary hypertension

B Fyntanidou', V Grosomanidis', K Kotzampassi², EThomaidou', G Mitos', Z Aidoni' ${ }^{1}$ C Skourtis ${ }^{1}$

'Department of Anaesthesiology - ICU, and 'Department of Surgery,

University of Thessaloniki Medical School, Thessaloniki, Greece

Critical Care 2010, 14(Suppl 1):P484 (doi: 10.1186/cc8716)

Introduction Pulmonary hypertension $(\mathrm{PH})$ is a life-threatening disease commonly seen in ICU septic patients and associated with poor outcome. New and better therapies are required, since the response to various agents such as NO, prostaglandins and phosphodiesterase inhibitors is usually partial and the mortality rate remains high. Inhaled drugs seem to be an attractive treatment option, since they are delivered directly to pulmonary resistance vessels. A new device (anesthetic conserving device -AnaConDa (ACD)) permitting direct administration of volatile anaesthetics - such as sevoflurane - to the breathing circuit of a conventional ICU ventilator in a safe and effective way has recently been introduced. The aim of the present study was to evaluate the efficacy and safety of sevoflurane administration via the ACD on a porcine model of acute $\mathrm{PH}$ during sepsis.

Methods PH was induced in 16 anaesthetized, mechanically ventilated swine $(25 \mathrm{~kg}$ ) by intravenous infusion of $0.5 \mathrm{mg} / \mathrm{kg}$ LPS (Escherichia coli, $111: B 4)$ in a period of 30 minutes. After LPS, animals were randomized into two groups. In group A sevoflurane was infused via the ACD, according to the manufacturer's recommendations in order to obtain an end-tidal concentration of $0.5 \%$, whereas group $B$ did not receive sevoflurane and served as control. Haemodynamic parameters (systemic and pulmonary) were recorded before (phase 0 ) and after the LPS (phase 1) and every 20 minutes for the next 2 hours (phases 2 to 7 ).

Results LPS was found to produce PH (phase 1) and to reduce arterial blood pressure in both groups. After sevoflurane infusion, both systolic (PAPs) and diastolic (PAPd) pulmonary pressures exhibited step-by-step reduction, which became statistically significant in phase 3 and thereafter, in relation to phase 1 values. On the contrary, in group B animals, not sevoflurane-treated, pulmonary pressures remained at high levels throughout the study period. Systemic arterial pressure exhibited an endotoxin-related reduction in both groups, which was not found to be affected by sevoflurane treatment.

Conclusions Sevoflurane administration via the ACD was found to reduce the pulmonary pressure in a porcine model of endotoxin-induced acute $\mathrm{PH}$ without any detrimental effects on the systemic circulation. This may represent a significant advance in the treatment of acute $\mathrm{PH}$. However, the potential clinical implications of the method merit further study.

P485

Does etomidate affect prognosis in septic shock patients treated with hydrocortisone?

B Jung, N Clavieras, G Chanques, M Cisse, S Jaber

Montpellier University Hospital, Montpellier, France

Critical Care 2010, 14(Suppl 1):P485 (doi: 10.1186/cc8717)

Introduction Etomidate is well tolerated and not inferior to ketamine but because it is implicated in critical illness-related corticosteroid insufficiency $(\mathrm{CIRCI})[1]$, its utilization in septic shock patients is controversial. In our ICU, the standard of care is to treat every septic shock patient with $200 \mathrm{mg}$ hydrocortisone for at least 5 days. The aim of our study was to compare prognosis of septic shock patients intubated with etomidate with patients intubated without etomidate.

Methods Observational study during a 2-year period including every septic shock patient. We compared the patients' prognosis when intubated with etomidate (E group) to patients intubated without (NE group). We recorded demographic characteristics, results of a cosyntropin test, dose and length of vasopressors and hydrocortisone and outcome. The main endpoint was mortality in the ICU. The secondary endpoints were the length of shock, the dose of vasopressors and hydrocortisone in the ICU and the plasma cortisol level.

Results We evaluated 62 patients in the E group and 31 in the NE group. Although SAPS II on admission was higher in the E group (54 (42 to 64) vs 
$\left.45(32 \text { to } 54)^{*}\right)$, the total dose ( $95 \mathrm{mg}$ ( 37 to 195) vs $66 \mathrm{mg}(31$ to 112$)$ ) and total duration ( 54 hours ( 36 to 91 ) vs 52 hours ( 31 to 81 )) of vasopressors from day 0 to day 5 was not different between groups. However, the cosyntropin responder rate was higher in the non-E group (40\% vs $18 \% *$ ), hydrocortisone treatment duration (168 hours (78 to 216) vs 96 hours (72 to 144$\left.)^{*}\right)$ and total dose of hydrocortisone $(1,200 \mathrm{mg}(500$ to 1,625$)$ vs $\left.750 \mathrm{mg}(350 \text { to } 1,125)^{*}\right)$ was higher in the E group than in the non-E group. Finally, mortality in the ICU was not different between groups (32\% vs $27 \%$ in the $E$ and the non-E groups, respectively). ${ }^{*} P<0.05$.

Conclusions In this study on septic shock treated with a short course of hydrocortisone, patients intubated with etomidate presented a CIRCI more frequently than patients intubated with another drug and needed a longer and higher dose of hydrocortisone treatment. However, length of shock, dose and duration of vasopressors and mortality were not different between groups. Etomidate-induced CIRCI when treated with hydrocortisone was not associated with a poorer outcome in our study. A comparison between etomidate and ketamine should be performed in this population.

Reference

1. Jabre et al:: Lancet 2009, 374:293-300.

P486

Use of methyl-naltrexone for the treatment of opioid-induced constipation in critical care patients

SB Sawh'1 , A Danga ${ }^{1}$, IP Selveraj ${ }^{2}$, A Cotton ${ }^{1}$, PB Patel $^{1}$

IImperial Healthcare NHS Trust, London, UK: ${ }^{2}$ Hillingdon Hospital NHS Trust,

Hillingdon, UK

Critical Care 2010, 14(Suppl 1):P486 (doi: 10.1186/cc8718)

Introduction Methyl-naltrexone (Relistor ${ }^{\oplus}$ ), a peripheral $\mu$-opioid receptor antagonist shown to induce laxation in patients receiving opioids, is licenced for use in palliative care; a similar benefit is proposed in critical care patients. Impaired gut motility and constipation are common issues in the critical care setting with contributing factors including trauma, surgery and use of opiate analgesics. The effects of methyl-naltrexone were studied on seven patients in whom conventional treatment for constipation with stimulant and osmotic laxatives, faecal softeners and suppositories failed to produce effects.

Methods Over a 6-week period, 15 critically ill patients with opiateinduced constipation $(\mathrm{OIC})$ of more than 4 days duration were prospectively studied. All patients were treated with conventional methods from admission to critical care, which included regular senna and sodium docusate, supplemented with glycerin suppositories and picolax for resistant constipation. Methyl-naltrexone was administered to seven patients at $0.15 \mu \mathrm{g} / \mathrm{kg}$ subcutaneously on alternate days until laxation occurred. The remainder of patients in the study continued with conventional therapy.

Results Six out of seven patients responded to methyl-naltrexone with laxation occurring between 12 and 24 hours. One patient did not respond due to faecal impaction but subsequently experienced laxation after manual disimpaction. Side effects were few (nausea 14\%, vomiting 14\%) and there was no increase in opiate requirement. Those patients treated by conventional means eventually produced laxation after a further 3 to 5 days.

Conclusions Previous studies have shown the earlier return of gut motility improves patient progress, facilitates earlier respiratory weaning, prevents debilitation and results in reduced length of stay. Methyl-naltrexone is a safe and efficacious drug for the treatment of OIC in critical care patients in whom conventional treatments prove unsuccessful. Side effects are few and opiate requirements remain unchanged. Further investigation to elucidate the cost-effectiveness of methyl-naltrexone use in OIC of the critically ill patient is warranted.

\section{References}

1. Artinian V, et al: Chest 2006, 129:960-967.

2. Mostafa SM, et al.: Br J Anaesth 2003, 91:815-819.

3. Thomas J, et al:: N Engl J Med 2008, 358:2332-2343.
P487

Safety and efficacy of a nurse-driven sedation protocol with daily sedation interruption in the ICU

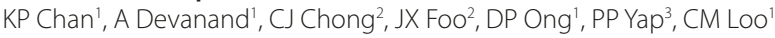

'Singapore General Hospital, Singapore; ${ }^{2}$ National University of Singapore,

Singapore; ${ }^{3}$ Mount Elizabeth Hospital, Singapore

Critical Care 2010, 14(Suppl 1):P487 (doi: 10.1186/cc8719)

Introduction Sedation algorithms have been shown to reduce the duration of mechanical ventilation in the ICU. We evaluated the effect of the implementation of a nurse-driven sedation protocol with daily sedation interruption in our ICU.

Methods We performed a before-and-after prospective study in our eightbed medical ICU over a 6-month period. All patients requiring mechanical ventilation and who were not receiving neuromuscular blocking agents were included. Prior to implementation, sedation was adjusted according to physician orders. During the intervention period, sedatives and analgesics were adjusted by nurses according to an algorithm utilizing the Sedation-Agitation Scale.

Results We included a total of 188 patients, 89 patients in the observational period and 99 patients during the intervention period. The duration of mechanical ventilation was similar (2 days in both groups). However, the ICU length of stay (LOS) was reduced by a day after implementation (3 days vs 2 days). Amongst patients who survived and who were mechanically ventilated for more than 4 days, the duration of mechanical ventilation and ICU LOS were reduced (7 days vs 6 days and 8 days vs 6 days, respectively). Fewer CT brains were performed for persistent coma after discontinuation of sedation in the intervention period (4.5\% vs 1.2\%). Complications such as extubation failure, tracheostomy and inadvertent removal of devices were similar in both groups.

Conclusions A nurse-driven sedation protocol is safe and may reduce duration of mechanical ventilation and ICU LOS, especially amongst patients who have been ventilated for more than 4 days.

\section{P488}

Effects of propofol and midazolam on indocyanine green elimination assessed with LiMON for early sepsis patients M Kargı, D Memis, N Sut

Trakya University, Edirne, Turkey

Critical Care 2010, 14(Suppl 1):P488 (doi: 10.1186/cc8720)

Introduction We aimed to select the sedative drug with the least impact on hepatic blood flow in sedation-administered patients. In our study, we aimed to establish whether or not midazolam and propofol affect liver function during sepsis. The hepatic blood flow is evaluated by the transcutaneous assessment of the indocyanine green plasma disappearance rate (ICG-PDR) in critically ill patients.

Methods Forty sepsis patients were included in the study with either the loading dose infusion of propofol $1 \mathrm{mg} / \mathrm{kg}$ over 15 minutes followed by a maintenance 1 to $3 \mathrm{mg} / \mathrm{kg} /$ hour ( $n=20$, Group P) or a loading dose of midazolam $0.2 \mathrm{mg} / \mathrm{kg}$ over 10 minutes followed by a maintenance 0.04 to $0.06 \mathrm{mg} / \mathrm{kg} / \mathrm{hour}$ ( $n=20$, Group M), 24 hours infusion. ICG elimination tests were conducted concurrently using the non-invasive liver function monitoring system (LiMON). A dose of $0.3 \mathrm{mg} / \mathrm{kg}$ ICG was given through a cubital fossa vein as a bolus and immediately flushed with $10 \mathrm{ml}$ normal saline. We calculated ICG-PDR. The ICG-PDR measurements were obtained at baseline (before start of the propofol or midazolam infusion) and were repeated at 24 hours. Biochemical and hemodynamic parameters, ICGPDR were recorded before start of the study and at 24 hours.

Results Biochemical and hemodynamic parameters did not differ significantly between the groups $(P<0.05)$. Group P compared with Group M; baseline ICG-PDR levels (23.67 \pm 12.02 vs $23.86 \pm 11.4$, respectively), and after the study ICG-PDR levels (26.04 \pm 13.60 vs $24.07 \pm 9.07$ ) did not differ in groups $(P>0.05)$. When we examined before and after ICG-PRD changes between groups, there was no significant difference $(P>0.05)$.

Conclusions In our study, we found that neither propofol nor midazolam infusion affected hepatic blood flow. 
P489

Assessment of correlation between Bispectral Index monitorization and sedation scales used in the intensive care unit

F Yaman, N Ozcan, A Ozcan, C Kaymak, H Basar

Ankara Training and Research Hospital, Ankara, Turkey

Critical Care 2010, 14(Suppl 1):P489 (doi: 10.1186/cc8721)

Introduction The level of sedation should be monitored as the depth of sedation can affect the rate of related complications, length of hospital stay and costs [1]. The recent study aims to monitor the depth of sedation of patients in the ICU using the Ramsay Sedation Scale (RSS), Richmond Agitation Sedation Scale (RASS), Sedation Agitation Scale (SAS) and Adaptation To Intensive Care Environment scale (ATICE) and to assess the correlation between these scales and Bispectral Index (BIS) scores and to compare the correlation coefficients of these scales with each other.

Methods After approval from the hospital ethics committee, 30 mechanically ventilated patients who required sedation in the ICU were enrolled into the study. Patients who are quadriplegic or need muscle paralysis are excluded. The choice of sedative agent and the dose regimen that will be used are left to consultant ICU staff different from the investigators. Demographic data and APACHE II scores of the patients were registered. BIS, RSS, RASS, SAS, ATICE scores, mean arterial pressure, heart rate, and peripheral oxygen saturation values $\left(\mathrm{SpO}_{2}\right)$ of the patients were registered before the initiation of the sedation and in 2 -hour intervals after initiation of the sedation.

Results Male/female ratio was 22/8, mean of ages and body weights of the patients were $57.3 \pm 15.8$ years and $69.6 \pm 14.3 \mathrm{~kg}$, respectively. The median of APACHE II scores was 14 (4 to 23). Mean arterial pressure, heart rate and $\mathrm{SpO}_{2}$ changes were insignificant after initiation of sedation compared with the beginning $(P>0.05)$. BIS, RASS, SAS scores were significantly lower, RSS scores were significantly higher in all intervals compared with the beginning $(P<0.05)$. Significant change was not observed for ATICE scores compared with the beginning value $(P>0.05)$. Statistically significant correlation was observed between all scoring systems and BIS values $(P<0.05)$. Significantly highest correlation was found between BIS-RSS and BIS-RASS ( $r=0.758$ and $r=0.750$, respectively), comparing correlation between BIS-SAS $(r=0.656)$ and BIS-ATICE $(r=0.565)(P<0.05)$. Also, correlation between BIS-SAS was significantly higher than BIS-ATICE correlation $(P<0.05)$.

Conclusions All scales assessed in this study showed significant correlation with BIS. The significantly highest correlation was found between BIS-RSS and BIS-RASS and lowest correlation between BIS-ATICE.

\section{References}

1. Jacobi J, et al:: Crit Care Med 2002, 30:119-141.

P490

Sedation and analgesia: early results from a web-based survey of UK practice

C Mckenzie, S Yassin, M Terblanche

Guy's and St Thomas'NHS Foundation Trust, London, UK

Critical Care 2010, 14(Suppl 1):P490 (doi: 10.1186/cc8722)

Introduction Evidence suggests prolonged sedation is associated with prolonged ventilation and mortality. Further evidence suggests that some sedatives and analgesics are more prone to cause oversedation than others, possibly due to disturbances in pharmacokinetic properties in the context of acute organ failure. Our aim was to describe current sedation and analgesic practice in UK ICUs, and to determine whether recent evidence has changed practice in recent years.

Methods We performed a web-based survey using a tool developed via a systematic, step-wise process following recognised practice for questionnaire design. These steps included question development and questionnaire formatting and testing before being published on the Zoomerang ${ }^{\mathrm{TM}}$ website. The sampling frame was pharmacists who are members of the United Kingdom Clinical Pharmacy Association Critical Care Group (UKCPA CCG). Participants were invited to complete the 15-minute questionnaire. Email reminders were sent to all members every 2 weeks. After 6 months a reminder email was sent to pharmacists in institutions that had not responded. Questions were split into four categories: pharmacist and ICU-specific data; current sedation practice; sedative choice; and analgesic choice.

Results The response rate was $68 \%$. Eighty-four per cent of respondents were senior pharmacists and $54 \%$ provided a service within a team. The median number of annual ICU admissions was 750 (IQR 635 to 865) and included a wide-ranging case mix. Forty-nine per cent of ICUs had a sedation policy, of which $76 \%$ included sedation holds and $87 \%$ used a sedation scoring system. Forty-three per cent assessed for delirium, but only $29 \%$ used a known delirium scoring system. Ninety-nine of the respondents used propofol (PPF) in the majority of patients, 56 midazolam (MDZ). Twenty-seven still used MDZ routinely in renal or hepatic failure, while 75 used MDZ where sedation was challenging.

Fifty-five used morphine, 37 fentanyl and 33 alfentanil as first-line opiate. Conclusions These data suggest that many ICUs do not routinely use sedation policies or assess patients for delirium. While PPF remains the first-line sedative for most ventilated patients, MDZ and morphine are still widely used despite evidence of prolonged effects in the presence of hepatic and renal failure.

\section{References}

1. McKenzie CA, et al.: Crit Care 2005, 9:32-36.

\section{P491}

Severe alcohol withdrawal syndrome in ICU: is propofol a safe option?

Jl Chico1, C Rivero², R Casado³, L Sayagues², E Saborido4, E Sanmartin², D Sander ${ }^{2}$, J Garcia-Allut ${ }^{2}$

${ }^{1}$ Hospital Montecelo, Pontevedra, Spain; ${ }^{2}$ Hospital Clinico, Santiago Compostela, Spain; ${ }^{3}$ Hospital Lozano Blesa, Zaragoza, Spain; ${ }^{4}$ Hospital Montecelo, Pontevedra, Spain

Critical Care 2010, 14(Suppl 1):P491 (doi: 10.1186/cc8723)

Introduction This study aims to compare the efficacy of midazolam or propofol in severe refractory AWS, to detail characteristics of patients and to test the usefulness of different scores (MELD, Child, APACHE II) in severe AWS.

Methods Patients admitted to the ICU during a 3-year period for refractory AWS after a failed treatment with benzodiazepines according to a symptom-triggered protocol in the general ward or emergency room were treated with neuroleptics and either midazolam or propofol infusion. Processes that could lead to a confounding delirium were excluded. We retrospectively analysed this cohort of patients.

Results Fifty patients were reviewed, $85.7 \%$ men. Mean age was 49 years. Thirty percent had been previously admitted to the ICU for the same reason. Main reason for alcohol withdrawal was abdominal pain (12.2\%). Mean alcohol ingestion was $150 \mathrm{~g} /$ day. Mean CHILD/MELD/APACHE score was $6 / 8.5 / 13$ (24.5\% patients had a Child score $\geq 7)$. Seventy-three percent of patients developed complications (especially respiratory infections in 19 patients). The most frequent agent found was MSSA and Gram-negative bacilli. Seizures accounted for $38 \%$ of patients and hepatic encephalopathy in two. We found no relationship between CHILD or MELD score and rate of intubation, length of stay or complications. Patients with APACHE II $>12$ at admission had a significant increase in risk of intubation (OR 31.2, $P<0.001)$. Patients with more than 12 days of stay had a significant risk of intubation (OR 49.7, $P<0.0001)$. Thirty-five patients received propofol as the first intravenous sedation and 14 midazolam. Only two patients with midazolam required a second intravenous sedation. Conversely, nine (26.47\%) patients initially with propofol required a second sedation with midazolam. The propofol group showed a significant increase in incidence of seizures ( $41.17 \%$ vs $21 \%$ in midazolam group) and infections ( $47.1 \%$ vs $28 \%$ ) independent of APACHE score. These findings were more remarkable in the subgroup that needed a second intravenous sedation (66\% seizures and $77 \%$ infection rate). This group had higher APACHE score and was associated with a greater likelihood of intubation and a significant 2-day increase in length of stay.

Conclusions Propofol has been proposed as an alternative to benzodiazepines in severe AWS, but it could increase the rate of complications (especially seizures and respiratory infections) that could lead to an increase in the intubation rate and length of stay. The APACHE score could be a predictor for the risk of complications and intubation. 
P492

Agitation and pain during physiotherapy in intensive care

K Everingham', L Salisbury², P Lapinlampi ${ }^{3}$, I Cornwall', F Frame ${ }^{4}$, T Walsh' 'NHS Lothian, Edinburgh, UK; ${ }^{2}$ University of Edinburgh, UK; ${ }^{3}$ GE Healthcare, Helsinki, Finland; ${ }^{4}$ University of Leicester, UK

Critical Care 2010, 14(Suppl 1):P492 (doi: 10.1186/cc8724)

Introduction The objective of this study was to identify the proportion of patients who experienced agitation and/or pain during physiotherapy sessions in intensive care (ICU). The prevalence and causes of distress in critically ill patients are poorly described. Identified stressors include physiotherapy $[1,2]$, but no standards for sedation/analgesia management during physiotherapy exist. Recent evidence supporting routine ICU management at lighter levels of sedation potentially increases the importance of interventions to avoid distress during interventions such as physiotherapy.

Methods A prospective observational study was undertaken. Fortynine patients admitted to the ICU requiring mechanical ventilation and physiotherapy were recruited into the study. A single session of physiotherapy was observed by an independent assessor. Agitation was measured using the Richmond Agitation Sedation Scale (RASS) and pain using the Behavioural Pain Scale (BPS). The RASS and BPS were collected immediately pre physiotherapy, after each intervention during physiotherapy and at 5 and 30 minutes post physiotherapy.

Results Sixteen participants (33\%) experienced agitation (RASS score 21) while 48 participants (98\%) experienced pain (BPS score $\geq 4$ ). Figure 1 shows the number of participants who experienced agitation and pain in relation to their RASS level prior to physiotherapy. Of interest, the independent assessor observed little communication, in regards to the management of sedation or pain at any stage during physiotherapy, between nursing and physiotherapy staff.

\begin{tabular}{|l|c|c|}
\hline \multicolumn{3}{|l|}{} \\
$\begin{array}{l}\text { The RASS level of } \\
\text { participants prior to } \\
\text { physiotherapy (number of } \\
\text { participants) }\end{array}$ & $\begin{array}{l}\text { Number (\%) of participants } \\
\text { who experienced agitation } \\
\text { during physiotherapy }\end{array}$ & $\begin{array}{l}\text { Number (\%) of participants who } \\
\text { experienced pain during } \\
\text { physiotherapy session }\end{array}$ \\
\hline$-5(\mathrm{n}=10)$ & $2(20 \%)$ & $10(100 \%)$ \\
\hline$-4(\mathrm{n}=6)$ & $1(17 \%)$ & $6(100 \%)$ \\
\hline$-3(\mathrm{n}=9)$ & $1(11 \%)$ & $9(100 \%)$ \\
\hline$-2(\mathrm{n}=5)$ & $2(40 \%)$ & $4(80 \%)$ \\
\hline$-1(\mathrm{n}=8)$ & $5(63 \%)$ & $8(100 \%)$ \\
\hline $0(\mathrm{n}=5)$ & $2(40 \%)$ & $5(100 \%)$ \\
\hline $1(\mathrm{n}=6)$ & $3(50 \%)$ & $6(100 \%)$ \\
\hline
\end{tabular}

Figure 1 (abstract P492). Agitation and pain in relation to prephysiotherapy RASS scores.

Conclusions Regardless of the RASS level pre physiotherapy, the majority of participants experienced pain. In contrast, only one-third of participants experienced agitation; this was more prevalent for those at a lighter sedation level. More routine use of RASS, BPS and communication with nursing staff should be undertaken during physiotherapy to ensure optimal levels of sedation and adequate levels of analgesia are achieved.

\section{References}

1. Novaes et al:: Intensive Care Med 1999, 25:1421-1426.

2. van de Leur et al:: Crit Care 2004, 8:R467-R473.

\section{P493}

Pain evaluation in the intensive care unit: listen to the patient, a new approach!

G Chanques', B Jung', M Cissé1, S De Lattre', E Viel'2, S Jaber

${ }^{1}$ Saint Eloi - Montpellier University Hospital, Montpellier, France; ${ }^{2}$ Carémeau -

Nimes University Hospital, Nimes, France

Critical Care 2010, 14(Suppl 1):P493 (doi: 10.1186/cc8725)

Introduction Contrary to wards that manage usually chronic or acute pain (algology, gerontology, surgery, recovery room, and so forth), a comparison of the five most popular self-report pain tools (vertical and horizontal Visual Analog Scale (VAS-V, VAS-H), 0 to 10 oral Numeric Rating
Scale (NRS-O), 0 to 10 visual enlarged NRS (NRS-V), Verbal Descriptor Scale (VDS)) has never been evaluated in an ICU setting.

Methods Consecutive patients admitted to a medical-surgical ICU during 1 year were included when alert (RASS >-2) and able to follow simple commands. Exclusion criteria: previous self-report pain assessment without the presence of an investigator. Pain assessment using the five scales in random order either at baseline (T1) and after (T2) administration of an analgesic, or during a nociceptive procedure, in absence of pain at baseline. Evaluated parameters: psychometric properties of scales (feasibility, validity, responsiveness and preference). Nonparametric tests were used for statistical analysis (Statview 5.0). Data are expressed as median (25th to 75 th).

Results One hundred patients were included 24 (12 to 96) hours after their admission to the ICU: age 58 (48 to 69) years, SAPS II 39 (32 to 48), SOFA 7 (3 to 9), medical admission (49\%), intubation before inclusion (76\%), at time to inclusion (50\%). At T1, $91 \%$ of patients were able to use at least one scale and $57 \%$ all five scales. The NRS-V had the lowest failure rate (9\%) compared with NRS-O (17\%), VDS (22\%), VAS-H (32\%) and VAS-V (34\%). Validity was measured in the 56 patients who were able to use the five scales both at T1 and T2. The pain intensity measured with the five scales changed significantly between T1 and T2 $(P<0.01)$, showing a good discriminative validity. The correlation between the five scales was important (Spearman's coefficients ranged from 0.75 to $0.96, P<0.001$ ). The responsiveness was very good for each of the five scales, either after an analgesia and a nociceptive procedure (effect size $>0.8$ ). Finally, the NRS- $V$ was considered respectively by 45,37 and $44 \%$ of patients as the easiest, most accurate and preferred scale. The preference for the other scales was $<12 \%$.

Conclusions The self-report of pain is feasible in ICU patients as soon as they are sufficiently alert (RASS >-2) and able to follow simple commands. The enlarged 0 to 10 Numeric Rating Scale is the easiest scale to use with the lowest failure rate and it is the preferred scale for the majority of critically ill patients who were able to communicate.

\section{P494}

\section{Pharmacokinetics of orally administered melatonin in critically ill} patients

B Moroni', G Mistraletti', RJ Reiter², R Esposti', E D'Angelo', P Formenti', D Palmisano', E Borotto', P Di Mauro', G lapichino

'Università degli Studi di Milano, Milan, Italy; '2University of Texas Health Science Center, San Antonio, TX, USA

Critical Care 2010, 14(Suppl 1):P494 (doi: 10.1186/cc8726)

Introduction Critically ill patients exhibit reduced melatonin secretion, both in nocturnal peaks and basal daytime levels. Oral melatonin supplementation may be useful for known sedative and antioxidant properties.

Methods Melatonin early enteral absorption and daily pharmacokinetics were determined in two cohorts of six high-risk patients in this prospective trial. During their third and fourth ICU day, they underwent two different sets of repeated blood samples to detect serum melatonin levels through radio-immunoassay. Cohort 1: samples taken at 20:00, 20:45, 21:30, 24:00, $03: 00,06: 00,14: 00,20: 00$ to describe the daily pharmacokinetics. Cohort $2: 20: 00,20: 05,20: 10,20: 20,20: 30,20: 45$ to study the early absorption. On ICU day 3, endogenous levels were measured, while the absorption of exogenous melatonin was determined on ICU day 4 after administration, at 20:00, of 3 mg melatonin.

Results All basal levels were below the expected values. Following enteral administration, pharmacological levels were already reached in 5 minutes, with a serum peak after 16 minutes (half-absorption time: 3 minutes 17 seconds). The maximum serum level observed was $11,040 \mathrm{pg} / \mathrm{ml}$ and the disappearance rate indicated a half-elimination time of 1 hour 34 minutes. Serum melatonin levels decreased significantly after midnight; pharmacological levels were maintained up to 10 hours following administration. No excessive sleepiness was reported in this patient group. Conclusions Critically ill patients exhibited reduced melatonin secretion, as reported in the literature. Despite the critical illness, the oral bioavailability was satisfactory: serum levels after oral administration showed basically unchanged intestinal absorption, while the disappearance rate was slower than reported elsewhere in healthy volunteers. 
P495

Manifestations and clinical course of delirium tremens in the ICU JA Camacho Oviedo, C Soriano Cuesta, J Manzanares Gomez, L Cachafeiro Fuciños, M Oliveros Fonseca, R Garcia Hernandez, M Jimenez Lendinez Hospital Universitario la Paz, Madrid, Spain

Critical Care 2010, 14(Suppl 1):P495 (doi: 10.1186/cc8727)

Introduction The aim of our study is to describe clinical progress, need for mechanical ventilation (MV), complications and mortality of patients with delirium tremens (DT) admitted to our ICU.

Methods Patients with a diagnosis of DT admitted to a medical ICU of a tertiary hospital from January 2001 to December 2008 were included. We recorded: admission diagnosis, pathologies associated with DT, APACHE II score, treatment, need for MV and duration, complications, length of stay in the ICU and total hospital stay, mortality and survival at 2 years.

Results There were 50 cases of DT. Median age 45 years, $96 \%$ male. Reasons for hospital admission: DT (68\%), seizures (36\%), sepsis (16\%), brain injury (10\%). Reasons for admission to the ICU: DT (54\%), DT and seizures (26\%), DT and brain injury (10\%), DT and sepsis (8\%), DT and other (2\%). Median APACHE II score was 9 (range: 8 to 13). Seventy-four percent of the patients were controlled with no need for MV with a midazolam infusion of 45 to $50 \mathrm{\mu g} / \mathrm{kg} / \mathrm{hour}$ and haloperidol of 17 to $30 \mu \mathrm{g} / \mathrm{kg} /$ hour. Twenty-eight per cent (14 patients) required MV, of them $78 \%$ had DT and other associated pathology on admission (Figure 1). From a total of 28 patients admitted with DT and no associated pathology, only four needed MV. The median time on MV was 2 days (range: 1 to 9). Complications were present in $22 \%$ : ventilation-associated pneumonia, and other infectious conditions. Total hospital length of stay 14 days ((SD: 11), 5.5 days in the ICU (SD: 9). The registered hospital mortality was $4 \%$. Rate of survival at 2 years was $84 \%$, from these $72 \%$ had continuous visits to emergency ( $\geq 5$ consultations in 2 years) for episodes related to alcohol withdrawal or complications associated with chronic alcohol abuse.

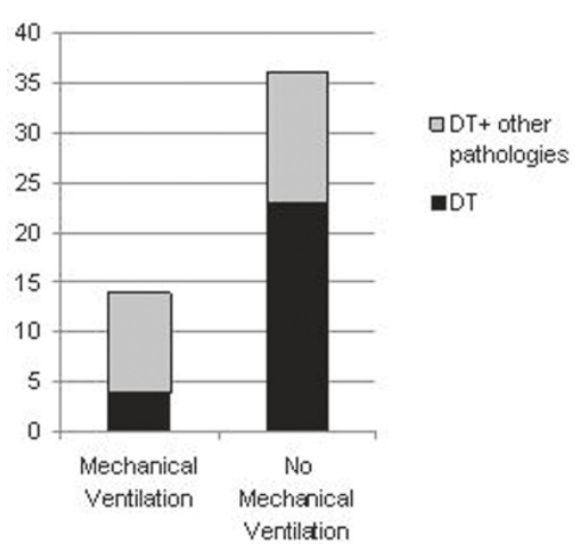

Figure 1 (abstract P495). Use of MV in patients admitted for DT.

Conclusions Even though our patients with DT are frequently admitted to the ICU, this is a pathology that most of the time has a benign course with low hospital mortality and high rate of survival at 2 years. These patients are regular users of the health system. The occurrence of complications and the need for MV in our patients were low, and were present in the group of DT with other diagnoses.

\section{P496}

Comparison of two methods for diagnosing delirium conducted in a mixed medical ICU and the concordance between two independent nurse assessors

S Heuer, J Nicholls, M Terblanche, D Treacher

Guy's and St Thomas' NHS Foundation Trust, London, UK

Critical Care 2010, 14(Suppl 1):P496 (doi: 10.1186/cc8728)

Introduction Delirium in ICU patients increases time on mechanical ventilation, is an independent risk factor for death and can cause cognitive impairment in survivors $[1,2]$. The two most commonly used validated assessment methods for diagnosing delirium in intensive care are the Confusion Assessment Method for ICU (CAM) and the Intensive Care Delirium Checklist Score (ISDCS) [3]. We wished to determine the prevalence of delirium in our unit, whether there was a difference in results between these methods of assessment and whether good agreement could be achieved between two trained assessors.

Methods We performed a prospective prevalence study in a single tertiary ICU. Patients were assessed between days 3 and 10 of their stay and tested provided their Richmond Agitation Sedation Score (RASS) was $\geq-3$ [1]. Each patient was independently assessed by the two trained assessors with the CAM and ISDCS, with randomisation of both the order of interview and the score used. Both sets of assessments were carried out within an hour of each other. Exclusions included readmissions, those who did not speak English, the deaf and registered psychiatric patients. The CAM was completed by the assessors and the ISDCS required observational answers from the bedside nurse.

Results We performed 104 assessments (208 tests) on 52 patients. Mean (SD) age, APACHE II and total SOFA on the day of admission were 67.4 (14.0), 14.0 (5.3) and 5.7 (2.7), respectively. Delirium was found in $37.5 \%$ of patients using the CAM, but only $14.4 \%$ using ISDCS. Inter-rater agreement for CAM and ISDCS was $86.5 \%$ ( $\mathrm{k} 71.4 \%$, SE $13.8 \%, P<0.001$ ) and $94.2 \%$ ( $\mathrm{k} 76.7 \%$, SE $13.8 \%, P<0.0001$ ), respectively. There was significant underscoring of the RASS by the bedside nurses. When the trained assessors found a difference, it was always lower than the score documented by the bedside nurse.

Conclusions There was good inter-rater agreement in the diagnosis of delirium between the two trained assessors, but the prevalence found was lower than previously reported [1] and varied considerably with the method used. The difference in results between the two scores may be due to a lack of discernment on the part of the bedside nurse of the ISDCS assessment process.

\section{References}

1. Ely et al:: Crit Care Med 2001, 29:1370-1379.

2. McNicoll et al:. J Am Geriatr Soc 2003, 51:591-598.

3. Delvin et al: Crit Care 2008, 12:1.

\section{P497}

\section{Quetiapine in prolonged ICU delirium}

MR Kasliwal, C McKenzie, NA Barrett

Guy's and St Thomas'NHS Foundation Trust, London, UK

Critical Care 2010, 14(Suppl 1):P497 (doi: 10.1186/cc8729)

Introduction Delirium affects up to $33 \%$ of acutely hospitalized patients. In ICU patients it can prolong the length of stay and increase mortality [1]. The optimal management of delirium requires a calm environment, sleep hygiene and correction of underlying factors (for example, infection). This can be a challenge in the ICU, and drug therapy, commonly haloperidol, clonidine, benzodiazepines or propofol, is often used [1]. Quetiapine, an atypical antipsychotic, has been used in acute delirium outside the ICU [2]. It has few extrapyramidal side effects, a short half-life and is mildly sedating. Our impression was that it had potential in the treatment of delirium.

Methods In a 30-bed tertiary ICU with more than 1,200 admissions annually we reviewed the notes of patients admitted from February 2008 to November 2009 who had a delirium treated with quetiapine. Patients were excluded if they were taking quetiapine prior to admission. The following data were recorded: Richmond Agitation and Sedation Score

Table 1 (abstract P497)

\begin{tabular}{lccc}
\hline Patient & RASSpre (D) & Q dose $(\mathbf{m g})$ & RASSpost (d) \\
\hline 1 & $+1(22)$ & $12.5 \mathrm{OD}$ & $0(5)$ \\
2 & $+3(21)$ & $50 \mathrm{BD}$ & $0(5)$ \\
3 & $+4(31)$ & $12.5 \mathrm{BD}$ & $0(3)$ \\
4 & $+4(28)$ & $12.5 \mathrm{BD}$ & $0(5)$ \\
5 & $+3(36)$ & $25 \mathrm{BD}$ & $0(6)$ \\
\hline
\end{tabular}

(D), days delirium present; $Q$, quetiapine; (d), days to achieve the RASS. 
(RASS), duration of delirium, agents used, patient demographics and adverse events.

Results Five patients met the inclusion criteria (Table 1). All were males, aged 37 to 85 years. All had a prolonged delirium prior to the commencement of quetiapine and all were on a combination of four drugs (clonidine, haloperidol, lorazepam and propofol) that were not controlling their delirium. At 3 to 7 days following commencement of quetiapine the RASS scores were 0 and other drugs were ceased. No adverse effects were noted.

Conclusions Quetiapine was successful in controlling prolonged ICU delirium and allowed weaning of other medications in these patients. It may be a useful delirium therapy. Further studies are required to demonstrate efficacy and safety.

\section{References}

1. Ely, et al:: JAMA 2004, 291:1753-1762.

2. Schwartz, et al.: Prim Care Companion J Clin Psychiatry 2000, 2:10-12.

\section{P498}

PREDICT, Prediction of Delirium in ICU Patients: development and validation of a prediction model

M Van den Boogaard, P Pickkers, HVan der Hoeven, R Donders,

TVan Achterberg, L Schoonhoven

Radboud University Medical Centre Nijmegen, the Netherlands

Critical Care 2010, 14(Suppl 1):P498 (doi: 10.1186/cc8730)

Introduction Delirium is a serious and frequent disorder in the ICU, but a prediction model for critically ill patients is lacking.

Methods We performed two prospective cohort studies in a 33-bed ICU for adult patients with use of the validated CAM-ICU, performed three times daily by well-trained ICU nurses. Twenty-three identified delirium risk factors were collected within 24 hours after admission and patients were followed during their ICU stay until death, discharge or development of delirium. Variables with $P>0.1$ in univariate logistic regression, or with a prevalence rate $<10 \%$ were excluded. Multivariate logistic regression analysis was followed by bootstrapping. Reduced coefficients were used on the second cohort for external validation. Also, nurses and physicians were asked to predict whether the patient would develop delirium during the ICU stay.

Results In the first study (February 2008 to February 2009) 2,116 consecutive patients and in the second study (May to September 2009) 748 patients were screened, of which 503 and 199 patients, respectively, were excluded for valid reasons. Ninety per cent of the total number of assessments that should have been performed was actually obtained and the Cohen's kappa for inter-rater reliability was 0.90 . Out of 1,613 patients, 411 developed delirium and in the second study 171 out of 549 patients. Eight risk factors were excluded because of a prevalence rate $<10 \%$ and one because of $P>0.1$. After multivariate logistic regression analysis, 10 risk factors were included in the prediction model (age, APACHE II score, coma, diagnose group, increased urea, infection, metabolic acidosis, morphine use, sedation use, emergency admission) of which the area under the receiver-operation characteristic curve (AUC) was 0.87 (95\% Cl 0.85 to 0.89 ) and after bootstrapping 0.86 . The cut-off point with the optimal predicted probability lies at 0.196 points. At this cut-off the sensitivity of the prediction rule is $80 \%$ and the specificity is $79 \%$. Validation of PREDICT on the second cohort resulted in an AUC of 0.89 (95\% Cl 0.86 to 0.92). The AUC of the prediction of nurses and physicians was significantly lower, both 0.59 ( $95 \% \mathrm{Cl} 0.49$ to 0.70 ).

Conclusions We have developed and validated a delirium prediction model (PREDICT) with a high AUC that can be used in all adult ICU patients within 24 hours after ICU admission to predict the chance of delirium during their ICU stay and to guide preventive interventions.

\section{P499}

\section{Hospital anxiety depression scale in our surgical ICU}

IF Filipovic-Grcic, DT Tonkovic, JG Grubisin, MP Peric, VM Majeric Kogler

University Clinical Hospital Center Zagreb, Croatia

Critical Care 2010, 14(Suppl 1):P499 (doi: 10.1186/cc8731)

Introduction All patients in the ICU go through mental, physical and emotional adjustment. They can experience exaggerated symptoms of anxiety and depression and it can delay their recovery [1]. Stress has a major role and there are so many triggers for it: the psychological and physical condition, surgery, pain, ICU environment and so forth. The aim of this study was to implement the Hospital Anxiety Depression Scale (HADS) in our everyday ICU practice and then to analyze the impact of the clinical picture (APACHE II score) and pain control (VAPS - visual analog pain scale) on the anxiety and depression symptoms (HADS) in surgical ICU patients. Methods With ethics approval and written consent, in this prospective study, to date are reported 70 patients. They all underwent major abdominal surgery and were discharged from the ICU after 24 to 30 hours. Patients with any mental disorder or neurological disease were excluded. On admission and discharge the VAPS, APACHE II and HADS were evaluated. HADS was evaluated again 1 month later (62 of 70 patients). Anxiety and depression are assessed as separate components, each with seven items that are rated from 0 to 3 [2]. A score $<7$ in a component is a normal result, 8 to 10 indicates mild symptoms, 11 to 14 moderate symptoms and 15 or more indicates severe symptoms [2]. The Mann-Whitney $\mathrm{U}$ test, $t$ test and correlations were used in statistical analysis.

Results Average age of the patients was $58.61 \pm 13.56$, range 24 to 78,37 male and 33 female. Average values on admission were: VAPS $3.02 \pm 0.50$, APACHE I| $10.31 \pm 2.79$, Anxiety $4.82 \pm 4.01$, Depression $3.91 \pm 3.23$, and on discharge were: VAPS $1.34 \pm 0.88$, APACHE $\| 5.25 \pm 2.78$, Anxiety $3.41 \pm$ 2.97, Depression $3.71 \pm 2.97$. One month later the values were: Anxiety $3.14 \pm 2.79$, Depression $1.27 \pm 0.57$

Patients with lower pain control had more exaggerated symptoms of anxiety and depression. After 1 month, seven patients had mild symptoms of anxiety, nine patients had mild symptoms of depression and four patients had moderate symptoms of depression.

Conclusions HADS has its role in everyday ICU practice. All patients with mild to severe symptoms of anxiety or depression should be further psychologically evaluated. There is a need to evaluate in our ICU the patients who are staying there a few days or more and compare it with the results of this study.

\section{References}

1. Sukantarat et al: Anaesthesia 2007, 62:239-243.

2. Zigmond et al: Acta Psych Scand 1983, 67:361-370.

\section{P500}

Fluid therapy change from synthetic colloids to only crystalloids in patients with severe sepsis

O Bayer, Y Sakr, B Kabisch, CS Hartog, N Riedemann, U Settmacher, KReinhart

Friedrich-Schiller-University Jena, Germany

Critical Care 2010, 14(Suppl 1):P500 (doi: 10.1186/cc8732)

Introduction After changing the standard resuscitation colloid on our ICU from 6\% HES 130/0.4 to 4\% gelatin, we found that both HES 130/0.4 and gelatin resulted in a high incidence of renal failure (43.2\% and $40.2 \%)$ in patients with severe sepsis [1]. We therefore changed from synthetic colloids to only crystalloids and herewith report on the effects.

Methods Before-after retrospective study in patients with severe sepsis on a surgical ICU. The synthetic colloids group (SynColl-Group) comprised patients who received either predominantly $6 \%$ HES 130 or predominantly gelatin between January 2005 and June 2006. The crystalloid group (CrysGroup) comprised patients from September 2008 to June 2009 who received only crystalloids. Acute renal failure (ARF) was defined as new need for renal replacement therapy (RRT) or at least a twofold increase in baseline creatinine.

Results Two hundred and five patients received synthetic colloids, and 141 received only crystalloids. There were minor baseline differences but SAPS II and SOFA scores, age and creatinine were similar at admission. Fluid therapy (median cumulative doses): patients in the SynCollGroup received 4 I (IQR 2 to 8.3 I) colloids and 27.9 I (IQR 15.7 to 48.3 I) crystalloids. Patients in the Crys-Group received only 25.0 I (IQR 13.1 to 52.2 I) crystalloids. Crystalloid volumes did not differ significantly between groups ( $P=0.605$ ). Median cumulative dose of HES was $50 \mathrm{ml} / \mathrm{kg}$ and of gelatin was $43.3 \mathrm{ml} / \mathrm{kg}$. Outcomes: patients in the SynColl-group had a higher incidence of ARF $(41.0 \%$ vs $23.4 \%, P=0.001)$ and increased need for RRT (35.1\% vs $22.0 \%, P=0.009)$. ICU mortality and hospital mortality did not differ between SynColl and Crys groups (31.2\% vs 30.5\% and 38\% 
vs $36.9 \%$, respectively). The ICU length of stay tended to be longer in the SynColl-Group ( 14 versus 10 days, $P=0.055$ ).

Conclusions In patients with severe sepsis, fluid therapy with synthetic colloids - gelatin or the third-generation 6\% HES 130/0.4 -considerably increases the incidence of ARF and need for RRT compared with crystalloids. These results confirm recent meta-analysis and RCTs, which demonstrated an increased incidence of ARF by synthetic colloids $[2,3]$.

\section{References}

1. Schabinski et al: Intensive Care Med 2009, 35:1539-1547.

2. Zarychanski et al:: Open Med 2009, 3:E196-E209.

3. Brunkhorst et al:: N Engl J Med 2008, 358:125-139.

\section{P501}

Effects of balanced and unbalanced colloid and crystalloid solutions on renal microvascular perfusion in endotoxemic rats

U Aksu', C Demirci-Tansel ${ }^{2}$, C Ince $^{1}$

'University of Amsterdam, the Netherlands; 'Istanbul University, Istanbul, Turkey

Critical Care 2010, 14(Suppl 1):P501 (doi: 10.1186/cc8733)

Introduction In this study we investigated the effects on renal microcirculatory perfusion of resuscitation with hydroxyethyl starch (HES 130/0.42) dissolved in a balanced Ringer's acetate salt solution (Plasma Volume Redibag ${ }^{\oplus}$, HES-RA) or hydroxyethyl starch (HES 130/0.4) dissolved in saline (Voluven ${ }^{\oplus}, \mathrm{HES}-\mathrm{NaCl}$ ) and compared this with resuscitation with saline solution $(0.9 \% \mathrm{NaCl}, \mathrm{NaCl})$ in an LPS-induced septic shock model in the rat.

Methods In 20 anesthetized, mechanically ventilated rats, endotoxic shock was induced by infusion of LPS (10 mg/kg over 30 minutes). When the mean arterial pressure (MAP) was decreased to $40 \mathrm{mmHg}$, fluid resuscitation was started in three groups of five rats each, and in one five-rat group no fluids were given. Renal cortical microvascular perfusion (RMP) was assessed at high temporal and spatial resolution using laser speckle imaging. Additional measurements included systemic hemodynamics, renal artery blood flow (RBF), blood gas analysis, creatinine clearance, and plasma ions and calculated renal oxygen delivery $\left(\mathrm{DO}_{2}\right)$. Time points were: baseline, during septic shock at a MAP of $40 \mathrm{mmHg}$, and at 30 minutes of resuscitation.

Results LPS infusion decreased MAP and RMP in all rats. Resuscitation with $\mathrm{NaCl}$ did not improve MAP $(P>0.05)$ and appeared to increase RMP heterogeneity $(0.95 \pm 0.08 \mathrm{AU}$ vs $1.83 \pm 0.13 \mathrm{AU} ; P<0.001)$, metabolic acidosis ( $7.1 \pm 0.06$ vs $6.93 \pm 0.05 \mathrm{pH} ; P<0.05$ and $11.36 \pm 1.1$ vs $15.9 \pm 1.4$ $\mathrm{mmol} / \mathrm{l}$ base excess; $P<0.05)$, and plasma chloride ion levels (110.3 \pm 0.7 vs $116.2 \pm 1.7 \mathrm{mmol} / / ; P<0.05)$. Fluid resuscitation did not affect systemic oxygenation parameters $\left(\mathrm{sO}_{2}, \mathrm{pO}_{2}, \mathrm{O}_{2} \mathrm{Hb}\right)$ and renal $\mathrm{DO}_{2}$. Resuscitation with $\mathrm{NaCl}$ increased plasma levels of $\mathrm{Na}^{2}$, tubular reabsorption of $\mathrm{Na}^{+}$, and creatinine clearance compared with resuscitation with the two colloid solutions. HES-NaCl and HES-RA provided similar effects on systemic and regional hemodynamic parameters. However, while HES-RA improved RBF $(2.3 \pm 0.4$ vs $5.7 \pm 0.2)$, HES-NaCl did not. NaCl resuscitation worsened the metabolic acidosis, which was not affected by the two colloid solutions. The plasma $\mathrm{HCO}_{3}{ }^{-}$level was increased by HES-RA resuscitation (13.18 \pm 2.1 vs $19.56 \pm 1 \mathrm{mmol} / 1 ; P<0.01$ ), whereas it was stable in the other groups.

Conclusions Balanced HES preparation (HES-RA, PlasmaVolume Redibag $^{\circledR}$ ) in endotoxemic rats showed acute beneficial effects on kidney microcirculation and on kidney function via bicarbonate balance whereas the $\mathrm{NaCl}$ treatment was harmful compared with both HES groups.

P502

Effects of balanced and unbalanced colloid and crystalloid solutions on renal oxygenation in a rat model of hemorrhagic shock and resuscitation

E Almac, U Aksu, C Ince

University of Amsterdam, the Netherlands

Critical Care 2010, 14(Suppl 1):P502 (doi: 10.1186/cc8734)

Introduction Hydroxyethyl starch (HES, $130 \mathrm{kD}$ ) dissolved either in saline (Voluven ${ }^{\oplus}$ ) or in a balanced Ringer's acetate solution (PlasmaVolume Redibag $\left.{ }^{\oplus}, \mathrm{PVR}\right)$ and Ringer's acetate (RA) and $0.9 \% \mathrm{NaCl}(\mathrm{NaCl})$ were investigated as regards their efficacy in restoring renal oxygenation in a rat model of hemorrhagic shock and resuscitation.
Methods In 22 anesthetized, mechanically ventilated rats, hemorrhagic shock was induced by withdrawing blood volume from the femoral artery until the mean arterial pressure (MAP $\mathrm{mmHg}$ ) was reduced to $40 \mathrm{mmHg}$. One hour after reaching a MAP of $40 \mathrm{mmHg}$, animals were resuscitated with either Voluven ${ }^{\oplus}(n=5), \operatorname{PVR}(n=6), \mathrm{RA}(n=5)$, or $\mathrm{NaCl}(n=6)$ until a target MAP of $80 \mathrm{mmHg}$ was reached. Oxygen tension in the renal cortex $\left(\mathrm{C}_{\mathrm{PPO}}, \mathrm{mmHg}\right)$, outer medulla $(\mathrm{M \mu PO}, \mathrm{mmHg})$, and renal vein were measured using oxygen-dependent quenched phosphorescent lifetimes of Oxyphor G2. Renal artery blood flow (RBF $\mathrm{ml} /$ minute) was measured. Renal oxygen delivery and renal oxygen consumption $\left(\mathrm{DO}_{2}\right.$ and $\mathrm{VO}_{2}$ $\mathrm{ml} \cdot$ minute/g) were calculated.

Results During hemorrhagic shock, $\mathrm{C} \mu \mathrm{PO}_{2}, \mathrm{M \mu PO}, \mathrm{RBF}_{2} \mathrm{DO}_{2}$, and $\mathrm{VO}_{2}$ decreased and $\mathrm{O}_{2} E R$ increased. During resuscitation, $\mathrm{C} \mu \mathrm{PO}_{2}$ and $\mathrm{M \mu PO}_{2}$ were increased in all groups $(P<0.001)$ although least prominent in the $\mathrm{NaCl}$ group. RBF was significantly increased during resuscitation in all groups (PVR; $5.1 \pm 0.2 P<0.001$, RA; $3.5 \pm 0.4 P<0.001$, Voluven ${ }^{\oplus} ; 3.4 \pm 0.4$ $P<0.01)$, except for the $\mathrm{NaCl}$ group, and $\mathrm{DO}$ was significantly increased in the PVR group $(0.20 \pm 0.02$ vs $0.39 \pm 0.06 ; P<0.01)$ and RA group $(0.22 \pm 0.03$ vs $0.41 \pm 0.06 ; P<0.01$ ), while this remained low in the other two groups. Fluid resuscitation was not able to restore $\mathrm{VO}_{2}$. After 1 hour of resuscitation $\mathrm{C} \mu \mathrm{PO}$, was significantly higher in the PVR group $(49.9 \pm 2.4 P<0.01)$ and the Voluven ${ }^{\oplus}$ group $(45.3 \pm 5.1 P<0.05)$ but not in the RA group $(40.6 \pm 2.2$ $P>0.05)$ compared with the $\mathrm{NaCl}$ group $(32.1 \pm 2.55)$. Furthermore, RBF was significantly higher in the PVR group $(5.1 \pm 0.2 P<0.01)$ compared with the NaCl group ( $2.4 \pm 0.5)$ after 1 hour of resuscitation.

Conclusions Our results suggest that both colloid solutions (Voluven ${ }^{\circledR}$ and PlasmaVolume Redibag ${ }^{\oplus}$ ) are preferred resuscitation fluids with respect to crystalloid solutions. Moreover, the balanced colloid solution (PVR) appears to restore renal blood flow and oxygenation more than the crystalloids or unbalanced colloids do in this rat model of hemorrhagic shock and resuscitation.

Acknowledgements This study was supported in part by a grant from Baxter Health Care.

P503

Effect of rapidly infused crystalloids on acid-base status of dehydrated patients in the emergency department

H Hasman, B Comert, O Cinar, A Uzun, L Yamenel

Gulhane Military Medical Academy, Ankara, Turkey

Critical Care 2010, 14(Suppl 1):P503 (doi: 10.1186/cc8735)

Introduction Dehydration is an important problem among patients admitted to the emergency department (ED). However, there has not yet been a consensus on the ideal fluid for these patients. This study was planned to evaluate the ideal crystalloid for the patients admitted to the ED who have symptoms of dehydration.

Methods We conducted a randomized controlled trial that included 90 dehydrated patients. Three groups of patients each included 30 randomized for one of the solutions Lactated Ringer's, $0.9 \% \mathrm{NaCl}$ or Isolyte ${ }^{\oplus}$ (Eczacıbassı-Baxter, Turkey). Solutions were infused at a rate of $20 \mathrm{ml} / \mathrm{kg} /$ hour for 2 hours. Venous blood sample $\mathrm{pH}, \mathrm{Na}^{+}, \mathrm{K}^{+}, \mathrm{Cl}^{-}$, and $\mathrm{HCO}_{3}^{-}$levels were evaluated at 0, 1, and 2 hours.

Results We detected a decrease in serum pH (7.406 to 7.365) and $\mathrm{HCO}_{3}$ (23.1 to 21.5) levels at the second hour in the $0.9 \% \mathrm{NaCl}$ group. However, in the Isolyte ${ }^{\oplus}$ group an increase in both serum $\mathrm{pH}(7.410$ to 7.434$)$ and $\mathrm{HCO}_{3}{ }^{-}$(23.4 to 24.4) levels were observed. In the Lactated Ringer's group,

Table 1 (abstract P503)

\begin{tabular}{lcc}
\hline & $\mathbf{p H}$ & $\mathrm{HCO}_{3}$ \\
\hline Lactated Ringer's & \\
$\quad$ hours & 7.396 & 22.8 \\
2 hours & 7.408 & 22.4 \\
$0.9 \%$ normal saline & & \\
$\quad$ hours & 7.406 & 23.1 \\
2 hours & 7.365 & 21.5 \\
|solyte & & \\
0 hours & 7.410 & 23.4 \\
2 hours & 7.434 & 24.4 \\
\hline
\end{tabular}


there was no significant change in serum $\mathrm{pH}$ and $\mathrm{HCO}_{3}$ - levels. All changes were in the physiological range (Table 1).

Conclusions All three crystalloid solutions can be safely used for dehydrated patients and the best metabolic profile can be maintained in patients who receive Isolyte ${ }^{\oplus}$.

P504

Impact of saline-based tetrastarch vs balanced tetrastarch and crystalloid solutions on hyperchloraemic acidosis in endotoxaemic sheep

TG Kampmeier', C Ertmer', S Rehberg', A Morelli², M Lange', C Höhn', HVan Aken ${ }^{1}$, M Westphal'

'University Hospital of Muenster, Germany; 'University of Rome 'La Sapienza', Rome, Italy

Critical Care 2010, 14(Suppl 1):P504 (doi: 10.1186/cc8736)

Introduction Septic shock is frequently associated with metabolic acidosis derived from increased lactic acid production and infusion of sodium chloride-based resuscitation fluids. Although the impact of hyperchloraemic acidosis on outcome is still not fully understood, avoiding such a condition is desirable. The hypothesis of the present study was that resuscitation with saline-based tetrastarch solutions results in higher plasma chloride and lower base excess levels as compared with either balanced tetrastarch or crystalloid solutions in ovine endotoxaemic shock.

Methods Thirty-one awake healthy ewes received a continuous infusion of Salmonella typhosa endotoxin started at $5 \mathrm{ng} / \mathrm{kg} /$ minute, which was doubled every hour until the mean arterial pressure (MAP) fell below $65 \mathrm{mmHg}$. Thereafter, sheep were randomized to receive either balanced 6\% HES 130/0.4; saline-based 6\% HES 130/0.4 (both HES solutions were infused up to $50 \mathrm{ml} / \mathrm{kg}$ ); balanced crystalloids; or no fluids. Fluid resuscitation was guided to optimize central venous pressure (8 to 12 $\mathrm{mmHg}$ ), pulmonary arterial occlusion pressure (12 to $15 \mathrm{mmHg}$ ) and mixed-venous oxygen saturation $(\geq 65 \%)$. Norepinephrine was infused to establish a MAP of $70 \pm 5 \mathrm{mmHg}$, if necessary. Haemodynamic variables and blood gas analyses were determined hourly throughout the study period. Animals surviving the 12-hour intervention period were deeply anaesthetized and killed.

Results Haemodynamic variables were comparable between groups. Plasma chloride concentrations were significantly higher, and oximetrycorrected base excess was lower in the saline-based tetrastarch group as compared with the balanced tetrastarch or balanced crystalloid group over the whole interventional period (each $P<0.05$ ). Whereas arterial $\mathrm{pCO}_{2}$ was lowest in the saline-based tetrastarch group (each $P<0.05$ ), there were no differences between treatment groups in arterial $\mathrm{pH}$.

Conclusions As compared with balanced tetrastarch or crystalloid solutions, saline-based tetrastarch infusion is associated with hyperchloraemic acidosis, which is compensated by hyperventilation in awake, endotoxaemic sheep.

\section{P505}

Are different hydroxyethyl starch solutions an independent risk factor for acute kidney injury in surgical critically ill patients? A retrospective analysis of 3,591 patients

C Ertmer1, S Rehberg1, A Morelli'2, TG Kampmeier1, J Wellmann', TVolkert', M Lange', AK Hüsing', H Van Aken', M Westphal'

'University of Muenster, Germany; ${ }^{2}$ University of Rome 'La Sapienza', Rome, Italy Critical Care 2010, 14(Suppl 1):P505 (doi: 10.1186/cc8737)

Introduction Acute kidney injury (AKI) is a common complication associated with increased mortality among ICU patients. The purpose of the present study was to retrospectively analyze the influence of different, saline-based hydroxyethyl starch (HES) solutions (tetrastarch, 6\% HES 130/0.4; pentastarch, $10 \%$ HES 200/0.5) on the risk of AKl in a cohort of surgical ICU patients.

Methods Data for 3,591 patients admitted to one of four ICUs of the University Hospital of Muenster from January 2000 until August 2008 were extracted from an electronic database and analyzed using univariate and multivariate logistic regression analyses. AKI was defined as grade I or higher according to the RIFLE classification.

Results Average amounts of colloid infusion per ICU day were 402 (231; $656)$ and $73(36 ; 172) \mathrm{ml} /$ day in patients treated with tetrastarch and

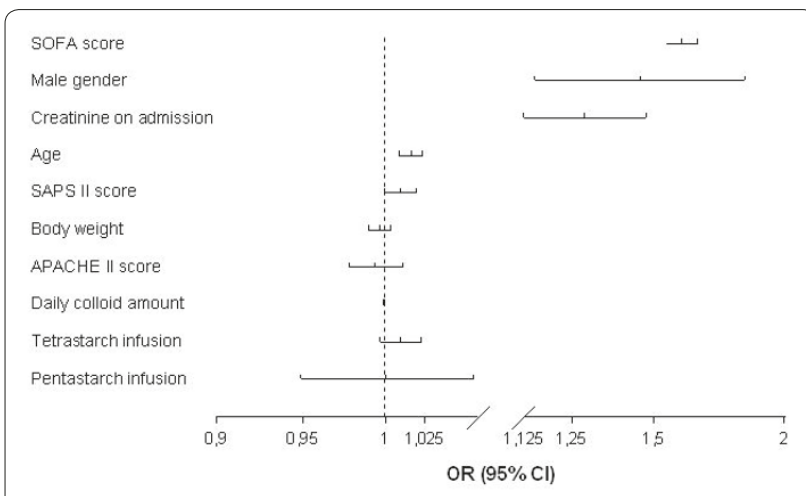

Figure 1 (abstract P505). Risk factors for acute renal failure.

pentastarch, respectively, whereas cumulative colloid amounts were 2,000 $(500 ; 3,500) \mathrm{ml}$ and $1,000(500 ; 2,000) \mathrm{ml}$. Maximum daily infusion rates of $6 \%$ HES 130/0.4 and 10\% HES 200/0.5 were below the pharmaceutically recommended daily dose limitations (that is, 50 and $20 \mathrm{ml} / \mathrm{kg} /$ day, respectively). The overall incidence of AKI was 20.4\%. Multivariate logistic regression analysis revealed SOFA score (OR 1.597; 95\% Cl 1.543, 1.653), male gender (OR $1.455 ; 95 \% \mathrm{Cl} 1.153,1.836)$, creatinine concentration on admission (OR 1.288; $95 \% \mathrm{Cl} 1.125,1.475)$, and patient age (OR 1.017; 95\% Cl 1.009, 1.024) as independent risk factors for AKI. See Figure 1.

Conclusions Neither of the investigated HES solutions (given within the recommended dose range) was an independent risk factor for AKI in the present cohort of surgical ICU patients.

\section{P506}

Role of early continuous venovenous hemodiafiltration in septic shock and multiorgan failure

M Soliman, A Battah, H Elsherif, A Elsherif, H Khaled Nagi

Cairo University, Cairo, Egypt

Critical Care 2010, 14(Suppl 1):P506 (doi: 10.1186/cc8738)

Introduction To evaluate early continuous venovenous hemodiafiltration (CVVHDF) in patients with refractory septic shock and multiorgan failure upon mortality and morbidity in the ICU.

Methods Forty patients were prospectively studied and randomly treated with either conventional treatment (20 patients; group II) or with early CVVHDF (less than 6 hours of maximal hemodynamic support) in addition to the conventional treatment (20 patients; group I). Metabolic acidosis, serum lactate and serum procalcitonin level (PCT) before and 5 days after CVVHDF were monitored to evaluate the outcome. APACHE II and $\triangle$ SOFA scoring systems were used before and 5 days after CVVHDF.

Results Compared with group II, patients of group I had lower mortality (55\% vs $70 \%)$ with an insignificant $P$ value $(P=0.54)$. Group I patients showed a nonsignificant $\triangle$ SOFA (5.95 \pm 4.39 vs $6.2 \pm 3.3$ in groups I and $\|$, respectively, $P=0.66$ ); regarding APACHE || scores, group I also showed statistically nonsignificant lower figures than group II (on admission APACHE II scores were $39.35 \pm 10.65$ vs $41.85 \pm 10$ in groups I and II, respectively, $P=0.45$, while on day 5 APACHE II scores were $34.8 \pm 10.6$ vs $36.1 \pm 10.9$ in groups I and II, respectively, $P=0.41$ ). Group I patients showed lower PCT on admission and day 5 than group II patients (on admission PCT level was $0.64 \pm 0.18$ vs $0.68 \pm 0.17$ in groups I and II, respectively, $P=$ $0.5)$ while the day $5 P C T$ level was $(0.51 \pm 0.15$ vs $0.52 \pm 0.17$ in groups $I$ and $\|$, respectively, $P=0.83$ ). Indicators of improvement showed a statistically significant difference between survivors and nonsurvivors in group | regarding serum lactate level at day $5(P<0.001)$, while other indicators as fever, renal profile, WBC count, metabolic acidosis, serum lactate level on admission and platelet count were statistically insignificant (on admission $P=0.2,0.55,0.45,0.41,0.65,0.55$, respectively, and on day $5 P=0.37,0.94$, $0.71,0.5,<0.001,0.88$, respectively). There was a significant statistical difference between survivors and nonsurvivors in group I considering the number of organ failures as less than or equal to three organs involved in comparison with more than three organs involved $(P=0.008)$. 
Conclusions Early CVVHDF may improve the prognosis of sepsis-related multiple organ failure. Continuous rising of the serum lactate level despite CVVHDF is associated with an increased mortality rate. Of all scoring systems used, SOFA maximum, $\triangle$ SOFA and day 5 APACHE II were the most accurate prognostic indicators for mortality.

\section{Reference}

1. Winchester et al.: Blood Purif 2003, 21:79-84.

\section{P507}

Effect of intravenous $\mathrm{H}_{2} \mathrm{~S}$ on porcine aortic occlusion-induced systemic inflammation and kidney ischemia/reperfusion injury

FWagner', F Simon', A Scheuerle', M Groeger', E Calzia', A Soell', O McCook', B Stahl', G Speit', M Georgieff', P Radermacher', C Szabo², H Schelzig'

'UIm University, UIm, Germany; 2University of Texas Medical Branch, Galveston, TX, USA

Critical Care 2010, 14(Suppl 1):P507 (doi: 10.1186/cc8739)

Introduction Both inhaled hydrogen sulfide $\left(\mathrm{H}_{2} \mathrm{~S}\right)$ [1] and intravenous $\mathrm{H}_{2} \mathrm{~S}$ donors protected against kidney ischemia/reperfusion (I/R) injury [2-4], but all these data originate from unresuscitated rodent models. Therefore, we investigated the effect of the $\mathrm{H}_{2} \mathrm{~S}$ donor $\mathrm{Na}_{2} \mathrm{~S}$ in a clinically relevant porcine model of aortic occlusion-induced renal I/R injury.

Methods Anesthetised and ventilated pigs received $\mathrm{Na}_{2} \mathrm{~S}(n=9)$ or vehicle $(n=10)$ for 2 hours before and 8 hours after 90 minutes of intra-aortic balloon occlusion-induced kidney ischemia. During reperfusion noradrenaline was titrated to keep blood pressure at baseline levels. Before $\mathrm{Na}_{2} \mathrm{~S}$, prior to aortic occlusion and at 1, 2, 4 and 8 hours of reperfusion, we measured renal blood flow and function (creatinine clearance and blood levels, fractional $\mathrm{Na}^{+}$excretion), blood cytokines (TNFa, IL-6, IL-1 $\beta$ ) and nitrates, renal tissue DNA damage (comet assay), HO-1 and caspase-3 expression (western blotting), and NF-KB activation (EMSA). Histological damage (glomerular tubularisation [5]) was assessed immediately post mortem.

Results $\mathrm{Na}_{2} \mathrm{~S}$ pretreatment was associated with a progressive fall in core temperature and significantly lower noradrenaline infusion rates needed to achieve the hemodynamic targets. While renal blood flow and fractional $\mathrm{Na}^{+}$ excretion were comparable, $\mathrm{Na}_{2} \mathrm{~S}$ attenuated the fall in creatinine clearance and the rise in creatinine blood levels, respectively, which coincided with significantly lower IL-6, IL-1 $\beta$, and nitrate blood levels. Kidney glomerular and tissue DNA damage were markedly attenuated, whereas NF-KB activation was significantly higher in the $\mathrm{Na}_{2} \mathrm{~S}$-treated animals.

Conclusions In a clinically relevant porcine model mimicking aortic cross-clamping-induced kidney I/R injury, $\mathrm{Na}_{2} \mathrm{~S}$ attenuated tissue injury and organ dysfunction as a result of reduced systemic inflammation and oxidative stress. The higher NF-KB activation and the unchanged fractional $\mathrm{Na}^{+}$excretion were most probably due to the drop in temperature [6] and the direct effect of $\mathrm{H}_{2} \mathrm{~S}$ on tubular $\mathrm{Na}^{+}$absorption [7], respectively.

Acknowledgements Supported by the DFG (SCHE 899/2-3) and Ikaria Inc. (Seattle, WA, USA).

\section{References}

1. J Am Soc Nephrol 2009, 20:1901-1905.

2. Lab Invest 2008, 88:1038-1048.

3. Eur J Pharmacol 2009, 606:205-209.

4. Am J Physiol Renal Physiol 2009, 297:F27-F25.

5. Nephron Exp Nephrol 2007, 105:e33-e40.

6. Am J Physiol Gastrointest Liver Physiol 2007, 292:G201-G207

7. J Pharmacol Exp Ther 2009, 329:1056-1062.

\section{P508}

Survival improvement in pigs with liver failure and superimposed sepsis by a new liver support system (Hepa Wash ${ }^{\oplus}$ )

A Al-Chalabi', E Matevossian', AK Preissel', H Yan', A Geiger', E Nairz', P Schimmel', C Schreiber', Z Yang', B Kreymann²

'Klinikum Rechts der Isar, München, Germany; 'Hepa Wash GmbH, Munich, Germany

Critical Care 2010, 14(Suppl 1):P508 (doi: 10.1186/cc8740)

Introduction Liver failure is often complicated by sepsis. Available liver support systems (LSS) failed to show survival benefits. Hepa Wash ${ }^{\oplus}(\mathrm{HW})$ is a newly developed LSS based on use of recycled albumin dialysate. The

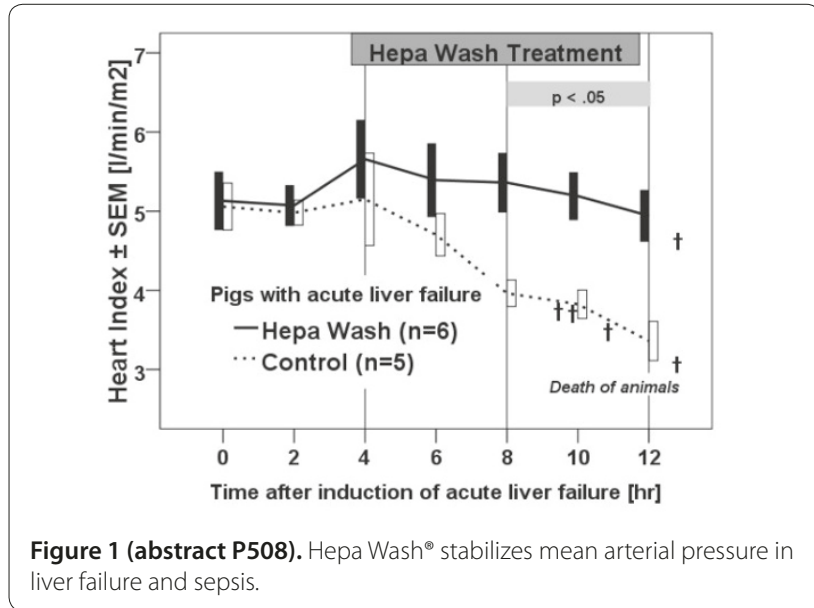

study aim is to evaluate its safety and efficacy in an animal model that combines liver failure with sepsis.

Methods Female German Landrace pigs ( $60 \mathrm{~kg})$ were randomly allocated to HW $(n=5)$ and control $(n=5)$ groups. On day 0 ischemic and cholestatic liver injury was induced by functional end-to-side portacaval shunt and bile duct ligation. Animals were returned then to the stall for observation. On day 3 the second phase of the experiment was started. Endotoxin was administered intravenously at an initial dose of $4 \mu \mathrm{g} / \mathrm{kg} / \mathrm{hour}$ with a stepwise twofold increment every hour and stopped after 7.5 hours. HW was started 2.5 hours after endotoxemia and continued for 7 hours.

Results On day 3, animals $(n=10)$ had hyperbilirubinemia $(5.2 \pm 0.3 \mathrm{mg} / \mathrm{dl}$; mean \pm SEM) and hyperammonemia $(264 \pm 38 \mu \mathrm{g} / \mathrm{dl})$. Endotoxemia in control animals resulted in severe multisystem organ failure and death (after $6.4 \pm 0.3$ hours) although they received only one-third of the total endotoxin dose on average. HW improved cardiovascular (Figure 1), cerebral, respiratory and renal parameters and eliminated surrogate parameters as bilirubin $(2.8 \pm 0.5$ vs $5.2 \pm 0.3 \mathrm{mg} / \mathrm{dl} P<0.05)$ and ammonia $(189 \pm 38$ vs $1,108 \pm 455 \mu \mathrm{g} / \mathrm{dl}, P<0.05)$. All animals in the HW group survived the 10 -hour observation period $(P<0.05)$. We did not notice any adverse effects during treatment.

Conclusions Hepa Wash ${ }^{\circledR}$ was safe and effective at improving survival in a swine model of liver failure and sepsis due to amelioration of the major organ functions and removal of protein-bound and water-soluble toxins.

\section{P509}

Increased glomerular filtration in the critically ill patient receiving anti-infective treatment

B Claus, K Colpaert, E Hoste, J Decruyenaere, J De Waele

Ghent University Hospital, Ghent, Belgium

Critical Care 2010, 14(Suppl 1):P509 (doi: 10.1186/cc8741)

Introduction Little is known about the occurrence of increased glomerular filtration rates (GFR) in critically ill patients. Increased GFR may in theory lead to subtherapeutic concentrations of life-saving drugs. The aim of this study was to evaluate the occurrence rate and pattern of increased GFR in critically ill patients receiving anti-infective treatment (AIT).

Methods Prospective evaluation of consecutive ICU patients during a 6-week period, treated with anti-infective drugs, either therapeutically or prophylactically; dialysed patients were excluded. Patient characteristics: retrieved from the computerized Intensive Care Information System. Surrogate measure for GFR: the 24-hour urinary creatinine clearance (Ccr) calculated per day of AIT. Increased GFR: Ccr > 120 ml/minute. Statistical analysis with SPSS 17.0: Student's $t$ test, chi-square or Fisher's exact test. Data expression: median and interquartile range (IQR). Multivariate analysis: logistic regression analysis with increased GFR as dependent variable.

Results One hundred and forty-one (94 male) patients were included; median age was 59.9 (50.3 to 70.1); 80 (56.7\%) were surgical ICU (SICU) patients. Most common infection types were pneumonia (59.2\%) and intra-abdominal infection (9.9\%). Prophylactic use was $17.6 \%$ of all AIT. Median length of stay 4.6 days; ICU mortality $5.7 \%$. Median Ccr in the 
whole patient group was $97.0 \mathrm{ml} /$ minute (57.5 to 164.5). In total, 753 AlT days were available for analysis in which 302 days of increased GFR were present (40.1\%). Seventy-six patients (53.9\%) had an episode of increased GFR at some time during AIT. In 31 patients (22.0\%) GFR was increased throughout the whole episode of AlT. Patients with increased GFR were significantly younger (54.2 vs $65.6, P<0.001)$ and there was a trend towards a higher incidence of increased GFR in patients treated for infections, in male and in SICU patients. Multivariate analysis showed that age (OR 0.93 per year), therapeutic administration of AIT (OR 5.49), and SICU admission (OR 2.27) were independently associated with increased GFR.

Conclusions Increased GFR were a frequent finding in critically ill patients receiving AIT, and was associated with younger age and SICU admission.

P510

Therapeutic plasma exchange in children with acute liver failure: assessment of laboratory parameters

D Demirkol, O Yanar, N Gerenli, A Citak, I Ozden, S Cantez, O Durmaz,

M Karabocuoglu

Istanbul University, Istanbul, Turkey

Critical Care 2010, 14(Suppl 1):P510 (doi: 10.1186/cc8742)

Introduction The aim of this study is to analyze the effect of therapeutic plasma exchange (TPE) on biochemical and coagulation parameters.

Methods We enrolled all children who were admitted to the pediatric ICU with diagnosis of acute liver failure and underwent TPE between December 2005 and December 2009. Serum prothrombin time, International Normalized Ratio, total and direct bilirubin, aminotransferases and serum ammonia values were recorded before and after TPE sessions.

Results Fourteen patients (male/female, 4/10, median age 8.5 years) underwent a total of 37TPE sessions. Acute liver failure was induced by viral hepatitis in five cases, Wilson disease in four cases, toxins in four cases and autoimmune hepatitis in one case. When compared with baseline, there were significant improvements in laboratory parameters (Table 1). Five of the 14 patients (35.7\%) died in the pediatric ICU, five patients underwent liver transplantation. Four patients (28.5\%) survived with extracorporeal liver support. No serious adverse effect of TPE was observed in the patients during or after completion of TPE.

Conclusions TPE is effective in improving liver biochemistry tests in children with liver failure. Expected changes in laboratory parameters after TPE must be considered especially in deciding for liver transplantation.

Reference

1. Singer AL, Olthoff KM, Haewen K, Rand E, Zamir G, Shaked A: Role of plasmapheresis in the management of acute hepatic failure in children. Ann Surg 2001, 234:418-424.

P511

New markers of inflammation-induced renal injury subside when endotoxin tolerance develops in humans as measured by urine proteomics

S Heemskerk, A Draisma, C Laarakkers, J Van der Hoeven, R Masereeuw, P Pickkers

Radboud University Nijmegen Medical Centre, Nijmegen, the Netherlands

Critical Care 2010, 14(Suppl 1):P511 (doi: 10.1186/cc8743)

Introduction Sepsis has been identified as the most common cause of renal injury in ICUs although the pathophysiology is not well understood.
No large clinical studies are available that show an improvement of renal function in patients with sepsis and this may be related to the lack of early diagnostic tests that indicate the onset of renal injury. The aim of the current study was to search for potential new early markers of renal injury during acute endotoxemia and to investigate whether renal injury can be ameliorated by the induction of lipopolysaccharide (LPS) tolerance.

Methods Five healthy males received intravenous bolus injections of $2 \mathrm{ng} / \mathrm{kg} /$ day Escherichia coli LPS for 5 consecutive days. We used surfaceenhanced laser desorption/ionization time-of-flight mass spectrometry (Seldi-TOF MS). This approach allows for rapid high-throughput profiling of multiple urine samples and detects low molecular weight biomarkers. Results Repeated LPS administrations induced a diminished glomerular filtration rate of $33 \pm 7 \%(P=0.02)$ on day 2 and an increase in serum creatinine of $11 \pm 3 \%(P=0.002)$ on day 3 , which was associated with the appearance of 15 peak intensities in the urinary protein profile including an increase in $\beta$-microglobulin levels $(P=0.04) 6$ hours after the first LPS administration. Four of the 15 peak intensities on day 1 correlated with serum creatinine levels on day $3 ; 3,950,4,445,6,723$ and $7,735 \mathrm{~m} / z(P=0.03$; $0.01 ; 0.02$ and 0.05 , respectively). With the development of LPS tolerance, renal function was restored, reflected by a decrease in serum creatinine and $\beta$-microglobulin levels to baseline $(P=0.2$ and 0.4 , respectively, between days 1 and 5$)$, and by attenuated peak intensities in the urinary protein profile ( $P<0.0001$ for all 15 peak intensities).

Conclusions In conclusion, renal injury occurs during repeated endotoxemia and can be predicted by new urinary markers using proteome research. The four markers that correlated with the extent of renal injury may represent potential new biomarkers for renal injury and need further identification. The inflammation-induced renal injury subsided when LPS tolerance developed after 5 consecutive days of LPS administrations

\section{P512}

Extraction and purification of 1-hydroxymidazolam glucuronide from ultrafiltrate

C Mckenzie', D Naughton², G Davies ${ }^{3}$, DTreacher'

'Guy's and St Thomas' NHS Foundation Trust, London, UK; ${ }^{2}$ Kingston

University, London, UK; ${ }^{3}$ King's College, London, UK

Critical Care 2010, 14(Suppl 1):P512 (doi: 10.1186/cc8744)

Introduction We developed a technique for detection of MDZ and 1-hydroxymidazolam glucuronide (1-OHMG). No commercial source of 1-OHMG prevented assay development. 1-OHMG had pharmacological activity and is renally excreted. We postulated that ultrafiltrate (UFR) would be rich in 1-OHMG. We describe a method to purify 1-OHMG from UFR.

Methods Ethics approval was granted. To purify, UFR was extracted on a C-18 column, it was washed and eluted. HPLC-MS separation was performed. The 1-OHMG (mol. wt 517/ 518 in MS) was desiccated and re-dissolved four times to maximise purity. Electrospray (ESI) mass spectrometry (MS) characterised 1-OHMG. The purity was calculated using NMR. A calibration plot of 1-OHMG, MDZ and DZP (internal standard) for HPLC-MS was performed.

Results 1-OHMG identity was confirmed using MS/MS (Figure 1). Two milligrams of 1-OHMG was purified from 5,000 ml UFR. The 1-OHMG was 98\% pure (NMR). The extinction coefficient was identical to MDZ. The calibration plot resulted in correlation of 0.912. The assay was applied into clinical practice, to report sera and UFR levels.

Table 1 (abstract P510). Biochemical and coagulation parameters before and after TPE

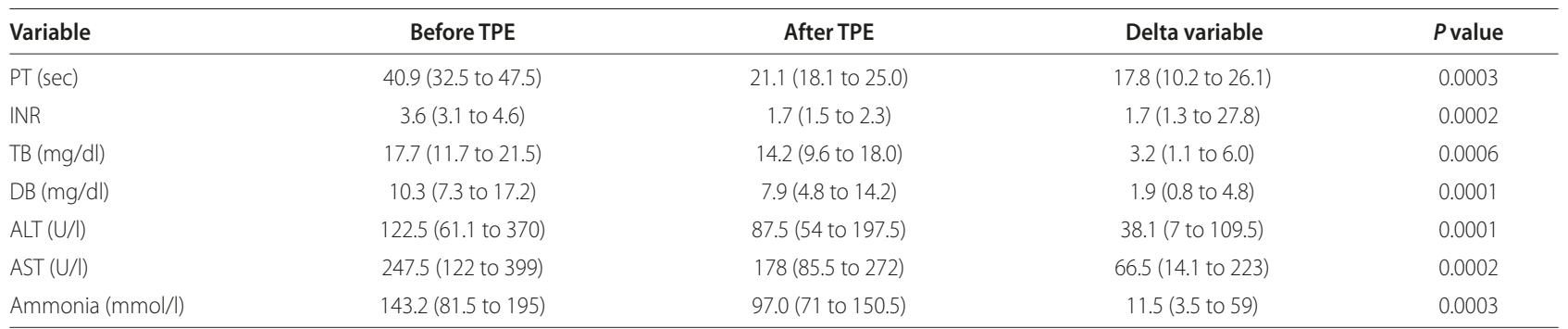




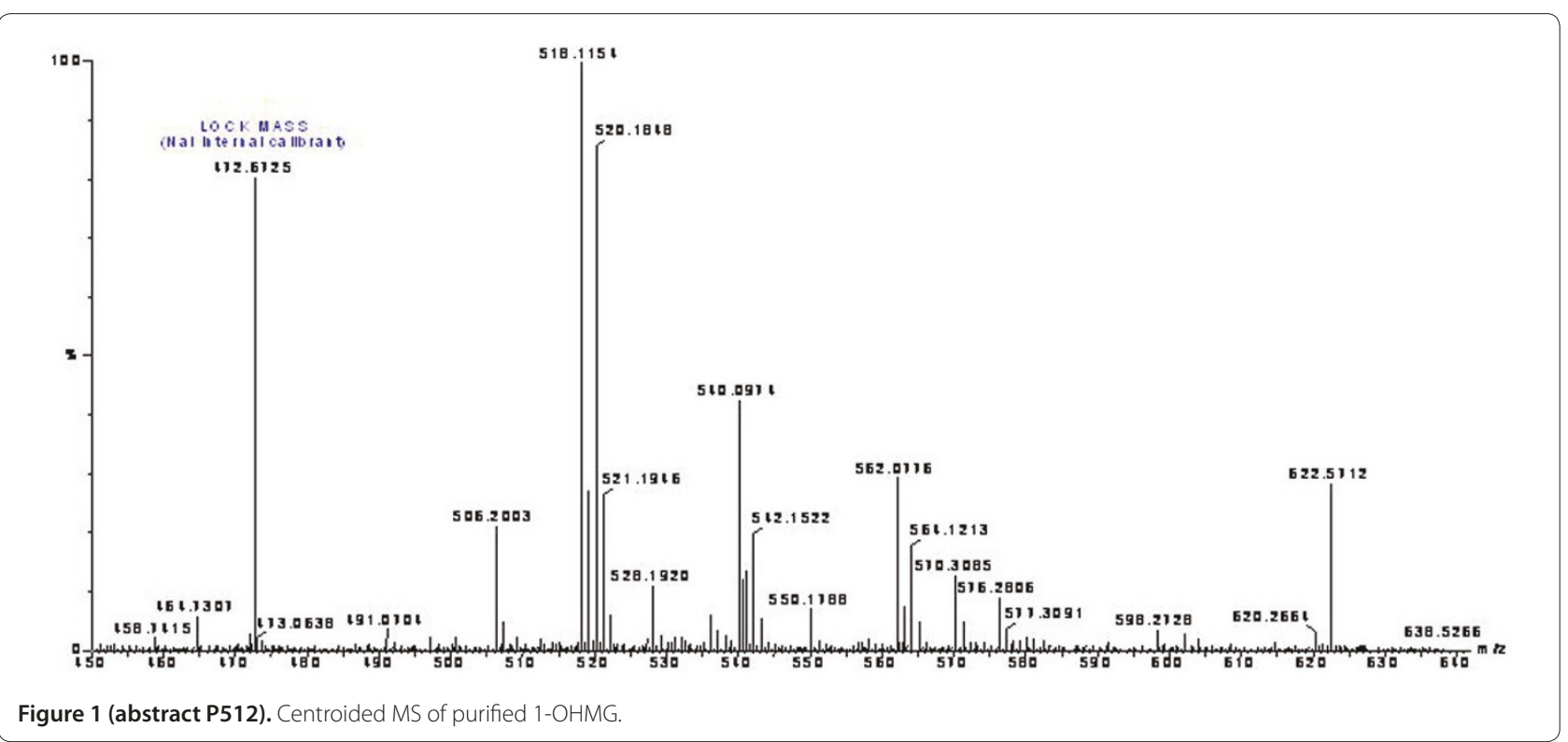

Conclusions We were able to extract and purify an active drug metabolite from UFR. Five litres of UFR resulted in $2 \mathrm{mg} 1-\mathrm{OHMG}$. This is a potentially rich source of drugs or drug metabolites, allowing pharmacokinetic studies greatly required in critical illness.

\section{Reference}

1. McKenzie et al:: Differentiating MDZ oversedation from neurological damage in the intensive care unit. Crit Care 2005, 9:32-33.

P513

Continuous renal replacement therapy: are we getting the dose right? S Martin, M Al-Haddad

Western Infirmary, Glasgow, UK

Critical Care 2010, 14(Suppl 1):P513 (doi: 10.1186/cc8745)

Introduction The Intensive Care Society recommends achieving a dose of $35 \mathrm{ml} / \mathrm{kg} /$ hour in $85 \%$ of patients receiving continuous renal replacement therapy (CRRT). However, a recent multicentre trial concluded there was no benefit of using $40 \mathrm{ml} / \mathrm{kg} /$ hour versus $25 \mathrm{ml} / \mathrm{kg} /$ hour. The extra cost of high-dose RRT has not been quantified. At our institution the target dose is $35 \mathrm{ml} / \mathrm{kg} /$ hour.

Methods Patients receiving renal replacement therapy from April to September 2009 were identified using Careview, an electronic patientcare record system. The effluent rate $(\mathrm{ml} /$ hour) was used to calculate the dose and adjusted for weight. A telephone survey of ICUs examined the dose and mode of CRRT used in Scotland.

Results Thirty-five patients, mean age 62, received CRRT with a mean duration of RRT of 78 hours. Twenty-three patients (66\%) were admitted

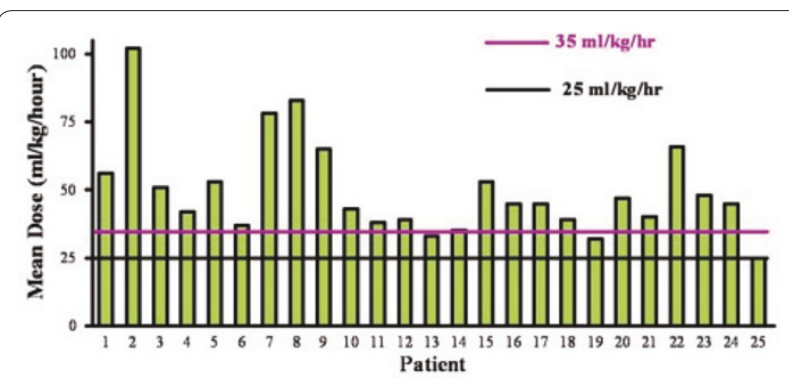

Figure 1 (abstract P513). Mean dose of RRT for each patient at Western Infirmary. with sepsis. Continuous venovenous haemodiafiltration was the initial mode in 31 patients (89\%). Ten patients were excluded from the dose analysis because no weight was documented. The mean dose actually delivered per patient was $50 \mathrm{ml} / \mathrm{kg} / \mathrm{hour}$. If $25 \mathrm{ml} / \mathrm{kg} /$ hour had been given then $40 \%$ less RRT fluid could have been used. The Scottish telephone survey revealed that one-third of Scottish ICUs routinely use $35 \mathrm{ml} / \mathrm{kg} /$ hour or more. If $25 \mathrm{ml} / \mathrm{kg} / \mathrm{hour}$ was used routinely in these units alone, then over $€ 24,000$ could be saved per annum.

Conclusions The dose of CRRT given for each patient at our institution currently exceeds the target dose by $43 \%$. Using $25 \mathrm{ml} / \mathrm{kg} /$ hour as the standard dose would provide a significant cost saving to the NHS in Scotland and to ICUs throughout Europe.

\section{Reference}

1. Bellomo R, Cass A, Cole L, et al:. Intensity of continuous renal-replacement therapy in critically ill patients. N Engl J Med 2009, 361:1627-1638.

P514

Use of CRRT-CVVH in a hemato-oncological ICU in patients treated with defibrotide for veno-occlusive disease in patients after allogeneic hematopoetic stem cell transplantation: single-center experience

M Navratil, Z Koristek, F Folber

Masaryk University Hospital, Brno, Czech Republic

Critical Care 2010, 14(Suppl 1):P514 (doi: 10.1186/cc8746)

Introduction Complex care in the hemato-oncological ICU sometimes requires the use of CRRT. As the hemato-oncological patients have some specifications we retrospectively followed up the results of our effort in patients with acute renal failure due to veno-occlusive disease (VOD) after allogeneic hematopoietic stem cell transplantation. VOD is one of the most severe early complications in this type of patients and its mortality is high. Methods From 1 January 2007 to 30 October 2009 we performed 94 procedures (one procedure $=24$ hours) in 15 patients with VOD and acute renal oligo-anuric failure. As a standard in our ICU we perform CVVH with post dilution on a Fresenius Multifiltrate machine. The key drug for the treatment of VOD is defibrotide and the patients were severely thrombocytopenic, we have not used any anticoagulation in the CVVH set. The dose of defibrotide ranged from 10 to $20 \mathrm{mg} / \mathrm{kg} /$ day according to the severity of thrombocytopenia and eventually hemorrhagic symptoms. Blood count, acid-base balance and biochemistry were monitored at least twice daily. The thrombocytopenia was corrected with the donor platelets and the platelet level was sustained at $20 \times 10^{9}$.

Results The introduction of CVH in all 15 patients dramatically helped to overcome the severe part of VOD until the defibrotide helped to restore 
the right function of vascular endothelium. None of the 15 patients died from VOD as a cause of death. Surprisingly, the median patency of the $\mathrm{CVVH}$ tubing set was 46 hours despite no anticoagulation in the tubing set. Defibrotide is efficient in preventing coagulation in the tubing set. No serious hemorrhagic events were observed. The fluent maintenance of fluid balance, the removal of waste products of metabolism and the maintenance of acid-base balance were very important for the patient in the critical point of advanced VOD.

Conclusions $\mathrm{CVVH}$ is an integral part of intensive care in hemato-oncology and the use of this therapeutic modality in patients with VOD with acute renal failure can significantly improve the results of our therapeutic efforts.

\section{P515}

Comparison of the efficacy and safety of two regional citrate anticoagulation protocols using acid citrate dextrose $A$ or Prismocitrate $10 / 2$, in patients with acute renal failure undergoing continuous venovenous haemodiafiltration

EL Ooi, TW Lim, N Lim

Changi General Hospital, Singapore

Critical Care 2010, 14(Suppl 1):P515 (doi: 10.1186/cc8747)

Introduction Continuous renal replacement therapy (CRRT) is the treatment of choice for acute renal failure in critically ill patients. Our study compared the efficacy and safety of regional citrate anticoagulation (RCA) with acid citrate dextrose A (ACD-A) against Prismocitrate 10/2 (Gambro). Use of Prismocitrate 10/2 eliminates the need for a separate infusion pump for ACD-A as it doubles as replacement fluid and anticoagulant. This removes a source of error in the calculation of fluid balance. The combination of tri-sodium citrate and citric acid in Prismocitrate 10/2 avoids the metabolic alkalosis associated with ACD-A $[1,2]$.

Methods This was a prospective sequential cohort study. All patients admitted to the surgical ICU who required CRRT for ARF were recruited. Group A, using ACD-A, was recruited from October 2007 to September 2008 ( $n=23$ ). Group B, using Prismocitrate 10/2, was recruited from October 2008 to September $2009(n=20)$. We evaluated the incidence of metabolic alkalosis and other biochemical changes, azotemia control, filter lifespan and complications.

Results The incidence of metabolic alkalosis in Group B from treatment day 2 onwards was significantly lower than Group A. The Group A median pH was 7.42 (6.81 to 7.62) compared with Group B pH 7.35 (7.2 to 7.49), $P=0.001$. The control of electrolytes and azotemia was not significantly different. The mean filter duration was 58.8 hours ( $95 \% \mathrm{Cl} 38.0$ to 79.6$)$ for Group A and 61.8 hours ( $95 \% \mathrm{Cl} 45.8$ to 77.8 ) for Group B ( $P=0.678)$. Longitudinal analysis revealed a statistically significant result for reduced metabolic alkalosis for Group B. (standard bicarbonate $P<0.001$, base excess $P<0.001$ ). Repeat treatment sessions also showed a statistically significant reduction of metabolic alkalosis using Prismocitrate 10/2 rather than ACD-A $(P=0.029)$.

Conclusions RCA with Prismocitrate 10/2 reduces the incidence of metabolic alkalosis associated with ACD-A. This regime is safe, feasible and improved patient safety, with no increase in complication rates. Our unit has now converted to Prismocitrate 10/2 for RCA.

\section{References}

1. Silverstein $\mathrm{FJ}$, et al: Metabolic alkalosis induced by regional citrate hemodialysis. ASAIO Trans 1989, 35:22-25.

2. Morgera S, et al:: Metabolic complications during regional citrate anticoagulation in continuous venovenous hemodialysis: a single center experience. Nephron Clin Pract 2004, 97:c31-c136.

P516

Addition of prostacyclin to heparin does not prolong circuit life during continuous haemofiltration

M Ostermann, L Tovey, H Dickie, R Beale

Guy's and St Thomas' Foundation Hospital, London, UK

Critical Care 2010, 14(Suppl 1):P516 (doi: 10.1186/cc8748)

Introduction Premature circuit clotting is a major problem during continuous venovenous haemofiltration $(\mathrm{CV} V \mathrm{H})$. Some studies have suggested that circuit life can be improved by adding epoprostenol to heparin.
Methods Retrospective review of mean circuit patency in patients on noncitrate-based anticoagulation regimens during CVH in a 30-bed multidisciplinary ICU between October 2008 and May 2009. Circuits which were discontinued electively were excluded from the analysis.

Results Patency data were available for 486 noncitrate circuits. There was no significant difference in mean circuit life between heparin given via circuit ( $n=169$ circuits; mean circuit life 19.8 hours) and epoprostenol alone ( $n=119$ circuits; mean circuit life 20.9 hours). In a small group of patients on heparin via the circuit, epoprostenol was added for patency reasons ( $n=19$ circuits). In this group, the mean circuit life was only 15 hours. Circuits of patients on systemic heparin $(n=100)$ lasted for a mean of 23.5 hours. In a small group of patients, epoprostenol was added via the circuit ( $n=32$ circuits). The mean circuit life was 28.9 hours $(P>0.05)$. Other types of noncitrate anticoagulation were activated protein $C$ and lepuridin, alone or in combination with heparin ( $n=47$ circuits).

Conclusions In our practice, anticoagulation with epoprostenol alone led to similar circuit survival as heparin administered via the circuit. Adding epoprostenol to heparin did not significantly prolong the mean circuit life. Further analysis of circuit patency in individual patients is needed to determine the characteristics of patients who may benefit and for whom the extra cost could be justified.

\section{P517}

Recurrent early filter clotting in regional citrate anticoagulated continuous venovenous hemodialysis due to undetected antibodies to heparin-platelet factor 4 complexes

T Slowinski, I Lieker, M Sander, D Khadzhynov, S Morgera, H Neumayer,

H Peters

Charité Campus Mitte, Berlin, Germany

Critical Care 2010, 14(Suppl 1):P517 (doi: 10.1186/cc8749)

Introduction We recently published a citrate anticoagulated continuous venovenous hemodialysis (citrate-CVVHD) protocol with variable treatment dose. The protocol was safe and easy to handle and the mean filter lifetime was 61.5 hours [1]. We here report five cases with unexplained recurrent early filter clotting beside effective citrate anticoagulation due to undetected antibodies to heparin-platelet factor 4 complexes (hep-PF4 $\mathrm{ab})$.

Methods Regionale citrate anticoagulation was performed with variable treatment dose using the MultiFiltrate ${ }^{\text {TM }}$ CRRT device (Fresenius, Germany) in CVVHD mode. Between May 2008 and August 2009 we detected five cases on the ICU. All had acute kidney injury with need for RRT. Underlying diseases were: three sepsis, one cardiac surgery, one abdominal surgery. All patients were anticoagulated with heparin or fractionated heparin for more than 10 days before initiation of citrate-CVHD. At the time of initiation of citrate-CVVHD, none of the patients had clinically evident signs of thomboembolism.

Results The mean filter lifetime before diagnosis of hep-PF4 ab was 8 (range 1.3 to 14 ) hours. Postfilter ionized calcium concentrations were always in the demanded range (mean 0.28 , range 0.24 to $0.35 \mathrm{mmol} / \mathrm{l}$ ). The mean thrombocyte count was $160 / \mathrm{nl}$ (range 48 to 215 ) at time of initiation of citrate-CVVHD. Thrombocyte counts fell to a mean 114/nl (range 36 to 177) within 5 days. Mean time to testing for and diagnosis of hep-PF4 ab was 6 (range 3 to 12) days. After positive testing (HIPA) for hep-PF4 ab, heparin was stopped and all patients received argatroban with a mean PTT of 83 (range 48 to 119) seconds. Under systemic anticoagulation with argatroban, the filter lifetime of citrate-CVVHD increased to a mean 58 (range 18 to 96) hours. Within 14 days after initiation of citrate-CVVHD, three patients had a diagnosis of a clinically significant thrombembolic event.

Conclusions In patients on the ICU with moderate-low thrombocyte count and without clinically evident signs of thomboembolism, often sepsis or the state of critical illness is assumed as the cause for decreasing platelet counts and tests for hep-PF4 ab are not performed. In cases of undetected and untreated hep-PF4 ab, filter clotting can occur beside effective citrate anticoagulation in CVVHD. In cases of unexplained recurrent early filter clotting in citrate-CVVHD, patients should be carefully examined for underlying hep-PF4 ab and heparin-induced thrombocytopenia.

\section{Reference}

1. Morgera et al:: Crit Care Med 2009, 37. 
P518

Pilot study on a regional citrate anticoagulated continuous venovenous hemodiafiltration protocol with variable treatment dose

T Slowinski, I Lieker, D Khadzhynov, M Sander, S Morgera, H Neumayer, H Peters

Charité Campus Mitte, Berlin, Germany

Critical Care 2010, 14(Suppl 1):P518 (doi: 10.1186/cc8750)

Introduction In order to add convective solute transport to a recently described citrate-CVVHD protocol, we established a citrate anticoagulated continuous venovenous hemodiafiltration (citrate-CVVHDF) protocol with variable treatment dose. Citrate-CVVHD has been shown to be safe and easy to handle [1]. Being based on dialysis, however, citrate-CVVHD primarily relies on diffusive solute transport. To also allow convective transport, we designed a new citrate-CVVHDF protocol with a postdilution hemofiltration dose.

Methods Prospective observational study. A CVVHDF-based citrate anticoagulation protocol on the Multifiltrate ${ }^{T M}$ CRRT device (Fresenius Medical Care (FMC), Germany) using a $4 \%$ trisodium citrate solution, the dialysate fluid $\mathrm{Ci}_{-}-\mathrm{Ca}^{\text {TM }}$ Dialysate K2 (FMC), and a continuous calcium chloride $(91 \mathrm{mmol} / \mathrm{l})$ infusion. For the filtration dose we used a standard bicarbonate-buffered substitution fluid (MultiBic ${ }^{\mathrm{TM}}$; FMC) in postdilution. Ten patients on the ICU with acute kidney injury and need for RRT were included. For variable doses, patients were divided into three groups according to their body weight $(1:<60 \mathrm{~kg}, 2: 60$ to $90 \mathrm{~kg}, 3:>90 \mathrm{~kg})$. The initial flows for dialysate/substitution fluid in the groups were 1,400/800, $1,800 / 1,000$, and 2,200/1,200 ml/hour, blood flow 80, 100, and $120 \mathrm{ml}$ minute, citrate flow 145, 175, and $220 \mathrm{ml} /$ hour, and calcium flow 32, 45, and $57 \mathrm{ml} /$ minute. Citrate flow was adjusted to postfilter ionized calcium (iCa) measurements, target range 0.25 to $0.35 \mathrm{mmol} / \mathrm{l}$. Calcium flow was adjusted to patients' systemic iCa. The treatment time was limited to 72 hours

Results Eight of 10 patients reached the maximum treatment time of 72 hours without clotting. In one patient the treatment had to be stopped after 50 hours because of central venous catheter flow problems and one patient died after 54 hours. Acid-base control: mean $(95 \% \mathrm{Cl}) \mathrm{pH}$; st-bicarbonate at 48 hours: 7.42 (7.38 to 7.44$) ; 24.6$ (21.3 to 27.8$) \mathrm{mmol} / \mathrm{l}$, and at 72 hours: 7.39 (7.35 to 7.43 ); 25.0 (21.3 to 27.6 ) mmol/l. Electrolyte control: mean $(95 \% \mathrm{Cl})$ s-sodium; s-potassium at 48 hours: 139 (137 to 141); 4.8 (4.4 to 5.2) $\mathrm{mmol} / \mathrm{l}$, and at 72 hours: 139 (137 to 142$) ; 4.9$ (4.4 to 5.4) $\mathrm{mmol} / \mathrm{l}$. Mean ( $95 \% \mathrm{Cl}$ ) treatment dose was 41 (37 to 46$) \mathrm{ml} / \mathrm{kg} / \mathrm{hour}$, mean (95\% Cl) s-urea at 48 hours: 51 (37 to 66) $\mathrm{mg} / \mathrm{dl}$, and at 72 hours: 50 (35 to 65$) \mathrm{mg} / \mathrm{dl}$.

Conclusions The citrate-CVVHDF protocol allows combined hemodialysis and hemofiltration in CRRT with variable treatment dose and with the advantages of regional citrate anticoagulation. In this pilot study, in 10 patients the filter run-time in citrate-CVVHDF was remarkable and metabolic control excellent.

\section{Reference}

1. Morgera et al:: Crit Care Med 2009, 37.

\section{P519}

Characteristics and outcome of patients treated with renal replacement therapy: single-center, 2-year experience B Kovacevic, S Gavrilovic, D Obradovic, M Kojicic, U Batranovic Institute for Pulmonary Diseases of Vojvodina, Sremska Kamenica, Serbia Critical Care 2010, 14(Suppl 1):P519 (doi: 10.1186/cc8751)

Introduction Continuous renal replacement therapy (CRRT) is frequently used among hemodynamically unstable critically ill patients with acute renal failure (ARF). However, data regarding management of these patients in Serbia are scarce. CRRT has been available at our institution since 2007. The objective of this study was to describe characteristics and outcome of patients treated with renal replacement therapy in a single tertiary-care center in Serbia.

Methods In this retrospective observational study we reviewed medical records of adult ( $\geq 18$ years) patients treated in the five-bed ICU at the Institute for Pulmonary Diseases in Sremska Kamenica, Serbia who underwent CRRT from April 2007 to November 2009.
Results From the total 314 patients treated in the ICU, 29 (9.2\%), 17 men, median age 54 years (interquartile range (IQR) 48 to 68), underwent CRRT. Patients were mostly nonsurgical ( $n=28,96 \%)$. Main admission diagnoses were septic shock $(n=11)$ and pneumonia $(n=9)$. The median APACHE II score was 27 (IQR 19 to 32), and SOFA day 1 was 10 (IQR 5 to 13). Seventeen patients (58.6\%) had chronic co-morbidities; Charlson score median 2 (IQR 0.75 to 3). Twenty-four patients required mechanical ventilation, and 13 were diagnosed with acute respiratory distress syndrome. Slow continuous ultrafiltration was used in four patients (13.7\%), hemodiafiltration (CVHDF) modality in 13 (44.8\%), hemodialysis (CVVHD) in 11 (37.9\%). Median time on CRRT was 3 days (IQR 2 to 4). Five patients (17\%) had a complete recovery of renal function at the time of ICU discharge. Median hospital length of stay was 12 days (IQR 5 to 20) and hospital mortality was 69\%.

Conclusions The need for CRRT in the observed population is substantial. Future studies are warranted to determine the impact of introduction of CRRT on hospital mortality in Eastern European countries and to provide future strategies for improving patient care in these settings.

\section{P520}

Renal function in living related kidney donors above the age of 70

$\checkmark$ Milic, N Vukovic, A Pavlovic, S Pavlovic

Clinical Center Nis, Serbia

Critical Care 2010, 14(Suppl 1):P520 (doi: 10.1186/cc8752)

Introduction Living related kidney donors are highly motivated for donation. Older donors may have increased risks in the perioperative and postoperative period. Age limits for donors was insisted on in the past. The glomerular filtration rate in older people is only slightly lower than in young individuals in the absence of primary renal disease or confounding cardiovascular disease. The aim of the study was to assess renal function of living related donors above the age of 70 in the early postoperative period.

Methods From March 2007 to November 2009, in Clinical Centre Nis, 25 living related donors donated their kidneys. The serum creatinine and calculated creatinine clearance (MDRD formula) were evaluated preoperatively, first, third and fifth postoperative days in the group under $(U)$ and in the group above (A) the age of 70 .

Results There were 16 donors in group $U$ (mean age 59, SD 5.38) and nine donors in group A (mean age 72.4, SD 1.88). The mean serum creatinine level in group $U$ was $64.66 \mathrm{mmol} / \mathrm{I}$ (SD 12.22), $120.2 \mu \mathrm{mol} / \mathrm{I}$ (SD 26.04), $120.91 \mu \mathrm{mol} / \mathrm{l}$ (SD 21.68) and $116.07 \mu \mathrm{mol} / \mathrm{l}$ (SD 21.35) preoperatively, on the first, third and fifth postoperative days, respectively. In group $A$ the mean serum creatinine level was $84.58 \mu \mathrm{mol} / \mathrm{I}$ (SD 23.63), $150.64 \mu \mathrm{mol} / \mathrm{l}$ (SD 38.67), 149.49 (SD 43.68) and $148.36 \mu \mathrm{mol} / \mathrm{I}$ (SD 39.08) preoperatively, first, third and fifth postoperative days, respectively. Calculated creatinine clearance in group $U$ was $102.62 \mathrm{ml} /$ minute $/ 1.73 \mathrm{~m}^{2}$ (SD 20.61), 50.44 $\mathrm{ml} /$ minute $/ 1.73 \mathrm{~m}^{2}$ (SD 10.28), $48.69 \mathrm{ml} /$ minute $/ 1.73 \mathrm{~m}^{2}$ (SD 7.88) and $51.62 \mathrm{ml} /$ minute/1.73 $\mathrm{m}^{2}$ (SD 8.96) preoperatively, first, third and fifth postoperative days, respectively, and in group A $78.11 \mathrm{ml} /$ minute/1.73 $\mathrm{m}^{2}$ (SD 23.31), $39.11 \mathrm{ml} /$ minute $/ 1.73 \mathrm{~m}^{2}$ (SD 10.35), $40.56 \mathrm{ml} /$ minute/1.73 $\mathrm{m}^{2}$ (SD 12.31) and $40.44 \mathrm{ml} /$ minute $/ 1.73 \mathrm{~m}^{2}$ (SD 11.36) preoperatively, first, third and fifth postoperative days, respectively. There is a statistically significant difference in serum creatinine level between groups $A$ and $U$ preoperatively $(P=0.039)$ and on the fifth postoperative day $(P=0.043)$, and there is a statistically significant difference in glomerular filtration rate between groups $\mathrm{A}$ and $\mathrm{U}$ preoperatively $(P=0.019)$, on the first $(P=0.018)$ and fifth postoperative days $(P=0.024)$.

Conclusions Deterioration of residual kidney function in the early postoperative period is higher in living donors above the age of 70. Patients over the age of 70 must be accepted with care for kidney donation.

P521

Augmented renal clearance in traumatic brain injury

A Udy, R Boots, S Senthuran, J Stuart, R Deans, M Lassig-Smith, J Lipman Royal Brisbane and Women's Hospital, Brisbane, Australia

Critical Care 2010, 14(Suppl 1):P521 (doi: 10.1186/cc8753)

Introduction Use of hypertonic saline and/or vasopressor infusion to achieve a desired cerebral perfusion pressure (CPP) in traumatic brain 
injury (TBI) is common. We hypothesised that the use of such therapies would significantly augment creatinine clearances $(\mathrm{CrCl})$ in this population. Methods Head-injured patients requiring hyperosmolar therapy using $3 \%$ or $20 \%$ saline solutions and/or norepinephrine infusion for the maintenance of a CPP $>60 \mathrm{mmHg}$ were recruited into the study. Additional management was consistent with local practice and in line with the Brain Trauma Foundation guidelines [1]. An 8-hour $\mathrm{CrCl}$, physiological variables, fluid balance, and medications were recorded daily during active management of CPP. A further $\mathrm{CrCl}$ was collected just prior to discharge (off CPP therapy), and if this was elevated, was repeated on the ward. Augmented renal clearance (ARC) was defined as a $\mathrm{CrCl}>160$ $\mathrm{ml} /$ minute $/ 1.73 \mathrm{~m}^{2}$ for males and $>150 \mathrm{ml} /$ minute $/ 1.73 \mathrm{~m}^{2}$ for females [2]. Results Twenty consecutive patients were enrolled. The average ICU length of stay was 15 days (Cl 95\% 11 to 18), and time to study entry averaged 2.3 days (CI 95\% 1.7 to 2.8). All patients received norepinephrine $(n=20), 85 \%$ $(n=17)$ received hypertonic saline, and therapy lasted on average 7.6 days (CI 95\% 5.6 to 9.5). ARC was demonstrated in 17 (85\%) patients at any point during active management of CPP. The mean maximum $\mathrm{CrCl}$ was $179 \mathrm{ml} /$ minute/1.73 $\mathrm{m}^{2}$ while on CPP therapy (Cl 95\% 159 to 198) returning to a mean $\mathrm{CrCl}$ of $111 \mathrm{ml} /$ minute $/ 1.73 \mathrm{~m}^{2}(\mathrm{Cl} 95 \% 91$ to 131, $P<0.001$ ) when measured in the ward. The mean $\mathrm{CrCl}$ in the ICU while not receiving CPP therapy was $150 \mathrm{ml} /$ minute $/ 1.73 \mathrm{~m}^{2}(\mathrm{Cl} 95 \% 134$ to $167, P=0.03)$. The mean time to reach peak $\mathrm{CrCl}$ while on active treatment was 4.7 days (Cl 95\% 3.0 to 6.4). Norepinephrine use, saline loading, mean arterial pressure, and central venous pressure, predicted $\mathrm{CrCl}$ on the day of measurement. Conclusions ARC is common in head-injured patients receiving active management of CPP and persists even after ceasing such therapy. This has significant implications for appropriate dosing of renally excreted drugs in this setting.

\section{References}

1. Bratton SL, et al: Guidelines for the management of severe traumatic brain injury. IX. Cerebral perfusion thresholds. J Neurotrauma 2007, 24(Suppl 1):S59-S64.

2. Stevens $L A$, et al:. Assessing kidney function - measured and estimated glomerular filtration rate. N Engl J Med 2006, 354:2473-2483.

\section{P522}

Pressure-controlled hemorrhagic shock in mice: a new model of acute kidney injury

N Mayeur', V Minville', A Jaafar², I Tack²

${ }^{1} \mathrm{CHU}$ Purpan, Toulouse, France; ${ }^{2} \mathrm{CHU}$ Ranqueil, Toulouse, France

Critical Care 2010, 14(Suppl 1):P522 (doi: 10.1186/cc8754)

Introduction At the present time, mice models have failed to reproduce human acute kidney injury (AKI) and new relevant models of AKI are required [1]. In this study we developed a model of AKI in mice based on pressure-controlled hemorrhagic shock that closely reproduces hypotension involved in most of human ischemic AKI.

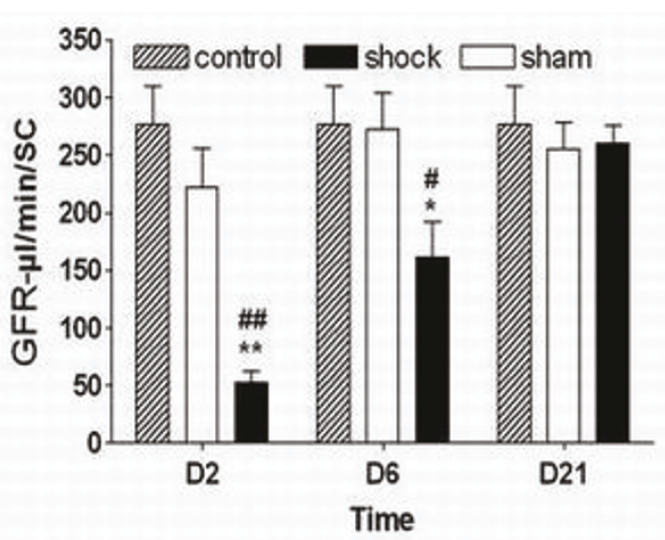

Figure 1 (abstract P522). Glomerular filtration rate 2 (D2), 6 (D6) and 21 (D21) days after the procedure.

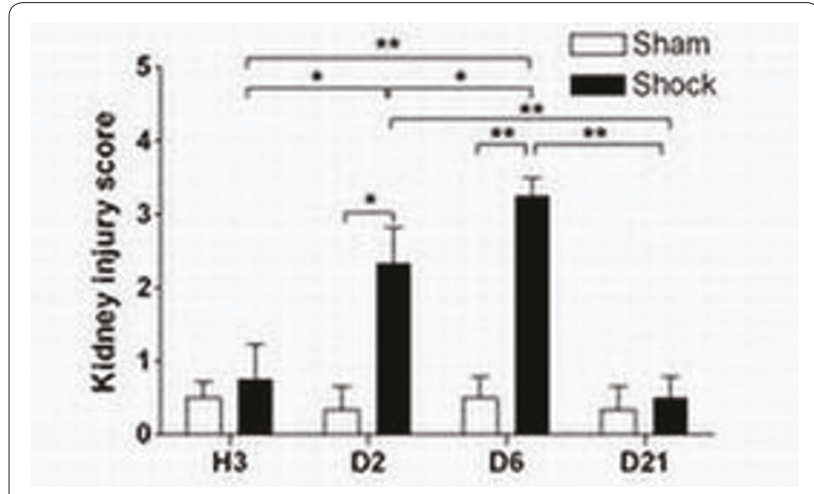

Figure 2 (abstract P522). Tubular injury score at H3, D2, D6 and D21. ${ }^{*} P<0.05$ and ${ }^{* *} P<0.01$.

Methods We first determined shock and resuscitation modalities in a C57/Bl6 mice population. A femoral arterial catheter was used for pressure control and bleeding and a central jugular catheter for anesthesia and resuscitation. We secondly explored renal repercussions of a 2 -hour shock duration at $35 \mathrm{mmHg}$ mean arterial pressure. We assessed successively the glomerular filtration rate (GFR), histological kidney injury score and performed real-time PCR after the procedure. The shock group was compared with sham mice and with a control group that underwent no procedure ( $n=5$ in each group).

Results The shock group GFR was decreased 2 (D2) and 6 (D6) days after shock when compared with control and sham (Figure 1). This AKI was intrinsic as showed by the $\mathrm{Na} / \mathrm{K}$ urinary ratio increase and the decrease in urine concentration ability. Tissular damage prevailed in the outer medulla with a maximal expression at D6 (paucicellular tubular epithelium, intratubular casts) (Figure 2). These lesions are associated with an increase of tissular KIM-1 and HIFs mRNA. Despite GFR normalization, the shock group showed discreet defect in urine concentration ability and a slight peritubular fibrosis 3 weeks after shock.

Conclusions This new model of AKI based on hypotension opens new perspectives in the field of short-term and long-term kidney function following AKI.

Reference

1. Rosen S, et al.: J Am Soc Nephrol 2008, 19.

\section{P523}

Continuous urinary electrolyte measurement in a swine model of mechanical ventilation

D Febres', T Langer', M Cressoni', A Protti', S Coppola', C Mietto',

A Santini', L Lombardi², M Lazzerini², P Cadringher', P Bertoli', L Gattinoni'

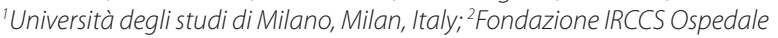
Maggiore Policlinico Mangiagallie Regina Elena, Milan, Italy; ${ }^{3}$ Università degli studi di Milano, Centro di Ricerche Chirurgiche Precliniche, Milan, Italy Critical Care 2010, 14(Suppl 1):P523 (doi: 10.1186/cc8755)

Introduction The kidney is the organ of the human body designed to sense and regulate intravascular volume. The KING (Kidney Instant Monitoring; Orvim, Paderno Dugnano, Italy) is a new device that allows continuous measurement of urinary electrolytes and real-time body response to changes in intrathoracic and intra-abdominal pressures and to lung injury.

Methods Sixteen pigs (weight $20 \pm 3 \mathrm{~kg}$ ) were anaesthetized, tracheotomized, catheterized and mechanically ventilated. Pigs were ventilated for approximately 10 hours with a TV of $10 \mathrm{ml} / \mathrm{kg}$, RR 15 breaths/minute, $\mathrm{F}_{1} \mathrm{O}_{2} 0.5$ and no PEEP. After TV was increased, pigs were divided into two groups: eight pigs with a lower TV and eight pigs with higher TV. A variable dead space was added to maintain normocapnia. Pigs were mechanically ventilated with the new TV up to 48 hours. The KING measured urinary output and urinary concentration of sodium and potassium every 10 minutes. $\mathrm{NaCl} 0.9 \%$ was exclusively infused. The average urinary concentration of each electrolyte was expressed as the total concentration 
for each electrolyte divided by the length of mechanical ventilation. Data were compared using the Wilcoxon test.

Results The higher TV group was ventilated with a TV of $890 \pm 251 \mathrm{ml}$ and a plateau pressure of $31 \pm 8 \mathrm{cmH}_{2} \mathrm{O}$ while the lower TV group had a TV of $472 \pm 262 \mathrm{ml}(P=0.006)$ and a plateau pressure of $17 \pm 9 \mathrm{cmH}_{2} \mathrm{O}$ $(P=0.02)$. The length of mechanical ventilation for the high TV group was $1,850 \pm 633$ minutes and for the low TV group was 3,341 \pm 749 minutes $(P=0.0006)$. The urinary output for the high TV was $37 \pm 16 \mathrm{ml} /$ hour and for the low TV was $52 \pm 19 \mathrm{ml} /$ hour $(P=0.10)$. The high TV group showed lower urinary sodium $(40 \pm 35 \mathrm{mEq} / \mathrm{l}$ vs $93 \pm 26 \mathrm{mEg} / \mathrm{l}, P=0.007)$, higher urinary potassium ( $52 \pm 12 \mathrm{mEq} / \mathrm{l}$ vs $35 \pm 23 \mathrm{mEq} / \mathrm{l}, P=0.04)$ and a lower urinary sodium/potassium ratio ( $0.77 \pm 0.7$ vs $3.86 \pm 3.3, P=0.001)$. Before TV was increased, urinary output, urinary concentrations of sodium, potassium and sodium/potassium ratio were not different.

Conclusions Data show that injurious ventilation is sensed by the kidney as a relative hypovolemia with reduction in sodium urinary output, increased excretion of potassium and inverted sodium/potassium ratio.

\section{P524}

Impact of positive fluid balance on survival in critically ill cancer patients

J Almeida, H Palomba, L Hajjar, VTorres, F Galas, M Ferreira, F Duarte, TTrielli, L Yu, J Otavio

Heart Institute, São Paulo, Brazil

Critical Care 2010, 14(Suppl 1):P524 (doi: 10.1186/cc8756)

Introduction Fluid overload has recently been linked to adverse outcomes in critically ill patients, but its impact on the outcomes of cancer patients admitted to ICUs has not been previously described. The aim of this study is analyze the impact of fluid overload on outcomes of patients with cancer needing intensive care.

Methods A total of 122 cancer patients admitted to the medical ICU in a 6-month period were prospectively evaluated for survival. Univariate and multivariate analysis were used to study ICU admission parameters associated with ICU mortality. Exclusion criteria were ICU stay <24 hours and chronic renal failure on dialysis.

Results Overall mortality was $15.5 \%(n=19)$. The mean age of all patients was $62.7 \pm 11.6$ years and $55 \%(n=68)$ were male. One hundred and six patients (86\%) had solid tumors and $16(14 \%)$ had hematologic malignancies. Postoperative care (49\%) and sepsis (38\%) were the major reasons for admission to the ICU. The mean APACHE II score value at ICU admission was $17.2 \pm 6.4$ and the mean Karnofsky score before ICU admission was $75.2 \pm 17.2$. At multivariate analysis, the following variables at ICU admission were significantly associated with ICU mortality in cancer patients: Lung Injury Score (LIS) >2 (OR 3.3; 95\% Cl 1.32 to 8.23) and positive fluid balance (for each $100 \mathrm{ml} / 24$ hours) (OR 1.03; $95 \% \mathrm{Cl} 1.01$ to 1.06 ).

Conclusions Fluid overload is independently associated with increased mortality in critically ill cancer patients. Further studies are necessary to determine the impact of positive fluid balance on acute organ dysfunction and overall prognosis of cancer patients.

\section{References}

1. Bouchard J, et al:: Fluid accumulation and acute kidney injury: consequence or cause. Curr Opin Crit Care 2009, 15:509-513.

2. Bouchard J, et al:. Fluid accumulation, survival and recovery of kidney function in critically ill patients with acute kidney injury. Kidney Int 2009, 76:422-427

P525

Fluid accumulation has an adverse effect on outcome of ICU patients with acute kidney injury

M Ostermann, H Cole, R Beale

Guy's and St Thomas' Foundation Hospital, London, UK

Critical Care 2010, 14(Suppl 1):P525 (doi: 10.1186/cc8757)

Introduction Management of acute kidney injury (AKI) is heterogeneous with little consensus about fluid therapy, vasopressors and diuretics. The aim was to analyse whether fluid management influences outcome in critically ill patients with AKI.
Table 1 (abstract P525). Mean cumulative fluid balance (SD) in ICU survivors and nonsurvivors

\begin{tabular}{lccc}
\hline Degree of AKI & Survivors & Nonsurvivors & $P$ value \\
\hline AKI I & $+2,527 \mathrm{ml}(118)$ & $+3,694 \mathrm{ml}(314)$ & $<0.0001$ \\
AKI II & $+4,348 \mathrm{ml}(929)$ & $+6,896 \mathrm{ml}(2,492)$ & 0.0007 \\
AKi III & $+3,228 \mathrm{ml}(173)$ & $+4,780 \mathrm{ml}(295)$ & $<0.0001$ \\
\hline
\end{tabular}

Methods Retrospective analysis of the data for 4,645 patients admitted to the multidisciplinary ICU at Guy's and St Thomas' Foundation Hospital between April 2004 and June 2009. AKI was defined according to the AKI network criteria, which distinguish between three different grades of AKI. Maximum degree of AKI and total cumulative fluid balance between ICU admission and the day of AKI were recorded.

Results A total of 1,225 patients (26.4\%) had AKI I, 29 patients (0.6\%) had AKI II and 1,183 patients (25.5\%) had AKI III of whom $89 \%$ were treated with renal replacement therapy. Two hundred and thirty-seven (5.1\%) patients had end-stage dialysis-dependent renal failure and 1,971 patients (42.4\%) had no AKI during their stay in the ICU. The ICU mortality was $15.6 \%$ in AKI I, $17.2 \%$ in AKI II and $34.9 \%$ in patients with AKI III (AKI I vS AKI III: $P<0.0001$ ). Cumulative fluid balance at diagnosis of AKI was significantly higher in patients who later died (Table 1).

Conclusions There is a correlation between fluid accumulation on the day of AKI and subsequent ICU outcome. Among patients with any degree of AKI, nonsurvivors had a higher cumulative fluid balance compared with ICU survivors. Future analysis needs to determine whether fluid accumulation was the cause of worse outcome, the result of more aggressive resuscitation or a marker of more severe AKI.

P526

Significant microalbuminuria, on admission to the ICU, may indicate AKI in patients with sepsis

S Basu', M Bhattacharya' ', T Chatterjee², S Todi', A Majumdar'

'AMRI Hospitals, Kolkata, India; '2 Jadavpur University, Kolkata, India

Critical Care 2010, 14(Suppl 1):P526 (doi: 10.1186/cc8758)

Introduction In sepsis, a generalised inflammatory onslaught causes systemic endothelial dysfunction; the glomerular manifestation of which is microalbuminuria. Recent histopathological evidence of extensive glomerular capillary infiltration by leucocytes in septic AKI suggests that endothelial dysfunction may play a particularly important role in this condition compared with patients with other causes of AKI. Markers of endothelial dysfunction such as microalbuminuria may therefore have a potential diagnostic role in septic AKI.

Methods Prospective observational study in a 20-bed ICU in a tertiarycare hospital. Microalbuminuria estimated as the spot urine albumincreatinine ratio (ACR, mg/g) was measured on ICU admission. Two hundred and sixty-six patients were recruited between January 2007 and December 2008. Patients with an ICU stay of less than 24 hours, pregnancy, menstruation, anuria, hematuria, proteinuria due to renal and post-renal structural diseases were excluded. Sepsis was diagnosed by the ACCP/ SCCM criteria; AKI was identified on admission using the RIFLE criteria.

Results Of the 266 patients studied, 130 patients had no AKI while 136 patients had AKI as classified by the RIFLE criteria. Of these 136 patients, 59 patients were in the Risk category, 62 in the Injury and 15 patients in the Failure, of which 26 patients (9.8\%) needed RRT. For the entire cohort, median age was 61.5 years, $61 \%$ were male, median APACHE II score was 15. Of these, 56 (21\%) of patients died on the ICU. ACR showed significant correlation with serum creatinine values $(P=0.0049$, Spearman rank correlation $(r)=0.173)$ and was negatively associated with estimated GFR $(P=0.0002, r=-0.225)$. The median level of ACR $(204.1 \mathrm{mg} / \mathrm{g}$, (IQR 119.7 to 402.0)) of patients with septic AKI $(n=66)$ on admission to the ICU was significantly greater $(P<0.0001)$ than the median ACR level of $67.1 \mathrm{mg} / \mathrm{g}$ (IQR 32.99 to 129.9) of patients who had AKI from other causes $(n=70)$. Patients of sepsis who had developed AKI also had higher ACR than those who did not $(n=51)$ (ACR $119.3 \mathrm{mg} / \mathrm{g}$ (IQR 37.02 to 354.3)) ( $P=0.0137)$

Conclusions Presence of significant microalbuminuria on admission to the ITU may be an early indicator of AKI in sepsis patients. 
P527

Neutrophil gelatinase-associated lipocalin as an early indicator for postoperative renal failure

CD Van der Marel, H De Geus, J Bakker

ErasmusMC, Rotterdam, the Netherlands

Critical Care 2010, 14(Suppl 1):P527 (doi: 10.1186/cc8759)

Introduction Neutrophil gelatinase-associated lipocalin plasma (pNGAL) and urine (UNGAL) concentrations can be used as early indicators for the development of acute kidney injury (AKI) [1]. As AKI is associated with higher risk of morbidity and mortality in ICU patients [2], higher pNGAL and UNGAL on admission might be relevant to the clinical course. No data on PNGAL and UNGAL as early indicators of postoperative AKI have been published. Methods We collected plasma and urine samples from patients admitted postoperatively following noncardiopulmonary bypass-related surgery. NGAL concentrations were measured using an immunofluorescence assay. Subsequently we compared the time points of maximum pNGAL, UNGAL and SCr concentrations in the group of patients developing postoperative AKI (Mann-Whitney U test). Data are presented as mean \pm SD.

Results One hundred and ninety-five consecutive patients (age: $58 \pm 16$ years; APACHE II $15 \pm 6$ ) were included. Twenty-two patients (age $63 \pm 17$; APACHE $\| 20 \pm 7$ ) developed AKI (AKI $1 n=14$; AKI $2 n=3$; AKI $3 n=5$ ). Time to reach peak ( $\left.T_{\text {max }}\right)$ concentrations for pNGAL, UNGAL and $\mathrm{SCr}$ are respectively 21 (21), 20 (22), 31 (32) for AKI 1, 39 (32), 24 (31), 54 (12) for AKI 2, and 17 (18), 26 (24), 34 (27) for AKI 3. Maximum concentrations of pNGAL $(\mathrm{ng} / \mathrm{ml}), \mathrm{uNGAL}(\mathrm{ng} / \mathrm{ml})$ and $\mathrm{SCr}(\mathrm{mmol} / \mathrm{l})$ are respectively 1,348 (1,569), 364 (220), 147 (38) for AKI 1, 424 (309), 949 (389), 175 (28) for AKI 2, and 2,375 (1,731), 726 (398), 265 (81). Figure 1 shows time course of pNGAL and UNGAL in patients developing AKI. $T_{\text {max }}$ of pNGAL and UNGAL was significantly earlier than $\mathrm{T}_{\max }$ of $\mathrm{SCr}$ (both $a<0.001$ ).

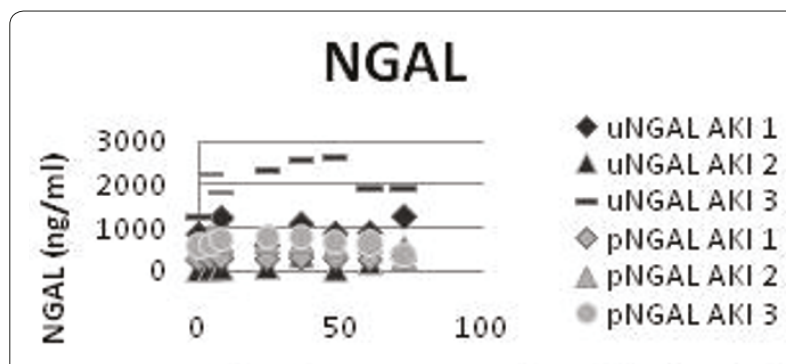

Time after postoperative admission (hr)

Figure 1 (abstract P527).

Conclusions In postoperative patients developing AKI, pNGAL and UNGAL reach peak concentrations 1 day earlier than $\mathrm{SCr}$. This might be of clinical importance as it provides us with an opportunity to prevent further deterioration of renal function at an earlier stage.

\section{References}

1. Mishra J: Lancet 2005, 365:1231-1238.

2. Metnitz PG: Crit Care Med 2002, 30:2051-2058

\section{P528}

Neutrophil gelatinase-associated lipocalin as an early indicator for mortality in adult critically ill patients with acute kidney injury

WP Ridder, H De Geus, J Bakker

Erasmus MC, Rotterdam, the Netherlands

Critical Care 2010, 14(Suppl 1):P528 (doi: 10.1186/cc8760)

Introduction Neutrophil gelatinase-associated lipocalin (NGAL) measured in plasma or urine has shown to be an excellent early predictor for the development of acute kidney injury (AKI) [1]. Furthermore, AKI is an independent risk factor for mortality in critically ill patients [2]. We therefore hypothesized that NGAL concentrations measured at ICU admission in plasma and urine are associated with mortality.

Methods We collected plasma and urine samples at ICU admission from all consecutive admitted patients $(n=700)$, of whom 171 during their first week of admittance, meeting criteria for any stage of AKI according to RIFLE classification for serum creatinine ( $\mathrm{sCr}$ ) increase only. NGAL concentrations were measured using a rapid immunofluorescence assay (Triage ${ }^{\circledast}$ meter plus; Biosite Inc., San Diego, CA, USA). The Mann-Whitney U test was used for comparison of mean NGAL concentrations in plasma and urine between survivors and nonsurvivors. Exclusion criteria were patients with a history of nephrectomy, chronic or end-stage renal disease, kidney transplant surgery and informed consent refusal $(n=36)$.

Results One hundred and seventy-one patients met criteria for AKI and were included in the statistical analysis. Median (25th to 75th percentile) age (years), APACHE II score after 24 hours and on admission sCr concentrations for survivors $(n=105)$ and nonsurvivors $(n=66)$ were respectively 59 (46 to 68) and 64 (53 to 73), 21 (15 to 26) and 26 (22 to 31 ), and 65 (60 to 80 ) and 80 (65 to 100) $\mu \mathrm{mol} / \mathrm{l}$. Median (25th to 75 th percentile) NGAL plasma and urine concentrations were respectively 300 (160 to 504$) \mathrm{ng} / \mathrm{ml}$ and $426(127$ to 1,543$) \mathrm{ng} / \mathrm{ml}$ for the survivors and 457 (281 to 991$) \mathrm{ng} / \mathrm{ml}$ and $1,189(381$ to 4,124$) \mathrm{ng} / \mathrm{ml}$ for the nonsurvivors. These differences for both plasma and urine NGAL values were statistically significant (both $P<0.005$ ).

Conclusions Plasma and urine NGAL concentrations at ICU admission are significantly higher in nonsurviving patients compared with concentrations in surviving patients. Therefore NGAL measured at admission might be an early indicator for mortality.

References

1. Mishra J: Lancet 2005, 365:1231-1238.

2. Metnitz PG: Crit Care Med 2002, 30:2051-2058.

\section{P529}

Urinary and plasma NGAL levels reflect the severity of acute kidney injury

K Bangert, LO Uttenthal

BioPorto Diagnostics A/S, Gentofte, Denmark

Critical Care 2010, 14(Suppl 1):P529 (doi: 10.1186/cc8761)

Introduction NGAL levels are dramatically increased in urine and plasma from patients with acute kidney injury (AKI). In this study we tested the clinical utility of NGAL in critically ill patients with many confounding conditions including sepsis. In addition, a new fully automated NGAL assay was validated with a selection of clinical samples from these patients.

Methods Plasma and urinary NGAL was monitored (daily to alternate days) in 135 consecutive patients admitted to intensive care. AKI was classified according to the RIFLE criteria. Three patients were excluded because of incomplete data. NGAL was determined with an ELISA kit (BioPorto Diagnostics). Differences between maximal NGAL levels in the patient were analyzed nonparametrically by the Kruskal-Wallis test. Data are reported as median (interquartile range) in $\mathrm{ng} / \mathrm{ml}$. A fully automated immunoturbidimetric NGAL test (The NGAL Test; BioPorto Diagnostics) was tested. The NGAL Test was validated by means of linearity studies with dilutions of calibrator material, determination of antigen excess zone and a method comparison with the ELISA kit on 40 urine and 40 plasma samples with NGAL levels from 25 to $2,995 \mathrm{ng} / \mathrm{ml}$ as measured by ELISA. Results Sixty-two patients did not have AKl; 15 were classified as risk (R), 11 as injury (I) and 44 as failure (F). The urinary NGAL was significantly $(P<0.0001)$ higher in patients with AKI (R 377 (102 to 830), I 519 (321 to 1,845) and $\mathrm{F} 2,747$ (559 to 7,621)) than in patients without AKI (51 (29 to 213)). Plasma NGAL levels were significantly $(P<0.0001)$ higher in patients with AKI (R 354 (259 to 511), I $546(331$ to 1,106) and F 1,062 (584 to 1,817)) than in patients without AKI (199 (128 to 308)). Linearity studies of The NGAL Test demonstrated a measuring range from 25 to $5,000 \mathrm{ng} / \mathrm{ml}$. No deleterious effect of antigen excess was seen up to a level of $40,000 \mathrm{ng} / \mathrm{ml}$. There was good agreement between the ELISA and The NGAL Test results, the Pearson coefficient of correlation being 0.98 for both urine and plasma samples.

Conclusions It is concluded that NGAL is dramatically increased in the urine and plasma of unselected critically ill patients with AKI and the degree of injury is reflected by the observed levels despite several confounding conditions. NGAL determination may therefore be useful for the diagnosis of renal injury and monitoring the management of patients admitted to intensive care. Validation of The NGAL Test demonstrates a performance that complies with the expectations for a fully automated clinical laboratory assay, which can thus be a valuable tool for the management of critically ill patients. 
P530

Economic impact and cost-effectiveness of urine neutrophil gelatinase-associated lipocalin following cardiac surgery

A Shaw', D Chalfin²

'Duke University Medical Center, Durham, NC, USA; ${ }^{2}$ Abbott Diagnostics,

Abbott Park, IL, USA

Critical Care 2010, 14(Suppl 1):P530 (doi: $10.1186 /$ cc8762)

Introduction Acute kidney injury (AKI) is common after cardiac surgery and recognition with specific biomarkers may improve outcome. We assessed the impact and cost-effectiveness (CE) of urine neutrophil gelatinase-associated lipocalin (UNGAL) for AKI diagnosis compared with current methods.

Methods We developed a decision analysis model to evaluate the CE of UNGAL plus standard diagnostic tests (for example, BUN, creatinine) compared with standard methods alone. The model was developed from the UK societal using costs per quality-adjusted life years (QALY). Model parameters were derived from the literature, UK economic data, and MD interviews. The base case was a 68-year-old male patient undergoing coronary artery bypass surgery. Sensitivity analyses were performed to determine how CE would vary with changes in the underlying model variables.

Results The base case yielded expected costs of $£ 4,244$ and 11.86 QALYS for NGAL compared with $£ 4,672$ and 11.79 QALYs without NGAL. The CE ratio for the NGAL strategy was $£ 358 / \mathrm{QALY}$ compared with $£ 396 / \mathrm{QALY}$ for the standard regimen. CE increased as treatment effect - defined as the ability to prevent AKI progression (Injury or Failure) - for the therapy triggered by an elevated NGAL level rose. Sensitivity analysis showed that the model was most responsive to the probability of developing AKI and least sensitive to the cost of the NGAL test. Probabilistic sensitivity analysis demonstrated that the NGAL strategy was the more cost-effective option at a willingness-to-pay threshold of $£ 30,000 / \mathrm{QALY}$ for each of the 1,000 simulations (Figure 1).

Conclusions Use of UNGAL appears to be cost-effective in the diagnosis of AKI after cardiac surgery.

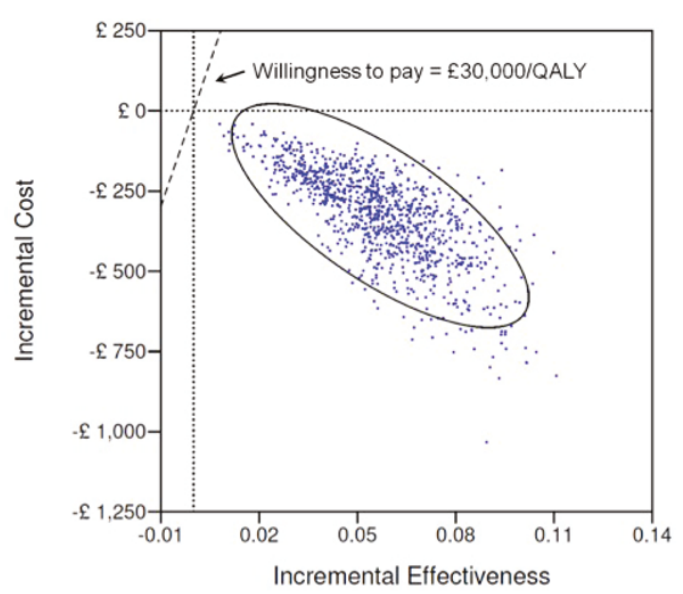

Figure 1 (abstract P530). Probabilistic sensitivity analysis of incremental CE of NGAL vs standard methods.

P531

Neutrophil gelatinase-associated lipocalin predicts acute kidney injury in critically ill patients

K Núñez, J Baldirà, M Cuartero, H Aguirre, M Rodríguez, P Vera, LL Zapata, A Roglan, J Ordóñez, AJ Betbesé

Hospital de la Santa Creu i Sant Pau, Barcelona, Spain

Critical Care 2010, 14(Suppl 1):P531 (doi: 10.1186/cc8763)

Introduction Plasma and urinary concentrations of neutrophil gelatinaseassociated lipocalin (NGAL) are early biomarkers of acute kidney injury (AKI) [1]. Most studies about the NGAL value to predict AKI were done in patients after cardiac surgery and not enough information about its usefulness in critically ill patients exists. To analyze the predictive value of plasma NGAL to predict AKI, evaluated by RIFLE score, in critically ill patients, particularly in those with severe sepsis and septic shock.

Methods NGAL was measured in whole-blood (Triage ${ }^{\oplus}$ NGAL Test; Inverness Medical, UK) at admission and after 48 hours in patients admitted to a general ICU. Patients were classified according to RIFLE score at admission and 24 and 48 hours thereafter and according to NGAL concentrations at admission using values suggested by Constatin and colleagues [2] ( $\leq 155$ or $>155 \mathrm{ng} / \mathrm{ml}$ ). NGAL values at admission were compared in patients under systemic inflammatory response syndrome (SIRS), severe sepsis and septic shock.

Results Sixty patients were included (28 female) with mean age $58 \pm$ 18.6 years, SAPS $\| 40.6 \pm 15.7$, SOFA $6.9 \pm 3.9$, length of ICU stay of $11.4 \pm$ 11.1 days and mortality of $23 \%$. Forty patients developed SIRS, six severe sepsis and 14 septic shock. Eleven patients developed AKI (RIFLE F), 10 of them at ICU admission; extracorporeal renal therapies were required in six cases. Thirty patients have NGAL $\leq 155 \mathrm{ng} / \mathrm{ml}$. When patients were classified according to RIFLE score at 24 hours of admission, NGAL values at admission were $180 \pm 170 \mathrm{ng} / \mathrm{ml}$ in 36 patients with RIFLE 0, $243 \pm 317$ $\mathrm{ng} / \mathrm{ml}$ in seven patients with RIFLE R, $303 \pm 134 \mathrm{ng} / \mathrm{ml}$ in four patients with RIFLE I and $536 \pm 421 \mathrm{ng} / \mathrm{ml}$ in nine patients with RIFLE $F(P=0.003)$. One patient was discharged before 24 hours (NGAL $<60 \mathrm{ng} / \mathrm{ml}$ ); whereas three patients who died before 24 hours had NGAL at admission of $578 \pm$ $350 \mathrm{ng} / \mathrm{ml}$. The area under the ROC curve of NGAL at admission for the prediction of AKI was 0.83 ( $95 \%$ confidence interval 0.70 to $0.97, P=0.001$ ), with an optimal cutoff value of $277 \mathrm{ng} / \mathrm{ml}$ with $73 \%$ sensitivity and $77 \%$ specificity. NGAL concentrations at admission were $160 \pm 159 \mathrm{ng} / \mathrm{ml}$ in 40 patients with SIRS, $335 \pm 206 \mathrm{ng} / \mathrm{ml}$ in six patients with severe sepsis and $540 \pm 347 \mathrm{ng} / \mathrm{ml}$ in 14 patients with septic shock $(P<0.001)$.

Conclusions Plasma NGAL concentrations measured at ICU admission appeared a useful predictor of AKI in critically ill patients; in addition, NGAL concentrations showed an increasing pattern from SIRS to severe sepsis and septic shock.

\section{References}

1. Misra J, et al.: Lancet 2005, 365:1231.

2. Constantin JM, et al.: J Crit Care 2009, in press.

\section{P532}

Study of serum vancomycin levels in different modalities of hemodialysis in Indian patients

C Sarkar, A Majumdar, S Todi

AMRI Hospitals, Kolkata, India

Critical Care 2010, 14(Suppl 1):P532 (doi: 10.1186/cc8764)

Introduction To evaluate the vancomycin trough level in patients on intermittent hemodialysis (IHD), slow low-efficiency dialysis (SLED) and continuous renal replacement therapy (CRRT) and to assess the appropriateness of an improvised protocol, which is to repeat the vancomycin dose $(15 \mathrm{mg} / \mathrm{kg})$ after 5 days on IHD, after 3 days on SLED and after 2 days in CRRT.

Methods This study was conducted at AMRI Hospitals, Kolkata between November 2008 and April 2009. Thirty-three patients were studied. Fifteen were on IHD, 15 were receiving SLED and three were on CRRT. Mean age for the IHD group was $56.09 \pm 11.4$, for SLED was $64.3 \pm 13.4$, and for CRRT was $68 \pm 8.34$. The male:female ratio was 2:1 for IHD, 1.5: 1 for SLED and 2:1 for CRRT. Patients with severe hepatic impairment, obesity, cystic fibrosis and neoplastic disorder were excluded from this study. The mean Charlson score was $3.6 \pm 4.8$ for the IHD group, $5.26 \pm 1.16$ for SLED and $8.6 \pm 11.2$ for CRRT, respectively. High-performance liquid chromatography was used for assay of vancomycin.

Results The mean vancomycin trough levels were $18 \pm 3.99$ on day 5 for patients on IHD, $15.3 \pm 4.1$ on day 3 for patients on SLED and $11.9 \pm 2.4$ for patients on CRRT. This prospective observational study is unique, in the sense that data are sparse regarding vancomycin levels, on various modalities of hemodialysis. The second and more important observation from this study was that our improvised dosing of vancomycin needs revision, since, of the patients on dialysis, $80 \%(12 / 15)$ of patients on IHD and $60 \%(9 / 15)$ of patients on SLED had higher than the recommended trough level $(15 \mu \mathrm{g} / \mathrm{ml})$, whereas for patients on CRRT the trough levels were subtherapeutic at 30 hours. 
Conclusions This pilot study underscores the need for more awareness, mandatory checking of vancomycin trough levels and a larger study in Indian patients to definitively determine dosage guidelines in this ethnic population.

\section{P533 \\ RIFLE criteria for acute kidney dysfunction following liver transplantation: incidence and risk factors}

A Kundakci, A Pirat, O Komurcu, A Torgay, H Karakayalı, G Arslan, M Haberal Baskent University Hospital, Ankara, Turkey

Critical Care 2010, 14(Suppl 1):P533 (doi: 10.1186/cc8765)

Introduction Acute renal dysfunction (ARD) is one of the most common complications of orthotopic liver transplantation (OLT), with an incidence ranging from 12 to $64 \%[1,2]$. These patients are often critically ill in the perioperative period with a number of other co-morbidities, resulting in an increased susceptibility to ARD. The RIFLE criteria were developed to provide a consensus definition for acute renal dysfunction in critically ill patients. Using the RIFLE criteria, we aimed to determine the incidence and risk factors for acute renal dysfunction in the early postoperative period.

Methods The records of 112 patients who underwent OLT, performed by the same team in the Baskent University Hospital, from January 2000 to February 2009, were retrospectively analyzed. Three levels of renal dysfunction, outlined in the RIFLE criteria, were investigated. Collected data included demographic features; co-morbidities; etiology of the liver failure; perioperative laboratory values; intraoperative hemodynamic parameters; use and volume of crystalloids, colloids, blood products, cell saver system, and albumin; portal vein clamping time; requirement for inotropes, vasopressors, and antihypertensive drugs; duration of anesthesia; and urine output.

Results ARD occurred in 64 of OLTs (57\%). Risk, injury, and failure frequencies were $19 \%, 11 \%$, and $28 \%$, respectively. Compared with those who did not have ARD postoperatively, those who did had significantly higher MELD scores ( $19 \pm 7$ vs $16 \pm 8 ; P=0.018)$, more frequently required inotropic agents intraoperatively (54\% vs $35 \% ; P=0.070$ ), received more colloids ( $300 \pm 433 \mathrm{ml}$ vs $105 \pm 203 \mathrm{ml} ; P=0.007$ ), had a longer portal vein clamping time $(88.0 \pm 42.0$ minutes vs $73.0 \pm 20.0$ minutes; $P=0.037)$ and had a higher incidence of intraoperative acidosis (64\% vs $44 \%$; $P=$ 0.047 ). Logistic regression analysis was performed using these data to find the predictors for development of ARD. ARD was found to be associated with MELD score (odds ratio $1.107,95 \% \mathrm{Cl} 1.022$ to $1.200, P=0.013$ ), portal vein clamping time (odds ratio $1.02095 \% \mathrm{Cl} 1.000$ to $1.040, P=0.053$ ) and intraoperative acidosis (odds ratio $3.610,95 \% \mathrm{Cl} 1.212$ to $10.753 P=0.021$ ). Conclusions Based on RIFLE criteria, more than one-half of the patients who underwent liver transplantation developed ARD postoperatively. A higher MELD score, longer portal vein clamping time, and occurrence of intraoperative acidosis were identified as the independent risk factors for development of ARD in this group of patients.

\section{P534}

Prophylactic use of laxative for constipation in critically ill patients Y Masri, J Abu Baker, R Ahmed

Rashid Hospital Trauma Center, Dubai, United Arab Emirates

Critical Care 2010, 14(Suppl 1):P534 (doi: 10.1186/cc8766)

Introduction The aim of this study was to evaluate the use of laxative prophylaxis for constipation in the ICU and the impact of early versus late bowel movement on patient outcome.

Methods After informed consent all adults in the ICU who were expected to stay on a ventilator for $>72$ hours were randomized to Control and Interventional groups. Patients who had major bowel surgery were excluded. The Control group did not receive any intervention for bowel movement for the first 72 hours, while the Interventional group received a prophylactic dose of Lactulose $20 \mathrm{cc}$ orally every 12 hours for the first 72 hours. After 72 hours the management of constipation in the both groups was left to the physician's discretion. Data were collected for age, sex, diagnosis, Simplified Acute Physiology Score (SAPS) II, doses of narcotics and sedatives, enteral nutrition, ventilator days, ICU days, bowel movement $<72$ hours and days of first bowel movement.
Results Over a 6-month period a total of 100 patients were enrolled, 50 patients in each Control and Interventional group. Mean age was 38.8 years in Control and 37.5 years in Interventional group. Both groups had a predominance of male patients. The most common diagnosis in both groups was polytrauma. Mean SAPS II for both was 35 . One hundred percent of patients were ventilated, with a mean dose of fentanyl (323.8 \pm $108.89 \mu \mathrm{g} /$ hour in Control and $345.83 \pm 94.43 \mu \mathrm{g} /$ hour in Interventional group) and a mean dose of midazolam (11.1 $\pm 4.04 \mathrm{mg} /$ hour in Control and $12.4 \pm 3.19 \mathrm{mg} /$ hour in Interventional group). Enteral nutrition was maintained and tolerated by $100 \%$ of patients. There were only two (4\%) patients in the Control group and nine (18\%) patients in the Interventional group who had bowel movement in $<72$ hours $(P<0.05)$. Twenty-three (46\%) patients in the Control and 29 (58\%) patients in the Interventional group had bowel movement after 5 days. Mean ventilator days were 16.19 and 17.36 days in the Control and Interventional groups. Subgroup analysis showed that the patients who moved their bowel in $<5$ days in both groups had mean ventilator days of 18.5 , while it was 15.88 days for the patients who moved their bowel after 5 days in both groups $(P<0.05)$. Forty-eight (96\%) patients in each group were discharged from the ICU. Mean ICU days for the Control group was $21.15 \pm 10.44$ and was $20.77 \pm$ 8.33 days for the Interventional group. A total of two (4\%) patients died in each group.

Conclusions Laxative prophylaxis can be used successfully to prevent constipation in ICU patients. Late bowel movement $>5$ days is associated with less ventilator days, compared with early $<5$ days bowel movement.

\section{P535}

Study of intra-abdominal hypertension in the medical intensive care unit

PTheerawit

Ramathibodi Hospital, Bangkok, Thailand

Critical Care 2010, 14(Suppl 1):P535 (doi: 10.1186/cc8767)

Introduction Intra-abdominal hypertension $(\mathrm{IAH})$ is associated with significant mortality in surgical and trauma patients. However, IAH can occur in medical patients as well. Thus, the main objective of this study was to assess whether the IAH during the first 24-hour period of admission was an independent predictor for 28-day mortality.

Methods We conducted a prospective observational study. All patients admitted to the medical ICU were enrolled in this study and underwent intra-abdominal pressure (IAP) measurement via the bladder. The primary outcome was the association between IAH and 28-day mortality rate.

Results Seventy-seven eligible patients were enrolled to this study. The incidence of IAH was 44\%. The mean age was $60.52 \pm 18.34$ years. In terms of 28-day mortality, there was no association between the presence of IAH and mortality outcome. However, regarding ICU mortality, nonsurvivors had significantly higher mean IAP within 24 hours of admission than survivors: 11.55 ( 8.0 to 20.3 ) vs 9.95 (3.0 to 27.0$)(P=0.041$ ).

Conclusions Neither the mean IAP nor the presence of IAH within the first 24 hours of admission was an independent predictor for 28-day mortality. Nonetheless, this study demonstrated the difference of IAP between survivors and nonsurvivors in terms of ICU mortality in the medical ICU.

\section{References}

1. Malbrain ML, Cheatham ML, Kirkpatrick A, et al.: Results from the International Conference of Experts on Intra-abdominal Hypertension and Abdominal Compartment Syndrome. I. Definitions. Intensive Care Med 2006, 32:1722-1732.

2. Malbrain ML, Chiumello D, Pelosi P, et al:. Incidence and prognosis of intraabdominal hypertension in a mixed population of critically ill patients: a multiple-center epidemiological study. Crit Care Med 2005, 33:315-322.

3. Malbrain ML, Chiumello D, Pelosi P, et al:: Prevalence of intra-abdominal hypertension in critically ill patients: a multicentre epidemiological study. Intensive Care Med 2004, 30: 822-829.

4. Malbrain ML: Abdominal pressure in the critically ill: measurement and clinical relevance. Intensive Care Med 1999, 25:1453-1458.

5. Gudmundsson FF, Viste A, Gislason H, Svanes K: Comparison of different methods for measuring intra-abdominal pressure. Intensive Care Med 2002, 28: 509-514. 
P536

Intra-abdominal pressure values of emergency department intensive care unit patients and clinical outcomes

O Demirci, S Girisgin, B Cander, E Dogan, D Dündar

Selcuk University Meram Faculty of Medicine, Konya, Turkey

Critical Care 2010, 14(Suppl 1):P536 (doi: 10.1186/cc8768)

Introduction Intra-abdominal hypertension (IAH) and abdominal compartment syndrome (ACS) have become serious causes of morbidity and mortality in critical surgical and medical patients, especially in the past 10 years. Increased IAP levels became one of the routine physiologic parameter measurement elements in critical patients and indicate prognosis. In this study, our aim was to observe IAP increase, APB decrease and their clinical manifestations in ICU patients.

Methods Eighty-nine ICU patients were included in this study. IAP levels were measured by infusing $25 \mathrm{ml}$ saline into the urinary bladder. The symphisis pubis plane affiliated as the zero point. Patients were divided into groups according to IAP and APB levels. Clinical follow-up, required medical care and survival were investigated.

Results Within the 89 patients, 36 of them diagnosed as IAH and 34 of them diagnosed as low APB. There were 25 patients that had both IAH and low APB. In these patients, during the first 2 days of the study, statistically significant SGOT, SGPT, $\mathrm{PO}_{2}$, urea and creatinine levels were found. We also determined increased positive inotropic support and ventilatory support need with increased sepsis and multiple organ failure incidence in these patients. Mortality rates were strongly related to IAH and low APB levels.

Conclusions In critically ill patients, IAP measurement, a very simple and valuable method, must be performed. IAH and low APB levels are indicators of high morbidity and mortality rates. Therefore, IAP measurement may become a routine element in follow-ups and survival rate determinations, in critically ill patients.

\section{References}

1. Cheatham M, Malbrain M: Cardiovascular implications of abdominal compartment syndrome. Acta Clin Belg Supp/ 2007, 1:98-112.

2. Cheatham M, Malbrain M: Intra-abdominal hypetension and the cardiovascular system. In Abdominal Compartment Syndrome. Edited by Ivatury R, Cheatham M, Malbrain M, Sugrue M. Georgetown, TX: Landes Biosciences; 2006:89-104

3. Malbrain ML, De laet I: Functional haemodynamics during intraabdominal hypertension: what to use and what not use. Acta Anaesthesiol Scand 2008, 52:576-577.

\section{P537}

Advanced haemodynamic assessment of patients with liver disease and abdominal compartment syndrome

D Joshi, S Saha, W Bernal, J Wendon, G Auzinger

King's College Hospital, London, UK

Critical Care 2010, 14(Suppl 1):P537 (doi: 10.1186/cc8769)

Introduction Intra-abdominal hypertension ( $\mathrm{AH},>12 \mathrm{mmHg}$ ) and abdominal compartment syndrome (ACS, >20 mmHg) frequently complicate the disease course in critically ill patients with liver disease. There is a paucity of data describing the circulatory changes observed during ACS outside experimental studies. The aim of this study was to investigate baseline haemodynamic variables in patients with ACS and their response following abdominal decompression.

Methods Patients admitted to the liver ICU of King's College Hospital with ACS were studied. Transpulmonary thermodilution cardiac output monitoring and calculation of volumetric indices of preload was performed with the PiCCO system. MAP, CVP, ITBVI, cardiac index (Cl), stroke volume index (SVI) and intraabdominal pressure (IAP) were analysed pre and post intervention.

Results Eighteen patients (nine female), median age 43 (range (R) 20 to 65) were studied. Ten patients had decompensated Budd-Chiari syndrome (BCS), eight underwent orthotopic liver transplantation and two had surgical shunt procedures. Eight patients had cirrhosis (PC) with organ failure and severe ascites necessitating therapeutic paracentesis. Pre-intervention IAP was raised (median 24, R 17 to 40). As a whole, volumetric markers of pre-load were low (median ITBVI 674, R 453 to 1,619) but improved (median ITBVI 763, R 512 to 1,110 ) post intervention ( $P=$ 0.02 ). Reduction in CVP post intervention was not statistically significant $(16, R \quad 0$ to 25 vs 14, R 5 to 29). Compared with the PC group, the BCS group had persistently low ITBVI (median 633, R 453 to 924) despite aggressive volume resuscitation prior to decompression (median positive fluid balance $10 \mathrm{I}, \mathrm{R} 0.5$ to 39). Abdominal decompression resulted in a reduction in IAP (median 15, R 6 to 31, $P<0.0001$ ), increase in preload (ITBVI) and improvement in $\mathrm{Cl}(3.9, \mathrm{R} 1.9$ to 7.4 vs $4.6, \mathrm{R} 2.7$ to $7.7, P<0.0001)$ and SVI (40, R 22 to 111 vs 44, 30 to 61, $P=0.001)$. There was an inverse relationship between IAP, CI $(P=0.003)$ and SVI $(P=0.004)$. ITBVI correlated with parameters of flow $(C \mid P<0.0001$ and SVI $P<0.0001)$, CVP did not.

Conclusions ACS leads to central hypovolaemia. Abdominal decompression restores preload in association with improvement in cardiac output. References

1. Cheatham et al:: Intensive Care Med 2007, 33:951-962.

2. Malbrain et al:. Intensive Care Med 2006, 32:1722-1732.

\section{P538}

Molecular adsorbent recirculating system in hepatic failure patients with a ventricular assist device

M Cristinar, J Thiranos, H Kremer, C Chalumeau, J Mazzucotelli

Nouvel Hopital Civil, Strasbourg, France

Critical Care 2010, 14(Suppl 1):P538 (doi: 10.1186/cc8770)

Introduction The study we performed focuses on the removal of bilirubin in hepatic failure patients with a ventricular assist device (VAD). Bilirubin is a risk factor revealing the severe hepatic insufficiency and the alteration of the clinical status. The mortality rate of patients developing liver failure in the cardiac ICU is very high. The molecular adsorbent recirculating system (MARS) is the hepatic failure treatment in patients with no other therapeutic options. It is a liver support system that uses an albuminenriched dialysate to facilitate the removal of albumin-bound toxins. Conventional hemodialysis techniques have little or no effect on liver detoxication and cannot improve these patients' prognosis. The study aims to prove the decrease of the mortality rate in hepatic failure patients with VAD as a result of using MARS.

Methods We conducted a retrospective study of 55 patients with VAD. They were hospitalized in our cardiac ICU between January 2000 and November 2009. All patients had a hepatic check-up when admitted to the ICU. The patients with altered liver function had at least one checkup per day. We had seven patients with hyperbilirubinaemia $>350$ umol// (the threshold for starting hepatic dialysis). Five patients received MARS therapy. Hepatic check-ups were performed at the beginning and at the end of the session.

Results Five patients were dialysed. Patient 1 - with a maximum serum bilirubin level of $350 \mu \mathrm{mol} / \mathrm{l}$, needed four cycles of MARS therapy; the patient survived. Patient 2 - max bilirubin 390 umol/l, 6 MARS; the patient survived. Patient 3 - max bilirubin 700 umol//, 3 MARS; the patient did not survive. Patient 4 - max bilirubin 680 Mmol/I, 3 MARS; the patient survived. Patient 5 - max bilirubin 500 Mmol/I, 15 MARS; the patient did not survive. The two patients that did not receive MARS therapy did not survive.

Conclusions MARS is a viable option for the treatment of hepatic failure patients and, in particular, for those with VAD. We consider that without MARS a VAD patient cannot survive if his serum bilirubin level exceeds $350 \mu \mathrm{mol} / \mathrm{l}$. Our hypothesis needs to be confirmed by multicenter randomized trials.

\section{References}

1. Saliba F, et al: The Molecular Adsorbent Recirculating System (MARS) in the intensive care unit: a rescue therapy for patients with hepatic failure. Crit Care 2006, 10:118.

2. El-Banayosy A, et al: Evaluation of MARS technique on patients suffering from hypoxic liver failure following cardiogenic shock. ASAIO J 2002, 48:139.

\section{P539}

Molecular adsorbent recirculating system in patients with acute liver failure: prospective follow-up

D Tran-Zweifel, B Goubaux, L Bornard, J Dellamonica, M Carles, O Perus, M Raucoules

Hopital de l'Archet II - CHU de Nice, France

Critical Care 2010, 14(Suppl 1):P539 (doi: 10.1186/cc8771)

Introduction A prospective observational study on molecular adsorbent recirculating system (MARS) therapy in a liver-disease-dedicated ICU. 
Methods From January 2004 to November 2009, all patients with an acute liver failure fulfilling MARS therapy criteria, in relation to two main indications: primary and secondary to chronic liver failure (ALF/ACLF = group 1) or intractable pruritus (IP = group 2), were included: Data were collected before and after each MARS procedure. APACHE and MELD scores [1] were used to predict the ICU mortality and the 90-day mortality, respectively.

Results Thirty-nine patients, APACHE $\|=41$ (21 to 86); MELD = 29 (14 to 47), having received 98 treatments (one to five treatments), were included. The overall mortality was $n=22(56 \%)$. Since 35 patients have been treated for ALF/ACLF, 15 underwent a liver transplantation (OLT) (see Figure 1). The 90-day mortality of the 20 patients from group 1 without OLT was 44\%, compared with $76 \%$ predicted by the MELD score. MARS was effective to decrease the bilirubin levels $-35 \%$ (of the pretreatment rate which was 30 times the baseline), $P<0.01$, the ammoniemia levels $-50 \%$ (of the pretreatment rate which was four times the baseline), $P<0.01$, and serum creatinine levels $-40 \%$ (of the pretreatment rate which was two times the baseline). The MARS therapy was also effective to improve the pruritus after two treatments in the group 2 patients. A single severe complication was noticed as hemorrhagic shock quickly resolved in one patient (group 1).

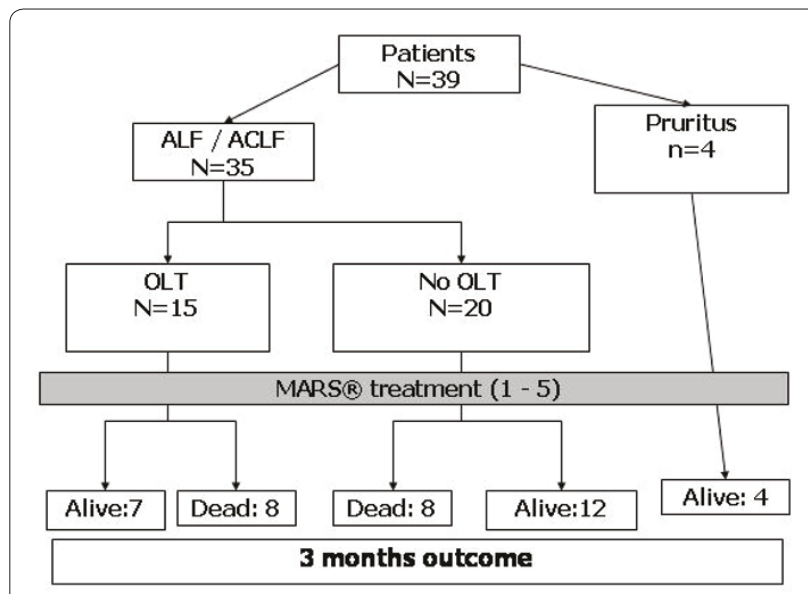

Figure 1 (abstract P539). Three-month outcome.

Conclusions MARS therapy improves liver function in patients with both acute and chronic liver failure, and allows those patients to wait until OLT, if necessary. Furthermore it also improves the 90-day survival of patients with ACLF having no OLT indication.

\section{References}

1. Hepatology 2001, 33:464-470

P540

A study of patients with cirrhosis admitted to nontransplant general intensive care in the UK: prevalence, case mix, outcomes and evaluation of critical illness and disease-specific scoring systems SJ Thomson', C Moran', ML Cowan', S Musa', R Beale², D Treacher², M Hamilton', M Grounds', TM Rahman

'St George's Hospital, London, UK; 'St Thomas' Hospital, London, UK Critical Care 2010, 14(Suppl 1):P540 (doi: 10.1186/cc8772)

Introduction Hospital admission rates attributable to cirrhosis are increasing in the UK. The evidence base for the outcome of critically ill patients with cirrhosis admitted to intensive care (ICU) is currently limited to data from tertiary transplant centres, which report mortality rates of between 45 and $74 \%$. We hypothesised that the disease cohort experienced in secondary care is less severe than that reported in the literature and would subsequently demonstrate lower mortality. We report the prevalence, case mix and outcomes of a dual-centre ICU study in London, UK.

Methods Data were collected prospectively from two large nontransplant general ICUs in London (St George's and St Thomas' Hospitals) over
20 months (November 2007 to June 2009). All ICU admissions were screened for cirrhosis. Clinical data were recorded to calculate and evaluate several critical illness and disease-specific scoring systems (APACHE II, SAPS II, SOFA, Child Pugh (CPS), MELD, UKELD, Glasgow Alcoholic Hepatitis Score and the Royal Free Hospital (RFH) score). Study endpoints were ICU and hospital mortality.

Results Cirrhosis accounted for 3.3\% (137/4,198) of ICU admissions. In 118 patients meeting inclusion criteria, ICU and hospital mortality rates were $38 \%$ and $47 \%$. Median (IQR) age was 50 (43 to 59) years, 68\% were male, median length of ICU stay was 4.5 ( 2 to 10 ) days and $72 \%$ had alcoholic cirrhosis. Median CPS was 10 (8 to 11) (45\% Grade A/B), MELD 18 (12 to 24) and APACHE II 16 (13 to 22). Hospital mortality was $24 \%$ in CPS grade $\mathrm{B}$ and $66 \%$ in grade C. After multivariate analysis, factors independently predictive of mortality were bilirubin, INR, urea, bicarbonate and $\mathrm{pO}_{2}$ / $\mathrm{FiO}_{2}$ ratio. AUROC for the scoring systems ranged from 0.76 (UKELD) to 0.81 (RFH) for the prediction of mortality. Renal failure (OR 3.4 (1.4 to 8.4)) and haemofiltration during admission (OR 9.6 (3.7 to 24.8)) had strong associations with mortality. Intubation for gastrointestinal bleeding $O R$ 0.4 (0.1 to 1.3) was associated with a trend towards better outcome than intubation for respiratory failure OR 2.7 (1.0 to 7.0).

Conclusions The mortality rates and disease stage reported here are lower than those described in the established literature. Data from specialist transplant centres should not be applied to the patient cohorts seen in general ICUs. We urge critical care physicians to carefully consider the individual clinical case when deciding on ICU admission and not apply potentially misrepresentative figures from previous datasets.

\section{P541}

\section{Acute liver failure: a European perspective}

J Wendon', W Bernal', P Laterre'2, F Nevens ${ }^{3}$, M Hudson $^{4}$, M Aldersly $^{3}$,

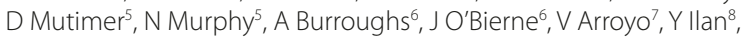
G Lalazar $^{8}, \mathrm{~K} \mathrm{Simpson}^{9}$, M Manns ${ }^{10}$, J Hadem ${ }^{10}$, F Larsen ${ }^{1}$

${ }^{1}$ King's College Hospital, London, UK; ${ }^{2}$ St Luc University Hospital, Brussels, Belgium: '3St James Hospital, Leeds, UK: ${ }^{4}$ University of Newcastle, UK; ${ }^{5}$ University of Birmingham, UK; ${ }^{6}$ Royal Free Hospital, London, UK; ${ }^{7}$ University of Barcelona, Spain; ${ }^{8}$ Hebrew University Hadassah Hospital, Jerusalem, Israel; ${ }^{9}$ Royal Infirmary Hospital, Edinburgh, UK; ${ }^{10}$ University of Hannover, Germany; "Rigshospital, Copenhagen, Denmark

Critical Care 2010, 14(Suppl 1):P541 (doi: 10.1186/cc8773)

Introduction Acute liver failure (ALF) is a rare disease with a spectrum of presentations from mild coagulopathy and altered conscious level to multiple organ failure and intracranial hypertension (ICH). A new group, Acute Liver Failure studies in Europe (ALSiE), has and wishes to further initiate collaboration between centres with clinical expertise in the management of ALF. It aims to establish a pan-European database of clinical and demographic data in ALF and initiate clinical studies.

Methods We describe the experience of 13 centres in seven countries over a 3-month period to 31 March 2009. ALF was defined as an INR >1.5 and encephalopathy in the absence of chronic liver disease. Results are presented as median and IQ range.

Results Eighty-five patients were treated, acetaminophen was the dominant aetiology in the UK, $60 \%$, and represented $34 \%$ in other centres. At presentation INR was 3.8 (2.1 to 6.5), hepatic encephalopathy (HE) grade I (0 to 2) was observed and 20\% required pressors. Grade III/IV coma was seen in $64 \%$ during their course and of these $25 \%$ developed ICH. Seventyone per cent required ventilation, 58\% renal replacement therapy and pressors in $65 \%$. Ninety per cent required management in a critical care environment. Overall survival was $75 \%$ - 42 cases fulfilled poor prognostic criteria (PPC), of whom 31 were transplanted, 28 (90\%) surviving to hospital discharge. Of the 11 remaining, four survived and seven died. Thirty-two out of 43 who did not fulfill PPC survived. Patients who died were older and had a predominant aetiology of hypoxic hepatitis. One organ support or less was associated with $70 \%$ survival with medical management alone. Two organ support or more was required in 55 patients and $27 \%$ survived with medical management, the remainder requiring transplantation or died.

Conclusions ALF has a high prevalence of progression to multiple organ failure requiring a multidisciplinary approach (critical care, transplant surgery and hepatology) to achieve optimal outcome. The development of ICH is $25 \%$ in grade III/IV HE. Despite this, outcomes are good. 
P542

Intracranial pressure monitoring in acute liver failure: a review of 10 years experience

M Peck, J Wendon, E Sizer, G Auzinger, W Bernal

King's College Hospital, London, UK

Critical Care 2010, 14(Suppl 1):P542 (doi: 10.1186/cc8774)

Introduction Intracranial hypertension (ICH) complicates around 25\% of grade III/IV encephalopathy in acute liver failure. Intracranial pressure (ICP) monitoring is controversial in this coagulopathic population.

Methods We examined the ITU charts and prospectively maintained an ITU database of all patients admitted with acute liver failure who underwent ICP monitoring from December 1998 to December 2008. Data are presented as median and range.

Results One hundred and fifteen patients were identified. Aetiology was acetaminophen (78), seronegative (14), drug induced (7), viral (14) and autoimmune (two). All patients had a Camino ICP bolt (subdural) inserted by the intensive care specialist. Coagulation support fresh frozen plasma $(10 \mathrm{ml} / \mathrm{kg})$ and platelets if the count was $<100$ were given. Recombinant factors were not given. Overall 66 survived and 49 died. Seventy patients underwent transplantation with $77 \%$ survival. Age was 31 (24 to 42),

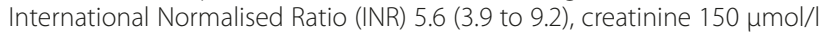
(105 to 143), Na 139 (135 to 143). INR pre insertion of the bolt was 3.5 (2.2 to 7.3), platelets 109 (51 to 137). At bolt insertion, pulse was 100 (89 to 111), MAP 80 (72 to 89), ICP 19.5 (12 to 25), CO 4.8 (4.3 to 5.5), lactate 3.5 (2.2 to 5.3), jugular venous saturation 76 (67 to 84 ), temperature 35.5 (34.8 to 36.4). Norepinephrine dose was $0.12 \mu \mathrm{g} / \mathrm{kg} /$ minute (0.02 to 0.4 ). ICP fell to 14 (9 to 19) at 6 hours and 13 ( 8 to 20) at 24 hours. Peak ICP was 30 (20 to 39 ), number of surges $>20$ per patient was 2 ( 0 to 7 ). Minimum cerebral perfusion pressure (CPP) was 45 (38 to 52). The pre-liver-transplant peak ICP was 27 (19 to 35), post was 23 (20 to 33). There were no correlates between clinical parameters, ICP or CPP at 0, 6, 12 or 24 hours. No difference in insertion ICP and CPP was found between groups. Sixteen patients had postmortem examination. Cerebral oedema was noted in seven, and in two there was evidence of an intracerebral bleed, fronto-parietal region (bolt site). Fifteen underwent CT scanning - of whom three had evidence of diffuse ischemia, oedema and tentorial herniation. One case had a small fronto-parietal bleed (survived). Mode of death was cerebral in 14 cases. In those who had a CPP $<4546 \%$ survived, whilst in those with a CPP $>45$ $66 \%$ survived.

Conclusions ICP monitoring is a relatively safe procedure in this high-risk cohort of patients. Three ICP bolt-related complications were observed, two died and one survived. In those who survived there were no clinically apparent neurological sequelae. Mortality is increased in those with CPP $<45$ but is not inevitable and should not preclude transplantation and/or aggressive medical management.

\section{P543}

\section{Microdialysis catheters in liver transplants detect vascular} complications and rejection

HH Haugaa, TI Tønnessen

Oslo University Hospital, Oslo, Norway

Critical Care 2010, 14(Suppl 1):P543 (doi: 10.1186/cc8775)

Introduction Following liver transplantation, hepatic artery stenosis and portal vein obstruction occur in 3 to $12 \%$ of the patients, and more frequently in children than adults. Today, the standard of care is Doppler ultrasound and liver enzyme assessment daily. Accordingly, detection of severe hypoperfusion may be delayed. The aim of the present study was to explore whether microdialysis catheters implanted in the left and right liver lobe, by measurement of glucose, lactate, pyruvate and glycerol every 2 hours, detected vascular complications and rejection.

Methods Seventy-three patients undergoing 82 liver transplantations were included. Nine of the patients were children. Two microdialysis catheters were inserted in the liver and one in subcutaneous tissue by a split needle technique. They were kept for as long as the catheters functioned, and maximally 4 weeks. Metabolic parameters (glucose, pyruvate, glycerol and lactate) were collected every 2 hours and measured bedside.

Results Median age of the patients was 52 years (6 months to 70 years). The median time for catheters inserted in the liver was 9.5 days, with a range from 0.5 to 26 days. Six patients had hepatic artery stenosis/ occlusion, and in five of them lactate increased to values $>10 \mathrm{mM}$ with a lactate/pyruvate ratio of several hundred and a concomitant decrease in glucose and increase in glycerol. In one patient (a 6-month-old child) lactate only increased to $2 \mathrm{mM}$ and the LP ratio to 20 . On the background of pathological metabolic values, all patients underwent immediate reoperation and blood clots were removed and the artery reanastomosed. In the child with low lactate values, the flow of the artery was less than 10\% of total hepatic blood flow, despite reanastomosis. Thus, the exceptionally high portal flow delivered enough oxygen to prevent major ischemia despite very low flow in the artery.

Thirteen patients had rejection verified by biopsy, and in six patients anti-rejection therapy was given based on liver function tests and clinical judgement. All patients had some increase in lactate during the period of rejection, but the increase was in all but four patients only $1 \mathrm{mM}$. In the remaining four the increase was 3 to $4 \mathrm{mM}$.

Conclusions By using microdialysis catheters measuring metabolic parameters, hepatic artery occlusions can be detected very rapidly. Patients with rejection show a small, but significant, increase in lactate.

\section{P544}

Drug-induced hepatic cholestasis in the ICU: a case-control study D Williamson', M Bédard Dufresne', V Bonhomme', M Albert', CVerdant', AJ Frenette

'Hôpital du Sacré-Coeur de Montréal, Canada; ${ }^{2}$ Centre Hospitalier HonoreMercier, St-Hyacinthe, Canada; ${ }^{3}$ Hopital Maisonneuve-Rosemont, Montreal, Canada

Critical Care 2010, 14(Suppl 1):P544 (doi: 10.1186/cc8776)

Introduction Liver dysfunction in critically ill patients represents a major concern. Many drugs used in the ICU have been associated with hepatotoxicity. Hepatotoxicity presents in three distinct patterns: cholestatic (alkaline phosphatase (AP) $\geq 2 \times U L N$ and ratio (ALT/ULN)/(AP/ $U L N$ ) $\leq 2$ ), hepatocellular (ALT $\geq 3 \times \cup L N$ and ratio $\geq 5$ ) and mixed (AP $\geq 2 \times$ ULN, ALT $\geq 3 \times$ ULN and ratio between 2 and 5). No published studies have assessed the drug-induced cholestastic pattern of hepatotoxicity in the ICU. The aim of this study was to assess whether use of pharmacological classes previously associated with cholestasis are associated with an increased risk of pure or mixed cholestasis in the ICU.

Methods A nested case-control study assessed the potential association between use of specified pharmacological classes and cholestasis. Cases were identified from a cohort of patients admitted $\geq 24$ hours in whom at least one value of AP $<240 \mathrm{IU} / \mathrm{I}$ had been obtained in the 72 hours following admission. We excluded patients with an identified cause of cholestasis as well as patients with bone metastasis and pregnant women. Each case the subject was matched to a control subject based on age, gender, and length of ICU stay and admission year. Exposure to antiepileptics, penicillins, cephalosporins, carbapenems, macrolid antibiotics and parenteral nutrition was collected and included in a multivariate conditional logistic regression analysis with known risk factors.

Results A total of 113 patients developed cholestasis between May 2001 and March 2009, of which 95 had no identified cause. We matched 95 cases with 95 controls and controlled for APACHE II score, sepsis, obesity, diabetes, length of stay and prior history of cholestasis. In multivariate logistic regression, parenteral nutrition (OR 6.26), sepsis (OR 4.05), and penicillins (OR 3.97) were independently associated with cholestasis.

Conclusions Cholestasis in the ICU often remains of unknown origin. In our study, sepsis, parenteral nutrition and penicillins were independently associated with the development of cholestasis in patients admitted to the ICU.

\section{P545}

\section{Diagnosis of acute cholecystitis in septic patients}

A loakimidou, M Moykas, A Theodotou, A Amygdalou, C Catsenos, K Mandragos

Korgialenio-Benakio Red Cross Hospital, Athens, Greece Critical Care 2010, 14(Suppl 1):P545 (doi: 10.1186/cc8777)

Introduction The aim of this retrospective observational study was to evaluate the usefulness of clinical, laboratory and imaging findings in the diagnosis of acute cholecystitis (AC) in septic patients. 
Methods Twelve out of 1,248 patients were included in the study. The patients had no intra-abdominal pathology on admission. Mean age was 59.08 (16.14) years. The most common ICU admission diagnosis was trauma (7/12). On admission, mean APACHIE II score was 17.916 (5.74) and SOFA score was 8.083 (3.369). On the day of diagnosis, SOFA score was 13 (4.03). Mean length of stay in the ICU before the diagnosis was 32.35 (13.76) days. Diagnosis was based on clinical, laboratory, U/S and $\mathrm{C} / \mathrm{T}$ criteria. Confirmation of the diagnosis was performed with an operative procedure: open cholecystectomy $(n=8)$, laparoscopy $(n=2)$, percutaneous cholecystostomy $(n=2)$.

Results Twelve of 1,248 patients (0.96\%) developed clinical, laboratory and imaging findings of AC. The AC was confirmed by an operative procedure in eight out of 12 patients (0.64\%), one calculus and seven acalculus. The mortality was $62.5 \%$. Necrotic areas of the gallbladder wall existed in three patients. Clinical diagnostic findings were: tenderness in the right upper quadrant of the abdomen and positive Murphy in 16.66\%; temperatures over $38.5^{\circ} \mathrm{C}$ in $50 \%$; leucocytosis in 58.33\%; metabolic acidosis in 50\%; elevation of ALT in 50\% and AST in 66.66\%; bilirubin >1.17 $\mathrm{mg} / \mathrm{dl}$ in 91.66\%; and an increase of alkaline phosphatase in $100 \%$ of patients with confirmed AC. There was one false negative $8 \%$ and three false positive $75 \%$ and one true negative $25 \%$ ultrasonography. The most common U/S finding was gallbladder distention $(n=8) 66.66 \%$ and sludge $(n=8) 66.66 \%$. Respectively, there were two false negative $25 \%$ and two false positive $50 \% \mathrm{C} / \mathrm{T}$ scans. Two patients underwent diagnostics laparoscopies that were true negative.

Conclusions There is not a specific diagnostic test for AC in trauma septic patients. Laparoscopy at the bedside in the ICU is an accurate and safe diagnostic tool.

Reference

1. Laurila et al:: Acta Anaesthesio/ Scand 2006, 50:173-179.

\section{P546}

Bleeding and pneumonia in intensive care unit patients given PPIs and H2RAs for prevention of stress ulcer: a metaanalysis

J Zhou, XWan, L Han

First Affiliated Hospital of Dalian Medical University, Dalian, China

Critical Care 2010, 14(Suppl 1):P546 (doi: 10.1186/cc8778)

Introduction To assess the efficacy and safety of proton pump inhibitor and histamine-2 receptor antagonist in ICU patients for stress ulcer prophylaxis.

Methods Trials were identified by performing a systematic search of MEDLINE, Ebsco and CNKI. Two reviewers assessed the quality of studies and extracted data independently. Disagreement was resolved by discussion. Revman 4.2.2 software developed by the Cochrane collaboration was used for meta-analysis.

Results Four trials involving 771 patients were included. Meta-analyses showed that the incidence of clinically important bleeding was significantly lower in the PPI group as compared with H2RA (OR 0.45, 95\% $\mathrm{Cl}(0.21,0.9), P=0.04)$. The incidence of nosocomial pneumonia (OR 1.03, $95 \% \mathrm{Cl}(0.63,1.70), P=0.89)$ and mortality $(\mathrm{OR} 1.17,95 \% \mathrm{Cl}(0.76,1.80), P=$ 0.47 ) had no significant difference.

Conclusions In comparison with H2RA, PPI is more effective in the prevention of stress ulcer bleeding (SUB) in patients in intensive care. There

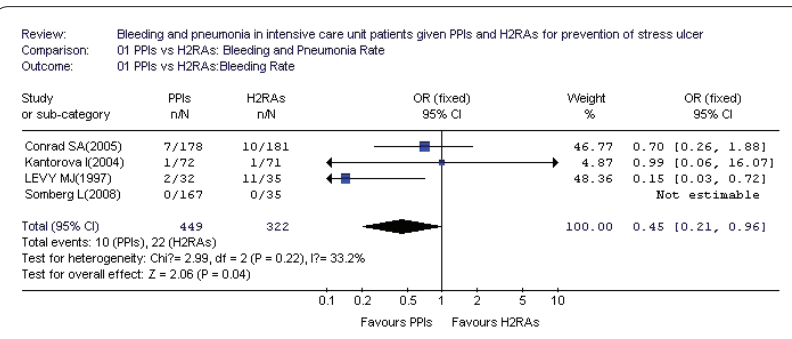

Figure 1 (abstract P546). PPIs vs H2RAs: bleeding rate.

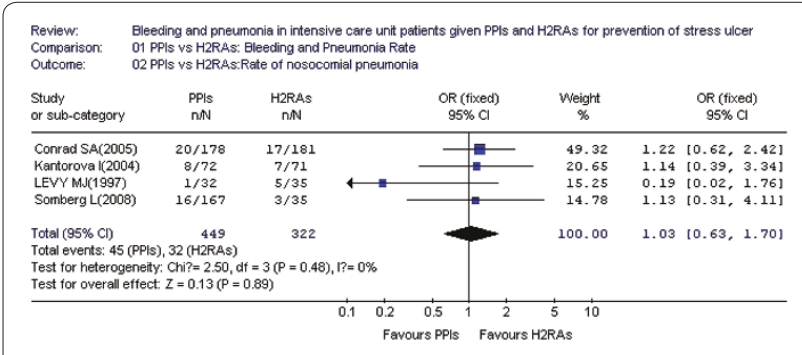

Figure 2 (abstract P546). PPIs vs H2RAs: rate of nosocomial pneumonia.

is no significant difference in the incidence of nosocomial pneumonia and mortality between the two groups. Because the randomized controlled trials are few and these findings are based on a small number of patients, firm conclusions cannot presently be proposed. More randomized, multicenter studies with sufficient sample size should be performed.

\section{Reference}

1. Stollman N, Metz DC: Pathophysiology and prophylaxis of stress ulcer in intensive care unit patients. J Crit Care 2005, 20:35-45

P547

Influence of septic shock on energy expenditure in critically ill ICU patients, estimated with indirect calorimetry

R Włodarski ${ }^{1}$, K Kusza $^{2}$

'Military Clinical Hospital, Bydgoszcz, Poland; ${ }^{2}$ University of Nicolaus

Copernicus, Collegium Medicum of Ludwik Rydygier, Bydgoszcz, Poland

Critical Care 2010, 14(Suppl 1):P547 (doi: 10.1186/cc8779)

Introduction Energy requirements of critically ill septic shock patients treated in the ICU are particularly difficult to determine. Recent research shows that energy expenditure $(\mathrm{EE})$ in those patients may be smaller than it was previously believed. EE values in this group are affected by the severity of the disease process as well as by administered treatment (sedation, inactivity of skeletal and respiratory muscles, fighting fever). The purpose of this study was to establish the influence of septic shock on EE in sedated and mechanically ventilated patients treated in the ICU. Using indirect calorimetry, we assessed EE in those patients and compared results with basal energy expenditure calculated according to the HarrisBenedict equation (BEE), and with EE values in patients subjected to general anesthesia.

Methods Two groups of patients were studied with regard to EE measured by means of indirect calorimetry. Group I consisted of 50 critically ill patients treated in the ICU for septic shock. They were evaluated using the APACHE II and SOFA scores, taking into account the 28-day mortality. EE was measured continuously over the first 24 hours of treatment by means of the Datex-Ohmeda M-COVX indirect calorimeter. Group II comprised 50 patients (ASA I and II), whose energy expenditure was measured under general anesthesia in surgical treatment of disc herniation. Energy expenditure measurements were taken with the use of the Datex-Ohmeda E-CAiOVX indirect calorimeter, adapted to work in an atmosphere containing anesthetic gases.

Results The mean EE value measured with a calorimeter (CEE) in septic shock patients (CEE group I) was 1,467 $\pm 304 \mathrm{kcal} /$ day or $20 \pm 4 \mathrm{kcal} / \mathrm{kg} /$ day. Their mean APACHE II score was 30.2, SOFA was 10.8. CEE in group I amounted to 67 to $137 \%$ of BEE (mean $101 \%$ of BEE) and to $121 \%$ of average CEE in anesthetized patients (CEE group II). Statistical dependence between mortality and CEE was demonstrated. CEE in surviving patients (APACHE: 26.8, SOFA: 8.1) amounted to 91 to $137 \%$ of BEE (mean 114\% of BEE) and to $139 \%$ of average CEE in group II. CEE in deceased patients (APACHE: 32.9, SOFA: 13) amounted to 67 to $121 \%$ of BEE (mean 94\% of $\mathrm{BEE}$ ) and to $111 \%$ of average CEE in group II.

Conclusions A decrease in the total metabolic rate of patients with severe sepsis and septic shock can constitute a prognostic indicator of heightened death risk in this group of ICU-treated patients. 
P548

Evaluation of a novel motility capsule for gastric emptying in a porcine model of acute lung injury

S Rauch, RM Muellenbach, A Johannes, B Zollhoefer, N Roewer

University Wuerzburg, Germany

Critical Care 2010, 14(Suppl 1):P548 (doi: 10.1186/cc8780)

Introduction The aim of this study was to evaluate a novel wireless motility capsule for gastric emptying in a large animal model of acute lung injury.

Methods All experimental procedures were approved by the Laboratory Animal Care and Use Committee of the District of Unterfranken, Germany and adhere to the NIH guidelines for ethical animal research. In seven anesthesized pigs, ventilated with high-frequency oscillation, a $\mathrm{pH}$, pressure and temperature sensing capsule (SmartPill'TM; SmartPill Corp., Buffalo, NY, USA) was positioned with a capsule delivery device (AdvanCE ${ }^{\text {TM}}$; US Endoscopy, Mentor, OH, USA) into the stomach. The capsule data were transmitted to a recorder attached to the abdomen. Gastric emptying of the capsule can be calculated by measuring the time required for the $\mathrm{pH}$ to the change from the acidic stomach to the alkaline duodenum as well as a change in pressure patterns. The location of the capsule was confirmed by autopsy after the animals had died due to the ARDS.

Results In total 8,640 datasets were obtained. The capsule pressure recordings ranged from 2 to $4 \mathrm{mmHg}(2.6 \pm 0.5 \mathrm{mmHg}$ (mean \pm SD)) and $\mathrm{pH}$ ranged from 2.3 to $5.6(3.7 \pm 1.6$ (mean \pm SD)). There was no change in pressure patterns or $\mathrm{pH}$ recordings greater than 6 during 24 hours. All animals had a gastroparesis with bloated stomach. All capsules were located in the stomach as indicated by the pressure and $\mathrm{pH}$ data and confirmed by autopsy.

Conclusions The preliminary data show that motility capsule technology has considerable potential for evaluating real-time gastric emptying.

\section{P549}

Preliminary results on the validation of a novel method for measuring intra-abdominal pressure and gastric residual volume M Malbrain', R Tepaske'2, J Valtysson'3 , P Palmers', A Van Ingelgem', N Van Regenmortel', I De laet', K Schoonheydt', H Dits'

'ZNA Stuivenberg, Antwerpen, Belgium; 'Academic Medical Centre, Amsterdam, the Netherlands; ${ }^{3}$ Akademiska Sjukhuset, Uppsala, Sweden

Critical Care 2010, 14(Suppl 1):P549 (doi: 10.1186/cc8781)

Introduction This study will look at the validation of a novel method combining measurement of gastric residual volume (GRV) and estimation of intra-abdominal pressure (IAP) via intragastric pressure (IGP).

Methods In total 88 paired IAP and GRV measurements were performed in the HOB position in 23 mechanically ventilated ICU patients. The IAP was estimated by the height of the urine column via the bladder (that is, IBP) using the FoleyManometer (Holtech Medical, Charlottenlund, Denmark) and via the stomach (that is, IGP) with the new device (Gastro PV; Holtech Medical). The GRV was measured with the new device (GRVprototype) and via the classic method (GRVclassic). The male-to-female ratio was 2:1, age $62.3 \pm 18.8$ (range 22 to 86 ), BMI $25.3 \pm 6.2$ (16.6 to 40.4), APACHE II score $21.3 \pm 4.1$ (13 to 30 ), SAPS II score $50.7 \pm 13.2$ (17 to 83 ), and SOFA score $10.1 \pm 2.8$ ( 6 to 17). The number of measurements in each patient was four (except one patient with five, one patient with one and one patient with two measurements). Statistical analysis was done with SPSS-13 software, values are expressed as mean \pm SD or median (and range).

Results The mean IBP was $11.3 \pm 4$ and mean IGP was $11.7 \pm 3.9$. Correlation between the IBP and IGP was significant but moderate $\left(R^{2}=\right.$ 0.37 ). Analysis according to Bland and Altman showed a bias and precision of 0.5 and $3 \mathrm{mmHg}$, respectively; however, the limits of agreement were large and ranged from -5.4 to $6.4 \mathrm{mmHg}$. The median GRVprototype was $100 \mathrm{ml}(0$ to 1,050$)$ and median GRVclassic was also $100 \mathrm{ml}(0$ to 1,250$)$. Correlation between the two methods was excellent $\left(R^{2}=0.89\right)$. Analysis according to Bland and Altman showed a bias and precision of -1.3 and $59.2 \mathrm{ml}$, respectively, and the limits of agreement ranged from -117 to 114 $\mathrm{mmHg}$. The median drainage time and return times were 3.1 minutes $(0.5$ to 15 ) and 2 minutes ( 0 to 21) for GRVprototype compared with 2 minutes (0.1 to 9) and 1 minute (0 to 8) for GRVclassic.

Conclusions The preliminary results of an ongoing multicentre pilot study show that the gastro PV is a good alternative to the standard method for measuring GRV. Because the nurse can perform other tasks during drainage and return of the GRV, and the fact that the system remains closed during measurement, this could be a major step forward in standardisation of GRV measurement. Furthermore it allows screening for intra-abdominal hypertension via IGP estimation.

Acknowledgements The gastro PV devices were provided by Holtech Medical, free of charge.

\section{P550}

Monitoring enteral nutrition efficacy in ICU patients: is there a place for the Brix value-derived fraction of enteral formula in gastric residual volume?

JS Stuer, M Casaer, D Lerouge, J Helderweirt, N Nijs, G Van den Berghe, IVanhorebeek

UZ Leuven, Belgium

Critical Care 2010, 14(Suppl 1):P550 (doi: 10.1186/cc8782)

Introduction Achieving nutritional targets by means of enteral nutrition in critically ill (ICU) patients has been shown to be difficult and unpredictable. In this regard, the amount of enteral formula lost in gastric residual volume (GRV) remains unknown. In previous studies performed in a non-ICU population, bedside monitoring of the specific gravity (or relative density) of GRV with a refractometer (expressed as Brix value) has been shown to be an accurate tool to measure the concentration of enteral nutrition (\% EF) in GRV [1,2]. The aim of this study is to evaluate the use of the Brix value in monitoring the concentration of enteral feeding in GRV of patients in the ICU.

Methods The specific gravity, $\mathrm{pH}$ and clinical aspect of GRV in fasting ICU patients were examined. The Brix value of gastric fluid from fasting critically ill patients was measured with a refractometer. One observer measured the Brix value of each sample in duplo. A third measurement was performed by an independent observer. The enteral nutrition was diluted in GRV in vitro to establish the relation between measured Brix value and \% EF by linear regression analysis. Factors influencing the measured Brix value were analyzed in a linear regression analysis.

Results The mean \pm SD Brix value for GRV of 51 fasting ICU patients was $2.9 \pm 1.1$ (ranging from 0.0 to 6.0). The inter-observer and intra-observer variabilities were 8.4 and $3.2 \%$. The relation between Brix value and \% EF was significantly affected by gastric fluid pH before mixing with nutrition. Analyzing all measurements with simple linear regression revealed the following linear relation $(P<0.0001)$ : \% $\mathrm{EF}=$ (Brix value $-2.368 / 0.185)$.

Conclusions The hand-held refractometer is a good tool for monitoring the overall nutritional efficacy in an enteral nutrition strategy measuring and discarding GRV. The linear relation between \% EF in GRV and measured Brix value described in earlier non-ICU studies was confirmed in ICU patients. However, the large baseline Brix variation in ICU patients and unpredictable impact of the $\mathrm{pH}$ of GRV makes the Brix value less suitable for the management of nutrition in individual patients.

\section{References}

1. Chang et al:: Clin Nutr 2005, 24:760-767.

2. Chang et al:: Clin Nutr 2004, 23:105-112.

\section{P551}

Initial gastric volume, nasogastric tube and pneumonia incidence in trauma patients

N Libert', E Pons-ukkola², T Leclerc², P Clapson², B Debien², JP Perez²

'HIA Val de Grâce, Paris, France; 'HIA Percy, Clamart, France

Critical Care 2010, 14(Suppl 1):P551 (doi: 10.1186/cc8783)

Introduction Gastric emptying is often delayed in major trauma patients. These patients are at risk of regurgitation and aspiration during rapid sequence induction or during coma. We hypothesized that an early nasogastric tube (NGT) would reduce the gastric residuals. We also checked whether gastric volume at admission was associated with aspiration pneumonia.

Methods Data from consecutive trauma patients that underwent a full body CT scan from January 2006 to August 2009 were retrospectively analyzed. Gastric gas, liquid/solid and total volume were measured using 3D contouring on the CT scan performed at admission. Secondarily referred trauma patients and patients hospitalized for less than 24 hours 
were excluded from the study. Onset time and occurrence of pneumonia were extracted from the hospital's database; patients who died during the first 48 hours were then excluded. Patients staying for 2 days or longer in the ICU were divided into two groups (with or without NGT) and analyzed separately.

Results One hundred and fifty-six patients were analyzed. CT scan showed a NGT in 30 patients (one malposition in the medium esophagus). Gastric gas, liquid/solid and total volume were not different in patients with and without NGT ( $Z$ test; gas: $54 \pm 147$ vs $95 \pm 168 \mathrm{ml}, P=0.179$; liquid/solid: $226 \pm 282$ vs $199 \pm 237, P=0.626$; Total: $280 \pm 311$ vs $295 \pm 320, P=0.824)$. Twenty out of 153 patients developed a pneumonia in the first 7 days, five of which with NGT and 15 without (chi-squared, $P=0.459$ ). There was no difference in gastric residuals between these patients and the 133 others (Mann-Whitney; Q1-median-Q3; Gas: 11-25-126 vs 14-43-90 ml, $P=1$; liquid/solid: $16-63-432$ vs 22-115-290 ml, $P=0.799$; Total: $29-252-$ 661 vs $68-186-371 \mathrm{ml}, P=0.873)$. Seventy-three patients were admitted to the ICU, of which 23 had a NGT and 20 developed pneumonia. There was no difference between gastric residuals of the 23 who had a NGT and the 50 others. A pneumonia developed in the first 3 days in $1 / 23$ patients with NGT vs 9/50 without NGT (chi-squared-Yates, $P=0.226$ ); in the first 7 days in 5/23 patients with NGT vs $15 / 50$ without NGT (chi-squared, $P=$ 0.462 ). There was no difference in gastric residuals between patients who developed early pneumonia (at 3 and 7 first days) and those who did not. There was no difference in ICU length of stay (10.8 vs 12.7 days, $P=0.825$ ) and onset time of pneumonia (4.2 vs 3.3 days, $P=0.234$ ).

Conclusions Our results suggest that gastric volume is high at admission of trauma patients, irrespective of NGT presence. In this study, pneumonia incidence was related neither with high gastric volume, nor with NGT usage.

\section{P552}

\section{Achieving nutritional targets in the ICU}

$\checkmark$ Gopal, S Hutchinson

Norfolk and Norwich University Hospital, Norwich, UK

Critical Care 2010, 14(Suppl 1):P552 (doi: 10.1186/cc8784)

Introduction McWhirter and Pennington stated in 1994 that 40\% of patients are malnourished on admission to hospital [1]. Malnutrition increases morbidity and mortality in the ICU. Early feeding improves outcome, length of stay and septic complications.

Methods We aimed to assess the standard of nutritional practice, and measure caloric achievement and caloric debt in the first 7 days of admission of critically ill patients. The European Society of Parenteral and Enteral Nutrition guidelines on enteral nutrition are set as the standard [2]. Between April and August 2009, all adult patients not fed orally within 3 days of admission to the ICU were included. The caloric target was $25 \mathrm{kcal} / \mathrm{kg} / \mathrm{day}$. All impediments to achievement of feeding targets were recorded.

Results Among 30 patients ( 16 surgical and 14 medical), 11 patients were fed early within 24 hours of admission, 19 patients were fed 24 hours after admission. Twenty-three patients were fed by nasogastric tube, three patients received small bowel feeding. Parenteral nutrition (PN) was used

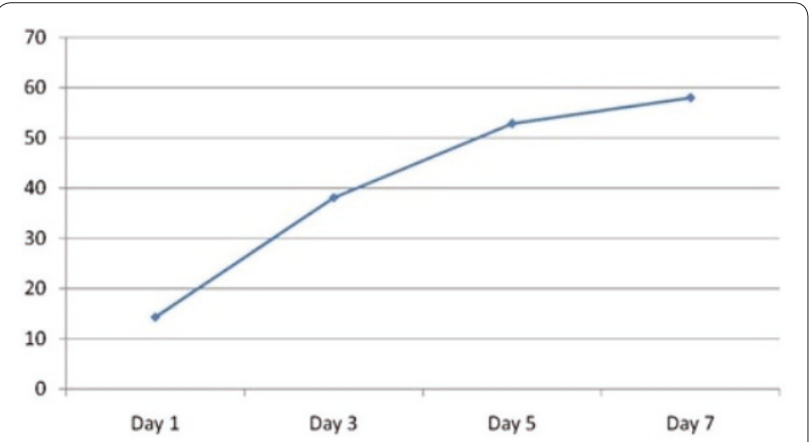

Figure 1 (abstract P552). $x$ axis: day since admission. y axis: percentage caloric target achieved. alone in two patients and as a supplement to enteral nutrition (EN) in one patient.

Conclusions Mean caloric achievement by day 7 was only $58.4 \%$ (Figure 1), well below target. The following recommendations have now been made to improve the nutritional practice. A higher threshold for residual gastric volumes has been adopted, an earlier aggressive use of prokinetic agents is recommended, strategies to access small bowel feeding earlier and PN is now considered within 24 hours if EN is contraindicated or as supplement to EN if the caloric target is not reached after 48 hours.

\section{References}

1. MCWhirter JP, Pennington CR: BMJ 1994, 308:945-948.

2. Kreymann KG, et al:: Clin Nutr 2006, 25:210.

P553

Evaluation of studies of nutritional support team: 2 years' experience A Ozcan, N Ozcan, T Cakan, C Kaymak, H Basar

Ankara Training and Research Hospital, Ankara, Turkey Critical Care 2010, 14(Suppl 1):P553 (doi: 10.1186/cc8785)

Introduction Nutritional screening and nutritional support have an important role to prevent malnutrition, which affects the prognosis and increases the morbidity and mortality of patients. We aimed to assess the patients who are consulted and followed by the nutritional support team during a 2-year period retrospectively.

Methods Demographic characteristics, subjective global assessment scores (SGA) of patients' type, route, duration of nutritional support and complications were assessed.

Results A total of 379 patients were consulted during the 2-year period. Two hundred and two of them (53.3\%) were male and 177 (46.7\%) were female and the mean value of ages was $63.5 \pm 17.9$ years ( 7 to 96 years, minimum to maximum). SGA scores were A in $35.1 \%(n=133), B$ in $26.6 \%$ $(n=101)$, and $C$ in $38.3 \%(n=145)$. In $31.9 \%(n=121)$ of the patients combined parenteral and enteral, in $29.6 \%(n=112)$ only parenteral, in $25.6 \%(n=97)$ only enteral and in $12.9 \%(n=49)$ oral nutritional support were administered. Combined parenteral and enteral nutritional support was applied via nasogastric tube and peripheral parenteral in 54.5\% $(n=66)$, nasogastric tube and santral parenteral in $29.8 \%(n=36)$, oral and santral in 5.8\% $(n=7)$, oral and peripheral parenteral in $6.6 \%(n=8)$, nasojejunal and peripheral parenteral in $1.7 \%(n=2)$ and gastrostomy and peripheral parenteral in $21.7 \%(n=2)$. Parenteral nutritional support was applied via peripheral route in $62.5 \%(n=70)$ and santral route in $37.5 \%$ $(n=42)$. Enteral nutrition was commonly applied by nasogastric tube in $87.6 \%(n=85)$ and gastrostomy tube in $10.3 \%(n=10)$. Nutritional support was applied during $18.2 \pm 23.7$ days ( 1 to 306 days). The most common complications were constipation (7.4\%), nasogastric tube displacement or obstruction (5.2\%), diarrhea (2.6\%), thrombophlebitis (3.4\%), aspiration (1.8\%) and hyperglycemia (1.6\%).

Conclusions Nutritional screening should be a component of physical examination in hospital and outpatients.

\section{P554}

Are we feeding our critically ill patients appropriately?

N Ramakrishnan, S Bhuvaneshwari, RVenkataraman, B Abraham

Apollo Hospitals, Chennai, India

Critical Care 2010, 14(Suppl 1):P554 (doi: 10.1186/cc8786)

Introduction Nutrition plays a significant part in the overall treatment plan for critically ill patients. In India, there are varying practices including use of kitchen feeds and/or commercial formula and also predominant use of bolus feeds. The multidisciplinary team in our critical care unit (CCU) includes a senior dietician and we wanted to review whether our standardized practice of routine screening for malnutrition using subjective global assessment, early use of nutrition (preferably within 24 hours) and continuous enteral feeding using commercial formula helped achieve nutritional goals.

Methods A retrospective chart review was conducted on 508 patients who received continuous enteral feeding on day 1 and day 5 of the ICU stay. Information on calories prescribed (using the Harris-Benedict equation) and delivered on day 1 and day 5 was collected. Achieving the nutritional goal was defined as successful delivery of $90 \%$ of prescribed 
Table 1 (abstract P556). Comparison of patients with APACHE II scores 15 to 25 in the two groups

\begin{tabular}{lcccccc}
\hline Group & $n$ & APACHE II scores & LOS ICU (days) & LOS hospital (days) & Treatment cost (¥10,000) & Mortality (\%) \\
\hline Up to nutrition goal & 28 & $17.58 \pm 3.02$ & $7.50 \pm 3.94$ & $8.42 \pm 3.45$ & $4.20 \pm 3.49$ & 0 \\
Not up to nutrition goal & 54 & $19.96 \pm 2.97$ & $13.11 \pm 9.80^{*}$ & $28.63 \pm 16.44^{*}$ & $9.42 \pm 5.67^{*}$ & 14.8 \\
\hline
\end{tabular}

*P $<0.05$.

calories. Data were analyzed using SPSS software and standard statistical methods.

Results On day $1,37.6 \%$ of patients achieved delivery of caloric goals, and on day 5 , this increased to $67.3 \%$. Fluid restriction was the single most common reason for not meeting caloric goals both on days 1 and 5 .

Conclusions Despite the availability of a clinical dietician and standardized nutritional practice, compliance of achieving caloric goals is low. Commercial formulas with a maximum of $2 \mathrm{kcal} / \mathrm{ml}$ are available in India, unlike the West where more calorie-dense formulas (up to 4.5 to $5 \mathrm{kcal} /$ $\mathrm{ml}$ ) are available. Availability of these higher calorie dense commercial formulas may help increase our rates of achieving nutritional goals as fluid restriction appears to be the single most common limiting factor.

\section{P555}

Effect of increased enteral nutrition on ICU-acquired infections: a multicenter observational study

DK Heyland'1 KE Stephens², AG Day', SA McClave ${ }^{3}$

'Clinical Evaluation Research Unit, Kingston General Hospital, Kingston,

Canada; ${ }^{2}$ Discovery Biometrics GlaxoSmithKline, Stevenage, UK; ${ }^{3}$ Department

of Medicine, Louisville School of Medicine, Louisville, KY, USA

Critical Care 2010, 14(Suppl 1):P555 (doi: 10.1186/cc8787)

Introduction The objective of this study was to evaluate the effect of increasing enteral nutrition (EN) adequacy on acquired infection in the ICU. Methods Prospective, multicenter, observational study in three medical/ surgical ICUs. We enrolled consecutive patients within 24 hours of admission to the ICU who were expected to stay for more than 24 hours. For this analysis, we excluded patients who were not mechanically ventilated in the ICU for more than 72 hours and who received parenteral nutrition. No interventions. Charts were reviewed to determine nutritional adequacy and clinical outcomes. Suspected infections were adjudicated by two or more clinicians to determine the presence or absence of infection (rated as either probable or possible infection).

Results A total of 598 patients were enrolled in the overall study; 206 were included in this analysis. The average patient age was 62.2 years; APACHE II score was 23.3; BMI 28.6; and 73\% were medical. Overall, patients received $48.8 \%$ (range 0 to $120 \%$ ) of their energy and $45.0 \%$ (range 0 to $120 \%$ ) of their protein requirements from $\mathrm{EN}$. On average, patients remained ventilated for 9.1 days (interquartile range (IQR) 6 to 11 ), in the ICU for 13.5 days (IQR, 7 to 14 ) and $25.2 \%$ and $21.8 \%$ developed an infection after 72 and 96 hours, respectively. Overall 28-day mortality was $29.1 \%$. In the overall analysis, greater amounts of energy and protein were consistently associated with a reduction in infection but only achieved levels near statistical significance when considering the incremental effect of 1,000 kcal/day and $30 \mathrm{~g} /$ day on patients with at least one probable infection after $>96$ hours (odds ratio (OR) $0.32,95 \%$ confidence interval $(\mathrm{Cl}), 0.10$ to $1.03, P=0.055$ and $\mathrm{OR}$ $0.41,95 \% \mathrm{Cl} 0.19$ to $0.89, P=0.024$ for energy and protein, respectively). In all cases, the OR was lower when considering infections that developed after 96 hours compared with infections that developed after 72 hours and when considering probable infections compared with all infections. There was a trend towards a reduction in pneumonia that developed after 96 hours with increasing amounts of energy (OR $0.40,95 \% \mathrm{Cl} 0.10$ to 1.53 $P=0.18$ ) and protein (OR $0.42,95 \% \mathrm{Cl} 0.16$ to $1.09, P=0.076)$.

Conclusions Increased EN adequacy was associated with a reduction in infectious complication, particularly after 96 hours of ICU admission.

\section{P556}

Impact of early enteral nutrition up to goal on the prognosis of patients with different severity of illness in the ICU

L Fang, W Fang, L Liu

Second Affiliated Hospital of Soochow University, Suzhou, Jiangsu, China

Critical Care 2010, 14(Suppl 1):P556 (doi: 10.1186/cc8788)

Introduction This study aimed to determine the value of early enteral nutrition up to goal on the prognosis of the patients with different severity of illness in the ICU.

Methods A total of 192 critically ill patients who met the criteria for enrollment were included in this study during the past 18 months in our ICU. They were divided by grade as the severity of illness according to APACHE II scores. Retrospective analysis on early enteral nutrition checked whether the third day of enteral nutrition supply has reached the 60\% nutrition goal (25 kcal/kg/day). So the patients were divided into two groups: up to goal and not up to goal. According to APACHE II scores, the patients were divided into three sub-groups with score inferior to 15 , between 15 and 25 and superior to 25.

Results When the scores of APACHE II were between 15 and 25, the LOS in the ICU $(t=3.966, P=0.000)$, in hospital $(t=8.165, P=0.000)$, the cost of medical care $(t=4.812, P=0.000)$ and the mortality $(t=5.421, P=0.038)$ were all significantly lower in patients with EEN up to goal. See Table 1.

Conclusions The EEN up to goal can significantly increase the survival rate of critically ill patients, shorten the LOS in hospital and reduce the cost of medical care, when the patients have APACHE II scores between 15 and 25. The clinical value of EEN up to goal varies depending on the severity of illness.

\section{Reference}

1. Nartindale RG, et al:: Guidelines for the provision and assessment of nutrition support therapy in the adult critically ill patient: Society of Critical Care Medicine and American Society for Parenteral and Enteral Nutrition: Executive Summary. Crit Care Med 2009, 37:1757-1761.

\section{P557}

Benefit of supplemental parenteral nutrition in the critically ill patient? Results of a multicenter observational study DK Heyland', NE Cahill1, M Wang 1', J Kutsogiannis², C Alberda³, L Gramlich² 'Clinical Evaluation Research Unit, Kingston General Hospital, Kingston, Canada; ${ }^{2}$ University of Alberta, Edmonton, Alberta, Canada; ${ }^{3}$ Alberta Health Services, Edmonton, Alberta, Canada Critical Care 2010, 14(Suppl 1):P557 (doi: 10.1186/cc8789)

Introduction Early enteral nutrition (EN) is the preferred strategy for feeding the critically ill; however, it is not always possible to achieve sufficient calories and protein with EN alone. The use of supplemental parenteral nutrition (PN) has been advocated as a strategy to avoid complications associated with protein/calorie deficit from inadequate enteral feeding. The purpose of this study was to evaluate the effect of this practice on nutritional and clinical outcomes.

Methods An international, observational study conducted in 2007 and again in 2008 examined nutrition practices in ICUs. Eligible patients were the mechanically ventilated who remained in the ICU for $>72$ hours and received early EN within 48 hours from admission. Data were collected on patient characteristics and daily nutrition practices for up to 12 days. Patient outcomes were recorded after 60 days. We compared the outcomes of patients who received early EN alone, early EN + early PN, and early EN + late PN (after 48 hours of admission). Regression analyses were conducted to determine the effect of increasing age, APACHE score, days in hospital prior to ICU admission, gastrointestinal dysfunction, nutritional strategy, and other baseline variables and their relationship to being alive and discharged within 60 days. 
Results A total of 2,920 patients from 260 ICUs were eligible for this study; 2,562 (87.7\%) in the Early EN group, 188 (6.4\%) in the Early PN group, and $170(5.8 \%)$ in the Late PN group. Adequacy of calories and protein was highest in the Early PN group ( $81.2 \%$ and $80.1 \%$, respectively) and lowest in the Early EN group (63.4\% and 59.3\%) $(P<0.0001)$. The 60-day mortality rate was $27.8 \%$ in the Early EN group, $34.6 \%$ in the Early PN group, and $35.3 \%$ in the Late PN group $(P=0.02)$. In a single predictor model, the proportion of patients discharged alive from hospital at 60 days was lower in the group that received Early PN (hazard ratio (HR) 0.75, 95\% confidence interval $(\mathrm{Cl}) 0.59$ to 0.96$)$ and Late $\mathrm{PN}(\mathrm{HR}=0.64,95 \% \mathrm{Cl} 0.51$ to 0.81$)(P=$ 0.0003) compared with Early EN. These findings persisted after adjusting for known confounders. In subgroup analyses, we could not demonstrate any benefit to supplemental $\mathrm{PN}$ in patients with $\mathrm{BMI}<20$.

Conclusions The supplemental use of PN may improve provision of calories and protein but is not associated with any clinical benefit.

\section{P558}

Role of fiber diet formula in surgical critically ill septic patients

K Chittawatanarat, P Pokawinpudisnun

Chaing Mai University, Chiang Mai, Thailand

Critical Care 2010, 14(Suppl 1):P558 (doi: 10.1186/cc8790)

Introduction Diarrhea commonly occurs in surgical critically ill septic patients who received broad-spectrum antibiotics. Some reports demonstrated that a fiber diet with prebiotic properties could decrease the risk of diarrhea in nonseptic patients. However, most reports excluded septic patients who received broad-spectrum antibiotics. The objective of this study was to compare risk of diarrhea and diarrhea score between fiber and nonfiber diets in surgical septic patients who received broadspectrum antibiotics.

Methods We conducted a prospective, randomized control, double-blind study on a general surgical ICU in a university hospital. The patients who received broad-spectrum antibiotics and no contraindication to enteral feeding were enrolled into two arms after informed consent. Allocation to intervention was randomized individually to receive fiber diet or nonfiber diet up to 14 days. Demographic data, disease severity, nutritional delivery and diarrhea score were recorded each day. Intention-to-treat analysis was performed after completed study.

Results Thirty-four patients were enrolled in the study (17 patients each in the fiber group and the nonfiber group). These two patient groups were similar in demographics, disease severity, nutritional status, cause of sepsis and total feeding per day. Although the proportion of patients who confronted with diarrhea score $>15$ was higher in the nonfiber group than the fiber group, these did not show statistical difference significantly (8/17 patients (47.06\%) vs 4/17 patients (23.53\%); $P=0.15)$. However; the group of the fiber diet had a lower mean diarrhea score (fiber vs nonfiber $=3.6 \pm 2.3$ vs $6.3 \pm 3.6 ; P=0.005$ ) and the generalized estimation equation model for repeated measurement (GEE) revealed a lower global diarrhea score in the fiber group than the nonfiber group about 3.03 (coefficient $-3.03(95 \% \mathrm{Cl}=-5.03$ to -0.92$) ; P=0.005)$. Cox proportional hazard ratio of fiber group was $0.45(95 \% \mathrm{Cl}=0.13$ to 1.49 ; $P=0.19)$ but no statistical difference.

Conclusions Enteral nutrition with a fiber diet formula could reduce the diarrhea score in surgical critically ill septic patients who received broadspectrum antibiotics.

\section{P559}

Effect of oral supplementation with immunonutrients on radiation-induced hematologic toxicity in cancer patients MC Calaguas

St Luke's Medical Center, Manila, Philippines

Critical Care 2010, 14(Suppl 1):P559 (doi: 10.1186/cc8791)

Introduction Radiation therapy in cancer patients causes hematologic abnormalities mainly attributed to an increased inflammatory state induced by radiation toxicity that are observed to be modulated by immunonutrition [1-3]. The objective of this study was to determine the effect of oral supplementation with immunonutrients on cancer patients undergoing radiation therapy on the following hematologic indices: hemoglobin, total WBC, neutrophil, lymphocyte, and platelet counts.
Methods Cancer patients on radiotherapy from 2007 to 2009 were randomly assigned to a study group $(n=24)$, which received standard diet plus oral supplementation with an immunonutrition regimen (= $200 \mathrm{ml}$ drink given twice per day providing $600 \mathrm{kcal}, 40 \mathrm{~g}$ protein, eicosapentanoic acid $=2 \mathrm{~g}$, antioxidants (Vit $\mathrm{A}=600 \mu \mathrm{g}$, Vit $\mathrm{C}=75.2 \mathrm{mg}$, Vit $\mathrm{E}=15 \mathrm{mg}$, B-carotene $=1,500 \mu \mathrm{g})$ ), and a control group $(n=32)$, which only had standard diet. The hematologic data - hemoglobin, total WBC count, neutrophil count, lymphocyte count, and platelet count - were taken before and after completion of the prescribed radiation dose. The results were analyzed using either paired or independent-samples $t$ test.

Results In the study group the blood indices were higher compared with the control group at the end of radiation therapy: hemoglobin in gm\% $(12.8>11.8, P=0.002)$, total $\operatorname{WBC}(7,276>4,877, P=0.0005)$, and neutrophil count $(5,363>3,315, P=0.0007)$. There were no differences in the lymphocyte and platelet counts. The WBC change in the study group followed a decreasing pattern $(-162.5)$ while in the control group it was an increasing pattern $(+1,657)$. This indicated lesser inflammation in the study group while there was higher inflammation in the control group $(P=0.004)$.

Conclusions Oral supplementation with immunonutrients in cancer patients undergoing radiation therapy resulted in lesser hematologic toxic changes in hemoglobin, total WBC, and neutrophil count.

References

1. Mell LK, et al: Int J Radiat Oncol Biol Phys 2008, 70:1431-1437.

2. Suchner U, et al:: Proc Nutr Soc 2000, 59:553-563.

3. Jho DH, et al:: Integr Cancer Ther 2004, 3:98-111.

\section{P560}

Impact of multichamber bag parenteral nutrition (OliClinomel) upon inflammatory markers (EPICOS Study)

A Pontes-Arruda', L Martins'1, S Lima', M Pinheiro²

${ }^{1}$ Hospital Fernandes Tavora, Fortaleza, Brazil;'2Laboratorio Evandro Pessoa, Fortaleza, Brazil

Critical Care 2010, 14(Suppl 1):P560 (doi: 10.1186/cc8792)

Introduction The use of a multichamber bag (MCB) as a way to deliver parenteral nutrition to critically ill patients has been associated by retrospective analysis with several benefits including lower bloodstream infection rates, and lower ICU stay [1]. This study aims to evaluate the effects of a MCT TPN system (considered a closed system) versus a conventional compounding system (COM, considered an open system) regardless of the type of lipid emulsion used.

Methods Serum samples were collected in a prospective multicenter open label study (NCT\#00798681) from 100 critical care patients receiving TPN with an olive oil-based lipid emulsion using either a premixed MCB and ready-to-use system ( $n=50$, OliClinomel; Baxter) or COM made either with an olive oil-based lipid emulsion (ClinOleic; Baxter) or with a LCT/MCT-based lipid emulsion (COM ClinOleic $n=25$, COM LCT/MCT $n=25$, total COM group $n=50$ ). All samples were collected at baseline, day 4 and day 7 (three samples per time point). Changes in the levels of inflammatory markers from baseline to day 7 were compared using analysis of covariance and $P<0.05$ was considered statistically significant. Results At baseline, demographic characteristics of the patients were balanced between the groups. Regardless of the type of lipid used, critically ill patients receiving TPN using a MCB system presented at day 4 lower levels of: TNFa (3.4 vs $8.2 \mathrm{pg} / \mathrm{ml})$, IL-6 (50.4 vs $62.0 \mathrm{pg} / \mathrm{ml})$, IL-8 (56.4 vs $93.7 \mathrm{pg} / \mathrm{ml})$, procalcitonin (PCT) (2.7 vs $7.6 \mathrm{ng} / \mathrm{ml}$ ), lymphocyte externalization of phosphatidylserine (5.2 vs 9.6\%), and resistin (9.1 vs 12.3 $\mathrm{ng} / \mathrm{ml}$ ) (all MCB vs COM and $P<0.05$ ). Statistically significant differences were maintained throughout day 7 . No differences were observed for the levels of C-reactive protein and IL-10.

Conclusions The administration of TPN using a MCB system led to a significant reduction in the inflammatory response when compared with COM, regardless of the type of lipid used. Particularly relevant are the differences in terms of PCT as an early marker of sepsis since COM TPN was associated in previous studies with higher bloodstream infection rates [1]. Reference

1. Wischmeyer et al:: Crit Care Med 2009, 37(Suppl). 
P561

Effect of $n-3$ fatty acids on cerebral markers and the inflammatory response in sepsis

CS Burkhart', M Siegemund', SP Strebel', LA Steiner ${ }^{2}$

'University Hospital Basel, Switzerland; ${ }^{2}$ CHUV et Université de Lausanne

Switzerland

Critical Care 2010, 14(Suppl 1):P561 (doi: 10.1186/cc8793)

Introduction Patients with sepsis are at high risk of developing sepsisassociated delirium (SAD), which is associated with increased mortality and may be an important risk factor for the development of long-term cognitive dysfunction. Mechanisms leading to SAD are unclear, therapy and prophylaxis unsatisfactory. Inflammation and its effects on the brain may play an important role. We hypothesized that $n-3$ fatty acids will have a beneficial effect on systemic inflammation and hence cerebral markers in sepsis.

Methods Randomized single-blind design. Patients $>18$ years admitted for treatment of sepsis. Exclusion criteria were thrombocytopenia, brain injury or tumors, intracranial infection and history of stroke. Patients received treatment according to the Surviving Sepsis Campaign and were randomly allocated to a group with or without daily supplementa intravenous n-3 fatty acids (Omegaven ${ }^{\mathrm{T}}$; Fresenius Kabi, $2 \mathrm{ml} / \mathrm{kg} /$ day). Daily screening for SAD was performed. CRP, IL-6, IL-8, S-100 $\beta$, and neuronspecific enolase (NSE) were monitored on days $1,2,3$, and 7 . The primary endpoint was the area under the curve of the S-100 $\beta$ time course.

Results Eighteen patients were included. SAPS || scores and baseline values for CRP, IL-6, IL-8, NSE and S-100 $\beta$ for patients with and without supplemental $n-3$ fatty acids were $51 \pm 14$ vs $57 \pm 22,241 \pm 65$ vs $218 \pm$ $106 \mathrm{mg} / \mathrm{l}, 2,054 \pm 2,822$ vs $734 \pm 1,467 \mathrm{pg} / \mathrm{ml}, 312 \pm 503 \mathrm{vs} 90 \pm 96 \mathrm{pg} / \mathrm{ml}$, $25 \pm 7 \mathrm{vs} 21 \pm 8 \mu \mathrm{g} / \mathrm{ml}$, and $0.57 \pm 0.90 \mathrm{vs} 0.24 \pm 0.24 \mu \mathrm{g} / \mathrm{ml}$, respectively. In agreement with earlier data, patients with SAD had higher values for $\mathrm{S}-100 \beta(P=0.019)[1]$. AUC for S-100 $\beta$ was $2.73 \pm 2.89$ vs $0.95 \pm 0.74(P=$ 0.14 ) for patients receiving supplemental $n-3$ fatty acids and controls, respectively. While none of the differences reached significance, IL-6 and IL-8 declined more rapidly in the $\mathrm{n}-3$ fatty acid-treated group whereas cerebral markers showed an opposite trend. The number of patients with $S A D$ and the duration of SAD were not different in both groups.

Conclusions Interpretation of the results is hampered by unevenly distributed baseline characteristics and more patients need to be investigated. In this small sample no beneficial effect of n-3 fatty acids on cerebral markers was found.

Reference

1. Pfister D, et al: Cerebral perfusion in sepsis-associated delirium. Crit Care 2008, 12:R63.

P562

Effects of an olive oil-based lipid emulsion (ClinOleic) versus LCT/ MCT-based lipid emulsions upon the inflammatory markers of critically ill patients (EPICOS Study)

S Lima', A Pontes-Arruda', L Martins', M Pinheiro²

'Hospital Fernandes Tavora, Fortaleza, Brazil;'2Laboratório Evandro Pessoa,

Fortaleza, Brazil

Critical Care 2010, 14(Suppl 1):P562 (doi: 10.1186/cc8794)

Introduction Olive oil-based lipid emulsions have been used for more than a decade to reduce the polyunsaturated fatty acid (PUFA) content in TPN, thus preventing the deleterious effects associated with PUFA excess. MCT/LCT-based lipid emulsions were recently associated with deleterious effects such as lipotoxicity, activation of inflammatory cascade and increased risk of cardiovascular disease [1]. This study aims to compare the effects of these two lipid emulsions upon several inflammatory markers in critically ill patients using TPN.

Methods Serum samples were collected in a prospective multicenter and open-label study (NCT\#00798681) from 100 critical care patients receiving TPN with an olive oil-based lipid emulsion using either a premixed readyto-use system (G1, $n=50$, OliClinomel; Baxter) or a compounded system $(G 2, n=25$, ClinOleic; Baxter), and the results were compared with those from patients receiving a compounded TPN made with an LCT/MCT lipid emulsion $(G 3, n=25)$. All samples were collected at baseline, day
4 and day 7 (three samples in each time point). Changes in the levels of inflammatory markers from baseline to day 7 were compared using analysis of covariance and $P<0.05$ was considered statistically significant. Results At baseline, demographic characteristics of the patients were balanced between the groups. Regardless of the delivery system used, patients receiving TPN with an olive oil-based lipid emulsion presented at day 4 lower levels of: IL-8 (54.4 vs 67.8 vs 120.7 pg/ml), TNFa (3.4 vs 3.5 vs $13.0 \mathrm{pg} / \mathrm{ml}$ ), lymphocyte phosphatidylserine externalization (PS) (5.2 vs 4.8 vs $14.7 \%$ ), and resistin (R) (9.1 vs 9.2 vs $15.4 \mathrm{ng} / \mathrm{ml}$ ) (all G1 vs G2 vs G3 and $P<0.05)$. No differences were observed in the levels of IL-10 and CRP. Statistically significant differences were maintained throughout day 7 for IL-8, TNFa, PS and R.

Conclusions The administration of an olive oil-based lipid emulsion within the TPN regimen led to a significant reduction in the inflammatory response when compared with a LCT/MCT-based lipid emulsion. Particularly relevant are the differences for resistin (an indicator of insulin resistance) and PS (an indicator of apoptosis).

\section{Reference}

1. Pontes-Arruda: Clin Nutr Supp/ 2009, 4:19.

\section{Abstract withdrawn}


P564

Abstract withdrawn

\section{P565}

Does a fish-oil-containing lipid emulsion improve liver function in comparison with a soybean oil lipid emulsion?

J Linke', H Topp', I Mueller', S Bark², N Mertes ${ }^{3}$, H Grimm $^{4}$, P Singer ${ }^{5}$ J Shaffer ${ }^{6}$

'Fresenius Kabi Deutschland GmbH, Oberursel, Germany; ${ }^{2}$ Visby Hospital, Visby, Sweden; ${ }^{3}$ Raphaelsklinik, Münster, Germany; ${ }^{4}$ Clinical Centre of Passau, Germany; ${ }^{5}$ General Intensive Care Department and Institute for Nutrition Research, Rabin MC, Petah Tikva, Israel; ${ }^{5}$ Salford Royal NHS Foundation Trust, Salford, UK

Critical Care 2010, 14(Suppl 1):P565 (doi: 10.1186/cc8797)

Introduction Liver function disturbances have been of concern in parenteral nutrition. The aim of the post hoc analyses of two pooled studies was to compare liver function parameters using fish-oil-containing vs soybean oil lipid emulsions.

Methods Two prospective, controlled, randomized, parallel-group, double-blind, multicenter studies compared SMOFlipid 20\%, a fishoil-containing lipid emulsion (SMOF: soybean oil $60 \mathrm{~g}$, medium-chain triglycerides $60 \mathrm{~g}$, olive oil $50 \mathrm{~g}$, fish oil $30 \mathrm{~g}$ per I), vs standard soybean oil emulsion (SO, $200 \mathrm{~g}$ soybean oil/I). The studies were as follows:
A: postsurgical adult patients, 5 days total parenteral nutrition, 100 SMOF vs 103 SO patients; B: adult patients receiving parenteral nutrition for 28 days, 22 SMOF vs 32 SO patients. Patients with data at baseline and study end were selected. The data were pooled and differences of laboratory data at 1 week minus baseline and study end minus baseline were calculated. Analyses of variance were applied using differences between 1 week/study end values and baseline values of bilirubin (BIL), AST and ALT as dependent variables and treatment group as independent variable. Covariates used: baseline values for BIL, AST, ALT and mean daily dose of fat per kg body weight.

Results Baseline values were not significantly different between the two treatment groups. The mean daily intake of fat $/ \mathrm{kg}$ bw after 1 week and study end was the same between both treatment groups. One-week baseline values of liver parameters (mean; SMOF group: BIL $-5.1^{*} \mu \mathrm{mol} / \mathrm{l}$, AST -5.4** U/I, ALT 0.6** U/l; SO group: BIL $-2.0^{*} \mu \mathrm{mol} / \mathrm{l}, \mathrm{AST} 0.6^{* *} \mathrm{U} / \mathrm{I}$, ALT 11.1 $\left.1^{* * *} \mathrm{U} / \mathrm{I}\right)$ were significantly different between treatment groups ( $P$ : $\left.0.035^{*}, 0.001^{* *}, 0.001^{* *}\right)$. Extending the analysis until 28 days did not alter the results.

Conclusions Infusion of SMOFlipid 20\% compared with a soybean oil standard lipid emulsion exerts a significant decrease in the values of BIL and AST. It also significantly attenuates the rise seen in ALT. These results indicate that SMOFlipid 20\% is promising in preventing parenteral nutrition-induced liver disturbances.

P566

Therapy of compartmentalization of the proinflammatory and anti-inflammatory cytokines in sepsis

EG Grigoryev

Kemerovo State Medical Academy, Kemerovo, Russia Federation

Critical Care 2010, 14(Suppl 1):P566 (doi: 10.1186/cc8798)

Introduction We supposed the diagnostic significance of the ratio between proinflammatory and anti-inflammatory cytokines in the peritoneal cavity and system blood flow in patients with abdominal sepsis. So we tried to correct such disbalance with glutamine intravenous and enteral supplementation.

Methods Prospective controlled randomized investigation of the patients with abdominal sepsis (excluding pancreatitis). Group 1 ( $n=16$ ): standard therapy. Group $2(n=11)$ : with intravenous infusion of glutamine (Dipeptiven; Fresenius Kabi, Germany). Group $3(n=11)$ : with intravenous infusion of glutamine and enteral supplementation of glutamine (Intestamine; Fresenius Kabi). The patients in groups were identical according to the severity of disease (APACHE II), the standard of intensive care, the volume of surgery care. We investigated the proinflammatory cytokines: TNFa, IL-1, IL-6, IL-8; and anti-inflammatory cytokines: soluble sTNF-RI and sTNF-RII, antagonist receptor $\mathrm{IL}-1, \mathrm{IL}-10$ in blood serum and in the peritoneal cavity during the initial 3 days of intensive care (ELISA; BD Biosciences Pharmingen, San Diego, CA, USA; EASIAs; Biosource, Nivelles, Belgium).

Results The probability of survival on day 28 was $73 \%$ in the standard therapy group, in the group with glutamine intravenous - $78 \%$, in the group with glutamine intravenous and enteral - $84 \%$. We did not fix the decrease of the duration of respiratory support in all of the groups. The duration of acute intestinal injury was significantly different (standard group 49 hours vs 38 hours in group with glutamine intravenous supplementation - 35 hours in group with intravenous and enteral glutamine supplementation). We investigated the prevalence of the concentration of proinflammatory cytokines in the peritoneal cavity and in blood serum according to the molar coefficient in the control group. The molar coefficient had a positive correlation with the SOFA scale. In group 2 (glutamine intravenous) the molar coefficients were decreased to the prevalence of anti-inflammatory cytokines (in serum on day 3, in peritoneal on day 2). In group 3 (glutamine intravenous and enteral) we investigated the significance difference and decrease of all cytokine levels in blood serum and in the peritoneal cavity.

Conclusions Intravenous and enteral supplementation of glutamine improved the cytokine balance in blood and the peritoneal compartment. 
P567

Intensive insulin therapy and brief hypoglycemia do not increase neurological injury markers in critically ill children

M Gielen, I Vanhorebeek, M Boussemaere, PJ Wouters, D Mesotten,

G Van den Berghe

Katholieke Universiteit Leuven, Belgium

Critical Care 2010, 14(Suppl 1):P567 (doi: 10.1186/cc8799)

Introduction Targeting glycemia to age-adjusted normal fasting blood glucose levels with intensive insulin therapy (IIT) improved short-term outcome of critically ill children as compared with conventional insulin therapy (CIT), but concomitantly increased the incidence of hypoglycemia [1]. Both hyperglycemia and hypoglycemia may adversely affect the developing brain of young children. We therefore studied the impact of targeting normoglycemia with IIT on circulating markers of brain injury in these patients.

Methods This was a pre-planned analysis of all 700 pediatric critically ill patients included in a prospective, randomized, controlled study on IIT [1]. Patients were randomly assigned to the target of normal for age fasting blood glucose levels ( 2.8 to $4.4 \mathrm{mmol} / \mathrm{l}$ for age $<1$ year and 3.9 to $5.6 \mathrm{mmol} / \mathrm{I}$ for age $\geq 1$ year) with insulin infusion throughout the ICU stay (IIT), or to insulin infusion only to prevent excessive hyperglycemia (CIT). Serum S100B and neuron-specific enolase (NSE), markers of astrocytic and neuronal damage, were measured by ELISA on fixed days $(n=700)$ and before and after hypoglycemia in a nested case-control design $(n=126)$.

Results Patients admitted to the ICU after cardiac surgery had higher admission S100B and NSE levels than patients in the other diagnostic categories $(P<0.0001)$, with the highest levels observed for patients with the highest Risk Adjustment for Congenital Heart Surgery (RACHS) score (both $P=0.002$ ). Admission levels of S100B and NSE were higher in patients needing intensive care for at least 3 days vs short-stay patients (both $P<0.0001)$ and in ICU nonsurvivors vs survivors $(P=0.002$ and $P$ $=0.0002$ ). IIT did not affect the levels of S100B or NSE as compared with CIT. Patients experiencing hypoglycemia at any time during the ICU stay revealed higher S100B and NSE levels on admission $(P<0.0001$ and $P=$ 0.0007) than those without hypoglycemia. In the nested case-control study, S100B and NSE decreased after hypoglycemia $(P=0.001$ and $P=$ 0.009 ) in the cases, unlike in the controls on matched days.

Conclusions IIT in the pediatric ICU did not evoke neurological damage, as evaluated by S100B and NSE, despite an increased incidence of brief hypoglycemia. Elevated markers in patients with hypoglycemia were not caused by hypoglycemia but explained by an increased risk of hypoglycemia in the most severely ill.

\section{References}

1. Vlasselaers D, et al: Lancet 2009, 373:547-556

\section{P568}

A cluster-controlled implementation project of intensive insulin therapy: effects on blood glucose control and incidence of severe hypoglycemia

R Harmsen', F Van Braam Houckgeest'2, J Van der Sluiijs', HVan den Oever', L Hofstra ${ }^{5}$, M Kuiper ${ }^{6}$, A Abu-Hanna', P Spronk', M Schultz'

'Academic Medical Center, Amsterdam, the Netherlands; ${ }^{2}$ Tergooi Hospitals, Hilversum, the Netherlands; ${ }^{3} \mathrm{MCH}$, The Hague, the Netherlands; ${ }^{4}$ Deventer Hospital, Deventer, the Netherlands; ${ }^{5}$ Scheper Hospital, Emmen, the Netherlands; ${ }^{6} M C L$, Leeuwarden, the Netherlands; ${ }^{7}$ Gelre Hospitals, Apeldoorn, the Netherlands

Critical Care 2010, 14(Suppl 1):P568 (doi: 10.1186/cc8800)

Introduction Intensive insulin therapy (IIT) is a complex intervention involving several steps that may all contain potential sources of variability. Changing practice in complex multidisciplinary environments is difficult. In addition, it is undecided whether IIT should be applied by nurses and physicians, or nurses alone. We implemented IIT using a conceptual implementation framework and compared overall blood glucose control and incidence of severe hypoglycemia (SH) (blood glucose level (BGL) $<40 \mathrm{mg} / \mathrm{dl}$ ) before and after implementation.
Methods Three ICUs developed and implemented an evidence-based guideline for IIT; three ICUs with no change in blood glucose control served as control ICUs. In the guideline ICUs, the IIT practice change comprised two periods: in the first year after implementation of the guideline, physicians and nurses used the guideline together; in the second year the guideline was applied solely by nurses. BGL collection and analysis included the 1 year before implementation of the guideline. Analysis was performed using statistical process control.

Results IIT guideline: the new guideline emphasized fast correction of high BGLs, acceptance of low BGLs and frequent BGL measurements (up to one/hour). Blood glucose control: BGLs of approximately 8,000 patients were collected and analyzed. The case mix was similar in guideline and control ICUs, and did not change over time. No change in overall blood glucose control or incidence of SH was observed in the control ICUs during the study duration. In the guideline ICUs, the median number of daily blood glucose measurements increased from 4 (3 to 7) per patient to 9 (5 to 12$)$ per patient $(P<0.001)$. Median morning $B G L$ declined in guideline ICUs, from 114 (96 to 139$) \mathrm{mg} / \mathrm{dl}$ to 100 (85 to 123$) \mathrm{mg} / \mathrm{dl}(P<0.001)$. The change in blood glucose control was accompanied by a rise in the incidence of $\mathrm{SH}$, from $7 \%$ to $13 \%(P<0.001)$. SH, however, did never cause short-term harm (no death or coma). Nurses alone performed equally well with regard to overall blood glucose control, and even showed a decline of incidence of $\mathrm{SH}$.

Conclusions Implementation of an evidence-based IIT guideline improves blood glucose control, with results comparable with the original studies on IIT in Leuven. The increase of incidence of SH is temporary, and improves when IIT is applied by nurses alone.

\section{P569}

Incidence of hypoglycemia in NICE sugar not so nice after consideration of the treatment period

JK Mader, G Bock, J Plank, G Köhler, TR Pieber, M Ellmerer

Medical University Graz, Austria

Critical Care 2010, 14(Suppl 1):P569 (doi: 10.1186/cc8801)

Introduction Hypoglycaemia is one of the major side effects when establishing glycaemic control in critically ill patients and may contribute to increased mortality rates. Until today, no comparable measure of hypoglycaemia frequency has been suggested. Current assessments depend either on the treatment period or the number of glucose measurements performed per patient. Therefore, we suggest an alternative method to present the hypoglycaemia incidence by number of episodes per patient normalized for a 24-hour treatment period.

Methods Two major studies performing glycaemic control in critically ill patients were chosen for comparison: the Leuven study with 1,200 patients at a medical ICU (605 standard care (STD), 595 tight glycaemic control (TGC); [1]) and the NICE study with 6,030 patients in a mixed ICU population (3,014 STD, 3,016 TGC; [2]). For both studies, rates of hypoglycaemia per patient per 24 hours were calculated based on published data.

Results Mean duration of treatment was 12.5 days for the Leuven study and 4.2 days for the NICE study. APACHE scores were 23 for Leuven and 21 for NICE, respectively. Number of hypoglycaemic events per study population was reported as follows: 19 (3.1\%) for STD and 111 (18.7\%) for TGC for the Leuven study and 15 (0.5\%) for STD and 206 (6.8\%) for TGC in the NICE study. When applying the new evaluation method, rates of hypoglycaemia per patient per 24 hours were $0.25 \%$ (STD) vs 1.49\% (TGC) for Leuven and $0.12 \%$ (STD) vs $1.63 \%$ (TGC) for NICE, respectively.

Conclusions The results reveal that the treatment period may have a significant impact on the reported rate of hypoglycemia. We conclude that reporting hypoglycaemic episodes in critically ill patients on a perpatient and per-24-hour basis may be a more adequate tool to accurately describe hypoglycaemia incidence which facilitates the comparison between different studies and study protocols.

\section{References}

1. Van den Berghe et al:: NEng/J Med 2006, 354:449-461.

2. NICE-SUGAR Study Investigators: N Eng/ J Med 2009, 360:1283-1297. 
P570

Comparison of a planned versus emergent approach implementation of tight glycemic control therapy on an ICU: an experimental design

M Luiking ${ }^{1}$, L Bras', R Van Linge ${ }^{2}$, M Grypdonck ${ }^{2}$

'St Antonius Ziekenhuis, Nieuwegein, the Netherlands; ${ }^{2}$ Universiteit Utrecht, the Netherlands

Critical Care 2010, 14(Suppl 1):P570 (doi: 10.1186/cc8802)

Introduction In this study the effectiveness of a hierarchic planned approach to implementation was compared with an emergent approach with a high degree of nurse participation. The innovation was a new tight glycemic control (TGC) therapy in the ICU. The implementation method to introduce a new evidence-based treatment to the ICU can make a difference to its effectiveness. New treatment procedures are very often strictly defined and introduced on the ICU in a top-down manner. On theoretical grounds, a bottom-up implementation method, with more participation and involvement of the ICU nurses, should be more effective.

Methods In an experiment a TGC protocol was introduced on a 24-bed ICU with 120 ICU nurses. In one nursing team the implementation was by means of the usual planned approach (PA). In the other team an emergent implementation approach (EA) was applied. During 7 months, 1,182 patients were admitted and randomly assigned to either team. The effectiveness of the implementation methods was measured at patientoutcome (glucose) level and on a process-execution (nurse compliance) level. The patient-outcome measurements were: mean glucose value, percentage of normal glucose values (defined as between 4.0 and $6.5 \mathrm{mmol} / \mathrm{l})$ and the time from admission to reach a normal glucose level $(6.5 \mathrm{mmol} / \mathrm{l})$. For the mean glucose value a difference was made between short-stay ( $<3$ days) and long-stay patients ( $\geq 3$ days). The compliance was expressed as compliance for safe, insulin treatment (the patient received the right amount of insulin) and compliance for efficient treatment (glucose measurements as well as insulin were according to the protocol).

Results The mean glucose for the short-stay patients was significantly lower in the EA than the PA team (6.42 vs $6.52 \mathrm{mmol} / \mathrm{l})$, no significant difference was found for the long-stay patients. The percentage of normal glucose values was $53.9 \%$ in the EA and $52.8 \%$ in the PA team (significant $P<0.001$ ). The time to reach normal glucose levels was 6 to 7 hours in the $E A$ and 10 to 11 hours in the PA team (significant, $P<0.05$ ). The compliance for safe insulin treatment was $91.3 \%$ in the EA and $79.0 \%$ in the PA team. The compliance for efficient treatment was $83.5 \%$ in the EA and $66.8 \%$ in the PA team.

Conclusions An emergent approach to implementation (with an increased participation of the ICU nurses) of a TGC resulted in better patient results and a higher compliance to the protocol.

\section{P571}

\section{Coagulation profile in hyperglycemic septic shock patients}

LC Sanches, D Lourenco, R Salomao, MA Noguti, M Brunialti, C Steula,

M Assuncao, F Machado

Federal University of São Paulo, Brazil

Critical Care 2010, 14(Suppl 1):P571 (doi: 10.1186/cc8803)

Introduction A hypercoagulable state is frequently found in sepsis patients as well as hyperglycemia [1]. The objective of this study was to determine the coagulation profile in hyperglycemic septic shock patients and the impact of glycemia control.

Methods Septic shock patients with less than 48 hours of organ dysfunction with normoglycemia ( $<150 \mathrm{mg} / \mathrm{dl}$, Group 1) or hyperglycemia (>200 mg/dl, Group 2) were included. Exclusion criteria were previous diabetes, coagulation diseases, use of heparin or fresh frozen plasma/ platelets, sepsis in the past 30 days or insulin use in the past 24 hours. The coagulation and inflammation profile was determined at inclusion and after 24 hours, only if the patients remained normoglycemic or achieved glycemic control with insulin infusion (Group 2). Results were expressed in median and interquartiles, $P<0.05$ was considered significant.

Results Forty-one patients were included, $59.7 \%$ male; mean APACHE ॥ score: $16.9 \pm 8.6, P=0.21$; mean SOFA score: $8.1 \pm 2.0$; mean number of organ dysfunctions: $3.6 \pm 0.9$, with a significant difference between the groups only regarding age (Group $1(n=21): 53.8 \pm 16.7$, Group 2 ( $n=$ 20): $68.8 \pm 16.4, P=0.006$ ). In both groups, a hypercoagulation profile was detected, with high levels of fragment $1+2(F 1+2)$, thrombin/antithrombin complex (TAT), D-dimer and tissue factor and with low levels of factor VII. Consumption of coagulation inhibitors (protein C (PC), antithrombin (AT), tissue factor pathway inhibitor) and increased fibrinolysis (plasminogen activator inhibitor 1 (PAl-1), tissue plasminogen activator (TPA) and plasminogen) were also found. However, at baseline no significant difference between groups was found except for a trend towards high levels of F1+2 $(P=0.08)$ and TAT $(P=0.058)$ in Group 2. After 24 hours higher levels of AT $(P=0.02), P C(P=0.02)$, plasminogen $(P=0.04)$ and a reduction in PAI-1 $(P=0.01)$ and TPA $(P=0.03)$ were detected in Group 2. Inflammatory parameters (IL-6, IL-8, IL-10, TNF) were similar at baseline, with decreased levels in both groups after 24 hours but the IL-6 $(P=0.02)$ and IL-8 $(P=0.0004)$ reduction was more pronounced in Group 2.

Conclusions Septic shock patients have a hypercoagulation pattern regardless of their glycemic status. However, glycemia control seems to be associated with reduction in inflammation and hypercoagulation parameters, possibly related to insulin infusion as glycemia levels did not differ between groups after 24 hours.

Acknowledgements Financial support from FAPESP.

Reference

1. Machado FR, et al:. Endocr Metab Immune Disord 2006, 26:175-182.

P572

Impact of glycemic control on survival in critical care patients with sepsis

RTiruvoipati, D Lewis, K Ong, M Gupta, K Haji, J Botha

Frankston Hospital, Frankston, Victoria, Australia

Critical Care 2010, 14(Suppl 1):P572 (doi: 10.1186/cc8804)

Introduction Several studies have shown an increased mortality and morbidity in various groups of patients with hyperglycemia requiring admission to the ICU. Contrary to these studies, a recent large multicentric international trial in ICU patients has shown an increased mortality in patients where normoglycemia was maintained. However, very few studies specifically evaluated the association between hyperglycemia and mortality in critical care patients with sepsis. Since hyperglycemia is known to be associated with immunosuppression and increased risk of life-threatening infections, we aimed to assess the impact of glycemic control (normoglycemia) in ICU patients with sepsis.

Methods We studied all patients admitted to our ICU with sepsis between July 2004 and May 2009. Patients were excluded if their blood sugar levels (BSL) were not measured at least once every 6 hours during their ICU stay. Patients were divided into two groups based on their glycemic control in the ICU: normoglycemic group (BSL 3.9 to 6.2 $\mathrm{mmol} / \mathrm{l}$ ) and conventional glucose control group (BSL 8 to $10 \mathrm{mmol} / \mathrm{l}$ ). BSLs were controlled using an infusion of actrapid insulin. Both groups were compared with regard to demographics, co-morbidities, severity of illness at ICU admission (APACHE III and SAPS II scores), ICU and hospital mortality and length of stay.

Results Over the 5-year study period a total of 314 patients were admitted with sepsis and 297 patients were included. On average every patient included in the study had BSL once every 4.2 hours throughout their ICU stay. Of these patients, 68 had normoglycemia (mean BSL 5.6) and 229 had conventional glucose control (mean BSL 8.5) during their ICU stay. There was no statistically significant difference between the groups in age, sex, APACHE III and SAPS II scores. The ICU mortality was significantly higher in the normoglycemic group $(30.8 \%$ vs $14.8 \% P<0.01$ ). There was no statistically significant difference in hospital mortality between both groups (33.8\% vs $25.3 \% P=0.16$ ). The ICU (median and IQR days: 1 ( 1 to 3 ) vs 4 ( 2 to 7$) P=<0.01$ ) and hospital duration of stay (6.0 (2.00 to 13.75) 6.0 (2.00 to 13.75$)$ vs 12.0 (7.00 to 27.00$) ; P<0.01$ ) were significantly higher in the conventional group.

Conclusions The ICU mortality in patients admitted to the ICU with sepsis who had normoglycemia was higher than in those patients who had conventional glucose control during their ICU stay. Aiming for a normal blood glucose levels is not indicated in patients with sepsis while they are in the ICU. 
P573

Therapeutic impact on intravenous insulin dosing with three new point-of-care glucose meters in an adult ICU

L Schmitz, M Suball, C Hanicq, T El-Mahi, V Piersoel, E Stevens, A Roman

CHU Saint-Pierre, Brussels, Belgium

Critical Care 2010, 14(Suppl 1):P573 (doi: 10.1186/cc8805)

Introduction Glycemic control is mandatory in the critically ill, because hypoglycemia and hyperglycemia are associated with increased mortality [1]. We compared in a prospective observational study three new pointof-care devices with the hexokinase reference method and we evaluated whether their results would modify insulin titration.

Methods Arterial blood glucose was simultaneously measured by the blood gas analyser RapidLab 1265, by three glucose meters (Accu-Chek performa, Precision XceedPro, Nova StatStrip), and with the hexokinase reference method. All values were duplicated and the average value of each was computed. Bland-Altman, Passing Bablok, Kanji [2] and modified Kanji approaches were performed. Biases were expressed as the glucose result of the point-of-care method minus the reference method. We evaluated the theoretical impact on insulin titration by comparing glucose meter results with the hexokinase reference method on a dynamic sliding scale targeting a glycemia of 80 to $130 \mathrm{mg} / \mathrm{dl}$.

Results A total of 156 matched analyses were done in 80 patients. The mean flash SOFA score was 4.5. The range of the reference glucose was 25 to $327 \mathrm{mg} / \mathrm{dl}$. The numbers of discrepancies of dosing insulin were respectively 8-5-6-14 at 0.1 U/hour, 0-6-3-6 at 0.2 U/hour and 2-3-0-0 at $0.3 \mathrm{U} /$ hour. None was greater than $0.3 \mathrm{U} /$ hour. Regarding the point-of-care results, total theoretical insulin dose changes were respectively: $-11.7 \mathrm{U}$, $-19.2 U,-22.2 \mathrm{U},+4.9 \mathrm{U}$ for all these measurements (devices order as in Methods). Table 1 presents the standard comparisons.

Table 1 (abstract P573)

\begin{tabular}{lccc}
\hline & Bias and 2SD & Passing reg & Cl of slope \\
\hline RapidLab & $-4.7 \pm 14.6$ & $Y=-4.8+1.1 X$ & 1.04 to 1.10 \\
AccuChek P & $-9.5 \pm 20.5$ & $Y=3.6+1.05 X$ & 1.00 to 1.11 \\
PXceed Pro & $-0.9 \pm 15.4$ & $Y=-4+1.03 X$ & 1.00 to 1.08 \\
Statstrip No & $1.2 \pm 18.1$ & $Y=-0.4+0.98 X$ & 0.93 to 1.03 \\
\hline
\end{tabular}

Conclusions Glucose monitoring with new glucose meters results has minor impact on intravenous insulin dose titration compared with the reference method.

\section{References}

1. Van den Berghe G: J Clin Endocrinol Metab 2009, 94:3163.

2. Kanji S: Crit Care Med 2005, 33:2778.

\section{P574}

Significance of blood glucose variability in the early phase for predicting survival in acutely ill patients with glucose intolerance M Hoshino', Y Haraguchi², I Mizushima ${ }^{3}$, M Sakai $^{4}$, S Kajiwara', M Takagi 'Shisei Hospital, Saitama, Japan; ${ }^{2}$ National Hospital Organization Disaster Medical Center, Tokyo, Japan; ${ }^{3}$ Nippon Engineering College, Tokyo, Japan;

${ }^{4}$ Tokyo Women's Medical University Hospital, Tokyo, Japan

Critical Care 2010, 14(Suppl 1):P574 (doi: 10.1186/cc8806)

Introduction Although high blood glucose (BG) causes high mortality in ICU patients, significance of BG variability is not elucidated. The purpose of the study was to clarify the significance of BG variability in the early phase for predicting survival and to suggest the importance of stabilizing $B G$ variability for mortality reduction.

Methods Strict BG control was performed by bedside-type artificial pancreas (AP), STG22. The number of the selected patients was 67, or patients with daily mean BG (BGm) below $200 \mathrm{mg} / \mathrm{dl}$ in early phase (3.2 \pm 2.5 days after admission). Studied items: (1) mortality, (2) BG parameters (mg/dl): BGm, daily standard deviation of BG (BGsd), daily maximal and minimal BG (BGmax, BGmin), and daily BG difference (BGd:BGmax BGmin), (3) accuracy (\%) of the BG parameters for predicting survival (AS),
(4) relationships among BG parameters, and (5) probable factors affecting BG variability (BGsd) including demographic data, SOFA score and MOF score (calculated using the criteria proposed by the Japanese Association for Critical Care Medicine, 1990).

Results (1) Mortality of the patients with BGsd below 14 mg/dl (group BGsd14b, $n=41$ ) was significantly lower than that with BGsd above $14 \mathrm{mg} / \mathrm{dl}$ (group BGsd14a, $n=26$ ) (17\% vs 46\%, $P<0.025)$. (2) AS (\%) was as follows: BGm <196 mg/dl (75\%), BGsd <17 mg/dl (73\%), BGmax <225 mg/dl (72\%), BGmin <172 mg/dl (72\%), and BGd <8 0 mg/dl (70\%). (3) Significant $(P<0.002)$ positive correlation was found between BGsd and BGd ( $r=0.87)$, BGsd and BGmax ( $r=0.79)$, and BGd and BGmax $(r=0.77)$. (4) There was a tendency that the ratio of the patients with MOF score above 3 was lower in group BGsd14b than that in group BGsd14a (7\% vs $23 \%, P<0.10)$, although there was no significant difference between the groups in BGm, demographic data, and SOFA score.

Conclusions First, high BG variability in the early phase, which appeared to accompany severe illness and could be evaluated by BGsd, BGd, and BGmax, was considered to be one of the significant risk factors as well as high BG or elevated BGm. Stabilizing BG variability from the early phase was suggested to be important for mortality reduction. Second, AP was reliable for elucidating the significance of strict BG control, and was considered to be a valuable therapeutic artificial organ for the better outcome of the ICU patients through the strict BG control.

\section{P575}

Blood glucose amplitude variation: effects of intensive insulin

therapy and relative association with mortality

E Bekaert, P Wouters, A Wilmer, G Van den Berghe, G Meyfroidt

UZ Leuven, Belgium

Critical Care 2010, 14(Suppl 1):P575 (doi: 10.1186/cc8807)

Introduction There is growing evidence that not only blood glucose (BG) level but also BG amplitude variation (BGAV) is associated with mortality in critically ill patients [1].

Methods Retrospective analysis of the data of the Leuven intensive insulin therapy (IIT) trial in 1,200 MICU patients, randomized to receive either IIT or conventional insulin therapy [2]. The hyperglycemic index (HGl) and hypoglycemic index (HoGl) were used as measures of BG level, the standard deviation of all $B G$ readings per patient (SD BG) as a measure of $B G A V$. The univariable effect of IIT on these indices was analyzed, the independent association with hospital mortality was assessed by multivariable logistic regression (MVR), corrected for baseline risks.

Results IIT reduced the median HGI from 3.2 to $0.8 \mathrm{mmol} / \mathrm{I}(P<0.0001)$, increased the median HoGl from 0.005 to $0.048 \mathrm{mmol} / \mathrm{l}(P<0.0001)$, and did not affect median SD BG (conventional: 2.12 ; IIT: $1.99 \mathrm{mmol} / \mathrm{I}(P=$ $0.161)$ ). The results of the MVR are summarized in Table $1 . \mathrm{HGl}, \mathrm{HoGl}$ and SD BG were independently associated with mortality.

Conclusions BGAV was associated with mortality in MICU patients, independent of baseline risks and BG level. IIT reduced $\mathrm{HGl}$, increased HoGl, and did not affect BGAV. Reducing BGAV, in addition to IIT, may theoretically increase its potential for clinical benefit.

\section{References}

1. Krinsley et al:: Crit Care Med 2008, 36:3008-3013.

2. Van den Berghe et al.: N Engl J Med 2006, 354:449-461.

Table 1 (abstract P575)

\begin{tabular}{lc}
\hline Risk & P value \\
\hline Malign & $<0.001$ \\
Female & 0.309 \\
BMI $>25$ & 0.044 \\
Age & 0.001 \\
Adm BG & 0.002 \\
HGl & $<0.001$ \\
HoGl & 0.003 \\
SD BG & 0.024 \\
\hline
\end{tabular}


P576

Prevention of fatal and disabling neuroglycopaenia: a comparison of arterial blood sampling methods

KA Brennan, D Turnbull

Sheffield Teaching Hospitals NHS Trust, Sheffield, UK

Critical Care 2010, 14(Suppl 1):P576 (doi: 10.1186/cc8808)

Introduction In 2008, the UK National Patient Safety Agency (NPSA) published a report following 42 incidents and two deaths where glucosecontaining flush solutions were attached to arterial lines. Deaths occurred when blood samples contaminated with the flush solution led to artificially high blood glucose readings, inappropriate insulin administration and iatrogenic neuroglycopaenia (the molar concentration of $5 \%$ glucose at $277 \mathrm{mmol} / \mathrm{l}$ is huge next to physiological blood glucose at $<11 \mathrm{mmol} / \mathrm{l})$. The NPSA sought a solution so we applied a bench model to test the performance of three open and three closed arterial line systems in preventing sample contamination.

Methods All arterial line systems were set up in a standard manner and pressurised to $300 \mathrm{mmHg}$ with $5 \%$ glucose used as the flush solution. This was connected to the radial artery using an $18 \mathrm{G}$ needle representing the radial cannula. The radial artery was simulated using a wide bore extension set with blood flow at $60 \mathrm{ml} /$ minute. Blood was simulated by the addition of red dye to Hartmann's solution. Increasing multiples of arterial line dead space were aspirated and discarded. Blood samples were then taken and the glucose concentration measured.

Results Significant glucose contamination ( $3 \mathrm{mmol} / \mathrm{I} \pm 3.4$ ) was detected in all open arterial line systems up to an aspiration volume of five times the dead space. No samples from the closed systems recorded glucose concentration $>1 \mathrm{mmol} / \mathrm{l}(0.2 \pm<0.1)$ (Figure 1).

Conclusions Recommended minimal discard volumes are inadequate in the presence of glucose as the flush solution. Human factors ensure that such errors can persist without a systems-based solution. Our study demonstrates that the closed loop arterial sampling system could be the universal solution sought by the NPSA.
P577

Impact of real-time continuous glucose monitoring on glucose variability in critically ill patients

R Brunner, R Kitzberger, W Miehsler, V Fuhrmann, C Madl, U Holzinger

Medical University of Vienna, Austria

Critical Care 2010, 14(Suppl 1):P57 (doi: 10.1186/cc8809)

Introduction Glucose variability has been found to be associated with mortality in critically ill patients, independent of the mean glucose concentration [1]. The aim of this analysis was to assess the impact of real-time continuous glucose monitoring (CGM) on glucose variability in critically ill patients receiving intensive insulin therapy (IIT).

Methods This is the post hoc analysis of a prospective, randomized, controlled trial [2]. Data of 124 patients admitted to the ICU either receiving IIT according to a real-time CGM system (Guardian ${ }^{\oplus}$; Medtronic, Northridge, CA, USA) $(n=63)$ or according to an algorithm $(n=61)$ with selective arterial blood glucose measurements (simultaneously blinded CGM) for 72 hours were analysed. Insulin infusion rates were guided according to the same algorithm in both groups. Mean glucose and standard deviation, as a marker of glucose variability, were calculated for the first 24 hours $\left(G u_{\text {MEAN } 1}, \mathrm{Glu}_{\mathrm{SD} 1}\right)$ and for the whole study period $\left(\mathrm{Glu} \mathrm{u}_{\mathrm{SD}}\right)$. Statistical comparison of parameters between study groups and between ICU survivors $(n=94)$ and nonsurvivors $(n=30)$ was performed using Student's t test.

Results The variability of sensor glucose during the entire study period was comparable between the real-time CGM group and controls (21.51 \pm 1.10 vs $23.44 \pm 1.26 \mathrm{mg} / \mathrm{dl} ; P=0.243)$. Although the mean sensor glucose during the first 24 hours in the ICU showed a trend towards lower values in the intervention group (real-time CGM vs control: Glu MEAN $104.40 \pm 2.00$ vs $109.23 \pm 1.96 \mathrm{mg} / \mathrm{dl} ; P=0.087$ ), variability of sensor glucose during the first 24 hours did not differ significantly (real-time CGM vs control: Glu 20.85 vs $18.46 \mathrm{mg} / \mathrm{dl} ; P=0.201$ ). Glu sD tended to be lower in ICU survivors (ICU survivors vs nonsurvivors: $21.61 \pm 8.60$ vs $25.10 \pm 10.55 \mathrm{mg} / \mathrm{dl} ; P=$ $0.071)$ whereas $\mathrm{Glu}_{\mathrm{SD} 1}(19.06 \pm 9.32$ vs $21.41 \pm 13.21 \mathrm{mg} / \mathrm{dl} ; P=0.282)$

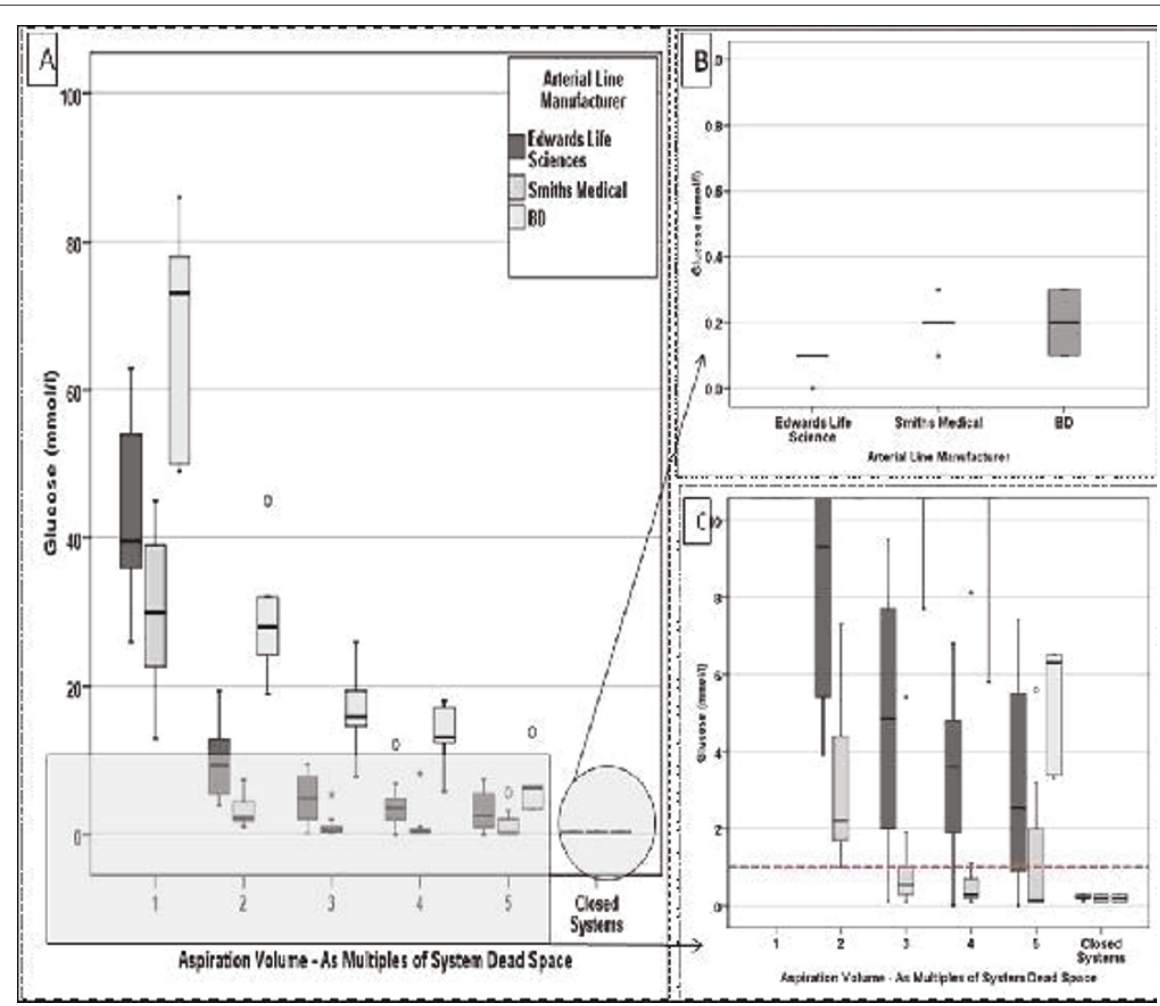

Figure 1 (abstract P576). 
and $\mathrm{Glu}_{\text {MEAN1 }}(106.39 \pm 14.83$ vs $108.00 \pm 18.44 \mathrm{mg} / \mathrm{dl} ; P=0.627)$ were not different between ICU survivors and nonsurvivors.

Conclusions IIT quided by real-time CGM did not result in reduced glucose variability compared with IIT according to an algorithm with selective arterial blood glucose measurements. Although mean sensor glucose during the first 24 hours tended to be lower in the real-time CGM group, glucose variability was not different between the two groups. $G \mathrm{u}_{\mathrm{SD}}$ tended to be lower in ICU survivors irrespective of glucose monitoring.

\section{References}

1. Egi M, et al.: Crit Care 2009, 13:302.

2. Holzinger U, et al:: Diab Care 2009, in press.

\section{P578}

Automated blood glucose monitor is insensitive to hematocrit variation and common saccharide interferents

S Hendee, S Vanslyke, J Faught, A Truong, W Patterson, RThompson Luminous Medical, Carlsbad, CA, USA

Critical Care 2010, 14(Suppl 1):P578 (doi: 10.1186/cc8810)

Introduction Luminous Medical is developing an automated, patientattached system (Argus Glucose Monitoring System) that uses a flowthrough glucose oxidase sensor to measure plasma glucose levels in whole blood. The Argus GMS will aid caregivers in achieving glycemic control in critical care patients. The FDA has recently indicated that ICU glucose monitors need to satisfy tighter accuracy requirements than those specified by ISO 15197 ( $\pm 15 \mathrm{mg} / \mathrm{dl}$ or $\pm 20 \%$ ). Luminous conducted a study to demonstrate Argus GMS accuracy under conditions of variable hematocrit, and in the presence of common saccharide interferents.

Methods Hematocrit Study Group: one unit of whole blood was collected under an IRB-approved protocol from two healthy volunteers. Blood samples were prepared at three hematocrit levels $(\sim 24,36$, and $48 \%)$. Three glucose levels were tested at each hematocrit: native glucose, native plus 100, and native plus $200 \mathrm{mg} / \mathrm{dl}$. Repeats were measured for each hematocrit/glucose combination ( $n=99$ total samples in this study group). Interferent Study Group: blood samples were prepared at native hematocrit ( 37\%) and native glucose level $(\sim 100 \mathrm{mg} / \mathrm{dl})$ ). Testing followed established interference testing guidelines [1]. Blood samples were spiked with concentrated interferent solution or with equal volume of normal saline. Testing alternated between interferent-containing and interferentfree samples ( $n=5$ test and five control samples for each interferent). Saccharide interferents included maltose $(55 \mathrm{mg} / \mathrm{dl})$, galactose $(15 \mathrm{mg} / \mathrm{dl})$, fructose $(18 \mathrm{mg} / \mathrm{dl})$, and xylose $(40 \mathrm{mg} / \mathrm{dl})$. In both study groups, blood samples were measured in vitro, in real time, using the Argus GMS in multisample mode. Plasma measurements from two YSI 2700 instruments were averaged to provide reference glucose values.
Results Hematocrit Study Group: the Argus GMS measured plasma glucose across a range of 50 to $350 \mathrm{mg} / \mathrm{dl}$ with RMS error of $5.5 \mathrm{mg} / \mathrm{dl}$ (mean error 2.6, 95\% Cl =-7.0 to 12.2, $R=0.998$ ). The Argus GMS showed no sensitivity to hematocrit variation. Interferent Study Group: the Argus GMS showed minimal glucose measurement bias for each of the four saccharide interferents (maltose $2.21 \mathrm{mg} / \mathrm{dl}$, galactose $4.09 \mathrm{mg} / \mathrm{dl}$, fructose $2.63 \mathrm{mg} / \mathrm{dl}$, and xylose $2.03 \mathrm{mg} / \mathrm{dl}$ ).

Conclusions The Argus GMS provides highly accurate glucose readings in whole blood samples with no observable sensitivity to hematocrit variation and with minimal influence from saccharide interferences. Argus demonstrated measurement accuracy within $\pm 10 \mathrm{mg} / \mathrm{dl}$ or $\pm 10 \%$ at $95 \%$ confidence limits.

\section{Reference}

1. Clinical and Laboratory Standards Institute: Interference Testing in Clinical Chemistry: Approved Guideline - Second Edition. Wayne, PA: Clinical and Laboratory Standards Institute; 2005.

\section{P579}

Performance of an automated blood glucose monitor in the OR/ICU M Higgins', G Bochicchio ${ }^{2}$, P Simpson ${ }^{3}$, J Leach ${ }^{3}$, A Kamath ${ }^{3}$, D Deyette 'Edwards Lifesciences, LLC, Irvine, CA, USA; ${ }^{2}$ University of Maryland School of Medicine, Baltimore, MD, USA; ${ }^{3}$ Dexcom, Inc., San Diego, CA, USA Critical Care 2010, 14(Suppl 1):P579 (doi: 10.1186/cc8811)

Introduction DexCom and Edwards Lifesciences collaborated to develop an intravenous blood glucose monitor for use in a critical care environment. The system provides automatic, real-time monitoring and trending of blood glucose (BG) values for critical care patients. The system is designed to address the needs of clinicians to monitor BG in critical care units. The sensor was also designed to accurately measure BG even in the presence of many drugs commonly used in the hospital.

Methods This was a blinded, multicenter study evaluating 17 adult patients presenting to the OR and ICU. They were monitored for up to 72 hours with reference samples taken at 4-hour intervals and measured with a YSI-2300 laboratory analyzer. Data are from initial roll-in patients used for training and assessing protocol complications. Reference blood samples were taken from either a peripheral vein, a central venous catheter or an arterial line. Glucose values were calculated prospectively but not displayed during the study.

Results Of 211 paired BG measurements, 94.3\% were within Clarke Error Grid A, 5.7\% in B and $0 \%$ in C, D and E (Figure 1a). The mean absolute relative difference was $6.5 \%$. No significant errors due to drugs were measured. These results compare favorably with a previous study of 50 subjects who were monitored continuously for up to 72 hours in a diabetic (a)

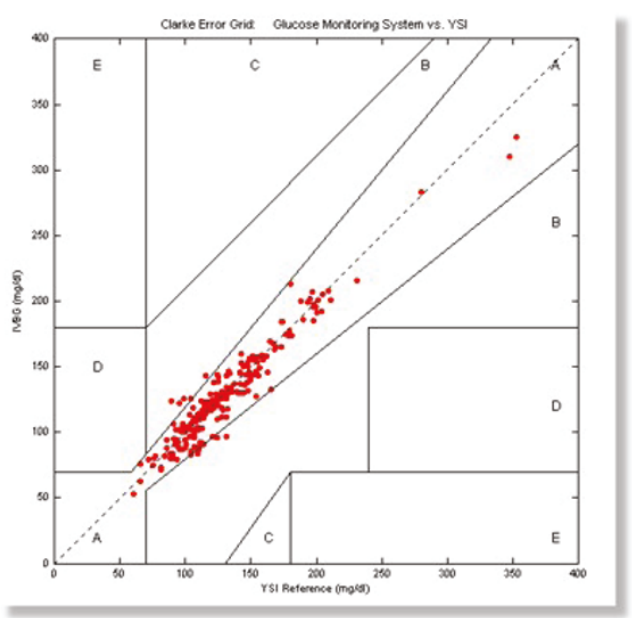

(b)

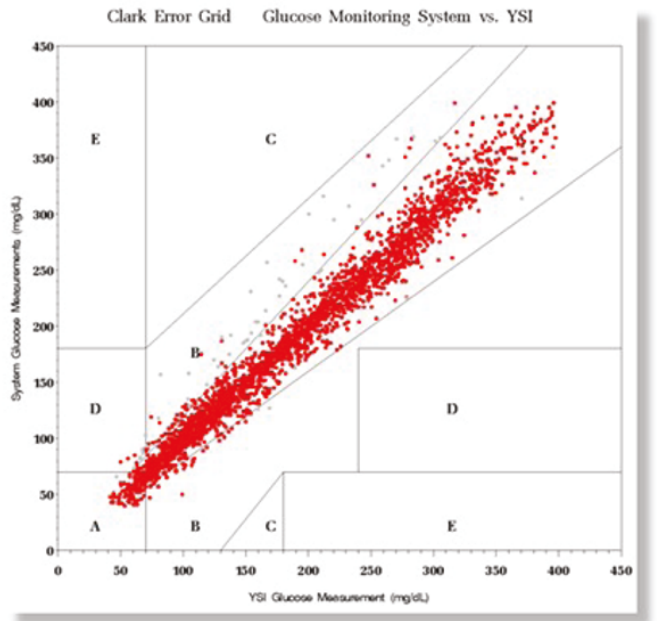

Figure 1 (abstract P579). Clarke Error Grid for (a) OR/ICU and (b) in-clinic. 
in-clinic study (Figure 1b) where large excursions of BG could safely be evaluated.

Conclusions The results of this study help illustrate the capability of the system to provide safe, accurate, automated glucose measurements in critical care patients.

P580

ICU glucose monitoring measured in plasma using mid-infrared spectroscopy

THeise', L Nosek1, J Gable², G Lim², C Calentine²

'Profil Institut für Stoffwechselforschung GmbH, Neuss, Germany; ${ }^{2} O p t i s S c a n$

Biomedical, Inc., Hayward, CA, USA

Critical Care 2010, 14(Suppl 1):P580 (doi: 10.1186/cc8812)

Introduction There are increasing calls for a highly accurate, automated system to enable tight glycemic control and avoid hypoglycemia in an ICU setting. OptiScan Biomedical has developed a glucose monitor based on mid-infrared spectroscopy that draws blood samples $(120 \mu \mathrm{l})$ and measures plasma glucose concentrations approximately every 15 minutes. The goal of this study was to validate the performance of the Optiscan model 5000 (OptiScanner) at different glycemic levels in a healthy diabetic patient population.

Methods Eighteen people (14 males, age 44 (18 to 64) years, BMI 29.0 (21.9 to 37.5$\left.) \mathrm{kg} / \mathrm{m}^{2}\right)$ with type $1(n=8)$ or type $2(n=10)$ diabetes were connected to an OptiScanner. Their blood glucose concentrations were kept in a euglycemic ( 75 to $180 \mathrm{mg} / \mathrm{dl})$, hypoglycemic $(<75 \mathrm{mg} / \mathrm{dl})$ and hyperglycemic (>180 mg/dl) range by intravenous administrations of insulin and glucose. Each OptiScanner blood sample was automatically withdrawn from a forearm vein. Blood samples for reference measurements using the YSI 2300 were withdrawn from the same arm within 60 seconds of the OptiScanner draw and analyzed immediately. A total of 250 paired data points in each glucose range will be collected in this ongoing study. Results The aggregate data points (374 paired readings between the OptiScanner and the YSI 2300) were within ISO standard, with $98.9 \%$ of the glucose values within $\pm 20 \%$ above $75 \mathrm{mg} / \mathrm{dl}$ and $\pm 15 \mathrm{mg} / \mathrm{dl}$ below this value. A Clark Error Grid analysis showed a total of 369 points (98.7\%) in zone A (Figure 1). Points outside A exceeded the A zone boundary by an average of $5.3 \%$ and a maximum of $8.7 \%$. The total coefficient for variance was $5.7 \%$. The total $r^{2}$ was 0.99

Conclusions These preliminary results show that the OptiScanner is highly accurate in a healthy diabetic population.

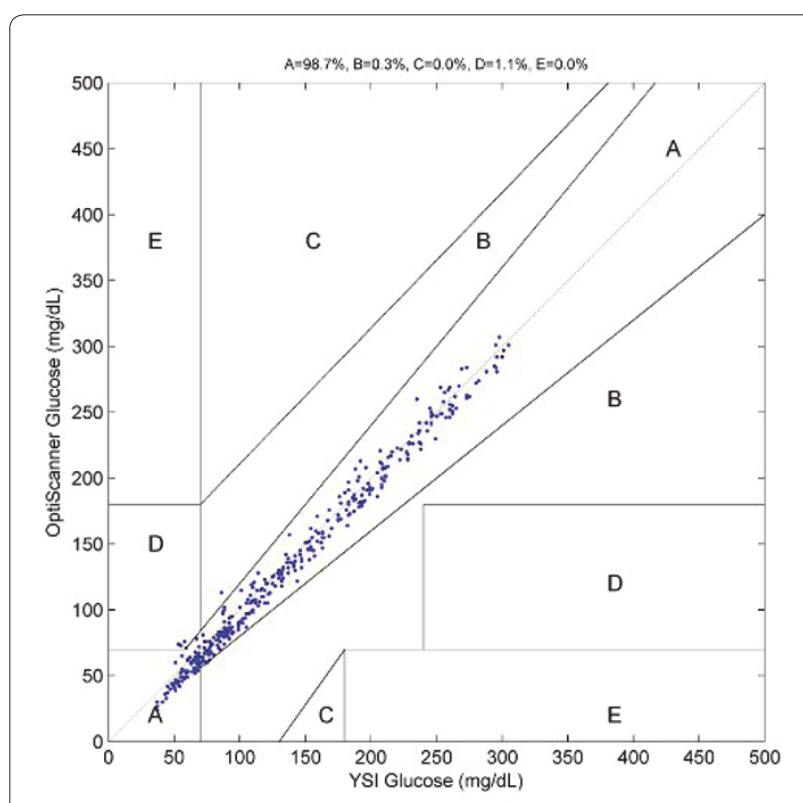

Figure 1 (abstract P580).
P581

Validation of $\mathrm{HbA} 1 \mathrm{c}$ as a diagnostic marker for diabetes in the critically ill

I Gornik, A Vujaklija-Brajković, V Gašparović

University Hospital Centre Zagreb, Croatia

Critical Care 2010, 14(Suppl 1):P581 (doi: 10.1186/cc8813)

Introduction Diabetes mellitus (DM) is a highly prevalent disease: about one-quarter of patients admitted to hospital is diagnosed with DM, but there are also a large number of patients who are undiagnosed. An acutely ill patient with hyperglycaemia and no history of diabetes may have stress hyperglycaemia or a first manifestation of diabetes, but it is virtually impossible to establish diagnosis based on measurements of glucose. $\mathrm{HbA1c}$ is widely used as a marker of glycaemic control and has been proposed and used, but not yet officially accepted, as a diagnostic marker for diabetes. We have tested its usefulness for diagnosing diabetes in the intensive care setting.

Methods We have included a cohort of adult patients admitted to a medical ICU. HbA1c was measured in all patients. Patients without hyperglycaemia during their illness and negative history of DM were used as negative controls; patients with known diagnosis of DM, impaired fasting glucose (IFG) or impaired glucose tolerance (IGT) were positive controls. All patients with negative history for DM and hyperglycaemia in ICU were called for measurement of fasting glucose and OGTT within 1 month after discharge.

Results There were 618 patients in the cohort: 138 with no hyperglycaemia, 79 with a history of IFG or IGT, 108 with a history of diabetes; 293 patients had hyperglycaemia during the ICU stay and no information about DM in the history. When analysing only positive and negative controls, a cut-off value of $6 \%$ showed $95 \%$ sensitivity and $93 \%$ specificity for pre-diabetes (IFG and IGT), cut-off of $7 \%$ had $91 \%$ sensitivity and $96 \%$ specificity for DM. Similar results were obtained for the group of patients with hyperglycaemia and no history of impaired glucose metabolism: cut-off of $6 \%$ had $97 \%$ sensitivity and $93 \%$ specificity for pre-diabetes, $7 \%$ cut-off had $95 \%$ sensitivity and $98 \%$ specificity for DM.

Conclusions HbAlc can be used for diagnosing diabetes and prediabetes in patients with critical care hyperglycaemia with high sensitivity and specificity. Since diabetes brings a burden of complications, impaired immune response, susceptibility to infections, and overall higher mortality, differentiating diabetics from patients without diabetes can be very valuable.

\section{P582}

Effect of intravenous glucocorticoids on the efficiency of tight glycaemic control

J Herbots' ', G Mwani'2, D Cottem', D Mesotten ${ }^{3}$

'UZ Leuven, Belgium; '2St-Jozef Ziekenhuis, Turnhout, Belgium; ${ }^{3} K U$ Leuven, Belgium

Critical Care 2010, 14(Suppl 1):P582 (doi: 10.1186/cc8814)

Introduction Intravenous glucocorticoids (GC) are often administered during critical illness in the context of post-transplant immunosuppression, ARDS, septic shock or severe inflammatory vasoplegia. As a counterregulatory hormone of insulin, GC may interfere substantially with tight glycaemic control to normal for age blood glucose levels by intensive insulin therapy. Therefore we hypothesized that the first administration of an intravenous GC gives rise to significant hyperglycaemia and that the effect is more pronounced for the synthetic GC.

Methods All patients admitted to the surgical and paediatric ICU between 1 July 2008 and 1 July 2009 were screened for intravenous glucocorticoid use in our patient data management system. In those patients, arterial blood glucose levels, all measured by blood gas analyzer, within the time frame of 48 hours surrounding the GC administration were retrieved. Up to two baseline blood glucose levels (before GC administration) and up to six blood glucose levels post administration were analysed by repeatedmeasures ANOVA. Data are expressed as mean \pm SD or as median (IQR). Results A total of 452 patients $-n=26$ ( $<1$ year), $n=76$ ( 1 to 17 years), $n=350$ (>17 years) - received intravenous GC: hydrocortisone 32\%, bolus methylprednisolone 52\%, drip methylprednisolone $4 \%$ and dexamethasone $12 \%$. Baseline blood glucose levels were $97 \pm 34 \mathrm{mg} / \mathrm{dl}$ (<1 year), 104 (89 to 132$) \mathrm{mg} / \mathrm{dl}$ (1 to 17 years), 136 (106 to 174$) \mathrm{mg} / \mathrm{dl}$ 
(>17 years). In the adult patients the first blood glucose measurement was 1.0 (0.4 to 2.0) hours after GC administration. Blood glucose levels decreased over time from baseline after $\mathrm{GC}$ administration $(P<0.0001)$. The sixth glycaemia was 110 (91 to 139) mg/dl taken 12.4 (9.6 to 16.5) hours after GC administration. This decrease of glycaemia was only present in patients who received $92 \pm 35 \mathrm{mg}$ hydrocortisone $(P=0.004)$ or 40 (20 to $125) \mathrm{mg}$ bolus methylprednisolone $(P<0.0001)$. In patients who received 113 (71 to 160) mg drip methylprednisolone $(P=0.14$ ) or 10 (5 to 12.5) mg dexamethasone $(P=0.44)$, blood glucose levels did not change over time. Conclusions We showed that, when a tight blood glucose protocol is implemented, intravenous GC administration does not necessarily lead to an increase in blood glucose level. The blood glucose profile after methylprednisolone or dexamethasone administration did not differ from the glycaemic response after hydrocortisone administration. However, avoiding hyperglycaemic peaks after the GC administration during tight glycaemic control requires an on average 2-hourly blood glucose measurement.

\section{P583}

Chronic critical illness: high levels of estrogens and mortality

G Bernardeschi, B Ferro, R Mori, A Abramo, F Forfori, F Giunta

Scuola di Specializzazione di Anestesia e Rianimazione, Università degli Studi di Pisa, Italy

Critical Care 2010, 14(Suppl 1):P583 (doi: 10.1186/cc8815)

Introduction Chronic critical illness is characterized by a severe metabolic disorder, caused by the loss of the physiologic hypothalamic and pituitary functions and the onset of the so-called wasting syndrome, as many studies have just confirmed [1]. In this study we assessed the correlation between the neuroendocrine pattern in chronic ICU patients and mortality.

Methods The patients enrolled were 25 (18 male and five female) with a mean age of 67 years and APACHE II score of $12 \pm 5$. We excluded females in a premenopausal state, patients with previous endocrine problems or in therapy with dopamine, high dose of cortisone or amiodarone. In these patients, we evaluate, on the seventh day of stay in the ICU (considered the chronic phase of critical illness), the mean value of four nocturnal hormonal measurements ( $\mathrm{LH}, \mathrm{FSH}$, estrogen, testosterone, DHEAS, androstenedione, androstenediol, progesterone, TSH, FT3, FT4, RT3, GH, IGF1, prolactin, cortisol). Furthermore, we observed hormonal patterns in people who died in the ICU.

Results We found statistical evidence in the correlation of high levels of estrogens, related to aromatase increased function [2], and the percentage of death. In the group of patients with estrogens more than $50 \mathrm{pg} / \mathrm{ml}$, six of them died, while if estrogens were normal or low, none died (Figure 1). Survivors and nonsurvivors did not differ by mechanism of injury or

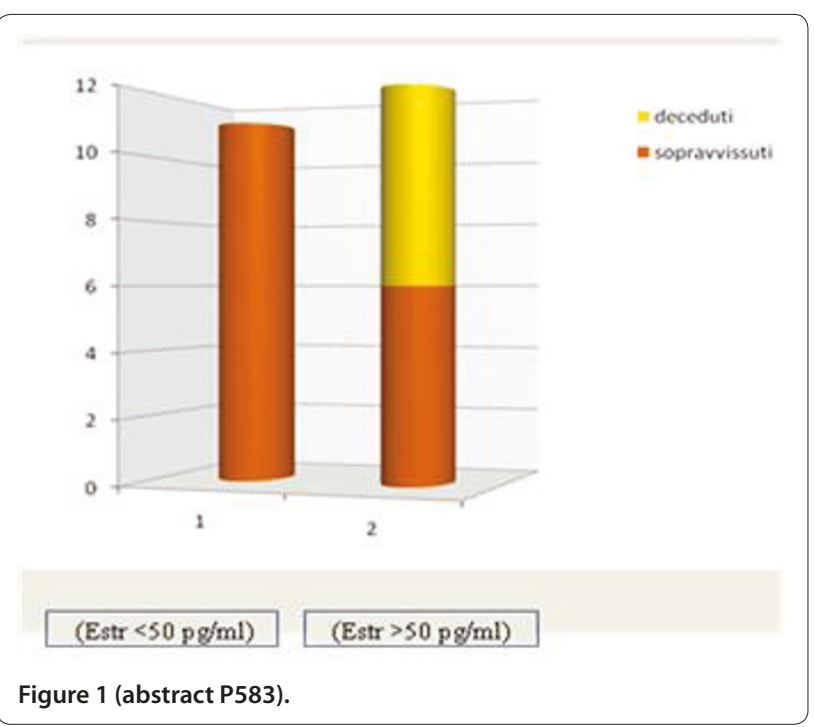

APACHE score (11.6 vs 11.8). Medium serum estradiol levels were $44.96 \mathrm{pg} /$ $\mathrm{ml}$ in survivors and $115.08 \mathrm{pg} / \mathrm{ml}$ in nonsurvivors.

Conclusions To date, the role of these hormones in critical illness pathophysiology and the increase of estrogen levels are still uncertain. Further studies are required.

References

1. Van den Berghe G: Frontiers Neuroendocrinol 2002, 23:370-391.

2. Spratt DI, et al:: Am J Physiol Endocrinol Metab 2006, 291:E631-E638.

\section{P584}

Estrogen decreases inflammation and apoptotic signaling in the heart following severe burn injury

JW Gatson, Q Zang, DL Maass, JP Minei, AH Idris, PE Pepe, JWigginton University of Texas Southwestern Medical Center at Dallas and the Parkland Health and Hospital System, Dallas, TX, USA

Critical Care 2010, 14(Suppl 1):P584 (doi: 10.1186/cc8816)

Introduction Patients with severe burn injury experience a rapid elevation in multiple circulating proinflammatory cytokines, with the levels correlating with both injury severity and outcome. Accumulations of these cytokines in animal models have been observed in remote organs, however data are lacking regarding early serial heart cytokine levels following burn injury, and the therapeutic effects of estrogen on these levels. Using an animal model, we studied the acute effects of a fullthickness third-degree burn on cardiac levels of IL-1 $\beta$, IL-6, IL-10, and TNFa. In addition, we analyzed the effect of estrogen on levels of cytochrome C, signifying apoptosis, and levels of NF-KB, signifying inflammation. Here, we hypothesized that acute estrogen treatment decreases inflammation in the heart and blocks the induction of apoptosis.

Methods In this study, 144 male rats received third-degree $40 \%$ total body surface area burns. Fifteen minutes following burn injury, the animals received a subcutaneous injection of either placebo or $17 \beta$-estradiol $(0.5 \mathrm{mg} / \mathrm{kg})$. The hearts were harvested at $0.5,1,2,4,6,8,12,18$, and 24 hours after injury, and the heart cytokine (IL-1 $\beta, I L-6, I L-10, T N F a)$ levels were measured using the ELISA method. In addition, we assessed the cytosolic levels of cytochrome $\mathrm{C}$ and NF-KB at the 24-hour time point using western blot analysis.

Results In the burned rats, $17 \beta$-estradiol significantly decreased the cardiac levels of TNFa ( 95\%), IL-6 ( 50\%), IL-1 $\beta(\sim 25 \%)$, and IL-10 ( 20\%), when compared with the placebo group. In addition, we determined that estradiol treatment restored cytosolic levels of NF-KB (65\% increase) at the 24-hour time point. Also, estrogen decreased cytosolic accumulation of cytochrome C (50\% reduction) at the 24-hour time point.

Conclusions Following severe burn injury, estrogens decrease cardiac inflammation and levels of the pro-apoptotic cytochrome C. In addition, estrogen signaling promotes cell survival, as indicated by an increase in NF-KB levels. Importantly, estrogen treatment following burn injury nearly ablated the deleterious burn-induced increase of TNFa in the heart to levels similar to unburned control animals. Elevated cardiac levels of TNFa have previously been linked to a poor outcome.

\section{P585}

Dexamethasone treatment during cardiac surgery and postoperative lactate levels

SC Schuit, J J Van Koesveld, AJ Bogers, J Bakker, RJ Van Thiel Erasmus MC, Rotterdam, the Netherlands

Critical Care 2010, 14(Suppl 1):P585 (doi: 10.1186/cc8817)

Introduction Glucocorticoids are known to have an anti-insulin action on glucose metabolism, leading to increased lactate production [1]. Alternatively, glucocorticoid-induced apoptosis is a well-recognized phenomenon initiated by mitochondrial dysfunction [2]. Increased lactate production follows loss of mitochondrial membrane potential during apoptosis. Both mechanisms may lead to clinically relevant hyperlactatemia following glucocorticoid administration during cardiac surgery requiring cardiopulmonary bypass.

Methods All adult patients undergoing cardiac surgery and cardiopulmonary bypass from 5 October through 21 November 2005 in a large academic teaching hospital in Rotterdam, the Netherlands were included in this study. Dexamethasone (60 to $80 \mathrm{mg}$ ) was given 


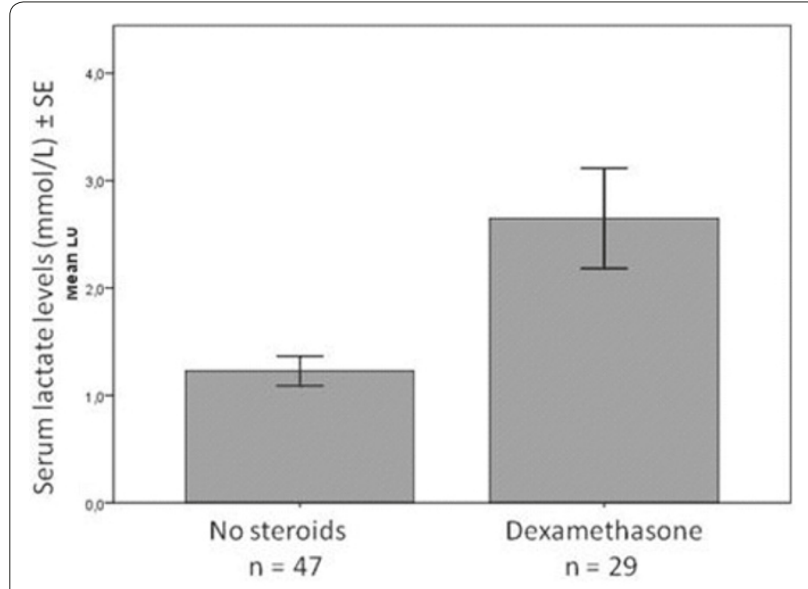

Figure 1 (abstract P585). Serum lactate levels in patients with and without dexamethasone during cardiopulmonary bypass.
Results Of the initial 293 patients, 89 were excluded for eating regular meals or having an initiation or withdrawal of statin during their ICU stay. Of the remaining 204 patients, 79 (38.7\%) had HTG, although guidelines for lipid intake were followed. Only three patients (1.5\%) had a combined fat intake (enteral and parenteral) between 1.51 and 1.71 $\mathrm{g} / \mathrm{kg} /$ day. Small positive correlations were observed with the intake of nutritional parenteral lipids (0.27), intake of all lipids given (0.20), intake of long-chain triglycerides (LCT) (0.15), and intake of parenteral LCT $(0.20)$. Medium positive correlations were observed with the amount received of lipids administered with continuous propofol $(0.40)$ and the amount of active principle of continuous propofol administered (0.42). In comparison with the control group $(n=81)$, patients with hepatic dysfunction ( $n=6)$, pancreatitis $(n=14)$, severe insulin resistance $(n=2)$, sepsis $(n=32)$ and dyslipidemia without statin $(n=7)$ had significantly higher mean values of TG (all $P<0.05$ ). Groups with cirrhosis and ascites $(n=3)$, diabetes mellitus $(n=11)$, chronic renal failure $(n=6)$ and patients with statin before and during hospitalization $(n=42)$ had similar levels of TG as the control group.

Conclusions When guidelines for fat intake in the ICU are followed, modest fat intake does not seem to explain HTG. Our results show that the amount of propofol given $(\mathrm{mg} / \mathrm{kg} /$ day) and some clinical factors might be correlated with HTG in the ICU.

\section{P587}

Septic patients show significantly lower 1,25-dihydroxy vitamin D levels than trauma patients

A Cecchi' ${ }^{1}$, S Dourr ${ }^{1}$, S Paladini' ${ }^{1}$ A Di Filippo ${ }^{2}$, M Ciapetti ${ }^{1}$, F Nencini ${ }^{1}$,

M Andreani ${ }^{3}$, G Messeri ${ }^{1}$, M Bonizzoli' ${ }^{1}$ J Parodo ${ }^{1}$, G Zagli ${ }^{1}$, A Peris ${ }^{1}$

${ }^{1}$ Careggi Teaching Hospital, Florence, Italy; ${ }^{2}$ Institute of Anesthesia, University of Florence, Italy; ${ }^{3}$ Postgraduate School of Anesthesia and Intensive Care, University of Florence, Italy

Critical Care 2010, 14(Suppl 1):P587 (doi: 10.1186/cc8819) thus received methylprednisolone, were excluded; a further two patients who received hydrocortisone for allergic reactions were also excluded. Data were incomplete for one patient, leaving a total of 76 patients for analysis. The mean lactate level was $1.2 \mathrm{mmol} / \mathrm{l}$ in the 47 patients who did not receive steroids and $2.6 \mathrm{mmol} / \mathrm{l}$ in the 29 patients who received dexamethasone $(P<0.0001$, Figure 1$)$. When adjusting for potential confounders such as age, glucose level, duration of cardiopulmonary bypass and preoperative NYHA heart failure classification, this difference remained significant $(P<0.0001)$

Conclusions Administration of dexamethasone during cardiac surgery requiring cardiopulmonary bypass is associated with a significant hyperlactatemia.

\section{References}

1. Plager JE, et al:: Endocrinology 1966, 78:1154-1158.

2. Distelhorst CW, et al:. Cell Death Diff 2002, 9:6-19.

\section{P586}

Risk factors for hypertriglyceridemia in the intensive care unit: an exploratory study

JC Devaud, P Voirol, P Marques-Vidal, L Tappy, N Rodondi, R Chiolero,

MM Berger, A Pannatier

University Hospital (CHUV), Lausanne, Switzerland

Critical Care 2010, 14(Suppl 1):P586 (doi: 10.1186/cc8818)

Introduction Despite guidelines recommending a daily fat intake of 0.7 to $1.5 \mathrm{~g} / \mathrm{kg} /$ day in the $\mathrm{ICU}$, subjects with hypertriglyceridemia $>2 \mathrm{mmol} / \mathrm{I}$ (HTG) are common. As there are limited data on risk factors for HTG in the ICU, we aimed to determine the factors related with HTG in the ICU.

Methods During 7 months, all consecutive patients staying $\geq 4$ days in an adult ICU from a university hospital were enrolled. Patients eating regular meals or having an initiation or withdrawal of statin during their ICU stay were excluded. Peak values of triglycerides (TG) were collected and the relationships between $\mathrm{log}-\mathrm{TG}$ and fat intake ( $\mathrm{g} / \mathrm{kg} /$ day) from nutritional (enteral and parenteral) and non-nutritional (propofol's emulsion) sources as well as propofol (mg/kg/day) were assessed using Pearson's correlation coefficients. Correlation was considered small for coefficients between 0.1 and 0.3 and medium for coefficients between 0.4 and 0.6. Nine groups of patients at risk of HTG were further compared with a control group (no risk factors for HTG) using Dunnett's test (significant if adjusted $P<0.05$ ).
Introduction It is known that 1,25-dihydroxyvitamin D (vitamin D) is involved in expression control of more than 200 genes. Vitamin D affects immunity, endothelial and mucosal functions as well as glucose and calcium metabolism. Moreover, its serum deficiency $(<20 \mathrm{ng} / \mathrm{ml})$ is reported to be common in hospitalized patients, especially among patients admitted to the ICU. Our aim was to evaluate vitamin D levels in a selected population of ICU patients and its correlation with admission pathology and outcome.

Methods Among all patients admitted to our general ICU (February to October 2009), 84 patients were studied, 53 admitted for major trauma and 31 patients admitted for severe sepsis/septic shock. Exclusion criteria were: age $<18$ years, malnutrition state $(\mathrm{BMI}<18)$, pregnancy, breastfeeding, chemotherapy and immunotherapy, every pathology affecting bone and calcium metabolism, vitamin D metabolism derangements for therapies, haematological and solid malignancies, HIV. Vitamin D levels were measured by radioimmunoassay and registered at admission as well demographic data, simplified acute physiology score (SAPS), injury severity score (ISS), length of stay (LOS), outcome. Data are expressed as the mean. Statistical analysis: Mann-Whitney $(P<0.05)$. The study was approved by the Internal Review Board, which waived the need for informed consent.

Results Vitamin D levels at admission to the ICU respectively were $14.1 \mathrm{ng} /$ $\mathrm{ml}$ in the sepsis group (age 61 years, SAPS 46.9) and $21.88 \mathrm{ng} / \mathrm{ml}$ in the trauma group (age 46.7 years, SAPS 36.2, ISS 26.8). To avoid the age-related bias, 23 patients older than 50 years were analysed. Vitamin D levels were found to be $20.9 \mathrm{ng} / \mathrm{ml}$ (mean age 66.6, SAPS 43.2, mean ISS 24) $(P=0.0195)$. No correlations with length of stay, duration of mechanical ventilation or outcome were found.

Conclusions We confirm a vitamin D inadequacy among patients admitted to the ICU, even in young trauma patients ( $>20$ and $<30 \mathrm{ng} / \mathrm{ml}$ ). However, septic patients showed a significantly lower vitamin D level than trauma patients with the same demographic/clinical characteristics (14.1 outcome need to be investigated with larger samples. vs $20.9 \mathrm{ng} / \mathrm{ml} ; P=0.0195)$. Correlations between vitamin D levels, LOS and 
P588

High prevalence of severe vitamin D deficiency in intensive care patients

JJ Weenink, H Oudemans-van Straaten, HT Yap, EH Slaats, PH Van der Voort OLVG hHospital, Amsterdam, the Netherlands

Critical Care 2010, 14(Suppl 1):P588 (doi: 10.1186/cc8820)

Introduction Vitamin D deficiency seems increasingly prevalent in the community, and deficiency has been reported in almost $60 \%$ of medical inpatients [1]. Pleiotropic effects of vitamin D like immunomodulation and effects on muscle strength may be of special importance to critically ill patients. Despite this knowledge, vitamin $\mathrm{D}$ deficiency has only been studied in small and selected groups of ICU patients [2]. Therefore, we prospectively determined the prevalence of vitamin $\mathrm{D}$ deficiency and related vitamin D status to outcome in a cohort of critically ill patients.

Methods This prospective observational cohort study was performed in a 20-bed general ICU of a teaching hospital in the city of Amsterdam between 1 March and 30 April 2009. We measured 25-hydroxyvitamin D (25-OH-D) on admission and after 48 hours in all consecutive patients admitted to our unit. Patients received enteral feeding. Additional vitamin D was not supplied. Vitamin D status was defined as: adequate: $>75 \mathrm{nmol} / \mathrm{l}$, insufficient: 50 to $75 \mathrm{nmol} / \mathrm{l}$, deficient: 25 to $50 \mathrm{nmol} / \mathrm{l}$, severely deficient: $<25 \mathrm{nmol} / \mathrm{l}$, undetectable: $<10 \mathrm{nmol}$ (to convert values for $25-\mathrm{OH}-\mathrm{D}$ to $\mathrm{ng} / \mathrm{ml}$, divide by 2.50 ). We compared observed and predicted mortality (APACHE IV), and standardized mortality ratio (SMR, observed/predicted number of nonsurvivors) between vitamin $D$ cohorts.

Results Vitamin D was measured in 111 patients at admission and in 44 after 48 hours. Mean 25-OH-D at admission was $31 \mathrm{nmol} / \mathrm{l}$. Thirteen percent of the patients had undetectable $25-\mathrm{OH}-\mathrm{D}$ levels, $49 \%$ was severely deficient and $85 \%$ had a vitamin D $<50$ nmol/l. Only two patients had an adequate 25-OH-D status. During the first 48 hours of ICU admission, vitamin D decreased in 24 of 44 patients. Mean change was $-2.3 \mathrm{nmol} / \mathrm{l}$ (range -32.5 to $+22.5 \mathrm{nmol} / \mathrm{l}$ ). Observed mortality was significantly lower than predicted in all patients $(P=0.02)$ and in patients with $25-\mathrm{OH}-\mathrm{D}$ $>25 \mathrm{nmol} / \mathrm{I}(P=0.04)$, but not in those $<25 \mathrm{nmol} / \mathrm{l}(P=0.21)$. See Table 1 .

Conclusions At the end of winter, one-half of our patients had severe vitamin D deficiency. In contrast to patients with severe deficiency, the cohort with vitamin D levels more than $25 \mathrm{nmol} / \mathrm{l}$ had a significant lower mortality than predicted by APACHE IV score. Measurements from a larger patient cohort are collected to further analyze this finding.

\section{References}

1. Thomas MK et al:: N Engl J Med 1998, 338:777-783.

2. Lee P: N Engl J Med 2009, 360:1912-1914.

P589

Vitamin $D$, parathyroid hormone and serum calcium levels and their association with hospital mortality in critically ill patients

K Amrein, S Amrein, A Holl, A Waltensdorfer, TR Pieber, H Dobnig

Medical University of Graz, Austria

Critical Care 2010, 14(Suppl 1):P589 (doi: 10.1186/cc8821)

Introduction Vitamin D deficiency is highly prevalent and has been associated with increased mortality in the general population $[1,2]$. Critically ill patients are especially prone to present with or develop low vitamin D levels due to their underlying disease, sunlight deprivation or longer hospital stays.

Methods Parathyroid hormone (PTH) and vitamin D status (25-hydroxyvitamin D (25(OH)D)) were assessed at different ICUs (medical, cardiac surgery, mixed surgical and neurological) in 404 patients (age $63 \pm 16$ years, 65\% male) from September 2008 until November 2009.
Results The mean serum 25(OH)D level was $19.6 \pm 11.2 \mathrm{ng} / \mathrm{ml}$. Normal $25(\mathrm{OH}) \mathrm{D}$ levels $(>30 \mathrm{ng} / \mathrm{ml}$ ) were found in $12.4 \%$ of all patients. The majority $(58.2 \%)$ were vitamin D deficient $(<20 \mathrm{ng} / \mathrm{ml})$, while $29.4 \%$ were considered vitamin D insufficient ( $>20$ and $<30 \mathrm{ng} / \mathrm{ml}$ ) by current definitions. Significant variations of vitamin $D$ levels occurred depending on season, with highest values found in August $(27.1 \pm 13.1 \mathrm{ng} / \mathrm{ml})$ and the lowest in March $(12.5 \pm 4.6 \mathrm{ng} / \mathrm{ml})$, respectively. Secondary hyperparathyroidism (PTH $>65 \mathrm{pg} / \mathrm{ml}$ ) was highly prevalent in medical and cardiac surgery patients (43.7\% and $37.9 \%$ ). In an age, sex and SAPS II score adjusted Cox-proportional hazards regression analysis model, patients with low PTH or serum calcium levels, as well as low 25(OH)D levels (cut-off $12 \mathrm{ng} / \mathrm{ml}$ ) were all at significantly greater risk for all-cause mortality. With all variables included into one model $(n=268$ patients) serum calcium remained an independent predictor of mortality, whereas PTH $\log (P=0.08)$ and $25(\mathrm{OH}) \mathrm{D}(P=0.16)$ levels suggested a trend.

Conclusions Vitamin D deficiency is often encountered in critically ill patients. Higher than the currently recommended daily dose of $200 \mathrm{IU}$ vitamin $D_{3}$ is probably required for achieving or maintaining normal $25(\mathrm{OH}) \mathrm{D}$ levels. Results of this study suggest that baseline parameters reflecting calcium/PTH status are associated with mortality. Whether a disturbance in calcium/vitamin $\mathrm{D}_{3}$ or PTH metabolism develops secondary to the medical condition of ICU patients or is a primary cause of mortality remains to be clarified in prospective analyses.

References

1. Dobnig H, et al: Arch Intern Med 2008, 168:1340-1349.

2. Melamed ML, et al.: Arch Intern Med 2008, 168:1629-1637.

\section{P590}

Effect of restoration of $250 \mathrm{OD}_{3}$ status in prolonged critical illness on serum innate immunity parameters LL-37 and SCD163

CM Ingels', SJ Van Cromphaut', PJ Wouters', HJ Moller', EVan Herck', I Jans', R Bouillon', G Van den Berghe

'University Hospitals Leuven, Belgium; '²Aarhus University, Aarhus, Denmark Critical Care 2010, 14(Suppl 1):P590 (doi: 10.1186/cc8822)

Introduction We previously observed that critically ill patients are vitD deficient. This status cannot be restored by increasing the normally recommended intravenous vitD dose. The antimicrobial peptide $L L-37$ is expressed in macrophages upon stimulation by $25 \mathrm{OHD}_{3}$. SCD163 is a soluble pattern-recognition receptor, which is a marker of macrophage activation and a predictor of mortality in SIRS. The aim of this study is to unravel the impact of $25 \mathrm{OHD}_{3}$ deficiency and normalization on innate immunity parameters - CRP, LL-37 and SCD163 - in critical illness.

Methods We randomly allocated patients upon ICU admission to receive either placebo $(n=13)$ or a 10 -day treatment of $15 \mu \mathrm{g} /$ day $250 \mathrm{HD}_{3}$ intravenously after a loading dose of $200 \mu \mathrm{g}$ intravenously $(n=11)$ on top of the normal vitD dose (200 IU). Patients with chronic bone or kidney disease, glucocorticoid treatment, age younger than 18 years, or with an anticipated ICU length of stay less than 10 days were excluded. On admission, patients were compared with healthy age-matched, gendermatched and BMI-matched controls $(n=24)$.

Results Administration of the $250 \mathrm{HD}$ regimen resulted in a rapid and sustained increase of serum $25 \mathrm{OHD}_{3}$ levels $(P<0.05$ vs placebo). $1,25(\mathrm{OH})_{2} D_{2}$ levels rose only transiently $\left(P^{3}=0.07\right.$ on day $1, P=0.08$ on day 2 vs placebo). $25 \mathrm{OHD}_{3}$ and $1,25(\mathrm{OH})_{2} \mathrm{D}_{3}$ levels were lower than those of healthy controls (sampled during summer). $25 \mathrm{OHD}_{3}$ treatment had no significant effect on CRP. On admission, LL-37 levels ( $26 \pm 6 \mu \mathrm{g} / \mathrm{l})$ were comparable with those of healthy controls $(22 \pm 2 \mu \mathrm{g} / \mathrm{l})$. The treatment tended to transiently increase LL-37 levels (change from baseline: $P=0.1$ on day 6 vs placebo). Serum sCD163 levels tended to be higher at baseline

Table 1 (abstract P588)

\begin{tabular}{lccccc}
\hline & Number of patients & Age (years) & Hospital mortality (\%) & APACHE IV p.m. (fraction) & SMR (fraction) \\
\hline All patients & 111 & 67 & 19 & 0.31 & $0.61\left(0.38\right.$ to $\left.0.93^{\mathrm{a}}\right)$ \\
$25-\mathrm{OH}-\mathrm{D}<25 \mathrm{nmol} / /$ & 54 & 68 & $24^{*}$ & 0.34 & $0.71\left(0.38\right.$ to $\left.1.21^{\mathrm{a}}\right)$ \\
$25-\mathrm{OH}-\mathrm{D}>25 \mathrm{nmol} / /$ & 57 & 67 & 14 & 0.29 & $0.48\left(0.21\right.$ to $\left.0.92^{\mathrm{a}}\right)$ \\
\hline
\end{tabular}

a95\% confidence interval (Fischer's exact). ${ }^{*} P=0.33$ compared with $>25$ nmol. p.m., predicted mortality. 
( $P=0.06$ vs healthy controls) and rose significantly over time $(P<0.05$ on day 5 and on day 10 vs day 0 ) but were not influenced by the treatment. Conclusions The preliminary results of this randomized placebocontrolled pilot study indicate that, in contrast to a high intravenous $500 \mathrm{IU}$ vitD supplement, intravenous $250 \mathrm{HD}$, treatment resulted in an immediate and persistent increase in serum $250 \mathrm{HD}_{3}$, but did not affect $1,25(\mathrm{OH})_{2} \mathrm{D}_{3}$ levels. Plasma LL-37 alterations in the studied prolonged critically ill patients hints at ameliorated LL-37 production provided that the $25 \mathrm{OHD}$ status is restored. Serum SCD163 and CRP levels evolved during the ICU stay, but were not significantly altered by $25 \mathrm{OHD}$ supplementation. The results of this pilot study warrant further research into the potential modulation of innate immune processes by $25 \mathrm{OHD}_{3}$ in the critically ill.

\section{P591}

Alterations in adipose tissue during critical illness: an adaptive and protective response?

L Langouche'1, SVander Perre', SThiessen', G Hermans'1, A D'Hoore', B Kola², M Korbonits², G Van den Berghe

'KU Leuven, Belgium; 'Barts and the London School of Medicine and DentistryQueen Mary University of London, UK

Critical Care 2010, 14(Suppl 1):P591 (doi: 10.1186/cc8823)

Introduction Critical illness is characterized by lean tissue wasting whereas adipose tissue is preserved. Obese critically ill patients may have a lower risk of death than nonobese patients, a recent observation that may suggest a protective role for adipose tissue during illness, a role which has not been previously investigated. We hypothesized that adipose tissue could function as a waste bin for potentially toxic metabolites, such as glucose, during critical illness.

Methods We studied adipose tissue biopsies from 61 of critically ill patients, more specifically morphology and the potential to take up and metabolize glucose, and compared this with biopsies from 20 matched controls.

Results Adipose tissue biopsies from critically ill patients revealed a higher number and a smaller size of adipocytes as compared with matched controls, coinciding with increased preadipocyte marker levels. Also, >95\% of adipose biopsies from critically ill patients displayed positive macrophage staining. Gene and protein expression of insulin-independent GLUTs, and tissue glucose content was increased. Glucokinase expression was upregulated whereas glycogen and glucose-6-phosphate levels were low. Acetyl-CoA-carboxylase protein level and activity of fatty-acid synthase were increased. A substantially increased activity of AMPK may play a crucial role.

Conclusions The larger number of small adipocytes in response to critical illness appears to have an increased ability to take up and metabolize glucose into fatty acids. Such changes may render adipose tissue biologically active as a functional waste bin for toxic metabolites during critical illness, which could contribute to survival.

\section{P592}

Impact of collection method and sample handling on measured levels of circulating ACTH

C Borin, D Mesotten, G Van den Berghe, HVervenne

KU Leuven, Belgium

Critical Care 2010, 14(Suppl 1):P592 (doi: 10.1186/cc8824)

Introduction Cortisol, which is produced and released by the adrenal gland under the regulation of the adrenocorticotropic hormone (ACTH), plays a crucial role in the survival mechanisms involved in critical illness. A dissociation between high plasma cortisol and low ACTH in patients has been observed in the more chronic phase of critical illness induced by severe sepsis and multiple trauma [1]. In order to assess the potential confounding impact of errors in the measurement of ACTH, we evaluated the stability of ACTH during sample processing.

Methods Two tests were performed. For the first test, two blood samples per patient were taken from 10 randomly selected patients in the surgical ICU. One sample was collected warm (that is, samples were collected at room temperature (RT), left for 60 to 90 minutes at RT followed by
24 hours at $4^{\circ} \mathrm{C}$, centrifuged (10 minutes, 1,000 rpm), and serum was stored at $-80^{\circ} \mathrm{C}$ ), the other sample was collected cold (that is, recommended collection procedure for $\mathrm{ACTH}$ detection: samples were collected on ice, centrifuged (10 minutes, $1,000 \mathrm{rpm})$, and plasma was stored at $-80^{\circ} \mathrm{C}$ ). For a second test, again, two blood samples were taken per patient $(n=$ 10) in the surgical ICU. One sample was kept frozen until ACTH testing (original), whereas the other sample was defrosted several times at RT before analysis (defrosted). ACTH levels were measured using an ACTH RIA kit (BRAHMS) and the different sampling methods and sample treatments were compared using Bland-Altman statistics.

Results No clinically relevant differences in ACTH levels were observed between cold $(28.2 \pm 22.3 \mathrm{pg} / \mathrm{ml})$ and warm $(24.3 \pm 20.6 \mathrm{pg} / \mathrm{ml})$ sampling (bias $3.8 \pm 7.3 \mathrm{pg} / \mathrm{ml}$ ) or between original $(22.1 \pm 10.8 \mathrm{pg} / \mathrm{ml}$ ) and defrosted $(22.3 \pm 10.5 \mathrm{pg} / \mathrm{ml}$ ) samples (bias $0.2 \pm 1.9 \mathrm{pg} / \mathrm{ml}$ ). Moreover, the differences in ACTH levels measured between the two collection methods or sample treatments were independent of the amount of ACTH present in the samples.

Conclusions We showed that warm collection or frequent defrosting of samples does not induce a clinically significant error in ACTH levels measured, implying that nonideally collected or treated samples can still be used for rough ACTH analysis.

\section{Reference}

1. Vermes et al.: J Clin Endocrinol Metab 1995, 80:1238-1242.

\section{P593}

Prognostic value of serum zinc levels in critically ill patients

B Cander, D Dundar, M Gul, S Girisgin

Selcuk University Meram Faculty of Medicine, Konya, Turkey

Critical Care 2010, 14(Suppl 1):P593 (doi: 10.1186/cc8825)

Introduction Zinc is a trace element that is required for normal function of many enzymes. Intact zinc homeostasis must be present for normal function of the immune system, oxidative stress responses. We explored the hypothesis that a decline in serum zinc concentrations among critically ill patients is related to mortality, length of stay in the ICU, APACHE $\|$ and SOFA scores.

Methods This prospective study was conducted in the ICU of the emergency department. All patients (>18 years) admitted to the ICU from September through November 2009 were enrolled into the study. Burn victims, alcoholics, patients with gastrointestinal fistulas and severe diarrhea were excluded from the study. Patients' demographic characteristics, APACHE and SOFA scores were recorded; blood samples were collected within 24 hours after admission and analyzed for serum zinc, copper, C-reactive protein and albumin levels. Patients were followed up for mortality and length of stay in the ICU for 28 days.

Results Thirty-six patients were enrolled during the study period, with median age 70.5 years (18 to 87 ). The male to female ratio was 1:1.1. Serum zinc levels correlated inversely with SOFA scores $(r=-0.41, P=$ 0.01 ). Patients with $\geq 8$ SOFA scores had significantly lower serum zinc levels compared with patients with <8 SOFA scores $(6.74 \pm 1.63 \mu \mathrm{mol} / \mathrm{l}$ $(n=11)$ and $9.17 \pm 2.76 \mu \mathrm{mol} / \mathrm{I}(n=25)$, respectively; $P<0.05)$. There was no significant difference between serum zinc levels of patients with $<25$ and $\geq 25$ APACHE $\|$ scores and also there was no significant difference between survivors and nonsurvivors. Serum zinc and copper levels had no significant relationship with length of stay in the ICU.

Conclusions Serum zinc concentrations correlated inversely with SOFA scores in this study. This result supports that organ failure and critical illness leads to a decline in serum zinc concentrations and that zinc administration may be beneficial for critically ill patients.

\section{References}

1. Cvijanovich NZ, et al: Zinc homeostasis in pediatric critical illness. Pediatr Crit Care Med 2009, 10:29-34.

2. Berger MM, et al:: Trace element requirements in critically ill burned patients. J Trace Elem Med Biol 2007, 21 (Suppl 1):44-48.

3. Heyland DK, et al: Zinc supplementation in critically ill patients: a key pharmaconutrient? J Parenter Enteral Nutr 2008, 32:509-519. 
P594

High stress during admission predicts the impact of a PICU follow-up clinic on parents' psychological outcome

G Colville, P Cream, S Kerry

St George's Hospital, London, UK

Critical Care 2010, 14(Suppl 1):P594 (doi: 10.1186/cc8826)

Introduction There are no formal reports of follow-up services in pediatric intensive care settings despite mounting evidence that parents report negative psychological symptoms for many months after a child's admission to the pediatric intensive care unit (PICU). The aim of this study was to establish the impact of a PICU follow-up clinic on parents' distress. Methods In this prospective, randomised controlled trial, parents of 133 children admitted to an eight-bed PICU for $>12$ hours were randomly allocated to either the intervention (follow-up clinic appointment offered 2 months after discharge) or control (no appointment) condition. Parents' baseline stress was assessed at discharge using the Parental Stressor Scale: PICU [1]. Five months later, post-traumatic stress, anxiety and depression were assessed by postal questionnaires (Impact of Event Scale [2] and Hospital Anxiety and Depression Scale [3]).

Results Outcome data were obtained for 105 parents. Whole group analyses revealed no significant differences in psychological outcome between the control and intervention groups. However, post hoc analyses revealed effects in favour of the intervention, for a sub-group of parents $(n=55)$ who had reported baseline stress scores above median during their child's hospitalisation. Within this sub-group, parents who received the intervention were less likely to report clinically significant levels of post-traumatic stress symptoms ( $25 \%$ vs $56 \%, P=0.018$ ) or depression (19\% vs $52 \%, P=0.009$ ) than controls.

Conclusions Whilst these results do not justify routine provision of PICU follow-up clinic appointments for all parents, they do suggest that rates of long-term distress in those who find the admission particularly traumatic could be reduced by a simple one-off intervention, which was feasible in a clinical setting.

\section{References}

1. Carter MC, Miles MS: The Parental Stressor Scale: Pediatric Intensive Care Unit. Matern Child Nurs J 1989, 18:187-198.

2. Horowitz M, Wilner N, Alvarez W: Impact of Event Scale: a measure of subjective stress. Psychosom Med 1979, 41:209-218.

3. Zigmond AS, Snaith RP: The hospital anxiety and depression scale. Acta Psychiatr Scand 1983, 67:361-370.

\section{P595}

Patient's contact person in the intensive care unit: is it really important?

AH Nielsen, CB Kancir

Regional Hospital West Jutland, Holstebro, Denmark

Critical Care 2010, 14(Suppl 1):P595 (doi: 10.1186/cc8827)

Introduction The concept of a patient's contact person was originally introduced in Denmark as a political goal. It was elevated into law in 2009. As an integrated part of the Danish Healthcare Quality Program, Danish hospitals must have programs allowing each patient a contact person, who should take an active part in treatment and care. However, the concept is still not well implemented on a broader scale. Implementing a patient's contact person concept in an ICU produces new obstacles. The ICU differs from other clinical settings in various ways. It is heavier staffed at all hours. The number of doctors is limited and nurses perform primary care. Each staff member has good insight in all patient cases, which are discussed at the daily conferences. So, is the patient's contact person really important in the ICU?

Methods Questionnaires were handed out to the close relatives of every patient who stayed more than 72 hours in the ICU. Ninety-four questionnaires have been returned with usable data. No attempt to retrieve questionnaires from nonrespondents has been made. All were returned in marked envelopes, the questionnaire itself being unmarked. The questionnaire contained two questions on the patient's contact person: 1. In your experience, did you have a contact person? 2. Do you think there is a need for a contact person? Both questions were simple yes/no answers, with the possibility of adding comments. The analysis focused on quantity and quality, as displayed in the remarks made.
Results The median age of the respondents was 56 years (range 27 to 81 years). On the first question, whether you had a contact person, 40 answered Yes and $54 \mathrm{No}$. On the second question, whether there is a need for a contact person, 55 answered Yes, 31 No and eight did not answer. Twenty-six and 23 answered respectively Yes and No in both questions. Analyzing the comments made on whether you had a contact person, a different picture emerges. Of 39 comments, four are indifferent and 35 are positive - feeling that they have been met in a competent and caring manner. The comments made on whether there is a need for a contact person reflect an important discussion: some wish for a contact person, arguing that it will make them feel safer when in distress. Others point out that it would be difficult and that the contact person might not be available when needed due to the rotation shift work of nurses and doctors.

Conclusions This study shows that there is still a place and need for improvement in the concept of a patient's contact person.

\section{P596}

Evaluating the quality of communication with patients' relatives in critical care

S Siddiqui, C Scott, D Bryden

Northern General Hospital, Sheffield, UK

Critical Care 2010, 14(Suppl 1):P596 (doi: 10.1186/cc8828)

Introduction Good communication with patients' relatives has humanitarian, professional and medico-legal benefits. The NHS Litigation Authority requires clear information documentation and monitoring that this is satisfactory [1]. This review was performed to ascertain relatives' opinions regarding the current standard of communication received from medical staff.

Methods A paper questionnaire was made available to relatives after discussion with a member of the medical staff. Data for the first month are discussed below, based on an average 50 admissions/month. The questionnaire was at the main reception desk, and staff also offered them to relatives after each professional contact. Closed questions with single answers chosen from five options (including one neutral option) were asked along with space for free comment.

Results Eighteen questionnaires were completed: to our knowledge no relatives refused completion. Thirteen out of 18 discussions took place between 8 am and $6 \mathrm{pm}$, Monday to Friday, three between $8 \mathrm{pm}$ and $6 \mathrm{am}$ weekdays, and two at the weekend. All 18/18 respondents felt the doctor had explained their role in care. Thirteen out of 18 discussions took place in the dedicated interview room, four at the bedside and one by telephone. Seventeen out of 18 rated the service as very good for information given, clarity and the effect of the exchange on overall impressions of care; one questionnaire rated the service as good.

Conclusions Relative communication is highly subjective but is known to influence impressions formed of overall quality of care. National audit frameworks emphasise objective quantifiable standards, which do not capture the quality of interaction [2]. We have monitored service quality based on subjective relative impressions, however the response rate $(<40 \%)$ has potential for a positive bias in our favour. Many discussions are complex and there is a possibility that questionnaires were not offered in certain situations. Additionally, despite full anonymity of the form, relatives may have been concerned about what was written impacting on their family member's care. It is known that benefits in the processes of clinical care leading to improvements in quality can arise purely from measurement; that is, performing service reviews such as this one [3]. If targets are to be used to benchmark quality of care in this area, there is a need for more consideration of the appropriate targets or alternatively an acceptance that there is the potential to have lower data capture in these areas.

\section{References}

1. NHSLA Risk Management Standards [http://www.nhsla.com/NR/rdonlyres/ EC2E2A20-C905-4A4A-9D71-AF989F736C7B/0/Introductiontomanual.pdf]

2. Humble SR et al.: Intensive Care Med 2007, 33:S209.

3. Wright J, Shojania KG: Br Med J 2009, 38:783-784. 
P597

Family satisfaction on the intensive care unit

C Meadows, A Bastin, S Kaul, S Finney

Royal Brompton Hospital, London, UK

Critical Care 2010, 14(Suppl 1):P597 (doi: 10.1186/cc8829)

Introduction Patient satisfaction data collation is a priority for the UK's Care Quality Commission. In the adult intensive care unit (AICU), patients are often sedated for prolonged periods. Family satisfaction data may be useful to gauge quality of service delivery. The Family Satisfaction-ICU (FS-ICU 34) questionnaire was developed and validated in the USA [1]. To our knowledge, we are the first group to use it in the UK.

Methods The FS-ICU 34 is an anonymised questionnaire. We adapted the American terminology for use in the UK. Inclusion criteria were: AICU admission of 5 days or more; presence of next-of-kin. Patients who died were excluded. It was handed personally to AICU patients' next-of-kin following discharge. Nursing staff from both the AICU and general wards assisted in returning completed questionnaires. Key areas of questioning using the FS-ICU were: perception of treatment of patient discomfort; coordination of AICU services; skill and competencies of AICU staff; consistency and frequency of communication; standard of family facilities; and emotional support. Relatives were asked to grade their answers on a five-point scale (excellent to poor).

Results Data from the first 4 months of this survey have now been analysed. One hundred per cent of patients' relatives who fulfilled the inclusion criteria received a questionnaire. The response rate was 68\%. The majority of respondents were satisfied with overall care and decision-making. Similarly to published data [2], families were most satisfied with nursing skill and competence ( $94.7 \%$ satisfied), and least satisfied with waiting room atmosphere (42.4\%) and frequency of communication with doctors (71.2\%). Conclusions The FS-ICU has enabled us to identify and target resources at key aspects of our service delivery. It has presented an opportunity for us to address misunderstandings and misconceptions regarding the ICU within our client base. This survey is unique in examining the links the ICU has with the community and informs the process of understanding that relationship.

\section{References}

1. Wall et al:: Crit Care Med 2007, 35:271-279.

2. Heyland et al:: Crit Care Med 2002, 30:1413-1418.

\section{P598}

Survey into bereavement of family members of patients who died in the intensive care unit

L Heijboer', J Van der Klink', J Hofhuis', A Hovingh', J Rommes',

M Westerman², P Spronk'

'Gelre Hospitals, Apeldoorn, the Netherlands; ${ }^{2} V U$ University, Amsterdam, the Netherlands

Critical Care 2010, 14(Suppl 1):P598 (doi: 10.1186/cc8830)

Introduction The death of a family member in the ICU is often sudden and unexpected and may have a strong impact. The care provided in the ICU differs from other hospital settings, resulting in special needs for family members of the dying patient. We studied the characteristics of bereavement, the need for follow-up bereavement service and to determine whether the information and care in the ICU is sufficient for relatives of deceased ICU patients.

Methods A structured telephone interview was conducted in a 10-bed mixed medical-surgical ICU. Patients and respondents were selected according to four criteria: (1) the patient had died between June 2008 and June 2009 in the ICU, (2) there had to be at least one relative, who (3) was involved during the ICU stay preceding death and (4) had sufficient knowledge of the Dutch language. For each deceased patient, one family member was identified as eligible for study participation. All eligible family members were first contacted by an ICU nurse to ask for agreement with the study. Furthermore, the structured interview was then emailed or sent to the family members so they would be able to read the questionnaire prior to the actual telephone interview. Three weeks after the initial telephone call by the nurses, the respondents were contacted for the structured telephone interview with specific attention to the use of new medication, work-related problems, resuming normal activities, money problems and sleeping problems.
Results A substantial portion of the respondents (37\%) complained about sleeping problems. A majority (77\%) was satisfied with the provided ICU care and the information provided. Almost all of the respondents (90\%) understood the fatal sequence of events during the dying process. Most common complaints concerned communication and the information provided. The need for a follow-up bereavement service was reported by $35 \%$ of the respondents. Reasons for such a service were firstly to deal with the death of the family member and secondly to answer remaining questions.

Conclusions This study demonstrates that most relatives had resumed their normal activities. In addition, there was a high level of satisfaction with the care provided in the ICU, although many considered a followup bereavement service potentially useful. The most frequent persisting bereavement characteristic was related to sleeping problems.

\section{P599}

Exploration of the needs and experiences of family members of a patient being admitted to a neurosurgery intensive care unit in Shiraz, Iran

S Gholamzadeh', S Gholamzadeh², H Sorbi Akbari

${ }^{1}$ Fatemeh Nursing College, Shiraz, Iran; ${ }^{2}$ Fars Medical Legal Organization,

Darab, Iran; ${ }^{3}$ Private Medical Office, Darab, Iran

Critical Care 2010, 14(Suppl 1):P599 (doi: 10.1186/cc8831)

Introduction Sudden hospitalization of a relative to a critical care setting is a catastrophic event for families. Nurses may not always assess the needs of relatives the way they themselves experience their needs to provide support. This qualitative study was conducted to explore the experiences of relatives of patients in adult neurosurgery ICUs in Shiraz, Iran.

Methods In this qualitative study, grounded theory was used to explore the experiences of 10 relatives of patients in neurosurgery ICUs. The data consisted of in-depth interviews and participant and nonparticipant observation. All data were recorded by writing in a notebook, and subsequent listening to the audio recordings and reading through the transcription of each interview was done.

Results Five major categories emerged from the data: information seeking, vigilant attendance, intense emotion, need for respect, and need to care about patient privacy. The most important needs of relatives were to have information about the condition of their loved one, talk to a doctor and their questions are answered honestly. The other theme was vigilant attendance. Many informants pointed out that they were willing to stay continuously inside the ICU to see what is going on and they wanted flexible open visiting. Also relatives showed the suffering they experience and their needs for support and empathy. The study also defined two new concepts in this area: need for respect and care about patient privacy.

Conclusions It is essential that intensive care nurses are able to identify the specific needs of family members to provide appropriate supportive interventions.

\section{References}

1. Engli M, Kirsivali-Farmer K: Needs of family of critically ill patients with and without acute brain injury. J Neurosci Nurs 1993, 25:78-85.

2. Curry $\mathrm{S}$ : Identifying family needs and stresses in the intensive care unit. $\mathrm{Br} \mathrm{J}$ Nurs 1995, 4:15-19.

\section{P600}

Deciding the end of life for patients with disorders of consciousness: a European survey

A Demertzi', D Ledoux', MA Bruno', A Vanhaudenhuyse', O Gosseries', A Soddu', M Boly', C Schnakers', G Moonen², S Laureys'

'Cyclotron Research Center, University of Liege, Belgium; ${ }^{2} \mathrm{CHU}$, Liege, Belgium Critical Care 2010, 14(Suppl 1):P600 (doi: 10.1186/cc8832)

Introduction Withdrawal of artificial nutrition and hydration (ANH) is ethically justified for patients with irreversible disorders of consciousness (coma, vegetative (VS), minimally conscious state (MCS)) [1]. However, the absence of definite ethical steps within the medical community makes objective end-of-life decisions difficult to take. The present survey aims to debrief attitudes towards end-of-life decisions in these patients in order to facilitate future guidelines. 
Methods A 16-item questionnaire around various issues on consciousness was presented to attendees at conferences in Europe. Data were obtained from 3,672 respondents ( $M$ age $36 \pm 16$ years, range 14 to 88; 55\% women; 34 EU countries) and were analyzed with SPSS v. 16.0.

Results Sixty-seven percent $(n=2,454)$ agreed with ANH withdrawal in chronic VS ( $31 \%, n=1,138$ disagreed; $2 \%, n=80$ no response). A significant agreement was expressed by nonreligious respondents (vs religious; $B=$ $0.70, P<0.001$ ) and nonmedical professionals (vs doctors; $B=0.34, P=$ 0.001). Significant disagreement was expressed by women (vs men; $B=$ $-0.25, P=0.004$ ), central and south Europeans (vs northern; $B=-0.85$, $P<0.001$ and $B=-1.23, P<0.001$, respectively) and those of higher age $(B=-0.008, P=0.01)$. Eighty percent $(n=2,956)$ did not wish to stay alive if themselves were in a permanent VS $(18 \%, n=625$ wished to stay alive; $2 \%, n=64$ no response). Seventy-eight percent considered that being in permanent VS is worse than death for the patient's family ( $55 \%$ considered it worse than death for patients themselves). Sixty-nine percent $(n=2,523)$ disagreed with ANH withdrawal in chronic MCS $(29 \%, n=1,073$ agreed; $2 \%, n=76$ no response). A significant disagreement was expressed by central and south Europeans (vs northern; $B=-0.58, P<0.001$ and $B=$ $-1.3, P<0.001$, respectively) and respondents of higher age $(B=-0.007$, $P=0.019) ;$ a significant agreement was expressed by non-religious respondents (vs religious; $B=0.65, P<0.001$ ). Sixty-four percent $(n=2,355)$ did not wish to be kept alive if themselves were in a permanent MCS (34\%, $n=1,248$ wished to stay alive; $2 \%, n=69$ no response). Forty percent considered that being in a MCS is worse than VS for the patient's family ( $50 \%$ considered it worse than VS for patients themselves).

Conclusions These findings raise important ethical issues concerning our care for patients with chronic disorders of consciousness. In light of high rates of diagnostic error in these patients [2], the necessity for adapted standards of care is warranted.

\section{References}

1. Royal College of Physicians: Clin Med 2003, 3:249-254.

2. Schnakers C, et al:: BMC Neurol 2009, 9:35.

\section{P601}

Evaluation of a new limitations and withdrawal of care framework order form in the intensive care unit

PB Sherren, L Turnbull, S Yoganathan

Queens Hospital, Romford, UK

Critical Care 2010, 14(Suppl 1):P601 (doi: 10.1186/cc8833)

Introduction The aim of this study was to demonstrate improvements in both staff and patient experiences with end-of-life care. The ICU is a setting where death is common; it has been suggested that $20 \%$ of patients in the US die on the ICU [1]. Given that the majority of ICU deaths involve the withholding or withdrawing of treatment [2], the importance of end-of-life care is clear. Despite this frequency, studies suggest that the current quality of end-of-life care is suboptimal on the ICU $[3,4]$. As a result, we developed a new framework to address this issue.

Methods We introduced our new framework over a 1-year period; we circulated questionnaires to the staff pre and post study to demonstrate any improvements in end-of-life care.

Results Our framework was found to be helpful by $97 \%$ of respondents and was associated with an improvement in communication and knowledge of end-of-life care. We discovered an increase in the number of staff who felt that patients, along with having their analgesia/sedation needs met, were now experiencing care that was more conducive to a good quality of dying. The number of staff who now felt confident in managing withdrawal of care trended towards a significant $P$ value. See Figure 1 for details.

Conclusions Quality of end-of-life care was improved with our new framework; however, further research is vital to ensure our patients receive the same kind of evidence-based medicine in their final hours as they did during their acute illness.

\section{References}

1. Angus DC, et al:: Crit Care Med 2004, 32:638-643.

2. Wood GG, et al:: Can J Anaesth 1995, 42:186-191.

3. Desbiens NA, et al: J Am Geriatr Soc 2000, 48:S183-S186.

4. Treece PD, et al:: Crit Care Med 2004, 32:1141-1147.

\begin{tabular}{|c|c|c|c|c|c|}
\hline & \multicolumn{2}{|c|}{$\begin{array}{l}\text { Pre framework } \\
\qquad \mathrm{n}=37\end{array}$} & \multicolumn{2}{|c|}{$\begin{array}{l}\text { Post framework } \\
\qquad N=33\end{array}$} & $\begin{array}{c}p- \\
\text { Value }\end{array}$ \\
\hline $\begin{array}{l}\text { Background knowledge on end of life care \% } \\
\text { (Standard deviation, SD) }\end{array}$ & \multicolumn{2}{|c|}{$\begin{array}{l}62.3 \\
(14.1)\end{array}$} & \multicolumn{2}{|c|}{$70.3(12.6)$} & 0.02 \\
\hline Clinical benefits and Staff satisfaction & Yes & No & Yes & No & $\begin{array}{c}p- \\
\text { Value }\end{array}$ \\
\hline $\begin{array}{l}\text { Is the current wethirawal practice meeting the } \\
\text { sedation'analgesia needs of the cying patients? }\end{array}$ & 21 & 16 & 27 & 6 & 0.04 \\
\hline $\begin{array}{l}\text { Is the current wrthdrawal practice conducive to a } \\
\text { good quality of life for the dying patient? }\end{array}$ & 15 & 22 & 23 & 10 & 0.02 \\
\hline $\begin{array}{l}\text { Do you feel supported during the withdrawal } \\
\text { process by } \\
\text { - Nursing staff? }\end{array}$ & 28 & 9 & 27 & 6 & 0.57 \\
\hline - Juzior Doctors? & 20 & 17 & 20 & 12 & 0.63 \\
\hline - Consultants? & 21 & 16 & 24 & 9 & 0.32 \\
\hline $\begin{array}{l}\text { Do you receive clear instructions on withdrawal, } \\
\text { either verbal or wnitten? }\end{array}$ & 16 & 21 & 29 & 4 & 0.0001 \\
\hline $\begin{array}{l}\text { Do you feel confident in the process of managing } \\
\text { withisawal of care on the ICU? }\end{array}$ & 23 & 14 & 26 & 7 & 0.06 \\
\hline
\end{tabular}

Figure 1 (abstract P601).

P602

End-of-life decisions in Hungarian intensive care units

L Zubek, G Élő, L Szabó

Semmelweis University, Budapest, Hungary

Critical Care 2010, 14(Suppl 1):P602 (doi: 10.1186/cc8834)

Introduction The acknowledgement of local practice with respect to endof-life decisions in accordance with laws and ethical principles is inevitable for intensive physicians in all countries. The first step for the required social dialogue is to search for local customs for harmonisation with ethical and legal regulations, as well as the interests of physicians and patients.

Methods In 2007 we performed the first Hungarian survey with the purpose to learn more about the local practice of end-of-life decisions. Questionnaires were sent out electronically to 743 registered members of the Hungarian Society of Anaesthesiology and Intensive Care. Respecting anonymity we have statistically evaluated 103 replies (response rate was $13.8 \%$ ) and compared with data from other European countries.

Results As expected, it turned out from the replies that the practice of domestic intensive care physicians is very paternal and this is promoted by legal regulations that share a similar character. Intensive care physicians generally make their decisions alone (3.75/5 points) without respecting the opinion of the patient (2.57/5 points), the relatives (2.14/5 points) or other medical personnel (2.37/5 points). Furthermore they prefer not to start a therapy rather than withdraw an ongoing treatment. Nevertheless, the frequency of end-of-life decisions ( 3 to $9 \%$ of ICU patients) is similar to other European countries.

Conclusions Hungarian intensive care physicians make end-of-life actions routinely. They usually decide based on their own opinion, slightly considering the opinion of nursing personnel, the patients or their relatives. They are not supported by the Hungarian legal regulation in making these decisions. Although the living will and the advanced directive are both acknowledged, they are not as widespread as required. Our study is the first step to commence a social dialogue, which is taken in the evolution of end-of-life decision-making procedures.

\section{References}

1. Van der Heide A, et al.: End of life decision making in six European countries: descriptive study. Lancet 2003, 362:345-350.

2. Sprung $\mathrm{CL}$, et al.: End-of-life practices in European intensive care units; the Ethicus Study. JAMA 2003, 290:790-797.

3. Vincent $J$ : Forgoing life support in western European intensive care units: the results of an ethical questionnaire. Crit Care Med 1999, 27:1626-1633.

Cite abstracts in this supplement using the relevant abstract number, e.g.: Zubek L, Élő G, Szabó L: End-of-life decisions in Hungarian intensive care units [abstract]. Critical Care 2010, 14(Suppl 1):P602. 